\title{
Ru-Based Catechothiolate Complexes Bearing an Unsaturated NHC Ligand: Effective Cross-Metathesis Catalysts for Synthesis of (Z)- $\alpha, \beta$-unsaturated Esters, Carboxylic Acids, and Primary, Secondary and Weinreb Amides
}

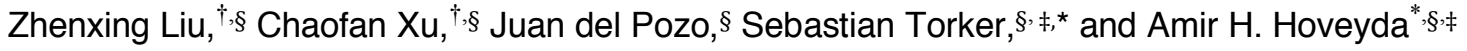 \\ ${ }^{\dagger}$ Department of Chemistry, Merkert Chemistry Center, Boston College, Chestnut Hill, Massachusetts 02467, USA \\ $\ddagger$ Supramolecular Science and Engineering Institute, University of Strasbourg, 67000 Strasbourg, France \\ e-mail: torker@unistra.fr, amir.hoveyda@bc.edu or ahoveyda@unistra.fr
}

\section{SUPPORTING INFORMATION}

\section{Table of Contents}

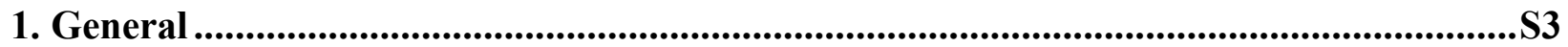

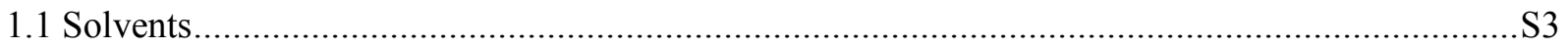

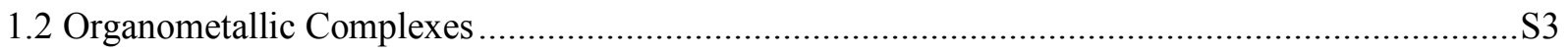

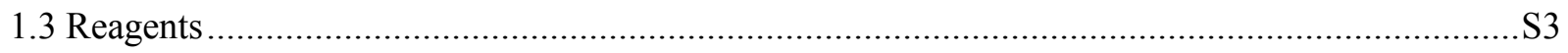

2. Preparation of Ru Complexes and Cross-Metathesis with (Z)-3-Hexene ..........................S6

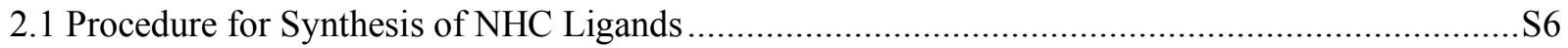

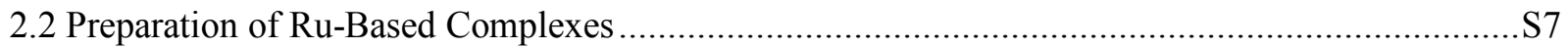

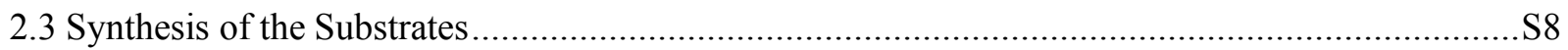

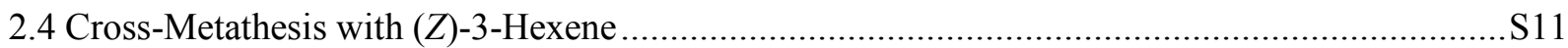

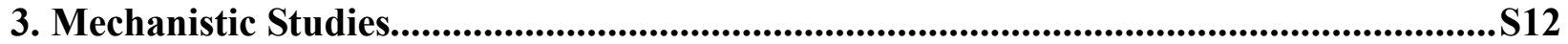

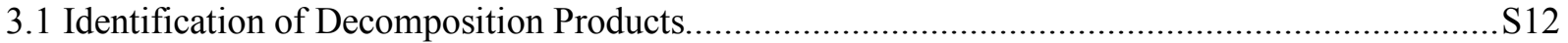

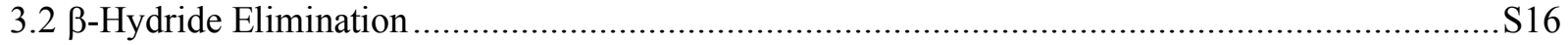

3.3 Cross-Metathesis Between (Z)-3-Hexene and Benzyl (Z)-but-2-enoate ...................................S17

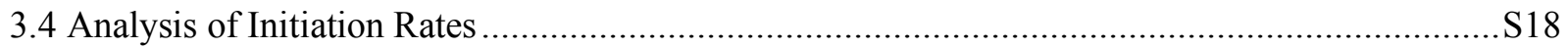

3.5 Relative Rates of Homo-Metathesis and Cross-Metathesis Reactions .......................................S19

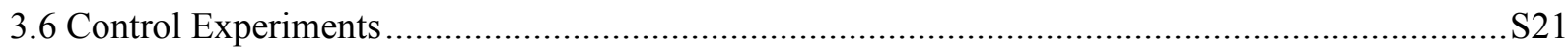

3.6.1 Homo-Metathesis vs. Cross-Metathesis Reactions...........................................................S2 1

3.6.2 Effect of A Lewis Base Additive on Homo-Metathesis of 8 ............................................S2 1 
4. Method Development ................................................................................................................S21

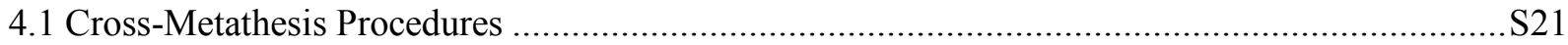

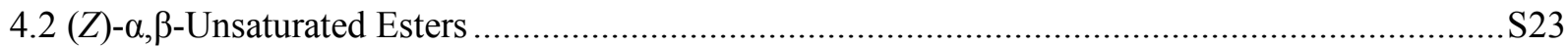

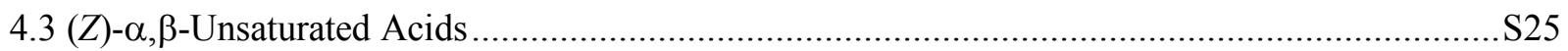

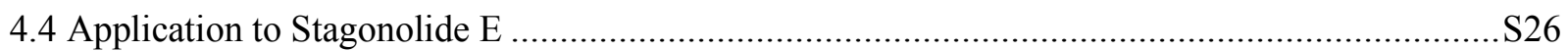

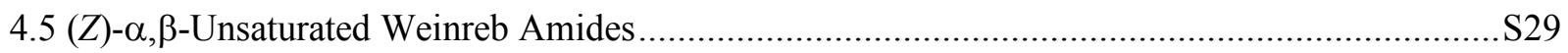

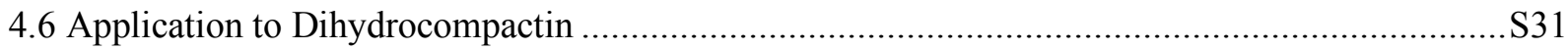

$4.7(Z)-\alpha, \beta$-Unsaturated Secondary and Primary Amides ........................................................... S33

5. Computational Studies..............................................................................................................S36

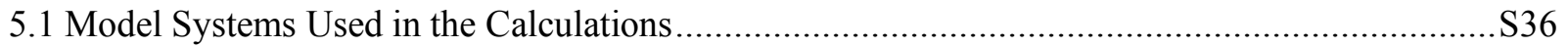

5.2 Competition Between Productive Olefin Metathesis and Decomposition Pathways ...................S36

5.2.1 Computationally Observed Difference between Ru-1c and Ru-1d ................................S37

5.2.2 Transition States for $\beta$-Hydride Elimination and Cyclopropanation .................................S38

5.2.3 Free Energy Surfaces With Smaller Model Systems Ru-1 $\mathbf{c}_{\text {model }}$ and $\mathbf{R u - 1 \mathbf { d } _ { \text { model } } \ldots \ldots . . . . . . . . . . . . . S 3 9}$

5.3 Origin of Reactivity Difference between Ru-1c and Ru-1d ...............................................S56

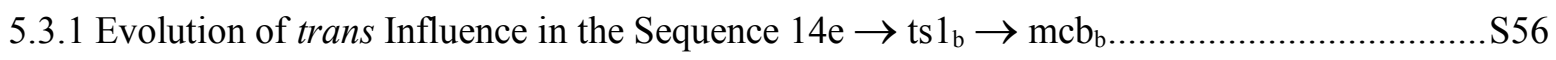

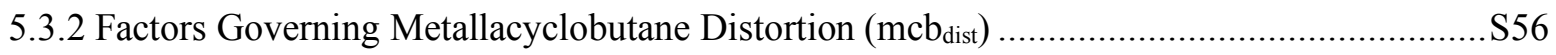

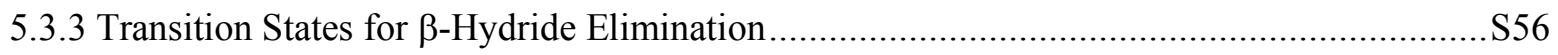

5.4 Comparison of $\mathrm{Ru}-\mathrm{S}($ trans $)$ and $\mathrm{Ru}-\mathrm{C}^{\mathrm{NHC}}$ Bond Lengths Based on X-Ray and DFT..................S62

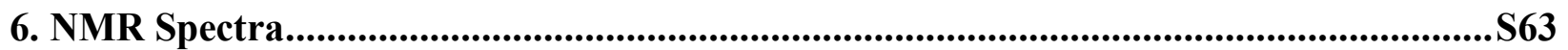

7.Coordinates After Optimization with $\omega-$ B97XD/Def2SVP THF(IEFPCM) ..............................S201

8. X-ray Structure of Ru-1c ..........................................................................................................S297

9. X-ray Structure of Ru-1d.........................................................................................................5327

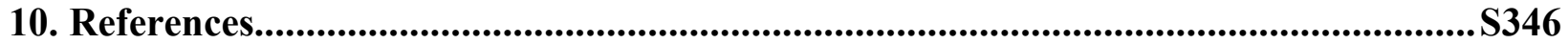




\section{General}

Unless otherwise noted, transformations were performed with distilled and degassed solvents under an atmosphere of dry $\mathrm{N}_{2}$, in oven $\left(135^{\circ} \mathrm{C}\right)$ or flame-dried glassware with standard dry box or vacuum line techniques. ${ }^{1} \mathrm{H}$ NMR spectra were recorded on a Varian Unity INOVA 400 (400 $\mathrm{MHz}), 500(500 \mathrm{MHz})$ or a $600(600 \mathrm{MHz})$ spectrometer. Chemical shifts are reported in ppm from tetramethylsilane with the solvent resonance resulting from incomplete deuterium incorporation as the internal standard $\left(\mathrm{CDCl}_{3}: \delta 7.26 \mathrm{ppm}, \mathrm{CD}_{2} \mathrm{Cl}_{2}: \delta 5.32 \mathrm{ppm}, \mathrm{C}_{6} \mathrm{D}_{6}: \delta 7.16\right.$ ppm, DMSO- $\left.d_{6}: \delta 2.50 \mathrm{ppm}\right)$. Data are reported as follows: chemical shift, multiplicity ( $\mathrm{s}=$ singlet, $\mathrm{d}=$ doublet, $\mathrm{t}=$ triplet, $\mathrm{q}=$ quartet, $\mathrm{p}=$ pentet, $\mathrm{br}=$ broad, $\mathrm{m}=$ multiplet), and coupling constants $(\mathrm{Hz})$, integration. ${ }^{13} \mathrm{C}$ NMR spectra were recorded on a Varian Unity INOVA 400 (100 $\mathrm{MHz}), 500(125 \mathrm{MHz})$, or $600(150 \mathrm{MHz})$ spectrometers with complete proton decoupling. Chemical shifts are reported in ppm from tetramethylsilane with the solvent resonance as the internal standard $\left(\mathrm{CDCl}_{3}: \delta 77.16 \mathrm{ppm}, \mathrm{CD}_{2} \mathrm{Cl}_{2}: \delta 53.84 \mathrm{ppm}, \mathrm{C}_{6} \mathrm{D}_{6}: \delta 128.06 \mathrm{ppm} ; \mathrm{DMSO}-d_{6}: \delta\right.$ $39.52 \mathrm{ppm})$. High-resolution mass spectrometry was performed on a Micromass LCT ESI-MS and JEOL Accu TOF Dart (positive mode) at the Boston College Mass Spectrometry Facility. Enantiomeric ratios were determined by HPLC analysis (high-performance liquid chromatography) with a Shimadzu chromatograph [Chiral Technologies Chiralcel OD-H (4.6 x $250 \mathrm{~mm})]$ in comparison with authentic racemic materials. Specific rotations were measured on a Rudolph Research Analytical Autopol IV Polarimeter. Melting points were determined using a Thomas Hoover Uni-melt capillary melting point apparatus. X-ray structures were obtained, as detailed in the cif file that has been provided, with a Microfocus sealed $\mathrm{Cu}$ tube from Incote. Infrared (IR) spectra were recorded on a Bruker FTIR Alpha (ATR Mode) spectrometer, $v_{\max }$ in $\mathrm{cm}^{-1}$. Bands are characterized as broad (br), strong (s), medium (m), or weak (w). Values for $E: Z$ ratios of products were determined by analysis of ${ }^{1} \mathrm{H}$ NMR spectra.

\subsection{Solvents}

Tetrahydrofuran (THF) was distilled over $\mathrm{Na}$ /benzophenone. $\mathrm{CH}_{2} \mathrm{Cl}_{2}$ and $\mathrm{Et}_{2} \mathrm{O}$ was purified under a positive pressure of dry $\mathrm{Ar}$ gas by a modified Innovative Technologies purification system. $\mathrm{CDCl}_{3}, \mathrm{C}_{6} \mathrm{D}_{6}, \mathrm{CD}_{2} \mathrm{Cl}_{2}$ and $\mathrm{DMSO}-d_{6}$ were purchased from Cambridge Isotope Laboratories and stored over activated $4 \AA$ molecular sieves prior to use. THF- $d_{8}$ was purchased from Oakwood company and distilled over $\mathrm{Na} /$ benzophenone. Purification of products was carried out with reagent grade solvents (Fisher).

\subsection{Organometallic Complexes}

Ru-1c was prepared according to a previously reported procedure. ${ }^{1}$

\subsection{Reagents}

Acetyl chloride: purchased from Fisher Scientific and used as received.

4-Allyl-1,2-dimethoxybenzene: purchased from Aldrich and used as received.

2-Allylphenol: purchased from Aldrich and used as received. 
4-Allylphenol: purchased from Aldrich and used as received.

8-Bromo-1-octene: purchased from Oakwood and used as received.

(E)-Buta-1,3-dien-1-ylbenzene: purchased from Aldrich and used as received.

(Z)-2-Butene: purchased from Aldrich and was dissolved in anhydrous THF and stored in the freezer at $-50{ }^{\circ} \mathrm{C}$; weight percent $(\mathrm{wt} \%)$ was calculated based on the ${ }^{1} \mathrm{H}$ NMR analysis of the mixture.

But-3-en-1-ylbenzene: purchased from Aldrich and used as received.

tert-Butylalcohol: purchased from Aldrich and used as received.

tert-Butyl 2,2,2-trichloroacetimidate: purchased from Oakwood and used as received.

n-Butyllithium (2.5 M in hexanes): purchased from Aldrich and used as received.

tert-Butyllithium (1.7 $\mathrm{M}$ in hexanes): purchased from Aldrich and used as received.

(Z)-2-Butenoic acid: purchased from Aurora and used as received.

2-Butynoic acid: purchased from Oakwood and used as received.

tert-Butyldimethylsilyl chloride (TBSCI): purchased from Oakwood and used as received.

Benylbromide: purchased from Aldrich and used as received.

3-Buten-1-ol: purchased from Aldrich and used as received.

Carbon tetrachloride: purchased from Acros and used after distillation over $\mathrm{CaH}_{2}$.

Dess-Martin periodinane: purchased from Oakwood and used as received.

2,3-Dichloro-5,6-dicyano-1,4-benzoquinone (DDQ): purchased from Alfa and used as received.

Diethyl allylphosphonate: purchased from Aldrich and used as received.

$N, N$-Diisopropylethylamine: purchased from Alfa and used as received.

$N$-(3-Dimethylaminopropyl)- $N$ '-ethylcarbodiimide hydrochloride (EDCI): purchased from Advanced ChemTech and used as received.

2,6-Dimethyloct-7-en-2-ol (dihydromyrcenol): purchased from Aldrich and used as received.

2-Fluoro-6-methylaniline: purchased from Oakwood and used as received.

Formic acid: purchased from Oakwood and used as received.

Glyoxal (40 wt\% in $\mathrm{H}_{2} \mathrm{O}$ ): purchased from Aldrich and used as received.

(Z)-3-Hexene: purchased from Alfa and used as received.

5-Hexenoic acid: purchased from Aldrich and used as received.

(S)-Hex-5-en-2-ol: purchased from Aldrich and used as received.

5-Hexen-1-ol: purchased from Aldrich and used as received.

First-generation phosphine-free ("Hoveyda-Grubbs") Ru complex: purchased from Materia and used as received.

Hydrogen chloride (2M in $\mathbf{E t}_{2} \mathbf{O}$ ): purchased from Acros and used as received. 
Hydroquinidine (anthraquinone-1,4-diyl) diether (DHQD) 2 AQN): purchased from Aldrich and used as received.

Hydroxybenzotriazole hydrate (HOBt): purchased from Advanced ChemTech and used as received.

Imidazole: purchased from Oakwood and used as received.

Isobutylamine: purchased from Aldrich and used as received.

Lithium aluminum hydride: purchased from Aldrich and used as received

Lithium perchlorate: purchased from Aldrich and used as received.

4-Methoxybenzylamine: purchased from Aldrich and used as received.

Methyl $N$-(triethylammoniumsulfonyl)carbamate (Burgess reagent): purchased from Oakwood and used as received.

8-Nonen-1-ol: purchased from TCI and used as received.

Paraformaldehyde: purchased from Fluka and used as received.

4-Penten-1-ol: purchased from Aldrich and used as received.

4-Pentenal: purchased from Alfa and used as received

Potassium carbonate: purchased from Fisher Scientific and used as received.

Potassium tert-butoxide: purchased from Aldrich and used as received.

Potassium bis(trimethylsilyl)amide: purchased from Aldrich and used as received.

Potassium ferricyanide: purchased from Aldrich and used as received.

Potassium osmate: purchased from Aldrich and used as received.

Silver chloride: purchased from Strem and used as received.

1-Tetradecene: purchased from Aldrich and used as received.

Tetrabutylammonium fluoride (1 M in THF): purchased from Oakwood and used as received. Trimethylsilyl chloride: purchased from Oakwood and used as received.

Undec-10-enal: purchased from Aldrich and used as received.

Benzyl pent-4-enoate: (from 4-pentenoic acid (Aldrich)) was prepared according to a reported procedures. $^{2}$

3,6-Dichlorobenzene-1,2-dithiol zinc salt: (from 3,6-dichlorobenzene-1,2-dithiol (Aldrich)) was prepared according to a reported procedure. ${ }^{3}$

(E)-Hepta-2,6-dien-1-ol: (from pent-4-enal (Aldrich)) was prepared according to a reported procedure. $^{4}$

(Z)-Hex-3-ene-3,4- $\boldsymbol{d}_{2}$ : (from 3-hexyne (Aldrich)) was prepared according to a reported procedure. $^{5}$

2-(Hex-5-en-1-yl)isoindoline-1,3-dione: (from phthalimide (Aldrich)) was prepared according to a reported procedure. ${ }^{6}$ 
(S)-1-((Hex-5-en-2-yloxy)methyl)-4-methoxybenzene: (from $(S)$-hex-5-en-2-ol (Aldrich))was prepared according to a reported procedure. ${ }^{7}$

$(\mathbf{2 E}, \mathbf{4 E})-8$-Iodoocta-2,4-diene: (from 5-chloropent-1-yne (Aldrich)) was prepared according to a reported procedure. ${ }^{8}$

$\boldsymbol{O}$-Trimethylsilyl hydroquinidine: (from hydroquinidine (Aldrich)) was prepared in analogy to a reported procedure. ${ }^{9}$

\section{Preparation of Ru Complexes and Cross-Metathesis with (Z)-3-Hexene}

\subsection{Procedure for Synthesis of NHC Ligands}

\section{$N^{1}, N^{2}$-Bis(2-fluoro-6-methylphenyl)ethane-1,2-diimine}

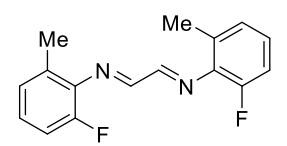

Based on a previously reported procedure ${ }^{10}$, a $250 \mathrm{~mL}$ round-bottom flask was charged with an aqueous solution of glyoxal (40 wt \%, $1.84 \mathrm{~mL}, 16 \mathrm{mmol}$ ), anhydrous $\mathrm{Na}_{2} \mathrm{SO}_{4}$ (13.6 g, $96 \mathrm{mmol}$, oven-dried overnight), and $\mathrm{CH}_{2} \mathrm{Cl}_{2}$ (40 $\mathrm{mL}$ ). The mixture was allowed to stir for $30 \mathrm{~min}$, after which 2-fluoro-6-methylaniline (4.00 g, $32 \mathrm{mmol})$, formic acid $(85 \mu \mathrm{L}, 2.24 \mathrm{mmol})$, and a second portion of anhydrous $\mathrm{Na}_{2} \mathrm{SO}_{4}(13.6 \mathrm{~g}$, $96 \mathrm{mmol}$, oven-dried overnight) were added. The heterogeneous mixture was allowed to stir vigorously for $12 \mathrm{~h}$, filtered to remove $\mathrm{Na}_{2} \mathrm{SO}_{4}$, and concentrated in vacuo. The residual (a mixture of solid and liquid) was filtered, and the solid was washed with hexanes $(10 \mathrm{~mL} \times 3)$ to afford $N^{1}, N^{2}$-bis(2-fluoro-6-methylphenyl)ethane-1,2-diimine as yellow solid (1.838 g, 6.75 mmol, 42\% yield). IR (neat): 2960 (w), 1607 (m), 1470 (m), 1258 (m), 1243 (m), 1191 (m), 1020 (m), 939 (m), 772 (s), $740(\mathrm{~m}) \mathrm{cm}^{-1} ;{ }^{1} \mathbf{H}$ NMR (400 MHz, $\left.\mathbf{C D C l}_{3}\right): \delta 8.39$ (t, $J=1.9 \mathrm{~Hz}$, 2H), 7.14-6.94 (m, 6H), $2.34(\mathrm{~s}, 6 \mathrm{H}) ;{ }^{13} \mathbf{C}$ NMR (150 MHz, CDCl $)$ : 165.1 (d, $J=6.5 \mathrm{~Hz}$ ), 152.7 (d, $J=248.5 \mathrm{~Hz}), 137.1(\mathrm{~d}, J=9.8 \mathrm{~Hz}), 134.9,126.8(\mathrm{~d}, J=8.5 \mathrm{~Hz}), 126.0(\mathrm{~d}, J=3.0 \mathrm{~Hz})$, 18.1 (d, $J=2.6 \mathrm{~Hz})$; ${ }^{19} \mathbf{F}$ NMR (376 $\left.\mathbf{M H z}, \mathbf{C D C l}_{3}\right)$ : $\delta-129.32$ to $-129.37(\mathrm{~m})$; HRMS[M+H] ${ }^{+}$: Calcd for $\mathrm{C}_{16} \mathrm{H}_{15} \mathrm{~F}_{2} \mathrm{~N}_{2}: 273.1198$, found: 273.1197 .

\section{1,3-Bis(2-fluoro-6-methylphenyl)-1H-imidazol-3-ium chloride}

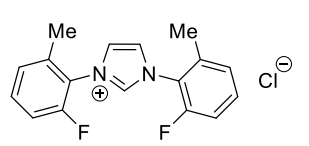

Based on a previously reported procedure ${ }^{11}$, an oven-dried 4-dram vial was charged with $N^{1}, N^{2}$-bis(2-fluoro-6-methylphenyl)ethane-1,2-diimine (838 mg, $3.08 \mathrm{mmol}, 1.0$ equiv.), paraformaldehyde (277.2 $\mathrm{mg}, 9.24 \mathrm{mmol}, 3.0$ equiv.), and toluene $(8.0 \mathrm{~mL})$. Under vigorous stirring, $\mathrm{HCl}\left(4.0 \mathrm{M}\right.$ in $\mathrm{Et}_{2} \mathrm{O}, 1.00 \mathrm{~mL}, 4.0 \mathrm{mmol}, 1.3$ equiv.) was slowly added in a dropwise fashion to the mixture with a total addition time of 30 min. The mixture was allowed to stir for $12 \mathrm{~h}$, after which the toluene supernatant was decanted. The solid residue was dried in vacuo and purified by silica gel chromatography (2\% to $4 \%$ to $8 \%$ $\mathrm{MeOH}$ in $\mathrm{CH}_{2} \mathrm{Cl}_{2}$ ) to afford 1,3-bis(2-fluoro-6-methylphenyl)-1H-imidazol-3-ium chloride as light purple solid (526 mg, 53\% yield). IR (neat): 3350 (br, m), 3024 (m), 2921 (m), 1547 (s), 1475 (s), 1278 (m), 1267 (m), 1229 (m), 1025 (m), 786 (s) cm ${ }^{-1} ;{ }^{1}$ H NMR (600 MHz, DMSO$\left.\boldsymbol{d}_{6}\right): \delta 10.24(\mathrm{~s}, 1 \mathrm{H}), 8.50(\mathrm{~s}, 2 \mathrm{H}), 7.66(\mathrm{q}, J=7.5 \mathrm{~Hz}, 2 \mathrm{H}), 7.50(\mathrm{t}, J=8.9 \mathrm{~Hz}, 2 \mathrm{H}), 7.43(\mathrm{~d}, J=$ $7.5 \mathrm{~Hz}, 2 \mathrm{H}), 2.31$ (s, 6H); ${ }^{13}$ C NMR (150 MHz, DMSO-d $)$ : $\delta 156.2$ (d, $\left.J=250.9 \mathrm{~Hz}\right), 140.2$, 
137.0, $132.6(\mathrm{~d}, J=8.7 \mathrm{~Hz}), 127.1(\mathrm{~d}, J=3.2 \mathrm{~Hz}), 125.0,121.8(\mathrm{~d}, J=12.7 \mathrm{~Hz}), 114.3(\mathrm{~d}, J=$ $18.9 \mathrm{~Hz}), 16.7 ;{ }^{19}$ F NMR (376 MHz, DMSO-d $)$ : $\delta-123.8$ (dd, $\left.J=9.6,5.7 \mathrm{~Hz}\right)$; HRMS[M-Cl] ${ }^{+}$: Calcd for $\mathrm{C}_{17} \mathrm{H}_{15} \mathrm{~F}_{2} \mathrm{~N}_{2}^{+}$: 285.1198, found: 285.1206.

\subsection{Preparation of Ru-Based Complexes}

Scheme S1
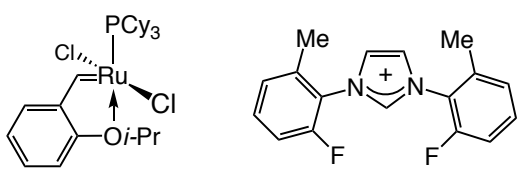

(2.0 equiv.)

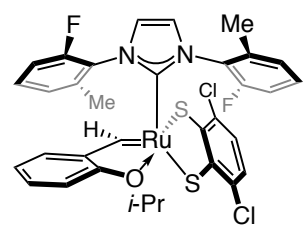

Ru-1c
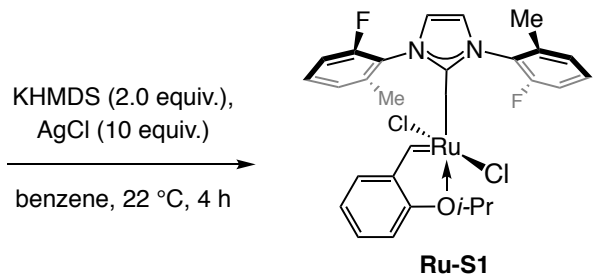

$\mathrm{THF}, 22^{\circ} \mathrm{C}, 2 \mathrm{~h}$

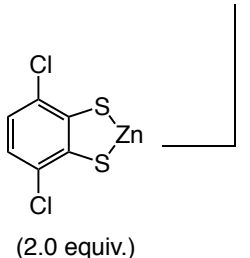

Ru-S1. In a $\mathrm{N}_{2}$-filled glovebox, a 6-dram vial was charged with potassium bis(trimethylsilyl)amide (82 mg, $0.411 \mathrm{mmol}, 2.0$ equiv.) and 1,3-bis(2-fluoro-6-methylphenyl)1H-imidazol-3-ium chloride (132 mg, $0.411 \mathrm{mmol}, 2.0$ equiv.) and $5 \mathrm{~mL}$ THF. The mixture was allowed to stir at $22{ }^{\circ} \mathrm{C}$ for $30 \mathrm{~min}$. First-generation phosphine-free Ru complex (123 mg, 0206 mmol, 1.0 equiv.) was added and the mixture was allowed to stir for $1 \mathrm{~h}$, after which silver chloride (286 mg, $2.06 \mathrm{mmol}, 10$ equiv.) was introduced and stirring was allowed to continue for an additional hour. Filtration and removal of the volatiles in vacuo afforded black oil, which was purified by silica gel chromatography $\left(20-100 \% \mathrm{Et}_{2} \mathrm{O}\right.$ in hexanes) to afford the product as green solid as a mixture of $N$-aryl rotamers $\left(86 \mathrm{mg}, 0.142 \mathrm{mmol}, 69 \%\right.$ yield). ${ }^{1} \mathbf{H}$ NMR (600 MHz, $\left.\mathbf{C}_{6} \mathbf{D}_{6}\right)(1: 1.5$ mixture of rotamers): $\delta 16.78(\mathrm{~s}, 0.37 \mathrm{H}), 16.72(\mathrm{~s}, 0.57 \mathrm{H}), 7.18(\mathrm{~d}, J=11.5 \mathrm{~Hz}$, $2 \mathrm{H}), 7.00(\mathrm{q}, J=7.4 \mathrm{~Hz}, 2 \mathrm{H}), 6.90-6.79(\mathrm{~m}, 4 \mathrm{H}), 6.73(\mathrm{t}, J=7.4 \mathrm{~Hz}, 1 \mathrm{H}), 6.44(\mathrm{~d}, J=8.0 \mathrm{~Hz}$, $1 \mathrm{H}), 6.28(\mathrm{~d}, J=5.3 \mathrm{~Hz}, 2 \mathrm{H}), 4.58(\mathrm{dt}, J=11.5,5.6 \mathrm{~Hz}, 1 \mathrm{H}), 2.37(\mathrm{~s}, 6 \mathrm{H}), 1.48$ (t, $J=5.5 \mathrm{~Hz}$, 6H); ${ }^{19}$ F NMR (470 MHz, $\mathbf{C D C l}_{3}$ ): $\delta-114.1$ (br), -115.3 (br); ${ }^{13} \mathbf{C}$ NMR (150 MHz, $\mathbf{C D}_{2} \mathbf{C l}_{2}$ ): (1:1.5 mixture of rotamers, all peaks are listed due to differentiation of rotamer peaks and C-F coupling peaks are non-trivial) $\delta 287.6,287.3,286.6,286.3,180.1,179.9,160.6,160.5,158.9$, $158.8,152.9,152.9,145.2,145.2,141.3,131.6,131.5,131.5,131.5,131.4,131.4,129.6,129.5$, $127.8,127.8,127.8,127.7,126.7,126.7,126.6,126.5,126.5,125.8,125.8,125.7,125.6,123.0$, $122.9,122.3,122.2,114.6,114.4,114.4,114.3,114.3,114.1,113.5,113.4,76.0,75.8,32.3,32.1$, 31.7, 27.6, 27.5, 27.4, 26.3, 26.2, 21.6, 21.5, 18.7, 18.6.

Ru-1d. A 1-dram vial containing a stir bar was charged with 3,6-dichlorobenzene-1,2-dithiol zinc salt (77.9 mg, 0.284 mmol, 2.0 equiv.) under $\mathrm{N}_{2}$ atm., and then a solution of Ru-S1 (86 mg, $0.142 \mathrm{mmol}, 1.0$ equiv.) in THF ( $2 \mathrm{~mL}$ ) was added. The mixture was allowed to stir for $2 \mathrm{~h}$ at $22{ }^{\circ} \mathrm{C}$, at which time the volatiles were removed in vacuo. The residual tetrahydrofuran was removed by co-evaporation with pentane. The resulting yellow solid was dissolved in $\mathrm{CH}_{2} \mathrm{Cl}_{2}$ 
and passed through a short column of celite $(2 \mathrm{~cm}$ in height) in a pipette $(\sim 0.5 \mathrm{~cm}$ in diameter) with $\mathrm{CH}_{2} \mathrm{Cl}_{2}$. After removal of the volatiles from the filtrate and co-evaporation with pentane, Ru-1d was isolated as brown solid and further crystallized from hexane/ $\mathrm{CH}_{2} \mathrm{Cl}_{2}$ (91 $\mathrm{mg}, 0.122$ mmol, 86\% yield). ${ }^{1} \mathbf{H}$ NMR (500 $\mathbf{~ M H z , ~} \mathbf{C D}_{\mathbf{2}} \mathbf{C l}_{2}$ ): (several rotamers in solution in ratios of 1:0.13:0.06:0.06; the major conformer are presented): $\delta 14.65(\mathrm{~s}, 1 \mathrm{H}), 7.31(\mathrm{t}, J=7.8 \mathrm{~Hz}, 1 \mathrm{H})$, $7.22(\mathrm{~d}, J=1.9 \mathrm{~Hz}, 1 \mathrm{H}), 7.13-7.06(\mathrm{~m}, 2 \mathrm{H}), 7.02(\mathrm{~d}, J=8.4 \mathrm{~Hz}, 1 \mathrm{H}), 6.98-6.92(\mathrm{~m}, 2 \mathrm{H}), 6.87$ $(\mathrm{dd}, J=17.1,7.7 \mathrm{~Hz}, 2 \mathrm{H}), 6.82(\mathrm{~d}, J=8.0 \mathrm{~Hz}, 1 \mathrm{H}), 6.77(\mathrm{~d}, J=8.0 \mathrm{~Hz}, 2 \mathrm{H}), 6.72$ (q, $J=7.2 \mathrm{~Hz}$, $1 \mathrm{H}), 6.18(\mathrm{t}, J=8.9 \mathrm{~Hz}, 1 \mathrm{H}), 5.31-5.24(\mathrm{~m}, 1 \mathrm{H}), 2.37(\mathrm{~s}, 3 \mathrm{H}), 2.19(\mathrm{~s}, 3 \mathrm{H}), 1.71(\mathrm{~d}, J=6.2 \mathrm{~Hz}$, $3 \mathrm{H}), 1.57(\mathrm{~d}, J=6.1 \mathrm{~Hz}, 3 \mathrm{H}) ;{ }^{19} \mathbf{F}$ NMR (470 MHz, $\left.\mathbf{C D}_{2} \mathbf{C l}_{2}\right): \delta-118.3$ (br), -124.1 (br); ${ }^{13} \mathbf{C}$ NMR (150 MHz, CD $\mathbf{C l}_{2}$ ): (major rotamer) $\delta 251.6$ (br), 189.5 (br), 158.1 (d, $J=249.0 \mathrm{~Hz}$ ), $157.6(\mathrm{~d}, J=249.3 \mathrm{~Hz}), 156.5,154.6,143.0,140.9,140.7,138.5,131.3,131.2(\mathrm{~d}, J=8.7 \mathrm{~Hz})$, $130.8(\mathrm{~d}, J=8.6 \mathrm{~Hz}), 129.7,128.2,126.4(\mathrm{~d}, J=3.3 \mathrm{~Hz}), 126.0(\mathrm{~d}, J=3.3 \mathrm{~Hz}), 124.8,124.3$, 123.1, 122.2, 121.6, 113.7 (d, $J=19.8 \mathrm{~Hz}), 113.4(\mathrm{~d}, J=19.9 \mathrm{~Hz}), 112.7,77.7,22.3(\mathrm{~d}, J=3.4$ $\mathrm{Hz}), 22.2(\mathrm{~d}, J=3.4 \mathrm{~Hz}), 22.1,19.3(\mathrm{~d}, J=2.2 \mathrm{~Hz}), 17.8(\mathrm{~d}, J=2.4 \mathrm{~Hz})$.

\subsection{Synthesis of the Substrates}

Benzyl (Z)-but-2-enoate (1a) An 8-dram vial was charged with potassium carbonate (152.0 mg, $1.1 \mathrm{mmol}, 1.1$ equiv.), (Z)-2-butenoic acid ( $86.1 \mathrm{mg}, 1.0 \mathrm{mmol}, 1.0$ equiv.) and $2.0 \mathrm{~mL} \mathrm{DMF}$, and the mixture was allowed to stir for $20 \mathrm{~min}$ at $22{ }^{\circ} \mathrm{C}$, after which benzyl bromide (188 $\mathrm{mg}, 1.1$ mmol, 1.1 equiv.) was added and the mixture was allowed to stir for $12 \mathrm{~h}$. The reaction was quenched by the addition of a saturated solution of $\mathrm{NH}_{4} \mathrm{Cl}(10 \mathrm{~mL})$ and the organic layer was washed with $\mathrm{Et}_{2} \mathrm{O}(50 \mathrm{~mL} \times 3)$. The combined organic layers were washed with brine, dried over $\mathrm{Na}_{2} \mathrm{SO}_{4}$ and concentrated in vacuo. The resulting black oil was purified by silica gel chromatography (2-10\% $\mathrm{Et}_{2} \mathrm{O}$ in hexanes) to afford 1a (169.5 $\mathrm{mg}, 0.96 \mathrm{mmol}, 96 \%$ yield) as colorless oil. The characterization data are consistent with those previously reported. ${ }^{12} \mathbf{1} \mathbf{H}$ NMR (400 MHz, CDCl $\mathbf{l}_{3}$ ): $\delta$ 7.41-7.30 (m, 5H), 6.36 (dq, $\left.J=11.5,7.3 \mathrm{~Hz}, 1 \mathrm{H}\right), 5.85$ (dq, $J=11.5,1.7$ $\mathrm{Hz}, 1 \mathrm{H}), 5.17(\mathrm{~s}, 2 \mathrm{H}), 2.16(\mathrm{dd}, J=7.3,1.8 \mathrm{~Hz}, 3 \mathrm{H}) ; \mathbf{H R M S}[\mathbf{M}+\mathbf{H}]^{+}$: Calcd for $\mathrm{C}_{11} \mathrm{H}_{13} \mathrm{O}_{2}$ : 177.0910, found: 177.0904 .

\section{tert-Butyl (Z)-but-2-enoate (S1)}

An 8-dram vial was charged with (Z)-2-butenoic acid (86.1 mg, $1.0 \mathrm{mmol}, 1.0$ $\mathrm{Me}{ }_{\mathrm{S} 1} \mathrm{CO}_{2}{ }^{t} \mathrm{Bu}$ equiv.), tert-butyl 2,2,2-trichloroacetimidate (327.8 mg, $1.5 \mathrm{mmol}, 1.5 \mathrm{equiv}$.) and

$\mathrm{CH}_{2} \mathrm{Cl}_{2}(5.0 \mathrm{~mL})$. The mixture was allowed to stir for $12 \mathrm{~h}$ after which the volatiles were removed in vacuo. The resulting yellow oil was purified by silica gel chromatography (2$10 \% \mathrm{Et}_{2} \mathrm{O}$ in hexanes) to afford $\mathbf{S 1}(116.6 \mathrm{mg}, 0.82 \mathrm{mmol}, 82 \%$ yield) as colorless oil. The characterization data are consistent with those previously reported. ${ }^{13}{ }^{1} \mathbf{H}$ NMR (400 MHz, $\left.\mathbf{C D C l}_{3}\right): \delta 6.22(\mathrm{dq}, J=11.5,7.2 \mathrm{~Hz}, 1 \mathrm{H}), 5.79-5.63(\mathrm{~m}, 1 \mathrm{H}), 2.18-2.02(\mathrm{~m}, 3 \mathrm{H}), 1.49$ (s, 9H); HRMS [M+H] $]^{+}$: Calcd for $\mathrm{C}_{8} \mathrm{H}_{15} \mathrm{O}_{2}:$ 143.1067, found: 143.1072.

(Z)-But-2-enoic acid (1b). A $100 \mathrm{~mL}$ round-bottom flask was charged with but-2-ynoic acid $(500.0 \mathrm{mg}, 5.95 \mathrm{mmol})$ and anhydrous $\mathrm{Et}_{2} \mathrm{O}(20 \mathrm{~mL})$; this was followed by the addition of $5 \%$ $\mathrm{Pd} / \mathrm{BaSO}_{4}(125 \mathrm{mg})$ and quinoline $(25 \mathrm{mg}, 0.19 \mathrm{mmol}, 3.2 \mathrm{~mol} \%$ ) The mixture was allowed to 
stir under $\mathrm{H}_{2}$ atm (balloon) at $22{ }^{\circ} \mathrm{C}$ for $1 \mathrm{~h}$. Reaction progress was monitored by ${ }^{1} \mathrm{H}$ NMR analysis $\left(\mathrm{CDCl}_{3}\right)$. Accordingly, when hydrogenation was found to be complete, the mixture was passed through a pad of Celite, and the filtrate was concentrated in vacuo, affording pale yellow oil, which was purified by silica gel chromatography (5-15\% EtOAc in hexanes) to afford benzyl (Z)-but-2-enoic acid (300.4 $\mathrm{mg}, 3.49 \mathrm{mmol}, 59 \%$ yield) in $>98: 2 \mathrm{Z}: E$ selectivity as colorless liquid. IR (neat): 3046 (w), 2944 (w), 1688 (s), 1641 (s), 1449 (m), 1227 (s), 820 (m), 727 (m), 415 (m) cm ${ }^{-1}$; ${ }^{1} \mathbf{H}$ NMR (600 MHz, CDCl $)$ ): $\delta 12.23$ (br, 1H), 6.47 (dq, $J=11.5,7.3$ $\mathrm{Hz}, 1 \mathrm{H}), 5.88-5.79(\mathrm{~m}, 1 \mathrm{H}), 2.16(\mathrm{dd}, J=7.3,1.8 \mathrm{~Hz}, 3 \mathrm{H}) .{ }^{13} \mathbf{C}$ NMR (150 MHz, CDCl 3$): \delta$ 172.6, 148.0, 120.4, 15.8. HRMS[M+H] $]^{+}$: Calcd for $\mathrm{C}_{4} \mathrm{H}_{7} \mathrm{O}_{2}:$ : 87.0446, Found: 87.0450.

(Z)- $\boldsymbol{N}$-methoxy- $\boldsymbol{N}$-methylbut-2-enamide (1c). A $100 \mathrm{~mL}$ round-bottom flask was charged with $N$-methoxy- $N$-methylbut-2-ynamide $(635.7 \mathrm{mg}, 5.00 \mathrm{mmol}), 5 \% \mathrm{Pd} / \mathrm{BaSO}_{4}(125 \mathrm{mg})$ and and quinoline (25 mg, $0.19 \mathrm{mmol}, 3.8 \mathrm{~mol} \%$ ). The mixture was allowed to stir under $\mathrm{H}_{2}$ atm (balloon) at $22{ }^{\circ} \mathrm{C}$. Reaction progress was monitored by ${ }^{1} \mathrm{H}$ NMR analysis $\left(\mathrm{CDCl}_{3}\right)$. Accordingly, when hydrogenation was found to be complete, the mixture was passed through a pad of Celite, and the filtrate was concentrated in vacuo, affording pale yellow oil, which was purified by silica gel chromatography (5-15\% EtOAc in hexanes) to afford (Z)- $N$-methoxy- $N$-methylbut-2-enamide (356.8 mg, $2.76 \mathrm{mmol}, 55 \%$ yield) in $>98: 2 \mathrm{Z}: E$ selectivity as colorless liquid. IR (neat): 2937 (w), 1656 (s), 1633 (m), 1441 (m), 1001 (m), 1274 (m), 818 (m), 456 (m) cm ${ }^{-1} ;{ }^{1}$ H NMR (500 MHz, CDCl $\mathbf{H}_{3}: \delta$ 6.18-6.16 (m, 2H), 3.62-3.58 (m, 3H), 3.15-3.12 (m, 3H), 2.04-2.02 (m, 3H); ${ }^{13}$ C NMR (125 MHz, $\left.\mathbf{C D C l}_{3}\right): \delta 167.6,141.8,119.1,61.4,32.0,15.3 ; \mathbf{H R M S}[\mathbf{M}+\mathbf{H}]^{+}:$Calcd for $\mathrm{C}_{6} \mathrm{H}_{12} \mathrm{NO}_{2}$ : 130.0868 , Found: 130.0863 .

(Z)- $N$-Benzylbut-2-enamide (1d). An 8-dram vial was charged with ( $Z$ )-2-butenoic acid (86.1 $\mathrm{mg}, 1.0 \mathrm{mmol}, 1.0$ equiv.) and $\mathrm{CH}_{2} \mathrm{Cl}_{2}(4.0 \mathrm{~mL})$. The following were subsequently added: benzylamine (96 mg, $0.9 \mathrm{mmol}, 0.9$ equiv.), $N$-(3-dimethylaminopropyl)- $N$ '-ethylcarbodiimide hydrochloride (EDCI, $229 \mathrm{mg}, 1.2 \mathrm{mmol}, 1.2$ equiv.), hydroxybenzotriazole hydrate (HOBt, 184 mg, $1.2 \mathrm{mmol}, 1.2$ equiv.), and $N, N$-diisopropylethylamine (310.2 mg, $2.4 \mathrm{mmol}, 2.4$ equiv.). The mixture was allowed to stir for $1 \mathrm{~h}$ and then the volatiles were removed in vacuo. The resulting yellow solid was purified by silica gel chromatography to afford 1d (119 mg, 0.68 mmol, 75\% yield) as colorless oil. IR (neat): 3288 (m, br), 3061 (m), 3027 (m), 2913 (w), 1658 (s), $1631(\mathrm{~s}), 1536(\mathrm{~s}), 1495(\mathrm{~m}), 1433(\mathrm{~m}), 1357(\mathrm{~m}), 1269(\mathrm{~m}), 1232(\mathrm{~m}), 1027$ (w), $809(\mathrm{~m})$, $743(\mathrm{~m}), 718(\mathrm{~m}) \mathrm{cm}^{-1} ;{ }^{1} \mathbf{H}$ NMR (400 MHz, $\left.\mathbf{C D C l}_{3}\right): \delta$ 7.36-7.27 (m, 5H), 6.19-6.07 (m, 1H), 5.79 (s, br, 1H), 5.73 (dt, $J=11.4,1.7 \mathrm{~Hz}, 1 \mathrm{H}), 4.48$ (t, $J=4.9 \mathrm{~Hz}, 2 \mathrm{H}), 2.15$ (dt, $J=7.2,1.8 \mathrm{~Hz}$, 3H); ${ }^{13}$ C NMR (125 MHz, $\left.\mathbf{C D C l}_{3}\right)$ : $\delta$ 166.6, 140.8, 138.5, 128.8, 128.0, 127.6, 123.0, 43.4, 15.3; HRMS[M+H] $]^{+}$: Calcd for $\mathrm{C}_{11} \mathrm{H}_{14} \mathrm{NO}: 176.1067$, found: 176.1072 .

(Z)-But-2-enamide (1e). A $50 \mathrm{~mL}$ round-bottom flask was charged with $5 \mathrm{~mL} \mathrm{CH}_{2} \mathrm{Cl}_{2} / \mathrm{H}_{2} \mathrm{O}$ (20/1) solution of (Z)- $N$-(4-methoxybenzyl)but-2-enamide (102.5 mg, $0.5 \mathrm{mmol}, 1.0$ equiv.), after which DDQ (170.2 $\mathrm{mg}, 0.75 \mathrm{mmol}, 1.5$ equiv.) was added and the mixture was allowed to stir for $4 \mathrm{~h}$ at $22{ }^{\circ} \mathrm{C}$. The reaction was quenched by the addition of a saturated solution of $\mathrm{Na}_{2} \mathrm{SO}_{3}$ $(20 \mathrm{~mL})$. The aqueous layer was washed with EtOAc $(20 \mathrm{~mL} \times 3)$, and the organic layers were 
combined, dried over $\mathrm{Na}_{2} \mathrm{SO}_{4}$, concentrated under reduced pressure, and the resulting purple solid was purified by silica gel chromatography (50-100\% EtOAc in hexanes) to afford 1e (37.4 mg, 0.44 mmol, 88\% yield) as off-white solid. M.p.: $115-116{ }^{\circ} \mathrm{C}$; IR (neat): 3393 (m), 3201 (m), 1668 (s), 1636 (m), 1610 (s), 1448 (m), 1366 (m), 1322 (m), 1260 (m), 817 (m), 709 (m); ${ }^{1} \mathbf{H}$ NMR (600 MHz, CDCl $\left.\mathbf{~}_{3}\right): \delta 6.17(\mathrm{dq}, J=11.7,7.2 \mathrm{~Hz}, 1 \mathrm{H}), 5.77(\mathrm{dd}, J=11.5,1.7 \mathrm{~Hz}, 1 \mathrm{H})$, 5.46 (br, 2H), 2.13 (dd, $J=7.2,1.2 \mathrm{~Hz}, 3 \mathrm{H}) ;{ }^{13} \mathbf{C}$ NMR (150 MHz, $\mathbf{C D C l}_{3}$ ): $\delta 168.66,141.75$, 122.28, 15.24; HRMS[M+H] $]^{+}$: Calcd for $\mathrm{C}_{4} \mathrm{H}_{8} \mathrm{NO}: 86.0600$, found: 86.0605.

\section{(Z)-N-(4-Methoxybenzyl)but-2-enamide (S2)}

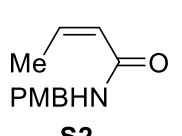

A $50 \mathrm{~mL}$ round-bottom flask was charged with $(Z)$-2-butenoic acid (86.1 $\mathrm{mg}, 1.0$ mmol, 1.0 equiv.) and $\mathrm{CH}_{2} \mathrm{Cl}_{2}(4.0 \mathrm{~mL})$. The following were subsequently added: 4methoxybenzylamine $\quad(123.5 \quad \mathrm{mg}, \quad 0.9 \quad \mathrm{mmol}, \quad 0.9 \quad$ equiv. $), \quad \mathrm{N}-(3-$ dimethylaminopropyl)- $N$ '-ethylcarbodiimide hydrochloride (EDCI, $229 \mathrm{mg}, 1.2 \mathrm{mmol}, 1.2$ equiv.), hydroxybenzotriazole hydrate (HOBt, $184 \mathrm{mg}, 1.2 \mathrm{mmol}, 1.2$ equiv.), and $N, N$ diisopropylethylamine (310.2 mg, $2.4 \mathrm{mmol}, 2.4$ equiv.). The mixture was allowed to stir for $1 \mathrm{~h}$ and the volatiles were removed in vacuo. The resulting yellow solid was purified by silica gel chromatography to afford S2 (149.6 mg, $0.73 \mathrm{mmol}, 81 \%$ yield) as off-white solid. M.p.: 76-77 ${ }^{\circ} \mathrm{C}$; IR (neat): 3298 (s), 3061 (w), 3039 (w), 2955 (w), 2916 (w), 1659 (m), 1624 (s), 1539 (s), 1513 (m), 1459 (m), 1247 (m), 1233 (m), $1218(\mathrm{~m}), 1175(\mathrm{~m}), 1108(\mathrm{~m}), 1027(\mathrm{~m}), 816$ $(\mathrm{m}), 696(\mathrm{~m}) \mathrm{cm}^{-1}{ }^{1} \mathbf{H}$ NMR (400 MHz, $\left.\mathbf{C D C l}_{3}\right): \delta$ 7.25-7.20 (m, 2H), 6.89-6.83 (m, 2H), 6.11 $(\mathrm{dq}, J=11.4,7.2 \mathrm{~Hz}, 1 \mathrm{H}), 5.70(\mathrm{dq}, J=11.4,1.7 \mathrm{~Hz}, 1 \mathrm{H}), 5.64$ (s, br, 1H), 4.41 (d, $J=5.7 \mathrm{~Hz}$, 2H), 3.80 (s, 3H), 2.15 (dd, $J=7.2,1.8 \mathrm{~Hz}, 3 \mathrm{H}) ;{ }^{13} \mathbf{C}$ NMR (100 MHz, CDCl 3 ): $\delta$ 166.5, 159.2, 140.7, 130.6, 129.4, 123.1, 114.2, 55.5, 42.9, 15.3; HRMS[M+H] $]^{+}$: Calcd for $\mathrm{C}_{12} \mathrm{H}_{16} \mathrm{NO}_{2}$ : 206.1176, found: 206.1174 .

\section{(Z)-N-Isobutylbut-2-enamide (S3)}

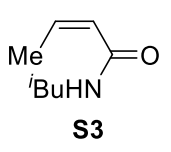

A $50 \mathrm{~mL}$ round-bottom flask was charged with $(Z)$-2-butenoic acid (86.1 $\mathrm{mg}, 1.0$ mmol, 1.0 equiv.) and $\mathrm{CH}_{2} \mathrm{Cl}_{2}(4.0 \mathrm{~mL})$. The following were then added sequentially: Isobutylamine (65.8 mg, $0.9 \mathrm{mmol}, 0.9$ equiv.), $N$-(3-dimethylaminopropyl)- $N{ }^{\prime}$ ethylcarbodiimide hydrochloride (EDCI, $229 \mathrm{mg}, 1.2 \mathrm{mmol}, 1.2$ equiv.), hydroxybenzotriazole hydrate (HOBt, $184 \mathrm{mg}, 1.2 \mathrm{mmol}, 1.2$ equiv.), and $N, N$-diisopropylethylamine (310.2 $\mathrm{mg}, 2.4$ mmol, 2.4 equiv.). The mixture was allowed to stir for $1 \mathrm{~h}$ and the volatiles were removed in vacuo. The resulting yellow oil was purified by silica gel chromatography (10-50\% EtOAc in hexanes) to afford $\mathbf{S 3}$ (91.5 mg, $0.65 \mathrm{mmol}, 72 \%$ yield) as off-white solid. M.p.: $45{ }^{\circ} \mathrm{C}$; IR (neat): 3292 (m, br), 3073 (w), 3028 (w), 2955 (m), 2924 (m), 2868 (m), 1658 (s), 1630 (s), 1541 (s), 1465 (m), $1434(\mathrm{~m}), 1386(\mathrm{~m}), 1268(\mathrm{~m}), 1232(\mathrm{~m}), 1157(\mathrm{~m}), 914(\mathrm{w}), 808(\mathrm{w}) \mathrm{cm}^{-1}$; ${ }^{1}$ H NMR (400 MHz, CDCl $)$ ): $\delta$ 6.13-5.99 (m, 1H), $5.71(\mathrm{dq}, J=11.4,1.7 \mathrm{~Hz}, 1 \mathrm{H}), 5.64(\mathrm{~s}, \mathrm{br}$, $1 \mathrm{H}), 3.10(\mathrm{td}, J=6.8,6.1,1.3 \mathrm{~Hz}, 2 \mathrm{H}), 2.10(\mathrm{dd}, J=7.2,1.7 \mathrm{~Hz}, 3 \mathrm{H}), 1.78(\mathrm{dp}, J=13.4,6.7 \mathrm{~Hz}$, 1H), 0.91 (dd, $J=6.7,1.1 \mathrm{~Hz}, 6 \mathrm{H}) ;{ }^{13} \mathbf{C}$ NMR (100 MHz, $\left.\mathbf{C D C l}_{3}\right): \delta 166.9,139.7,123.5,46.7$, 28.7, 20.3, 15.1; HRMS[M+H] $]^{+}$: Calcd for $\mathrm{C}_{8} \mathrm{H}_{16} \mathrm{NO}$ : 142.1226, found: 142.1229 . 


\subsection{Cross-Metathesis with (Z)-3-Hexene}

Benzyl ( $Z$ )-pent-2-enoate (2a). In a glove box, an oven-dried vial equipped with a magnetic stir bar was charged with $(Z)$-3-hexene $(84.2 \mathrm{mg}, 1.00 \mathrm{mmol}, 10.0$ equiv.) and benzyl (Z)-but-2enoate $(17.6 \mathrm{mg}, 0.100 \mathrm{mmol}, 1.00$ equiv.) in THF $(200 \mu \mathrm{L})$. To this mixture was added a THF solution $(400 \mu \mathrm{L})$ of Ru-1d (3.7 mg, $0.005 \mathrm{mmol}, 5.0 \mathrm{~mol} \%)$. The mixture was allowed to stir for $8 \mathrm{~h}$ at $22{ }^{\circ} \mathrm{C}$, after which the reaction was quenched by exposing the solution to air and the addition of undistilled $\mathrm{Et}_{2} \mathrm{O}$. Removal of the volatiles in vacuo afforded black oil, which was purified by silica gel chromatography (5-15\% EtOAc in hexanes) to afford 2a (14.8 $\mathrm{mg}, 0.078$ mmol, 78\% yield) in >98:2 Z:E selectivity as colorless oil. IR (neat): 3035 (w), 2964 (w), 1719 (s), 1642 (m), 1166 (s), 821 (m), $696(\mathrm{~m}) \mathrm{cm}^{-1} ;{ }^{1} \mathbf{H}$ NMR (400 MHz, $\left.\mathbf{C D C l}_{3}\right): \delta 7.39-7.30(\mathrm{~m}$, $5 \mathrm{H}), 6.25(\mathrm{dt}, J=11.5,7.5 \mathrm{~Hz}, 1 \mathrm{H}), 5.80(\mathrm{dt}, J=11.5,1.7 \mathrm{~Hz}, 1 \mathrm{H}), 5.16(\mathrm{~s}, 2 \mathrm{H}), 2.68$ (pd, $J=$ 7.5, $1.7 \mathrm{~Hz}, 2 \mathrm{H}), 1.06(\mathrm{t}, J=7.6 \mathrm{~Hz}, 3 \mathrm{H}) .{ }^{13} \mathbf{C}$ NMR (100 MHz, $\left.\mathbf{C D C l}_{3}\right): \delta 166.3,152.8,136.3$, 128.7, 128.3, 128.3, 118.9, 65.8, 22.7, 13.6. HRMS[M+H] $]^{+}$: Calcd for $\mathrm{C}_{12} \mathrm{H}_{15} \mathrm{O}_{2}: 191.1430$, Found: 191.1425. The same procedure was employed for the reaction of Ru-1c.

(Z)-Pent-2-enoic acid (3a). The same procedure as described above was followed. The resulting black oil residue was purified by silica gel chromatography (5-10\% EtOAc in hexanes) to afford olefin 3a $(7.0 \mathrm{mg}, 0.070 \mathrm{mmol}, 70 \%$ yield) in $98: 2 \mathrm{Z:E}$ selectivity as colorless oil. The corresponding spectral data are consistent with those previously reported. ${ }^{14}{ }^{1} \mathrm{H}$ NMR (400 MHz, $\left.\mathbf{C D C l}_{3}\right): \delta$ carboxylic acid proton is invisible, $6.35(\mathrm{dt}, J=11.5,7.5 \mathrm{~Hz}, 1 \mathrm{H}), 5.76(\mathrm{dt}, J=11.5$, $1.7 \mathrm{~Hz}, 1 \mathrm{H}), 2.67(\mathrm{pd}, J=7.6,1.7 \mathrm{~Hz}, 2 \mathrm{H}), 1.06(\mathrm{t}, J=7.6 \mathrm{~Hz}, 3 \mathrm{H})$. Carboxylic acid proton was not observable. ${ }^{13} \mathbf{C}$ NMR (100 $\left.\mathbf{~ M H z}, \mathbf{C D C l}_{3}\right): \delta 172.3,155.0,118.7,22.8,13.5$. The same procedure was employed for the reaction of Ru-1c.

(Z)-N-Methoxy- $N$-methylpent-2-enamide (5a). The same procedure as described above was followed. The resulting black oil residue was purified by silica gel chromatography (10-20\% EtOAc in hexanes) to afford $\mathbf{5 a}(9.7 \mathrm{mg}, 0.068 \mathrm{mmol}, 68 \%$ yield) in $>98: 2 \mathrm{Z}: E$ selectivity as colorless oil. IR (neat): 2958 (m), 2923 (s), 2852 (m), 1643 (w), 800 (m) $\mathrm{cm}^{-1} ;{ }^{1}$ H NMR (400 MHz, $\left.\mathbf{C D C l}_{3}\right): \delta 6.16(\mathrm{~d}, J=11.6 \mathrm{~Hz}, 1 \mathrm{H}), 6.05(\mathrm{dt}, J=11.6,7.2 \mathrm{~Hz}, 1 \mathrm{H}), 3.63(\mathrm{~s}, 3 \mathrm{H}), 3.16(\mathrm{~s}$, $3 \mathrm{H}), 2.57(\mathrm{p}, J=7.5 \mathrm{~Hz}, 2 \mathrm{H}), 1.00(\mathrm{t}, J=7.6 \mathrm{~Hz}, 3 \mathrm{H}) ;{ }^{13} \mathbf{C} \mathbf{N M R}\left(\mathbf{1 0 0} \mathbf{M H z}, \mathbf{C D C l}_{\mathbf{3}}\right): \delta 167.5$, 148.9, 117.6, 61.5, 32.2, 22.5, 13.8; HRMS[M+H] ${ }^{+}$: Calcd for $\mathrm{C}_{7} \mathrm{H}_{14} \mathrm{NO}_{2}:$ : 144.1019, Found: 144.1023. The same procedure was employed for the reaction of Ru-1c.

(Z)- $N$-Benzylpent-2-enamide (6a). The same procedure as described above was followed. The resulting black oil residue was purified by silica gel chromatography (10-20\% EtOAc in hexanes) to afford olefin product $(15.9 \mathrm{mg}, 0.084 \mathrm{mmol}, 84 \%$ yield) in $>98: 2 \mathrm{Z:E}$ selectivity as colorless oil. IR (neat): 3285 (s), 3063 (w), 1671 (w), 1628 (s), 1552 (s), 1276 (s), 820 (m), 693 (m) cm ${ }^{1} \mathbf{H}$ NMR (500 MHz, $\left.\mathbf{C D C l}_{3}\right): \delta 7.30(\mathrm{~m}, 5 \mathrm{H}), 6.00(\mathrm{dt}, J=11.4,7.4 \mathrm{~Hz}, 1 \mathrm{H}), 5.77(\mathrm{br}, 1 \mathrm{H}), 5.67$ $(\mathrm{d}, J=11.4 \mathrm{~Hz}, 1 \mathrm{H}), 4.47(\mathrm{~d}, J=5.8 \mathrm{~Hz}, 2 \mathrm{H}), 3.60-2.45(\mathrm{~m}, 2 \mathrm{H}), 1.05(\mathrm{t}, J=7.6 \mathrm{~Hz}, 3 \mathrm{H}) ;{ }^{13} \mathbf{C}$ NMR (125 MHz, $\left.\mathbf{C D C l}_{3}\right): \delta 166.5,147.9,138.5,128.8,128.0,127.6,121.5,43.5,22.4,13.9$; HRMS[M+H] $]^{+}$: Calcd for $\mathrm{C}_{12} \mathrm{H}_{16} \mathrm{NO}$ : 190.1232, Found: 190.1229. The same procedure was employed for the reaction of $\mathbf{R u - 1 c}$. 
(Z)-Pent-2-enamide (7a). The same procedure as described above was followed. The resulting black oil was purified by silica gel chromatography (50 100\% ethyl acetate in hexanes) and filtered through a small plug of activated charcoal to afford product in >98:2 Z:E selectivity as colorless oil (4.4 $\mathrm{mg}, 0.0444 \mathrm{mmol}, 89 \%$ yield). The characterization data are consistent with those previously reported. ${ }^{15}{ }^{1} \mathbf{H}$ NMR (500 MHz, $\left.\mathbf{C D C l}_{3}\right): \delta 6.04(\mathrm{dt}, J=11.5,7.4 \mathrm{~Hz}, 1 \mathrm{H}), 5.71$ $(\mathrm{d}, J=11.5 \mathrm{~Hz}, 1 \mathrm{H}), 5.52(\mathrm{~d}, J=84.1 \mathrm{~Hz}, 2 \mathrm{H}), 2.65(\mathrm{p}, J=8.5,8.0 \mathrm{~Hz}, 2 \mathrm{H}), 1.04$ (t, $J=7.6 \mathrm{~Hz}$, $3 \mathrm{H}$ ); HRMS[2M+H] $]^{+}$: Calcd for $\mathrm{C}_{10} \mathrm{H}_{19} \mathrm{~N}_{2} \mathrm{O}_{2}$ : 199.1441, found: 199.1438. The same procedure was employed for the reaction of $\mathbf{R u - 1 c}$.

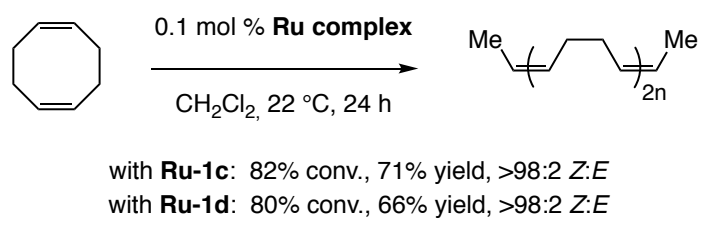

Polymerization of cyclooctdiene. In an $\mathrm{N}_{2}$-filled glovebox, an oven-dried vial equipped with a magnetic stir bar was charged with 1,5-cyclooctadiene (108 $\mathrm{mg}, 1.00 \mathrm{mmol}$, after purification by passing through a plug of basic alumina). A solution of Ru-1d $(0.70 \mathrm{mg}, 0.001 \mathrm{mmol})$ in $\mathrm{CH}_{2} \mathrm{Cl}_{2}$ $(0.2 \mathrm{~mL})$ was added and the resulting solution was allowed to stir at $22{ }^{\circ} \mathrm{C}$ for $24 \mathrm{~h}$, after which $\mathrm{MeOH}(2.0 \mathrm{~mL})$ was added, causing the polymer to precipitate. The polymer was washed again with $\mathrm{MeOH}(2.0 \mathrm{~mL})$ and dried in vacuo $\left(1.0 \times 10^{-1}\right.$ torr $)$ to afford polycyclooctadiene $(71.3 \mathrm{mg}$, $66 \%$ yield). The characterization data are consistent with those previously reported. ${ }^{16}$

\section{Mechanistic Studies}

\subsection{Identification of Decomposition Products}

In a $\mathrm{N}_{2}$-filled glovebox, an oven-dried vial equipped with a magnetic stir bar was charged with a THF solution $(200 \mu \mathrm{L}$, pre-made stock solution) of benzyl (Z)-but-2-enoate (17.6 mg, 0.100 mmol, 1.00 equiv.), (Z)-hex-3-ene (84.2 mg, $1.00 \mathrm{mmol}, 10.0$ equiv.) and a THF solution (400 $\mu \mathrm{L}$, pre-made stock solution) of Ru-1c (3.7 mg, $0.005 \mathrm{mmol}, 1.00$ equiv.). The mixture was allowed to stir at $22{ }^{\circ} \mathrm{C}$, while aliquots were removed and analyzed by GC-MS after 1, 2, 4 and 9 h. The same procedure was employed for Ru-1d. 


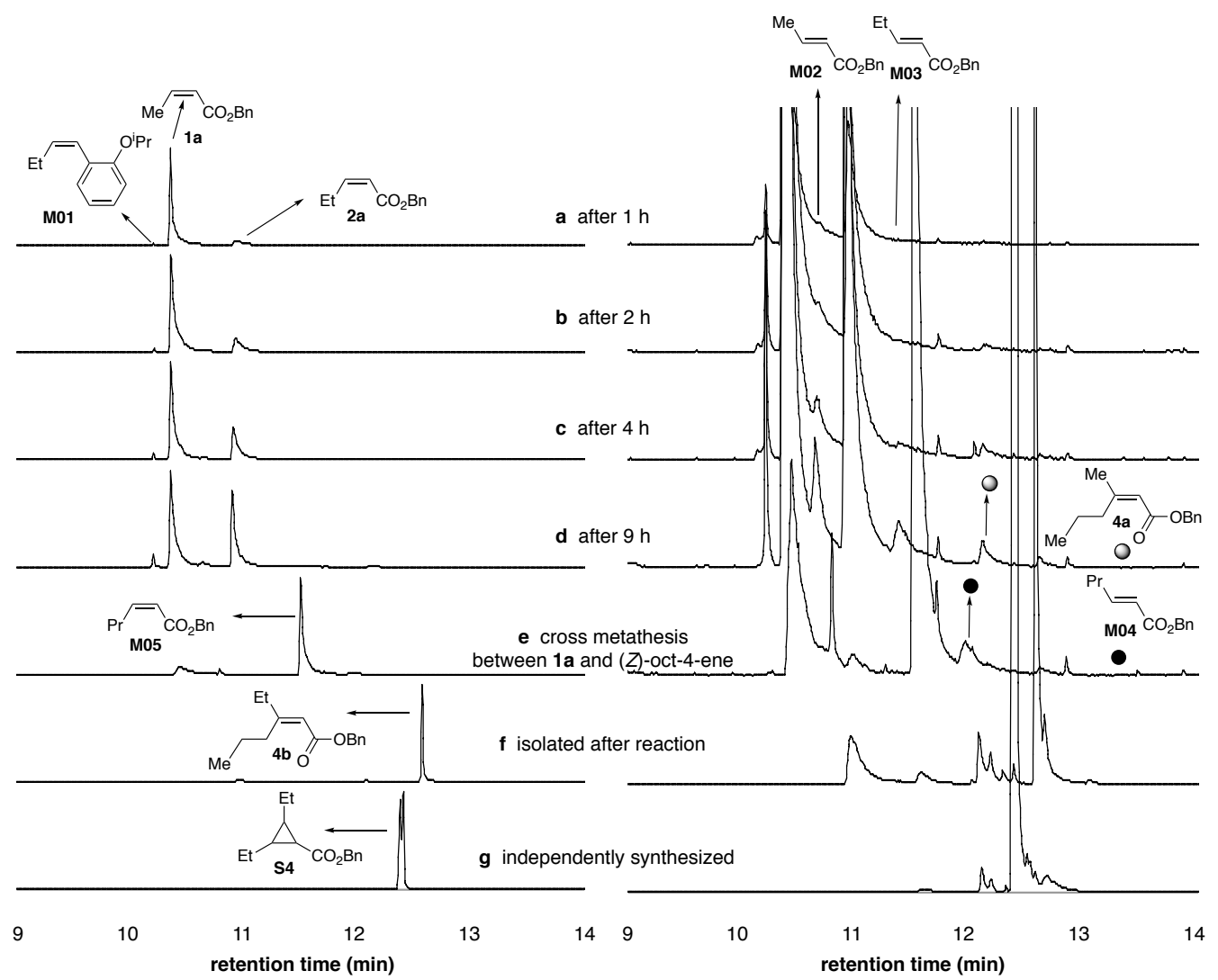

Fig. S1. Identification of decomposition products generated by a reaction carried out in the presence of Ru-1c (GCMS analysis; unscaled GC traces (left); intensity scaled by a factor of 15 (right)).

Analysis: In the case of the reaction carried out with Ru-1c, $<10 \%$ 2a was detected after $1 \mathrm{~h}$ (GC-MS; Fig. S1a). Conversion reached nearly 50\% after 9 h (Fig. S1d). Based on MS analysis and an independently prepared sample of M05 (Fig. S3 and Fig. S1e), we confirmed the formation of isomerization byproducts M02, M03, and M04. Importantly, we confirmed the formation of trisubstituted enoates $\mathbf{4 a}$ and $\mathbf{4 b}$ ( 70:30; see DFT Section for further analysis). We were unable to detect cyclopropanation product after the reaction, which we independently synthesized as mixture of two isomers through cyclopropane formation method with a Rh-based complex (Fig. S1g). 


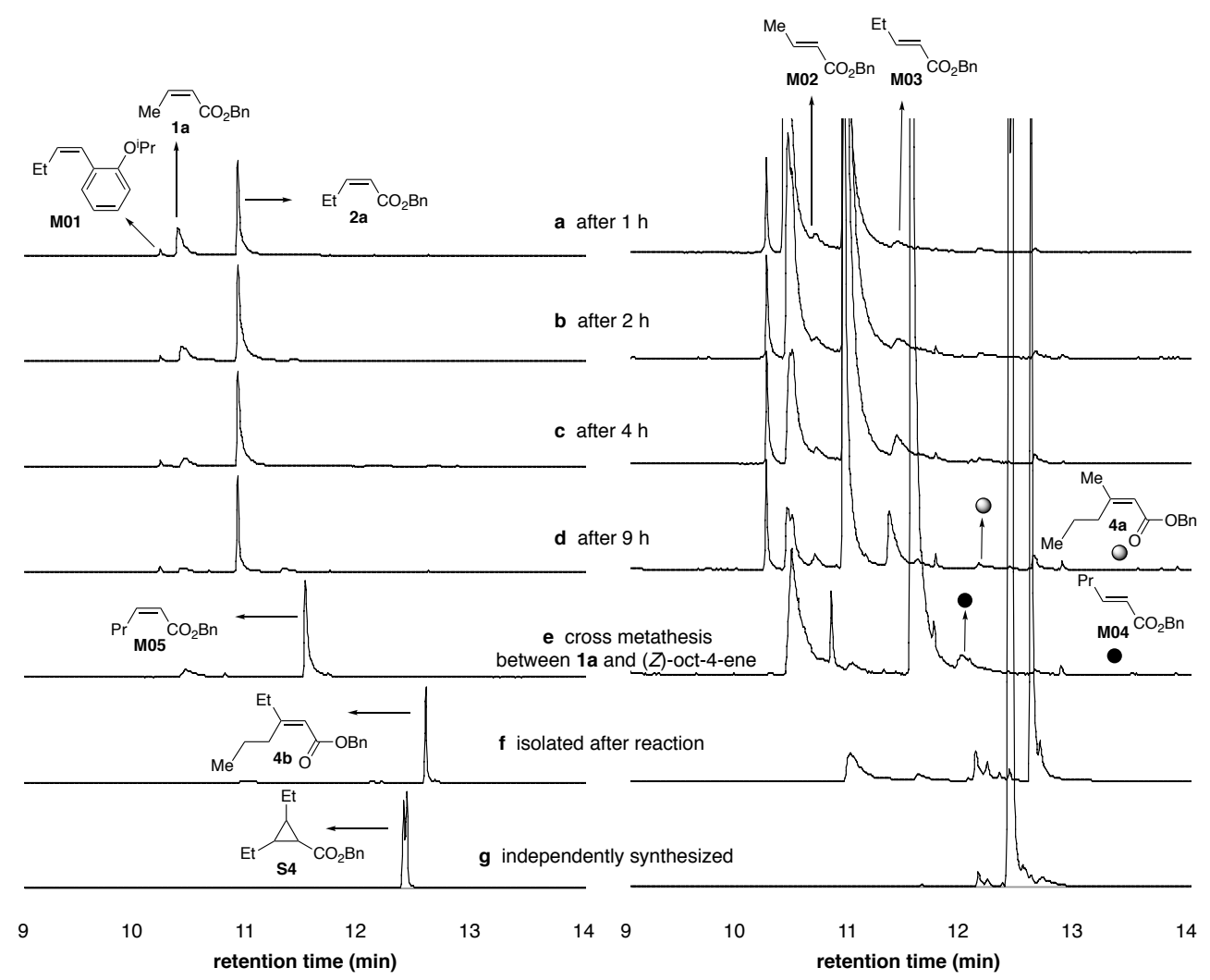

Fig. S2. Identification of decomposition products generated by a reaction carried out in the presence of Ru-1d (GCMS analysis; unscaled GC traces (left); intensity scaled by a factor of 15 (right)).

Analysis: In the case of the reaction carried out with Ru-1d, (Z)-but-2-enoate 1a was converted to 2a after $1 \mathrm{~h}$ (GC analysis; left trace, Fig. S2a). Apart from the efficiency difference, the other notable distinction relative to the transformation with $\mathbf{R u - 1 c}$ is the reversal in $4 \mathbf{a}: 4 \mathbf{b}$ ratio derived ( $20: 80$, Fig. S2d). Because the concentration of (Z)-pent-2-enoate 2a increases as the reaction progresses, increasing amounts of $\mathbf{4 b}$ are generated (right trace, Fig. S2d; compared to Fig. S2a). Subjection of $\mathbf{4 b}$ to GC-MS confirmed that $\mathbf{4 b}$ is the same species that is produced in the course of the reaction (Fig. S2f). The stereochemical identity of $\mathbf{4 b}$ was ascertained through appropriate nOe experiments (Fig. S4). The resulting data (Et group and alkenyl protons in cis relationship) are consistent with the pathway predicted by DFT studies. The structure of $\mathbf{4 a}$ was confirmed by MS fragment analysis (Fig. S3). 


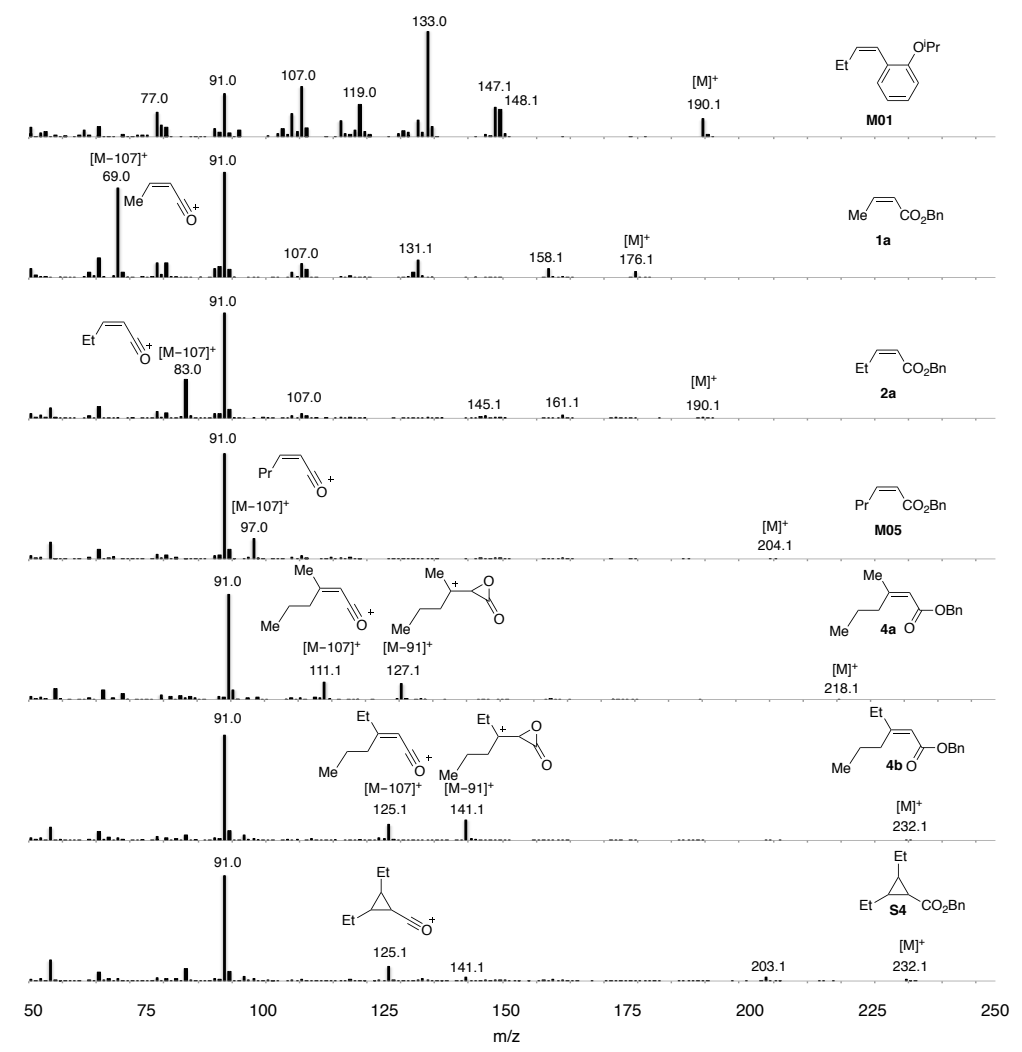

Fig. S3. Mass spectra and proposed fragmentation for the most relevant species and byproducts observed during CM between (Z)-hex-3-ene and benzyl (Z)-but-2-enoate.

Analysis: All compounds display a peak corresponding to their molecular weight $[\mathrm{M}]^{+}$. All spectra display a peak at $\mathrm{m} / \mathrm{z}=91$, which corresponds to the tropylium ion derived from the benzyl protecting group. All benzyl ester substrates show a peak at $[\mathrm{M}-107]^{+}$, which is the

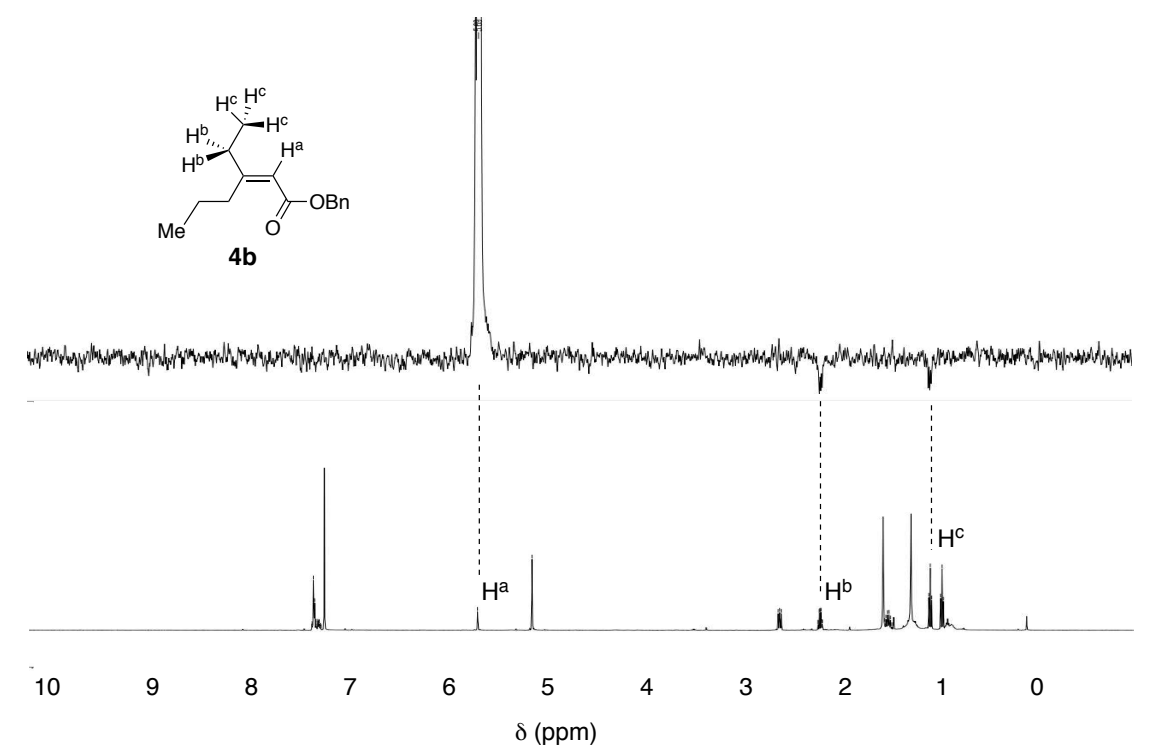

Fig. S4. Determination of the stereochemical identity of $\mathbf{4 b}$ (nOe experiments recorded in $\mathrm{CDCl}_{3}$ at $500 \mathrm{MHz}$ ). 
acylium ion after loss of benzyloxide. The spectra containing trisubstituted enoates $\mathbf{4 a}$ and $\mathbf{4 b}$ contain an additional peak at $\mathrm{m} / \mathrm{z}=[\mathrm{M}-91]^{+}$, which is either absent or far weaker in the spectra of the other benzyl esters. We propose that appearance of this peak depends on the possible formation of a stabilized tertiary carbocation.

Benzyl (Z)-3-ethylhex-2-enoate (4b). IR (neat): 2961 (m), 2925 (s), 1717 (s), 1142 (m) cm ; $^{-1}$ ${ }^{1}$ H NMR (500 MHz, CDCl 3$): \delta 7.39-7.29(\mathrm{~m}, 5 \mathrm{H}), 5.69$ (s, 1H), $5.13(\mathrm{~s}, 2 \mathrm{H}), 2.60$ (t, J= 7.5 Hz, 2H), 2.21-2.16 (m, 2H), 1.53-1.45 (m, 2H), $1.06(\mathrm{t}, J=7.4 \mathrm{~Hz}, 3 \mathrm{H}), 0.94(\mathrm{t}, J=7.4 \mathrm{~Hz}, 3 \mathrm{H})$; ${ }^{13}$ C NMR (125 MHz, $\left.\mathbf{C D C l}_{3}\right): \delta$ 166.9, 166.6, 136.6, 128.7, 128.3, 128.2, 114.1, 65.6, 34.5, 31.4, 22.1, 14.4, 12.2; HRMS[M+H] ${ }^{+}$: Calcd for $\mathrm{C}_{15} \mathrm{H}_{21} \mathrm{O}_{2}: 233.1536$, Found: 233.1537.

\section{Benzyl 2,3-diethylcyclopropane-1-carboxylate (S4)}

In a $\mathrm{N}_{2}$-filled glovebox, an oven-dried vial equipped with a magnetic stir bar was charged with $\mathrm{CO}_{2} \mathrm{Bn}$
$\mathrm{mg}, 2.50 \mathrm{mmol}, 5.00$ equiv. $)$ in $\mathrm{CH}_{2} \mathrm{Cl}_{2}(300 \mu \mathrm{L})$. To this solution was added
$\mathrm{Rh}$
$\mathrm{Rh}(\mathrm{OAc})_{4}(2.2 \mathrm{mg}, 0.005 \mathrm{mmol}, 5.0 \mathrm{~mol} \%)$, causing significant $\mathrm{N}_{2}$ evolution. After
five min the vessel was sealed, and the mixture was allowed to stir for $12 \mathrm{~h}$ at $22{ }^{\circ} \mathrm{C}$. The reaction was quenched by the addition of undistilled $\mathrm{Et}_{2} \mathrm{O}$ and the volatiles were removed in vacuo. The resulting green oil was purified by silica gel chromatography (5-15\% EtOAc in hexanes) to afford S4 (diastereomeric mixture; $34.3 \mathrm{mg}, 0.148 \mathrm{mmol}, 30 \%$ yield) as colorless oil. IR (neat): 2962 (m), 2930 (w), 1723 (s), 1455 (m), 1164 (s), 1141 (s), 736 (m), 696 (m) cm ; $^{-1}$ ${ }^{1}$ H NMR (400 MHz, CDCl $)$ : $\delta$ 7.39-7.29 (m, 5H), 5.10-5.09 (m, 2H), 1.72-1.64 (m, 2H), 1.49-1.12 (m, 5H), 1.02-0.99 (m, 3H), 0.94-0.90 (m, 3H); ${ }^{13} \mathbf{C}$ NMR (100 MHz, CDCl $): \delta$ $174.8,172.2,136.7,136.5,128.7,128.6,128.3,128.2,128.1,128.1,66.2,65.8,30.3,27.7,26.6$, 20.7, 20.2, 15.8, 14.2, 14.1; HRMS[M+H] ${ }^{+}$: Calcd for $\mathrm{C}_{15} \mathrm{H}_{21} \mathrm{O}_{2}: 233.1536$, Found: 233.1531 .

\section{2 $\beta$-Hydride Elimination}

In a $\mathrm{N}_{2}$-filled glovebox, an oven-dried vial equipped with a magnetic stir bar was charged with (Z)-hex-3-ene-3,4- $d_{2}\left(86.2 \mathrm{mg}, 1.00 \mathrm{mmol}, 10.0\right.$ equiv.) and benzyl (Z)-but-2-enoate-3,4- $d_{2}(17.8$ $\mathrm{mg}, 0.100 \mathrm{mmol}, 1.00$ equiv.), and THF $(200 \mu \mathrm{L})$. To this mixture was added Ru-1c $(3.7 \mathrm{mg}$, $0.005 \mathrm{mmol}, 5.0 \mathrm{~mol} \%$; stock solution in THF, $400 \mu \mathrm{L}$ ), and was allowed to stir for $8 \mathrm{~h}$ at $22^{\circ} \mathrm{C}$. The reaction was quenched by the addition of undistilled $\mathrm{Et}_{2} \mathrm{O}$ and the volatiles were removed in vacuo to leave behind black oil, which was purified by silica gel chromatography (5-15\% EtOAc in hexanes) to afford $\mathbf{2 a}$ or $\mathbf{2 a}-\boldsymbol{d}_{\mathbf{2}}(15.4 \mathrm{mg}, 0.080 \mathrm{mmol}, 80 \%$ yield) in $>98: 2 \mathrm{Z:E}$ ratio and $95 \%$ deuterium incorporation as colorless oil. The same procedure was used for a reaction with Ru-1d.

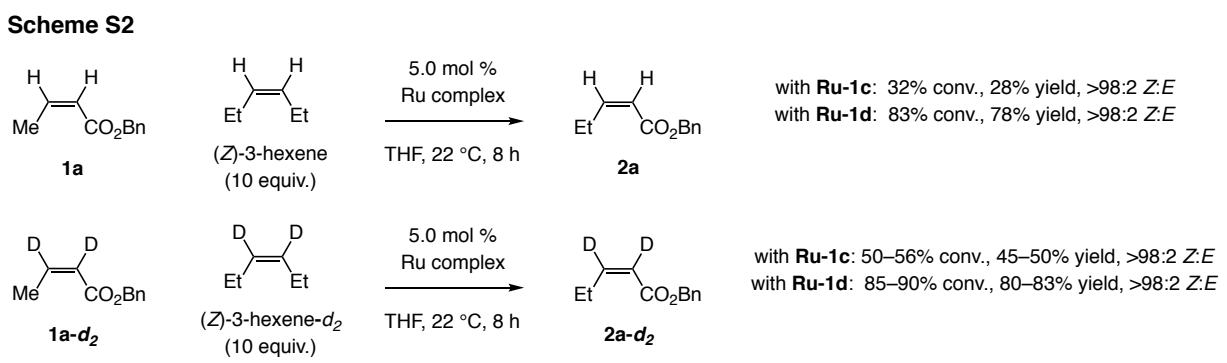


Analysis: With Ru-1c, for reaction of deuterium-labeled substrates with $\mathbf{R u - 1 c}$, there was a boost in efficiency (from 32\% to 50-56\% conv.), whereas in the case of Ru-1d conversion was high regardless of whether deuterium-labeled alkenes were used or not ( 83 vs. $85-90 \%$ conv.). These data indicate that when the rate of decomposition by $\beta$-hydride elimination reduced, there is diminished gap between the efficiency of cross-metathesis reactions performed in the presence of Ru-1c and Ru-1d.

Benzyl ( $\boldsymbol{Z}$ )-pent-2-enoate-2,3- $\boldsymbol{d}_{\mathbf{2}}\left(\mathbf{1} \mathbf{a}-\boldsymbol{d}_{2}\right)$. To a solution of benzyl but-2-ynoate (870.4 $\mathrm{mg}, 5.00$ mmol, 1.0 equiv.) in $20 \mathrm{~mL}$ anhydrous $\mathrm{Et}_{2} \mathrm{O}$ was added $5 \% \mathrm{Pd} / \mathrm{BaSO}_{4}(150 \mathrm{mg})$, quinoline (30 $\mathrm{mg}, 0.23 \mathrm{mmol}, 4.6 \mathrm{~mol} \%$ ) under $\mathrm{D}_{2}$ atm. (balloon) at $22{ }^{\circ} \mathrm{C}$. Reaction progress was monitored by analyzing the composition of an aliquot ( ${ }^{1} \mathrm{H}$ NMR spectroscopy). Once hydrogenation was complete, the solution was allowed to pass through a pad of Celite. The volatiles were removed in vacuo, and the resulting pale yellow oil was purified by silica gel chromatography (5-15\% EtOAc in hexanes) to afford $\mathbf{1 a - d _ { 2 }}(540 \mathrm{mg}, 3.03 \mathrm{mmol}, 61 \%$ yield $)$ in $>98: 2 \mathrm{Z}: E$ selectivity and 96\% D incorporation as colorless oil. IR (neat): 3034 (w), 1713 (s), 1620 (m), 1251 (s), 1128 (s), 1087 (m), 696 (m) cm ${ }^{-1} ;{ }^{1} \mathbf{H}$ NMR (500 MHz, $\left.\mathbf{C D C l}_{3}\right): \delta 7.40-7.30$ (m, 5H), 5.17 (s, 2H), 2.15 (s, 3H); ${ }^{13} \mathbf{C}$ NMR (100 MHz, CDCl 3$): \delta 166.4,145.5-145.2(\mathrm{~m}), 136.3,128.7,128.3,128.2$, 120.3-119.9 (m), 65.8, 15.4; HRMS[M+H] $]^{+}$: Calcd for $\mathrm{C}_{11} \mathrm{H}_{11} \mathrm{D}_{2} \mathrm{O}_{2}$ : 179.1036, Found: 179.1043.

Benzyl (Z)-pent-2-enoate-2,3- $\boldsymbol{d}_{\mathbf{2}}\left(\mathbf{2 a -} \boldsymbol{d}_{\mathbf{2}}\right)$. IR (neat): 3034 (w), 2964 (w), 2934 (w), 1717 (s), 1618 (m), 1243 (s), 1141 (s), 1104 (m), 697 (w) cm ${ }^{-1} ;{ }^{1} \mathbf{H}$ NMR (500 MHz, CDCl 3 ): $\delta$ $7.38-7.32(\mathrm{~m}, 5 \mathrm{H}), 5.16(\mathrm{~s}, 2 \mathrm{H}), 2.67(\mathrm{q}, J=7.4 \mathrm{~Hz}, 2 \mathrm{H}), 1.05(\mathrm{t}, J=7.6 \mathrm{~Hz}, 3 \mathrm{H}) ;{ }^{13} \mathbf{C}$ NMR (125 MHz, $\left.\mathbf{C D C l}_{3}\right): \delta 166.3,152.5-152.2$ (m), 136.4, 128.7, 128.3, 128.3, 118.8-118.4 (m), 65.8, 22.5, 13.6; HRMS[M+H] ${ }^{+}$: Calcd for $\mathrm{C}_{12} \mathrm{H}_{13} \mathrm{D}_{2} \mathrm{O}_{2}$ : 193.1192, Found: 193.1209.

\subsection{Cross-Metathesis Between $(Z)-3-H e x e n e$ and Benzyl $(Z)$-but-2-enoate}

In a glove box, a pair of oven-dried NMR tubes were charged with a THF- $d_{8}$ solution $(200 \mu \mathrm{L}$, pre-made stock) of benzyl (Z)-but-2-enoate (17.6 mg, $0.100 \mathrm{mmol}, 1.00$ equiv.), (Z)-hex-3-ene (84.2 mg, $1.00 \mathrm{mmol}, 10.0$ equiv.), and anthracene (internal standard, $8.9 \mathrm{mg}, 0.050 \mathrm{mmol}, 0.500$ equiv.). One sample was diluted with THF- $d_{8}(400 \mu \mathrm{L})$ and the corresponding ${ }^{1} \mathrm{H}$ NMR spectrum

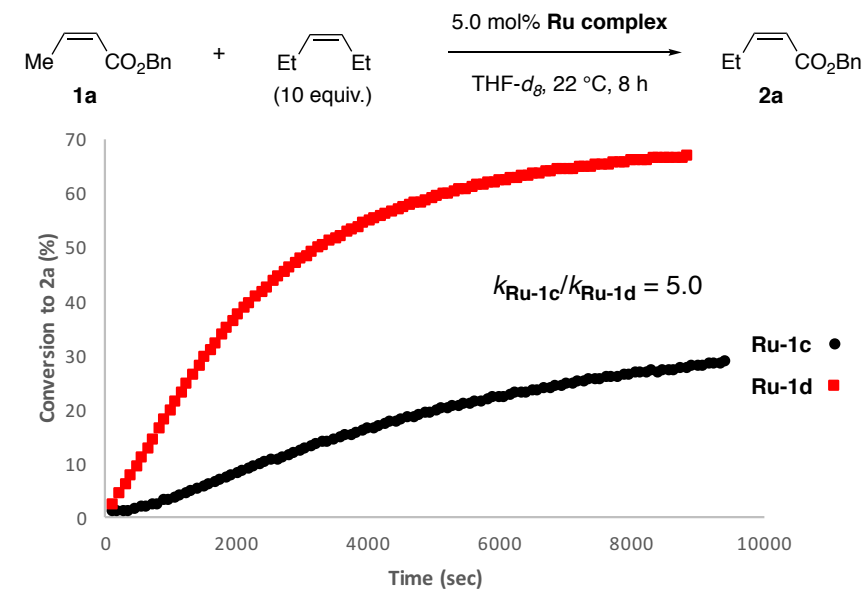

Fig. S5. Reaction profile for cross-metathesis between (Z)-hex-3-ene and benzyl (Z)-but-2-enoate. 
was recorded $\left(t_{0}\right)$. To the other sample was added a THF- $d_{8}$ solution (400 $\mu \mathrm{L}$, pre-made stock solution) of Ru-1c (3.7 mg, $0.005 \mathrm{mmol}, 5.0 \mathrm{~mol} \%$; through a syringe and with shaking). Reaction progress was monitored by ${ }^{1} \mathrm{H}$ NMR analysis for $8 \mathrm{~h}$ at $25{ }^{\circ} \mathrm{C}$. The same protocol was followed for a similar experiment with $\mathbf{R u - 1 d}$.

Analysis: A significant rate difference can be observed when cross-metathesis between benzyl (Z)-but-2-enoate and (Z)-hex-3-ene catalyzed by Ru-1c and Ru-1d is monitored by ${ }^{1} \mathrm{H}$ NMR over time (Fig. S5). The rate for cross metathesis to product is five times faster with Ru-1d as compared to $\mathbf{R u - 1 c}$.

\subsection{Analysis of Initiation Rates}

With benzyl $(Z)$-but-2-enoate: In a glovebox, an oven-dried vial equipped with a magnetic stir bar was charged with a THF solution $(100 \mu \mathrm{L}$, pre-made stock solution) of anthracene (internal standard, $0.891 \mathrm{mg}, 0.005 \mathrm{mmol}, 1.00$ equiv.) and a THF solution of Ru-1c (3.7 mg, $0.005 \mathrm{mmol}$, 1.00 equiv.; stock solution, $400 \mu \mathrm{L}$ ). The mixture was allowed to stir for five min at $22{ }^{\circ} \mathrm{C}$, after which an aliquot was removed and diluted with $\mathrm{CD}_{2} \mathrm{Cl}_{2}(600 \mu \mathrm{L})$ and the corresponding ${ }^{1} \mathrm{H}$ NMR spectrum was recorded $\left(t_{0}\right)$. A solution of benzyl $(Z)$-but-2-enoate $(17.6 \mathrm{mg}, 0.100 \mathrm{mmol}$, 20.0 equiv., $100 \mu \mathrm{L}$ THF) was added and the resulting solution was allowed to stir for $8 \mathrm{~h}$ at $22{ }^{\circ} \mathrm{C}$. Reaction progress was again analyzed as described above $\left({ }^{1} \mathrm{H} \mathrm{NMR} ; t_{\mathrm{end}}\right)$. The same protocol was used for the corresponding experiment with Ru-1d.

With $\left(Z\right.$ )-hex-3-ene: In a $\mathrm{N}_{2}$-filled glovebox, an oven-dried NMR tube equipped with a septum was charged with a thf- $d_{8}$ solution $(100 \mu \mathrm{L}$, pre-made stock solution) of anthracene (internal standard, $0.891 \mathrm{mg}, 0.005 \mathrm{mmol}, 1.00$ equiv.) and a THF- $d_{8}$ solution (400 $\mu \mathrm{L}$, pre-made stock solution) of Ru-1c (3.7 mg, $0.005 \mathrm{mmol}, 1.0$ equiv.). Then, the reaction vessel was sealed and taken out the glovebox, and a ${ }^{1} \mathrm{H}$ NMR spectrum of the sample was acquired $\left({ }^{1} \mathrm{H} \mathrm{NMR}\right.$ at $\left.\mathrm{t}_{0}\right)$. A THF- $d_{8}$ solution (100 $\mu \mathrm{L}$, pre-made stock solution) of (Z)-hex-3-ene (84.2 mg, $1.00 \mathrm{mmol}, 200$ equiv.) was added into the NMR tube through the septum via syringe and stirred manually. The reaction was then monitored by ${ }^{1} \mathrm{H}$ NMR for $8 \mathrm{~h}$ at $25^{\circ} \mathrm{C}$. The same procedure was employed for Ru-1d.

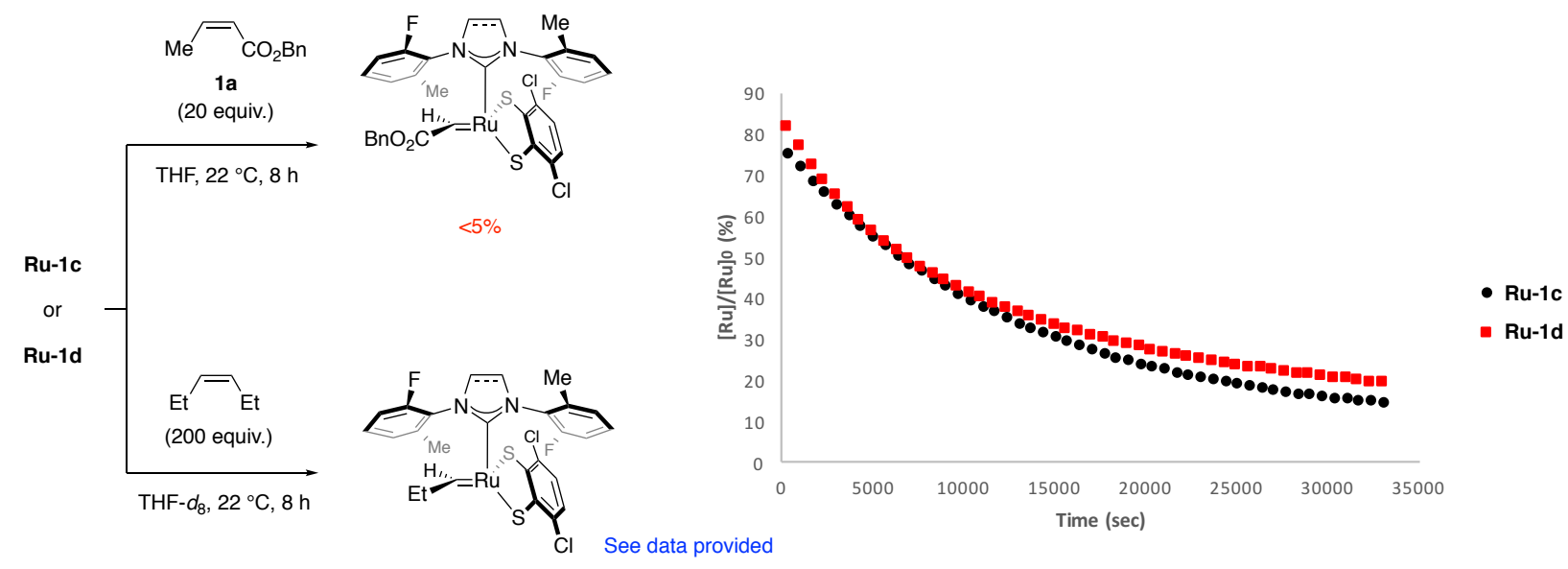

Fig. S6. Initiation studies with benzyl (Z)-but-2-enoate and (Z)-hex-3-ene. 
Analysis: Regardless of the complex used, whereas there is no initiation with electron-deficient benzyl (Z)-but-2-enoate, there is facile transformation with the more electron-rich ( $Z$ )-hex-3-ene. Furthermore, the kinetic profile indicates that reaction with $(Z)$-hex-3-ene occurs at a similar rate when $\mathbf{R u - 1 c}$ or $\mathbf{R u - 1 d}$ is used. These data reveal that a difference in the rate of catalyst initiation is not the reason for the observed difference in olefin metathesis efficiency (Fig. S6).

\subsection{Relative Rates of Homo-Metathesis and Cross-Metathesis Reactions}
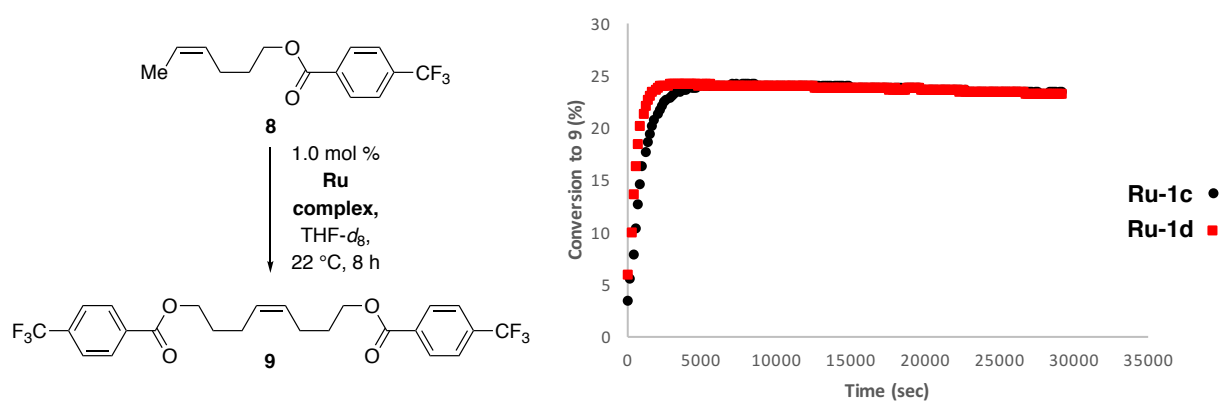

Fig. S7. Progress of a homo-metathesis reaction (by ${ }^{19} \mathrm{~F}$ NMR spectroscopy).

Analysis: Regardless of whether Ru-1c or Ru-1d is used, homo-metathesis of electron-rich substrate $\mathbf{8}$ proceeds at a similar rate.
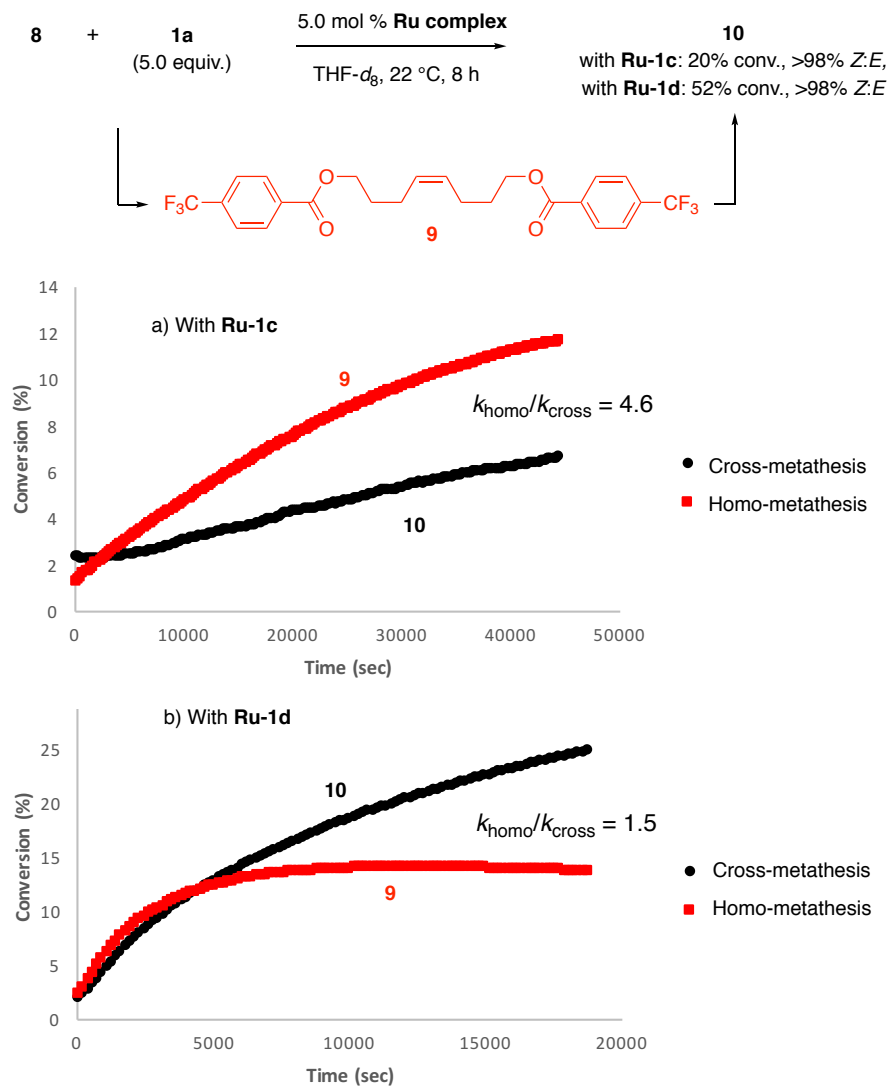

Fig. S8. Progress of a homo-metathesis vs. cross-metathesis (by ${ }^{19}$ F NMR spectroscopy). 
Analysis: For cross-metathesis between $\mathbf{8}$ and ester 1a, the rates for homo-metathesis (8 to 9 ) are faster than cross-metathesis ( 8 to $\mathbf{1 0}$ ) for both $\mathrm{Ru}$ complexes. However, the rate difference for Ru-1c $\left(k_{\text {homo }} / k_{\text {cross }}=4.6\right)$ is larger than Ru-1d $\left(k_{\text {homo }} / k_{\text {cross }}=1.5\right)$. Considering compound 1a is a more challenging coupling partner (more electron-deficient than 8), the greater chemo-selectivity for the more electron-rich olefin suggests that Ru-1c is less reactive than Ru-1d. Besides, the initial rates of both homo-metathesis and cross metathesis with $\mathbf{R u - 1 d}$ are faster than the rates with Ru-1c. This further verify that $\mathbf{R u - 1 c}$ is less reactive than $\mathbf{R u - 1 d}$, especially towards the metathesis with electron-deficient substrates.

(Z)-Hex-4-en-1-yl 4-(trifluoromethyl) benzoate (8). To a solution of 4-(trifluoromethyl) benzoic acid (570.4 mg, $3.0 \mathrm{mmol}, 1.0$ equiv.), EDCI (690.1 mg, $3.6 \mathrm{mmol}, 1.2$ equiv.), and DMAP (36.7 mg, $0.30 \mathrm{mmol}, 0.100$ equiv.) in $\mathrm{CH}_{2} \mathrm{Cl}_{2}$ (4.5 mL) was added (Z)-hex-4-en-1-ol (300.5 mg, $3.0 \mathrm{mmol}, 1.0$ equiv.). After $12 \mathrm{~h}$, the volatiles were removed in vacuo, and the resulting gray oil residue was purified by silica gel chromatography (5-15\% EtOAc in hexanes) to afford (Z)-hex-4-en-1-yl 4-(trifluoromethyl) benzoate (365.4 mg, $1.34 \mathrm{mmol}, 45 \%$ yield) in $>98: 2 Z$ : $E$ ratio as colorless oil. IR (neat): 2690 (w), 2033 (w), 1726 (s), 1326 (s), 1276 (s), 1131 (m), $863(\mathrm{w}), 704(\mathrm{w}) \mathrm{cm}^{-1} ;{ }^{\mathbf{1}} \mathbf{H}$ NMR (500 MHz, $\left.\mathbf{C D C l}_{3}\right): \delta 8.16(\mathrm{~d}, J=8.4 \mathrm{~Hz}, 2 \mathrm{H}), 7.71$ $(\mathrm{d}, J=8.2 \mathrm{~Hz}, 2 \mathrm{H}), 5.56-5.49(\mathrm{~m}, 1 \mathrm{H}), 5.44-5.39(\mathrm{~m}, 1 \mathrm{H}), 4.36(\mathrm{t}, J=6.6 \mathrm{~Hz}, 2 \mathrm{H}), 2.22(\mathrm{q}, J=$ $7.2 \mathrm{~Hz}, 2 \mathrm{H}), 1.86$ (p, $J=6.9 \mathrm{~Hz}, 2 \mathrm{H}), 1.61$ (d, $\left.J=6.7 \mathrm{~Hz}, 3 \mathrm{H}) ;{ }^{13} \mathbf{C ~ N M R ~ ( 1 2 5 ~ M H z , ~ C D C l} \mathbf{3}\right): \delta$ $165.5,134.5$ (q, $J=32.8 \mathrm{~Hz}), 130.1,129.0,125.5$ (q, $J=3.8 \mathrm{~Hz}), 125.3,123.8(\mathrm{q}, J=273.4 \mathrm{~Hz})$, 65.2, 28.6, 23.4, 12.9. ${ }^{19}$ F NMR (564 MHz, THF- $\left.d_{8}\right): \delta-64.1$ (s); HRMS[M+H] ${ }^{+}$: Calcd for $\mathrm{C}_{14} \mathrm{H}_{16} \mathrm{O}_{2} \mathrm{~F}_{3}: 273.1097$, Found: 273.1099.

(Z)-Oct-4-ene-1,8-diyl bis(4-(trifluoromethyl) benzoate (9). Colorless oil. IR (neat): 2958 (w), 1722 (s), 1324 (s), 1273 (s), 1125 (s), 1100 (s), 862 (w), 704 (w) cm ${ }^{-1}$; ${ }^{1}$ H NMR (500 MHz, $\left.\mathbf{C D C l}_{3}\right): \delta 8.13(\mathrm{~d}, J=8.2 \mathrm{~Hz}, 4 \mathrm{H}), 7.68(\mathrm{~d}, J=8.3 \mathrm{~Hz}, 4 \mathrm{H}), 5.48(\mathrm{~m}, 2 \mathrm{H}), 4.34(\mathrm{t}, J=6.6 \mathrm{~Hz}$, 4H), 2.22 (q, $J=7.1 \mathrm{~Hz}, 4 \mathrm{H}), 1.85$ (p, $J=6.8 \mathrm{~Hz}, 4 \mathrm{H}) ;{ }^{13} \mathbf{C}$ NMR (125 MHz, CDCl $\left.\mathbf{3}\right): \delta 165.5$, 134.5 (q, $J=32.3 \mathrm{~Hz}), 133.7,130.0,129.6,125.5$ (q, $J=3.8 \mathrm{~Hz}), 123.8$ (q, $J=273.0 \mathrm{~Hz}), 65.1$, 28.7, 23.8; ${ }^{19}$ F NMR (564 MHz, THF-d $)$ : $\delta-64.1$ (s); HRMS[M+H] ${ }^{+}$: Calcd for $\mathrm{C}_{24} \mathrm{H}_{23} \mathrm{O}_{4} \mathrm{~F}_{6}$ : 489.1495, Found: 489.1499.

(Z)-6-(Benzyloxy)-6-oxohex-4-en-1-yl 4-(trifluoromethyl) benzoate (10). Colorless oil. IR (neat): 2957 (w), 1718 (s), 1324 (s), 1273 (s), 1161 (s), 1120 (s), 863 (m), 698 (m) cm ${ }^{-1}$; ${ }^{1} \mathbf{H}$ NMR (500 MHz, CDCl$): ~ \delta 8.15(\mathrm{~d}, J=8.1 \mathrm{~Hz}, 2 \mathrm{H}), 7.70(\mathrm{~d}, J=8.1 \mathrm{~Hz}, 2 \mathrm{H}), 7.54-7.29(\mathrm{~m}$, $5 \mathrm{H}), 6.30(\mathrm{dt}, J=11.5,7.6 \mathrm{~Hz}, 1 \mathrm{H}), 5.90(\mathrm{dd}, J=11.5,1.8 \mathrm{~Hz}, 1 \mathrm{H}), 5.14(\mathrm{~s}, 2 \mathrm{H}), 4.38(\mathrm{t}, J=6.5$ $\mathrm{Hz}, 2 \mathrm{H}), 2.86(\mathrm{q}, J=7.4 \mathrm{~Hz}, 2 \mathrm{H}), 1.96(\mathrm{p}, J=7.1 \mathrm{~Hz}, 2 \mathrm{H}) ;{ }^{13} \mathbf{C}$ NMR (125 MHz, CDCl $\left.\mathbf{3}\right): \delta$ $166.0,165.5,149.2,136.2,134.5$ (q, $J=32.6 \mathrm{~Hz}), 133.7,130.1,128.7,128.3,128.3,125.5$ (q, $J$ $=3.7 \mathrm{~Hz}), 123.8(\mathrm{q}, \mathrm{J}=273.0 \mathrm{~Hz}), 120.7,66.0,65.0,28.2,25.8 ;{ }^{19} \mathbf{F}$ NMR (564 MHz, THF-d $)$ : $\delta$-64.1 (s); HRMS[M+H] $]^{+}$: Calcd for $\mathrm{C}_{21} \mathrm{H}_{20} \mathrm{O}_{4} \mathrm{~F}_{3}$ : 393.1308, Found: 393.1319 . 


\subsection{Control Experiments}

\subsubsection{Homo-Metathesis vs. Cross-Metathesis Reactions}
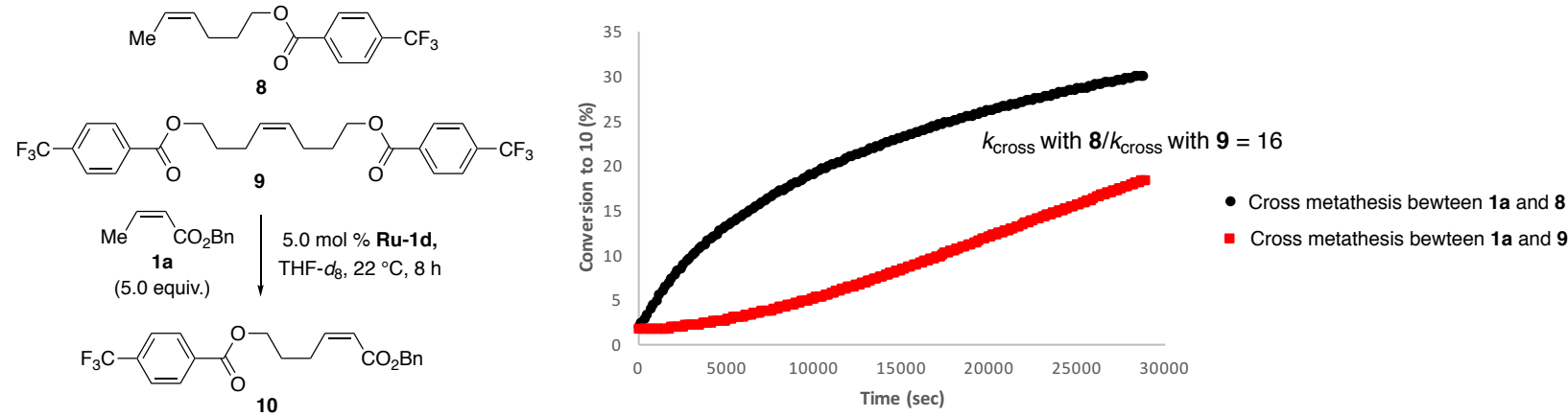

Fig. S9. Relatives rates of homo-metathesis and cross-metathesis (by ${ }^{19} \mathrm{~F}$ NMR spectroscopy).

Analysis: The data in Fig. S9 illustrate that cross-metathesis between benzyl (Z)-but-2-enoate (1a) is more efficient with enoate 8 than alkyl-substituted olefin 9 . The initial rate of the former reaction is 16 times faster than the cross-metathesis with $\mathbf{9}$, indicating that it is unlikely that $\mathbf{1 0}$ is generated by the reaction that involves Ru-1d and 9 at the early stages of the process (initial rates).

\subsubsection{Effect of A Lewis Base Additive on Homo-Metathesis of 8}
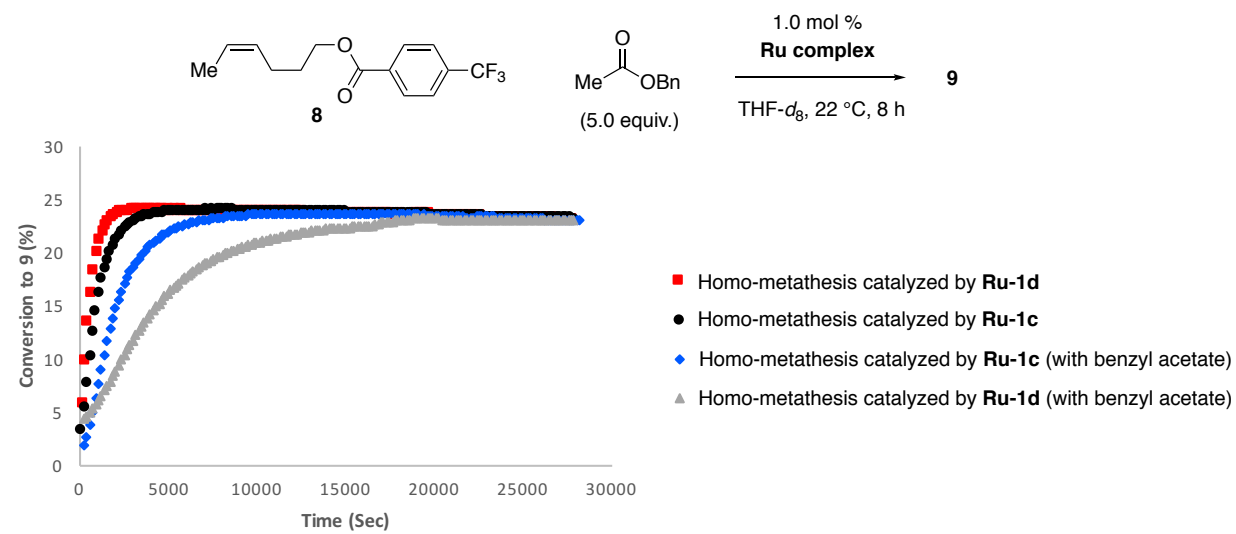

Fig. S10. Examination of the effect of a Lewis base on the rate of cross-metathesis (by ${ }^{19} \mathrm{~F}$ NMR spectroscopy).

Analysis: The presence of a carboxylic ester is detrimental to the rate of homo-metathesis, but more so when Ru-1d is used (vs. Ru-1c). This may be attributed to higher Lewis acidity of Ru1d.

\section{Method Development}

\subsection{Cross-Metathesis Procedures}

Condition A: In a $\mathrm{N}_{2}$-filled glove box, an oven-dried vial equipped with a magnetic stir bar was charged with a THF solution of the alkene substrate $(0.050 \mathrm{mmol})$ and the appropriate amount of Z-2-butene (used as received) and a THF solution of Ru-1d (1.0 mol \%; $0.50 \mathrm{M})$. The mixture was allowed to stir for $1 \mathrm{~h}$ at $22{ }^{\circ} \mathrm{C}$, after which the volatiles were removed in vacuo (100 torr, 2 
min). The flask containing the residue was then charged with the $\alpha, \beta$-unsaturated alkene, a THF solution of Ru-1d $(5.0 \mathrm{~mol} \%$; $0.25 \mathrm{M}$ ), the system was placed under 100 torr $\mathrm{h}$ vacuum for $1 \mathrm{~h}$, and the solution was allowed to stir for $19 \mathrm{~h}$ at $22{ }^{\circ} \mathrm{C}$. The reaction was quenched by the addition of undistilled $\mathrm{Et}_{2} \mathrm{O}$ and the volatiles were removed in vacuo. The resulting residue (typically black oil) was purified by silica gel chromatography to afford the desired product.

Condition B: In a $\mathrm{N}_{2}$-filled glove box, an oven-dried vial equipped with a magnetic stir bar was charged with a THF solution of the alkene substrate $(0.050 \mathrm{mmol})$ and the appropriate amount of $Z$-2-butene (used as received) and a THF solution of Ru-1d (1.0 mol \%; $0.50 \mathrm{M})$. The mixture was allowed to stir for $1 \mathrm{~h}$ at $22{ }^{\circ} \mathrm{C}$, after which the volatiles were removed in vacuo (100 torr, 2 $\mathrm{min})$. The flask containing the residue was charged with the $\alpha, \beta$-unsaturated alkene, a THF solution of Ru-1d (4.0 mol \%; $0.25 \mathrm{M}$ ), the system was placed under 100 torr vacuum for $1 \mathrm{~h}$, and the solution was allowed to stir for $8 \mathrm{~h}$ at $22{ }^{\circ} \mathrm{C}$. The flask containing the residue was again charged with a THF solution of Ru-1d (4.0 mol \%; $0.25 \mathrm{M})$, the system was placed under 100 torr vacuum for $1 \mathrm{~h}$, and the solution was allowed to stir for $12 \mathrm{~h}$ at $22{ }^{\circ} \mathrm{C}$. The reaction was quenched by the addition of undistilled $\mathrm{Et}_{2} \mathrm{O}$ and the volatiles were removed in vacuo. The resulting residue (typically black oil) was purified by silica gel chromatography to afford the desired product.

Condition C: In a glove box, an oven-dried vial equipped with a magnetic stir bar was charged with a THF solution of the alkene substrate $(0.050 \mathrm{mmol})$ and a THF solution of Ru-1d (4.0 mol \%; $0.25 \mathrm{M})$. The system was placed under 100 torr vacuum for $1 \mathrm{~h}$ and the solution was allowed to stir for $8 \mathrm{~h}$ at $22{ }^{\circ} \mathrm{C}$. The mixture was again charged with a THF solution of Ru-1d $(4.0 \mathrm{~mol} \% ; 0.25 \mathrm{M})$, and the system was placed under 100 torr vacuum for $1 \mathrm{~h}$, and the solution was allowed to stir for $12 \mathrm{~h}$ at $22{ }^{\circ} \mathrm{C}$. The reaction was quenched by the addition of undistilled $\mathrm{Et}_{2} \mathrm{O}$ and the volatiles were removed in vacuo. The resulting residue (typically black oil) was purified by silica gel chromatography.

Condition D: In a glove box, an oven-dried vial equipped with a magnetic stir bar was charged with a THF solution of the alkene substrate $(0.050 \mathrm{mmol})$ and the appropriate amount of Z-2butene (used as received) and a THF solution of Ru-1d $(2.0 \mathrm{~mol} \% ; 0.50 \mathrm{M})$. The mixture was allowed to stir for $1 \mathrm{~h}$ at $22{ }^{\circ} \mathrm{C}$, after which the volatiles were removed in vacuo (100 torr, $2 \mathrm{~min}$ ). The flask containing the residue was charged with the $\alpha, \beta$-unsaturated alkene, a THF solution of Ru-1d (4.0 mol \%; $0.25 \mathrm{M}$ ), the system was placed under 100 torr vacuum for $1 \mathrm{~h}$, and the solution was allowed to stir for $8 \mathrm{~h}$ at $22{ }^{\circ} \mathrm{C}$. The flask containing the residue was again charged with a THF solution of Ru-1d $(4.0 \mathrm{~mol} \%$; $0.25 \mathrm{M})$, the system was placed under 100 torr vacuum for $1 \mathrm{~h}$, and the solution was allowed to stir for $12 \mathrm{~h}$ at $22{ }^{\circ} \mathrm{C}$. The reaction was quenched by the addition of undistilled $\mathrm{Et}_{2} \mathrm{O}$ and the volatiles were removed in vacuo. The resulting residue (typically black oil) was purified by silica gel chromatography to afford the desired product. 


\section{$4.2(Z)-\alpha, \beta$-Unsaturated Esters}

Benzyl (Z)-9-bromonon-2-enoate (2b). Colorless oil. IR (neat): 2930 (m), 2855 (m), 1719 (s), 1642 (m), 1164 (s), 817 (m), 737 (m), 697 (m) cm ${ }^{-1}$; ${ }^{1}$ H NMR (400 MHz, CDCl $)$ ): $\delta$ 7.39-7.30 $(\mathrm{m}, 5 \mathrm{H}), 6.25(\mathrm{dt}, J=11.5,7.6 \mathrm{~Hz}, 1 \mathrm{H}), 5.83(\mathrm{dt}, J=11.5,1.7 \mathrm{~Hz}, 1 \mathrm{H}), 5.16(\mathrm{~s}, 2 \mathrm{H}), 3.39(\mathrm{t}, J=$ $6.9 \mathrm{~Hz}, 2 \mathrm{H}), 2.67$ (qd, $J=7.5,1.7 \mathrm{~Hz}, 2 \mathrm{H}), 1.85$ (p, $J=14.3,6.9 \mathrm{~Hz}, 2 \mathrm{H}), 1.51-1.31(\mathrm{~m}, 6 \mathrm{H})$; ${ }^{13}$ C NMR (100 MHz, $\left.\mathbf{C D C l}_{3}\right): \delta 166.3,151.1,136.3,128.7,128.4,128.3,119.7,76.8,65.9,34.0$, 32.9, 29.1, 28.9, 28.5, 28.1; HRMS[M+H] $]^{+}$: Calcd for $\mathrm{C}_{16} \mathrm{H}_{22} \mathrm{BrO}_{2}: 325.0804$, found: 325.0803 .

Benzyl (Z)-5-phenylpent-2-enoate (2c). Colorless oil. IR (neat): 3086 (w), 3063 (w), 3029 (m), 2926 (m), 1718 (s), 1643 (m), 1496 (m), 1454 (m), 1414 (m), 1213 (m), 1173 (s), 1157 (s), 1080 (m), 1003 (m), 735 (m), 697 (m); ${ }^{1} \mathbf{H}$ NMR (600 MHz, CDCl $): \delta 7.40-7.31$ (m, 5H), 7.27 (d, $J$ $=6.9 \mathrm{~Hz}, 2 \mathrm{H}), 7.19(\mathrm{t}, J=6.9 \mathrm{~Hz}, 3 \mathrm{H}), 6.28(\mathrm{dt}, J=11.5,7.5 \mathrm{~Hz}, 1 \mathrm{H}), 5.84(\mathrm{~d}, J=11.5 \mathrm{~Hz}, 1 \mathrm{H})$, 5.15 (s, 2H), $3.00(\mathrm{q}, J=7.6 \mathrm{~Hz}, 2 \mathrm{H}), 2.76(\mathrm{t}, J=7.7 \mathrm{~Hz}, 2 \mathrm{H}) ;{ }^{13} \mathbf{C}$ NMR (150 MHz, CDCl$): \delta$ $166.2,149.8,141.2,136.3,128.7,128.6,128.5,128.4,128.3,126.2,120.2,65.9,35.2,30.7$; HRMS $[\mathbf{M}+\mathbf{H}]^{+}$: Calcd for $\mathrm{C}_{18} \mathrm{H}_{19} \mathrm{O}_{2}: 267.1385$, found: 267.1384 .

Benzyl (Z)-7-(1,3-dioxoisoindolin-2-yl)hept-2-enoate (2d). Colorless oil. IR (neat): 2939 (w), 1704 (s), 1395 (m), 1163 (m), 719 (m) cm ${ }^{-1} ;{ }^{1} \mathbf{H}$ NMR (400 MHz, CDCl $)$ ): $\delta$ 7.87-7.80 (m, 2H), $7.74-7.68(\mathrm{~m}, 2 \mathrm{H}), 7.40-7.27(\mathrm{~m}, 5 \mathrm{H}), 6.22(\mathrm{dt}, J=11.5,7.6 \mathrm{~Hz}, 1 \mathrm{H}), 5.83(\mathrm{dt}, J=11.5,1.7 \mathrm{~Hz}$, $1 \mathrm{H}), 5.14(\mathrm{~s}, 2 \mathrm{H}), 3.69(\mathrm{t}, J=7.2 \mathrm{~Hz}, 2 \mathrm{H}), 2.72(\mathrm{qd}, J=7.5,1.7 \mathrm{~Hz}, 2 \mathrm{H}), 1.77-1.66(\mathrm{~m}, 2 \mathrm{H})$, 1.55-1.46 (m, 2H); ${ }^{13} \mathbf{C}$ NMR (100 MHz, $\left.\mathbf{C D C l}_{3}\right): \delta 168.5,166.2,150.3,136.3,134.0,132.3$, 128.7, 128.4, 128.3, 123.3, 120.1, 65.9, 37.9, 28.6, 28.4, 26.4; HRMS[M+H] $]^{+}$: Calcd for $\mathrm{C}_{22} \mathrm{H}_{22} \mathrm{O}_{4} \mathrm{~N}: 364.1549$, found: 364.1554 .

Benzyl (Z)-10-hydroxydec-2-enoate (2e). Colorless oil. IR (neat): 2927 (m), 1719 (s), 1642 (m), 1165 (s), 737 (m), 697 (m) cm ${ }^{-1}$; ${ }^{1} \mathbf{H}$ NMR (400 MHz, CDCl $)$ ): $\delta$ 7.40-7.29 (m, 5H), 6.25 $(\mathrm{dt}, J=11.5,7.6 \mathrm{~Hz}, 1 \mathrm{H}), 5.82(\mathrm{dt}, J=11.5,1.7 \mathrm{~Hz}, 1 \mathrm{H}), 5.16(\mathrm{~s}, 2 \mathrm{H}), 3.63(\mathrm{t}, J=6.6 \mathrm{~Hz}, 2 \mathrm{H})$, $2.66(\mathrm{qd}, J=7.5,1.7 \mathrm{~Hz}, 2 \mathrm{H}), 1.60-1.51(\mathrm{~m}, 2 \mathrm{H}), 1.49-1.40(\mathrm{~m}, 2 \mathrm{H}), 1.37-1.28(\mathrm{~m}, 6 \mathrm{H}) ;{ }^{13} \mathrm{C}$ NMR (100 MHz, $\left.\mathbf{C D C l}_{3}\right): \delta$ 166.3, 151.4, 136.3, 128.7, 128.3, 128.3, 119.5, 65.8, 63.1, 32.9, 29.3, 29.2, 29.2, 29.0, 25.7; HRMS[M+H] ${ }^{+}$: Calcd for $\mathrm{C}_{17} \mathrm{H}_{25} \mathrm{O}_{3}: 277.1812$, found: 277.1804 .

Benzyl (Z)-12-oxododec-2-enoate (2f). Colorless oil. IR (neat): 2925 (m), 2854 (m), 1720 (s), 1642 (m), 1163 (s), 737 (m), $687(\mathrm{~m}) \mathrm{cm}^{-1}$; ${ }^{1} \mathbf{H}$ NMR (400 MHz, CDCl $)$ ): $\delta 9.76$ (t, $J=1.9 \mathrm{~Hz}$, $1 \mathrm{H}), 7.40-7.29(\mathrm{~m}, 5 \mathrm{H}), 6.25$ (dt, $J=11.5,7.5 \mathrm{~Hz}, 1 \mathrm{H}), 5.82(\mathrm{dt}, J=11.5,1.7 \mathrm{~Hz}, 1 \mathrm{H}), 5.16$ (s, $2 \mathrm{H}), 2.66(\mathrm{qd}, J=7.5,1.7 \mathrm{~Hz}, 2 \mathrm{H}), 2.41(\mathrm{td}, J=7.4,1.9 \mathrm{~Hz}, 2 \mathrm{H}), 1.68-1.57(\mathrm{~m}, 2 \mathrm{H}), 1.48-1.38$ (m, 2H), 1.36-1.24 (m, 8H); ${ }^{13} \mathbf{C}$ NMR (100 MHz, $\left.\mathbf{C D C l}_{3}\right): \delta 203.0,166.3,151.4,136.3,128.7$, 128.3, 128.3, 119.5, 65.8, 44.0, 29.4, 29.3, 29.3, 29.3, 29.2, 29.1, 22.2; HRMS[M+H] ${ }^{+}$: Calcd for $\mathrm{C}_{19} \mathrm{H}_{27} \mathrm{O}_{3}$ : 303.196, found: 303.1972 .

(Z)-7-(benzyloxy)-7-oxohept-5-enoic acid (2g). Colorless oil. IR (neat): 1707 (s), 1415 (m), 1173 (m), 1154 (m), 738 (m), 697 (m) cm ${ }^{-1} ;{ }^{1} \mathbf{H}$ NMR (400 MHz, CDCl $)$ ): $\delta$ 7.41-7.29 (m, 5H), $6.23(\mathrm{dt}, J=11.5,7.6 \mathrm{~Hz}, 1 \mathrm{H}), 5.87$ (dt, $J=11.5,1.7 \mathrm{~Hz}, 1 \mathrm{H}), 5.15$ (s, 2H), 2.73 (qd, $J=7.5,1.7$ $\mathrm{Hz}, 2 \mathrm{H}), 2.39$ (t, $J=7.6 \mathrm{~Hz}, 2 \mathrm{H}), 1.80$ (p, $J=7.6 \mathrm{~Hz}, 2 \mathrm{H}) ;{ }^{13} \mathbf{C}$ NMR (100 MHz, CDCl$): ~ \delta$ 
178.0, 166.1, 149.4, 136.2, 128.7, 128.4, 128.4, 120.6, 66.0, 33.2, 28.4, 24.0; HRMS[M+H] ${ }^{+}$: Calcd for $\mathrm{C}_{14} \mathrm{H}_{17} \mathrm{O}_{4}: 249.1127$, found: 249.1127 .

Benzyl (2Z,4E)-5-phenylpenta-2,4-dienoate (2h). Colorless oil. IR (neat): 1709 (m), 1622 (m), 1164 (s), 999 (m), 959 (m), 755 (m), 696 (m) cm ${ }^{-1} ;{ }^{1}$ H NMR (400 MHz, CDCl $)$ ): $\delta 8.14$ (ddd, $J$ $=15.7,11.4,1.1 \mathrm{~Hz}, 1 \mathrm{H}), 7.51-7.27(\mathrm{~m}, 10 \mathrm{H}), 6.86-6.74(\mathrm{~m}, 2 \mathrm{H}), 5.78(\mathrm{dt}, J=11.2,0.9 \mathrm{~Hz}$, 1H), 5.22 (s, 2H); ${ }^{13} \mathbf{C}$ NMR (100 MHz, $\left.\mathbf{C D C l}_{3}\right): \delta$ 166.4, 145.4, 141.6, 136.4, 136.3, 129.2, $128.9,128.7,128.4,128.4,127.7,125.1,117.3,66.1 ; \mathbf{H R M S}[\mathbf{M}+\mathbf{H}]^{+}$: Calcd for $\mathrm{C}_{18} \mathrm{H}_{17} \mathrm{O}_{2}$ : 265.1229, found: 265.1220 .

Benzyl (Z)-4-(1H-indol-3-yl)but-2-enoate (2i). Colorless oil. IR (neat): 3408 (m), 3054 (m), 3031 (m), 2951 (w), 1708 (m), 1637 (m), 1454 (m), 1411 (m), 1367 (m), 1190 (m), 1159 (s), 1123 (m), 1091 (m), 815 (m), 739 (s), 696 (m) cm ${ }^{-1} ;{ }^{1}$ H NMR (400 MHz, CDCl $)$ ): $\delta 7.97$ (s, br, $1 \mathrm{H}), 7.59(\mathrm{~d}, \mathrm{~J}=7.9 \mathrm{~Hz}, 1 \mathrm{H}), 7.44-7.30(\mathrm{~m}, 6 \mathrm{H}), 7.21(\mathrm{t}, J=7.4 \mathrm{~Hz}, 1 \mathrm{H}), 7.11(\mathrm{t}, J=7.5 \mathrm{~Hz}$, $1 \mathrm{H}), 7.03(\mathrm{~d}, J=1.9 \mathrm{~Hz}, 1 \mathrm{H}), 6.50(\mathrm{dt}, J=11.4,7.4 \mathrm{~Hz}, 1 \mathrm{H}), 5.91(\mathrm{dt}, J=11.4,1.7 \mathrm{~Hz}, 1 \mathrm{H})$, 5.24 (s, 2H), $4.17(\mathrm{~d}, \mathrm{~J}=7.4 \mathrm{~Hz}, 2 \mathrm{H}) ;{ }^{13} \mathbf{C}$ NMR (100 MHz, $\left.\mathbf{C D C l}_{3}\right): \delta 166.4,149.5,136.5$, 136.3, 128.7, 128.4, 128.3, 127.4, 122.3, 121.9, 119.6, 119.2, 119.1, 113.9, 111.3, 66.0, 25.4; HRMS[M+H] $]^{+}$: Calcd for $\mathrm{C}_{19} \mathrm{H}_{18} \mathrm{NO}_{2}: 292.1332$, found:292.1336.

Benzyl (Z)-4-(3,4-dimethoxyphenyl)but-2-enoate (2j). Colorless oil. IR (neat): 1717 (m), 1514 (m), 1261 (m), 1236 (m), 1156 (s), 1029 (m) cm ${ }^{-1} ;{ }^{1} \mathbf{H}$ NMR (600 MHz, CDCl $): \delta 7.42-$ $7.31(\mathrm{~m}, 5 \mathrm{H}), 6.82-6.72(\mathrm{~m}, 3 \mathrm{H}), 6.38(\mathrm{dt}, J=11.4,7.6 \mathrm{~Hz}, 1 \mathrm{H}), 5.90(\mathrm{~d}, J=11.4 \mathrm{~Hz}, 1 \mathrm{H}), 5.21$ $(\mathrm{s}, 2 \mathrm{H}), 3.97(\mathrm{~d}, J=7.6 \mathrm{~Hz}, 2 \mathrm{H}), 3.85(\mathrm{~d}, J=11.9 \mathrm{~Hz}, 6 \mathrm{H}) ;{ }^{13} \mathbf{C}$ NMR (100 MHz, CDCl$): \delta$ $166.3,149.2$, 149.1, 147.8, 136.2, 132.0, 128.7, 128.4, 128.4, 120.6, 119.5, 112.0, 111.5, 66.1, 56.1, 56.0, 34.9; HRMS[M+H] $]^{+}$: Calcd for $\mathrm{C}_{19} \mathrm{H}_{21} \mathrm{O}_{4}: 313.1440$, found: 313.1434 .

Benzyl (Z)-4-(4-hydroxyphenyl)but-2-enoate (2k). Colorless oil. IR (neat): 3428 (br), 3066 (w), 3033 (w), 2956 (w), 1716 (m), 1694 (m), 1641 (m), 1512 (s), 1442 (m), 1412 (m), 1210 (s), 1190 (s), 1158 (s), 824 (m), 752 (m), 736 (m), $697(\mathrm{~m}) \mathrm{cm}^{-1} ;{ }^{1} \mathbf{H}$ NMR (400 MHz, CDCl $): \delta$ 7.44-7.31 (m, 5H), 7.14-7.03 (m, 2H), 6.88-6.71 (m, 2H), $6.36(\mathrm{dt}, J=11.4,7.6 \mathrm{~Hz}, 1 \mathrm{H}), 5.89$ $(\mathrm{dt}, J=11.4,1.8 \mathrm{~Hz}, 1 \mathrm{H}), 5.21(\mathrm{~s}, 2 \mathrm{H}), 4.79(\mathrm{~s}, \mathrm{br}, 1 \mathrm{H}), 3.96(\mathrm{dd}, J=7.6,1.7 \mathrm{~Hz}, 2 \mathrm{H}) ;{ }^{13} \mathbf{C} \mathbf{N M R}$ (150 MHz, $\mathbf{C D C l}_{3}$ ): $\delta 166.36,154.22,149.27,136.16,131.64,129.91,128.73,128.40,128.37$, 119.42, 115.59, 66.08, 34.46; HRMS[M+H] ${ }^{+}$: Calcd for $\mathrm{C}_{17} \mathrm{H}_{17} \mathrm{O}_{3}: 269.1178$, found: 269.1177 .

tert-Butyl (Z)-6-hydroxyhex-2-enoate (2l). Colorless oil. IR (neat): 2933 (m), 1713 (m), 1367 (m), 1219 (m), 1149 (s), 820 (m) cm ${ }^{-1}$; ${ }^{1} \mathbf{H}$ NMR (400 MHz, CDCl 3 ): $\delta 6.11$ (dt, $J=11.5,8.3 \mathrm{~Hz}$, $1 \mathrm{H}), 5.78(\mathrm{dt}, J=11.5,1.3 \mathrm{~Hz}, 1 \mathrm{H}), 3.61(\mathrm{q}, J=6.1 \mathrm{~Hz}, 2 \mathrm{H}), 2.75-2.66(\mathrm{~m}, 3 \mathrm{H}), 1.71(\mathrm{dt}, J=$ 12.3, 6.1 Hz, 2H), 1.48 (s, 9H); ${ }^{13} \mathbf{C}$ NMR (100 MHz, $\left.\mathbf{C D C l}_{3}\right): \delta 166.9,147.6,122.8,80.9,61.0$, 31.2, 28.3, 24.8; HRMS[M+H] $]^{+}$: Calcd for $\mathrm{C}_{10} \mathrm{H}_{19} \mathrm{O}_{3}:$ : 187.1334, found: 187.1332.

tert-Butyl (Z)-8-hydroxy-4,8-dimethylnon-2-enoate (2m). Colorless oil. IR (neat): 2970 (m), 2933 (m), 1715 (m), 1367 (m), 1153 (s), 824 (m) cm ${ }^{-1}$; ${ }^{1}$ H NMR (400 MHz, CDCl 3 ): $\delta 5.84$ (dd, $J=11.6,10.2 \mathrm{~Hz}, 1 \mathrm{H}), 5.62(\mathrm{dd}, J=11.6,0.9 \mathrm{~Hz}, 1 \mathrm{H}), 3.53-3.40(\mathrm{~m}, 1 \mathrm{H}), 1.48$ (s, 9H), 1.40$1.23(\mathrm{~m}, 5 \mathrm{H}), 1.19(\mathrm{~s}, 6 \mathrm{H}), 1.00(\mathrm{~d}, J=6.7 \mathrm{~Hz}, 3 \mathrm{H}) ;{ }^{13} \mathbf{C}$ NMR (100 MHz, CDCl $): \delta 166.2$, 
154.3, 120.5, 80.2, 71.1, 44.0, 37.5, 32.5, 29.5, 29.3, 28.4, 22.1, 20.6; HRMS[M+H] ${ }^{+}$: Calcd for $\mathrm{C}_{15} \mathrm{H}_{29} \mathrm{O}_{3}: 257.2117$, found: 257.2111.

Benzyl (Z)-6-hydroxyhex-2-enoate (2n). In a $\mathrm{N}_{2}$-filled glove box, an oven-dried vial equipped with a magnetic stir bar was charged with (Z)-hex-4-en-1-ol (5.0 mg, $0.050 \mathrm{mmol})$, benzyl (Z)but-2-enoate $(44.1 \mathrm{mg}, 0.25 \mathrm{mmol})$ and a thf solution of Ru-1d (1.9 mg, $0.0025 \mathrm{mmol})$, the system was placed under 100 torr vacuum for $1 \mathrm{~h}$, and then the solution was allowed to stir for 7 $\mathrm{h}$ at $22{ }^{\circ} \mathrm{C}$. The reaction was quenched by the addition of undistilled $\mathrm{Et}_{2} \mathrm{O}$ and the volatiles were removed in vacuo. The resulting residue (typically black oil) was purified by silica gel chromatography to afford $\mathbf{2 n}$ in $>98: 2 \mathrm{Z}: E$ ratio as colorless oil (5.7 $\mathrm{mg}, 0.026 \mathrm{mmol}, 52 \%$ yield). IR (neat): 3465 (br, m), 2937 (m), 2875 (m), 1718 (s), 1641 (m), 1454 (m), 1415 (m), 815 (m), $737(\mathrm{~m}), 697(\mathrm{~m}) \mathrm{cm}^{-1} ;{ }^{1} \mathbf{H}$ NMR (500 MHz, $\left.\mathbf{C D C l}_{3}\right): \delta$ 7.41-7.31 (m, 5H), 6.31-6.23 (m, 1H), $5.91(\mathrm{dd}, \mathrm{J}=11.5,1.4 \mathrm{~Hz}, 1 \mathrm{H}), 5.17(\mathrm{~s}, 2 \mathrm{H}), 3.62$ (s, 2H), 2.75 (q, J = 8.1, $7.5 \mathrm{~Hz}, 2 \mathrm{H}), 2.39$ (s, 1H), 1.73 (p, J = 5.9 Hz, 2H); ${ }^{13} \mathbf{C}$ NMR (150 MHz, $\left.\mathbf{C D C l}_{3}\right): \delta 167.0,145.0,138.1,128.9,128.1$, 127.8, 123.5, 60.1, 43.7, 30.6, 24.7; HRMS[M+H] ${ }^{+}$: Calcd for $\mathrm{C}_{13} \mathrm{H}_{17} \mathrm{O}_{3}$ : 221.1178, found: 221.1176 .

\section{$4.3(Z)-\alpha, \beta$-Unsaturated Acids}

(Z)-12-(Ferrenyloxy)dodec-2-enoic acid (3b). Colorless oil. IR (neat): 2922 (m), 2851 (m), 1707 (s), 1690 (s), 821 (m), 502 (w), 485 (w) cm ${ }^{-1} ;{ }^{1} \mathbf{H}$ NMR (600 MHz, CDCl $)$ ): $\delta$ carboxylic acid proton is invisible, 6.36-6.32 (m, $1 \mathrm{H}), 5.79(\mathrm{~d}, J=11.4 \mathrm{~Hz}, 1 \mathrm{H}), 5.00-4.62(\mathrm{~m}, 2 \mathrm{H})$, $4.51-4.26(\mathrm{~m}, 2 \mathrm{H}), 4.20(\mathrm{~m}, 5 \mathrm{H}), 2.66(\mathrm{q}, J=7.4 \mathrm{~Hz}, 2 \mathrm{H}), 1.72(\mathrm{p}, J=6.8 \mathrm{~Hz}, 2 \mathrm{H}), 1.45-1.35$ $(\mathrm{m}, 14 \mathrm{H}) ;{ }^{13} \mathbf{C}$ NMR (150 MHz, $\left.\mathbf{C D C l}_{3}\right): \delta$ 172.0, 170.7, 153.5, 119.0, 71.7, 71.3, 70.3, 69.9, 64.5, 29.6, 29.4, 29.4, 29.3, 29.3, 29.1, 29.0, 26.2; HRMS[M+H] $]^{+}$Calcd for $\mathrm{C}_{23} \mathrm{H}_{31} \mathrm{FeO}_{4}$ : 427.1567, found: 427.1565 .

(Z)-5-Phenylpent-2-enoic acid (3c). Colorless oil. IR (neat): 2925 (m), 1692 (s), 1638 (m), 1435 (m), 1240 (m), 698 (m) cm ${ }^{-1}$; ${ }^{1} \mathbf{H}$ NMR (600 MHz, CDCl $): \delta 11.39$ (br, 1H), $7.30(\mathrm{t}, J=$ $7.6 \mathrm{~Hz}, 2 \mathrm{H}), 7.24-7.18(\mathrm{~m}, 3 \mathrm{H}), 6.38(\mathrm{dt}, J=11.5,7.5 \mathrm{~Hz}, 1 \mathrm{H}), 5.82$ (d, $J=11.5 \mathrm{~Hz}, 1 \mathrm{H}), 3.03-$ $2.99(\mathrm{~m}, 2 \mathrm{H}), 2.79(\mathrm{t}, J=7.7 \mathrm{~Hz}, 2 \mathrm{H}) ;{ }^{13} \mathbf{C}$ NMR (150 MHz, $\left.\mathbf{C D C l}_{3}\right): \delta$ 171.4, 152.1, 141.1, 128.58, 128.59, 126.2, 119.7, 35.1, 30.8; HRMS[M+H] $]^{+}$: Calcd for $\mathrm{C}_{11} \mathrm{H}_{13} \mathrm{O}_{2}$ : 177.0916, found: 177.0909 .

(Z)-4-(1H-indol-3-yl)but-2-enoic acid (3d). Colorless oil. IR (neat): 3119 (w), 2982 9m), 2930 (w), 2906 (w), 1487 (w), 1391 (m),1369 (m), 1269 (m), 1152 (m), 1100 (m), 1027 (s), 963 (s), 882(m), $797(\mathrm{~m}), 740(\mathrm{~m}) \mathrm{cm}^{-1} ;{ }^{1} \mathbf{H}$ NMR (600 MHz, $\left.\mathbf{C D C l}_{3}\right): \delta 7.98(\mathrm{~s}, 1 \mathrm{H}), 7.61(\mathrm{~d}, J=7.8 \mathrm{~Hz}$, $1 \mathrm{H}), 7.37(\mathrm{~d}, J=8.1 \mathrm{~Hz}, 3 \mathrm{H}), 7.21(\mathrm{t}, \mathrm{J}=7.5 \mathrm{~Hz}, 1 \mathrm{H}), 7.13(\mathrm{t}, \mathrm{J}=7.5 \mathrm{~Hz}, 1 \mathrm{H}), 7.05(\mathrm{~s}, 1 \mathrm{H}), 6.60$ $(\mathrm{dt}, J=11.5,7.4 \mathrm{~Hz}, 1 \mathrm{H}), 5.90(\mathrm{~d}, J=11.4 \mathrm{~Hz}, 1 \mathrm{H}), 4.17(\mathrm{~d}, J=7.3 \mathrm{~Hz}, 2 \mathrm{H}) ;{ }^{13}$ C NMR (100 MHz, $\left.\mathbf{C D C l}_{3}\right): \delta 169.6,151.4,136.3,127.2,122.2,121.8,119.5,118.8,118.2,113.5,111.1$, 25.3; HRMS[M+H] $]^{+}$: Calcd for $\mathrm{C}_{12} \mathrm{H}_{12} \mathrm{O}_{2}: 202.0863$, found:202.0866.

(Z)-12-Oxododec-2-enoic acid (3e). Colorless oil. IR (neat): 3064 (m), 2923 (s), 2854 (m), $1734(\mathrm{w}), 1658$ (m), $1457(\mathrm{w}), 699(\mathrm{w}) \mathrm{cm}^{-1} ;{ }^{1} \mathbf{H}$ NMR (400 MHz, $\mathbf{C D C l}_{3}$ ): $\delta$ carboxylic acid 
proton is invisible, 9.77-9.76 (m, $1 \mathrm{H}), 6.35(\mathrm{dt}, J=11.4,7.5 \mathrm{~Hz}, 1 \mathrm{H}), 5.79(\mathrm{~d}, J=12.0 \mathrm{~Hz}, 1 \mathrm{H})$, 2.68-2.63 (m, 2H), 2.44-2.40 (m, 2H), 1.64-1.59 (m, 2H), 1.46-1.41 (m, 2H), 1.35-1.27 (m, 8H); ${ }^{13} \mathbf{C}$ NMR (100 MHz, $\left.\mathbf{C D C l}_{3}\right): \delta 203.1,170.9,153.5,118.9,44.0,29.9,29.4,29.28,29.27$, 29.2, 29.0, 22.2; HRMS[M+H] $]^{+}$: Calcd for $\mathrm{C}_{12} \mathrm{H}_{23} \mathrm{O}_{2}: 199.1698$, found: 199.1688.

(Z)-8-Hydroxy-4,8-dimethylnon-2-enoic acid (3f). Colorless oil. IR (neat): 2966 (m), 2930 (m), 1696 (s), 1640 (m), 1376 (m), 1227 (m), 830 (m) cm ${ }^{-1} ;{ }^{1}$ H NMR (500 MHz, CDCl $): \delta$ carboxylic acid proton is invisible, $6.12-6.02(\mathrm{~m}, 1 \mathrm{H}), 5.75(\mathrm{~d}, J=11.6 \mathrm{~Hz}, 1 \mathrm{H}), 3.53(\mathrm{br}, 1 \mathrm{H})$, 1.53-1.23 (m, 7H), 1.20 (s, 6H), $1.02(\mathrm{~d}, J=6.6 \mathrm{~Hz}, 3 \mathrm{H}) ;{ }^{13} \mathbf{C}$ NMR (125 MHz, CDCl $): \delta 170.8$, 158.6, 117.8, 71.2, 43.9, 37.5, 32.8, 29.5, 29.2, 22.1, 20.5; HRMS[M+H-H $\mathbf{H} \mathbf{O}]^{+}$: Calcd for $\mathrm{C}_{11} \mathrm{H}_{19} \mathrm{O}_{2}$ : 183.1385 , found: 183.1384 .

\subsection{Application to Stagonolide $E$}

\section{Scheme S3}

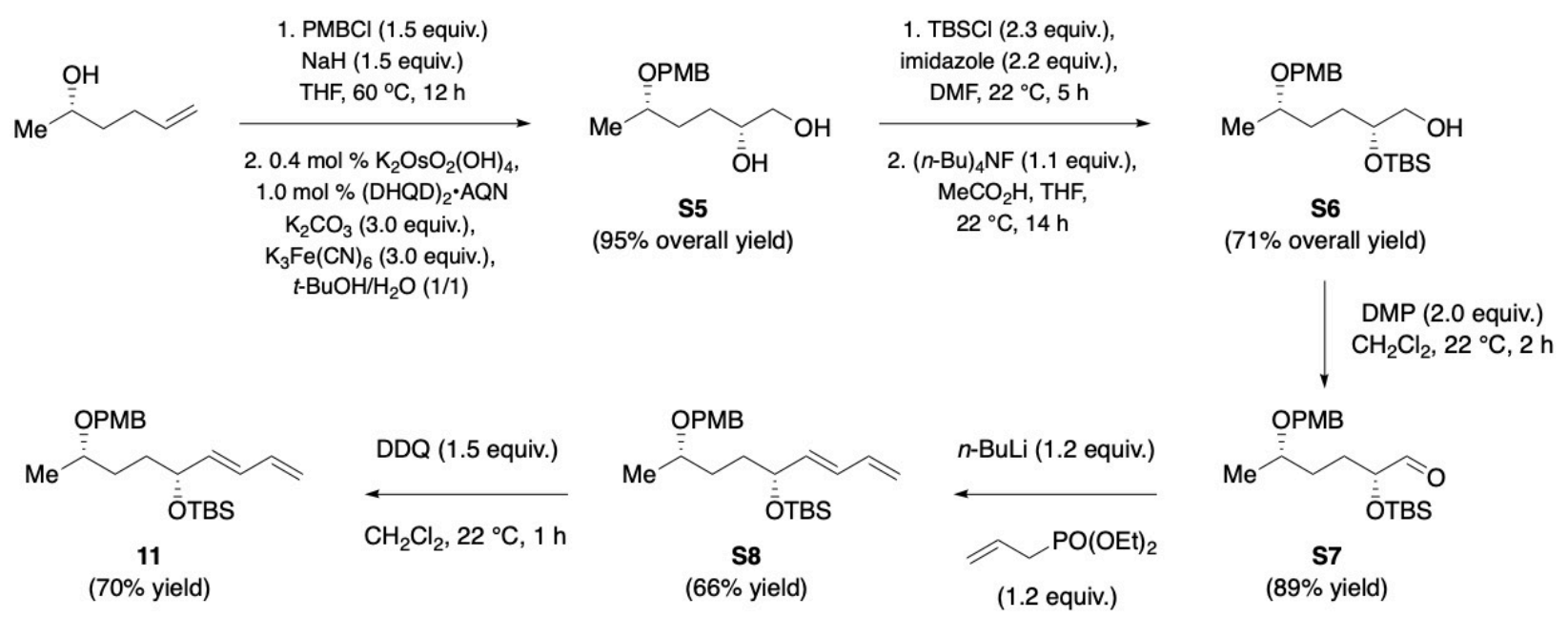

(2R,5S)-5-((4-Methoxybenzyl)oxy)hexane-1,2-diol (S5). Based on a reported procedure, ${ }^{7}$ a 50 $\mathrm{ml}$ round-bottom flask equipped with a stir bar was charged with $p$-methoxybenzyl chloride (234.9 mg, $1.5 \mathrm{mmol}, 1.5$ equiv.), $\mathrm{NaH}$ (60 mg, $1.5 \mathrm{mmol}, 1.5$ equiv.) and $10 \mathrm{~mL}$ THF. The mixture was allowed to stir for 0.5 at $22{ }^{\circ} \mathrm{C} \mathrm{h}$, after which $(S)$-hex-5-en-2-ol (100.2 mg, 1.0 mmol, 1.0 equiv.) was added to the mixture. The mixture was allowed to stir at $60{ }^{\circ} \mathrm{C}$ for $12 \mathrm{~h}$, after which excess ethanolamine $(5 \mathrm{~mL})$ was added. The mixture was allowed to stir for anther 5 $\mathrm{h}$, and then the mixture was charged with $\mathrm{Et}_{2} \mathrm{O} /$ hexane $(20 \mathrm{~mL} / 10 \mathrm{~mL})$ and a saturated solution of $\mathrm{NH}_{4} \mathrm{Cl}(20 \mathrm{~mL})$ were added. The organic layer was separated and the aqueous layer was washed with $\mathrm{Et}_{2} \mathrm{O}(20 \mathrm{~mL} \times 3)$. The combined organic layers were dried over $\mathrm{MgSO}_{4}$, and the volatiles were removed in vacuo. The resulting yellow oil was used without further purification. A $100 \mathrm{~mL}$ round-bottom flask was charged with (DHQD)AQN (8.6 mg, $0.010 \mathrm{mmol}, 1.0 \mathrm{~mol} \%$ ), potassium ferricyanide (990 mg, $3.0 \mathrm{mmol}, 3.0$ equiv.), potassium osmate $(1.4 \mathrm{mg}, 0.004 \mathrm{mmol}$, $0.4 \mathrm{~mol} \%$ ), potassium carbonate (420 mg, $3.0 \mathrm{mmol}, 3.0$ equiv.), and $t-\mathrm{BuOH} / \mathrm{H}_{2} \mathrm{O}(1 / 1,10 \mathrm{~mL})$. The mixture was allowed to cool to $0{ }^{\circ} \mathrm{C}$ and $(S)-1-(($ hex-5-en-2-yloxy)methyl)-4methoxybenzene (220.3 mg, $1.0 \mathrm{mmol}, 1.0$ equiv.) was added, and then allowed to stir for $12 \mathrm{~h}$ at 
$0{ }^{\circ} \mathrm{C}$. Sodium thiosulfate $(800 \mathrm{mg})$ was added slowly and the suspension was allowed to warm to $22{ }^{\circ} \mathrm{C}$ with vigorous stirring. Ethyl acetate $(50 \mathrm{~mL})$ was added and the organic layer was separated. The aqueous layer was washed with EtOAc $(40 \mathrm{~mL} \times 3)$. The combined organic layers were dried over $\mathrm{MgSO}_{4}$, and concentrated in vacuo. The resulting yellow oil was purified by silica gel chromatography (50-100\% EtOAc in hexanes) to afford S5 as colorless oil (241.5 mg, $0.95 \mathrm{mmol}, 95 \%$ yield) in 90:10 d.r. The characterization data are consistent with those previously reported. ${ }^{17}[\alpha]_{\mathbf{D}}{ }^{20}+33.8\left(\mathrm{c} 0.3, \mathrm{CHCl}_{3}\right) ;{ }^{1} \mathbf{H} \mathbf{~ N M R}\left(\mathbf{6 0 0} \mathbf{M H z}, \mathbf{C D C l}_{3}\right): \delta$ 7.31-7.21 $(\mathrm{m}, 2 \mathrm{H}), 6.88(\mathrm{~d}, J=8.6 \mathrm{~Hz}, 2 \mathrm{H}), 4.55(\mathrm{~d}, J=11.2 \mathrm{~Hz}, 1 \mathrm{H}), 4.36(\mathrm{~d}, J=11.2 \mathrm{~Hz}, 1 \mathrm{H}), 3.80$ (s, $3 \mathrm{H}), 3.69-3.51(\mathrm{~m}, 3 \mathrm{H}), 3.42(\mathrm{t}, J=11.3 \mathrm{~Hz}, 1 \mathrm{H}), 1.70-1.56(\mathrm{~m}, 3 \mathrm{H}), 1.54-1.47(\mathrm{~m}, 1 \mathrm{H}), 1.21(\mathrm{~d}$, $J=6.1 \mathrm{~Hz}, 3 \mathrm{H})$; HRMS[M+H] $]^{+}$: Calcd for $\mathrm{C}_{14} \mathrm{H}_{23} \mathrm{O}_{4}: 255.1591$, found: 255.1587 .

(2R,5S)-2-((tert-Butyldimethylsilyl)oxy)-5-((4-methoxybenzyl)oxy)hexan-1-ol (S6). A 100 $\mathrm{mL}$ round-bottom flask was charged with $(2 R, 5 S)-5-((4-m e t h o x y b e n z y l)$ oxy $)$ hexane-1,2-diol (456.8 $\mathrm{mg}, 1.8 \mathrm{mmol}, 1.0$ equiv.), and THF were added $(10 \mathrm{ml})$; this was followed by imidazole (157 mg, $4.1 \mathrm{mmol}, 2.3$ equiv.), and $\mathrm{TBSCl}$ (603 mg, $4.0 \mathrm{mmol}, 2.2$ equiv.). The resulting mixture was allowed to stir for $12 \mathrm{~h}$ at $22{ }^{\circ} \mathrm{C}$. After complete consumption of the starting material, acetic acid $(0.5 \mathrm{~mL})$ and tetrabutylammonium fluoride $(1.0 \mathrm{M}$ in THF, $2.3 \mathrm{~mL}, 2.3$ mmol, 2.3 equiv.) were added, and the resulting solution was allowed to stir for $14 \mathrm{~h}$ at $22{ }^{\circ} \mathrm{C}$. The reaction was quenched by the addition of a saturated solution of $\mathrm{NH}_{4} \mathrm{Cl}$ (dropwise). The mixture was washed with EtOAc $(50 \mathrm{~mL} \times 3)$, and the combined organic layers were dried over $\mathrm{MgSO}_{4}$ and the volatiles were removed in vacuo, leaving behind a red oil, which was purified by silica gel chromatography (5-10\% EtOAc in hexanes) to afford $\mathbf{S 6}$ as colorless oil (470 mg, 1.28 mmol, 71\% yield). $[\alpha]_{\mathbf{D}}{ }^{20}+3.7\left(c 0.43, \mathrm{CHCl}_{3}\right)$; IR (neat): $2952(\mathrm{~m}), 2928(\mathrm{~m}), 2856(\mathrm{~m}), 1613$ (m), 1513 (m), 1462 (m), 1373 (m), 1301 (m), 1246 (s), 1172 (m), 1036 (s), 957 (m), 833 (s), $774(\mathrm{~s}) \mathrm{cm}^{-1}$; ${ }^{1} \mathbf{H}$ NMR (400 MHz, $\left.\mathbf{C D C l}_{3}\right): \delta$ 7.29-7.22 (m, 2H), 6.90-6.84 (m, 2H), $4.50(\mathrm{~d}, J=$ $11.4 \mathrm{~Hz}, 1 \mathrm{H}), 4.37(\mathrm{~d}, J=11.3 \mathrm{~Hz}, 1 \mathrm{H}), 3.80(\mathrm{~s}, 3 \mathrm{H}), 3.76-3.66(\mathrm{~m}, 1 \mathrm{H}), 3.59-3.50(\mathrm{~m}, 1 \mathrm{H})$, $3.50-3.40(\mathrm{~m}, 2 \mathrm{H}), 1.86(\mathrm{t}, J=6.3 \mathrm{~Hz}, 1 \mathrm{H}), 1.70-1.37(\mathrm{~m}, 4 \mathrm{H}), 1.18(\mathrm{~d}, J=6.1 \mathrm{~Hz}, 3 \mathrm{H}), 0.90(\mathrm{~s}$, 9H), 0.07 (s, 6H); ${ }^{13} \mathbf{C}$ NMR (100 MHz, $\left.\mathbf{C D C l}_{3}\right): \delta$ 159.2, 131.2, 129.4, 113.9, 74.5, 72.9, 70.1, $66.3,55.4,32.2,29.9,26.0,19.8,18.3,-4.3,-4.4 ; \mathbf{H R M S}[\mathbf{M}+\mathbf{H}]^{+}$: Calcd for $\mathrm{C}_{20} \mathrm{H}_{37} \mathrm{O}_{4} \mathrm{Si}$ : 369.2456, found: 369.2446 .

(2R,5S)-2-((tert-Butyldimethylsilyl)oxy)-5-((4-methoxybenzyl)oxy)hexanal (S7). A $100 \mathrm{~mL}$ flask was charged with (2R,5S)-2-((tert-butyldimethylsilyl)oxy)-5-((4methoxybenzyl)oxy)hexan-1-ol (222.2 mg, $0.6 \mathrm{mmol}, 1.0$ equiv.), and $\mathrm{CH}_{2} \mathrm{Cl}_{2}$ (5.0 mL). DessMartin periodinane (509 mg, $1.2 \mathrm{mmol}, 2.0$ equiv.) was then added, and the resulting mixture was allowed to stir for $2 \mathrm{~h}$ at $22{ }^{\circ} \mathrm{C}$, after which it was added to an aqueous solution of $10 \%$ $\mathrm{Na}_{2} \mathrm{SO}_{3}$ and a saturated solution of aqueous $\mathrm{NaHCO}_{3}(10 \mathrm{~mL} / 10 \mathrm{~mL})$. The resulting biphasic mixture was allowed to stir until both layers became colorless. The two layers were separated and the aqueous layer was washed with EtOAc $(50 \mathrm{~mL} \times 3)$. The combined organic layers were washed with brine, dried over $\mathrm{Na}_{2} \mathrm{SO}_{4}$ and the volatiles were removed in vacuo. The yellow oil, was purified by silica gel chromatography (5-10\% EtOAc in hexanes) to afford $\mathbf{S 7}$ as colorless oil (196 mg, $0.534 \mathrm{mmol}, 89 \%$ yield). $[\alpha]_{\mathbf{D}}{ }^{\mathbf{2 0}}+9.0$ (c 0.28, $\left.\mathrm{CHCl}_{3}\right)$; IR (neat): 2954 (m), 2929 
(m), $2856(\mathrm{~m}), 1729(\mathrm{~m}), 1612(\mathrm{~m}), 1513(\mathrm{~m}), 1463(\mathrm{~m}), 1301(\mathrm{~m}), 1248(\mathrm{~s}), 1212(\mathrm{~m}), 1036(\mathrm{~m})$, 939 (w), 835 (s), 778 (m) cm ${ }^{-1}$; ${ }^{1}$ H NMR (400 MHz, CDCl $)$ ): $\delta 9.57$ (d, $J=1.6$ Hz, 1H), 7.25 (d, $J=7.1 \mathrm{~Hz}, 2 \mathrm{H}), 6.87(\mathrm{~d}, J=8.5 \mathrm{~Hz}, 2 \mathrm{H}), 4.49(\mathrm{~d}, J=11.4 \mathrm{~Hz}, 1 \mathrm{H}), 4.36(\mathrm{~d}, J=11.4 \mathrm{~Hz}, 1 \mathrm{H})$, $3.92(\mathrm{td}, J=5.9,5.2,3.2 \mathrm{~Hz}, 1 \mathrm{H}), 3.80(\mathrm{~s}, 3 \mathrm{H}), 3.50(\mathrm{dt}, J=11.4,5.3 \mathrm{~Hz}, 1 \mathrm{H}), 1.86-1.46(\mathrm{~m}$, $4 \mathrm{H}), 1.18(\mathrm{~d}, J=6.1 \mathrm{~Hz}, 3 \mathrm{H}), 0.92(\mathrm{~s}, 9 \mathrm{H}), 0.06(\mathrm{~d}, J=7.5 \mathrm{~Hz}, 6 \mathrm{H}) ;{ }^{13} \mathbf{C}$ NMR (100 MHz, $\left.\mathbf{C D C l}_{3}\right): \delta 204.2,159.2,131.1,129.4,113.9,77.6,73.7,70.0,55.4,31.4,28.5,25.9,19.6,18.3,-$ 4.5, -4.8; HRMS[M+H] $]^{+}$: Calcd for $\mathrm{C}_{20} \mathrm{H}_{35} \mathrm{O}_{4} \mathrm{Si}: 367.1950$, found: 367.1949 .

tert-Butyl(((5R,8S,E)-8-((4-methoxybenzyl)oxy)nona-1,3-dien-5-yl)oxy)dimethylsilane (S8). An 8-dram vial was charged with diethyl allylphosphonate (22 mg, $0.12 \mathrm{mmol}, 1.2$ equiv.) and THF $(1.0 \mathrm{~mL})$. The solution was allowed to cool to $-78{ }^{\circ} \mathrm{C}$, after which it was charged with $n$ BuLi (1.7 M in hexane, $53 \mu \mathrm{L}, 0.12 \mathrm{mmol}, 1.2$ equiv.). After $15 \mathrm{~min}$, a solution of $(2 R, 5 S)-2-((t$ butyldimethylsilyl)oxy)-5-((4-methoxybenzyl)oxy)hexanal (36 mg, $0.1 \mathrm{mmol}, 1.0$ equiv.) in HMPA (43 mg, $0.24 \mathrm{mmol}, 2.4$ equiv.), and THF (2.0 mL) were added. The mixture was allowed to stir for $2 \mathrm{~h}$ at $-78{ }^{\circ} \mathrm{C}$ and then stir for $12 \mathrm{~h}$ at $22{ }^{\circ} \mathrm{C}$. The reaction was quenched by addition of a saturated solution of $\mathrm{NH}_{4} \mathrm{Cl}(10 \mathrm{~mL})$. The organic layer was separated, and the aqueous layer was washed with $\mathrm{Et}_{2} \mathrm{O}(10 \mathrm{~mL} \times 3)$. The combined organic ether layers were washed with brine, dried over $\mathrm{Na}_{2} \mathrm{SO}_{4}$ and the volatiles were removed in vacuo. The resulting yellow oil was purified by silica gel chromatography (2-5\% Et $\mathrm{H}_{2} \mathrm{O}$ in hexanes) to afford $\mathbf{S 8}$ as colorless oil (26.9 mg, 0.066 mmol, 66\% yield). $[\alpha]_{\mathbf{D}}{ }^{20}-4.2$ (c 0.15, $\left.\mathrm{CHCl}_{3}\right)$; IR (neat): $2952(\mathrm{~m}), 2928(\mathrm{~m}), 2855$ (m), 1612 (m), 1512 (m), 1462 (m), 1372 (m), 1339 (m), 1246 (s), 1206 (m), 1067 (s), 1003 (m), 950 (m), 833 (s), 773 (s) cm ${ }^{-1}$; ${ }^{1}$ H NMR (400 MHz, $\left.\mathbf{C D C l}_{3}\right): \delta 7.28-7.22(\mathrm{~m}, 2 \mathrm{H}), 6.87(\mathrm{~d}, J=$ $8.5 \mathrm{~Hz}, 2 \mathrm{H}), 6.31$ (dt, $J=16.9,10.3 \mathrm{~Hz}, 1 \mathrm{H}), 6.11(\mathrm{dd}, J=15.2,10.5 \mathrm{~Hz}, 1 \mathrm{H}), 5.64$ (dd, $J=15.2$, $6.4 \mathrm{~Hz}, 1 \mathrm{H}), 5.16(\mathrm{~d}, J=16.9 \mathrm{~Hz}, 1 \mathrm{H}), 5.05(\mathrm{~d}, J=10.9 \mathrm{~Hz}, 1 \mathrm{H}), 4.48(\mathrm{~d}, J=11.4 \mathrm{~Hz}, 1 \mathrm{H}), 4.38$ $(\mathrm{d}, J=11.4 \mathrm{~Hz}, 1 \mathrm{H}), 4.11(\mathrm{q}, J=6.0 \mathrm{~Hz}, 1 \mathrm{H}), 3.80(\mathrm{~s}, 3 \mathrm{H}), 3.48$ (q, $J=5.9 \mathrm{~Hz}, 1 \mathrm{H}), 1.72-1.39$ $(\mathrm{m}, 4 \mathrm{H}), 1.17(\mathrm{~d}, J=6.1 \mathrm{~Hz}, 3 \mathrm{H}), 0.90(\mathrm{~s}, 9 \mathrm{H}), 0.03(\mathrm{~d}, J=7.7 \mathrm{~Hz}, 6 \mathrm{H}) ;{ }^{13} \mathbf{C}$ NMR (100 MHz, $\left.\mathbf{C D C l}_{3}\right): \delta 159.2,137.6,136.8,131.3,130.1,129.3,116.7,113.9,74.4,73.0,70.0,55.4,34.1$, 32.0, 26.1, 19.8, 18.4, -4.2, -4.7; HRMS[M+H] $]^{+}$: Calcd for $\mathrm{C}_{23} \mathrm{H}_{42} \mathrm{NO}_{3} \mathrm{Si}$ : 408.2929, found: 408.2937.

(2S,5R,E)-5-((tert-Butyldimethylsilyl)oxy)nona-6,8-dien-2-ol (11). An 8-dram vial was charged with $\mathrm{CH}_{2} \mathrm{Cl}_{2} / \mathrm{H}_{2} \mathrm{O} \quad(20 / 1, \quad 5.0 \mathrm{~mL})$ solution of tert-butyl $(((5 R, 8 S, E)-8-((4-$ methoxybenzyl)oxy)nona-1,3-dien-5-yl)oxy)dimethylsilane (170 mg, $0.44 \mathrm{mmol}, 1.0$ equiv.), and DDQ (148 mg, $0.65 \mathrm{mmol}, 1.5$ equiv.) was then added. The mixture was allowed to stir for $1 \mathrm{~h}$ at $22{ }^{\circ} \mathrm{C}$, after which the reaction was quenched by the addition of a saturated solutuion of $\mathrm{Na}_{2} \mathrm{SO}_{3}(50 \mathrm{~mL})$. The organic layer was separated, and the aqueous layer was washed with EtOAc $(50 \mathrm{~mL} \times 3)$. The organic layers were then combined, dried over $\mathrm{Na}_{2} \mathrm{SO}_{4}$, and concentrated under reduced pressure. The resulting black oil was purified by silica gel chromatography (5-10\% EtOAc in hexanes) to afford 11 as colorless oil (83 $\mathrm{mg}, 0.308 \mathrm{mmol}, 70 \%$

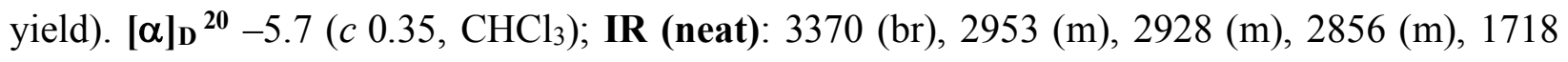
(w), 1471 (m), 1375 (m), 1251 (m), $1074(\mathrm{~m}), 1005$ (m), 833 (s), 774 (s), 669 (m) cm ${ }^{-1}$; ${ }^{1}$ H NMR (500 MHz, $\left.\mathbf{C D C l}_{3}\right): \delta 6.32(\mathrm{dt}, J=16.9,10.3 \mathrm{~Hz}, 1 \mathrm{H}), 6.14(\mathrm{dd}, J=15.7,10.6 \mathrm{~Hz}, 1 \mathrm{H}), 5.66$ (dd, 
$J=15.2,6.6 \mathrm{~Hz}, 1 \mathrm{H}), 5.17(\mathrm{~d}, J=17.2 \mathrm{~Hz}, 1 \mathrm{H}), 5.06(\mathrm{~d}, J=10.1 \mathrm{~Hz}, 1 \mathrm{H}), 4.20(\mathrm{q}, J=5.9 \mathrm{~Hz}$, $1 \mathrm{H}), 3.80(\mathrm{dt}, J=11.0,5.3 \mathrm{~Hz}, 1 \mathrm{H}), 1.70-1.56(\mathrm{~m}, 2 \mathrm{H}), 1.49(\mathrm{~m}, 2 \mathrm{H}), 1.18(\mathrm{~d}, J=6.2 \mathrm{~Hz}, 3 \mathrm{H})$, 0.90 (s, 9H), 0.05 (d, $J=11.7 \mathrm{~Hz}, 6 \mathrm{H}) ;{ }^{13} \mathbf{C}$ NMR (125 MHz, $\left.\mathbf{C D C l}_{3}\right): \delta$ 137.2, 136.7, 130.4, $116.9,73.1,68.1,34.7,34.3,26.0,23.6,18.4,-4.2,-4.6$; HRMS[M+H] $]^{+}$: Calcd for $\mathrm{C}_{15} \mathrm{H}_{31} \mathrm{O}_{2} \mathrm{Si}$ : 271.2088, found: 271.2079 .

(2Z,4E,6R,9S)-6-((tert-Butyldimethylsilyl)oxy)-9-hydroxydeca-2,4-dienoic acid (3g). In a glovebox, an oven-dried vial equipped with a magnetic stir bar was sequentially charged with (2S,5R,E)-5-((t-butyldimethylsilyl)oxy)nona-6,8-dien-2-ol (13.5 mg, $0.05 \mathrm{mmol}), Z$-butene (THF solution, 12 wt \%, $115 \mathrm{mg}, 0.25 \mathrm{mmol})$, and Ru-1d (0.8 mg, $0.001 \mathrm{mmol}$, in $200 \mu \mathrm{L}$ THF). The mixture was allowed to stir for $1 \mathrm{~h}$ at $22{ }^{\circ} \mathrm{C}$, after which the volatiles were removed in vacuo (100 torr, $2 \mathrm{~min}$ ). The vessel was then charged with (in this precise order) (Z)-but-2-enoic acid (21.5 mg, $0.25 \mathrm{mmol}$ ), a solution of Ru-1d (1.5 mg, $0.002 \mathrm{mmol}$ in $200 \mu \mathrm{L}$ THF), and subjected to 100 torr vacuum for $1 \mathrm{~h}$. The resulting solution was allowed to stir for $8 \mathrm{~h}$ at $22{ }^{\circ} \mathrm{C}$. The mixture was then charged with a solution of Ru-1d (1.5 mg, $0.002 \mathrm{mmol}$ in $200 \mu \mathrm{L} \mathrm{THF})$ and subjected to 100 torr vacuum for $30 \mathrm{~min}$, and allowed to stir for $12 \mathrm{~h}$ at $22{ }^{\circ} \mathrm{C}$. At this point, the reaction was quenched by the addition of undistilled $\mathrm{Et}_{2} \mathrm{O}$ while the mixture was exposed to air, and the volatiles were removed in vacuo. The resulting black oil was purified by silica gel chromatography (10\% to $50 \%$ EtOAc in hexanes) and filtered through a small plug of activated charcoal to afford product in $>98: 2 \mathrm{Z}: E$ ratio as colorless oil $(8.3 \mathrm{mg}, 0.0265 \mathrm{mmol}, 53 \%$ yield $)$. The characterization data are consistent with those previously reported. ${ }^{18}{ }^{1} \mathbf{H}$ NMR (600 MHz, $\left.\mathbf{C D C l}_{3}\right): \delta 7.46(\mathrm{dd}, J=15.2,11.6 \mathrm{~Hz}, 1 \mathrm{H}), 6.66(\mathrm{t}, J=11.4 \mathrm{~Hz}, 1 \mathrm{H}), 6.05(\mathrm{dd}, J=15.3,5.9 \mathrm{~Hz}$, $1 \mathrm{H}), 5.67(\mathrm{~d}, J=11.3 \mathrm{~Hz}, 1 \mathrm{H}), 4.33(\mathrm{q}, J=5.7 \mathrm{~Hz}, 1 \mathrm{H}), 3.80(\mathrm{dt}, J=12.2,6.0 \mathrm{~Hz}, 1 \mathrm{H}), 1.76-$ $1.57(\mathrm{~m}, 3 \mathrm{H}), 1.51(\mathrm{dt}, J=19.6,9.5 \mathrm{~Hz}, 2 \mathrm{H}), 1.19(\mathrm{~d}, J=6.2 \mathrm{~Hz}, 3 \mathrm{H}), 0.91(\mathrm{~s}, 9 \mathrm{H}), 0.07(\mathrm{~s}, 3 \mathrm{H})$, 0.05 (s, 3H); HRMS[M+H] $]^{+}$: Calcd for $\mathrm{C}_{16} \mathrm{H}_{31} \mathrm{O}_{4} \mathrm{Si}$ : 315.1986 , found: 315.1999 .

\section{$4.5(Z)-\alpha, \beta$-Unsaturated Weinreb Amides}

(Z)- $\mathrm{N}$-Methoxy- $\mathrm{N}$-methyl-5-phenylpent-2-enamide (5b). Colorless oil. IR (neat): 3026 (brs), 1655 (s), 1394 (m), 1347 (m), 1178 (w), 1000 (m), 794 (m), 637 (m) cm ${ }^{-1} ;{ }^{1}$ H NMR (500 MHz, $\left.\mathbf{C D C l}_{3}\right): \delta 8.41-6.81(\mathrm{~m}, 5 \mathrm{H}), 6.25(\mathrm{~d}, J=10.7 \mathrm{~Hz}, 1 \mathrm{H}), 6.13(\mathrm{dt}, J=11.7,7.3 \mathrm{~Hz}, 1 \mathrm{H}), 3.61(\mathrm{~s}$, $3 \mathrm{H}), 3.20(\mathrm{~s}, 3 \mathrm{H}), 2.97(\mathrm{q}, J=6.9 \mathrm{~Hz}, 2 \mathrm{H}), 2.78(\mathrm{t}, J=7.6 \mathrm{~Hz}, 2 \mathrm{H}) .{ }^{13} \mathbf{C}$ NMR (125 MHz, $\left.\mathbf{C D C l}_{3}\right): \delta$ 167.6, 146.2, 141.6, 128.7, 128.4, 126.0, 118.9, 61.6, 35.4, 32.2, 30.6. HRMS $[\mathbf{M}+\mathbf{H}]^{+}$: Calcd for $\mathrm{C}_{13} \mathrm{H}_{18} \mathrm{NO}_{2}: 220.1338$, found: 220.1339 .

(Z)- $\mathrm{N}$-Methoxy- $\mathrm{N}$-methyldodec-2-enamide (5c). Colorless oil. IR (neat): 2855 (m), 1631 (s), 1513 (s), 1394 (m), 998 (m), 727 (m) cm ${ }^{-1} ;{ }^{1} \mathbf{H}$ NMR (600 MHz, CDCl 3 ,) $\delta 6.22$ (d, J=11.4 Hz, $1 \mathrm{H}), 6.11(\mathrm{dt}, J=11.6,7.3 \mathrm{~Hz}, 1 \mathrm{H}), 3.67(\mathrm{~s}, 3 \mathrm{H}), 3.21(\mathrm{~s}, 3 \mathrm{H}), 2.61(\mathrm{q}, J=7.1 \mathrm{~Hz}, 2 \mathrm{H}), 1.43$ (p, $J=7.4 \mathrm{~Hz}, 2 \mathrm{H}), 1.34-1.25(\mathrm{~m}, 12 \mathrm{H}), 0.87(\mathrm{t}, J=7.0 \mathrm{~Hz}, 3 \mathrm{H}) .{ }^{13} \mathbf{C}$ NMR (150 MHz, CDCl $\left.\mathbf{3}\right) \delta$ 168.1, 147.9, 118.1, 61.6, 32.1, 29.9, 29.7, 29.6, 29.53, 29.49, 29.46, 29.3, 22.8, 14.3. HRMS[M] ${ }^{+}$: Calcd for $\mathrm{C}_{14} \mathrm{H}_{28} \mathrm{NO}_{2}: 242.2120$, found: 242.2118 .

(Z)- $\mathrm{N}$-Methoxy- $\mathrm{N}$-methyl-12-oxododec-2-enamide (5d). Colorless oil. IR (neat): 2924 (s), 2854 (m), 1723 (m), 1656 (s), 997 (m), 433 (w) cm ${ }^{-1} ;{ }^{1} \mathbf{H}$ NMR (600 MHz, CDCl $)$ ): $\delta 9.76$ (s, 
$1 \mathrm{H}), 6.23(\mathrm{~d}, J=10.3 \mathrm{~Hz}, 1 \mathrm{H}), 6.11(\mathrm{dt}, J=11.7,7.4 \mathrm{~Hz}, 1 \mathrm{H}), 3.67(\mathrm{~s}, 3 \mathrm{H}), 3.21(\mathrm{~s}, 3 \mathrm{H}), 2.61(\mathrm{~d}$, $J=7.2 \mathrm{~Hz}, 2 \mathrm{H}), 2.41(\mathrm{t}, J=6.8 \mathrm{~Hz}, 2 \mathrm{H}), 1.62(\mathrm{p}, J=7.7 \mathrm{~Hz}, 2 \mathrm{H}), 1.43(\mathrm{p}, J=7.3 \mathrm{~Hz}, 2 \mathrm{H})$, 1.34-1.29 (m, 8H); ${ }^{13} \mathbf{C}$ NMR (150 MHz, $\left.\mathbf{C D C l}_{3}\right): \delta 203.1,167.8,147.8,118.4,61.6,44.1,29.9$, 29.4, 29.4, 29.4, 29.3, 29.2, 22.2, 15.4; HRMS[M] ${ }^{+}$: Calcd for $\mathrm{C}_{14} \mathrm{H}_{26} \mathrm{NO}_{3}$ : 256.1913, found: 256.1926 .

(Z)-12-(Methoxy(methyl)amino)-12-oxododec-10-enoic acid (5e). Colorless oil. IR (neat): 2924 (s), 2854 (m), 1733 (m), 1708 (s), 1656 (m), 1179 (m), 999 (m), 724 (m) cm ${ }^{-1}$; ${ }^{1}$ H NMR $\left(\mathbf{5 0 0} \mathbf{~ M H z}, \mathbf{C D C l}_{3}\right) \delta$ carboxylic acid proton is too broad to be visible, $6.23(\mathrm{~d}, J=9.2 \mathrm{~Hz}, 1 \mathrm{H})$, $6.11(\mathrm{dt}, J=11.6,7.3 \mathrm{~Hz}, 1 \mathrm{H}), 3.68(\mathrm{~s}, 3 \mathrm{H}), 3.21(\mathrm{~s}, 3 \mathrm{H}), 2.60(\mathrm{q}, J=7.5 \mathrm{~Hz}, 2 \mathrm{H}), 2.33(\mathrm{t}, J=$ $7.4 \mathrm{~Hz}, 2 \mathrm{H}), 1.66-1.60$ (m, 2H), 1.46-1.40 (m, 2H), 1.34-1.31 (m, 8H). ${ }^{13} \mathrm{C}$ NMR (125 MHz, $\left.\mathbf{C D C l}_{3}\right) \delta 178.8,167.8,147.9,118.1,61.6,34.0,32.2,29.9,29.3,29.3,29.2,29.3,29.1,24.8$; HRMS[M] ${ }^{+}$: Calcd for $\mathrm{C}_{14} \mathrm{H}_{26} \mathrm{NO}_{4}: 272.1862$, found: 272.1857 .

(Z)-7-(1,3-Dioxoisoindolin-2-yl)- $N$-methoxy- $N$-methylhept-2-enamide (5f). Colorless oil. IR (neat): 2925 (brs), 2854 (w), 1708 (s), 1655 (m), 1395 (m), 794 (w), 720 (w) cm ${ }^{-1}$; ${ }^{1}$ H NMR (600 MHz, CDCl $\mathbf{l}_{3} \delta$ 7.85-7.82 (m, 2H), 7.71-7.69 (m, 2H), $6.24(\mathrm{~d}, J=10.6 \mathrm{~Hz}, 1 \mathrm{H}), 6.08(\mathrm{dt}$, $J=11.7,7.4 \mathrm{~Hz}, 1 \mathrm{H}), 3.70(\mathrm{t}, J=7.2 \mathrm{~Hz}, 2 \mathrm{H}), 3.67(\mathrm{~s}, 3 \mathrm{H}), 3.20(\mathrm{~s}, 3 \mathrm{H}), 2.68(\mathrm{q}, J=7.1 \mathrm{~Hz}, 2 \mathrm{H})$, $1.73(\mathrm{p}, J=7.5 \mathrm{~Hz}, 2 \mathrm{H}), 1.51(\mathrm{p}, J=7.5 \mathrm{~Hz}, 2 \mathrm{H}) .{ }^{13} \mathbf{C}$ NMR (150 MHz, $\left.\mathbf{C D C l}_{3}\right) \delta 168.5,146.8$, $134.0,132.3,125.7,123.3,118.8,61.7,38.1,32.1,28.6,28.4,26.7 . \mathbf{H R M S}[\mathbf{M}+\mathbf{H}]^{+}$: Calcd for $\mathrm{C}_{17} \mathrm{H}_{21} \mathrm{~N}_{2} \mathrm{O}_{4}$ : 317.1501, found: 317.1503 .

(Z)-4-(3,4-Dimethoxyphenyl)- $\boldsymbol{N}$-methoxy- $\boldsymbol{N}$-methylbut-2-enamide (5g). Colorless oil. IR (neat): 2966 (w), 1617 (s), 1430 (m), 1347 (m), 961 (m), 696 (m) cm ${ }^{-1}$; ${ }^{1}$ H NMR (600 MHz, $\left.\mathbf{C D C l}_{3}\right) \delta 6.81-6.80(\mathrm{~m}, 3 \mathrm{H}), 6.31(\mathrm{~d}, J=10.0 \mathrm{~Hz}, 1 \mathrm{H}), 6.29-6.15(\mathrm{~m}, 1 \mathrm{H}), 3.93(\mathrm{~d}, J=6.8 \mathrm{~Hz}$, 2H), $3.86(\mathrm{~d}, J=7.4 \mathrm{~Hz}, 6 \mathrm{H}), 3.70(\mathrm{~s}, 3 \mathrm{H}), 3.25$ (s, 3H). ${ }^{13} \mathbf{C}$ NMR (150 MHz, CDCl $) \delta 167.5$, 149.1 147.6, 145.7, 132.8, 120.6, 118.2, 112.2, 111.5, 61.7, 56.1, 56.0, 35.0, 32.2. HRMS[M] ${ }^{+}$: Calcd for $\mathrm{C}_{14} \mathrm{H}_{24} \mathrm{NO}_{4}: 266.1393$, found: 266.1402 .

(Z)-8-Hydroxy- $\boldsymbol{N}$-methoxy- $\boldsymbol{N}$-4,8-trimethylnon-2-enamide (5h). Colorless oil. IR (neat): 3440 (brs), 2965 (m), 2934 (m), 1652 (s), 1629 (m), 999 (m), 806 (m) cm ${ }^{-1}$; ${ }^{1}$ H NMR (600 MHz, $\left.\mathbf{C D C l}_{3}\right): \delta 6.19(\mathrm{~d}, J=9.8 \mathrm{~Hz}, 1 \mathrm{H}), 5.84(\mathrm{t}, J=10.9 \mathrm{~Hz}, 1 \mathrm{H}), 3.68$ (s, 3H), 3.46 (brs, 1H), 3.21 (s, $3 \mathrm{H}), 1.63-1.32(\mathrm{~m}, 7 \mathrm{H}), 1.19(\mathrm{~s}, 3 \mathrm{H}), 1.17(\mathrm{~s}, 3 \mathrm{H}), 1.00(\mathrm{~d}, J=6.6 \mathrm{~Hz}, 3 \mathrm{H}) ;{ }^{13} \mathbf{C}$ NMR (150 MHz, $\left.\mathbf{C D C l}_{3}\right): \delta 167.8,153.1,117.2,70.9,61.6,43.7,37.4,32.5,32.2,30.0,28.9,21.9,20.8$; HRMS $[\mathbf{M}+\mathbf{H}]^{+}$: Calcd for $\mathrm{C}_{13} \mathrm{H}_{26} \mathrm{NO}_{3}: 244.1913$, found: 244.1902 .

(2Z,4E)- $N$-Methoxy- $N$-methyl-5-phenylpenta-2,4-dienamide (5i). Colorless oil. IR (neat): 3060 (brs), 2934 (w), 1643 (s), 1617 (m), 1295 (m), 696 (m), 655 (w) cm ${ }^{-1}$; ${ }^{1}$ H NMR (500 MHz, $\left.\mathbf{C D C l}_{3}\right) \delta 8.20(\mathrm{dd}, J=15.5,11.5 \mathrm{~Hz}, 1 \mathrm{H}), 7.50(\mathrm{~d}, J=8.1 \mathrm{~Hz}, 2 \mathrm{H}), 7.40-7.13(\mathrm{~m}, 3 \mathrm{H}), 6.76(\mathrm{~d}$, $J=15.8 \mathrm{~Hz}, 1 \mathrm{H}), 6.68(\mathrm{t}, J=11.3 \mathrm{~Hz}, 1 \mathrm{H}), 6.23(\mathrm{~d}, J=11.3 \mathrm{~Hz}, 1 \mathrm{H}), 3.70(\mathrm{~s}, 3 \mathrm{H}), 3.25(\mathrm{~s}, 3 \mathrm{H})$; ${ }^{13}$ C NMR (125 MHz, $\left.\mathbf{C D C l}_{3}\right) \delta 167.5,143.1,140.4,136.7,128.78,128.75,127.6,125.7,115.7$, 61.8, 32.4; HRMS[M+H] ${ }^{+}$: Calcd for $\mathrm{C}_{13} \mathrm{H}_{16} \mathrm{NO}_{2}$ : 218.1181, found: 218.1190 . 


\subsection{Application to Dihydrocompactin}

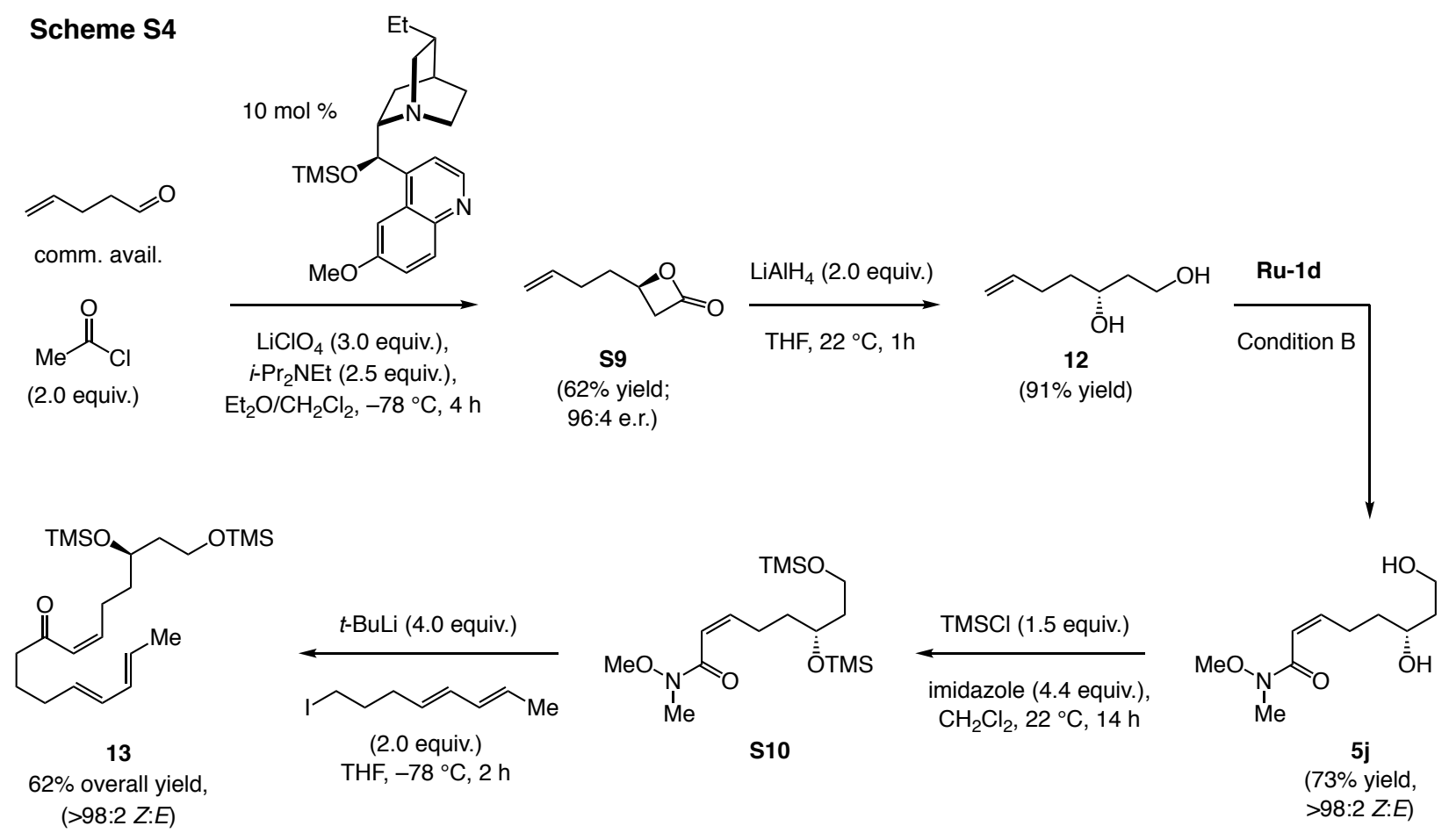

(R)-4-(But-3-en-1-yl)oxetan-2-one (S9). Based on a previously repotred procedure, ${ }^{9}$ an 8-dram vial equipped with a magnetic stir bar was charged with $O$-trimethylsilyl quinidine (39.6 $\mathrm{mg}$, $0.10 \mathrm{mmol}, 0.10$ equiv.), lithium perchlorate $\left(53.2 \mathrm{mg}, 0.50 \mathrm{mmol}, 0.50\right.$ equiv.), and $\mathrm{Et}_{2} \mathrm{O}$ (1.0 $\mathrm{mL}$ ). The solution was allowed to stir at $22{ }^{\circ} \mathrm{C}$ until it became homogenous, at which point $\mathrm{CH}_{2} \mathrm{Cl}_{2}(2.0 \mathrm{~mL})$ was added and the mixture was allowed to cool to $-78{ }^{\circ} \mathrm{C}$. Diisopropylethylamine (323 mg, $2.5 \mathrm{mmol}, 2.5$ equiv.) and 4-pentenal (84.12 mg, $1.0 \mathrm{mmol}, 1.0$ equiv.) were then added sequentially, after which acetyl chloride $(157 \mathrm{mg}, 2.0 \mathrm{mmol}, 2.0$ equiv., in $0.5 \mathrm{~mL} \mathrm{CH} \mathrm{Cl}_{2}$ ) was slowly introduced over $1 \mathrm{~h}\left(\right.$ at $\left.-78{ }^{\circ} \mathrm{C}\right)$. The mixture was allowed to stir for $12 \mathrm{~h}$ at $-78^{\circ} \mathrm{C}$, after which it was charged with $\mathrm{Et}_{2} \mathrm{O}(10 \mathrm{~mL})$ and the mixture was allowed to warm to room temperature. The solution was filtered and the volatiles were removed in vacuo to afford yellow oil, which was purified by silica gel chromatography (10-20\% $\mathrm{Et}_{2} \mathrm{O}$ in hexanes) to

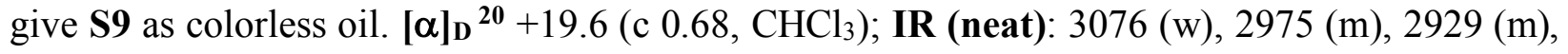
1815 (s), 1640 (m), 1442 (m), 1411 (m), 1376 (m), 1302 (m), 1202 (m), 1120 (m), 994 (m), 960 (m), 914 (m), 853 (m), 824 (m), 780 (m), 526 (m) cm ${ }^{-1} ;{ }^{1} \mathbf{H}$ NMR (400 MHz, CDCl $): \delta 5.81$ (ddt, $J=16.8,10.2,6.6 \mathrm{~Hz}, 1 \mathrm{H}), 5.14-5.00(\mathrm{~m}, 2 \mathrm{H}), 4.53(\mathrm{dtd}, J=7.6,5.8,4.3 \mathrm{~Hz}, 1 \mathrm{H}), 3.52(\mathrm{dd}$, $J=16.3,5.8 \mathrm{~Hz}, 1 \mathrm{H}), 3.08$ (dd, $J=16.3,4.3 \mathrm{~Hz}, 1 \mathrm{H}), 2.32-2.10(\mathrm{~m}, 2 \mathrm{H}), 2.05-1.94(\mathrm{~m}, 1 \mathrm{H})$, 1.91-1.80 (m, 1H); ${ }^{\mathbf{1 3}} \mathbf{C}$ NMR (150 MHz, CDCl $\left.\mathbf{3}\right): \delta 168.2,136.5,116.2,70.8,43.0,34.0,29.3$. HRMS[M+H] $]^{+}$: Calcd for $\mathrm{C}_{7} \mathrm{H}_{11} \mathrm{O}_{2}$ : 127.0754 , found: 127.0760 . Enantiomeric purity was determined by $\mathrm{GC}$ analysis [CDB/DM column, flow rate $0.2 \mathrm{~mL} / \mathrm{min}$, method: $100{ }^{\circ} \mathrm{C}$ for 100 $\min ]$. 


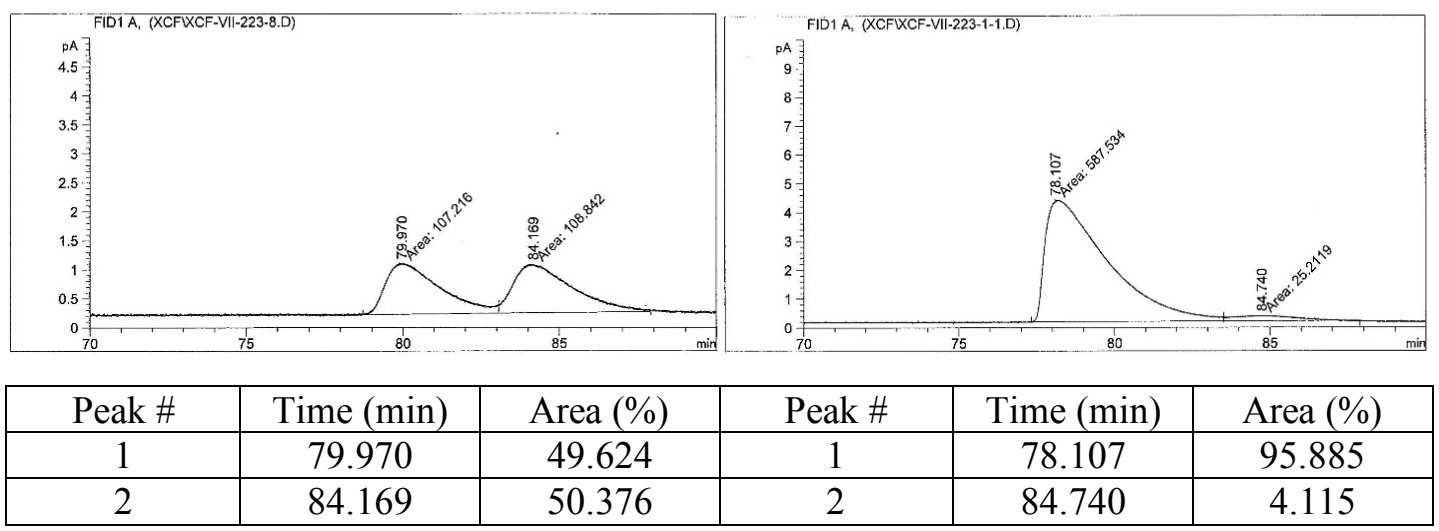

(R)-Hept-6-ene-1,3-diol (12). An 8-dram vial equipped with a magnetic stir bar was charged with lithium aluminum hydride $(30.0 \mathrm{mg}, 0.80 \mathrm{mmol}, 2.0$ equiv. $)$ and THF $(1.0 \mathrm{ml})$. The mixture was allowed to cool to $0{ }^{\circ} \mathrm{C}$ and $(R)$-4-(but-3-en-1-yl)oxetan-2-one (50.0 mg, $0.40 \mathrm{mmol}, 1.0$ equiv.in $1.0 \mathrm{~mL} \mathrm{THF}$ ) was added. The mixture was allowed to warm to $22^{\circ} \mathrm{A}$ saturated solution of potassium sodium tartrate $(5.0 \mathrm{~mL})$ and $\mathrm{Et}_{2} \mathrm{O}(5.0 \mathrm{~mL})$ were added, and the resulting mixture was allowed to stir for $0.5 \mathrm{~h}$ at $22{ }^{\circ} \mathrm{C}$. The organic and aqueous layers were separated and the aqueous layer was washed with EtOAc $(20 \mathrm{~mL} \times 3)$. The combined organic layers were washed with brine, dried over $\mathrm{Na}_{2} \mathrm{SO}_{4}$ and concentrated in vacuo. The resulting yellow oil was purified by silica gel chromatography (20-100\% EtOAc in hexanes) to afford $\mathbf{1 2}$ as colorless oil (47.1 mg,

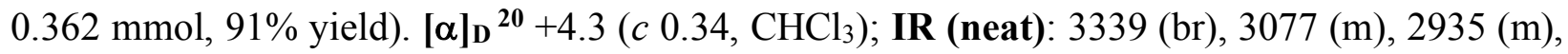
1640 (m), 1442 (m), 1331 (m), 1057 (m), 995 (m), 911 (m) cm ${ }^{-1} ;{ }^{1}$ H NMR (400 MHz, CDCl $)$ : $\delta 5.85$ (ddt, $J=17.0,10.3,6.8 \mathrm{~Hz}, 1 \mathrm{H}), 5.06$ (dt, $J=17.1,1.5 \mathrm{~Hz}, 1 \mathrm{H}), 5.02-4.96$ (m, 1H), 3.98$3.77(\mathrm{~m}, 3 \mathrm{H}), 2.33(\mathrm{~d}, J=3.6 \mathrm{~Hz}, 1 \mathrm{H}), 2.26-2.09(\mathrm{~m}, 3 \mathrm{H}), 1.76-1.56(\mathrm{~m}, 4 \mathrm{H}) ;{ }^{13} \mathbf{C}$ NMR (150 MHz, $\left.\mathbf{C D C l}_{3}\right): \delta 138.5,115.2,72.0,62.0,38.5,36.9,30.1 ; \mathbf{H R M S}[\mathbf{M}+\mathbf{H}]^{+}:$Calcd for $\mathrm{C}_{7} \mathrm{H}_{15} \mathrm{O}_{2}$ : 131.1067, found: 131.1066 .

$(\boldsymbol{R}, Z)-6,8-D i h y d r o x y-N$-methoxy- $N$-methyloct-2-enamide (5j). Colorless oil. $[\boldsymbol{\alpha}]_{\mathrm{D}}{ }^{\mathbf{2 0}}-84.9$ (c 0.5, $\mathrm{CHCl}_{3}$ ); IR (neat): 3381 (br), 2934 (m), 1647 (s), 1617 (s), 1440 (m), 1398 (m), 1362 (m), $1180(\mathrm{~m}), 1103$ (m), 1059 (s), 1028 (m), 996 (m), 796 (m) cm ${ }^{-1} ;{ }^{1} \mathbf{H}$ NMR (600 MHz, CDCl $): \delta$ $6.35(\mathrm{~d}, J=10.9 \mathrm{~Hz}, 1 \mathrm{H}), 6.09(\mathrm{td}, J=11.3,6.4 \mathrm{~Hz}, 1 \mathrm{H}), 5.37(\mathrm{~s}, 1 \mathrm{H}), 3.87-3.77(\mathrm{~m}, 3 \mathrm{H}), 3.69$ $(\mathrm{s}, 3 \mathrm{H}), 3.44(\mathrm{~s}, 1 \mathrm{H}), 3.23(\mathrm{~s}, 3 \mathrm{H}), 3.08$ (q, $J=10.9,10.2 \mathrm{~Hz}, 1 \mathrm{H}), 2.21$ (dq, $J=10.5,4.9 \mathrm{~Hz}, 1 \mathrm{H})$, $1.71(\mathrm{~m}, 2 \mathrm{H}), 1.62(\mathrm{~m}, 1 \mathrm{H}), 1.58-1.50(\mathrm{~m}, 1 \mathrm{H}) ;{ }^{13} \mathbf{C}$ NMR (125 MHz, $\left.\mathbf{C D C l}_{3}\right): \delta$ 168.0, 145.9, 120.1, 70.1, 62.4, 61.7, 38.1, 35.8, 32.1, 25.2; HRMS[M+H] $]^{+}$: Calcd for $\mathrm{C}_{10} \mathrm{H}_{20} \mathrm{NO}_{4}$ : 218.1387, found: 218.1381 .

$(\boldsymbol{R}, Z)-N$-Methoxy- $N$-methyl-6,8-bis((trimethylsilyl)oxy)oct-2-enamide (S10). An 8-dram vial was charged with a $\mathrm{CH}_{2} \mathrm{Cl}_{2}(1 \mathrm{~mL})$ solution of $(R, Z)$-6,8-dihydroxy- $N$-methoxy- $N$-methyloct-2enamide ( $9.0 \mathrm{mg}, 0.04 \mathrm{mmol}), \mathrm{TMSCl}(22.6 \mathrm{mg}, 0.2 \mathrm{mmol}, 5.0$ equiv.), and imidazole (12.0 mg, $0.18 \mathrm{mmol}, 4.4$ equiv.). The mixture was allowed to stir for $12 \mathrm{~h}$ at $22{ }^{\circ} \mathrm{C}$, after which the volatiles were directly removed in vacuo. The resulting yellow oil was purified by chromatography with a 150 mesh neutral $\mathrm{Al}_{2} \mathrm{O}_{3}$ column $(2-5 \%$ EtOAc in hexanes) to afford $\mathbf{S 1 0}$ as colorless oil (12.6 mg, $0.035 \mathrm{mmol}, 87 \%$ yield). $[\alpha]_{\mathbf{D}}{ }^{\mathbf{2 0}}-12.0$ (c $\left.0.34, \mathrm{CDCl}_{3}\right)$; IR (neat): 
2955 (m), 1660 (m), 1426 (m), 1344 (m), 1249 (m), 1178 (m), 1092 (m), 1043 (m), 1002 (m), $880(\mathrm{~m}), 838$ (s), $748(\mathrm{~m}) \mathrm{cm}^{-1} ;{ }^{1} \mathbf{H}$ NMR (500 MHz, $\left.\mathbf{C D C l}_{3}\right): \delta 6.23(\mathrm{~d}, J=11.0 \mathrm{~Hz}, 1 \mathrm{H}), 6.11$ $(\mathrm{dt}, J=11.6,7.3 \mathrm{~Hz}, 1 \mathrm{H}), 3.83(\mathrm{p}, J=6.5 \mathrm{~Hz}, 1 \mathrm{H}), 3.67(\mathrm{~s}, 3 \mathrm{H}), 3.63(\mathrm{t}, J=6.6 \mathrm{~Hz}, 2 \mathrm{H}), 3.20(\mathrm{~s}$, $3 \mathrm{H}), 2.66$ (q, $J=7.2 \mathrm{~Hz}, 2 \mathrm{H}), 1.75-1.62(\mathrm{~m}, 2 \mathrm{H}), 1.60-1.55(\mathrm{~m}, 2 \mathrm{H}), 0.11(\mathrm{~s}, 9 \mathrm{H}), 0.10(\mathrm{~s}, 9 \mathrm{H})$; ${ }^{13}$ C NMR (150 MHz, $\mathbf{C D}_{2} \mathbf{C l}_{2}$ ): $\delta$ 167.7, 147.1, 118.7, 69.5, 61.8, 59.6, 40.5, 37.6, 25.7, 0.5, -0.4; HRMS[M+H] $]^{+}$: Calcd for $\mathrm{C}_{16} \mathrm{H}_{36} \mathrm{NO}_{4} \mathrm{Si}_{2}: 362.2177$, found: 362.2164 .

$(R, 6 Z, 12 E, 14 E)-1,3-B i s((t r i m e t h y l s i l y l) o x y) h e x a d e c a-6,12,14-t r i e n-8-o n e \quad(13)$. An 6-dram vial was charged with a THF solution $(1.0 \mathrm{~mL})$ of $(2 E, 4 E)-8$-iodoocta-2,4-diene $(16.5 \mathrm{mg}, 0.07$ mmol, 2.0 equiv.). The solution was allowed to cool to $-78{ }^{\circ} \mathrm{C}$, after which, $t$-BuLi $(1.7 \mathrm{M}$ in hexane, $82 \mu \mathrm{L}, 0.14 \mathrm{mmol}, 4.0$ equiv.) was added, and the mixture was allowed to stir for $1 \mathrm{~h}$ (at $\left.-78 \quad{ }^{\circ} \mathrm{C}\right)$. A THF solution $(2.0 \mathrm{~mL})$ of $(R, Z)-N$-methoxy- $N$-methyl-6,8bis((trimethylsilyl)oxy)oct-2-enamide $(12.6 \mathrm{mg}, 0.035 \mathrm{mmol}, 1.0$ equiv.) was added and the mixture was allowed to stir for $2 \mathrm{~h}$ at $-78{ }^{\circ} \mathrm{C}$. The reaction was then quenched by the addition of a saturated solution of aqueous $\mathrm{NH}_{4} \mathrm{Cl}$. The aqueous layer was washed with EtOAc $(10 \mathrm{~mL} \times 3)$, and the combined organic layers were washed with brine, dried over $\mathrm{Na}_{2} \mathrm{SO}_{4}$, and concentrated in vacuo. The resulting yellow oil was purified by chromatography with a 150 mesh neutral $\mathrm{Al}_{2} \mathrm{O}_{3}$ column (1-5\% EtOAc in hexanes) to afford 13 as colorless oil (9.3 $\mathrm{mg}, 0.0228 \mathrm{mmol}, 65 \%$ yield). The characterization data are consistent with those previously reported. ${ }^{9}{ }^{1} \mathbf{H}$ NMR (500 MHz, CDCl $\left.\mathbf{l}_{3}\right): \delta .17-5.96(\mathrm{~m}, 4 \mathrm{H}), 5.66-5.55(\mathrm{~m}, 1 \mathrm{H}), 5.54-5.47(\mathrm{~m}, 1 \mathrm{H}), 3.90-3.78(\mathrm{~m}, 1 \mathrm{H})$, $3.63(\mathrm{t}, J=6.8 \mathrm{~Hz}, 2 \mathrm{H}), 2.65(\mathrm{q}, J=8.7 \mathrm{~Hz}, 2 \mathrm{H}), 2.44$ (t, $J=7.3 \mathrm{~Hz}, 2 \mathrm{H}), 2.13-2.04(\mathrm{~m}, 2 \mathrm{H})$, $1.69(\mathrm{~m}, 7 \mathrm{H}), 1.53-1.49(\mathrm{~m}, 2 \mathrm{H}), 0.12(\mathrm{~s}, 9 \mathrm{H}), 0.10(\mathrm{~s}, 9 \mathrm{H}) ; \mathbf{H R M S}[\mathbf{M}+\mathbf{H}]^{+}$: Calcd for $\mathrm{C}_{22} \mathrm{H}_{43} \mathrm{O}_{3} \mathrm{Si}_{2}: 411.2745$, found: 411.2761 .

\section{7 (Z)- $\alpha, \beta$-Unsaturated Secondary and Primary Amides}

Benzyl ( $\boldsymbol{Z}$ )-6-(benzylamino)-6-oxohex-4-enoate (6b). Colorless oil. IR (neat): 3304 (br), 3063 (m), 3031 (m), 2947 (m), 2922 (m), 1733 (s), 1661 (m), 1633 (m), 1537 (m), 1497 (m), 1454 (m), 1424 (m), 1381 (m), 1235 (m), 1155 (m), 737 (m), 697 (m) cm ${ }^{-1} ;{ }^{1}$ H NMR (500 MHz, CDCl $)$ : $\delta 7.39-7.25(\mathrm{~m}, 10 \mathrm{H}), 6.40(\mathrm{~s}, \mathrm{br}, 1 \mathrm{H}), 5.95(\mathrm{dt}, J=10.5,7.6 \mathrm{~Hz}, 1 \mathrm{H}), 5.77(\mathrm{~d}, J=11.4 \mathrm{~Hz}, 1 \mathrm{H})$, $5.10(\mathrm{~s}, 2 \mathrm{H}), 4.49(\mathrm{~d}, J=5.7 \mathrm{~Hz}, 2 \mathrm{H}), 2.91(\mathrm{q}, J=7.1 \mathrm{~Hz}, 2 \mathrm{H}), 2.55(\mathrm{t}, J=6.8 \mathrm{~Hz}, 2 \mathrm{H}) ;{ }^{13} \mathbf{C}$ NMR (100 MHz, $\left.\mathbf{C D C l}_{3}\right): \delta$ 173.2, 166.2, 141.7, 138.5, 136.0, 128.8, 128.7, 128.4, 128.0, 127.6, 124.2, 66.6, 43.5, 33.5, 24.2; HRMS[M+H] $]^{+}$: Calcd for $\mathrm{C}_{20} \mathrm{H}_{22} \mathrm{O}_{3} \mathrm{~N}: 324.1600$, found: 324.1597 .

(Z)- $\mathrm{N}$-(4-Methoxybenzyl)-5-phenylpent-2-enamide (6c). Colorless oil. IR (neat): 3297 (m), 3026 (m), 2928 (m), 2835 (w), 1658 (s), 1628 (m), 1512 (s), 1453 (m), $1356(\mathrm{~m}), 1245(\mathrm{~m}), 1175$ (m), $1033(\mathrm{~m}), 817$ (m), 699 (m) cm ${ }^{-1} ;{ }^{1} \mathbf{H}$ NMR (600 MHz, CDCl $): \delta 7.26(\mathrm{t}, J=7.5 \mathrm{~Hz}, 2 \mathrm{H})$, $7.19(\mathrm{~m}, 5 \mathrm{H}), 6.86(\mathrm{~d}, J=8.6 \mathrm{~Hz}, 2 \mathrm{H}), 5.99(\mathrm{dt}, J=11.5,7.5 \mathrm{~Hz}, 1 \mathrm{H}), 5.70(\mathrm{~d}, J=11.5 \mathrm{~Hz}, 1 \mathrm{H})$, $5.52(\mathrm{~s}, 1 \mathrm{H}), 4.36(\mathrm{~d}, J=5.7 \mathrm{~Hz}, 2 \mathrm{H}), 3.80(\mathrm{~s}, 3 \mathrm{H}), 2.98(\mathrm{q}, J=7.4 \mathrm{~Hz}, 2 \mathrm{H}), 2.77$ (t, $J=7.5 \mathrm{~Hz}$, 2H); ${ }^{13} \mathbf{C}$ NMR (125 MHz, $\left.\mathbf{C D C l}_{3}\right): \delta$ 166.3, 159.2, 144.0, 141.4, 130.5, 129.4, 128.7, 128.5, 126.1, 123.1, 114.2, 55.4, 42.9, 35.4, 30.5; HRMS[M+H] $]^{+}$: Calcd for $\mathrm{C}_{19} \mathrm{H}_{22} \mathrm{NO}_{2}$ : 296.1645, found: 296.1648 . 
(Z)- $\mathrm{N}$-Benzyl-6-hydroxyhex-2-enamide (6d). Colorless oil. IR (neat): 3285 (br), 3064 (m), 3029 (m), 2870 (m), 1656 (s), 1629 (m), $1544(\mathrm{~m}), 1496$ (m), 1454 (m), 1426 (m), 1272 (m), 1242 (m), 1071 (m), 1029 (m), 811 (m), 739 (m), 698 (m) cm-1; ${ }^{\mathbf{1}} \mathbf{H}$ NMR (400 MHz, CDCl $): \delta$ $7.38-7.27$ (m, 5H), 6.01 (dt, $J=11.4,8.6 \mathrm{~Hz}, 1 \mathrm{H}), 5.93$ (brs, $1 \mathrm{H}), 5.82$ (d, $J=11.5 \mathrm{~Hz}, 1 \mathrm{H}), 4.49$ $(\mathrm{d}, J=5.7 \mathrm{~Hz}, 2 \mathrm{H}), 3.88(\mathrm{t}, J=6.3 \mathrm{~Hz}, 1 \mathrm{H}), 3.60$ (q, $J=5.2 \mathrm{~Hz}, 2 \mathrm{H}), 2.88-2.70(\mathrm{~m}, 2 \mathrm{H}), 1.81-$ 1.61 (m, 2H); ${ }^{13} \mathbf{C}$ NMR (100 MHz, $\left.\mathbf{C D C l}_{3}\right): \delta 167.0,145.1,138.1,128.9,128.1,127.8,123.5$, 60.1, 43.7, 30.6, 24.8; HRMS[M+H] ${ }^{+}$: Calcd for $\mathrm{C}_{13} \mathrm{H}_{18} \mathrm{NO}_{2}: 220.1338$, found: 220.1334 .

(Z)-6-Hydroxy- $\boldsymbol{N}$-(4-methoxybenzyl)hex-2-enamide (6e). Colorless oil. IR (neat): 3291 (br), 2933 (m), 1656 (m), 1612 (s), 1586 (m), 1543 (m), 1512 (s), 1463 (m), 1301 (m), 1245 (s), 1175 (m), $1033(\mathrm{~m}), 813(\mathrm{~m}) \mathrm{cm}^{-1}$; ${ }^{1} \mathbf{H}$ NMR (600 MHz, $\left.\mathbf{C D C l}_{3}\right): \delta 7.21$ (d, $\left.J=8.3 \mathrm{~Hz}, 2 \mathrm{H}\right), 6.86(\mathrm{~d}, J$ $=8.3 \mathrm{~Hz}, 2 \mathrm{H}), 6.02-5.95(\mathrm{~m}, 2 \mathrm{H}), 5.80(\mathrm{~d}, J=11.4 \mathrm{~Hz}, 1 \mathrm{H}), 4.40(\mathrm{~d}, J=5.6 \mathrm{~Hz}, 2 \mathrm{H}), 4.02(\mathrm{~s}$, $1 \mathrm{H}), 3.79(\mathrm{~s}, 3 \mathrm{H}), 3.58(\mathrm{~d}, J=5.4 \mathrm{~Hz}, 2 \mathrm{H}), 2.74(\mathrm{q}, J=7.8 \mathrm{~Hz}, 2 \mathrm{H}), 1.70(\mathrm{p}, J=5.8 \mathrm{~Hz}, 2 \mathrm{H}) ;{ }^{13} \mathrm{C}$ NMR (125 MHz, CDCl $)$ ): $\delta$ 166.9, 159.3, 144.8, 130.2, 129.5, 123.6, 114.3, 60.1, 55.5, 43.2, 30.6, 24.7; HRMS[M+H] $]^{+}$: Calcd for $\mathrm{C}_{14} \mathrm{H}_{20} \mathrm{NO}_{3}$ : 250.1438 , found: 250.1447 .

(Z)- $N$-Benzyl-7-(1,3-dioxoisoindolin-2-yl)hept-2-enamide (6f). Colorless oil. IR (neat): 3360 (br), 3061 (w), 3029 (w), 2928 (m), 2857 (w), 1770 (m), 1707 (s), 1662 (m), 1531 (m), 1436 (m), 1396 (m), 1370 (m), 1336 (m), 1235 (m), 1040 (m), 719 (m), 699 (m) cm ${ }^{-1} ;{ }^{1}$ H NMR (500 MHz, CDCl $\left._{3}\right): \delta$ 7.84-7.75 (m, 2H), 7.71-7.63 (m, 2H), 7.29-7.20 (m, 5H), 5.99-5.89 (m, 1H), $5.78(\mathrm{~s}$, br, $1 \mathrm{H}), 5.68(\mathrm{~d}, J=11.4 \mathrm{~Hz}, 1 \mathrm{H}), 4.43(\mathrm{~d}, J=5.4 \mathrm{~Hz}, 2 \mathrm{H}), 3.64(\mathrm{t}, J=7.0 \mathrm{~Hz}, 2 \mathrm{H}), 2.70(\mathrm{q}, J=$ $7.0 \mathrm{~Hz}, 2 \mathrm{H}), 1.68(\mathrm{p}, J=6.2 \mathrm{~Hz}, 2 \mathrm{H}), 1.46(\mathrm{p}, J=7.4 \mathrm{~Hz}, 2 \mathrm{H}) ;{ }^{13} \mathbf{C}$ NMR (100 MHz, CDCl $\left.\mathbf{3}\right): \delta$ 168.6, 166.3, 145.2, 138.5, 134.0, 132.3, 128.8, 128.1, 127.6, 123.3, 122.7, 43.5, 37.9, 28.3, 28.3, 26.6; HRMS[M+H] $]^{+}$: Calcd for $\mathrm{C}_{22} \mathrm{H}_{23} \mathrm{O}_{3} \mathrm{~N}_{2}$ : 363.1709 , found: 363.1712 .

(Z)- $\mathrm{N}$-Benzyl-4-(3,4-dimethoxyphenyl)but-2-enamide (6g). Colorless oil. IR (neat): 3305 (br), 3062 (w), 3029 (w), 2932 (w), 1659 (m), 1631 (m), 1590 (m), 1512 (s), 1453 (m), 1259 (m), $1233(\mathrm{~m}), 1188(\mathrm{~m}), 1027(\mathrm{~m}), 809(\mathrm{~m}), 756(\mathrm{~m}), 699(\mathrm{~m}) \mathrm{cm}^{-1} ;{ }^{1} \mathbf{H}$ NMR (500 MHz, CDCl$): \delta$ $7.32(\mathrm{dq}, J=15.0,8.0 \mathrm{~Hz}, 5 \mathrm{H}), 6.83-6.75(\mathrm{~m}, 3 \mathrm{H}), 6.17(\mathrm{dt}, J=11.3,7.6 \mathrm{~Hz}, 1 \mathrm{H}), 5.81(\mathrm{~s}, 1 \mathrm{H})$, $5.76(\mathrm{~d}, J=12.3 \mathrm{~Hz}, 1 \mathrm{H}), 4.51(\mathrm{~d}, J=5.7 \mathrm{~Hz}, 2 \mathrm{H}), 4.02(\mathrm{~d}, J=7.6 \mathrm{~Hz}, 2 \mathrm{H}), 3.85(\mathrm{~s}, 6 \mathrm{H}) ;{ }^{13} \mathrm{C}$ NMR (125 MHz, CDCl 3 ): $\delta$ 166.2, 149.2, 147.7, 144.7, 138.4, 132.8, 128.9, 128.0, 127.7, 122.0, 120.6, 112.2, 111.5, 56.1, 56.0, 43.6, 34.6; HRMS[M+H] $]^{+}$: Calcd for $\mathrm{C}_{19} \mathrm{H}_{22} \mathrm{NO}_{3}: 312.1600$, found: 312.1594 .

(Z)-4-(3,4-Dimethoxyphenyl)- $\boldsymbol{N}$-(4-methoxybenzyl)but-2-enamide (6h). Colorless oil. IR (neat): 3301 (br), 2998 (w), 2934 (m), 1659 (m), 1628 (m), 1589 (m), 1512 (s), 1463 (m), 1235 (m), 1175 (m), 1153 (m), 1139 (m), 1028 (m), 815 (m) cm ${ }^{-1} ;{ }^{1}$ H NMR (500 MHz, CDCl $): \delta$ $7.24(\mathrm{~d}, J=8.5 \mathrm{~Hz}, 2 \mathrm{H}), 6.87(\mathrm{~d}, J=8.5 \mathrm{~Hz}, 2 \mathrm{H}), 6.82-6.76(\mathrm{~m}, 3 \mathrm{H}), 6.16(\mathrm{dt}, J=11.3,7.6 \mathrm{~Hz}$, $1 \mathrm{H}), 5.74(\mathrm{~d}, J=11.3 \mathrm{~Hz}, 2 \mathrm{H}), 4.44(\mathrm{~d}, J=5.6 \mathrm{~Hz}, 2 \mathrm{H}), 4.01(\mathrm{~d}, J=7.5 \mathrm{~Hz}, 2 \mathrm{H}), 3.85(\mathrm{~s}, 6 \mathrm{H})$, 3.80 (s, 3H); ${ }^{13} \mathbf{C}$ NMR (125 MHz, $\left.\mathbf{C D C l}_{3}\right): \delta$ 166.2, 159.2, 149.2, 147.6, 144.5, 132.8, 130.4, $129.4,122.1,120.5,114.3,112.1,111.5,56.1,56.0,55.5,43.1,34.6$; HRMS [M+H] ${ }^{+}:$Calcd for $\mathrm{C}_{20} \mathrm{H}_{24} \mathrm{NO}_{4}: 342.1700$, found: 342.1697 . 
(Z)-4-(3,4-Dimethoxyphenyl)- $\boldsymbol{N}$-isobutylbut-2-enamide (6i). Colorless oil. IR (neat): 3305 (m), 2959 (m), 2929 (m), 2870 (w), 1654 (m), 1625 (m), 1589 (m), 1547 (m), 1514 (m), 1416 (m), 1261 (s), 1234 (m), 1172 (m), 1025 (m), 810 (m), 760 (m) cm ${ }^{-1} ;{ }^{1}$ H NMR (400 MHz, $\left.\mathbf{C D C l}_{3}\right): \delta 6.81-6.76(\mathrm{~m}, 3 \mathrm{H}), 6.12(\mathrm{dt}, J=11.3,7.6 \mathrm{~Hz}, 1 \mathrm{H}), 5.75(\mathrm{dt}, J=11.3,1.6 \mathrm{~Hz}, 1 \mathrm{H})$, 5.56 (br, 1H), 3.98 (dd, $J=7.6,1.5 \mathrm{~Hz}, 2 \mathrm{H}), 3.85(\mathrm{~d}, J=3.5 \mathrm{~Hz}, 6 \mathrm{H}), 3.15$ (t, $J=6.5 \mathrm{~Hz}, 2 \mathrm{H})$, $1.81(\mathrm{dp}, J=13.4,6.7 \mathrm{~Hz}, 1 \mathrm{H}), 0.93(\mathrm{~d}, J=6.7 \mathrm{~Hz}, 6 \mathrm{H}) ;{ }^{13} \mathbf{C}$ NMR (100 MHz, CDCl 3$): \delta 166.5$, 149.2, 147.6, 143.9, 132.9, 122.4, 120.5, 112.1, 111.5, 56.1, 56.0, 46.8, 34.6, 28.7, 20.3; HRMS[M+H] $]^{+}$: Calcd for $\mathrm{C}_{16} \mathrm{H}_{24} \mathrm{NO}_{3}: 278.1756$, found: 278.1763 .

(Z)-6-Hydroxyhex-2-enamide (7a). Off-white solid. M.p.: 73-74 ${ }^{\circ} \mathrm{C}$; IR (neat): 3337 (br), 2928 (m), 2873 (m), 1664 (m), 1603 (m), 1439 (m), 1328 (m), 1259 (m), 1171 (m), 1063 (m), $811(\mathrm{~m}) \mathrm{cm}^{-1} ;{ }^{1} \mathbf{H}$ NMR (400 MHz, $\left.\mathbf{C D C l}_{3}\right): \delta 6.05$ (dt, $\left.J=11.5,8.6 \mathrm{~Hz}, 1 \mathrm{H}\right), 5.88(\mathrm{~d}, J=11.5$ $\mathrm{Hz}, 1 \mathrm{H}), 5.69$ (br, 2H), $3.72(\mathrm{~s}, \mathrm{br}, 1 \mathrm{H}), 3.59(\mathrm{t}, J=5.5 \mathrm{~Hz}, 4 \mathrm{H}), 2.82-2.63(\mathrm{~m}, 2 \mathrm{H}), 1.71(\mathrm{dt}, J=$ 13.1, 6.0 Hz, 2H); ${ }^{13} \mathbf{C}$ NMR (150 $\left.\mathbf{M H z}, \mathbf{C D C l}_{3}\right): \delta$ 169.1, 146.1, 122.7, 60.2, 30.6, 24.7; HRMS[M+H] $]^{+}$: Calcd for $\mathrm{C}_{6} \mathrm{H}_{12} \mathrm{NO}_{2}: 130.0863$, found: 130.0868 .

(Z)-4-(2-Hydroxyphenyl)but-2-enamide (7b). Off-white solid. M.p.: $121{ }^{\circ} \mathrm{C}$; IR (neat): 3360 (br), 3204 (m), 3031 (m), 2924 (m), 1696 (s), 1667 (m), 1600 (s), 1570 (m), 1489 (s), 1467 (m), 1270 (m), 1245 (m), 1118 (m), 807 (m), 757 (s), 748 (m), 709 (m) cm ${ }^{-1}$; ${ }^{1}$ H NMR (400 MHz, CDCl $\left._{3}\right): \delta 9.23(\mathrm{~s}, 1 \mathrm{H}), 7.20-7.08(\mathrm{~m}, 2 \mathrm{H}), 6.89(\mathrm{~d}, J=8.5 \mathrm{~Hz}, 1 \mathrm{H}), 6.81(\mathrm{t}, J=7.4 \mathrm{~Hz}, 1 \mathrm{H})$, $6.21(\mathrm{dt}, J=11.1,9.1 \mathrm{~Hz}, 1 \mathrm{H}), 5.84(\mathrm{~d}, J=11.4 \mathrm{~Hz}, 1 \mathrm{H}), 5.63(\mathrm{~s}, \mathrm{br}, 2 \mathrm{H}), 3.85$ (d, $J=9.1 \mathrm{~Hz}$, 2H); ${ }^{13} \mathbf{C}$ NMR (100 MHz, $\left.\mathbf{C D C l}_{3}\right): \delta$ 170.1, 156.4, 145.4, 130.8, 128.7, 122.2, 119.8, 119.7, 117.1, 31.6; HRMS[M+H] $]^{+}$: Calcd for $\mathrm{C}_{10} \mathrm{H}_{12} \mathrm{NO}_{2}$ : 178.0863, found: 178.0853.

(Z)-4-(4-Hydroxyphenyl)but-2-enamide (7c). Off-white solid. M.p.: $121{ }^{\circ} \mathrm{C}$; IR (neat): 3359 (m), 3200 (m), 3031 (m), 2924 (m), 1697 (m), 1668 (m), 1644 (m), 1600 (s), 1570 (m), 1490 (m), 1467 (m), 757 (m), 748 (m) cm ${ }^{-1}$; ${ }^{1} \mathbf{H}$ NMR (400 MHz, CDCl $)$ : $\delta 9.23$ (s, 1H), 7.19-7.07 (m, $2 \mathrm{H}), 6.89(\mathrm{~d}, J=8.2 \mathrm{~Hz}, 1 \mathrm{H}), 6.81(\mathrm{t}, J=7.3 \mathrm{~Hz}, 1 \mathrm{H}), 6.26-6.15(\mathrm{~m}, 1 \mathrm{H}), 5.84(\mathrm{~d}, J=11.5 \mathrm{~Hz}$, 1H), 5.64 (s, br, 1H), 3.85 (d, $J=9.1 \mathrm{~Hz}, 2 \mathrm{H}) ;{ }^{13}$ C NMR (150 MHz, CD $\mathbf{M}_{3}$ OD): $\delta$ 171.6, 156.8, 146.1 132.1, 130.5, 122.2, 116.3, 34.9; HRMS[M+H] $]^{+}$: Calcd for $\mathrm{C}_{10} \mathrm{H}_{12} \mathrm{NO}_{2}$ : 178.0863, found: 178.0860 .

(Z)-6-Hydroxyhex-2-enenitrile (14). In a $\mathrm{N}_{2}$-filled glovebox, a 4-dram vial was charged with $0.5 \mathrm{~mL} \mathrm{CH} \mathrm{Cl}_{2}$ solution of (Z)-5-phenylpent-2-enamide (4.4 mg, $0.034 \mathrm{mmol}, 1.0$ equiv.). The Burgess reagent (16.2 $\mathrm{mg}, 0.068 \mathrm{mmol}, 2.0$ equiv.) was added, and the resulting mixture was allowed to stir for $1 \mathrm{~h}$ at $22{ }^{\circ} \mathrm{C}$. The volatiles were removed under reduced pressure, leaving behind a black oil, which was purified by silica gel chromatography (10-50\% EtOAc in hexanes) to afford 14 as colorless oil $(3.2 \mathrm{mg}, 0.029 \mathrm{mmol}, 86 \%$ yield). The characterization data are consistent with those previously reported. ${ }^{19}{ }^{1} \mathbf{H}$ NMR (600 $\left.\mathbf{M H z}, \mathbf{C D C l}_{3}\right): \delta 6.54$ (dt, $J=10.7$, $7.6 \mathrm{~Hz}, 1 \mathrm{H}), 5.35$ (d, $J=10.9 \mathrm{~Hz}, 1 \mathrm{H}), 3.71$ (q, $J=6.1 \mathrm{~Hz}, 2 \mathrm{H}), 2.54$ (q, $J=7.5 \mathrm{~Hz}, 2 \mathrm{H}), 1.76$ (p, $J=6.6 \mathrm{~Hz}, 2 \mathrm{H})$; HRMS[M+H] $]^{+}$: Calcd for $\mathrm{C}_{6} \mathrm{H}_{10} \mathrm{NO}: 112.0757$, found: 112.0762 . 


\section{Computational Studies}

DFT studies ${ }^{20}$ were performed with the Gaussian 09/Gaussian 16 suite of programs. ${ }^{21}$ Geometries were optimized with the $\omega$ B97XD functional and the Def2SVP basis set. ${ }^{22}$ The effect of a polar reaction medium (tetrahydrofuran, THF) was approximated by means of an integral equation formalism variant of the polarizable continuum model (IEFPCM). ${ }^{23}$ Stationary points were probed through vibrational analysis and Gibbs free energy corrections were performed under standard conditions $(298.15 \mathrm{~K}, 1.0 \mathrm{~atm})$. Transition states have been verified through Intrinsic Reaction Coordinate calculations (IRC) employing the L(ocal) Q(uadratic) A(approximation) method, ${ }^{24}$ followed by optimization of the end-points with the abovementioned optimization method. We also probed the performance of various density functionals through single point energy calculations at the geometries optimized with the level described above by means of the SMD solvation mode ${ }^{25}$ with THF as solvent and the larger Def2TZVPP ${ }^{22}$ basis set. Since the correct density functional is not known, we tested several of the state-of-the-art approaches that have been developed over the past decade: ${ }^{20,26} \mathrm{MN} 15,{ }^{20 \mathrm{i}}$ M06, ${ }^{27} \omega \mathrm{B} 97 \mathrm{XD}{ }^{28}$ and PBE0-D3BJ ${ }^{206},{ }^{29}$ and PBE0. ${ }^{29} \mathrm{We}$ only report the

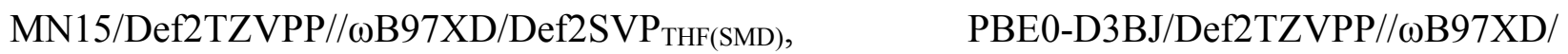

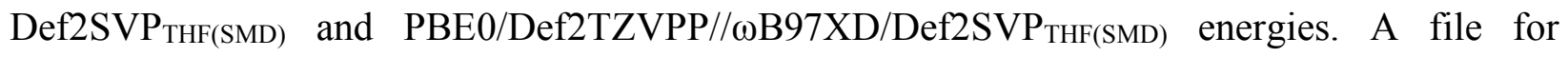
convenient viewing of computed geometries with the program Mercury 3.3 is appended as separate "coordinates.xyz" file in Section $7 .{ }^{30}$

\subsection{Model Systems Used in the Calculations}

To simplify the calculations, we used the following model systems (Scheme S5). In Ru1c and Ru-1d the $\mathrm{Cl}$ substituents on the dithiolate ligand have been replaced by hydrogens. In addition, the calculations have been carried out with truncated dithiolate ligands (Ru-1 $\mathbf{c}_{\text {model }}$ and

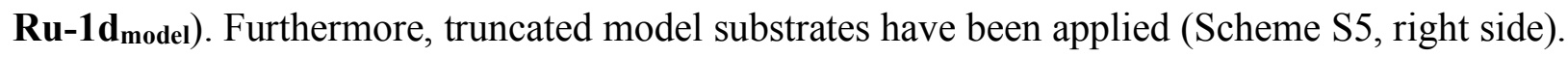

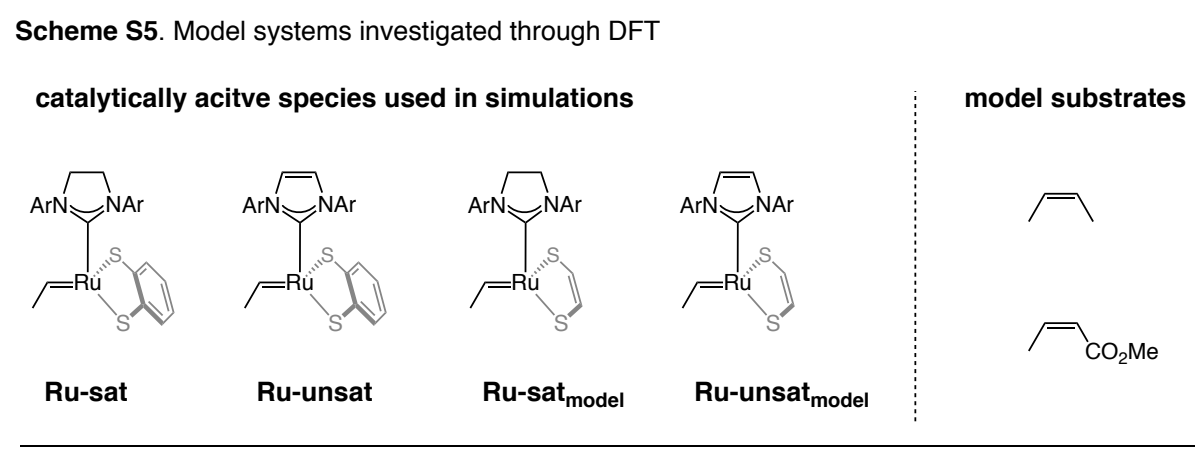

$\mathrm{Ar}=$ 2-fluoro,6-methyl phenyl

\subsection{Competition Between Productive Olefin Metathesis and Decomposition Pathways}

For insight regarding the rates of productive and nonproductive pathways, as well as various modes of decomposition with complexes Ru-1c and Ru-1d, the processes listed below 
$(\mathrm{i}-\mathrm{v})$ were investigated by DFT (optimization with $\omega-\mathrm{B} 97 \mathrm{XD} / \mathrm{Def} 2 \mathrm{SVP} \mathrm{P}_{\mathrm{THF}(\mathrm{IEFPCM}) \text { ). Please note }}$ that only the structures associated with Ru-1c are shown in Figs. S11-1 to S11-5.

Distortion of trimethyl substituted metallacyclobutane $\mathbf{m c b}_{\mathbf{a}}$ (relevant to homometathesis) to generate $\mathbf{m c b}(\mathbf{d i s t})_{\mathbf{a}}$ via ts(dist) a and subsequent decomposition by $\beta$-hydride elimination $\left(\rightarrow \mathbf{t s}(\mathbf{B H E})_{\mathbf{a}} \rightarrow \operatorname{prod}(\mathbf{B H E})_{\mathbf{a}}\right)$ and cyclopropantion $\left(\rightarrow \mathbf{t s}(\mathbf{C P})_{\mathbf{a}}\right.$ $\rightarrow \operatorname{prod}(\mathbf{C P})$ a; Fig. S11-1).

(ii) Nonproductive olefin metathesis, which is also relevant to homometathesis $\left(\mathbf{1 4 e} \rightarrow \mathbf{t s} \mathbf{1}_{\mathbf{a}}\right.$ $\rightarrow$ mcb $_{\mathbf{a}} \rightarrow$ ts $_{\mathbf{a}} \rightarrow$ 14e; Fig. S11-2).

(iii) $\quad$ Productive cross-metathesis, $\left(\mathbf{1 4 e} \rightarrow \mathbf{t s}_{\mathbf{b}} \rightarrow \mathbf{m c b}_{\mathbf{b}} \rightarrow \mathbf{t s}_{\mathbf{b}} \rightarrow \mathbf{1 4 e}\right.$; Fig. S11-3).

(iv) Nonproductive olefin metathesis with methyl-(Z)-but-2-enoate $\left(\mathbf{1 4} \mathbf{e} \rightarrow \mathbf{t s 1}_{\mathbf{c}} \rightarrow \mathbf{m c b}_{\mathbf{c}} \rightarrow\right.$ $\mathbf{t s} \mathbf{2}_{\mathbf{c}} \rightarrow \mathbf{1 6 e}$ ), which involves the key metallacyclobutane intermediate (i.e., $\mathbf{m c b} \mathbf{c}$ ), responsible for catalyst decomposition (Fig. S11-4).

(v) Distortion of trisubstituted metallacyclobutane mcbs, bearing an ester group in $\alpha$ position, to generate $\mathbf{m c b}(\mathbf{d i s t})_{\mathbf{c}}$ via ts(dist)c and subsequent decomposition by $\beta$ hydride elimination $\left(\rightarrow \operatorname{ts}(\mathbf{B H E})_{\mathbf{c}} \rightarrow \operatorname{prod}(\mathbf{B H E})_{\mathbf{c}}\right)$ and cyclopropantion $\left(\rightarrow \operatorname{ts}(\mathbf{C P})_{\mathbf{c}} \rightarrow\right.$ $\operatorname{prod}(\mathbf{C P})_{\mathbf{c}}$; see Fig. S11-5).

The corresponding free energy surfaces for Ru-1c (blue curve) and Ru-1d (red curve) associated with $\mathrm{i}-\mathrm{v}$ are summarized in the diagrams below with three different levels of theory,

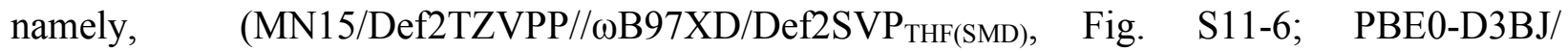

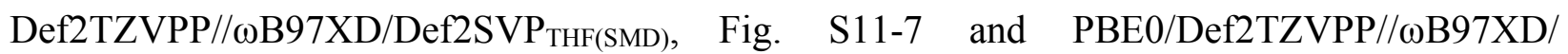

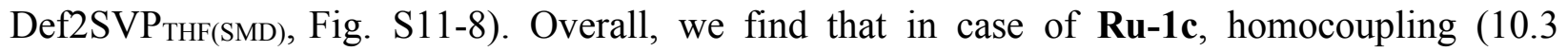
$\mathrm{kcal} / \mathrm{mol}$ for ts $\mathbf{1}_{\mathbf{a}}$; blue curve in Fig. S11-6) is favored by nearly $3 \mathrm{kcal} / \mathrm{mol}$ over productive cross-metathesis $\left(13.4 \mathrm{kcal} / \mathrm{mol}\right.$ for ts $\left.\mathbf{1}_{\mathbf{b}}\right)$, whereas the barriers for metallacyclobutane distortion (ts(dist) $)_{\mathbf{a}}$ and ts(dist) $)$, the entry points to decomposition, are predicted to be notably higher in energy (24.4 and $18.0 \mathrm{kcal} / \mathrm{mol}$, respectively; Fig. S11-6). Comparison of the latter two barriers reveals a major distinction, which might be the reason why stereoretentive cross-metathesis with $\alpha, \beta$-unsaturated carboxylic acid derivatives are challenging. Specifically, we find that in ts(dist) $)_{\mathbf{a}}$ the mcb $\mathrm{C} \alpha$ bearing a methyl substituent shifts into a trans position with respect to $\mathrm{S}($ cis) (cis to the NHC), giving rise to unfavorable trans influence. In contrast, trans influence is

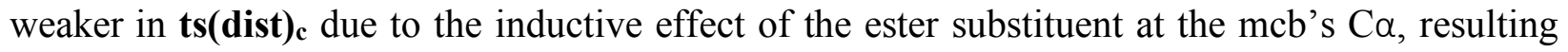
in a lower barrier to its formation. That ts(dist)c is only $4.5 \mathrm{kcal} / \mathrm{mol}$ higher in energy than ts1 $\mathbf{b}_{\mathbf{b}}$ likely renders mcb distortion competitive with cross-metathesis, especially if one considers errors associated with DFT calculations ( $\sim 2 \mathrm{kcal} / \mathrm{mol})$.

\subsubsection{Computationally Observed Difference between Ru-1c and Ru-1d}

Despite the experimentally observed differences between Ru-1c and Ru-1d, the corresponding free energy surface associated with Ru-1d is quantitatively similar to the one corresponding to Ru-1c ( $<1-2 \mathrm{kcal} / \mathrm{mol}$ energy difference corresponds to the error of the DFT method; red curve, Fig. S11-6). This renders direct comparison between saturated and unsaturated NHC ligands challenging based on DFT calculations, a fact that has been pointed out 
previously. ${ }^{31}$ While Tolman electronic parameters (TEP), ${ }^{31 c, 32}$ which represent a measure of the combined $\sigma$-donor and $\pi$-acceptor properties, are similar for the corresponding saturated and unsaturated NHCs, $\mathrm{pK}_{\mathrm{a}}$ measurements ${ }^{33}$ and ${ }^{31} \mathrm{P}$ and ${ }^{79} \mathrm{Se}$ NMR chemical shifts of carbenephosphinide $^{34}$ and carbene-selenium ${ }^{35}$ adducts suggest that the saturated variants are stronger $\sigma$ donors as well as better $\pi$-acceptors. Furthermore, experimentally determined redox potentials for $\mathrm{Ir}^{36}$ and $\mathrm{Ru}^{37}$ complexes have led to the conclusion that saturated NHCs could be stronger or weaker overall electron donors than their unsaturated counterparts. Still, consistent with the potentially increased $\sigma$-donor strength of saturated NHCs is an expanded $\mathrm{N}-\mathrm{C}-\mathrm{N}$ angle, which raises the energy of the filled $\mathrm{sp}^{2}$ orbital. ${ }^{33}$ Additionally, investigations regarding the steric properties between SIMes and IMes do not provide a conclusive measure of any reactivity differences that might arise from minor size differences (e.g., buried volume ${ }^{38}$ or repulsiveness ${ }^{39}$ ). Nonetheless, based on structural parameters ( $\mathrm{Ru}-\mathrm{S}($ trans $)$ and $\mathrm{Ru}-\mathrm{C}^{\mathrm{NHC}}$ bond lengths) it might be suggested that the variations between transformations performed with Ru-1c and Ru-1d are rooted in a subtle but significant difference in the degree of trans influence - stronger in Ru-1c than in Ru-1d (see Sections 5.3 and 5.4, below).

\subsubsection{Transition States for $\beta$-Hydride Elimination and Cyclopropanation}

Unlike the abovementioned experimental results (no observation of a cyclopropanation

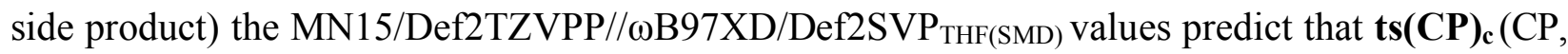
cyclopropanation) is $0.8 \mathrm{kcal} / \mathrm{mol}$ lower in energy than ts(BHE)c (i.e., $13.1 \mathrm{vs} .13 .9 \mathrm{kcal} / \mathrm{mol}$, respectively for Ru-1c; Fig. S11-6). Nonetheless, the calculations show that $\beta$-hydride elimination is $15.7 \mathrm{kcal} / \mathrm{mol}$ more exergonic $(-11.4 \mathrm{vs}$. $4.2 \mathrm{kcal} / \mathrm{mol}$, respectively for $\operatorname{prod}(B H E)_{c}$ and $\operatorname{prod}(\mathbf{C P})_{\mathbf{c}}$; Fig. S11-6). A similar qualitative trend is predicted at the PBE0-

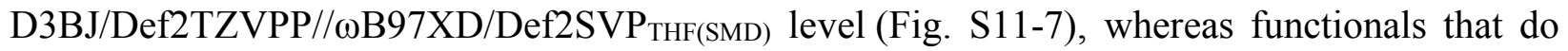

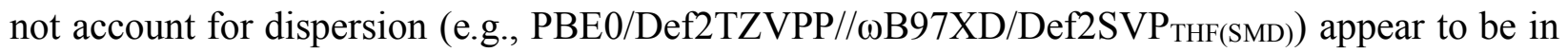
line with the experimental trend $\left(27.7\right.$ and $28.5 \mathrm{kcal} / \mathrm{mol}$ for $\mathbf{t s}(\mathbf{B H E})_{\mathbf{c}}$ and $\mathbf{t s}(\mathbf{C P})_{\mathbf{c}}$, respectively; Fig. S11-8).

While correct modeling of dispersion interactions is crucial for achieving appropriate predictions of weak, non-covalent interactions in the gas phase, ${ }^{40,20 b, 27}$ there have been recent concerns regarding the attenuation of dispersion interactions in solution, which render definitive statements on the relative energy of $\mathbf{t s}(\mathbf{B H E})_{\mathbf{c}}$ and $\mathbf{t s}(\mathbf{C P})_{\mathbf{c}}$ based on the present DFT data challenging. ${ }^{41}$ Comparison of transition state structures for $\operatorname{ts}(\mathbf{B H E})_{\mathbf{c}}$ and $\mathbf{t s}(\mathbf{C P})_{\mathbf{c}}$ reveals that ts(CP)c might be stabilized to a larger degree by attractive dispersion forces due to a more favored face-to-face $\pi-\pi$ stacking interaction, ${ }^{42}$ which is largely absent in ts(BHE)c (Scheme S6). Hence, if dispersion is disregarded (i.e., by applying the PBE0 functional; Fig. S11-8) the relative order between $\mathbf{t s}(\mathbf{B H E})_{\mathbf{c}}$ and $\mathbf{t s}(\mathbf{C P})_{\mathbf{c}}$ is indeed reversed. 


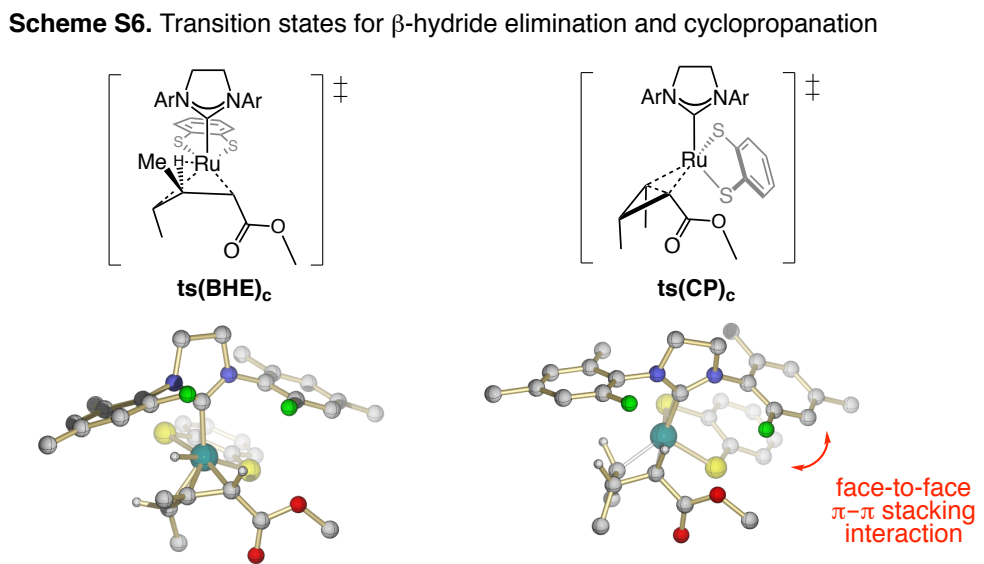

\subsubsection{Free Energy Surfaces With Smaller Model Systems Ru-1 $\mathrm{c}_{\text {model }}$ and Ru-1d $\mathrm{d}_{\text {model }}$}

To test the influence of dispersion further, we investigated the free energy surfaces with

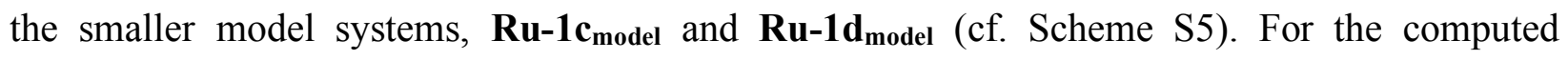
structures see Figs. S12-1 to S12-5. The corresponding free energy surfaces for Ru-1 $\mathbf{c}_{\text {model }}$ (blue curve) and Ru-1d $\mathbf{d}_{\text {model }}$ (red curve) are shown below with three different levels of theory

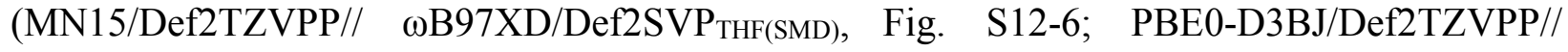
$\omega B 97 X D /$ Def2SVP ThF(SMD), Fig. S12-7 and PBE0/Def2TZVPP// $\omega$ B97XD/Def2SVP ThF(SMD), Fig. S12-8). As might be expected, ts(BHE) is predicted to be favored over $\mathbf{t s}(\mathbf{C P})_{\mathbf{c}}$ with all density functionals examined, suggesting that dispersion interactions in solution should probably be taken with caution. 


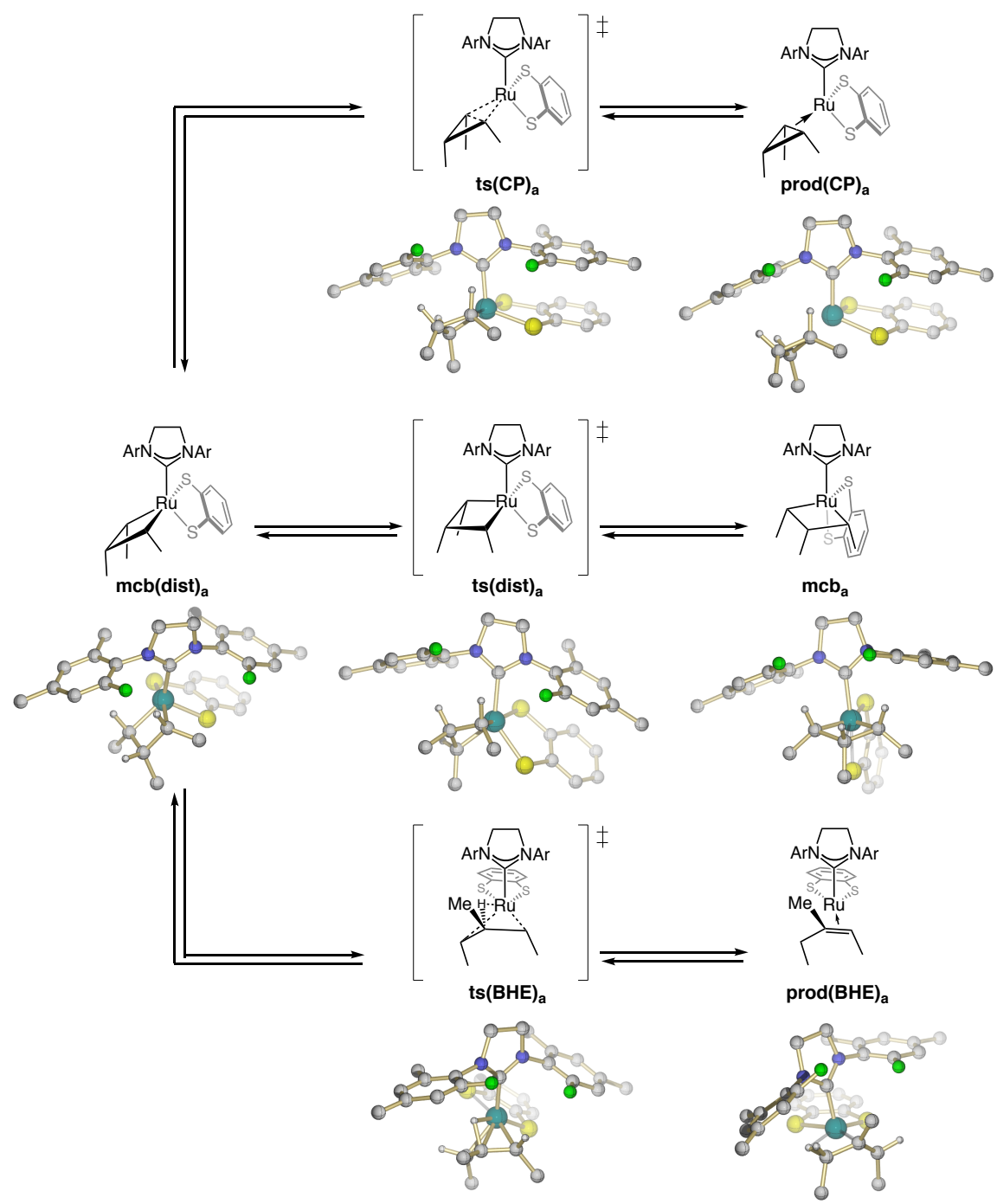

Fig. S11-1. Computed structures ( $\omega B$ B7XD/Def2SVP $\left.\mathrm{CH}_{2 \mathrm{Cl}(\mathrm{IEFPCM})}\right)$ for decomposition of $\mathbf{m c b}$ a through $\beta$-hydride elimination and cyclopropanation. ts(CP) and $\operatorname{prod}(\mathbf{C P})$, transition state and product for cyclopropanation; ts(BHE) and prod(BHE), transition state and product for $\beta$-hydride elimination; mcb, metallacyclobutane; ts(dist), transition state for metallacyclobutane distortion; mcb(dist), distorted mcb after structural distortion. 


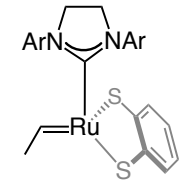

$14 \mathrm{e}$

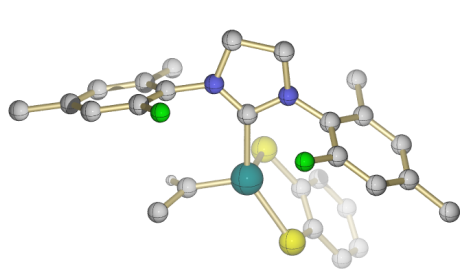

$\uparrow \mid$

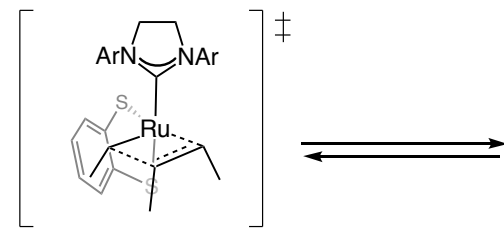

ts1

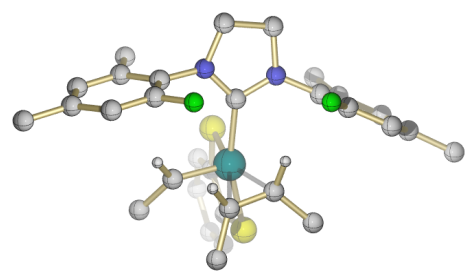

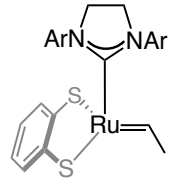

$14 \mathrm{e}$
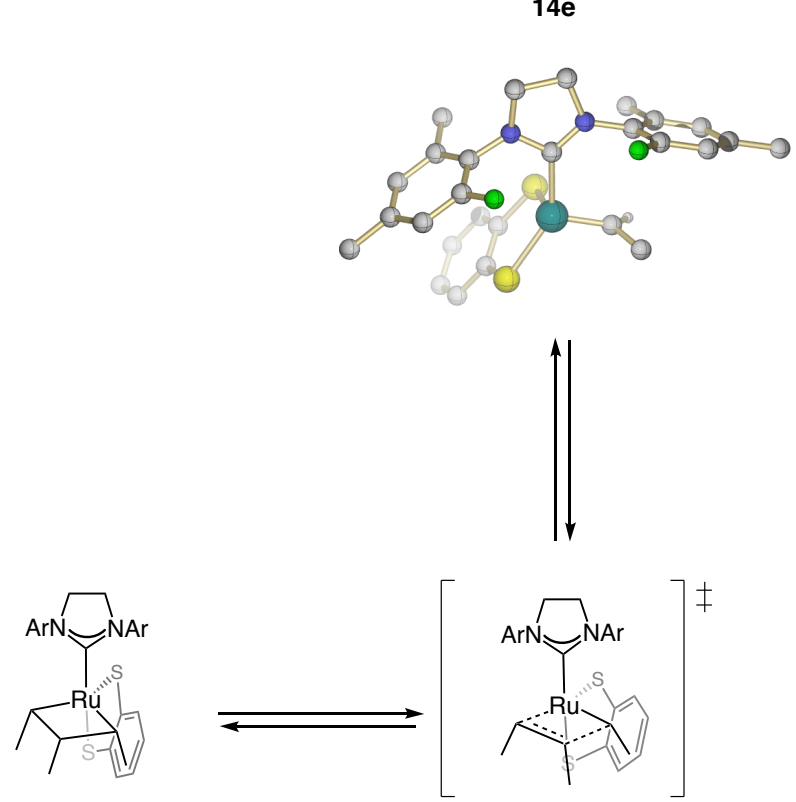

$\mathbf{m c b}_{\mathbf{a}}$

ts2
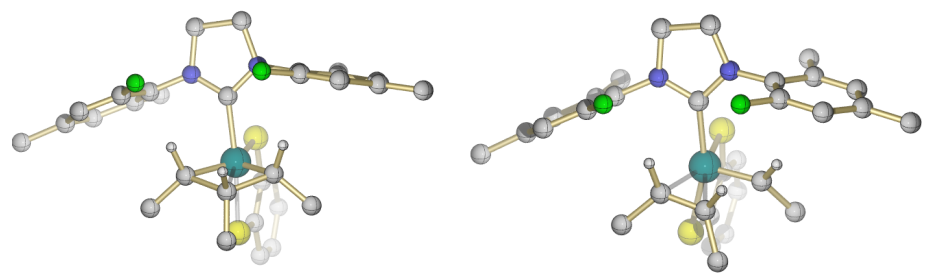

Fig. S11-2. Computed structures ( $\omega B$ B7XD/Def2SVP $\left.\mathrm{CH}_{2 \mathrm{CI}(\mathrm{IEFPCM})}\right)$ for non-productive OM with cis-butene; 14e, 14electron intermediate; mcb, metallacyclobutane; ts1 and ts2, transition states for mcb formation and breakage. ts, transition state; mcb, metallacyclobutane. 


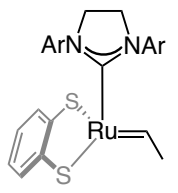

$14 \mathrm{e}$

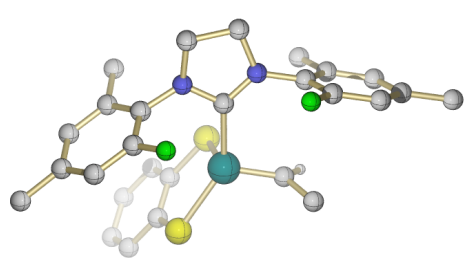

$$
\uparrow
$$

ts $1_{b}$
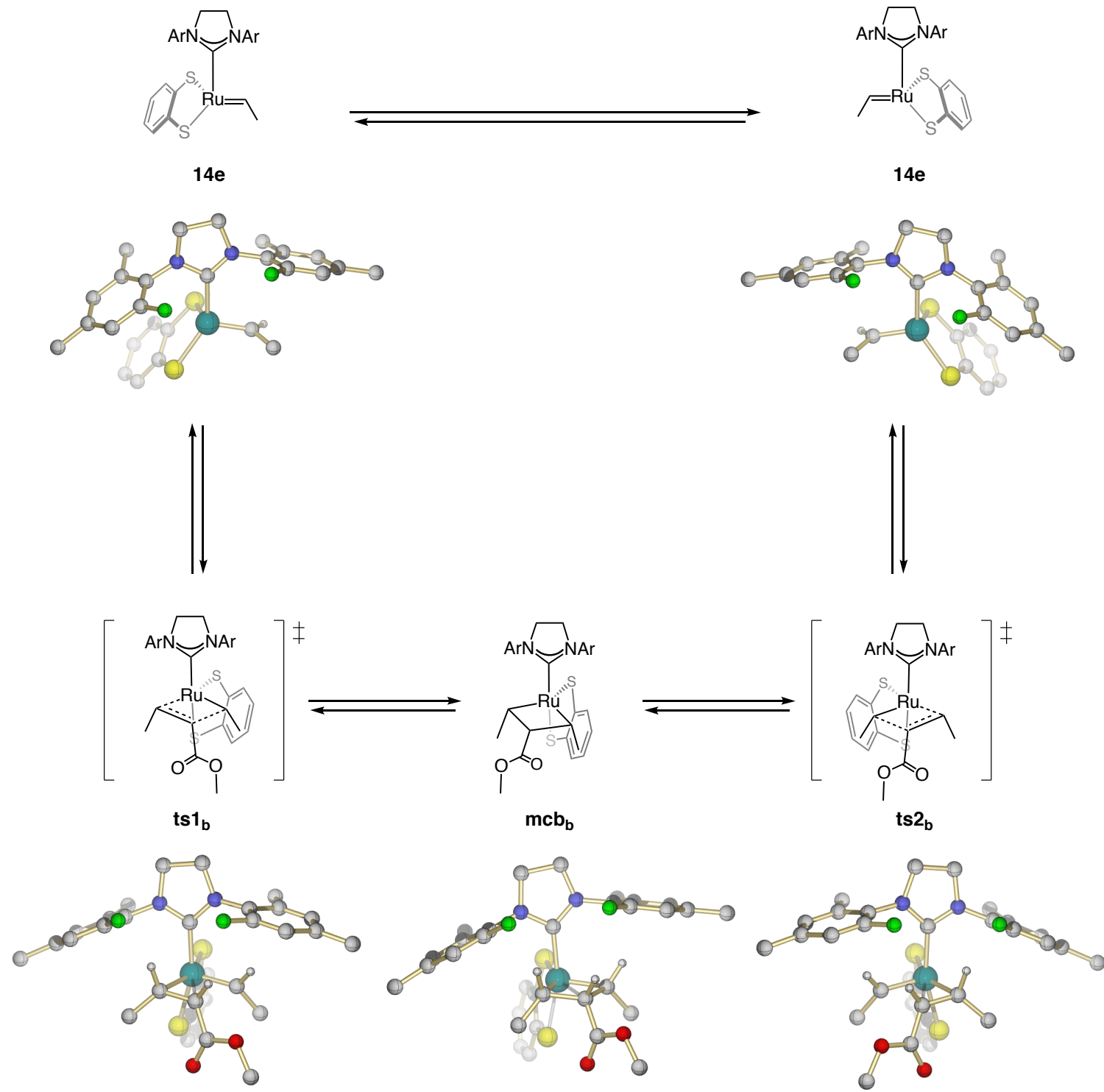

$14 \mathrm{e}$
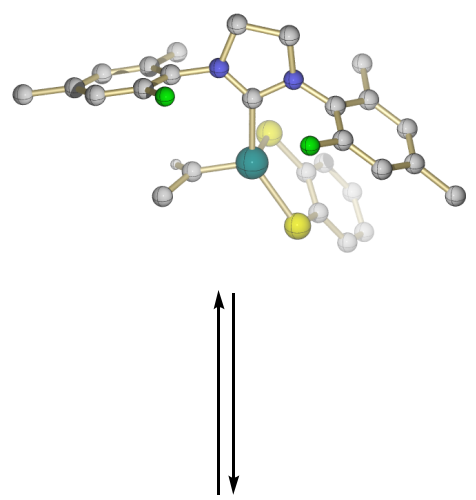

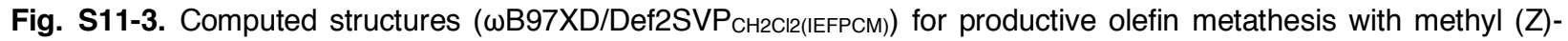
but-2-enoate. 14e, 14-electron intermediate; mcb, metallacyclobutane; ts1 and ts2, transition states for mcb formation and breakage. 


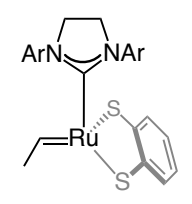

$14 \mathrm{e}$

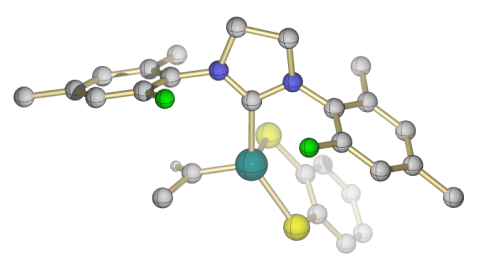

$\uparrow$

ts $1_{c}$
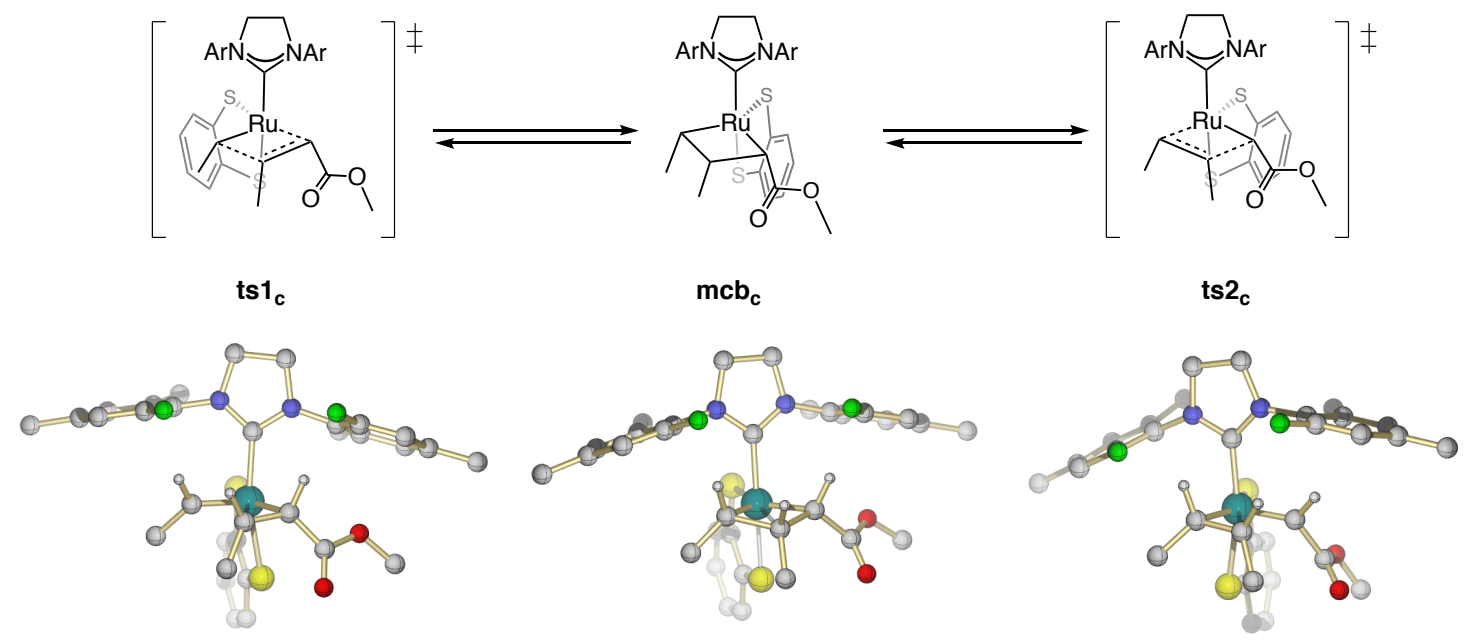

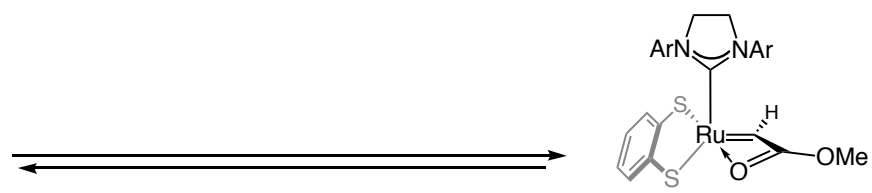

$16 e$

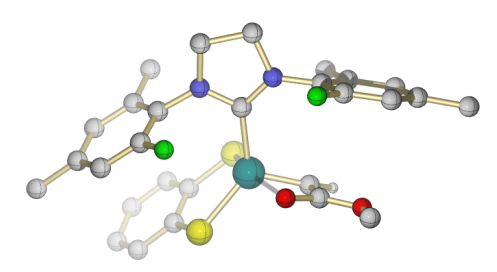

$\uparrow \mid$

Fig. S11-4. Computed structures ( $\left.\omega \mathrm{B} 97 \mathrm{XD} / \mathrm{Def}_{2} \mathrm{SVP}_{\mathrm{CH} 2 \mathrm{CI}(\mathrm{IEFPCM})}\right)$ for non-productive olefin metathesis with methyl (Z)-but-2-enoate. 14e, 14-electron intermediate; $\mathbf{m c b}$, metallacyclobutane; ts1 and ts2, transition states for mcb formation and breakage; 16e, 16-electron intermediate. 


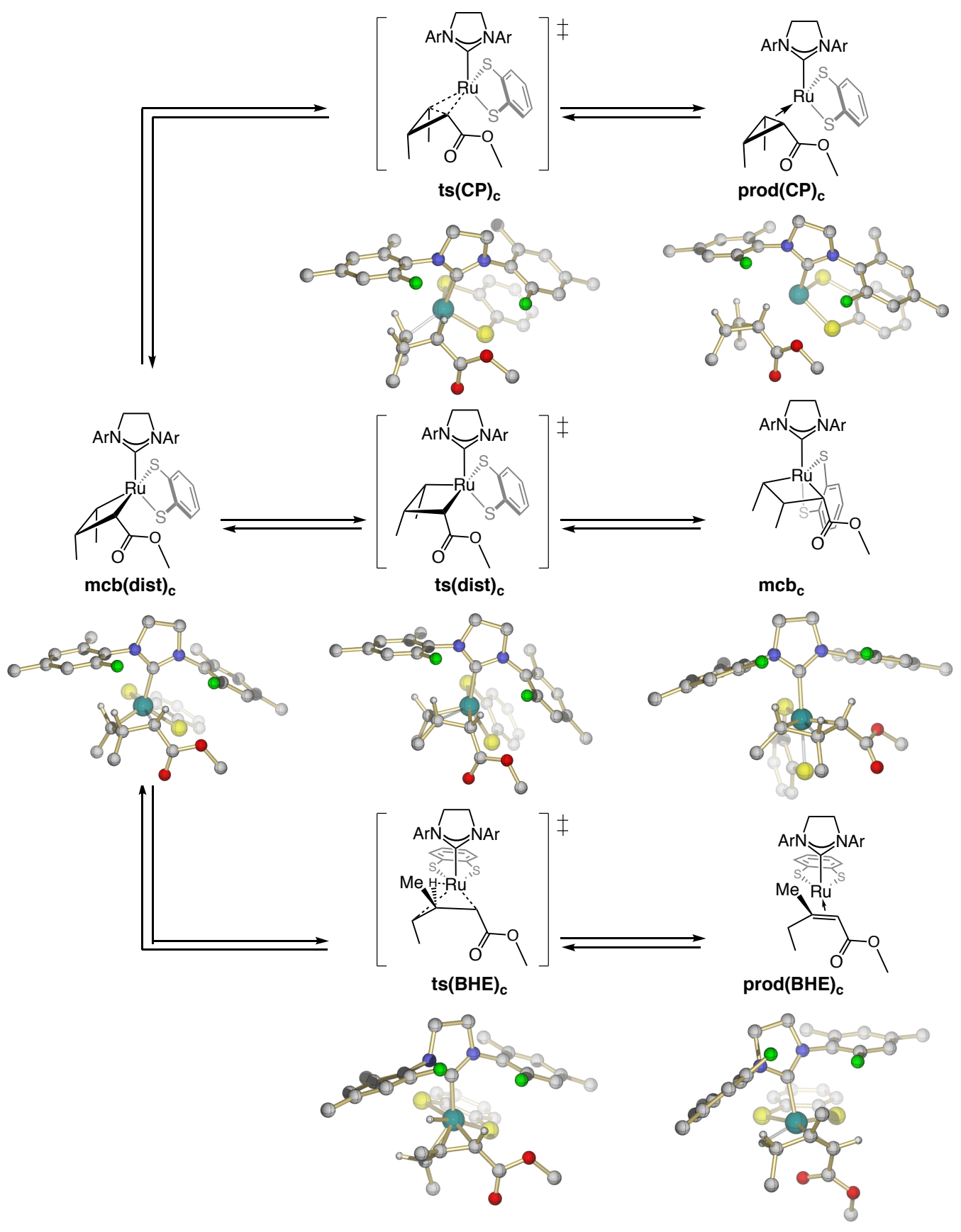

Fig. S11-5. Computed structures ( $\omega B$ B7XD/Def2SVP $\left.\mathrm{CH}_{2 \mathrm{CI}(\mathrm{IEFPCM})}\right)$ for decomposition of $\mathbf{m c b}_{\mathbf{c}}$ through $\beta$-hydride elimination and cyclopropanation. ts(CP) and $\operatorname{prod}(\mathbf{C P})$, transition state and product for cyclopropanation; ts(BHE) and prod(BHE), transition state and product for $\beta$-hydride elimination; mcb, metallacyclobutane; ts(dist), transition state for metallacyclobutane distortion; mcb(dist), distorted mcb after structural distortion. 


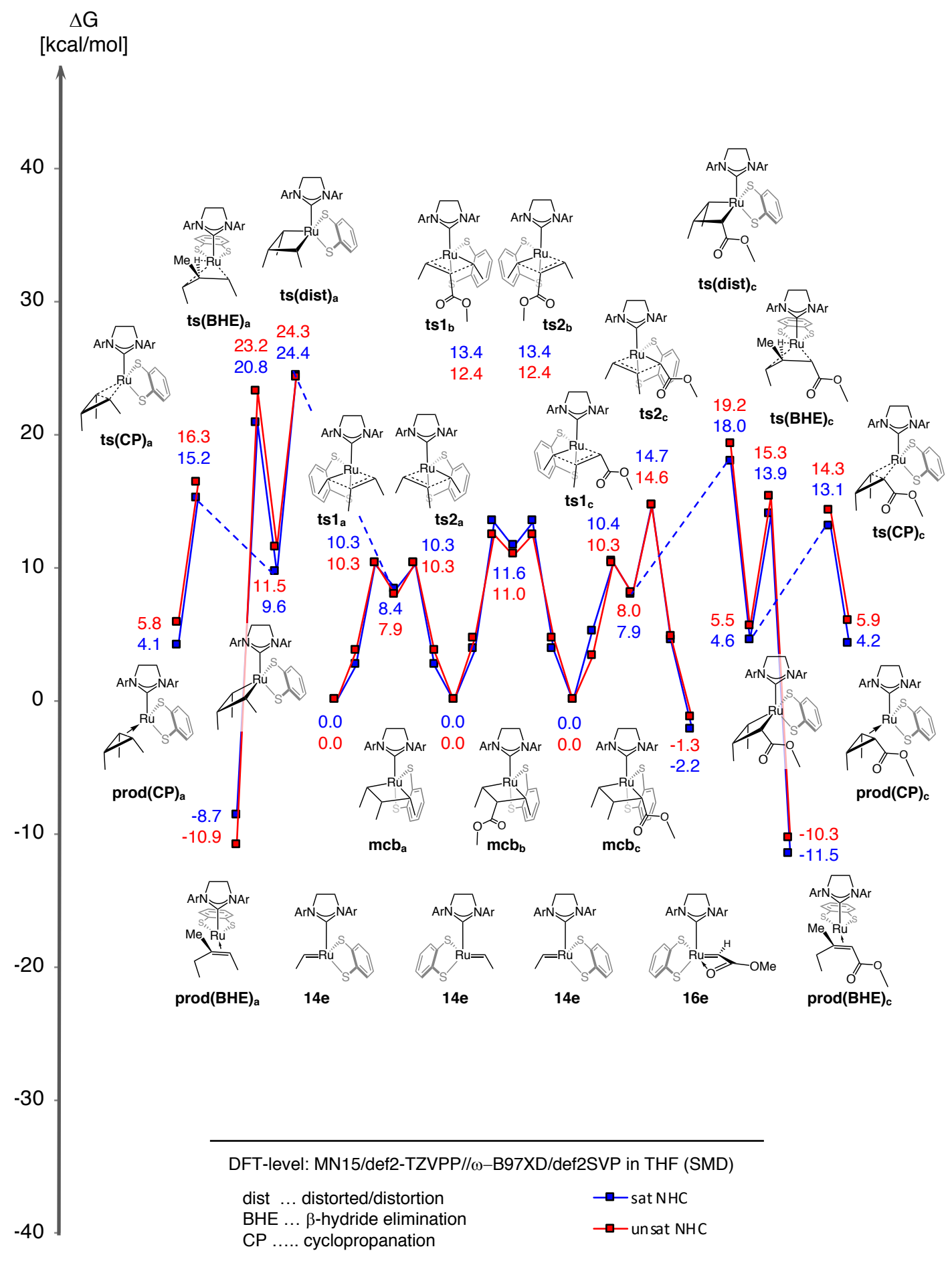

Fig. S11-6. Free energy surface ( $\Delta \mathrm{G}$ in $\mathrm{kcal} / \mathrm{mol}$ relative to 14e) for nonproductive olefin metathesis with cis-butene (index a), productive and nonproductive olefin metathesis with methyl (Z)-but-2-enoate (indices b and c), as well as decomposition through $\beta$-hydride elimination and cyclopropanation at the MN15/Def2TZVPP// $\omega B$ 97XD/ Def2SVP $\mathrm{CH}_{\mathrm{C} 2 \text { I2(SMD) }}$ level. ts(CP) and prod(CP), transition state and product for cyclopropanation; ts(BHE) and prod(BHE), transition state and product for $\beta$-hydride elimination; ts(dist), transition state for metallacyclobutane distortion; 14e, 14-electron intermediate; mcb, metallacyclobutane; ts1 and ts2, transition states for mcb formation and breakage; 16e, 16-electron intermediate. 


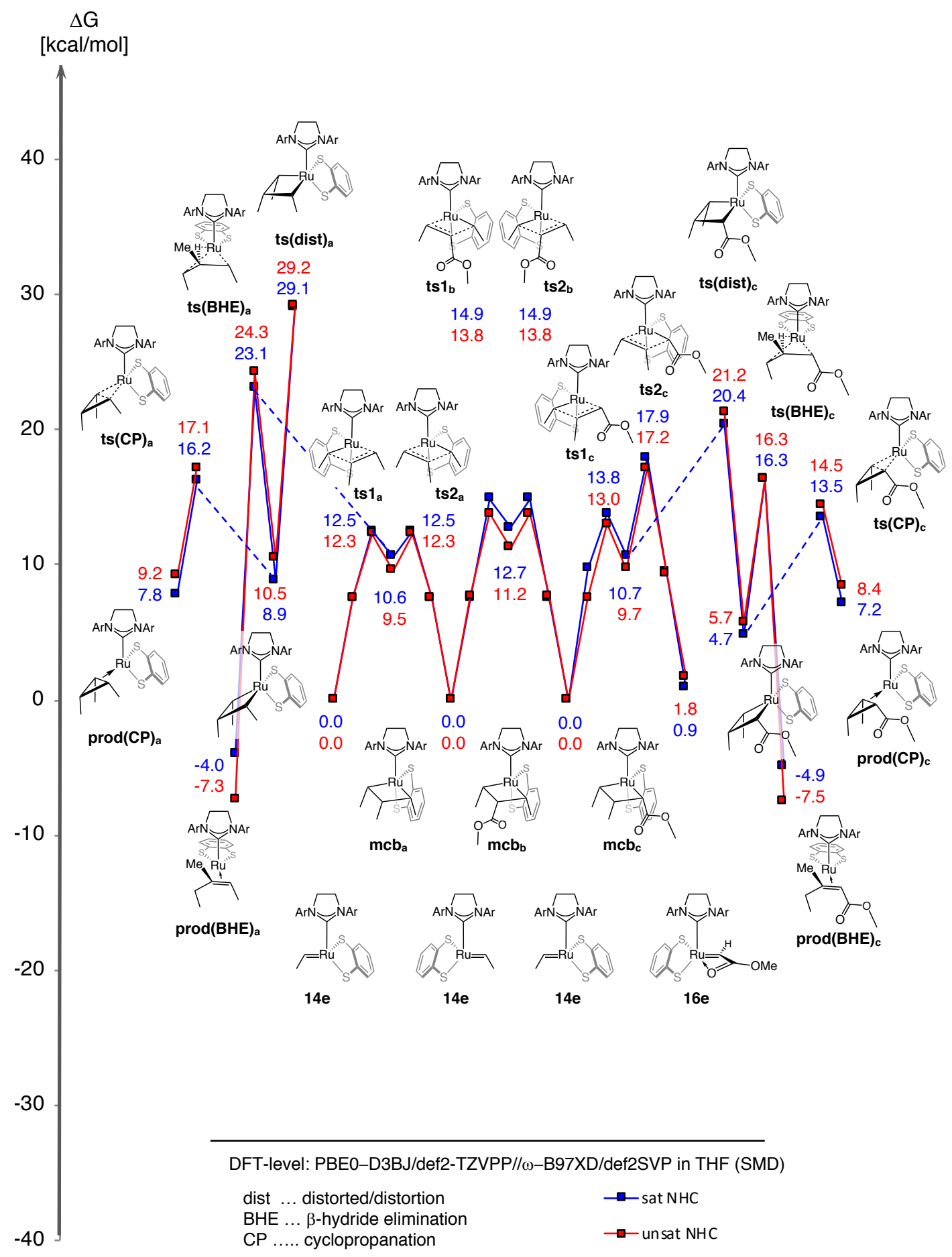

Fig. S11-7. Free energy surface ( $\Delta \mathrm{G}$ in $\mathrm{kcal} / \mathrm{mol}$ relative to $14 \mathrm{e}$ ) for nonproductive olefin metathesis with cis-butene (index a), productive and nonproductive olefin metathesis with methyl (Z)-but-2-enoate (indices b and c), as well as decomposition through $\beta$-hydride elimination and cyclopropanation at the PBE0-D3BJ/Def2TZVPP//

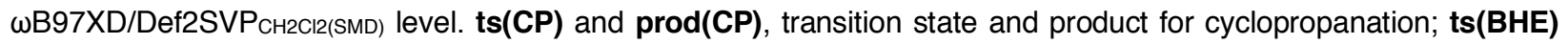
and prod(BHE), transition state and product for $\beta$-hydride elimination; ts(dist), transition state for metallacyclobutane distortion; 14e, 14-electron intermediate; mcb, metallacyclobutane; ts1 and ts2, transition states for mcb formation and breakage; 16e, 16-electron intermediate. 


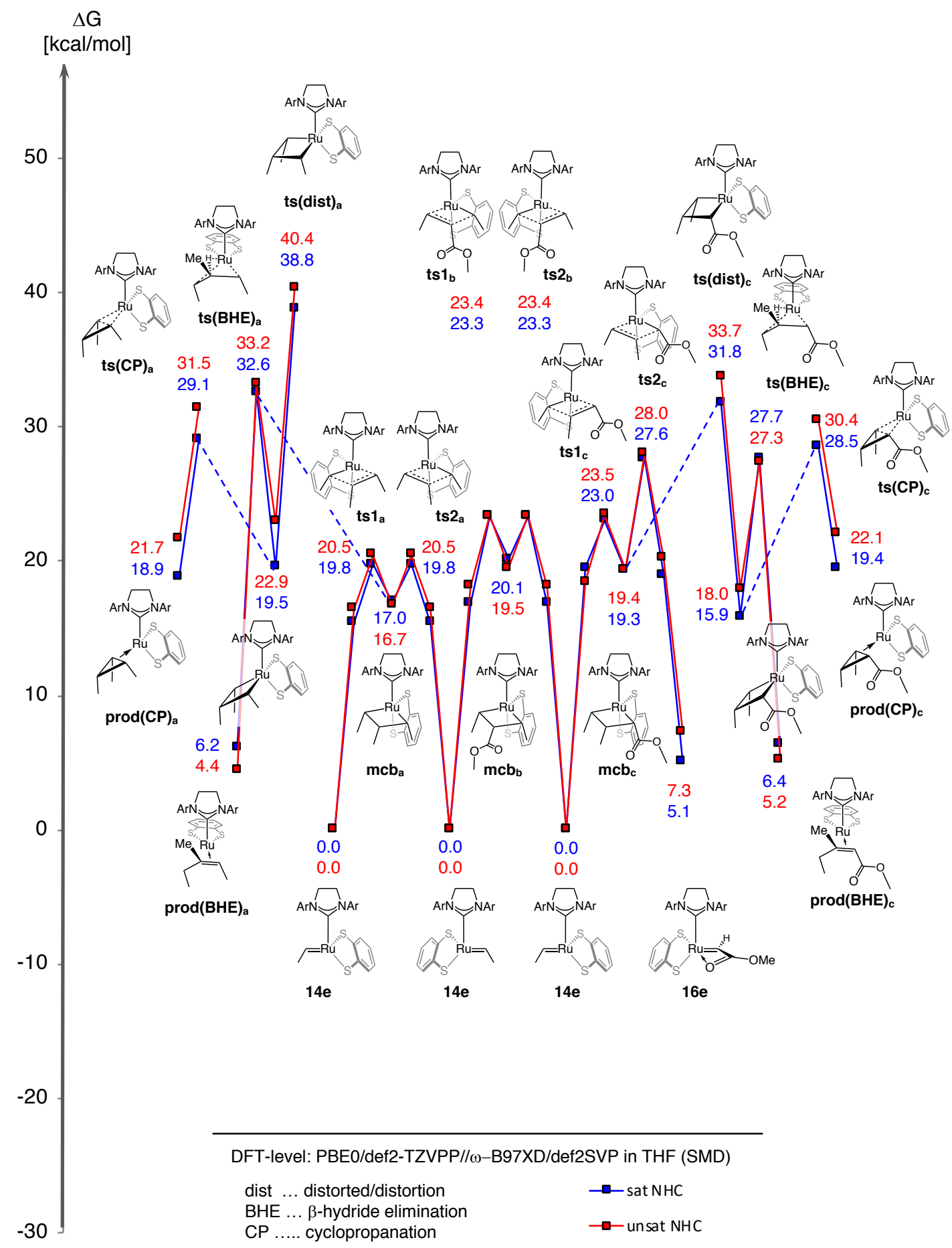

Fig. S11-8. Free energy surface ( $\Delta \mathrm{G}$ in $\mathrm{kcal} / \mathrm{mol}$ relative to $14 \mathrm{e})$ for nonproductive olefin metathesis with cis-butene (index a), productive and nonproductive olefin metathesis with methyl (Z)-but-2-enoate (indices b and c), as well as

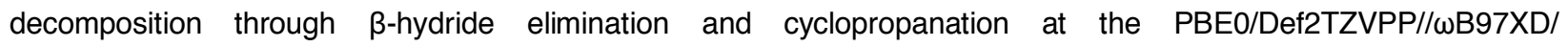

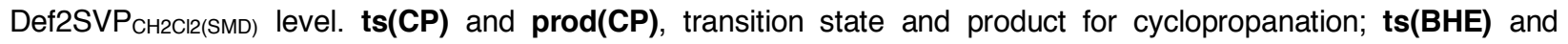
$\operatorname{prod}(\mathrm{BHE})$, transition state and product for $\beta$-hydride elimination; ts(dist), transition state for metallacyclobutane distortion; 14e, 14-electron intermediate; mcb, metallacyclobutane; ts1 and ts2, transition states for mcb formation and breakage; 16e, 16-electron intermediate. 


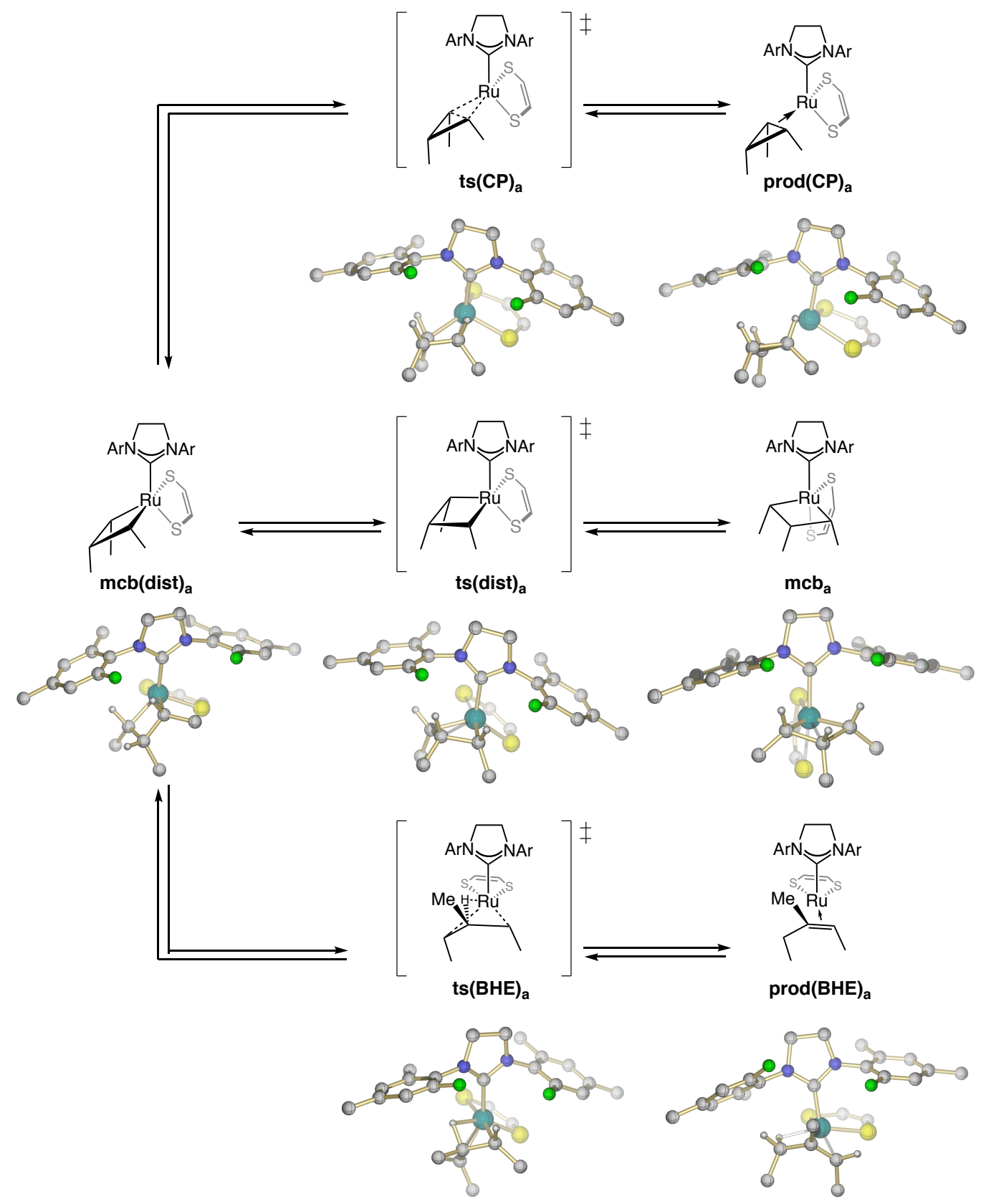

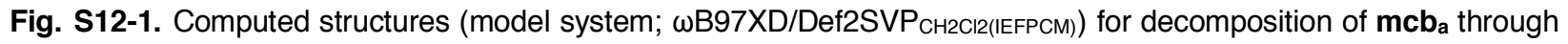
$\beta$-hydride elimination and cyclopropanation. ts(CP) and $\operatorname{prod}(\mathrm{CP})$, transition state and product for cyclopropanation; ts(BHE) and prod(BHE), transition state and product for $\beta$-hydride elimination; mcb, metallacyclobutane; ts(dist), transition state for metallacyclobutane distortion; $\mathbf{m c b}(\mathbf{d i s t})$, mcb after structural distortion. 


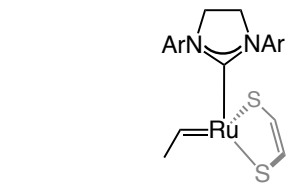

$14 \mathrm{e}$
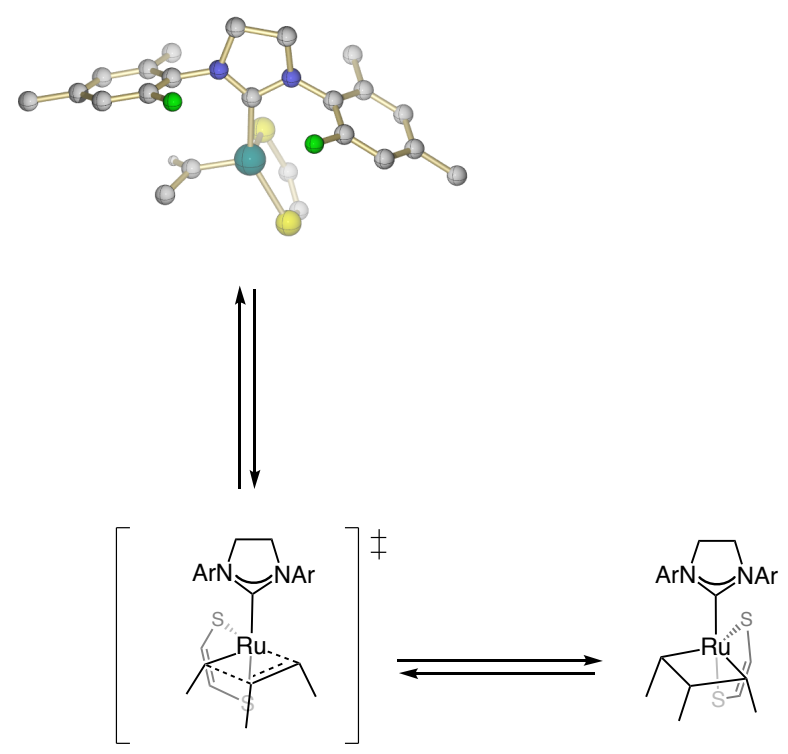

ts 1

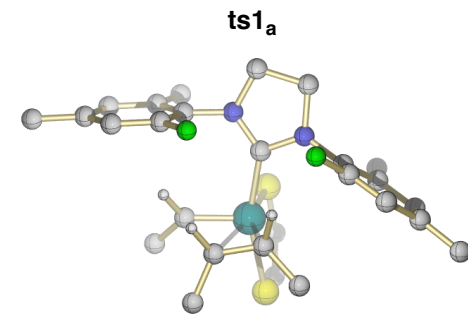

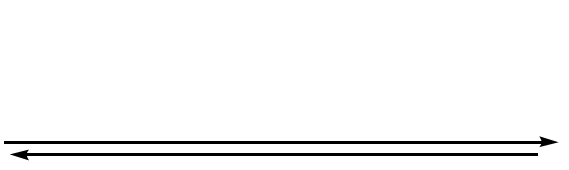

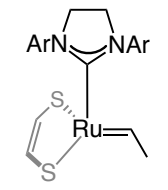

$14 \mathrm{e}$

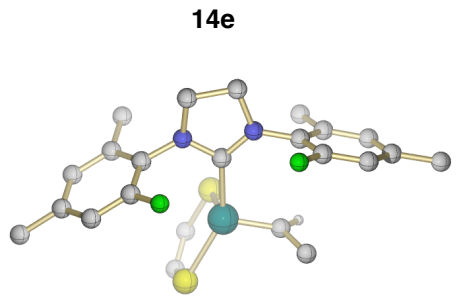

$\uparrow$
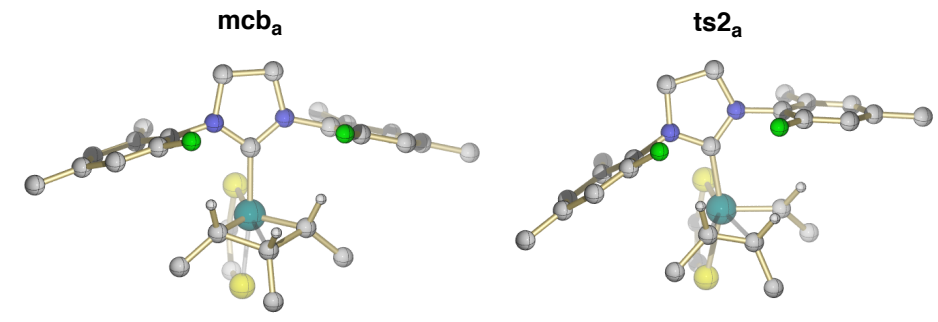

Fig. S12-2. Computed structures (model system; $\omega B 97 X \mathrm{XD} /$ Def2SVP $\mathrm{CH}_{\mathrm{CH} \text { (IIEFPM) }}$ ) for nonproductive olefin metathesis with cis-butene. 14e, 14-electron intermediate; mcb, metallacyclobutane; ts1 and ts2, transition states for mcb formation and breakage. 


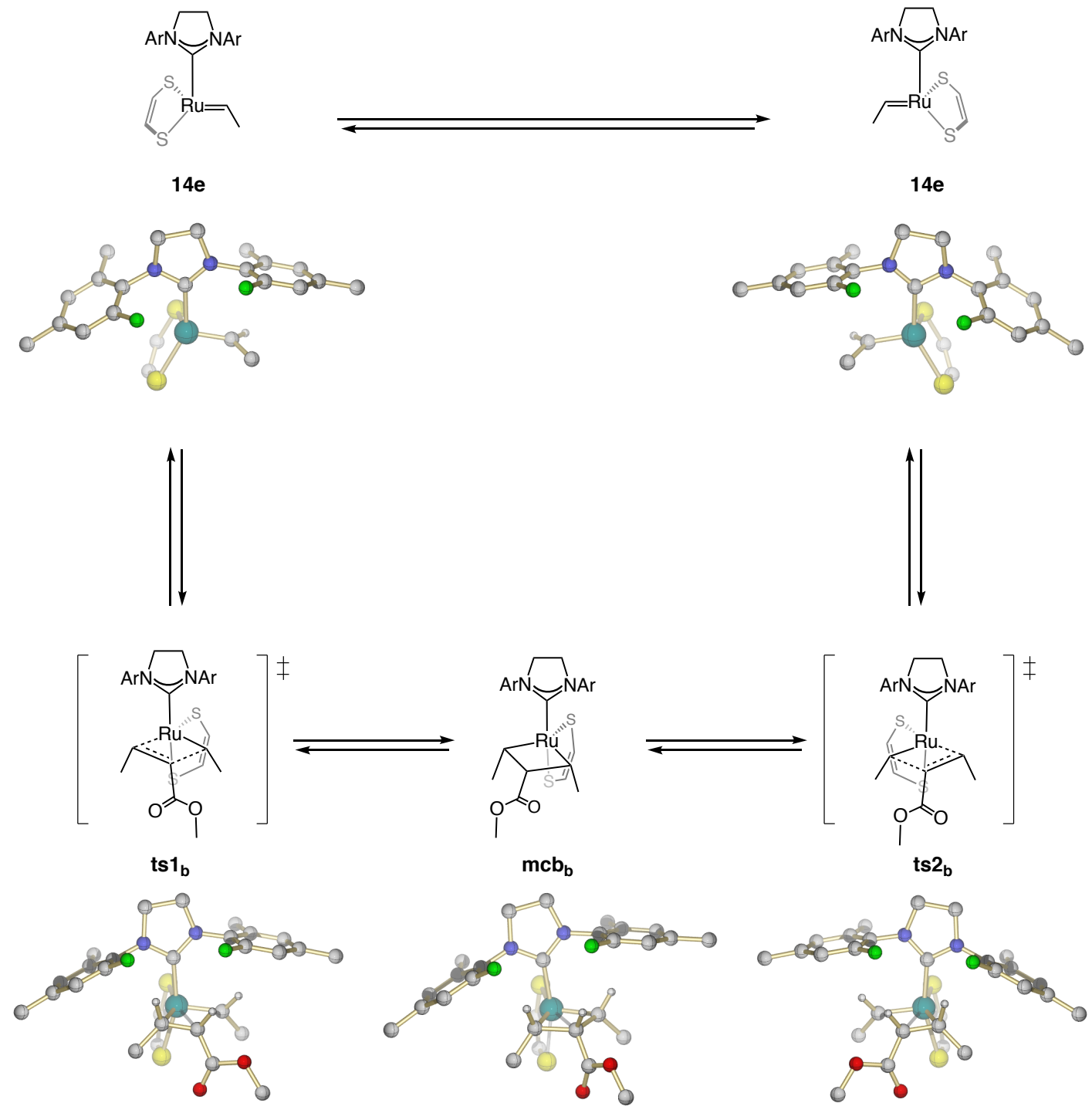

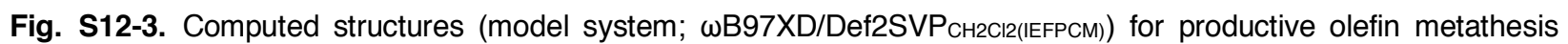
with methyl (Z)-but-2-enoate. 14e, 14-electron intermediate; mcb, metallacyclobutane; ts1 and ts2, transition states for mcb formation and cleavage. 

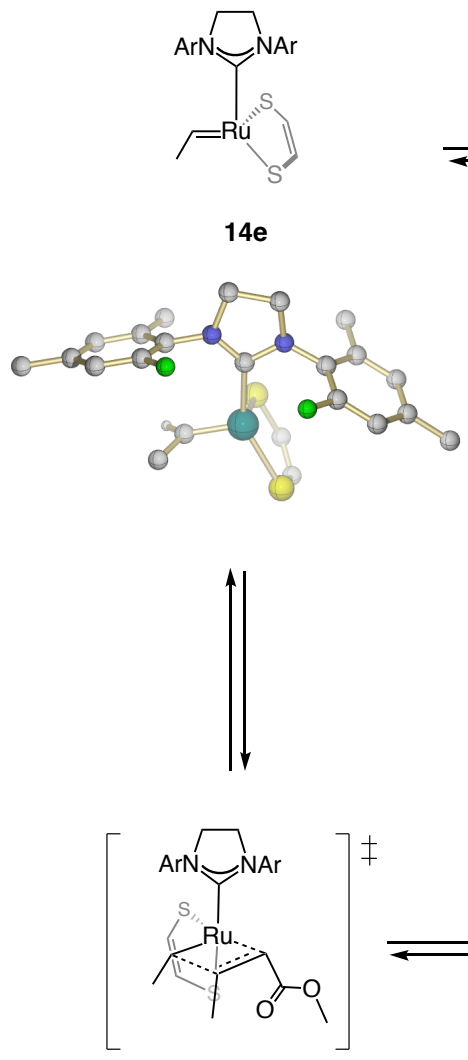

ts $1_{c}$
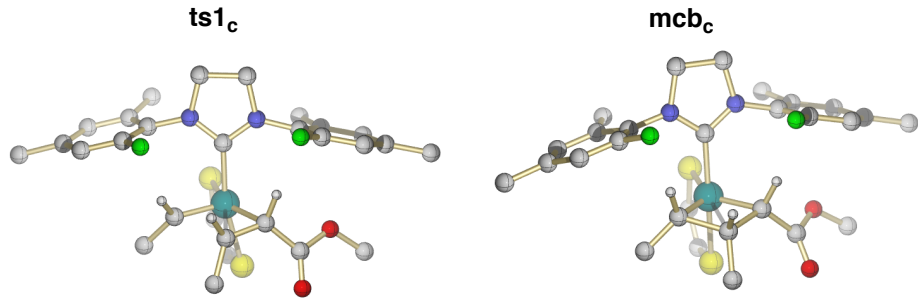
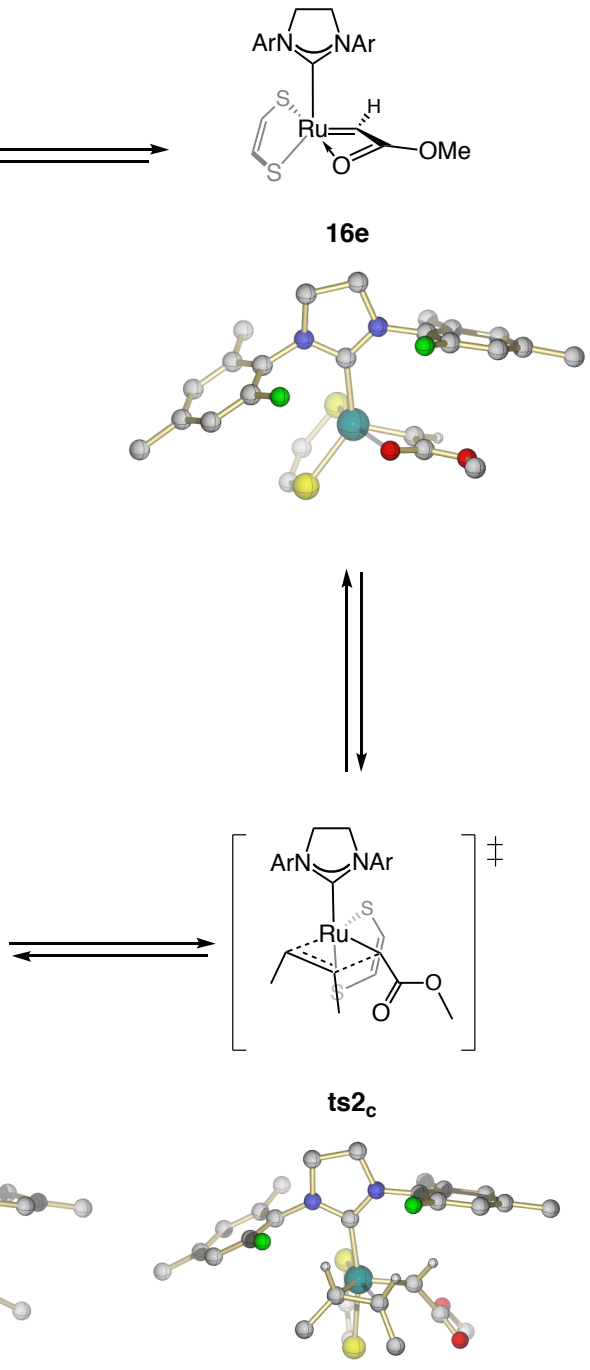

Fig. S12-4. Computed structures (model system; $\omega B$ 97XD/Def2SVPCH2CI2(IEFPCM)) for nonproductive OM with methyl (Z)-but-2-enoate. 14e, 14-electron intermediate; mcb, metallacyclobutane; ts1 and ts2, transition states for mcb formation and breakage; 16e, 16-electron intermediate. 


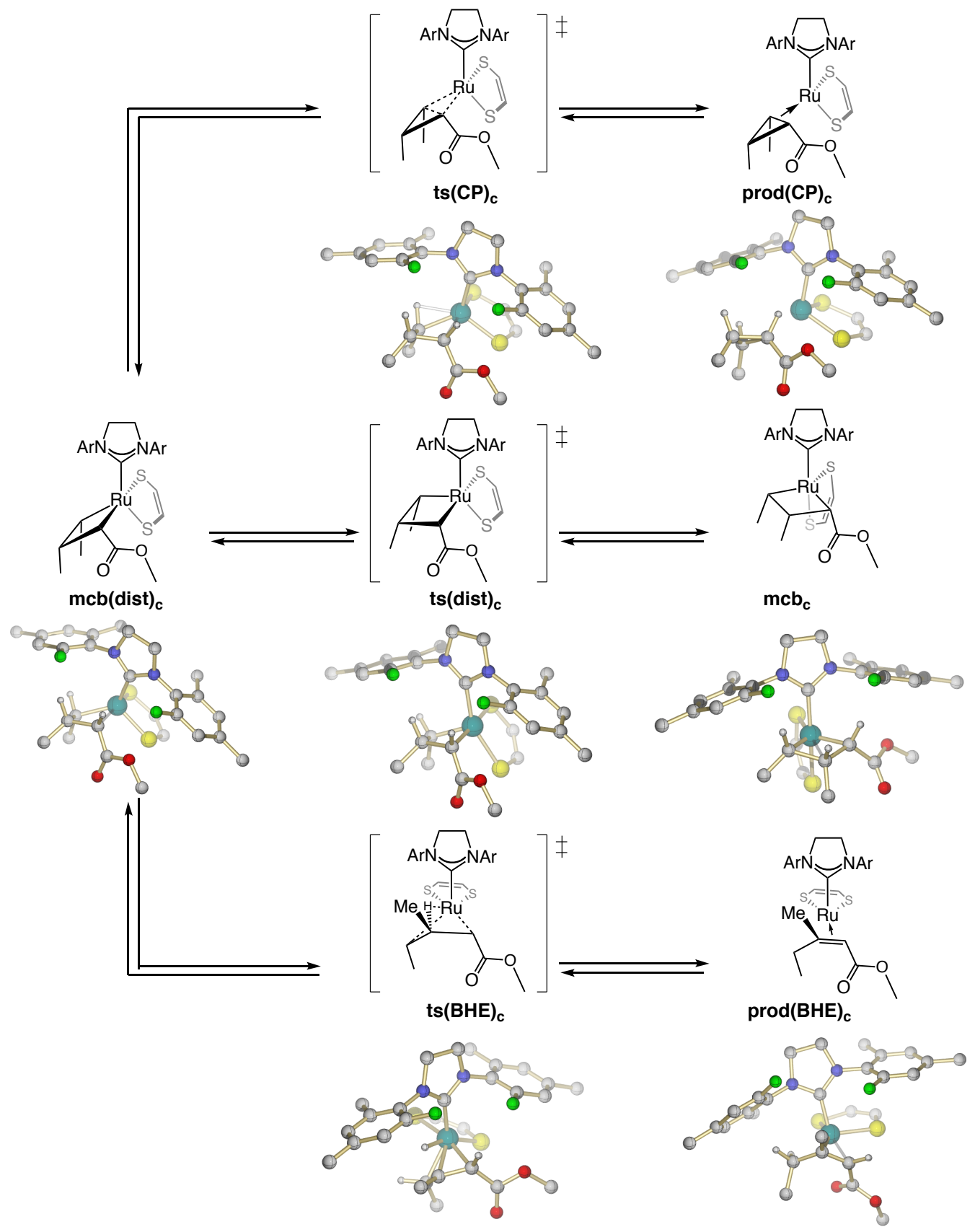

Fig. S12-5. Computed structures (model system; $\omega B 97 X D / D e f 2 S V P_{\mathrm{CH}_{2 C I}(\mathrm{IEFPCM})}$ ) for decomposition of mcb through $\beta$-hydride elimination and cyclopropanation. ts(CP) and $\operatorname{prod}(\mathrm{CP})$, transition state and product for cyclopropanation; ts(BHE) and prod(BHE), transition state and product for $\beta$-hydride elimination; mcb, metallacyclobutane; ts(dist), transition state for metallacyclobutane distortion; $\mathbf{m c b}(\mathbf{d i s t})$, mcb after structural distortion. 


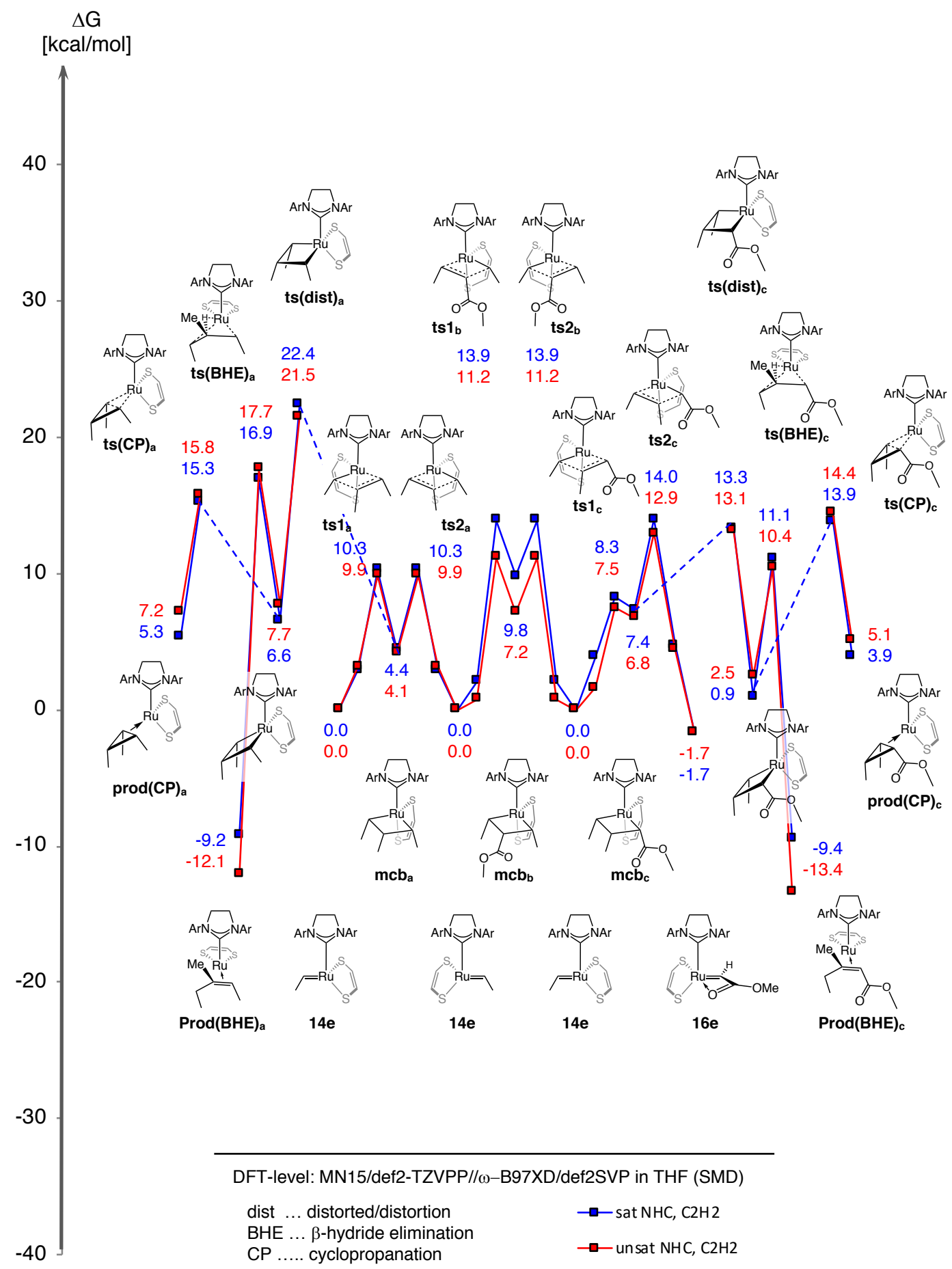

Fig. S12-6. Free energy surface (model system; $\Delta \mathrm{G}$ in $\mathrm{kcal} / \mathrm{mol}$ relative to $14 \mathrm{e}$ ) for nonproductive olefin metathesis with cis-butene (index a), productive and nonproductive olefin metathesis with methyl (Z)-but-2-enoate (indices $b$ and c), as well as decomposition through $\beta$-hydride elimination and cyclopropanation at the MN15/Def2TZVPP// $\omega B$ 97XD/ Def2SVP ${ }_{\mathrm{CH} 2 \mathrm{Cl} 2(\mathrm{SMD})}$ level. ts(CP) and prod(CP), transition state and product for cyclopropanation; ts(BHE) and $\operatorname{prod}(\mathrm{BHE})$, transition state and product for $\beta$-hydride elimination; ts(dist), transition state for metallacyclobutane distortion; 14e, 14-electron intermediate; mcb, metallacyclobutane; ts1 and ts2, transition states for mcb formation and breakage; 16e, 16-electron intermediate. 


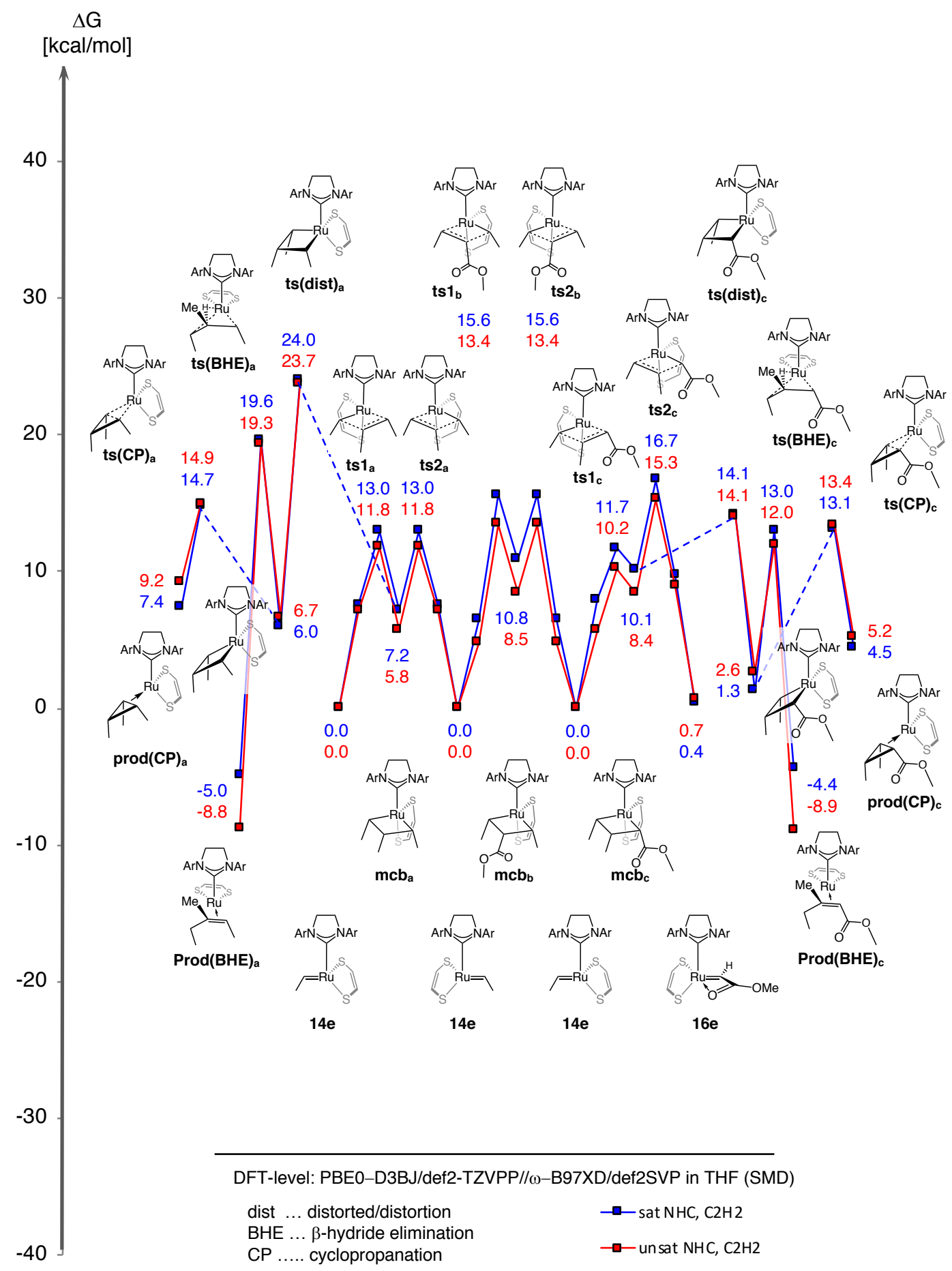

Fig. S12-7. Free energy surface (model system; $\Delta \mathrm{G}$ in $\mathrm{kcal} / \mathrm{mol}$ relative to $14 \mathrm{e}$ ) for nonproductive olefin metathesis with cis-butene (index a), productive and nonproductive olefin metathesis with methyl (Z)-but-2-enoate (indices $b$ and c), as well as decomposition through $\beta$-hydride elimination and cyclopropanation at the PBE0-

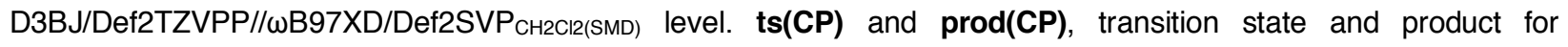
cyclopropanation; ts(BHE) and prod(BHE), transition state and product for $\beta$-hydride elimination; ts(dist), transition state for metallacyclobutane distortion; 14e, 14-electron intermediate; mcb, metallacyclobutane; ts1 and ts2, transition states for mcb formation and breakage; 16e, 16-electron intermediate. 


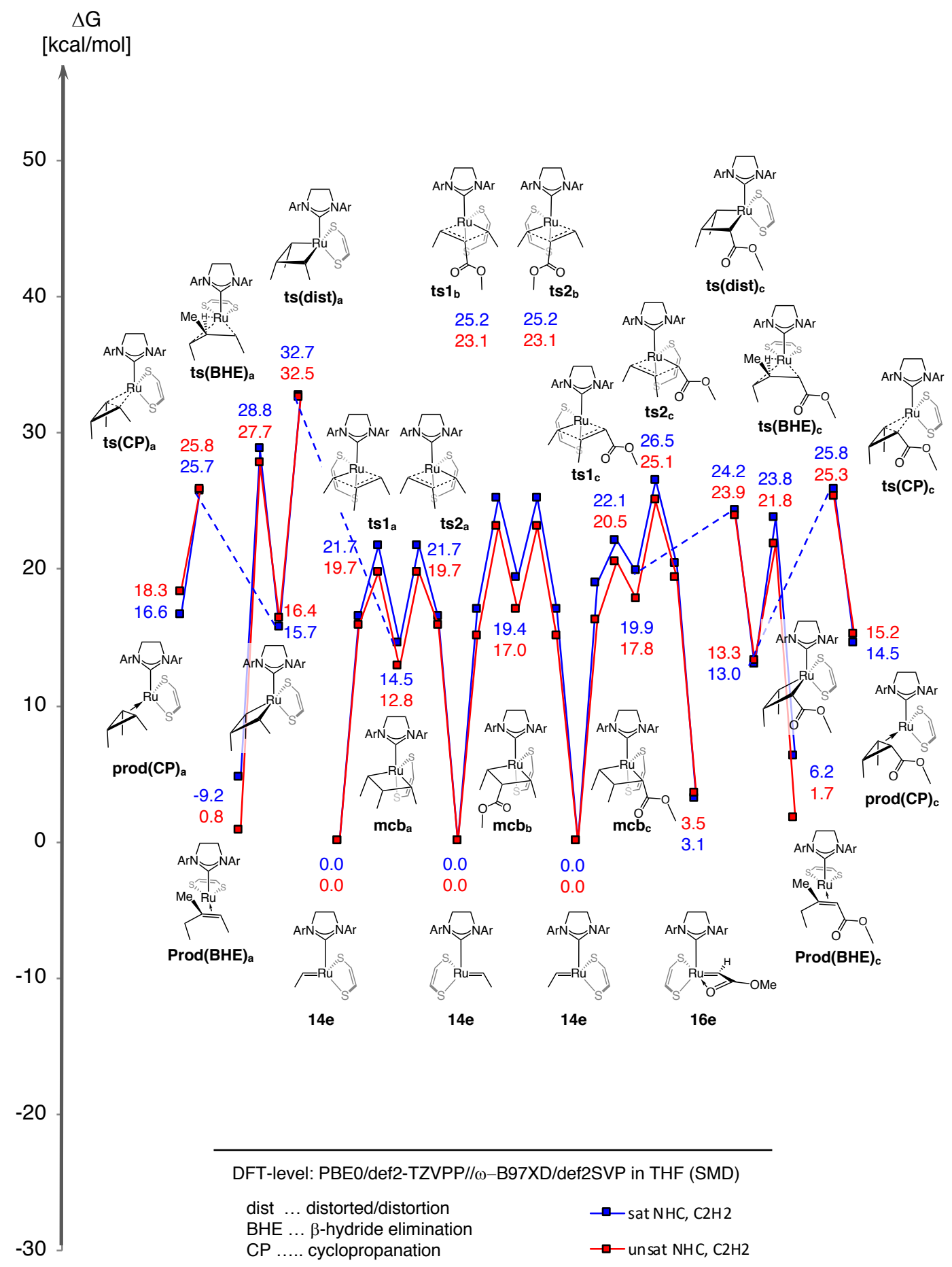

Fig. S12-8. Free energy surface (model system; $\Delta \mathrm{G}$ in $\mathrm{kcal} / \mathrm{mol}$ relative to $14 \mathrm{e}$ ) for nonproductive olefin metathesis with cis-butene (index a), productive and nonproductive olefin metathesis with methyl (Z)-but-2-enoate (indices $b$ and c), as well as decomposition through $\beta$-hydride elimination and cyclopropanation at the PBE0/Def2TZVPP//WB97XD/ Def2SVP ${ }_{\mathrm{CH} 2 \mathrm{Cl} 2(\mathrm{SMD})}$ level. ts(CP) and $\operatorname{prod}(\mathbf{C P})$, transition state and product for cyclopropanation; ts(BHE) and $\operatorname{prod(BHE),~transition~state~and~product~for~} \beta$-hydride elimination; ts(dist), transition state for metallacyclobutane distortion; 14e, 14-electron intermediate; mcb, metallacyclobutane; ts1 and ts2, transition states for mcb formation and breakage; 16e, 16-electron intermediate. 


\subsection{Origin of Reactivity Difference between Ru-1c and Ru-1d}

\subsubsection{Evolution of trans Influence in the Sequence $14 \mathrm{e} \rightarrow \mathbf{t s}_{\mathbf{b}} \rightarrow$ mcb $_{\mathbf{b}}$}

Analysis of bond lengths in the DFT optimized structures ( $\omega$ B97XD/Def2SVP $\left.{ }_{\text {THF(SMD) }}\right)$ supports the hypothesis that the saturated NHC ligand in Ru-1c does exert a stronger trans influence (Fig. S13-1a). While trans influence is avoided in the 14-electron intermediate (14e), as indicated by the small $\mathrm{C}^{\mathrm{NHC}}-\mathrm{Ru}-\mathrm{S}($ trans $)$ angle $\left(143.9^{\circ}\right)$, the $\mathrm{Ru}-\mathrm{S}($ trans $)$ bond length increases during transition from $\mathbf{1 4 e} \rightarrow \mathbf{t s}_{\mathbf{b}} \rightarrow \mathbf{m c b}_{\mathbf{b}}(2.336 \rightarrow 2.405 \rightarrow 2.423 \AA)$. While a similar trend is observed for $\mathbf{R u - 1 d}(2.330 \rightarrow 2.400 \rightarrow 2.418 \AA$, values in grey box $)$, the $\mathrm{Ru}-$ $\mathrm{S}$ (trans) bond length is shorter by $0.005 \AA$ on average. In contrast, the $\mathrm{Ru}-\mathrm{C}^{\mathrm{NHC}}$ bond lengths are longer in the intermediates and transition states derived from Ru-1d $(2.062 \rightarrow 2.103 \rightarrow 2.122 \AA$ vs $2.041 \rightarrow 2.094 \rightarrow 2.105 \AA$ for Ru-1c). A similar trend is observed with the smaller model systems (Ru-1 $\mathbf{c}_{\text {model }}$ and Ru-1d $\mathbf{d}_{\text {model, }}$ Fig. S13-1b).

\subsubsection{Factors Governing Metallacyclobutane Distortion (mcb dist )}

The structural distortion from $\mathbf{m c b}_{\mathbf{a}} \rightarrow \mathbf{t s}(\mathbf{d i s t})_{\mathbf{a}} \rightarrow \mathbf{m c b}(\mathbf{d i s t})_{\mathbf{a}}$ involves the interconversion of two metallacyclobutane intermediates, which can both be described by a trigonal bipyramidal geometry (TBP). This process is likely facilitated by lowering of trans influence that exists between the $\mathrm{NHC}$ ligand $\left(\mathrm{C}^{\mathrm{NHC}}\right)$ and the sulfur in trans position ( $\mathrm{S}($ trans $)$ ). While the

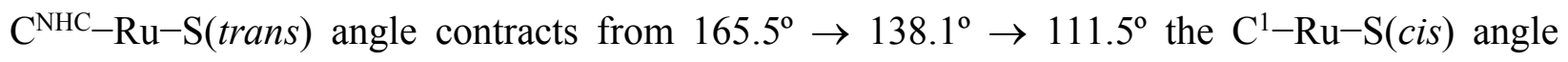
expands $\left(126.9^{\circ} \rightarrow 160.1^{\circ} \rightarrow 166.1^{\circ}\right.$; Fig. S13-2a). Similar trends are observed for the sequence $\mathbf{m c b}_{\mathbf{c}} \rightarrow \mathbf{t s}(\mathbf{d i s t})_{\mathbf{c}} \rightarrow \mathbf{m c b}(\mathbf{d i s t})_{\mathbf{c}}$, with the distinction that the $\mathrm{C}^{1-}-\mathrm{Ru}-\mathrm{S}($ cis $)$ angle can adopt an almost perfectly linear geometry $\left(140.4^{\circ} \rightarrow 179.1^{\circ} \rightarrow 173.8^{\circ}\right.$; Fig. S13-2b). This highlights the reduced trans influence between $\mathrm{C}^{1}$ and $\mathrm{S}($ trans $)$ and underscores the greater propensity of $\mathbf{m c b}_{\mathbf{c}}$ to undergo rearrangement. For the corresponding structural parameters in the smaller model systems (Ru-1 $\mathbf{c}_{\text {model }}$ and Ru-1d $\mathbf{d}_{\text {model }}$ ) (see Fig. 13-3).

\subsubsection{Transition States for $\boldsymbol{\beta}$-Hydride Elimination}

The geometries for transition states for $\beta$-hydride elimination are displayed in Fig. S13-4. For example, during transition from $\mathbf{m c b}(\text { dist })_{\mathbf{a}} \rightarrow \mathbf{t s}(\mathbf{B H E})_{\mathbf{a}}$ the $\mathrm{C}^{3}-\mathrm{Ru}-\mathrm{C}^{\mathrm{NHC}}$ angle undergoes a minor expansion $\left(121.5^{\circ} \rightarrow 131.0^{\circ}\right.$; Fig. S13-4a) through which an open ligation site for transfer of the $\beta$-hydride is created. Since ts(BHE) is structurally related to the distorted metallacyclobutane $\mathbf{m c b}(\mathbf{d i s t}) \mathbf{c}$, the transition state for $\beta$-hydride elimination is lowered in energy because the trans influence between $\mathrm{C}^{1}$ and $\mathrm{S}($ cis $)$ is weaker (Fig. S13-4b). For the corresponding structural parameters in the smaller model systems (Ru-1 $\mathbf{c}_{\text {model }}$ and Ru-1d $\mathbf{d}_{\text {model }}$ ), see Fig. S13-5. 
a Change of structural parameters during productive $\mathrm{OM}$

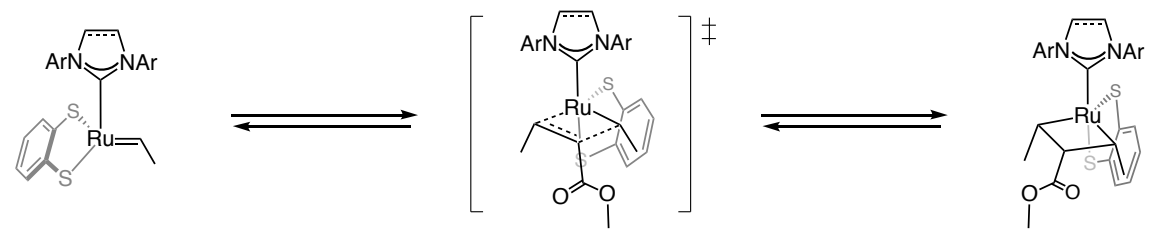

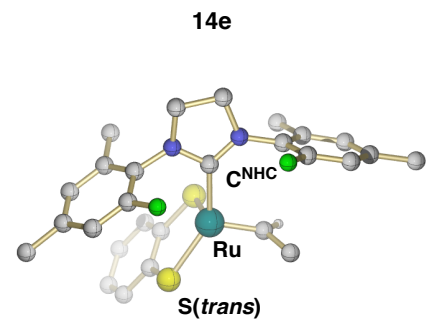

$\mathrm{Ru}-\mathrm{C}^{\mathrm{NHC}}$ $2.041 \AA$

Ru-S(trans) .......... $2.336 \AA$ $\mathrm{C}^{\mathrm{NHC}}-\mathrm{Ru}-\mathrm{S}\left(\right.$ trans) $\ldots 143.9^{\circ}$

$\mathrm{Ru}-\mathrm{C}^{\mathrm{NHC}} \ldots \ldots \ldots \ldots . . . . .2 .062 \AA$

Ru-S(trans) .......... $2.330 \AA$

$\mathrm{C}^{\mathrm{NHC}}-\mathrm{Ru}-\mathrm{S}\left(\right.$ trans) $\ldots 148.8^{\circ}$

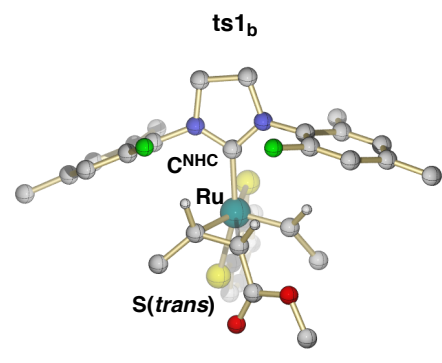

$\mathrm{Ru}-\mathrm{C}^{\mathrm{NHC}}$

$\mathrm{Ru}-\mathrm{S}($ trans)........... $2.405 \AA$ $\mathrm{C}^{\mathrm{NHC}}-\mathrm{Ru}-\mathrm{S}\left(\right.$ trans)... $160.9^{\circ}$

$\mathrm{Ru}-\mathrm{C}^{\mathrm{NHC}} \ldots \ldots \ldots \ldots . .2 .103 \AA$

$\mathrm{Ru}-\mathrm{S}$ (trans).......... $2.400 \AA$

$\mathrm{C}^{\mathrm{NHC}}-\mathrm{Ru}-\mathrm{S}\left(\right.$ trans)... $162.2^{\circ}$

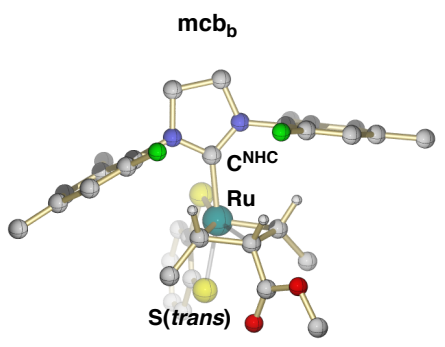

$\mathrm{Ru}-\mathrm{C}^{\mathrm{NHC}}$ $2.105 \AA$

Ru-S(trans) .......... $2.423 \AA$ $\mathrm{C}^{\mathrm{NHC}}-\mathrm{Ru}-\mathrm{S}\left(\right.$ trans)... $163.5^{\circ}$

$\mathrm{Ru}-\mathrm{C}^{\mathrm{NHC}} \ldots \ldots \ldots \ldots . .122 \AA$

$\mathrm{Ru}-\mathrm{S}($ trans) .......... $2.418 \AA$ $\mathrm{C}^{\mathrm{NHC}}-\mathrm{Ru}-\mathrm{S}\left(\right.$ trans)... $166.4^{\circ}$

b Change of structural parameters during productive OM (model system)<smiles></smiles>

model_14e

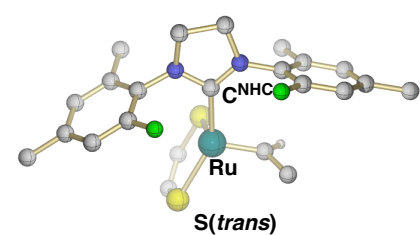

$\mathrm{Ru}-\mathrm{C}^{\mathrm{NHC}}$ .. $2.052 \AA$

Ru-S(trans) ........... $2.342 \AA$

$\mathrm{C}^{\mathrm{NHC}}-\mathrm{Ru}-\mathrm{S}\left(\right.$ trans) $\ldots 148.1^{\circ}$

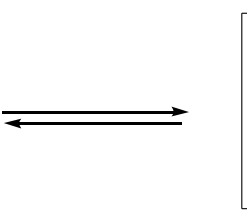

L

model_ts $1_{b}$

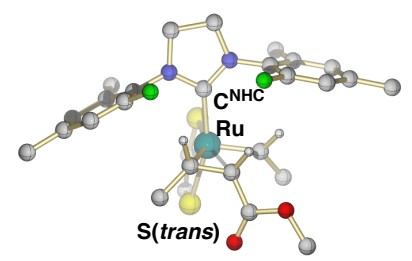

$\mathrm{Ru}-\mathrm{C}^{\mathrm{NHC}}$

$2.091 \AA$

$\mathrm{Ru}-\mathrm{S}($ trans)

$2.410 \AA$

$\mathrm{C}^{\mathrm{NHC}}-\mathrm{Ru}-\mathrm{S}\left(\right.$ trans) $\ldots 160.2^{\circ}$

$\mathrm{Ru}-\mathrm{C}^{\mathrm{NHC}} \ldots \ldots \ldots \ldots . . . .2 .104 \AA$

$\mathrm{Ru}-\mathrm{S}($ trans) ........... $2.399 \AA$

$\mathrm{C}^{\mathrm{NHC}}-\mathrm{Ru}-\mathrm{S}\left(\right.$ trans) $\ldots 162.5^{\circ}$

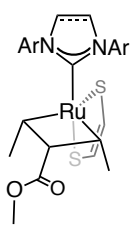

model_mcb $b_{b}$

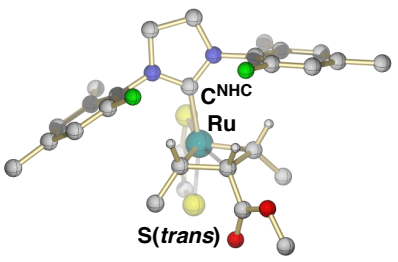

$\mathrm{Ru}-\mathrm{C}^{\mathrm{NHC}}$ $2.107 \AA$

$\mathrm{Ru}-\mathrm{S}$ (trans) ........... 2.421

$\mathrm{C}^{\mathrm{NHC}}-\mathrm{Ru}-\mathrm{S}\left(\right.$ trans) $\ldots 164.0^{\circ}$

$\mathrm{Ru}-\mathrm{C}^{\mathrm{NHC}} \ldots \ldots \ldots \ldots . . .2 .125 \AA$

$\mathrm{Ru}-\mathrm{S}($ trans) $\ldots \ldots \ldots \ldots . .2 .415 \AA$ $\mathrm{C}^{\mathrm{NHC}}-\mathrm{Ru}-\mathrm{S}\left(\right.$ trans) $\ldots \quad 167.0^{\circ}$

Fig. S13-1. Evolution of trans influence during productive olefin metathesis (index b) for real system (top) and smaller

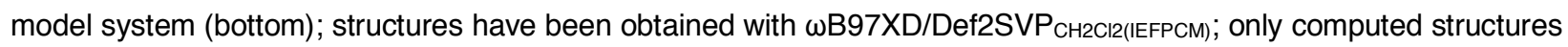
for complexes bearing saturated $\mathrm{NHC}$ ligands are displayed; bond lengths $(\AA)$ and angles $\left(^{\circ}\right)$ for corresponding analogues with unsaturated $\mathrm{NHC}$ are given in grey boxes. 14e, 14-electron intermediate; ts1, transition states for mcb formation; mcb, metallacyclobutane. 
a Change of structural parameters during metallacyclobutane distortion of $\mathbf{~ m c b}_{\mathbf{a}}$

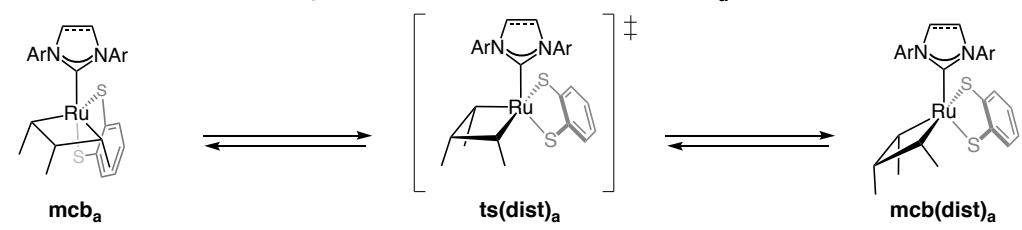

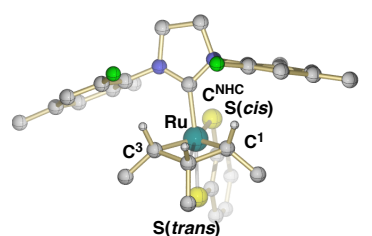

$\mathrm{Ru}-\mathrm{C}^{\mathrm{NHC}}$

$2.096 \AA$

$\mathrm{Ru}-\mathrm{S}($ trans).......... $2.425 \AA$ $\mathrm{C}^{\mathrm{NHC}}-\mathrm{Ru}-\mathrm{S}\left(\right.$ trans)... $165.6^{\circ}$

$\mathrm{C}^{1}-\mathrm{Ru}-\mathrm{S}\left(\right.$ cis)......... $126.9^{\circ}$

$\mathrm{C}^{3}-\mathrm{Ru}-\mathrm{C}^{\mathrm{NHC}} \ldots \ldots \ldots . . .89 .9^{\circ}$

$\mathrm{Ru}-\mathrm{C}^{\mathrm{NHC}} \ldots \ldots \ldots \ldots . . . . .2 .106 \AA$

$\mathrm{Ru}-\mathrm{S}$ (trans)........... $2.424 \AA$

$\mathrm{C}^{\mathrm{NHC}}-\mathrm{Ru}-\mathrm{S}\left(\right.$ trans)... $169.1^{\circ}$

$\mathrm{C}^{1}-\mathrm{Ru}-\mathrm{S}(\mathrm{cis}) \ldots \ldots \ldots .133 .3^{\circ}$

$\mathrm{C}^{3}-\mathrm{Ru}-\mathrm{C}^{\mathrm{NHC}}$......... $90.2^{\circ}$

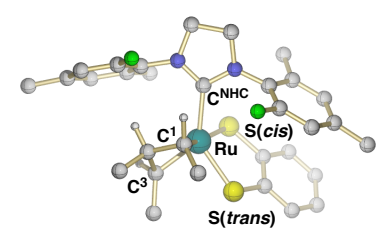

$\mathrm{Ru}-\mathrm{C}^{\mathrm{NHC}}$

$2.154 \AA$

$\mathrm{Ru}-\mathrm{S}($ trans).......... $2.309 \AA$

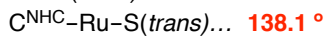

$\mathrm{C}^{1}-\mathrm{Ru}-\mathrm{S}$ (cis)......... 160.1 ${ }^{\circ}$

$\mathrm{C}^{3}-\mathrm{Ru}-\mathrm{C}^{\mathrm{NHC}} \ldots \ldots \ldots 130.6^{\circ}$

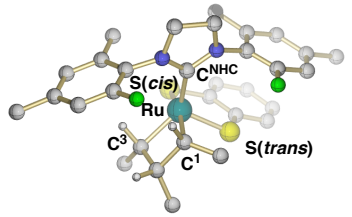

$\mathrm{Ru}-\mathrm{C}^{\mathrm{NHC}}$ $1.994 \AA$

$\mathrm{Ru}-\mathrm{S}$ (trans).......... 2.200 $\AA$ $\mathrm{C}^{\mathrm{NHC}}-\mathrm{Ru}-\mathrm{S}$ (trans)... $111.5^{\circ}$

$\mathrm{C}^{1}-\mathrm{Ru}-\mathrm{S}\left(\right.$ cis)......... $166.1^{\circ}$

$\mathrm{C}^{3}-\mathrm{Ru}-\mathrm{C}^{\mathrm{NHC}} \ldots \ldots \ldots 121.5^{\circ}$

$\mathrm{Ru}-\mathrm{C}^{\mathrm{NHC}} \ldots \ldots \ldots \ldots . . . .2 .009 \AA$

Ru-S(trans)........... 2.200 ^ $\mathrm{C}^{\mathrm{NHC}}-\mathrm{Ru}-\mathrm{S}\left(\right.$ trans)... $110.4^{\circ}$ $\mathrm{C}^{1}-\mathrm{Ru}-\mathrm{S}($ cis $) \ldots \ldots \ldots .166 .3^{\circ}$ $\mathrm{C}^{3}-\mathrm{Ru}-\mathrm{C}^{\mathrm{NHC}} \ldots \ldots \ldots 121.6^{\circ}$

b Change of structural parameters during metallacyclobutane distortion of $\mathbf{~ m c b}_{\mathbf{c}}$

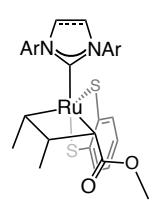

$\mathrm{mcb}_{\mathrm{c}}$

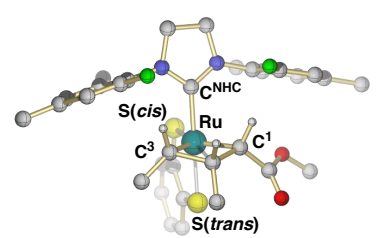

$\mathrm{Ru}-\mathrm{C}^{\mathrm{NHC}}$ $2.101 \AA$

Ru-S(trans)........... $2.422 \AA$

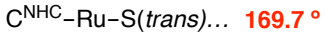

$\mathrm{C}^{1}-\mathrm{Ru}-\mathrm{S}($ cis $) \ldots \ldots \ldots . . .140 .4^{\circ}$

$\mathrm{C}^{3}-\mathrm{Ru}-\mathrm{C}^{\mathrm{NHC}}$......... $91.0^{\circ}$

$\mathrm{Ru}-\mathrm{C}^{\mathrm{NHC}} \ldots \ldots \ldots \ldots . . . . .2 .110 \AA$

Ru-S(trans)............ $2.416 \AA$

$\mathrm{C}^{\mathrm{NHC}}$-Ru-S(trans)... $170.1^{\circ}$

$\mathrm{C}^{1}-\mathrm{Ru}-\mathrm{S}$ (cis)......... $141.8^{\circ}$

$\mathrm{C}^{3}-\mathrm{Ru}-\mathrm{C}^{\mathrm{NHC}}$......... $90.7^{\circ}$
$\mathrm{Ru}-\mathrm{C}^{\mathrm{NHC}} \ldots \ldots \ldots \ldots . . . . .2 .169 \AA$

$\mathrm{Ru}-\mathrm{S}($ trans)........... $2.310 \AA$

C $136.9^{\circ}$

$\mathrm{C}^{1}-\mathrm{Ru}-\mathrm{S}(\mathrm{cis})$......... $158.9^{\circ}$

$\mathrm{C}^{3}-\mathrm{Ru}-\mathrm{C}^{\mathrm{NHC}}$.......... $132.2^{\circ}$

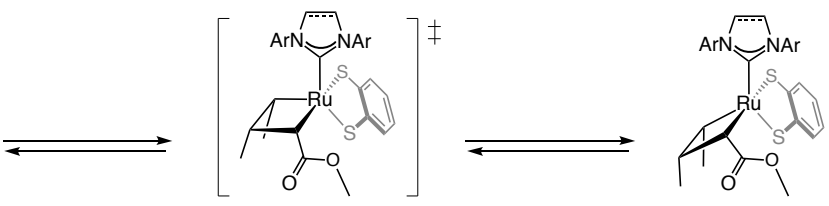

ts(dist)

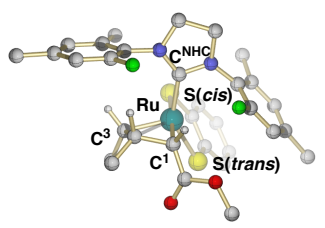

$\mathrm{Ru}-\mathrm{C}^{\mathrm{NHC}}$

.. $2.053 \AA$

Ru-S(trans)........... $2.321 \AA$

$\mathrm{C}^{\mathrm{NHC}}-\mathrm{Ru}-\mathrm{S}$ (trans)... $143.5^{\circ}$

$\mathrm{C}^{1}-\mathrm{Ru}-\mathrm{S}$ (cis)......... $179.1^{\circ}$

$\mathrm{C}^{3}-\mathrm{Ru}-\mathrm{C}^{\mathrm{NHC}}$........... $107.2^{\circ}$

$\mathrm{Ru}-\mathrm{C}^{\mathrm{NHC}} \ldots \ldots \ldots \ldots . . . . .2 .052 \AA$

Ru-S(trans)........... $2.309 \AA$

$\mathrm{C}^{\mathrm{NHC}}-\mathrm{Ru}-\mathrm{S}$ (trans)... $140.2^{\circ}$

$\mathrm{C}^{1}-\mathrm{Ru}-\mathrm{S}\left(\right.$ cis)......... $178.0^{\circ}$

$\mathrm{C}^{3}-\mathrm{Ru}-\mathrm{C}^{\mathrm{NHC}} \ldots \ldots \ldots 106.5^{\circ}$

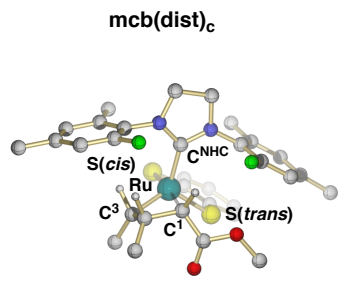

$\mathrm{Ru}-\mathrm{C}^{\mathrm{NHC}} \ldots \ldots \ldots \ldots . . . . .2 .000 \AA$

Ru-S(trans)........... $2.223 \AA$

$\mathrm{C}^{\mathrm{NHC}}-\mathrm{Ru}-\mathrm{S}\left(\right.$ trans)... $114.3^{\circ}$

$\mathrm{C}^{1}-\mathrm{Ru}-\mathrm{S}\left(\right.$ cis)........ $173.8^{\circ}$

$\mathrm{C}^{3}-\mathrm{Ru}-\mathrm{C}^{\mathrm{NHC}} \ldots \ldots \ldots 115.8^{\circ}$

$\mathrm{Ru}-\mathrm{C}^{\mathrm{NHC}} \ldots \ldots \ldots \ldots . . . . .2 .012 \AA$

Ru-S(trans)........... $2.226 \AA$ $\mathrm{C}^{\mathrm{NHC}}-\mathrm{Ru}-\mathrm{S}\left(\right.$ trans)... $113.6^{\circ}$ $\mathrm{C}^{1}-\mathrm{Ru}-\mathrm{S}\left(\right.$ cis)......... $175.2^{\circ}$ $\mathrm{C}^{3}-\mathrm{Ru}-\mathrm{C}^{\mathrm{NHC}} \ldots \ldots \ldots 114.8^{\circ}$

Fig. S13-2. Geometrical changes during metallacyclobutane distortion for the actual system starting from mcba (top)

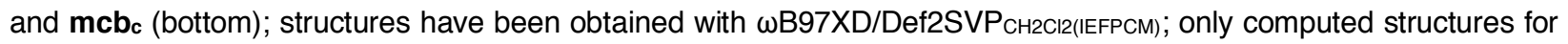
complexes bearing saturated NHC ligands are displayed; bond lengths $(\AA)$ and angles $\left(^{\circ}\right)$ for corresponding analogues with unsaturated NHC are given in grey boxes. mcb, metallacyclobutane; ts(dist), transition states for mcb distortion; $\mathbf{m c b}$ (dist), metallacyclobutane after structural distortion. 
a Change of structural parameters during metallacyclobutane distortion of $\mathbf{~ m c b}_{\mathbf{a}}$ (model system)
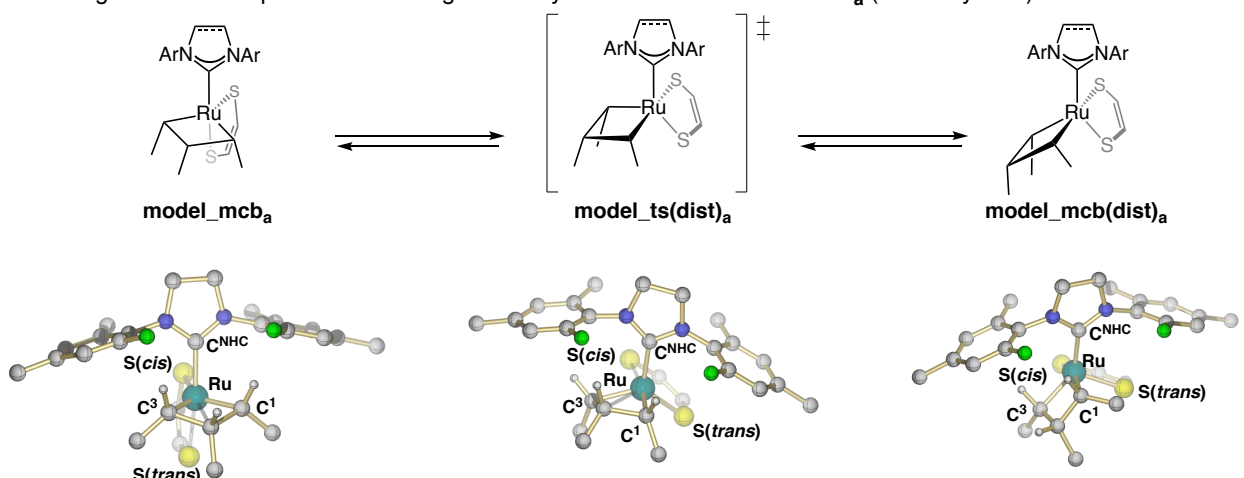

$\mathrm{Ru}-\mathrm{C}^{\mathrm{NHC}}$

$\mathrm{Ru}-\mathrm{C}^{\mathrm{NHC}}$

$\mathrm{Ru}-\mathrm{C}^{\mathrm{NHC}}$ $2.007 \AA$

Ru-S(trans).......... $2.427 \AA$

Ru-S(trans).

$\mathrm{Ru}-\mathrm{S}$ (trans).......... 2.196

$\mathrm{C}^{\mathrm{NHC}}-\mathrm{Ru}-\mathrm{S}\left(\right.$ trans)... $168.5^{\circ}$

$\mathrm{C}^{\mathrm{NHC}}$-Ru-S(trans) $124.8^{\circ}$

$\mathrm{C}^{1}-\mathrm{Ru}-\mathrm{S}$ (cis)......... $133.2^{\circ}$

$\mathrm{C}^{1}-\mathrm{Ru}-\mathrm{S}$ (cis)......... $171.7^{\circ}$

$\mathrm{C}^{\mathrm{NHC}}-\mathrm{Ru}-\mathrm{S}$ (trans)... $108.9^{\circ}$

$\mathrm{C}^{3}-\mathrm{Ru}-\mathrm{C}^{\mathrm{NHC}}$..........991.0

$\mathrm{C}^{3}-\mathrm{Ru}-\mathrm{C}^{\mathrm{NHC}}$..........107.9 ${ }^{\circ}$

$\mathrm{C}^{1}-\mathrm{Ru}-\mathrm{S}\left(\right.$ cis)........ $161.8^{\circ}$

$\mathrm{C}^{3}-\mathrm{Ru}-\mathrm{C}^{\mathrm{NHC}}$.......... $122.3^{\circ}$

\begin{tabular}{|c|c|c|}
\hline$\ldots \ldots . . .2 .105 \AA$ & $\mathrm{Ru}-\mathrm{C}^{\mathrm{NHC}}$. & $\mathrm{Ru}-\mathrm{C}^{\mathrm{NHC}}$. \\
\hline $\mathrm{Ru}-\mathrm{S}($ trans)........... $2.422 \AA$ & $\mathrm{Ru}-\mathrm{S}($ trans)........... $2.283 \AA$ & $\mathrm{Ru}-\mathrm{S}($ trans)... \\
\hline $\mathrm{C}^{\mathrm{NHC}}-\mathrm{Ru}-\mathrm{S}($ trans $) \ldots 1^{169.7^{\circ}}$ & $\mathrm{C}^{\mathrm{NHC}}-\mathrm{Ru}-\mathrm{S}\left(\right.$ trans)$\ldots \quad 123.7^{\circ}$ & $\mathrm{C}^{\mathrm{NHC}}-\mathrm{Ru}-\mathrm{S}($ trans $) \ldots 108.5^{\circ}$ \\
\hline $\mathrm{C}^{1}-\mathrm{Ru}-\mathrm{S}($ cis $) \ldots \ldots \ldots \quad 136.8^{\circ}$ & $\mathrm{C}^{1}-\mathrm{Ru}-\mathrm{S}($ cis $) \ldots \ldots \ldots \quad 171.3^{\circ}$ & $\mathrm{C}^{1}-\mathrm{Ru}-\mathrm{S}($ cis $) \ldots \ldots \ldots . \quad 162.6^{\circ}$ \\
\hline $\mathrm{C}^{3}-\mathrm{Ru}-\mathrm{C}^{\mathrm{NHC}} \ldots \ldots \ldots . . .90 .7^{\circ}$ & $\mathrm{C}^{3}-\mathrm{Ru}-\mathrm{C}^{\mathrm{NHC}}$. & $\mathrm{C}^{3}-\mathrm{Ru}-\mathrm{C}^{\mathrm{NHC}}$ \\
\hline
\end{tabular}

b Change of structural parameters during metallacyclobutane distortion of $\mathbf{~ m c b}_{\mathbf{c}}$ (model system)

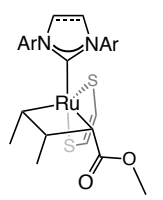

model_mcb

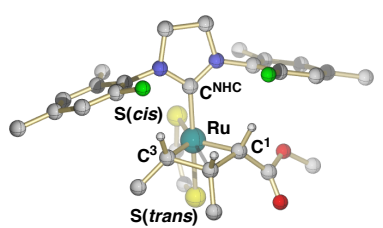

$\mathrm{Ru}-\mathrm{C}^{\mathrm{NHC}}$

$2.102 \AA$

$\mathrm{Ru}-\mathrm{S}($ trans)........... $2.418 \AA$

$\mathrm{C}^{\mathrm{NHC}}-\mathrm{Ru}-\mathrm{S}\left(\right.$ trans)... $170.0^{\circ}$

$\mathrm{C}^{1}-\mathrm{Ru}-\mathrm{S}(\mathrm{cis}) \ldots \ldots \ldots \mathbf{1 3 7 . 6}^{\circ}$

$\mathrm{C}^{3}-\mathrm{Ru}-\mathrm{C}^{\mathrm{NHC}}$

$90.4^{\circ}$

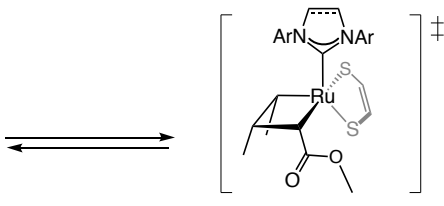

model_ts(dist)

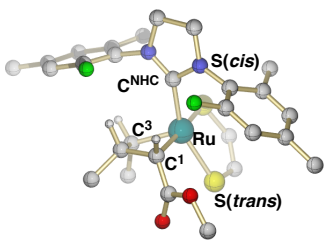

$\mathrm{Ru}-\mathrm{C}^{\mathrm{NHC}}$

$2.068 \AA$

$\mathrm{Ru}-\mathrm{S}$ (trans)........... $2.349 \AA$

$\mathrm{C}^{\mathrm{NHC}}-\mathrm{Ru}-\mathrm{S}\left(\right.$ trans)... $156.2^{\circ}$

$\mathrm{C}^{1}-\mathrm{Ru}-\mathrm{S}\left(\right.$ cis)......... $175.3^{\circ}$

$\mathrm{C}^{3}-\mathrm{Ru}-\mathrm{C}^{\mathrm{NHC}}$

$102.1^{\circ}$

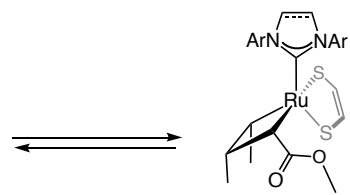

model_mcb(dist)c

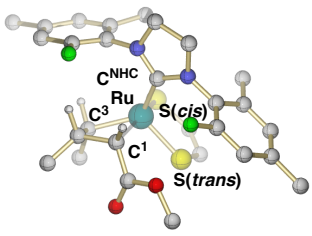

$\mathrm{Ru}-\mathrm{C}^{\mathrm{NHC}}$ $2.006 \AA$

Ru-S(trans)........... $2.202 \AA$ $\mathrm{C}^{\mathrm{NHC}}-\mathrm{Ru}-\mathrm{S}\left(\right.$ trans)... $110.5^{\circ}$

$\mathrm{C}^{1}-\mathrm{Ru}-\mathrm{S}\left(\right.$ cis)......... $165.1^{\circ}$

$\mathrm{C}^{3}-\mathrm{Ru}-\mathrm{C}^{\mathrm{NHC}}$.......... $121.4^{\circ}$

\begin{tabular}{|c|c|c|}
\hline $\mathrm{Ru}-\mathrm{C}^{\mathrm{NHC}} \ldots \ldots \ldots \ldots \ldots .2 .109 \AA$ & $\ldots \ldots . .2 .079 \AA$ & $\mathrm{Ru}-\mathrm{C}^{\mathrm{NHC}}$ \\
\hline $\mathrm{Ru}-\mathrm{S}($ trans $) \ldots \ldots \ldots \ldots . . .2 .415 \AA$ & $\mathrm{Ru}-\mathrm{S}($ trans).......... $2.340 \AA$ & $\mathrm{Ru}-\mathrm{S}($ trans).. \\
\hline $\mathrm{C}^{\mathrm{NHC}}-\mathrm{Ru}-\mathrm{S}\left(\right.$ trans) $\ldots \quad 170.0^{\circ}$ & $\mathrm{C}^{\mathrm{NHC}}-\mathrm{Ru}-\mathrm{S}($ trans $) \ldots 157.3^{\circ}$ & $\mathrm{C}^{\mathrm{NHC}}-\mathrm{Ru}-\mathrm{S}($ trans $) \ldots 111.4^{\circ}$ \\
\hline $\mathrm{C}^{1}-\mathrm{Ru}-\mathrm{S}($ cis $) \ldots \ldots \ldots \quad 142.3^{\circ}$ & $\mathrm{C}^{1}-\mathrm{Ru}-\mathrm{S}($ cis $) \ldots \ldots \ldots . \quad 173.4^{\circ}$ & $\mathrm{C}^{1}-\mathrm{Ru}-\mathrm{S}($ cis $) \ldots \ldots \ldots . \quad 169.2^{\circ}$ \\
\hline $\mathrm{C}^{3}-\mathrm{Ru}-\mathrm{C}^{\mathrm{NHC}}$ & $\mathrm{C}^{3}-\mathrm{Ru}-\mathrm{C}^{\mathrm{NHC}}$ & $\mathrm{C}^{3}-\mathrm{Ru}-\mathrm{C}^{\mathrm{NHC}}$ \\
\hline
\end{tabular}

Fig. S13-3. Geometrical changes during metallacyclobutane distortion for the model system starting from mcba (top) and $\mathbf{m c b}_{\mathbf{c}}$ (bottom); structures have been obtained with $\omega \mathrm{B} 97 \mathrm{XD} /$ Def2SVP $_{\mathrm{CH} 2 \mathrm{CI}(\mathrm{IEFPCM})}$; only computed structures for complexes bearing saturated NHC ligands are displayed; bond lengths $(\AA)$ and angles $\left({ }^{\circ}\right)$ for corresponding analogues with unsaturated $\mathrm{NHC}$ are given in grey boxes. mcb, metallacyclobutane; ts(dist), transition states for mcb distortion; mcb(dist), metallacyclobutane after structural distortion. 


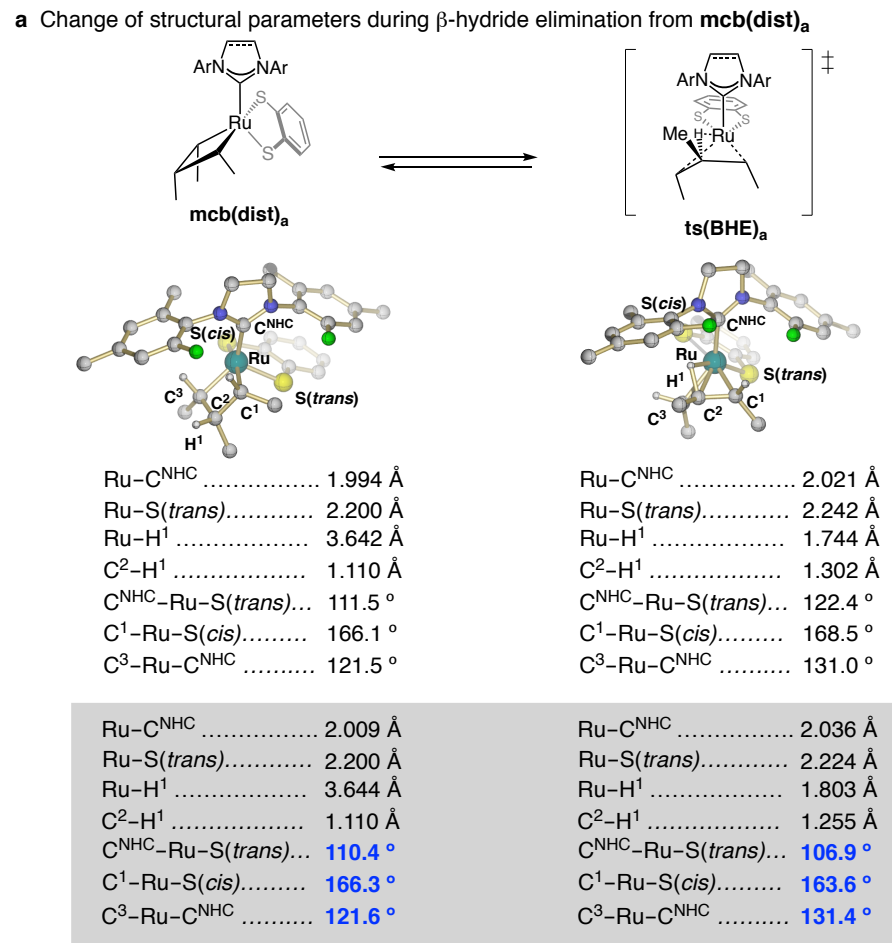

b Change of structural parameters during $\beta$-hydride elimination from $\mathbf{m c b}(\mathbf{d i s t}) \mathbf{c}$
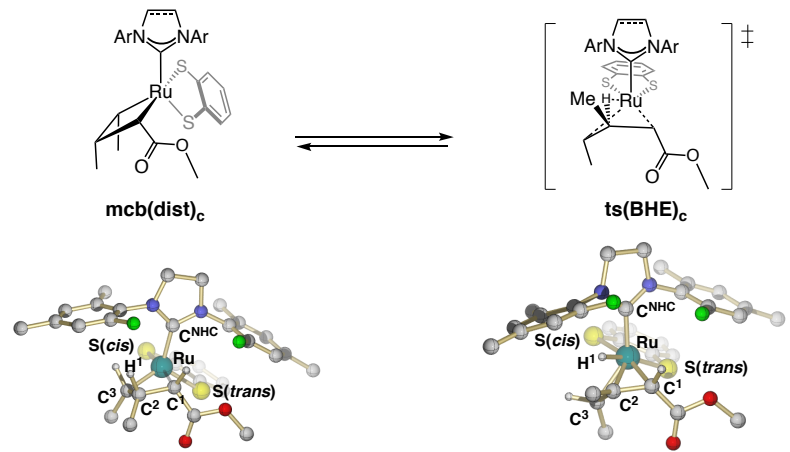

$\mathrm{Ru}-\mathrm{C}^{\mathrm{NHC}} \ldots \ldots \ldots \ldots . \ldots 2.000 \AA$

$\mathrm{Ru}-\mathrm{S}($ trans) $\ldots \ldots \ldots \ldots .2 .223 \AA$

$\mathrm{Ru}-\mathrm{H}^{1} \ldots \ldots \ldots \ldots \ldots . . . . .197 \AA$

$\mathrm{C}^{2}-\mathrm{H}^{1} \ldots \ldots \ldots \ldots \ldots . . . . .1 .095 \AA$

$\mathrm{C}^{\mathrm{NHC}}-\mathrm{Ru}-\mathrm{S}\left(\right.$ trans)... $114.3^{\circ}$

$\mathrm{C}^{1}-\mathrm{Ru}-\mathrm{S}\left(\right.$ cis) $\ldots \ldots \ldots .173 .8^{\circ}$

$\mathrm{C}^{3}-\mathrm{Ru}-\mathrm{C}^{\mathrm{NHC}} \ldots \ldots \ldots .115 .8^{\circ}$

$\mathrm{Ru}-\mathrm{C}^{\mathrm{NHC}} \ldots \ldots \ldots \ldots . . . . .2 .019 \AA$

Ru-S(trans)........... 2.254 А

$\mathrm{Ru}-\mathrm{H}^{1} \ldots \ldots \ldots \ldots \ldots . . .695 \AA$

$\mathrm{C}^{2}-\mathrm{H}^{1} \ldots \ldots \ldots \ldots \ldots . . . . . .1 .393 \AA$

$\mathrm{C}^{\mathrm{NHC}}-\mathrm{Ru}-\mathrm{S}\left(\right.$ trans)... $118.8^{\circ}$

$\mathrm{C}^{1}-\mathrm{Ru}-\mathrm{S}$ (cis)......... $167.6^{\circ}$

$\mathrm{C}^{3}-\mathrm{Ru}-\mathrm{C}^{\mathrm{NHC}} \ldots \ldots \ldots 130.4^{\circ}$

\begin{tabular}{|c|c|}
\hline $\mathrm{Ru}-\mathrm{C}^{\mathrm{NHC}}$. & $\mathrm{Ru}-\mathrm{C}^{\mathrm{NHC}}$ \\
\hline $\mathrm{Ru}-\mathrm{S}(\operatorname{trans}) .$. & $\mathrm{Ru}-\mathrm{S}($ trans)... \\
\hline $\mathrm{Ru}-\mathrm{H}^{1} \ldots \ldots \ldots \ldots \ldots . . . . . .208 \AA$ & ... $1.727 \AA$ \\
\hline …........... $1.096 \AA$ & $\ldots \ldots \ldots \ldots .1 .336 \AA$ \\
\hline $\mathrm{C}^{\mathrm{NHC}}-\mathrm{Ru}-\mathrm{S}\left(\right.$ trans) $\ldots 1^{113.6^{\circ}}$ & $\mathrm{C}^{\mathrm{NHC}}-\mathrm{Ru}-\mathrm{S}\left(\right.$ trans) $\ldots 100.8^{\circ}$ \\
\hline $\mathrm{C}^{1}-\mathrm{Ru}-\mathrm{S}($ cis $) \ldots \ldots \ldots . . .175 .2^{\circ}$ & $\mathrm{C}^{1}-\mathrm{Ru}-\mathrm{S}(\mathrm{cis}) \ldots \ldots \ldots \quad 160.7^{\circ}$ \\
\hline $\mathrm{C}^{3}-\mathrm{Ru}-\mathrm{C}^{\mathrm{NHC}}$ & $\mathrm{C}^{3}-\mathrm{Ru}-\mathrm{C}^{\mathrm{NHC}}$ \\
\hline
\end{tabular}

Fig. S13-4. Geometrical changes during $\beta$-hydride elimination for real system starting from $\mathbf{m c b}(\mathbf{d i s t}) \mathrm{a}$ (top) and mcb(dist)c (bottom); structures have been obtained with $\omega B$ 97XD/Def2SVPCH2CI2 (IEFPCM); only computed structures for complexes bearing saturated NHC ligands are displayed; bond lengths ( $\AA$ ) and angles $\left(^{\circ}\right)$ for analogues with unsaturated NHC are given in grey boxes. mcb(dist), metallacyclobutane after structural distortion; ts(BHE), transition state for $\beta$-hydride elimination. 
a Change of structural parameters during $\beta$-hydride elimination from mcb(dist)a (model system)
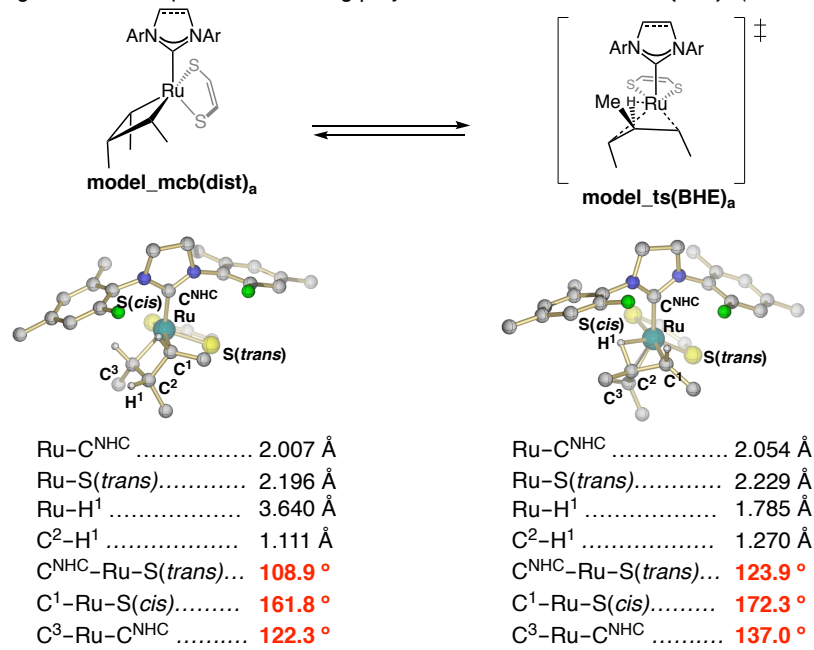

$\mathrm{Ru}-\mathrm{C}^{\mathrm{NHC}}$..............2.054

$\mathrm{Ru}-\mathrm{S}$ (trans).......... $2.229 \AA$

$\mathrm{Ru}-\mathrm{H}^{1} \ldots \ldots \ldots \ldots \ldots . . . . . .1 .785 \AA$

$\mathrm{C}^{2}-\mathrm{H}^{1} \ldots \ldots \ldots \ldots \ldots . .270 \AA$

$\mathrm{C}^{\mathrm{NHC}}-\mathrm{Ru}-\mathrm{S}\left(\right.$ trans) ... $123.9^{\circ}$

$\mathrm{C}^{1}-\mathrm{Ru}-\mathrm{S}(\mathrm{cis}) \ldots \ldots \ldots .172 .3^{\circ}$

$\mathrm{C}^{3}-\mathrm{Ru}-\mathrm{C}^{\mathrm{NHC}} \ldots \ldots \ldots 122.3^{\circ}$

$\mathrm{C}^{3}-\mathrm{Ru}-\mathrm{C}^{\mathrm{NHC}} \ldots \ldots \ldots 137.0^{\circ}$

\begin{tabular}{|c|c|c|}
\hline $\mathrm{Ru}-\mathrm{C}^{\mathrm{NHC}}$ & $2.019 \AA$ & $\mathrm{Ru}-\mathrm{C}^{\mathrm{NHC}}$ \\
\hline $\mathrm{Ru}-\mathrm{S}($ trans)............ & $2.197 \AA$ & $\mathrm{Ru}-\mathrm{S}($ trans)... \\
\hline $\mathrm{Ru}-\mathrm{H}^{1} \ldots \ldots \ldots \ldots \ldots \ldots$ & $3.640 \AA$ & $\ldots \ldots \ldots . .1 .758 \AA$ \\
\hline $\mathrm{C}^{2}-\mathrm{H}^{1} \ldots \ldots \ldots \ldots \ldots$ & $1.111 \AA$ & $\mathrm{C}^{2}-\mathrm{H}^{1} \ldots \ldots \ldots \ldots$ \\
\hline $\mathrm{C}^{\mathrm{NHC}}-\mathrm{Ru}-\mathrm{S}($ trans) $\ldots$ & $108.5^{\circ}$ & $\mathrm{C}^{\mathrm{NHC}}-\mathrm{Ru}-\mathrm{S}\left(\right.$ trans) $\ldots 1^{118.6^{\circ}}$ \\
\hline $\mathrm{C}^{1}-\mathrm{Ru}-\mathrm{S}($ cis $) \ldots \ldots \ldots$ & $162.6^{\circ}$ & $\mathrm{C}^{1}-\mathrm{Ru}-\mathrm{S}(\mathrm{cis}) \ldots \ldots \ldots \quad 169.6^{\circ}$ \\
\hline $\mathrm{C}^{3}-\mathrm{Ru}-\mathrm{C}^{\mathrm{NHC}} \ldots \ldots \ldots$ & $122.8^{\circ}$ & $\mathrm{C}^{3}-\mathrm{Ru}-\mathrm{C}^{\mathrm{NHC}} \ldots \ldots \ldots 137.4^{\circ}$ \\
\hline
\end{tabular}

b Change of structural parameters during $\beta$-hydride elimination from $\mathbf{m c b}$ (dist)c (model system)

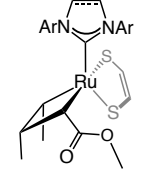

model_mcb(dist)c

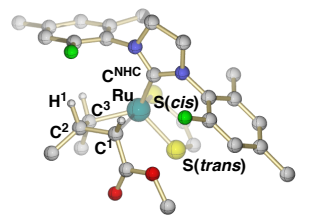

$\mathrm{Ru}-\mathrm{C}^{\mathrm{NHC}}$

$\mathrm{Ru}-\mathrm{S}($ trans)

$2.006 \AA$

$\mathrm{Ru}-\mathrm{H}^{1} \ldots \ldots \ldots \ldots \ldots . .3 .136 \AA$

$\mathrm{C}^{2}-\mathrm{H}^{1} \ldots \ldots \ldots \ldots \ldots . . .1 .095 \AA$

$\mathrm{C}^{\mathrm{NHC}}-\mathrm{Ru}-\mathrm{S}\left(\right.$ trans)... $110.5^{\circ}$

$\mathrm{C}^{1}-\mathrm{Ru}-\mathrm{S}(\mathrm{cis}) \ldots \ldots \ldots .165 .1^{\circ}$

$\mathrm{C}^{3}-\mathrm{Ru}-\mathrm{C}^{\mathrm{NHC}} \ldots \ldots \ldots .121 .4^{\circ}$

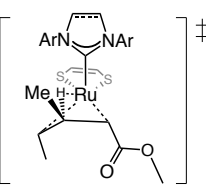

model_ts(BHE)

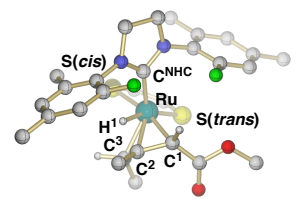

$\mathrm{Ru}-\mathrm{C}^{\mathrm{NHC}}$

Ru-S(trans) ............. $2.247 \AA$

$\mathrm{Ru}-\mathrm{H}^{1} \ldots \ldots \ldots \ldots \ldots . . . . . .1 .706 \AA$

$\mathrm{C}^{2}-\mathrm{H}^{1} \ldots \ldots \ldots \ldots \ldots . . . . . . . .1 .374 \AA$

$\mathrm{C}^{\mathrm{NHC}}-\mathrm{Ru}-\mathrm{S}\left(\right.$ trans)... $122.6^{\circ}$

$\mathrm{C}^{1}-\mathrm{Ru}-\mathrm{S}(\mathrm{cis}) \ldots \ldots \ldots . .168 .0^{\circ}$

$\mathrm{C}^{3}-\mathrm{Ru}-\mathrm{C}^{\mathrm{NHC}} \ldots \ldots \ldots .133 .3^{\circ}$

$\mathrm{Ru}-\mathrm{C}^{\mathrm{NHC}}$

Ru-S(trans)........... 2.247 $\AA$

$\mathrm{Ru}-\mathrm{H}^{1} \ldots \ldots \ldots \ldots \ldots . . . . . .1 .722 \AA$

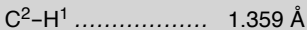

$\mathrm{C}^{\mathrm{NHC}}-\mathrm{Ru}-\mathrm{S}\left(\right.$ trans)... $103.0^{\circ}$

$\mathrm{C}^{1}-\mathrm{Ru}-\mathrm{S}(\mathrm{cis}) \ldots \ldots \ldots .159 .7^{\circ}$

$\mathrm{C}^{3}-\mathrm{Ru}-\mathrm{C}^{\mathrm{NHC}} \ldots \ldots \ldots . .134 .1^{\circ}$

Fig. S13-5. Geometrical changes during $\beta$-hydride elimination for model system starting from $\mathbf{m c b}(\mathbf{d i s t})_{\mathbf{a}}$ (top) and

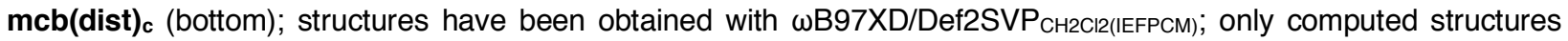
for complexes bearing saturated NHC ligands are displayed; bond lengths $(\AA)$ and angles $\left({ }^{\circ}\right)$ for corresponding analogues with unsaturated $\mathrm{NHC}$ are given in grey boxes. $\mathbf{m c b}$ (dist), metallacyclobutane after structural distortion; ts(BHE), transition state for $\beta$-hydride elimination. 


\subsection{Comparison of Ru-S(trans) and $\mathrm{Ru}-\mathrm{C}^{\mathrm{NHC}}$ Bond Lengths Based on X-Ray and DFT}

To secure further evidence for the stronger trans influence exerted by a saturated NHC ligand, we analyzed the $\mathrm{Ru}-\mathrm{S}\left(\right.$ trans) and $\mathrm{Ru}-\mathrm{C}^{\mathrm{NHC}}$ bond lengths in the $\mathrm{X}$-ray structures obtained for Ru-1c and Ru-1d (Fig. S14-2). We find that the $\mathrm{Ru}-\mathrm{C}^{\mathrm{NHC}}$ bond lengths in the unsaturated system (2.055 and $2.062 \AA$; Fig. S14-2a) are approximately $0.02 \AA$ shorter than those in the complex bearing the saturated NHC (2.083, 2.073 and $2.096 \AA$; Fig. S14-2b). The $\mathrm{Ru}-\mathrm{S}($ trans $)$ bond lengths are also shorter (2.265 and $2.274 \AA$ vs. 2.299, 2.283 and $2.283 \AA$; Fig. S14-2). The shorter $\mathrm{Ru}-\mathrm{C}^{\mathrm{NHC}}$ bond lengths in the $\mathrm{X}$-ray structure of Ru-1d appear to contradict the findings obtained in Section 5.3. Nonetheless, DFT optimized structures of the catalyst precursors reveal a longer $\mathrm{Ru}-\mathrm{C}^{\mathrm{NHC}}$ bond length for Ru-1d compared to those for Ru-1c (2.040 vs. $2.023 \AA$; Fig. S14-1). The discrepancy between the structures obtained through X-ray and DFT can likely be attributed to interference by crystal packing forces (Fig. S14-2). While there appears to be facile $\pi-\pi$ stacking between the aryl unit on the dithiolate ligand and an N-aryl group within the NHC ligand in Ru-1d (small $\mathrm{C}^{\mathrm{NHC}}-\mathrm{Ru}-\mathrm{S}$ (trans) angle; Fig. S14-2a), the aryl-aryl association in Ru1c is disrupted by either the isopropoxy group (mode $\mathbf{A}$ ), the 2-fluoro,6-methyl phenyl moiety (mode B), or the aromatic ring of the benzylidene $(\operatorname{mode} \mathbf{C})$ of a neighboring molecule in the crystal (Fig. S14-2b).
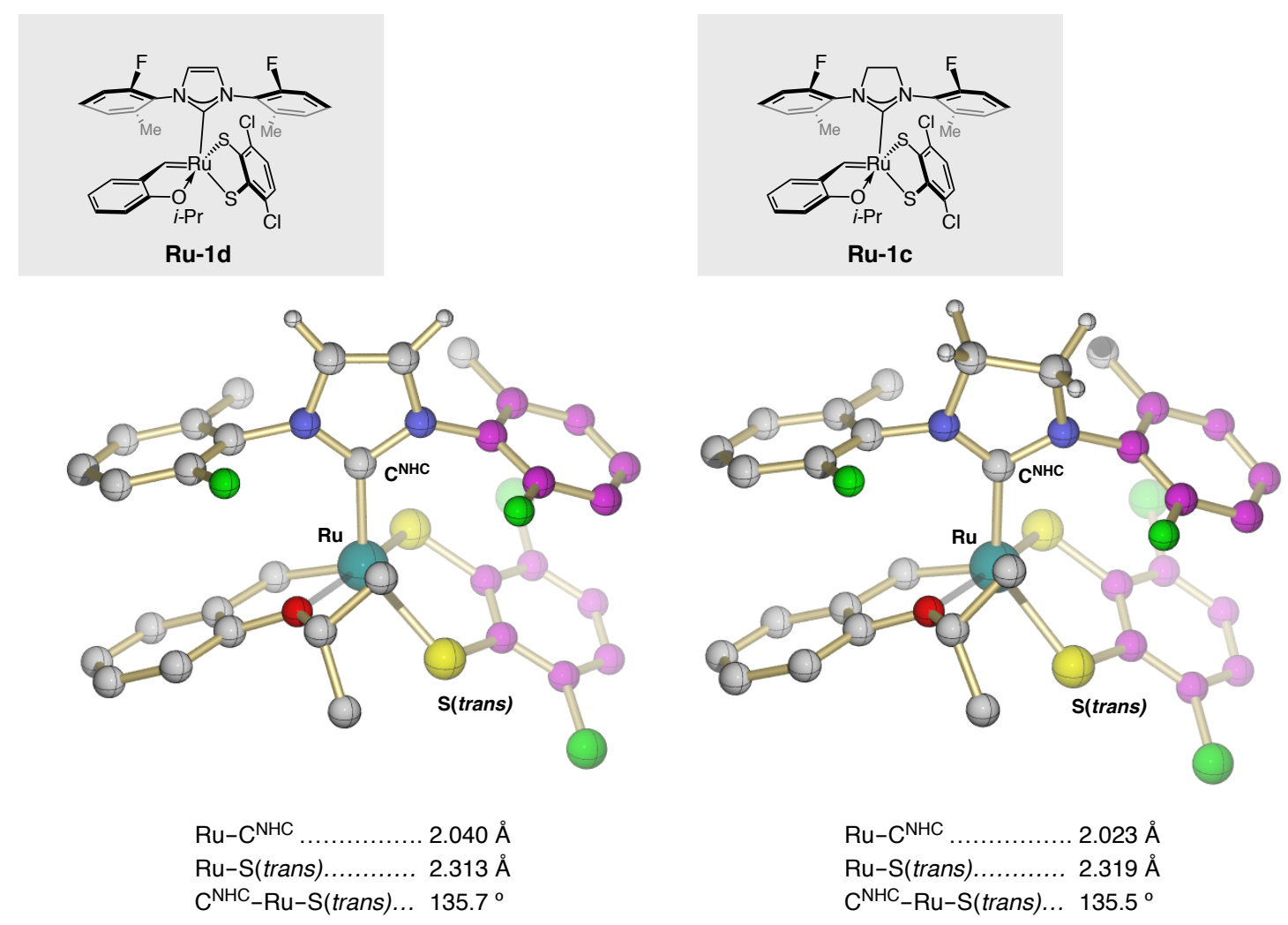

Fig. S14-1. Computed structures ( $\omega B$ 97XD/Def2SVP $\left.{ }_{\mathrm{CH} 2 \mathrm{CI}(\mathrm{IEFPCM})}\right)$ of catalyst precursors Ru-1d (left) and Ru-1c (right). 
a Packing in crystal structure of Ru-1d

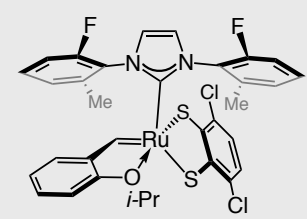

Ru-1d
$\mathrm{Ru}-\mathrm{C}^{\mathrm{NHC}}$

$\mathrm{Ru}-\mathrm{S}$ (trans).

$\mathrm{C}^{\mathrm{NHC}}-\mathrm{Ru}-\mathrm{S}\left(\right.$ trans)... $139.6 \& 142.4^{\circ}$

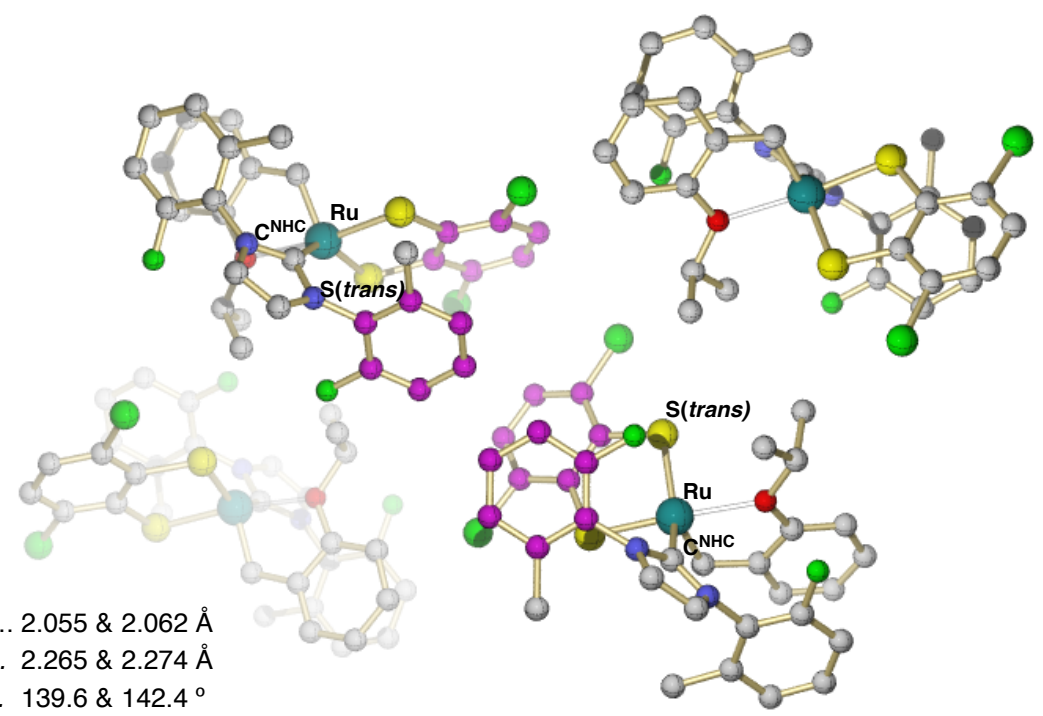

b Packing in crystal structure of Ru-1c

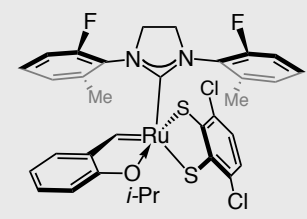

Ru-1c

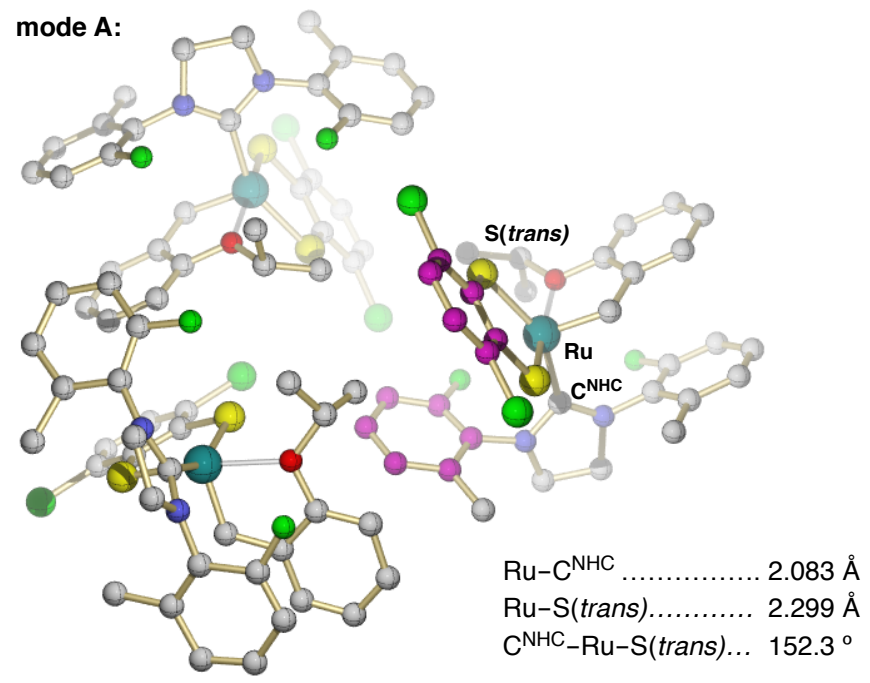

mode $B$ :

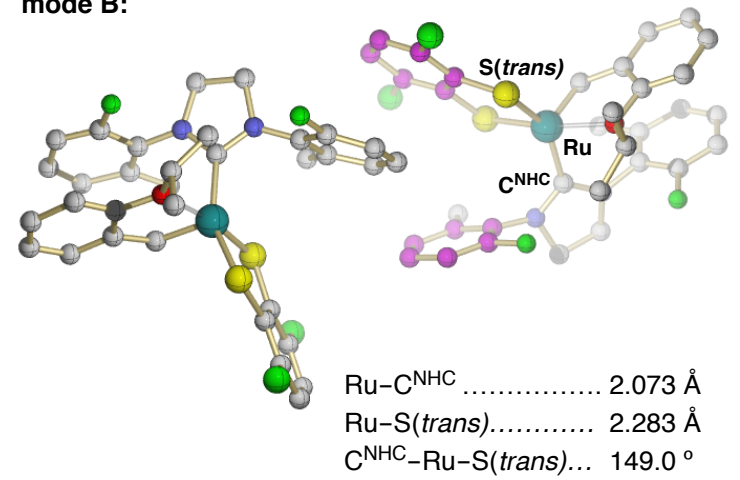

mode C:

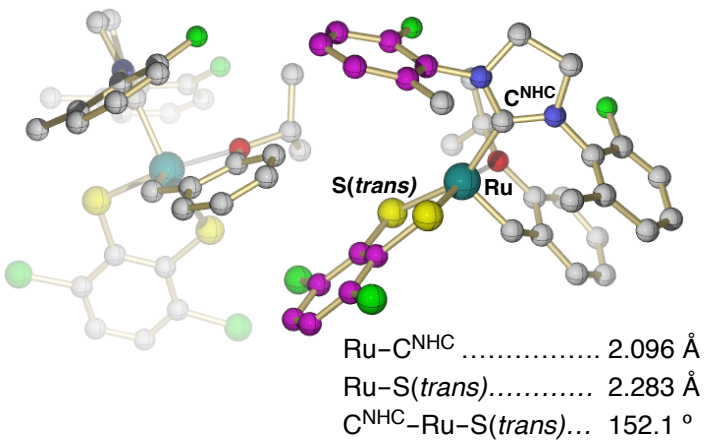

Fig. S14-2. Crystal packing in complexes Ru-1d (a) and Ru-1c (b).

\section{NMR Spectra}




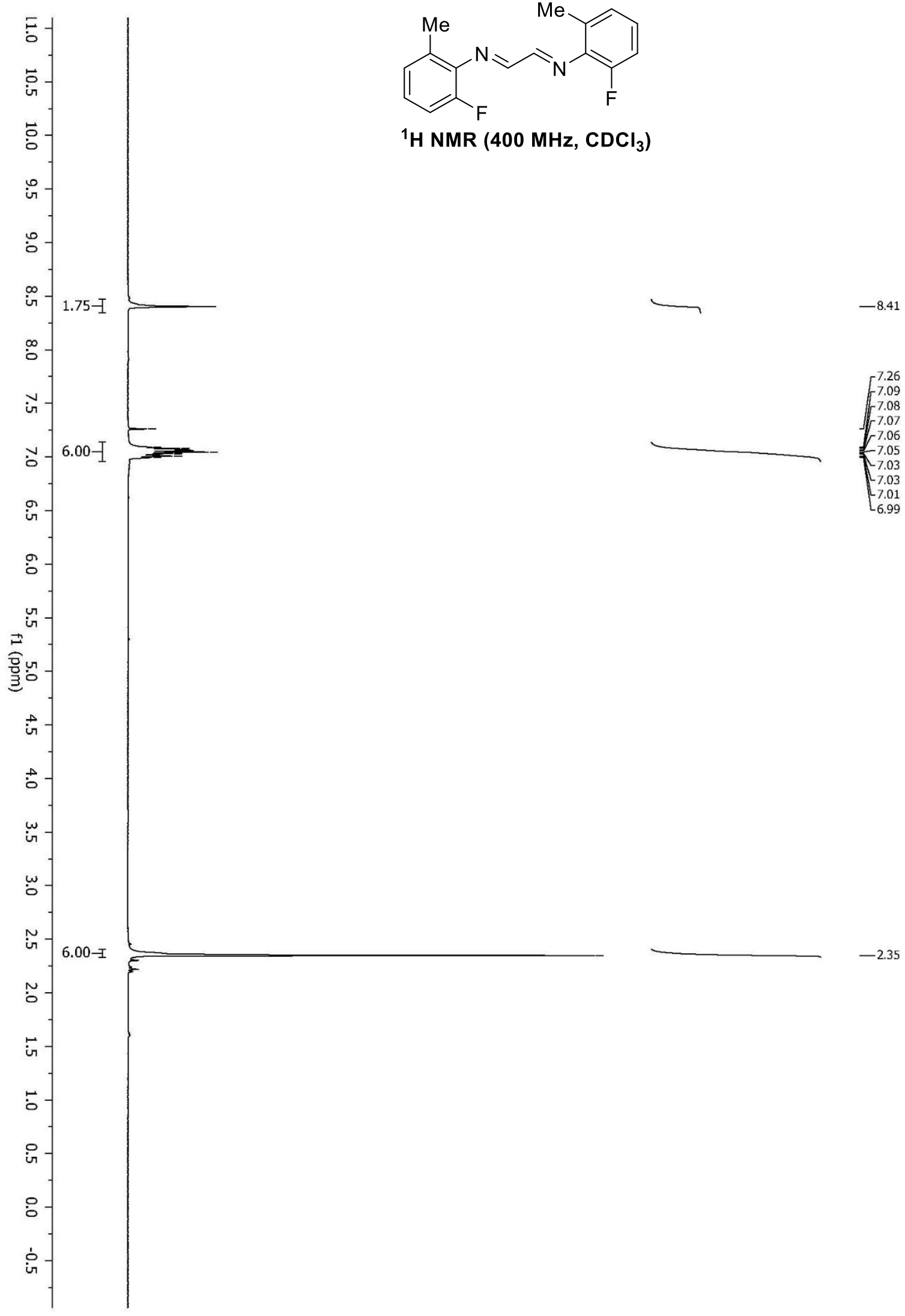



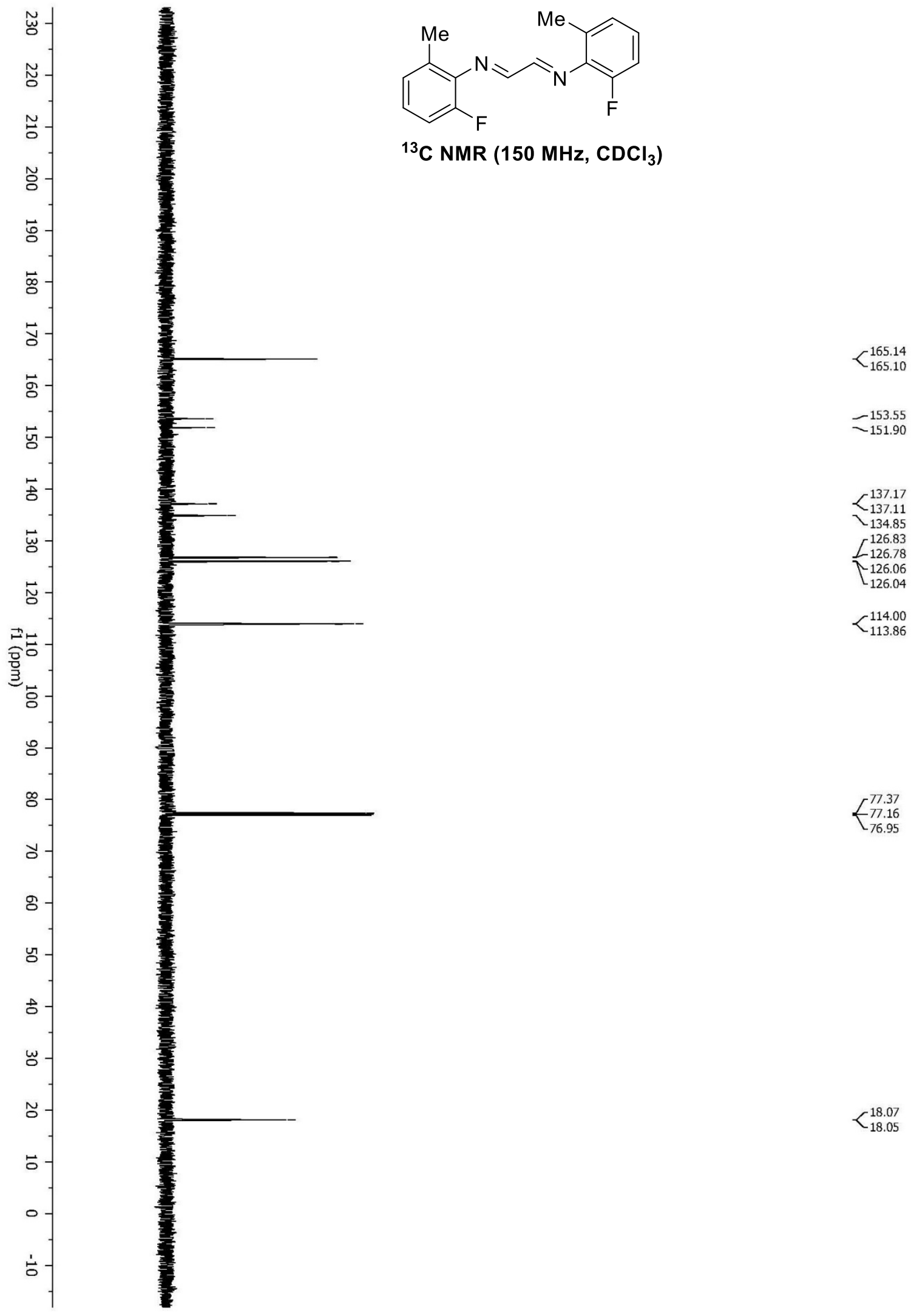

${ }^{13} \mathrm{C}$ NMR (150 MHz, $\left.\mathrm{CDCl}_{3}\right)$ 


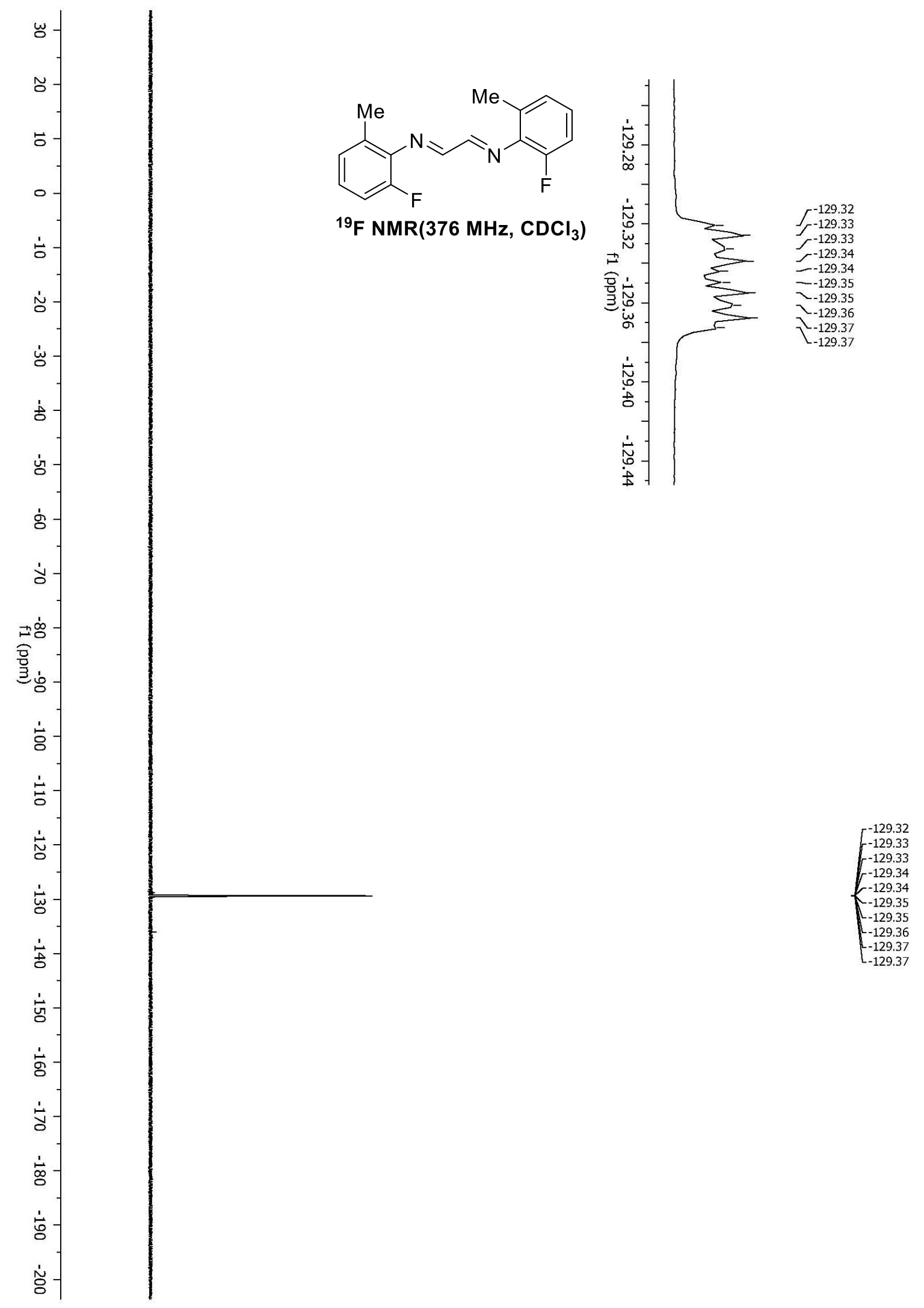




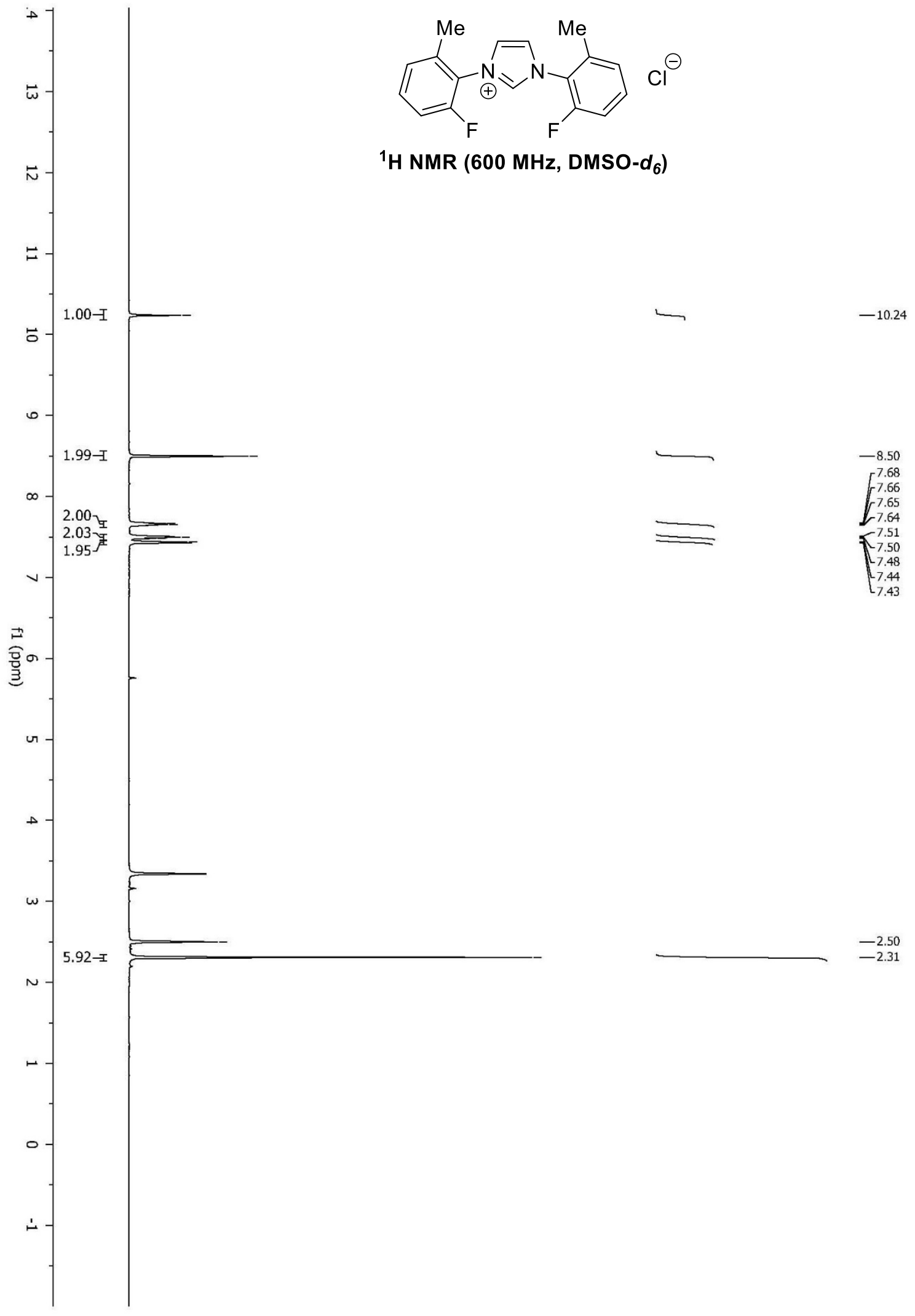


Lin \& Xu et al., Supporting Information, Page S68

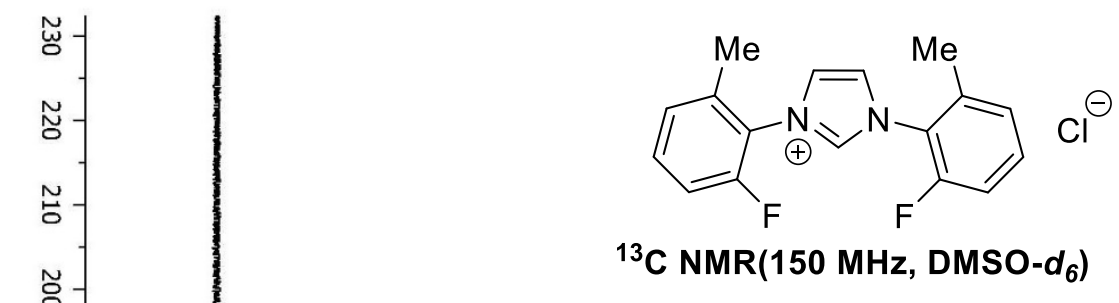

-157.05
-155.38
$\sigma^{140.24}$
$\digamma_{137.01}^{132.58}$
$\Gamma_{132.52}^{132.13}$
$\Gamma_{127.11}^{127.13}$
-125.03
$\tau_{121.83}^{121.74}$
$<_{114.25}^{114.37}$

39.94
39.80
39.66
39.52
39.38
39.24
39.10




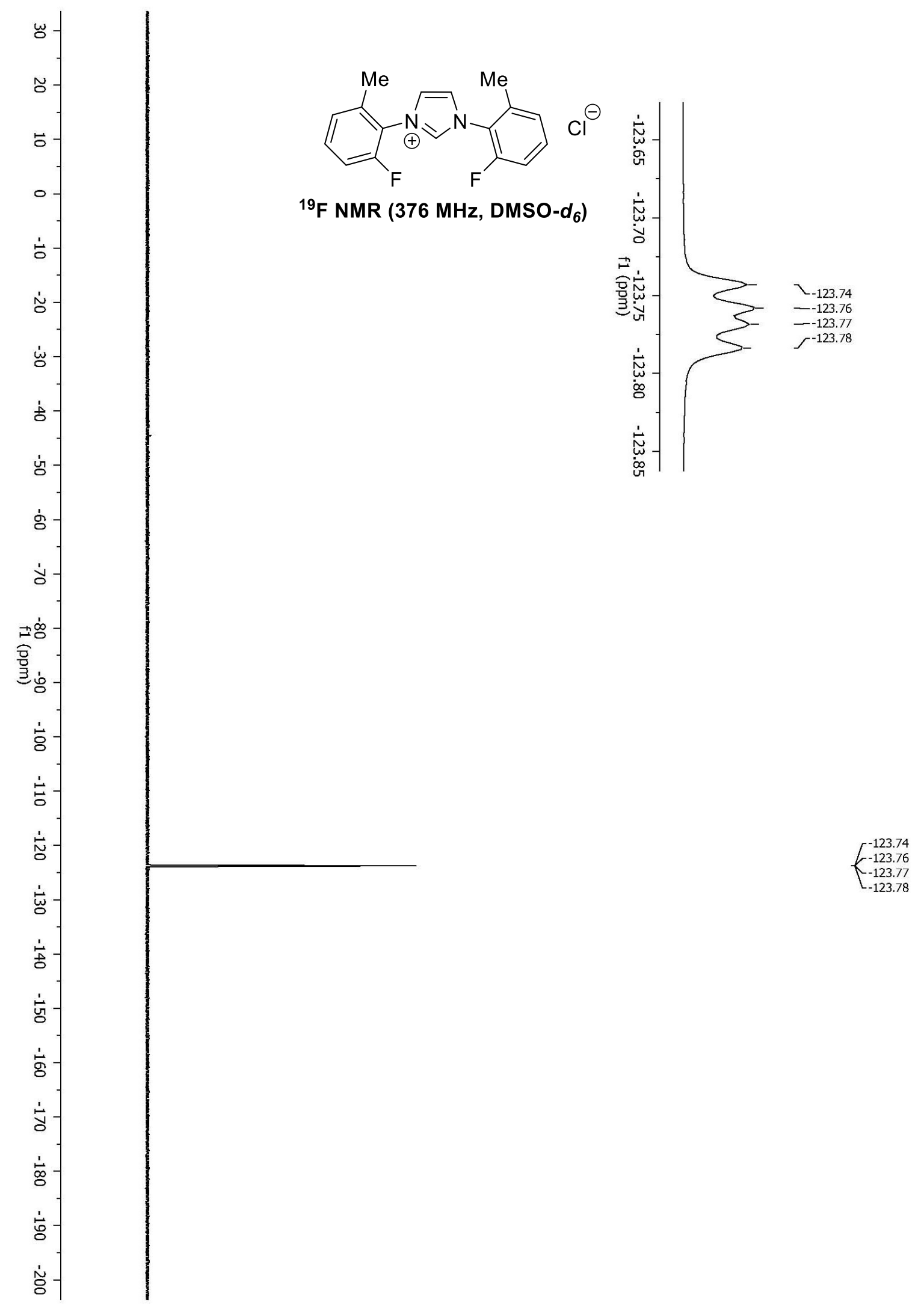




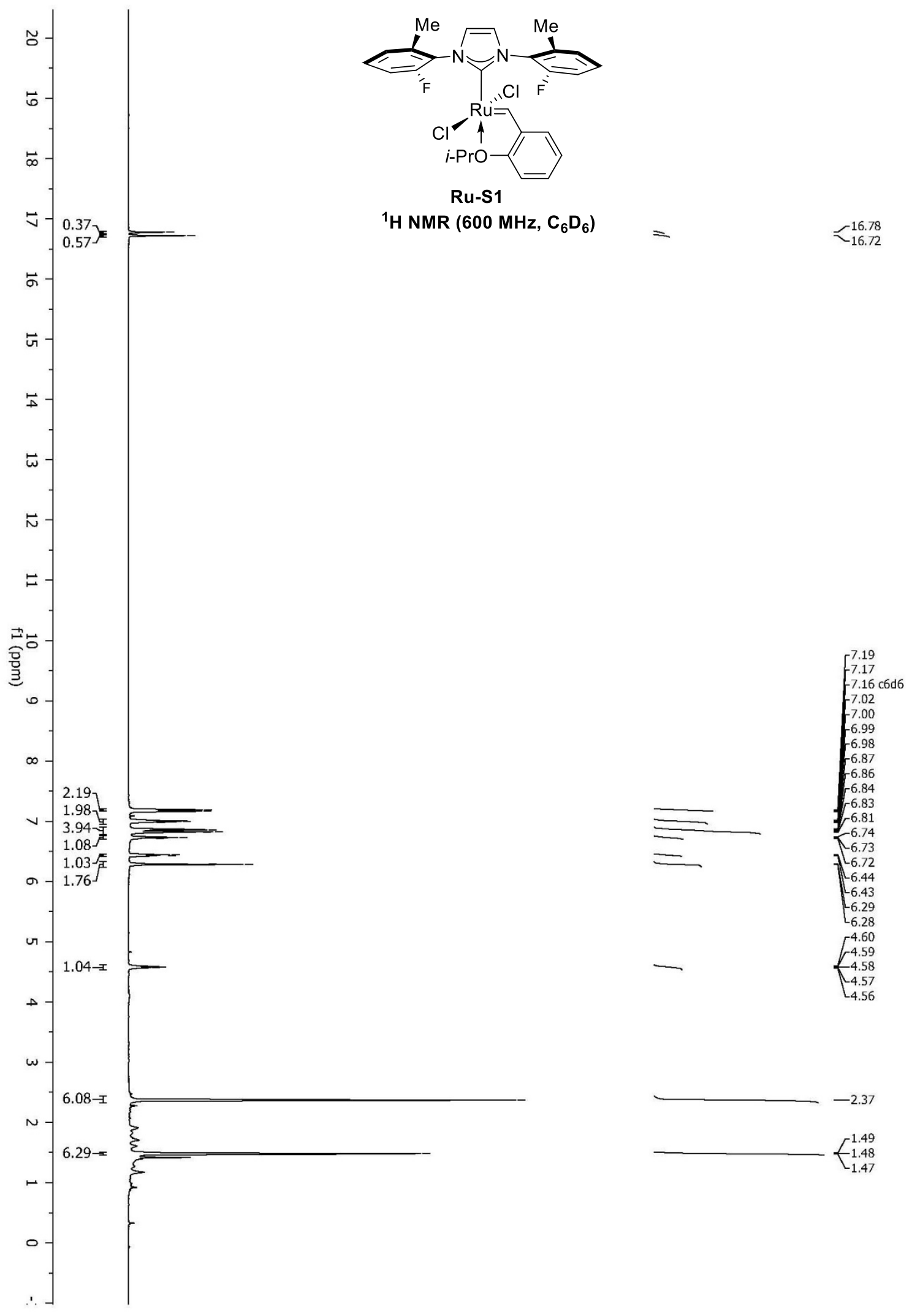




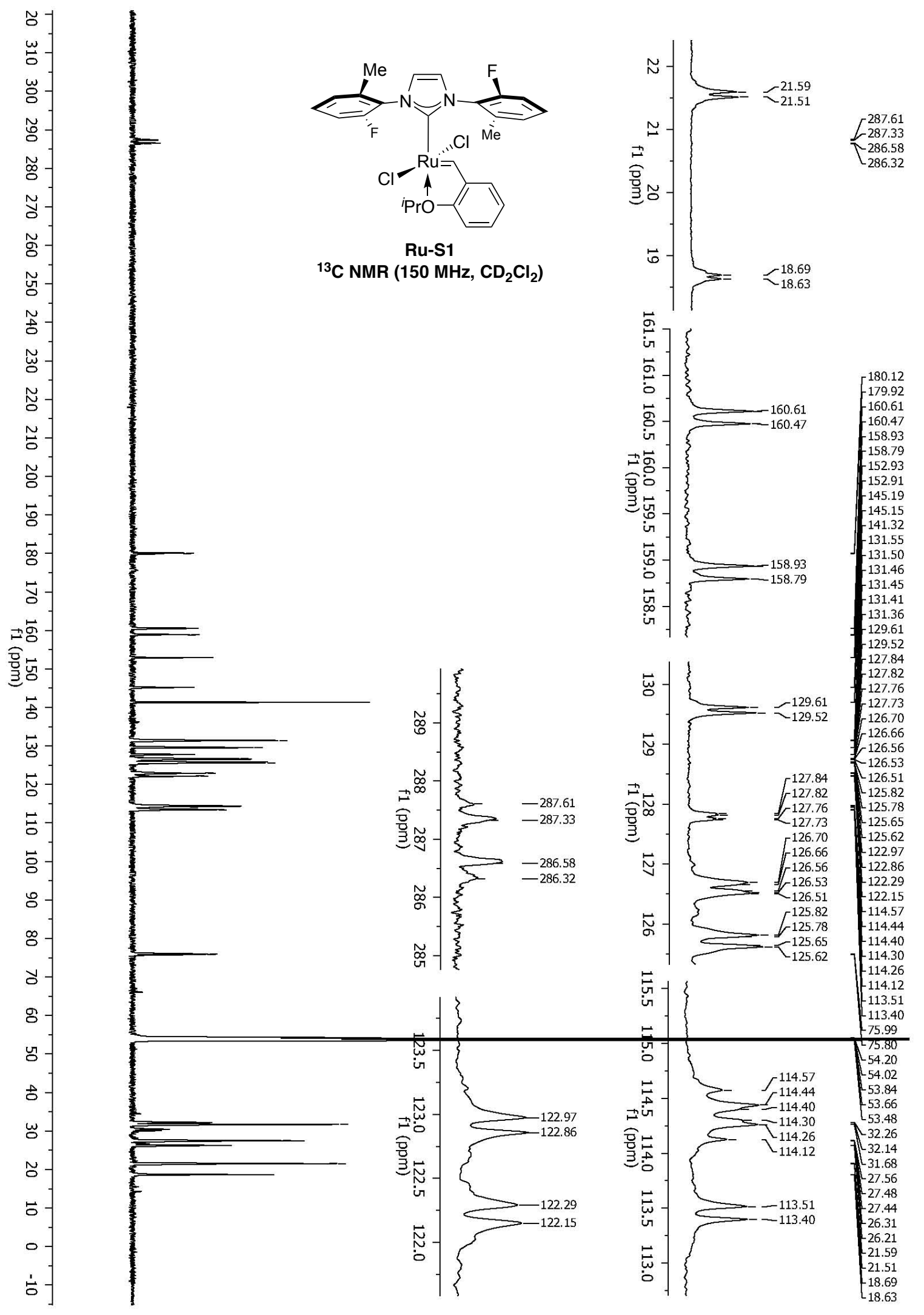



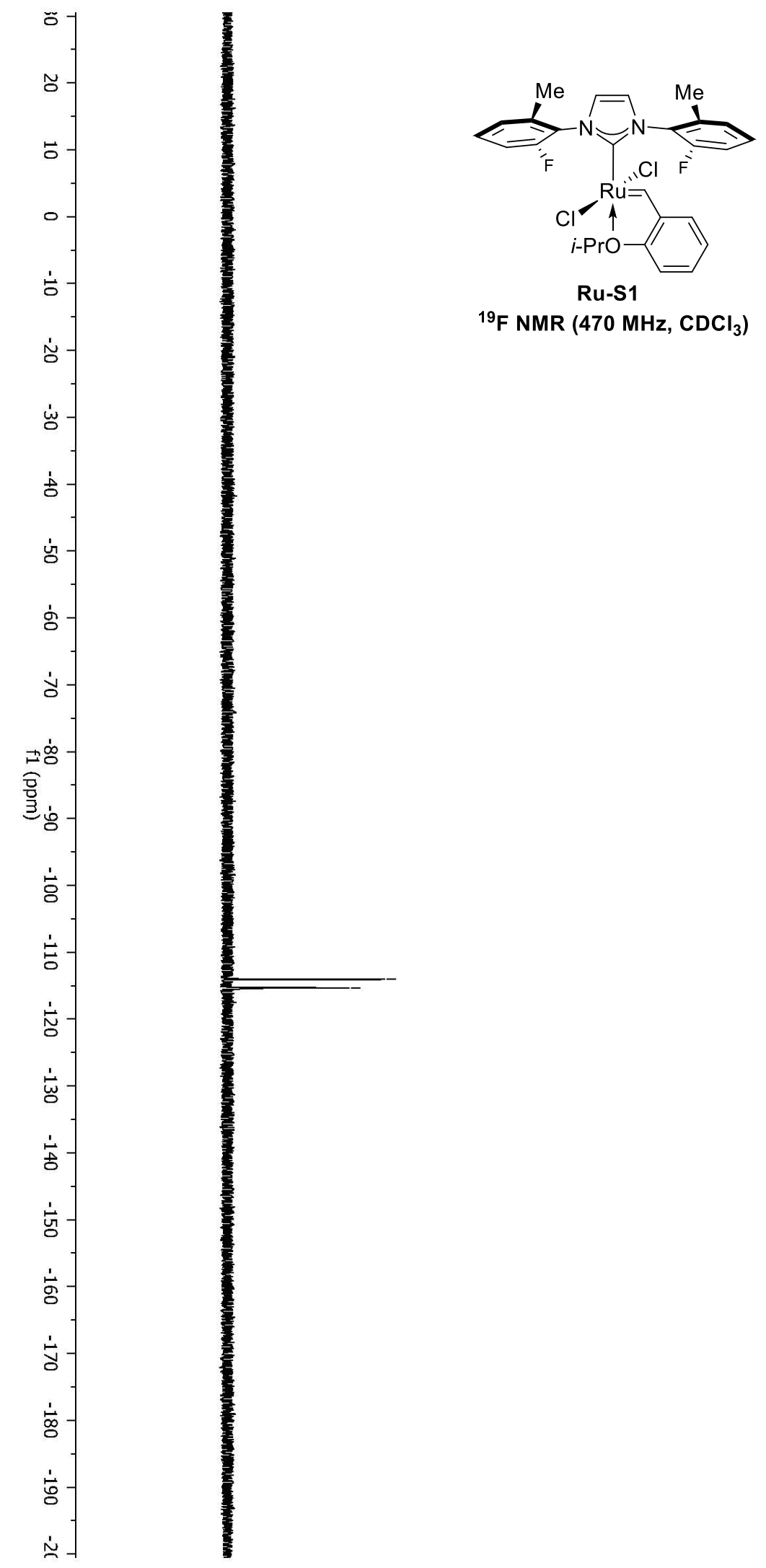

$=-114.07$ 


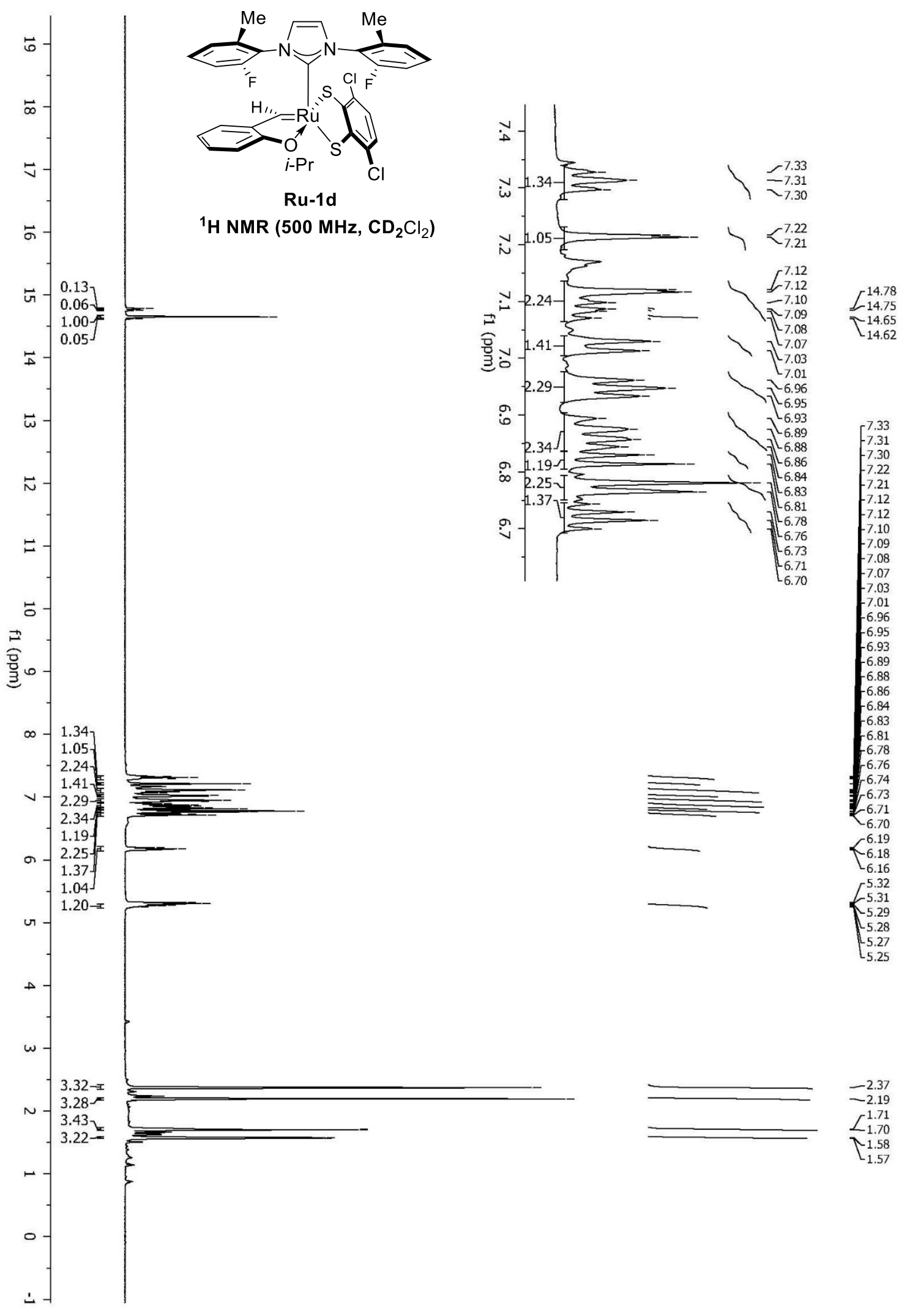




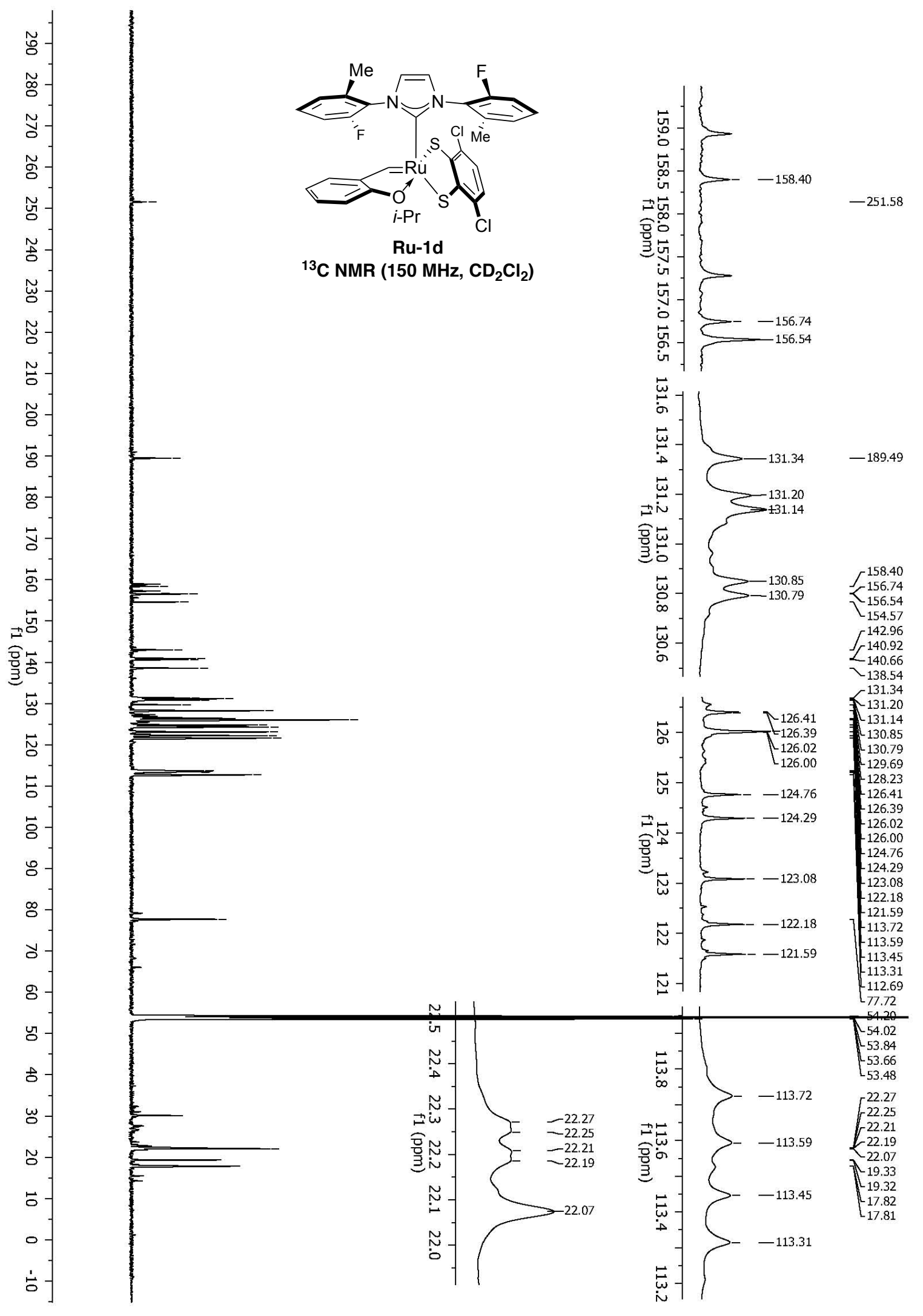




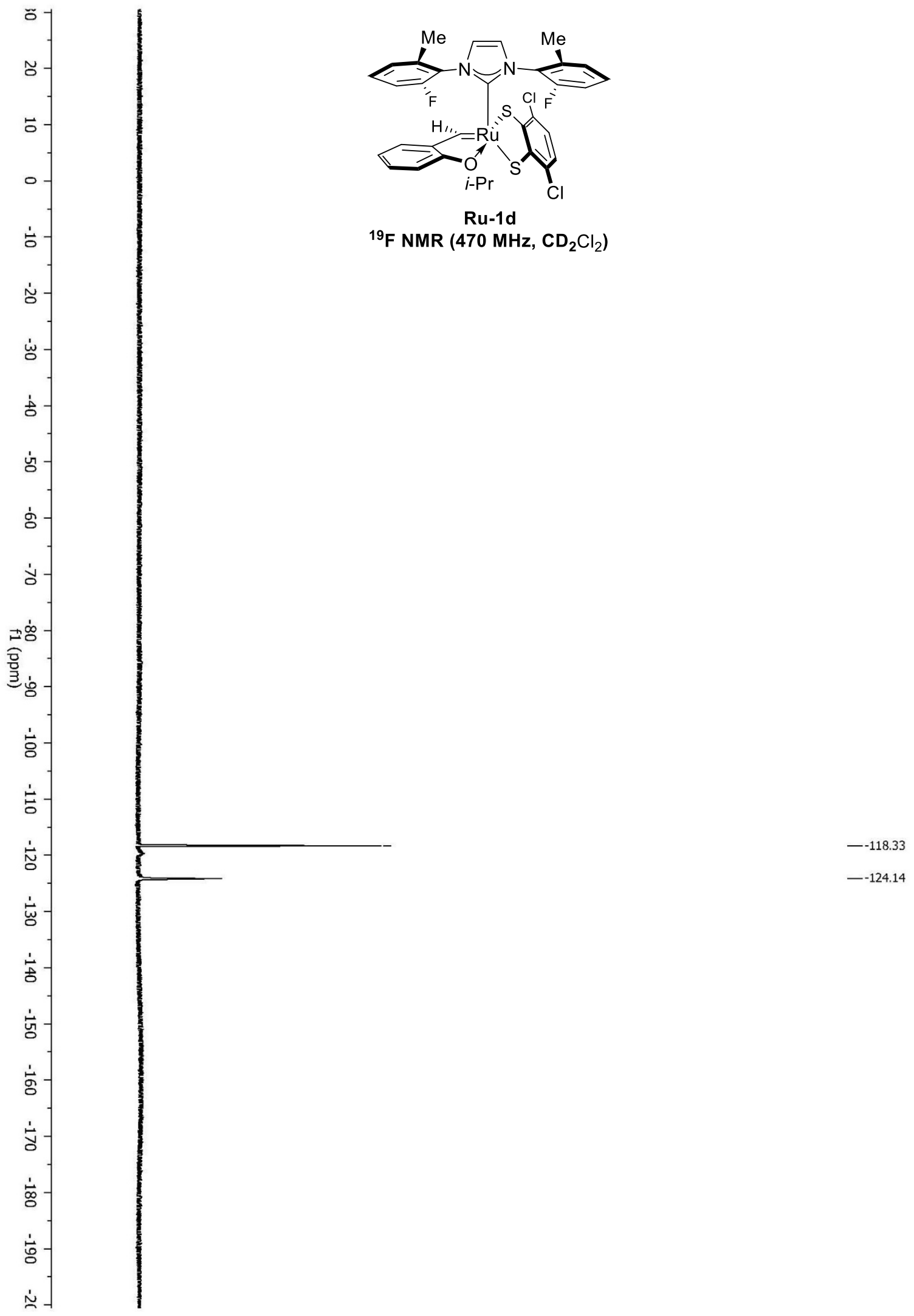




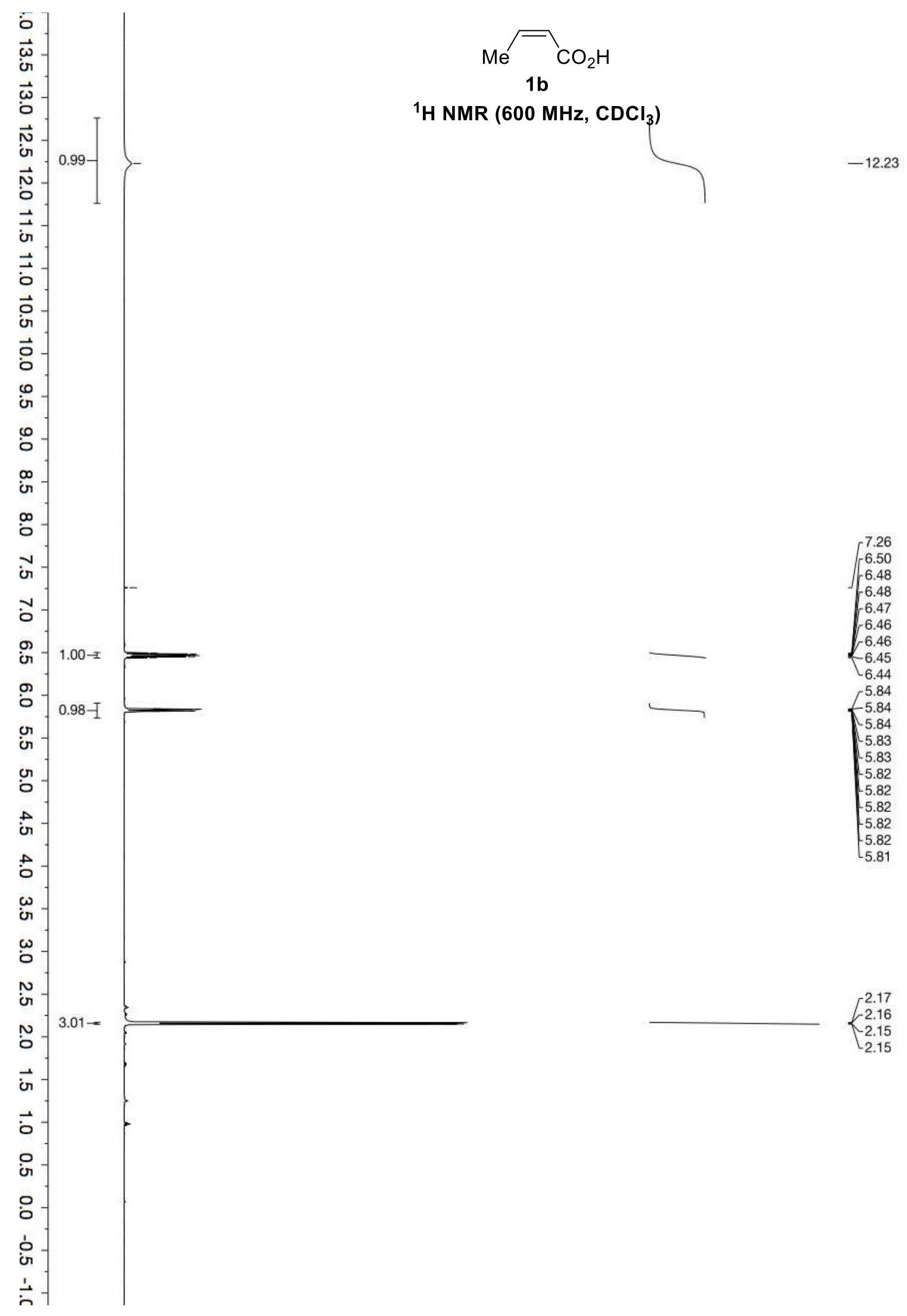




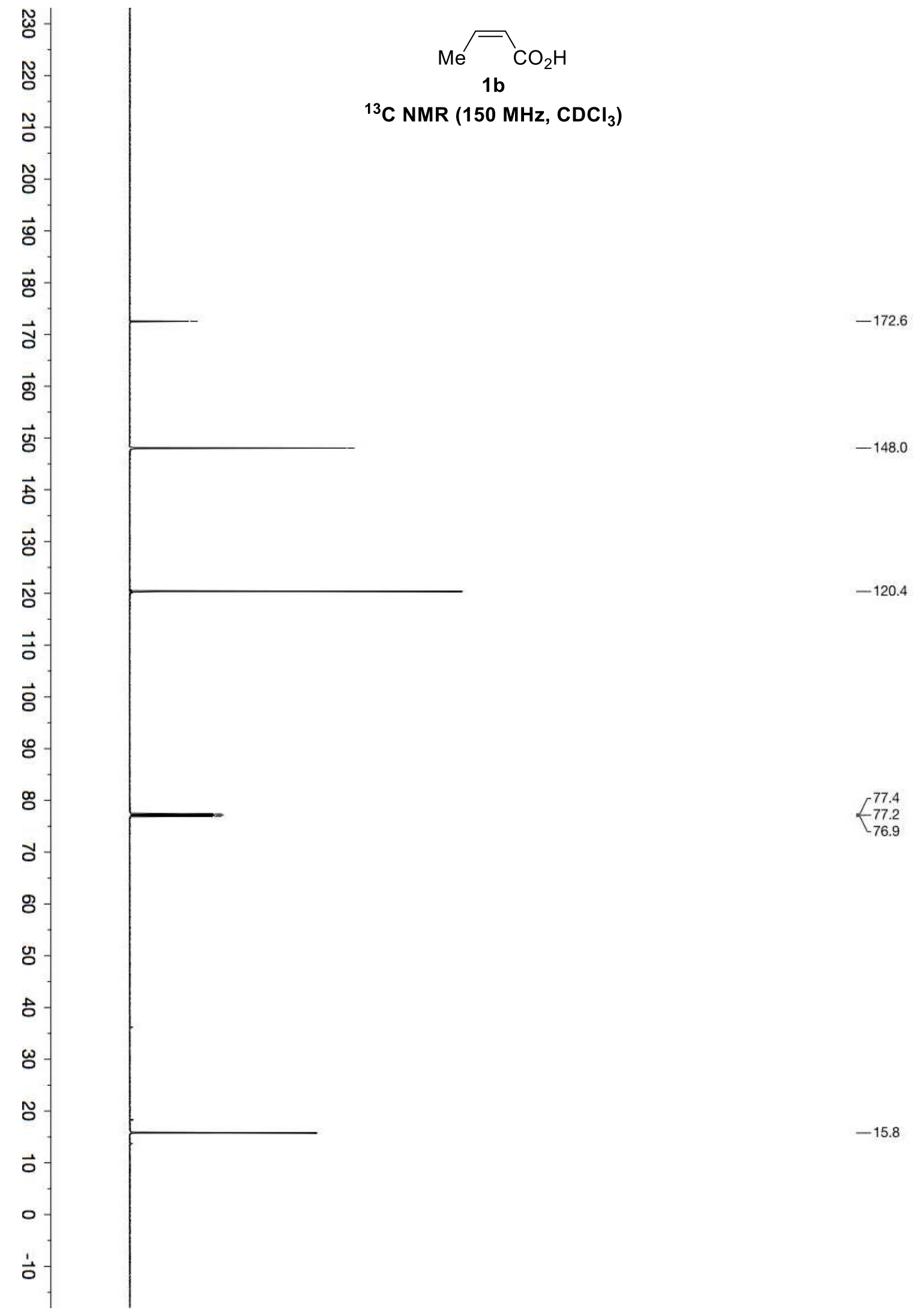




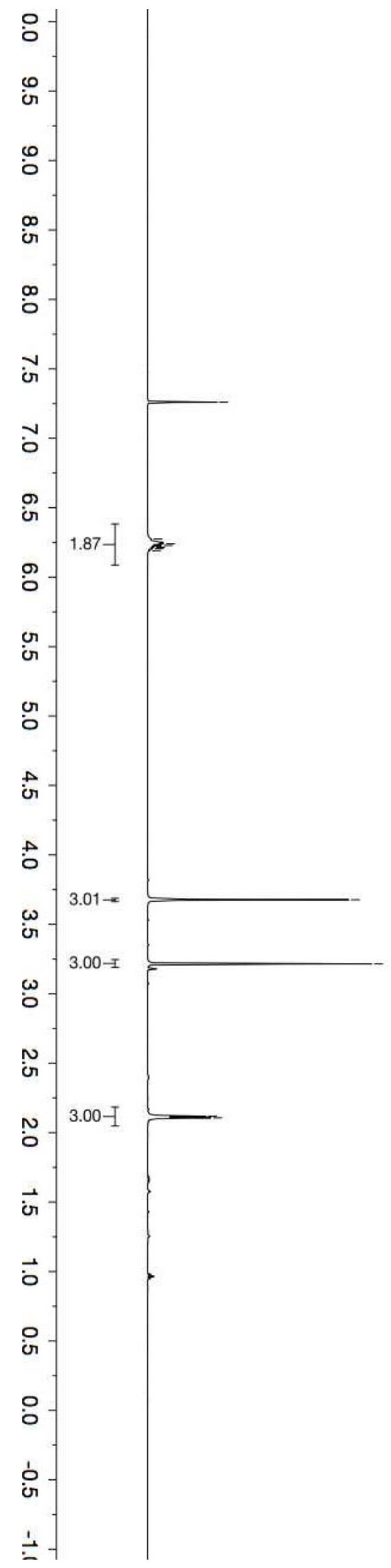

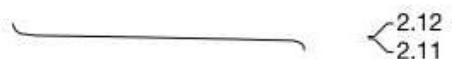




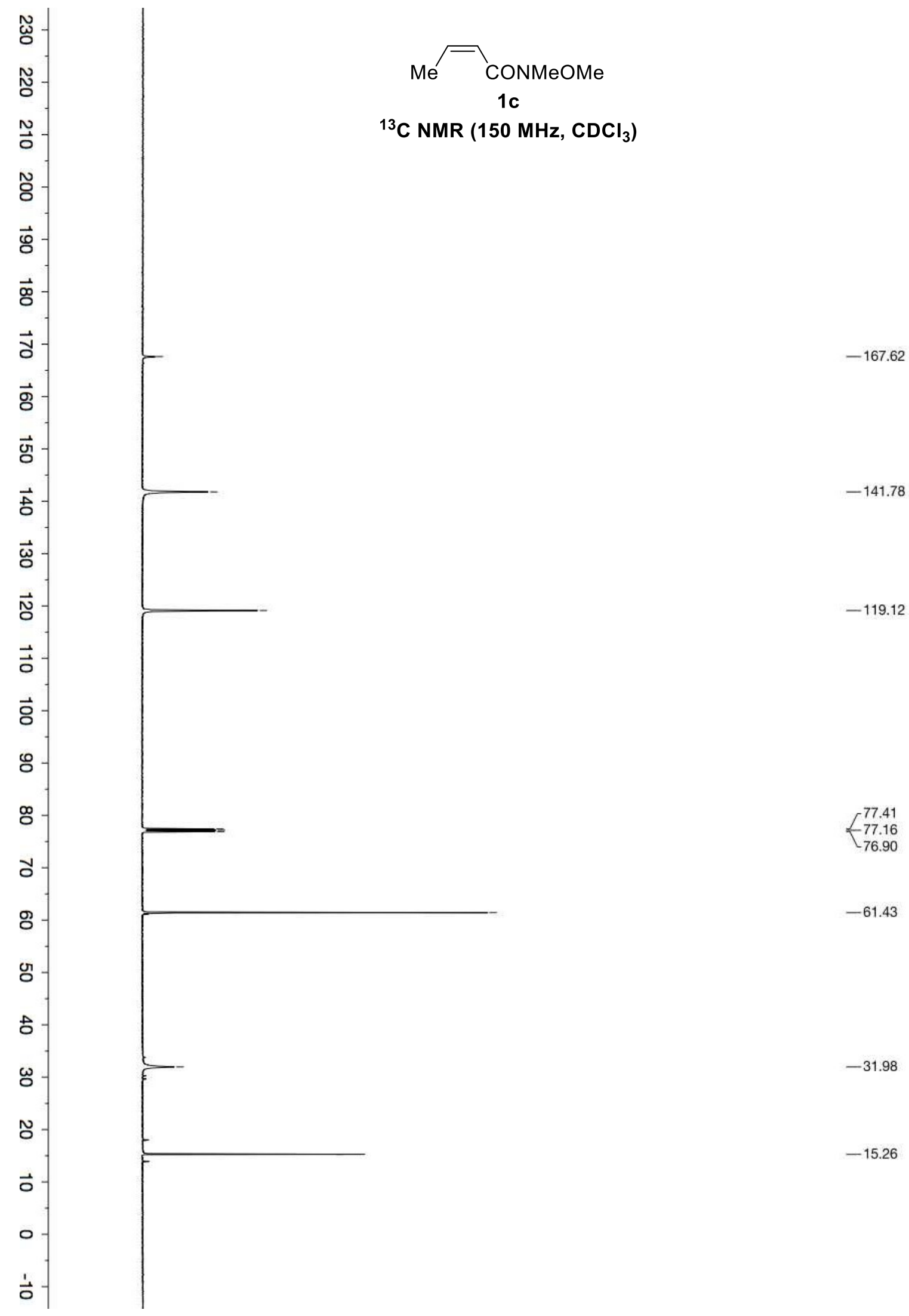



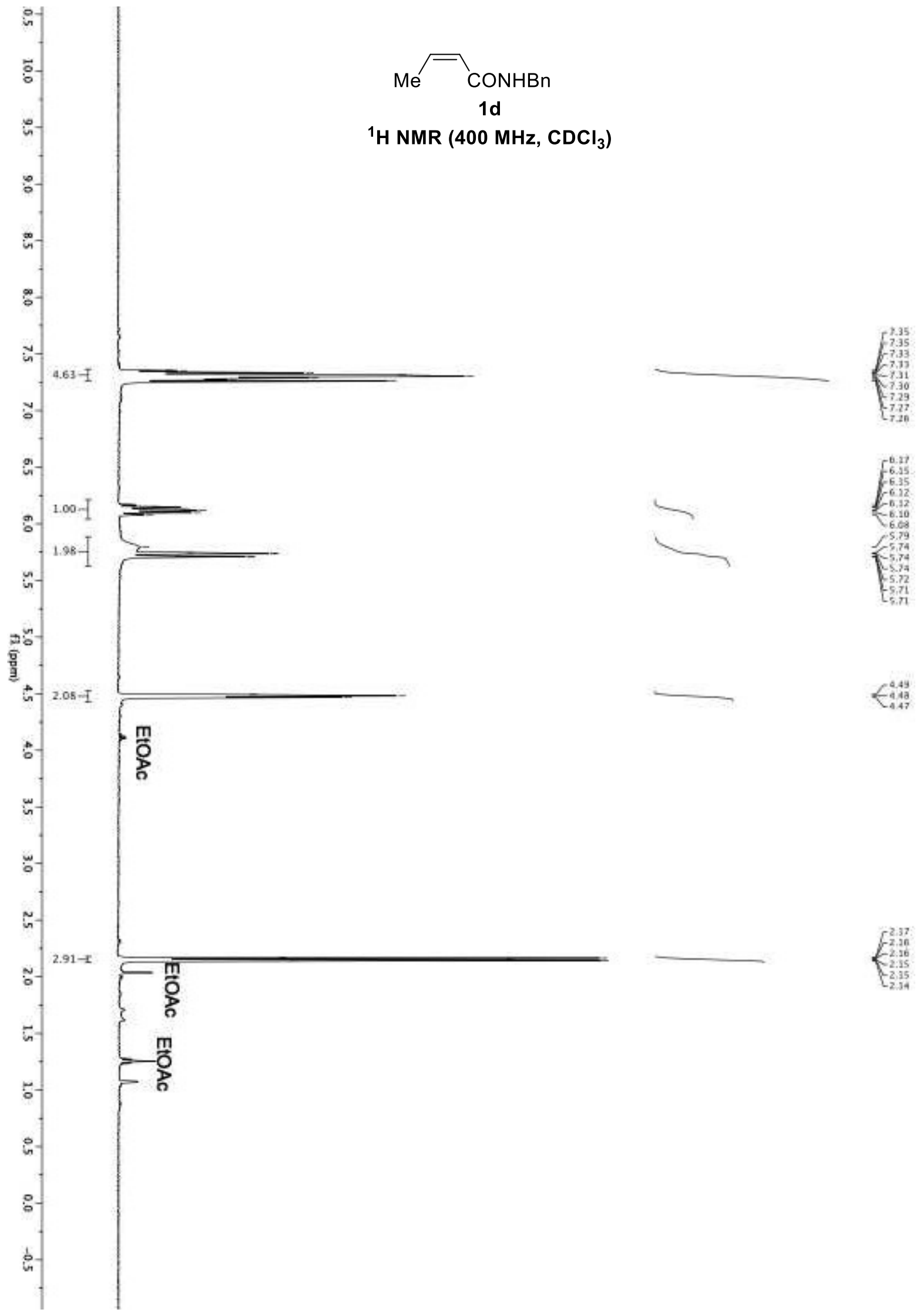


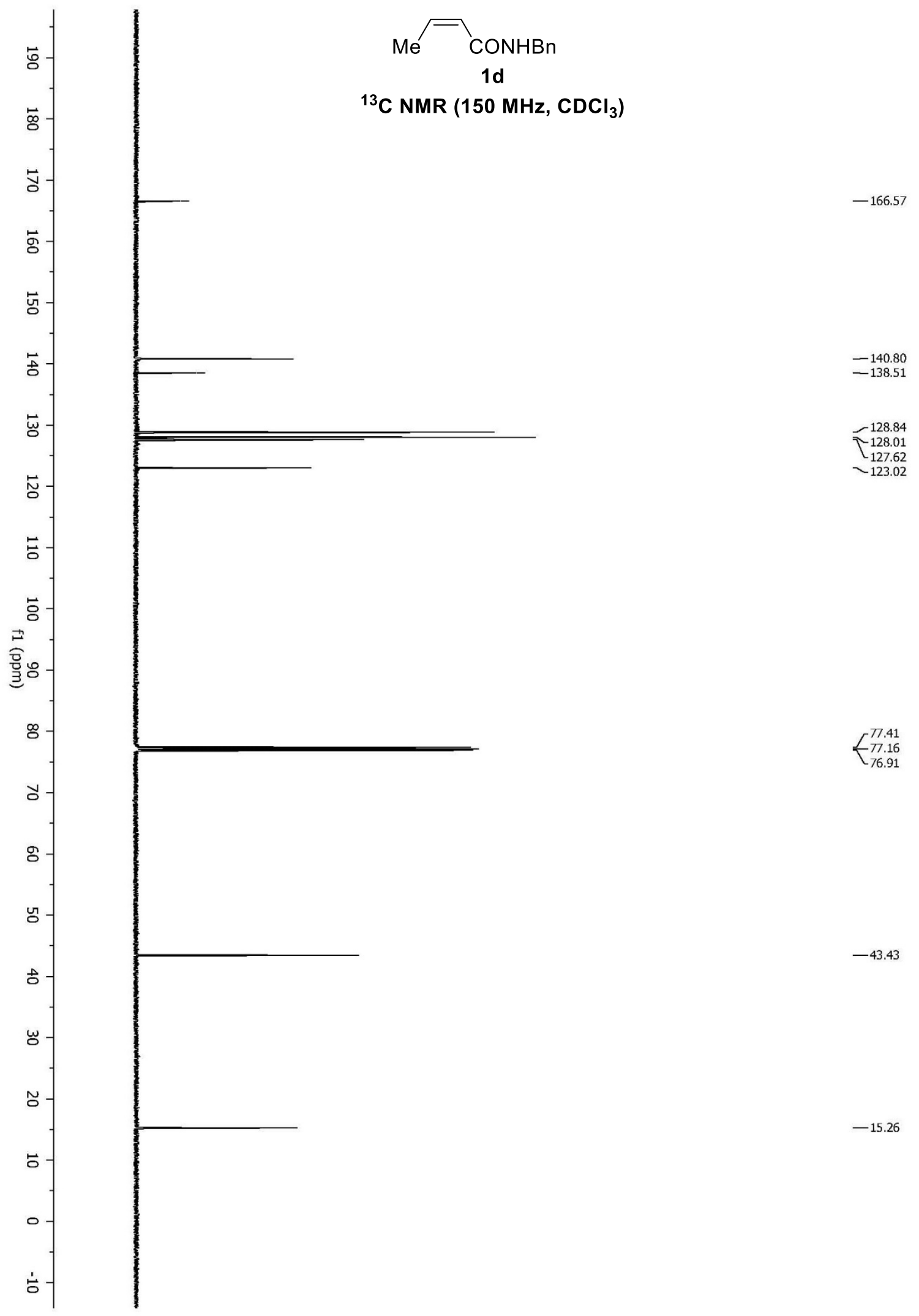




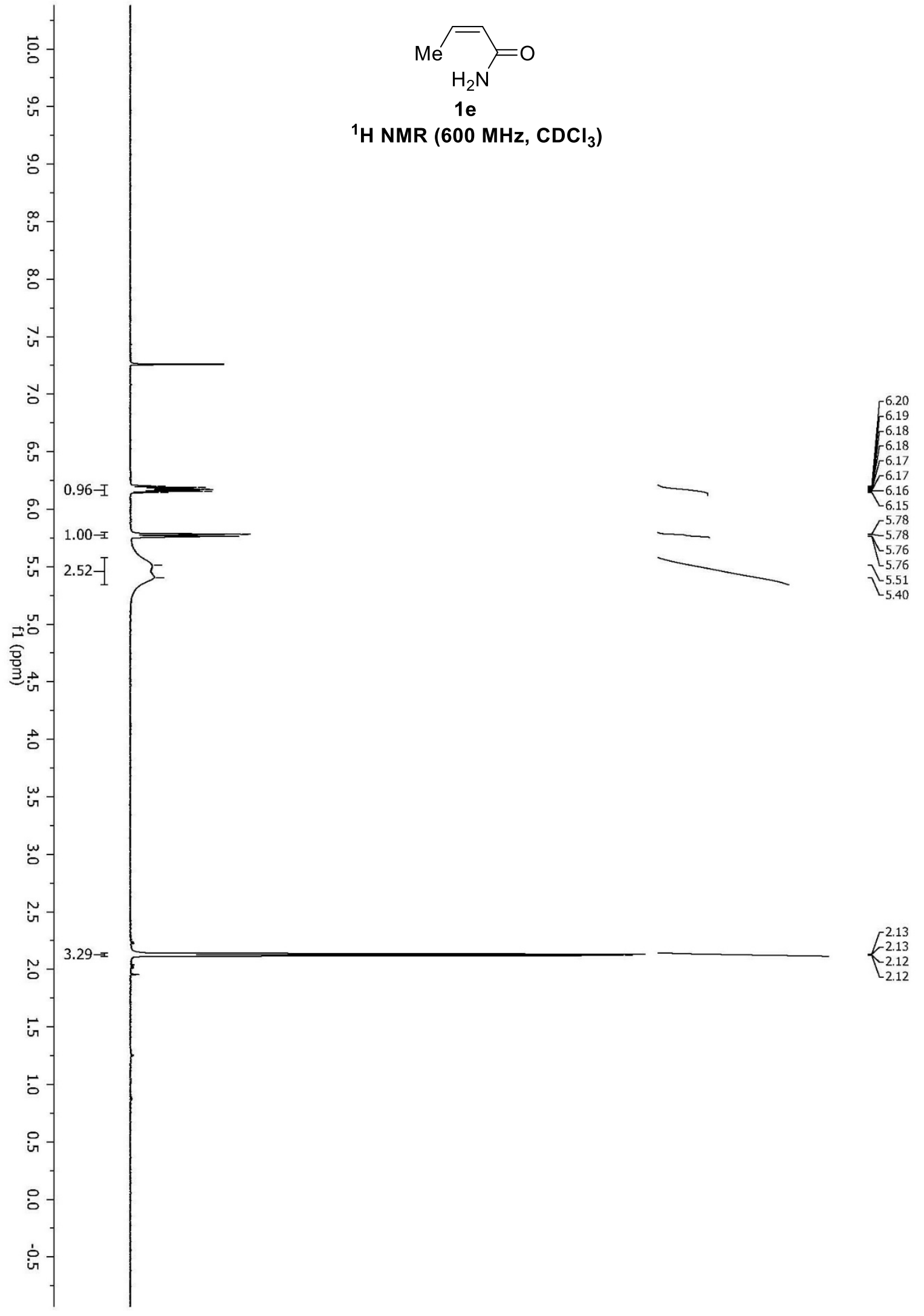




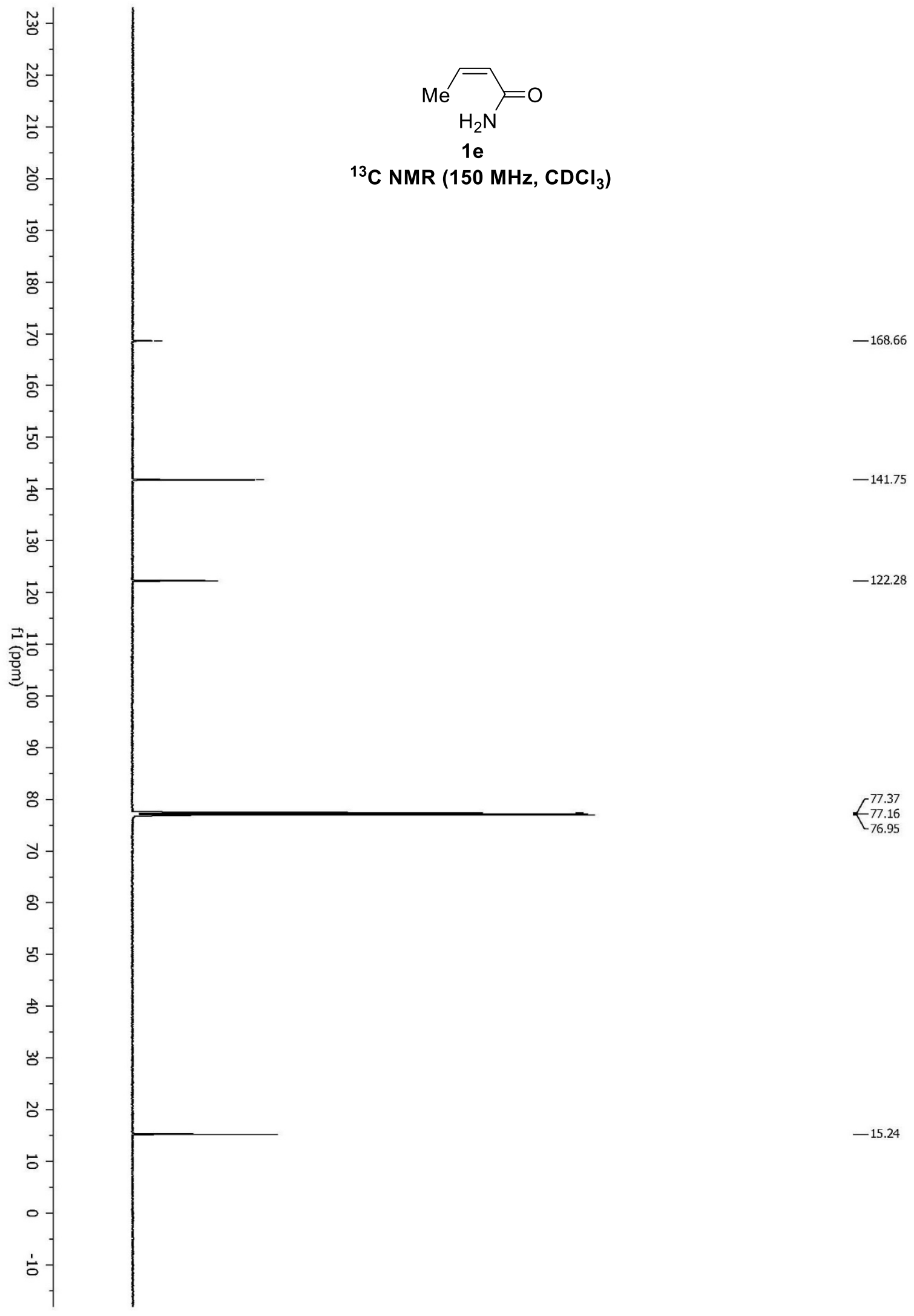



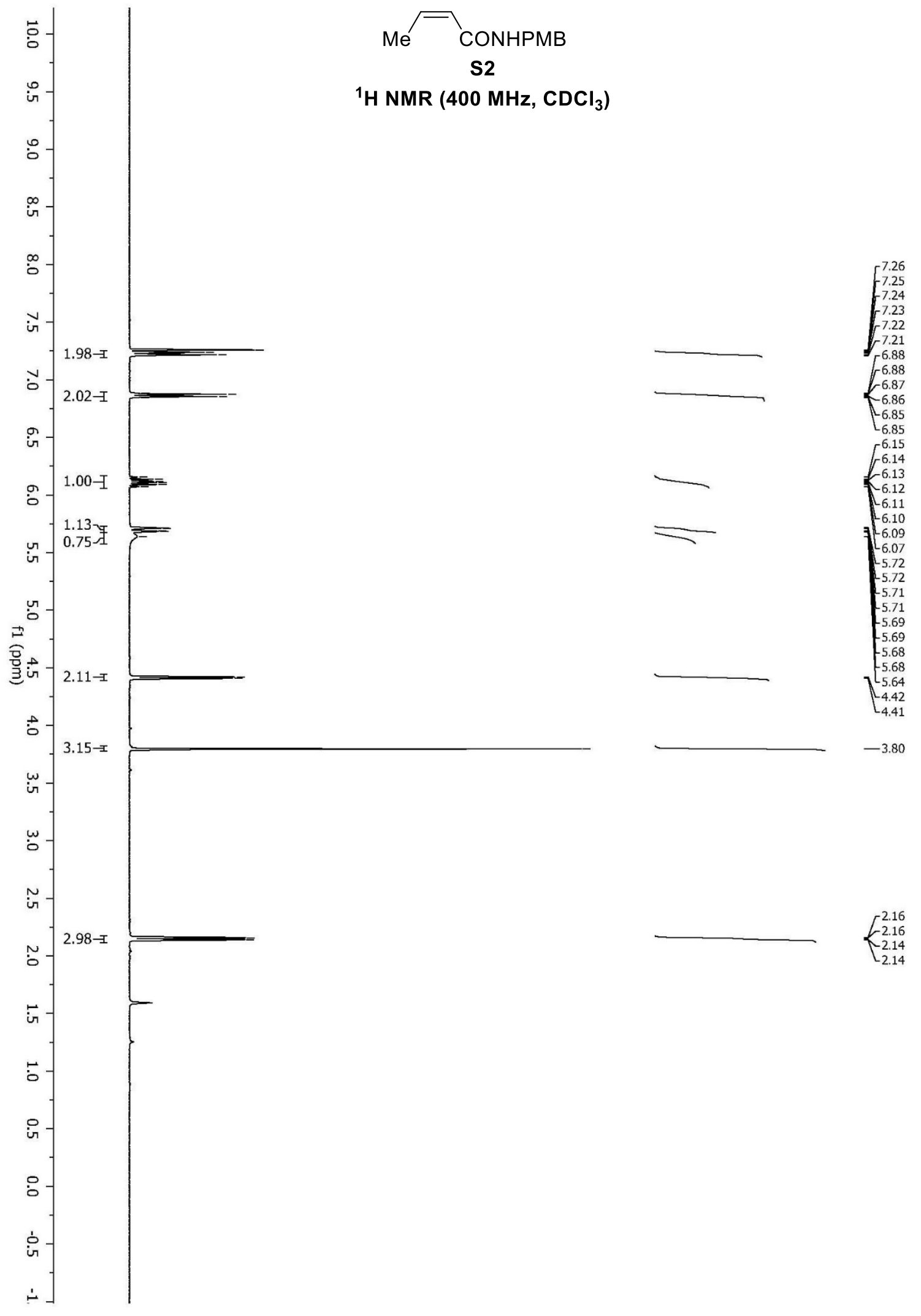


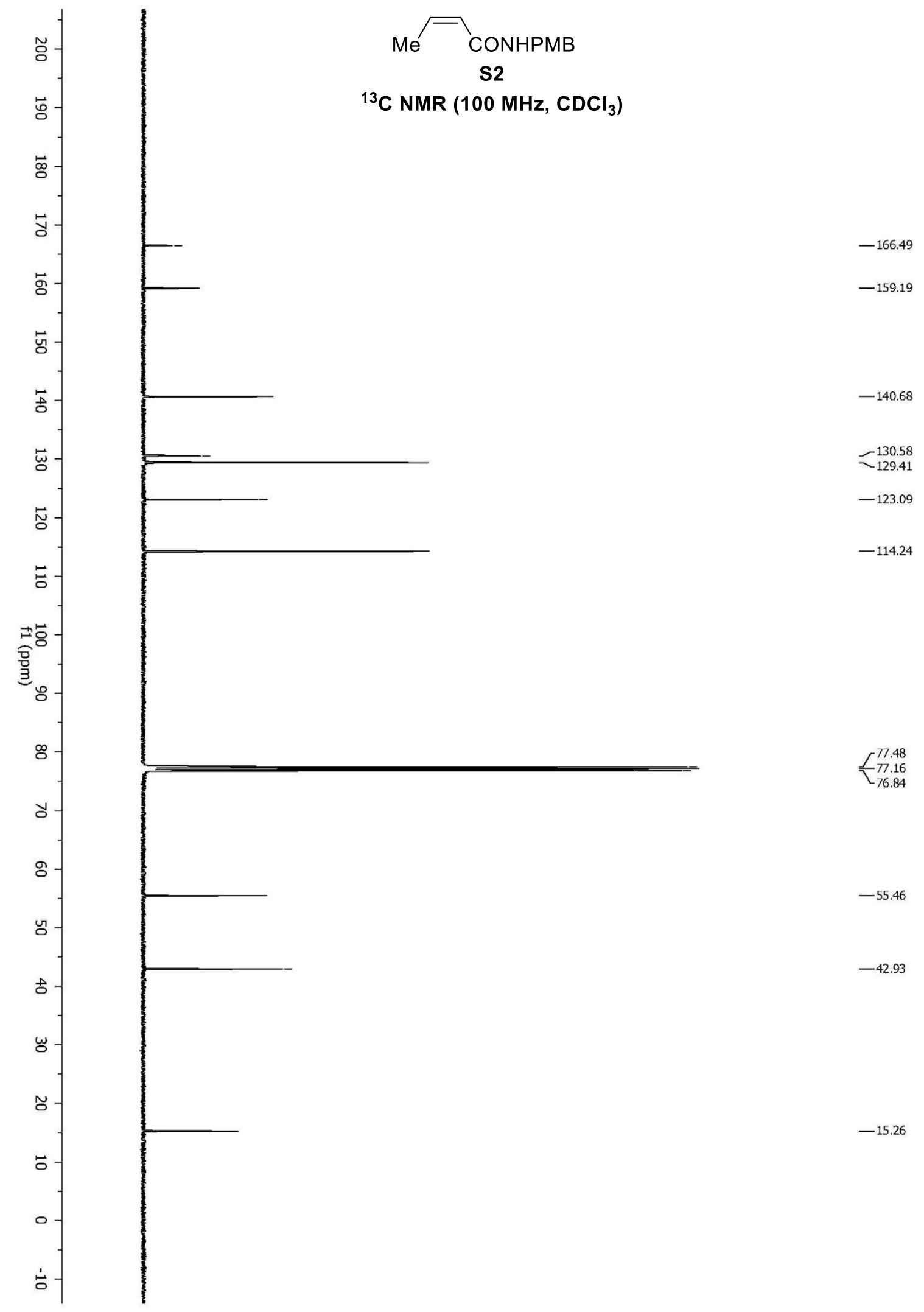




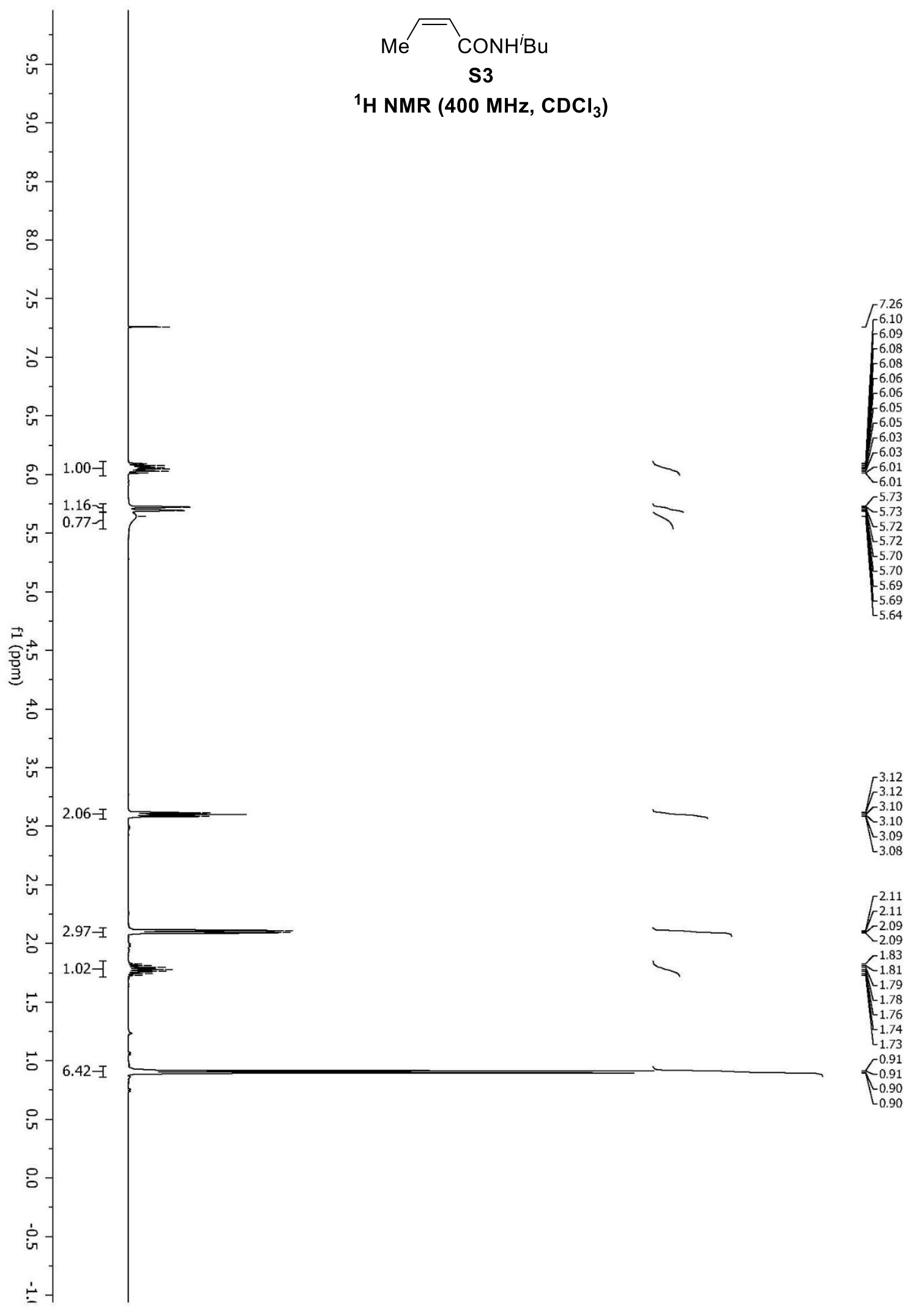




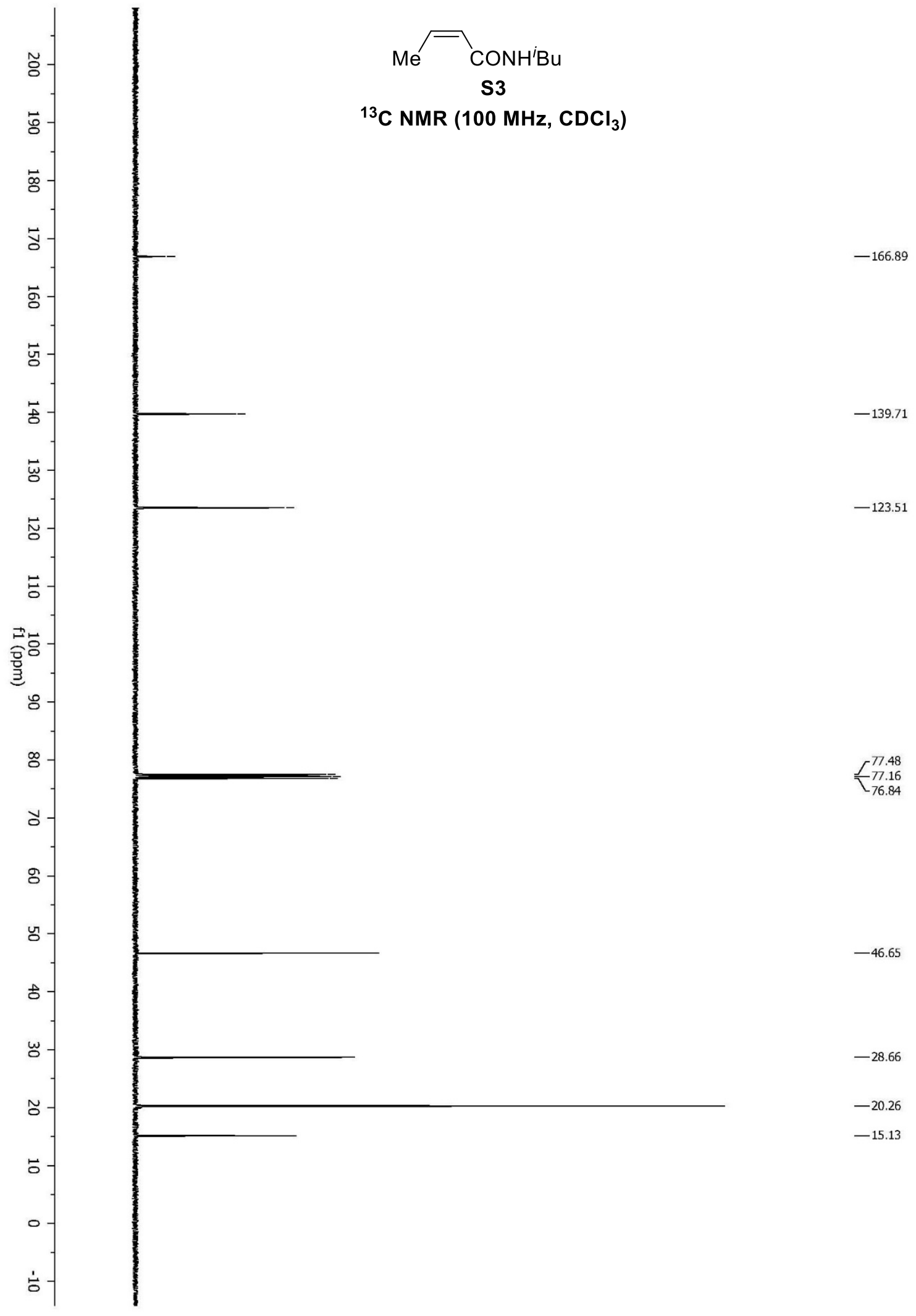



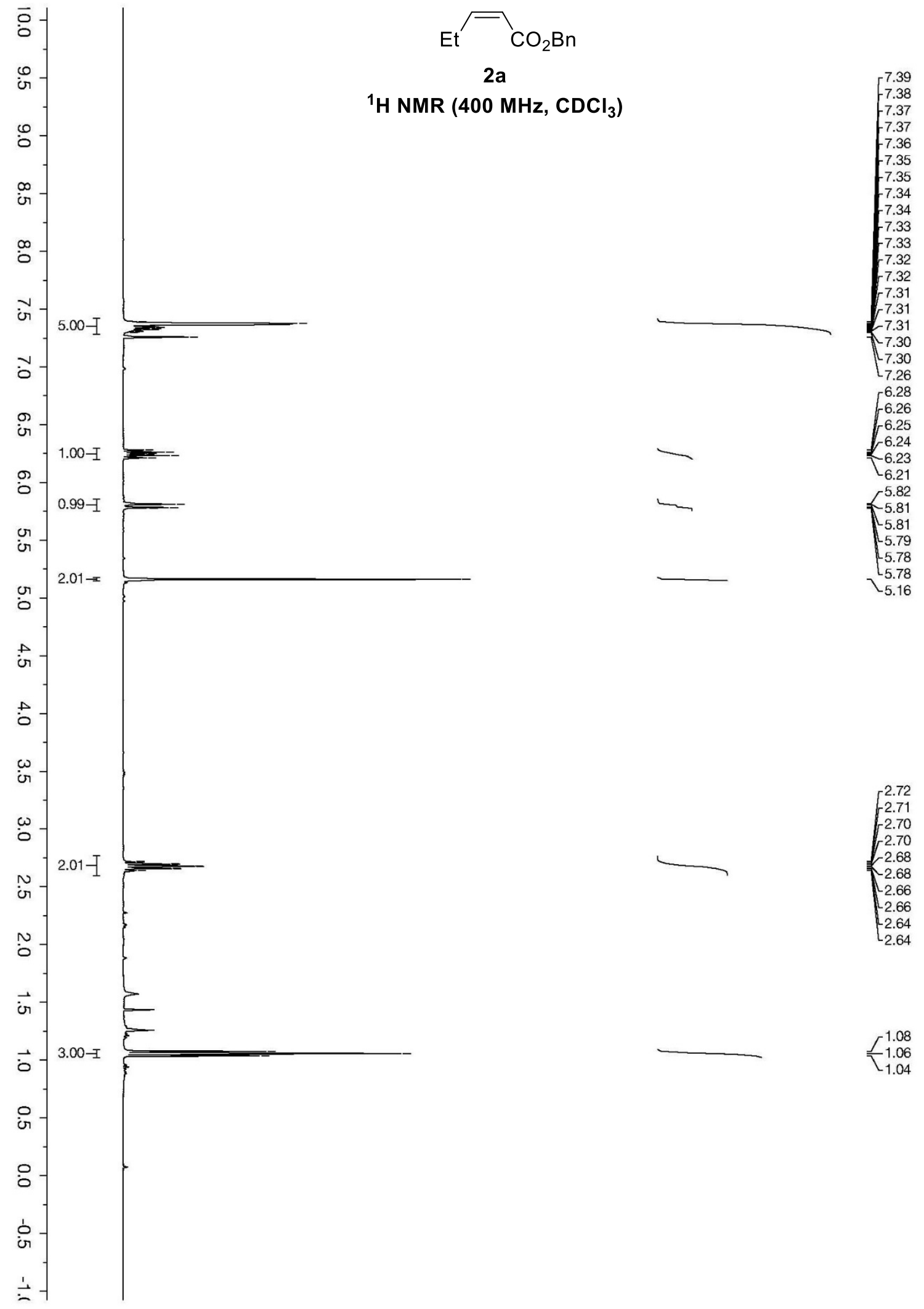


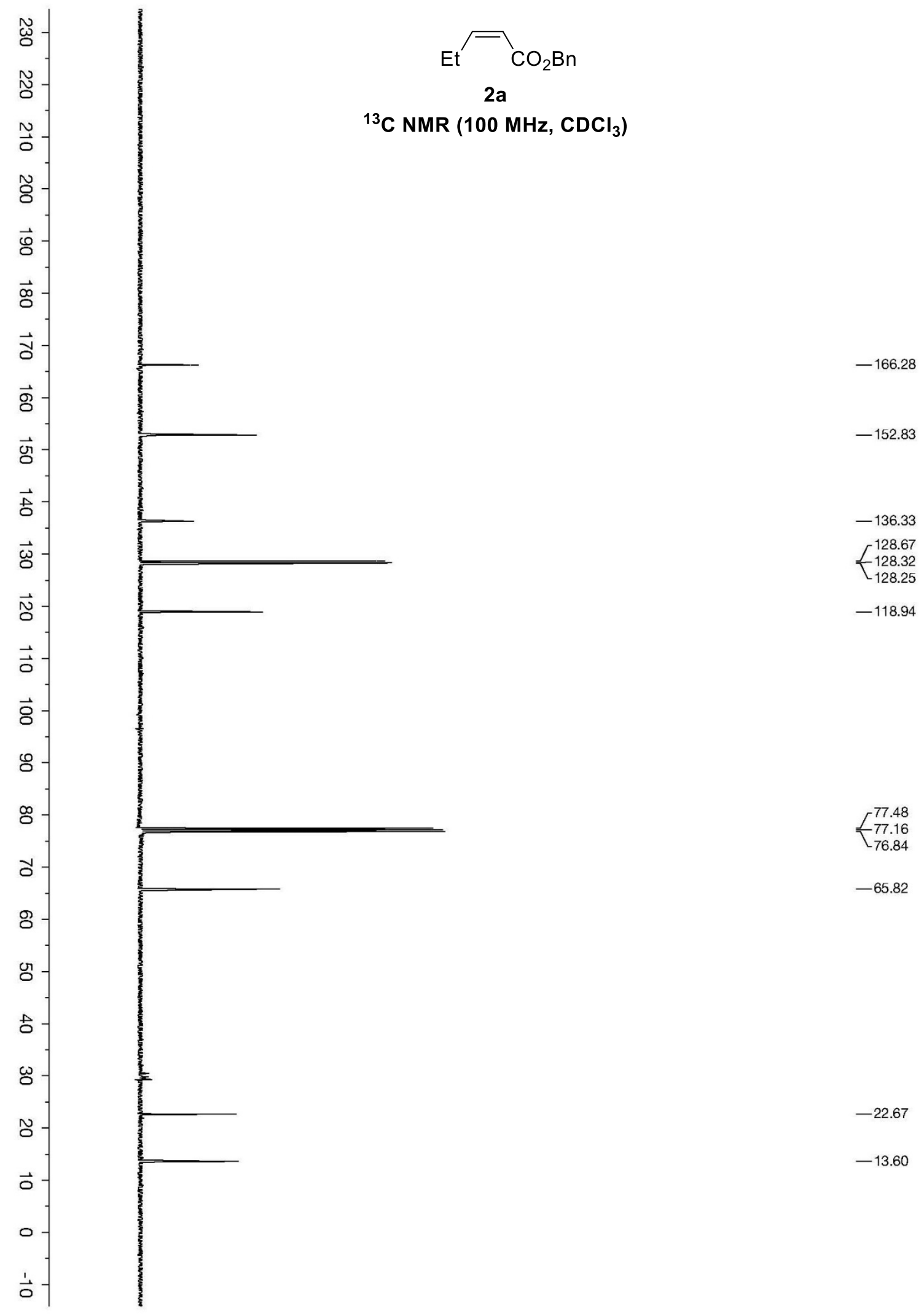




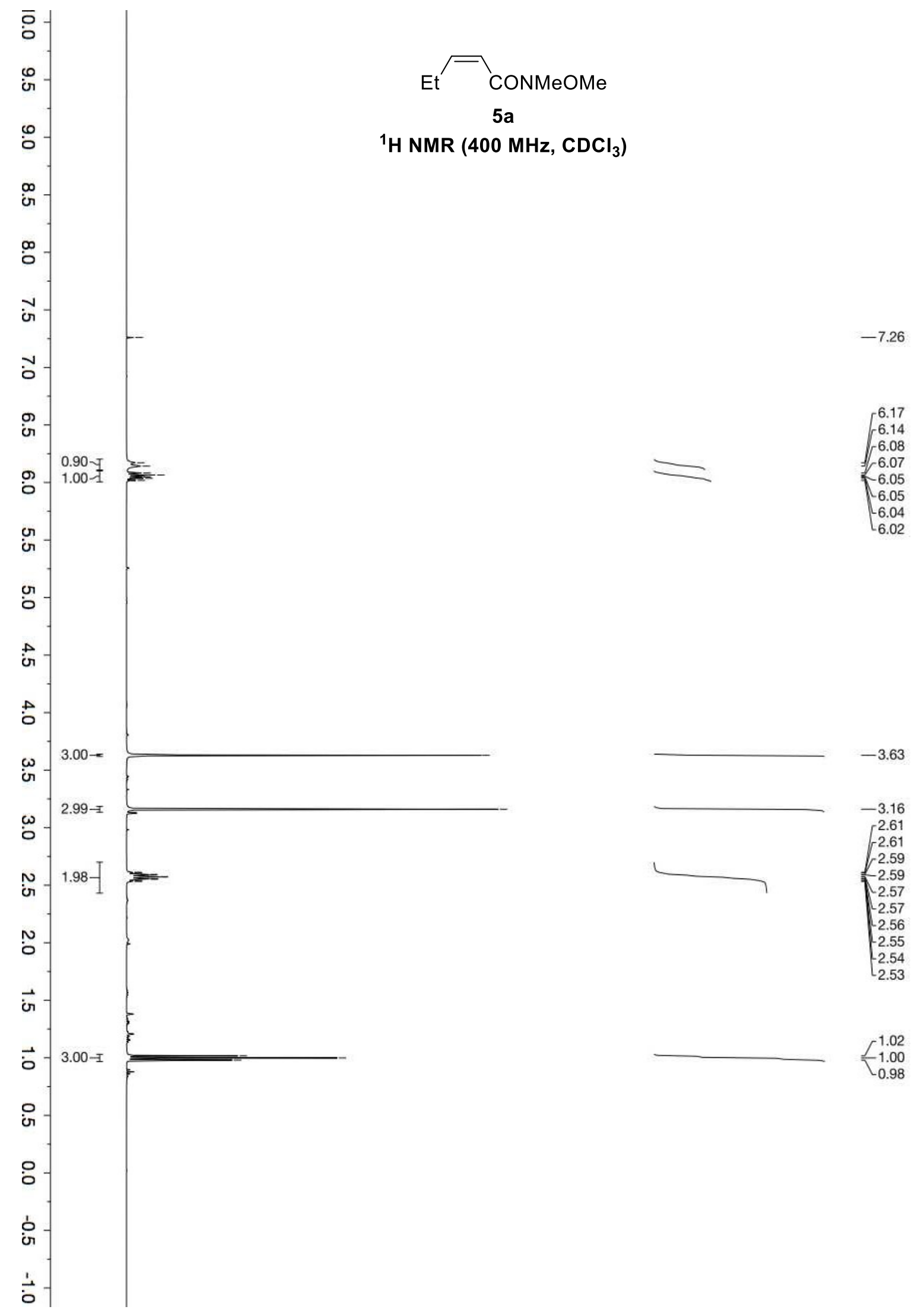




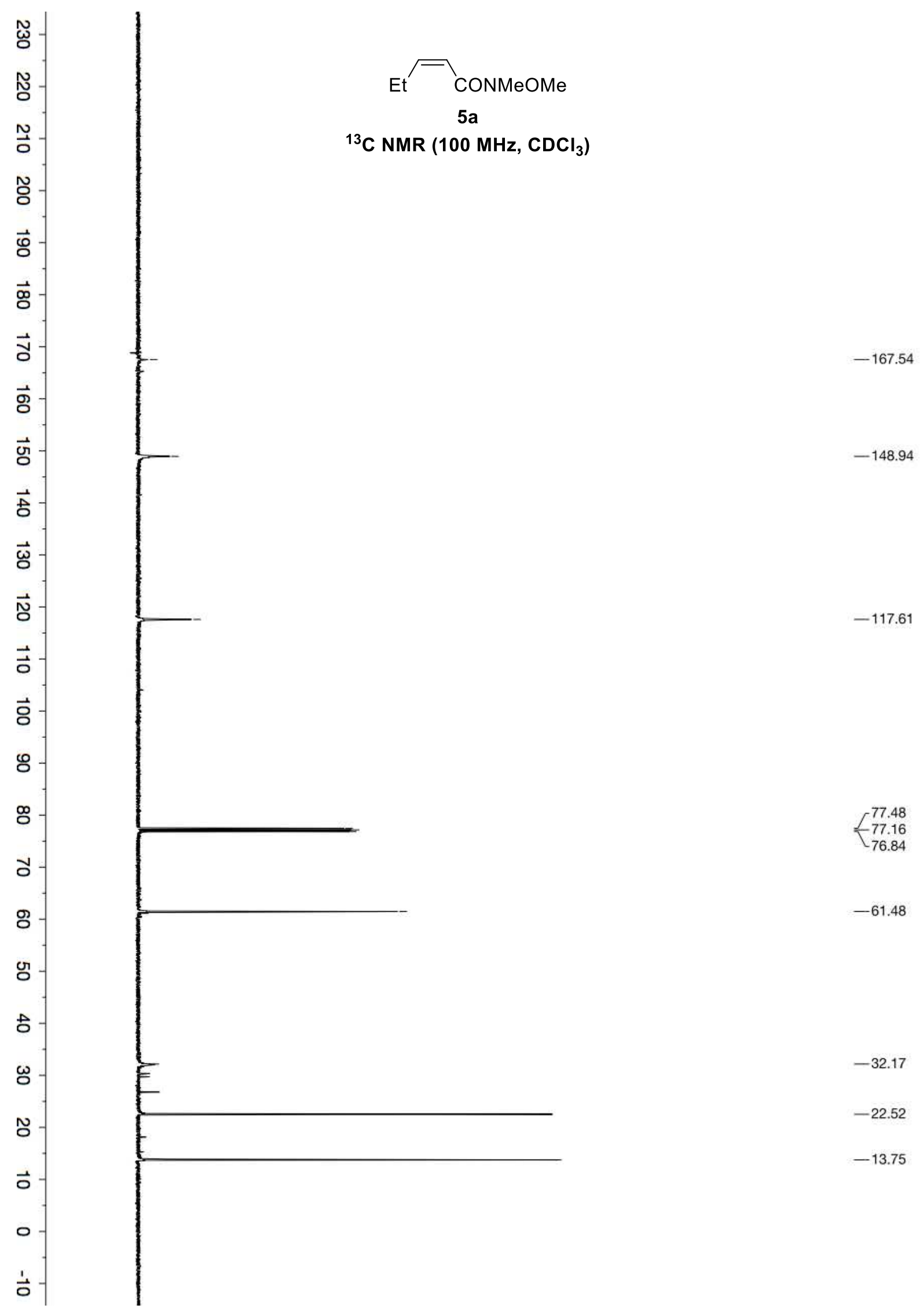




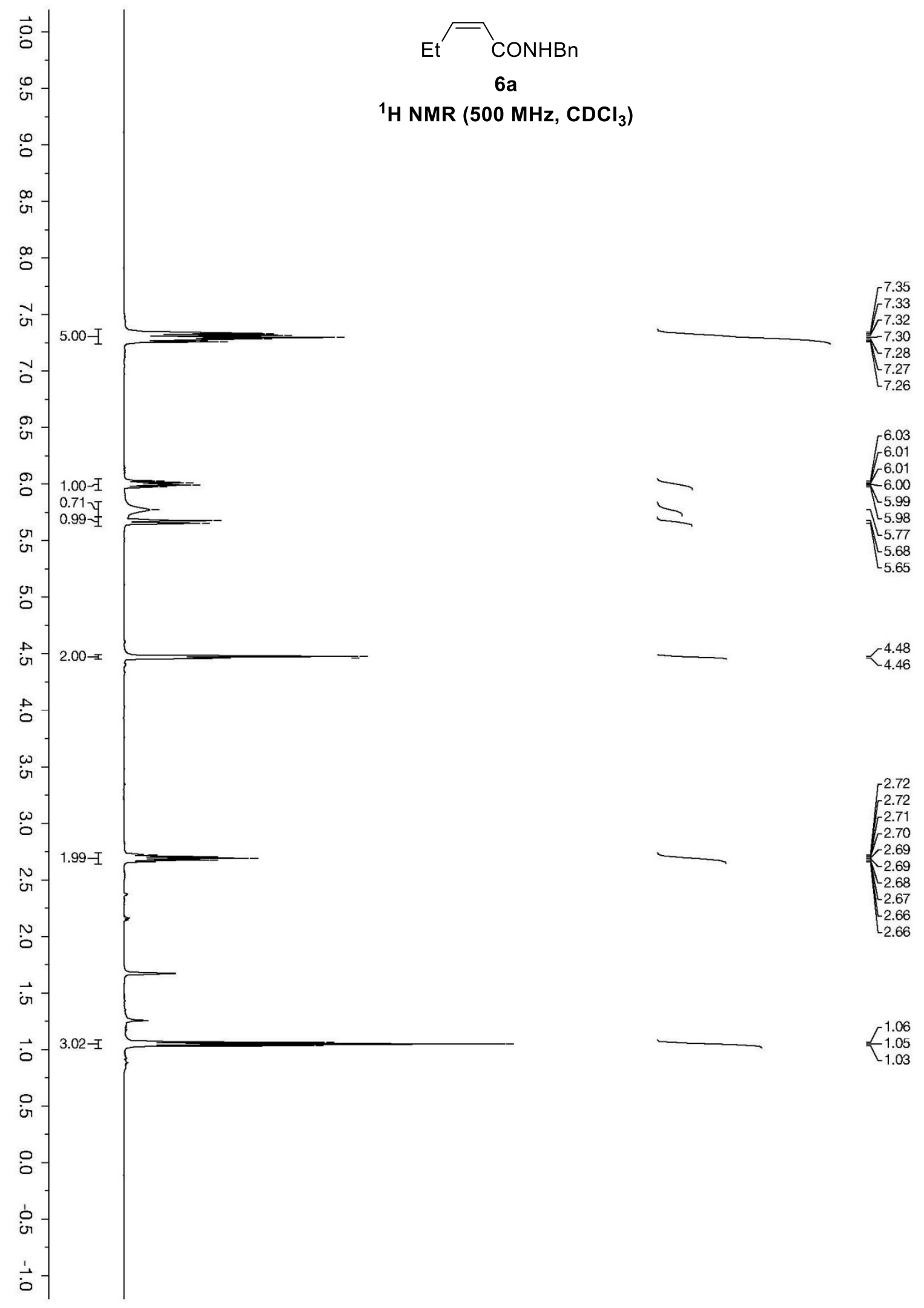




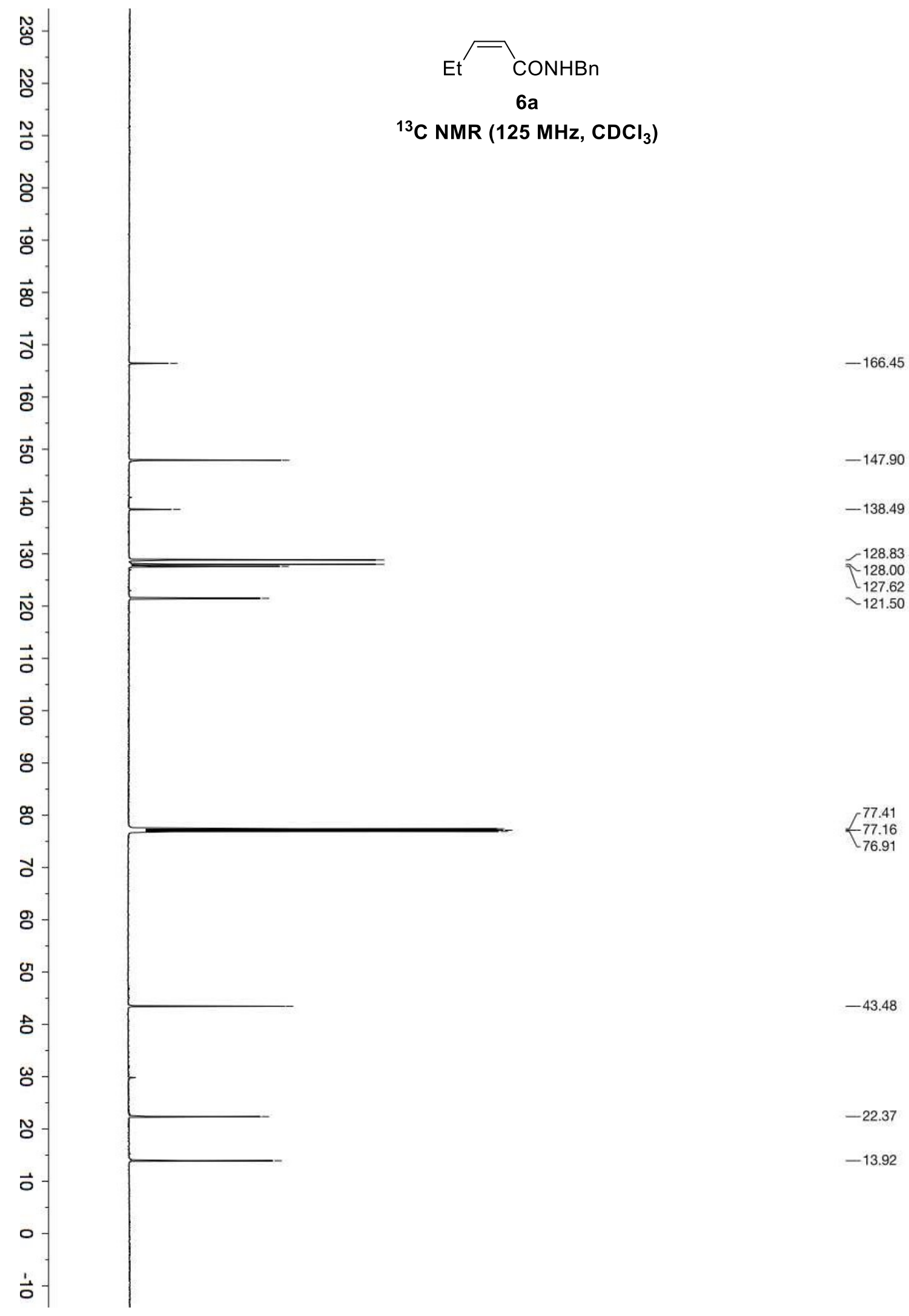




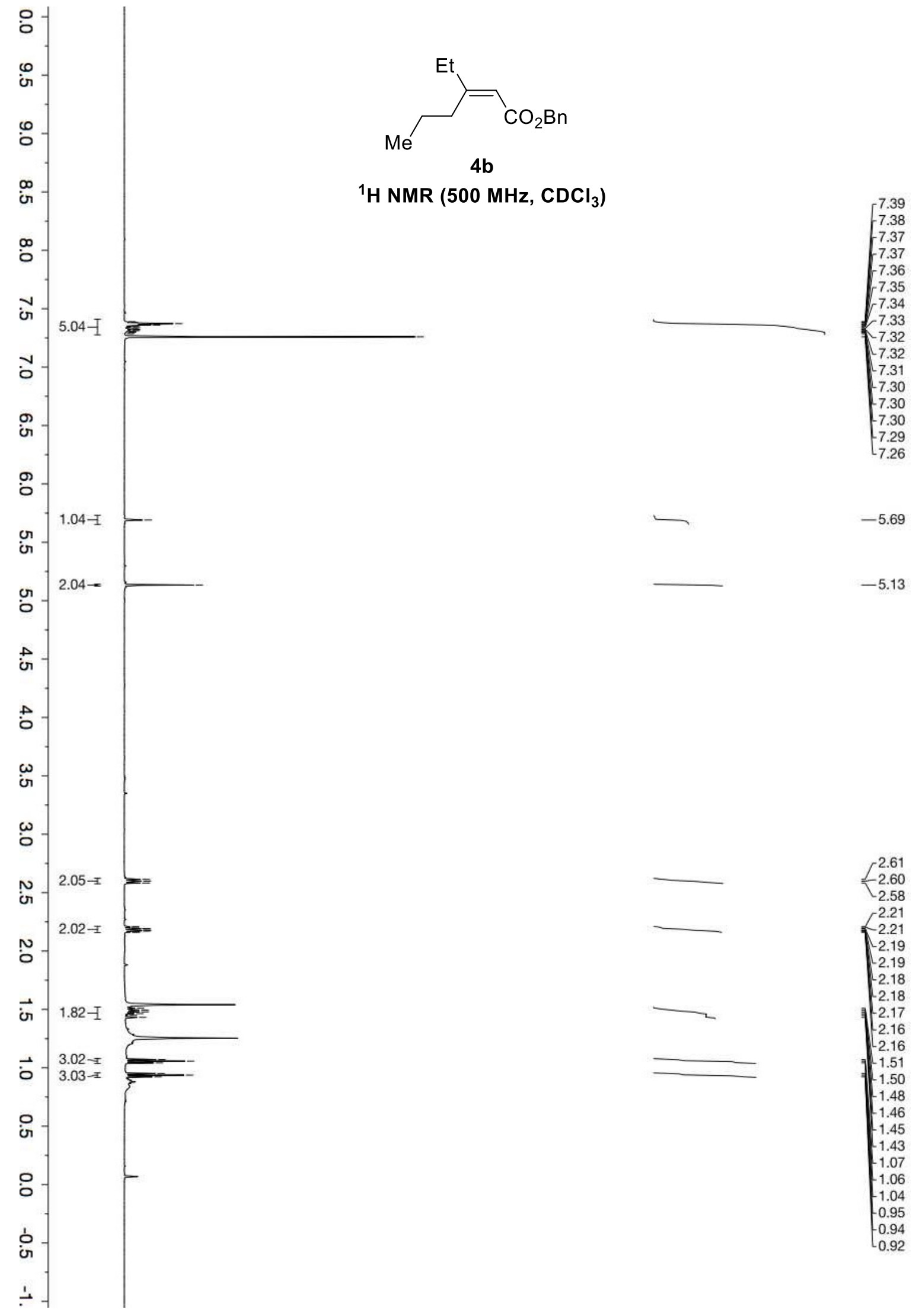




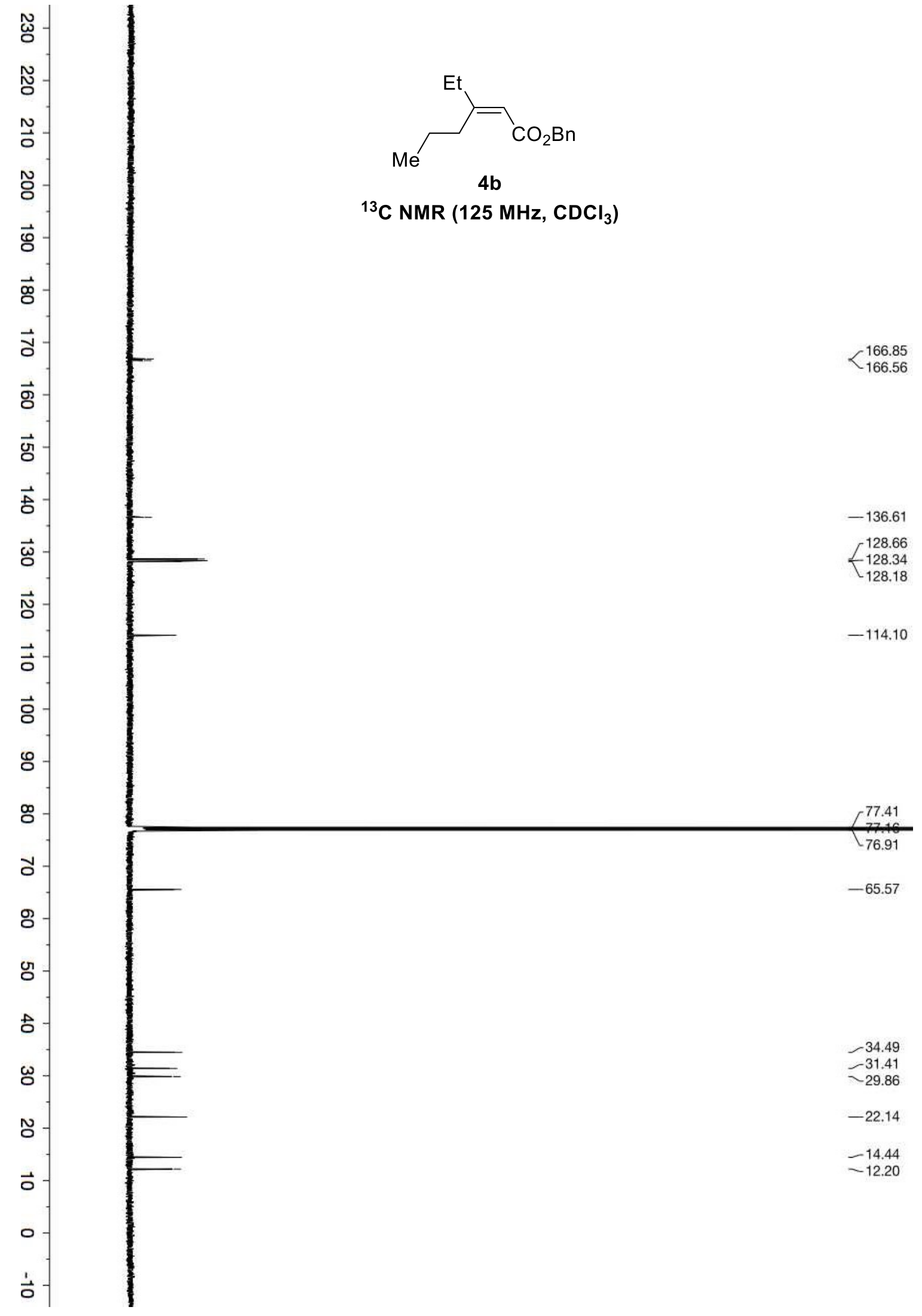




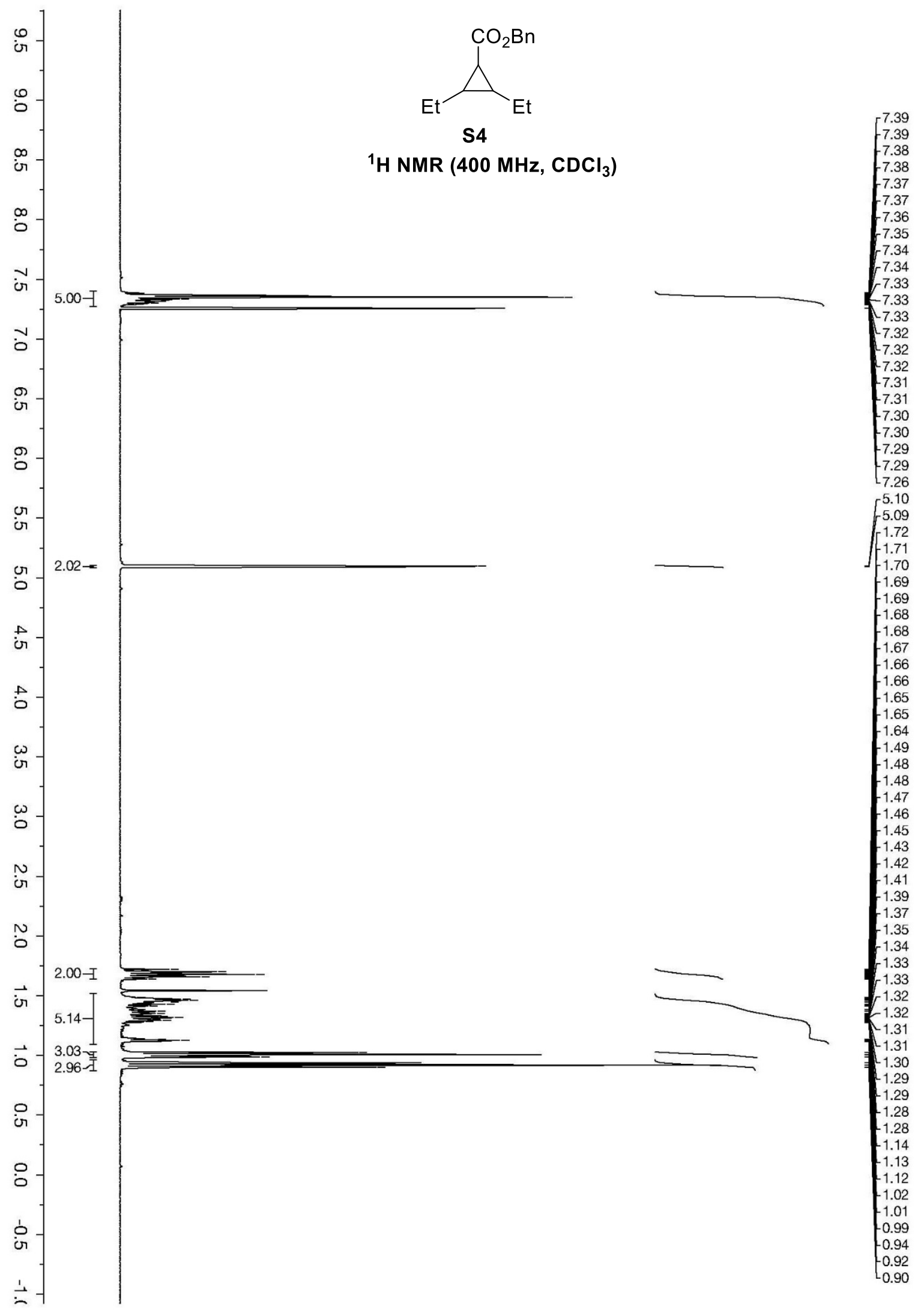




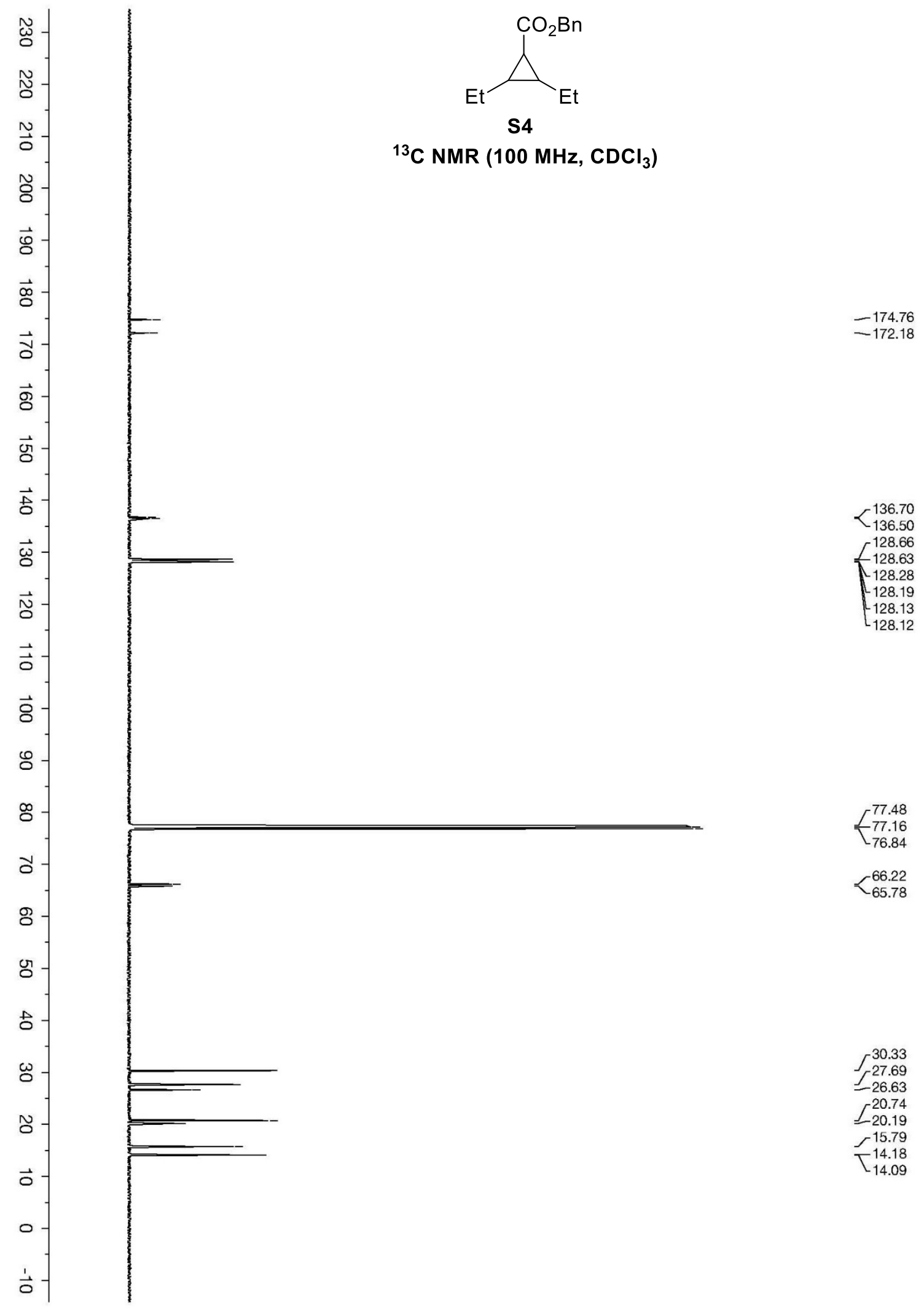




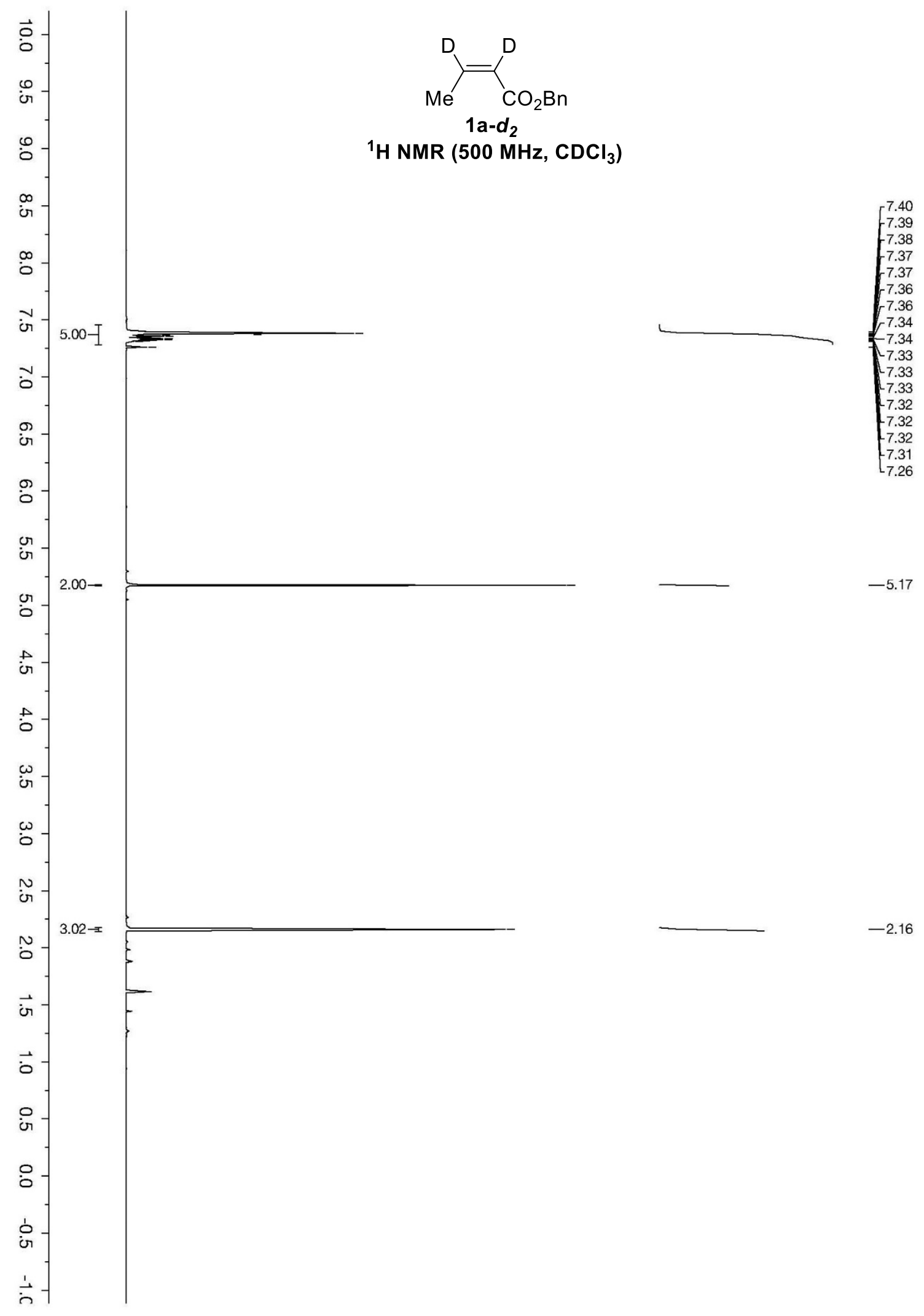




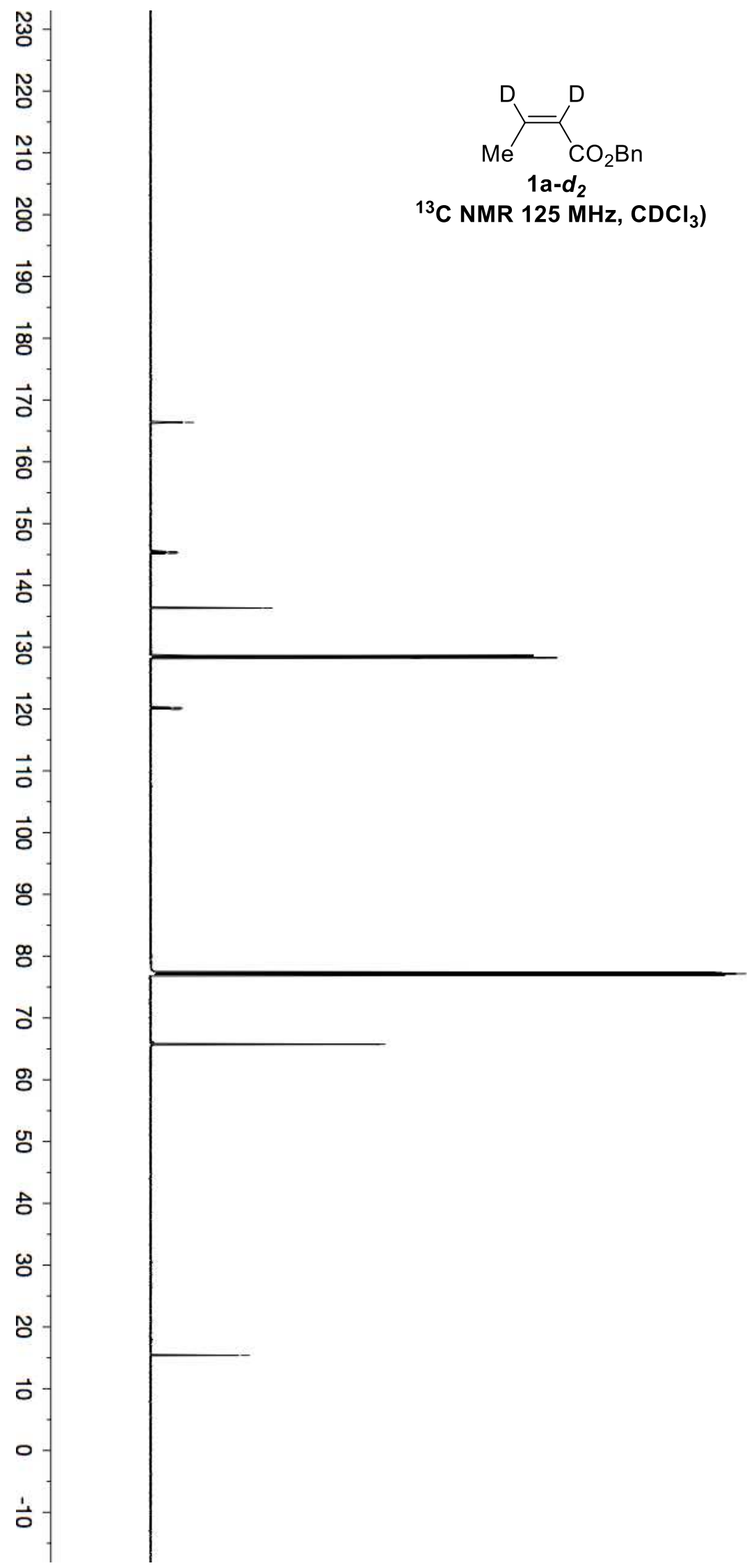




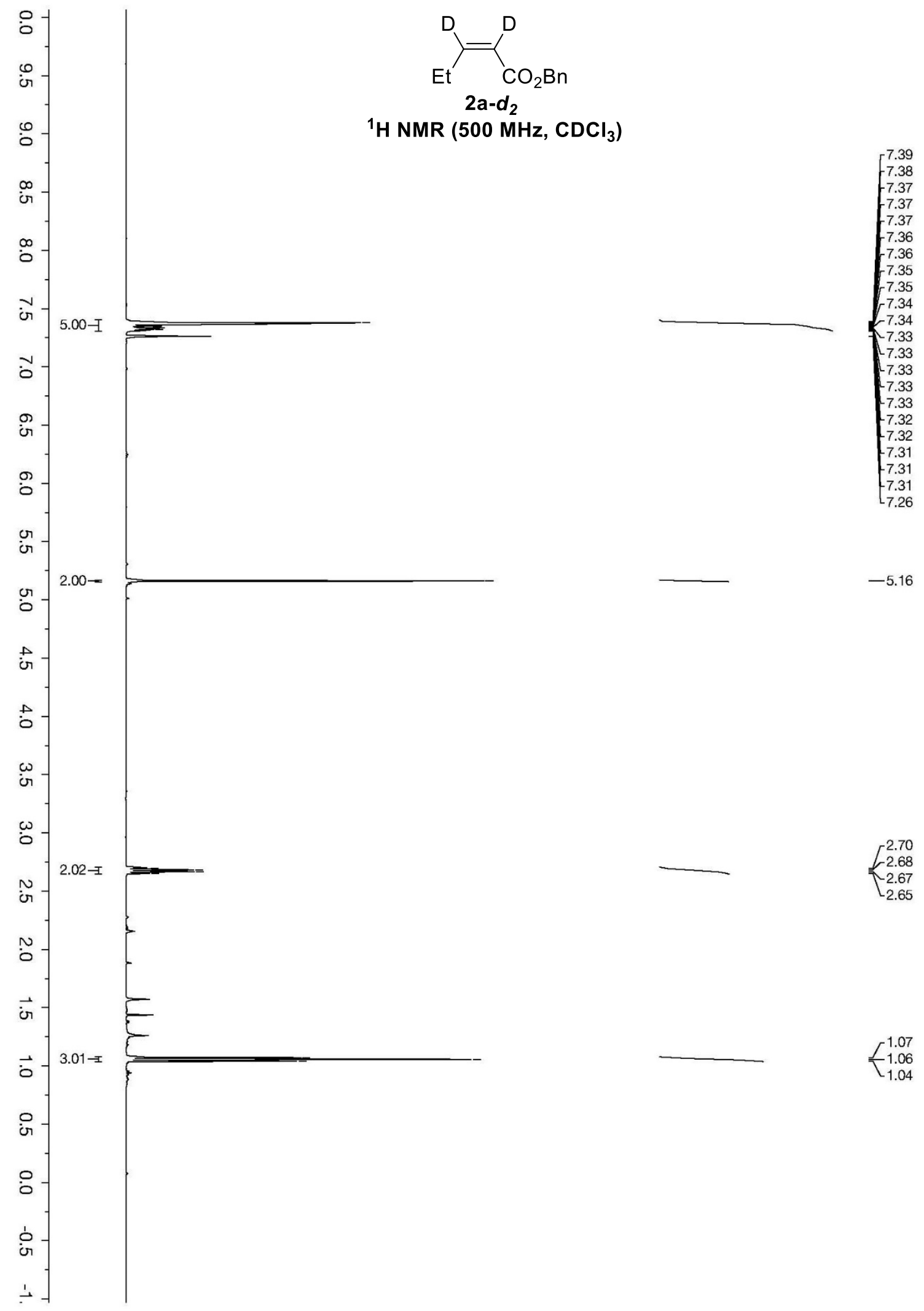




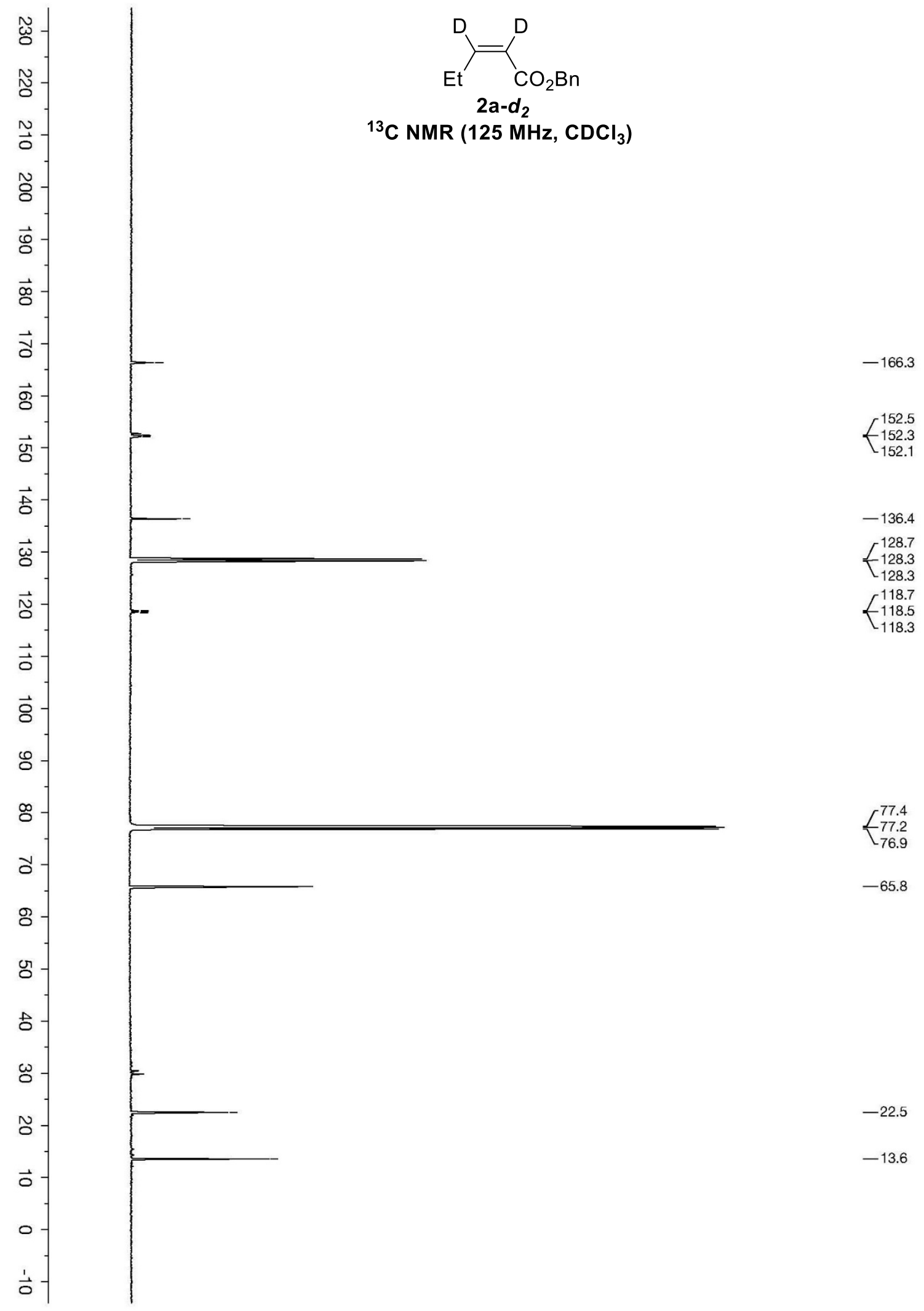




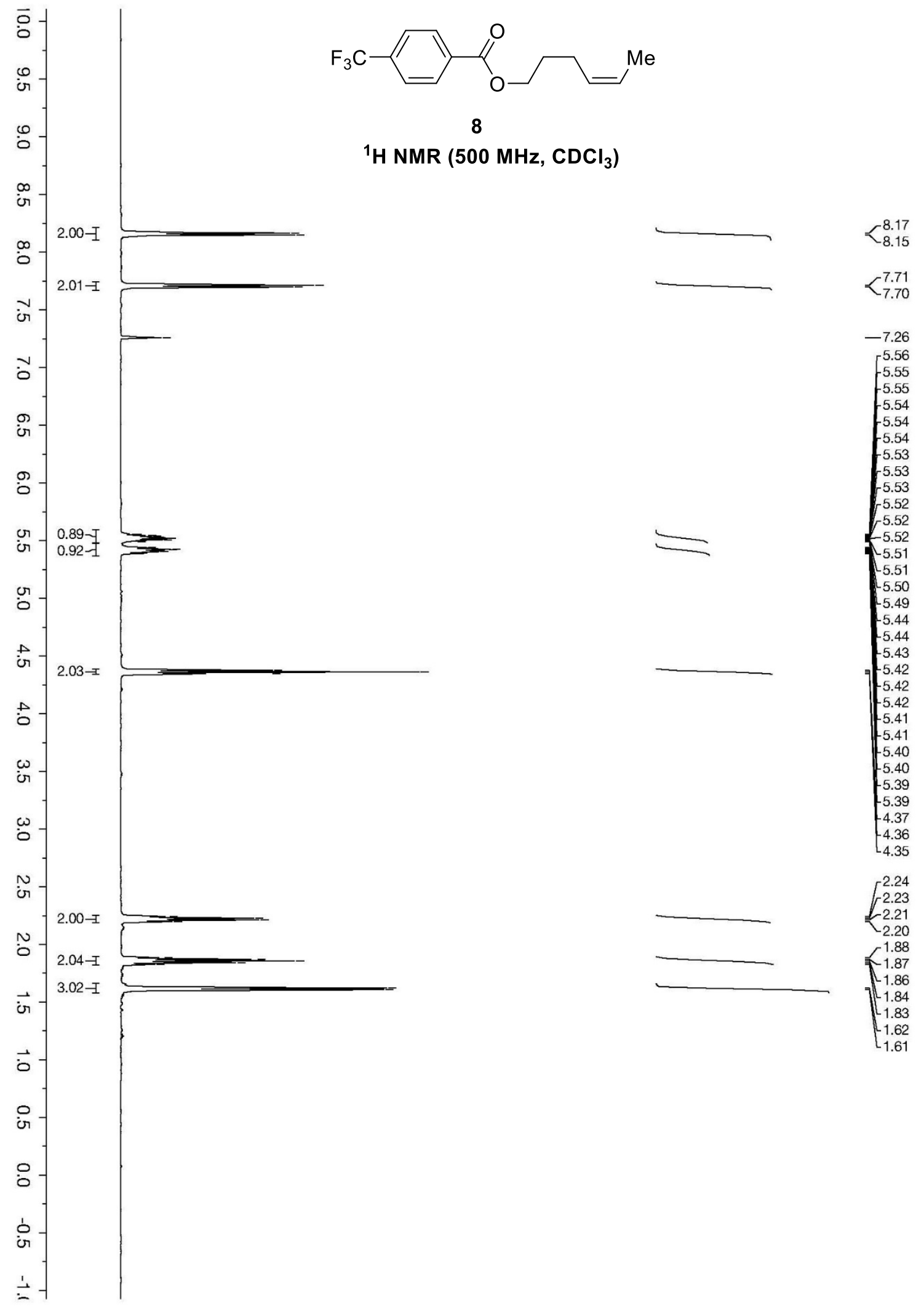




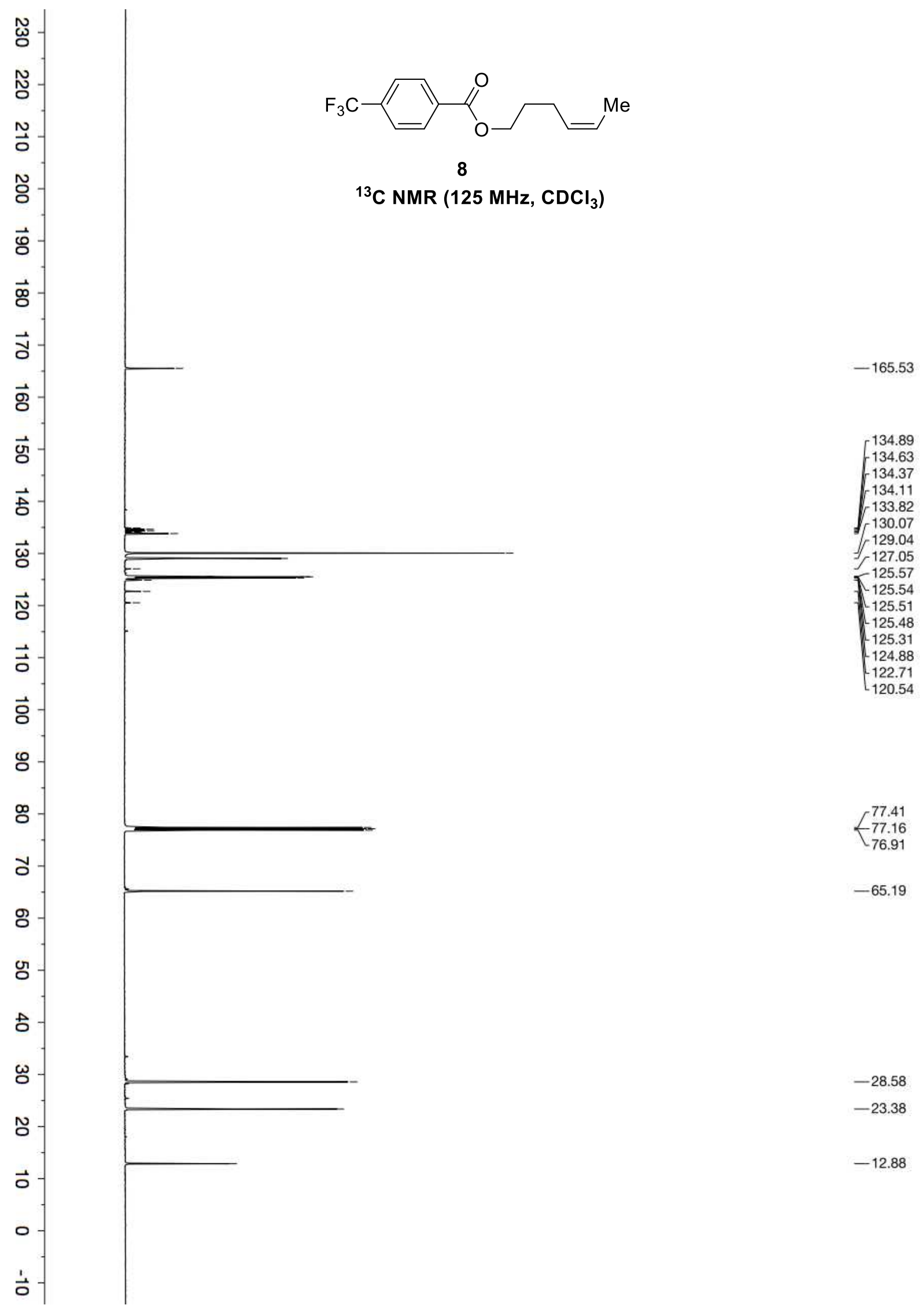




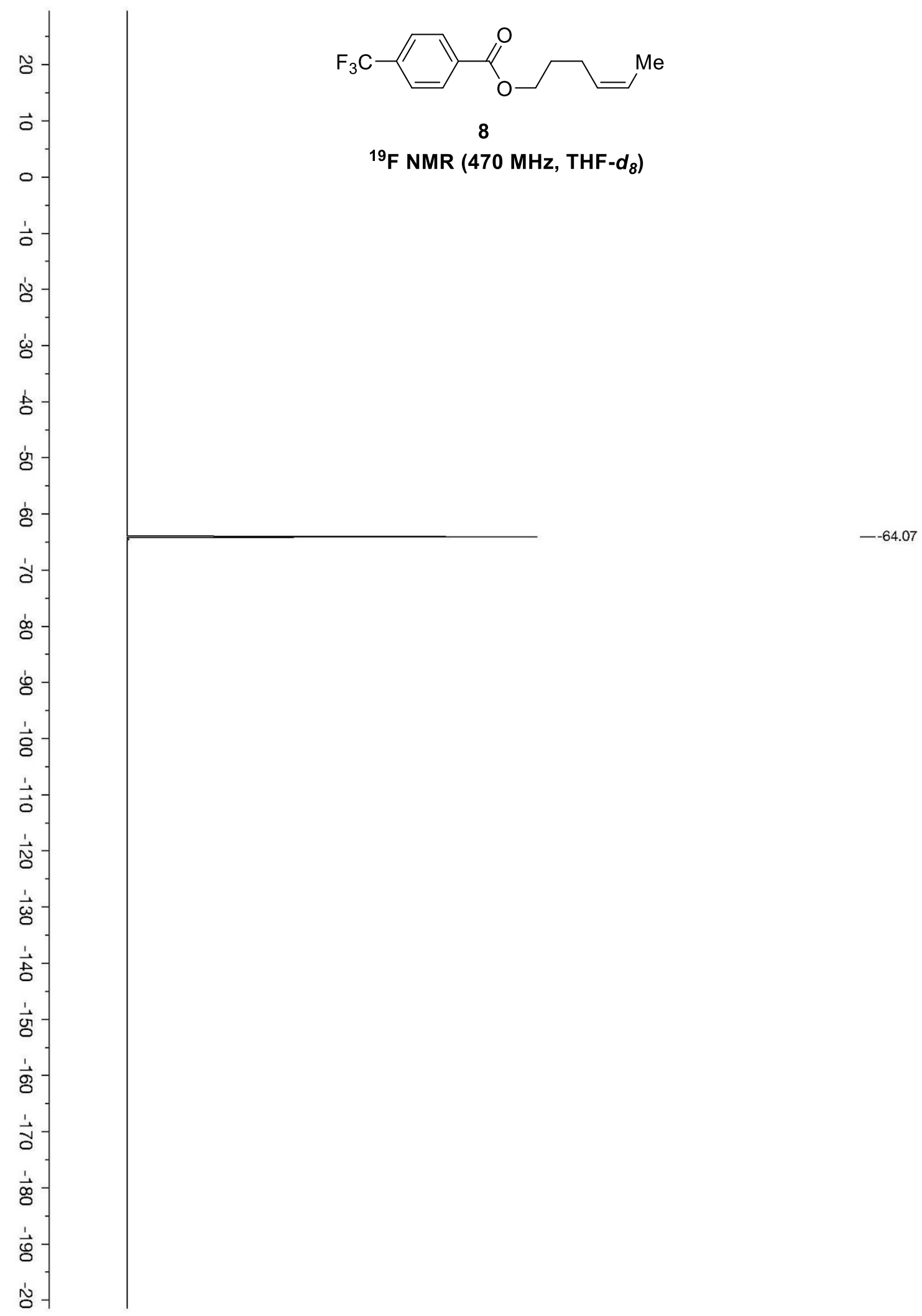




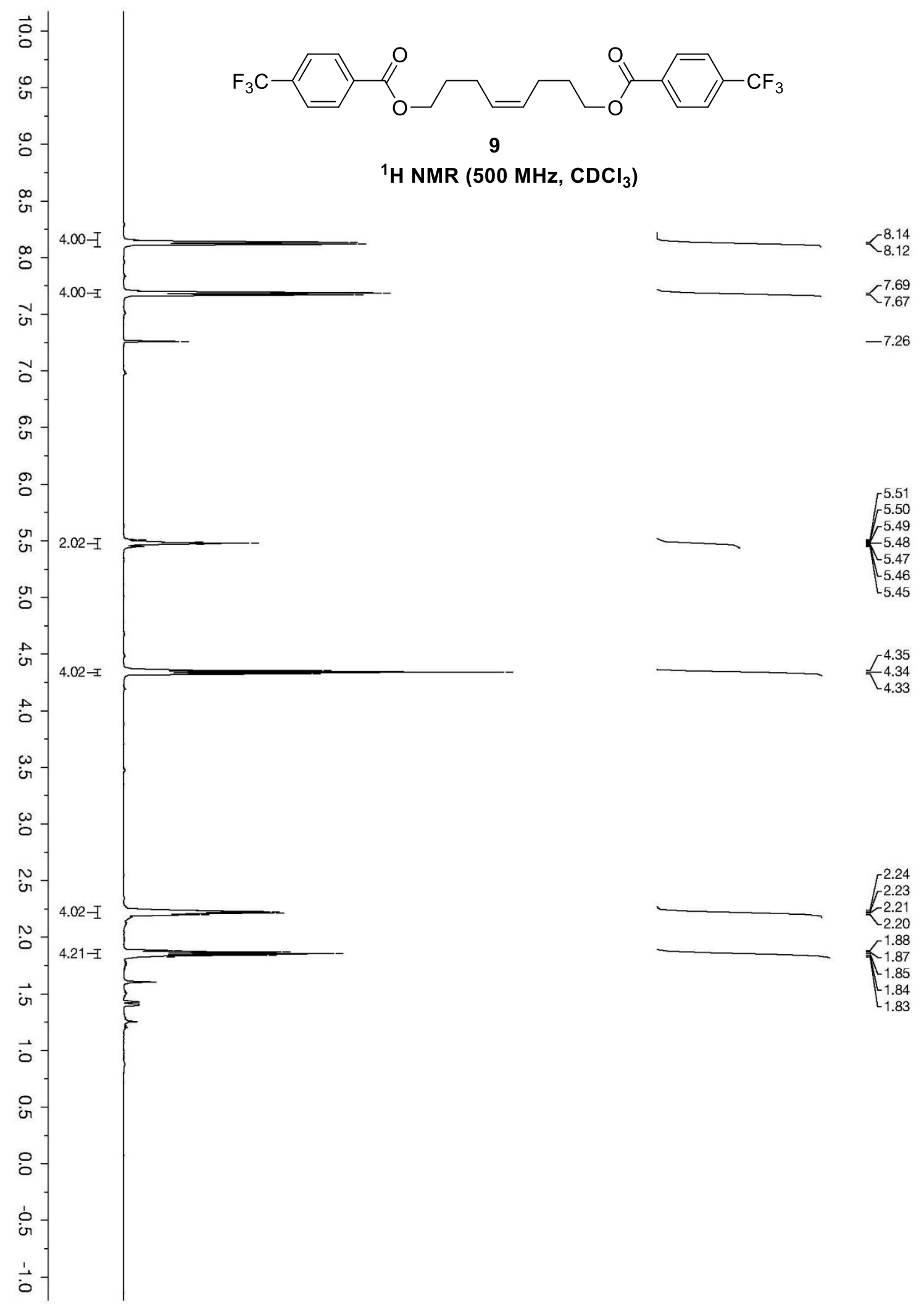


Lin \& Xu et al., Supporting Information, Page S106

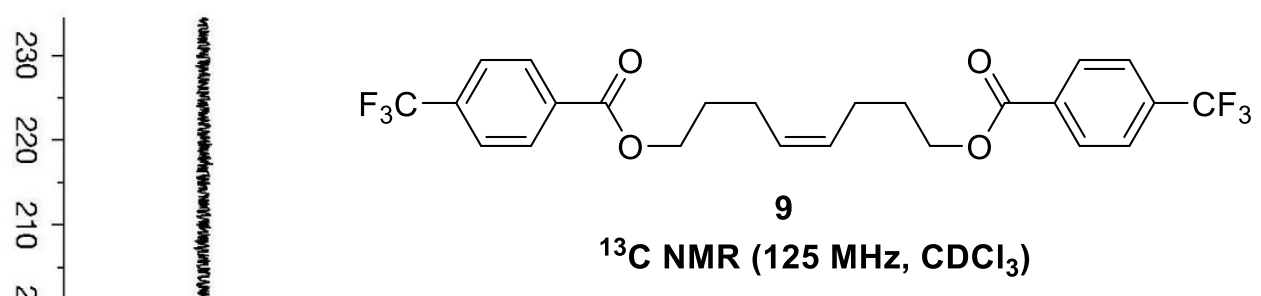

$-165.47$

134.93
134.68
134.42
134.16
133.69
-130.03
-129.57
-127.00
-125.58
125.55
125.52
125.49
124.83
122.66
120.50

$\left\{\begin{array}{l}77.41 \\ 77.16 \\ 76.90\end{array}\right.$

$-65.07$

$-28.67$ 


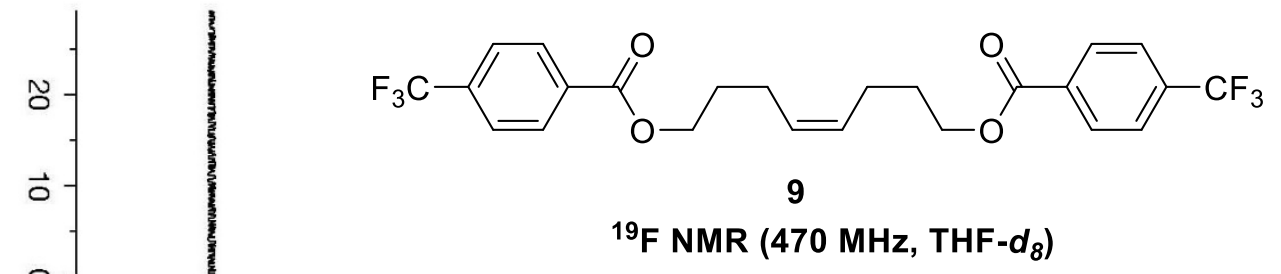




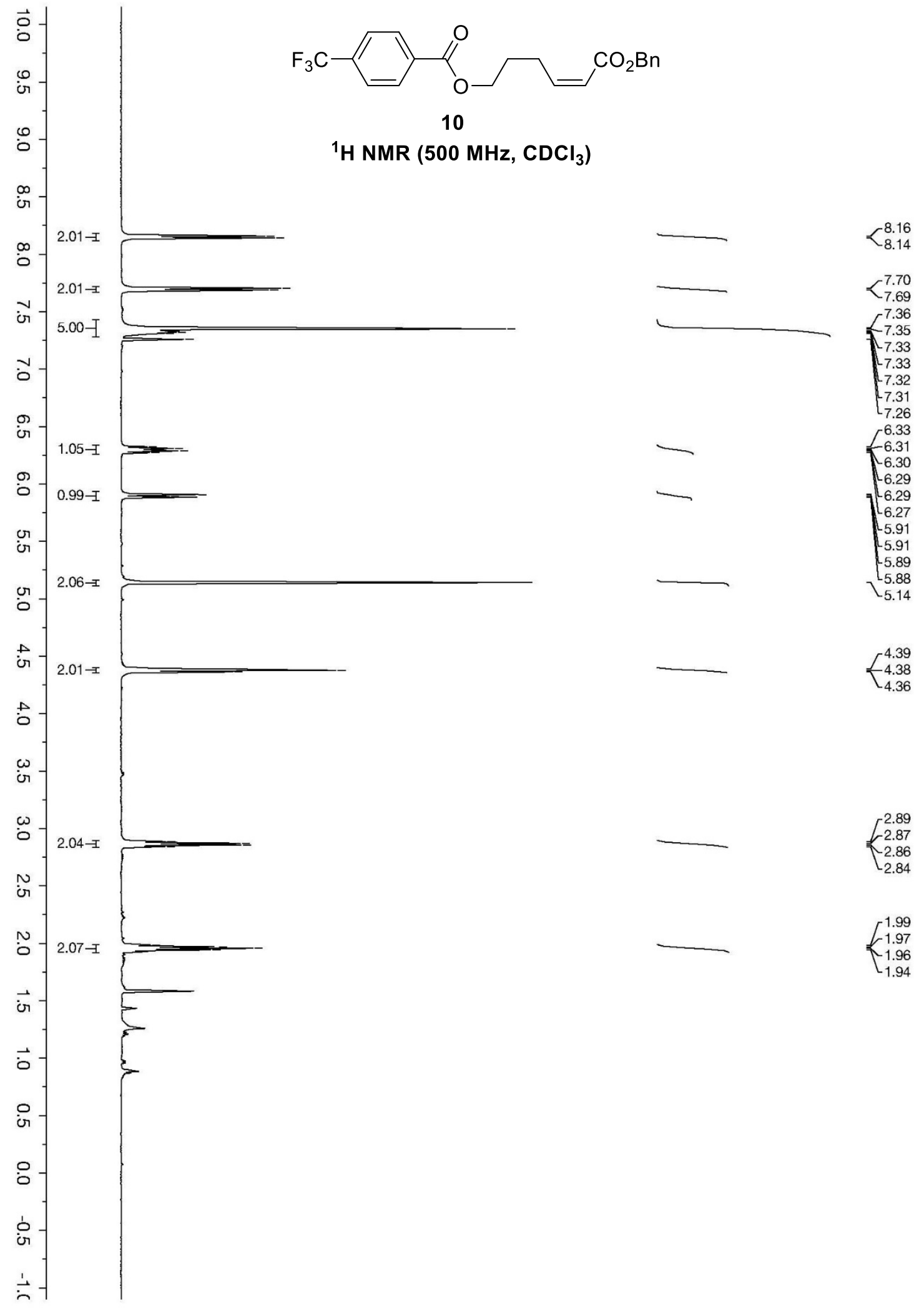




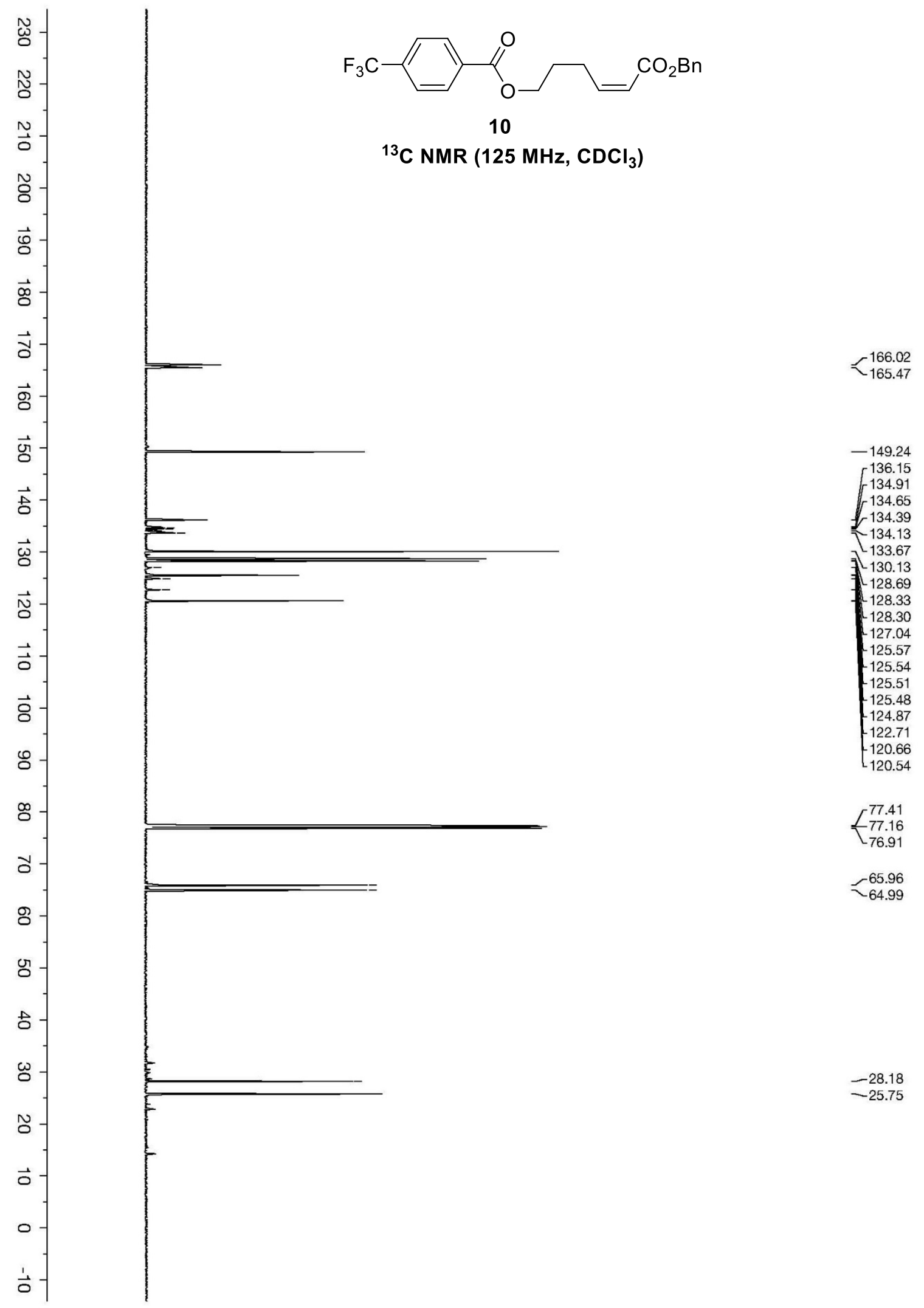




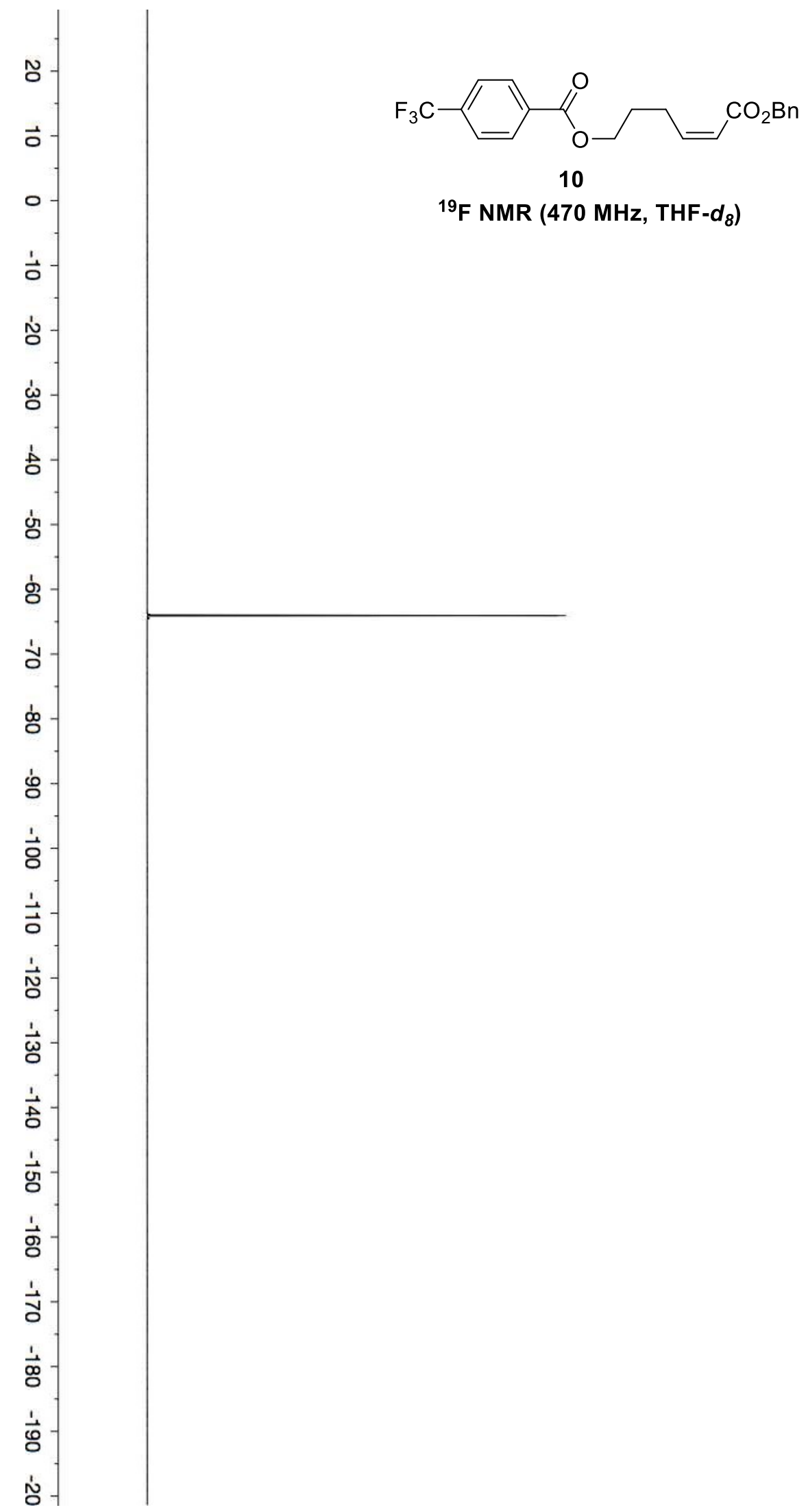


Lin \& Xu et al., Supporting Information, Page S111

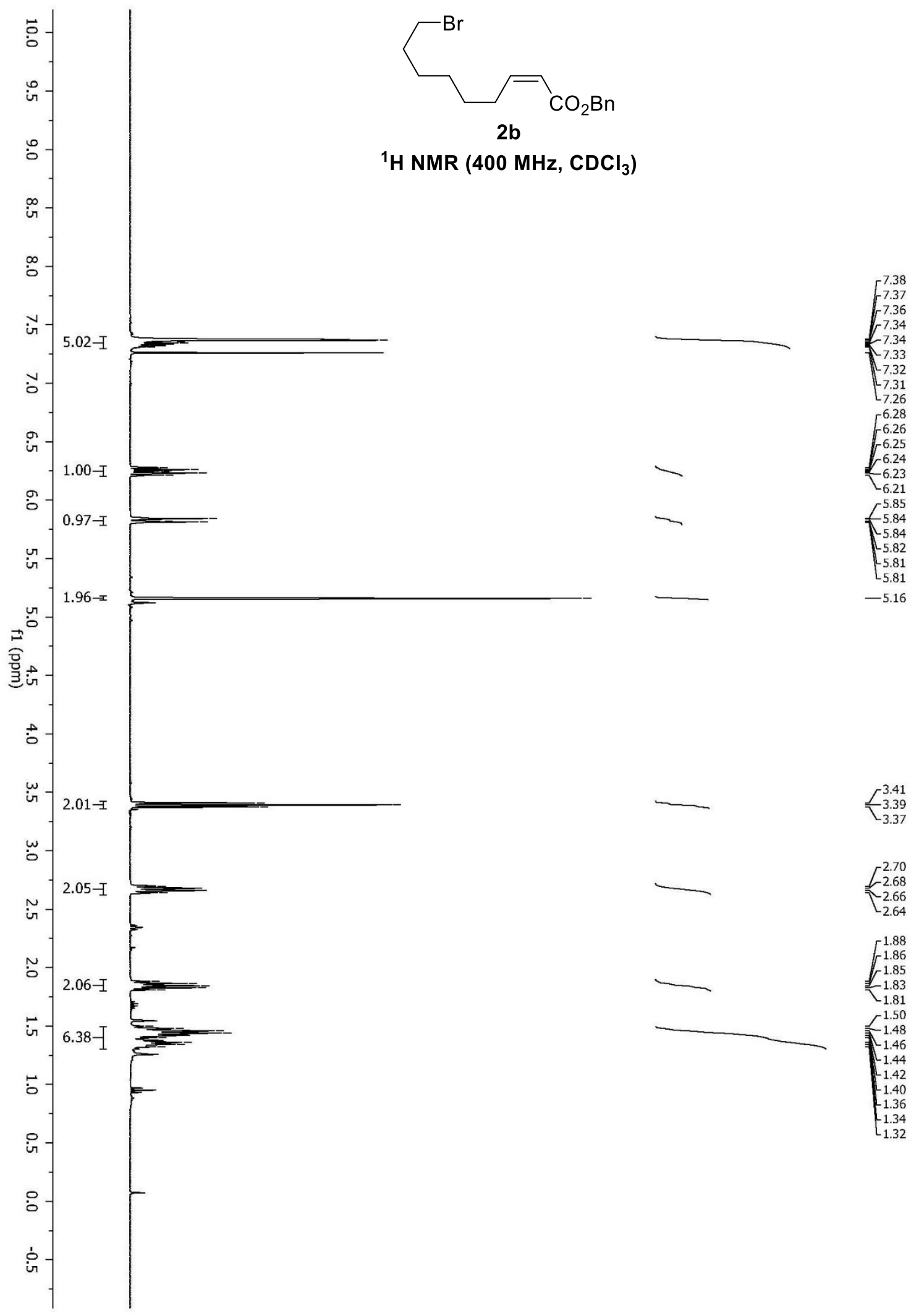




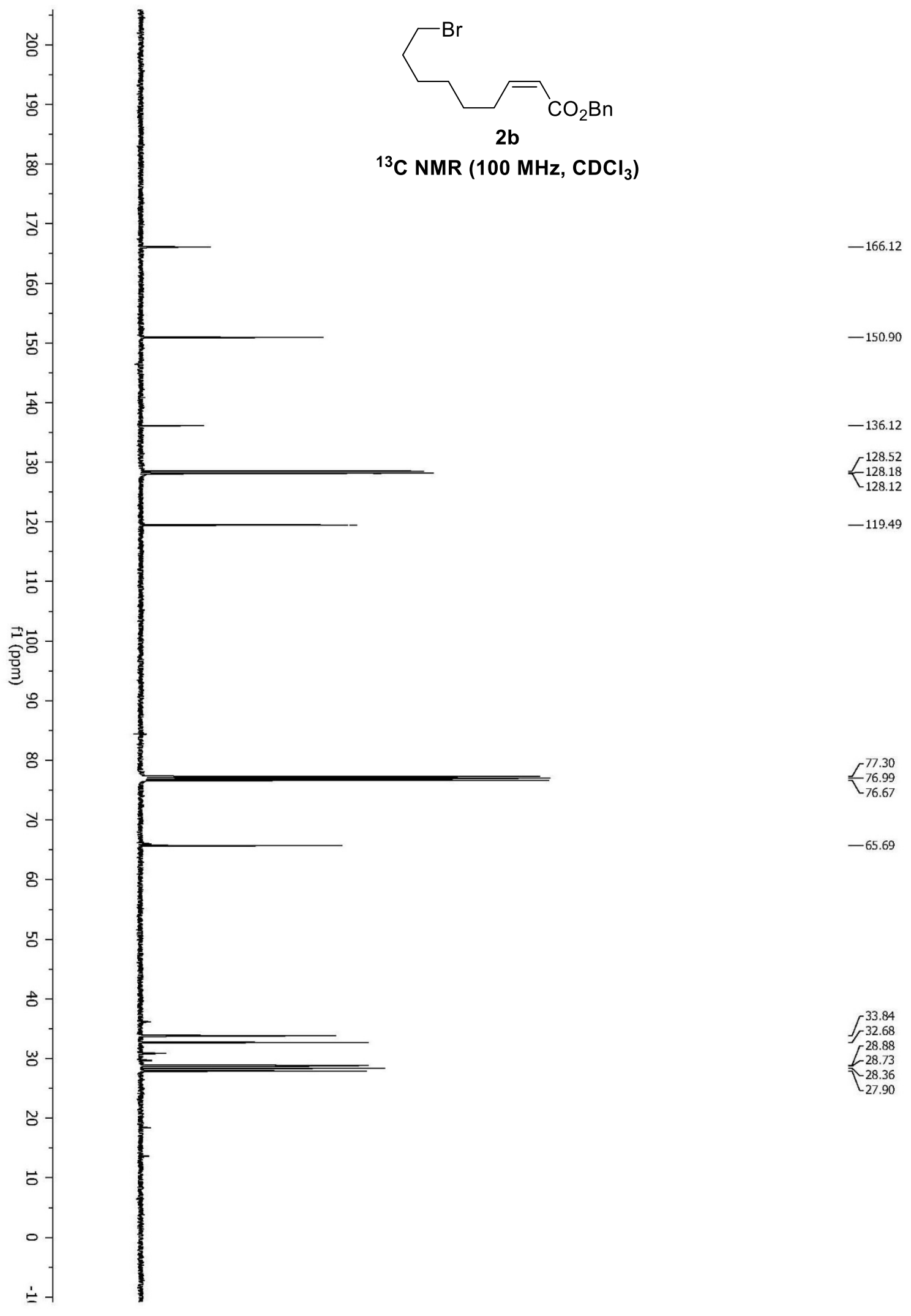




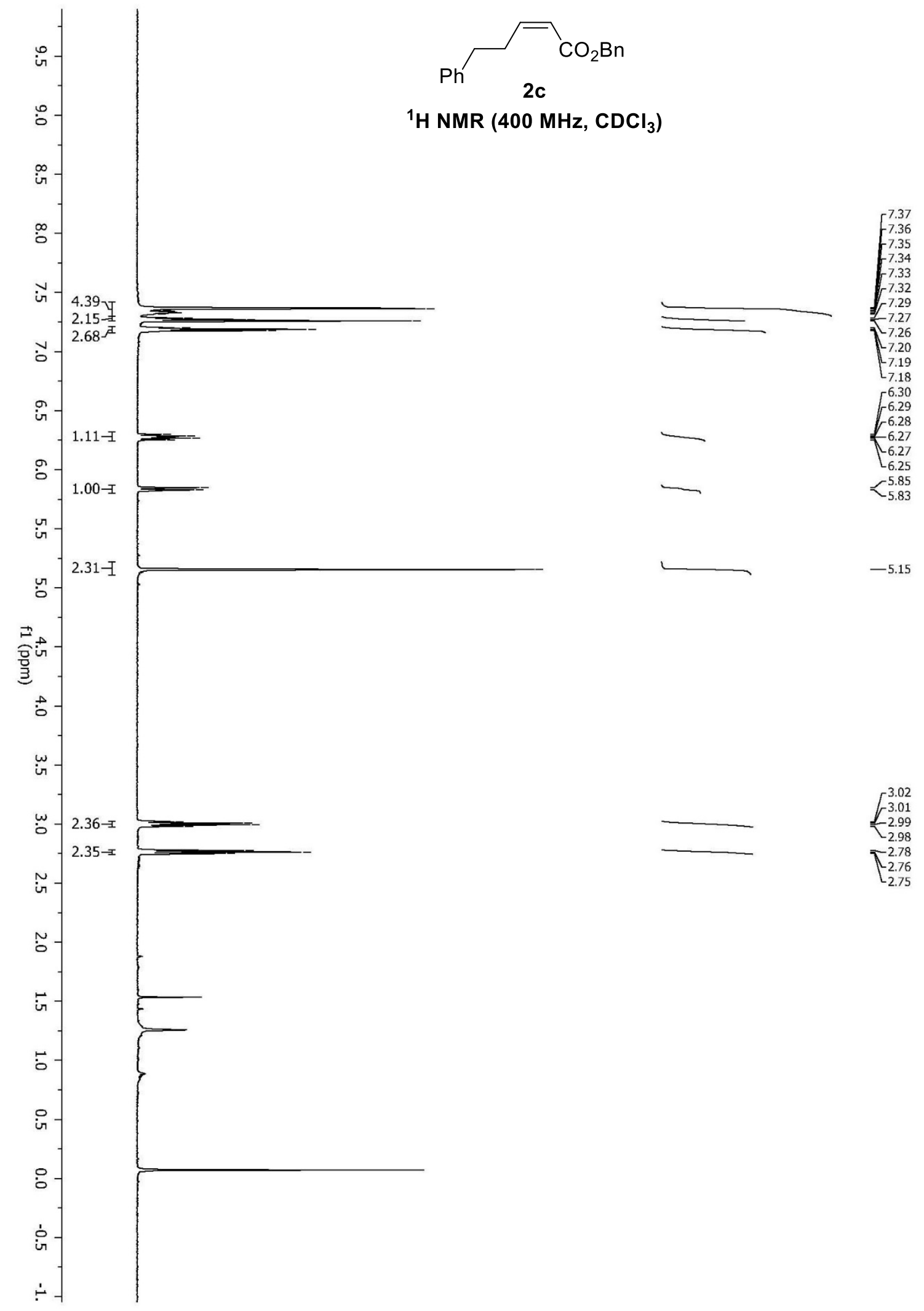




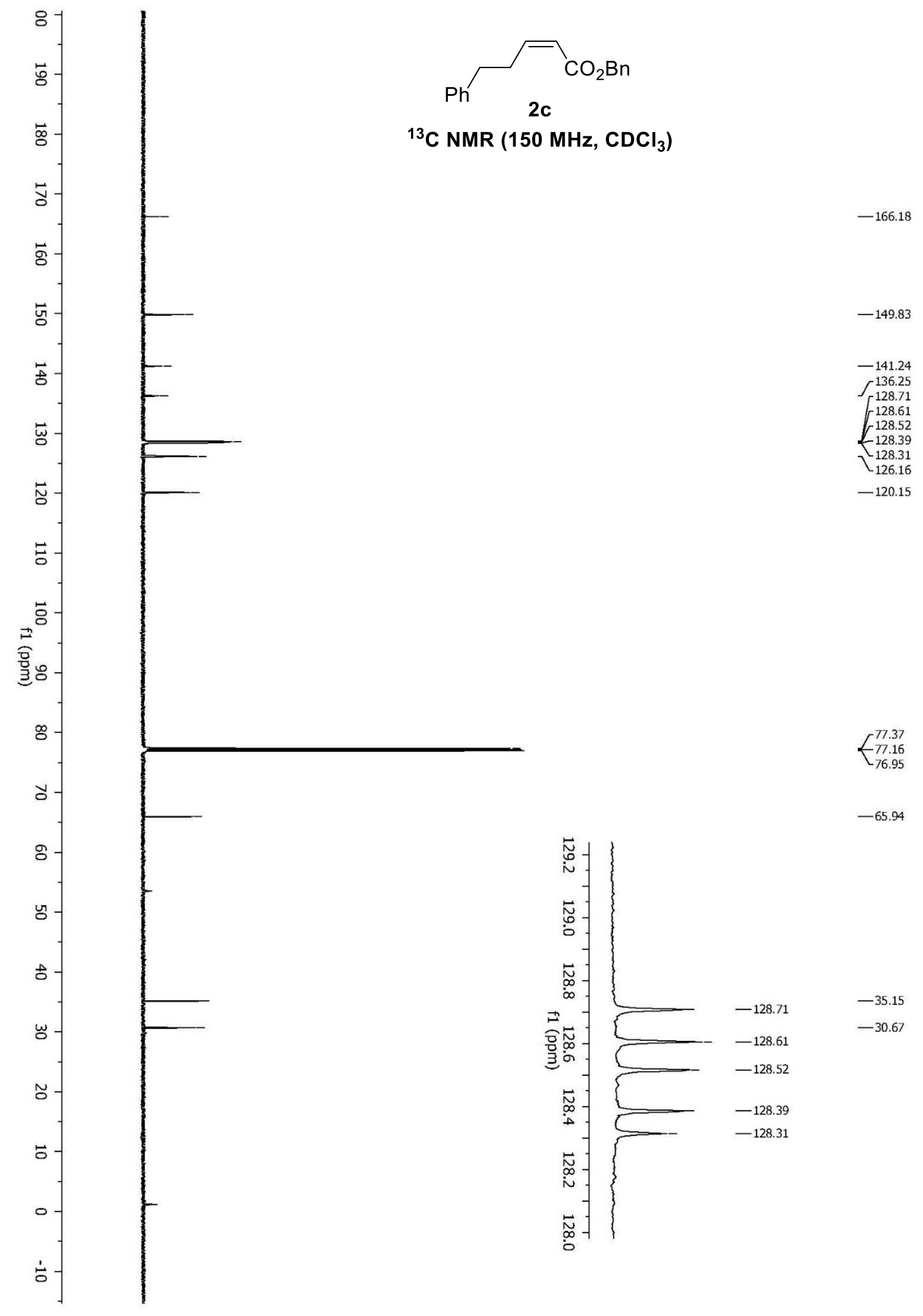




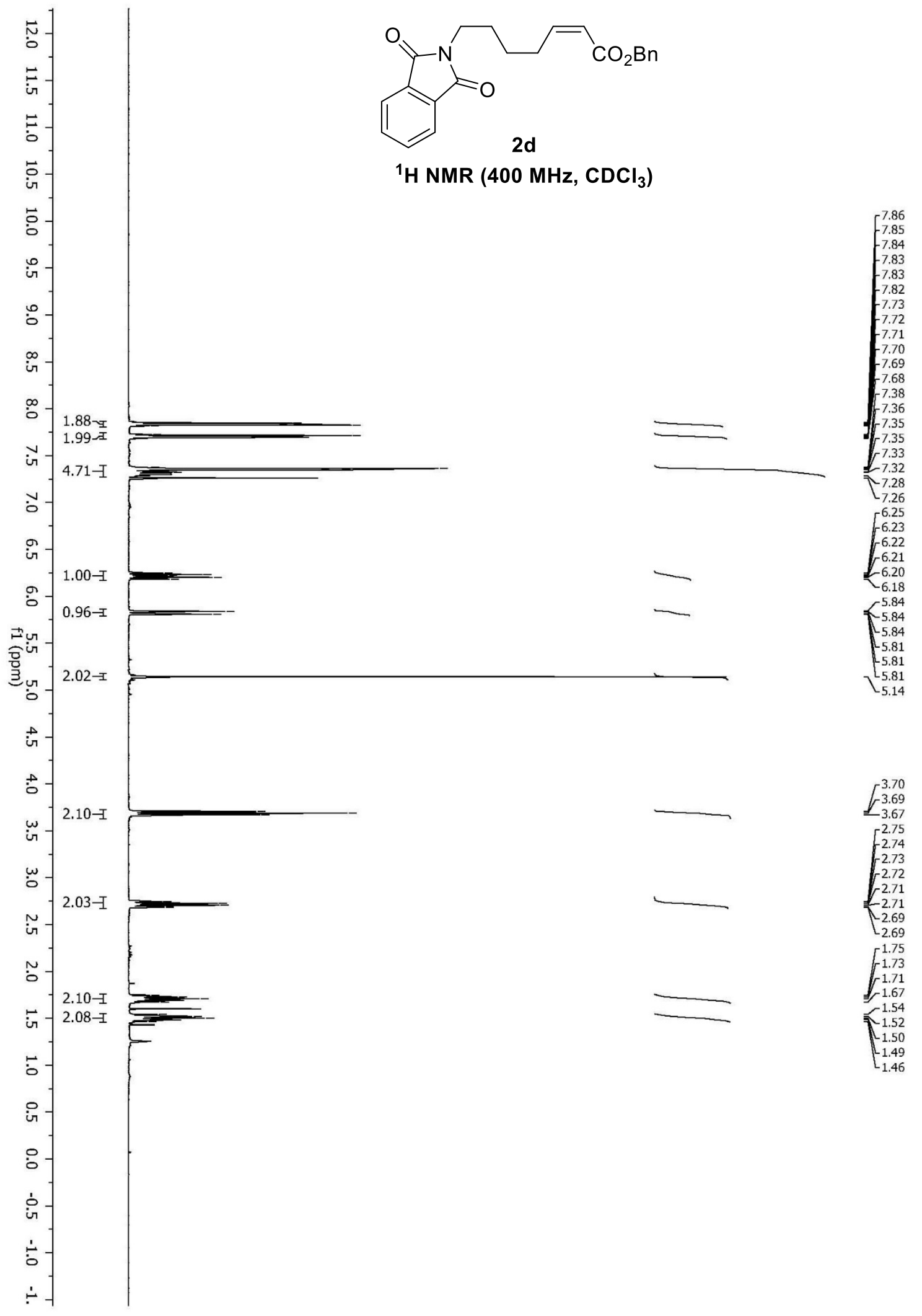




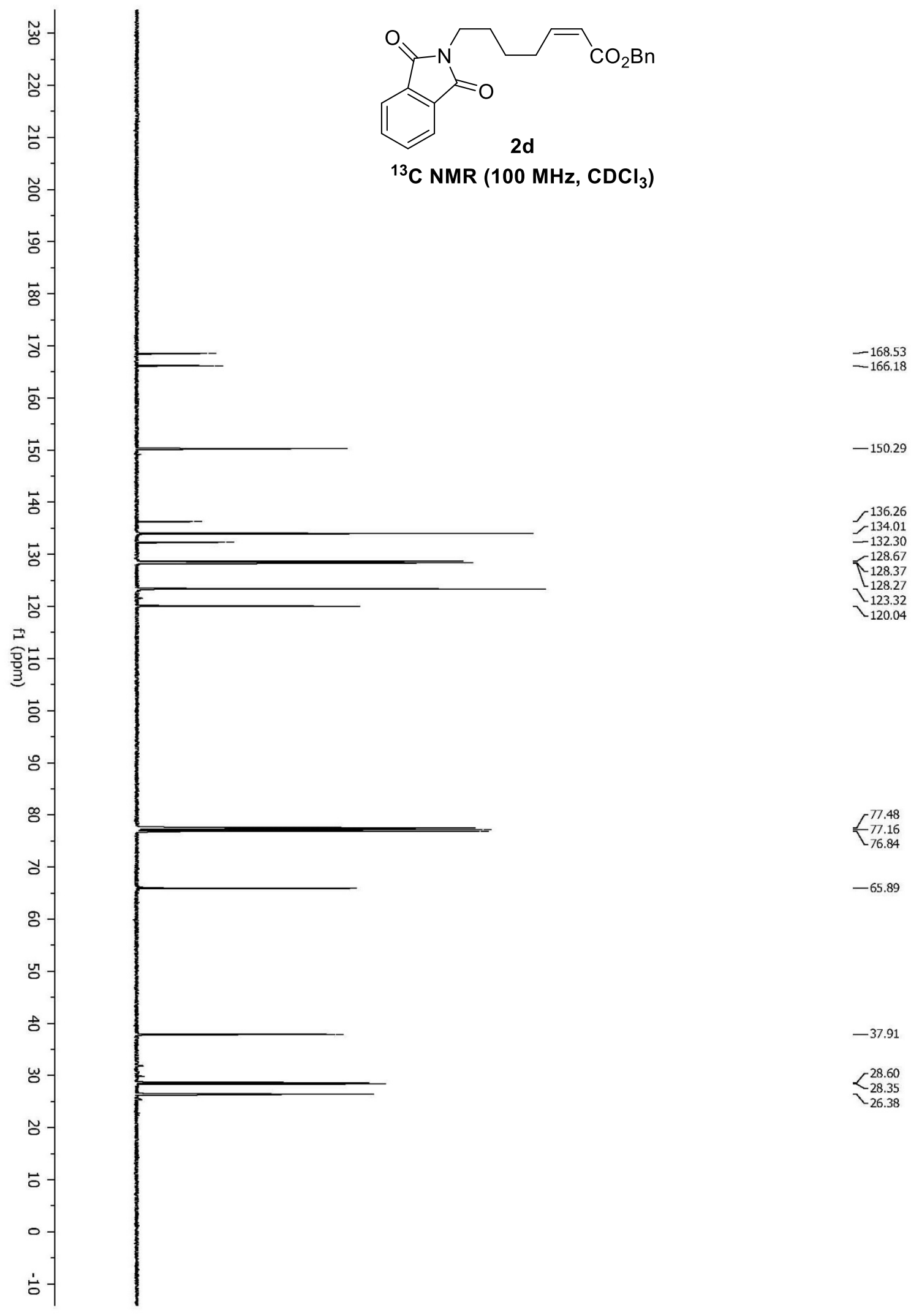




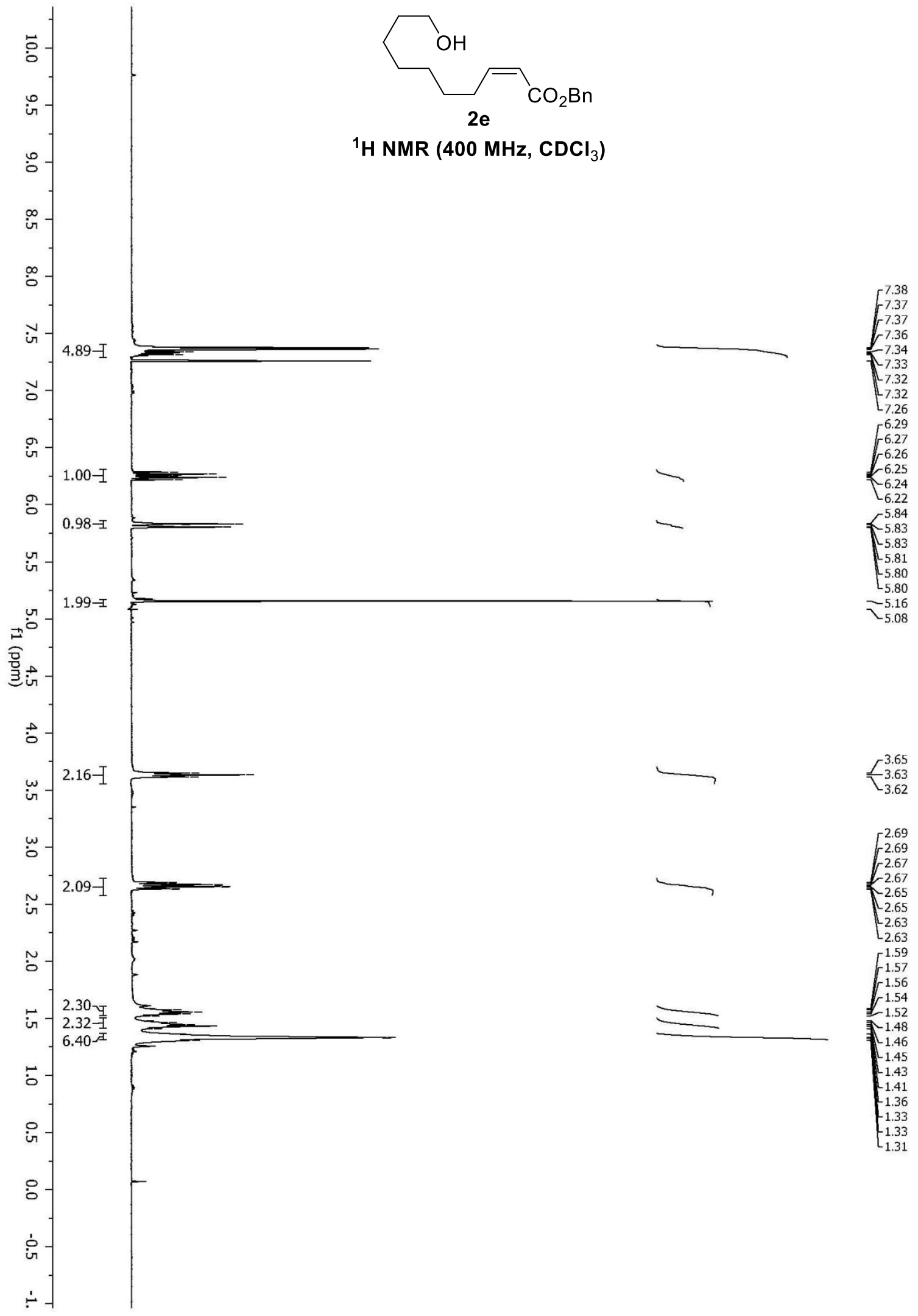




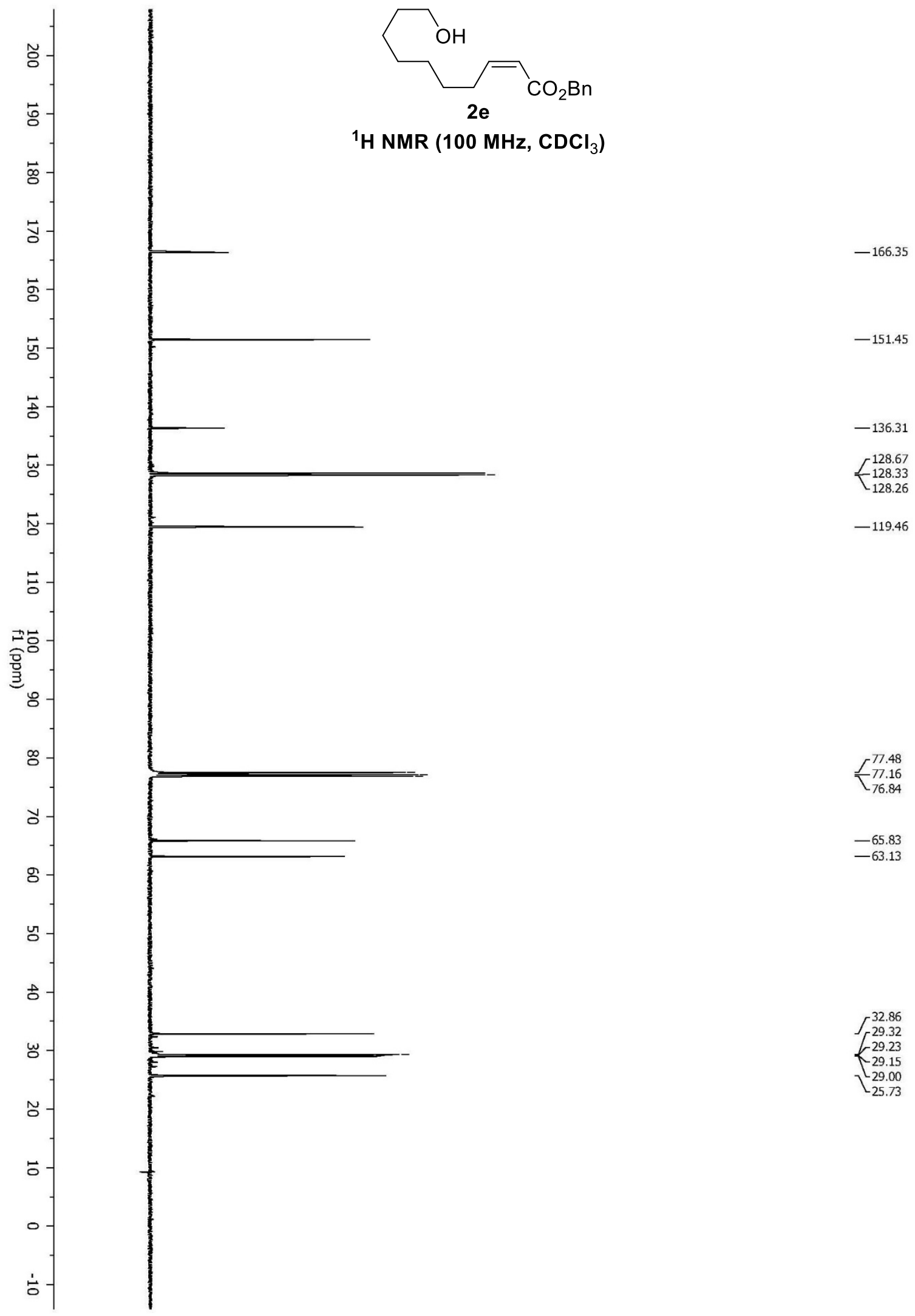




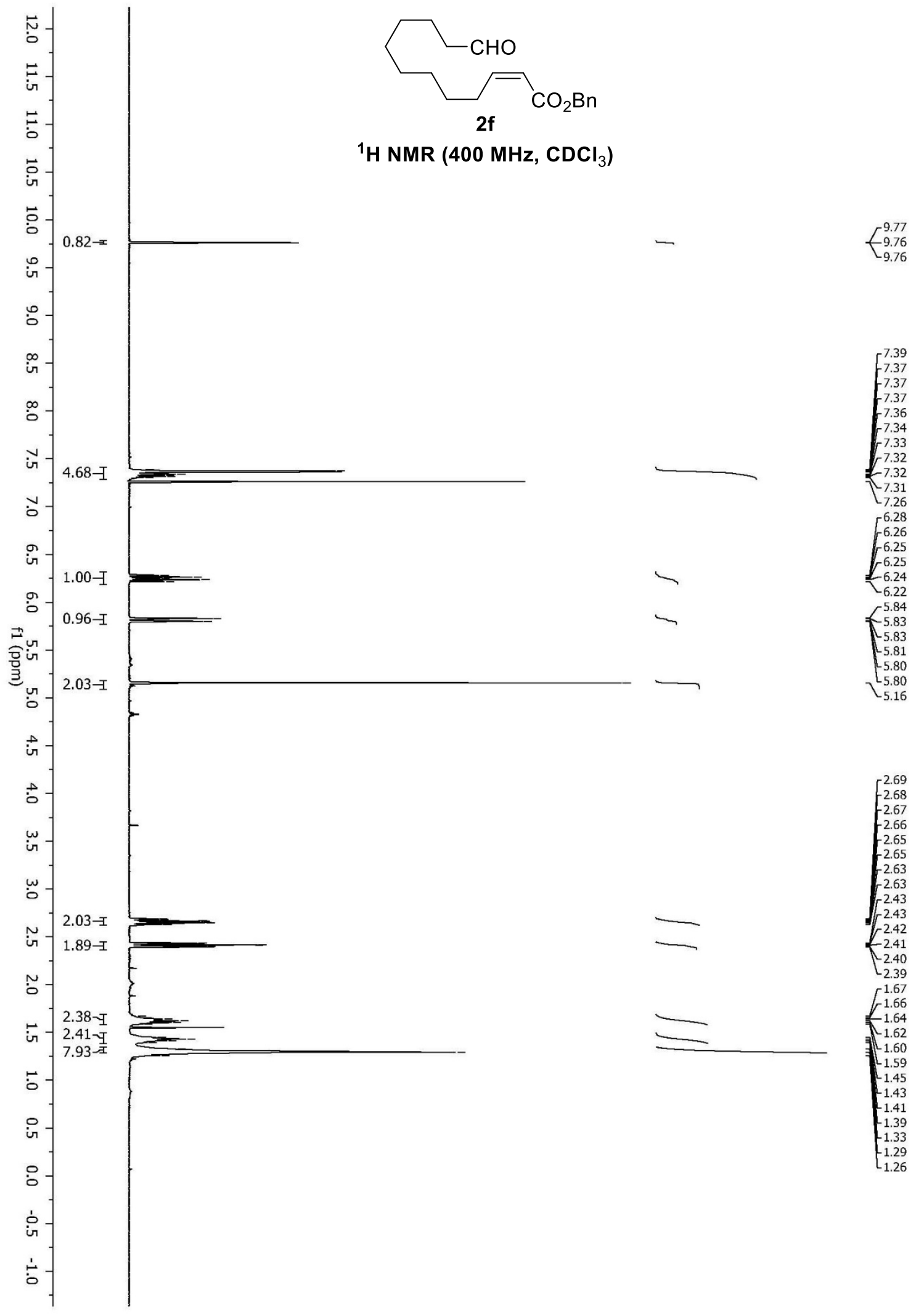




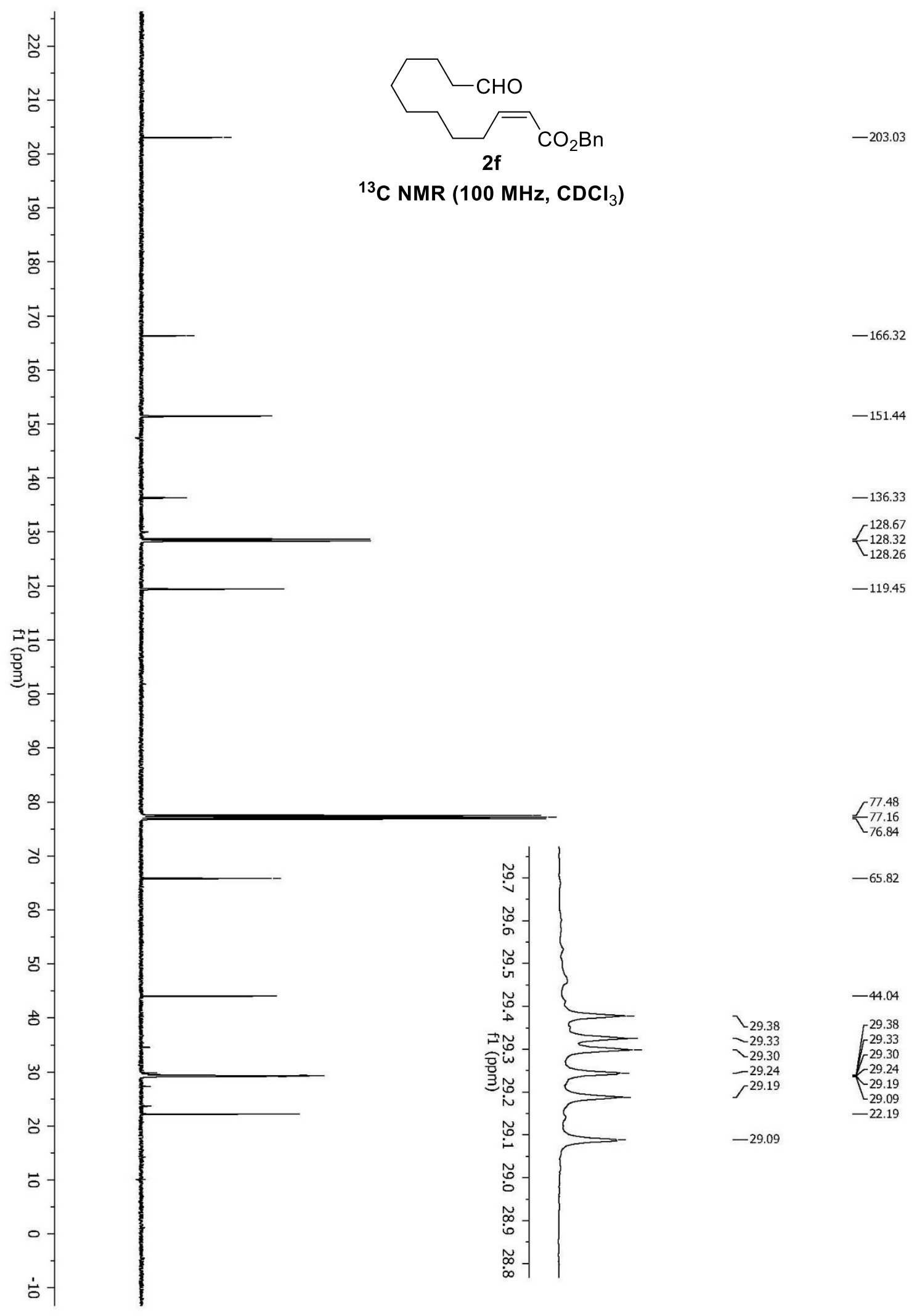




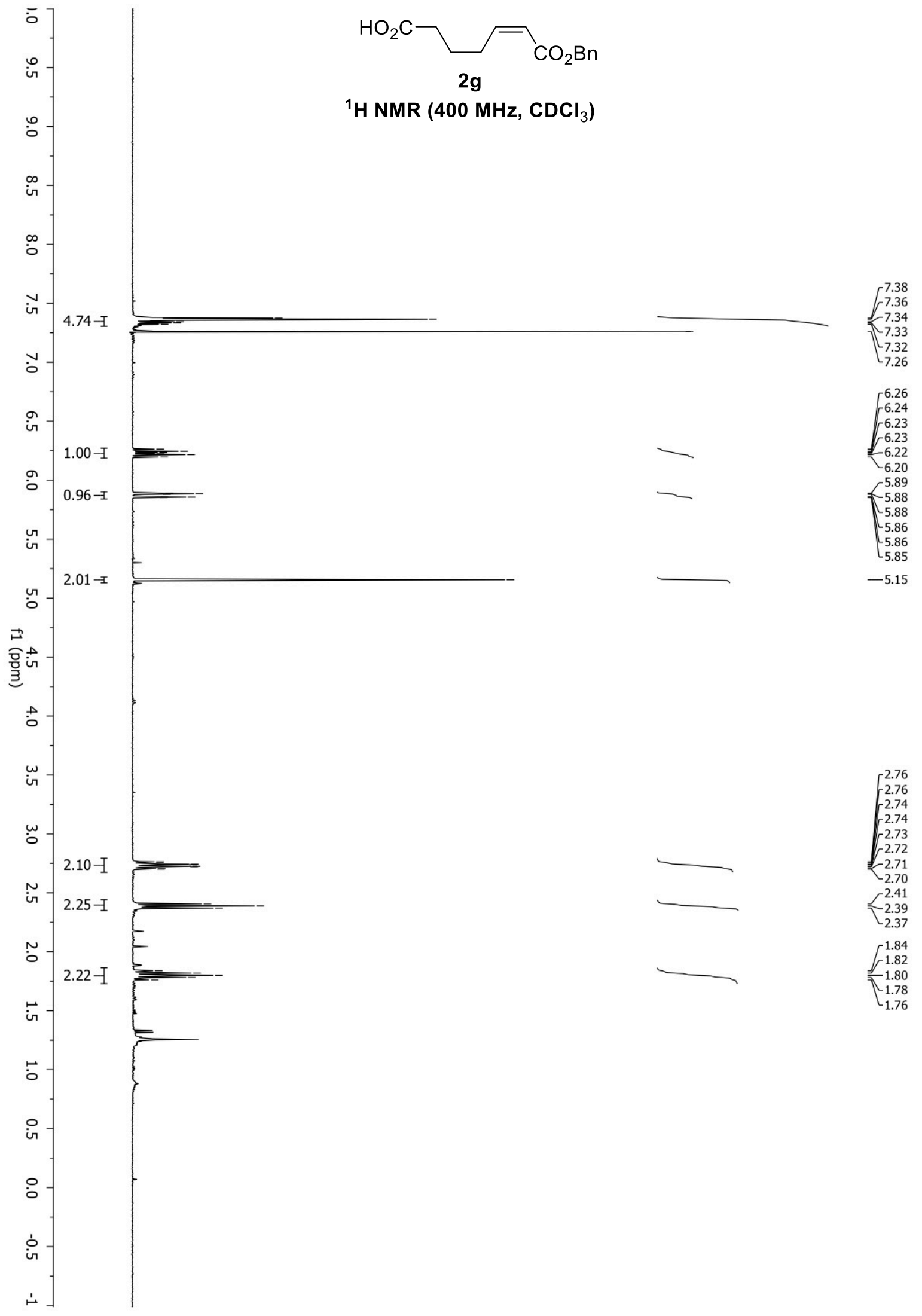




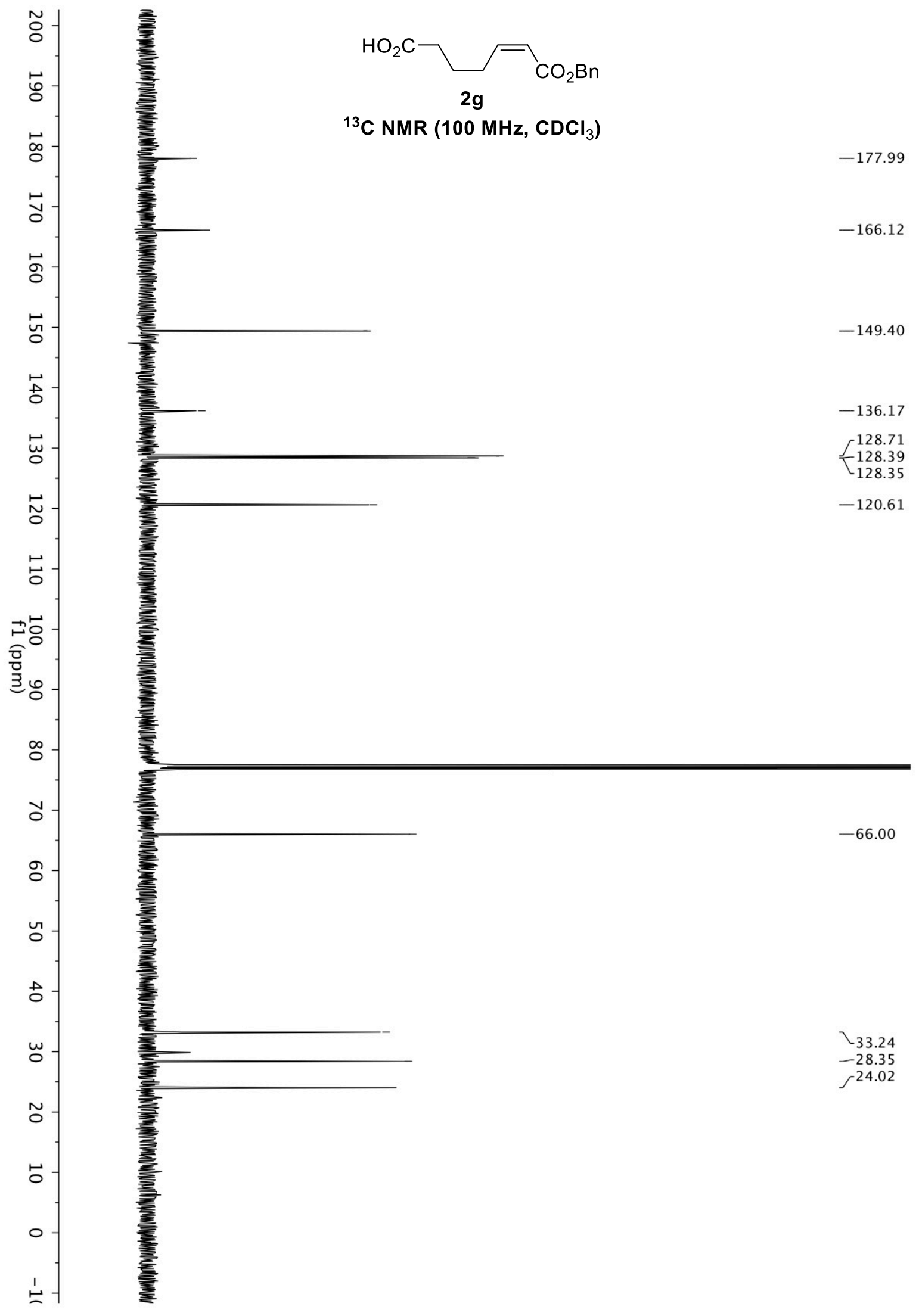




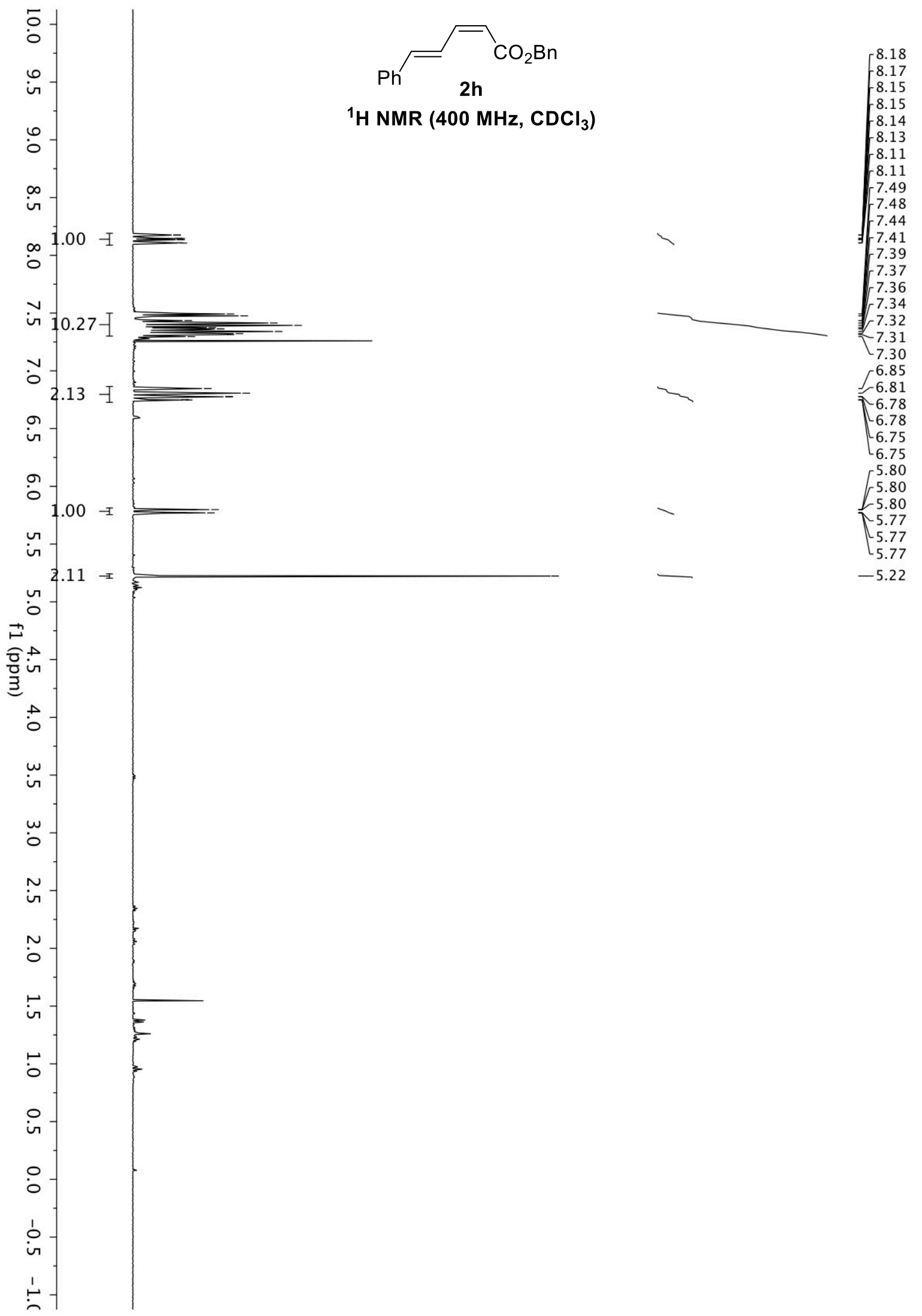




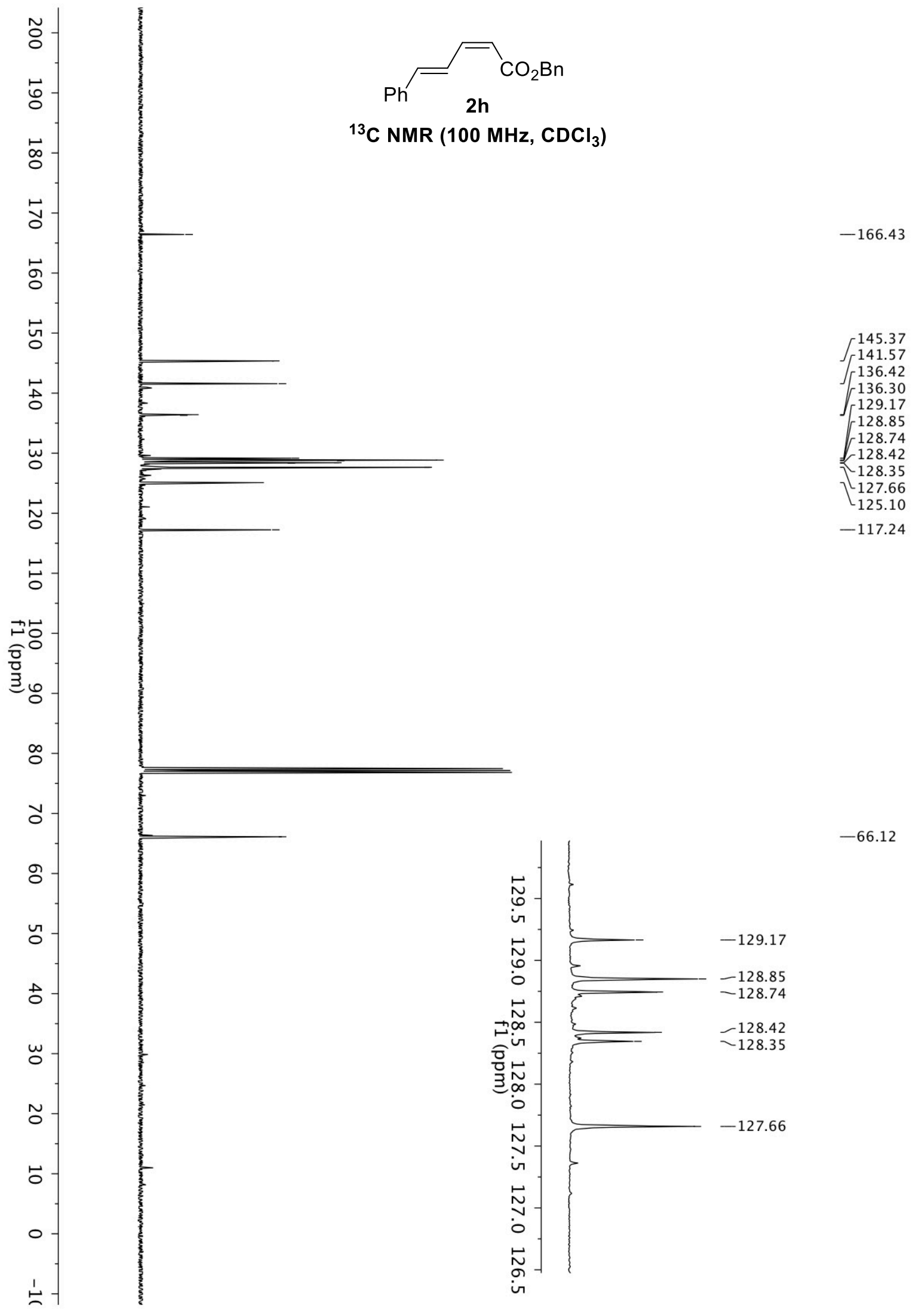




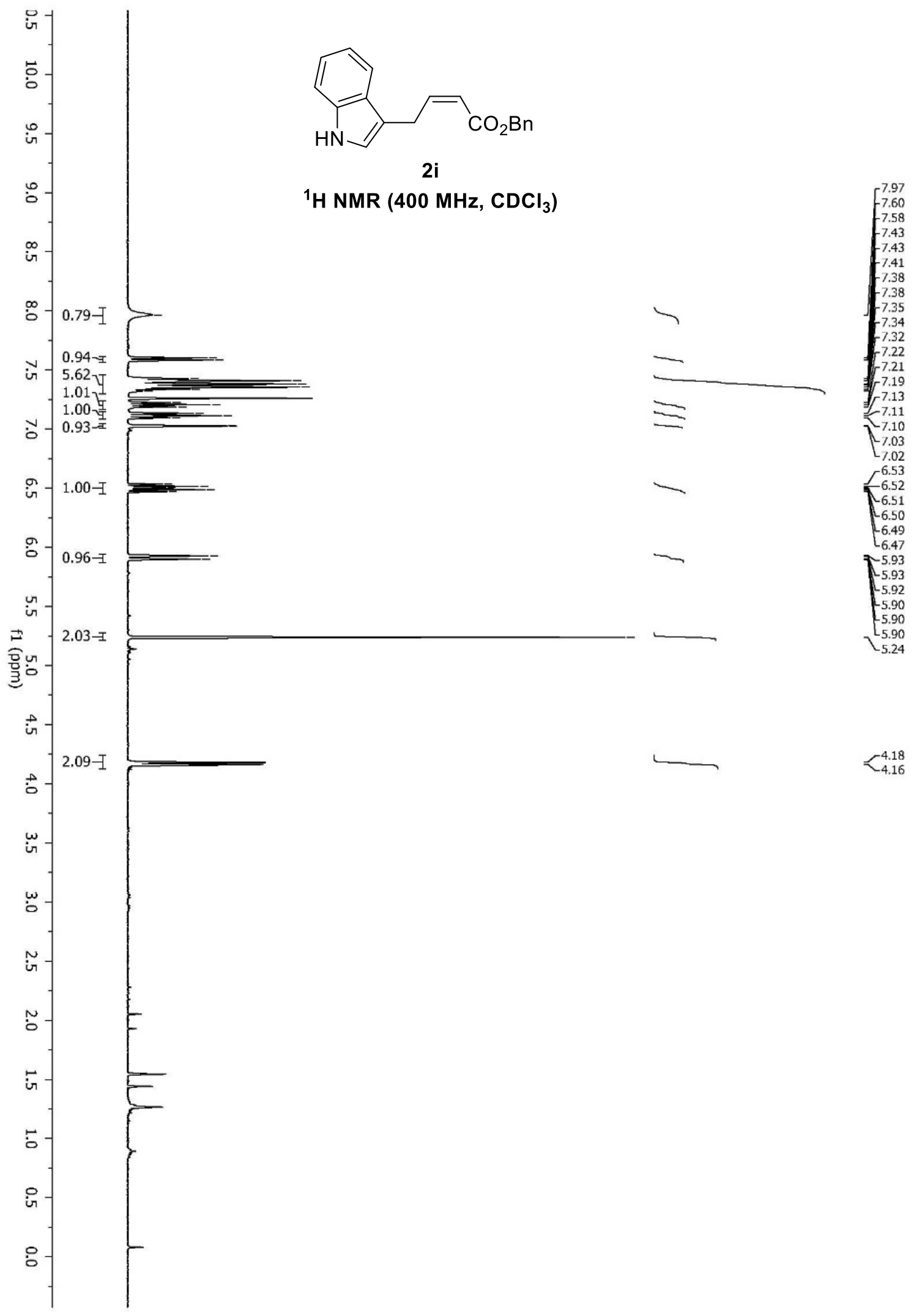




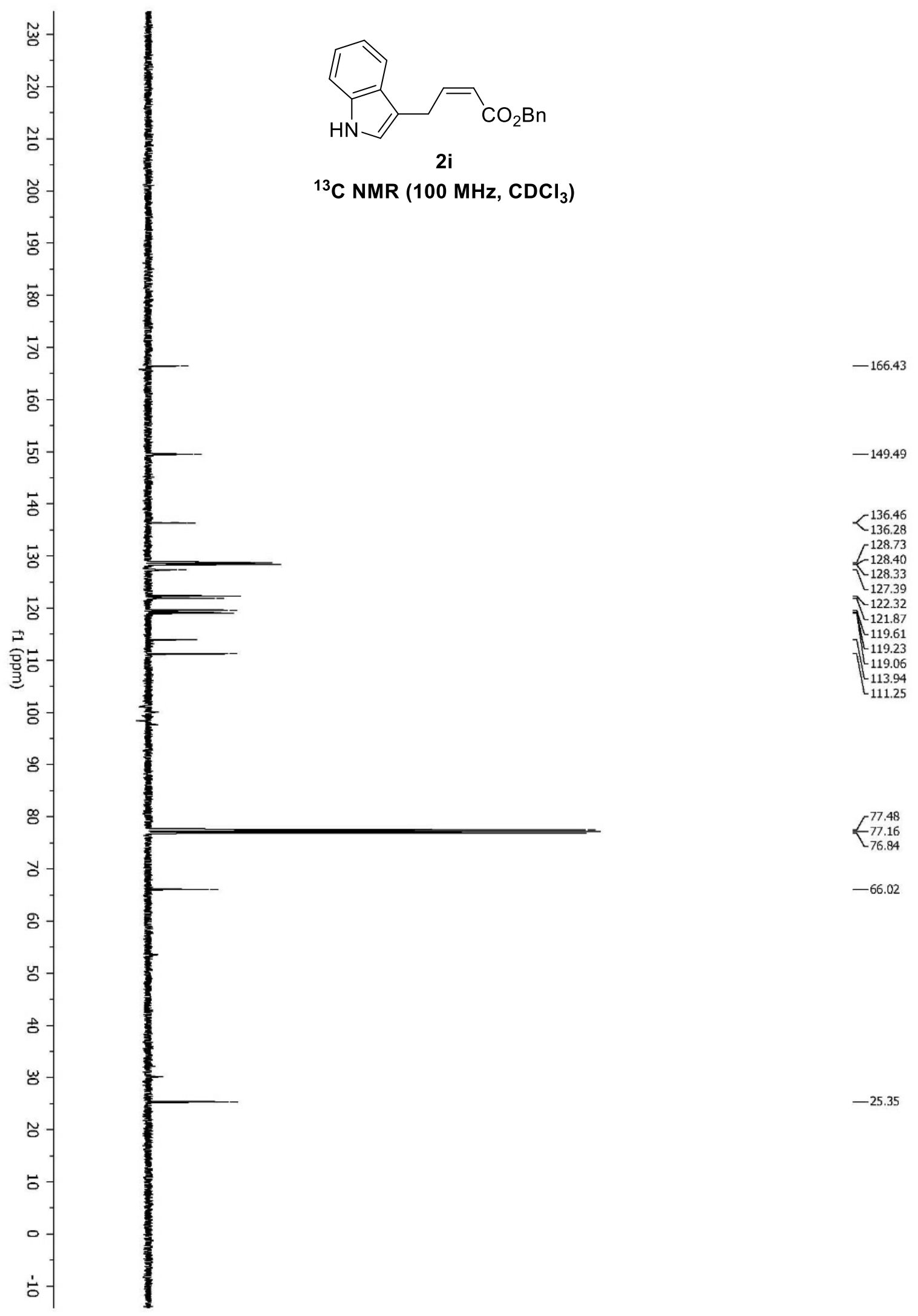




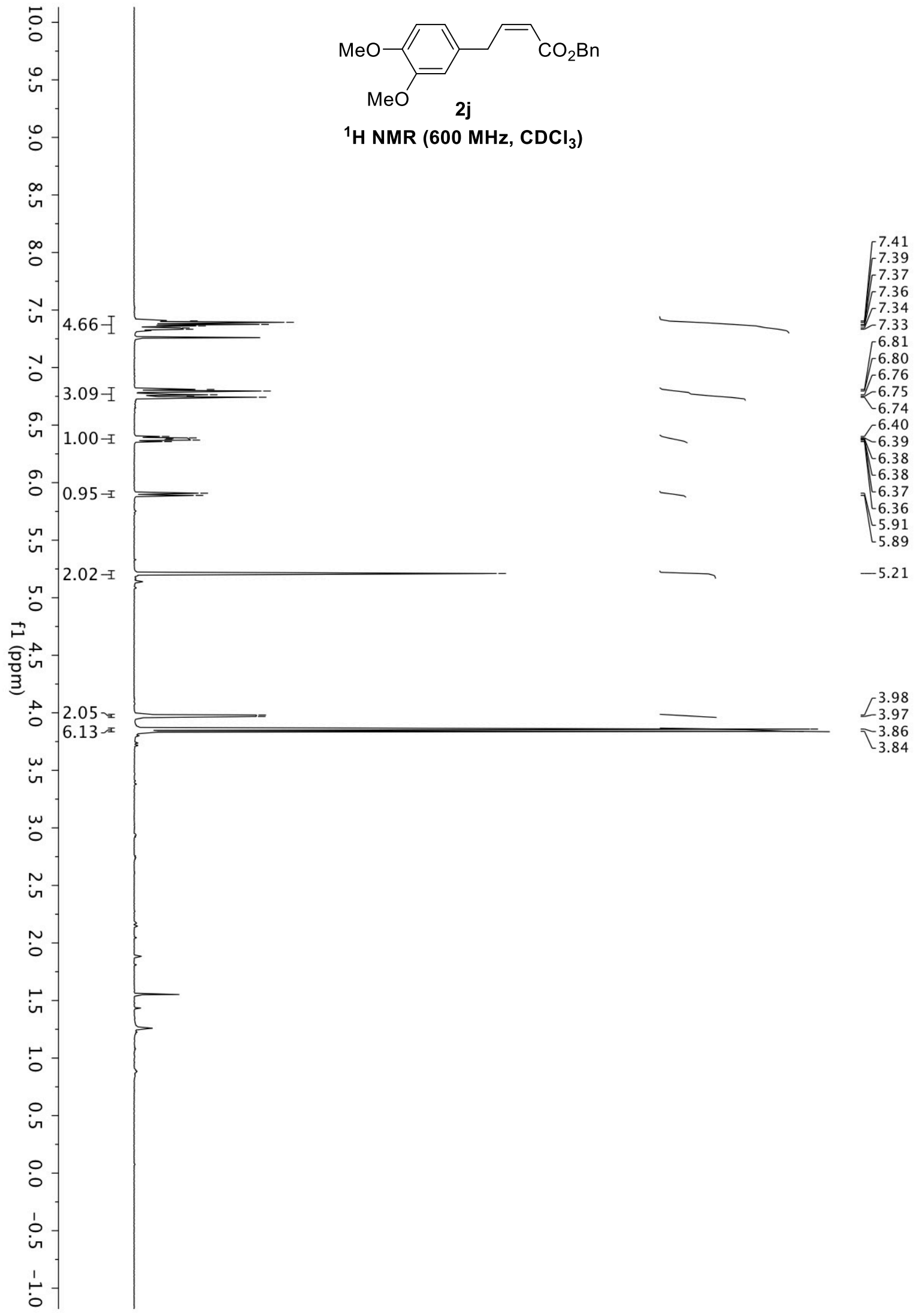




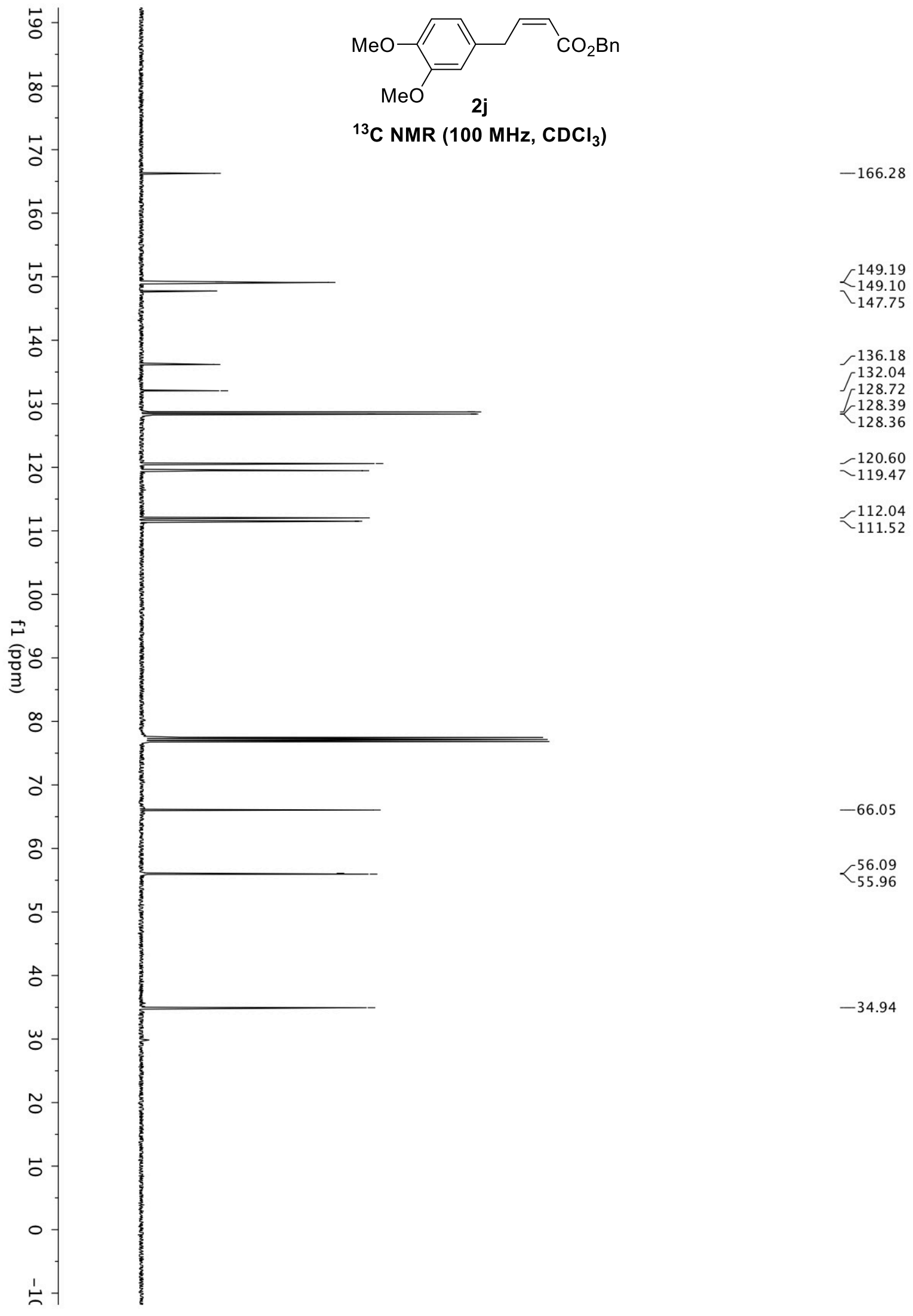




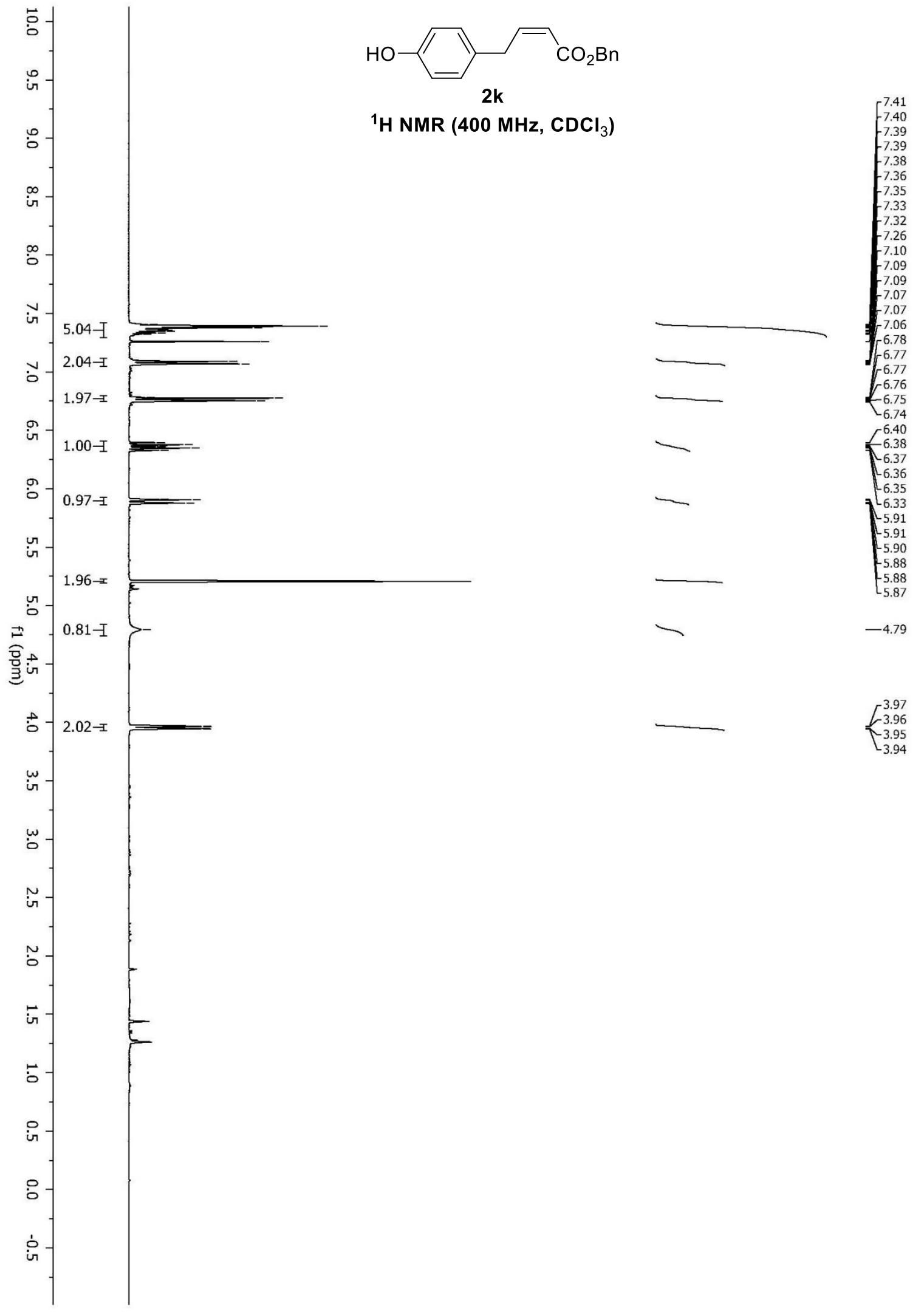




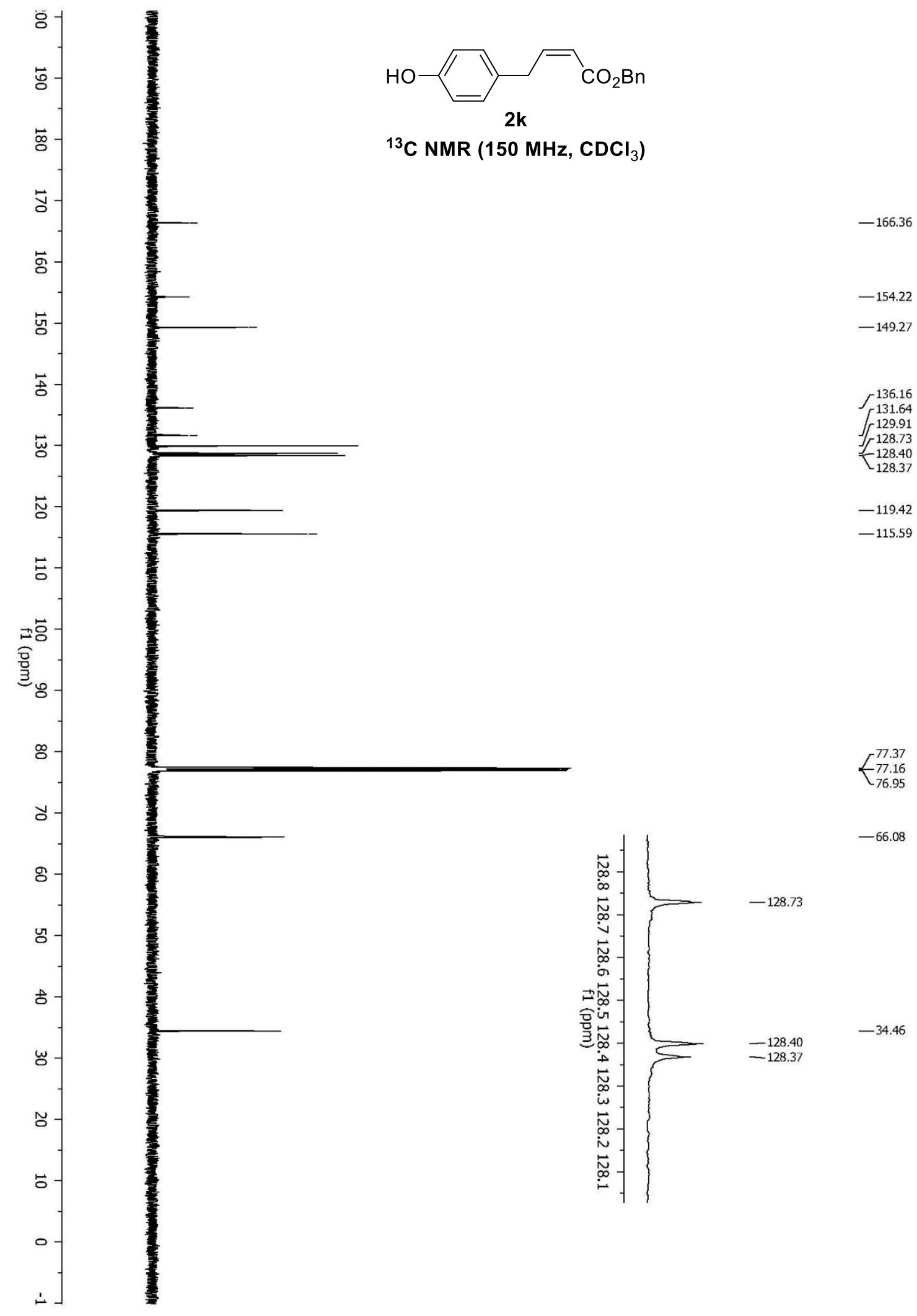




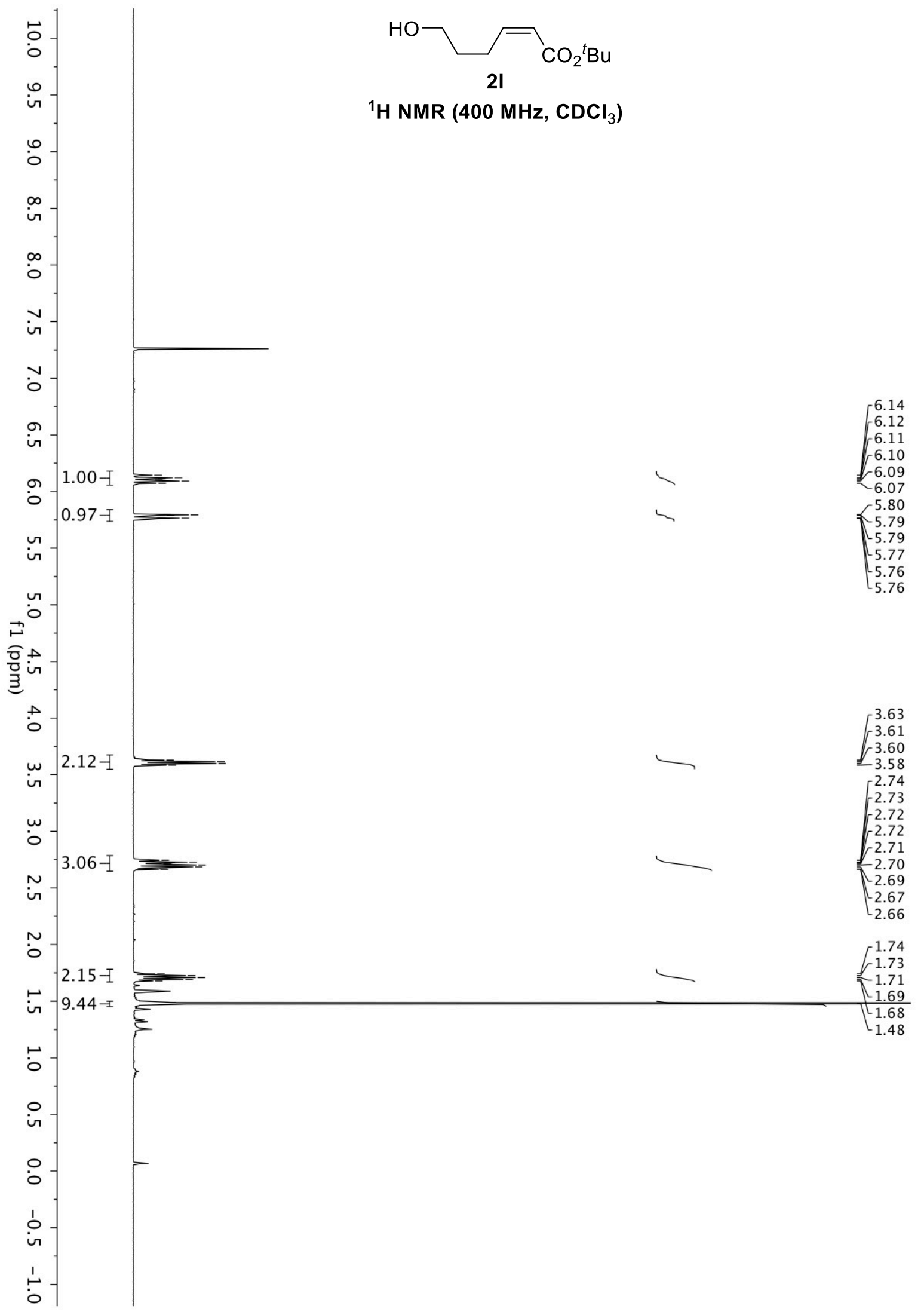




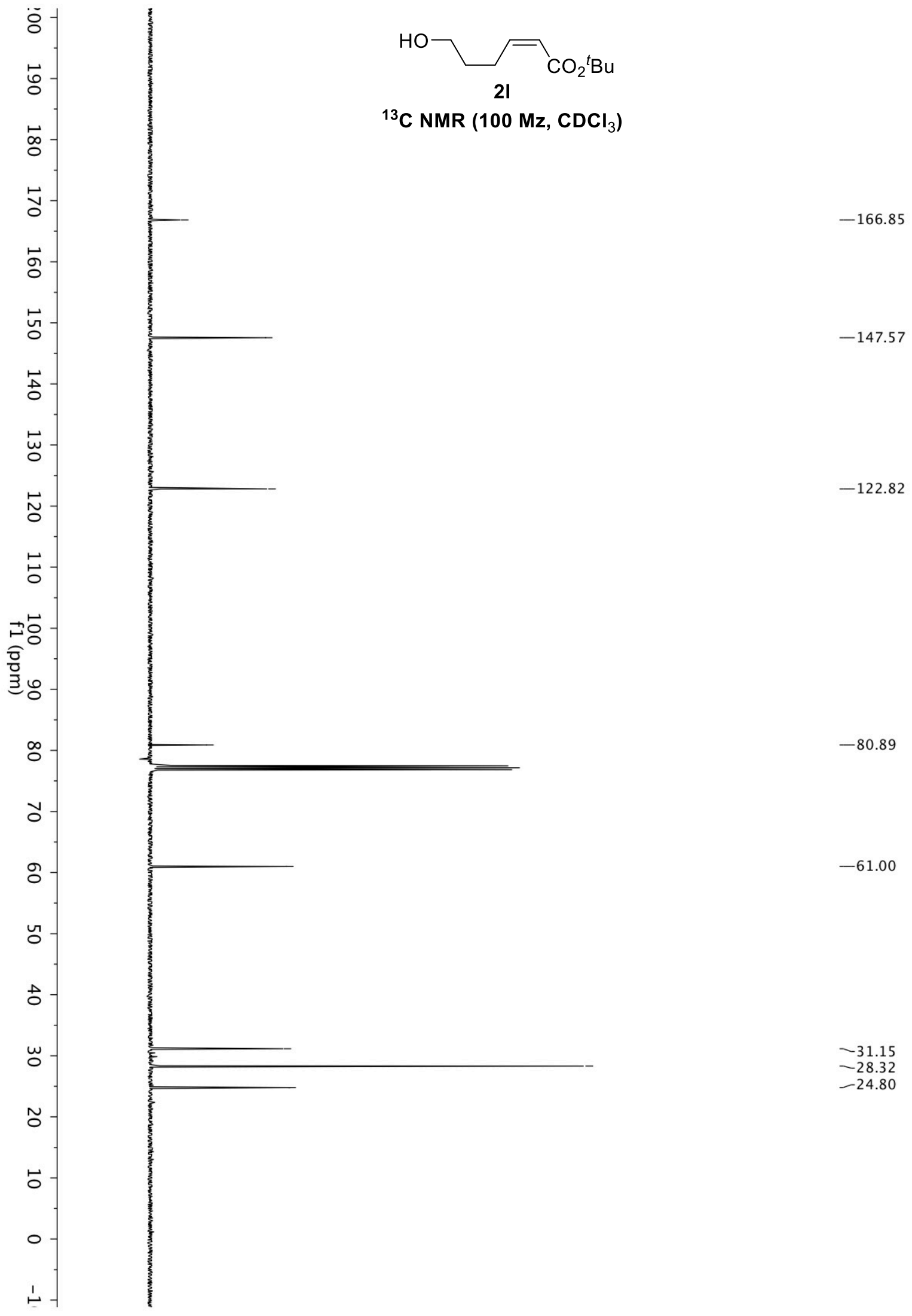




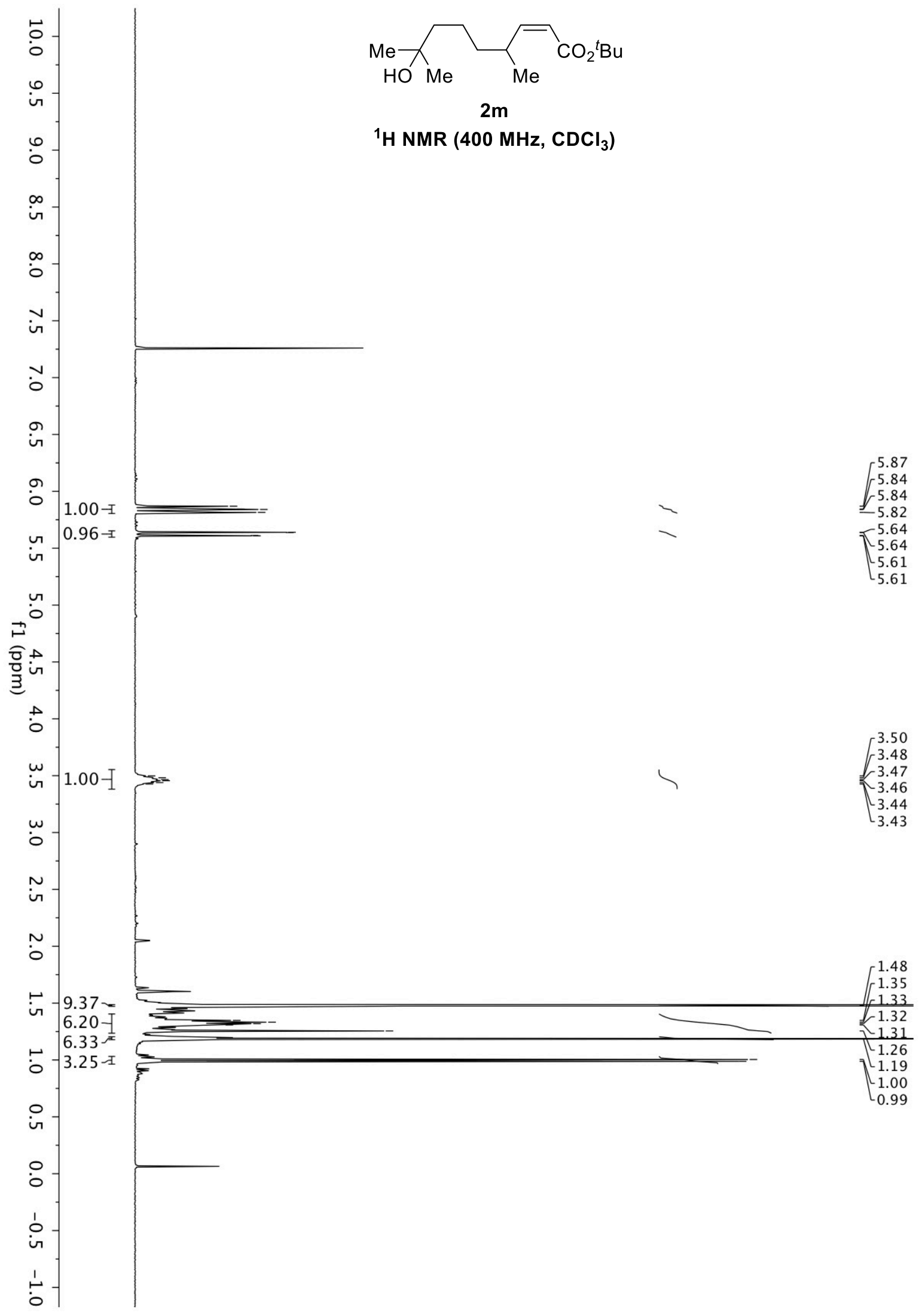




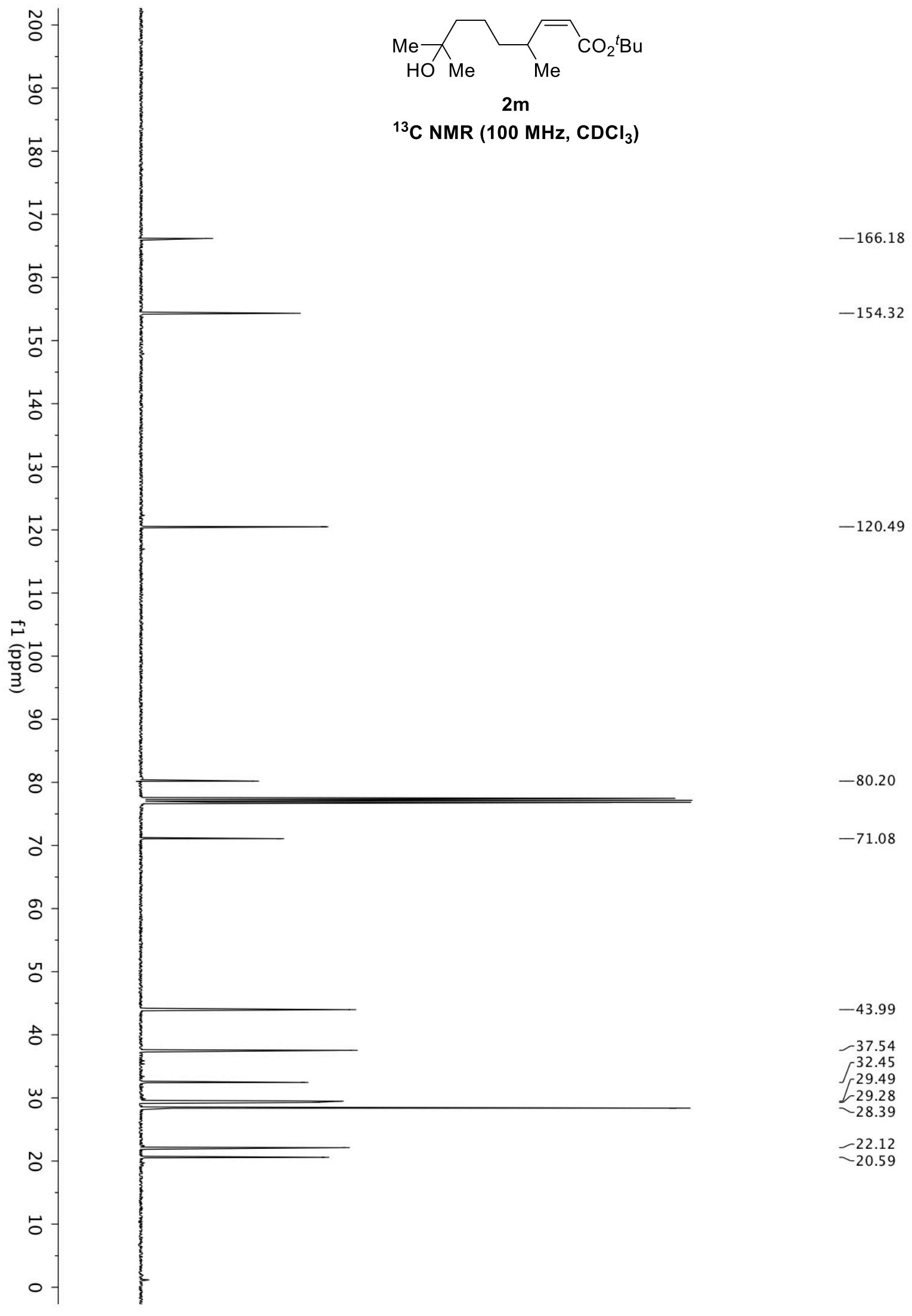



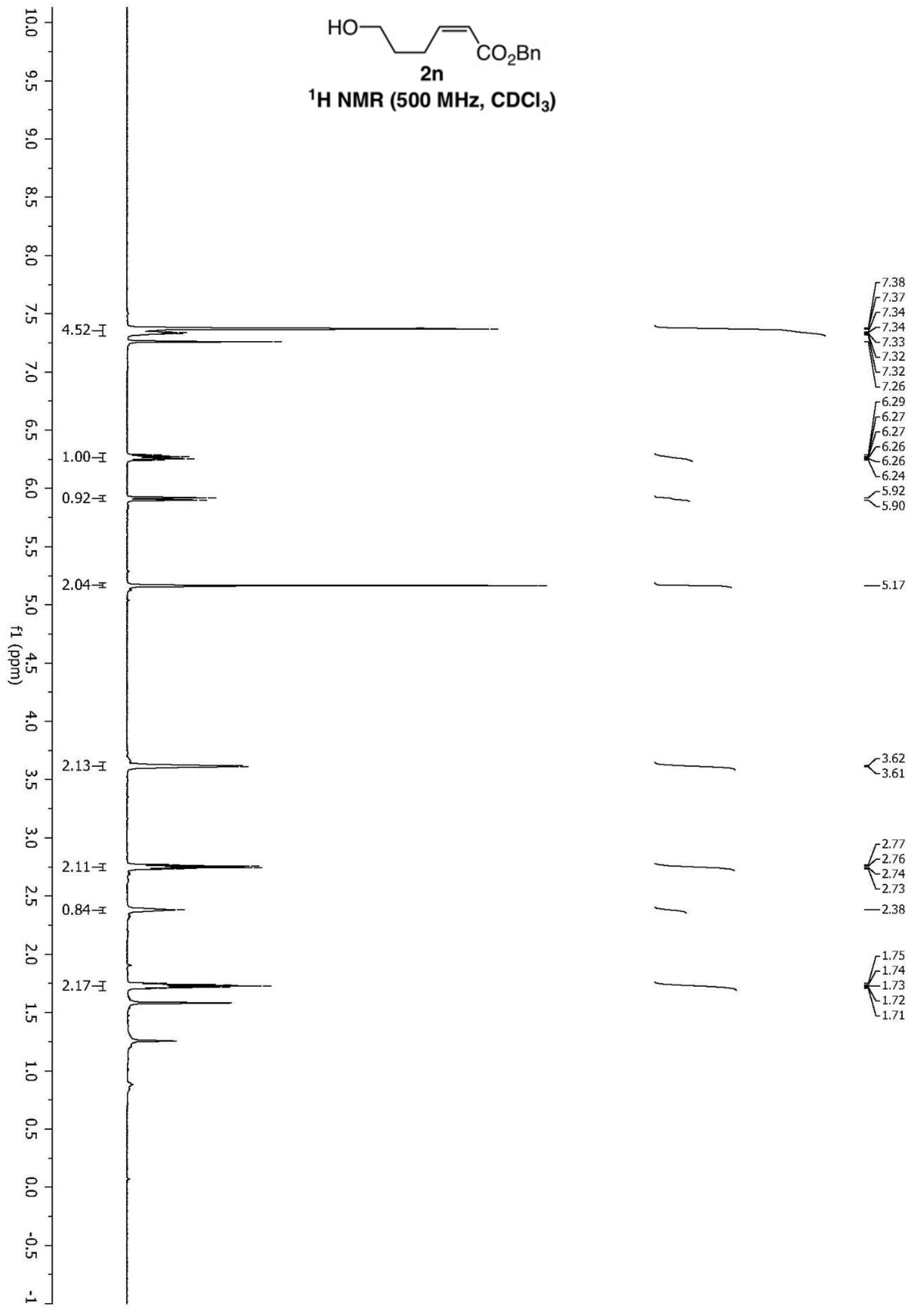


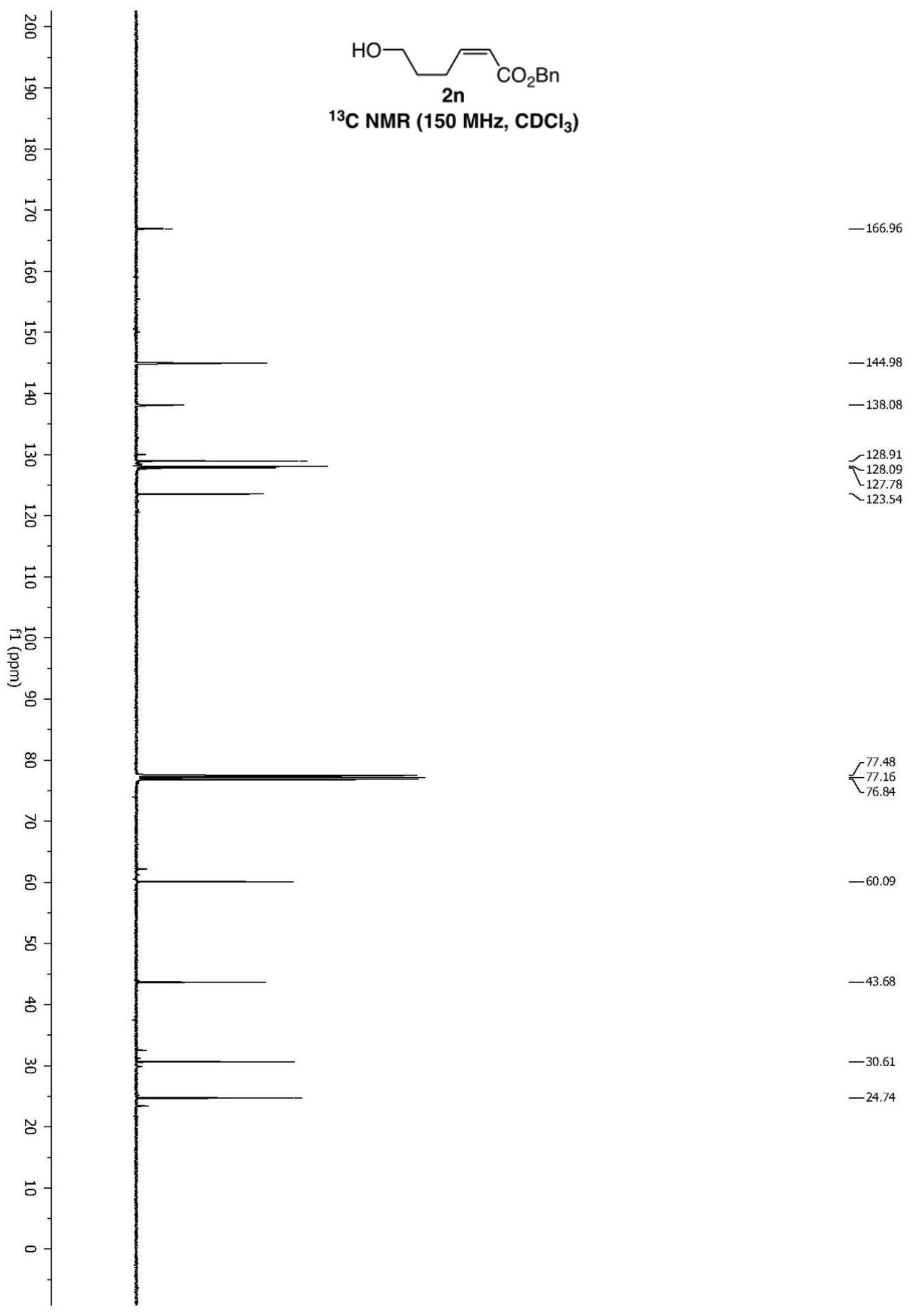




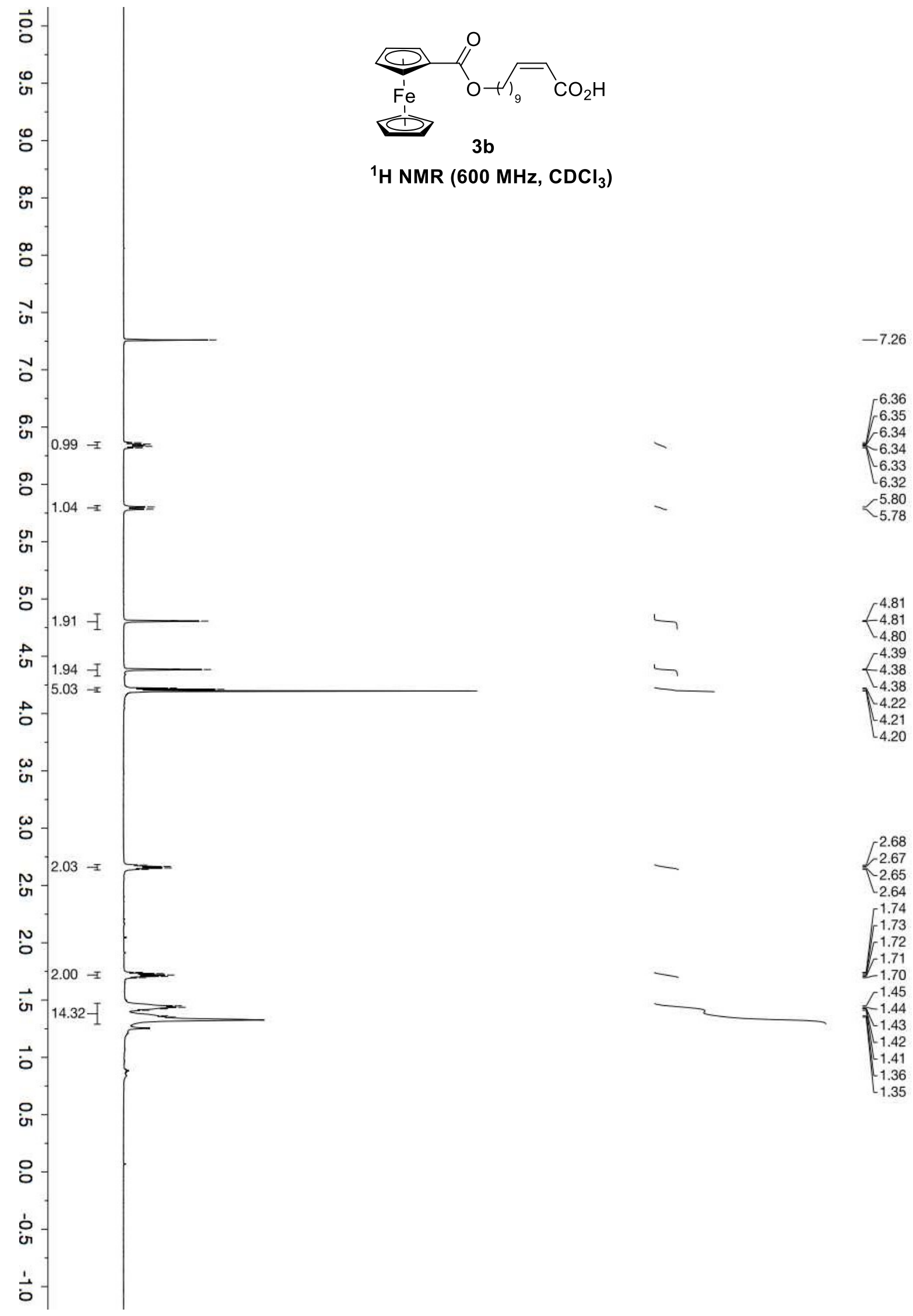




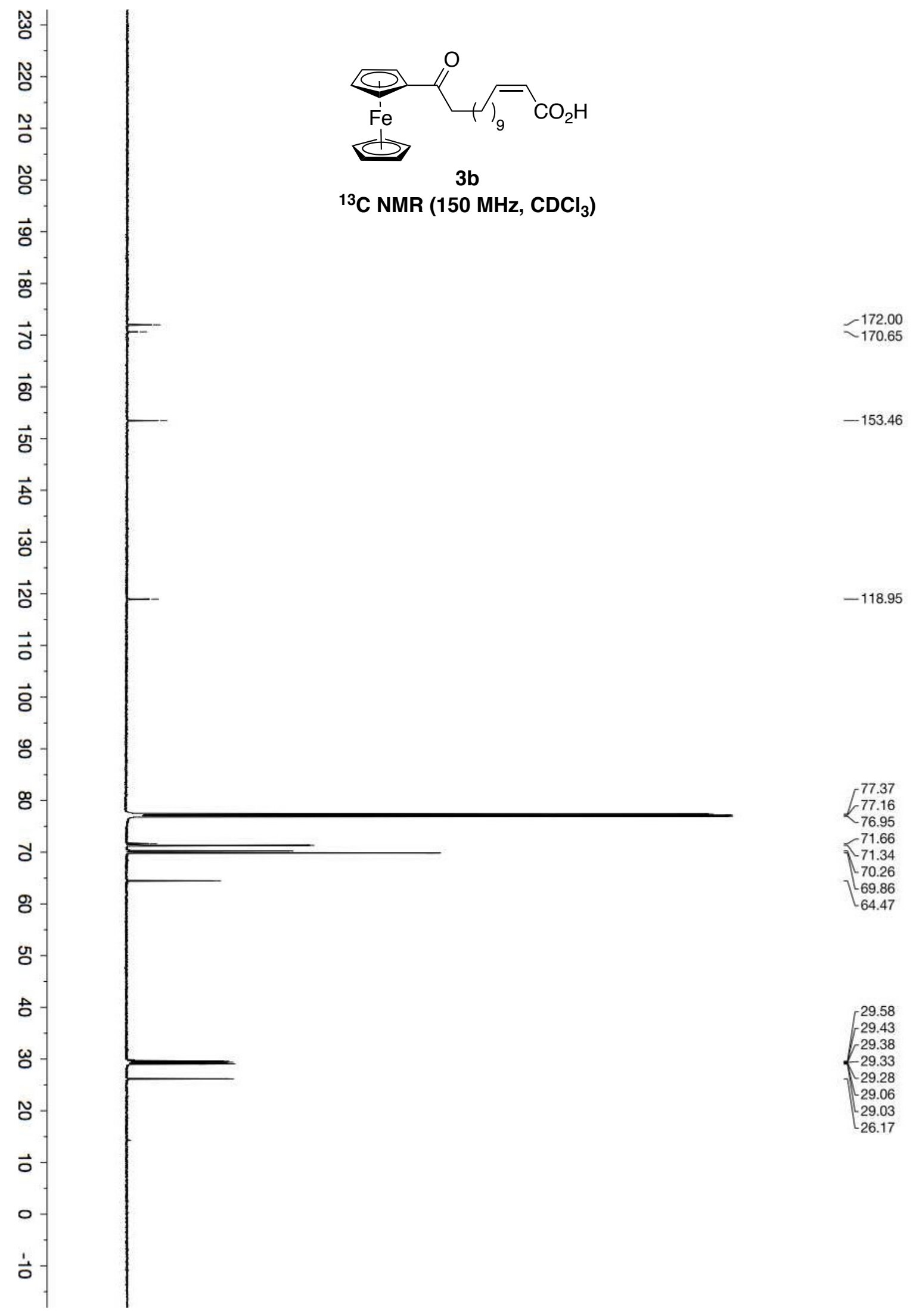




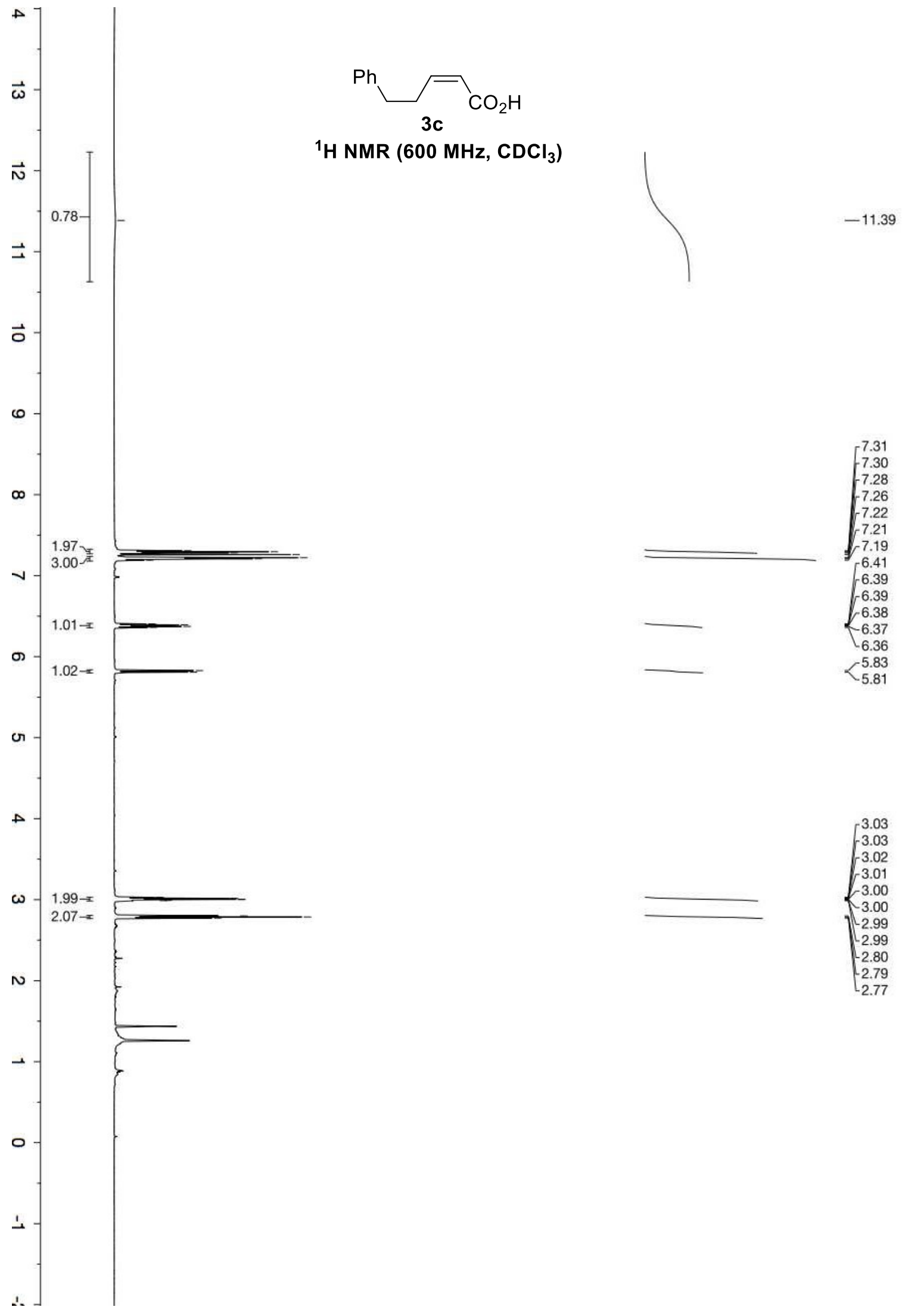




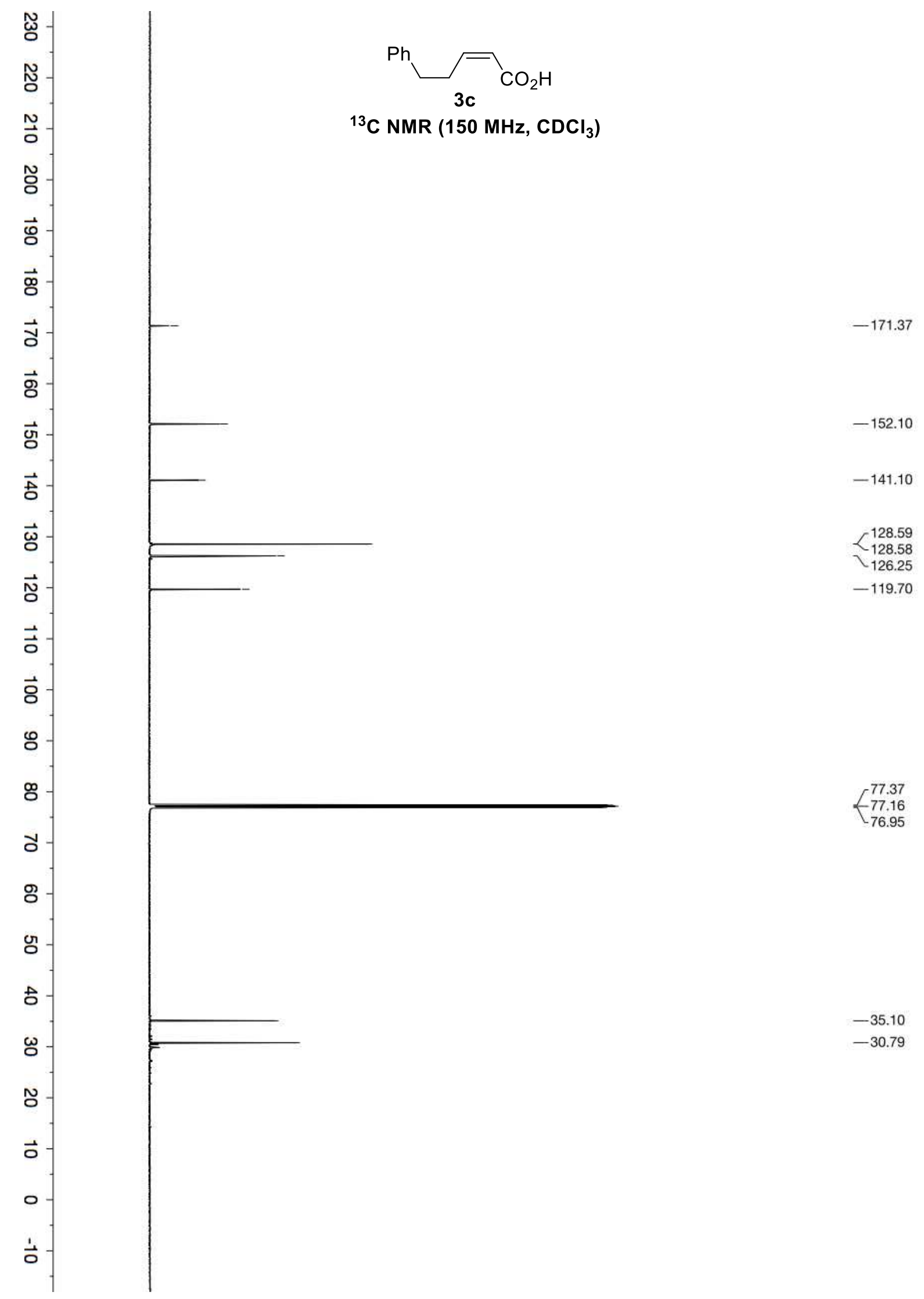




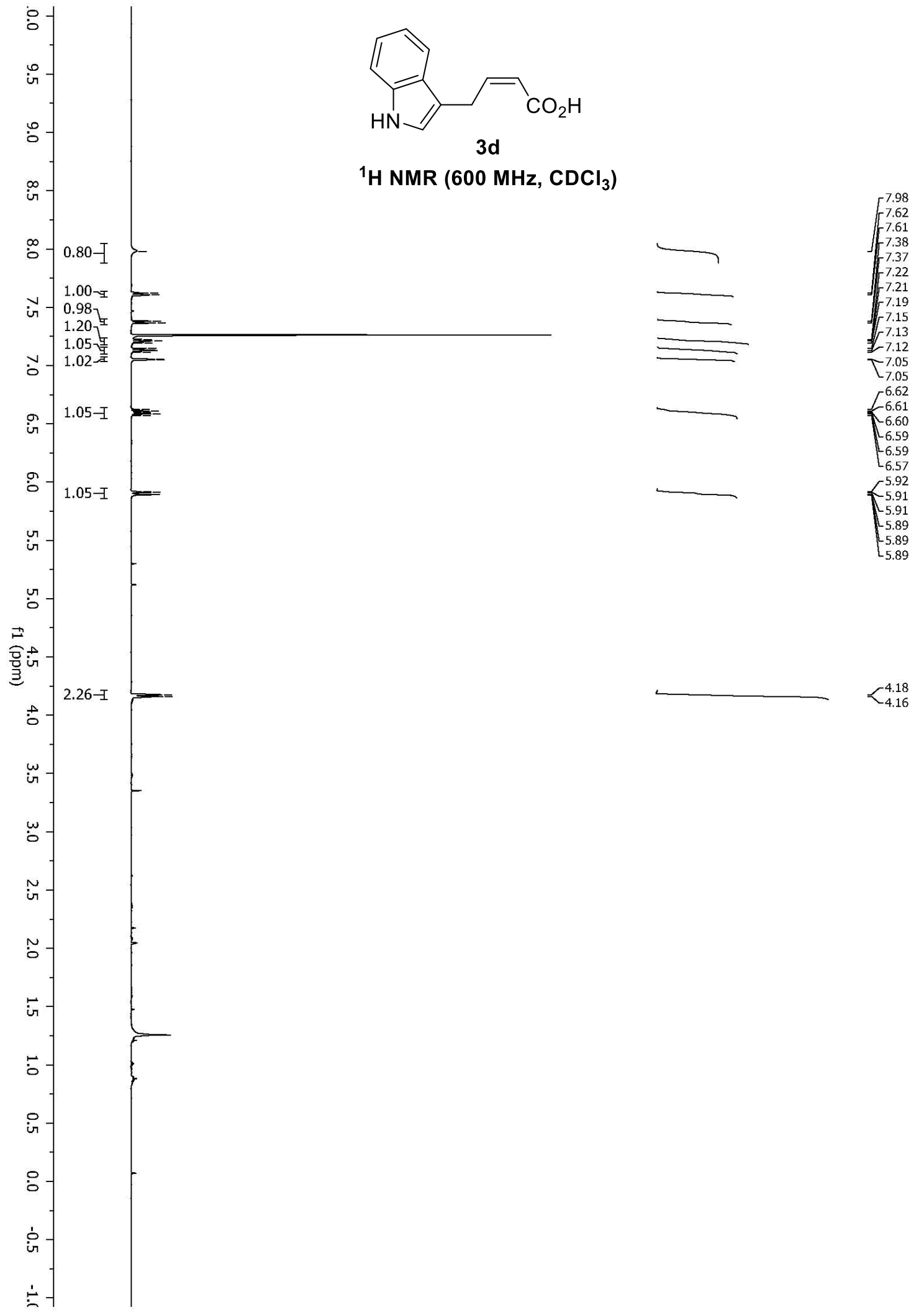




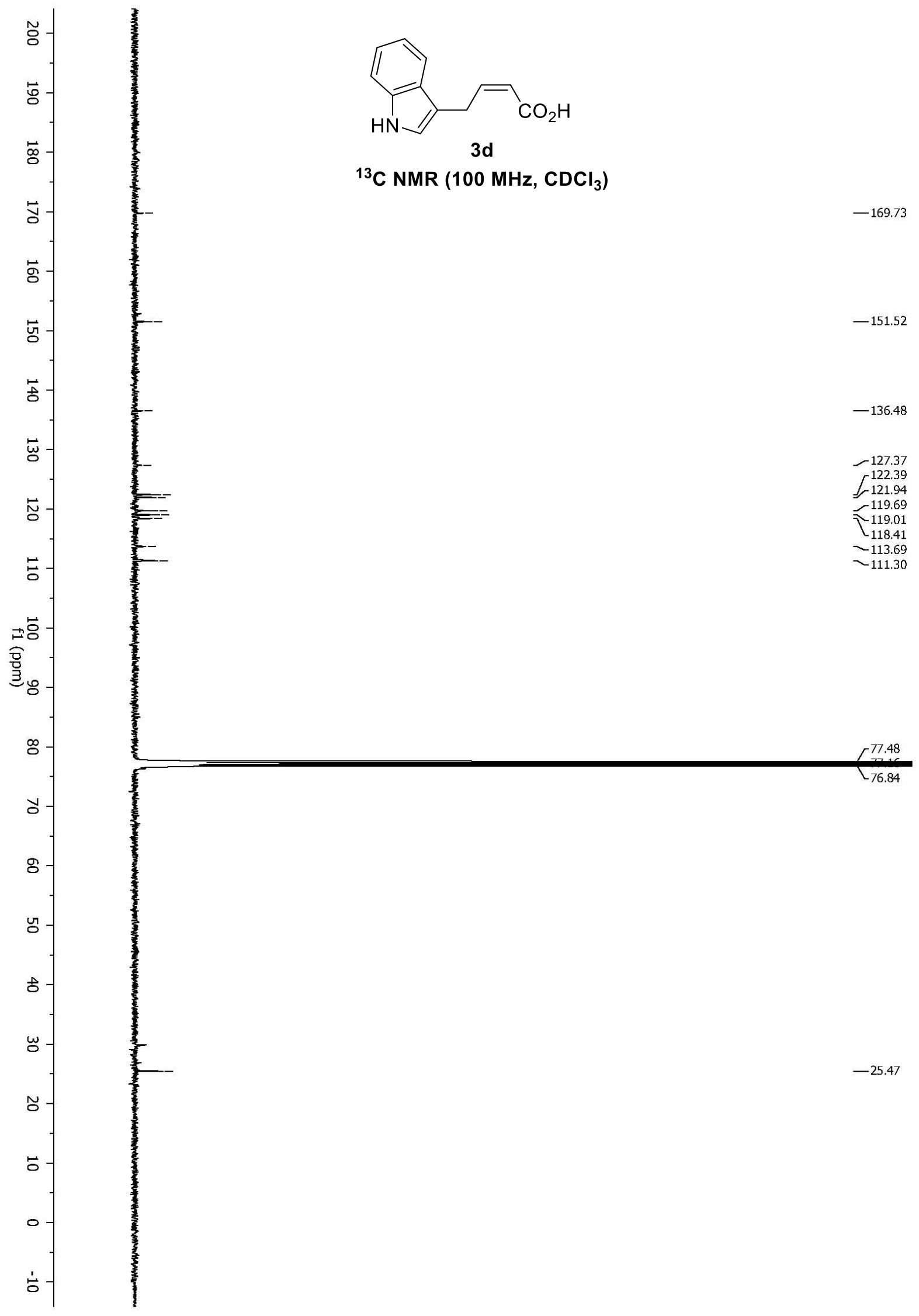




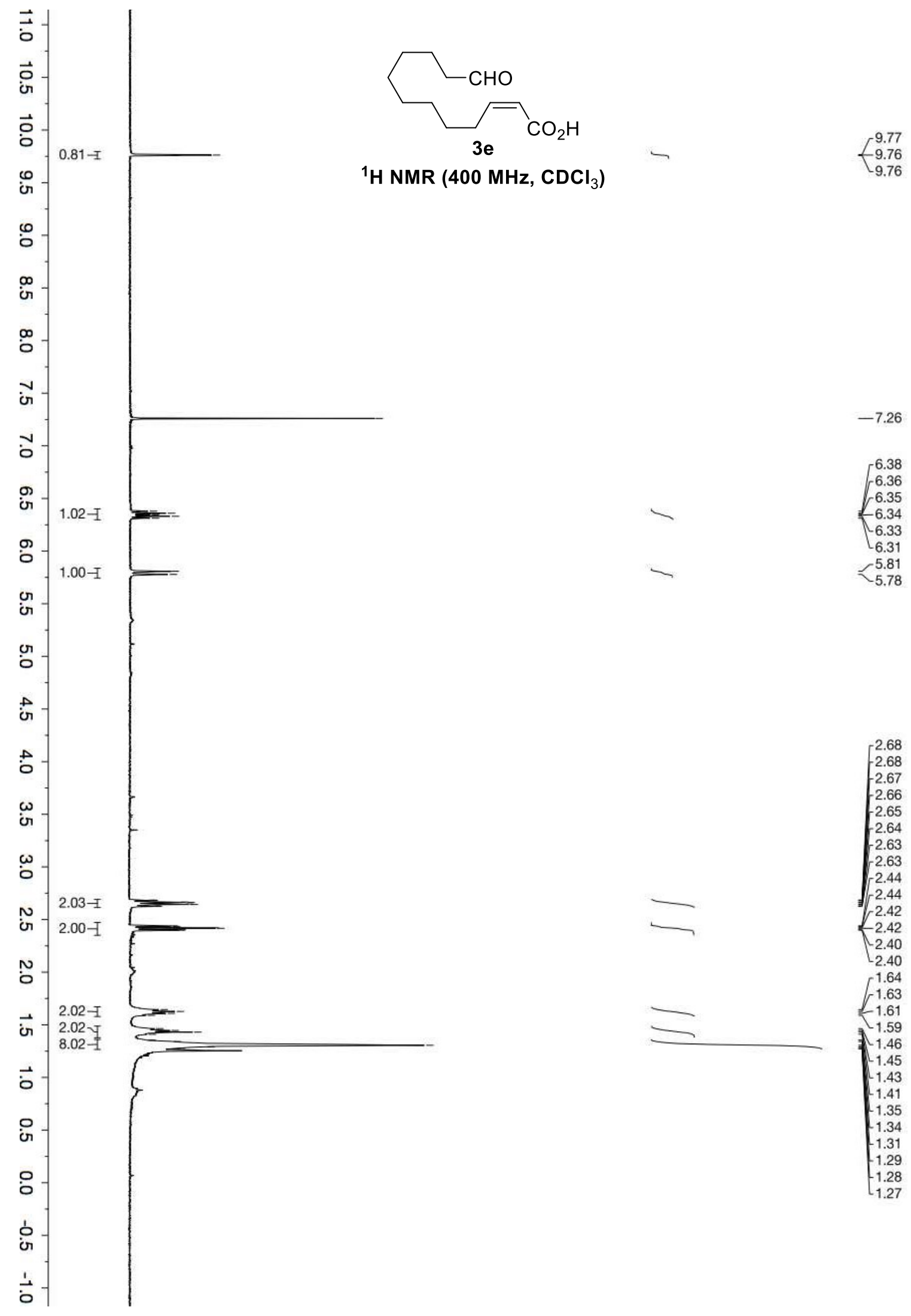




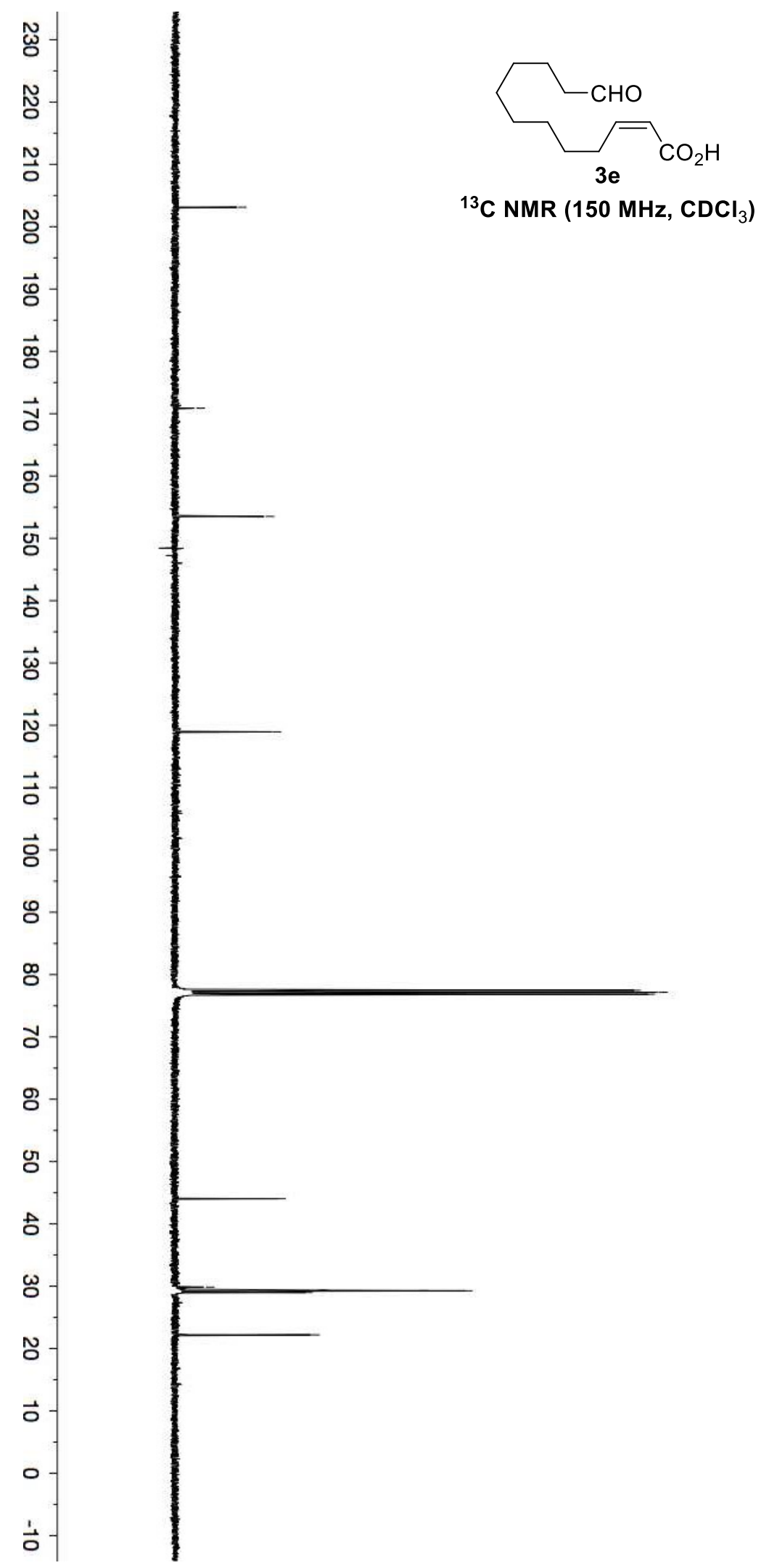




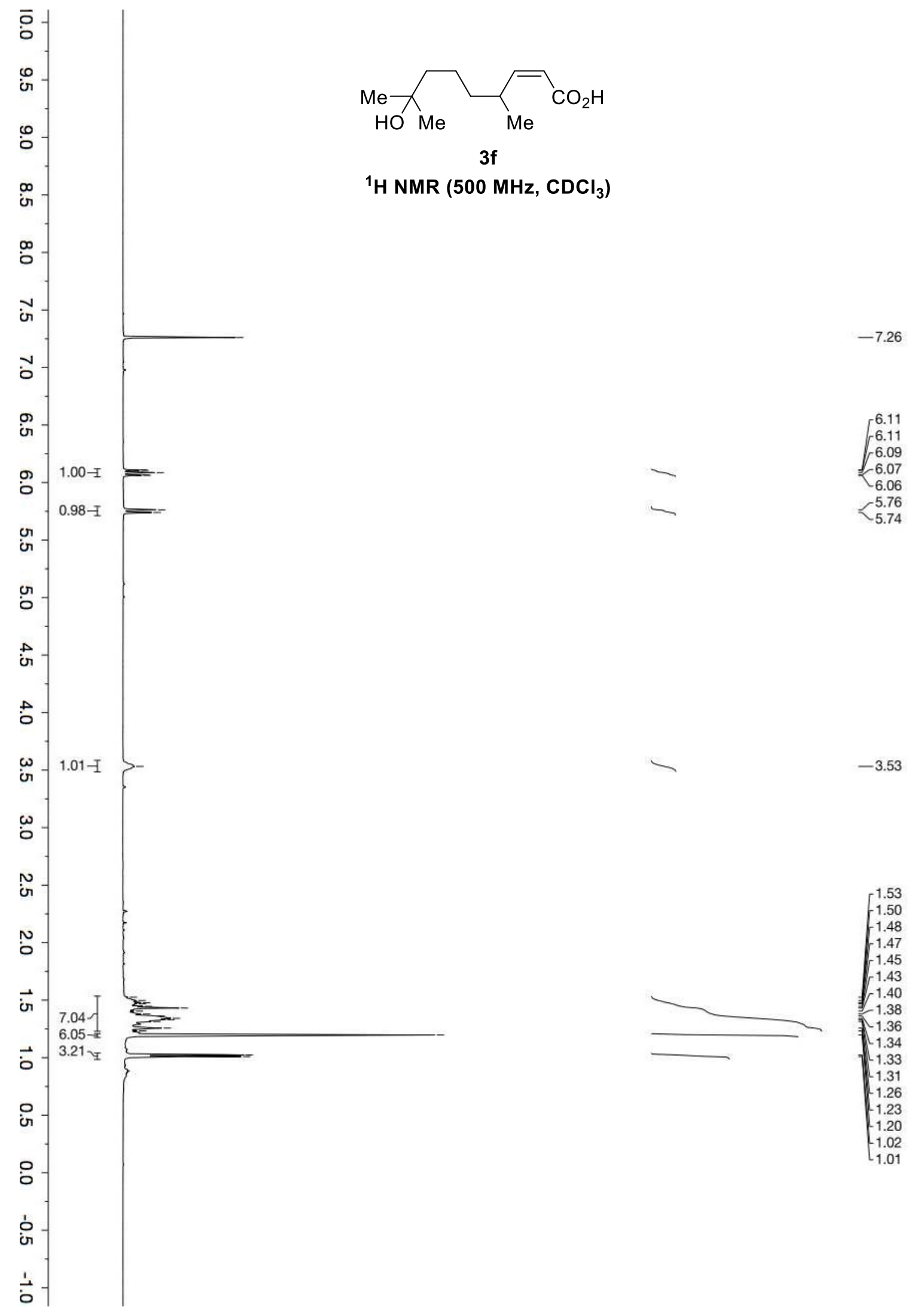




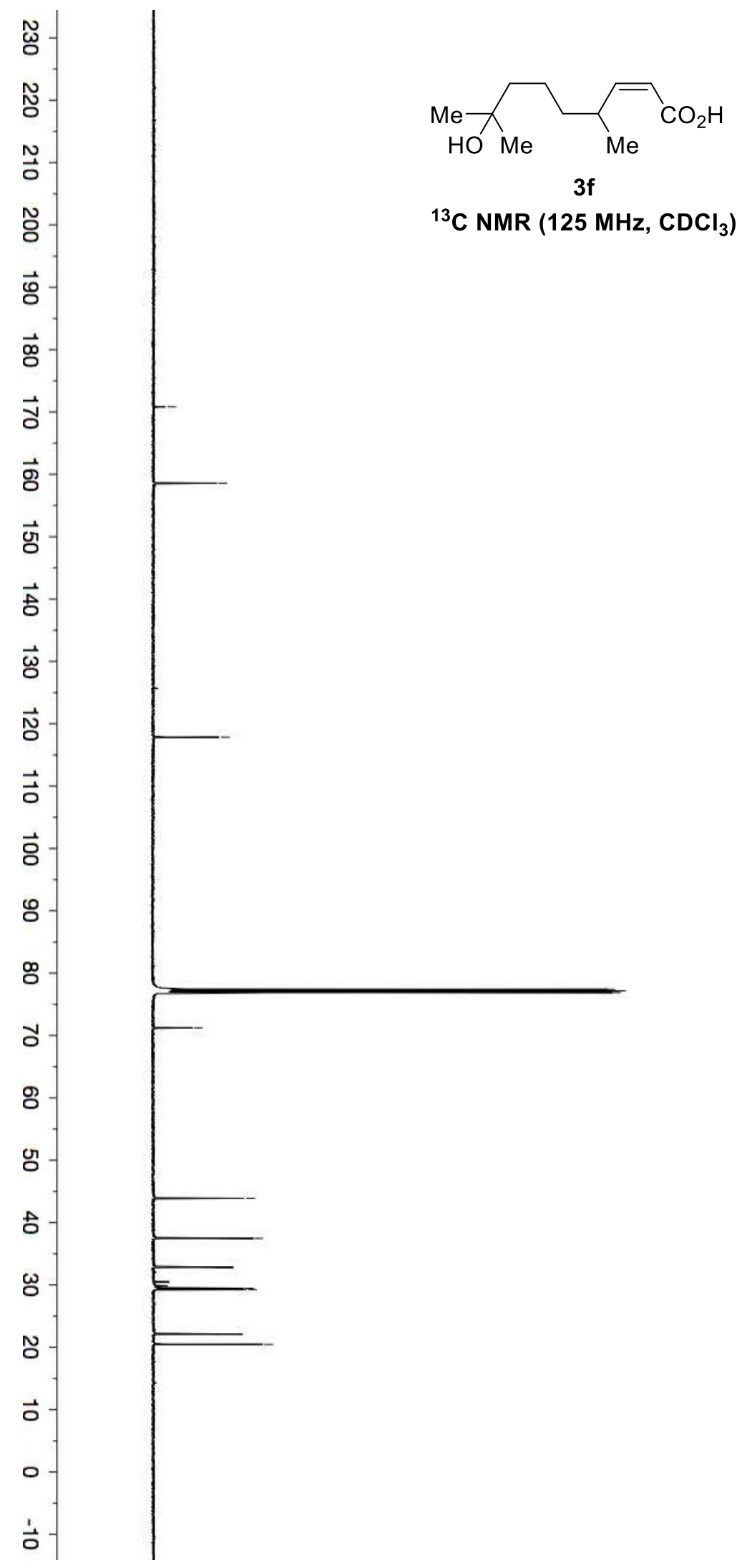




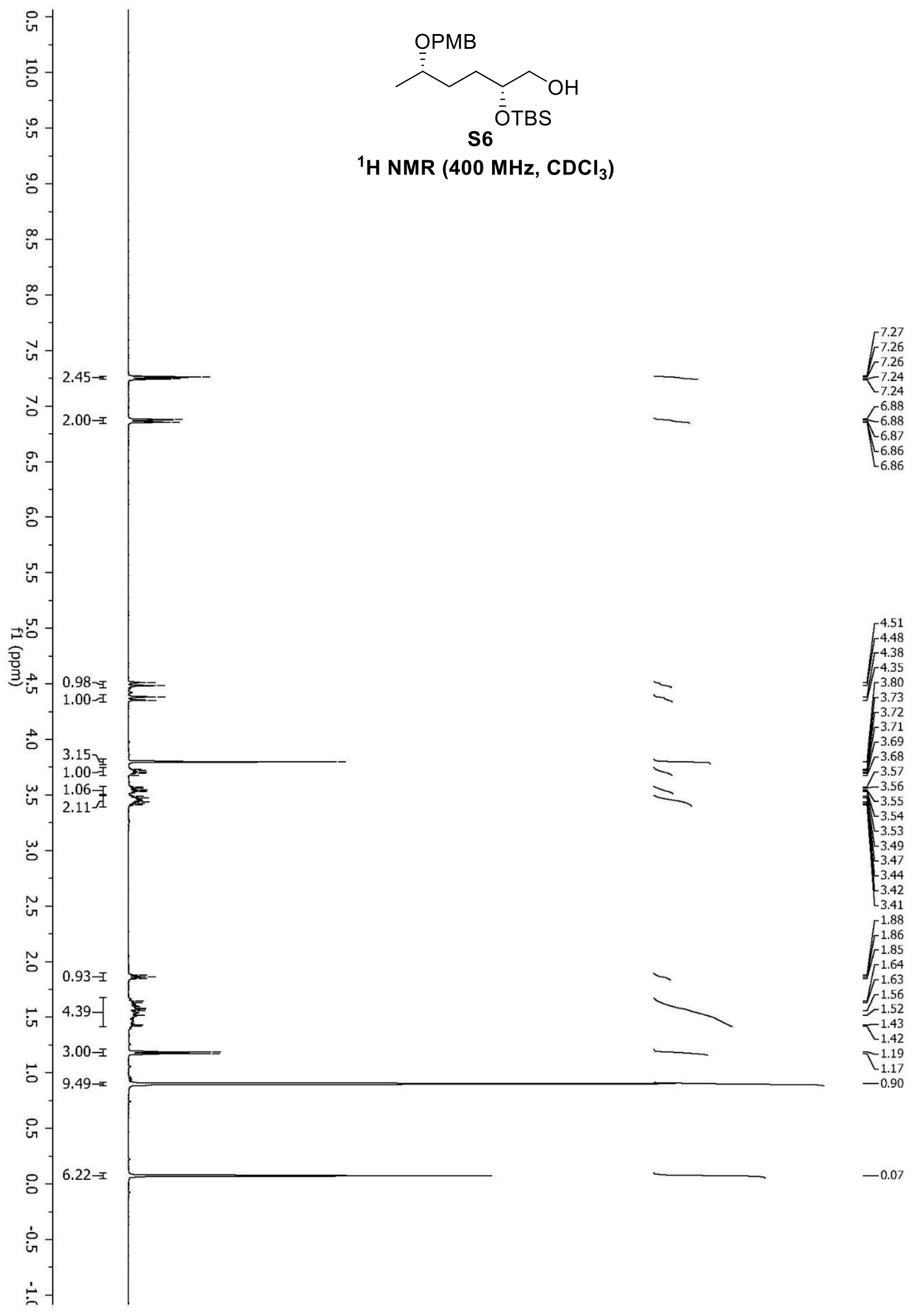



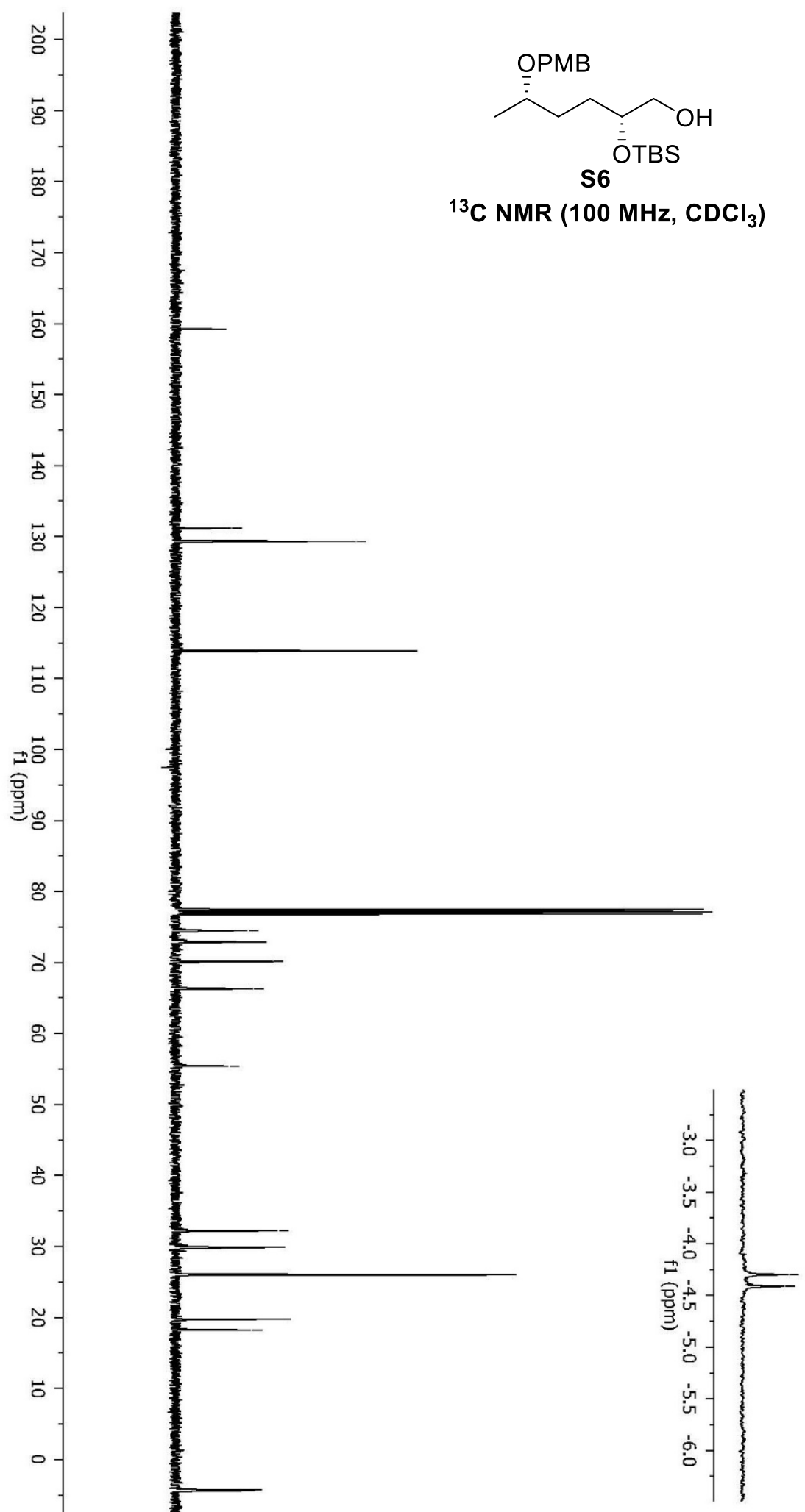

$-55.43$

\&

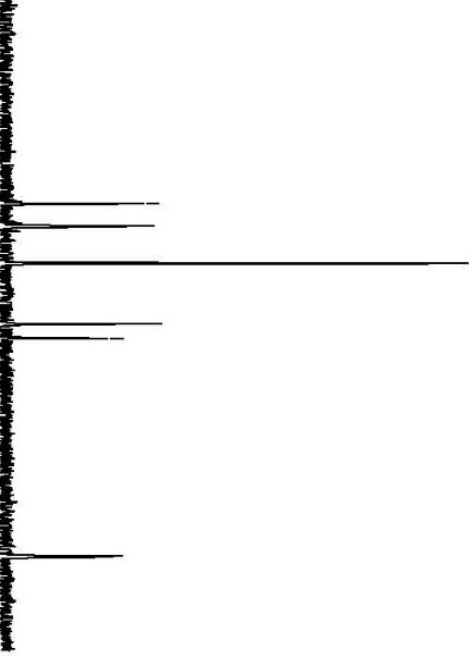

$=-4.30$ 


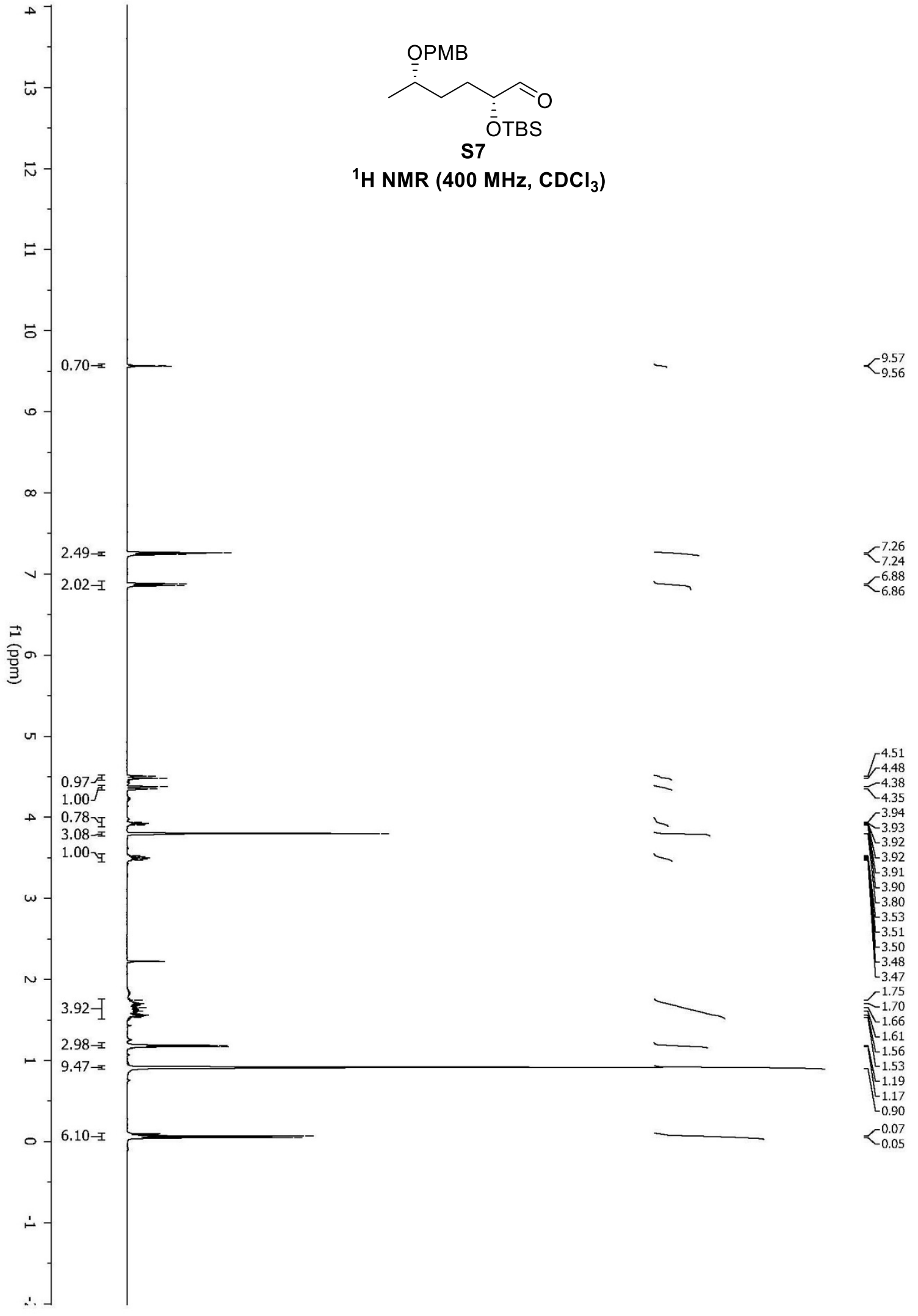




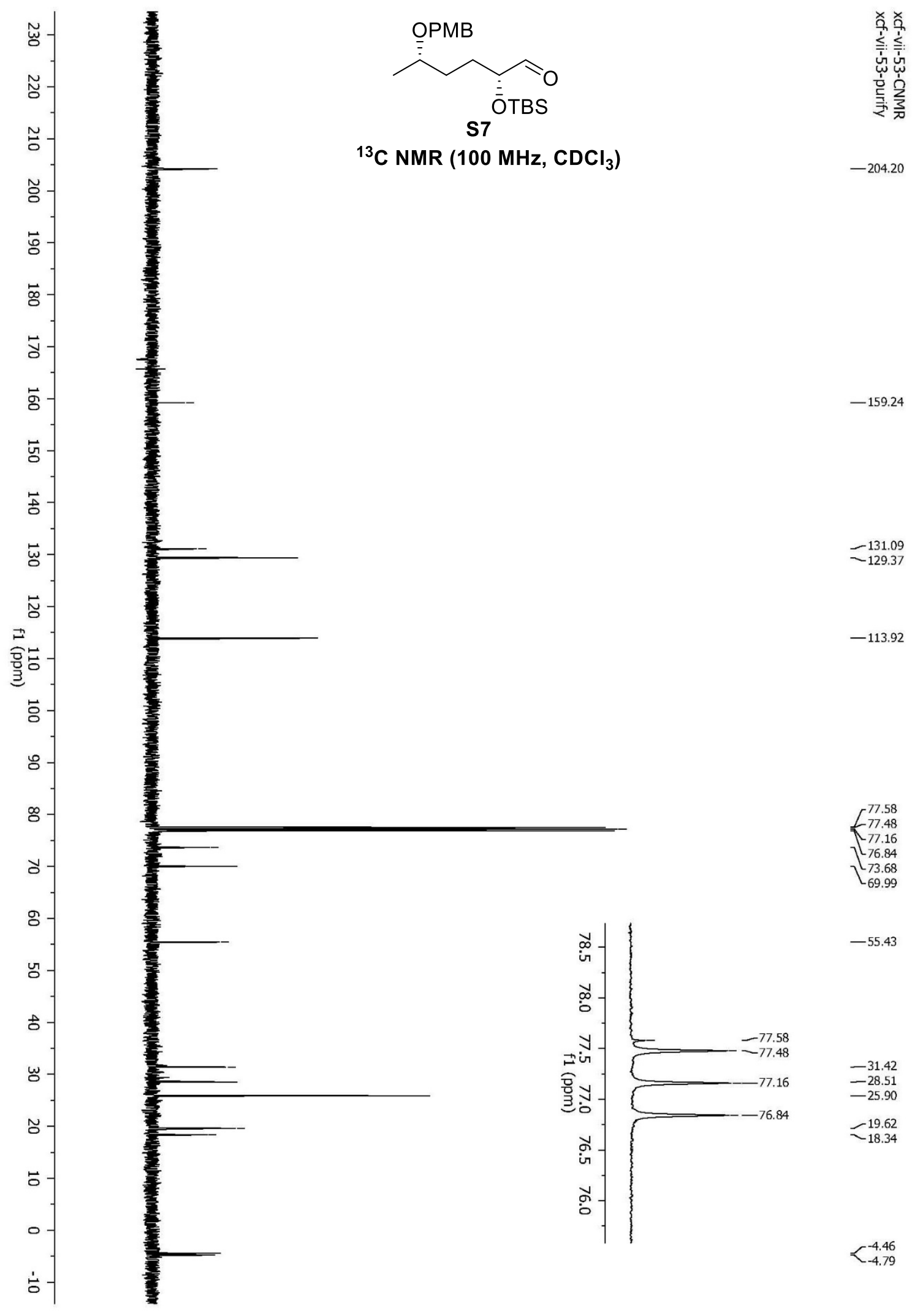




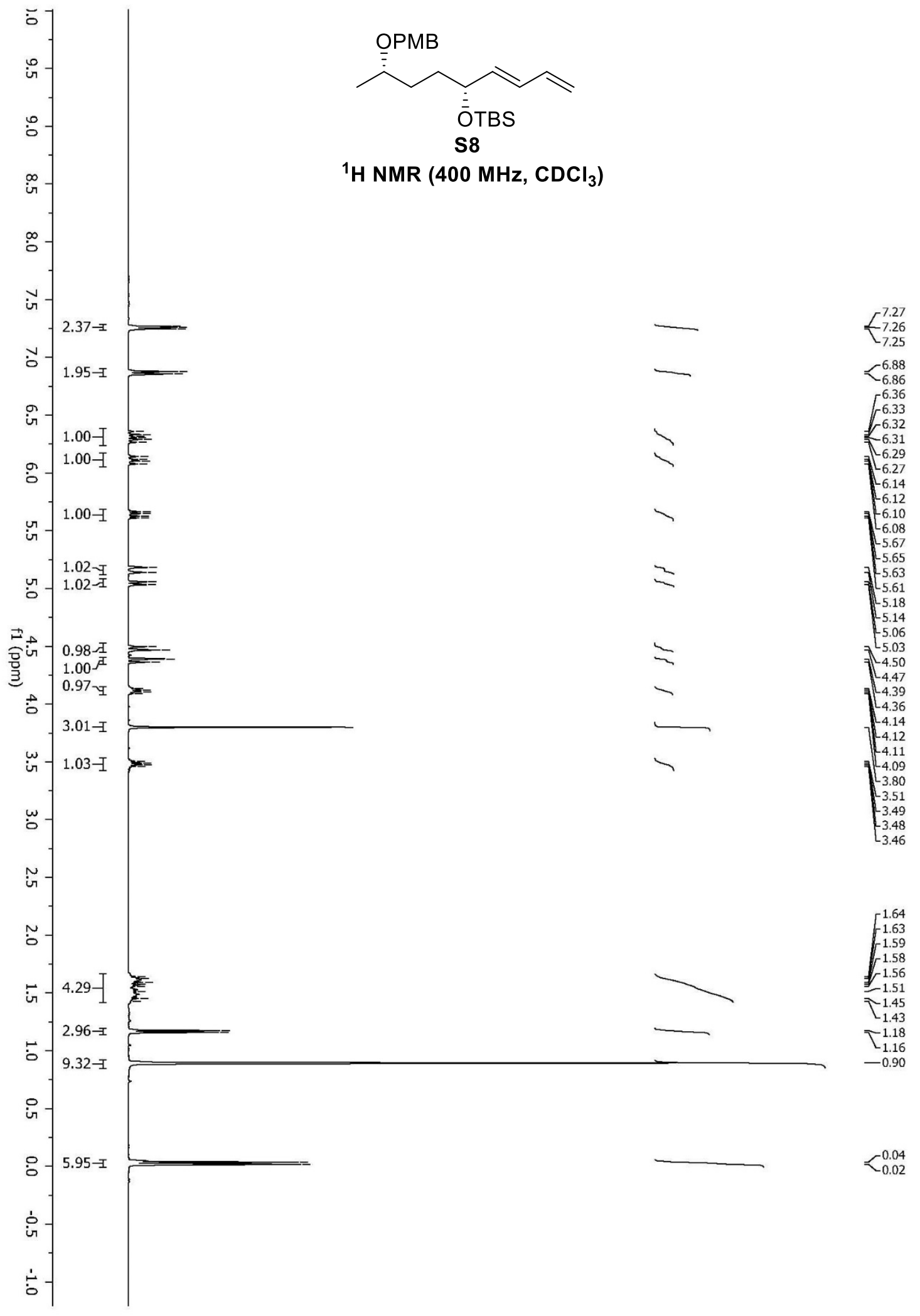




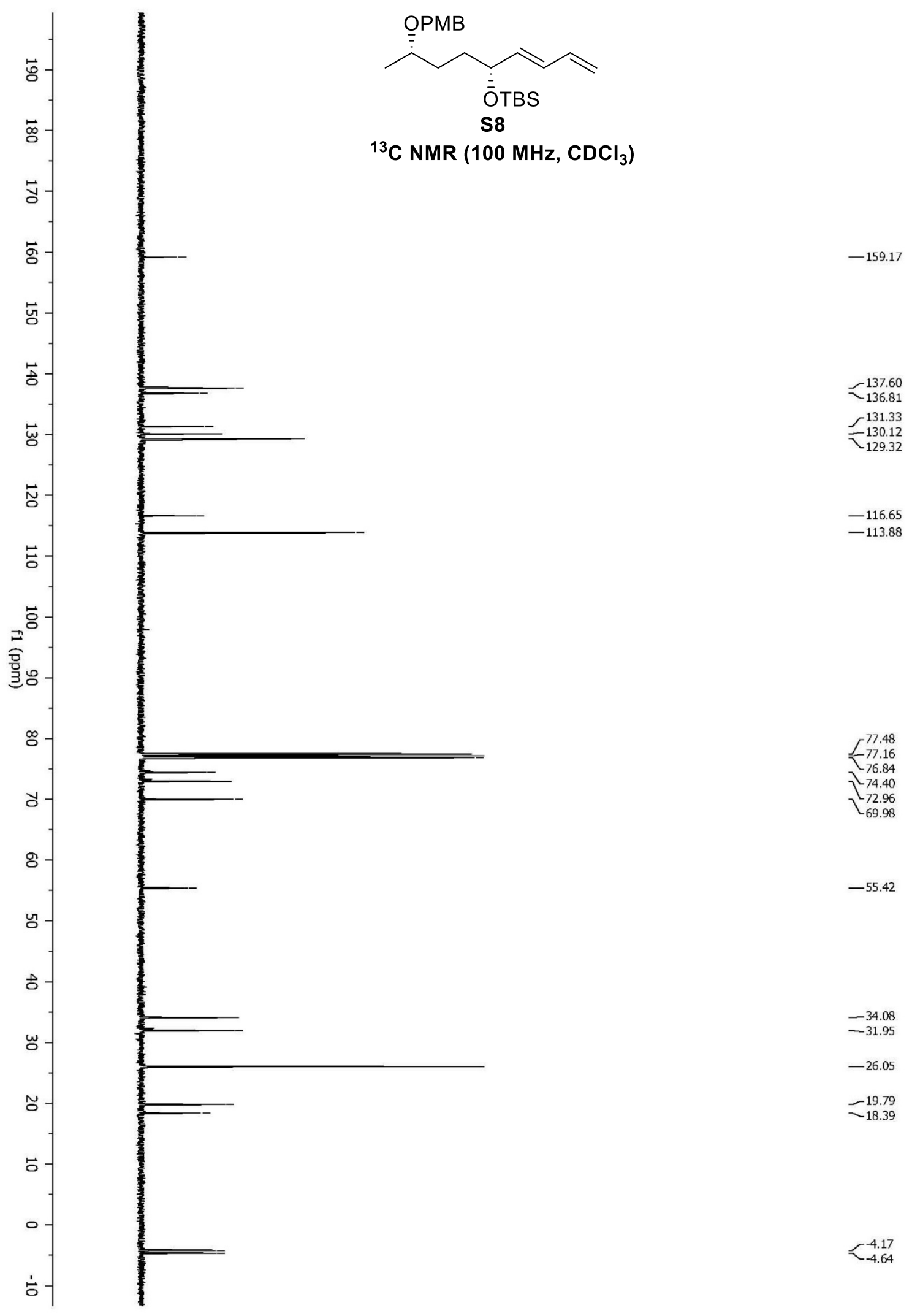




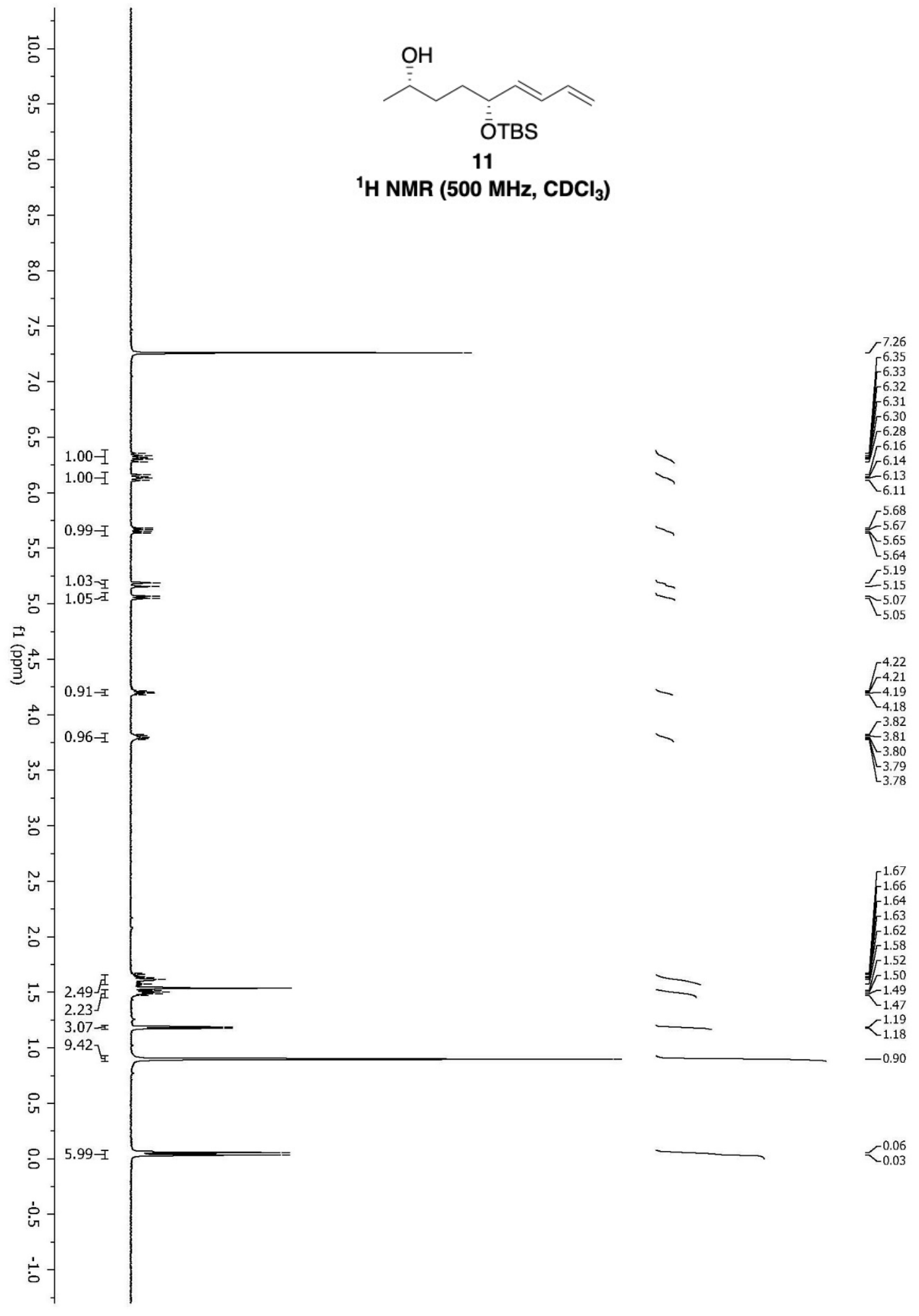




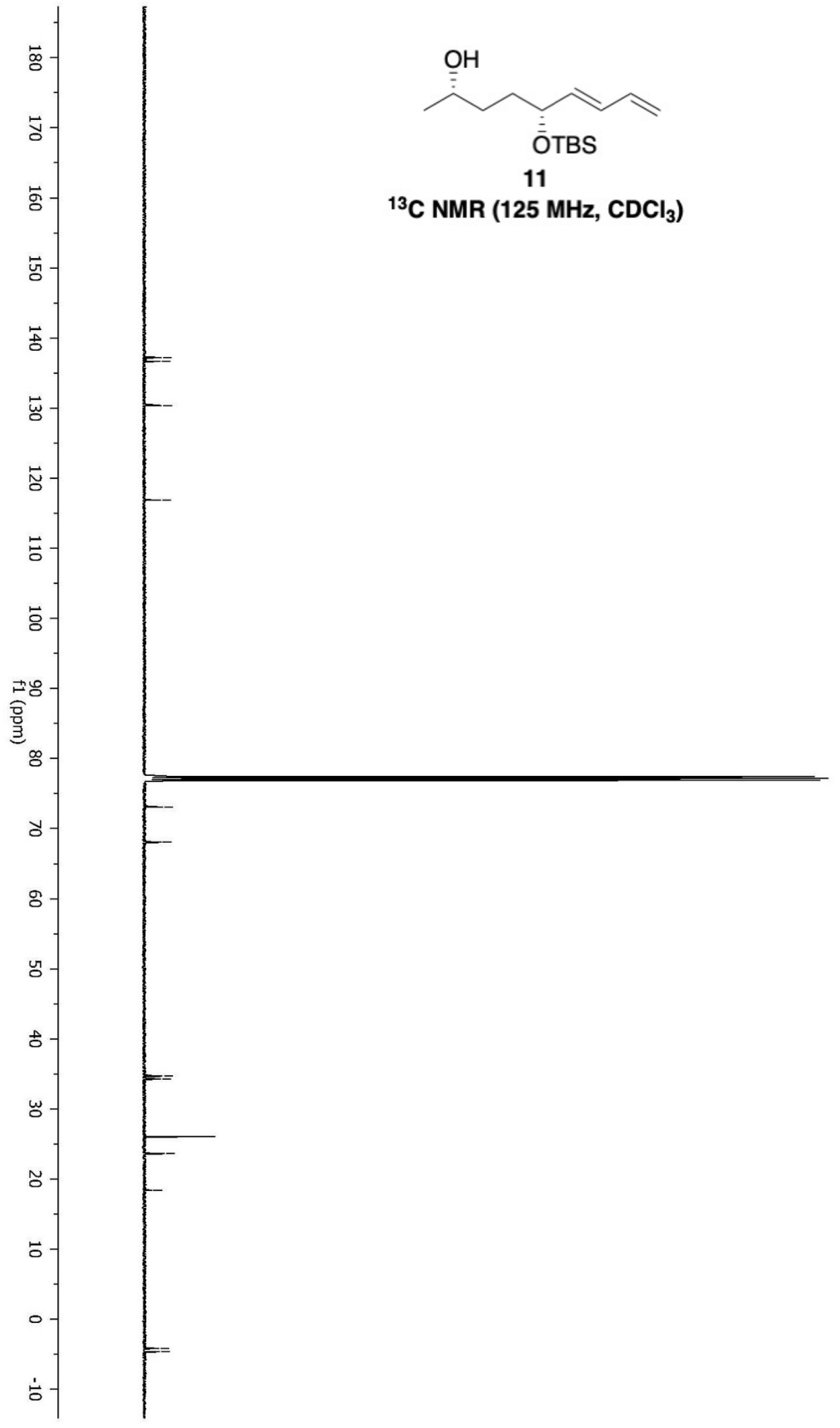

$$
\begin{aligned}
& \chi_{136.70}^{137.21} \\
& -130.36
\end{aligned}
$$$$
-116.88
$$ 


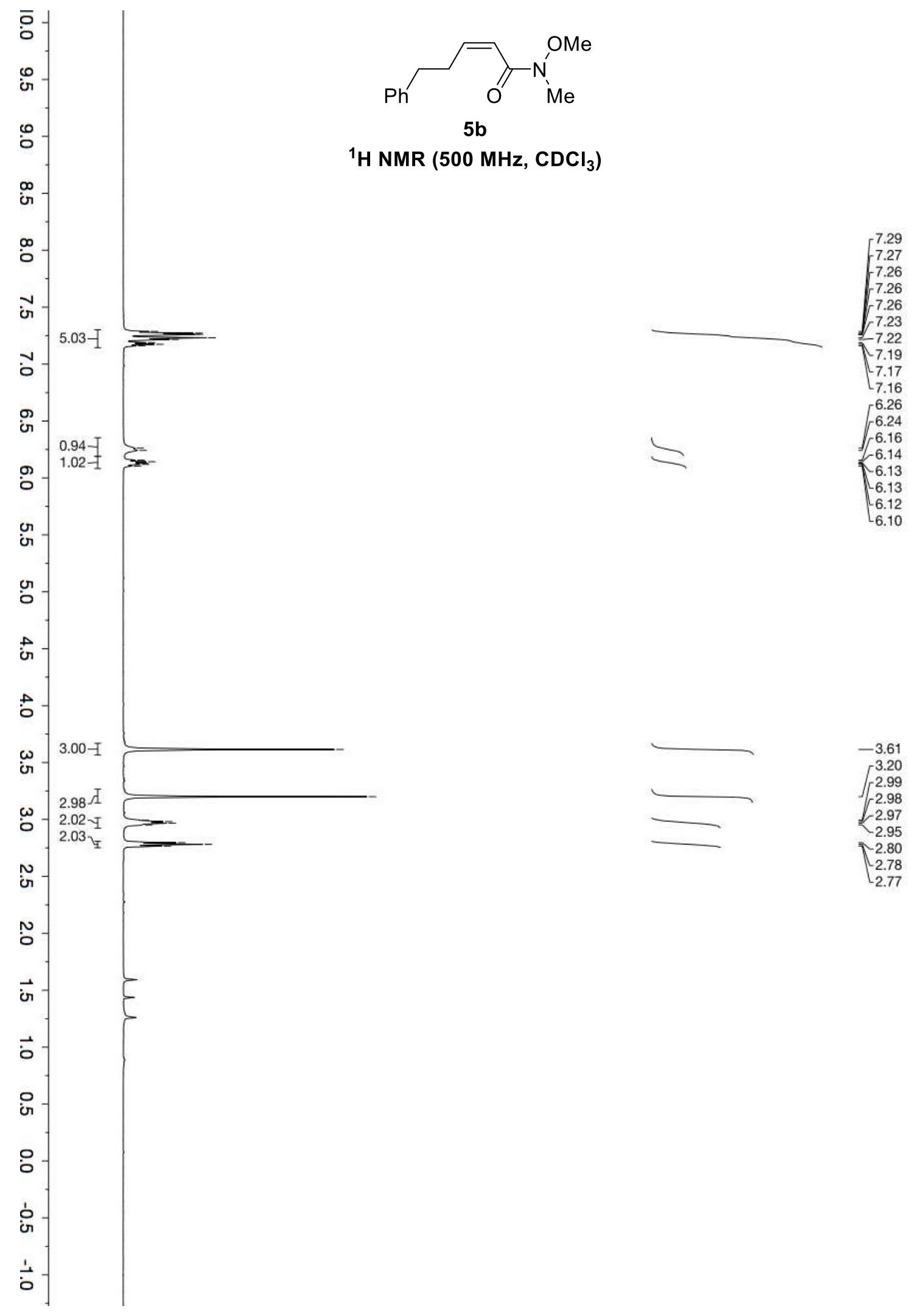




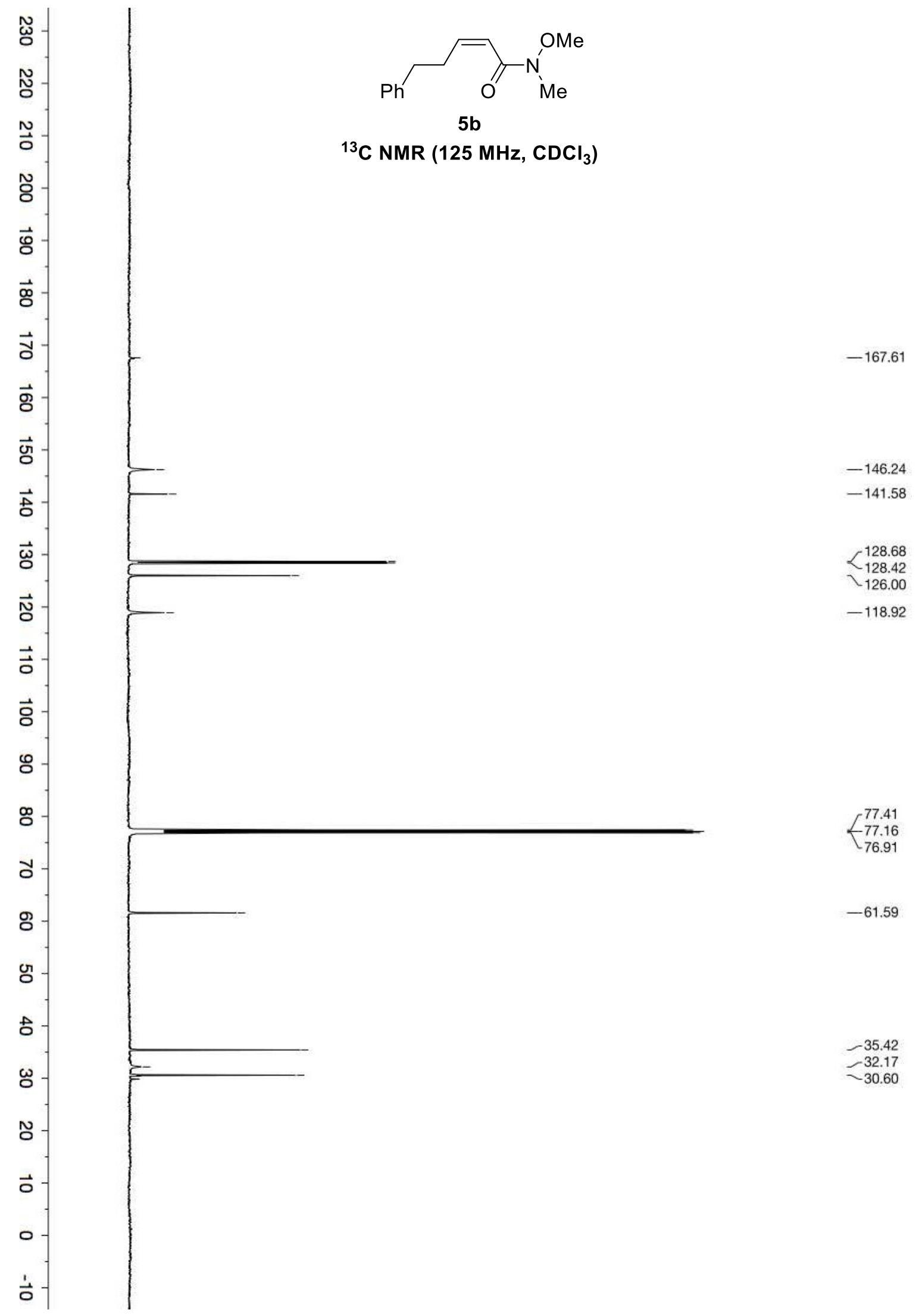




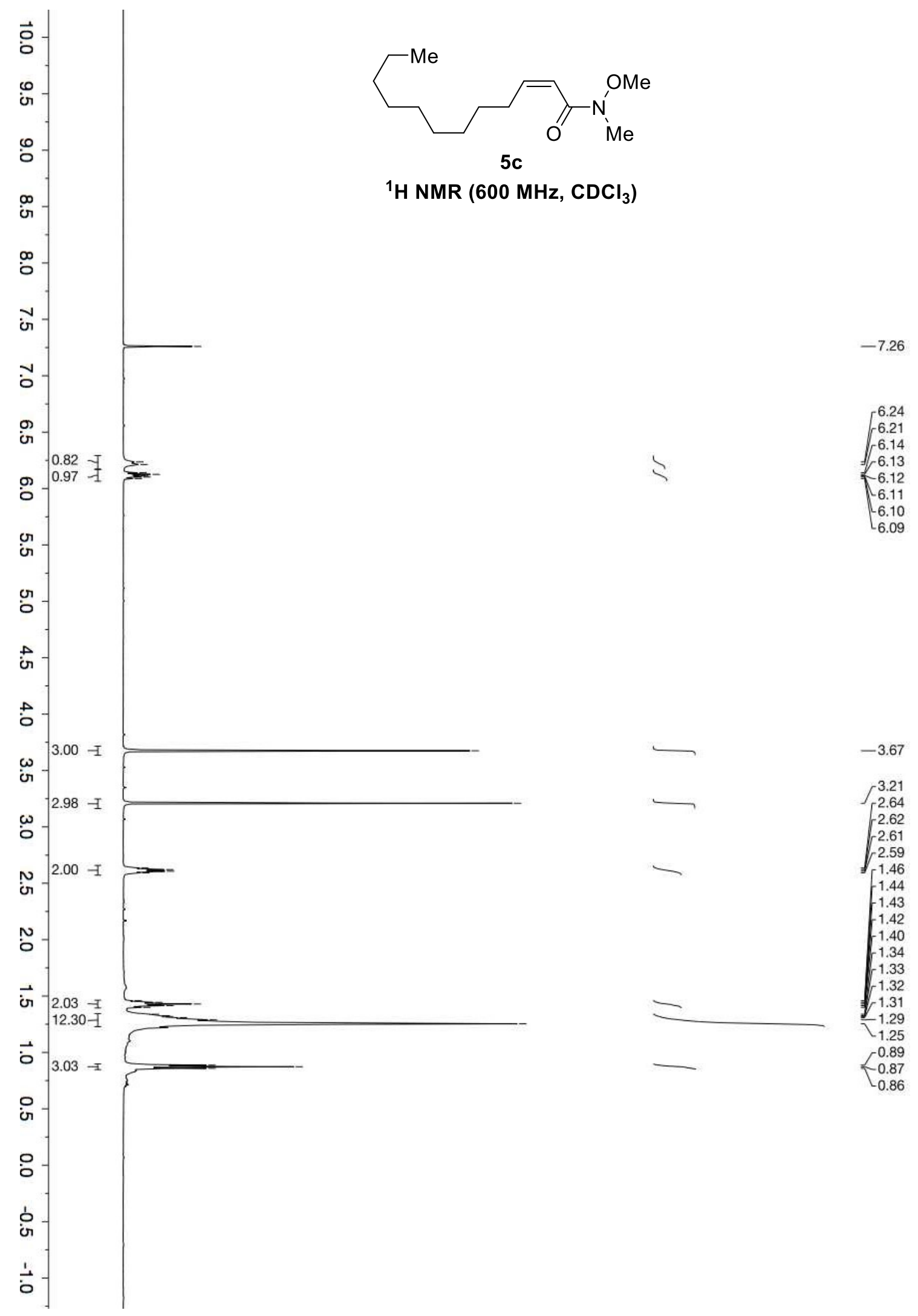




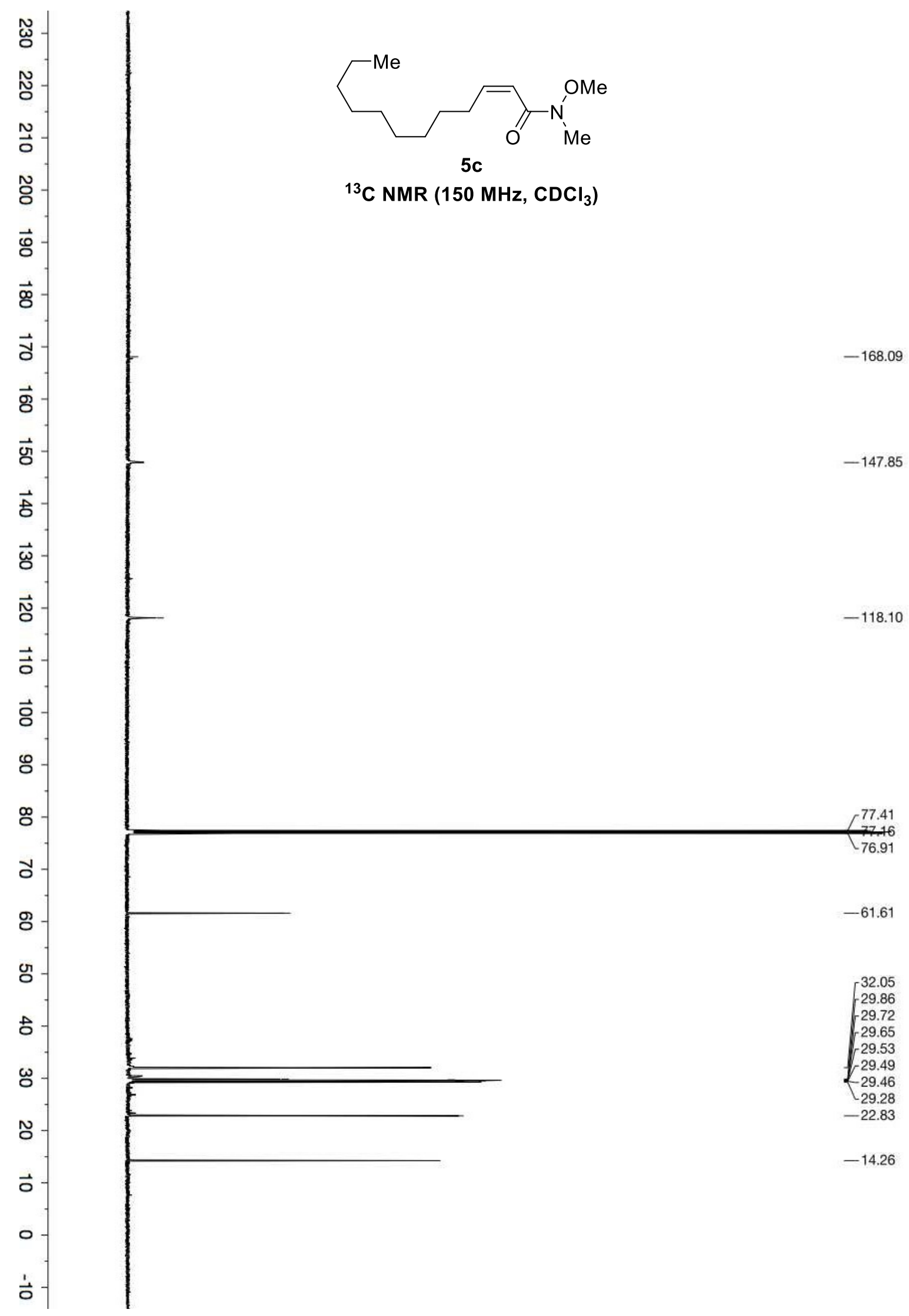




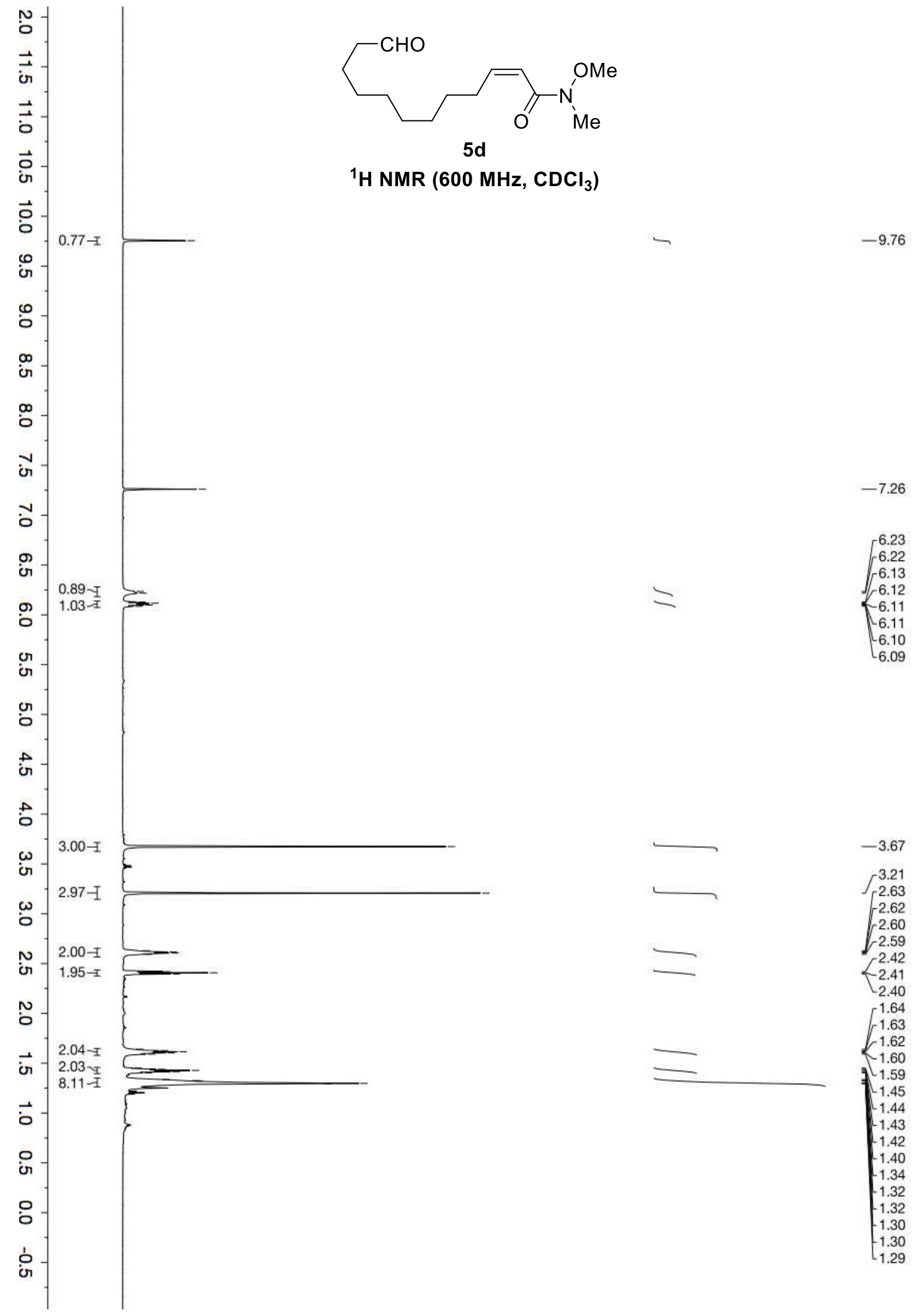




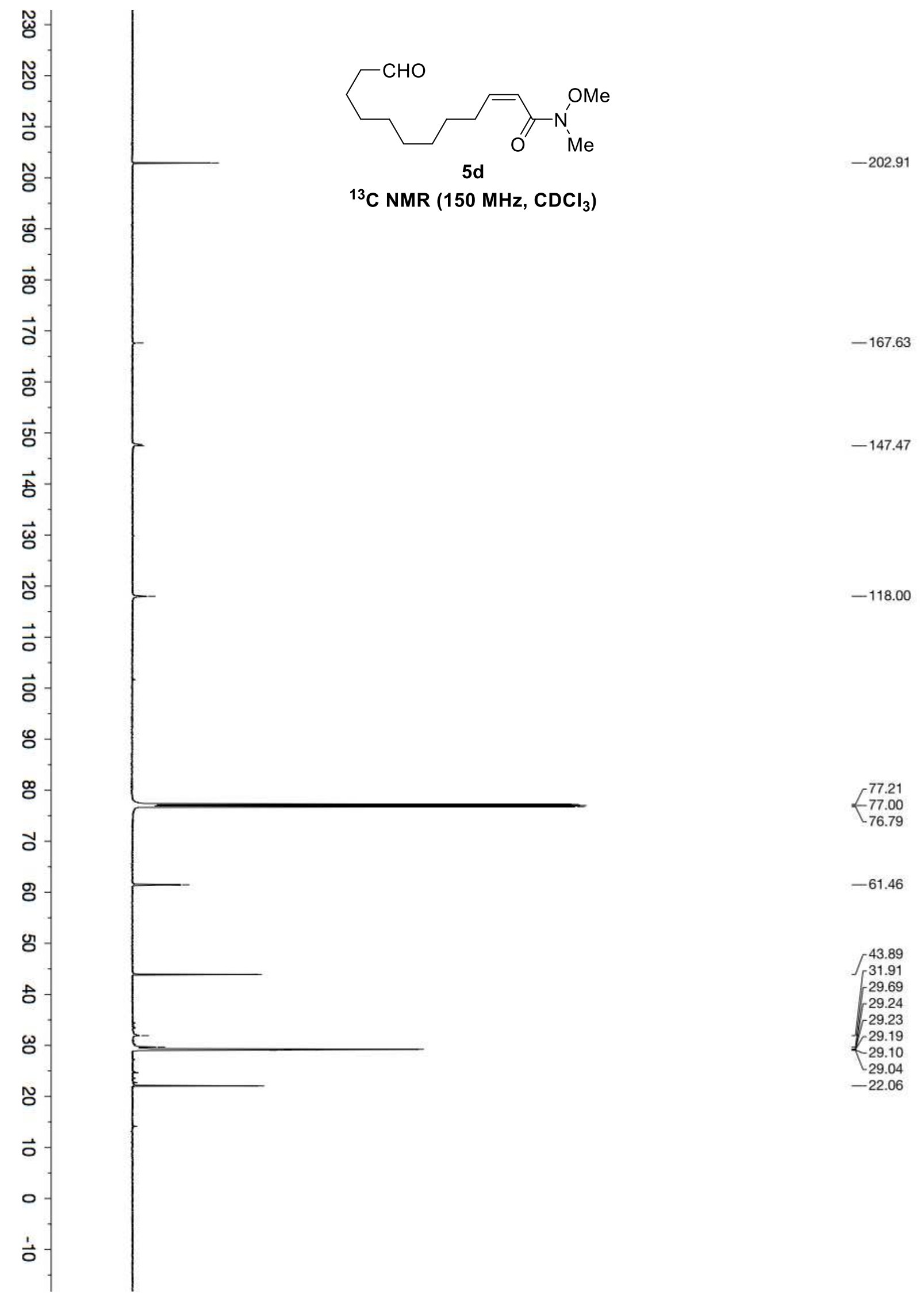




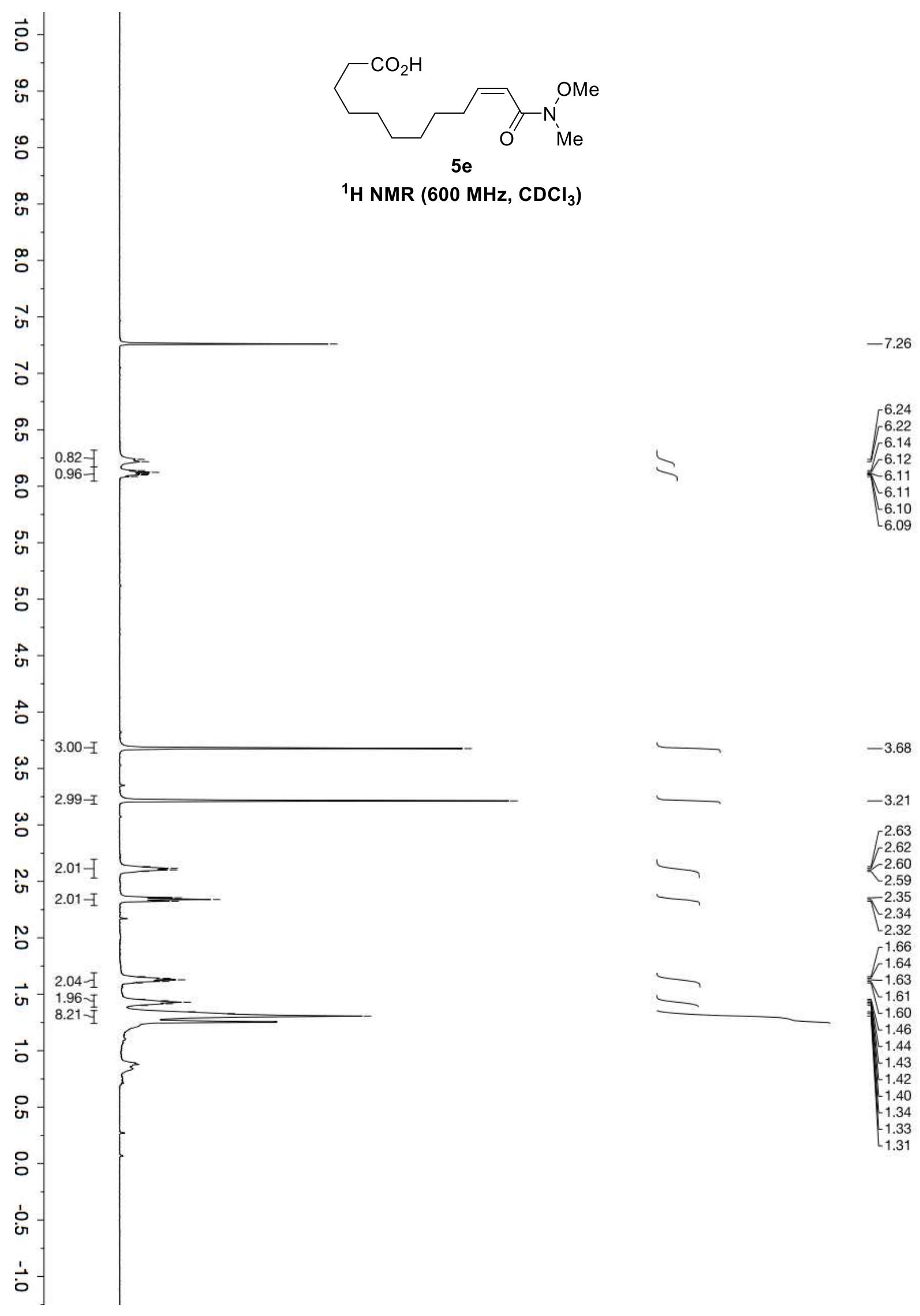




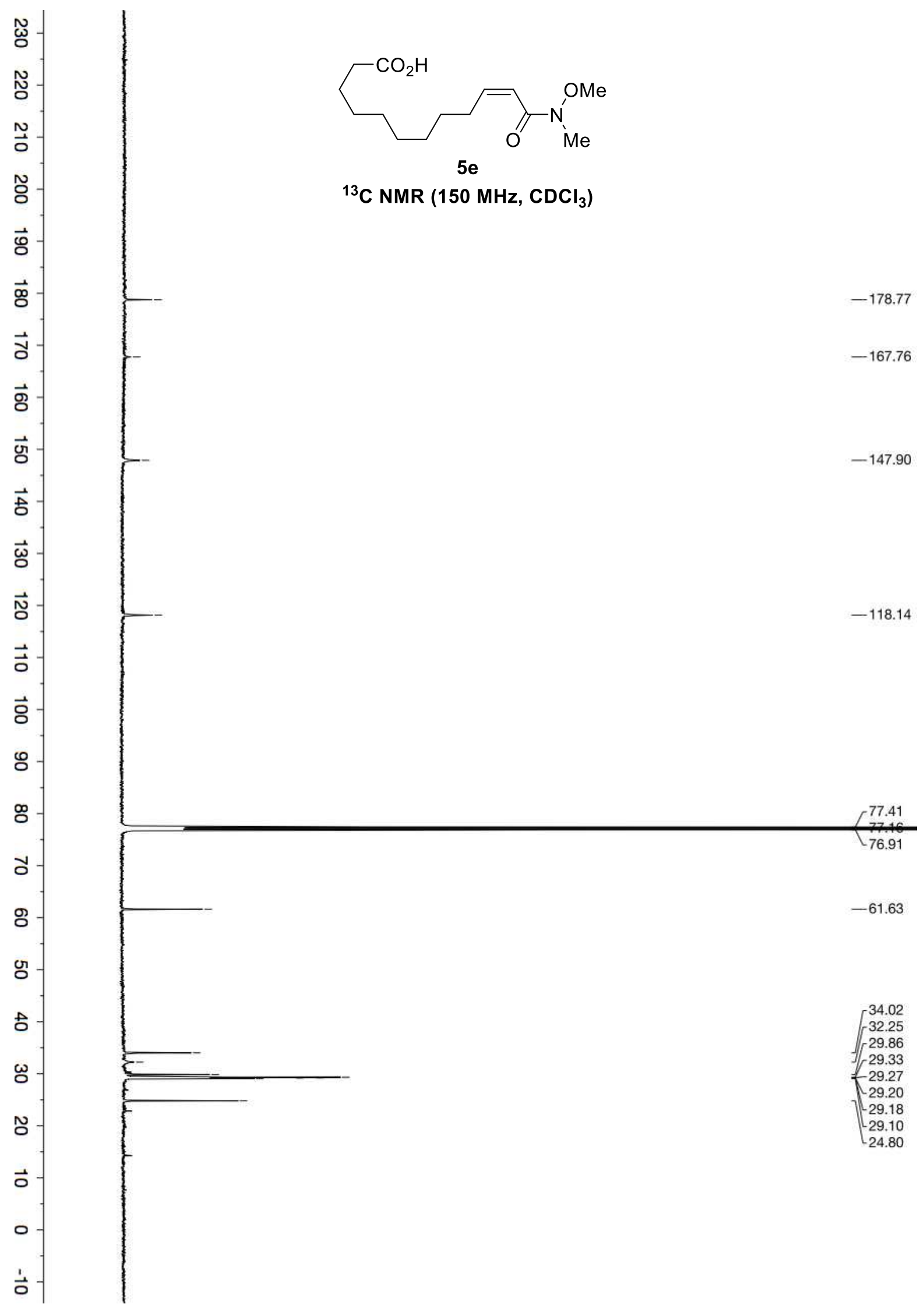




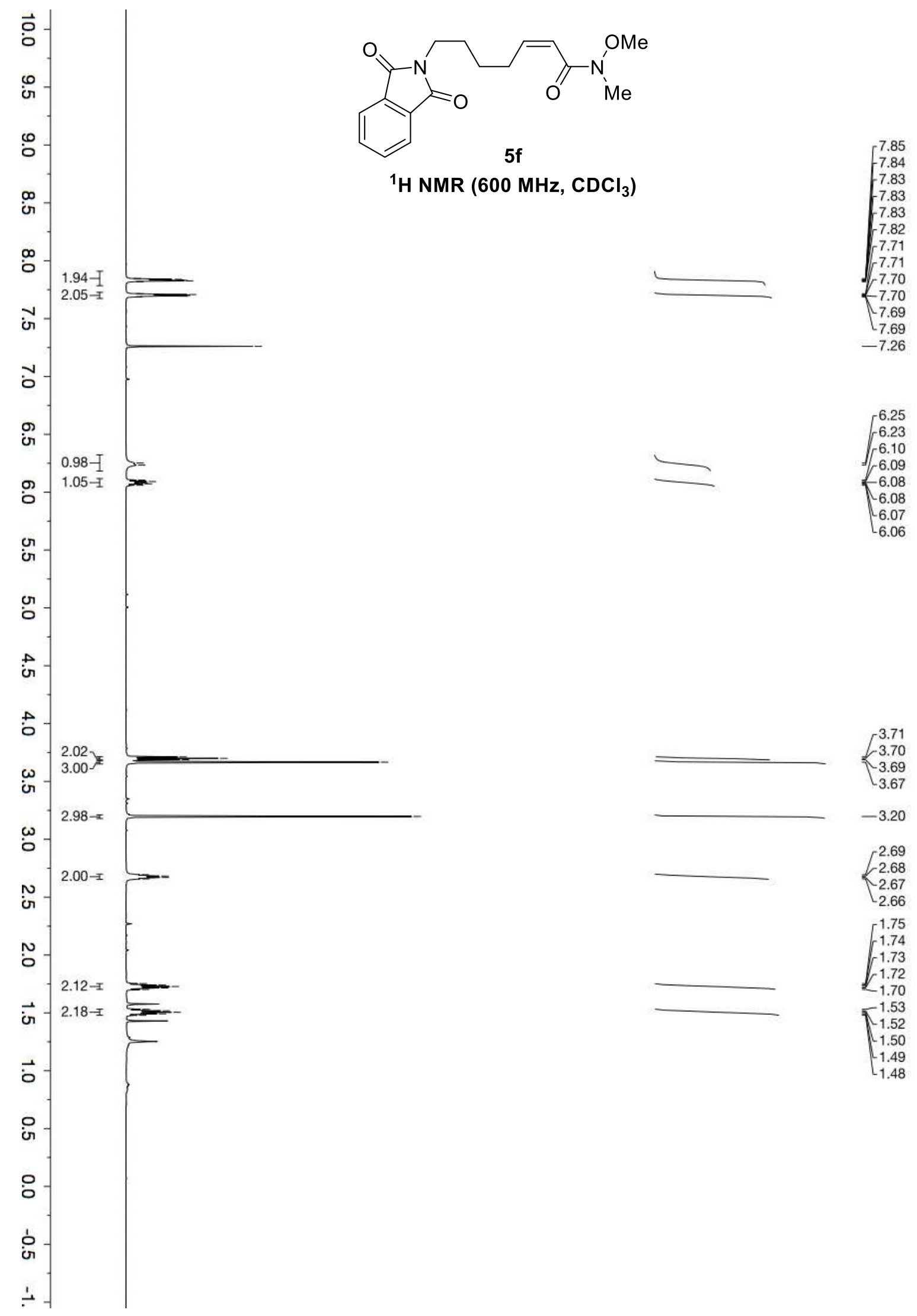




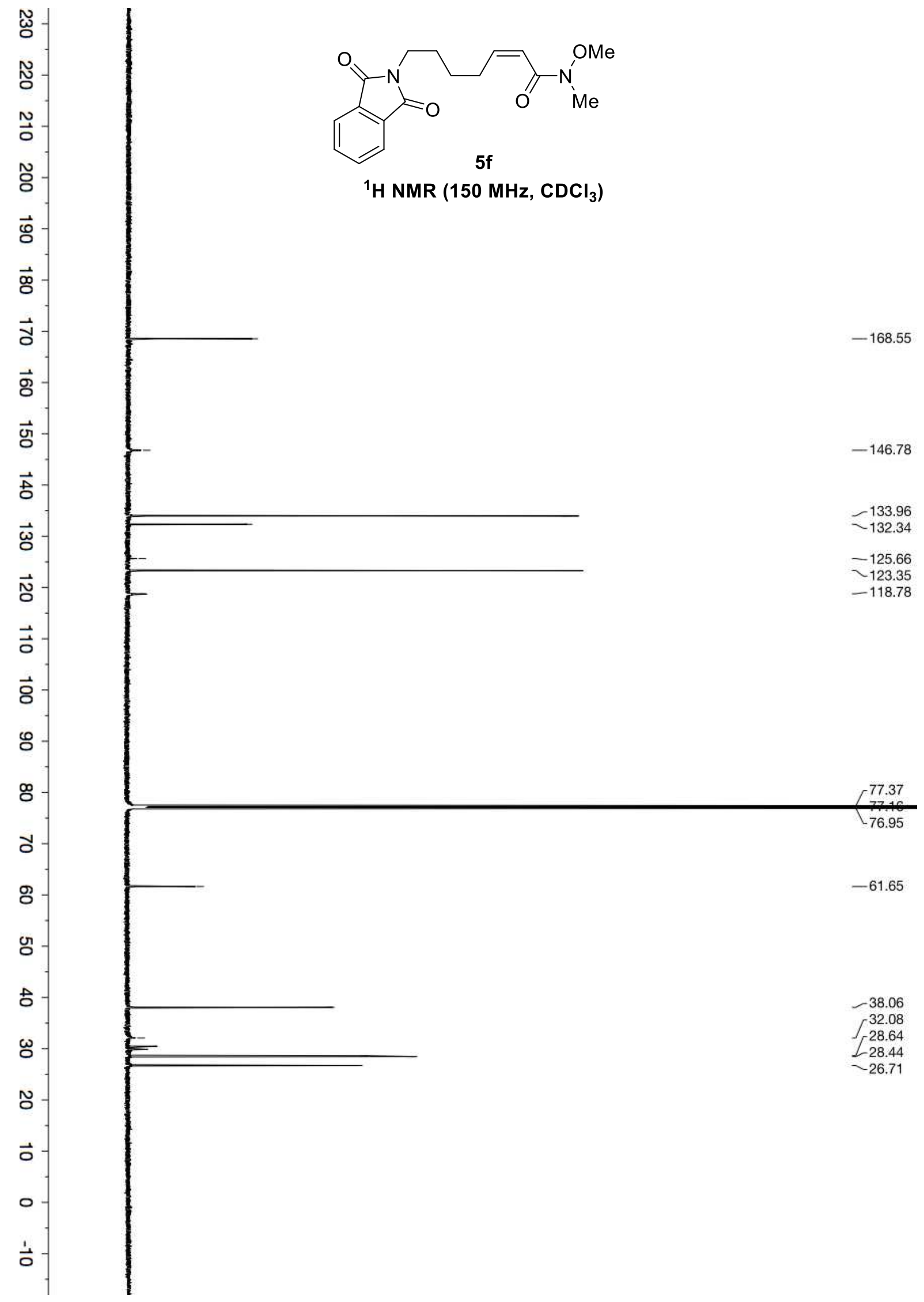




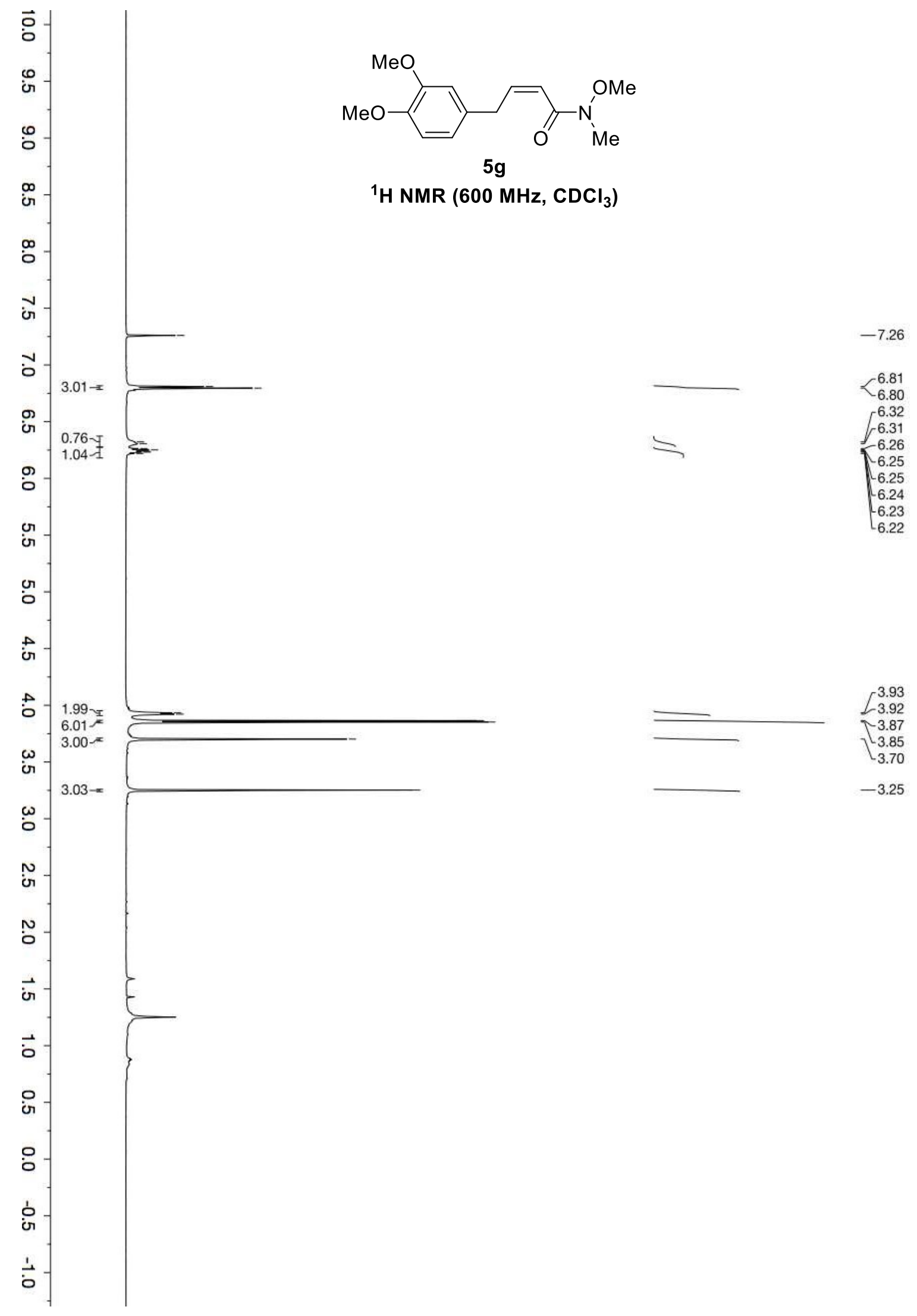




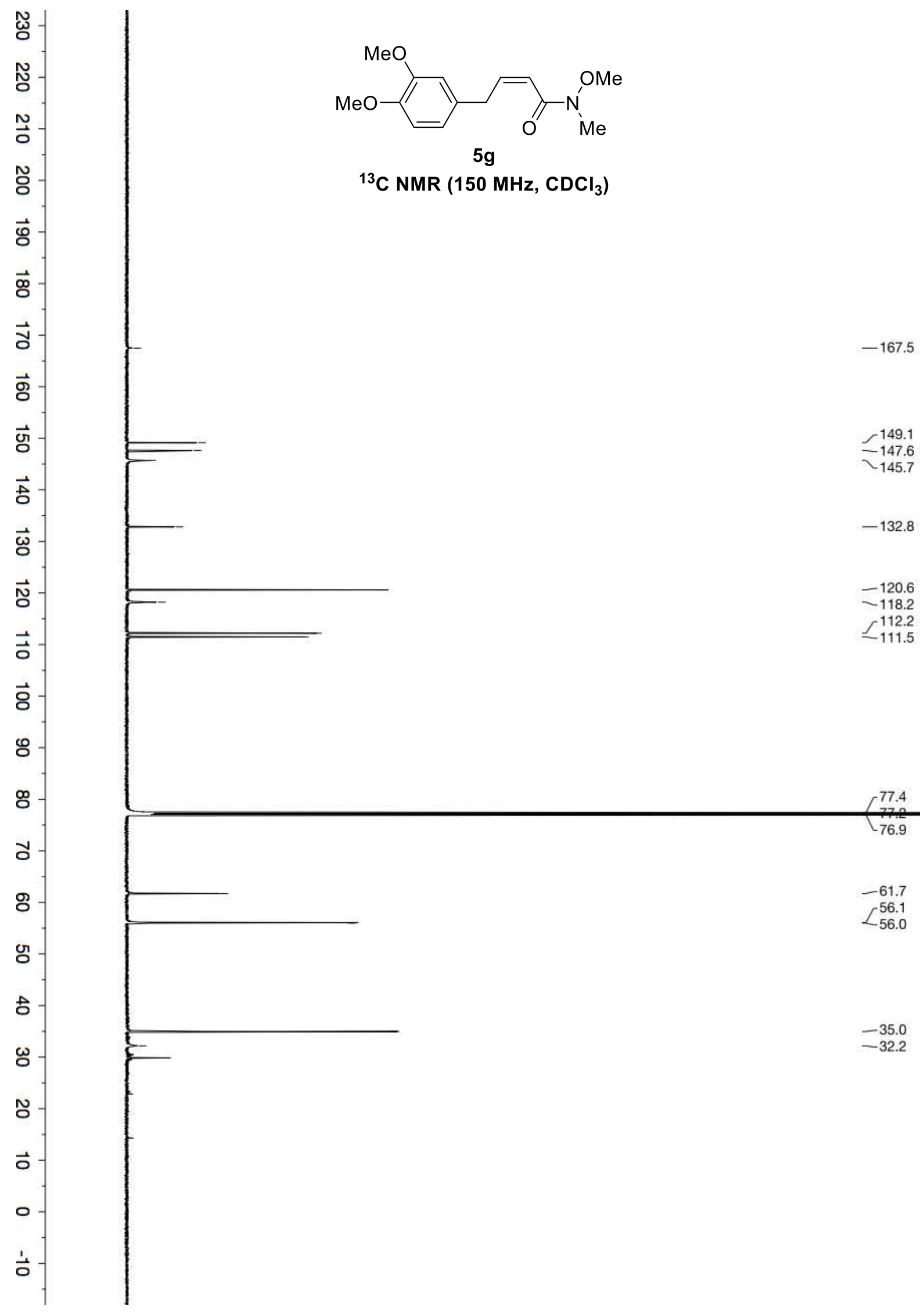




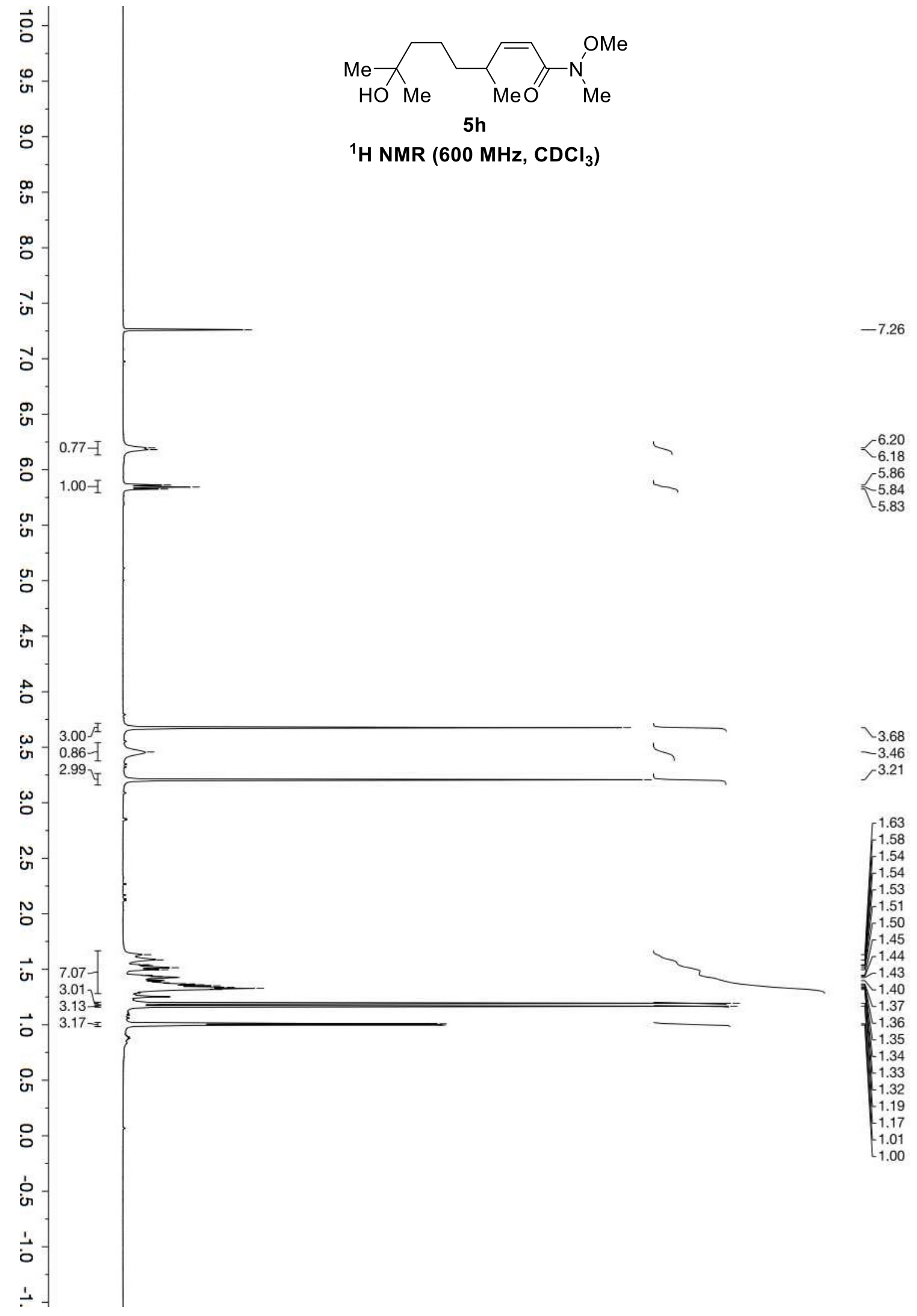




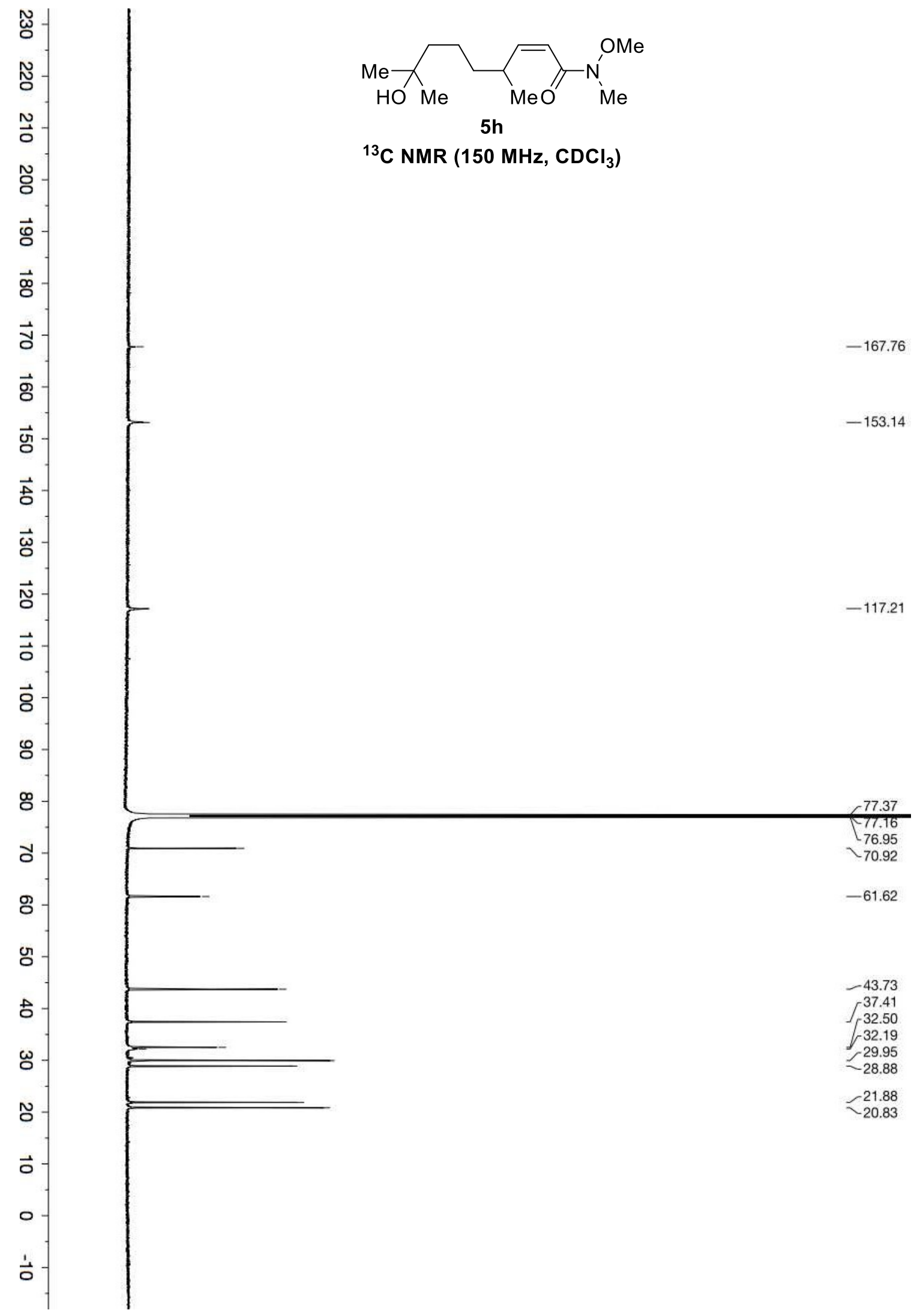




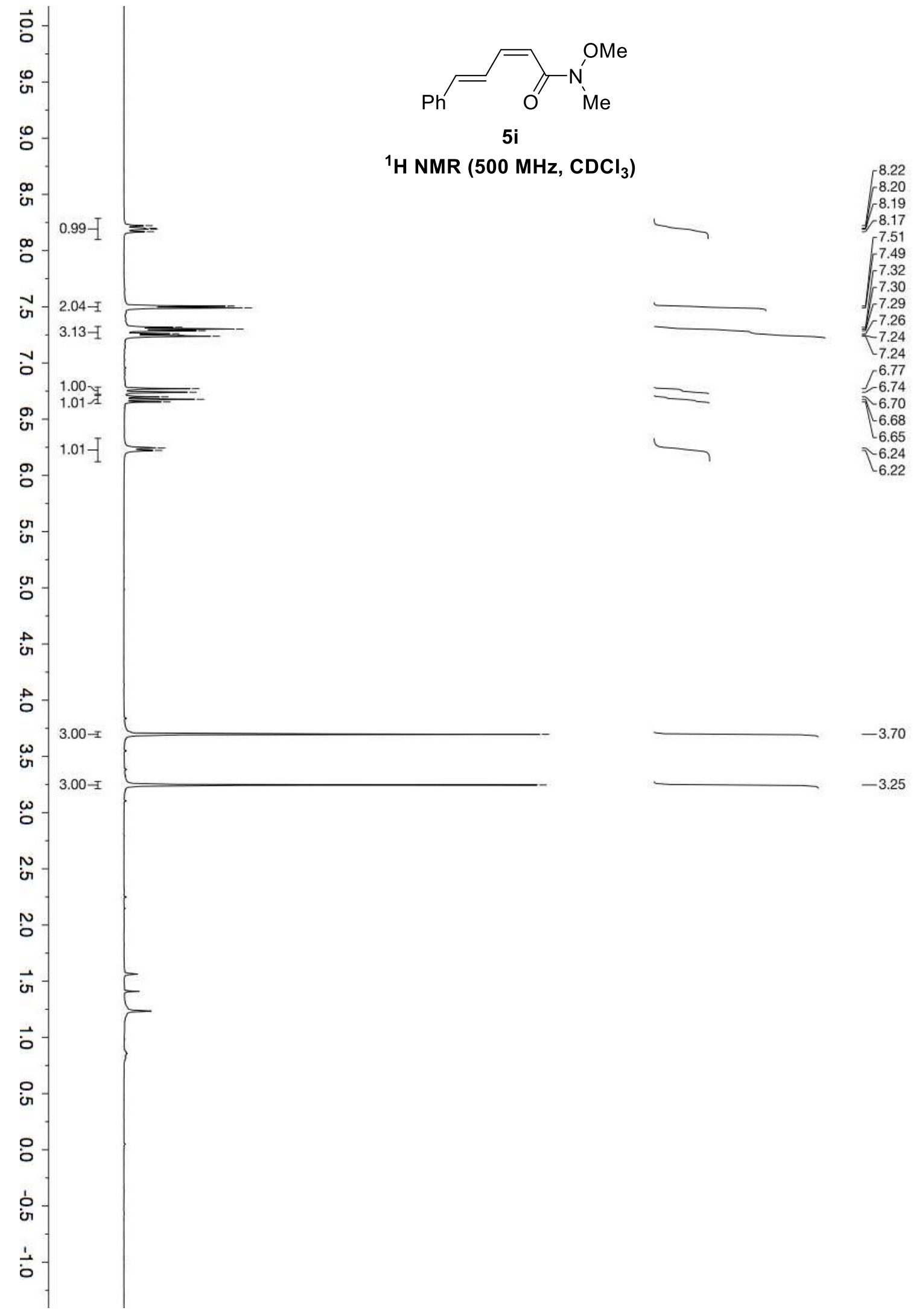




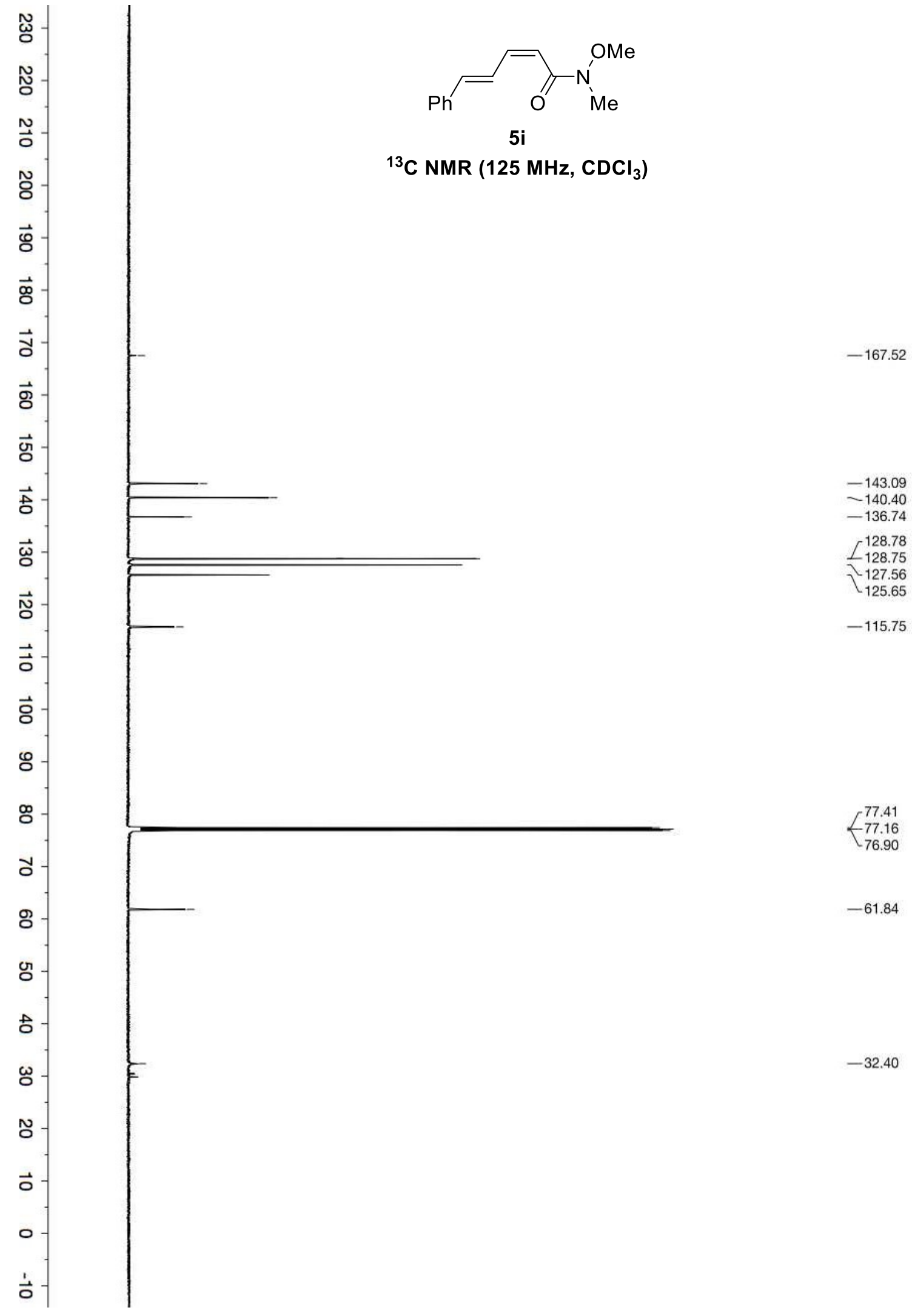



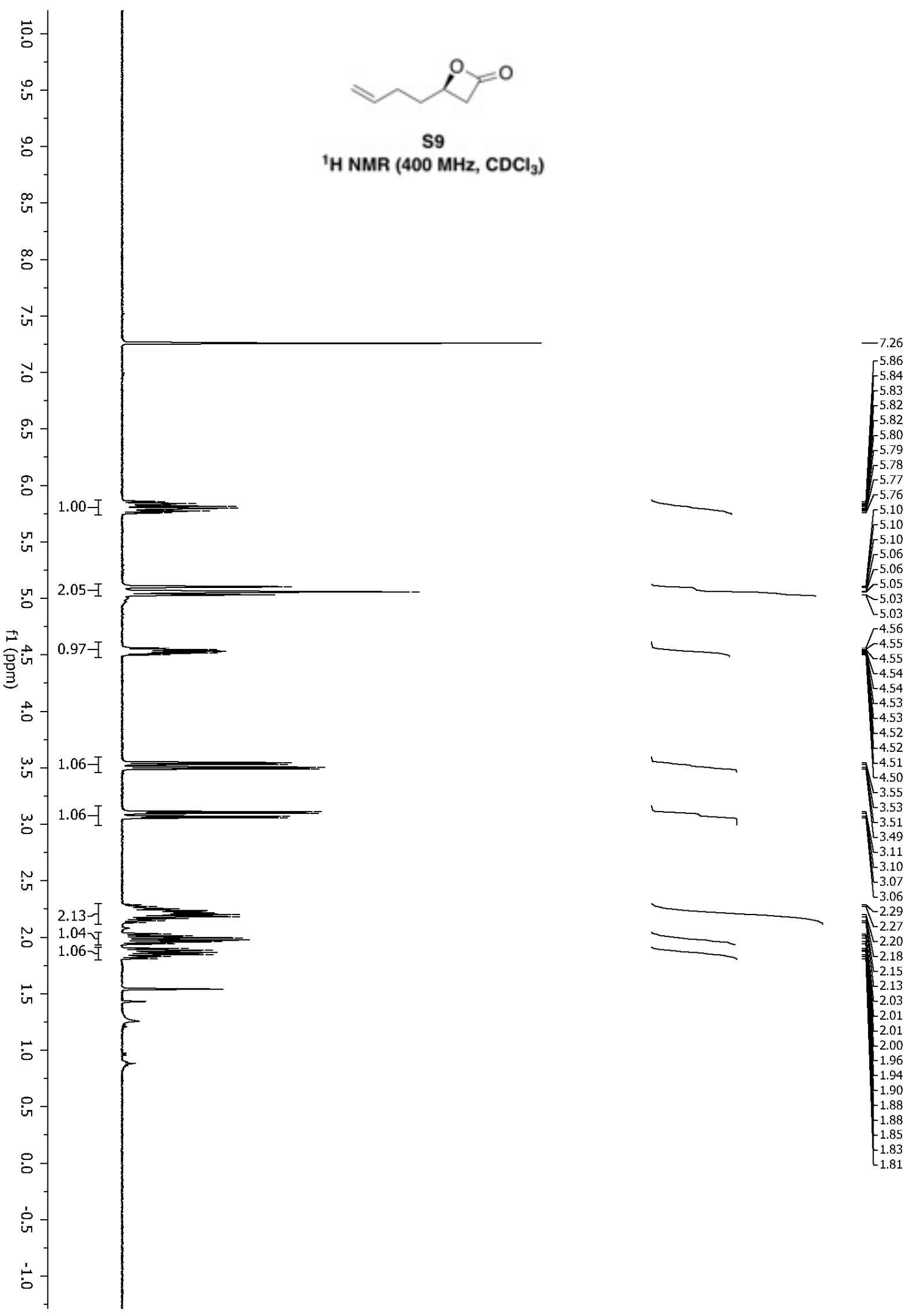


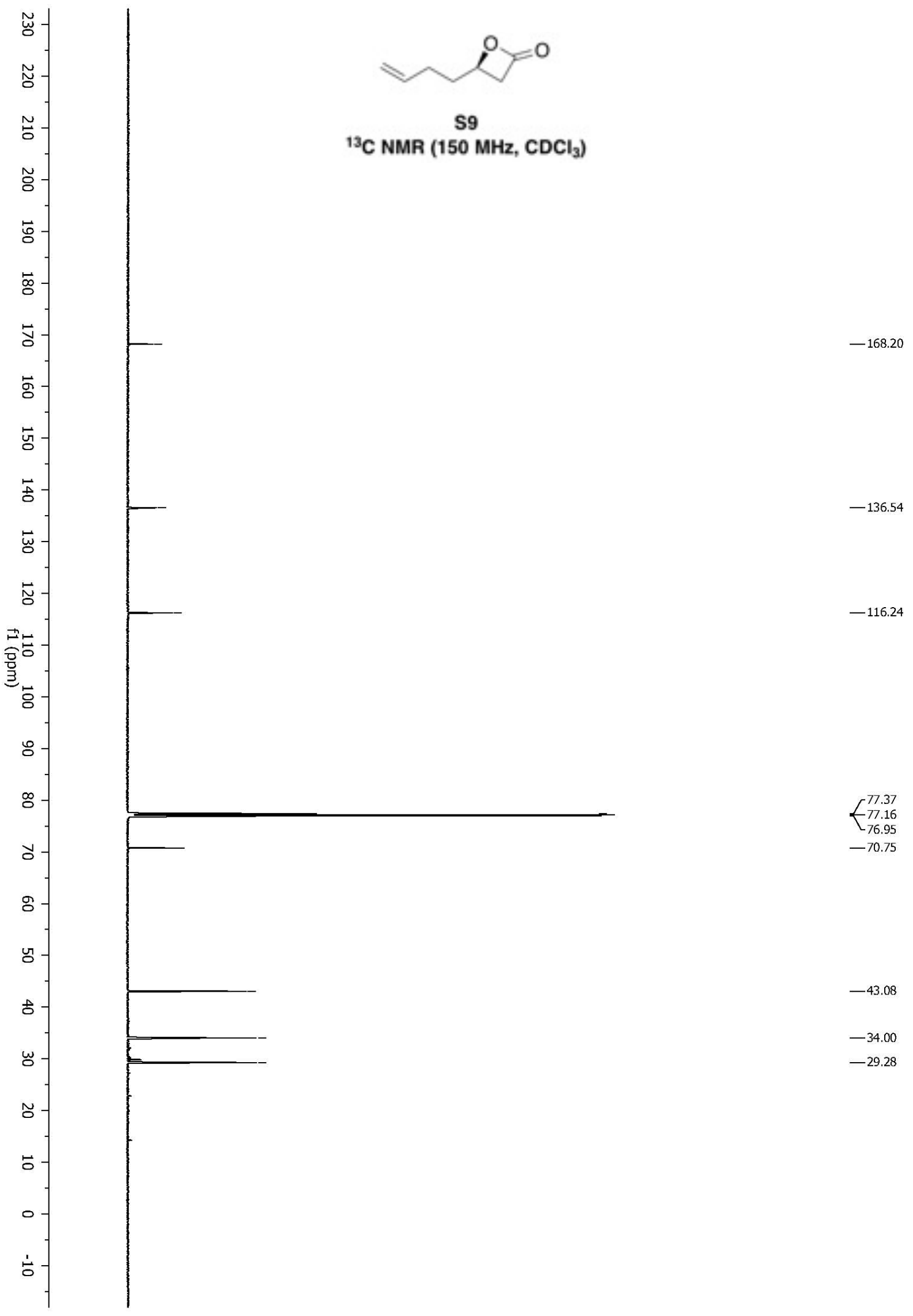




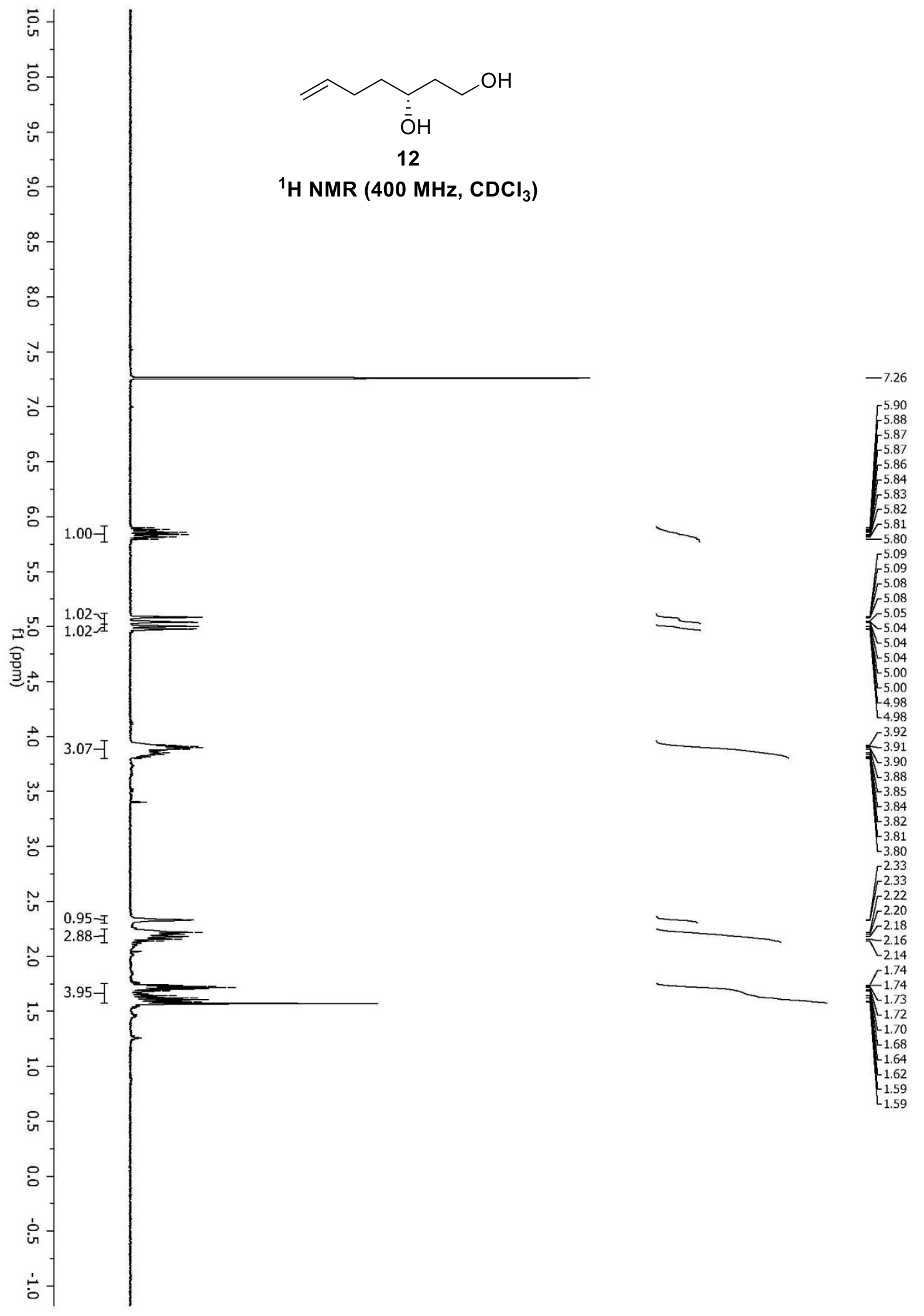




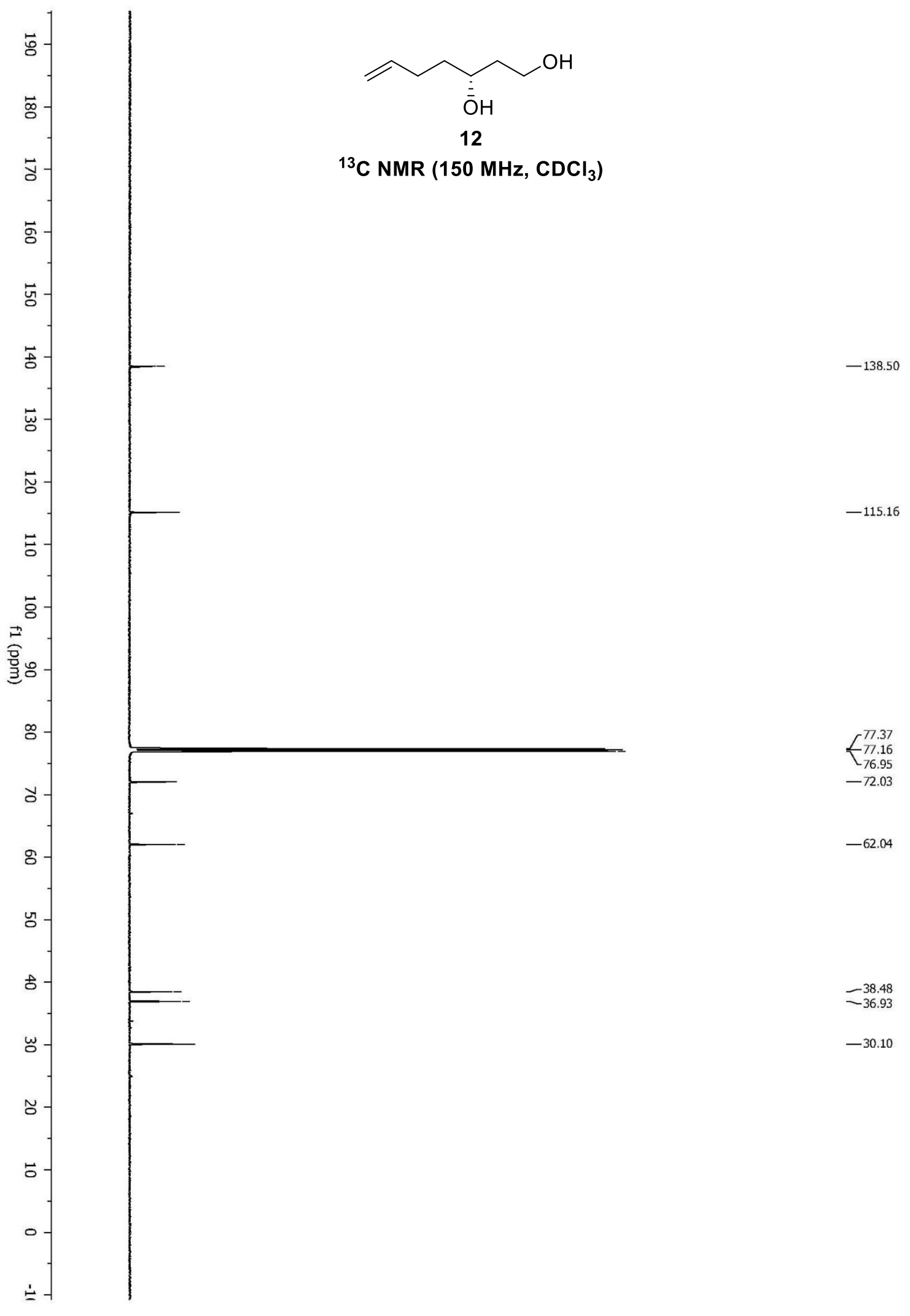




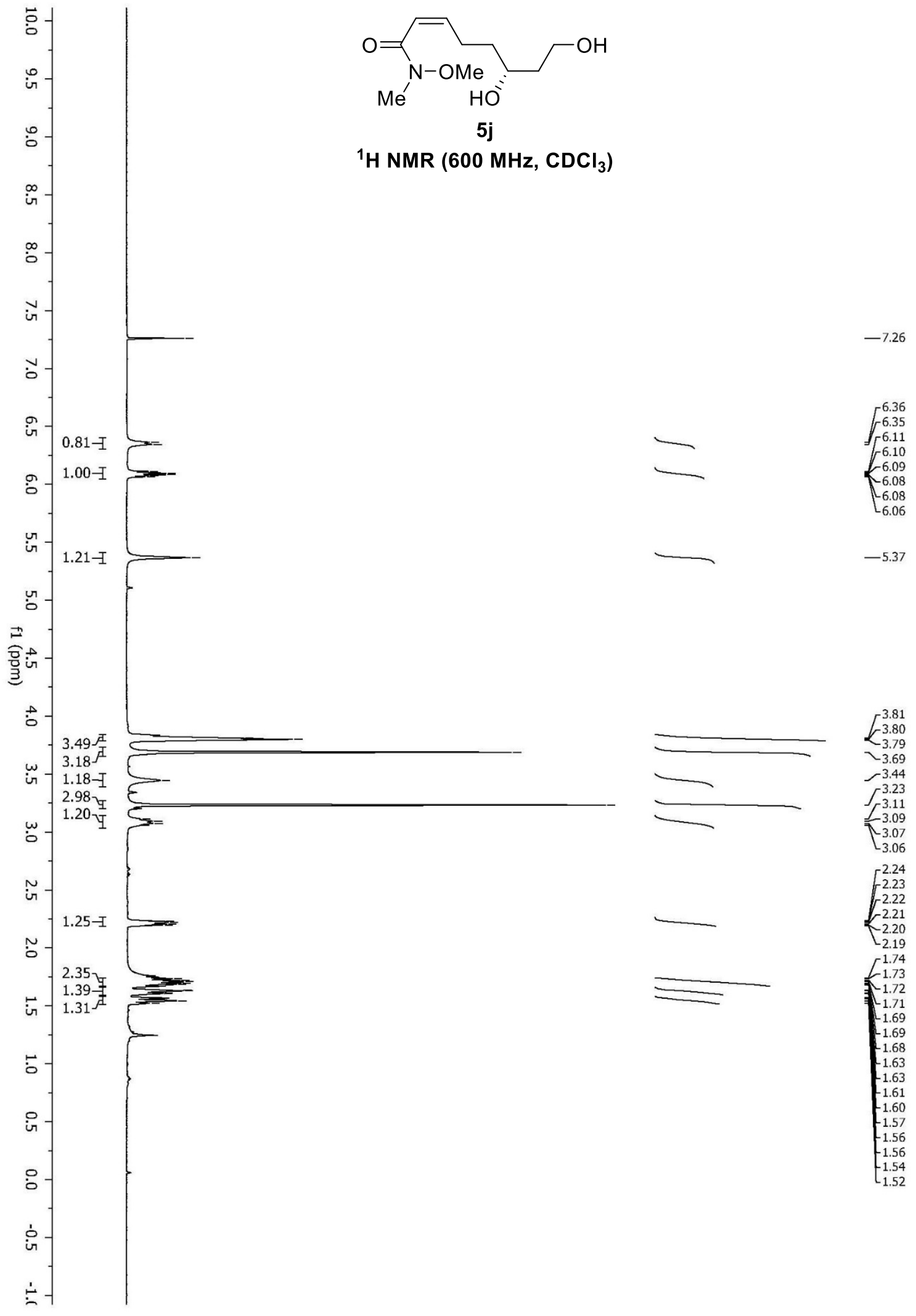




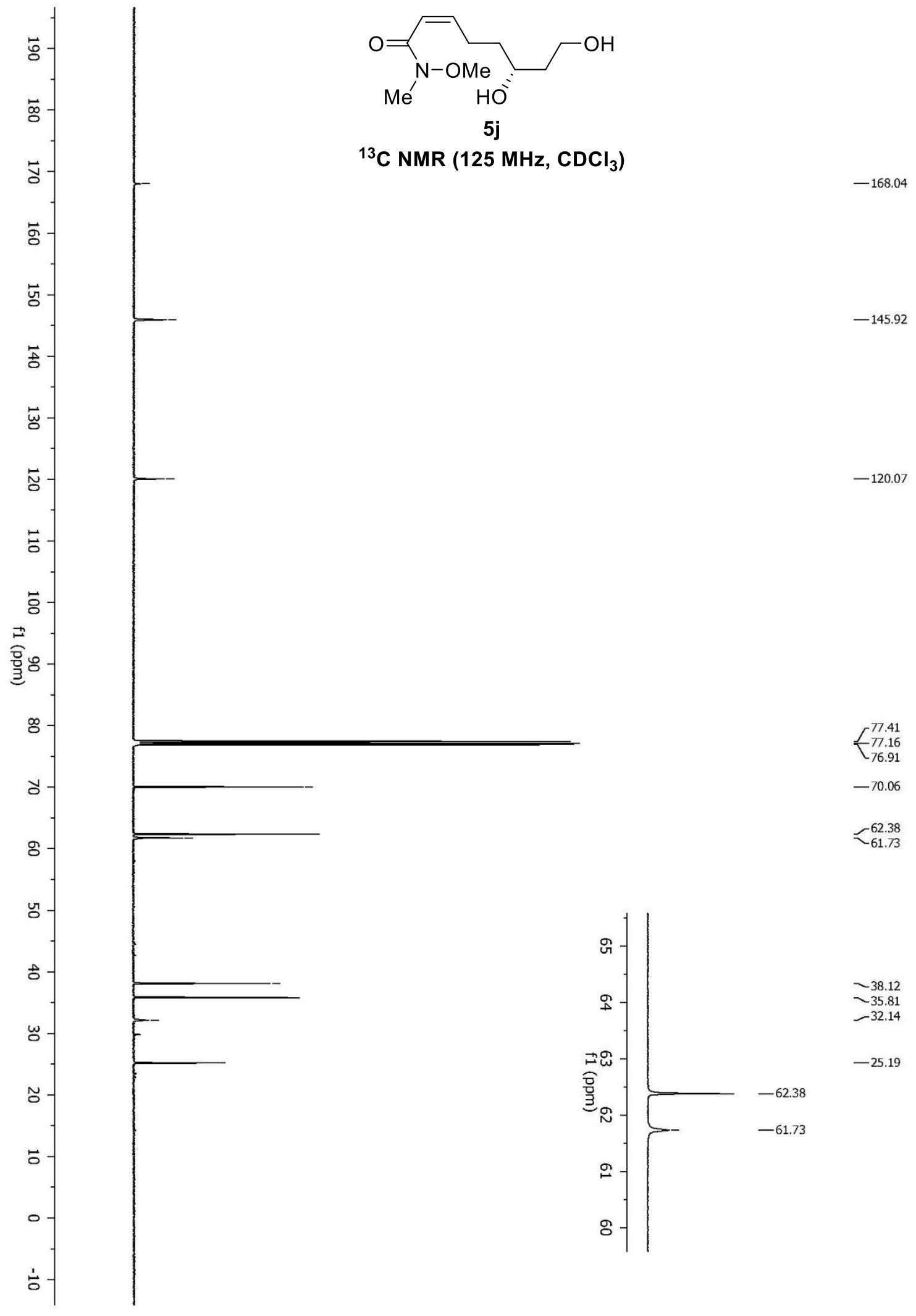




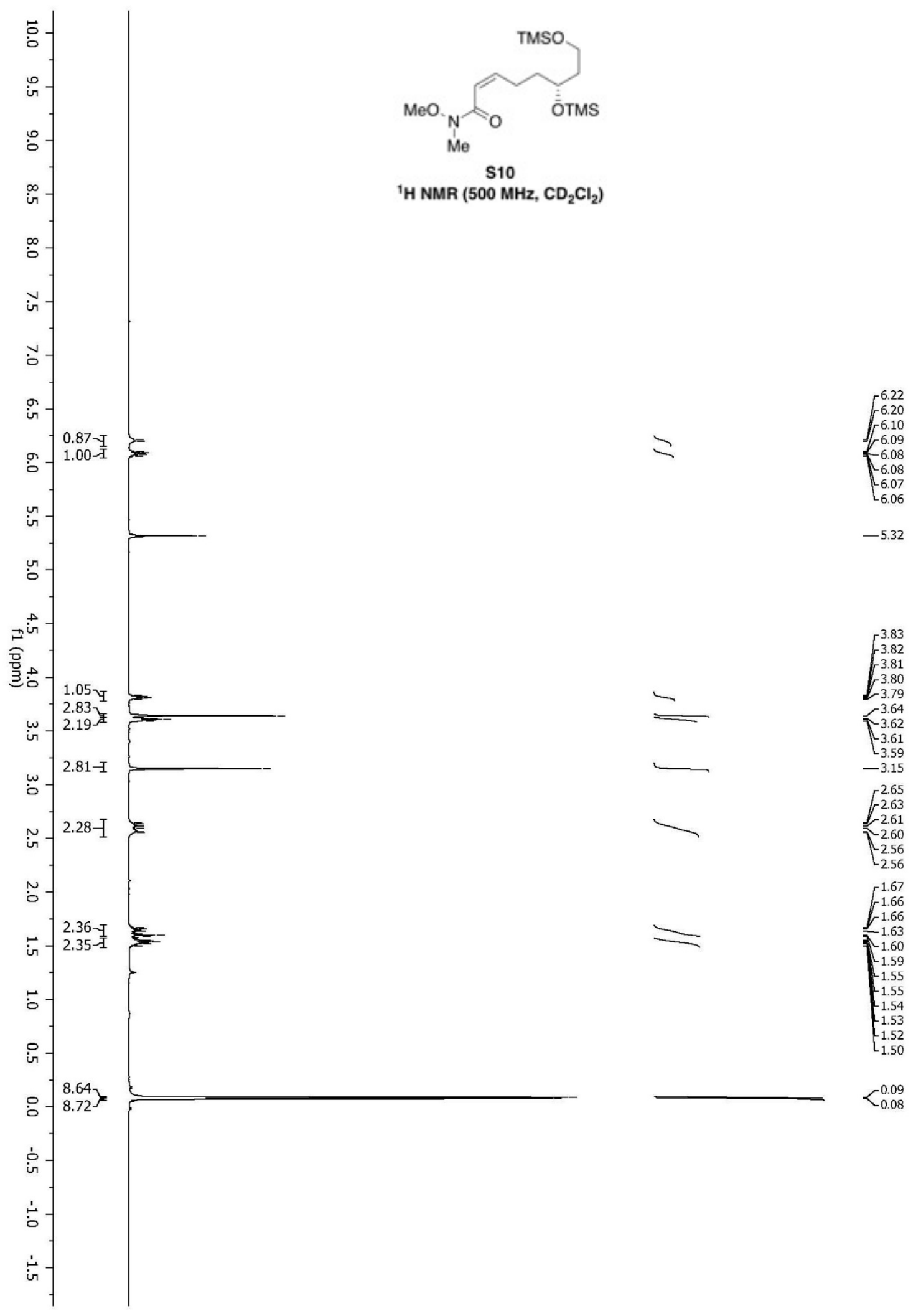




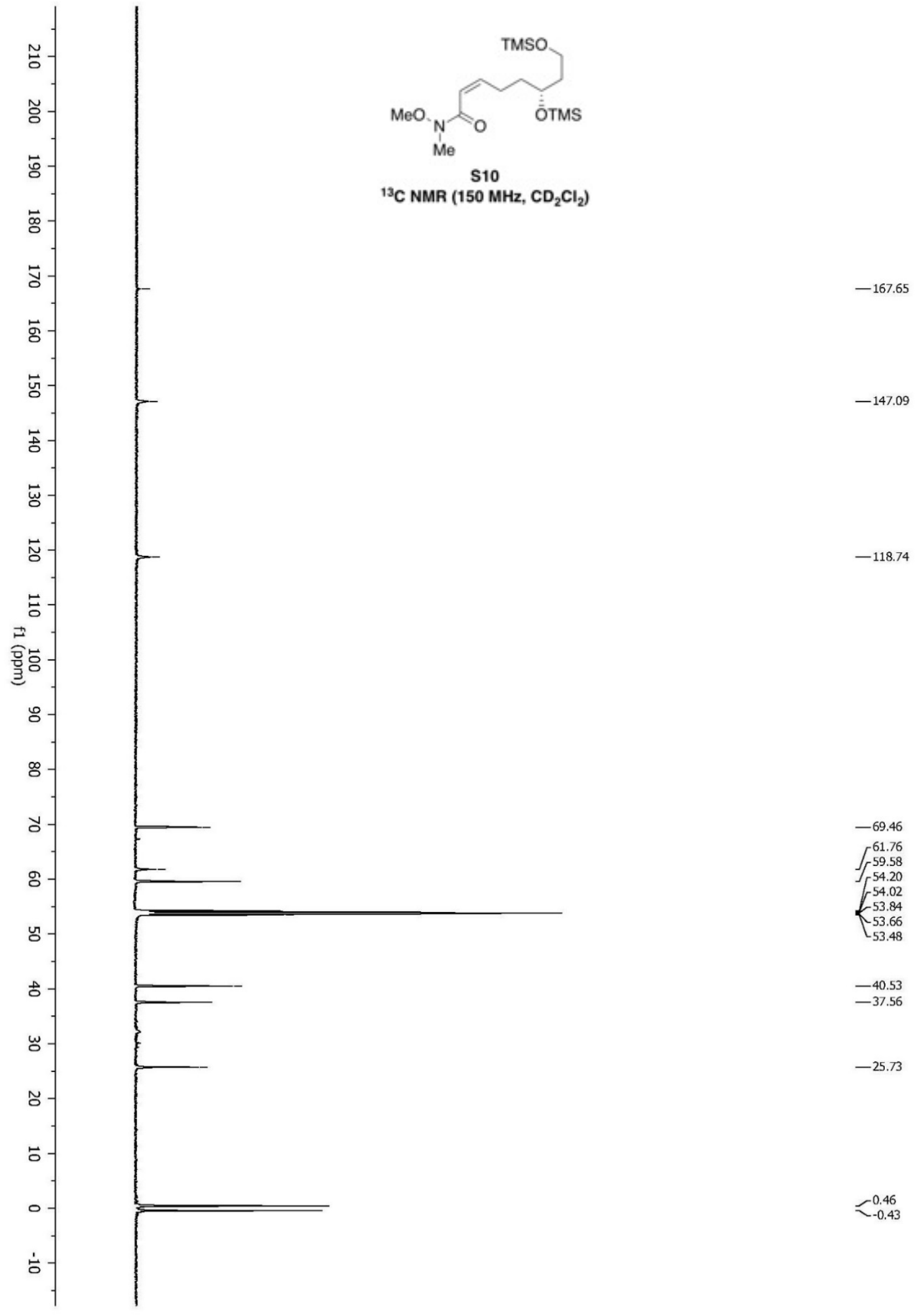




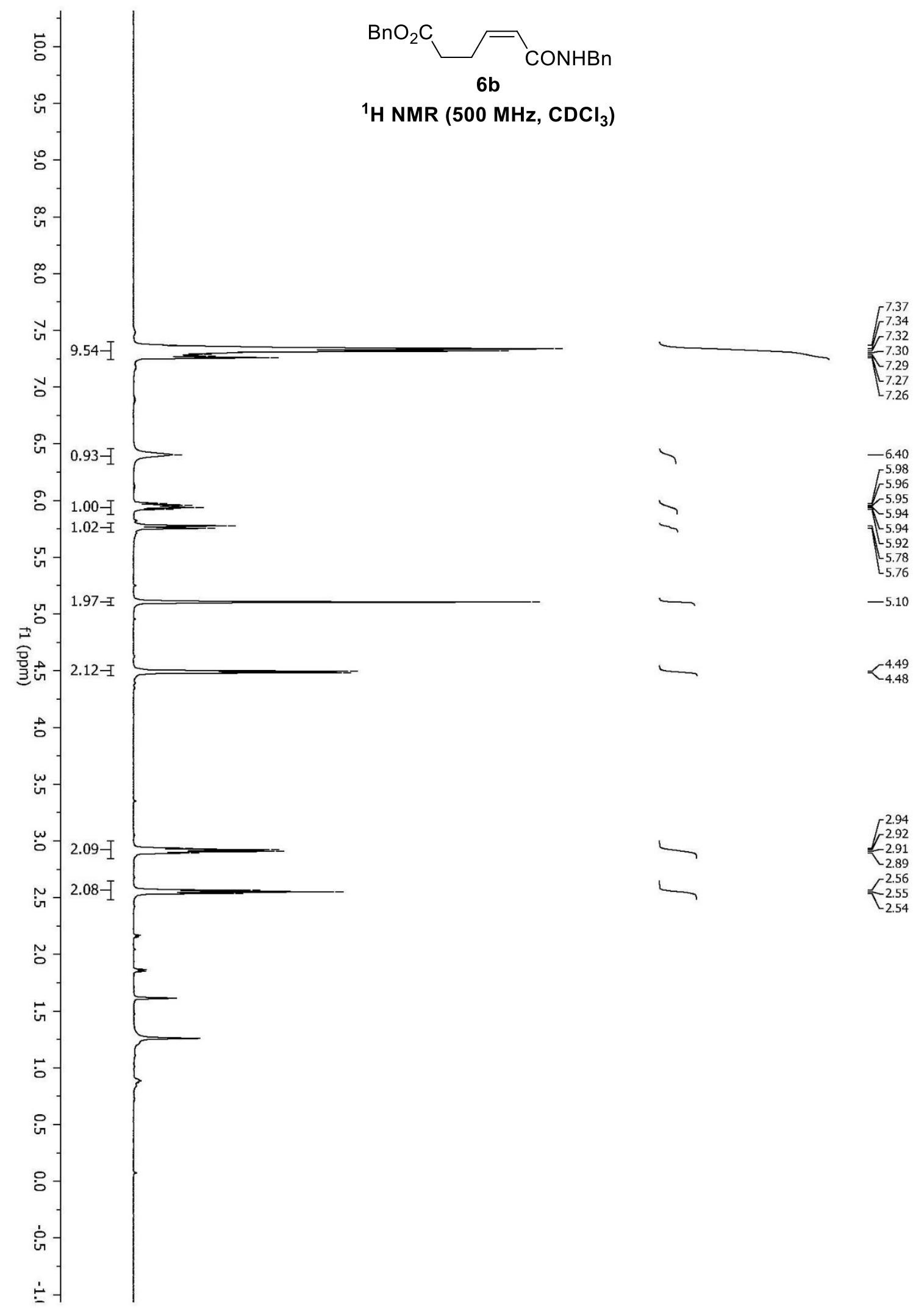




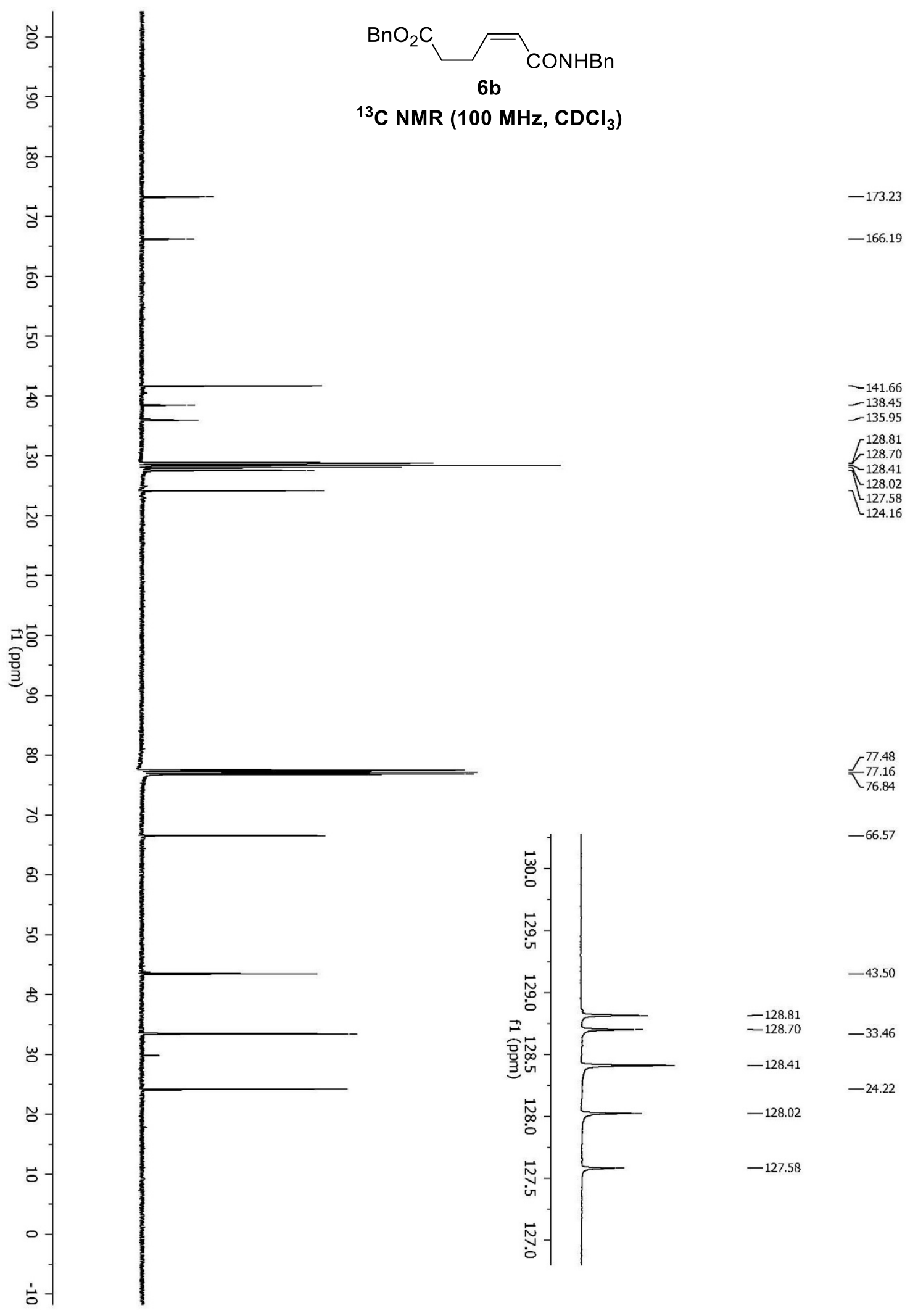




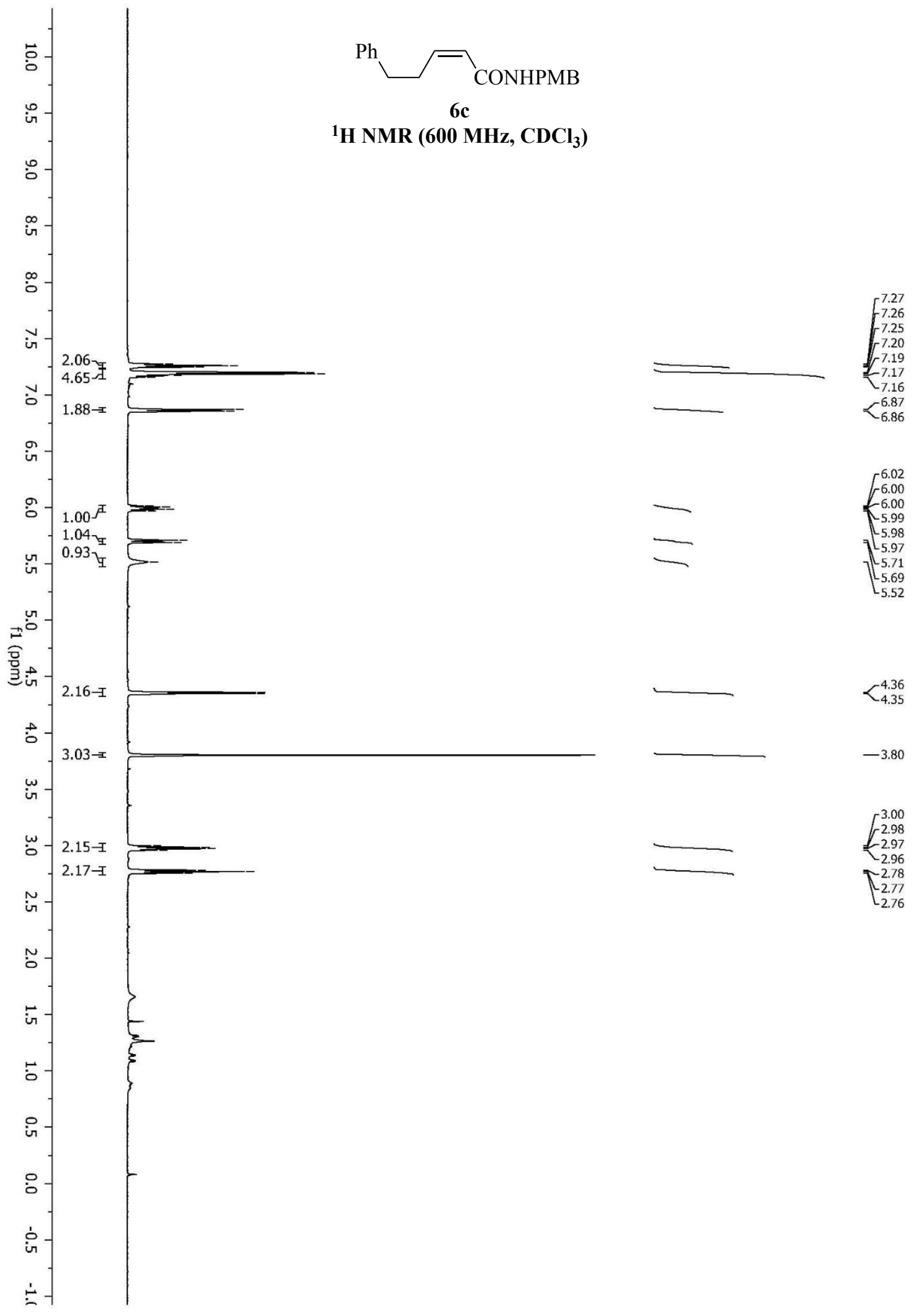




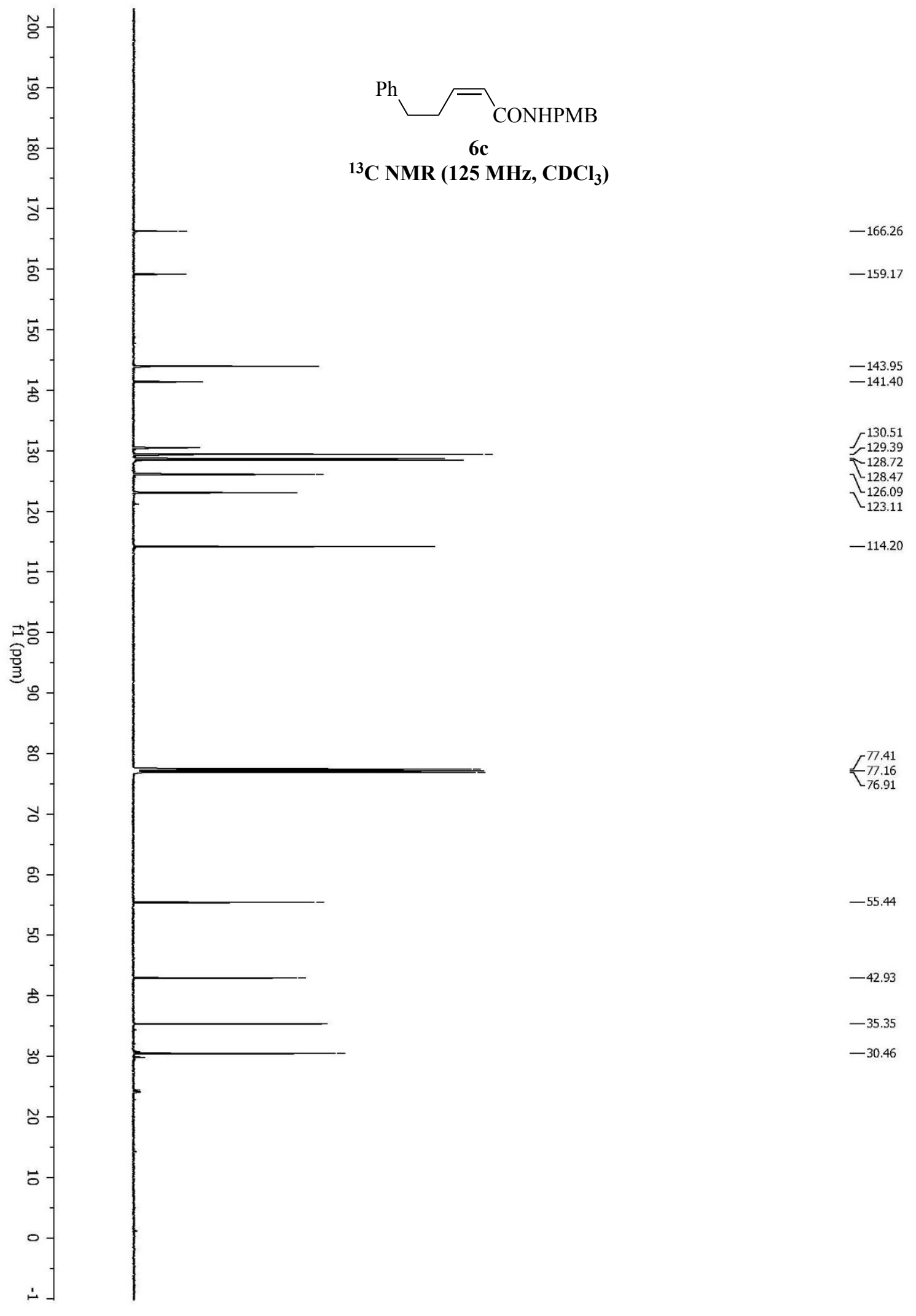




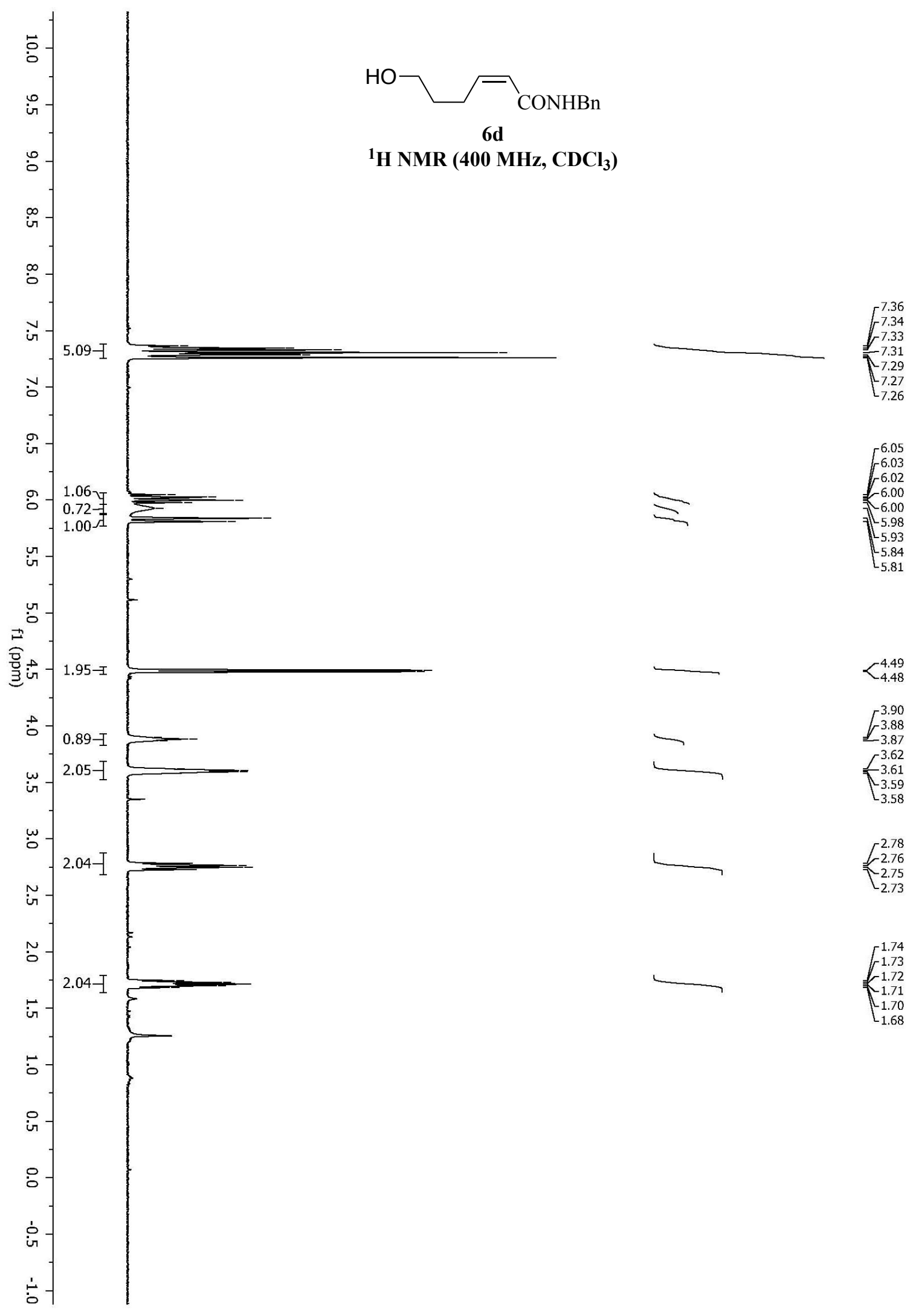




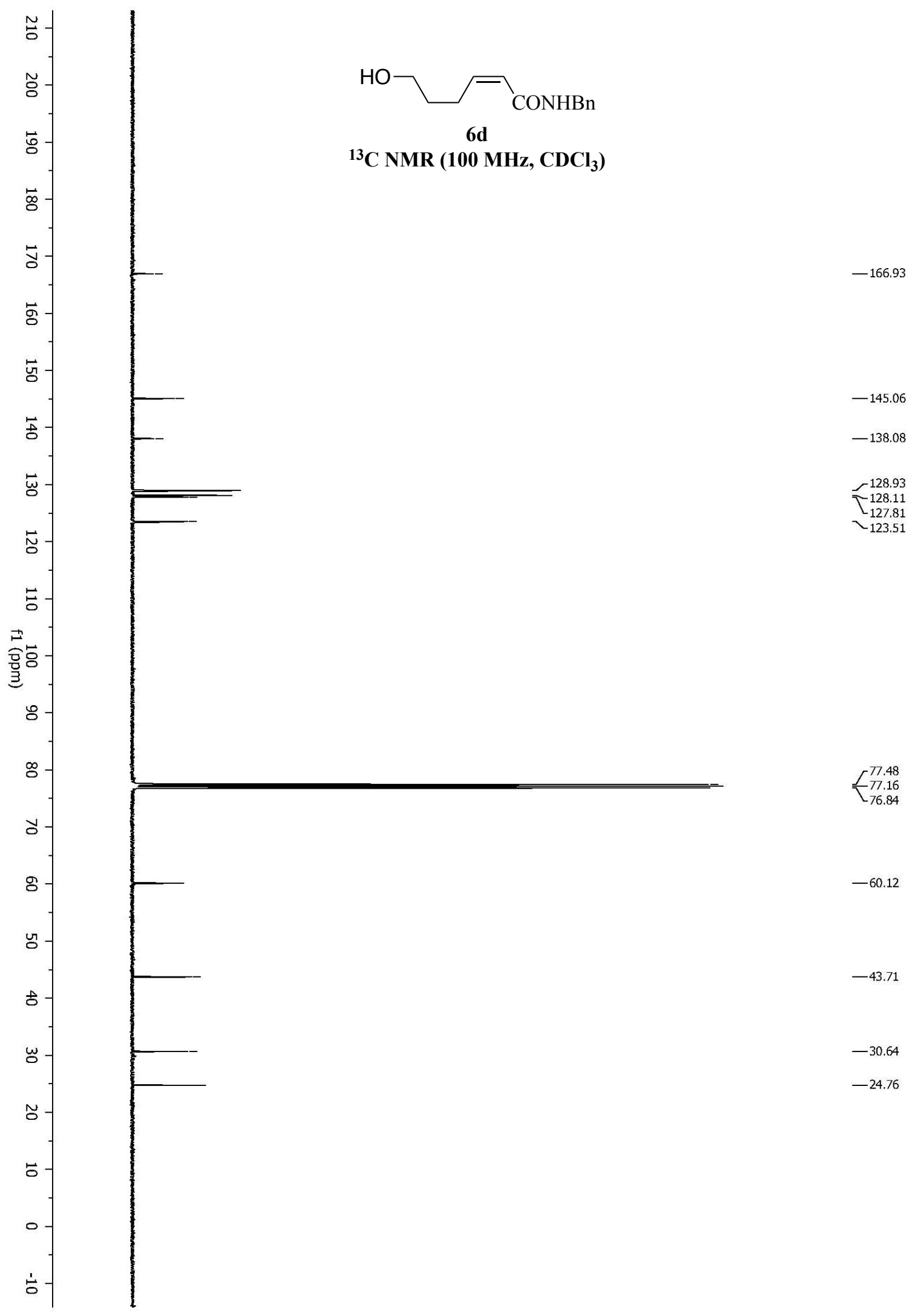




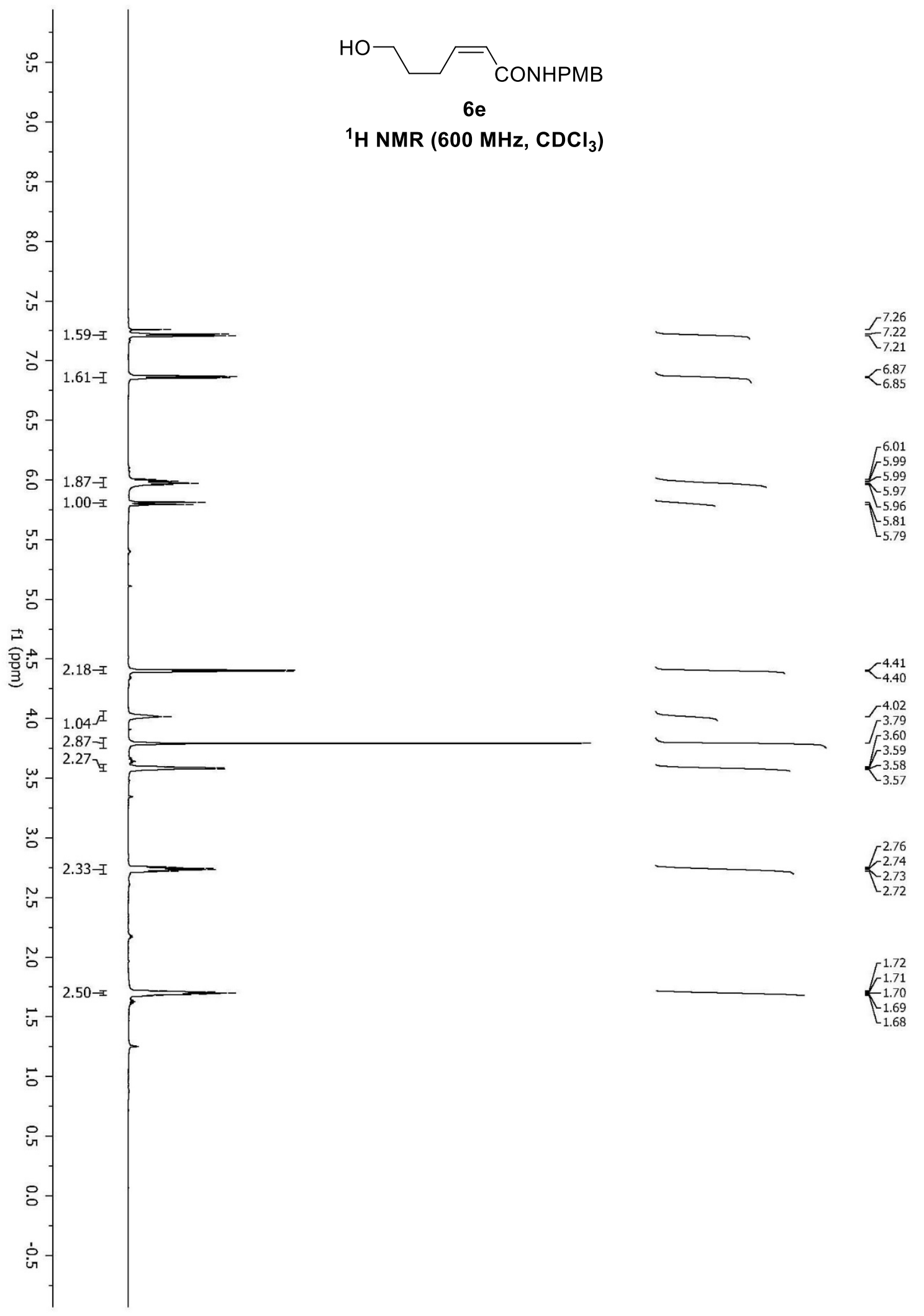




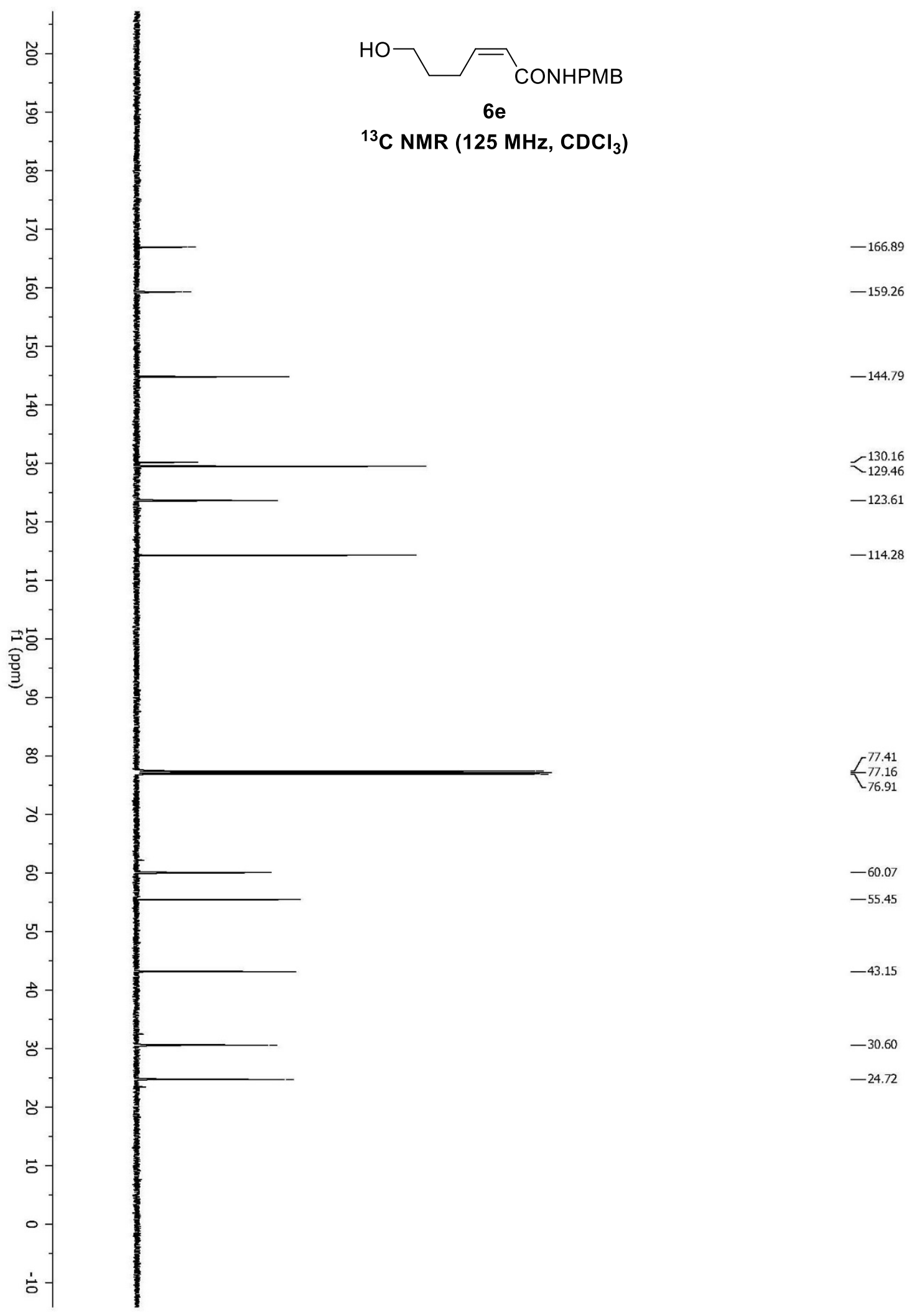




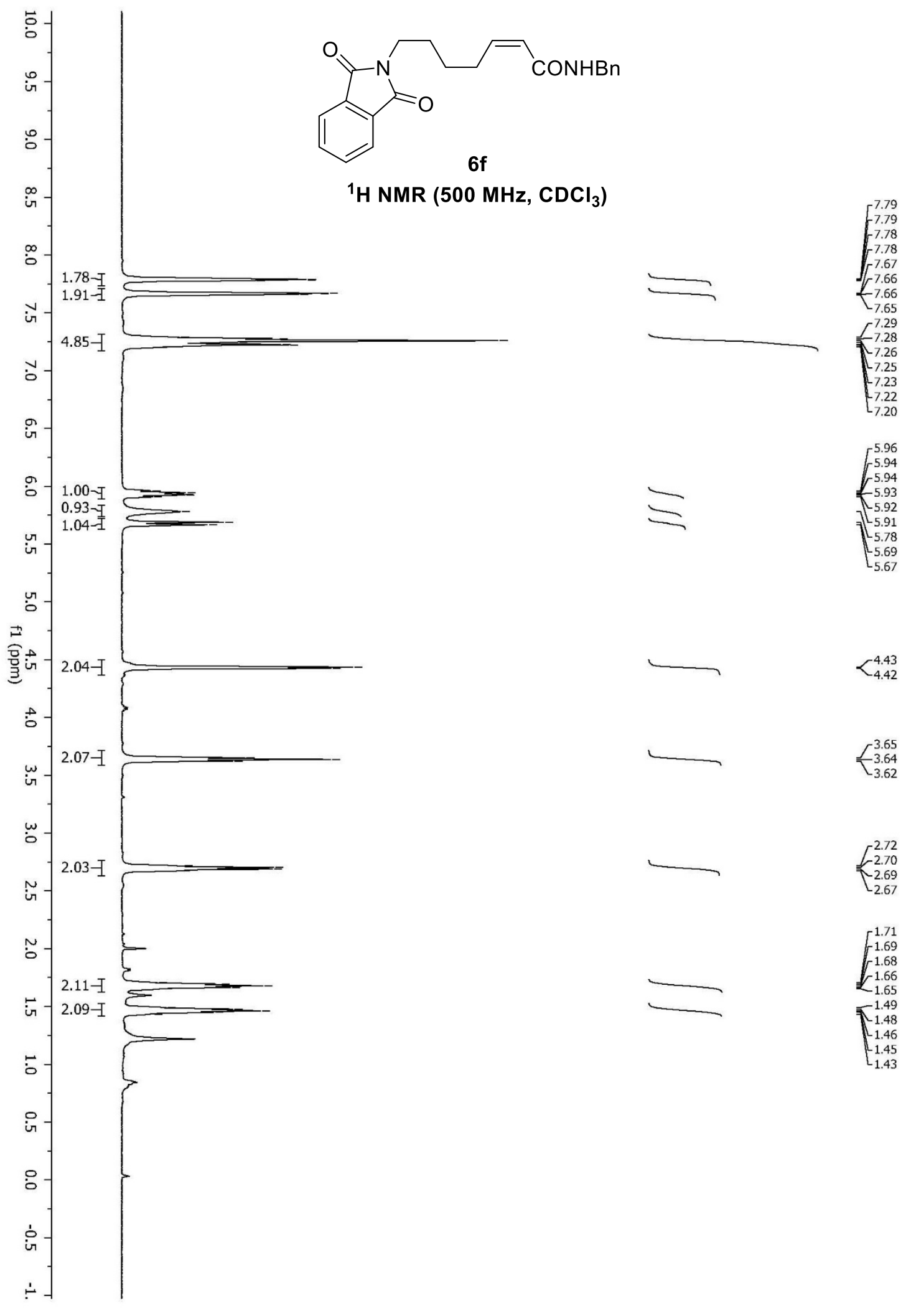




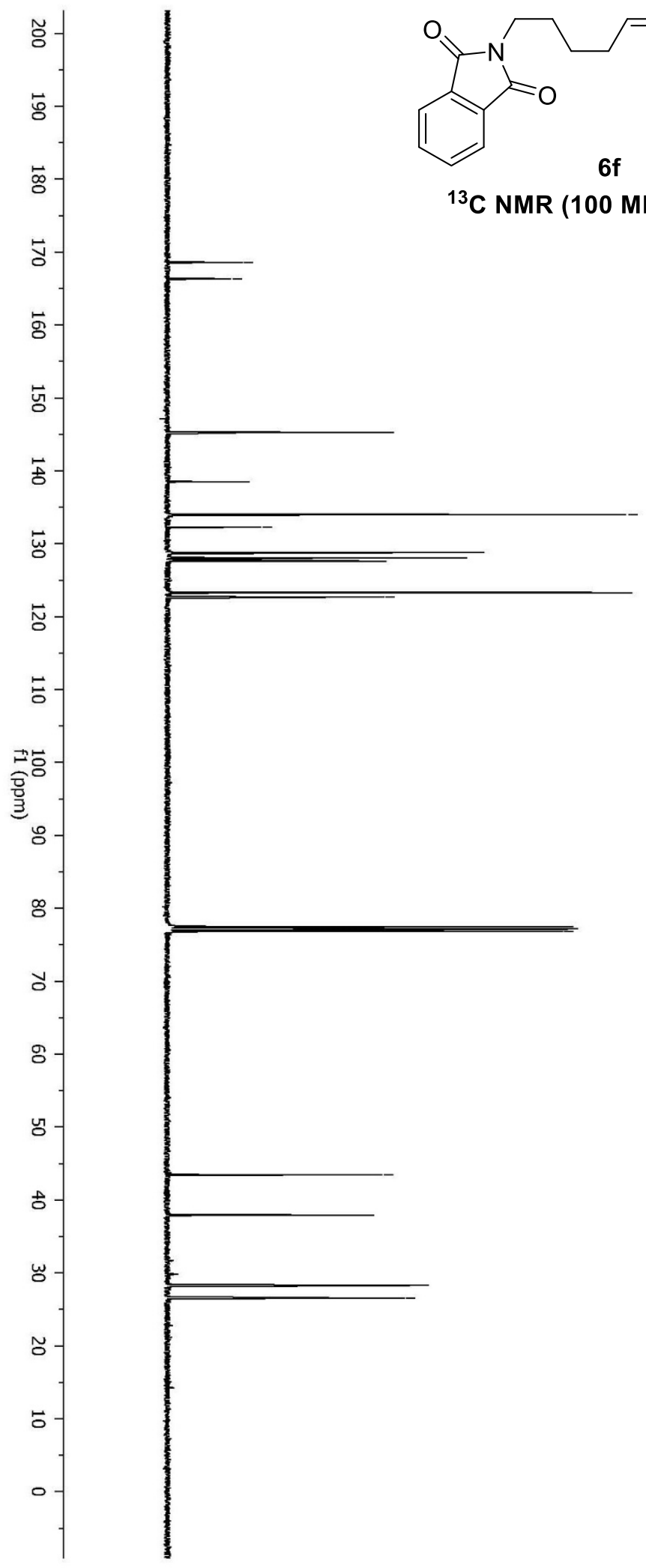<smiles>CC(C)(C)OC(=O)NOCc1ccccc1</smiles>

-168.56
-16633

$-145.22$

$-138.47$

133.99

132.30

128.82

128.06

127.62

$\chi_{122.66}^{123.30}$
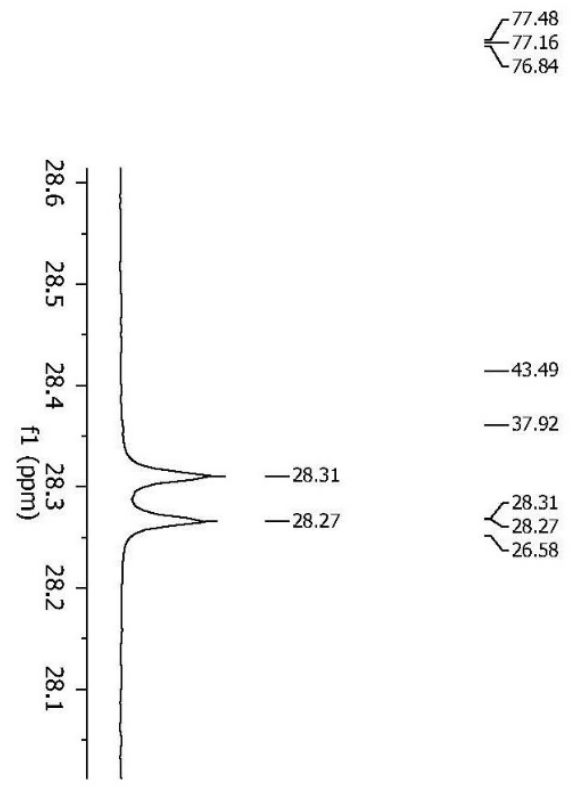

$-43.49$

$-37.92$

$\mathcal{L}_{28.27}^{28.31}$

ᄂ26.58 


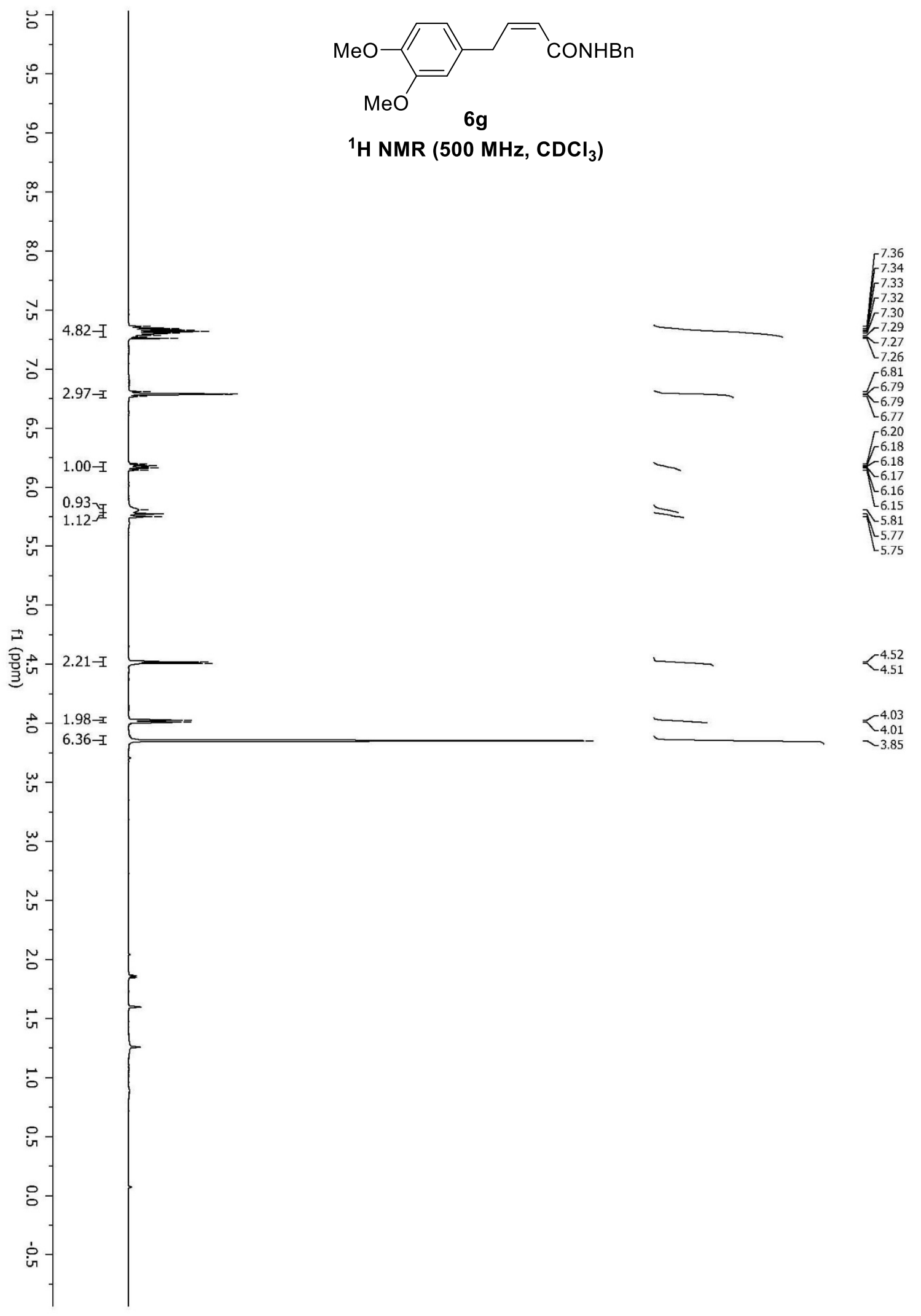




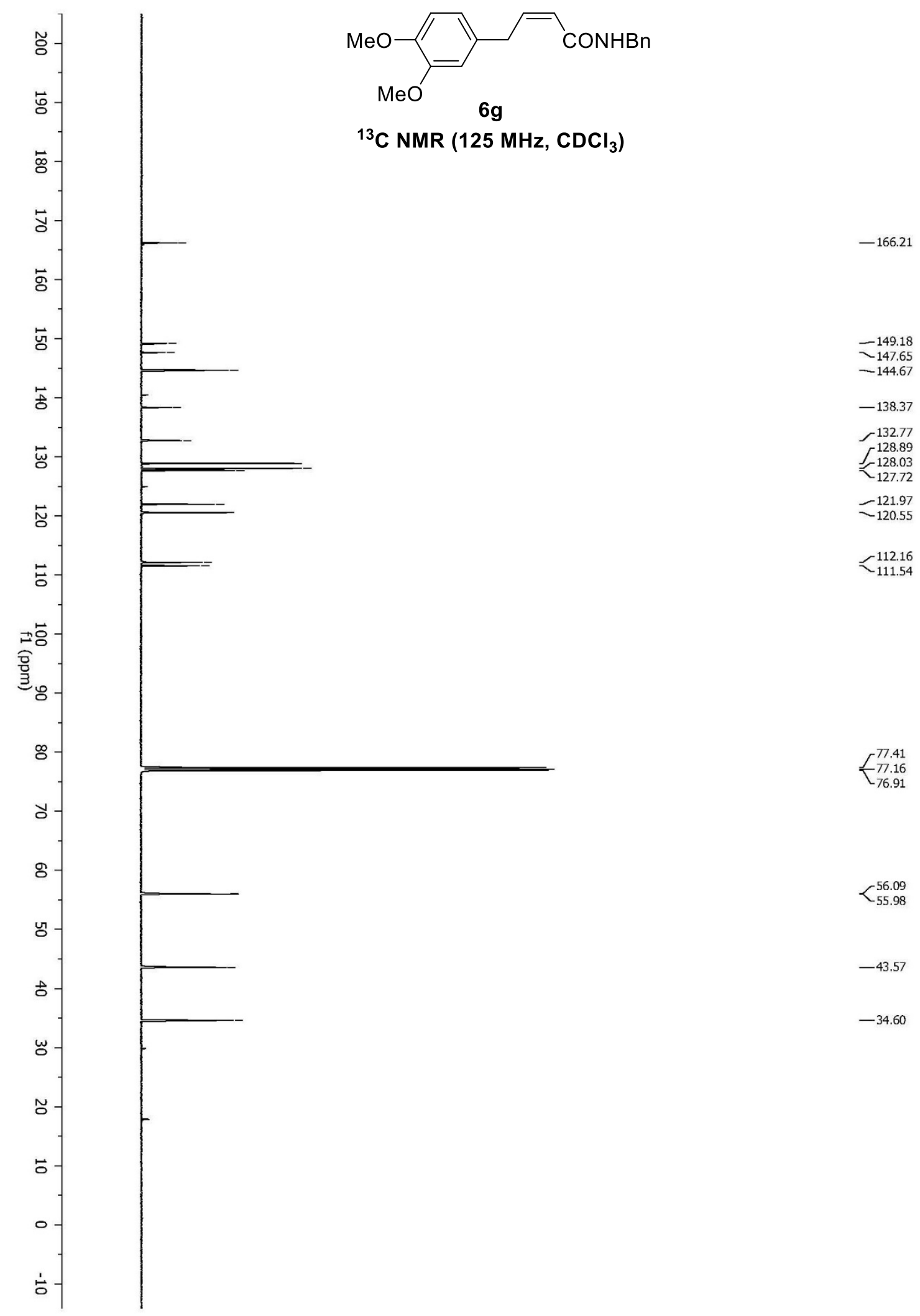




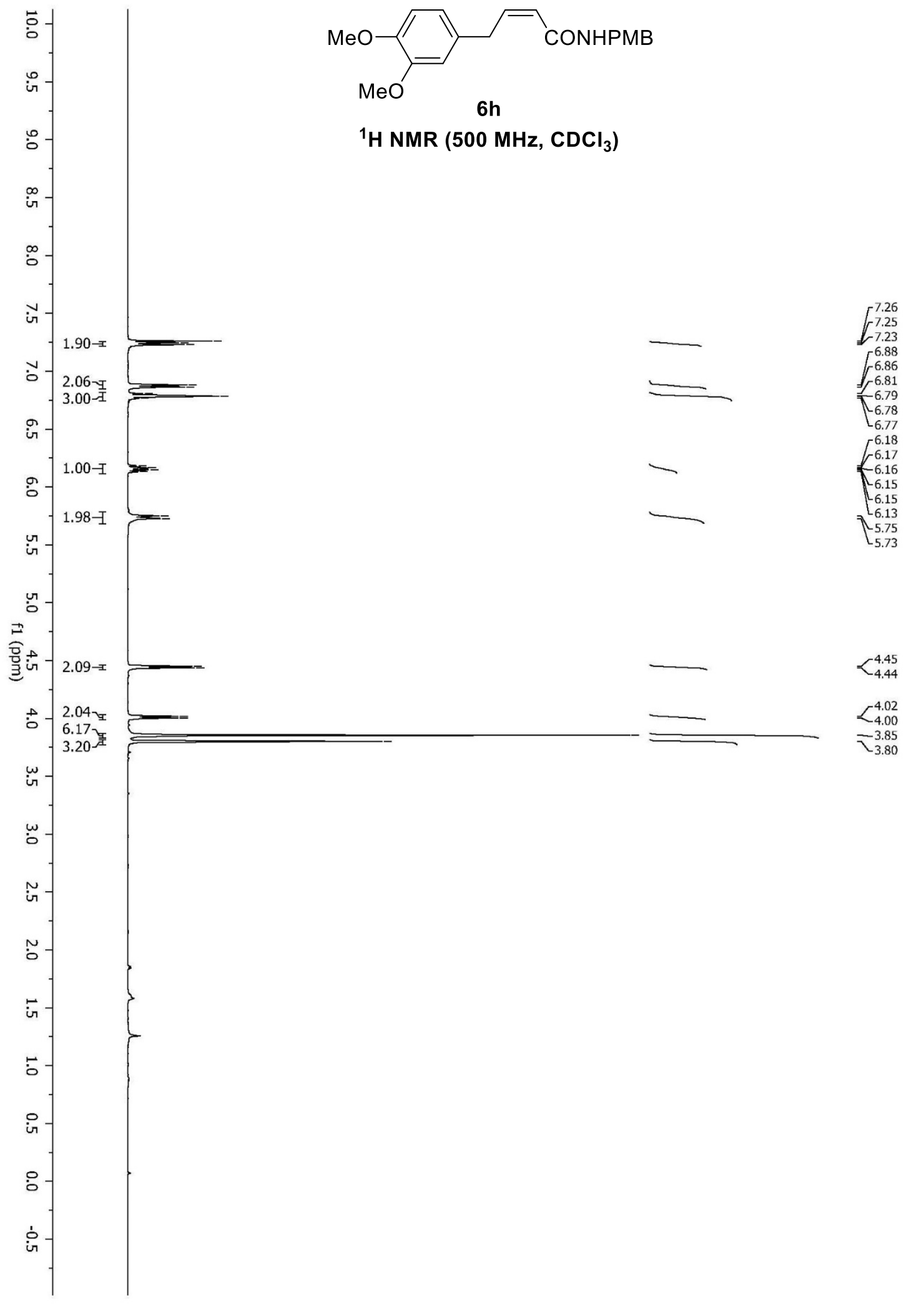




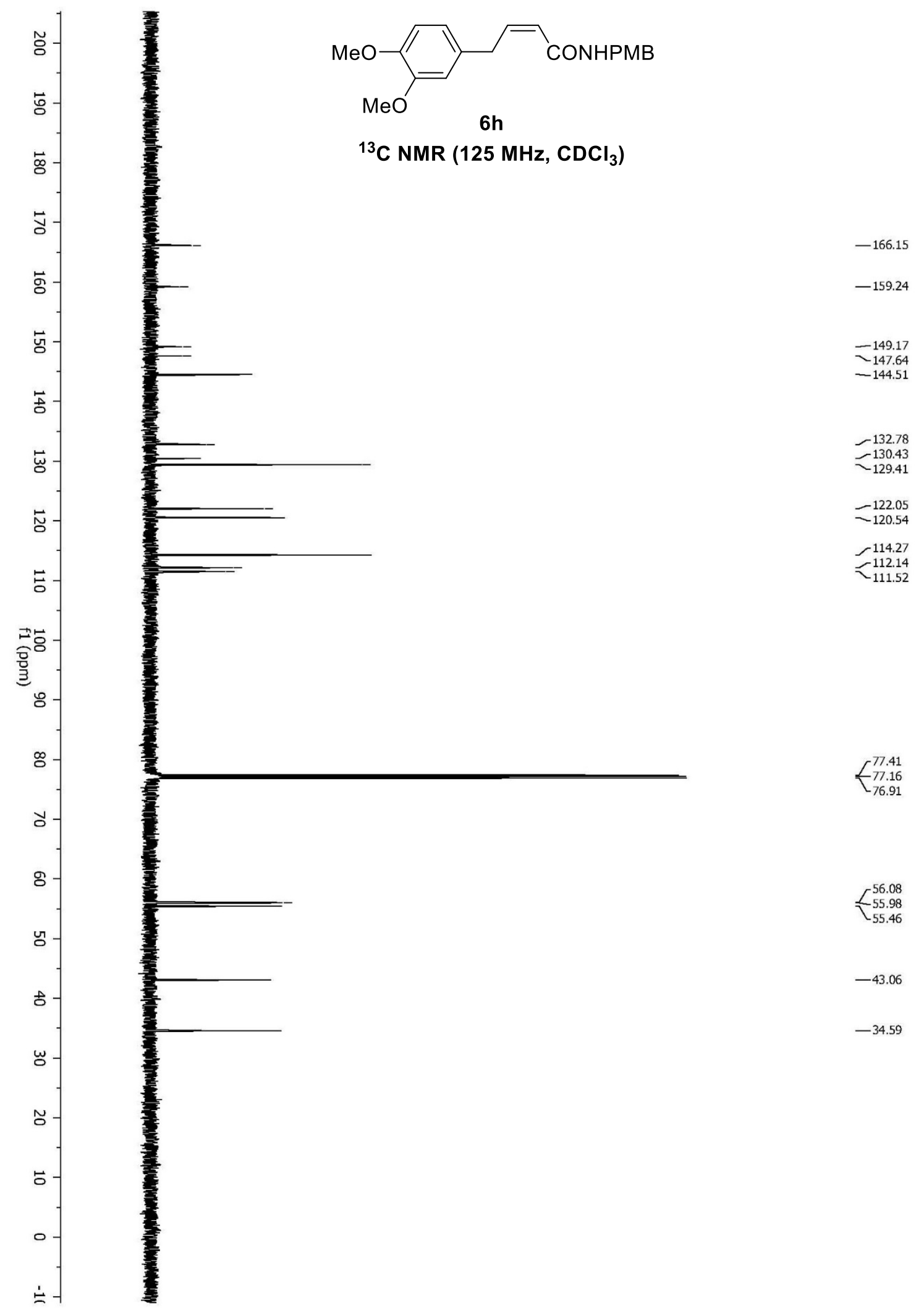




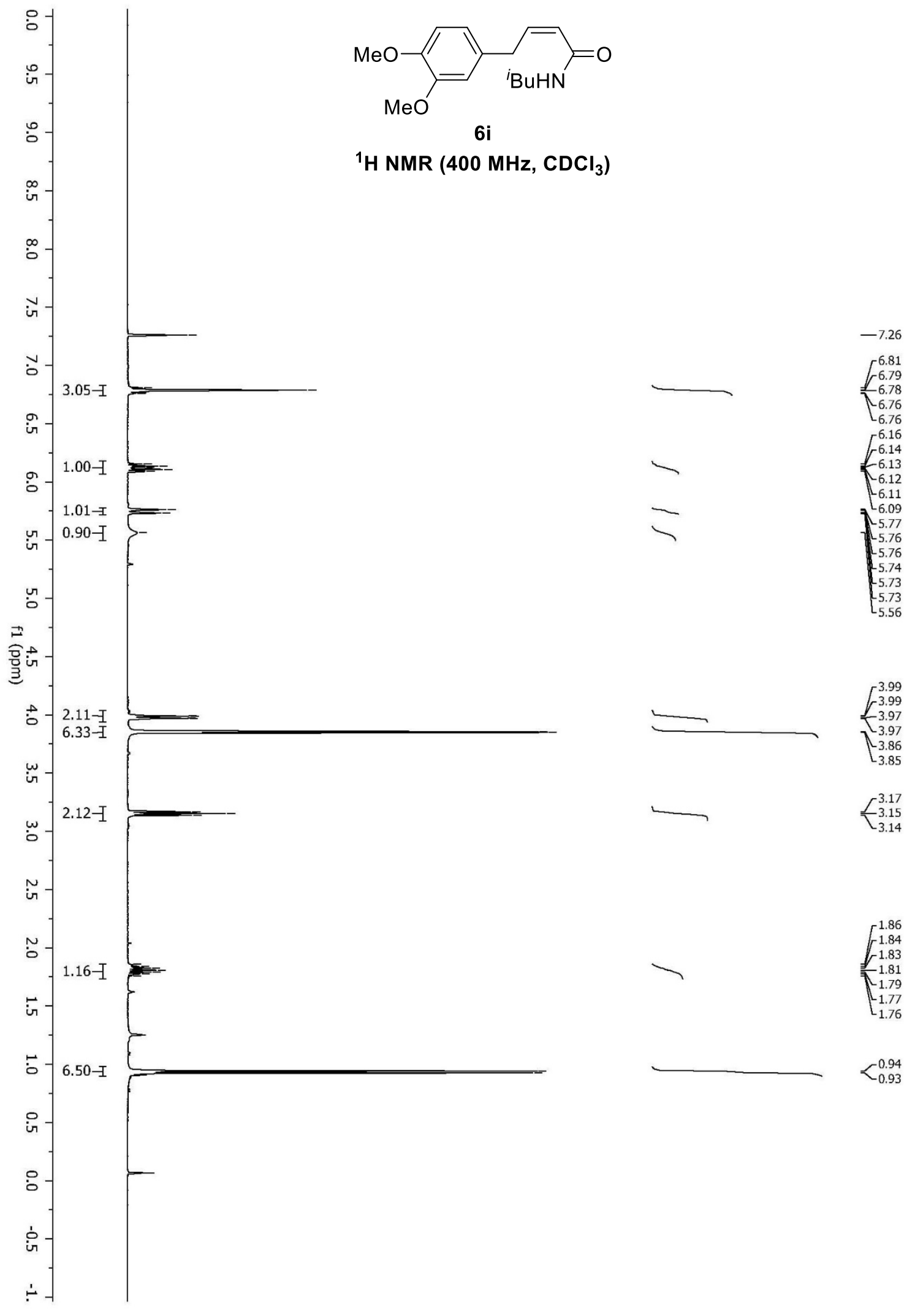




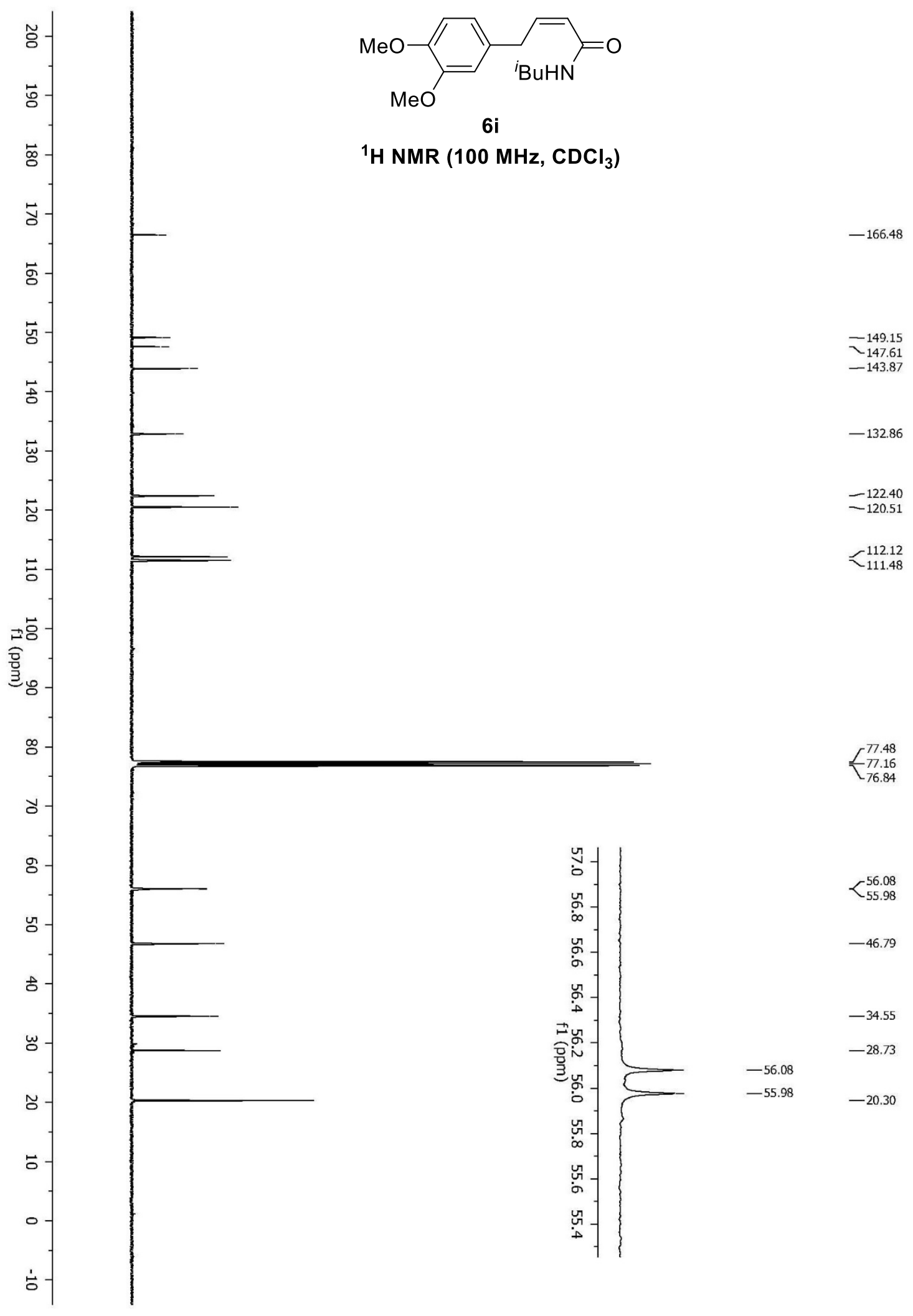




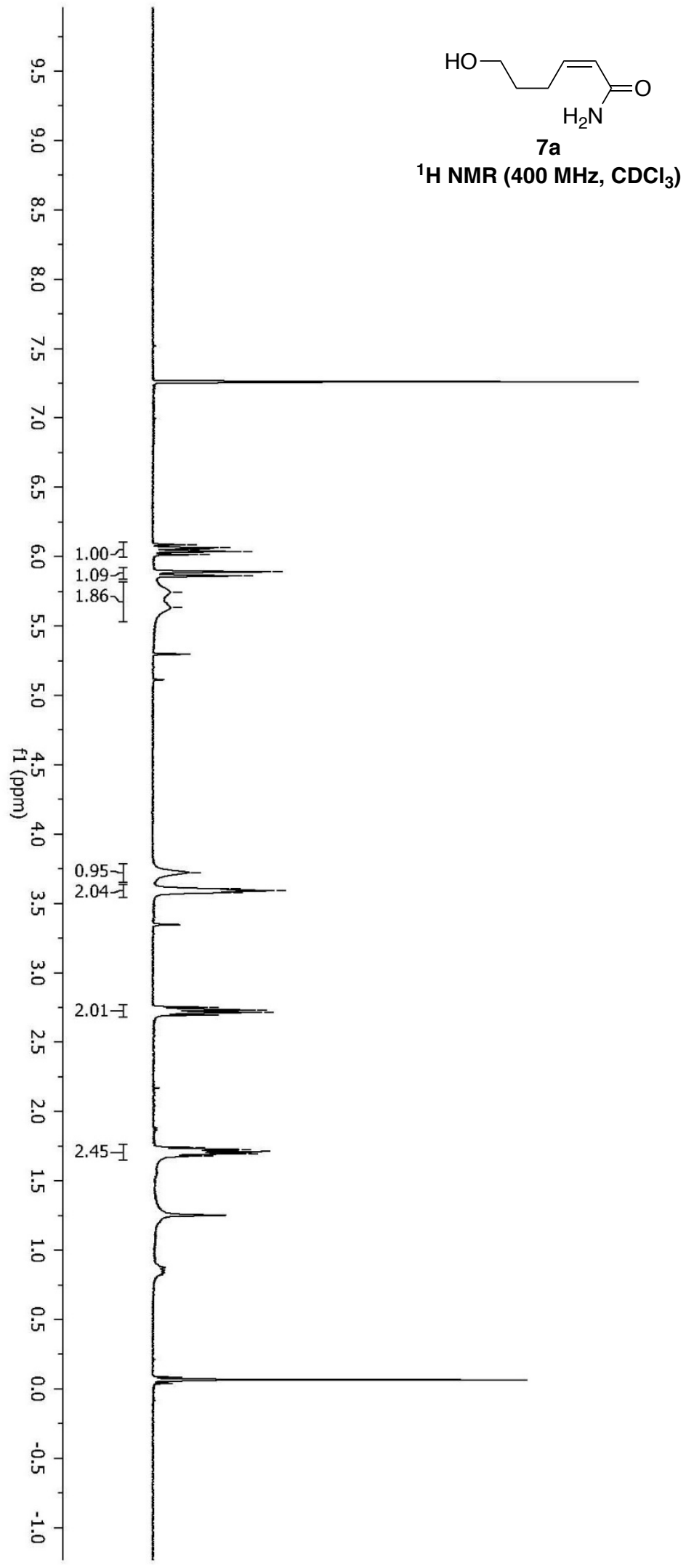




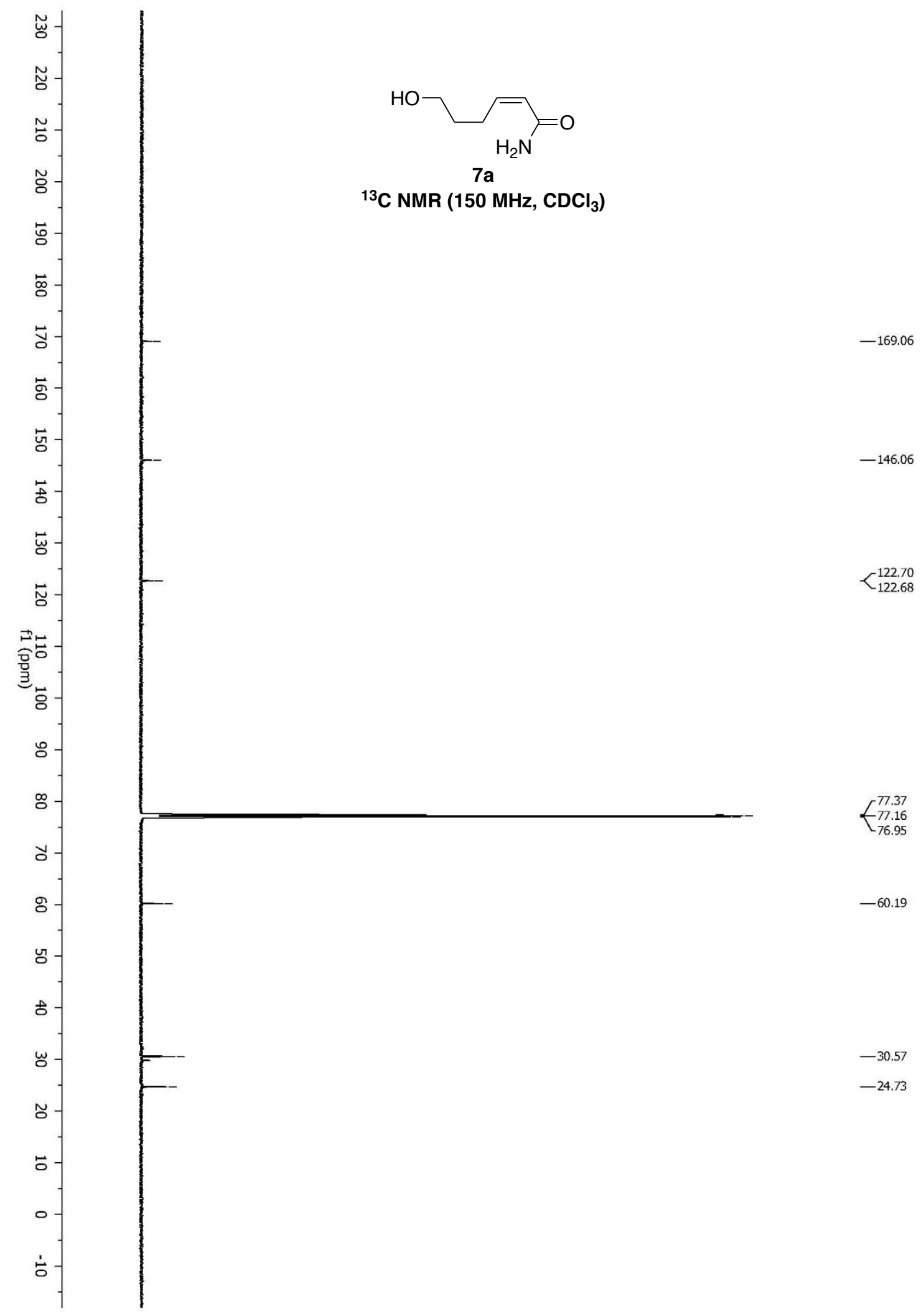




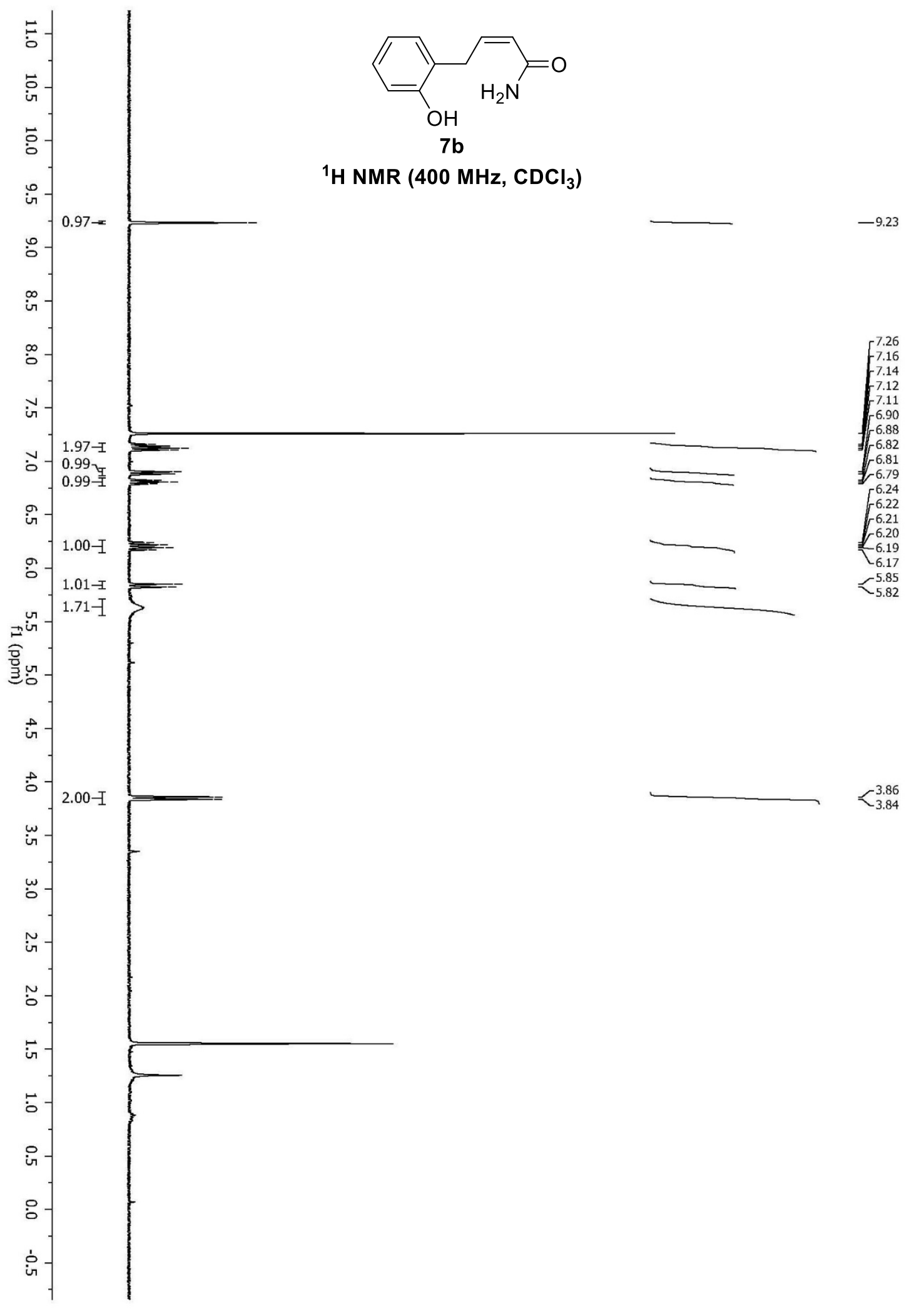




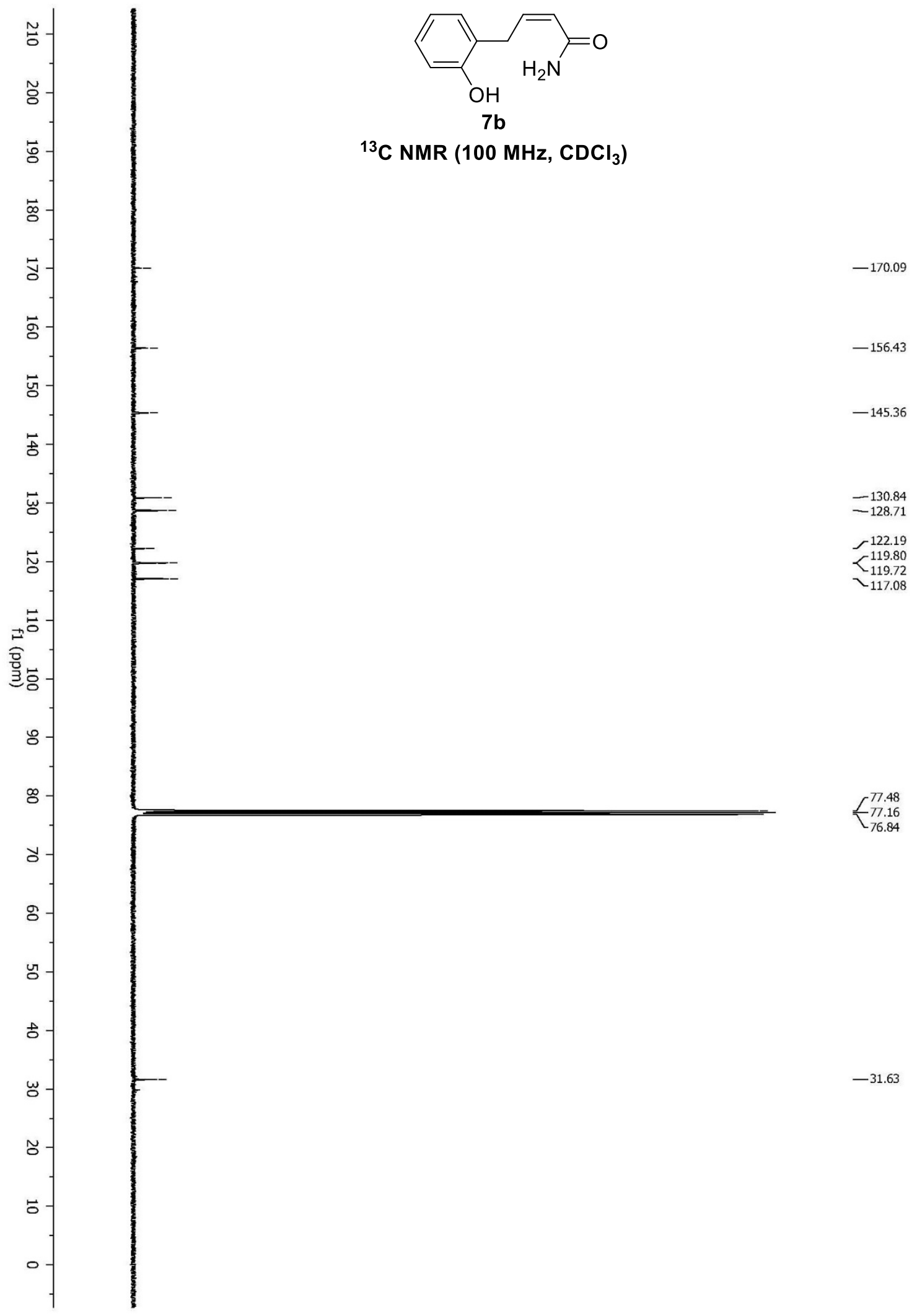



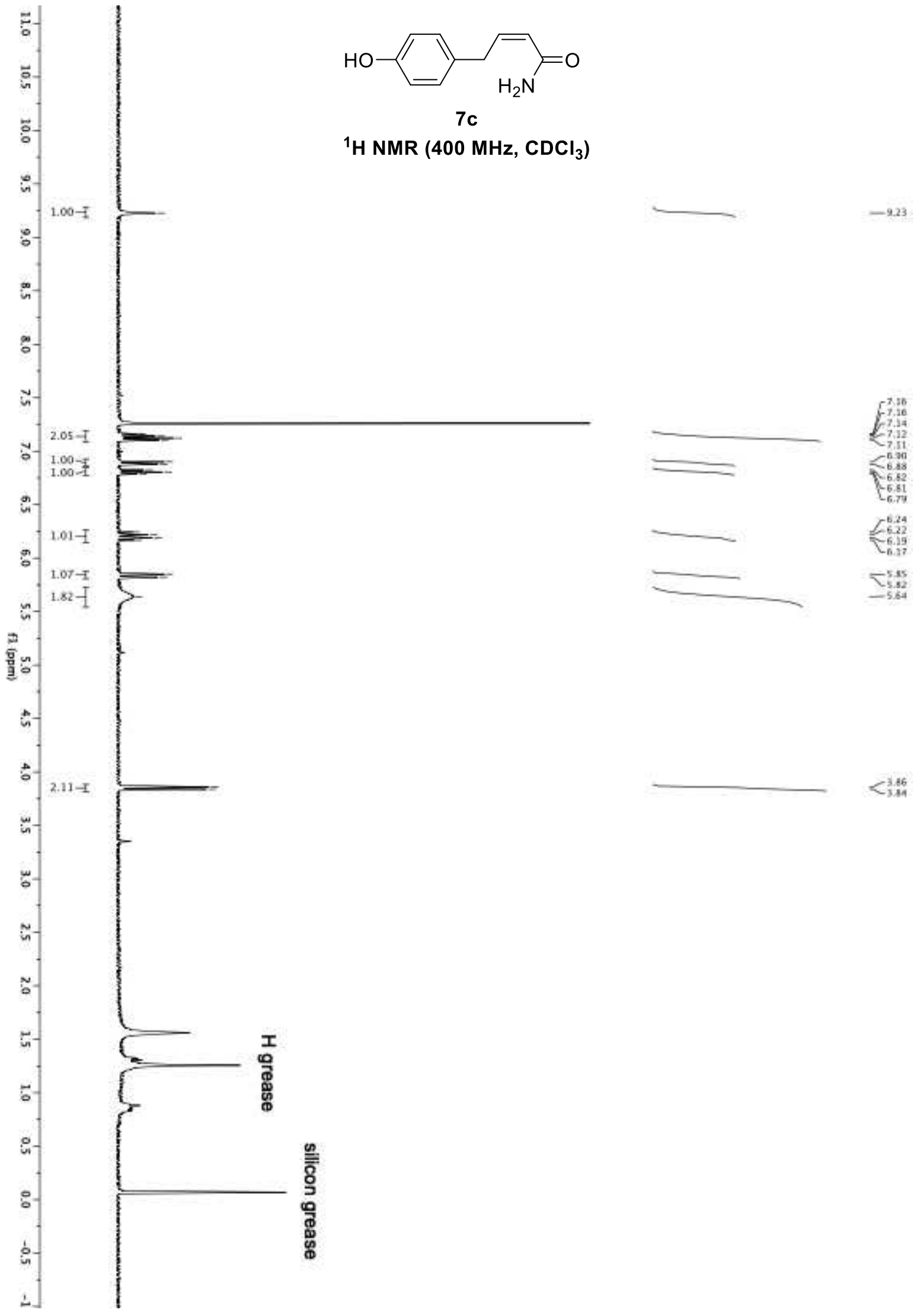


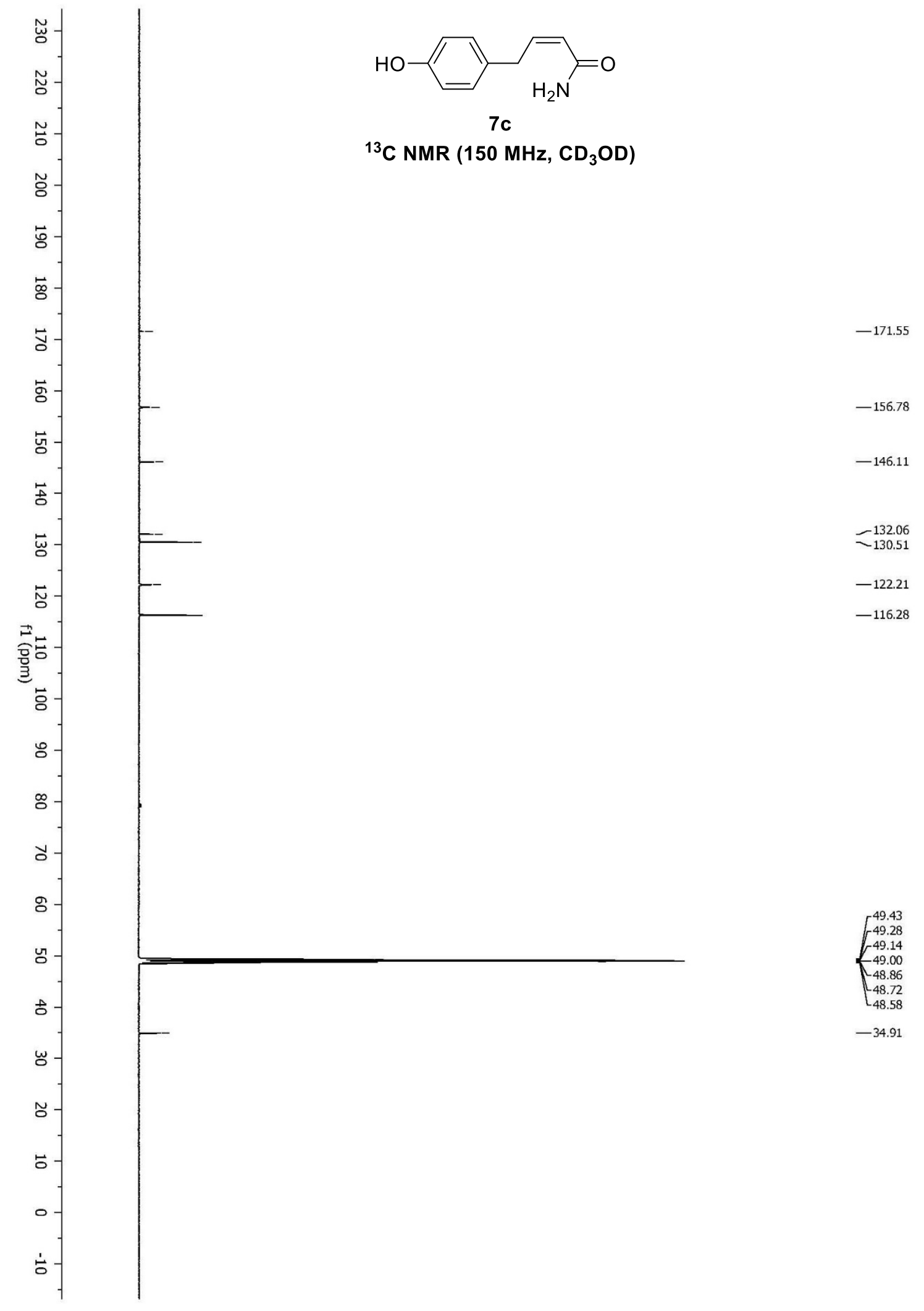




\section{Coordinates After Optimization with $\omega-$ B97XD/Def2SVPTHF(IEFPCM)}

12

cis butene / electronic energy: $-\mathbf{1 5 7 . 0 6 0 3 9 5 7 5 9}$ a.u. / lowest freq: $80.71 \mathrm{~cm}-1$

$\begin{array}{lllll}C & 0.669406 & 0.663584 & 0.000003\end{array}$

$\begin{array}{llll}C & -0.669408 & 0.663584 & -0.000001\end{array}$

$\begin{array}{llll}\text { H } & 1.169481 & 1.639591 & 0.000003\end{array}$

$\begin{array}{llll}\text { C } & 1.587618 & -0.522192 & 0.000000\end{array}$

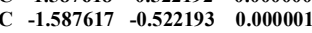

H $1.051663-1.480972 \quad 0.000020$

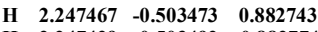

$\begin{array}{llll}\text { H } & 2.247429 & -0.503492 & -0.882774\end{array}$

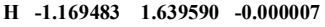

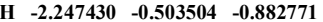

$\begin{array}{llll}\text { H } & -2.247465 & -0.503468 & 0.882745\end{array}$

$\begin{array}{llll}H & -1.051656 & -1.480969 & 0.000031\end{array}$

15

cis_methylbutenoate / electronic energy: $\mathbf{- 3 4 5 . 4 2 6 4 0 4 2 4 8}$ a.u. / lowest freq: $44.60 \mathrm{~cm}-1$

$\begin{array}{llll}C & \mathbf{1 . 9 9 9 7 8 0} & \mathbf{0 . 6 8 9 6 3 9} & \mathbf{0 . 0 0 0 0 0 2}\end{array}$

$\begin{array}{llll}C & 0.689296 & 0.981132 & 0.000003\end{array}$

$\begin{array}{llll}\text { H } & \mathbf{2 . 6 8 1 0 2 3} & \mathbf{1 . 5 4 8 9 9 9} & \mathbf{0 . 0 0 0 0 0 5}\end{array}$

$\begin{array}{llll}C & 2.664993 & -0.646662 & -0.000006\end{array}$

$\begin{array}{llll}C & -0.420233 & 0.000062 & 0.000002\end{array}$

$\begin{array}{llll}\text { H } & 1.946992 & -1.472253 & -0.000006\end{array}$

$\begin{array}{llll}\text { H } & 3.321455 & -0.730489 & 0.881052\end{array}$

$\begin{array}{llll}\text { H } & 3.321449 & -0.730481 & -0.881068\end{array}$

$\begin{array}{llll}0 & -1.599640 & 0.634433 & -0.000005\end{array}$

$\begin{array}{llll}C & -2.562750 & -0.6378876 & -0.000005\end{array}$

H $-3.616437-0.507202-0.000015$

$\begin{array}{llll}C & -3.616437 & 0.507202 & -0.000015\end{array}$

\begin{tabular}{llll}
\hline & -2.793271 & -0.818980 & 0.893472
\end{tabular}

\begin{tabular}{llll}
\hline & -2.793260 & -0.819000 & -0.893463
\end{tabular}

\begin{tabular}{llll}
\hline & -0.334036 & -1.207414 & 0.000007
\end{tabular}

$\begin{array}{lllll}\text { H } & 0.374942 & 2.027074 & 0.000005\end{array}$

sat prod_CP_a / electronic energy: $-\mathbf{2 4 0 1 . 7 5 1 9 3 8 2 4}$ a.u. / lowest freq: $22.98 \mathrm{~cm}-1$

Ru $0.38091 \overline{5}-\mathbf{- 0 . 2 7 6 5 9 2}-\mathbf{0 . 7 9 7 9 1 8}$

$\begin{array}{llll}\mathrm{S} & -\mathbf{0 . 8 8 2 5 8 8} & \mathbf{1 . 0 4 6 9 1 3} & -\mathbf{2 . 1 5 8 1 0 3}\end{array}$

$\begin{array}{llll}\text { C } & -2.513685 & \mathbf{0 . 3 7 4 7 3 9} & \mathbf{- 2 . 0 8 2 1 1}\end{array}$

$\begin{array}{llll}C & -2.723870 & -0.869458 & -1.470373\end{array}$

$\begin{array}{llll}C & -1.365996 & -1.715294 & -0.729824\end{array}$

$\begin{array}{lllll}\text { C } & 0.295986 & 0.866693 & 0.747660\end{array}$

$\begin{array}{lrrr}\mathrm{N} & \mathbf{- 0 . 7 3 4 0 1 2} & \mathbf{1 . 2 5 5 1 9 2} & \mathbf{1 . 5 3 7 4 2 4}\end{array}$

$\begin{array}{llll}N & 1.394793 & 1.548589 & 1.185612\end{array}$

$\begin{array}{llll}\text { C } & -\mathbf{0 . 2 9 9 1 5 1} & \mathbf{2 . 0 8 0 7 3 7} & \mathbf{2 . 6 6 8 3 9 2}\end{array}$

$\begin{array}{llll}C & \mathbf{1 . 1 0 2 3 2 2} & \mathbf{2 . 5 1 1 3 4 1} & \mathbf{2 . 2 4 4 6 1 9}\end{array}$

C $-\mathbf{- 2 . 0 5 1 2 4 7} \quad \mathbf{0 . 7 3 2 8 0 6} \quad \mathbf{1 . 5 4 0 9 9 3}$

$\begin{array}{llll}C & -2.299081 & -0.498463 & 2.141556\end{array}$

$\begin{array}{llll}C & -3.129073 & 1.471699 & 1.02824\end{array}$

$\begin{array}{llll}C & -3.569798 & -1.043799 & 2.216873\end{array}$

$\begin{array}{llll}C & -4.409912 & 0.923288 & 1.11408\end{array}$

$\begin{array}{llll}\text { C } & -4.648218 & -0.329152 & 1.689489\end{array}$

$\begin{array}{llll}\text { H } & -3.701519 & -\mathbf{2 . 0 2 3 6 7 2} & \mathbf{2 . 6 7 9 4 0 4}\end{array}$

$\begin{array}{llll}\text { H } & -5.249849 & 1.483338 & 0.695624\end{array}$

$\begin{array}{lllll}\text { C } & -6.033399 & -0.915967 & 1.693610\end{array}$

$\begin{array}{llll}\text { F } & -1.266913 & -1.170320 & 2.663878\end{array}$

\begin{tabular}{lllll}
\hline & -2.895471 & 2.819194 & 0.406242
\end{tabular}

H $\quad-2.122130 \quad 2.751600 \quad-0.373599$

H $\quad-\mathbf{3 . 8 1 7 9 2 4} \quad \mathbf{3 . 2 0 6 1 3 8} \quad \mathbf{- 0 . 0 4 5 7 5 5}$

H $\quad-\mathbf{2 . 5 5 1 8 6 0} \quad 3.551145 \quad 1.154816$

$\begin{array}{llll}C & 2.658533 & 1.496766 & 0.550804\end{array}$

$\begin{array}{llll}C & 3.703362 & 0.828732 & 1.203115\end{array}$

$\begin{array}{llll}C & 2.925695 & \mathbf{2 . 1 2 3 9 3 2} & -0.677970\end{array}$

$\begin{array}{llll}C & 4.96585 & 2.123932 & -0.677970\end{array}$

$\begin{array}{llll}C & 4.965871 & 0.7061031 & 0.658252\end{array}$

C $5.223477 \quad 1.267863-0.599716$

$\begin{array}{llll}\text { C } & 5.223477 & 1.267863 & -0.599716\end{array}$

$\begin{array}{llll}\text { H } & 5.737156 & 0.168690 & 1.213149\end{array}$

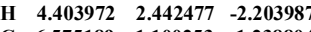

C $\quad 6.575189 \quad 1.100253 \quad-1.238804$

\begin{tabular}{llll}
$C$ & $\mathbf{1 . 9 0 5 4 3 8}$ & $\mathbf{2 . 9 8 1 4 4 8}$ & -1.378585 \\
\hline
\end{tabular}

$\begin{array}{lllll}\text { H } & 1.663660 & 2.573771 & -2.371119\end{array}$

$\begin{array}{lllll}\text { H } & \mathbf{0 . 9 6 7 0 3 4} & \mathbf{3 . 0 6 2 8 6 9} & \mathbf{- 0 . 8 1 7 5 2}\end{array}$

$\begin{array}{llll}\text { H } & \mathbf{2 . 3 1 2 8 3 0} & 3.992061 & \mathbf{- 1 . 5 2 9 2 9 5}\end{array}$

$\begin{array}{llll}\text { F } & 3.451326 & \mathbf{0 . 2 7 9 7 4 0} & \mathbf{2 . 3 9 4 9 8 5}\end{array}$

$\begin{array}{llll}C & \mathbf{2 . 7 2 8 9 4 7} & \mathbf{- 2 . 0 3 8 0 2 7} & \mathbf{- 0 . 6 9 5 6 3 8}\end{array}$

$\begin{array}{llll}\text { H } & 3.014405 & -1.008009 & -0.948359\end{array}$

$\begin{array}{llll}\text { C } & \mathbf{2 . 0 3 7 6 9 4} & \mathbf{- 2 . 2 2 5 4 9 4} & \mathbf{0 . 6 7 9 6 3 3}\end{array}$

$\begin{array}{llll}C & 3.492798 & -2.579173 & \mathbf{0 . 4 7 8 7 5 3}\end{array}$

$\begin{array}{lllll}H & 1.889551 & -1.316194 & 1.27084\end{array}$

C $\quad \begin{array}{llll}1.034141 & -3.314664 & 0.994819\end{array}$

$\begin{array}{llll}\text { H } & 4.202112 & -1.878838 & 0.927660\end{array}$

$\begin{array}{llll}C & 3.962638 & -4.014136 & \mathbf{0 . 5 3 6 6 1 9}\end{array}$

$\begin{array}{llll}C & -3.603687 & 1.066459 & -2.632658\end{array}$

$\begin{array}{llll}C & -3.603687 & 1.066459 & -2.632658\end{array}$

$\begin{array}{llll}C & -4.8798182 & 0.515640 & -2.594308\end{array}$

C $-5.083670-0.738838-3.02694$

C $-5.083670-0.738838-2.006061$

$\begin{array}{llll}C & -6.084182 & -1.177025 & -1.977673 \\ C & -4.012375 & -1.422414 & -1.43724\end{array}$

$\begin{array}{llll}C & -4.012375 & -1.422414 & -1.443724\end{array}$

$\begin{array}{llll}-4.166924 & -2.390772 & -0.959932\end{array}$

$\begin{array}{llll}-3.438899 & 2.045017 & -3.092129\end{array}$

$\begin{array}{llll}\text { H } & -6.798316 & -0.139525 & 1.837077\end{array}$

$\begin{array}{llll}-6.152418 & -1.674364 & 2.479800\end{array}$

$\begin{array}{llll}\text { H } & -6.235860 & -1.400757 & 0.724908\end{array}$ 
$\begin{array}{llll}\text { H } & 7.380260 & 1.187175 & -0.495474\end{array}$ $\begin{array}{llll}\text { H } & 6.743139 & 1.848234 & -2.02525\end{array}$

$\begin{array}{llll}\text { H } & 6.660156 & 0.103343 & -1.699639\end{array}$

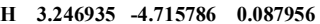

$\begin{array}{llll}\text { H } & 4.2420216 & -4.326940 & \mathbf{1 . 5 8 0 4 1 8}\end{array}$

$\begin{array}{llll}4 & 4.120216 & -4.326940 & 1.580418\end{array}$

$\begin{array}{cccc}\text { H } & 4.919992 & -4.130247 & \mathbf{0 . 0 0 5 5 9 6}\end{array}$

\begin{tabular}{llll} 
H & 0.045526 & -2.884239 & 1.196806 \\
\hline & 1.347884 & -3.871269 & 1.89225
\end{tabular}

$\begin{array}{llll}\text { H } & \mathbf{1 . 3 4 7 8 8 4} & \mathbf{- 3 . 8 7 1 2 6 9} & \mathbf{1 . 8 9 2 2 2 5}\end{array}$

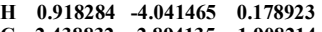

C $\quad 2.438832-2.894135-1.908214$

$\begin{array}{lllll}\text { H } & \mathbf{1 . 7 3 8 5 3 2} & \mathbf{- 2 . 3 9 8 0 7 9} & \mathbf{- 2 . 5 9 6 1 3 1}\end{array}$

$\begin{array}{llll}\text { H } & 1.999551 & -3.865426 & -1.644919\end{array}$

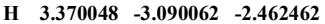

$\begin{array}{llll}\text { H } & 1.124284 & 3.537579 & 1.840788\end{array}$

H $\quad \mathbf{1 . 8 3 6 6 0 6} \quad \mathbf{2 . 4 3 9 9 7 0} \quad \mathbf{3 . 0 5 7 9 6 3}$

\begin{tabular}{llll}
$\mathrm{H}$ & 1.836606 & 2.439970 & 3.057963 \\
\hline & -0.981020 & 2.927807 & 2.821583
\end{tabular}

$\begin{array}{llll}H & -0.981020 & 2.927807 & 2.821583\end{array}$

$\begin{array}{llll}H & -0.288177 & 1.473936 & 3.58884\end{array}$

74

sat_ts_CP_a / electronic energy: $-\mathbf{2 4 0 1 . 7 3 8 6 9 2 6 9}$ a.u. / lowest freq: $-\mathbf{2 3 2 . 6 3} \mathrm{cm}-1$

Ru $\quad 0.334088 \quad-0.770678 \quad-0.518291$

$\begin{array}{lllll}S & -0.806866 & 0.221355 & -2.32780\end{array}$

$\begin{array}{llll}C & -2.497504 & -0.226860 & -2.128714\end{array}$

$\begin{array}{llll}\text { C } & -2.860111 & -1.158245 & -1.143993\end{array}$

$\begin{array}{llll}S & -1.620450 & -1.837430 & -0.090992\end{array}$

$\begin{array}{llll}C & 0.477247 & 0.917116 & 0.446397\end{array}$

$\begin{array}{lrl}\mathrm{N} & \mathbf{- 0 . 5 1 1 3 4 7}\end{array}$

$\begin{array}{cccc}\text { N } & 1.616583 & 1.652004 & 0.596504 \\ \text { C } & -0.014163 & \mathbf{2}\end{array}$

$\begin{array}{llll}\text { C } & \mathbf{- 0 . 0 1 4 1 6 3} & \mathbf{2 . 7 8 3 3 7 9} & \mathbf{1 . 7 9 2 0 8 6}\end{array}$

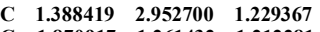

$\begin{array}{llll}C & -1.870917 & 1.261432 & 1.212281\end{array}$

$\begin{array}{lllll}\text { C } & -2.220639 & 0.355097 & \mathbf{2 . 2 0 8 7 1 5}\end{array}$

$\begin{array}{llll}\text { C } & -2.887512 & 1.884680 & 0.469772\end{array}$

$\begin{array}{llll}C & -3.535638 & 0.001020 & 2.460805\end{array}$

$\begin{array}{llll}C & -4.211852 & 1.530738 & 0.731807\end{array}$

\begin{tabular}{llll}
$C$ & -4.211852 & 1.530738 & 0.731807 \\
\hline
\end{tabular}

$\begin{array}{lllll}C & -4.554314 & 0.587727 & 1.706568\end{array}$

$\begin{array}{llll}\text { H } & -3.748136 & -0.736337 & 3.236976\end{array}$

$\begin{array}{llll}\text { H } & \mathbf{- 5 . 0 0 5 0 2 9} & \mathbf{1 . 9 9 4 3 1 5} & \mathbf{0 . 1 3 9 9 5 3}\end{array}$

$\begin{array}{llll}\text { C } & -5.990030 & 0.184790 & 1.905766\end{array}$

$\begin{array}{llll}\text { F } & -1.244344 & -0.192317 & 2.941021\end{array}$

$\begin{array}{llll}\text { C } & -\mathbf{2 . 5 5 0 8 2 0} & \mathbf{2 . 9 1 3 6 4 0} & \mathbf{- 0 . 5 7 1 8 9 2}\end{array}$

$\begin{array}{llll}\text { H } & -1.792025 & \mathbf{2 . 5 2 3 1 7 9} & \mathbf{- 1 . 2 6 6 1 8 2}\end{array}$

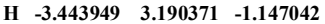

$\begin{array}{llll}\text { H } & -\mathbf{2 . 1 4 7 6 0 1} & \mathbf{3 . 8 3 0 5 3 3} & \mathbf{- 0 . 1 1 2 3 7 6}\end{array}$

$\begin{array}{llll}\text { C } & \mathbf{2 . 9 0 2 7 1 1} & \mathbf{1 . 3 6 6 6 6 3} & \mathbf{0 . 0 7 9 6 4 0}\end{array}$

$\begin{array}{llll}\text { C } & 3.910627 & 1.016817 & \mathbf{0 . 9 8 5 6 3 6}\end{array}$

$\begin{array}{llll}C & 3.237391 & \mathbf{1 5 0 6 4 1 2} & \mathbf{- 1 . 2 7 5 9 1 7}\end{array}$

$\begin{array}{llll}C & \mathbf{5 . 2 0 7 8 4 8} & \mathbf{0 . 7 4 8 5 2 5} & \mathbf{0 . 5 9 5 0 0 8}\end{array}$

$\begin{array}{llll}C & 4.550818 & 1.217631 & -1.669996\end{array}$

$\begin{array}{llll}C & 5.540525 & 0.834676 & -0.763141\end{array}$

$\begin{array}{llll}\text { H } & 5.944255 & 0.463766 & 1.348633\end{array}$

$\begin{array}{lllll}\text { H } & 4.807069 & 1.311944 & -2.728372\end{array}$

$\begin{array}{llll}\text { C } & 6.940413 & 0.517540 & -1.215394\end{array}$

C $\quad 2.226232 \quad 1.940870 \quad-2298233$

\begin{tabular}{llll}
$C$ & 2.22632 & 1.940870 & -2.298233 \\
\hline & 1.863027 & 1.077369 & -2.877222
\end{tabular}

\begin{tabular}{llll} 
H & 1.863027 & 1.077369 & -2.877222 \\
\hline & 1.346489 & 2.408534 & -1.839254
\end{tabular}

$\begin{array}{lllll}\text { H } & 1.346489 & 2.408534 & \mathbf{- 1 . 8 3 9 2 5 4}\end{array}$

$\begin{array}{llll}\text { H } & \mathbf{2 . 6 7 7 0 1 3} & \mathbf{2 . 6 4 8 2 1 2} & \mathbf{- 3 . 0 0 7 8 7 5}\end{array}$

$\begin{array}{lllll}\text { F } & 3.586108 & 0.919586 & 2.279366\end{array}$

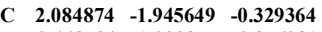

$\begin{array}{llll}\text { H } & \mathbf{2 . 4 4 8 7 8 4} & \mathbf{- 1 . 0 2 9 8 7 7} & \mathbf{- 0 . 8 7 6 9 8 1}\end{array}$

$\begin{array}{llll}\text { C } & 1.345161 & -1.789913 & 1.382479\end{array}$

$\begin{array}{llll}C & \mathbf{2 . 7 6 5 2 4 8} & \mathbf{- 2 . 1 4 5 4 9 3} & \mathbf{0 . 9 8 4 5 0 6}\end{array}$

$\begin{array}{llll}\text { H } & 1.298856 & -0.873718 & 1.974448\end{array}$

C $0.493589-2898521 \quad 1.955294$

$\begin{array}{llll}C & 0.493589 & -2.898521 & 1.955294\end{array}$

$\begin{array}{llll}\text { H } & 3.457992 & -1.335631 & 1.223312\end{array}$

$\begin{array}{llll}\text { C } & 3.373520 & -3.482739 & \mathbf{1 . 3 5 4 1 7 2}\end{array}$

$\begin{array}{llll}C & -3.501637 & 0.349818 & -2.924080\end{array}$

$\begin{array}{llll}C & -4.834883 & -0.005308 & -2.750753\end{array}$

$\begin{array}{lllll}\text { H } & \mathbf{- 5 . 6 0 4 3 3 2} & \mathbf{0 . 4 5 4 2 1 6} & \mathbf{- 3 . 3 7 5 9 3 7}\end{array}$

$\begin{array}{lllll}\text { C } & -5.189184 & -0.950064 & -1.779632\end{array}$

\begin{tabular}{lllll} 
H & -6.235989 & -1.230025 & -1.639595 \\
\hline
\end{tabular}

$\begin{array}{llll}\text { C } & -4.204941 & -1.519151 & -0.980028\end{array}$

H $-4.473173-2.238770-0.201638$

$\begin{array}{llll}-3.43173 & -2.238770 & -0.201638\end{array}$

$\begin{array}{llll}4 & -3.222546 & 1.088247 & \mathbf{- 3 . 6 8 0 4 5 9}\end{array}$

$\begin{array}{llll}-1 & -6.663625 & 1.050844 & 1.833597\end{array}$

$\begin{array}{llll}\text { H } & -6.145982 & -0.297823 & \mathbf{2 . 8 8 0 3 9 8}\end{array}$

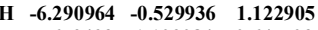

H $\quad \begin{array}{llll}\text { H.679423 } & 1.102084 & -0.647703\end{array}$

$\begin{array}{llll}\text { H } & 7.077391 & 0.730455 & -2.283771\end{array}$

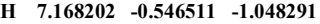

$\begin{array}{llll}\text { H } & \mathbf{2 . 7 1 5 3 4 5} & \mathbf{- 4 . 3 3 1 6 8 5} & \mathbf{1 . 1 2 6 3 8 5}\end{array}$

$\begin{array}{llll}\text { H } & 3.594992 & \mathbf{3 . 5 1 4 7 8 1} & \mathbf{2 . 4 3 1 6 7 4}\end{array}$

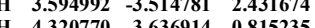

\begin{tabular}{lllll}
\hline & 4.320770 & -3.636914 & 0.815235 \\
\hline
\end{tabular}

\begin{tabular}{llll} 
H & -0.485812 & -2.512174 & 2.260952 \\
\hline & 0.988417 & -3.328723 & 2.842329
\end{tabular}

$\begin{array}{llll}\text { H } & 0.988417 & -3.328723 & 2.842329\end{array}$

$\begin{array}{lllll}\text { H } & 0.319684 & -3.719567 & 1.244008\end{array}$

$\begin{array}{lllll}\text { C } & 1.883355 & -3.154251 & -1.223547\end{array}$

$\begin{array}{lllll}\text { H } & \mathbf{1 . 3 4 2 4 6 0} & \mathbf{- 2 . 9 0 7 4 0 1} & \mathbf{- 2 . 1 4 8 0 2 4}\end{array}$

$\begin{array}{llll}\text { H } & \mathbf{1 . 3 1 9 4 0 6} & \mathbf{- 3 . 9 4 2 2 2 2} & \mathbf{- 0 . 7 0 5 7 5 3}\end{array}$

$\begin{array}{llll}\text { H } & \mathbf{2 . 8 6 3 7 9 7} & \mathbf{- 3 . 5 7 4 0 4 1} & \mathbf{- 1 . 5 0 2 6 0 2}\end{array}$

$\begin{array}{lllll}\text { H } & \mathbf{1 . 4 4 7 9 8 3} & \mathbf{3 . 7 5 2 4 7 8} & \mathbf{0 . 4 7 2 4 2 8}\end{array}$

$\begin{array}{llll}\text { H } & \mathbf{2 . 1 4 0 9 1 8} & 3.147792 & \mathbf{2 . 0 0 4 4 4 9}\end{array}$

$\begin{array}{lll}0.140918 & 3.147792 & 2.004449\end{array}$

\begin{tabular}{llll} 
H & $-\mathbf{0 . 6 5 1 6 4 5}$ & $\mathbf{3 . 6 6 2 1 1 6}$ & $\mathbf{1 . 6 2 9 6 0 4}$ \\
\hline & -0.009791 & $\mathbf{2 . 5 5 2 7 0 3}$ & $\mathbf{2 . 8 7 0 0 9 4}$
\end{tabular}

$\begin{array}{llll}\text { H } & -0.009791 & 2.552703 & 2.870094\end{array}$ 
74

sat_prod_BHE_a / electronic energy: - 2401.77602218 a.u. / lowest freq: $21.47 \mathrm{~cm}-1$

$\begin{array}{llll}S & 0.347291 & -2.463656 & -0.438398\end{array}$

$\begin{array}{llll}C & -0.445828 & 0.541435 & -0.658145\end{array}$

$\begin{array}{llll}\text { Ru } & -0.198878 & -0.617275 & 0.935626\end{array}$

$\begin{array}{llll}C & -\mathbf{2 . 4 8 3 7 3 4} & \mathbf{- 0 . 4 4 4 5 5 5} & \mathbf{1 . 7 7 4 5 6 4}\end{array}$

$\begin{array}{llll}C & -0.360015 & 0.333230 & 2.935242\end{array}$

$\begin{array}{llll}\text { S } & 2.057925 & \mathbf{- 0 . 5 5 6 9 1 3} & \mathbf{1 . 4 2 6 3 7 0}\end{array}$

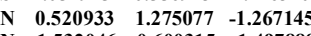

$\begin{array}{llll}N & -1.532046 & 0.600315 & -1.497888\end{array}$

$\begin{array}{llll}C & 1.818092 & 1.614360 & -0.808479\end{array}$

$\begin{array}{llll}C & 0.058167 & 1.978985 & -2.464433\end{array}$

C $-\mathbf{- 1 . 2 2 3 6 7 5} \quad 1.240573 \quad-2.782045$

$\begin{array}{llll}C & -2.894713 & 0.282604 & -1.256834\end{array}$

$\begin{array}{llll}C & 4.359727 & 2.450608 & 0.054501\end{array}$

$\begin{array}{llll}\text { C } & 1.968740 & 2.592688 & 0.174522\end{array}$

$\begin{array}{llll}C & \mathbf{2 . 9 6 0 5 2 3} & \mathbf{1 . 0 7 5 7 2 2} & \mathbf{- 1 . 4 1 1 6 6 2}\end{array}$

$\begin{array}{llll}C & 4.213801 & 1.502981 & \mathbf{- 0 . 9 5 9 7 1 7}\end{array}$

$\begin{array}{llll}\text { C } & 3.207261 & 3.009302 & 0.621593\end{array}$

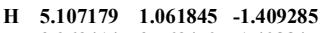

$\begin{array}{llll}\text { H } & 3.263414 & 3.763470 & 1.408845\end{array}$

$\begin{array}{llll}\text { C } & \mathbf{- 5 . 6 4 6 8 0 1} & \mathbf{- 0 . 1 1 0 5 8 3} & \mathbf{- 0 . 7 2 9 5 4 4}\end{array}$

$\begin{array}{lllll}C & \mathbf{- 3 . 7 6 4 3 3 8} & \mathbf{1 . 3 4 9 6 5 3} & \mathbf{- 0 . 9 8 3 9 6 1}\end{array}$

$\begin{array}{llll}\text { C } & -\mathbf{3 . 4 4 9 4 0 0} & \mathbf{- 0 . 9 9 8 6 7 7} & \mathbf{- 1 . 3 7 5 1 3 4}\end{array}$

$\begin{array}{llll}C & -4.810832 & -1.168894 & -1.084209\end{array}$

$\begin{array}{llll}9 & 1.181503 & -0.703489\end{array}$

\begin{tabular}{llll}
\hline-3.261943 & 2.591783 & -0.996595
\end{tabular}

$\begin{array}{llll}\text { C } & -2.636614 & -2.185719 & -1.796743\end{array}$

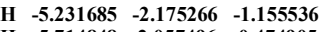

H $\quad \begin{array}{llll}-5.714848 & \mathbf{2 . 0 5 7 4 9 6} & \mathbf{- 0 . 4 7 4 9 0 5}\end{array}$

$\begin{array}{llll}\text { C } & -7.098804 & -0.330453 & -0.402177\end{array}$

$\begin{array}{lllll}\text { H } & -7.743363 & 0.322496 & -1.009094\end{array}$

$\begin{array}{llll}\text { H } & -1.686300 & -1.892305 & -2.256269\end{array}$

$\begin{array}{llll}H & -7.297769 & -0.095245 & 0.654710\end{array}$

$\begin{array}{llll}4 & -7.297769 & -0.095245 & 0.654710\end{array}$

\begin{tabular}{llll}
4 & -7.297769 & -0.095245 & 0.654710 \\
\hline & -3.208693 & -1.372265 & -0.579993 \\
\hline
\end{tabular}

\begin{tabular}{llll} 
H & -3.208155 & -2.802140 & -2.504724 \\
\hline
\end{tabular}

$\begin{array}{llll}H & -2.390882 & -2.822503 & -0.932614\end{array}$

$\begin{array}{lllll}\text { F } & 0.867339 & 3.133013 & 0.708244\end{array}$

C $\quad \mathbf{5 . 7 2 1 3 6 0} \quad \mathbf{2 . 8 5 1 1 6 5} \mathbf{0 . 5 5 6 8 0 6}$

$\begin{array}{llll}\text { H } & 6.511907 & 2.550379 & -0.144086\end{array}$

H $\quad \begin{array}{llll}5.789234 & 3.937863 & 0.711461\end{array}$

$\begin{array}{llll}\text { H } & \mathbf{5 . 9 3 0 1 3 0} & \mathbf{2 . 3 6 9 3 4 8} & \mathbf{1 . 5 2 5 3 2 0}\end{array}$

$\begin{array}{llll}\text { C } 2.849064 & 0.059546 & -2.513744\end{array}$

H $2.9539040 .540823-3.500162$

$\begin{array}{llll}\text { H } & \mathbf{2 . 9 5 3 9 0 4} & \mathbf{0 . 5 4 0 8 2 3} & \mathbf{- 3 . 5 0 0 1 6 2}\end{array}$

$\begin{array}{llll}\text { H } & 3.641312 & \mathbf{- 0 . 6 9 6 4 5 9} & \mathbf{- 2 . 4 2 0 8 3 8}\end{array}$

$\begin{array}{llll}H & \mathbf{1 . 8 8 1 6 2 4} & \mathbf{- 0 . 4 5 8 3 0 0} & \mathbf{- 2 . 4 8 1 4 1 9}\end{array}$

$\begin{array}{lllll}\text { C } & \mathbf{2 . 1 0 3 7 5 2} & \mathbf{- 2 . 5 1 5 4 9 1} & \mathbf{- 0 . 4 9 2 1 8 4} \\ \text { C } & \mathbf{2 . 7 6 4 5 8 5} & \mathbf{- 3 . 4 0 2 2 2 1} & \mathbf{- 1 . 3 6 0 6 2 7}\end{array}$

$\begin{array}{llll}C & 2.863308 & -1.674152 & 0.336734\end{array}$

$\begin{array}{llll}C & 4.152456 & -3.451790 & -1.404130\end{array}$

$\begin{array}{llll}\text { H } & 2.170072 & -4.051062 & -2.009329\end{array}$

$\begin{array}{llll}\text { C } & 4.266231 & -1.729182 & 0.283655\end{array}$

$\begin{array}{llll}C & 4.908966 & -2.610257 & -0.576844\end{array}$

$\begin{array}{llll}\text { H } & 4.650820 & -4.144273 & -2.086634\end{array}$

H $4.847180-1.057495 \quad 0.920986$

$\begin{array}{llll}\text { H } & \mathbf{6 . 0 0 0 5 5 2} & \mathbf{- 2 . 6 4 1 2 6 2} & \mathbf{- 0 . 6 1 1 0 2 7}\end{array}$

$\begin{array}{llll}\text { H } & -3.304131 & 0.036130 & 1.229657\end{array}$

$\begin{array}{llll}C & -1.494921 & 0.618590 & \mathbf{2 . 1 6 7 2 3 5}\end{array}$

$\begin{array}{llll}\text { H } & 0.274018 & 1.208061 & 3.120091\end{array}$

$\begin{array}{lrrr}C & \mathbf{- 0 . 1 5 6 4 1 0} & \mathbf{- 0 . 7 6 4 4 4 5} & \mathbf{3 . 9 6 2 2 9 6}\end{array}$

$\begin{array}{llll}C & -1.858799 & \mathbf{2 . 0 5 2 4 9 9} & \mathbf{1 . 8 7 9 0 1 6}\end{array}$

$\begin{array}{llll}\text { H } & \mathbf{- 2 . 6 9 3 9 6 1} & \mathbf{2 . 3 4 9 1 1 1} & \mathbf{2 . 5 3 6 2 4 7}\end{array}$

$\begin{array}{llll}\text { H } & -1.012376 & 2.718919 & 2.080303\end{array}$

$\begin{array}{llll}\text { H } & -\mathbf{2} .177346 & \mathbf{2 . 2 1 2 6 7 6} & \mathbf{0 . 8 4 4 9 6 0}\end{array}$

$\begin{array}{llll}\text { H } & -2.034108 & -1.136402 & 0.940470\end{array}$

$\begin{array}{llll}\text { C } & -3.081714 & -1.392497 & 2.812864\end{array}$

H $0.894406 \quad-0.776243 \quad 4.279173$

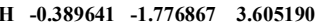

$\begin{array}{llll}\text { H } & -\mathbf{0 . 7 8 4 7 6 7} & \mathbf{- 0 . 5 7 1 5 2 7} & \mathbf{4 . 8 4 8 3 7 3}\end{array}$

$\begin{array}{llll}\text { H } & -2.353182 & -2.101997 & 3.218892\end{array}$

$\begin{array}{llll}\text { H } & -3.894709 & -1.972916 & 2.353412\end{array}$

$\begin{array}{llll}\text { H } & -3.504688 & -0.816644 & 3.649067\end{array}$

$-3.504688-0.816644-3.649067$

\begin{tabular}{llll} 
H & -0.110255 & $\mathbf{3 . 0 4 2 6 2 4}$ & $\mathbf{- 2 . 2 2 6 6 7 8}$ \\
\hline & -0.803549 & 1.914479 & -3.267228
\end{tabular}

\begin{tabular}{llll}
$H$ & 0.803549 & 1.914479 & -3.267228 \\
\hline & -1.085987 & 0.466671 & -3.555179
\end{tabular}

$\begin{array}{lllll}\text { H } & -1.085987 & 0.466671 & \mathbf{3 . 5 5 5 1 7 9}\end{array}$

$\begin{array}{llll}\text { H } & -2.034668 & 1.907902 & -3.096432\end{array}$

74

sat_ts_BHE_a / electronic energy: $\mathbf{- 2 4 0 1 . 7 2 1 4 1 9 5 1}$ a.u. / lowest freq: $\mathbf{- 8 9 . 9 8} \mathbf{c m}-1$

$0.618166-1.990351-1.198663$

$\begin{array}{llll}C & -0.660460 & 0.821849 & -0.681397\end{array}$

$\begin{array}{llll}\text { Ru } & -0.017284 & -0.548159 & 0.657753\end{array}$

$\begin{array}{llll}C & -1.073032 & -2.000280 & 1.767510\end{array}$

$\begin{array}{llll}C & -\mathbf{0 . 7 5 4 8 5 2} & \mathbf{0 . 3 6 2 9 8 8} & \mathbf{2 . 4 4 7 1 0 3}\end{array}$

$\begin{array}{llll}S & 2.126403 & -0.685146 & 1.299317\end{array}$

$\begin{array}{llll}\text { N } & 0.221672 & 1.699379 & -1.20828\end{array}$

N $-1.847440 \quad 1.029294-1.289742$

$\begin{array}{llll}C & 1.522892 & 1.961138 & -0.706716\end{array}$

$\begin{array}{llll}C & -0.393352 & 2.650183 & -2.13686\end{array}$

$\begin{array}{llll}C & -1.775690 & \mathbf{2 . 0 4 4 4 0 4} & \mathbf{- 2 . 3 4 9 7 6}\end{array}$

$\begin{array}{llll}\text { C } & -3.114615 & 0.464768 & -0.992575\end{array}$

$\begin{array}{llll}\text { C } & \mathbf{4 . 0 4 2 2 3 8} & \mathbf{2 . 4 9 7 0 1 0} & \mathbf{0 . 4 0 6 3 4 3}\end{array}$

$\begin{array}{llll}\text { C } & \mathbf{1 . 6 5 4 1 3 0} & \mathbf{2 . 6 9 8 3 1 8} & \mathbf{0 . 4 7 1 7 3 0}\end{array}$

$\begin{array}{llll}\text { C } \quad 2.673118 & 1.529260 & -1.379235\end{array}$ 
$\begin{array}{llll}C & 3.915821 & 1.804830 & -0.80115\end{array}$ $\begin{array}{llll}\text { C } & \mathbf{2 . 8 8 5 2 6 8} & 2.964677 & \mathbf{1 . 0 4 0 2 8 3}\end{array}$

$\begin{array}{llll}\text { H } & 4.816992 & 1.444642 & -1.30388\end{array}$

$\begin{array}{llll}\text { H } & \mathbf{2 . 9 3 0 4 7 2} & \mathbf{3 . 5 2 1 8 8 8} & \mathbf{1 . 9 7 7 8 6 7}\end{array}$

$\begin{array}{llll}C & -5.754934 & -0.385495 & -0.490563\end{array}$

C $-4.035588 \quad 1.274424-0.317539$

$\begin{array}{llll}C & -4.035588 & 1.274424 & -0.317539\end{array}$

C $-3.529985-0.796694-1.442411$

$\begin{array}{llll}C & -4.842595 & -1.200706 & -1.164336\end{array}$

$\begin{array}{llll}\text { C } & -5.335300 & 0.881158 & -0.066639\end{array}$

$\begin{array}{llll}\text { F } & -\mathbf{3 . 6 2 1 9 5 3} & \mathbf{2 . 4 7 1 6 3 5} & \mathbf{0 . 1 1 0 2 5 8}\end{array}$

$\begin{array}{llll}C & -2.594938 & -1.705062 & -2.182202\end{array}$

$\begin{array}{llll}\text { H } & -5.164709 & -2.187741 & -1.505968\end{array}$

$\begin{array}{llll}\text { H } & -\mathbf{6 . 0 0 1 4 3 9} & \mathbf{1 . 5 5 6 3 9 0} & \mathbf{0 . 4 7 3 3 1 1}\end{array}$

$\begin{array}{llll}\text { C } & -7.166216 & \mathbf{- 0 . 8 3 7 8 1 1} & \mathbf{- 0 . 2 2 9 0 9 3}\end{array}$

$\begin{array}{llll}\text { H } & -7.877763 & -0.254117 & -0.833471\end{array}$

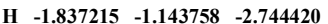

$\begin{array}{llll}\text { H } & -7.436612 & -\mathbf{0 . 6 8 9 7 5 5} & \mathbf{0 . 8 2 6 6 8 0}\end{array}$

$\begin{array}{llll}\text { H } & -7.301910 & -\mathbf{1 . 8 9 9 2 2 9} & \mathbf{- 0 . 4 7 5 1 4 8}\end{array}$

\begin{tabular}{lllll}
\hline & -3.146974 & -2.356172 & -2.872559
\end{tabular}

$\begin{array}{llll}\text { H } & -\mathbf{2 . 0 4 5 8 4 0} & -\mathbf{2 . 3 4 6 5 3 8} & -\mathbf{1 . 4 7 5 6 2 9}\end{array}$

$\begin{array}{llll}\text { F } & 0.545412 & 3.141262 & 1.072297\end{array}$

$\begin{array}{lllll}\text { C } & 5.391943 & \mathbf{2 . 7 0 6 7 6 0} & \mathbf{1 . 0 3 8 1 9 7}\end{array}$

H $\quad 6.197899 \quad 2.643112 \quad 0.294383$

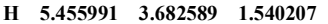

$\begin{array}{lllll}\text { H } & 5.578049 & 1.931888 & 1.799301\end{array}$

$\begin{array}{lllll}\text { C } & \mathbf{2 . 5 6 6 5 7 5} & \mathbf{0 . 7 9 0 5 9 6} & \mathbf{- 2 . 6 8 2 7 1 8}\end{array}$

$\begin{array}{llll}\text { H } & 2.383169 & 1.491796 & -3.513505\end{array}$

$\begin{array}{llll}\text { H } & 3.495631 & \mathbf{0 . 2 4 6 8 2 9} & \mathbf{- 2 . 8 9 8 8 6 1}\end{array}$

$\begin{array}{llll}\text { H } & \mathbf{1 . 7 3 9 4 3 6} & \mathbf{0 . 0 6 7 2 5 3} & \mathbf{- 2 . 6 5 5 6 1 3}\end{array}$

$\begin{array}{llll}C & \mathbf{2 . 3 3 9 3 9 2} & \mathbf{- 2 . 2 0 5 0 3 1} & \mathbf{- 0 . 9 8 0 6 8 3}\end{array}$

$\begin{array}{llll}C & 3.097018 & -2.929828 & -1.925714\end{array}$

$\begin{array}{llll}C & 3.013287 & -1.648025 & 0.125234\end{array}$

$\begin{array}{llll}C & 4.465786 & -3.094202 & -1.773342\end{array}$

$\begin{array}{llll}\text { H } & \mathbf{2} 586517 & \mathbf{3} 360493 & -2.790869\end{array}$

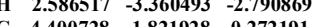

$\begin{array}{llll}\text { C } & 4.400728 & -1.821928 & 0.272191 \\ \text { C } & \mathbf{1 . 1 2 7 3 1 4} & -2.53782 & -0.667914\end{array}$

$\begin{array}{llll}C & \mathbf{5 . 1 2 7 3 1 4} & \mathbf{- 2 . 5 3 7 8 2 6} & \mathbf{- 0 . 6 6 7 9 1 4}\end{array}$

$\begin{array}{rrrr}\text { H } & \mathbf{5 . 0 2 9 2 7 6} & \mathbf{- 3 . 6 5 9 2 5 2} & \mathbf{- 2 . 5 1 9 9 6 1} \\ \text { H } & \mathbf{4 . 9 0 1 4 4 5} & \mathbf{- 1 . 3 7 5 9 9 7} & \mathbf{1 . 1 3 5 7 0 8}\end{array}$

$\begin{array}{llll}\text { H } & \mathbf{6 . 2 0 5 0 7 2} & -\mathbf{2 . 6 6 6 4 5 6} & \mathbf{- 0 . 5 4 6 6 1 5}\end{array}$

$\begin{array}{lllll}\text { H } & \mathbf{- 1 . 6 9 4 9 9 1} & \mathbf{- 2 . 6 4 2 8 7 4} & \mathbf{1 . 1 2 8 5 7 8}\end{array}$

$\begin{array}{lllll}\text { C } & -1.706132 & -0.689591 & 2.007167\end{array}$

$\begin{array}{llll}\text { H } & -1.211982 & 1.352256 & 2.296553\end{array}$

$\begin{array}{lllll}\text { C } & \mathbf{0 . 0 2 2 5 9 4} & \mathbf{0 . 3 6 4 9 8 7} & \mathbf{3 . 7 4 7 0 0 7}\end{array}$

$\begin{array}{llll}C & -3.205006 & -0.571824 & 2.244025\end{array}$

\begin{tabular}{llll}
\hline & $\mathbf{- 3 . 3 9 8 2 0 9}$ & $\mathbf{- 0 . 9 1 6 6 9 9}$ & $\mathbf{3 . 2 7 1 3 5 7}$
\end{tabular}

\begin{tabular}{llll}
\hline & -3.534777 & $\mathbf{0 . 4 7 2 0 7 6}$ & $\mathbf{2 . 1 7 0 4 6 6}$
\end{tabular}

$\begin{array}{llll}\text { H } & \mathbf{- 3 . 7 9 5 9 6 7} & \mathbf{- 1 . 1 8 9 7 2 9} & \mathbf{1 . 5 5 2 2 3 2}\end{array}$

$\begin{array}{llll}\text { H } & -1.756740 & -0.446130 & 0.729628\end{array}$

$\begin{array}{llll}\text { C } & -0.309897 & -2.832616 & \mathbf{2 . 7 6 8 5 0 3}\end{array}$

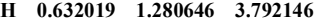

$\begin{array}{lllll}\text { H } & 0.717457 & \mathbf{- 0 . 4 7 5 9 7 1} & \mathbf{3 . 8 6 2 3 0 1}\end{array}$

$\begin{array}{lllll}\text { H } & -0.645425 & 0.375108 & 4.627046\end{array}$

$\begin{array}{llll}\text { H } & 0.355359 & -2.256862 & 3.416188\end{array}$

\begin{tabular}{llll}
\hline & 0.355359 & -2.256862 & 3.416188 \\
\hline & 0.292995 & -3.587233 & 2.244452
\end{tabular}

$\begin{array}{llll}\text { H } & 0.292995 & \mathbf{3 . 5 8 7 2 3 3} & \mathbf{2 . 2 4 4 4 5 2}\end{array}$

\begin{tabular}{rrrr}
\hline & -1.033819 & $\mathbf{- 3 . 3 6 9 5 6 1}$ & $\mathbf{3 . 4 0 7 1 4 0}$
\end{tabular}

$\begin{array}{llll}\text { H } & \mathbf{- 0 . 4 3 5 5 8 7} & \mathbf{3 . 6 4 7 2 7 3} & \mathbf{- 1 . 6 7 0 8 7 5}\end{array}$

\begin{tabular}{lllll} 
H & $\mathbf{0 . 1 8 9 6 3 9}$ & $\mathbf{2 . 7 2 1 6 6 8}$ & $\mathbf{- 3 . 0 6 4 6 5 4}$ \\
\hline & -1.881547 & $\mathbf{1 . 5 5 6 8 1 5}$ & $\mathbf{3 . 3 3 1 3 4 6}$
\end{tabular}

$\begin{array}{lllll}\text { H } & -1.881547 & 1.556815 & -3.331346\end{array}$

$\begin{array}{llll}\text { H } & -2.582350 & 2.779928 & -2.237170\end{array}$

74

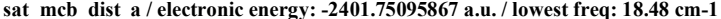

Ru $0.3 \overline{2} 913 \overline{8} \quad-\mathbf{0 . 6 7 4 6 3 6} \quad \mathbf{0 . 2 5 9 7 1 0}$

$-0.636710-1.937297-1.552174$

$\begin{array}{llll}C & -2.310555 & -2.080078 & -1.053704\end{array}$

$\begin{array}{llll}C & -2.712543 & -1.661778 & 0.227068\end{array}$

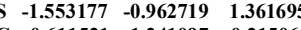

$\begin{array}{llll}C & 0.611521 & 1.241097 & -0.215061\end{array}$

$\begin{array}{llll}\mathrm{N} & -\mathbf{0 . 3 2 8 8 9 5} & \mathbf{2 . 2 0 0 1 2 9} & \mathbf{- 0 . 3 1 1 7 2 0}\end{array}$

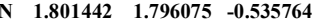

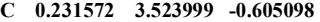

$\begin{array}{llll}\text { C } & \mathbf{1 . 7 0 3 1 2 4} & \mathbf{3 . 2 1 5 4 4 4} & \mathbf{- 0 . 8 8 8 1 8 3}\end{array}$

$\begin{array}{lrrr}C & -1.734154 & 2.028947 & -0.212280\end{array}$

$\begin{array}{llll}C & -2.387140 & \mathbf{2 . 3 4 1 1 8 3} & \mathbf{0 . 9 7 6 4 5 1}\end{array}$

C $-2.488204-1.601002-1.317192$

$\begin{array}{llll}C & -3.751763 & \mathbf{2 . 1 6 9 5 0 5} & \mathbf{1 . 1 3 6 3 8 8}\end{array}$

$\begin{array}{llll}C & \mathbf{- 3 . 8 6 4 2 3 6} & \mathbf{1 . 4 3 1 9 4 1} & \mathbf{- 1 . 1 5 1 7 1 4}\end{array}$

$\begin{array}{llll}C & -4.508313 & 1.691868 & 0.062472\end{array}$

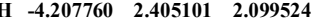

$\begin{array}{llll}\text { H } & -4.454528 & 1.077355 & -2.000559\end{array}$

$\begin{array}{llll}\text { C } & -\mathbf{5 . 9 8 3 1 8 9} & \mathbf{1 . 4 3 7 0 2 9} & \mathbf{0 . 2 1 2 4 3 2}\end{array}$

$\begin{array}{lllll}\text { F } & -1.664560 & 2.812968 & 1.996937\end{array}$

C $-1.821348 \quad 1.329629 \quad-2.635795$

\begin{tabular}{llll}
$C$ & -1.821348 & 1.329629 & -2.635795 \\
\hline & -1.263508 & 0.380934 & -2.590624
\end{tabular}

$\begin{array}{lll}-1.263508 & 0.380934-2.590624\end{array}$

$\begin{array}{llll}\text { H } & -2.565124 & 1.253982 & -3.439541\end{array}$

$\begin{array}{lllll}\text { H } & -1.105980 & 2.123554 & -2.897509\end{array}$

$\begin{array}{llll}\text { C } & 3.011892 & 1.082339 & -0.714900\end{array}$

$\begin{array}{llll}C & 4.031169 & 1.207173 & 0.231057\end{array}$

$\begin{array}{llll}C & 3.224209 & 0.268522 & -1.841322\end{array}$

$\begin{array}{llll}C & 5.209065 & 0.485641 & 0.148344\end{array}$

$\begin{array}{llll}C & 4.416863 & -0.455880 & -1.920838\end{array}$

$\begin{array}{llll}C & 5.406797 & -0.377395 & -0.934861\end{array}$

\begin{tabular}{llll}
\hline & 5.961699 & 0.606730 & 0.929468
\end{tabular}

$\begin{array}{lllll}H & 5.579841 & -1.102200 & -2.786898\end{array}$ 
$\begin{array}{llll}C & 6.654549 & -1.213434 & -1.026743\end{array}$ $\begin{array}{llll}C & 2.179754 & 0.162493 & -2.917716\end{array}$

$\begin{array}{llll}\text { H } & 1.355446 & -0.499102 & -2.608097\end{array}$

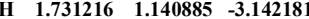

$\begin{array}{lllll}\text { H } & \mathbf{2} & .612655 & -0.244445 & -3.840344\end{array}$

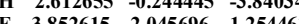

$\begin{array}{llll}\text { F } 3.852615 & 2.045696 & 1.254463\end{array}$

$\begin{array}{llll}\text { C } & 1.864086 & -1.851801 & 0.88748\end{array}$

$\begin{array}{llll}\text { H } & 2.559580 & -1.330423 & 0.188923\end{array}$

$\begin{array}{llll}C & \mathbf{1 . 2 8 5 8 4 6} & \mathbf{- 0 . 0 1 4 7 1 8} & \mathbf{2 . 0 9 2 0 2 3}\end{array}$

$\begin{array}{llll}\text { C } & 2.037340 & -1.343778 & 2.305749\end{array}$

$\begin{array}{llll}\text { H } & \mathbf{2 . 0 2 1 3 3 1} & \mathbf{0 . 7 5 3 4 3 3} & \mathbf{1 . 8 2 3 6 4 5}\end{array}$

$\begin{array}{llll}\text { C } & 0.489975 & 0.535702 & 3.262574\end{array}$

$\begin{array}{llll}\text { H } & 3.107195 & -1.190798 & 2.55830\end{array}$

$\begin{array}{llll}\text { C } & \mathbf{1 . 4 3 8 3 7 2} & \mathbf{- 2 . 2 6 7 6 2 5} & \mathbf{3 . 3 5 9 4 2}\end{array}$

$\begin{array}{llll}C & -3.285733 & -2.610241 & -1.921721\end{array}$

$\begin{array}{llll}C & -4.612165 & -2.715661 & -1.526738\end{array}$

$\begin{array}{llll}\text { H } & -5.352998 & -3.122097 & -2.219391\end{array}$

$\begin{array}{llll}C & -5.000650 & -2.303676 & -0.243825\end{array}$

$\begin{array}{llll}C & -6.043296 & -2.389261 & 0.070675\end{array}$

$\begin{array}{llll}C & -4.053552 & -1.780695 & 0.625472\end{array}$

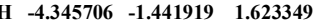

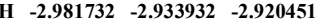

$\begin{array}{llll}\text { H } & -6.534779 & 1.744629 & -0.687497\end{array}$

$\begin{array}{llll}\text { H } & -6.402565 & 1.966808 & 1.078461\end{array}$

\begin{tabular}{cccc} 
H & $\mathbf{- 6 . 1 6 2 3 5 1}$ & $\mathbf{0 . 3 5 9 3 3 6}$ & $\mathbf{0 . 3 5 3 9 4 7}$ \\
\hline & $\mathbf{7 . 5 1 0 0 8 5}$ & -0.715008 & -0.550695
\end{tabular}

$\begin{array}{lll}-7.510085 & -0.715008-0.550695\end{array}$

$\begin{array}{llll}\text { H } & 6.912674 & -1.431212 & \mathbf{- 2 . 0 7 2 1 7 3}\end{array}$

H $6.505315-2.177391 \quad-0.514740$

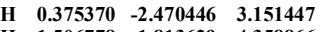

H $\quad 1.506778-1.813629 \quad 4.358866$

$\begin{array}{lrrr}\text { H } & 1.969190 & -3.231069 & 3.394829\end{array}$

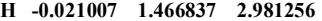

$\begin{array}{llll}\text { H } & 1.152703 & \mathbf{0 . 7 6 2 4 5 4} & \mathbf{4 . 1 1 8 6 1 1}\end{array}$

$\begin{array}{llll}H & -0.281647 & -0.159135 & 3.626541\end{array}$

\begin{tabular}{llll}
\hline & -0.281647 & -0.159135 & 3.626541 \\
$C$ & 1.837130 & -3.337545 & 0.601757
\end{tabular}

$\begin{array}{llll}\text { C } & 1.837130 & -3.337545 & \mathbf{0 . 6 0 1 7 5 7}\end{array}$

$\begin{array}{llll}\text { H } & 1.754632 & -3.541107 & -0.473311\end{array}$

$\begin{array}{lllll}\text { H } & \mathbf{0 . 9 9 9 6 5 2} & \mathbf{- 3 . 8 3 6 7 5 3} & \mathbf{1 . 1 0 9 2 2 6} \\ \text { H } & \mathbf{2 . 7 7 0 7 4 5} & \mathbf{- 3 . 7 9 5 7 6 8} & \mathbf{0 . 9 7 6 4 4 5}\end{array}$

H $\quad \begin{array}{llll}1.973179 & 3.361214 & -1.945743\end{array}$

$\begin{array}{lllll}\text { H } & \mathbf{2 . 3 8 7 5 3 1} & \mathbf{3 . 8 1 1 0 7 0} & \mathbf{- 0 . 2 6 9 3 3 4}\end{array}$

H $\quad-\mathbf{0 . 2 8 3 8 4 5} \quad 3.974867-1.464703$

H $\quad 0.097480 \quad 4.184088 \quad 0.264476$

74

sat ts PTR a / electronic energy: $-\mathbf{2 4 0 1 . 7 1 6 7 5 3 4 5}$ a.u. / lowest freq: $-\mathbf{1 4 4 . 9 1} \mathrm{cm}-1$

$\begin{array}{llll}\mathrm{Ru}^{-} \mathbf{0 . 1 9 2 6 0 3} & \mathbf{- 0 . 8 4 1 1 5 9} & \mathbf{- 0 . 0 5 3 3 2 5}\end{array}$

S $-1.095493-0.200125-1.854286$

$\begin{array}{llll}C & -2.677124 & -0.916233 & -1.605452\end{array}$

$\begin{array}{llll}\text { C } & -2.868743 & -1.835245 & -0.558329\end{array}$

$\begin{array}{lllll}\text { S } & -1.565411 & -2.213201 & 0.54551\end{array}$

$\begin{array}{llll}C & \mathbf{0 . 6 8 6 4 4 3} & 1.253705 & 0.019910\end{array}$

$\begin{array}{llll}\text { N } & -0.205501 & 2.211866 & 0.330874\end{array}$

$\begin{array}{llll}\text { N } & \mathbf{1 . 8 8 4 6 4 9} & \mathbf{1 . 8 4 8 7 8 1} & \mathbf{- 0 . 1 1 0 8 0 8}\end{array}$

$\begin{array}{lllll}\text { C } & \mathbf{0 . 4 0 8 7 2 3} & \mathbf{3 . 5 2 5 5 0 5} & \mathbf{0 . 5 5 7 7 3 0}\end{array}$

C $1.833304 \quad 3.309025 \quad 0.054563$

C 1.8333043 .3010250 .054563

$\begin{array}{llll}C & -1.587294 & 2.012633 & 0.59278\end{array}$

$\begin{array}{llll}\text { C } & -1.984713 & 1.456339 & 1.806799\end{array}$

$\begin{array}{llll}\text { C } & -2.570024 & \mathbf{2 . 3 8 0 1 3 6} & -\mathbf{0 . 3 4 0 7 1 0}\end{array}$

$\begin{array}{llll}C & -3.307157 & 1.151269 & \mathbf{2 . 0 8 4 0 7 1}\end{array}$

$\begin{array}{llll}C & -3.902102 & 2.081635 & -0.051428\end{array}$

$\begin{array}{llll}\text { C } & -4.286772 & 1.442218 & 1.132238\end{array}$

$\begin{array}{llll}\text { H } & -3.553761 & 0.679664 & 3.036841\end{array}$

\begin{tabular}{llll}
\hline & -4.666558 & $\mathbf{2 . 3 3 2 9 0 2}$ & $\mathbf{- 0 . 7 9 0 9 7 0}$
\end{tabular}

$\begin{array}{llll}C & -5.620249 & 1.042783 & 1.354510\end{array}$

$\begin{array}{llll}C & -5.720249 & 1.042783 & 1.354510\end{array}$

$\begin{array}{llll}C & -5.72052665 & 1.214310 & 2.731487\end{array}$

$\begin{array}{llll}\text { C } & -2.203674 & 3.088273 & -1.614974\end{array}$

$\begin{array}{llll}\text { H } & -1.205500 & 2.795477 & -1.964436\end{array}$

$\begin{array}{llll}\text { H } & -2.926049 & 2.856916 & -2.409135\end{array}$

$\begin{array}{lllll}\text { H } & -2.212063 & 4.180599 & -1.464725\end{array}$

$\begin{array}{llll}\text { C } & 3.104243 & 1.214640 & \mathbf{- 0 . 4 5 2 4 0 6}\end{array}$

$\begin{array}{llll}C & 4.101071 & 1.124766 & 0.521983\end{array}$

$\begin{array}{llll}\text { C } & 3.348178 & 0.683819 & -1.728170\end{array}$

$\begin{array}{llll}\text { C } & 5.310119 & 0.492947 & 0.293193\end{array}$

C $5.5667480 .033720-1.94$

$\begin{array}{llll}C & 4.566748 & 0.033720 & -1.949340\end{array}$

$\mathrm{C}$
$\mathbf{4}$

$\begin{array}{llll}\text { H } & 6.044979 & 0.441945 & 1.098409\end{array}$

$\begin{array}{lllll}\text { H } & 4.757289 & -0.393617 & -2.937222\end{array}$

$\begin{array}{lllll}C & 6.830668 & -0.822945 & -1.231097\end{array}$

$\begin{array}{llll}C & 2.320055 & 0.789620 & -2.817941\end{array}$

$\begin{array}{lllll}\text { H } & 1.553479 & 0.007539 & \mathbf{- 2 . 7 0 2 8 7 4}\end{array}$

$\begin{array}{lllll}\text { H } & \mathbf{1 . 7 9 6 3 7 7} & \mathbf{1 . 7 5 5 7 1 4} & \mathbf{- 2 . 7 8 5 5 2 8}\end{array}$

$\begin{array}{lllll}\text { H } & \mathbf{2 . 7 8 5 2 7 3} & \mathbf{0 . 6 7 3 6 1 0} & \mathbf{- 3 . 8 0 5 2 8 9}\end{array}$

$\begin{array}{llll}F & 3.849910 & 1.636613 & 1.731428\end{array}$

$\begin{array}{llll} & 3.849910 & 1.636613 & -3.8051428\end{array}$

C $1.379517-2.467606-0.054446$

$\begin{array}{lllll}\text { H } & 1.921549 & -2.431653 & -1.014478\end{array}$

$\begin{array}{llll}\text { C } & 1.022825 & -0.845373 & 1.850784\end{array}$

$\begin{array}{llll}\text { C } & 2.105530 & -1.616564 & 1.043719\end{array}$

$\begin{array}{llll}\text { H } & 1.406700 & 0.135303 & 2.163854\end{array}$

C $\quad 0.414493 \quad-1.554153 \quad 3.041992$

$\begin{array}{llll}\text { H } & \mathbf{2 . 7 6 9 7 3 3} & -\mathbf{0 . 9 0 5 8 2 2} & \mathbf{0 . 5 4 5 6 7 5}\end{array}$

$\begin{array}{llll}\text { C } & 3.033179 & -2.464332 & 1.931213\end{array}$

$\begin{array}{llll}\text { C } & -3.760067 & -0.556833 & -2.425778\end{array}$

C $-5.012202-1.124202-2.232881$

$\begin{array}{lllll}C & -5.012202 & -1.124202 & -2.232881 \\ H & -5.846256 & -0.839020 & -2.878019\end{array}$ 


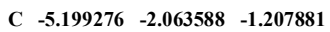
H $\quad-6.181168-2.516858-1.052116$ $\begin{array}{llll}C & -4.140604 & -2.415469 & -0.382400\end{array}$ $\begin{array}{llll}\text { H } & -4.287733 & -3.140246 & 0.422638\end{array}$ $\begin{array}{llll}\text { H } & -3.603557 & 0.178894 & \mathbf{- 3 . 2 1 9 1 3 6}\end{array}$ $\begin{array}{llll}-6.414817 & 1.795950 & 0.955823\end{array}$ \begin{tabular}{llll}
4 & -6.414817 & 1.795950 & 0.955823 \\
\hline & -5.939050 & 0.893787 & 2.420757
\end{tabular} $\begin{array}{lllll}\text { H } & -5.939050 & 0.893787 & 2.420757\end{array}$ $\begin{array}{llll}\text { H } & \mathbf{- 5 . 9 2 4 8 8 3} & \mathbf{0 . 0 9 3 7 7 5} & \mathbf{0 . 8 3 2 8 1 6}\end{array}$ $\begin{array}{llll}\text { H } & 7.640249 & -0.484191 & -0.570538\end{array}$ $\begin{array}{lllll}\text { H } & 7.153334 & -0.695835 & -2.273789\end{array}$ H $\quad 6.689383-1.901773-1.058517$ H $\quad 2.486693 \quad-3.233581 \quad 2.492296$

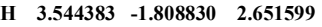
$\begin{array}{llll}\text { H } & 3.794898 & -2.961152 & 1.312403\end{array}$ $\begin{array}{llll}\text { H } & -\mathbf{0 . 4 3 6 3 2 1} & \mathbf{- 0 . 9 7 6 0 4 0} & \mathbf{3 . 4 2 4 8 6 3}\end{array}$ H $\quad 1.148064 \quad-1.642957 \quad 3.863124$ $\begin{array}{lllll}\text { H } & 0.051863 & -2.564450 & \mathbf{2 . 8 0 6 7 6 4}\end{array}$ $\begin{array}{llll}\text { C } & \mathbf{1 . 0 4 9 2 1 8} & \mathbf{- 3 . 8 9 8 4 4 3} & \mathbf{0 . 3 0 2 3 4 1}\end{array}$

$\begin{array}{llll}\text { H } & \mathbf{0 . 3 8 7 8 8 7} & \mathbf{- 4 . 3 2 9 9 6 8} & \mathbf{- 0 . 4 6 2 8 4 1}\end{array}$

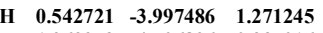
H $\quad \begin{array}{llll}\text { 1.969273 } & -4.506826 & 0.337016\end{array}$ $\begin{array}{llll}\text { H } & \mathbf{2 . 0 2 2 6 1 2} & \mathbf{3 . 7 9 5 4 6 4} & \mathbf{- 0 . 9 1 4 9 7 7}\end{array}$ H $\quad \mathbf{2 . 5 9 4 5 1 0} \quad \mathbf{3 . 6 4 4 3 2 1} \mathbf{0 . 7 7 0 2 1 2}$ $\begin{array}{lllll}\text { H } & -\mathbf{0 . 1 2 7 3 0 3} & 4.307923 & \mathbf{0 . 0 0 4 0 2 5}\end{array}$ $\begin{array}{llll}\text { H } & 0.368897 & 3.768520 & 1.630825\end{array}$ 62

sat_14e1 / electronic energy: -2244.66194430 a.u. / lowest freq: $19.44 \mathrm{~cm}-1$

S $0.751619-1.626323 \quad 1.094274$

$\begin{array}{lllll}C & -0.853320 & 0.960647 & 0.464477\end{array}$

$\begin{array}{llll}\text { Ru } & \mathbf{0 . 0 1 1 4 2 3} & \mathbf{- 0 . 4 5 6 2 8 6} & \mathbf{- 0 . 7 2 3 3 5 4}\end{array}$

C $-1.384994 \quad-1.515916 \quad-1.110362$

$\begin{array}{llll}\text { S } & 1.984431 & -1.049467 & -1.825414\end{array}$

$\begin{array}{lllll}\text { N } & -2.109428 & 1.186086 & 0.874191\end{array}$

$\begin{array}{lllll}\text { N } & -0.070946 & 1.937943 & 0.964047\end{array}$

C $-3.249567 \quad 0.412787 \quad 0.544485$

$\begin{array}{lllll}C & -3.24967 & 0.412787 & 0.544485\end{array}$

$\begin{array}{llll}C & -2.227484 & 2.371909 & 1.734263\end{array}$

$\begin{array}{llll}C & -0.833203 & 2.998062 & 1.630346\end{array}$

$\begin{array}{llll}C & 1.270382 & 2.122236 & 0.530968\end{array}$

$\begin{array}{llll}\text { H } & \mathbf{- 0 . 8 2 5 3 0 8} & \mathbf{3 . 9 0 8 0 1 9} & \mathbf{1 . 0 1 0 1 6 7}\end{array}$

\begin{tabular}{llll}
\hline & $-\mathbf{0 . 3 9 9 3 7 0}$ & $\mathbf{3 . 2 3 9 8 8 8}$ & $\mathbf{2 . 6 0 9 7 3 1}$
\end{tabular}

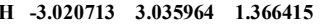

$\begin{array}{llll}\text { C } & -5.562403 & -1.007732 & -0.183995\end{array}$

$\begin{array}{llll}\text { C } & -4.087310 & 0.861811 & \mathbf{- 0 . 4 7 7 5 3 7}\end{array}$

$\begin{array}{llll}C & \mathbf{- 3 . 5 7 8 9 7 7} & \mathbf{- 0 . 7 6 0 8 8 5} & \mathbf{1 . 2 3 4 9 4 0}\end{array}$

$\begin{array}{llll}C & -3.578977 & -0.760885 & 1.234940 \\ \text { C } & -4.728663 & -1.456676 & 0.844998\end{array}$

$\begin{array}{rrrr}C & -4.728663 & -1.456676 & 0.844998 \\ \text { C } & -\mathbf{5 . 2 3 2 1 1 0} & \mathbf{0 . 1 7 9 4 9 5} & \mathbf{- 0 . 8 4 9 0 0 2}\end{array}$

$\begin{array}{llll}\text { H } & -\mathbf{4 . 9 8 4 0 5 6} & \mathbf{- 2 . 3 8 0 6 2 4} & \mathbf{1 . 3 6 9 9 3 8}\end{array}$

$\begin{array}{llll}\text { H } & -5.847004 & 0.571615 & -1.661223\end{array}$

$\begin{array}{llll}C & \mathbf{3 . 8 7 2 5 3 3} & \mathbf{2 . 4 2 3 8 9 9} & \mathbf{- 0 . 4 8 3 8 6 0}\end{array}$

$\begin{array}{llll}C & \mathbf{1 . 5 0 1 1 7 7} & \mathbf{2 . 6 3 8 1 7 7} & \mathbf{- 0 . 7 4 6 1 0 9}\end{array}$

$\begin{array}{llll}\text { C } & \mathbf{2 . 3 6 6 5 9 6} & \mathbf{1 . 7 9 3 4 7 5} & \mathbf{1 . 3 4 4 5 6 1}\end{array}$

$\begin{array}{llll}C & 3.649025 & 1.942180 & 0.811524\end{array}$

$\begin{array}{llll}C & 2.773904 & 2.794043 & -1.264579\end{array}$

$\begin{array}{llll}C & 2.773904 & 2.794043 & -1.264579\end{array}$

$\begin{array}{llll}\text { F } & 0.442575 & 2.966855 & \mathbf{- 1 . 4 9 6 3 6 8} \\ \text { C } & 2.152262 & 1.27003 & 2.735284\end{array}$

$\begin{array}{llll}\text { C } & \mathbf{2 . 1 5 2 2 6 2} & 1.270903 & \mathbf{2 . 7 3 5 2 8 4}\end{array}$

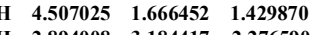

$\begin{array}{lllll}\text { H } & \mathbf{2 . 8 9 4 0 0 8} & \mathbf{3 . 1 8 4 4 1 7} & \mathbf{- 2 . 2 7 6 5 9 0}\end{array}$

$\begin{array}{llll}C & 5.266759 & \mathbf{2 . 5 0 6 5 2 6} & \mathbf{- 1 . 0 4 3 1 6 8}\end{array}$

$\begin{array}{lllll}\text { H } & 5.354805 & 3.305217 & -1.792547\end{array}$

H $3.110259 \quad 1.129545 \quad 3.251993$

$\begin{array}{llll}\text { H } & -2.478332 & \mathbf{2 . 0 6 5 6 3 5} & \mathbf{2 . 7 6 1 7 0 1}\end{array}$

$\begin{array}{llll}\text { H } & \mathbf{5 . 5 2 8 2 3 8} & \mathbf{1 . 5 5 7 1 4 8} & \mathbf{- 1 . 5 3 7 2 3 7}\end{array}$

$\begin{array}{llll}\text { H } & 6.008559 & 2.683002 & -0.252063\end{array}$

\begin{tabular}{llll}
$H$ & 6.008559 & 2.683002 & -0.252063 \\
\hline
\end{tabular}

$\begin{array}{lllll}H & 1.628777 & 0.302840 & \mathbf{2 . 6 8 8 5 3 9}\end{array}$

$\begin{array}{llll}\text { H } & 1.534833 & 1.957709 & 3.333628\end{array}$

$\begin{array}{llll} & -3.749589 & 1.982049 & -1.122311\end{array}$

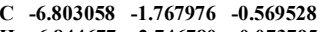

$\begin{array}{lllll}\text { H } & -6.844677 & -2.746780 & -0.073795\end{array}$

$\begin{array}{lllll}\text { H } & -7.706848 & -1.205837 & -0.287051\end{array}$

$\begin{array}{llll}\text { H } & -6.846464 & -1.928590 & -1.656754\end{array}$

$\begin{array}{llll}\text { C } & -2.696739 & -1.269581 & 2.338196\end{array}$

$\begin{array}{llll}\text { C } & -1.796138 & -1.747422 & \mathbf{1 . 9 2 1 9 4 1}\end{array}$

$\begin{array}{lll}-1.796138 & -1.747422 & 1.921941\end{array}$

\begin{tabular}{lllll}
\hline & -2.352438 & -0.454867 & $\mathbf{2 . 9 9 1 1 4 5}$
\end{tabular}

\begin{tabular}{llll}
\hline & -3.223889 & -2.010366 & 2.953099
\end{tabular}

$\begin{array}{llll}C & \mathbf{2 . 3 5 3 7 6 8} & \mathbf{- 2 . 2 2 7 7 2 9} & \mathbf{0 . 6 4 7 7 4 8}\end{array}$

$\begin{array}{llll}C & 3.106435 & -2.947054 & 1.589672\end{array}$

$\begin{array}{llll}C & 2.886921 & -1.993089 & -0.631028\end{array}$

$\begin{array}{llll}C & 4.369255 & -3.433234 & 1.268777\end{array}$

$\begin{array}{llll}\text { H } & \mathbf{2 . 6 8 6 7 2 4} & \mathbf{- 3 . 1 1 9 7 4 4} & \mathbf{2 . 5 8 4 7 2 8}\end{array}$

$\begin{array}{llll}C & 4.158644 & -2.497467 & \mathbf{- 0 . 9 4 6 2 0 7}\end{array}$

$\begin{array}{llll}C & 4.898114 & -3.209123 & -0.006729\end{array}$

$\begin{array}{llll}C & 4.943058 & -3.989445 & 2.013682\end{array}$

$\begin{array}{llll}\mathrm{H} & 4.943058 & -3.989445 & 2.013682\end{array}$

\begin{tabular}{lllll} 
H & 4.567676 & -2.317897 & -1.944124 \\
\hline
\end{tabular}

$\begin{array}{llll}H & 5.888284 & -3.589551 & -0.267812\end{array}$

$\begin{array}{lllll}\text { H } & -1.627855 & -2.439286 & -0.556881\end{array}$

$\begin{array}{lllll}C & -2.179802 & -1.275574 & -2.358322\end{array}$

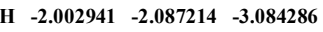

$\begin{array}{llll}\text { H } & -3.256591 & -1.277543 & -2.120843\end{array}$

$\begin{array}{llll}\text { H } & -1.942175 & -0.316082 & -2.848020\end{array}$

74

sat_pc1_a / electronic energy: $-\mathbf{2 4 0 1 . 7 4 9 9 9 2 3 0}$ a.u. / lowest freq: $10.99 \mathrm{~cm}-1$

S $\begin{array}{llll}0.353549 & 1.733810 & -1.393445\end{array}$

C $-0.369468-1.091101-0.768617$ 
$\begin{array}{lllll}\text { Ru } & 0.034471 & 0.465834 & 0.549556\end{array}$ $\begin{array}{llll}C & 0.652193 & -1.170076 & 2.067929\end{array}$

$\begin{array}{llll}\text { C } & -1.674441 & 1.044901 & 0.878871\end{array}$

$\begin{array}{llll}\text { S } & 1.271117 & 2.153655 & 1.665330\end{array}$

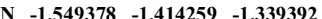

$\begin{array}{llll}\mathrm{N} & \mathbf{0 . 5 7 7 7 5 1} & -1.849621 & -1.367465\end{array}$

C $-2.824264-1.064761-0.829361$

C $-1.456950-2.531472-2.286872$

$\begin{array}{llll}C & 1.912855 & -2.054356 & \mathbf{- 0 . 9 2 1 4 9 4}\end{array}$

H $\quad 0.379421 \quad-3.739915 \quad-2.306882$

H $\quad 0.422204-2.342546 \quad-3.414831$

$\begin{array}{lrrr}\text { H } & -\mathbf{1 . 9 4 0 0 7 1} & \mathbf{- 3 . 4 2 3 1 1 8} & \mathbf{- 1 . 8 5 7 8 0 2} \\ \mathrm{C} & \mathbf{- 5 . 3 5 5 3 5 0} & \mathbf{- 0 . 5 0 7 8 6 3} & \mathbf{0 . 2 5 9 5 8 7}\end{array}$

$\begin{array}{llll}C & -3.324052 & -1.780189 & 0.260163\end{array}$

$\begin{array}{llll}C & -3.324052 & -1.780189 & 0.260163\end{array}$

$\begin{array}{llll}C & -3.615593 & -0.067810 & -1.411994\end{array}$

$\begin{array}{llll}C & -4.871389 & 0.191477 & -0.850316\end{array}$

$\begin{array}{llll}C & -4.560446 & -1.516451 & 0.819124\end{array}$

$\begin{array}{lllll}\text { H } & -5.490301 & 0.975646 & -1.293814\end{array}$

H $-4.893246 \quad-2.097021 \quad 1.681486$

$\begin{array}{llll}\text { C } & 4.484128 & -2.677175 & 0.061358\end{array}$

$\begin{array}{llll}C & \mathbf{2 . 1 3 7 0 9 1} & \mathbf{- 3 . 1 3 2 4 5 8} & \mathbf{- 0 . 0 5 2 7 6 5}\end{array}$

$\begin{array}{llll}C & 3.019377 & -1.326649 & -1.377248\end{array}$

$\begin{array}{llll}C & 4.282068 & -1.642652 & -0.850894\end{array}$

$\begin{array}{llll}C & 3.380813 & -3.450158 & 0.449482\end{array}$

$\begin{array}{llll}C & 1.081165 & -3.876073 & 0.30635\end{array}$

$\begin{array}{llll}C & \mathbf{2 . 9 0 8 8 3 0} & \mathbf{- 0 . 2 6 4 5 3 4} & \mathbf{- 2 . 4 3 2 9 4 7}\end{array}$

$\begin{array}{llll}\text { H } & 5.142405 & -1.060298 & -1.190937\end{array}$

$\begin{array}{llll}\text { H } & 3.479471 & -4.287881 & 1.142098\end{array}$

$\begin{array}{llll}\text { C } & 5.847264 & -2.973591 & 0.624961\end{array}$

$\begin{array}{llll}\text { H } & 6.090143 & -4.042101 & 0.528825\end{array}$

H $3.673626 \quad-0.429200 \quad-3.206018$

$\begin{array}{llll}\text { H } & -1.960204 & -2.284347 & -3.231244\end{array}$

$\begin{array}{llll}\text { H } & 5.883351 & -2.725799 & 1.697254\end{array}$

\begin{tabular}{llll} 
H & $\mathbf{5 . 8 8 3 3 5 1}$ & $\mathbf{- 2 . 7 2 5 7 9 9}$ & $\mathbf{1 . 6 9 7 2 5 4}$ \\
\hline & 6.628472 & -2393001 & $\mathbf{0 . 1 1 6 6 4 1}$
\end{tabular}

\begin{tabular}{ccccc} 
H & 6.628472 & -2.393001 & 0.116641 \\
\hline
\end{tabular}

\begin{tabular}{lllll} 
H & 3.068669 & 0.733099 & -1.999795 \\
\hline
\end{tabular}

$\begin{array}{crrrr}\text { H } & \mathbf{1 . 9 1 8 3 6 2} & \mathbf{- 0 . 2 4 9 4 7 3} & \mathbf{- 2 . 9 0 0 6 3 7} \\ \text { F } & \mathbf{- 2 . 5 5 3 1 0 6} & \mathbf{- 2 . 7 3 5 8 0 1} & \mathbf{0 . 7 9 3 8 3 4}\end{array}$

\begin{tabular}{llll}
\hline & -6.695358 & -0.183810 & 0.863792
\end{tabular}

$\begin{array}{llll}\text { H } & \mathbf{- 6 . 5 6 8 7 2 1} & 0.333769 & \mathbf{1 . 8 2 7 5 7 9}\end{array}$

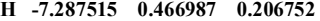

$\begin{array}{llll}\text { H } & -7.273043 & -1.098939 & 1.059016\end{array}$

$\begin{array}{llll}\text { C } & -\mathbf{3 . 0 9 4 6 1 7} & \mathbf{0 . 7 2 4 2 4 7} & \mathbf{- 2 . 5 7 5 8 9 3}\end{array}$

$\begin{array}{llll}\text { H } & -2.786966 & 0.066258 & -3.402524\end{array}$

$\begin{array}{llll}\text { H } & -3.855864 & 1.419582 & -2.952568\end{array}$

$\begin{array}{lllll}\text { H } & -2.204407 & 1.301551 & -2.276882\end{array}$

$\begin{array}{lllll}\text { C } & \mathbf{1 . 0 7 6 6 1 9} & \mathbf{3 . 2 5 2 0 2 5} & \mathbf{- 0 . 8 6 7 5 7 5}\end{array}$

$\begin{array}{lllll}C & 1.267203 & 4.293284 & -1.792887\end{array}$

$\begin{array}{llll}\text { C } & 1.472900 & 3.435844 & 0.470367\end{array}$

$\begin{array}{llll}C & 1.845007 & 5.496835 & -1.405336\end{array}$

$\begin{array}{lllll}\text { H } & 0.953569 & 4.142818 & \mathbf{- 2 . 8 2 9 5 3 9}\end{array}$

$\begin{array}{lllll}\text { C } & \mathbf{2 . 0 5 1 3 7 2} & \mathbf{4 . 6 6 0 3 2 0} & \mathbf{0 . 8 4 9 7 2 7}\end{array}$

$\begin{array}{llll}\text { C } & 2.239068 & 5.681346 & -0.075127\end{array}$

\begin{tabular}{cccc} 
H & 1.985707 & $\mathbf{6 . 2 9 4 0 0 2}$ & -2.139134 \\
\hline
\end{tabular}

\begin{tabular}{cccc}
\hline & $\mathbf{2 . 3 5 3 7 8 7}$ & $\mathbf{4 . 8 0 0 7 9 7}$ & $\mathbf{1 . 8 9 0 9 6 7}$
\end{tabular}

$\begin{array}{cccc}\text { H } & \mathbf{2 . 6 9 1 0 9 2} & \mathbf{6 . 6 2 4 5 2 9} & \mathbf{0 . 2 4 0 6 8 6}\end{array}$

$\begin{array}{llll}C & \mathbf{2 . 0 5 0 1 0 2} & \mathbf{- 0 . 9 9 1 3 9 5} & \mathbf{2 . 5 9 7 3 1 3}\end{array}$

$\begin{array}{lllll}\text { H } & 0.499782 & -2.085901 & 1.497431\end{array}$

$\begin{array}{llll}\text { C } & -0.496658 & -0.601901 & 2.578988\end{array}$

$\begin{array}{llll}\text { H } & -2.414701 & 0.443479 & 1.435371\end{array}$

$\begin{array}{llll}\text { C } & -2.213598 & 2.412946 & \mathbf{0 . 6 2 5 3 3 9}\end{array}$

$\begin{array}{lllll}\text { H } & -\mathbf{3 . 2 2 2 6 7 7} & \mathbf{2 . 3 4 2 1 3 4} & \mathbf{0 . 1 8 4 8 1 4}\end{array}$

$\begin{array}{llll}\text { H } & -2.341097 & 2.889393 & 1.615782\end{array}$

$\begin{array}{llll}\text { H } & -1.574117 & 3.054172 & 0.010174\end{array}$

$\begin{array}{llll}\text { H } & \mathbf{2 . 7 8 4 0 4 5} & \mathbf{- 0 . 9 3 4 0 5 7} & \mathbf{1 . 7 8 0 0 8 8}\end{array}$

$\begin{array}{llll}\text { H } & \mathbf{2 . 3 1 4 5 3 1} & \mathbf{- 1 . 8 7 0 6 6 2} & \mathbf{3 . 2 0 9 3 6 7}\end{array}$

$\begin{array}{llll}\text { H } & \mathbf{2 . 1 6 0 4 0 4} & \mathbf{- 0 . 0 9 1 2 0 4} & \mathbf{3 . 2 1 3 8 0 1}\end{array}$

$\begin{array}{llll}\text { H } & -1.427298 & -1.116751 & 2.324778\end{array}$

$\begin{array}{llll}\text { C } & \mathbf{- 0 . 5 8 8 4 4 7} & \mathbf{0 . 2 8 7 6 8 0} & \mathbf{3 . 7 8 7 1 5 3}\end{array}$

$\begin{array}{lllll}\text { H } & 0.332956 & 0.852904 & 3.972274\end{array}$

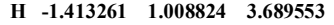

$\begin{array}{llll}\text { H } & -0.797276 & -0.330710 & 4.676424\end{array}$

74

sat ts1 a / electronic energy: -2401.74131860 a.u. / lowest freq: $-\mathbf{1 1 9 . 8 2} \mathrm{cm}-1$

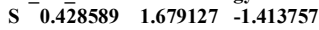

$\begin{array}{llll}C & -0.490804 & -1.112160 & -0.735872\end{array}$

$\begin{array}{lllll}\text { Ru } & 0.130037 & 0.430026 & 0.511223\end{array}$

$\begin{array}{llll}\text { C } & 0.620431 & -1.055469 & 2.030178\end{array}$

$\begin{array}{llll}\text { C } & -1.544602 & 0.935362 & 1.125511\end{array}$

$\begin{array}{llll}\mathrm{S} & \mathbf{1 . 5 3 8 9 0 4} & \mathbf{2 . 0 8 2 2 6 4} & \mathbf{1 . 5 7 0 4 1 2}\end{array}$

$\begin{array}{llll}\text { N } & -1.684497 & -1.402995 & -1.301246\end{array}$

$\begin{array}{llll}\text { N } & 0.409189 & -1.957412 & -1.298831\end{array}$

$\begin{array}{llll}C & -2.947824 & -0.944492 & -0.852530\end{array}$

$\begin{array}{llll}C & -1.653373 & -2.558768 & -2.204965\end{array}$

$\begin{array}{llll}C & -0.159172 & -2.841263 & -2.321588\end{array}$

$\begin{array}{llll}C & \mathbf{1 . 7 5 1 8 7 0} & -\mathbf{2 . 1 7 6 2 7 1} & \mathbf{- 0 . 8 8 5 1 4 7}\end{array}$

$\begin{array}{llll}\text { H } & 0.092799 & -3.889356 & -2.109720\end{array}$

$\begin{array}{lllll}\text { H } & 0.248868 & -2.581240 & -3.310610\end{array}$

$\begin{array}{llll}\text { H } & \mathbf{- 2 . 2 0 7 0 7 0} & \mathbf{- 3 . 3 9 7 4 7 9} & \mathbf{- 1 . 7 5 5 4 5 1}\end{array}$

$\begin{array}{llll}C & -5.435470 & -0.114165 & 0.161072\end{array}$

$\begin{array}{llll}\text { C } & -\mathbf{3 . 5 6 3 8 1 7} & -1.612061 & \mathbf{0 . 2 0 4 3 6 8}\end{array}$

$\begin{array}{llll}C & -3.605298 & 0.140148 & -1.451117\end{array}$

$\begin{array}{llll}C & -4.835963 & 0.538784 & -0.923635\end{array}$ 


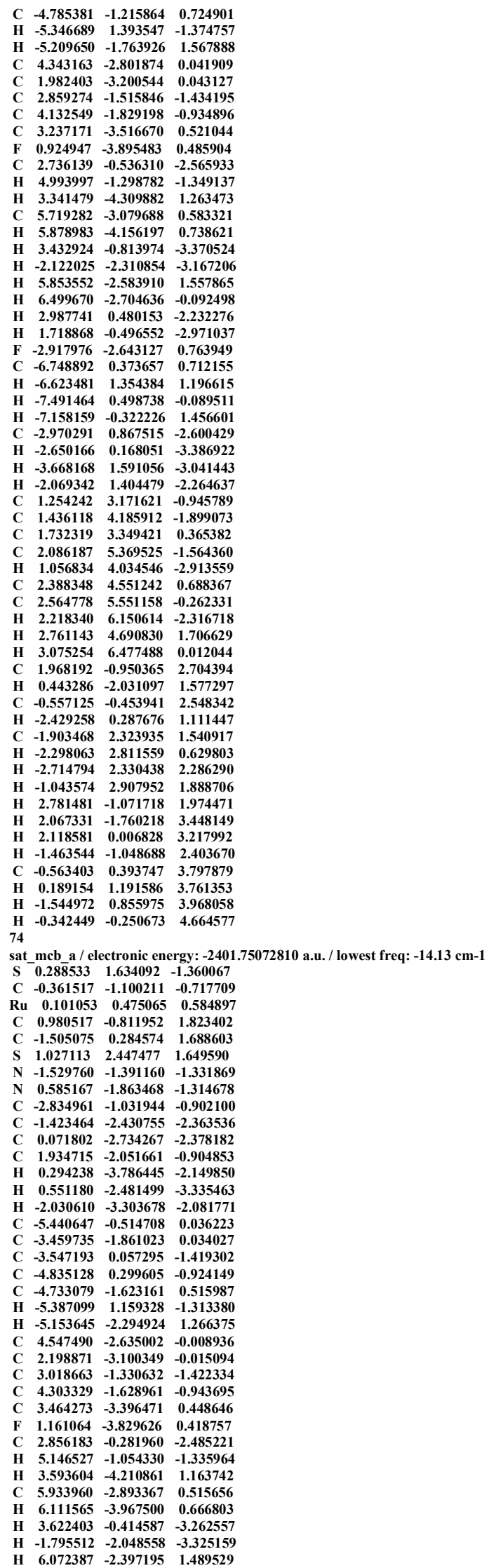


$\begin{array}{llll}\text { H } & 6.700829 & -2.505078 & -0.168234\end{array}$ $\begin{array}{llll}\text { H } & 2.975174 & 0.724252 & -2.057827\end{array}$

$\begin{array}{llll}\text { H } & 1.863948 & -0.314633 & -2.949229\end{array}$

$\begin{array}{llll}\text { F } & -2.763084 & -2.898916 & 0.514405\end{array}$

$\begin{array}{llll}\text { C } & -6.837275 & -0.227655 & 0.519199\end{array}$

$\begin{array}{llll}\text { H } & -7.009168 & 0.853339 & 0.618054\end{array}$

$\begin{array}{lll}0.618054 \\ 0.615168 & -0.196307\end{array}$

$\begin{array}{lll}-7.57975 & -0.615168 & -0.196307\end{array}$

C $-2.059696 \quad 0.960599-2.464776$

$\begin{array}{lll}0.960599 & -2.464776\end{array}$

$\begin{array}{llll}\text { H } & -2.140029 & 0.475165 & -3.009562\end{array}$

$\begin{array}{llll}\text { H } & -3.731332 & 1.274859 & -3.180911\end{array}$

$\begin{array}{llll}\text { H } & -2.534474 & 1.861038 & -1.997767\end{array}$

$\begin{array}{llll}C & 0.949891 & 3.232994 & -0.986231 \\ C\end{array}$

$\begin{array}{llll}\text { C } & 1.157588 & 4.147489 & \mathbf{- 2 . 0 2 9 4 4 6}\end{array}$

$\begin{array}{llll}\text { C } & 1.252984 & 3.588933 & 0.339468\end{array}$

C $1.659643 \quad 5.418033 \quad-1.768004$

$\begin{array}{llll}\text { H } & 0.919763 & 3.850055 & \mathbf{- 3 . 0 5 4 6 6 8}\end{array}$

$\begin{array}{llll}\text { C } & 1.759752 & 4.879322 & 0.586887\end{array}$

$\begin{array}{llll}C & 1.960507 & \mathbf{5 . 7 8 2 3 8 9} & \mathbf{- 0 . 4 5 0 3 3 0}\end{array}$

$\begin{array}{lllll}\text { H } & 1.816722 & 6.123678 & -2.586804\end{array}$

$\begin{array}{lllll}\text { H } & 1.995373 & 5.163339 & 1.615894\end{array}$

$\begin{array}{llll}\text { H } & 2.356084 & 6.777510 & -0.233090\end{array}$

$\begin{array}{llll}C & \mathbf{2 . 2 9 7 8 3 4} & \mathbf{- 0 . 5 7 7 4 0 7} & \mathbf{2 . 5 2 1 4 0 1}\end{array}$

$\begin{array}{lllll}\text { H } & 0.911311 & -1.855372 & 1.511267\end{array}$

$\begin{array}{llll}\text { C } & -0.370639 & -0.437564 & 2.579789\end{array}$

\begin{tabular}{llll}
\hline H & -2.222895 & -0.469741 & 1.366012
\end{tabular}

$\begin{array}{llll}\text { C } & \mathbf{- 2 . 2 4 7 5 4 2} & \mathbf{1 . 4 5 6 3 8 7} & \mathbf{2 . 2 8 7 1 6 0}\end{array}$

$\begin{array}{llll}\text { H } & -2.874283 & 1.900142 & 1.498422\end{array}$

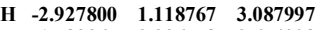

$\begin{array}{llll}\text { H } & -1.583365 & 2.236558 & 2.674908\end{array}$

$\begin{array}{llll}\text { H } & 3.106055 & -0.739706 & 1.791256\end{array}$

H $\quad 2.444674 \quad-1.308851 \quad 3.335021$

$\begin{array}{llll}\text { H } & \mathbf{2 . 4 0 4 9 2 4} & \mathbf{0 . 4 3 6 2 9 9} & \mathbf{2 . 9 2 3 3 3 8}\end{array}$

$\begin{array}{llll}H & 0.826506 & -1.429910 & 2.698706\end{array}$

\begin{tabular}{lrrr}
$H$ & -0.826506 & -1.429910 & 2.698706 \\
\hline
\end{tabular}

\begin{tabular}{llll} 
C & $-\mathbf{0 . 1 5 8 5 0 0}$ & $\mathbf{0 . 2 1 8 4 9 3}$ & $\mathbf{3 . 9 3 9 8 9 8}$ \\
\hline & -1.116761 & 0.319431 & 4.467727
\end{tabular}

\begin{tabular}{lrrr}
$\mathrm{H}$ & $-\mathbf{1 . 1 1 6 7 6 1}$ & $\mathbf{0 . 3 1 9 4 3 1}$ & 4.467727 \\
\hline & 0.501221 & -0.402611 & 4.561675
\end{tabular}

$\begin{array}{cccc}\text { H } & 0.501221 & -\mathbf{0 . 4 0 2 6 1 1} & 4.561675 \\ \text { H } & 0.289586 & 1.216912 & 3.855608\end{array}$

H

sat_ts2_a / electronic energy: $-\mathbf{2 4 0 1 . 7 4 1 3 1 8 6 0}$ a.u. / lowest freq: $-\mathbf{1 1 9 . 8 2} \mathbf{c m}-1$

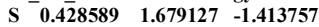

$\begin{array}{llll}C & -0.490804 & -1.112160 & -0.735872\end{array}$

$\begin{array}{llll}\text { Ru } & 0.130037 & 0.430026 & 0.511223\end{array}$

$\begin{array}{llll}\text { C } & 0.620431 & -1.055469 & 2.030178\end{array}$

$\begin{array}{llll}C & -1.544602 & 0.935362 & 1.125511\end{array}$

$\begin{array}{llll}\text { S } & \mathbf{1 . 5 3 8 9 0 4} & \mathbf{2 . 0 8 2 2 6 4} & \mathbf{1 . 5 7 0 4 1 2}\end{array}$

N $-1.684497-1.402995-1.301246$

N $0.409189-1.957412-1.298831$

$\begin{array}{llll}C & -2.947824 & -0.944492 & -0.852530\end{array}$

$\begin{array}{lllll}C & -1.653373 & -2.558768 & -2.204965\end{array}$

$\begin{array}{llll}C & -0.159172 & -2.841263 & -2.321588\end{array}$

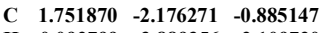

$\begin{array}{llll}\text { H } & 0.092799 & -3.889356 & -2.109720\end{array}$

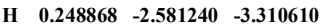

$\begin{array}{llll}\text { H } & -2.207070 & -3.397479 & -1.755451\end{array}$

$\begin{array}{llll}C & \mathbf{- 5 . 4 3 5 4 7 0} & \mathbf{- 0 . 1 1 4 1 6 5} & \mathbf{0 . 1 6 1 0 7 2}\end{array}$

$\begin{array}{lllll}\text { C } & -3.563817 & -1.612061 & 0.204368\end{array}$

$\begin{array}{llll}C & -3.605298 & 0.140148 & -1.451117\end{array}$

$\begin{array}{llll}C & -4.835963 & 0.538784 & -0.923635\end{array}$

$\begin{array}{llll}C & -4.785381 & -1.215864 & 0.724901\end{array}$

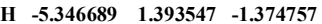

$\begin{array}{llll}\text { H } & -5.209650 & -1.763926 & 1.567888\end{array}$

$\begin{array}{llll}\text { C } & 4.343163 & -2.801874 & 0.041909\end{array}$

$\begin{array}{lllll}C & \mathbf{1 . 9 8 2 4 0 3} & \mathbf{- 3 . 2 0 0 5 4 4} & \mathbf{0 . 0 4 3 1 2 7}\end{array}$

$\begin{array}{llll}C & \mathbf{2 . 8 5 9 2 7 4} & -\mathbf{1 . 5 1 5 8 4 6} & \mathbf{- 1 . 4 3 4 1 9 5}\end{array}$

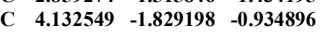

$\begin{array}{llll}\text { C } & 3.237171 & -3.516670 & 0.521044\end{array}$

$\begin{array}{llll}\text { F } & \mathbf{0 . 9 2 4 9 4 7} & \mathbf{- 3 . 8 9 5 4 8 3} & \mathbf{0 . 4 8 5 9 0 4}\end{array}$

$\begin{array}{llll}C & \mathbf{2 . 7 3 6 1 3 9} & \mathbf{- 0 . 5 3 6 3 1 0} & \mathbf{- 2 . 5 6 5 9 3 3}\end{array}$

$\begin{array}{llll}\text { H } & 4.993997 & -1.298782 & -1.349137\end{array}$

$\begin{array}{llll}\text { H } & 3.341479 & -4.309882 & 1.263473\end{array}$

$\begin{array}{llll}\text { C } & \mathbf{5 . 7 1 9 2 8 2} & \mathbf{- 3 . 0 7 9 6 8 8} & \mathbf{0 . 5 8 3 3 2 1}\end{array}$

H $\quad 5.878983 \quad-4.156197 \quad 0.738621$

$\begin{array}{lrrr}\text { H } & \mathbf{3 . 4 3 2 9 2 4} & \mathbf{- 0 . 8 1 3 9 7 4} & \mathbf{- 3 . 3 7 0 5 2 4}\end{array}$

$\begin{array}{lllll}\text { H } & -2.122025 & -2.310854 & -3.167206\end{array}$

$\begin{array}{llll}\text { H } & \mathbf{5 . 8 5 3 5 5 2} & \mathbf{- 2 . 5 8 3 9 1 0} & \mathbf{1 . 5 5 7 8 6 5}\end{array}$

$\begin{array}{lllll}\text { H } & 6.499670 & -2.704636 & -0.092498\end{array}$

$\begin{array}{llll}\text { H } & 2.987741 & 0.480153 & -2.232276\end{array}$

$\begin{array}{llll}\text { H } & 1.718868 & -0.496552 & -2.971037\end{array}$

$\begin{array}{llll}\text { F } & -2.917976 & -2.643127 & 0.763949\end{array}$

$\begin{array}{llll}\text { C } & -6.748892 & 0.373657 & 0.712155\end{array}$

\begin{tabular}{ccccc} 
H & -6.623481 & 1.354384 & 1.196615 \\
\hline & -7.491464 & 0.498738 & -0.089511
\end{tabular}

\begin{tabular}{llll}
\hline & -7.491464 & 0.498738 & -0.089511
\end{tabular}

$\begin{array}{llll}\text { H } & -7.158159 & -0.322226 & 1.456601\end{array}$

$\begin{array}{llll}\text { C } & -2.970291 & 0.867515 & -2.600429\end{array}$

$\begin{array}{llll}\text { H } & -2.650166 & 0.168051 & -3.386922\end{array}$

$\begin{array}{llll}\text { H } & \mathbf{- 3 . 6 6 8 1 6 8} & \mathbf{1 . 5 9 1 0 5 6} & \mathbf{- 3 . 0 4 1 4 4 3}\end{array}$

$\begin{array}{llll}\text { H } & -2.069342 & 1.404479 & -2.264637\end{array}$

$\begin{array}{llll}\text { C } & \mathbf{1 . 2 5 4 2 4 2} & \mathbf{3 . 1 7 1 6 2 1} & \mathbf{- 0 . 9 4 5 7 8 9}\end{array}$

$\begin{array}{llll}C & 1.436118 & 4.185912 & -1.899073\end{array}$

C $1.732319 \quad 3.349421 \quad 0.365382$

$\begin{array}{llll}C & 2.086187 & 5.369525 & 0.365382\end{array}$

$\begin{array}{llll}C & \mathbf{2 . 0 8 6 1 8 7} & \mathbf{5 . 3 6 9 5 2 5} & -\mathbf{1 . 5 6 4 3 6 0}\end{array}$

$\begin{array}{llll}\text { H } & 1.056834 & 4.034546 & -2.913559\end{array}$ 


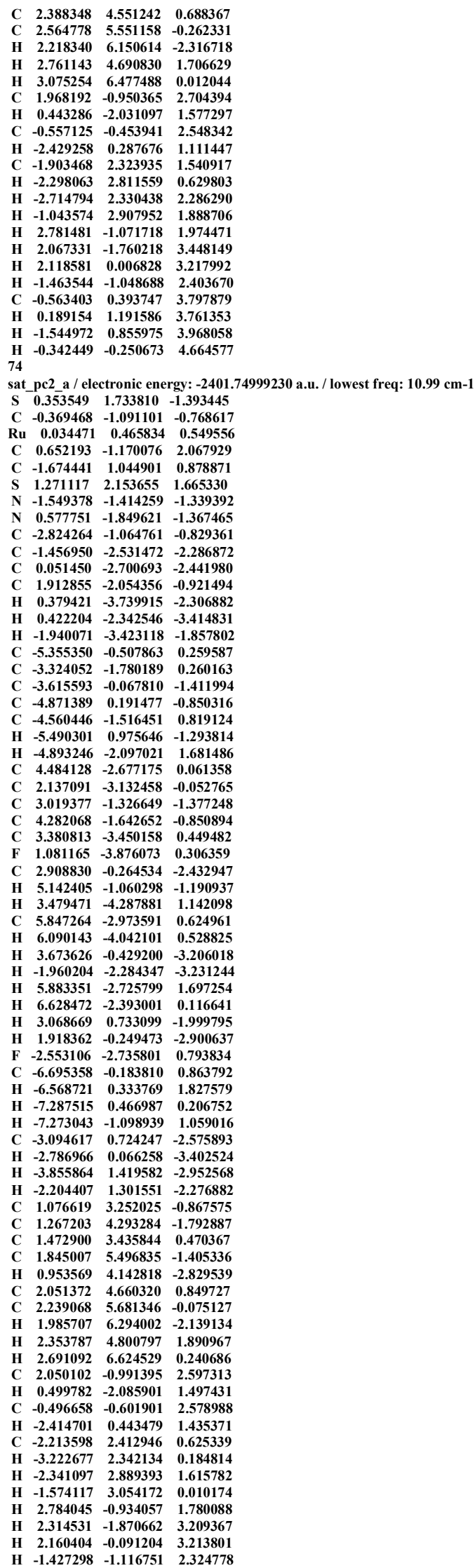


$\begin{array}{lrrr}C & -\mathbf{0 . 5 8 8 4 4 7} & \mathbf{0 . 2 8 7 6 8 0} & \mathbf{3 . 7 8 7 1 5 3}\end{array}$ $\begin{array}{llll}\mathrm{H} & 0.332956 & 0.852904 & 3.97227\end{array}$ $\begin{array}{llll}\text { H } & -1.413261 & 1.008824 & 3.689553\end{array}$

$\begin{array}{llll}\text { H } & -0.797276 & -0.330710 & 4.676424\end{array}$

at_14e1 / electronic energy: -2244.66194430 a.u. / lowest freq: $19.44 \mathrm{~cm}-1$

$\begin{array}{llll}S & 0.751619 & -1.626323 & 1.094274\end{array}$

$\begin{array}{llll}C & \mathbf{- 0 . 8 5 3 3 2 0} & \mathbf{0 . 9 6 0 6 4 7} & \mathbf{0 . 4 6 4 4 7 7}\end{array}$

$\begin{array}{llll}\text { Ru } \quad \mathbf{0 . 0 1 1 4 2 3} & \mathbf{- 0 . 4 5 6 2 8 6} & \mathbf{- 0 . 7 2 3 3 5 4}\end{array}$

$\begin{array}{lrrr}\text { Ru } & 0.011423 & -0.456286 & -0.723354 \\ C & -1.384994 & -1.515916 & -1.110362\end{array}$

$\begin{array}{lllll}\text { S } & 1.984431 & -1.049467 & -1.825414\end{array}$

$\begin{array}{llll}\text { N } & -2.109428 & 1.186086 & 0.874191\end{array}$

$\begin{array}{llll}N & -0.070946 & 1.937943 & 0.964047\end{array}$

$\begin{array}{llll}\text { C } & -\mathbf{3 . 2 4 9 5 6 7} & \mathbf{0 . 4 1 2 7 8 7} & \mathbf{0 . 5 4 4 4 8 5}\end{array}$

$\begin{array}{llll}\text { C } & -2.227484 & 2.371909 & 1.734263\end{array}$

$\begin{array}{llll}C & -0.833203 & 2.998062 & 1.630346\end{array}$

$\begin{array}{llll}C & 1.270382 & \mathbf{2 . 1 2 2 2 3 6} & \mathbf{0 . 5 3 0 9 6 8}\end{array}$

$\begin{array}{lllll}\text { H } & \mathbf{- 0 . 8 2 5 3 0 8} & \mathbf{3 . 9 0 8 0 1 9} & \mathbf{1 . 0 1 0 1 6 7}\end{array}$

$\begin{array}{llll}\text { H } & -\mathbf{0 . 3 9 9 3 7 0} & \mathbf{3 . 2 3 9 8 8 8} & \mathbf{2 . 6 0 9 7 3 1}\end{array}$

H $-\mathbf{3 . 0 2 0 7 1 3} \quad 3.035964 \quad 1.366415$

$\begin{array}{llll}C & -5.562403 & -1.007732 & -0.183995\end{array}$

$\begin{array}{llll}C & -4.087310 & \mathbf{0 . 8 6 1 8 1 1} & \mathbf{- 0 . 4 7 7 5 3 7}\end{array}$

$\begin{array}{llll}\text { C } & \mathbf{- 3 . 5 7 8 9 7 7} & \mathbf{- 0 . 7 6 0 8 8 5} & \mathbf{1 . 2 3 4 9 4 0}\end{array}$

$\begin{array}{llll}\text { C } & -4.728663 & -1.456676 & 0.844998\end{array}$

C $\begin{array}{rrrr}-5.232110 & 0.179495 & -0.849002\end{array}$

\begin{tabular}{lll}
-1.369938 \\
\hline
\end{tabular}

$\begin{array}{llll}\text { H } & -5.847004 & 0.571615 & -1.661223\end{array}$

$\begin{array}{llll}\text { C } & \mathbf{3 . 8 7 2 5 3 3} & \mathbf{2 . 4 2 3 8 9 9} & \mathbf{- 0 . 4 8 3 8 6 0}\end{array}$

$\begin{array}{llll}C & 1.501177 & 2.638177 & -0.746109\end{array}$

$\begin{array}{llll}C & 2.366596 & 1.793475 & 1.344561\end{array}$

$\begin{array}{llll}C & 3.649025 & 1.942180 & 0.811524\end{array}$

$\begin{array}{llll}C & 2.773904 & 2.794043 & -1.264579\end{array}$

$\begin{array}{llll}\text { F } & 0.442575 & 2.966855 & -1.496368\end{array}$

$\begin{array}{llll}\text { C } \quad 2.152262 & 1.270903 & 2.735284\end{array}$

$\begin{array}{llll}\text { H } & \mathbf{4} & \mathbf{1 5 0 7 0 2 5} & \mathbf{1}\end{array}$

$\begin{array}{llll}\mathrm{H} & \mathbf{4 . 5 0 7 0 2 5} & 1.666452 & \mathbf{1 . 4 2 9 8 7 0}\end{array}$

$\begin{array}{llll}\text { H } & \mathbf{2 . 8 9 4 0 0 8} & 3.184417 & \mathbf{2 . 2 7 6 5 9 0}\end{array}$

$\begin{array}{llll}C & 5.266759 & 2.506526 & -1.043168\end{array}$

H $\quad \mathbf{5 . 3 5 4 8 0 5} \quad \mathbf{3 . 3 0 5 2 1 7} \quad \mathbf{- 1 . 7 9 2 5 4 7}$

H $3.110259 \quad 1.129545 \quad 3.251993$

$\begin{array}{llll}\text { H } & -2.478332 & 2.065635 & 2.761701\end{array}$

H $\quad 5.528238 \quad 1.557148 \quad-1.537237$

$\begin{array}{llll}\text { H } & 6.008559 & \mathbf{2 . 6 8 3 0 0 2} & \mathbf{- 0 . 2 5 2 0 6 3}\end{array}$

$\begin{array}{lllll}\text { H } & 1.628777 & 0.302840 & 2.688539\end{array}$

H $\quad \mathbf{1 . 5 3 4 8 3 3} \quad 1.957709 \quad 3.333628$

$\begin{array}{llll}1.534853 & 1.95790 & 1.322311\end{array}$

$\begin{array}{llll}C & -6.803058 & -1.767976 & -0.569528\end{array}$

$\begin{array}{llll}\text { H } & \mathbf{- 6 . 8 4 4 6 7 7} & \mathbf{- 2 . 7 4 6 7 8 0} & \mathbf{- 0 . 0 7 3 7 9 5}\end{array}$

$\begin{array}{llll}\text { H } & -7.706848 & -1.205837 & -0.287051\end{array}$

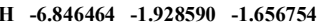

$\begin{array}{llll}\text { C } & \mathbf{- 2 . 6 9 6 7 3 9} & \mathbf{- 1 . 2 6 9 5 8 1} & \mathbf{2 . 3 3 8 1 9 6}\end{array}$

$\begin{array}{llll}\text { H } & -1.796138 & -1.747422 & 1.921941\end{array}$

$\begin{array}{llll}\text { H } & \mathbf{- 2 . 3 5 2 4 3 8} & \mathbf{- 0 . 4 5 4 8 6 7} & \mathbf{2 . 9 9 1 1 4 5}\end{array}$

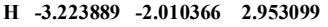

$\begin{array}{llll}\text { C } & 2.353768 & -2.227729 & 0.647748\end{array}$

$\begin{array}{llll}C & 3.106435 & -2.947054 & \mathbf{1 . 5 8 9 6 7 2}\end{array}$

$\begin{array}{llll}C & \mathbf{2 . 8 8 6 9 2 1} & \mathbf{- 1 . 9 9 3 0 8 9} & \mathbf{- 0 . 6 3 1 0 2 8}\end{array}$

$\begin{array}{llll}C & 4.369255 & -3.433234 & 1.268777\end{array}$

$\begin{array}{lllll}\text { H } & \mathbf{2 . 6 8 6 7 2 4} & \mathbf{- 3 . 1 1 9 7 4 4} & \mathbf{2 . 5 8 4 7 2 8}\end{array}$

$\begin{array}{lrrr}C & 4.158644 & -2.497467 & -0.946207\end{array}$

$\begin{array}{llll}C & 4.898114 & -3.209123 & -0.006729\end{array}$

$\begin{array}{llll}\text { H } & 4.943058 & -3.989445 & 2.013682\end{array}$

H $4.567676-2317897-1.944124$

H $\quad \begin{array}{llll}5.888284 & -3.589551 & -0.267812\end{array}$

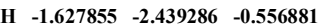

$\begin{array}{llll}C & -2.179802 & -1.275574 & -2.358322\end{array}$

$\begin{array}{llll}\text { H } & -2.002941 & -\mathbf{2 . 0 8 7 2 1 4} & \mathbf{- 3 . 0 8 4 2 8 6}\end{array}$

$\begin{array}{llll}\text { H } & -3.256591 & -1.277543 & -2.120843\end{array}$

H $-1.942175-0.316082-2.848020$

77

sat pc1 b / electronic energy: $-\mathbf{2 5 9 0 . 1 1 8 0 3 3 9 8}$ a.u. / lowest freq: $15.20 \mathrm{~cm}-1$

$\begin{array}{llll}0.473371 & 1.368362 & -1.834544\end{array}$

$\begin{array}{llll}C & 0.018932 & -1.393337 & -0.788218\end{array}$

$\begin{array}{llll}\text { Ru } & 0.034809 & 0.430199 & 0.253658\end{array}$

$\begin{array}{rrrr}\text { Ru } & 0.034809 & 0.430199 & 0.253658 \\ C & 0.762447 & -0.793519 & 2.009029\end{array}$

$\begin{array}{llll}C & -1.781197 & 0.756609 & 0.261514\end{array}$

$\begin{array}{llll}\text { S } & 0.725195 & 2.510608 & 1.162766\end{array}$

N $-1.042153-1.999924-1.357021$

N $1.113420 \quad-2.099718-1.151505$

$\begin{array}{llll}C & -2.405656 & -1.730825 & -1.085267\end{array}$

$\begin{array}{llll}C & \mathbf{- 0 . 7 0 7 9 9 0} & \mathbf{- 3 . 2 3 9 0 2 8} & \mathbf{- 2 . 0 6 9 2 8 2}\end{array}$

C $\quad 0.817374-3.233463 \quad-2.036579$

$\begin{array}{llll}\text { C } & \mathbf{2 . 4 3 1 0 4 0} & -\mathbf{1 . 9 7 1 7 1 8} & \mathbf{- 0 . 6 3 2 8 6 7}\end{array}$

H $1.237925-\mathbf{4} .160230-1.623365$

$\begin{array}{lllll}\text { H } & \mathbf{1 . 2 6 6 3 5 7} & \mathbf{- 3 . 0 6 0 3 8 4} & \mathbf{- 3 . 0 2 6 3 4 3}\end{array}$

H $-1.141481-4.100811-1.539541$

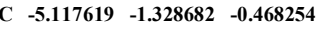

$\begin{array}{llll}C & -2.981785 & -2.279855 & 0.062317\end{array}$

$\begin{array}{llll}C & -3.205083 & -0.989473 & -1.963562\end{array}$

$\begin{array}{llll}\text { C } & -4.552093 & -0.801303 & -1.632861\end{array}$

$\begin{array}{llll}C & -4.309527 & -2.084732 & 0.391388\end{array}$

H $\quad-5.178145-0.210570-2.306215$

$\begin{array}{lll}-5.178145 & -0.210570 & -2.306215\end{array}$

$\begin{array}{lrrr}\text { H } & -4.702910 & -2.521087 & 1.311291 \\ \text { C } & 5.000253 & -1.890043 & 0.530049\end{array}$ 
$\begin{array}{llll}C & 2.779723 & -2.781044 & 0.457923\end{array}$

$\begin{array}{llll}C & 3.429319 & -1.185383 & -1.222099\end{array}$

C $4.687644-1.139423-0.602375$

$\begin{array}{llll}\text { C } & \mathbf{4 . 0 2 3 9 5 1} & \mathbf{- 2 . 7 5 0 9 3 8} & \mathbf{1 . 0 5 0 7 9 5}\end{array}$

$\begin{array}{llll}\text { F } & 1.847256 & -3.612864 & 0.944128\end{array}$

C $3.224970-0.459478-2.520908$

H $5.457629-0.502499-1.045512$

$\begin{array}{cccc}\text { H } & \mathbf{4 . 2 1 9 6 9 6} & \mathbf{- 3 . 3 8 7 8 6 7} & \mathbf{1 . 9 1 5 1 8 0}\end{array}$

$\begin{array}{llll}\text { C } & 6.350226 & -1.793156 & 1.187017\end{array}$

$\begin{array}{llll}\text { H } & 6.791756 & -2.789329 & 1.337769\end{array}$

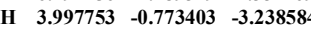

H $\quad-1.116495 \quad-3.220033 \quad-3.088969$

H $\quad 6.261182 \quad-1.321330 \quad 2.177870$

$\begin{array}{llll}\text { H } & \mathbf{7 . 0 4 5 9 2 2} & -\mathbf{1 . 1 9 1 7 8 3} & \mathbf{0 . 5 8 7 0 4 6}\end{array}$

$\begin{array}{llll}\text { H } & 3.309993 & 0.627113 & -2.382427\end{array}$

$\begin{array}{lllll}\text { H } & \mathbf{2 . 2 3 7 4 8 3} & \mathbf{- 0 . 6 5 3 8 5 4} & \mathbf{- 2 . 9 5 2 9 4 4}\end{array}$

$\begin{array}{cccc}\text { F } & -2.197531 & -2.989528 & 0.882613\end{array}$

$\begin{array}{llll}\text { C } & -6.566050 & -1.099151 & -0.130157\end{array}$

$\begin{array}{llll}\text { H } & \mathbf{- 6 . 6 6 4 5 3 1} & \mathbf{- 0 . 5 8 1 3 5 6} & \mathbf{0 . 8 3 5 8 6 5}\end{array}$

$\begin{array}{llll}\text { H } & -\mathbf{7 . 0 6 7 2 0 9} & \mathbf{- 0 . 4 9 3 3 9 6} & \mathbf{- 0 . 8 9 6 5 7 2}\end{array}$

$\begin{array}{lllll}\text { H } & -7.102812 & -\mathbf{2 . 0 5 5 8 3 7} & \mathbf{- 0 . 0 4 2 2 8 0}\end{array}$

$\begin{array}{llll}\text { C } & -2.604499 & -0.379459 & -3.196710\end{array}$

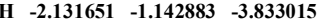

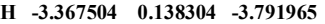

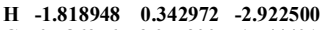

$\begin{array}{llll}C & 0.786956 & 3.077300 & -1.544401\end{array}$

$\begin{array}{llll}C & \mathbf{0 . 9 3 7 4 5 2} & \mathbf{3 . 9 5 4 9 4 5} & \mathbf{- 2 . 6 3 2 3 8 5}\end{array}$

$\begin{array}{llll}\text { C } & \mathbf{0 . 8 8 6 7 6 8} & \mathbf{3 . 5 7 4 0 4 7} & \mathbf{- 0 . 2 3 2 8 8 8}\end{array}$

$\begin{array}{llll}C & 1.187163 & 5.306331 & -2.427296\end{array}$

$\begin{array}{lllll}\text { H } & \mathbf{0 . 8 5 2 0 8 6} & \mathbf{3 . 5 5 7 8 4 2} & \mathbf{- 3 . 6 4 7 5 8 5}\end{array}$

$\begin{array}{llll}C & 1.136636 & 4.945062 & -0.040160\end{array}$

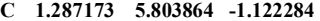

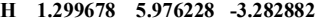

\begin{tabular}{lllll}
\hline & 1.212192 & 5.330208 & 0.980261
\end{tabular}

\begin{tabular}{llll}
$\mathrm{H}$ & 1.212192 & 5.330208 & 0.980261 \\
\hline & 1.478918 & 6.865590 & -0.950698
\end{tabular}

$\begin{array}{llll}\text { H } & 1.478918 & \mathbf{6 . 8 6 5 5 9 0} & \mathbf{- 0 . 9 5 0 6 9 8} \\ \text { C } & 2.056141 & -0.284294 & 2.586325\end{array}$

$\begin{array}{llll}C & 2.056141 & -0.284294 & \mathbf{2 . 5 8 6 3 2 5}\end{array}$

$\begin{array}{rrrrr}\text { H } & 0.811018 & -\mathbf{1 . 8 1 6 5 6 2} & \mathbf{1 . 6 3 7 8 4 4} \\ \text { C } & \mathbf{- 0 . 5 2 7 2 8 1} & \mathbf{- 0 . 3 6 7 3 6 3} & \mathbf{2 . 3 1 1 7 9 9}\end{array}$

$\begin{array}{rrrr}\text { C } & \mathbf{- 0 . 5 2 7 2 8 1} & \mathbf{- 0 . 3 6 7 3 6 3} & \mathbf{2 . 3 1 1 7 9 9} \\ \text { H } & \mathbf{- 2 . 4 8 3 2 5 2} & \mathbf{0 . 1 7 9 2 8 5} & \mathbf{0 . 8 8 0 2 5 4}\end{array}$

$\begin{array}{llll}\text { H } & -\mathbf{2 . 4 8 3 2 5 2} & \mathbf{0 . 1 7 9 2 8 5} & \mathbf{0 . 8 8 0 2 5 4} \\ \text { C } & -\mathbf{0 . 8 7 3 7 1 8} & \mathbf{0 . 7 0 0 3 2 2} & \mathbf{3 . 2 8 4 1 0 7}\end{array}$

$\begin{array}{llll}C & -2.459996 & 1.937933 & -0.336298\end{array}$

$\begin{array}{llll}\text { H } & -3.470928 & 1.673572 & -0.685144\end{array}$

$\begin{array}{llll}\text { H } & -2.588308 & 2.647201 & 0.504832\end{array}$

H $-1.894862 \quad 2.449880-1.122342$

\begin{tabular}{llll}
\hline & -0.123827 & 1.280858 & 4.032659
\end{tabular}

\begin{tabular}{llll}
\hline & -2.194533 & 0.941564 & 3.262407
\end{tabular}

$\begin{array}{lllll}\text { C } & -2.663559 & 1.985277 & 4.100474\end{array}$

$\begin{array}{llll}\text { H } & -3.743616 & 2.053905 & 3.931138\end{array}$

$\begin{array}{llll}\text { H } & -2.180143 & 2.937683 & 3.839953\end{array}$

$\begin{array}{llll}\text { H } & -2.461832 & 1.762626 & 5.157960\end{array}$

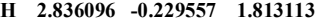

$\begin{array}{llll}\text { H } & 2.405167 & -1.008643 & 3.342201\end{array}$

$\begin{array}{llll}H & 1.942566 & 0.694411 & 3.060611\end{array}$

\begin{tabular}{llll}
\hline & -1.345637 & -1.064128 & 2.124382
\end{tabular}

77

sat_ts1_b / electronic energy: -2590.10241942 a.u. / lowest freq: $\mathbf{- 1 4 4 . 9 4} \mathrm{cm}-1$

$\begin{array}{llll}S & 0.832650 & 1.359557 & -1.796507\end{array}$

$\begin{array}{llll}C & -0.223177 & -1.294461 & -0.920022\end{array}$

$\begin{array}{llll}\text { Ru } & 0.178313 & 0.428603 & 0.200180\end{array}$

$\begin{array}{llll}\text { C } & 0.410221 & -0.814058 & 1.941613\end{array}$

$\begin{array}{lllll}\text { C } & -1.587385 & 0.992816 & 0.461750\end{array}$

$\begin{array}{llll}\text { S } & -1.378096 & \mathbf{2 . 2 3 8 0 8 5} & \mathbf{1 . 2 3 4 4 4 3}\end{array}$

$\begin{array}{llll}\mathrm{N}-1.311751 & -1.690580 & -1.612132\end{array}$

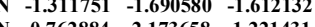

C $0.762884-2.173658-1.221431$

$\begin{array}{llll}C & -2.632131 & -1.218847 & -1.410050\end{array}$

$\begin{array}{llll}C & -1.120391 & -2.924396 & -2.383976\end{array}$

$\begin{array}{llll}\text { C } & 0.356746 & -3.233990 & -2.150333\end{array}$

$\begin{array}{llll}C & \mathbf{2 . 0 3 0 2 2 9} & \mathbf{- 2 . 2 8 4 9 0 0} & \mathbf{- 0 . 5 8 4 5 3 5}\end{array}$

$\begin{array}{llll}\text { H } & 0.517112 & -4.220849 & -1.693513\end{array}$

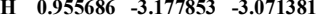

H $-1.786028-3.712315-2.001909$

$\begin{array}{llll}C & -5.242756 & -0.340713 & -0.851799\end{array}$

C $-3.381042-1.771128-0.370405$

$\begin{array}{llll}C & -3.381042 & -1.771128 & -0.370405\end{array}$

$\begin{array}{llll}C & -3.208943 & -0.231360 & -2.218640\end{array}$

$\begin{array}{llll}C & -4.507589 & 0.191906 & -1.916879\end{array}$

$\begin{array}{llll}C & -4.664213 & -1.348775 & -0.071640\end{array}$

$\begin{array}{lllll}\text { H } & -4.960033 & 0.971613 & -2.534807\end{array}$

$\begin{array}{llll}\text { H } & -5.193949 & -1.801809 & 0.768134\end{array}$

$\begin{array}{lllll}\text { C } & 4.457458 & -2.658141 & 0.804522\end{array}$

$\begin{array}{llll}C & \mathbf{2 . 1 2 8 3 4 1} & \mathbf{- 3 . 1 3 7 5 8 3} & \mathbf{0 . 5 2 3 6 5 0}\end{array}$

$\begin{array}{llll}C & 3.201294 & -1.687631 & -1.068985\end{array}$

C $4.388110-1.868311-0.342177$

$\begin{array}{llll}\text { C } & 4.388110 & -1.868311 & -0.342177 \\ \text { C } & 3.298952 & -3.324664 & 1.227619\end{array}$

$\begin{array}{llll}C & 3.298952 & -3.324664 & 1.227619\end{array}$

$\begin{array}{lllll}\text { F } & 1.024135 & -3.792194 & 0.912185\end{array}$

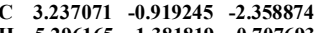

$\begin{array}{llll}\text { H } & 5.296165 & -1.381819 & -0.707693\end{array}$

$\begin{array}{llll}\text { H } & 3.299472 & \mathbf{- 3 . 9 8 3 5 8 5} & \mathbf{2 . 0 9 7 7 8 9}\end{array}$

$\begin{array}{llll}\text { C } & 5.738237 & -2.808962 & 1.579627\end{array}$

$\begin{array}{llll}\text { H } & \mathbf{5 . 9 6 1 3 4 4} & \mathbf{- 3 . 8 6 8 5 2 8} & \mathbf{1 . 7 7 3 2 0 1}\end{array}$

H $4.025028-1.326721 \quad-3.009664$

$\begin{array}{llll}H & -1.359448 & -2.754182 & -3.443302\end{array}$

$\begin{array}{llll}4 & 5.655924 & -2.708656 & -3.557110\end{array}$

$\begin{array}{llll}\text { H } & \mathbf{5 . 6 5 5 9 2 4} & \mathbf{- 2 . 3 0 8 6 5 6} & \mathbf{2 . 5 5 7 1 1 0}\end{array}$

$\begin{array}{llll}\text { H } & 6.588551 & -2.366312 & 1.044284\end{array}$ 
$\begin{array}{lllll}\text { H } & 3.458390 & 0.141946 & -2.177522\end{array}$ $\begin{array}{llll}H \quad 2.278170 & -0.961142 & -2.887170\end{array}$ $\begin{array}{llll}\text { F } & -2.806261 & -2.710735 & 0.391440\end{array}$

$\begin{array}{llll}\text { C } & -6.619262 & 0.175159 & -0.528509\end{array}$

$\begin{array}{lllll}\mathrm{H} & -6.566358 & 0.933679 & 0.268580\end{array}$

$\begin{array}{llll}-7.086269 & 0.645625 & -1.404290\end{array}$

\begin{tabular}{llll}
\hline & -7.086269 & 0.645625 & -1.404290 \\
\hline & -7.276755 & -0.630325 & -0.172724
\end{tabular}

$\begin{array}{llll}H & -7.276755 & -0.630325 & -0.172724\end{array}$

$\begin{array}{llll}\text { C } & -2.422915 & 0.380449 & -3.341660\end{array}$

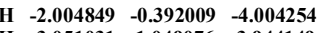

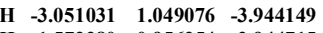

$\begin{array}{lllll}\text { H } & -1.572280 & 0.956254 & -2.944715\end{array}$

$\begin{array}{llll}C & 1.613565 & 2.902315 & -1.431622\end{array}$

$\begin{array}{llll}C & \mathbf{2 . 0 0 9 3 0 0} & \mathbf{3 . 7 4 3 2 4 0} & \mathbf{- 2 . 4 8 3 5 2 1}\end{array}$

$\begin{array}{llll}C & \mathbf{1 . 8 4 4 6 3 4} & \mathbf{3 . 2 8 3 6 8 1} & \mathbf{- 0 . 0 9 8 4 5 6}\end{array}$

C $2.632198 \quad 4.958986-2.222550$

$\begin{array}{llll}\text { H } & \mathbf{1 . 8 2 2 1 7 5} & \mathbf{3 . 4 3 1 0 8 5} & \mathbf{- 3 . 5 1 4 7 3 2}\end{array}$

$\begin{array}{llll}\text { C } & \mathbf{2 . 4 7 6 3 4 3} & 4.517073 & \mathbf{0 . 1 4 8 3 6 2}\end{array}$

$\begin{array}{llll}\text { C } & \mathbf{2 . 8 6 6 4 3 2} & \mathbf{5 . 3 4 5 3 9 4} & \mathbf{- 0 . 8 9 7 6 4 6}\end{array}$

$\begin{array}{llll}\text { H } & \mathbf{2 . 9 3 5 2 1 7} & \mathbf{5 . 6 0 6 1 3 5} & \mathbf{- 3 . 0 4 8 8 0 7}\end{array}$

$\begin{array}{llll}\text { H } & 2.656481 & 4.817297 & 1.183981\end{array}$

$\begin{array}{llll}\text { H } & 3.354181 & 6.299043 & -0.681976\end{array}$

$\begin{array}{llll}\text { C } & \mathbf{1 . 5 6 8 6 7 1} & \mathbf{- 0 . 5 7 2 2 4 8} & \mathbf{2 . 8 7 9 5 3 5}\end{array}$

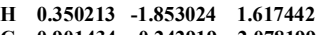

$\begin{array}{llll}\text { C } & \mathbf{- 0 . 9 0 1 4 3 4} & \mathbf{- 0 . 2 4 2 9 1 9} & \mathbf{2 . 0 7 8 1 9 9}\end{array}$

$\begin{array}{llll}\text { H } & -2.461294 & \mathbf{0 . 3 4 6 6 3 6} & \mathbf{0 . 3 2 9 4 3 5}\end{array}$

$\begin{array}{llll}\mathrm{C} & -1.272259 & 0.712361 & 3.171994\end{array}$

$\begin{array}{llll}\text { C } & \mathbf{- 2 . 0 0 0 8 8 4} & \mathbf{2 . 4 0 8 2 5 9} & \mathbf{0 . 6 9 3 3 6 9}\end{array}$

$\begin{array}{llll}\text { H } & -2.153483 & \mathbf{2 . 8 3 2 7 0 5} & \mathbf{- 0 . 3 1 6 3 5 7}\end{array}$

$\begin{array}{llll}\text { H } & -2.963754 & 2.467172 & 1.223532\end{array}$

$\begin{array}{llll}\text { H } & -1.231490 & 3.011460 & 1.189444\end{array}$

$\begin{array}{llll}\text { O } & \mathbf{- 0 . 5 3 4 2 8 9} & \mathbf{1 . 4 0 8 4 0 6} & \mathbf{3 . 8 2 0 7 0 3}\end{array}$

$\begin{array}{llll}\text { O } & -2.598331 & 0.684762 & 3.359129\end{array}$

$\begin{array}{llll}\text { C } & -\mathbf{3 . 1 1 7 2 8 8} & \mathbf{1 . 5 4 9 3 2 0} & \mathbf{4 . 3 5 9 9 1 0}\end{array}$

$\begin{array}{llll}\text { H } & -4.196635 & 1.367763 & 4392343\end{array}$

\begin{tabular}{llll}
$\mathrm{H}$ & -4.196635 & 1.367763 & 4.392343 \\
\hline & -2.918371 & 2.600250 & 4.105352
\end{tabular}

$\begin{array}{llll}\text { H } & -2.918371 & 2.600250 & 4.105352\end{array}$

$\begin{array}{llll}\text { H } & -2.666484 & \mathbf{1 . 3 3 0 3 0 2} & \mathbf{5 . 3 3 7 7 5 6}\end{array}$

\begin{tabular}{lllll} 
H & $\mathbf{2 . 5 1 7 0 3 9}$ & $\mathbf{- 0 . 7 7 1 5 3 7}$ & $\mathbf{2 . 3 6 0 0 3 3}$ \\
\hline & $\mathbf{1 . 5 0 0 5 9 4}$ & -1.282113 & $\mathbf{3}$
\end{tabular}

$\begin{array}{cccc}\text { H } & 1.500594 & -1.282113 & 3.721955 \\ \text { H } & 1.589167 & 0.444261 & 3.282251\end{array}$

$\begin{array}{llll}-1.724759 & -0.921459 & 1.847893\end{array}$

77

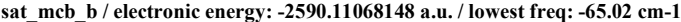

$\begin{array}{llll}0.914686 & 1.576435 & -1.603225\end{array}$

$\begin{array}{llll}C & -0.321487 & -1.006360 & -1.148346\end{array}$

$\begin{array}{llll}\text { Ru } & 0.199342 & 0.481823 & 0.245583\end{array}$

$\begin{array}{llll}C & 0.441628 & -0.958381 & 1.620500\end{array}$

$\begin{array}{lrrr}C & -1.584094 & 0.819019 & 0.957582\end{array}$

$\begin{array}{llll}\mathrm{S} & 1.403055 & \mathbf{2 . 1 6 4 8 6 9} & \mathbf{1 . 5 0 6 2 2 5}\end{array}$

N $-1.413336-1.152952-1.926660$

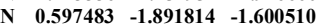

$\begin{array}{llll}C & -2.707536 & -0.648489 & -1.638631\end{array}$

$\begin{array}{llll}C & -1.303637 & -2.236072 & -2.912645\end{array}$

$\begin{array}{llll}C & 0.151578 & -2.671706 & -2.761054\end{array}$

\begin{tabular}{llll}
$C$ & 0.151578 & -2.671706 & -2.761054 \\
\hline
\end{tabular}

$\begin{array}{llll}C & 1.803641 & -2.278617 & -0.951375\end{array}$

$\begin{array}{llll}\text { C } & 0.258308 & -3.747238 & -2.563982\end{array}$

$\begin{array}{llll}\text { H } & 0.765021 & -2.420036 & -3.639530\end{array}$

$\begin{array}{lllll}\text { H } & -2.015761 & -3.038084 & -2.666867\end{array}$

$\begin{array}{llll}\text { C } & \mathbf{- 5 . 2 9 0 8 4 1} & \mathbf{0 . 2 1 7 4 1 1} & \mathbf{- 0 . 9 4 5 0 6 1}\end{array}$

$\begin{array}{llll}C & \mathbf{- 3 . 5 0 9 7 0 9} & \mathbf{- 1 . 3 7 2 8 3 2} & \mathbf{- 0 . 7 5 2 4 9 4}\end{array}$

$\begin{array}{lllll}C & -3.216083 & 0.518051 & -2.220314\end{array}$

$\begin{array}{llll}C & -4.501613 & 0.932596 & -1.850274\end{array}$

$\begin{array}{llll}C & -4.779170 & -0.963245 & -0.391539\end{array}$

$\begin{array}{lllll}\text { H } & -4.899211 & 1.851994 & -\mathbf{2 . 2 8 7 6 4 9}\end{array}$

$\begin{array}{llll}\text { H } & -\mathbf{5 . 3 5 1 0 2 5} & \mathbf{- 1 . 5 5 7 8 3 4} & 0.323020\end{array}$

$\begin{array}{llll}C & 4.072721 & -\mathbf{3 . 2 2 4 1 9 3} & \mathbf{0 . 4 3 5 0 6 6}\end{array}$

$\begin{array}{lrrr}C & \mathbf{1 . 7 2 8 8 3 6} & \mathbf{- 3 . 3 3 9 0 8 8} & \mathbf{- 0 . 0 3 8 8 1 7}\end{array}$

$\begin{array}{llll}\text { C } & \mathbf{3 . 0 6 3 3 9 1} & -\mathbf{1 . 7 4 0 3 6 1} & -\mathbf{1 . 2 4 2 9 8 1}\end{array}$

$\begin{array}{llll}C & 4.169740 & -\mathbf{2 . 2 1 4 1 9 8} & \mathbf{- 0 . 5 2 1 7 6 1}\end{array}$

$\begin{array}{llll}\text { C } & \mathbf{2 . 8 2 0 1 5 7} & -\mathbf{3 . 8 1 0 4 8 1} & \mathbf{0 . 6 6 0 8 5 2}\end{array}$

$\begin{array}{lllll}\text { F } & 0.530162 & -3.902187 & 0.170023\end{array}$

$\begin{array}{llll}\text { C } & 3.274171 & -0.717489 & -2.322640\end{array}$

$\begin{array}{llll}\text { H } & 5.148824 & -1.776623 & -0.733668\end{array}$

$5.148824-1.77$

$\begin{array}{llll}\text { H } & \mathbf{2 . 6 8 5 0 7 1} & -4.621587 & \mathbf{1 . 3 7 8 5 4 2}\end{array}$

$\begin{array}{llll}\text { C } & 5.272384 & -3.669897 & 1.226298\end{array}$

$\begin{array}{llll}\text { H } & 5.258484 & -4.755615 & 1.397649\end{array}$

$\begin{array}{lllll}\text { H } & 4.077239 & -1.049664 & -2.996863\end{array}$

$\begin{array}{lllll}\text { H } & -1.538698 & -1.861773 & -3.918549\end{array}$

H $\quad 5.279386 \quad-3.181277 \quad 2.213674$

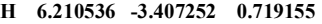

H $3.573327 \quad 0.248600 \quad-1.890769$

$\begin{array}{llll}\text { H } & \mathbf{2 . 3 6 4 2 8 8} & -0.542384 & \mathbf{- 2 . 9 0 6 5 2 4}\end{array}$

F $-2.993053-2.476598-0.196822$

\begin{tabular}{llll}
$F$ & -2.993053 & -2.476598 & -0.196822 \\
\hline
\end{tabular}

$\begin{array}{llll}C & -6.676673 & 0.681632 & -0.585175\end{array}$

$\begin{array}{llll}\text { H } & \mathbf{- 6 . 8 1 4 8 1 1} & 1.747558 & \mathbf{- 0 . 8 1 0 5 9 9}\end{array}$

$\begin{array}{llll}\text { H } & -7.431894 & 0.116913 & -1.154532\end{array}$

$\begin{array}{llll}\text { H } & -6.884082 & 0.521986 & 0.482386\end{array}$

$\begin{array}{llll}\text { C } & -\mathbf{2 . 3 8 2 7 9 1} & \mathbf{1 . 3 2 9 8 6 2} & \mathbf{- 3 . 1 6 9 0 5 5}\end{array}$

$\begin{array}{lllll}\text { H } & -1.763237 & 0.690776 & -3.813449\end{array}$

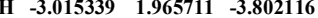

H $-1.689112 \quad 1.974396 \quad-2.607469$

$\begin{array}{llll}C & 1893655 & 2.955145 & -1.089789\end{array}$

$\begin{array}{llll}C & \mathbf{2 . 4 6 6 9 1 9} & \mathbf{3 . 7 9 0 5 4 4} & \mathbf{- 2 . 0 6 0 1 4 8} \\ \text { C } & \mathbf{2 . 0 8 7 7 1 6} & \mathbf{3 . 2 1 1 6 9 9} & \mathbf{0 . 2 7 8 5 7 5}\end{array}$

$\begin{array}{llll}\text { C } \quad 2.087716 & 3.211699 & 0.278575\end{array}$ 
$\begin{array}{llll}C & 3.229702 & 4.890262 & -1.682740\end{array}$ $\begin{array}{llll}C & 2.305643 & 3.569011 & -3.118824\end{array}$

$\begin{array}{llll}\text { C } & 2.862977 & 4.331037 & \mathbf{0 . 6 4 1 7 1 8}\end{array}$

C $\quad \mathbf{3 . 4 2 6 2 5 2} \quad \mathbf{5 . 1 5 7 9 1 8} \quad \mathbf{- 0 . 3 2 2 3 6 4}$

H $3.670632 \quad 5.537448 \quad-2.444097$

$\begin{array}{lll}3.018542 & 4.540375 & 1.703297\end{array}$

$\begin{array}{llll}3 & 3.018542 & 4.540375 & 1.70329\end{array}$

$\begin{array}{llll}\text { H } & 4.024194 & 6.019025 & -0.01391\end{array}$

$\begin{array}{llll}C & 1.537122 & -0.986364 & 2.660212\end{array}$

$\begin{array}{rrrr}\text { H } & \mathbf{0 . 2 5 1 7 9 3} & \mathbf{- 1 . 9 7 2 4 7 7} & \mathbf{1 . 2 6 3 7 9 9} \\ \text { C } & \mathbf{- 0 . 9 8 2 4 4 8} & \mathbf{- 0 . 3 3 5 0 3 7} & \mathbf{1 . 9 4 7 6 4 8}\end{array}$

$\begin{array}{llll}\text { H } & -2.429008 & 0.371444 & 0.433410\end{array}$

$\begin{array}{llll}\text { C } & -1.112748 & 0.158748 & 3.370028\end{array}$

$\begin{array}{llll}\text { C } & -2.020768 & 2.134701 & 1.563348\end{array}$

$\begin{array}{llll}\text { H } & -2.279594 & 2.814558 & 0.737767\end{array}$

$\begin{array}{llll}\text { H } & -2.934868 & 1.989922 & 2.164386\end{array}$

$\begin{array}{llll}\text { H } & -1.246354 & \mathbf{2 . 6 0 9 5 1 1} & \mathbf{2 . 1 7 3 6 4 3}\end{array}$

\begin{tabular}{lllll}
\hline & -0.365998 & 0.896894 & 3.95551
\end{tabular}

\begin{tabular}{llll}
\hline & -2.232710 & -0.331624 & 3.905576
\end{tabular}

$\begin{array}{llll}C & -2.533498 & 0.076808 & 5.234845\end{array}$

$\begin{array}{llll}\text { H } & \mathbf{- 3 . 4 7 7 7 9 6} & \mathbf{- 0 . 4 1 1 2 8 9} & \mathbf{5 . 4 9 6 7 9 4}\end{array}$

$\begin{array}{llll}\text { H } & -2.643439 & 1.168975 & 5.287879\end{array}$

$\begin{array}{llll}\text { H } & -1.740084 & -0.236751 & 5.927135\end{array}$

$\begin{array}{llll}\text { H } & \mathbf{2 . 4 5 8 7 4 6} & -\mathbf{1 . 3 2 4 1 0 0} & \mathbf{2 . 1 6 1 7 4 0}\end{array}$

H $\quad 1.304291 \quad-1.723174 \quad 3.448163$

$\begin{array}{llll}\text { H } & 1.724923 & -0.013268 & 3.122430\end{array}$

\begin{tabular}{llll}
\hline & -1.690147 & -1.154328 & 1.786777
\end{tabular}

77

sat_ts2_b / electronic energy: $-\mathbf{2 5 9 0 . 1 0 2 4 1 9 4 2}$ a.u. / lowest freq: $\mathbf{- 1 4 4 . 9 4} \mathrm{cm}-1$

$\begin{array}{llll}\mathrm{S} & \mathbf{0 . 8 3 2 6 5 0} & \mathbf{1 . 3 5 9 5 5 7} & \mathbf{- 1 . 7 9 6 5 0 7}\end{array}$

$\begin{array}{llll}C & -0.223177 & -1.294461 & -0.920022\end{array}$

$\begin{array}{llll}\text { Ru } & 0.178313 & 0.428603 & 0.200180\end{array}$

$\begin{array}{llll}\text { C } & 0.410221 & -0.814058 & 1.941613\end{array}$

$\begin{array}{lrrr}C & -1.587385 & 0.992816 & 0.461750\end{array}$

$\begin{array}{llll}C & 1.378096 & \mathbf{2 . 2 3 8 0 8 5} & \mathbf{1 . 2 3 4 4 4 3}\end{array}$

$\mathrm{N}-1.311751-1.690580-1.612132$

$\begin{array}{llll}\mathrm{N} & -1.311751 & -1.690580 & -1.612132 \\ \mathrm{~N} & 0.762884 & -2.173658 & -1.221431\end{array}$

N $0.762884-2.173658-1.221431$

$\begin{array}{llll}C & -2.632131 & -1.218847 & -1.410050\end{array}$

$\begin{array}{llll}C & -1.120391 & -2.924396 & -2.383976\end{array}$

C $\quad 0.356746-3.233990 \quad-2.150333$

$\begin{array}{llll}C & 2.030229 & -2.284900 & -0.584535\end{array}$

$\begin{array}{lllll}\text { H } & 0.517112 & -4.220849 & -1.693513\end{array}$

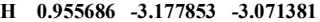

H $\quad-1.786028-3.712315-2.001909$

$\begin{array}{llll}C & -5.242756 & -0.340713 & -0.851799\end{array}$

$\begin{array}{llll}C & -3.381042 & -1.771128 & -0.370405\end{array}$

$\begin{array}{llll}C & -3.208943 & -0.231360 & -2.218640\end{array}$

$\begin{array}{llll}C & -4.507589 & 0.191906 & -1.916879\end{array}$

$\begin{array}{llll}C & -4.664213 & -1.348775 & -0.071640\end{array}$

$\begin{array}{lllll}\text { H } & -4.960033 & 0.971613 & -2.534807\end{array}$

$\begin{array}{llll}\text { H } & -5.193949 & -1.801809 & 0.768134\end{array}$

$\begin{array}{llll}\text { C } & 4.457458 & -2.658141 & 0.804522\end{array}$

$\begin{array}{llll}C & \mathbf{2 . 1 2 8 3 4 1} & \mathbf{- 3 . 1 3 7 5 8 3} & \mathbf{0 . 5 2 3 6 5 0}\end{array}$

$\begin{array}{llll}C & 3.201294 & -1.687631 & -1.068985\end{array}$

$\begin{array}{llll}C & \\ C & 4.388110 & -1.868311 & -0.342177\end{array}$

$\begin{array}{llll}C & 4.388110 & -1.868311 & -0.342177\end{array}$

$\begin{array}{llll}\text { C } & 3.298952 & -3.324664 & 1.227619\end{array}$

$\begin{array}{llll}\text { F } & 1.024135 & -3.792194 & 0.912185\end{array}$

$\begin{array}{lllll}C & 3.237071 & -0.919245 & -2.358874\end{array}$

$\begin{array}{llll}C & 5.296165 & -1.381819 & -0.707693\end{array}$

$\begin{array}{lllll}\text { H } & \mathbf{3 . 2 9 9 4 7 2} & \mathbf{- 3 . 9 8 3 5 8 5} & \mathbf{2 . 0 9 7 7 8 9}\end{array}$

$\begin{array}{llll}\text { C } & \mathbf{5 . 7 3 8 2 3 7} & \mathbf{- 2 . 8 0 8 9 6 2} & \mathbf{1 . 5 7 9 6 2 7}\end{array}$

$\begin{array}{llll}\text { H } & 5.961344 & \mathbf{- 3 . 8 6 8 5 2 8} & \mathbf{1 . 7 7 3 2 0 1}\end{array}$

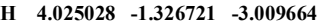

$\begin{array}{llll}\text { H } & \mathbf{- 1 . 3 5 9 4 4 8} & \mathbf{- 2 . 7 5 4 1 8 2} & \mathbf{- 3 . 4 4 3 3 0 2}\end{array}$

\begin{tabular}{llll}
\hline & 5.655924 & -2.308656 & $\mathbf{2 . 5 5 7 1 1 0}$
\end{tabular}

\begin{tabular}{llll} 
H & $\mathbf{6 . 5 8 8 5 5 1}$ & $\mathbf{- 2 . 3 6 6 3 1 2}$ & $\mathbf{1 . 0 4 4 2 8 4}$ \\
\hline & $\mathbf{3 . 4 5 8 3 9 0}$ & $\mathbf{0 . 1 4 1 9 4 6}$ & -2.177522
\end{tabular}

$\begin{array}{rrrr}\text { H } & 3.458390 & \mathbf{0 . 1 4 1 9 4 6} & -\mathbf{2 . 1 7 7 5 2 2} \\ \text { H } & \mathbf{2 . 2 7 8 1 7 0} & -\mathbf{0 . 9 6 1 1 4 2} & \mathbf{- 2 . 8 8 7 1 7 0}\end{array}$

$\begin{array}{llll}\text { F } & -\mathbf{2 . 8 0 6 2 6 1} & -\mathbf{2 . 7 1 0 7 3 5} & 0.391440\end{array}$

$\begin{array}{llll}\text { C } & -6.619262 & 0.175159 & -0.528509\end{array}$

$\begin{array}{lllll}\text { H } & -6.566358 & 0.933679 & 0.268580\end{array}$

$\begin{array}{llll}\text { H } & -7.086269 & 0.645625 & -1.404290\end{array}$

$\begin{array}{llll}\text { H } & -7.276755 & -0.630325 & -0.172724\end{array}$

C $-2.422915 \quad 0.3804$

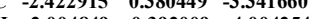

$\begin{array}{llll}\mathrm{H} & -2.004849 & -0.392009 & -4.004254\end{array}$

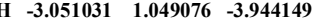

$\begin{array}{llll}\text { H } & -1.572280 & 0.956254 & -2.944715\end{array}$

C $\quad 1.613565 \quad 2.902315-1.431622$

$\begin{array}{llll}C & \mathbf{2 . 0 0 9 3 0 0} & \mathbf{3 . 7 4 3 2 4 0} & \mathbf{- 2 . 4 8 3 5 2 1}\end{array}$

$\begin{array}{lllll}C & \mathbf{1 . 8 4 4 6 3 4} & \mathbf{3 . 2 8 3 6 8 1} & \mathbf{- 0 . 0 9 8 4 5 6}\end{array}$

$\begin{array}{lllll}C & 2.632198 & 4.958986 & \mathbf{- 2 . 2 2 2 5 5 0}\end{array}$

$\begin{array}{lllll}\text { H } & 1.822175 & \mathbf{3 . 4 3 1 0 8 5} & \mathbf{- 3 . 5 1 4 7 3 2}\end{array}$

$\begin{array}{llll}C & 2.476343 & 4.517073 & 0.148362\end{array}$

C $2.866432 \quad 5.345394 \quad-0.897646$

$\begin{array}{llll}\text { C } & 2.866432 & 5.345394 & \mathbf{- 0 . 8 9 7 6 4 6}\end{array}$

$\begin{array}{lllll}\text { H } & \mathbf{2 . 9 3 5 2 1 7} & \mathbf{5 . 6 0 6 1 3 5} & \mathbf{- 3 . 0 4 8 8 0 7}\end{array}$

$\begin{array}{lllll}\text { H } & \mathbf{2 . 6 5 6 4 8 1} & 4.817297 & \mathbf{1 . 1 8 3 9 8 1}\end{array}$

$\begin{array}{llll}\text { H } & \mathbf{3 . 3 5 4 1 8 1} & \mathbf{6 . 2 9 9 0 4 3} & \mathbf{- 0 . 6 8 1 9 7 6}\end{array}$

$\begin{array}{llll}\text { C } & \mathbf{1 . 5 6 8 6 7 1} & \mathbf{- 0 . 5 7 2 2 4 8} & \mathbf{2 . 8 7 9 5 3 5}\end{array}$

$\begin{array}{llll}\text { H } & 0.350213 & -1.853024 & 1.617442\end{array}$

$\begin{array}{lrrr}\text { C } & \mathbf{- 0 . 9 0 1 4 3 4} & \mathbf{- 0 . 2 4 2 9 1 9} & \mathbf{2 . 0 7 8 1 9 9}\end{array}$

$\begin{array}{llll}\text { H } & -2.461294 & 0.346636 & 0.329435\end{array}$

$\begin{array}{llll}\text { C } & -1.272259 & 0.712361 & 3.171994\end{array}$

C $-2.00084 \quad 2.408259 \quad 0.693369$

\begin{tabular}{llll}
$C$ & -2.000884 & 2.408259 & 0.693369 \\
\hline
\end{tabular}

$\begin{array}{lllll}\text { H } & -2.153483 & 2.832705 & -0.316357\end{array}$ 


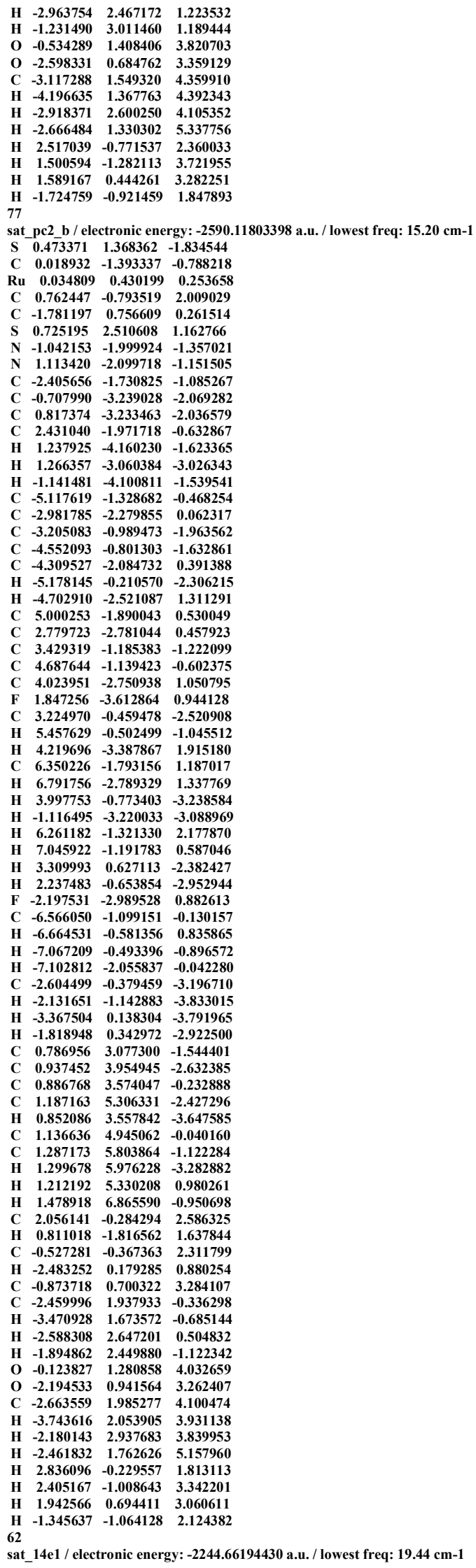




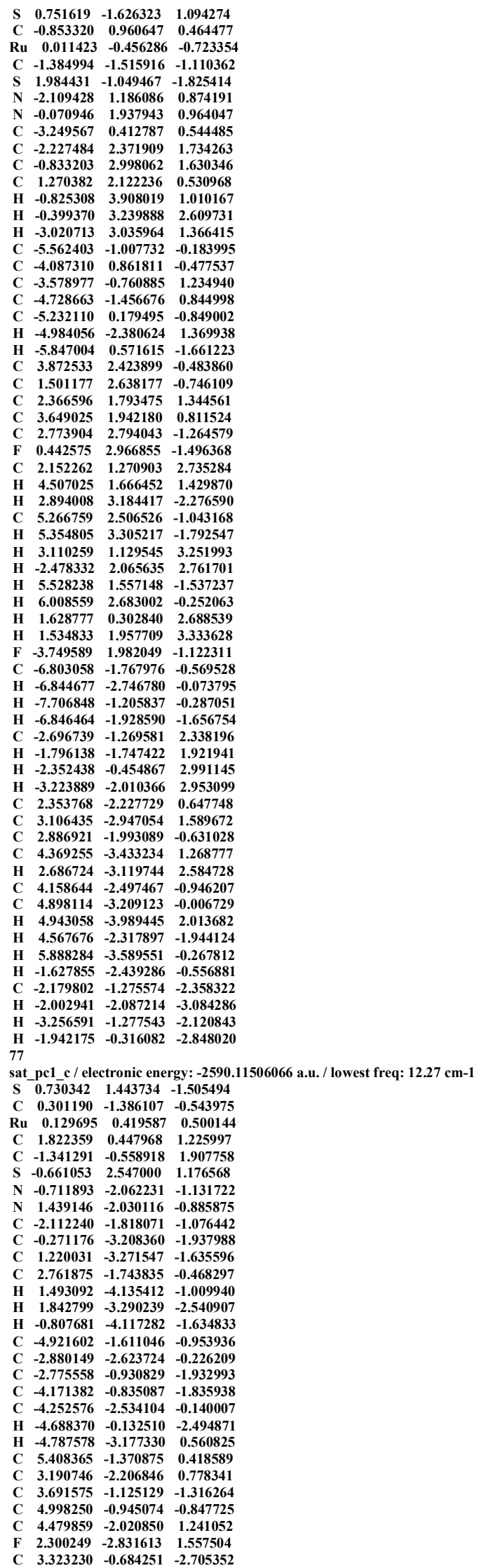


$\begin{array}{llll}\text { H } & 5.721855 & -0.451868 & -1.502132\end{array}$

$\begin{array}{llll}\text { H } & 4.747812 & -\mathbf{2 . 3 8 6 3 3 5} & \mathbf{2 . 2 3 3 9 4 5}\end{array}$

$\begin{array}{llll}\text { C } & \mathbf{6 . 8 0 8 6 5 3} & \mathbf{- 1 . 1 1 3 2 2 9} & \mathbf{0 . 9 0 5 8 2 7}\end{array}$

$\begin{array}{llll}\text { H } & 7.132279 & -1.879504 & 1.623520\end{array}$

H $3.889773-1.264867 \quad-3.449642$

- $-0.485650-3.017280-3.000966$

\begin{tabular}{llll} 
H & $\mathbf{- 0 . 4 8 5 6 5 0}$ & $\mathbf{- 3 . 0 1 7 2 8 0}$ & $\mathbf{- 3 . 0 0 0 9 6 6}$ \\
\hline & 6.862193 & -0.138224 & $\mathbf{1 . 4 1 6 1 0 8}$
\end{tabular}

\begin{tabular}{llll}
\hline & 6.862193 & -0.138224 & 1.416108
\end{tabular}

$\begin{array}{llll}\text { H } & 7.524960 & -1.089084 & 0.073165\end{array}$

$\begin{array}{llll}\text { H } & 3.567671 & 0.377236 & -2.849851\end{array}$

$\begin{array}{lllll}\text { H } & 2.251928 & -0.804934 & -2.904399\end{array}$

$\begin{array}{llll}\text { F } & -2.233638 & -3.507541 & 0.548270\end{array}$

$\begin{array}{llll}\text { C } & -6.417169 & -1.471775 & -0.861659\end{array}$

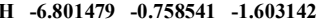

H $\quad-6.915673-2.439555-1.021563$

$\begin{array}{llll}\text { H } & -6.711764 & -1.116475 & 0.138122\end{array}$

$\begin{array}{llll}C & -2.041915 & -0.108299 & -2.952046\end{array}$

$\begin{array}{llll}\text { H } & -\mathbf{1 . 0 5 6 5 5 1} & -\mathbf{0 . 5 2 9 7 0 7} & \mathbf{- 3 . 1 8 8 0 3 3}\end{array}$

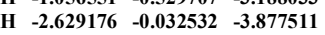

$\begin{array}{lllll}\text { H } & -1.859031 & 0.907365 & -2.572522\end{array}$

$\begin{array}{lllll}C & 0.529987 & 3.173371 & -1.238242\end{array}$

$\begin{array}{llll}C & 0.977134 & 4.087577 & -2.209010\end{array}$

$\begin{array}{lrrrr}C & \mathbf{- 0 . 0 8 3 1 8 7} & \mathbf{3 . 6 5 3 1 3 6} & \mathbf{- 0 . 0 6 6 6 3 3}\end{array}$

$\begin{array}{llll}C & 0.811511 & \mathbf{5 . 4 5 5 2 9 9} & \mathbf{- 2 . 0 2 9 4 1 0}\end{array}$

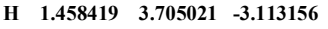

$\begin{array}{lrrr}C & -0.243646 & 5.040460 & 0.102732\end{array}$

$\begin{array}{llll}C & 0.196599 & 5.934098 & \mathbf{- 0 . 8 6 5 8 7 4}\end{array}$

H $\quad 1.164215 \quad 6.152320 \quad-2.793070$

H $\quad \begin{array}{rrrr}-0.720282 & 5.410036 & \mathbf{1 . 0 1 4 4 9 5}\end{array}$

$\begin{array}{lllll}\text { H } & \mathbf{0 . 0 6 4 0 5 1} & \mathbf{7 . 0 0 7 8 1 1} & \mathbf{- 0 . 7 1 4 3 9 9}\end{array}$

$\begin{array}{llll}C & \mathbf{2 . 8 6 2 1 5 0} & \mathbf{1 . 4 9 7 2 1 7} & \mathbf{1 . 0 4 6 3 7 7}\end{array}$

$\begin{array}{llll}\text { H } & 2.158030 & -0.287915 & 1.973830\end{array}$

$\begin{array}{llll}\text { C } & -\mathbf{0 . 1 4 3 8 5 8} & \mathbf{- 0 . 5 5 2 7 7 2} & \mathbf{2 . 6 0 6 2 9 0}\end{array}$

$\begin{array}{llll}\text { H } & -1.615950 & -1.468882 & 1.383180\end{array}$

$\begin{array}{llll}C & -2.512385 & 0.310344 & 2.220272\end{array}$

$\begin{array}{llll}\text { C } & -2.512385 & 0.310344 & 2.220272\end{array}$

$\begin{array}{llll}C & 0.185016 & 0.303916 & 3.796408\end{array}$

\begin{tabular}{cccc} 
H & $\mathbf{- 0 . 3 0 8 6 1 8}$ & $\mathbf{1 . 2 7 9 9 6 3}$ & $\mathbf{3 . 7 5 9 0 2 1}$ \\
\hline
\end{tabular}

$\begin{array}{lrrr}\text { H } & -0.154915 & -0.213758 & 4.708826\end{array}$

$\begin{array}{llll}\text { H } & \mathbf{1 . 2 7 1 0 8 9} & \mathbf{0 . 4 4 7 6 7 3} & \mathbf{3 . 8 9 1 0 7 6}\end{array}$

$\begin{array}{lllll}\text { O } & -2.693225 & 0.933743 & 3.239153\end{array}$

\begin{tabular}{llll}
\hline & -3.395891 & 0.280865 & 1.219673
\end{tabular}

$\begin{array}{llll}C & -4.535947 & 1.110938 & 1.344852\end{array}$

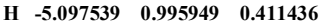

$\begin{array}{llll}\text { H } & -4.234407 & 2.159930 & \mathbf{1 . 4 7 7 9 9 8}\end{array}$

$\begin{array}{lrrr}\text { H } & \mathbf{- 5 . 1 5 5 5 2 0} & \mathbf{0 . 8 0 4 8 8 2} & \mathbf{2 . 2 0 0 4 7 4}\end{array}$

$\begin{array}{llll}\text { H } & \mathbf{3 . 8 2 2 8 1 2} & \mathbf{1 . 0 1 4 3 2 7} & \mathbf{0 . 7 9 4 8 8 3}\end{array}$

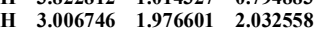

$\begin{array}{llll}\text { H } & 2.621751 & 2.267966 & 0.307631\end{array}$

$\begin{array}{llll}\text { H } & 0.449438 & -1.468019 & 2.521524\end{array}$

77

sat_ts1_c / electronic energy: $\mathbf{- 2 5 9 0 . 1 0 8 2 0 6 6 0}$ a.u. / lowest freq: $-\mathbf{1 1 8 . 5 5} \mathbf{c m}-1$

$S \quad \begin{array}{llll}0.884937 & 1.327379 & -1.464069\end{array}$

$\begin{array}{llll}C & 0.257480 & -1.459458 & -0.406940\end{array}$

$\begin{array}{llll}\text { Ru } & 0.081762 & 0.417792 & 0.491913\end{array}$

$\begin{array}{llll}C & 1.564131 & 0.135632 & 1.569461\end{array}$

$\begin{array}{lrrr}C & -1.449158 & -0.314830 & \mathbf{1 . 8 3 3 1 7 6}\end{array}$

$\begin{array}{llll}\text { S } & \mathbf{- 0 . 5 5 4 6 4 5} & \mathbf{2 . 6 7 3 7 8 8} & \mathbf{1 . 0 6 4 9 3 0}\end{array}$

$\begin{array}{llll}\text { N } & -0.775039 & -2.169556 & -0.921516\end{array}$

$\begin{array}{llll}\text { N } & \mathbf{1 . 3 7 7 9 9 1} & -\mathbf{2 . 1 6 2 7 9 0} & \mathbf{- 0 . 7 0 4 1 4 2}\end{array}$

$\begin{array}{llll}C & -2.159902 & -1.848991 & -0.961672\end{array}$

$\begin{array}{lllll}C & \mathbf{- 0 . 3 7 9 0 8 2} & \mathbf{- 3 . 4 1 6 5 7 7} & \mathbf{- 1 . 5 8 7 2 7 2}\end{array}$

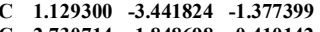

$\begin{array}{llll}C & 2.730714 & -1.848698 & -0.410142\end{array}$

$\begin{array}{lllll}\text { H } & 1.457559 & -\mathbf{4} .271934 & \mathbf{- 0 . 7 3 4 3 2 7}\end{array}$

$\begin{array}{llll}\text { H } & \mathbf{1 . 6 9 4 2 7 8} & \mathbf{- 3 . 4 9 2 4 7 2} & \mathbf{- 2 . 3 1 9 4 9 7}\end{array}$

$\begin{array}{llll}\text { H } & -0.889025 & -4.272355 & -1.123463\end{array}$

$\begin{array}{llll}C & -4.954785 & -1.490342 & -1.051560\end{array}$

$\begin{array}{llll}C & \mathbf{- 3 . 0 2 1 2 1 9} & \mathbf{- 2 . 5 1 8 6 8 7} & \mathbf{- 0 . 0 8 6 7 1 2}\end{array}$

$\begin{array}{llll}C & -2.718850 & -1.026600 & -1.948972\end{array}$

$\begin{array}{llll}C & -4.109104 & -0.851461 & -1.958282\end{array}$

$\begin{array}{llll}C & -4.390345 & -2.351275 & -0.102745\end{array}$

$\begin{array}{lllll}\text { H } & -4.543930 & -0.198316 & -2.719372\end{array}$

$\begin{array}{llll}\text { H } & -5.002737 & -2.889010 & 0.623314\end{array}$

$\begin{array}{llll}C & 5.424569 & -1.362834 & 0.264043\end{array}$

$\begin{array}{llll}\text { C } & 5.424569 & -1.362834 & 0.264043 \\ \text { C } & 3.286147 & -2.308444 & 0.788099\end{array}$

C $3.286147-2.3$

$\begin{array}{llll}C & 3.570105 & -1.214105 & -1.335914\end{array}$

$\begin{array}{llll}C & 4.898382 & -0.962926 & -0.965377\end{array}$

$\begin{array}{llll}C & 4.596875 & -2.067504 & 1.148468\end{array}$

$\begin{array}{llll}\text { F } & \mathbf{2 . 4 9 0 9 3 1} & \mathbf{- 2 . 9 8 0 9 0 9} & \mathbf{1 . 6 3 2 4 2 9}\end{array}$

$\begin{array}{llll}\text { C } & 3.108807 & -0.878345 & -2.726891\end{array}$

$\begin{array}{llll}\text { H } & 5.546975 & -0.446594 & -1.677805\end{array}$

$\begin{array}{llll}\text { H } & 4.961260 & -2.429133 & 2.111803\end{array}$

C $6.846443-1.051415 \quad 0.646188$

\begin{tabular}{llll} 
C & 6.846443 & -1.051415 & 0.646188 \\
\hline
\end{tabular}

\begin{tabular}{llll} 
H & 7.343683 & -1.932932 & $\mathbf{1 . 0 7 6 1 5 3}$ \\
\hline & 3.580375 & -1.568308 & -3.44495
\end{tabular}

$\begin{array}{llll}\text { H } & 3.580375 & -1.568308 & \mathbf{- 3 . 4 4 4 9 6 5}\end{array}$

$\begin{array}{lllll}\text { H } & -0.665189 & -3.379167 & -2.649525\end{array}$

$\begin{array}{llll}\text { H } & 6.874631 & -0.256456 & 1.407730\end{array}$

$\begin{array}{llll}\text { H } & 7.429910 & -0.709744 & -0.219060\end{array}$

H $3.400023 \quad 0.144163 \quad-3.001261$

$\begin{array}{llll}\text { H } & \mathbf{2 . 0 2 0 7 8 2} & \mathbf{- 0 . 9 5 4 7 3 6} & \mathbf{- 2 . 8 3 4 0 1 2}\end{array}$

$\begin{array}{llll}\text { F } & -2.476134 & -3.346611 & 0.817719\end{array}$

$\begin{array}{llll}\text { C } & -6.443845 & -1.273149 & -1.080268\end{array}$

C $-6.738582-1.273149-1.08$

$\begin{array}{lllll}\text { H } & -6.736582 & -0.597472 & -1.895052 \\ \text { H } & -6.978989 & -2.225513 & -1.212942\end{array}$ 
$\begin{array}{llll}\text { H } & -6.791630 & -0.834698 & -0.132102\end{array}$ $\begin{array}{llll}\text { C } & -1.865718 & -0.358509 & -2.987283\end{array}$ $\begin{array}{llll}\text { H } & -1.007593 & -0.984287 & -3.272087\end{array}$ $\begin{array}{llll}\text { H } & -2.452327 & -0.136619 & -3.888431\end{array}$ $\begin{array}{llll}\text { H } & -1.443899 & 0.581935 & -2.603137\end{array}$ C $0.794978 \quad 3.085581-1.300008$ $\begin{array}{llll}\text { C } & 0.794978 & 3.085581 & -1.300008\end{array}$ $\begin{array}{llll}C & 1.353610 & 3.898945 & -2.298572\end{array}$ $\begin{array}{llll}C & 0.163090 & 3.672096 & -0.190607\end{array}$ $\begin{array}{llll}C & 1.288004 & 5.284791 & -2.203825\end{array}$

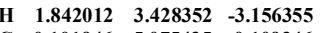
$\begin{array}{llll}C & 0.101946 & \mathbf{5 . 0 7 5 4 3 5} & \mathbf{- 0 . 1 0 9 3 4 6}\end{array}$ $\begin{array}{lllll}C & 0.657483 & 5.874372 & -1.102129\end{array}$ $\begin{array}{lllll}\text { H } & \mathbf{1 . 7 2 7 6 9 9} & \mathbf{5 . 9 0 6 6 6 8} & \mathbf{- 2 . 9 8 6 9 1 2}\end{array}$ $\begin{array}{lrrr}\text { H } & -\mathbf{0 . 3 8 9 5 0 7} & \mathbf{5 . 5 3 3 0 6 1} & \mathbf{0 . 7 5 3 2 3 1}\end{array}$

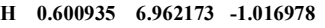

$\begin{array}{llll}\text { C } & 2.524323 & 1.198593 & 1.976019\end{array}$

$\begin{array}{llll}\text { H } & \mathbf{1 . 9 0 2 3 1 9} & \mathbf{- 0 . 8 5 7 5 8 8} & \mathbf{1 . 8 7 9 8 3 3}\end{array}$

$\begin{array}{llll}\text { C } & \mathbf{- 0 . 3 0 6 9 8 2} & \mathbf{- 0 . 3 0 7 5 8 7} & \mathbf{2 . 6 7 7 6 6 9}\end{array}$

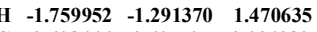

$\begin{array}{llll}\text { C } & -2.608446 & 0.605707 & 2.024289\end{array}$ $\begin{array}{llll}\text { C } & -\mathbf{0 . 1 0 5 7 5 8} & \mathbf{0 . 6 1 5 3 6 9} & \mathbf{3 . 8 5 3 6 4 7}\end{array}$ \begin{tabular}{llll} 
H & -0.276047 & 1.665871 & 3.593423 \\
\hline
\end{tabular} $\begin{array}{llll}\text { H } & -\mathbf{0 . 8 3 0 3 3 1} & \mathbf{0 . 3 4 1 7 8 8} & 4.635463\end{array}$ $\begin{array}{lllll}\text { H } & 0.904186 & 0.504810 & 4.270787\end{array}$ $\begin{array}{llll}\text { O } & -2.842765 & 1.273843 & 3.003149\end{array}$ $\begin{array}{llll}0 & -3.420403 & 0.560452 & 0.965745\end{array}$ $\begin{array}{llll}C & -4.557129 & 1.403616 & \mathbf{0 . 9 9 0 0 2 4}\end{array}$ $\begin{array}{llll}\text { H } & -5.057500 & \mathbf{1 . 2 6 3 8 4 0} & \mathbf{0 . 0 2 5 5 6 3}\end{array}$ $\begin{array}{llll}\text { H } & -4.256397 & \mathbf{2 . 4 5 4 2 9 8} & \mathbf{1 . 1 1 0 9 0 3}\end{array}$ $\begin{array}{llll}\text { H } & -5.235431 & 1.128107 & 1.810788\end{array}$

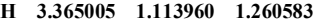
$\begin{array}{llll}\text { H } & 2.941265 & 1.009653 & 2.978353\end{array}$

$\begin{array}{llll}\text { H } & \mathbf{2 . 1 1 0 2 7 8} & \mathbf{2 . 2 1 0 8 4 4} & \mathbf{1 . 9 0 7 3 1 9}\end{array}$

$\begin{array}{llll}\text { H } & 0.126813 & -1.302045 & 2.815563\end{array}$

sat_mcb_c / electronic energy: - -2590.11664431 a.u. / lowest freq: $16.66 \mathrm{~cm}-1$

$\begin{array}{llll}S & 0.233975 & 1.405554 & -1.525364\end{array}$

$\begin{array}{llll}\text { C } & 0.480030 & -1.339592 & -0.589594\end{array}$

$\begin{array}{llll}\text { Ru } & 0.139535 & 0.422807 & 0.502537\end{array}$

$\begin{array}{llll}C & 1.506111 & -0.079440 & 1.801919\end{array}$

$\begin{array}{lrrr}\text { C } & -1.165890 & -\mathbf{0 . 4 5 6 2 6 8} & 1.722704\end{array}$

$\begin{array}{llll}S & -0.384692 & 2.618516 & 1.381192\end{array}$

$\begin{array}{llll}N & -0.468636 & -2.113950 & -1.161058\end{array}$

$\begin{array}{lllll}N & 1.672007 & -1.830888 & -1.003380\end{array}$

$\begin{array}{llll}C & -1.882712 & -2.031685 & -1.029721\end{array}$

$\begin{array}{llll}C & 0.061158 & -3.212735 & -1.977820\end{array}$

C $\quad \begin{array}{lll}\text { 1.569158 } & -\mathbf{3 . 0 3 0 2 0 0} & -\mathbf{1 . 8 4 3 4 6 4}\end{array}$

$\begin{array}{lllll}C & 2.979337 & -1.393921 & -0.654287\end{array}$

$\begin{array}{llll}\text { H } & 2.059167 & -3.879999 & -1.346168\end{array}$

$\begin{array}{lllll}\text { H } & \mathbf{2 . 0 7 0 3 5 2} & \mathbf{- 2 . 8 5 9 7 8 9} & \mathbf{- 2 . 8 0 7 4 7 0}\end{array}$

$\begin{array}{llll}\text { H } & -0.290216 & -4.176960 & -1.583538\end{array}$

$\begin{array}{llll}\text { C } & -4.684218 & -2.095553 & -0.715734\end{array}$

$\begin{array}{llll}C & -2.502324 & -2.826085 & -0.058512\end{array}$

C $-2.692020-1.308989-1.914025$

C $-4.080761-1.347743-1.72499$

$\begin{array}{llll}C & -3.868245 & -2.862122 & 0.126567\end{array}$

$\begin{array}{llll}\text { H } & -4.711220 & -0.770089 & -2.406330\end{array}$

H $\quad-4.283752 \quad-3.484573 \quad 0.921089$

$\begin{array}{lllll}\text { C } & 5.590991 & -0.684937 & 0.143774\end{array}$

$\begin{array}{llll}C & 3.623482 & -2.023085 & 0.417386\end{array}$

$\begin{array}{llll}C & 3.698460 & -0.453528 & -1.403379\end{array}$

$\begin{array}{llll}C & 4.986286 & -0.104271 & -0.971110\end{array}$

$\begin{array}{llll}\text { C } & 4.892597 & -1.681845 & 0.838123\end{array}$

$\begin{array}{llll}\text { F } & \mathbf{2 . 9 5 3 4 5 5} & \mathbf{- 2 . 9 7 8 2 0 2} & \mathbf{1 . 0 7 7 9 1 1}\end{array}$

$\begin{array}{llll}\text { C } & 3.162649 & 0.142390 & -2.674754\end{array}$

$\begin{array}{llll}\text { H } & \mathbf{5 . 5 3 9 8 3 8} & \mathbf{0 . 6 4 2 9 9 8} & -\mathbf{1 . 5 4 5 8 9 5}\end{array}$

H $\quad 5.323647 \quad-2.191283 \quad 1.701743$

$\begin{array}{llll}\text { C } & 6.955926 & -0.251524 & 0.605996\end{array}$

$\begin{array}{llll}\text { H } & 7.544722 & -1.104426 & 0.972148\end{array}$

$\begin{array}{llll}\text { H } & 3.883325 & -0.019495 & -3.490094\end{array}$

$\begin{array}{llll}\text { H } & -0.294662 & -3.114367 & \mathbf{- 3 . 0 1 4 2 7 3}\end{array}$

$\begin{array}{lllll}\text { H } & 6.867848 & 0.468160 & 1.435463\end{array}$

$\begin{array}{rrrr}H & 7.515690 & \mathbf{0 . 2 3 9 1 6 1} & \mathbf{- 0 . 2 0 1 7 2 4}\end{array}$

$\begin{array}{cccc}\text { H } & 7.515690 & \mathbf{0 . 2 3 9 1 6 1} & -0.201724 \\ \text { H } & \mathbf{3 . 0 1 2 4 0 2} & \mathbf{1 . 2 2 5 4 5 1} & \mathbf{- 2 . 5 6 5 7 8 8}\end{array}$

$\begin{array}{rrrr}\text { H } & 3.012402 & 1.225451 & -2.565788 \\ \text { H } & \mathbf{2 . 1 9 8 7 1 4} & -\mathbf{0 . 2 9 2 6 1 3} & -\mathbf{2 . 9 6 2 8 0 4}\end{array}$

$\begin{array}{lrrr}\text { H } & \mathbf{2 . 1 9 8 7 1 4} & \mathbf{- 0 . 2 9 2 6 1 3} & \mathbf{- 2 . 9 6 2 8 0 4} \\ \text { F } & -\mathbf{1 . 7 1 6 6 4 1} & \mathbf{- 3 . 5 6 5 5 0 1} & \mathbf{0 . 7 4 0 0 6 2}\end{array}$

$\begin{array}{llll}C & -6.175614 & -2.083146 & -0.516337\end{array}$

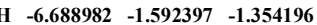

H $\quad-6.574101-3.103198 \quad-0.414030$

$\begin{array}{llll}\text { H } & -6.435791 & -\mathbf{1 . 5 3 8 9 5 7} & \mathbf{0 . 4 0 5 2 1 7}\end{array}$

$\begin{array}{llll}C & -2.128799 & -0.530377 & -3.068661\end{array}$

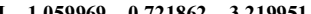

$\begin{array}{ll}-1.059969-0.721862 & -3.219951\end{array}$

$\begin{array}{lll}-1.059969 & -0.721862-3.219951 \\ -1.669532 & -0.783206 & -3.992380\end{array}$

$\begin{array}{llll}H & -2.239333 & 0.549699 & -2.895461\end{array}$

$\begin{array}{lllll}C & -0.125983 & 3.125406 & -1.310634\end{array}$

$\begin{array}{lllll}C & -0.137341 & 3.959590 & -2.438507\end{array}$

$\begin{array}{lllll}C & \mathbf{- 0 . 3 9 6 7 9 9} & \mathbf{3 . 6 4 8 6 1 3} & \mathbf{- 0 . 0 3 5 7 2 7}\end{array}$

$\begin{array}{llll}C & -0.412947 & 5.316101 & -2.309171\end{array}$

$\begin{array}{lrrrr}\text { H } & \mathbf{0 . 0 7 2 0 0 5} & \mathbf{3 . 5 2 9 4 3 9} & \mathbf{- 3 . 4 2 1 8 7 1}\end{array}$

$\begin{array}{llll}\text { C } & \mathbf{- 0 . 6 7 5 4 6 0} & \mathbf{5 . 0 2 4 5 1 2} & \mathbf{0 . 0 7 7 4 2 3}\end{array}$

$\begin{array}{llll}C & -0.682343 & 5.847626 & -1.042187\end{array}$

$\begin{array}{llll}\text { H } & -0.419058 & 5.959279 & -3.191980\end{array}$

$\begin{array}{lllll}\text { H } & -0.885206 & 5.441146 & 1.065864\end{array}$ 
$\begin{array}{lllll}\text { H } & -0.898364 & 6.912545 & -0.92825\end{array}$

$\begin{array}{llll}\text { C } & 2.489077 & 0.889046 & \mathbf{2 . 4 0 8 9 1 5}\end{array}$

$\begin{array}{llll}\text { H } & 1.983538 & -1.045311 & 1.64704\end{array}$

$\begin{array}{llll}\text { C } & \mathbf{0 . 1 0 8 0 0 8} & -\mathbf{0 . 3 9 5 3 3 1} & \mathbf{2 . 6 1 9 7 5 6}\end{array}$

$\begin{array}{llll}\text { H } & -1.419078 & -1.488313 & 1.477397\end{array}$

$\begin{array}{llll}\text { C } & -2.433400 & 0.254548 & 2.097086\end{array}$

$\begin{array}{llll}\text { C } & -2.433400 & 0.254548 & 2.09708 \\ \text { C } & 0.015918 & 0.410586 & 3.912250\end{array}$

$\begin{array}{lllll}\text { H } & \mathbf{0 . 1 4 2 5 7 7} & \mathbf{1 . 4 8 0 1 5 7} & \mathbf{3 . 7 2 8 6 6 4}\end{array}$

$\begin{array}{llll}\text { H } & \mathbf{- 0 . 8 2 6 3 0 9} & \mathbf{0 . 0 5 0 9 1 7} & \mathbf{4 . 5 1 3 6 0 6}\end{array}$

$\begin{array}{llll}\text { H } & 0.941564 & 0.288220 & 4.490124\end{array}$

\begin{tabular}{lllll}
\hline & -2.770817 & $\mathbf{0 . 6 5 9 0 0 2}$ & $\mathbf{3 . 1 8 2 8 6 7}$
\end{tabular}

$\begin{array}{llll}\text { O } & -\mathbf{3 . 2 3 1 3 0 1} & \mathbf{0 . 3 0 8 2 6 1} & \mathbf{1 . 0 2 6 8 2}\end{array}$

$\begin{array}{llll}\text { C } & -4.499885 & 0.914171 & 1.196373\end{array}$

$\begin{array}{llll}\text { H } & -\mathbf{4 . 3 8 8 7 3 4} & \mathbf{1 . 9 7 3 0 9 9} & \mathbf{1 . 4 7 0 4 0 1}\end{array}$

$\begin{array}{llll}H & -5.3887398 & 0.401046 & 1.976084\end{array}$

\begin{tabular}{llll}
\hline H & -5.007136 & 0.827121 & 0.229142
\end{tabular}

\begin{tabular}{lrrr} 
H & $\mathbf{5 . 0 0 7 1 3 6}$ & $\mathbf{0 . 8 2 7 1 2 1}$ & $\mathbf{0 . 2 2 9 1 4}$ \\
\hline & 3.29485 & $\mathbf{1 . 0 4 3 2 4 7}$ & $\mathbf{1 . 6 7 3 7 5 1}$
\end{tabular}

\begin{tabular}{llll} 
H & $\mathbf{3 . 2 9 4 8 6 5}$ & $\mathbf{1 . 0 4 3 2 4 7}$ & $\mathbf{1 . 6 7 3 7 5}$ \\
\hline & $\mathbf{2 . 9 5 5 0 8 3}$ & $\mathbf{0 . 4 6 4 9 7 8}$ & $\mathbf{3 . 3 1 4 3 3 1}$
\end{tabular}

$\begin{array}{cccc}\text { H } & \mathbf{2 . 9 5 5 0 8 3} & \mathbf{0 . 4 6 4 9 7 8} & \mathbf{3 . 3 1 4 3 3 1} \\ \text { H } & \mathbf{2 . 0 4 4 5 4 5} & \mathbf{1 . 8 6 2 6 3 1} & \mathbf{2 . 6 4 2 9 1 6}\end{array}$

$\begin{array}{lllll}\text { H } & 0.295380 & -1.453262 & 2.846249\end{array}$

77

sat_ts2_c / electronic energy: $-\mathbf{2 5 9 0 . 1 0 2 1 8 6 9 1}$ a.u. / lowest freq: $-196.85 \mathrm{~cm}-1$

S $1.013730 \quad 1.053742-1.441808$

$\begin{array}{llll}C & -0.156312 & -1.645727 & -0.428406\end{array}$

$\begin{array}{llll}\text { Ru } & 0.126513 & 0.209523 & 0.502586\end{array}$

$\begin{array}{rr}-0.923049 & 2.322135\end{array}$

$\begin{array}{llll}\text { C } & -1.649051 & 0.699664 & 0.680349\end{array}$

\begin{tabular}{llll}
\hline & 0.974859 & 2.206405 & 1.549928
\end{tabular}

N $-1.268533 \quad-2.290934-0.835210$

N $\quad 0.874784-2.492735 \quad-0.659362$

$\begin{array}{llll}\text { C } & -2.592285 & -1.791275 & -0.829960\end{array}$

$\begin{array}{llll}C & -1.031853 & -3.640124 & -1.362532\end{array}$

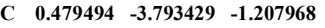

$\begin{array}{llll}C & 2.255540 & -2.229948 & -0.457468\end{array}$

$\begin{array}{llll}\text { H } & 0.759594 & -4.594718 & -0.508858\end{array}$

$\begin{array}{llll}\text { H } & 0.990605 & -3.975755 & -2.164470\end{array}$

$\begin{array}{llll}\text { H } & -1.598445 & -4.378153 & -0.777680\end{array}$

$\begin{array}{llll}\text { C } & \mathbf{- 5 . 2 2 0 6 3 0} & \mathbf{- 0 . 8 0 7 8 9 6} & \mathbf{- 0 . 6 7 7 7 3 1}\end{array}$

$\begin{array}{llll}C & -3.465271 & -2.199883 & 0.178919\end{array}$

$\begin{array}{llll}C & -3.052128 & -0.908838 & -1.816840\end{array}$

$\begin{array}{llll}C & -4.361100 & -0.425612 & -1.712798\end{array}$

$\begin{array}{llll}\text { C } & -4.759042 & -1.721782 & 0.278352\end{array}$

$\begin{array}{llll}\text { H } & -4.718805 & 0.280940 & -2.465783\end{array}$

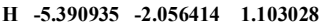

$\begin{array}{llll}C & 4.992534 & -1.754907 & 0.019732\end{array}$

$\begin{array}{llll}\text { C } & \mathbf{2 . 8 6 0 1 9 3} & \mathbf{- 2 . 5 7 5 4 3 4} & \mathbf{0 . 7 5 3 4 0 5}\end{array}$

$\begin{array}{lrrr}C & 3.065109 & -1.735212 & -1.493387\end{array}$

$\begin{array}{llll}C & 4.415001 & -1.490442 & -1.225621\end{array}$

$\begin{array}{llll}C & 4.196359 & -2.329182 & 1.016062\end{array}$

$\begin{array}{llll}\text { F } & 2.127611 & -3.195408 & 1.685429\end{array}$

$\begin{array}{llll}\text { C } & 2.522860 & -1.541214 & -2.882410\end{array}$

$\begin{array}{lllll}\text { H } & 5.039754 & -1.082830 & -2.024490\end{array}$

$\begin{array}{llll}\text { H } & \mathbf{4 . 6 0 4 8 1 0} & \mathbf{- 2 . 6 0 2 6 8 5} & \mathbf{1 . 9 9 0 6 4 8}\end{array}$

$\begin{array}{llll}\text { H } & 4.604810 & -2.602685 & 1.990648\end{array}$

$\begin{array}{llll}\text { C } & 6.436613 & -1.430955 & 0.291361\end{array}$

$\begin{array}{llll}\text { H } & 6.889038 & -2.160354 & \mathbf{0 . 9 7 7 8 1 4}\end{array}$

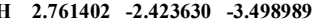

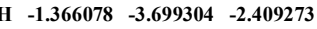

$\begin{array}{lllll}\text { H } & \mathbf{6 . 5 2 3 0 7 2} & \mathbf{- 0 . 4 3 8 2 7 7} & \mathbf{0 . 7 6 1 1 3 3}\end{array}$

$\begin{array}{llll}\text { H } & \mathbf{7 . 0 2 4 4 2 6} & \mathbf{- 1 . 4 1 1 2 6 1} & \mathbf{- 0 . 6 3 6 5 4 0}\end{array}$

H $\quad 2.977471 \quad-0.664321 \quad-3.361423$

$\begin{array}{llll}\text { H } & \mathbf{1 . 4 3 5 0 7 3} & \mathbf{- 1 . 4 0 2 2 6 8} & \mathbf{- 2 . 8 8 7 5 6 2}\end{array}$

$\begin{array}{lllll}\text { F } & -\mathbf{3 . 0 0 5 4 8 1} & \mathbf{- 3 . 0 4 8 4 1 4} & \mathbf{1 . 1 0 7 3 6 4}\end{array}$

$\begin{array}{llll}\text { C } & -6.619431 & -0.261113 & -0.581251\end{array}$

$\begin{array}{llll}\text { H } & -6.815325 & 0.481657 & -1.365746\end{array}$

$\begin{array}{llll}\text { H } & -7.362762 & -1.067042 & -0.678455\end{array}$

\begin{tabular}{llll}
\hline & -6.784060 & 0.218432 & 0.395159
\end{tabular}

$\begin{array}{llll}\text { C } & -2.133608 & -0.456455 & -2.914520\end{array}$

$\begin{array}{llll}\text { H } & -1.592246 & -1.305270 & -3.358173\end{array}$

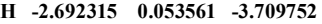

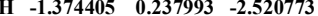

$\begin{array}{llll}\text { C } & 1.427314 & 2.740858 & -1.109392\end{array}$

$\begin{array}{llll}C & 1.776180 & 3.581688 & -2.177024\end{array}$

$\begin{array}{llll}C & \mathbf{1 . 3 9 6 9 7 1} & \mathbf{3 . 2 4 8 1 0 2} & \mathbf{0 . 2 0 0 6 5 7}\end{array}$

$\begin{array}{llll}C & 2.089777 & 4.918686 & -1.954691\end{array}$

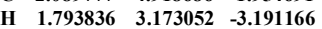

$\begin{array}{llll}\text { C } & 1.721040 & \mathbf{4 . 5 9 9 8 6 7} & \mathbf{0 . 4 1 1 5 2 6}\end{array}$

$\begin{array}{llll}C & \mathbf{2 . 0 6 0 9 8 5} & \mathbf{5 . 4 2 8 8 3 3} & \mathbf{- 0 . 6 5 2 0 6 6}\end{array}$

$\begin{array}{llll}\text { H } & \mathbf{2 . 3 5 4 8 8 5} & \mathbf{5 . 5 6 4 2 2 7} & \mathbf{- 2 . 7 9 5 1 7 2}\end{array}$

$\begin{array}{lllll}H & 1.693358 & 4.996751 & 1.429780\end{array}$

$\begin{array}{llll}\text { H } & \mathbf{2 . 3 0 0 5 6 1} & \mathbf{6 . 4 7 8 6 4 3} & \mathbf{- 0 . 4 6 6 9 6 7}\end{array}$

$\begin{array}{llll}C & 1.371701 & -0.560837 & 3.309540\end{array}$

$\begin{array}{llll}C & 0.37171450 & -1.971276 & 2.020330\end{array}$

$\begin{array}{llll}\mathrm{H} & 0.331450 & -1.971276 & 2.020330\end{array}$

\begin{tabular}{llll}
$C$ & -1.047478 & -0.413337 & 2.436598 \\
\hline
\end{tabular}

$\begin{array}{llll}\text { H } & -2.544968 & 0.080155 & 0.554063\end{array}$

$\begin{array}{llll}\text { C } & -2.057985 & 2.145192 & 0.821808\end{array}$

$\begin{array}{llll}\text { C } & -1.429840 & 0.526867 & 3.551441\end{array}$

\begin{tabular}{llll} 
H & -0.766691 & 1.399538 & 3.600423 \\
\hline
\end{tabular}

$\begin{array}{llll}\text { H } & -2.455452 & 0.893732 & 3.440383\end{array}$

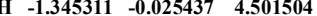

$\begin{array}{lllll}\text { O } & -2.685606 & 2.598331 & 1.749236\end{array}$

$\begin{array}{lllll}O & -1.720517 & \mathbf{2 . 8 4 4 5 2 4} & -0.254610\end{array}$

$\begin{array}{llll}C & -1.720517 & 2.844524 & -0.254610\end{array}$

$\begin{array}{llll}C & -1.979205 & 4.240658 & -0.225269\end{array}$

$\begin{array}{llll}\text { H } & -1.464164 & 4.701751 & 0.629160\end{array}$ 
$\begin{array}{llll}\text { H } & -\mathbf{3 . 0 5 7 8 9 7} & \mathbf{4 . 4 4 0 1 6 5} & -\mathbf{0 . 1 5 2 3 5 7}\end{array}$ $\begin{array}{llll}\text { H } & -1.577137 & 4.642623 & -1.161419\end{array}$ $\begin{array}{llll}\text { H } & \mathbf{2 . 3 6 6 6 6 7} & \mathbf{- 0 . 7 3 5 6 4 8} & \mathbf{2 . 8 7 7 1 2 3}\end{array}$ $\begin{array}{llll}\text { H } & 1.279952 & -1.203683 & 4.201947\end{array}$ $\begin{array}{llll}\text { H } & 1.328073 & 0.485720 & 3.632465\end{array}$ $\begin{array}{llll}\text { H } & -1.821502 & -1.137337 & \mathbf{2 . 1 7 0 9 4 6}\end{array}$

77

sat pc2 c / electronic energy: $-\mathbf{2 5 9 0 . 1 1 2 9 9 2 3 3 ~ a . u . ~ / ~ l o w e s t ~ f r e q : ~} \mathbf{1 7 . 2 9} \mathrm{cm}-1$

S $\mathbf{0 . 8 5 6 0 0 2} \quad 1.315636-1.215393$

$\begin{array}{llll}C & -0.463432 & -1.486083 & -0.465692\end{array}$

$\begin{array}{lllll}\text { Ru } & 0.094427 & 0.170763 & 0.676455\end{array}$

$\begin{array}{llll}\text { C } & 0.146897 & -1.427327 & 2.425966\end{array}$

$\begin{array}{llll}\text { C } & -1.460147 & 1.129114 & 0.679771\end{array}$

$\begin{array}{lllll}\text { S } & 1.670680 & 1.408793 & 1.875823\end{array}$

N $-1.692878-1.935144 \quad-0.776797$

$\begin{array}{lllll}N & 0.418105 & -2.392648 & -0.933915\end{array}$

$\begin{array}{llll}C & -2.921785 & -1.257927 & -0.591079\end{array}$

$\begin{array}{llll}C & -1.695193 & -3.231204 & -1.467598\end{array}$

$\begin{array}{llll}C & -0.204921 & -\mathbf{3 . 5 5 1 3 1 8} & -\mathbf{1 . 5 8 1 0 7 0}\end{array}$

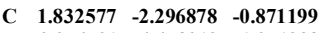

H $\quad 0.074701-4.473012-1.051223$

H $\quad 0.138868-3.633374 \quad-2.622782$

H $\quad-2.247236-3.971216 \quad-0.871210$

$\begin{array}{llll}C & -5.381049 & 0.026902 & -0.167661\end{array}$

$\begin{array}{llll}C & -3.737365 & -1.592370 & 0.488444\end{array}$

$\begin{array}{llll}\text { C } & -3.364101 & -0.303345 & -1.516678\end{array}$

$\begin{array}{llll}C & -4.587050 & 0.329715 & -1.278142\end{array}$

$\begin{array}{llll}C & -4.944795 & -0.960224 & 0.724770\end{array}$

$\begin{array}{llll}\text { H } & -4.925353 & 1.093867 & -1.981783\end{array}$

H $\quad-5.533803 \quad-1.244916 \quad 1.598680$

C $\quad 4.627551 \quad-2.116822 \quad-0.622801$

$\begin{array}{llll}C & \mathbf{2 . 5 1 0 2 7 0} & \mathbf{- 2 . 8 8 5 5 4 0} & \mathbf{0 . 1 9 8 2 8 7}\end{array}$

$\begin{array}{llll}C & 2.576081 & -1.688165 & -1.894065\end{array}$

$\begin{array}{llll}C & 3.962280 & -1.596193 & -1.736879\end{array}$

C $3.962280-1.596193-1.736879$

$\begin{array}{llll}C & 3.882034 & -2.793614 & 0.349100\end{array}$

$\begin{array}{llll}\text { F } & 1.803101 & -3.582076 & 1.095141\end{array}$

$\begin{array}{llll}C & 1.920472 & -1.214373 & \mathbf{- 3 . 1 6 0 7 5 0}\end{array}$

$\begin{array}{lllll}\text { H } & 4.543716 & -1.101224 & -2.518918\end{array}$

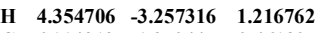

$\begin{array}{lllll}\text { C } & 6.114219 & -1.950445 & -0.461307\end{array}$

$\begin{array}{llll}\text { H } & 6.565471 & -2.824064 & 0.029601\end{array}$

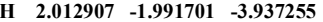

$\begin{array}{llll}\text { H } & -2.190609 & -3.138173 & -2.445128\end{array}$

$\begin{array}{llll}\text { H } & 6.332429 & -1.070136 & 0.163954\end{array}$

$\begin{array}{llll}\text { H } & 6.608262 & -1.799558 & -1.430791\end{array}$

$\begin{array}{llll}\text { H } & \mathbf{2 . 4 0 4 1 1 0} & \mathbf{- 0 . 3 0 3 3 2 2} & \mathbf{- 3 . 5 3 5 6 6 8}\end{array}$

$\begin{array}{llll}\text { H } & \mathbf{0 . 8 5 5 6 5 9} & \mathbf{- 0 . 9 9 5 2 3 4} & \mathbf{- 3 . 0 1 7 4 8 8}\end{array}$

$\begin{array}{llll}\text { F } & -3.320488 & -2.548413 & 1.328479\end{array}$

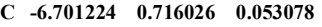

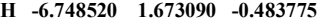

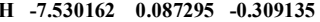

$\begin{array}{llll}\text { H } & -6.877273 & 0.907330 & 1.121029\end{array}$

$\begin{array}{llll}C & -2.517948 & 0.026200 & -2.712955\end{array}$

C -2.517938 - $0.026200-2.71255$

$\begin{array}{llll}C & -2.5176234 & -0.879777 & -3.285633\end{array}$

$\begin{array}{llll}\text { H } & -3.039872 & 0.722480 & -3.381266\end{array}$

$\begin{array}{lllll}\text { H } & -1.576479 & 0.500191 & -2.398965\end{array}$

$\begin{array}{llll}C & 1.931621 & 2.569390 & -0.611950\end{array}$

$\begin{array}{llll}C & \mathbf{2 . 4 5 4 8 2 9} & \mathbf{3 . 5 3 1 8 2 9} & \mathbf{- 1 . 4 9 2 5 0 9}\end{array}$

$\begin{array}{llll}C & 2.294123 & 2.606657 & 0.744231\end{array}$

$\begin{array}{llll}C & 3.328485 & 4.511470 & -1.035806\end{array}$

$\begin{array}{llll}\text { H } & 2.166779 & 3.498439 & \mathbf{- 2 . 5 4 6 8 0 4}\end{array}$

$\begin{array}{llll}\text { C } & 3.173745 & 3.604519 & 1.196340\end{array}$

C 3.6903

$\begin{array}{llll}C & 3.690325 & 4.548186 & \mathbf{0 . 3 1 6 1 6 2}\end{array}$

$\begin{array}{llll}\text { H } & 3.726550 & \mathbf{5 . 2 5 2 3 3 9} & \mathbf{- 1 . 7 3 3 1 7 6}\end{array}$

$\begin{array}{lllll}\text { H } & \mathbf{3 . 4 4 7 3 6 8} & \mathbf{3 . 6 3 0 7 7 7} & \mathbf{2 . 2 5 4 4 7 0}\end{array}$

$\begin{array}{cccc}\text { H } & 4.374414 & 5.316995 & \mathbf{0 . 6 8 2 6 9 0}\end{array}$

$\begin{array}{llll}\text { C } & 1.382637 & -1.450498 & 3.283924\end{array}$

$\begin{array}{llll}\text { H } & -0.009123 & -2.342961 & 1.851253\end{array}$

$\begin{array}{llll}\text { C } & \mathbf{- 0 . 9 5 7 3 3 6} & -\mathbf{0 . 6 3 3 1 8 4} & \mathbf{2 . 6 4 0 0 2 4}\end{array}$

$\begin{array}{llll}\text { H } & \mathbf{- 2 . 3 6 6 7 5 4} & \mathbf{0 . 8 8 0 7 1 7} & \mathbf{1 . 2 5 9 8 4 3}\end{array}$

$\begin{array}{llll}\text { C } & -1.585250 & 2.497191 & 0.084065\end{array}$

\begin{tabular}{llll}
$C$ & -1.106953 & 0.380426 & 3.740295 \\
\hline
\end{tabular}

$\begin{array}{lll}-1.106954 & 0.380426 & 3.740295\end{array}$

$\begin{array}{lllll}\text { H } & -0.156544 & 0.857765 & 4.008050\end{array}$

$\begin{array}{llll}\mathrm{H} & -1.811003 & 1.174241 & \mathbf{3 . 4 5 2 9 9 7}\end{array}$

$\begin{array}{llll}H & -1.517380 & -0.112161 & 4.637656\end{array}$

$\begin{array}{lllll}\text { O } & -1.178830 & 3.492884 & 0.635640\end{array}$

\begin{tabular}{lllll}
\hline & -2.273419 & 2.523025 & -1.054197
\end{tabular}

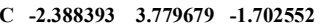

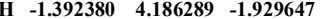

$\begin{array}{lllll}\text { H } & -2.934559 & 4.498330 & -1.075478\end{array}$

$\begin{array}{llll}\text { H } & -2.939421 & 3.595023 & -2.631199\end{array}$

$\begin{array}{llll}4 & 2.292820 & -1.614050 & 2.692745\end{array}$

\begin{tabular}{llll}
\hline & 2.292820 & -1.614050 & 2.692745 \\
\hline
\end{tabular}

\begin{tabular}{llll} 
H & $\mathbf{1 . 2 9 2 9 2 5}$ & $\mathbf{- 2 . 2 9 2 1 8 5}$ & $\mathbf{3 . 9 9 1 2 5 1}$ \\
\hline & $\mathbf{1 . 5 1 6 9 1 7}$ & -0.530165 & 3.864186
\end{tabular}

$\begin{array}{llll}\text { H } & 1.516917 & \mathbf{- 0 . 5 3 0 1 6 5} & \mathbf{3 . 8 6 4 1 8 6}\end{array}$

$\begin{array}{llll}\text { H } & -1.896967 & -0.994132 & 2.219512\end{array}$

65

sat_14e2 / electronic energy: -2433.03474668 a.u. / lowest freq: $11.72 \mathrm{~cm}-1$

S $1.041507 \quad 1.157786-1.732126$

$\begin{array}{llll}C & -0.559448 & -1.146654 & -0.260380\end{array}$

$\begin{array}{llll}\text { Ru } & -0.057551 & \mathbf{0 . 7 5 8 5 6 7} & \mathbf{0 . 2 5 0 5 5 6}\end{array}$

$\begin{array}{llll}\text { C } & -1.512410 & \mathbf{1 . 8 1 7 3 7 1} & \mathbf{0 . 0 0 4 5 3 8}\end{array}$

$\begin{array}{llll}\text { S } & 1.720248 & 1.645229 & 1.362454\end{array}$

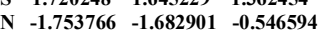


$\begin{array}{llll}N & 0.360855 & -2.125560 & -0.267270\end{array}$ $\begin{array}{llll}C & -2.983734 & -0.991275 & -0.67456\end{array}$

$\begin{array}{llll}C & -1.675541 & -3.127643 & -0.80822\end{array}$

$\begin{array}{llll}C & -0.239442 & -3.458637 & -0.399746\end{array}$

$\begin{array}{llll}C & 1.713911 & -1.957839 & 0.139338\end{array}$

$\begin{array}{llll}\text { H } & -0.185909 & -3.982461 & 0.566981\end{array}$

H $0.294233-4.055358-1.150967$

$233-4.055358-1.150967$

C -5.45301

C -5.45301

$\begin{array}{lllll}C & -3.919548 & -1.087234 & 0.356143\end{array}$

$\begin{array}{llll}C & -3.296952 & -0.244346 & -1.818705\end{array}$

$\begin{array}{llll}C & -4.526871 & 0.419393 & -1.859050\end{array}$

$\begin{array}{llll}C & -5.142619 & -0.443112 & 0.302589\end{array}$

$\begin{array}{llll}\text { H } & -4.770309 & 1.017060 & -2.741096\end{array}$

$\begin{array}{llll}\text { H } & -5.837926 & -0.547061 & 1.137697\end{array}$

$\begin{array}{llll}\text { C } & 4.340795 & -1.547942 & 1.040790\end{array}$

$0099 \quad 1.497843$

$\begin{array}{llll}C & 2.762761 & -1.919113 & -0.795205\end{array}$

$\begin{array}{llll}C & 4.057605 & -1.708899 & -0.321265\end{array}$

C $3.295705 \quad-1.641537 \quad 1.961570$

$\begin{array}{llll}\text { F } & 0.999315 & -1.921862 & \mathbf{2 . 3 7 1 6 0 4}\end{array}$

$\begin{array}{llll}C & 2.483269 & -2.083328 & -2.261117\end{array}$

H $4.875553-1.650082-1.043740$

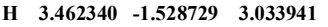

C 5.741868 -1.237491 1.492333

$\begin{array}{llll}\text { H } & \mathbf{5 . 8 8 0 9 3 2} & -\mathbf{1 . 4 4 4 4 5 4} & \mathbf{2 . 5 6 2 0 4 2}\end{array}$

$\begin{array}{llll}\text { H } & 3.412422 & -\mathbf{2 . 0 3 2 6 8 8} & -\mathbf{2 . 8 4 3 3 1 1}\end{array}$

H $\begin{array}{llll}-1.870204 & -3.322003 & -1.874360\end{array}$

$\begin{array}{llll}\text { H } & 5.956604 & -0.170144 & 1.322549\end{array}$

H $\quad 6.482713-1.816950 \quad 0.923154$

H $\quad 1.808683-1.283998 \quad-2.603912$

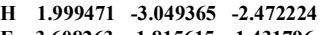

$\begin{array}{llll}\text { F } & -3.608263 & -1.815615 & 1.431796\end{array}$

$\begin{array}{llll}\text { C } & -6.753231 & \mathbf{1 . 0 9 5 6 5 2} & \mathbf{- 0 . 8 6 4 6 1 2}\end{array}$

$\begin{array}{llll}\text { H } & -6.936728 & 1.517264 & -1.861746\end{array}$

\begin{tabular}{llll}
\hline & -7.600970 & 0.447656 & $\mathbf{- 0 . 5 9 8 2 1 0}$
\end{tabular}

$\begin{array}{lllll}H & -6.737805 & 1.927855 & -0.143430\end{array}$

$\begin{array}{llll}C & \mathbf{- 2 . 3 0 9 8 6 8} & \mathbf{- 0 . 1 3 1 2 4 9} & \mathbf{- 2 . 9 4 4 5 7 0}\end{array}$

$\begin{array}{llll}\text { H } & -1.844260 & -1.100192 & -3.175448\end{array}$

$\begin{array}{llll}\text { H } & -2.790663 & 0.251632 & -3.853693\end{array}$

$\begin{array}{lllll}\text { H } & -1.496365 & 0.557833 & -2.669669\end{array}$

$\begin{array}{llll}C & \mathbf{2 . 6 6 4 1 5 9} & \mathbf{1 . 6 3 4 0 9 6} & \mathbf{- 1 . 2 3 2 9 6 4}\end{array}$

$\begin{array}{llll}\text { C } & 3.684763 & 1.760959 & -2.189312\end{array}$

$\begin{array}{llll}\text { C } & 2.959631 & 1.875526 & 0.118778\end{array}$

$\begin{array}{llll}C & 4.973452 & 2.118957 & -1.809365\end{array}$

$\begin{array}{lllll}\text { H } & \mathbf{3 . 4 5 7 2 6 8} & \mathbf{1 . 5 6 2 1 8 4} & \mathbf{- 3 . 2 4 0 0 4 9}\end{array}$

C $4.257786 \quad 2.252261 \quad 0.491600$

$\begin{array}{llll}C & \mathbf{5 . 2 6 2 7 9 5} & \mathbf{2 . 3 6 8 0 6 2} & \mathbf{- 0 . 4 6 3 0 5 7}\end{array}$

$\begin{array}{llll}\text { H } & 5.757583 & \mathbf{2 . 2 0 3 2 8 5} & \mathbf{- 2 . 5 6 5 2 7 8}\end{array}$

$\begin{array}{lllll}\text { H } & \mathbf{4 . 4 7 5 8 7 5} & \mathbf{2 . 4 3 8 3 4 1} & \mathbf{1 . 5 4 6 5 9 2}\end{array}$

$\begin{array}{lllll}\text { H } & 6.273764 & \mathbf{2 . 6 4 8 1 7 4} & \mathbf{- 0 . 1 5 8 6 2 4}\end{array}$

$\begin{array}{llll}\text { H } & -1.808679 & 2.559915 & -0.742188\end{array}$

$\begin{array}{lllll}C & -2.187006 & 1.569408 & 1.269258\end{array}$

\begin{tabular}{lll}
$C$ & -187006 & 1.569408 \\
\hline & 1.26958
\end{tabular}

\begin{tabular}{llll}
$\mathrm{O}$ & -1.588251 & 0.824035 & 2.060693 \\
\hline
\end{tabular}

$\begin{array}{llll}O & -3.370808 & 2.082997 & 1.516237\end{array}$

$\begin{array}{llll}\text { C } & \mathbf{- 3 . 9 9 6 2 2 0} & \mathbf{1 . 7 2 5 2 0 6} & \mathbf{2 . 7 4 8 4 7 4}\end{array}$

$\begin{array}{llll}\text { H } & \mathbf{- 3 . 4 3 9 7 2 9} & \mathbf{2 . 1 4 7 7 1 8} & \mathbf{3 . 5 9 6 0 2 9}\end{array}$

$\begin{array}{llll}\text { H } & -\mathbf{4 . 0 4 2 7 3 2} & \mathbf{0 . 6 3 3 0 7 0} & \mathbf{2 . 8 5 2 2 8 0}\end{array}$

$\begin{array}{llll}\text { H } & -5.004659 & 2.148768 & 2.708934\end{array}$

77

sat ts PTR c / electronic energy: $-\mathbf{2 5 9 0 . 0 9 8 2 2 0 1 5}$ a.u. / lowest freq: $-192.38 \mathrm{~cm}-1$

Ru ${ }^{-} \mathbf{0 . 0 1 4 7 5 0} \quad 0.011600 \quad-0.527834$

S $0.479873-2.280844-0.483899$

$\begin{array}{llll}C & 2.827038 & -1.329501 & -1.535920\end{array}$

$\begin{array}{llll}\text { S } & 2.070417 & 0.241487 & -1.580252\end{array}$

$\begin{array}{llll}C & -0.886965 & -0.214096 & 1.302511\end{array}$

$\begin{array}{llll}\text { N } & -0.075321 & -0.134976 & 2.372387\end{array}$

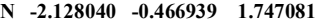

$\begin{array}{llll}\text { C } & -\mathbf{0 . 7 7 0 6 9 4} & -\mathbf{0 . 2 5 0 6 8 0} & \mathbf{3 . 6 5 3 9 6 1}\end{array}$

$\begin{array}{llll}C & -2.204341 & -0.571992 & 3.213618\end{array}$

C $1.295223 \quad 0.220232 \quad 2.246394$

C $1.6273401 .572238 \quad 2.120280$

C $1.627340-1.572238 \quad 2.120280$

C $2.318717-0.742048 \quad 2.211795$

$\begin{array}{llll}C & 2.913597 & 1.991661 & \mathbf{1 . 8 3 4 4 6 1}\end{array}$

$\begin{array}{llll}C & 3.615065 & -0.310750 & 1.923460\end{array}$

$\begin{array}{llll}\text { C } & 3.923217 & 1.034765 & 1.697745\end{array}$

$\begin{array}{llll}\text { H } & 3.106719 & 3.056990 & 1.704168\end{array}$

$\begin{array}{llll}\text { H } & 4.409961 & -1.057867 & 1.854232\end{array}$

$\begin{array}{llll}\text { C } & 5.312978 & 1.441174 & 1.290222\end{array}$

$\begin{array}{lllll}\text { F } & 0.661630 & 2.477017 & \mathbf{2 . 2 8 9 8 4 4}\end{array}$

C $2.046438-2.186400 \quad 2.523906$

$\begin{array}{llll}C & 2.046438 & -2.186400 & 2.523906\end{array}$

$\begin{array}{lllll}\text { H } & 0.992233 & -2.444287 & \mathbf{2 . 3 6 9 8 9 1}\end{array}$

$\begin{array}{lllll}\text { H } & \mathbf{2 . 6 5 4 2 9 6} & \mathbf{- 2 . 8 4 4 1 2 5} & \mathbf{1 . 8 8 7 5 5 5}\end{array}$

$\begin{array}{llll}\text { H } & 2.306747 & -2.393929 & 3.574643\end{array}$

$\begin{array}{llll}\text { C } & -3.305792 & -0.601120 & 0.967821\end{array}$

$\begin{array}{llll}C & -4.171009 & 0.493893 & 0.880252\end{array}$

$\begin{array}{llll}\text { C } & -3.661621 & -1.796069 & 0.329397\end{array}$

$\begin{array}{llll}\text { C } & -\mathbf{5 . 3 4 0 6 9 5} & \mathbf{0 . 4 6 4 2 5 9} & \mathbf{0 . 1 4 6 3 3 9}\end{array}$

$\begin{array}{llll}C & -4.848745 & -1.822678 & -0.414277\end{array}$

$\begin{array}{llll}\text { C } & -5.690222 & -0.713903 & -0.526418\end{array}$

$\begin{array}{llll}\text { H } & -5.964511 & 1.358832 & 0.101604\end{array}$

$\begin{array}{rrrr}H & -5.123367 & -2.751092 & -0.921541\end{array}$ 


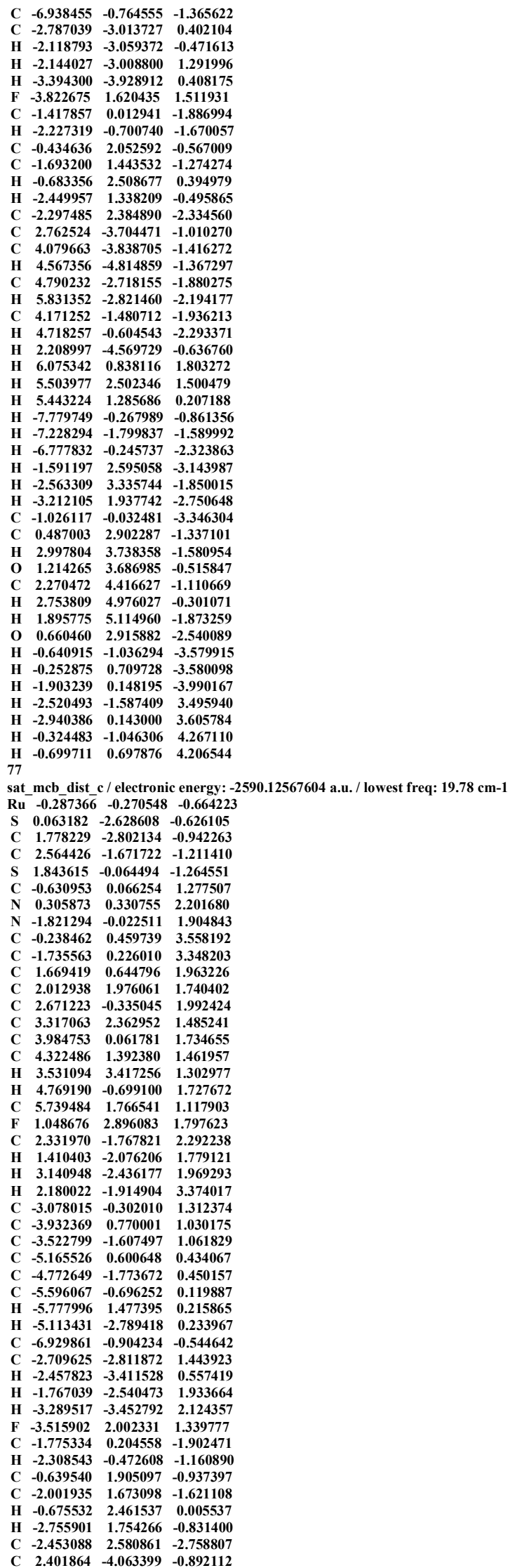


$\begin{array}{llll}\text { C } & 3.767968 & -4.188069 & -1.103755\end{array}$ $\begin{array}{llll}\text { H } & 4.235810 & -5.174142 & -1.057902\end{array}$ $\begin{array}{llll}\text { C } & 4.547514 & -3.052480 & -1.368585\end{array}$

$\begin{array}{llll}\text { H } & \mathbf{5 . 6 2 3 4 0 0} & \mathbf{- 3 . 1 4 8 8 0 4} & -1.529312\end{array}$

C $3.947117-1.802196-1.420221$

$\begin{array}{llll}\text { H } & 4.544245 & -0.907117 & -1.614478\end{array}$

$\begin{array}{lllll}\text { H } & \mathbf{4 . 5 4 4 2 4 5} & \mathbf{- 0 . 9 0 7 1 1 7} & -1.614478 \\ \text { H } & \mathbf{1 . 7 9 5 2 2 0} & \mathbf{- 4 . 9 4 6 3 7 4} & \mathbf{- 0 . 6 7 5 4 4 1}\end{array}$

$\begin{array}{llll}\text { H } & 6.462051 & 1.153737 & 1.674914\end{array}$

$\begin{array}{llll}\text { H } & 5.941603 & 2.825472 & 1.330062\end{array}$

$\begin{array}{llll}\text { H } & 5.925215 & 1.601232 & 0.044345\end{array}$

$\begin{array}{llll}\text { H } & -7.743330 & -0.502008 & 0.077775\end{array}$

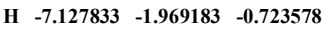

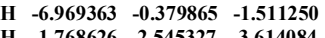

$\begin{array}{llll}-1.407567 & 3.622955 & -2.405660\end{array}$

\begin{tabular}{llll}
\hline & -2.497567 & 3.622955 & -2.405660
\end{tabular}

$\begin{array}{llll}H & -3.463092 & 2.298641 & \mathbf{- 3 . 0 9 5 3 8 6}\end{array}$

$\begin{array}{llll}C & -1.808484 & -0.343203 & -3.302796 \\ C & 0.409983 & 2.452146 & -1.816944\end{array}$

$\begin{array}{llll}\text { C } & 0.409983 & 2.452146 & -1.816944\end{array}$

$\begin{array}{lllll}\text { H } & \mathbf{3 . 0 1 3 4 4 2} & \mathbf{2 . 7 1 5 1 6 7} & \mathbf{- 2 . 3 0 2 3 1 8}\end{array}$

$\begin{array}{lllll}\text { O } & 1.353737 & 3.117777 & -1.120921\end{array}$

C $\quad 2.480004 \quad 3.564161 \quad-1.849512$

H $3.134061 \quad 4.070292-1.130092$

H $\quad 2.188756 \quad 4.264770 \quad-2.645705$

$\begin{array}{llll}\text { O } & 0.490003 & 2.334992 & -3.025223\end{array}$

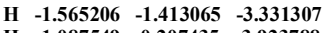

$\begin{array}{llll}\text { H } & -1.087549 & 0.207435 & \mathbf{- 3 . 9 2 3 7 8 8}\end{array}$

$\begin{array}{lllll}\text { H } & \mathbf{- 2 . 8 0 9 7 1 5} & \mathbf{- 0 . 1 9 4 1 8 8} & \mathbf{- 3 . 7 4 2 1 0 6}\end{array}$

$\begin{array}{lllll}\text { H } & -2.113384 & -0.647910 & 3.898287\end{array}$

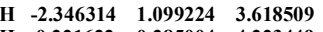

$\begin{array}{llll}\text { H } & 0.221622 & -0.285004 & 4.223449\end{array}$

$\begin{array}{llll}\text { H } & -0.017913 & 1.461469 & 3.952649\end{array}$

77

sat ts_BHE_c / electronic energy: $\mathbf{- 2 5 9 0 . 0 9 9 3 0 3 8 0}$ a.u. / lowest freq: $\mathbf{- 4 5 8 . 9 4} \mathbf{c m}-1$

$\begin{array}{llll}\mathrm{S} & \mathbf{0 . 2 8 8 0 7 3} & \mathbf{2 . 3 8 5 8 5 6} & \mathbf{- 0 . 8 9 3 7 6 0}\end{array}$

$\begin{array}{llll}\text { C } & -0.727157 & 0.318516 & 1.140377\end{array}$

$\begin{array}{llll}\text { Ru } & -0.092558 & 0.018662 & -0.752819\end{array}$

$\begin{array}{llll}\text { C } & -1.185524 & -0.137508 & -2.557391\end{array}$

$\begin{array}{llll}C & -0.645858 & -2.055289 & -1.039354\end{array}$

$\begin{array}{llll}\mathrm{S} & \mathbf{2 . 1 1 7 3 0 0} & -\mathbf{0 . 2 0 7 5 1 7} & -1.136114\end{array}$

$\begin{array}{lllll}\mathrm{N} & 0.159594 & 0.314990 & 2.157042\end{array}$

$\begin{array}{lrrr}\text { N } & -1.923799 & 0.687184 & 1.645074\end{array}$

$\begin{array}{lrrr}\text { C } & 1.466738 & -0.233667 & 2.115255\end{array}$

$\begin{array}{llll}\text { C } & \mathbf{- 0 . 4 4 8 3 6 5} & \mathbf{0 . 6 0 1 9 4 4} & \mathbf{3 . 4 5 8 2 3 5}\end{array}$

$\begin{array}{llll}\text { C } & -1.862828 & 1.024287 & 3.073779\end{array}$

$\begin{array}{llll}C & -3.201324 & 0.626382 & 1.028660\end{array}$

$\begin{array}{llll}\text { C } & 3.991379 & -1.444800 & \mathbf{1 . 9 8 6 9 7 2}\end{array}$

$\begin{array}{llll}C & 1.602839 & -1.622642 & 2.075092\end{array}$

$\begin{array}{llll}\text { C } & 2.610444 & 0.572339 & 2.143465\end{array}$

$\begin{array}{llll}C & 3.857466 & -0.056479 & 2.076964\end{array}$

$\begin{array}{llll}C & \mathbf{2 . 8 3 7 6 5 5} & \mathbf{- 2 . 2 3 7 4 9 2} & \mathbf{2 . 0 0 0 6 0 8}\end{array}$

$\begin{array}{llll}\text { H } & 4.756178 & 0.565929 & 2.069735\end{array}$

$\begin{array}{lrrr}\text { H } & \mathbf{2 . 8 8 7 7 6 0} & \mathbf{- 3 . 3 2 5 9 4 4} & \mathbf{1 . 9 4 0 8 4 0}\end{array}$

$\begin{array}{llll}\text { C } & -5.848513 & 0.412830 & 0.082706\end{array}$

$\begin{array}{llll}C & -5.848513 & 0.412830 & 0.082706\end{array}$

$\begin{array}{llll}C & -4.012974 & -0.468259 & 1.345495 \\ C & -3.736567 & 1.654590 & 0.237833\end{array}$

$\begin{array}{llll}C & -3.736567 & 1.654590 & 0.237833\end{array}$

$\begin{array}{lllll}\text { C } & -5.047509 & 1.514441 & \mathbf{- 0 . 2 3 2 9 2 2}\end{array}$

$\begin{array}{llll}\text { C } & \mathbf{- 5 . 3 1 5 4 4 6} & \mathbf{- 0 . 5 8 9 8 7 0} & \mathbf{0 . 8 9 9 4 1 2}\end{array}$

$\begin{array}{llll}\text { F } & -3.489213 & -1.438466 & 2.101291\end{array}$

$\begin{array}{llll}C & -2.931142 & 2.869671 & -0.111986\end{array}$

$\begin{array}{llll}\text { H } & -5.462340 & 2.310307 & -0.856914\end{array}$

$\begin{array}{llll}\text { H } & -5.892792 & -1.473248 & 1.178015\end{array}$

$\begin{array}{llll}\text { C } & -7.241590 & \mathbf{0 . 2 8 7 0 1 6} & \mathbf{- 0 . 4 7 1 8 2 1}\end{array}$

$\begin{array}{llll}\text { H } & -7.886492 & -0.306716 & 0.190311\end{array}$

$\begin{array}{llll}\text { H } & \mathbf{- 3 . 5 7 3 7 7 0} & \mathbf{3 . 7 5 8 0 6 0} & \mathbf{- 0 . 1 7 4 1 9 8}\end{array}$

$\begin{array}{lllll}\text { H } & -\mathbf{7 . 2 1 7 0 6 7} & \mathbf{- 0 . 2 1 8 2 6 9} & \mathbf{- 1 . 4 5 0 5 1 6}\end{array}$

$\begin{array}{llll}H & -7.704192 & 1.272397 & -0.620717\end{array}$

$\begin{array}{lllll}\text { H } & -2.443349 & 2.738540 & -1.090282\end{array}$

$\begin{array}{llll}\text { H } & -2.130731 & 3.053466 & 0.615665\end{array}$

$\begin{array}{llll}\text { F } & 0.494521 & -2.367115 & 2.100115\end{array}$

$\begin{array}{llll}\text { C } & 5.346251 & -2.082323 & 1.834308\end{array}$

$\begin{array}{llll}\text { H } & 6.144157 & -1.426423 & \mathbf{2 . 2 0 8 5 9 3}\end{array}$

H $5.403191-3.0419$

\begin{tabular}{llll} 
H & $\mathbf{5 . 5 5 1 4 3 4}$ & $\mathbf{- 2 . 2 8 3 6 3 0}$ & $\mathbf{0 . 7 7 0 5 1 0}$ \\
\hline
\end{tabular}

$\begin{array}{llll}\text { C } & 2.498557 & 2.068487 & \mathbf{2 . 2 2 8 7 0 2}\end{array}$

$\begin{array}{llll}\text { H } & 2.469667 & \mathbf{2 . 3 9 7 5 8 6} & \mathbf{3 . 2 8 0 6 1 2}\end{array}$

$\begin{array}{lllll}\text { H } & \mathbf{3 . 3 6 1 6 7 4} & \mathbf{2 . 5 4 9 4 8 7} & \mathbf{1 . 7 4 8 5 9 4}\end{array}$

H $\quad \mathbf{1 . 5 8 6 2 5 3} \quad 2.426455 \quad 1.732235$

$\begin{array}{lllll}C & 2.018813 & 2.530294 & -1.104854\end{array}$

$\begin{array}{llll}C & 2.629120 & 3.801407 & -1.132394\end{array}$

$\begin{array}{llll}C & 2.837729 & 1.391161 & -1.220742\end{array}$

$\begin{array}{llll}C & 4.003671 & 3.929290 & -1.264607\end{array}$

$\begin{array}{lllll}\text { H } & 4.001446 & 4.691209 & -1.038088\end{array}$

$\begin{array}{llll}\text { H } & 2.001446 & 4.691209 & -1.038088\end{array}$

$\begin{array}{llll}\text { C } 4.230373 & 1.530550 & -1.353251 \\ \text { C } 4.814259 & 2.788627 & -1.373752\end{array}$

$\begin{array}{llll}\text { C } & 4.814259 & 2.788627 & -1.373752\end{array}$

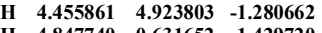

$\begin{array}{lllll}\text { H } & 4.847740 & 0.631652 & -1.429720\end{array}$

$\begin{array}{lllll}\text { H } & 5.896900 & \mathbf{2 . 8 8 9 7 6 9} & \mathbf{- 1 . 4 7 5 4 3 0}\end{array}$

$\begin{array}{llll}\text { C } & \mathbf{- 0 . 3 3 5 8 4 4} & \mathbf{- 0 . 2 8 5 1 3 7} & \mathbf{- 3 . 7 9 4 3 6 8}\end{array}$

$\begin{array}{lllll}\text { H } & -1.941057 & 0.648137 & \mathbf{- 2 . 6 9 2 9 4 0}\end{array}$

$\begin{array}{llll}\text { C } & -1.667888 & -1.177439 & -1.637503\end{array}$

$\begin{array}{llll}C & -0.975747 & -2.550853 & -0.120187\end{array}$

C $0.308234-2.904646-1.766160$

$\begin{array}{llll}C & 0.308234 & -2.904646 & -1.766160\end{array}$

$\begin{array}{llll}C & -3.138871 & -1.544941 & -1.576904\end{array}$ 
$\begin{array}{llll}\text { H } & -3.332320 & -2.216344 & -2.427080\end{array}$ $\begin{array}{lllll}\text { H } & \mathbf{- 3 . 3 7 3 0 9 7} & \mathbf{- 2 . 0 9 0 2 4 6} & \mathbf{- 0 . 6 5 4 5 5 2}\end{array}$ $\begin{array}{llll}\text { H } & -3.798797 & -0.670422 & -1.664851\end{array}$

$\begin{array}{lllll}O & 0.405908 & -3.078444 & -2.963635\end{array}$

$\begin{array}{llll}\text { O } & 1.118730 & -3.537311 & -0.897403\end{array}$

\begin{tabular}{llll} 
C & 2.135014 & -4.349696 & -1.453471 \\
\hline
\end{tabular}

$\begin{array}{lllll}\text { H } & \mathbf{2 . 8 1 3 2 9 8} & -\mathbf{3 . 7 5 2 5 4 1} & \mathbf{- 2 . 0 8 0 1 8 2}\end{array}$

$\begin{array}{cccc}\text { H } & \mathbf{1 . 7 1 0 1 5 0} & \mathbf{- 5 . 1 5 8 6 6 9} & \mathbf{- 2 . 0 6 5 6 6 4} \\ \text { H } & \mathbf{2 . 6 8 7 6 6 6} & \mathbf{- 4 . 7 7 3 4 6 0} & \mathbf{- 0 . 6 0 7 2 9 4}\end{array}$

$\begin{array}{llll}\text { H } & 0.096748 & 0.689735 & -4.058187\end{array}$

$\begin{array}{llll}\text { H } & -0.991311 & -0.597929 & -4.626791\end{array}$

$\begin{array}{lllll}\text { H } & 0.459233 & -1.026329 & -3.695806\end{array}$

\begin{tabular}{rrrr} 
H & -1.769131 & $-\mathbf{0 . 2 0 7 5 8 4}$ & $-\mathbf{0 . 6 4 3 3 1 8}$ \\
\hline & -0.429882 & -0.304932 & 4.08387
\end{tabular}

$\begin{array}{llll}\text { H } & 0.107616 & 1.394581 & 3.977117\end{array}$

\begin{tabular}{llll} 
H & 0.107616 & 1.394581 & 3.97711 \\
\hline$H$ & -2.036249 & 2.103115 & 3.204400
\end{tabular}

$\begin{array}{llll}\text { H } & -2.036249 & 2.103115 & 3.204400 \\ \text { H } & -2.634380 & 0.477889 & 3.631528\end{array}$

sat_prod_BHE_c / electronic energy: $\mathbf{- 2 5 9 0 . 1 4 6 2 6 3 2 0}$ a.u. / lowest freq: $21.60 \mathrm{~cm}-1$

$\begin{array}{llll}S & 0.273155 & -0.801956 & -2.342432\end{array}$

$\begin{array}{llll}C & -0.410281 & 1.097221 & -0.089751\end{array}$

$\begin{array}{llll}\text { Ru } & -\mathbf{0 . 2 8 3 2 6 4} & \mathbf{- 0 . 9 1 0 7 6 4} & \mathbf{- 0 . 0 2 9 0 7 4}\end{array}$

$\begin{array}{llll}C & \mathbf{- 2 . 6 2 9 2 9 7} & \mathbf{- 1 . 4 5 0 0 3 4} & \mathbf{0 . 6 6 9 4 4 0}\end{array}$

$\begin{array}{lllll}\text { C } & -0.438621 & -1.917041 & 1.921483\end{array}$

$\begin{array}{llll}\text { S } & 2.011877 & -1.137349 & 0.337441\end{array}$

$\mathrm{N} \quad \mathbf{0 . 5 5}$

$\begin{array}{lrrrr}N & -1.488703 & 1.784478 & -0.591224\end{array}$

$\begin{array}{llll}\text { C } & 1.824412 & 1.902860 & 0.718919\end{array}$

$\begin{array}{llll}C & \mathbf{0 . 0 8 5 6 8 9} & \mathbf{3 . 4 0 5 3 3 7} & \mathbf{- 0 . 1 0 1 3 4 3}\end{array}$

$\begin{array}{llll}C & -1.174540 & 3.182615 & -0.905870\end{array}$

$\begin{array}{llll}C & -2.860971 & 1.419434 & -0.614250\end{array}$

$\begin{array}{llll}C & 4.310590 & 1.777613 & 2.022937 \\ C & 1.914814 & 1.686091 & 2.094047\end{array}$

$\begin{array}{llll}C & 2.996212 & 2.120335 & -0.014819\end{array}$

$\begin{array}{llll}C & 2.99212 & 2.120335 & -0.014819\end{array}$

$\begin{array}{llll}\text { C } & 4.221825 & 2.044091 & \mathbf{0 . 6 5 5 7 7 5} \\ \text { C } & 3.126010 & 1.607557 & \mathbf{2 . 7 5 1 7 3 4}\end{array}$

\begin{tabular}{llll} 
C & 3.126010 & 1.607557 & 2.751734 \\
\hline$H$ & 5.140750 & 2.183727 & 0.080300
\end{tabular}

\begin{tabular}{cccc} 
H & $\mathbf{5 . 1 4 0 7 5 0}$ & $\mathbf{2 . 1 8 3 7 2 7}$ & $\mathbf{0 . 0 8 0 3 0 0}$ \\
\hline & $\mathbf{3}$ & $\mathbf{2}$
\end{tabular}

$\begin{array}{lllll}\text { H } & 3.137964 & \mathbf{1 . 4 0 9 8 8 9} & \mathbf{3 . 8 2 5 3 6 9}\end{array}$

$\begin{array}{llll}\text { C } & \mathbf{- 5 . 6 2 0 8 2 7} & \mathbf{0 . 8 2 9 8 3 2} & \mathbf{- 0 . 4 3 2 5 0 4}\end{array}$

$\begin{array}{llll}\text { C } & -\mathbf{3 . 6 5 5 6 8 0} & \mathbf{1 . 8 7 7 6 8 8} & \mathbf{0 . 4 4 6 7 3 4}\end{array}$

$\begin{array}{llll}C & -3.495639 & 0.741360 & -1.663307\end{array}$

$\begin{array}{llll}C & -4.860069 & 0.442159 & -1.535577\end{array}$

$\begin{array}{llll}\text { C } & -4.999182 & 1.587135 & 0.569602\end{array}$

$\begin{array}{llll}\text { F } & -\mathbf{3 . 0 7 0 9 3 1} & \mathbf{2 . 6 3 3 1 2 4} & \mathbf{1 . 3 8 7 2 8 0}\end{array}$

$\begin{array}{llll}\text { C } & -2.763908 & 0.322655 & -2.903085\end{array}$

$\begin{array}{lllll}\text { H } & \mathbf{- 5 . 3 4 4 8 9 5} & \mathbf{- 0 . 1 0 8 9 6 3} & \mathbf{- 2 . 3 4 5 6 8 0}\end{array}$

$\begin{array}{llll}\text { H } & \mathbf{- 5 . 5 4 8 0 1 9} & \mathbf{1 . 9 5 7 0 7 2} & \mathbf{1 . 4 3 7 3 8 7}\end{array}$

$\begin{array}{llll}\text { C } & -\mathbf{7 . 0 7 4 1 0 9} & \mathbf{0 . 4 5 9 9 4 6} & \mathbf{- 0 . 3 1 0 6 1 0}\end{array}$

$\begin{array}{llll}\text { H } & -7.682949 & \mathbf{1 . 3 3 0 7 1 2} & \mathbf{- 0 . 0 2 6 6 8 0}\end{array}$

$\begin{array}{llll}\text { H } & -3.388289 & 0.499891 & \mathbf{- 3 . 7 8 9 8 8 2}\end{array}$

$\begin{array}{llll}\text { H } & -7.212210 & -0.303791 & 0.470719\end{array}$

$\begin{array}{llll}\text { H } & -7.466492 & \mathbf{0 . 0 5 3 2 3 4} & \mathbf{- 1 . 2 5 2 1 7 8}\end{array}$

$\begin{array}{llll}\text { H } & -2.530179 & -0.753552 & -2.875935\end{array}$

$\begin{array}{llll}4 & -1.811678 & 0.852409 & -3.016003\end{array}$

\begin{tabular}{ccccc}
\hline H & -1.811678 & $\mathbf{0 . 8 5 2 4 0 9}$ & $\mathbf{- 3 . 0 1 6 0 0 3}$ \\
\hline & 0.782122 & 1.525148 & 2.788418
\end{tabular}

\begin{tabular}{llll} 
F & $\mathbf{0 . 7 8 2 1 2 2}$ & $\mathbf{1 . 5 2 5 1 4 8}$ & $\mathbf{2 . 7 8 8 4 1 8}$ \\
\hline C & 5.64548 & $\mathbf{1 . 6 5 7 3 1 1}$ & $\mathbf{2 . 7 0 7 1 0 0}$
\end{tabular}

$\begin{array}{lllll}\text { C } & \mathbf{5 . 6 4 5 4 4 8} & \mathbf{1 . 6 4 7 3 1 1} & \mathbf{2 . 7 0 7 1 0 0}\end{array}$

$\begin{array}{lllll}\text { H } & \mathbf{6 . 4 5 6 5 7 4} & \mathbf{2 . 0 5 0 9 7 7} & \mathbf{2 . 0 8 5 9 0 1}\end{array}$

$\begin{array}{llll}\text { H } & 5.653751 & 2.172549 & 3.673337\end{array}$

$\begin{array}{lllll}\text { H } & 5.872162 & 0.588271 & 2.908639\end{array}$

$\begin{array}{llll}\text { C } & \mathbf{2 . 9 4 7 8 7 6} & \mathbf{2 . 4 1 9 8 0 8} & -1.487642\end{array}$

\begin{tabular}{ccccc} 
H & 3.055214 & 3.501891 & -1.669424 \\
\hline & 3.76091 & 1.908468 & -2.010620
\end{tabular}

$\begin{array}{lllll}\text { H } & 3.768091 & \mathbf{1 . 9 0 8 4 6 8} & \mathbf{- 2 . 0 1 0 6 2 0}\end{array}$

$\begin{array}{lllll}\text { H } & \mathbf{2 . 0 0 3 3 0 0} & \mathbf{2 . 0 8 3 6 8 3} & \mathbf{- 1 . 9 3 4 6 0 4}\end{array}$

$\begin{array}{llll}C & 2.035451 & -0.748486 & -2.387144\end{array}$

$\begin{array}{llll}C & \mathbf{2 . 6 9 6 9 4 7} & \mathbf{- 0 . 5 5 2 9 9 8} & \mathbf{- 3 . 6 1 0 8 8 0}\end{array}$

$\begin{array}{llll}C & \mathbf{2 . 7 9 7 3 2 0} & \mathbf{- 0 . 9 1 1 9 1 5} & \mathbf{- 1 . 2 1 8 1 2 7}\end{array}$

$\begin{array}{llll}\text { C } & 4.086464 & -0.527261 & -3.678126\end{array}$

$\begin{array}{llll}\text { H } & \mathbf{2 . 1 0 2 9 8 0} & \mathbf{- 0 . 4 1 8 7 5 5} & \mathbf{- 4 . 5 1 9 0 1 2}\end{array}$

$\begin{array}{llll}C & 4.198689 & -0.877243 & -1.293470\end{array}$

$\begin{array}{llll}C & 4.842813 & -0.692299 & -2.512212\end{array}$

$\begin{array}{lllll}\text { H } & 4.581298 & -0.375514 & -4.640309\end{array}$

$\begin{array}{llll}\text { H } & 4.778873 & \mathbf{- 0 . 9 8 5 4 8 2} & -\mathbf{0 . 3 7 3 1 9 8}\end{array}$

$\begin{array}{lllll}\text { H } & 5.934355 & -0.668603 & -2.554186\end{array}$

$\begin{array}{llll}C & -3.316412 & -2.811610 & 0.748581\end{array}$

$\begin{array}{llll}\text { H } & \mathbf{- 3 . 3 9 5 8 8 0} & \mathbf{- 0 . 6 7 0 5 4 6} & \mathbf{0 . 7 1 5 9 8 0}\end{array}$

$\begin{array}{lrrr}\text { C } & -1.574672 & -1.116881 & 1.694726\end{array}$

$\begin{array}{lllll}\text { H } & 0.180931 & -1.694960 & 2.792998\end{array}$

$\begin{array}{llll}C & -0.118970 & -3.166961 & 1.220331\end{array}$

$\begin{array}{llll}C & -1.932353 & -0.098963 & 2.744406\end{array}$

$\begin{array}{llll}\text { H } & -2.777815 & -0.479669 & 3.341285\end{array}$

$\begin{array}{llll}\text { H } & -1.090059 & 0.094927 & 3.417917\end{array}$

$\begin{array}{llll}\text { H } & -2.239325 & 0.852702 & 2.301629\end{array}$

\begin{tabular}{llll}
\hline & $-\mathbf{0 . 3 5 0 2 9 2}$ & $-\mathbf{3 . 2 9 0 9 0 2}$ & $\mathbf{0 . 0 1 8 8 1 6}$
\end{tabular}

$\begin{array}{llll}\text { O } & \mathbf{0 . 5 0 8 3 3 8} & \mathbf{- 4 . 0 7 3 0 7 5} & \mathbf{1 . 9 3 6 7 9 7}\end{array}$

$\begin{array}{llll}\text { C } & \mathbf{1 . 0 4 2 0 4 5} & \mathbf{- 5 . 1 9 7 2 1 4} & \mathbf{1 . 2 3 7 8 7 0}\end{array}$

$\begin{array}{lllll}\text { H } & 1.761179 & -4.858242 & 0.479867\end{array}$

$\begin{array}{llll}\text { H } & \mathbf{0 . 2 3 9 8 4 0} & \mathbf{- 5 . 7 6 8 9 2 5} & \mathbf{0 . 7 5 2 8 8 5}\end{array}$

$\begin{array}{llll}\text { H } & 1.543267 & \mathbf{- 5 . 8 1 1 3 6 8} & \mathbf{1 . 9 9 2 2 8 5}\end{array}$

$\begin{array}{llll}\text { H } & -4.094756 & -2.881030 & -0.024531\end{array}$

$\begin{array}{llll}\text { H } & -3.800922 & -2.919017 & 1.730402\end{array}$

$\begin{array}{llll}\text { H } & -2.623146 & -3.647254 & 0.606157\end{array}$

$\begin{array}{llll}\text { H } & -2.211574 & -1.340352 & -0.396967\end{array}$

$\begin{array}{llll}\text { H } & -\mathbf{0 . 1 0 7 2 0 8} & \mathbf{3 . 8 8 1 4 8 3} & \mathbf{0 . 8 7 4 4 0 4}\end{array}$ 
$\begin{array}{lllll}\text { H } & 0.840199 & 3.995251 & -0.635876\end{array}$ H $\quad-1.006712 \quad 3.288428-1.990223$ $\begin{array}{llll}\text { H } & -1.994337 & 3.848916 & -0.612418\end{array}$ 77

sat ts CP c / electronic energy: $-\mathbf{2 5 9 0 . 1 0 8 4 0 5 1 4}$ a.u. / lowest freq: $-\mathbf{2 3 6 . 1 3} \mathrm{cm}-1$

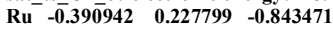

S $1.522825 \quad 1.446611-0.836807$

$\begin{array}{llll}C & 2.795275 & 0.448594 & -1.536836\end{array}$

$\begin{array}{lllll}C & \mathbf{2 . 4 7 3 3 8 2} & \mathbf{- 0 . 8 0 0 9 2 9} & \mathbf{- 2 . 0 8 7 0 4 3}\end{array}$

$\begin{array}{llll}C & 0.798716 & -1.345971 & -2.085391\end{array}$

$\begin{array}{lllll}C & \mathbf{- 0 . 4 3 5 4 7 1} & \mathbf{- 0 . 8 1 6 3 1 8} & \mathbf{0 . 8 0 5 3 0 8}\end{array}$

$\begin{array}{llll}N & -1.568820 & -1.357934 & 1.339412\end{array}$

N $\quad 0.579530-1.176447 \quad 1.627359$

$\begin{array}{llll}C & -1.320025 & -2.182039 & 2.523638\end{array}$

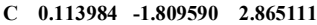

$\begin{array}{llll}C & -2.851555 & -1.403065 & 0.744586\end{array}$

$\begin{array}{llll}C & -3.890747 & -0.694249 & 1.357631\end{array}$

$\begin{array}{llll}C & -3.152447 & -2.211864 & -0.363813\end{array}$

$\begin{array}{llll}C & -5.189001 & -0.711691 & \mathbf{0 . 8 8 4 6 4 1}\end{array}$

$\begin{array}{llll}C & -4.464515 & -2.207002 & -0.853798\end{array}$

$\begin{array}{llll}C & -5.487821 & -1.467832 & -0.255627\end{array}$

$\begin{array}{llll}\text { H } & -5.951519 & -\mathbf{0 . 1 2 5 3 7 4} & \mathbf{1 . 4 0 0 7 4 3}\end{array}$

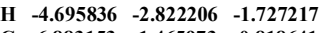

$\begin{array}{llll}C & -6.883153 & -1.465973 & -0.818641\end{array}$

$\begin{array}{llll}\text { F } & -3.599125 & 0.045483 & 2.432259\end{array}$

$\begin{array}{llll}C & -2.105134 & -3.062685 & -1.027543\end{array}$

$\begin{array}{llll}\text { H } & -2.534207 & -4.027149 & -1.331210\end{array}$

$\begin{array}{lllll}\text { H } & -1.716721 & -2.573835 & -1.934341\end{array}$

$\begin{array}{llll}\text { H } & -1.244175 & -3.247999 & -0.372737\end{array}$

$\begin{array}{llll}\text { C } & \mathbf{1 . 9 6 5 4 5 8} & \mathbf{- 0 . 8 8 5 2 4 0} & \mathbf{1 . 5 2 9 1 2}\end{array}$

$\begin{array}{llll}C & \mathbf{2 . 4 5 8 5 1 6} & \mathbf{0 . 3 0 3 4 3 0} & \mathbf{2 . 0 6 2 6 7 5}\end{array}$

$\begin{array}{llll}C & \mathbf{2 . 8 7 2 1 8 0} & \mathbf{- 1 . 8 4 2 4 8 7} & \mathbf{1 . 0 4 7 8 8 2}\end{array}$

$\begin{array}{llll}\text { C } & \mathbf{3 . 8 0 9 2 0 8} & \mathbf{0 . 6 0 6 6 4 7} & \mathbf{2 . 0 7 3 2 5 9}\end{array}$

$\begin{array}{llll}C & 4.234945 & -1.535258 & \mathbf{1 . 0 6 6 2 4 4}\end{array}$

$\begin{array}{llll}C & 4.234945 & -1.535258 & 1.066244\end{array}$

C $4.719613 \quad-0.319959-1.557687$

$\begin{array}{llll}\text { H } & 4.135746 & 1.562990 & 2.486096\end{array}$

$\begin{array}{llll}\text { H } & 4.942499 & -2.269413 & 0.673089\end{array}$

$\begin{array}{llll}\text { C } & 6.187848 & 0.001677 & 1.502771\end{array}$

$\begin{array}{llll}\text { C } & \mathbf{2 . 3 8 2 3 6 7} & \mathbf{- 3 . 1 7 2 0 2 7} & \mathbf{0 . 5 4 8 5 1 2}\end{array}$

$\begin{array}{llll}\text { H } & 3.215830 & -3.777199 & 0.168747\end{array}$

$\begin{array}{llll}\text { H } & \mathbf{1 . 8 8 7 2 5 6} & \mathbf{- 3 . 7 4 1 5 7 8} & \mathbf{1 . 3 5 1 3 8 5}\end{array}$

$\begin{array}{lllll}\text { H } & 1.651104 & -3.029910 & -0.261331\end{array}$

$\begin{array}{llll}\text { F } & \mathbf{1 . 5 9 2 2 4 9} & \mathbf{1 1 6 4 5 4 7} & \mathbf{2 . 5 9 8 7 4 5}\end{array}$

$\begin{array}{llll}C & -2.228029 & 1.263508 & -1.368368\end{array}$

\begin{tabular}{llll} 
C & -2.228029 & 1.263508 & -1.368368 \\
\hline
\end{tabular}

$\begin{array}{llll}C & -2.532573 & 0.186614 & -1.417410\end{array}$

$\begin{array}{llll}C & -1.716688 & 1.897582 & 0.271499\end{array}$

$\begin{array}{llll}C & -3.063289 & 2.046764 & -0.424573\end{array}$

$\begin{array}{llll}\text { H } & -1.770378 & 1.343599 & 1.208191\end{array}$

$\begin{array}{llll}\text { C } & -\mathbf{3 . 6 4 3 8 5 0} & \mathbf{3 . 3 9 3 4 6 7} & \mathbf{- 0 . 7 9 8 3 6 9}\end{array}$

$\begin{array}{llll}\text { H } & -3.809223 & 1.430768 & 0.082894\end{array}$

$\begin{array}{lrrr}\text { C } & 4.124821 & 0.893551 & -1.544238\end{array}$

$\begin{array}{lllll}C & 5.128737 & 0.100998 & -2.088574\end{array}$

$\begin{array}{llll}\text { H } & 6.161986 & 0.456429 & -2.091155\end{array}$

$\begin{array}{llll}\text { H } & 6.161986 & 0.456429 & -2.091155 \\ C & 4.814100 & -1.155364 & -2.619994\end{array}$

\begin{tabular}{llll}
$C$ & 4.814100 & -1.155364 & -2.619994 \\
\hline & 5.600771 & -1.787939 & -3.038064
\end{tabular}

$\begin{array}{llll}\text { C } & 5.600771 & -1.787939 & \mathbf{- 3 . 0 3 8 0 6 4}\end{array}$

$\begin{array}{llll}C & 3.496790 & -1.601545 & -2.618096\end{array}$

$\begin{array}{lllll}\text { H } & 3.247031 & -2.582049 & -3.032354\end{array}$

$\begin{array}{llll}\text { H } & 4.364270 & 1.865548 & -1.104443\end{array}$

$\begin{array}{llll}\text { H } & 6.800947 & -0.908553 & 1.562030\end{array}$

$\begin{array}{llll}\text { H } & 6.486283 & 0.679343 & 2.314967\end{array}$

$\begin{array}{lllll}\text { H } & 6.425527 & 0.497902 & 0.548157\end{array}$

H $\quad-7.097781-0.502757-1.307471$

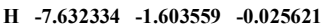

$\begin{array}{llll}\text { H } & -7.016400 & -2.259692 & -1.565686\end{array}$

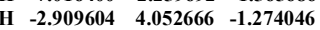

$\begin{array}{llll}\text { H } & -4.019065 & 3.900694 & 0.102848\end{array}$

$\begin{array}{lllll}\text { H } & -4.493458 & 3.254671 & -1.483774\end{array}$

$\begin{array}{lllll}C & -1.839455 & 1.893463 & -2.690795\end{array}$

$\begin{array}{lllll}\text { O } & \mathbf{- 0 . 6 8 0 5 7 5} & \mathbf{3 . 9 3 8 0 9 6} & \mathbf{- 0 . 4 9 1 8 8 8}\end{array}$

$\begin{array}{llll}\text { C } & -\mathbf{0 . 8 3 2 0 0 4} & \mathbf{3 . 0 8 6 6 4 7} & \mathbf{0 . 3 5 6 6 7 0}\end{array}$

$\begin{array}{lllll}\text { O } & -0.210653 & 3.124002 & 1.536193\end{array}$

$\begin{array}{llll}C & 0.766355 & 4.136268 & 1.715436\end{array}$

\begin{tabular}{llll} 
H & 0.317318 & 5.135607 & 1.62521 \\
\hline
\end{tabular}

$\begin{array}{lllll}H & 1.174103 & 3.991589 & 2.72111\end{array}$

$\begin{array}{llll}H & 1.565868 & 4.029281 & 0.968615\end{array}$

$\begin{array}{lllll}\text { H } & -1.166517 & 1.253913 & -3.279296\end{array}$

$\begin{array}{lllll}\text { H } & -1.348741 & 2.859828 & -2.521838\end{array}$

$\begin{array}{llll}\text { H } & -2.754277 & 2.060409 & -3.282820\end{array}$

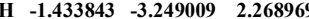

$\begin{array}{llll}\text { H } & -2.029441 & -1.936890 & 3.324629\end{array}$

$\begin{array}{lllll}\text { H } & 0.173635 & -1.084282 & 3.692455\end{array}$

$\begin{array}{lllll}\text { H } & 0.737079 & -2.677144 & 3.119480\end{array}$

sat_prod_CP_c / electronic energy: $-\mathbf{2 5 9 0 . 1 2 2 4 3 8 6 5}$ a.u. / lowest freq: $21.68 \mathrm{~cm}-1$

Ru $\quad-0.314852 \quad-0.019036-0.837672$

$\begin{array}{lllll}S & 1.357375 & 1.498455 & -0.792416\end{array}$

$\begin{array}{llll}C & 2.747794 & 0.706267 & -1.53248\end{array}$

$\begin{array}{llll}C & \mathbf{2 . 5 9 1 8 2 4} & \mathbf{- 0 . 5 5 5 5 1 4} & \mathbf{- 2 . 1 2 4 6 3}\end{array}$

$\begin{array}{llll}\text { S } & 0.995111 & -1.307844 & -2.171787\end{array}$

$\begin{array}{llll}C & -0.197928 & -1.097933 & 0.759178\end{array}$

$\begin{array}{llll}\text { N } & -1.278948 & -1.761624 & 1.266758\end{array}$

$\begin{array}{lll}0.856731 & -1.434821 & 1.540946\end{array}$

$\begin{array}{lrrr}N & 0.856731 & -1.434821 & 1.540946 \\ C & -\mathbf{0 . 9 5 1 0 3 2} & -2.648228 & 2.381863\end{array}$ 
$\begin{array}{llll}C & 0.462251 & -2.194189 & 2.731283\end{array}$ $\begin{array}{llll}C & -2.570845 & -1.769141 & 0.688333\end{array}$ $\begin{array}{llll}C & -3.599561 & -1.082985 & 1.346119\end{array}$ $\begin{array}{llll}C & -2.876767 & -2.468050 & -0.491291\end{array}$ $\begin{array}{llll}C & -4.892574 & -1.035107 & 0.863073\end{array}$ C $-4.183164-2.389735-0.992836$ $\begin{array}{llll}C & -4.183164 & -2.389735 & -0.992836\end{array}$ $\begin{array}{llll}C & -5.195231 & -1.682015 & -0.342584\end{array}$ $\begin{array}{llll}\mathrm{H} & -5.651495 & -0.485208 & 1.42299\end{array}$

$\begin{array}{lllll}\text { H } & -4.416675 & -2.918777 & -1.920346\end{array}$ $\begin{array}{llll}\text { C } & \mathbf{- 6 . 5 8 0 4 7 8} & -\mathbf{1 . 5 8 8 4 0 7} & \mathbf{- 0 . 9 2 1 8 8 5}\end{array}$ $\begin{array}{llll}\text { F } & \mathbf{- 3 . 3 0 0 2 7 1} & \mathbf{- 0 . 4 3 5 4 3 9} & \mathbf{2 . 4 7 5 9 5 6}\end{array}$ $\begin{array}{llll}\text { C } & -1.844733 & -3.292410 & -1.214397\end{array}$

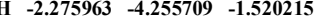
$\begin{array}{llll}\text { H } & -1.503294 & -2.782031 & -2.128037\end{array}$

$\begin{array}{llll}\text { H } & \mathbf{- 0 . 9 5 8 1 1 7} & \mathbf{- 3 . 4 8 2 7 7 6} & \mathbf{- 0 . 5 9 6 8 6 5}\end{array}$

$\begin{array}{llll}\text { C } & 2.170842 & -0.904481 & 1.488722\end{array}$

$\begin{array}{llll}C & \mathbf{2 . 4 3 1 6 3 2} & \mathbf{0 . 3 3 1 7 2 5} & \mathbf{2 . 0 7 4 6 1 5}\end{array}$

$\begin{array}{llll}\text { C } & 3.237711 & -1.646305 & 0.958662\end{array}$

$\begin{array}{llll}C & 3.701247 & \mathbf{0 . 8 8 3 2 8 5} & \mathbf{2 . 1 0 7 0 2 6}\end{array}$

C $\quad 4.518219-1.090715 \quad 1.000560$ $\begin{array}{llll}C & 4.767339 & 0.168769 & 1.554774\end{array}$ H $\quad 3.843135 \quad 1.865772 \quad 2.561158$

$\begin{array}{llll}\text { H } & 5.348935 & -1.654069 & 0.568478\end{array}$

$\begin{array}{llll}\text { C } & \mathbf{6 . 1 4 9 4 4 1} & \mathbf{0 . 7 6 1 2 2 8} & \mathbf{1 . 5 1 6 7 7 8}\end{array}$

$\begin{array}{llll}\text { C } & 2.995772 & -3.007414 & 0.370666\end{array}$ $\begin{array}{llll}\text { H } & 3.914979 & \mathbf{- 3 . 4 1 0 3 7 4} & \mathbf{- 0 . 0 7 4 0 8 9}\end{array}$ $\begin{array}{llll}\text { H } & \mathbf{2 . 6 4 9 4 0 2} & \mathbf{- 3 . 7 1 7 9 8 9} & \mathbf{1 . 1 3 8 2 8 2}\end{array}$

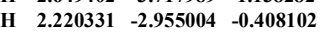

$\begin{array}{lllll}\text { F } & \mathbf{1 . 4 1 6 0 6 7} & \mathbf{0 . 9 9 5 0 8 5} & \mathbf{2 . 6 3 4 0 7 1}\end{array}$

$\begin{array}{llll}C & -3.044857 & 1.579952 & -1.093287\end{array}$

$\begin{array}{llll}\text { H } & -3.221065 & 0.500485 & -1.177444\end{array}$

$\begin{array}{llll}\text { C } & -2.286314 & 1.952390 & 0.193914\end{array}$

$\begin{array}{llll}\text { C } & -3.788572 & 2.223661 & 0.019901\end{array}$

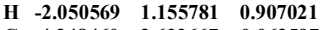

$\begin{array}{llll}C & -4.348460 & 3.622667 & -0.063597\end{array}$

\begin{tabular}{llll}
\hline & -4.401087 & 1.546539 & 0.620062
\end{tabular}

$\begin{array}{llll}C & 4.009802 & 1.317887 & -1.524692\end{array}$

C $\quad \begin{array}{rlll}5.105387 & 0.678033 & -2.091595\end{array}$

H $\quad 6.084481 \quad 1.162798-2.080993$

$\begin{array}{llll}C & \mathbf{4 . 9 5 4 8 6 9} & \mathbf{- 0 . 5 9 1 6 9 7} & \mathbf{- 2 . 6 6 2 9 6 0}\end{array}$

H $\quad \begin{array}{llll}5.816387 & -1.102779 & \mathbf{- 3 . 0 9 9 0 6 6}\end{array}$

$\begin{array}{llll}C & 3.706455 & -1.203088 & -2.678785\end{array}$

$\begin{array}{llll}\text { H } & 3.582204 & -2.194148 & -3.123914\end{array}$

$\begin{array}{lllll}\text { H } & 4.124503 & 2.297898 & -1.053576\end{array}$

$\begin{array}{llll}\text { H } & \mathbf{6 . 9 2 1 6 2 2} & -\mathbf{0 . 0 1 2 9 4 5} & \mathbf{1 . 6 3 0 0 2 8}\end{array}$

\begin{tabular}{cccc}
\hline H & 6.291207 & 1.515030 & 2.303734 \\
\hline
\end{tabular}

\begin{tabular}{llll} 
H & 6.317334 & 1.252942 & 0.545054 \\
\hline
\end{tabular}

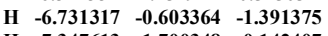

H $\quad-7.347613 \quad-1.700348 \quad-0.142407$

$\begin{array}{llll}\text { H } & -6.748025 & -2.355264 & -1.689919\end{array}$

H $\quad \begin{array}{llll}-3.693787 & 4.303001 & -0.620601\end{array}$

$\begin{array}{lllll}\text { H } & -4.493149 & \mathbf{4 . 0 4 1 3 5 5} & \mathbf{0 . 9 4 3 4 9 7}\end{array}$

$\begin{array}{llll}\text { H } & -5.328996 & \mathbf{3 . 5 9 7 5 8 0} & -0.562527\end{array}$

C $-2.792789 \quad 2.248307-2.423309$

\begin{tabular}{llll}
$C$ & -5.328996 & 3.597580 & -0.562527 \\
\hline & -1.296823 & 4.078307 & -2.423309
\end{tabular}

$\begin{array}{llll}\text { C } & -1.296823 & 4.071594 & -0.422048\end{array}$

$\begin{array}{llll}\text { C } & -1.360981 & 3.111860 & 0.311327\end{array}$

$\begin{array}{llll}\text { O } & -\mathbf{0 . 6 0 4 5 2 6} & 2.979915 & 1.401962\end{array}$

$\begin{array}{llll}\text { C } & \mathbf{0 . 3 6 3 5 3 7} & \mathbf{3 . 9 8 8 4 5 5} & \mathbf{1 . 6 4 5 8 3 7}\end{array}$

$\begin{array}{llll}\text { H } & -0.113408 & 4.974452 & 1.738805\end{array}$

$\begin{array}{lllll}\text { H } & \mathbf{0 . 8 5 7 4 8 9} & 3.712909 & \mathbf{2 . 5 8 2 8 4 8}\end{array}$

$\begin{array}{cccc}\text { H } & 1.099489 & 4.012465 & 0.830135\end{array}$

H $-1.954849 \quad 1.761148 \quad-2.943919$

\begin{tabular}{lllll} 
H & $\mathbf{- 2 . 5 4 1 6 7 5}$ & $\mathbf{3 . 3 0 8 5 8 6}$ & $\mathbf{- 2 . 3 1 1 2 6 2}$ \\
\hline
\end{tabular}

$\begin{array}{llll}\text { H } & \mathbf{- 3 . 6 8 6 9 1 7} & \mathbf{2 . 1 5 5 9 5 9} & \mathbf{- 3 . 0 5 8 2 1 7}\end{array}$

$\begin{array}{llll}H & \mathbf{0 . 9 8 8 0 1 6} & \mathbf{- 3 . 7 0 0 2 4 3} & \mathbf{2 . 0 5 2 7 0 6}\end{array}$

\begin{tabular}{llll}
$H$ & -1.659945 & -2.517565 & 3.210307 \\
\hline
\end{tabular}

$\begin{array}{llll}\text { H } & 0.481400 & -1.534368 & 3.614065\end{array}$

$\begin{array}{llll}\text { H } & 1.151894 & -3.030161 & 2.909242\end{array}$

68

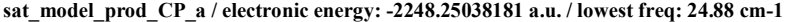

Ru $\quad 0.120222 \quad 0.027021 \quad 1.041338$

$\begin{array}{llll}S & -0.477826 & -1.733241 & 2.367070\end{array}$

$\begin{array}{llll}C & -2.129179 & -1.396795 & \mathbf{2 . 8 4 5 4 5 0}\end{array}$

$\begin{array}{llll}C & -2.129179 & -1.396795 & 2.845450 \\ \text { C } & -2.703113 & -\mathbf{0 . 2 1 7 2 0 1} & \mathbf{2 . 5 5 4 2 1 5}\end{array}$

$\begin{array}{llll}\text { S } & -1.813263 & 1.011769 & 1.680411\end{array}$

$\begin{array}{llll}C & -0.128832 & -0.894353 & -0.631437\end{array}$

N $-1.241403-1.165358-1.355514$

N $\quad 0.902680-1.535343-1.250256$

$\begin{array}{llll}\text { C } & -\mathbf{0 . 9 5 4 1 3 1} & -\mathbf{1 . 8 7 6 0 8 4} & -2.604257\end{array}$

C $\quad 0.497541-2.321081-2.413112$

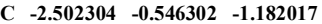

C $\quad-2.684344 \quad 0.761440 \quad-1.628591$

$\begin{array}{llll}C & -3.580310 & -1.227194 & -0.603861\end{array}$

$\begin{array}{llll}C & -3.888496 & \mathbf{1 . 4 2 8 7 6 5} & -\mathbf{1 . 4 9 7 4 3 0}\end{array}$

$\begin{array}{llll}C & -4.797187 & -0.551542 & -0.472656\end{array}$

$\begin{array}{cccc}C & -4.797187 & -0.551542 & -0.472656 \\ \text { C } & -4.968120 & \mathbf{0 . 7 6 8 8 8 3} & \mathbf{- 0 . 9 0 0 5 8 6}\end{array}$

$\begin{array}{lllll}\text { H } & \mathbf{- 3 . 9 7 0 7 5 9} & \mathbf{2 . 4 5 8 0 3 8} & \mathbf{- 1 . 8 5 1 7 4 4}\end{array}$

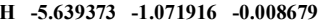

$\begin{array}{llll}\text { C } & -6.276049 & 1.484273 & -0.688596\end{array}$

$\begin{array}{llll}\text { F } & -1.644209 & 1.390203 & -2.190626\end{array}$

$\begin{array}{llll}\text { C }-3.413237 & -2.646938 & -0.141466\end{array}$

$\begin{array}{llll}C & -3.458433 & -2.771499 & 0.388168\end{array}$

$\begin{array}{llll}\text { H } & -2.458433 & -2.771499 & 0.388168 \\ \text { H } & -4.228888 & -2.939233 & 0.532658\end{array}$ 
$\begin{array}{llll}\text { H } & -3.417532 & -3.342346 & -0.996899\end{array}$

$\begin{array}{llll}\text { C } & 2.259289 & -1.339310 & -0.895568\end{array}$

$\begin{array}{llll}C & 2.988855 & -0.372535 & -1.602063\end{array}$

$\begin{array}{llll}\text { C } & 2.910946 & -2.073948 & 0.106663\end{array}$

C $\quad 4.308124 \quad-0.080656-1.322990$

C $4.244111-1.754549-0.404351$

$\begin{array}{llll}\text { C } & 4.244111 & -1.754549 & 0.404351 \\ \text { C } 4.952192 & -0.770554 & -0.285659\end{array}$

$\begin{array}{llll}\text { C } & 4.952192 & -0.770554 & \mathbf{0 . 2 8 5 6 5 9}\end{array}$

$\begin{array}{lllll}\text { H } & 4.822844 & 0.686923 & -1.903931\end{array}$

$\begin{array}{lllll}\text { H } & 4.747177 & -2.313758 & 1.197595 \\ \text { C } & \mathbf{6 . 3 7 8 2 3 2} & \mathbf{- 0 . 4 4 1 1 1 9} & \mathbf{0 . 0 6 3 4 6 5}\end{array}$

$\begin{array}{llll}\text { C } & \mathbf{2 . 2 3 5 0 7 5} & \mathbf{- 3 . 2 1 3 1 6 1} & \mathbf{0 . 8 1 7 1 0 0}\end{array}$

$\begin{array}{llll}\text { H } & 2.304660 & -3.092125 & 1.907539\end{array}$

$\begin{array}{llll}\text { H } & 1.173727 & -3.291749 & 0.554855\end{array}$

$\begin{array}{llll}\text { H } & 2.732978 & -4.160060 & 0.557786\end{array}$

$\begin{array}{llll}\text { F } & \mathbf{2 . 3 5 9 6 2 7} & \mathbf{0 . 3 0 0 8 6 2} & \mathbf{2 . 5 7 1 1 0 7}\end{array}$

C $2.083683 \quad 2.046207 \quad 0.768205$

$\begin{array}{llll}\text { C } & 2.083683 & 2.046207 & 0.768205\end{array}$

$\begin{array}{llll}\mathrm{H} & \mathbf{2 . 4 8 1 2 0 3} & \mathbf{1 . 0 2 5 8 3 3} & \mathbf{0 . 6 8 5 3 4 4} \\ \mathrm{C} & \mathbf{0 . 9 6 9 6 1 6} & \mathbf{2 . 4 3 0 3 3 7} & -0.243147\end{array}$

$\begin{array}{llll}\text { C } & 0.969616 & 2.430337 & \mathbf{- 0 . 2 4 2 1 4 7}\end{array}$

$\begin{array}{llll}C & \mathbf{2 . 3 9 8 0 9 2} & \mathbf{2 . 8 8 6 9 7 6} & \mathbf{- 0 . 4 3 3 9 5 4}\end{array}$

$\begin{array}{lllll}\text { H } & 0.662399 & 1.654210 & -0.951374\end{array}$

$\begin{array}{llll}\text { C } & -\mathbf{0 . 1 1 5 4 6 2} & \mathbf{3 . 4 4 8 1 9 1} & \mathbf{0 . 0 3 8 3 0 1}\end{array}$

H $2.936586 \quad 2.369273 \quad-1.231885$

$\begin{array}{llll}\text { C } & \mathbf{2 . 7 7 3 4 9 3} & \mathbf{4 . 3 4 5 7 3 3} & \mathbf{- 0 . 3 1 7 0 5 6}\end{array}$

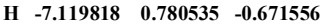

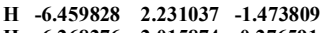

$\begin{array}{lllll}H & -6.268276 & 2.015874 & 0.276591\end{array}$

$\begin{array}{lllll}\text { H } & \mathbf{7 . 0 1 3 8 1 0} & \mathbf{- 0 . 4 3 6 0 4 7} & \mathbf{- 0 . 8 3 4 3 1 7}\end{array}$

$\begin{array}{rrrrr}\text { H } & 6.793445 & -1.163396 & \mathbf{0 . 7 7 8 8 2 0} \\ \text { H } & \mathbf{6 . 4 4 3 6 2 9} & \mathbf{0 . 5 6 0 6 3 7} & \mathbf{0 . 5 1 5 7 6 2}\end{array}$

\begin{tabular}{lllll}
\hline H & $\mathbf{2 . 2 3 7 3 3 6}$ & $\mathbf{4 . 8 6 1 1 9 4}$ & $\mathbf{0 . 4 9 0 9 1 5}$
\end{tabular}

$\begin{array}{lllll}\text { H } & 2.549948 & 4.878486 & -1.254273\end{array}$

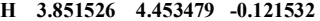

$\begin{array}{llll}\text { H } & -1.103034 & 2.970189 & 0.049840\end{array}$

$\begin{array}{llll}H & -0.125724 & 4.219677 & -0.747413\end{array}$

H $-0.125724 \quad 4.219677-0.747413$

$\begin{array}{llll}\text { H } & 0.014174 & 3.956931 & 1.003446\end{array}$

\begin{tabular}{llll} 
C & 2.164276 & 2.597543 & 2.172971 \\
\hline & 1.698817 & 1.909495 & 2.83807
\end{tabular}

$\begin{array}{lllll}\text { H } & \mathbf{1 . 6 9 8 8 1 7} & \mathbf{1 . 9 0 9 4 9 5} & \mathbf{2 . 8 9 3 8 0 7} \\ \text { H } & \mathbf{1 . 6 6 4 1 5 8} & \mathbf{3 . 5 6 9 2 9 8} & \mathbf{2 . 2 7 7 4 4 6}\end{array}$

$\begin{array}{lllll}\text { H } & \mathbf{3 . 2 1 7 3 0 7} & \mathbf{2 . 7 3 1 8 4 4} & \mathbf{2 . 4 6 5 1 9 6}\end{array}$

$\begin{array}{llll}\text { H } & \mathbf{0 . 5 8 4 8 6 7} & \mathbf{- 3 . 3 9 8 2 5 2} & \mathbf{- 2 . 1 9 8 0 1 4}\end{array}$

H $\quad 1.127933-2.089132 \quad-3.282803$

$\begin{array}{lllll}\text { H } & -1.643650 & -2.720191 & -2.741757\end{array}$

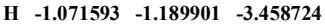

$\begin{array}{llll}\text { H } & -2.657285 & -2.187744 & 3.386413\end{array}$

\begin{tabular}{llll}
\hline & -3.730967 & 0.024174 & 2.840296
\end{tabular}

68

sat_model_ts_CP_a / electronic energy: $\mathbf{- 2 2 4 8 . 2 3 8 5 2 7 2 0}$ a.u. / lowest freq: $\mathbf{- 2 3 8 . 7 0} \mathbf{c m}-1$

$\mathrm{Ru}^{-} \mathbf{0 . 0 4 1 2 8 5} \quad-\mathbf{0 . 4 7 3 1 7 1} \quad-\mathbf{0 . 9 3 6 6 9 7}$

$\begin{array}{lllll}S & -0.382310 & 1.104425 & -2.637616\end{array}$

$\begin{array}{llll}C & -2.026891 & 0.803805 & -3.145010\end{array}$

$\begin{array}{lllll}C & -2.741403 & -0.202749 & -2.614668\end{array}$

$\begin{array}{llll}\text { S } & -2.036379 & -1.254572 & -1.405576\end{array}$

$\begin{array}{lrrrr}C & 0.057909 & 0.815799 & 0.533498\end{array}$

N $-1.008594 \quad 1.302524 \quad 1.213168$

\begin{tabular}{llll}
$\mathrm{N}$ & -1.008594 & 1.302524 & 1.213168 \\
\hline
\end{tabular}

\begin{tabular}{llll}
$\mathrm{N}$ & 1.152409 & 1.457177 & 1.032994 \\
\hline $\mathrm{C}$ & -0.642321 & $\mathbf{2}$
\end{tabular}

$\begin{array}{lllll}\mathrm{C} & \mathbf{- 0 . 6 4 2 3 2 1} & \mathbf{2 . 2 0 1 5 1 7} & \mathbf{2 . 3 0 9 2 5 7}\end{array}$

$\begin{array}{llll}C & 0.827876 & 2.486282 & 2.021716\end{array}$

$\begin{array}{llll}C & -2.352629 & 0.866189 & 1.109486\end{array}$

$\begin{array}{llll}C & -2.771639 & -0.241958 & 1.842644\end{array}$

$\begin{array}{llll}\text { C } & -3.290096 & 1.569759 & 0.342441\end{array}$

$\begin{array}{llll}C & -4.071119 & -0.713246 & \mathbf{1 . 7 9 8 5 5 5}\end{array}$

$\begin{array}{llll}\text { C } & -4.603299 & 1.092497 & 0.299149\end{array}$

$\begin{array}{llll}\text { C } & -\mathbf{5 . 0 0 7 9 7 4} & \mathbf{- 0 . 0 4 6 4 7 7} & \mathbf{1 . 0 0 1 9 1 6}\end{array}$

$\begin{array}{llll}\text { H } & -4.337961 & -1.598380 & 2.379245\end{array}$

$\begin{array}{llll}\text { H } & -\mathbf{5 . 3 3 4 2 4 1} & \mathbf{1 . 6 2 7 2 9 7} & \mathbf{- 0 . 3 1 3 0 5 9}\end{array}$

$\begin{array}{llll}\text { C } & -6.414126 & -0.570678 & 0.881998\end{array}$

$\begin{array}{llll}\text { F } & -1.875859 & -0.872982 & 2.613445\end{array}$

C $\quad \mathbf{- 2 . 8 7 7 6 7 7} \mathbf{2 . 8 0 6 9 2 1} \mathbf{- 0 . 4 0 2 7 7 0}$

H $\quad \mathbf{- 1 . 9 6 9 0 5 2} \quad \mathbf{2 . 6 1 9 3 4 6} \quad \mathbf{- 0 . 9 9 2 7 7 6}$

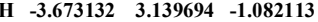

$\begin{array}{llll}\text { H } & -2.661206 & 3.631518 & 0.296010\end{array}$

$\begin{array}{llll}\text { C } & \mathbf{2 . 5 0 3 3 1 7} & 1.226549 & \mathbf{0 . 6 7 9 3 2 2}\end{array}$

$\begin{array}{llll}\text { C } & 3.290276 & 0.461967 & 1.549714\end{array}$

$\begin{array}{llll}C & 3.106281 & 1.775077 & -0.461283\end{array}$

$\begin{array}{llll}C & 4.611665 & 0.157406 & 1.292813\end{array}$

C $\quad \begin{array}{llll}4.446902 & \mathbf{1 . 4 5 8 7 7 1} & \mathbf{- 0 . 7 2 2 5 5 0}\end{array}$

$\begin{array}{llll}\text { C } & 5.207505 & 0.651811 & 0.123974\end{array}$

$\begin{array}{llll}\text { H } & 5.165780 & -0.461547 & 2.000915\end{array}$

H $\quad 4.911553 \quad 1.871984-1.621630$

$\begin{array}{llll}\text { C } & \mathbf{6 . 6 4 1 4 0 4} & \mathbf{0 . 3 1 8 2 2 9} & \mathbf{- 0 . 1 8 7 9 5 5}\end{array}$

$\begin{array}{llll}C & 2361059 & 2.692409 & -1.387416\end{array}$

H $2.253520 \quad 2.238897 \quad-2.383543$

\begin{tabular}{llll} 
H & $\mathbf{2 . 2 5 3 5 2 0}$ & $\mathbf{2 . 2 3 8 8 9 7}$ & $\mathbf{- 2 . 3 8 3 5 4 3}$ \\
\hline & 1 &
\end{tabular}

\begin{tabular}{llll}
$H$ & 1.351972 & 2.916014 & $\mathbf{- 1 . 0 2 2 9 0 2}$ \\
\hline & 2.913313 & 3.635716 & -1.509475
\end{tabular}

$\begin{array}{llll}\text { H } & 2.913313 & 3.635716 & -1.509475\end{array}$

$\begin{array}{lllll}\text { F } & 2.718638 & -0.002237 & \mathbf{2 . 6 6 8 2 6 4}\end{array}$

$\begin{array}{lllll}C & 1.594570 & -1.862960 & -0.654391\end{array}$

$\begin{array}{lllll}\text { H } & \mathbf{2 . 1 0 1 5 8 4} & \mathbf{- 0 . 8 5 4 9 1 1} & \mathbf{- 0 . 7 6 5 5 0 0}\end{array}$

$\begin{array}{llll}\text { C } & 0.347244 & -2.165739 & 0.702099\end{array}$

$\begin{array}{llll}\text { C } & \mathbf{1 . 8 2 8 4 2 5} & \mathbf{- 2 . 4 9 7 1 5 5} & \mathbf{0 . 6 7 6 3 6 4}\end{array}$

$\begin{array}{llll}\text { H } & 0.085862 & -1.493445 & 1.519627\end{array}$

$\begin{array}{llll}\text { C } & -0.632311 & -3.307285 & 0.566204\end{array}$

\begin{tabular}{llll} 
C & -0.632311 & -3.307285 & 0.566204 \\
\hline
\end{tabular}

$\begin{array}{llll}\text { H } & 2.380040 & -1.847889 & 1.359713 \\ \text { C } & 2.315398 & -3.926958 & 0.785431\end{array}$ 


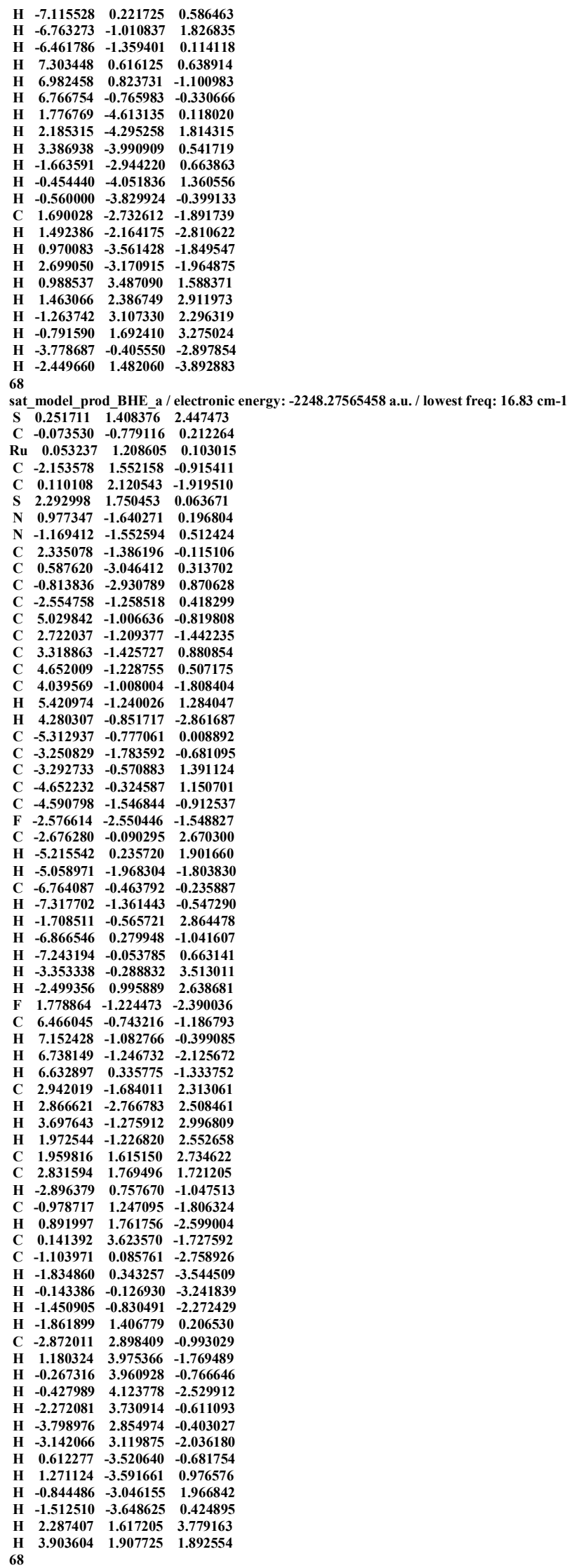


sat_model_ts_BHE_a / electronic energy: $\mathbf{- 2 2 4 8 . 2 2 6 4 8 6 0 2}$ a.u. / lowest freq: $-\mathbf{9 9 . 6 0} \mathrm{cm}-\mathbf{1}$

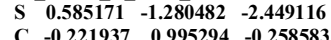

$\begin{array}{llll}C & -0.221937 & 0.995294 & -\mathbf{0 . 2 5 8 5 8 3}\end{array}$

$\begin{array}{llll}\text { Ru } & \mathbf{0 . 2 2 9 2 5 9} & \mathbf{- 1 . 0 0 2 3 9 0} & \mathbf{- 0 . 0 9 7 3 5 5} \\ \mathrm{C} & -0.784167 & -2.781322 & \mathbf{0 . 4 5 7 0 8 4}\end{array}$

$\begin{array}{llll}C & \mathbf{- 0 . 7 8 4 1 6 7} & \mathbf{- 2 . 7 8 1 3 2 2} & \mathbf{0 . 4 5 7 0 8 4}\end{array}$

$\begin{array}{llll}C & -0.321885 & -0.916045 & 1.971763\end{array}$

$\begin{array}{lrrr}\text { S } & \mathbf{2 . 2 9 9 9 2 4} & \mathbf{- 1 . 7 9 4 3 9 7} & \mathbf{0 . 1 3 6 1 7 7} \\ \mathrm{N} & \mathbf{0 . 7 6 7 7 0 6} & \mathbf{1 . 9 1 4 3 0 8} & -0.316679\end{array}$

$\begin{array}{llll}\mathrm{N} & \mathbf{0 . 7 6 7 7 0 6} & \mathbf{1 . 9 1 4 3 0 8} & \mathbf{- 0 . 3 1 6 6 7 9} \\ \mathrm{N} & -1.390093 & \mathbf{1 . 6 6 3 9 0 2} & -0.352757\end{array}$

$\begin{array}{lllll}\mathrm{N} & -1.390093 & 1.663902 & -0.352757\end{array}$

$\begin{array}{lllll}C & \mathbf{2 . 1 2 8 9 3 9} & \mathbf{1 . 6 6 3 8 1 0} & \mathbf{- 0 . 0 0 1 6 4 6}\end{array}$

$\begin{array}{llll}C & 0.276234 & 3.295068 & -0.300690\end{array}$

$\begin{array}{llll}C & -1.210189 & 3.106273 & -0.571500\end{array}$

$\begin{array}{llll}C & -2.715176 & 1.160739 & -0.314710\end{array}$

$\begin{array}{llll}C & 4.790389 & 1.158777 & \mathbf{0 . 7 3 4 6 5 0}\end{array}$

$\begin{array}{llll}C & 2.503174 & 1.552056 & 1.337890\end{array}$

$\begin{array}{llll}C & 3.109680 & 1.577378 & -0.997013\end{array}$

$\begin{array}{lllll}C & 4.427491 & 1.320619 & -0.605728\end{array}$

$\begin{array}{llll}\text { C } & 3.805975 & 1.291873 & 1.720374\end{array}$

$\begin{array}{llll}\text { H } & 5.196409 & 1.237205 & -1.378095\end{array}$

$\begin{array}{lllll}\text { H } & 4.039549 & 1.191142 & 2.781906\end{array}$

$\begin{array}{llll}C & -5.427157 & 0.411115 & -0.131739\end{array}$

$\begin{array}{llll}C & -3.491239 & 1.466006 & 0.809047\end{array}$

$\begin{array}{llll}\text { C } & \mathbf{- 3 . 3 1 8 6 9 0} & \mathbf{0 . 4 8 5 3 7 7} & \mathbf{- 1 . 3 8 5 4 0 9} \\ \text { C } & -4.61721 & \mathbf{0 . 3 5}\end{array}$

$\begin{array}{llll}C & -4.661721 & 0.106753 & -1.259358\end{array}$

$\begin{array}{llll}\text { C } & -4.822913 & 1.115135 & 0.916612\end{array}$

$\begin{array}{llll}\text { F } & \mathbf{- 2 . 9 0 3 7 0 9} & \mathbf{2 . 1 1 1 1 2 3} & \mathbf{1 . 8 2 2 5 7 2}\end{array}$

$\begin{array}{llll}\text { C } & -2.550576 & \mathbf{0 . 1 5 2 2 6 8} & \mathbf{- 2 . 6 2 9 7 7 7}\end{array}$

$\begin{array}{lllll}\text { H } & -5.129125 & -0.430718 & -2.088473\end{array}$

$\begin{array}{llll}\text { H } & -5.369757 & 1.375045 & 1.824916\end{array}$

$\begin{array}{lllll}\text { C } & \mathbf{- 6 . 8 7 4 5 2 4} & \mathbf{0 . 0 0 9 8 2 1} & \mathbf{- 0 . 0 3 5 9 4 9}\end{array}$

$\begin{array}{lllll}\text { H } & -7.527041 & 0.896485 & -0.059138\end{array}$

$\begin{array}{lllll}\text { H } & -1.732156 & \mathbf{0 . 8 6 2 5 7 6} & -\mathbf{2 . 8 0 7 9 6 3}\end{array}$

$\begin{array}{llll}\text { H } & -7.074192 & -0.516460 & 0.909113\end{array}$

$\begin{array}{llll}\text { H } & -7.165500 & -0.648123 & -0.865524\end{array}$

$\begin{array}{llll}\text { H } & -3.212432 & 0.142651 & -3.505888\end{array}$

$\begin{array}{llll}\text { H } & -2.091258 & -0.843932 & -2.538793\end{array}$

$\begin{array}{lllll}\text { F } & \mathbf{1 . 5 6 2 1 0 7} & \mathbf{1 . 6 9 8 7 0 8} & \mathbf{2 . 2 7 5 2 5 4}\end{array}$

$\begin{array}{llll}\text { C } & 6.206958 & 0.823680 & 1.118069\end{array}$

$\begin{array}{lllll}\text { H } & 6.917342 & 1.121264 & 0.334697\end{array}$

$\begin{array}{llll}\text { H } & 6.496552 & 1.316902 & 2.056822\end{array}$

$\begin{array}{llll}\text { H } & 6.314356 & -0.262209 & 1.270666\end{array}$

$\begin{array}{llll}\text { C } & \mathbf{2 . 7 3 7 7 1 2} & \mathbf{1 . 7 4 7 3 6 0} & \mathbf{- 2 . 4 4 1 9 3 4}\end{array}$

$\begin{array}{lllll}\text { H } & 2.287720 & 2.735785 & -2.623718\end{array}$

$\begin{array}{lllll}\text { H } & 3.619135 & 1.649827 & \mathbf{- 3 . 0 8 9 0 8 8}\end{array}$

$\begin{array}{lllll}\text { H } & \mathbf{1 . 9 9 7 3 4 7} & \mathbf{0 . 9 8 8 7 2 5} & \mathbf{- 2 . 7 3 8 1 7 4}\end{array}$

$\begin{array}{llll}\text { C } 2.155397 & -1.976722 & -2.539984\end{array}$

$\begin{array}{llll}C & \mathbf{2 . 8 9 5 7 6 0} & \mathbf{- 2 . 2 2 8 9 2 6} & \mathbf{- 1 . 4 2 7 5 4 4}\end{array}$

$\begin{array}{llll}\text { H } & -1.441326 & -3.096010 & -0.367291\end{array}$

$\begin{array}{llll}\text { C } & -1.373539 & -1.662415 & 1.222517\end{array}$

$\begin{array}{llll}\text { H } & -\mathbf{0 . 7 2 5 4 5 9} & \mathbf{0 . 0 5 8 0 3 2} & \mathbf{2 . 2 8 8 0 6 6}\end{array}$

$\begin{array}{llll}\text { C } & 0.539149 & -1.481774 & 3.084294\end{array}$

$\begin{array}{llll}C & -2.834843 & -1.694386 & 1.654670\end{array}$

$\begin{array}{llll}\text { H } & -2.938832 & -2.486172 & 2.412698\end{array}$

$\begin{array}{llll}\text { H } & -\mathbf{3 . 1 3 3 4 5 9} & -\mathbf{0 . 7 4 5 2 9 0} & \mathbf{2 . 1 1 5 6 5 0}\end{array}$

$\begin{array}{llll}\text { H } & -3.517040 & -1.918550 & 0.820591\end{array}$

$\begin{array}{llll}\text { H } & -1.529082 & -0.923156 & 0.201677\end{array}$

$\begin{array}{llll}\text { C } & -\mathbf{0 . 1 1 7 9 2 9} & \mathbf{- 3 . 9 8 8 6 5 1} & \mathbf{1 . 0 7 3 3 4 0}\end{array}$

$\begin{array}{llll}\text { H } & \mathbf{1 . 1 1 0 3 4 6} & \mathbf{- 0 . 6 5 6 4 4 6} & \mathbf{3 . 5 3 4 3 1 5}\end{array}$

$\begin{array}{llll}\text { H } & 1.277751 & -2.222447 & 2.750461\end{array}$

\begin{tabular}{cccc} 
H & $-\mathbf{0 . 0 5 9 3 5 2}$ & $\mathbf{- 1 . 9 4 2 5 1 9}$ & $\mathbf{3 . 8 9 1 4 2 6}$ \\
\hline & 0.580574 & -3.754246 & 1.880255
\end{tabular}

\begin{tabular}{cccc} 
H & 0.580574 & -3.754246 & 1.880255 \\
\hline & 0.52826 & -4.55460 & 0.305025
\end{tabular}

$\begin{array}{llll}\text { H } & \mathbf{0 . 4 2 8 7 2 6} & -4.554160 & 0.305025\end{array}$

$\begin{array}{lllll}\text { H } & -\mathbf{0 . 8 9 6 7 3 3} & -\mathbf{4 . 6 5 7 1 9 5} & \mathbf{1 . 4 8 3 4 1 5}\end{array}$

$\begin{array}{lllll}\text { H } & \mathbf{0 . 4 6 9 7 1 5} & 3.745101 & \mathbf{0 . 6 8 6 0 0 4}\end{array}$

$\begin{array}{lllll}\text { H } & \mathbf{0 . 7 8 0 1 4 3} & \mathbf{3 . 9 0 1 0 2 6} & \mathbf{- 1 . 0 6 5 4 2 0}\end{array}$

\begin{tabular}{lllll} 
H & -1.488037 & 3.354315 & -1.608295 \\
\hline
\end{tabular}

$\begin{array}{cccc}\text { H } & -1.845685 & 3.686598 & 0.109246\end{array}$

$\begin{array}{lllll}\text { H } & \mathbf{2 . 5 5 3 9 9 8} & -\mathbf{2 . 2 0 4 7 4 4} & \mathbf{- 3 . 5 3 4 1 1 3}\end{array}$

$\begin{array}{llll}\text { H } & 3.886800 & -2.687997 & -1.483217\end{array}$

68

sat model meb dist a / electronic energy: $-\mathbf{2 2 4 8 . 2 5 3 8 5 8 6 0}$ a.u. / lowest freq: $19.19 \mathrm{~cm}-1$

$\mathrm{Ru}^{-0.194691} \mathbf{0 . 8 6 7 1 7 1}-\mathbf{0 . 0 2 6 3 5 6}$

$\begin{array}{llll}\mathrm{S} & -\mathbf{0 . 0 1 3 4 4 2} & \mathbf{2 . 0 2 9 8 5 9} & \mathbf{- 2 . 1 1 7 2 4 1}\end{array}$

$\begin{array}{lrrr}\mathrm{S} & \mathbf{- 0 . 0 1 3 4 4 2} & \mathbf{2 . 0 2 9 8 5 9} & \mathbf{- 2 . 1 1 7 2 4 1} \\ \mathrm{C} & \mathbf{1 . 5 3 4 9 3 0} & \mathbf{2 . 7 9 4 5 0 7} & \mathbf{- 1 . 9 7 1 9 1 3}\end{array}$

$\begin{array}{llll}\text { C } & 1.534930 & 2.794507 & -1.971913 \\ \text { C } & \mathbf{2 . 2 8 1 2 3 1} & \mathbf{2 . 6 8 0 1 0 1} & \mathbf{- 0 . 8 5 1 9 9 6}\end{array}$

$\begin{array}{lllll}\mathrm{S} & \mathbf{1 . 7 2 1 7 0 6} & \mathbf{1 . 7 8 0 9 3 9} & \mathbf{0 . 5 3 5 7 4 2}\end{array}$

$\begin{array}{llll}C & 0.022590 & -1.127268 & -0.062099\end{array}$

$\begin{array}{llll}N & \mathbf{1 . 1 4 4 5 4 8} & \mathbf{- 1 . 8 7 2 2 1 6} & \mathbf{- 0 . 0 2 2 8 9 2}\end{array}$

$\begin{array}{lllll}\mathrm{N} & \mathbf{- 1 . 0 2 5 8 8 7} & \mathbf{- 1 . 9 7 7 7 1 1} & \mathbf{- 0 . 1 4 8 2 4 1}\end{array}$

$\begin{array}{lllll}C & \mathbf{0 . 8 8 2 2 3 5} & \mathbf{- 3 . 3 1 4 4 8 9} & \mathbf{- 0 . 1 0 6 4 2 4}\end{array}$

$\begin{array}{llll}C & \mathbf{- 0 . 6 4 4 9 6 6} & \mathbf{- 3 . 3 8 7 9 5 7} & \mathbf{- 0 . 0 4 7 5 7 0}\end{array}$

$\begin{array}{llll}\text { C } & \mathbf{2 . 4 8 2 3 3 8} & -\mathbf{1 . 4 0 5 1 9 7} & \mathbf{- 0 . 0 7 9 7 9 7}\end{array}$

$\begin{array}{llll}C & 3.276412 & -1.461169 & 1.063338\end{array}$

$\begin{array}{llll}C & 3.046947 & -0.948905 & -1.280761\end{array}$

$\begin{array}{llll}C & 4.581818 & -1.001562 & 1.081586\end{array}$

$\begin{array}{llll}C & 4.365477 & \mathbf{- 0 . 4 8 9 0 9 8} & \mathbf{- 1 . 2 5 9 4 3 4}\end{array}$

$\begin{array}{llll}C & 5.139124 & -0.488747 & -0.093962\end{array}$

$\begin{array}{llll}\text { H } & 5.148851 & -1.050529 & 2.012938\end{array}$

$\begin{array}{llll}\text { H } & 4.804461 & -0.115662 & -2.188214 \\ C & 6.537980 & 0.067222 & -0.099477\end{array}$

$\begin{array}{llll}C & 6.537980 & 0.067222 & -0.099477\end{array}$

$\begin{array}{llll}\text { F } & 2.752480 & -1.979381 & 2.180195\end{array}$

$\begin{array}{llll}\text { C } & \mathbf{2 . 2 3 8 1 9 9} & -\mathbf{0 . 9 3 6 8 6 0} & -2.545686\end{array}$

$\begin{array}{llll}C & 1.493050 & -0.126255 & -2.516818\end{array}$ 
$\begin{array}{llll}\text { H } & \mathbf{2 . 8 8 2 8 2 2} & \mathbf{- 0 . 7 8 1 5 1 4} & \mathbf{- 3 . 4 2 0 4 0 8}\end{array}$ $\begin{array}{llll}\text { H } & \mathbf{1 . 6 8 9 2 5 7} & -1.880662 & -2.682349\end{array}$ $\begin{array}{llll}C & -2.369392 & -1.570310 & -0.327572\end{array}$

$\begin{array}{llll}\text { C } & \mathbf{- 3 . 2 6 2 7 4 0} & \mathbf{- 1 . 6 1 6 0 9 4} & \mathbf{0 . 7 4 3 9 4 6}\end{array}$

$\begin{array}{llll}C & -2.826108 & -1.113872 & -1.577793\end{array}$

C $-4.555326-1.129984-0.648558$

$\begin{array}{lllll}C & -4.555326 & -1.129984 & 0.648558\end{array}$

$\begin{array}{llll}C & -4.131314 & -0.623925 & -1.667400\end{array}$

$\begin{array}{llll}\text { C } & \mathbf{- 4 . 9 9 8 3 9 2} & \mathbf{- 0 . 6 0 1 4 6 9} & \mathbf{- 0 . 5 6 8 4 7 6}\end{array}$

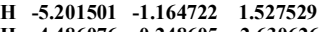

$\begin{array}{llll}\text { H } & -4.486076 & -0.248605 & -2.630626\end{array}$

$\begin{array}{llll}C & -6.374903 & -0.006096 & -0.689373\end{array}$

$\begin{array}{llll}C & -1.919903 & -1.147255 & -2.776954\end{array}$

H

$492995-1.006640-3.70$

\begin{tabular}{llll} 
H & -2.492995 & -1.006640 & -3.702187 \\
\hline
\end{tabular}

$\begin{array}{lll}-2.844427 & -2.127791 & 1.904539\end{array}$

$\begin{array}{llll}\text { C } & -1.881099 & 1.767312 & \mathbf{0 . 6 9 2 0 6 1}\end{array}$

$\begin{array}{llll}\text { H } & -2.496655 & 0.945697 & \mathbf{0 . 2 5 7 2 4 4}\end{array}$

$\begin{array}{lllll}\text { C } & \mathbf{- 0 . 6 3 2 1 3 7} & \mathbf{0 . 4 1 7 1 8 7} & \mathbf{2 . 0 4 4 8 8 4}\end{array}$

$\begin{array}{llll}C & -1.664137 & 1.553697 & \mathbf{2 . 1 7 8 5 8 0}\end{array}$

$\begin{array}{llll}\text { H } & -1.172425 & -0.539081 & \mathbf{2 . 0 7 4 1 7 9}\end{array}$

\begin{tabular}{llll} 
C & 0.462129 & 0.337198 & 3.094371 \\
\hline
\end{tabular}

$\begin{array}{llll}\text { H } & -2.595484 & 1.223495 & 2.685836\end{array}$

$\begin{array}{llll}\text { C } & -1.166871 & 2.800890 & 2.900938\end{array}$

H $7.020886-\mathbf{- 0 . 0 7 4 0 9 3}-\mathbf{- 1 . 0 7 6 5 3 4}$

H $\quad \begin{array}{llll}\text { 7.163671 } & -\mathbf{0 . 4 0 4 2 7 7} & \mathbf{0 . 6 7 0 8 6 7}\end{array}$

$\begin{array}{lllll}\text { H } & 6.518598 & 1.149669 & 0.105346\end{array}$

$\begin{array}{llll}\text { H } & \mathbf{- 7 . 0 6 5 5 1 5} & \mathbf{- 0 . 4 2 9 1 8 6} & \mathbf{0 . 0 5 2 7 4 7}\end{array}$

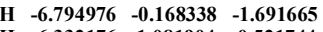

$\begin{array}{llll}\text { H } & -6.332176 & 1.081904 & -0.521744\end{array}$

$\begin{array}{llll}\text { H } & -\mathbf{0 . 2 5 0 3 4 1} & \mathbf{3 . 1 9 3 6 1 5} & \mathbf{2 . 4 3 1 3 9 5}\end{array}$

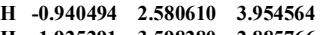

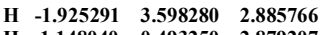

$\begin{array}{llll}\text { H } & 1.148040 & -\mathbf{0 . 4 9 3 2 5 0} & \mathbf{2 . 8 7 9 2 0 7}\end{array}$

$\begin{array}{llll}\text { H } & 0.034991 & 0.162737 & 4.099820\end{array}$

\begin{tabular}{llll}
\hline & $\mathbf{1 . 0 6 7 6 2 7}$ & $\mathbf{1 . 2 5 3 8 2 2}$ & $\mathbf{3 . 1 5 3 7 6 8}$
\end{tabular}

$\begin{array}{llll}\text { C } & -2.366712 & 3.108439 & 0.184913\end{array}$

$\begin{array}{lllll}\text { H } & \mathbf{- 2 . 5 6 2 4 9 3} & \mathbf{3 . 0 8 5 3 9 8} & \mathbf{- 0 . 8 9 4 4 8 9}\end{array}$

$\begin{array}{llll}\text { H } & -1.638714 & 3.908607 & 0.380707\end{array}$

$\begin{array}{llll}\text { H } & -3.303430 & 3.378896 & 0.706838\end{array}$

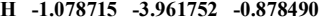

$\begin{array}{llll}\text { H } & -1.012284 & \mathbf{- 3 . 8 1 2 0 7 5} & \mathbf{0 . 8 9 8 7 0 7}\end{array}$

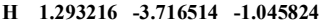

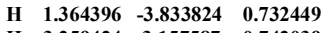

$\begin{array}{lllll}\text { H } & 3.259424 & 3.157587 & -0.742039\end{array}$

$\begin{array}{llll}H & 1.888746 & 3.392862 & -2.817209\end{array}$

68

sat_model_ts_PTR_a / electronic energy: $\mathbf{- 2 2 4 8 . 2 2 6 4 3 8 9 2}$ a.u. / lowest freq: $-\mathbf{1 7 6 . 3 0} \mathbf{c m}-1$

$\begin{array}{llll}\text { Ru } & -0.236949 & -0.904113 & -0.064279\end{array}$

$\begin{array}{llll}S & -0.000216 & -0.868660 & -2.492341\end{array}$

$\begin{array}{llll}\text { C } & -1.387716 & -1.738314 & -3.049793\end{array}$

$\begin{array}{llll}C & -2.317746 & -2.251465 & -2.211355\end{array}$

$\begin{array}{llll}S & -2.147211 & -2.096724 & -0.486703\end{array}$

$\begin{array}{llll}C & -2.147211 & -2.096724 & -0.486703\end{array}$

$\begin{array}{llll}\mathrm{C} & 0.143381 & 1.080307 & -0.047738\end{array}$

$\begin{array}{llll}\mathrm{N} & -0.883997 & 1.951474 & 0.000986\end{array}$

N $\quad \begin{array}{llll}1.277914 & 1.804114 & \mathbf{- 0 . 1 4 2 6 9 9}\end{array}$

$\begin{array}{lllll}\text { C } & -\mathbf{0 . 4 7 6 9 3 1} & 3.356957 & -0.033654\end{array}$

C $\quad \mathbf{1 . 0 4 9 0 6 1} \quad \mathbf{3 . 2 5 4 4 0 4} \mathbf{- 0 . 1 1 5 0 3 6}$

$\begin{array}{llll}C & -2.240774 & 1.577113 & 0.181823\end{array}$

$\begin{array}{llll}C & -2.717452 & 1.362557 & 1.475759\end{array}$

$\begin{array}{llll}\text { C } & -\mathbf{3 . 1 1 9 1 5 5} & \mathbf{1 . 4 3 4 2 1 2} & \mathbf{- 0 . 9 0 2 3 7 6}\end{array}$

$\begin{array}{llll}C & -4.011582 & 0.943162 & 1.726951\end{array}$

$\begin{array}{llll}\text { C } & -4.429465 & 1.024708 & -0.642307\end{array}$

$\begin{array}{llll}\text { C } & -4.886072 & 0.754670 & 0.651466\end{array}$

$\begin{array}{lllll}\text { H } & -4.324583 & 0.771687 & \mathbf{2 . 7 5 8 5 3 1}\end{array}$

$\begin{array}{llll}\text { H } & \mathbf{- 5 . 1 1 4 2 9 0} & \mathbf{0 . 8 9 4 8 0 1} & \mathbf{- 1 . 4 8 4 1 9 6}\end{array}$

$\begin{array}{llll}\text { C } & -6.281984 & 0.244715 & 0.888269\end{array}$

$\begin{array}{llll}\text { F } & -1.892021 & 1.581548 & 2.503549\end{array}$

$\begin{array}{llll}C & -2.646850 & 1.705702 & -2.301411\end{array}$

$\begin{array}{llll}\text { H } & -1.720647 & 1.149635 & -2.512060\end{array}$

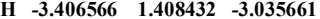

$\begin{array}{llll}\text { H }-2.437580 & \mathbf{2} 777504 & \mathbf{2 . 4 4 7 5 3 7}\end{array}$

C $2.608992 \quad 1.314552-0.153083$

$\begin{array}{llll}C & 2.608992 & 1.314552 & -0.153083\end{array}$

$\begin{array}{llll}C & 3.290281 & 1.214321 & 1.065146\end{array}$

$\begin{array}{llll}C & 3.296139 & 1.005857 & -1.333878\end{array}$

$\begin{array}{llll}\text { C } & 4.578977 & 0.730446 & 1.164717\end{array}$

$\begin{array}{llll}C & 4.605014 & 0.513499 & -1.230018\end{array}$

$\begin{array}{lllll}\text { C } & \mathbf{5 . 2 5 3 5 4 8} & \mathbf{0 . 3 5 3 9 0 6} & -\mathbf{0 . 0 0 5 0 3 7}\end{array}$

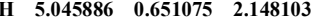

$\begin{array}{llll}\text { H } & \mathbf{5 . 1 3 5 3 2 6} & \mathbf{0 . 2 5 3 7 4 1} & \mathbf{- 2 . 1 4 9 9 9 9}\end{array}$

$\begin{array}{llll}C & 6.647479 & -0.207070 & 0.078990\end{array}$

C $2.679702 \quad 1.209337 \quad-2.688274$

\begin{tabular}{llll}
$C$ & 2.679702 & 1.209337 & -2.688274 \\
\hline & 2.566651 & 0.250645 & -3.213860
\end{tabular}

$1.683102-1.661446-2.623$

$\begin{array}{llll}\text { H } & 1.683102 & 1.661446 & -2.623639\end{array}$

\begin{tabular}{lllll}
\hline & 3.325107 & 1.855948 & $\mathbf{- 3 . 3 0 1 1 5 1}$
\end{tabular}

F $\quad 2.646252 \quad 1.588240 \quad 2.178631$

$\begin{array}{llll}\text { C } & 1.476978 & -1.877509 & 0.142060\end{array}$

$\begin{array}{llll}\text { H } & 2.323693 & -1.394293 & -0.368298\end{array}$

$\begin{array}{llll}\text { C } & \mathbf{- 0 . 2 7 2 2 4 1} & \mathbf{- 1 . 0 8 7 9 7 2} & \mathbf{1 . 9 5 9 0 8 6}\end{array}$

$\begin{array}{llll}C & 1.275294 & -1.385519 & 1.614978\end{array}$

$\begin{array}{lllll}\text { H } & -0.316293 & -0.156091 & 2.537919\end{array}$

C $-1.042902-2.179620 \quad 2.670411$

$\begin{array}{rrrr}C & -1.042902 & -2.179620 & 2.670411 \\ H & 1.827701 & -\mathbf{0 . 4 5 8 8 2 6} & \mathbf{1 . 7 6 4 4 8 5}\end{array}$ 


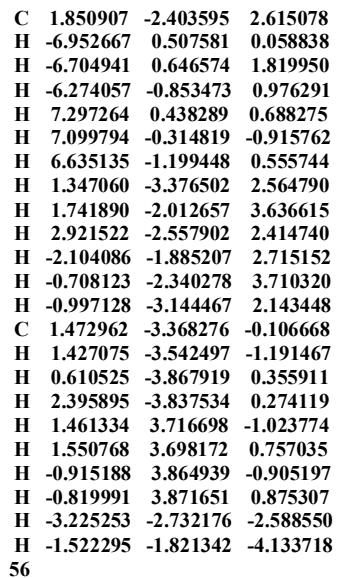

sat model 14e1 / electronic energy: -2091.16423387 a. / lowest freq: $21.22 \mathrm{~cm}-1$

$\begin{array}{llll}S & -0.664 \overline{109} & 1.799643 & 1.689243\end{array}$

$\begin{array}{llll}C & 0.227217 & \mathbf{- 0 . 8 6 2 9 0 8} & \mathbf{0 . 2 9 0 2 4 4}\end{array}$

$\begin{array}{lllll}\text { Ru } & \mathbf{- 0 . 2 1 1 1 9 7} & \mathbf{1 . 0 1 6 7 6 8} & \mathbf{- 0 . 4 0 7 6 8 0}\end{array}$

$\begin{array}{llll}C & \mathbf{1 . 4 2 4 5 0 0} & \mathbf{1 . 7 3 3 3 3 1} & \mathbf{- 0 . 5 9 2 1 7 1}\end{array}$

S $\quad-1.816541 \quad 2.506135-1.237129$

$\begin{array}{llll}\mathrm{N} & \mathbf{1 . 3 9 4 5 3 7} & -1.490357 & \mathbf{0 . 5 0 8 4 4 0}\end{array}$

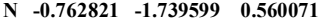

$\begin{array}{llll}\text { C } & 2.692215 & -0.958779 & 0.309200\end{array}$

C $\quad \mathbf{1 . 2 3 0 0 6 6} \quad-\mathbf{2 . 8 7 0 1 0 0} \quad \mathbf{0 . 9 8 5 0 7 1}$

$\begin{array}{lrrr}C & -0.273548 & -3.096949 & 0.823069\end{array}$

$\begin{array}{llll}C & -2.121849 & -1.504494 & 0.220277\end{array}$

$\begin{array}{llll}\text { H } & -0.511958 & -\mathbf{3 . 7 4 8 6 1 1} & \mathbf{- 0 . 0 3 2 2 2 6}\end{array}$

H $\quad-0.741515-3.519510 \quad 1.722198$

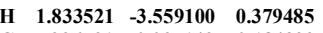

$\begin{array}{llll}\text { C } & 5.296721 & -0.025149 & -0.184392\end{array}$

$\begin{array}{llll}C & 3.376869 & -1.295342 & -0.859417\end{array}$

$\begin{array}{llll}C & 3.324750 & -0.154868 & 1.266493\end{array}$

$\begin{array}{llll}C & 4.615864 & 0.308757 & 0.990896\end{array}$

C $4.660794-0.850792-1.119572$

$\begin{array}{llll}C & 4.660794 & -0.850792 & -1.119572\end{array}$

$\begin{array}{llll}\text { H } & 5.109873 & 0.948403 & 1.726569\end{array}$

\begin{tabular}{llll}
\hline & 5.145706 & -1.137539 & -2.054519
\end{tabular}

$\begin{array}{llll}\text { C } & -4.775562 & -1.028160 & -0.572668\end{array}$

$\begin{array}{llll}C & -2.504038 & -1.562566 & -1.121264\end{array}$

$\begin{array}{llll}C & \mathbf{3 . 0 9 2 8 4 0} & -\mathbf{1 . 2 3 6 1 3 9} & \mathbf{1 . 1 9 7 0 0 7}\end{array}$

$\begin{array}{llll}C & -4.404104 & -1.000505 & 0.776958\end{array}$

$\begin{array}{llll}\text { C } & -\mathbf{3 . 8 0 3 5 7 2} & \mathbf{- 1 . 3 2 2 9 9 5} & -1.533072\end{array}$

$\begin{array}{llll}\text { F } & -1.567554 & -1.843708 & -2.034834\end{array}$

$\begin{array}{llll}C & -2.709277 & -1.176576 & 2.647422\end{array}$

C $-2.702703-0.780341 \quad 1.531268$

$\begin{array}{llll}C & -5.163703 & -0.780341 & 1.531268\end{array}$

\begin{tabular}{llll}
\hline & -4.041065 & -1.365544 & -2.597421
\end{tabular}

$\begin{array}{llll}C & -6.188122 & -0.714724 & -0.986696\end{array}$

$\begin{array}{llll}\text { H } & -6.453201 & -1.217490 & -1.926953\end{array}$

$\begin{array}{lllll}\text { H } & \mathbf{- 3 . 5 9 1 6 1 0} & \mathbf{- 1 . 0 1 8 3 3 4} & \mathbf{3 . 2 8 0 8 9 4}\end{array}$

$\begin{array}{llll}\text { H } & \mathbf{1 . 5 6 0 8 9 3} & \mathbf{- 2 . 9 4 6 6 6 4} & \mathbf{2 . 0 3 2 5 9 4}\end{array}$

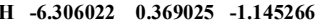

$\begin{array}{lllll}\text { H } & -6.908420 & -1.015852 & -0.213549\end{array}$

$\begin{array}{llll}\text { H } & -2.005546 & -0.344624 & 2.808233\end{array}$

$\begin{array}{llll}\text { H } & -2.212824 & -2.102676 & 2.973809\end{array}$

$\begin{array}{llll}\text { F } & 2.751193 & -2.062144 & -1.757262\end{array}$

$\begin{array}{llll}\text { C } & \mathbf{6 . 6 9 1 4 5 3} & \mathbf{0 . 4 7 6 1 1 0} & \mathbf{- 0 . 4 4 7 6 8 5}\end{array}$

$\begin{array}{llll}\text { H } & 7.005422 & 1.207051 & 0.309065\end{array}$

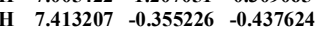

$\begin{array}{lllll}\text { H } & 6.758790 & 0.953220 & -1.436573\end{array}$

$\begin{array}{llll}\text { C } & 2.611588 & 0.215799 & \mathbf{2 . 5 3 4 5 5 5}\end{array}$

$\begin{array}{llll}\text { H } & 1.814016 & 0.947250 & 2.328842\end{array}$

$\begin{array}{llll}\text { H } & 2.131703 & -0.659479 & 2.996298\end{array}$

\begin{tabular}{llll}
\hline & 3.305502 & 0.655838 & 3.262345
\end{tabular}

C $3.3055020 .655838-3.262345$

\begin{tabular}{lrrr} 
C & -1.811696 & 3.103380 & 1.419988 \\
\hline
\end{tabular}

$\begin{array}{llll}C & -2.286639 & 3.405858 & 0.200804\end{array}$

$\begin{array}{llll}\text { H } & \mathbf{1 . 9 0 2 6 8 2} & \mathbf{2 . 3 5 7 6 5 0} & \mathbf{0 . 1 8 2 2 6 8}\end{array}$

$\begin{array}{llll}\text { C } & \mathbf{2 . 1 4 3 6 4 7} & \mathbf{1 . 6 7 7 1 1 5} & \mathbf{- 1 . 9 0 6 1 9 4}\end{array}$

$\begin{array}{llll}\text { H } & \mathbf{2 . 1 5 8 5 7 3} & \mathbf{2 . 6 8 0 1 6 7} & \mathbf{- 2 . 3 6 6 1 6 3}\end{array}$

H $3.191768 \quad 1.374421 \quad-1.749241$

$\begin{array}{lllll}\text { H } & 1.688401 & 0.975310 & -2.624380\end{array}$

$\begin{array}{llll}\text { H } & -2.999965 & 4.223089 & 0.057677\end{array}$

$\begin{array}{llll}H & -2.122544 & 3.655846 & 2.312637\end{array}$

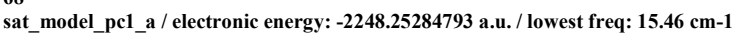

S $0.170256-1.639551 \quad-2.041910$

$\begin{array}{llll}C & -0.067712 & -0.949120 & -0.50097\end{array}$

$\begin{array}{llll}\text { Ru } & 0.015319 & 1.008833 & 0.209268\end{array}$

$\begin{array}{llll}\text { C } & \mathbf{0 . 8 8 4 5 3 3} & \mathbf{0 . 1 1 5 1 0 4} & \mathbf{2 . 1 4 1 7 2 0}\end{array}$

$\begin{array}{llll}C & -1.781565 & 1.352223 & 0.381919\end{array}$

$\begin{array}{lllll}\text { S } & 0.870706 & 3.170209 & 0.699995\end{array}$

$\begin{array}{llll}N & -1.155223 & -1.674923 & -0.838608\end{array}$

$\begin{array}{llll}N & 1.011813 & -1.690191 & -0.844872\end{array}$

$\begin{array}{llll}C & -011813 & -1.690191 & -0.844872\end{array}$

$\begin{array}{lrrr}\text { C } & -2.490950 & -1.389444 & -0.464648 \\ \text { C } & -0.842832 & -3.017642 & -1.341256\end{array}$ 
$\begin{array}{llll}C & 0.676246 & -2.968595 & -1.480980\end{array}$ $\begin{array}{llll}C & \mathbf{2 . 3 7 1 0 2 7} & \mathbf{- 1 . 4 1 8 8 3 7} & \mathbf{- 0 . 5 3 2 2 2 2}\end{array}$

$\begin{array}{llll}\text { H } & \mathbf{1 . 1 7 8 6 4 2} & \mathbf{- 3 . 7 9 8 1 3 3} & \mathbf{- 0 . 9 6 4 7 8}\end{array}$

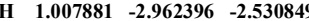

H $\quad-1.177186-3.774634-0.615477$

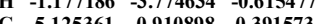

$\begin{array}{llll}\text { C } & -5.125361 & -0.910898 & 0.391573 \\ \text { C } & -2.928140 & -1.776553 & 0.803098\end{array}$

$\begin{array}{llll}C & -2.928140 & -1.776553 & 0.803098\end{array}$

$\begin{array}{llll}C & -3.397319 & -0.784027 & -1.343264\end{array}$

$\begin{array}{llll}C & -4.702134 & -0.552971 & -0.892018\end{array}$

$\begin{array}{llll}C & -4.214930 & -1.540641 & 1.249925\end{array}$

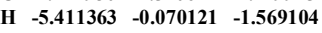

$\begin{array}{llll}\text { H } & -4.495823 & -1.848781 & 2.258761\end{array}$

$\begin{array}{llll}\text { C } & 5.051773 & -1.011750 & 0.249526\end{array}$

$\begin{array}{llll}C & 2.895984 & -1.978678 & 0.640380\end{array}$

C $3.243684 \quad-0.733233 \quad-1.388581$

$\begin{array}{llll}C & 4.561748 & -0.518728 & -0.959557\end{array}$

$\begin{array}{llll}C & 4.198585 & -1.782527 & 1.050541\end{array}$

$\begin{array}{llll}\text { F } & \mathbf{2 . 0 8 8 0 3 7} & \mathbf{- 2 . 7 4 0 8 5 1} & \mathbf{1 . 3 9 0 7 3 8}\end{array}$

$\begin{array}{llll}\text { C } & \mathbf{2 . 8 4 1 0 4 6} & \mathbf{- 0 . 3 0 1 8 5 5} & \mathbf{- 2 . 7 6 9 7 4 9}\end{array}$

H $\quad \begin{array}{llll}\text { H.233335 } & 0.040566 & -1.616265\end{array}$

$\begin{array}{llll}\text { H } & 4.535777 & -2.228051 & 1.988036\end{array}$

$\begin{array}{llll}\text { C } & \mathbf{6 . 4 6 0 9 7 0} & \mathbf{- 0 . 7 3 1 1 0 7} & \mathbf{0 . 6 9 6 1 4 7}\end{array}$

$\begin{array}{lllll}\text { H } & 6.926647 & -1.625846 & 1.133645\end{array}$

$\begin{array}{llll}\text { H } & 3.484832 & -0.804293 & -3.508130\end{array}$

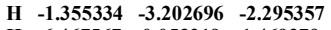

$\begin{array}{llll}\text { H } & 6.467567 & 0.053219 & 1.469379\end{array}$

$\begin{array}{llll}\text { H } & 7.086153 & -0.384387 & -0.137555\end{array}$

$\begin{array}{lllll}\text { H } & \mathbf{2 . 9 6 2 5 1 8} & \mathbf{0 . 7 8 2 8 1 1} & \mathbf{- 2 . 8 9 4 3 3 2}\end{array}$

$\begin{array}{llll}\text { H } & \mathbf{1 . 7 9 4 1 7 2} & -\mathbf{0 . 5 3 7 0 6 2} & \mathbf{- 2 . 9 8 8 1 7 3}\end{array}$

$\begin{array}{llll}\text { F } & -2.049067 & -2.372323 & 1.618485\end{array}$

$\begin{array}{llll}\text { C } & -6.526307 & -0.622192 & 0.860416\end{array}$

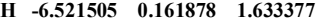

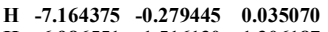

$\begin{array}{llll}\text { H } & -6.986551 & -1.516130 & 1.306187\end{array}$

$\begin{array}{llll}\text { C } & -\mathbf{2 . 9 5 1 7 0 8} & \mathbf{- 0 . 3 5 8 5 7 1} & \mathbf{- 2 . 7 1 2 0 2 9}\end{array}$

$\begin{array}{llll}\text { H } & -2.474964 & -1.189404 & -3.253537\end{array}$

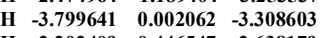

$\begin{array}{lllll}\text { H } & -2.202409 & 0.446547 & \mathbf{- 2 . 6 3 8 1 7 9}\end{array}$

$\begin{array}{llll}\text { C } & 0.561178 & 3.344612 & -1.999321\end{array}$

$\begin{array}{llll}C & 0.850278 & 3.982140 & -0.849840\end{array}$

$\begin{array}{llll}C & 2.170937 & 0.744574 & \mathbf{2 . 6 0 9 2 3 3}\end{array}$

$\begin{array}{llll}\text { H } & 0.973647 & -0.941629 & 1.892686\end{array}$

$\begin{array}{llll}\text { C } & -0.393751 & 0.539872 & \mathbf{2 . 4 5 5 9 3 4}\end{array}$

$\begin{array}{llll}\text { H } & -2.434531 & 0.819832 & 1.096094\end{array}$

$\begin{array}{llll}C & -2.529439 & \mathbf{2 . 4 6 5 1 6 4} & \mathbf{- 0 . 2 7 1 3 2 2}\end{array}$

$\begin{array}{llll}\text { H } & -3.437681 & 2.065242 & -0.754979\end{array}$

$\begin{array}{llll}\text { H } & -2.880928 & \mathbf{3 . 1 3 6 6 3 5} & \mathbf{0 . 5 3 4 3 2 1}\end{array}$

$\begin{array}{lllll}\text { H } & -1.942752 & 3.051005 & -0.987368\end{array}$

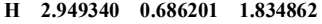

$\begin{array}{llll}\text { H } & 2.541184 & \mathbf{0 . 1 8 7 5 4 2} & \mathbf{3 . 4 8 7 2 8 8}\end{array}$

H $2.053036 \quad 1.797951 \quad 2.890507$

$\begin{array}{llll}\text { H } & -1.173608 & -\mathbf{0 . 2 2 3 8 6 3} & 2.391741\end{array}$

$\begin{array}{llll}C & -0.741443 & 1.710507 & 3.334269\end{array}$

\begin{tabular}{lrrr}
$C$ & -0.741443 & 1.710507 & 3.334269 \\
\hline & 0.008757 & 2.509486 & 3.292731
\end{tabular}

\begin{tabular}{ccccc}
$\mathrm{C}$ & $\mathbf{0 . 0 0 8 7 5 7}$ & $\mathbf{2 . 5 0 9 4 8 6}$ & $\mathbf{3 . 2 9 2 7 3 1}$ \\
\hline
\end{tabular}

$\begin{array}{cccc}\text { H } & -1.712394 & \mathbf{2 . 1 4 5 0 3 2} & \mathbf{3 . 0 5 3 8 8 0} \\ \text { H } & -\mathbf{0 . 8 2 5 6 1 6} & \mathbf{1 . 3 6 9 5 6 9} & \mathbf{4 . 3 8 0 1 8 4}\end{array}$

$\begin{array}{llll}\text { H } & \mathbf{1 . 1 0 1 2 2 1} & \mathbf{5 . 0 4 8 2 2 2} & \mathbf{- 0 . 8 4 6 2 5 4}\end{array}$

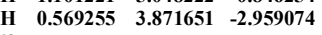

68

sat model ts1 a / electronic energy: $\mathbf{- 2 2 4 8 . 2 4 3 2 8 1 1 2}$ a.u. / lowest freq: $-\mathbf{1 3 9 . 1 1} \mathrm{cm}-1$

$\begin{array}{llll}-0.018964 & 1.703279 & -2.083492\end{array}$

$\begin{array}{llll}C & -0.139656 & -0.876241 & -0.617983\end{array}$

$\begin{array}{lllll}\text { Ru } & 0.078655 & 1.040066 & 0.139481\end{array}$

$\begin{array}{llll}\text { C } & 0.986158 & 0.184386 & \mathbf{1 . 9 2 0 2 2 7}\end{array}$

$\begin{array}{llll}\text { C } & -1.651602 & 1.258913 & 0.776340\end{array}$

$\begin{array}{lllll}\text { S } & \mathbf{1 . 0 6 5 8 9 3} & \mathbf{3 . 2 0 7 7 9 7} & \mathbf{0 . 5 4 2 6 1 2}\end{array}$

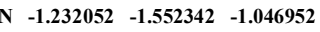

N $0.936627-1.599453-1.016963$

C $\quad-\mathbf{2 . 5 4 7 1 8 3}-\mathbf{- 1 . 3 4 8 7 5 0}-\mathbf{- 0 . 5 5 8 6 9 2}$

$\begin{array}{llll}\text { C } & \mathbf{- 0 . 9 2 4 7 2 4} & \mathbf{- 2 . 8 4 5 2 4 2} & \mathbf{- 1 . 6 6 9 4 1 1}\end{array}$

C $\quad 0.594162-2.791157-1.800031$

$\begin{array}{llll}\text { C } & \mathbf{2 . 2 8 2 2 1 4} & -1.437918 & -0.585166\end{array}$

$1.088289-3.681027-1.387044$

\begin{tabular}{llll} 
H & $\mathbf{1 . 0 8 8 2 8 9}$ & $\mathbf{- 3 . 6 8 1 0 2 7}$ & $\mathbf{- 1 . 3 8 7 0 4 4}$ \\
\hline & $\mathbf{0 . 9 2 6 7 7 3}$ & -2.662416 & -2.841634
\end{tabular}

\begin{tabular}{llll}
$H$ & 0.926773 & -2.662416 & -2.841634 \\
\hline
\end{tabular}

$\begin{array}{llll}\text { H } & -1.261531 & -3.663694 & -1.014295\end{array}$

$\begin{array}{llll}\text { C } & -5.102993 & -0.994985 & 0.561200\end{array}$

$\begin{array}{llll}C & -2.875065 & -1.869329 & 0.691888\end{array}$

$\begin{array}{llll}C & -3.528076 & -0.664235 & -1.290635\end{array}$

$\begin{array}{llll}C & -4.787125 & -0.494337 & -0.708670\end{array}$

$\begin{array}{llll}\text { C } & -4.124982 & -1.701988 & 1.266144\end{array}$

$\begin{array}{llll}\mathrm{C} & -\mathbf{5 . 5 5 2 4 7 0} & 0.051873 & -1.266534\end{array}$

$\begin{array}{llll}\text { H } & -4.316625 & -2.117333 & 2.257020\end{array}$

$\begin{array}{llll}\text { H } & -4.316625 & -2.117333 & \mathbf{2 . 2 5 7 0 2 0} \\ \text { C } & \mathbf{4 . 9 2 0 2 4 4} & -1.329657 & 0.414013\end{array}$

$\begin{array}{llll}C & 4.920244 & -1.329657 & 0.414013\end{array}$

$\begin{array}{llll}C & 2.684514 & -2.176983 & 0.535022\end{array}$

$\begin{array}{lllll}C & 3.247777 & -0.684819 & -1.266537\end{array}$

$\begin{array}{llll}C & 4.545253 & -0.633167 & -0.735004\end{array}$

$\begin{array}{llll}C & 3.964424 & -\mathbf{2 . 1 3 3 2 8 2} & \mathbf{1 . 0 4 8 6 5 6}\end{array}$

$\begin{array}{llll}\text { F } & \mathbf{1 . 7 6 9 6 2 9} & \mathbf{- 2 . 9 4 9 7 2 1} & \mathbf{1 . 1 3 6 9 8 8}\end{array}$

$\begin{array}{lllll}\text { C } & \mathbf{2 . 9 4 6 4 3 3} & \mathbf{0 . 0 3 4 8 2 7} & \mathbf{- 2 . 5 4 9 4 7 2}\end{array}$

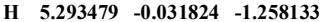

$\begin{array}{llll}\text { H } \quad 4.202964 & -2.717299 & 1.939378\end{array}$

C $6.308727-1.2087530 .981071$

$\begin{array}{llll}\text { C } & 6.308727 & -1.208753 & 0.981071 \\ \text { H } & 6.655093 & -2.162254 & 1.404338\end{array}$ 
$\begin{array}{llll}\text { H } & 3.770601 & -0.106029 & -3.263212\end{array}$

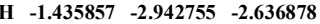
$\begin{array}{llll}\text { H } & 6.324263 & -0.463502 & 1.792291\end{array}$ $\begin{array}{llll}\text { H } & \mathbf{7 . 0 2 8 2 5 6} & -\mathbf{0 . 8 8 3 1 3 2} & \mathbf{0 . 2 1 7 6 5 0}\end{array}$ H $2.825476 \quad 1.113487 \quad-2.371494$ - $2.011607-0.313540-3.003505$ $\begin{array}{llll}\text { H } & 2.011607 & -0.313540 & \mathbf{- 3 . 0 0 3 5 0 5}\end{array}$ $\begin{array}{llll}\text { F } & -1.922785 & -2.512391 & 1.379479\end{array}$ $\begin{array}{llll}\text { C } & \mathbf{- 6 . 4 7 0 0 7 3} & \mathbf{- 0 . 7 6 8 2 3 7} & \mathbf{1 . 1 4 9 7 9 1}\end{array}$ $\begin{array}{llll}\text { H } & -6.648603 & 0.305695 & 1.312470\end{array}$ $\begin{array}{llll}\text { H } & -7.254747 & \mathbf{- 1 . 1 2 8 5 3 5} & \mathbf{0 . 4 6 8 0 0 7}\end{array}$ $\begin{array}{llll}\text { H } & -6.587326 & -1.282462 & 2.112813\end{array}$ $\begin{array}{llll}C & -3.205198 & -0.102745 & -2.644745\end{array}$ \begin{tabular}{llll} 
H & -2.818441 & $-\mathbf{0 . 8 8 3 1 8 9}$ & $\mathbf{- 3 . 3 1 7 4 9 0}$ \\
\hline & -4.095203 & $\mathbf{0 . 3 4 1 9 7 9}$ & $\mathbf{- 3 . 1 0 8 4 4 8}$
\end{tabular} $\begin{array}{llll}\text { H } & -2.422471 & 0.667732 & -2.564924 \\ \text { C } & 0.500091 & 3.380747 & -2.110439\end{array}$ $\begin{array}{llll}\text { C } & 0.500091 & 3.380747 & -2.110439\end{array}$ $\begin{array}{lllll}C & 0.951588 & 4.004919 & -1.008604\end{array}$ $\begin{array}{llll}\text { C } & \mathbf{2 . 3 1 0 8 7 0} & \mathbf{0 . 7 4 8 3 9 6} & \mathbf{2 . 3 7 8 3 1 2}\end{array}$ $\begin{array}{llll}\text { H } & 1.009703 & -0.896729 & 1.779793\end{array}$ $\begin{array}{llll}\text { C } & \mathbf{- 0 . 2 6 7 0 1 5} & \mathbf{0 . 6 5 9 5 6 4} & \mathbf{2 . 3 9 8 4 0 2}\end{array}$ $\begin{array}{llll}\text { H } & -2.326986 & 0.434713 & 1.037318\end{array}$ $\begin{array}{llll}\text { C } & -2.369458 & 2.567217 & 0.844326\end{array}$ $\begin{array}{llll}\text { H } & -2.994243 & 2.607177 & -0.067798\end{array}$ $\begin{array}{llll}\text { H } & -\mathbf{3 . 0 6 1 7 8 4} & \mathbf{2 . 6 0 4 8 5 1} & \mathbf{1 . 7 0 1 3 7 1}\end{array}$ $\begin{array}{llll}\text { H } & -1.701556 & 3.435745 & 0.841750\end{array}$ $\begin{array}{llll}\text { H } & 3.088575 & \mathbf{0 . 5 8 4 5 4 4} & \mathbf{1 . 6 1 8 5 4 9}\end{array}$ $\begin{array}{llll}\text { H } & \mathbf{2 . 6 3 2 2 6 4} & \mathbf{0 . 2 2 8 6 0 6} & \mathbf{3 . 2 9 7 9 5 5}\end{array}$ $\begin{array}{lllll}\text { H } & \mathbf{2 . 2 6 7 7 6 5} & \mathbf{1 . 8 2 4 0 4 7} & \mathbf{2 . 5 8 6 4 4 7}\end{array}$ $\begin{array}{llll}\text { H } & -1.011821 & -0.128017 & 2.546302\end{array}$ $\begin{array}{llll}\text { C } & -\mathbf{0 . 3 9 2 1 1 8} & \mathbf{1 . 8 0 9 4 0 2} & \mathbf{3 . 3 6 8 7 5 4}\end{array}$ H $\quad 0.176662 \quad 2.690771 \quad 3.047849$ H $\quad-1.439104 \quad 2.109881 \quad 3.509243$ $\begin{array}{llll}\text { H } & -\mathbf{0 . 0 0 4 3 5 0} & 1.486773 & 4.349207\end{array}$ $\begin{array}{llll}\text { H } & 1.281728 & 5.048386 & -1.056802\end{array}$ \begin{tabular}{llll}
\hline & 1.281728 & 5.048386 & -1.056802
\end{tabular} $\begin{array}{lllll}H & 0.441174 & 3.889656 & -3.077957\end{array}$ 68

sat_model_mcb_a / electronic energy: $-\mathbf{2 2 4 8 . 2 5 4 2 6 9 4 4}$ a.u. / lowest freq: $19.08 \mathrm{~cm}-1$ $\begin{array}{lllll}S & 0.085791 & 1.033547 & \mathbf{2 . 2 4 2 5 0 1}\end{array}$

$\begin{array}{llll}C & 0.022832 & -1.020682 & 0.195482\end{array}$

$\begin{array}{llll}\text { Ru } & \mathbf{- 0 . 0 2 2 3 0 6} & \mathbf{1 . 0 6 1 2 9 9} & \mathbf{- 0 . 0 2 6 4 0 2}\end{array}$

$\begin{array}{llll}\text { C } & -1.356073 & 0.910788 & -1.486621\end{array}$

$\begin{array}{lrrrr}C & 1.348384 & 1.012153 & -1.443465\end{array}$

$\begin{array}{llll}\mathrm{S} & \mathbf{- 0 . 1 7 6 9 0 4} & \mathbf{3 . 4 7 3 7 6 9} & \mathbf{0 . 1 9 1 0 3 0}\end{array}$

$\begin{array}{llll}\text { N } & 1.110881 & -1.807489 & 0.374655\end{array}$

$\begin{array}{llll}\mathrm{N} & -1.110881 & -1.807489 & \mathbf{0 . 3 7 4 6 5 5}\end{array}$

$\begin{array}{lrrr}\mathrm{N} & -1.053802 & -1.823042 & \mathbf{0 . 3 7 7 2 4 4} \\ \mathrm{C} & 2.481686 & -1.464942 & \mathbf{0 . 2 2 1 2 7 7}\end{array}$

$\begin{array}{llll}C & 2.481686 & -1.464942 & 0.221277\end{array}$

$\begin{array}{llll}\text { C } & 0.800835 & -3.219472 & 0.626149\end{array}$

$\begin{array}{llll}C & -0.723142 & -3.221111 & 0.675527\end{array}$

$\begin{array}{llll}\text { C } & -2.431031 & -1.498383 & 0.238773\end{array}$

$\begin{array}{llll}\text { H } & -1.173180 & -3.886465 & -0.074961\end{array}$

$\begin{array}{llll}\text { H } & -1.121726 & -3.498463 & 1.663154\end{array}$

H $\quad \mathbf{1 . 2 0 0 9 2 7} \quad \mathbf{- 3 . 8 4 2 4 7 5}-\mathbf{- 0 . 1 8 7 4 4 1}$

$\begin{array}{llll}\text { C } & 5.206972 & -0.900742 & -0.243035\end{array}$

$\begin{array}{llll}\text { C } & 3.077680 & -1.664487 & -1.030062\end{array}$

$\begin{array}{llll}C & 3.292824 & -1.057388 & 1.286825\end{array}$

$\begin{array}{llll}C & 4.639232 & -0.762138 & 1.022523\end{array}$

$\begin{array}{llll}\text { C } & 4.402603 & -1.380252 & -1.286722\end{array}$

H $\quad 5.266860 \quad-0.425005 \quad 1.851777$

$\begin{array}{llll}\text { H } & 4.797136 & -1.531902 & -2.293084\end{array}$

$\begin{array}{llll}\text { C } & -5.170934 & -0.983631 & -0.201462\end{array}$

$\begin{array}{llll}\text { C } & \mathbf{- 3 . 0 4 6 9 1 6} & \mathbf{- 1 . 7 5 8 2 9 1} & \mathbf{- 0 . 9 9 1 3 3 9}\end{array}$

$\begin{array}{llll}C & -3.228563 & -1.051390 & 1.299932\end{array}$

$\begin{array}{llll}C & -4.582453 & -0.784638 & 1.047093\end{array}$

$\begin{array}{llll}\text { C } & -4.379887 & -1.499787 & -1.236733\end{array}$

$\begin{array}{llll}C & -2.289732 & -2.258997 & -1.977830\end{array}$

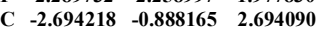

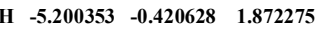

$\begin{array}{llll}\text { H } & -4.789649 & -1.700293 & -2.228243\end{array}$

$\begin{array}{llll}\text { C } & \mathbf{- 6 . 6 1 7 7 8 4} & -\mathbf{0 . 6 5 0 4 6 7} & \mathbf{- 0 . 4 4 7 0 6 7}\end{array}$

$\begin{array}{lllll}\text { H } & -7.108638 & \mathbf{- 1 . 4 2 3 5 9 5} & \mathbf{- 1 . 0 5 5 4 4 4}\end{array}$

$\begin{array}{llll}\text { H } & -3.386935 & -1.343685 & 3.416407\end{array}$

$\begin{array}{llll}\text { H } & \mathbf{1 . 2 6 3 4 9 7} & \mathbf{- 3 . 5 4 6 9 1 1} & \mathbf{1 . 5 6 8 5 5 5}\end{array}$

$1.704700-0.301907-0.993729$

$\begin{array}{llll}\text { H } & -6.704700 & 0.301907 & \mathbf{- 0 . 9 9 3 7 2 9}\end{array}$

$\begin{array}{llll}-7.170372 & -0.545426 & 0.496253\end{array}$

$\begin{array}{llll}\text { H } & -2.586284 & 0.176083 & 2.946208\end{array}$

$\begin{array}{llll}\text { H } & -1.703596 & -1.342863 & 2.813070\end{array}$

$\begin{array}{lllll}\text { F } & 2.306828 & -2.128779 & -2.024016\end{array}$

C $\quad 6.650494-0.562173-0.501241$

$\begin{array}{llll}\text { H } & 7.165687 & \mathbf{- 0 . 2 6 4 3 7 3} & \mathbf{0 . 4 2 1 5 6 2}\end{array}$

H $7.184573 \quad-1.421538 \quad-0.933672$

$\begin{array}{lllll}\text { H } & 6.731591 & 0.266893 & -1.220864\end{array}$

C $2.785976-0.981040-2.698968$

\begin{tabular}{llll} 
C & 2.785976 & -0.981040 & $\mathbf{2 . 6 9 8 9 6 8}$ \\
\hline & 1.752322 & -1.334628 & 2.786633
\end{tabular}

\begin{tabular}{lllll} 
H & $\mathbf{1 . 7 5 2 3 2 2}$ & -1.334628 & $\mathbf{2 . 7 8 6 6 3 3}$ \\
\hline & 3.427970 & -1.586856 & 3.355885
\end{tabular}

$\begin{array}{llll}\text { H } & 3.427970 & -1.586856 & \mathbf{3 . 3 5 5 8 8 5}\end{array}$

$\begin{array}{llll}\text { H } & \mathbf{2 . 8 0 5 4 5 9} & \mathbf{0 . 0 5 4 7 0 3} & \mathbf{3 . 0 6 5 9 4 0}\end{array}$

$\begin{array}{lllll}\text { C } & \mathbf{0 . 0 0 9 7 9 0} & 2.705317 & \mathbf{2 . 7 8 2 5 1 0}\end{array}$

C $\quad \mathbf{- 0 . 0 9 6 8 5 6} \quad \mathbf{3 . 7 2 1 5 9 7} \mathbf{1 . 9 0 9 3 5 5}$

$\begin{array}{lllll}C & -2.552416 & 1.813217 & -1.668419\end{array}$

$\begin{array}{lllll}\text { H } & -1.628701 & -0.111689 & -1.747989\end{array}$

$\begin{array}{llll}\text { C } & -\mathbf{0 . 0 0 4 5 3 8} & \mathbf{1 . 2 6 1 2 7 5} & \mathbf{- 2 . 2 6 4 2 0 6}\end{array}$

$\begin{array}{lllll}\text { H } & 1.711571 & 0.018992 & -1.703683\end{array}$

$\begin{array}{llll}C & 1.715933 & 0.018992 & -1.703683\end{array}$

$\begin{array}{llll}C & 2.473933 & 2.009665 & -1.578635 \\ H & 3.256181 & 1.739481 & -\mathbf{0 . 8 5 2 1 7 7}\end{array}$ 


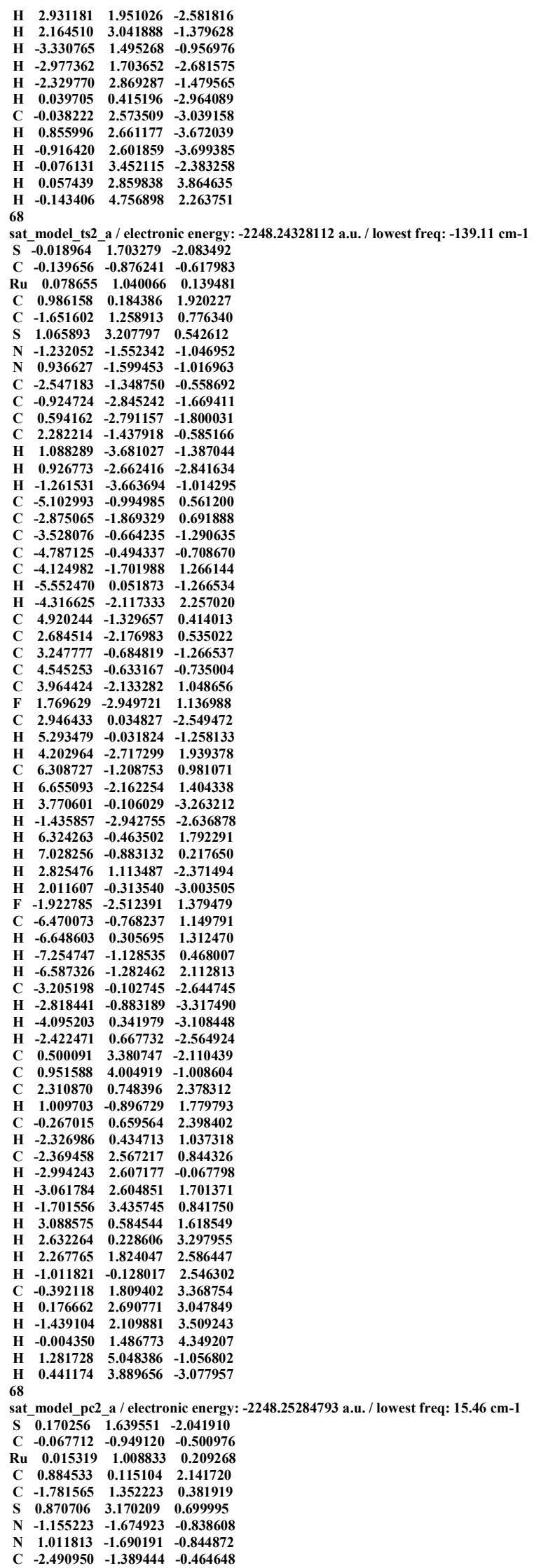


$\begin{array}{llll}C & -0.842832 & -3.017642 & -1.341256\end{array}$ $\begin{array}{llll}C & 0.676246 & -2.968595 & -1.48098\end{array}$

$\begin{array}{llll}\text { C } & 2.371027 & -1.418837 & -0.532222\end{array}$

H $\quad 1.178642 \quad-3.798133 \quad-0.964787$

H $\quad \begin{array}{llll}1.007881 & -2.962396 & -2.530849\end{array}$

H $-1.177186 \quad-3.774634 \quad-0.615477$

$\begin{array}{llll}C & -2.928140 & -1.776553 & 0.803098\end{array}$

$\begin{array}{llll}C & -3.397319 & -0.784027 & -1.343264\end{array}$

$\begin{array}{llll}C & -4.702134 & -0.552971 & -0.892018\end{array}$

$\begin{array}{llll}C & -4.214930 & -1.540641 & 1.249925\end{array}$

H $\quad-5.411363 \quad-0.070121 \quad-1.569104$

$\begin{array}{llll}\text { H } & -4.495823 & -1.848781 & 2.258761\end{array}$

$\begin{array}{llll}\text { C } & 5.051773 & -1.011750 & 0.249526\end{array}$

$\begin{array}{llll}\text { C } & 2.895984 & -1.978678 & 0.640380\end{array}$

$\begin{array}{llll}\text { C } & 3.243684 & -0.733233 & -1.388581\end{array}$

$\begin{array}{llll}C & 4.561748 & -0.518728 & -0.959557\end{array}$

$\begin{array}{llll}C & 4.198585 & -1.782527 & 1.050541\end{array}$

F $\quad \mathbf{2 . 0 8 8 0 3 7} \quad \mathbf{- 2 . 7 4 0 8 5 1} \quad \mathbf{1 . 3 9 0 7 3 8}$

$\begin{array}{llll}C & 2.841046 & -0.301855 & -2.769749\end{array}$

H $\quad 5.233335 \quad 0.040566-1.616265$

$\begin{array}{llll}\text { H } & 4.535777 & -2.228051 & 1.988036\end{array}$

$\begin{array}{llll}\text { C } & 6.460970 & -0.731107 & 0.696147\end{array}$

$\begin{array}{llll}\text { H } & 6.926647 & -1.625846 & 1.133645\end{array}$

\begin{tabular}{llll} 
H & $\mathbf{3 . 4 8 4 8 3 2}$ & $\mathbf{- 0 . 8 0 4 2 9 3}$ & $\mathbf{- 3 . 5 0 8 1 3 0}$ \\
\hline & -1.355334 & $-\mathbf{3 . 2 0 2 6 9 6}$ & $\mathbf{- 2 . 2 9 5 3 5 7}$
\end{tabular}

\begin{tabular}{lrrrr} 
H & -1.355334 & -3.202696 & -2.295357 \\
\hline & 6.467567 & 0.053219 & 1.469379
\end{tabular}

\begin{tabular}{lllll} 
H & $\mathbf{6 . 4 6 7 5 6 7}$ & $\mathbf{0 . 0 5 3 2 1 9}$ & $\mathbf{1 . 4 6 9 3 7 9}$ \\
\hline & $\mathbf{7 . 0 8 6 1 5 3}$ & -0.384387 & -0.13755
\end{tabular}

$\begin{array}{ccccc}\text { H } & \mathbf{7 . 0 8 6 1 5 3} & \mathbf{- 0 . 3 8 4 3 8 7} & \mathbf{- 0 . 1 3 7 5 5 5} \\ \text { H } & \mathbf{2 . 9 6 2 5 1 8} & \mathbf{0 . 7 8 2 8 1 1} & \mathbf{- 2 . 8 9 4 3 3 2}\end{array}$

$\begin{array}{ccccc}\text { H } & \mathbf{2 . 9 6 2 5 1 8} & \mathbf{0 . 7 8 2 8 1 1} & \mathbf{- 2 . 8 9 4 3 3 2} \\ \text { H } & \mathbf{1 . 7 9 4 1 7 2} & \mathbf{- 0 . 5 3 7 0 6 2} & \mathbf{- 2 . 9 8 8 1 7 3}\end{array}$

$\begin{array}{llll}\text { F } & -2.049067 & -2.372323 & 1.618485\end{array}$

$\begin{array}{llll}\text { C } & -6.526307 & -0.622192 & 0.860416\end{array}$

$\begin{array}{llll}\text { H } & -6.521505 & 0.161878 & 1.633377\end{array}$

$\begin{array}{llll}H & -7.164375 & -0.279445 & 0.035070\end{array}$

\begin{tabular}{llll}
4 & -7.164375 & -0.279445 & 0.035070 \\
\hline & -6.986551 & -1.516130 & 1.306187
\end{tabular}

$\begin{array}{llll}\text { H } & -6.986551 & -1.516130 & 1.306187 \\ \text { C } & -2.951708 & -0.358571 & -2.712029\end{array}$

$\begin{array}{llll}C & -2.951708 & -0.358571 & -2.712029\end{array}$

$\begin{array}{ccccc}\text { H } & -2.474964 & -1.189404 & -3.253537 \\ \text { H } & -3.799641 & 0.002062 & -3.308603\end{array}$

$\begin{array}{llll}\text { H } & -\mathbf{2 . 2 0 2 4 0 9} & \mathbf{0 . 4 4 6 5 4 7} & \mathbf{- 2 . 6 3 8 1 7 9}\end{array}$

$\begin{array}{llll}\text { C } & 0.561178 & 3.344612 & -1.999321\end{array}$

$\begin{array}{lllll}C & 0.850278 & 3.982140 & \mathbf{- 0 . 8 4 9 8 4 0}\end{array}$

$\begin{array}{llll}C & 2.170937 & 0.744574 & \mathbf{2 . 6 0 9 2 3 3}\end{array}$

$\begin{array}{llll}\text { H } & 0.973647 & -0.941629 & 1.892686\end{array}$

$\begin{array}{llll}C & -0.393751 & 0.539872 & 2.455934\end{array}$

$\begin{array}{llll}\text { H } & -2.434531 & 0.819832 & 1.096094\end{array}$

$\begin{array}{lllll}C & -2.529439 & \mathbf{2 . 4 6 5 1 6 4} & \mathbf{- 0 . 2 7 1 3 2 2}\end{array}$

$\begin{array}{llll}\text { H } & -3.437681 & 2.065242 & -0.754979\end{array}$

$\begin{array}{rrrr}\text { H } & -2.880928 & \mathbf{3 . 1 3 6 6 3 5} & \mathbf{0 . 5 3 4 3 2 1}\end{array}$

$\begin{array}{llll}\text { H } & -\mathbf{1 . 9 4 2 7 5 2} & 3.051005 & -0.987368\end{array}$

$\begin{array}{ccccc}\text { H } & \mathbf{2 . 9 4 9 3 4 0} & \mathbf{0 . 6 8 6 2 0 1} & \mathbf{1 . 8 3 4 8 6 2}\end{array}$

$\begin{array}{lllll}\text { H } & 2.541184 & 0.187542 & 3.487288\end{array}$

$\begin{array}{lllll}\text { H } & 2.053036 & \mathbf{1 . 7 9 7 9 5 1} & \mathbf{2 . 8 9 0 5 0 7}\end{array}$

$\begin{array}{llll}\text { H } & -1.173608 & -0.223863 & 2.391741\end{array}$

\begin{tabular}{rrrr} 
H & -1.173608 & -0.223863 & 2.391741 \\
\hline
\end{tabular}

$\begin{array}{rrrr}\text { C } & -0.741443 & 1.710507 & 3.334269\end{array}$

$\begin{array}{rrrrr}\text { H } & 0.008757 & \mathbf{2 . 5 0 9 4 8 6} & \mathbf{3 . 2 9 2 7 3 1} \\ \text { H } & -\mathbf{1 . 7 1 2 3 9 4} & \mathbf{2 . 1 4 5 0 3 2} & \mathbf{3 . 0 5 3 8 8 0}\end{array}$

$\begin{array}{cccc}\text { H } & -1.712394 & \mathbf{2 . 1 4 5 0 3 2} & \mathbf{3 . 0 5 3 8 8 0} \\ \text { H } & -\mathbf{0 . 8 2 5 6 1 6} & \mathbf{1 . 3 6 9 5 6 9} & \mathbf{4 . 3 8 0 1 8 4}\end{array}$

$\begin{array}{llll}\text { H } & \mathbf{1 . 1 0 1 2 2 1} & \mathbf{5 . 0 4 8 2 2 2} & \mathbf{- 0 . 8 4 6 2 5 4}\end{array}$

$\begin{array}{llll}\text { H } & 0.569255 & 3.871651 & -2.959074\end{array}$

56

sat model 14e1 / electronic energy: -2091.16423387 a.u. / lowest freq: $21.22 \mathrm{~cm}-1$

$\begin{array}{llll}S & -0.664109 & 1.799643 & 1.689243\end{array}$

$\begin{array}{llll}C & 0.227217 & -0.862908 & 0.290244\end{array}$

C $1.22721797,0.016768-0.407680$

$\begin{array}{llll}\text { Ru } & -0.211197 & 1.016768 & -0.407680\end{array}$

$\begin{array}{llll}C & \mathbf{1 . 4 2 4 5 0 0} & \mathbf{1 . 7 3 3 3 3 1} & \mathbf{- 0 . 5 9 2 1 7 1}\end{array}$

S $-1.816541-2.506135-1.23712$

$\begin{array}{llll}N & 1.394537 & -1.490357 & 0.508440\end{array}$

$\begin{array}{llll}\mathrm{N} & \mathbf{- 0 . 7 6 2 8 2 1} & \mathbf{- 1 . 7 3 9 5 9 9} & \mathbf{0 . 5 6 0 0 7 1}\end{array}$

$\begin{array}{llll}\text { C } & \mathbf{2 . 6 9 2 2 1 5} & \mathbf{- 0 . 9 5 8 7 7 9} & \mathbf{0 . 3 0 9 2 0 0}\end{array}$

C $\quad \mathbf{1 . 2 3 0 0 6 6} \quad-\mathbf{2 . 8 7 0 1 0 0} \quad \mathbf{0 . 9 8 5 0 7 1}$

$\begin{array}{llll}\text { C } & -\mathbf{0 . 2 7 3 5 4 8} & -\mathbf{3 . 0 9 6 9 4 9} & \mathbf{0 . 8 2 3 0 6 9}\end{array}$

$\begin{array}{llll}C & -2.121849 & -1.504494 & 0.220277\end{array}$

$\begin{array}{llll}\text { H } & -\mathbf{0 . 5 1 1 9 5 8} & -\mathbf{3 . 7 4 8 6 1 1} & -\mathbf{0 . 0 3 2 2 2 6}\end{array}$

$\begin{array}{llll}\text { H } & -0.741515 & -3.519510 & 1.722198\end{array}$

$\begin{array}{llll}\text { H } & 1.833521 & -3.559100 & 0.379485\end{array}$

$\begin{array}{llll}\text { C } & \mathbf{5 . 2 9 6 7 2 1} & \mathbf{- 0 . 0 2 5 1 4 9} & \mathbf{- 0 . 1 8 4 3 9 2}\end{array}$

$\begin{array}{llll}C & 3.376869 & -1.295342 & -0.859417\end{array}$

$\begin{array}{llll}C & 3.324750 & -0.154868 & 1.266493\end{array}$

$\begin{array}{llll}\text { C } & 4.615864 & 0.308757 & 0.990896\end{array}$

$\begin{array}{llll}C & 4.660794 & -0.850792 & -1.119572\end{array}$

$\begin{array}{llll}\text { H } & 5.109873 & 0.948403 & 1.726569\end{array}$

H $5.145706 \quad-1.137539 \quad-2.054519$

$\begin{array}{llll}C & -4.775562 & -1.028160 & -0.572668\end{array}$

$\begin{array}{llll}C & -2.504038 & -1.562566 & -1.121264\end{array}$

$\begin{array}{llll}C & -3.092840 & -1.236139 & 1.197007\end{array}$

$\begin{array}{llll}C & -4.404104 & -1.000505 & 0.776958\end{array}$

$\begin{array}{llll}C & -3.803572 & -1.322995 & -1.533072\end{array}$

$\begin{array}{llll}\text { F } & -1.567554 & -1.843708 & -2.034834\end{array}$

$\begin{array}{llll}\text { C } & \mathbf{- 2 . 7 0 9 2 7 7} & \mathbf{- 1 . 1 7 6 5 7 6} & \mathbf{2 . 6 4 7 4 2 2}\end{array}$

$\begin{array}{lllll}\text { H } & -\mathbf{5 . 1 6 3 7 0 3} & -\mathbf{0 . 7 8 0 3 4 1} & \mathbf{1 . 5 3 1 2 6 8}\end{array}$

$\begin{array}{llll}\text { H } & -4.041065 & -1.365544 & -2597421\end{array}$

C $-6.188122-0.714724-0.986696$

$\begin{array}{llll}\text { C } & \mathbf{- 6 . 1 8 8 1 2 2} & \mathbf{- 0 . 7 1 4 7 2 4} & \mathbf{- 0 . 9 8 6 6 9 6}\end{array}$

$\begin{array}{llll}\text { H } & -6.453201 & -1.217490 & -1.926953\end{array}$ 
$\begin{array}{llll}\text { H } & -3.591610 & -1.018334 & 3.280894\end{array}$ $\begin{array}{llll}\text { H } & 1.560893 & -2.946664 & 2.032594\end{array}$ $\begin{array}{llll}\text { H } & -6.306022 & 0.369025 & -1.145266\end{array}$ $\begin{array}{llll}\text { H } & -6.908420 & -1.015852 & -0.213549\end{array}$ $\begin{array}{llll}\text { H } & -2.005546 & -0.344624 & 2.808233\end{array}$ I $-2.212824-2.102676 \quad 2.973809$ $\begin{array}{llll}\text { H } & -2.212824 & -2.102676 & 2.973809 \\ \text { F } & 2.751193 & -2.062144 & -1.757262\end{array}$ $\begin{array}{llll} & 2.751193 & -2.062144 & -1.757262\end{array}$ \begin{tabular}{llll} 
C & 6.691453 & 0.476110 & -0.447685 \\
\hline
\end{tabular} $\begin{array}{llll}\text { H } & 7.005422 & 1.207051 & \mathbf{0 . 3 0 9 0 6 5}\end{array}$

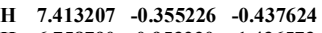
$\begin{array}{lllll}\text { H } & 6.758790 & \mathbf{0 . 9 5 3 2 2 0} & -\mathbf{1 . 4 3 6 5 7 3}\end{array}$ $\begin{array}{llll}\text { C } & \mathbf{2 . 6 1 1 5 8 8} & \mathbf{0 . 2 1 5 7 9 9} & \mathbf{2 . 5 3 4 5 5 5}\end{array}$ \begin{tabular}{llll} 
H & $\mathbf{1 . 8 1 4 0 1 6}$ & $\mathbf{0 . 9 4 7 2 5 0}$ & $\mathbf{2 . 3 2 8 8 4 2}$ \\
\hline & 2.131703 & -0.659479 & $\mathbf{2 . 9 9 6 2 9 8}$
\end{tabular} H $\quad \mathbf{2 . 1 3 1 7 0 3} \quad \mathbf{- 0 . 6 5 9 4 7 9} \quad \mathbf{2 . 9 9 6 2 9 8}$

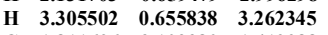
$\begin{array}{llll}C & -1.811696 & 3.103380 & 1.419988\end{array}$ $\begin{array}{llll}\text { C } & -2.286639 & 3.405858 & 0.200804\end{array}$ $\begin{array}{llll}\text { H } & \mathbf{1 . 9 0 2 6 8 2} & \mathbf{2 . 3 5 7 6 5 0} & \mathbf{0 . 1 8 2 2 6 8}\end{array}$ $\begin{array}{llll}C & 2.143647 & 1.677115 & -1.906194\end{array}$ $\begin{array}{lllll}\text { H } & \mathbf{2 . 1 5 8 5 7 3} & \mathbf{2 . 6 8 0 1 6 7} & \mathbf{- 2 . 3 6 6 1 6 3}\end{array}$ H $3.191768 \quad 1.374421 \quad-1.749241$ H $\quad$\begin{tabular}{llll}
\hline .688401 & 0.975310 & -2.624380
\end{tabular} $\begin{array}{llll}\text { H } & -2.999965 & 4.223089 & 0.057677\end{array}$ $\begin{array}{llll}\text { H } & -2.122544 & 3.655846 & 2.312637\end{array}$

71

sat_model_pc1 b / electronic energy: -2436.62140760 a.u. / lowest freq: $17.76 \mathrm{~cm}-1$ $\begin{array}{llll}\mathrm{S} & \mathbf{- 0 . 2 5 0 7 2 1} & \mathbf{0 . 2 1 9 7 3 2} & \mathbf{2 . 7 2 5 0 9 3}\end{array}$

$\begin{array}{llll}C & -0.224409 & -1.324630 & 0.178664\end{array}$

$\begin{array}{llll}\text { Ru } & -0.005695 & 0.740640 & 0.463194\end{array}$

$\begin{array}{lllll}C & -0.922558 & 0.933712 & -1.592716\end{array}$

$\begin{array}{llll}\text { C } & 1.838852 & 0.835350 & 0.481018\end{array}$ $\begin{array}{lrrrr}\mathrm{S} & \mathbf{- 0 . 4 3 2 0 7 8} & \mathbf{2 . 9 9 1 5 0 2} & \mathbf{1 . 0 9 1 7 0 0}\end{array}$

$\begin{array}{llll}\text { N } & 0.752967 & -2.255508 & 0.170740\end{array}$

$\begin{array}{llll}\text { N }-1.400491 & -1.992777 & 0.148911\end{array}$

C $2.125893-2.004066-0.070851$

$\begin{array}{llll}C & 2.125893 & -2.004066 & -0.070851 \\ C & 0.260289 & -3.628852 & 0.011895\end{array}$

$\begin{array}{llll}\text { C } & 0.260289 & -3.628852 & 0.011895\end{array}$

$\begin{array}{llll}C & -1.246668 & -3.453080 & 0.167550\end{array}$

$\begin{array}{llll}C & -2.689253 & -1.453732 & -0.118751\end{array}$

H $-1.814525-3.910013-0.653864$

H $-\mathbf{- 1 . 6 2 7 7 3 3}-\mathbf{- 3 . 8 5 5 7 4 3} \quad \mathbf{1 . 1 1 8 5 5 8}$

$\begin{array}{lllll}\text { H } & 0.541499 & \mathbf{- 4 . 0 1 1 8 8 7} & \mathbf{- 0 . 9 8 1 2 5 8}\end{array}$

$\begin{array}{llll}\text { C } & 4.826124 & -1.537351 & -0.696778\end{array}$

$\begin{array}{llll}C & 2.542297 & -1.777640 & -1.384601\end{array}$

$\begin{array}{lllll}\text { C } & 3.080547 & -2.032194 & 0.952264\end{array}$

$\begin{array}{llll}C & 4.418444 & -1.794097 & 0.615603\end{array}$

$\begin{array}{llll}\text { C } 3.862054 & -1.533716 & -1.713406\end{array}$

$\begin{array}{llll}\text { H } & \mathbf{5 . 1 6 7 1 3 5} & -\mathbf{1 . 8 0 0 0 4 2} & \mathbf{1 . 4 1 1 6 1 8}\end{array}$

H $\quad 4.126692-1.341917-2.754842$

$\begin{array}{llll}C & -5.223453 & -0.472988 & -0.879988\end{array}$

$\begin{array}{llll}\text { C } & -3.109887 & -1.436662 & -1.456261\end{array}$

$\begin{array}{llll}\text { C } & -\mathbf{3 . 5 9 9 8 5 4} & \mathbf{- 1 . 0 5 8 9 4 6} & \mathbf{0 . 8 6 9 3 1 0}\end{array}$

$\begin{array}{llll}C & -4.842104 & -0.549892 & 0.458991\end{array}$

$\begin{array}{llll}C & -4.337451 & -0.951993 & -1.854631\end{array}$

C $-2.264865-1.907767-2.384392$

$\begin{array}{llll}\mathrm{F} & -2.264865 & -1.907767 & -2.384392 \\ C & -3.315505 & -1.21051 & 2.335767\end{array}$

$\begin{array}{llll}\text { C } & -3.315505 & -1.212051 & 2.335767\end{array}$

$\begin{array}{lllll}\text { H } & \mathbf{- 5 . 5 4 4 3 2 4} & \mathbf{- 0 . 2 2 0 7 8 3} & \mathbf{1 . 2 2 9 3 3 4}\end{array}$

$\begin{array}{lllll}\text { H } & -4.589422 & -0.944159 & -2.916545\end{array}$

$\begin{array}{llll}\text { C } & -6.549299 & 0.110114 & -1.286894\end{array}$

$\begin{array}{llll}\text { H } & -7.056123 & -0.529781 & -2.023780\end{array}$

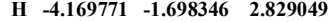

$\begin{array}{llll}\text { H } & 0.695907 & -4.289434 & 0.773629\end{array}$

$\begin{array}{lllll}\text { H } & -6.405955 & 1.096984 & -1.754325\end{array}$

$\begin{array}{lllll}\text { H } & -7.214694 & 0.240098 & -0.423058\end{array}$

$\begin{array}{lllll}\text { H } & -3.158535 & -0.232466 & 2.808744\end{array}$

$\begin{array}{llll}\text { H } & -2.410090 & -1.801108 & 2.520094\end{array}$

$\begin{array}{llll}\text { F } & 1.612040 & -1.763449 & -2.346956\end{array}$

$\begin{array}{llll}\text { C } & 6.269128 & -1.268023 & -1.028697\end{array}$

$\begin{array}{lllll}\text { H } & \mathbf{6 . 3 8 4 5 6 4} & \mathbf{- 0 . 2 7 7 0 8 0} & \mathbf{- 1 . 4 9 3 2 7 6}\end{array}$

$\begin{array}{llll}\text { H } & 6.901490 & -1.301654 & -0.131635\end{array}$

$\begin{array}{llll}\text { H } & 6.650555 & -2.009945 & -1.746578\end{array}$

$\begin{array}{llll}\text { C } & \mathbf{2 . 6 5 4 5 6 0} & \mathbf{- 2 . 2 6 0 9 4 5} & \mathbf{2 . 3 7 3 5 6 3}\end{array}$

$\begin{array}{llll}\text { H } & 2.126399 & -3.220094 & 2.484505\end{array}$

H $3.519896 \quad-2.267229 \quad 3.048844$

$\begin{array}{llll}\text { H } & \mathbf{1 . 9 5 8 9 6 0} & \mathbf{- 1 . 4 6 9 7 7 9} & \mathbf{2 . 6 9 6 5 5 7}\end{array}$

$\begin{array}{llll}\text { C } & \mathbf{- 0 . 3 8 8 2 2 5} & \mathbf{1 . 7 6 7 9 0 6} & \mathbf{3 . 5 2 5 3 9 8}\end{array}$

$\begin{array}{llll}C & \mathbf{- 0 . 4 5 3 7 1 9} & \mathbf{2 . 9 2 3 0 9 0} & \mathbf{2 . 8 3 7 5 3 0}\end{array}$

$\begin{array}{llll}C & -2.159482 & 1.792478 & -1.616506\end{array}$

$\begin{array}{lllll}\text { H } & -1.082443 & -0.087817 & -1.936535\end{array}$

\begin{tabular}{llll} 
C & 0.403141 & 1.352717 & -1.685954 \\
\hline
\end{tabular}

$\begin{array}{lllll}\text { H } & \mathbf{2 . 4 4 8 1 1 5} & \mathbf{0 . 6 6 8 9 5 0} & \mathbf{- 0 . 4 1 9 3 8 5}\end{array}$

$\begin{array}{llll}\text { C } & 0.851743 & 2.761542 & -1.827074\end{array}$

\begin{tabular}{llll}
$C$ & $\mathbf{2 . 6 8 4 8 5 6}$ & $\mathbf{1 . 3 5 2 3 0 6}$ & $\mathbf{1 . 5 8 9 7 1 4}$ \\
\hline & $\mathbf{3 . 5 8 9 5 9 5}$ & $\mathbf{0 . 7 3 1 9 7 8}$ & $\mathbf{1 . 7 0 0 4 2 6}$
\end{tabular}

$\begin{array}{lllll}\text { H } & 3.58559 & 0.731978 & 1.700426\end{array}$

$\begin{array}{llll}\text { H } & 3.031345 & 2.348094 & 1.251538\end{array}$

$\begin{array}{llll}\text { H } & 2.173091 & 1.472982 & 2.550281\end{array}$

$\begin{array}{lllll}\text { O } & 0.161186 & 3.733762 & -2.022403\end{array}$

$\begin{array}{lllll}O & 2.190427 & 2.838715 & -1.746071\end{array}$

$\begin{array}{llll}C & 2.756191 & 4.137546 & -1.802965\end{array}$

$\begin{array}{llll}\text { H } & 3.837577 & 4.006006 & -1.687463\end{array}$

$\begin{array}{llll}\text { H } & 2.364142 & 4.766456 & -0.990805\end{array}$

$\begin{array}{lllll}\text { H } & 2.536790 & 4.622026 & -2.765093\end{array}$

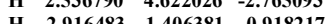

$\begin{array}{llll}\text { H } & -2.916483 & 1.406381 & \mathbf{- 0 . 9 1 8 2 1 7} \\ \text { H } & -2.599911 & 1.743279 & -2.627319\end{array}$ 
$\begin{array}{lrrr}\text { H } & -1.944158 & 2.836825 & -1.374997\end{array}$ $\begin{array}{lllll}\text { H } & 1.136323 & 0.629754 & -2.046615\end{array}$ $\begin{array}{llll}\text { H } & -0.419751 & 1.752964 & 4.619634\end{array}$ $\begin{array}{llll}\text { H } & -\mathbf{0 . 5 3 9 1 2 9} & \mathbf{3 . 8 8 2 3 0 1} & \mathbf{3 . 3 5 8 7 6 5}\end{array}$ 71

sat_model ts1 b / electronic energy: $-\mathbf{2 4 3 6 . 6 0 5 3 9 6 5 1}$ a.u. / lowest freq: $-\mathbf{1 3 9 . 6 9} \mathbf{c m}-1$ $S$ 0.388694 $-0.194041-2.769079$

C $0.160897-1.364808-0.044293$

$\begin{array}{lllll}\text { Ru } & \mathbf{0 . 1 4 8 2 3 7} & \mathbf{0 . 6 4 0 2 2 0} & \mathbf{- 0 . 6 3 6 9 0 3}\end{array}$

$\begin{array}{llll}C & 0.837137 & 1.169064 & 1.327058\end{array}$

$\begin{array}{lllll}C & -1.683845 & 0.977318 & -0.425384\end{array}$

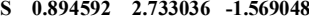

$\begin{array}{llll}N & -0.821453 & -2.291667 & 0.003647\end{array}$

$\begin{array}{llll}\mathrm{N} & 1.322322 & -2.029208 & 0.172772\end{array}$

$\begin{array}{llll}C & -2.204724 & -2.007230 & 0.118051\end{array}$

$\begin{array}{llll}\text { C } & -\mathbf{0 . 3 5 8 7 6 2} & -\mathbf{3 . 6 3 7 3 2 8} & \mathbf{0 . 3 6 3 6 2 5}\end{array}$

$\begin{array}{llll}C & \mathbf{1 . 1 5 8 5 5 1} & \mathbf{- 3 . 4 7 7 6 3 9} & \mathbf{0 . 3 3 7 2 9 7}\end{array}$

$\begin{array}{llll}\text { C } & \mathbf{2 . 5 9 9 6 7 0} & -\mathbf{1 . 4 5 9 9 3 2} & \mathbf{0 . 4 3 3 5 4 5}\end{array}$

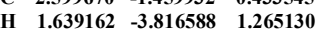

H $\quad \begin{array}{lll}1.627681 & -4.004645 & -0.507723\end{array}$

$\begin{array}{llll}\text { H } & -0.738748 & -3.907364 & 1.360852\end{array}$

$\begin{array}{llll}\text { C } & -4.916636 & -1.362220 & 0.485329\end{array}$

$\begin{array}{llll}\text { C } & -2.712848 & -1.672972 & 1.374715\end{array}$

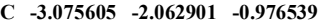

C $-4.419251-1.730198-\mathbf{- 0 . 7 6 9 0 6 0}$

C -4.04

H $-5.102223-1.756637-1.621932$

H $-4.376521 \quad-1.069285 \quad 2.579335$

$\begin{array}{llll}C & \mathbf{5 . 0 9 1 4 3 3} & \mathbf{- 0 . 3 6 5 6 4 0} & \mathbf{1 . 1 8 0 1 7 4}\end{array}$

$\begin{array}{llll}C & 2.935778 & -1.221155 & 1.772965\end{array}$

$\begin{array}{llll}\text { C } & 3.573312 & -1.229985 & -0.546862\end{array}$

$\begin{array}{llll}C & 4.793859 & -0.663086 & -0.149152\end{array}$

$\begin{array}{llll}\text { C } & 4.140647 & -0.674704 & 2.162243\end{array}$

$\begin{array}{llll}\text { F } & 2.030405 & -1.531868 & \mathbf{2 . 7 1 2 0 6 0}\end{array}$

$\begin{array}{llll}\text { F } 2.030405 & -1.531868 & 2.712060 \\ \text { C } & 3.374034 & -1.613152 & -1.985052\end{array}$

\begin{tabular}{llll} 
C & 3.374034 & -1.613152 & -1.985052 \\
\hline & 5.545114 & -0.463908 & -0.917930
\end{tabular}

$\begin{array}{llll}\text { H } & 5.545114 & -0.463908 & \mathbf{0 . 9 1 7 9 3 0}\end{array}$

$\begin{array}{llll}\text { H } & 4.326365 & -0.491558 & 3.222013\end{array}$

$\begin{array}{llll}\text { C } & 6.393412 & 0.282755 & 1.565092\end{array}$

$\begin{array}{llll}\text { H } & 6.807412 & -0.163319 & 2.480759\end{array}$

$\begin{array}{llll}\text { H } & 4.211327 & \mathbf{- 2 . 2 4 3 2 8 8} & \mathbf{- 2 . 3 2 0 1 3 5}\end{array}$

$\begin{array}{lllll}\text { H } & \mathbf{- 0 . 7 2 5 6 2 5} & \mathbf{- 4 . 3 7 8 8 7 8} & \mathbf{- 0 . 3 5 9 2 7 7}\end{array}$

$\begin{array}{llll}\text { H } & 6.240963 & 1.355680 & 1.762426\end{array}$

$\begin{array}{lllll}\text { H } & 7.140298 & 0.193082 & 0.764880\end{array}$

$\begin{array}{llll}\text { H } & 3.342982 & -0.719329 & -2.624555\end{array}$

(2.625

F $-1.859782-1.621717-2.140849$

C $-6.359334-0.974631 \quad 0.668918$

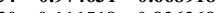

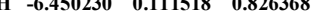

$\begin{array}{llll}\text { H } & -6.961321 & -1.238700 & -0.210804\end{array}$

$\begin{array}{llll}-6.794616 & -1.468976 & 1.549373\end{array}$

\begin{tabular}{llll}
$C$ & -2.557689 & -2.434025 & -2.335796 \\
\hline & -2.040844 & -3.405292 & -2.312309
\end{tabular}

H $-3.374788-2.495657-3.066025$

$-1.826021-1.689138-2.684906$

$\begin{array}{llll}\text { H } & -1.826021 & -1.689138 & -2.684906 \\ \text { C } & 0.788727 & 1.17348 & -3.792520\end{array}$

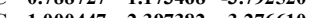

$\begin{array}{llll}\text { C } & \mathbf{1 . 0 0 0 4 4 7} & \mathbf{2 . 3 9 7 3 8 2} & \mathbf{- 3 . 2 7 6 6 1 0}\end{array}$

$\begin{array}{llll}C & 1.962217 & 2.164130 & 1.487997\end{array}$

$\begin{array}{llll}\text { H } & 1.020141 & 0.248629 & 1.881352\end{array}$

$\begin{array}{llll}C & \mathbf{- 0 . 5 6 5 8 1 6} & \mathbf{1 . 4 9 5 3 4 7} & \mathbf{1 . 3 3 2 4 2 5}\end{array}$

$\begin{array}{llll}\text { H } & -2.359941 & 0.362189 & 0.178208\end{array}$

$\begin{array}{llll}\text { C } & -1.077644 & 2.897042 & 1.459331\end{array}$

$\begin{array}{llll}C & -2.456323 & 2.004158 & -1.185143\end{array}$

$\begin{array}{lllll}\text { H } & -2.928002 & 1.447881 & -2.016415\end{array}$

$\begin{array}{llll}\text { H } & -3.274671 & 2.418749 & -0.575433\end{array}$

H $-1.834201 \quad 2.797836-1.611907$

\begin{tabular}{llll}
\hline & -0.501456 & 3.917779 & 1.182709
\end{tabular}

\begin{tabular}{llll}
\hline & -2.319832 & $\mathbf{2 . 8 7 8 4 3 3}$ & $\mathbf{1 . 9 6 2 7 8 2}$
\end{tabular}

$\begin{array}{llll}\text { C } & -2.951436 & 4.138949 & 2.137819\end{array}$

$\begin{array}{llll}\text { H } & -\mathbf{3 . 9 2 7 9 6 2} & \mathbf{3 . 9 3 3 5 6 1} & \mathbf{2 . 5 8 8 6 2 9}\end{array}$

H $-3.080796 \quad 4.644277 \quad 1.169982$

$\begin{array}{llll}\text { H } & -2.357308 & 4.785025 & 2.798854\end{array}$

$\begin{array}{llll}\text { H } & \mathbf{2 . 8 8 8 7 9 0} & \mathbf{1 . 7 4 5 7 9 4} & \mathbf{1 . 0 6 9 0 1 3}\end{array}$

$\begin{array}{llll}\text { H } & \mathbf{2 . 1 3 7 5 8 2} & \mathbf{2 . 3 4 0 3 5 6} & \mathbf{2 . 5 6 3 5 8 2}\end{array}$

$\begin{array}{llll}\text { H } & \mathbf{1 . 7 5 3 9 6 8} & \mathbf{3 . 1 2 3 8 5 0} & \mathbf{1 . 0 0 6 8 5 4}\end{array}$

\begin{tabular}{lllll}
\hline & -1.196968 & 0.792428 & 1.879172
\end{tabular}

$\begin{array}{llll}\text { H } & \mathbf{1 . 2 6 6 9 6 7} & \mathbf{3 . 2 3 5 5 4 8} & \mathbf{- 3 . 9 2 9 8 1 5}\end{array}$

$\begin{array}{llll}\text { H } & 0.865219 & 0.971107 & -4.865315\end{array}$

71

sat_model_mcb_b / electronic energy: -2436.61404309 a.u. / lowest freq: $15.11 \mathrm{~cm}-1$

$\begin{array}{llll}S & -0.559842 & -0.735026 & 2.674749\end{array}$

$\begin{array}{llll}C & -0.095962 & -1.364459 & -0.130845\end{array}$

$\begin{array}{llll}\text { Ru } & \mathbf{- 0 . 1 7 5 7 3 8} & \mathbf{0 . 5 1 2 2 2 8} & \mathbf{0 . 8 2 2 8 2 0}\end{array}$

$\begin{array}{lllr}\text { Ru } & -0.15738 & 0.512228 & \mathbf{0 . 8 2 2 8 2 0} \\ C & -\mathbf{0 . 8 3 5 5 6 0} & \mathbf{1 . 3 0 7 5 3 1} & \mathbf{- 0 . 8 9 2 8 7 5}\end{array}$

$\begin{array}{llll}C & \mathbf{1 . 6 3 0 9 4 1} & \mathbf{1 . 1 2 1 0 3 2} & \mathbf{0 . 3 7 7 6 4 2}\end{array}$

$\begin{array}{lrrr}C & -\mathbf{0 . 8 3 4 3 3 8} & \mathbf{2 . 4 0 8 6 3 7} & \mathbf{2 . 1 7 5 8 5 5}\end{array}$

$\begin{array}{llll}\text { N } & 0.919189 & -2.238215 & -0.297392\end{array}$

$\begin{array}{llll}\text { N } & -1.226327 & -2.015794 & -0.495132\end{array}$

$\begin{array}{llll}C & 2.298681 & -1.910141 & -0.325992\end{array}$

$\begin{array}{llll}\text { C } & 0.511661 & -3.535538 & -0.852492\end{array}$

$\begin{array}{llll}C & -1.007030 & -3.400981 & -0.926355\end{array}$

$\begin{array}{llll}C & -2.510308 & -1.443206 & -0.713768\end{array}$

$\begin{array}{llll}\text { H } & -1.400070 & -3.550144 & -1.941910\end{array}$

$\begin{array}{llll}\text { H } & -1.530726 & -4.092176 & -0.249352\end{array}$ 
$\begin{array}{lllll}\text { H } & 0.973601 & -3.681767 & -1.840155\end{array}$ $\begin{array}{llll}C & 5.010249 & -1.201622 & -0.54349\end{array}$

$\begin{array}{llll}C & 2.830451 & -1.409499 & -1.516248\end{array}$

$\begin{array}{llll}C & 3.143942 & \mathbf{- 2 . 0 9 5 4 8 8} & \mathbf{0 . 7 7 4 2 2 3}\end{array}$

$\begin{array}{llll}C & 4.487405 & -1.725501 & 0.643294\end{array}$

$\begin{array}{llll}C & 4.487405 & -1.725501 & 0.643294\end{array}$

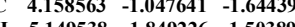

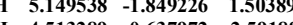

$\begin{array}{llll} & 4.513289 & -0.637972 & -2.591885\end{array}$

$\begin{array}{llll}C & -4.999817 & -0.285181 & -1.366633\end{array}$

$\begin{array}{llll}C & -2.784247 & -0.942424 & -1.992997\end{array}$

$\begin{array}{llll}C & -3.545048 & -1.454046 & 0.230543\end{array}$

$\begin{array}{llll}C & -4.763616 & -0.850357 & -0.113692\end{array}$

\begin{tabular}{llll}
$C$ & -3.987008 & -0.357659 & -2.332220 \\
\hline
\end{tabular}

$\begin{array}{llll}\text { C } & -3.410697 & -2.125666 & 1.567248\end{array}$

$\begin{array}{llll}\text { H } & \mathbf{- 5 . 5 6 2 2 1 4} & \mathbf{- 0 . 8 3 9 2 1 9} & \mathbf{0 . 6 3 2 7 1 5}\end{array}$

$\begin{array}{llll}H & -4.122793 & 0.039325 & -3.339801\end{array}$

$\begin{array}{llll}\text { C } & -6.302136 & 0.399894 & -1.681123\end{array}$

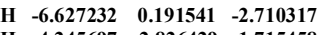

$\begin{array}{llll}\text { H } & -4.245697 & -2.826429 & 1.715458\end{array}$

$\begin{array}{lllll}\text { H } & 0.838846 & -4.352142 & -0.193684\end{array}$

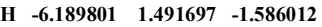

$\begin{array}{llll}\text { H } & -7.097533 & 0.085806 & -0.991956\end{array}$

$\begin{array}{llll}\text { H } & -3.440602 & -1.384127 & 2.378403\end{array}$

$\begin{array}{llll}\text { H } & -2.464552 & -2.669115 & 1.663011\end{array}$

$\begin{array}{llll} & 1.998103 & -1.229988 & -2.550113\end{array}$

$\begin{array}{llll}C & 6.464234 & -0.829325 & -0.655755\end{array}$

$\begin{array}{llll}\text { H } & \mathbf{6 . 9 3 7 0 6 2} & \mathbf{- 0 . 7 5 3 6 8 8} & \mathbf{0 . 3 3 2 5 0 1}\end{array}$

$\begin{array}{llll}\text { H } & 7.013615 & -1.587749 & -1.235528\end{array}$

$\begin{array}{lllll}\text { H } & 6.588578 & 0.132004 & -1.174499\end{array}$

$\begin{array}{llll}C & 2.600644 & -2.635739 & \mathbf{2 . 0 6 5 0 1 8}\end{array}$

$\begin{array}{llll}\text { H } & \mathbf{2 . 0 6 5 9 3 8} & \mathbf{- 3 . 5 8 4 2 5 6} & \mathbf{1 . 9 0 6 6 9 5}\end{array}$

H $3.406019 \quad-2.809644 \quad 2.790092$

H $1.877947-1.929442 \quad 2.501160$

$\begin{array}{lrrr}H & 1.877947 & -1.929442 & 2.501160\end{array}$

$\begin{array}{llll}C & -1.054685 & 0.364055 & 3.950681\end{array}$

$\begin{array}{llll}C & -1.157965 & 1.686140 & 3.721838\end{array}$

$\begin{array}{llll}C & -1.874492 & 2.398289 & -1.015436\end{array}$

$\begin{array}{lllll}\text { H } & -\mathbf{0 . 9 5 4 8 9 1} & \mathbf{0 . 5 9 9 2 1 0} & \mathbf{- 1 . 7 1 5 1 1 5}\end{array}$

$\begin{array}{lllll}\text { C } & \mathbf{0 . 7 2 0 5 7 0} & \mathbf{1 . 6 6 9 1 0 6} & \mathbf{- 0 . 8 5 5 4 3 8}\end{array}$

$\begin{array}{llll}\text { H } & \mathbf{2 . 3 1 9 7 0 5} & \mathbf{0 . 3 8 6 0 8 8} & \mathbf{- 0 . 0 4 0 0 3 3}\end{array}$

$\begin{array}{llll}\text { C } & \mathbf{0 . 9 7 6 7 8 7} & 3.149805 & -1.009334\end{array}$

$\begin{array}{llll}C & \mathbf{2 . 4 2 2 1 6 6} & \mathbf{2 . 1 1 6 3 9 1} & \mathbf{1 . 1 9 9 1 4 3}\end{array}$

$\begin{array}{llll}\text { H } & 2.881009 & 1.569697 & 2.036587\end{array}$

\begin{tabular}{cccc} 
H & $\mathbf{3 . 2 4 5 4 8 9}$ & $\mathbf{2 . 5 3 8 1 7 0}$ & $\mathbf{0 . 5 9 6 7 4 2}$ \\
\hline & $\mathbf{1 . 5 1 0 5 1 3}$ & $\mathbf{2 . 9 2 6 1 8 3}$ & $\mathbf{1 . 6 0 7 4 8 0}$
\end{tabular}

\begin{tabular}{cccc} 
H & $\mathbf{1 . 8 1 0 5 1 3}$ & $\mathbf{2 . 9 2 6 1 8 3}$ & $\mathbf{1 . 6 0 7 4 8 0}$ \\
\hline & $\mathbf{0 . 5 1 7 0 4 0}$ & $\mathbf{4} 039556$ & -0.34420
\end{tabular}

\begin{tabular}{lllll}
\hline & 0.517040 & 4.039556 & -0.344420
\end{tabular}

$\begin{array}{lllll}\text { O } & 1.840542 & 3.349252 & -2.007904\end{array}$

$\begin{array}{llll}C & 2.215656 & 4.697850 & -2.262076\end{array}$

$\begin{array}{lllll}\text { H } & \mathbf{2 . 9 3 4 2 7 2} & \mathbf{4 . 6 6 4 9 2 0} & -\mathbf{3 . 0 8 7 3 7 7}\end{array}$

$\begin{array}{llll}\text { H } & 2.680342 & 5.144059 & -1.371945\end{array}$

$\begin{array}{lllll}\text { H } & 1.339313 & \mathbf{5 . 2 9 7 2 1 4} & \mathbf{- 2 . 5 4 5 7 6 7}\end{array}$

$\begin{array}{lllll}\text { H } & -2.862662 & 1.930215 & -0.888464\end{array}$

\begin{tabular}{llll}
\hline & -1.852883 & 2.846339 & -2.024098
\end{tabular}

\begin{tabular}{llll}
\hline & -1.852883 & 2.846339 & -2.024098 \\
\hline & -1.764086 & 3.190284 & -0.269229
\end{tabular}

\begin{tabular}{llll} 
H & -1.764086 & 3.190284 & $\mathbf{- 0 . 2 6 9 2 2 9}$ \\
\hline & 1.146489 & 1.142525 & -1.714882
\end{tabular}

$\begin{array}{llll}H & 1.146489 & 1.142525 & -1.714882\end{array}$

$\begin{array}{llll}\text { H } & -1.272625 & -0.089801 & 4.921661 \\ \text { H } & -1.470073 & \mathbf{2 . 3 6 2 9 9 6} & \mathbf{4 . 5 2 4 7 1 2}\end{array}$

71

sat_model_ts2_b / electronic energy: $-\mathbf{2 4 3 6 . 6 0 5 3 9 6 5 1}$ a.u. / lowest freq: $-\mathbf{1 3 9 . 6 9} \mathrm{cm}-1$

$\begin{array}{llll}S & 0.388694 & -0.194041 & -2.769079\end{array}$

$\begin{array}{llll}C & 0.160897 & -1.364808 & -0.044293\end{array}$

$\begin{array}{lllll}\text { Ru } & 0.148237 & 0.640220 & -0.636903\end{array}$

$\begin{array}{lllll}\text { C } & 0.837137 & 1.169064 & 1.327058\end{array}$

$\begin{array}{llll}C & -1.683845 & 0.977318 & -\mathbf{0 . 4 2 5 3 8 4}\end{array}$

$\begin{array}{lllll}C & 0.894592 & \mathbf{2 . 7 3 3 0 3 6} & -\mathbf{1 . 5 6 9 0 4 8}\end{array}$

$\begin{array}{lllll}\text { N } & -\mathbf{0 . 8 2 1 4 5 3} & \mathbf{- 2 . 2 9 1 6 6 7} & \mathbf{0 . 0 0 3 6 4 7}\end{array}$

$\begin{array}{llll}\text { N } & \mathbf{1 . 3 2 2 3 2 2} & \mathbf{- 2 . 0 2 9 2 0 8} & \mathbf{0 . 1 7 2 7 7 2}\end{array}$

$\begin{array}{llll}C & -2.204724 & -2.007230 & 0.118051\end{array}$

$\begin{array}{llll}C & -0.358762 & -3.637328 & 0.363625\end{array}$

$\begin{array}{llll}\text { C } & \mathbf{1 . 1 5 8 5 5 1} & \mathbf{- 3 . 4 7 7 6 3 9} & \mathbf{0 . 3 3 7 2 9 7}\end{array}$

$\begin{array}{llll}\text { C } & \mathbf{2 . 5 9 9 6 7 0} & -\mathbf{1 . 4 5 9 9 3 2} & \mathbf{0 . 4 3 3 5 4 5}\end{array}$

H $1.639162-3.816$

$\begin{array}{lll}1.639162 & -3.816588 & 1.265130\end{array}$

\begin{tabular}{llll}
\hline & $\mathbf{1 . 6 2 7 6 8 1}$ & $\mathbf{- 4 . 0 0 4 6 4 5}$ & $\mathbf{- 0 . 5 0 7 7 2 3}$ \\
\hline
\end{tabular}

\begin{tabular}{llll}
\hline & -0.738748 & -3.907364 & 1.360852
\end{tabular}

$\begin{array}{llll}C & -4.916636 & -1.362220 & \mathbf{0 . 4 8 5 3 2 9}\end{array}$

$\begin{array}{llll}C & -2.712848 & -1.672972 & 1.374715\end{array}$

$\begin{array}{llll}C & -3.075605 & -2.062901 & -0.976539\end{array}$

$\begin{array}{llll}\text { C } & -4.419251 & -1.730198 & -0.769060\end{array}$

$\begin{array}{llll}\text { C } & -4.040605 & -1.344490 & 1.577918\end{array}$

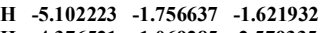

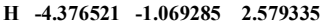

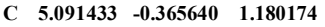

$\begin{array}{lrrr}C & 5.091433 & -0.365640 & 1.180174 \\ \text { C } & \mathbf{2 . 9 3 5 7 7 8} & -1.221155 & \mathbf{1 . 7 7 2 9 6 5}\end{array}$

$\begin{array}{llll}C & 3.573312 & -1.229985 & \mathbf{- 0 . 5 4 6 8 6 2}\end{array}$

$\begin{array}{llll}C & 4.793859 & \mathbf{- 0 . 6 6 3 0 8 6} & \mathbf{- 0 . 1 4 9 1 5 2}\end{array}$

$\begin{array}{lllll}\text { C } & 4.140647 & -0.674704 & 2.162243\end{array}$

$\begin{array}{llll}\text { F } & \mathbf{2 . 0 3 0 4 0 5} & \mathbf{- 1 . 5 3 1 8 6 8} & \mathbf{2 . 7 1 2 0 6 0}\end{array}$

$\begin{array}{llll}C & 3.374034 & -1.613152 & -1.985052\end{array}$

$\begin{array}{llll}\text { H } & \mathbf{5 . 5 4 5 1 1 4} & \mathbf{- 0 . 4 6 3 9 0 8} & \mathbf{- 0 . 9 1 7 9 3 0}\end{array}$

$\begin{array}{llll}\text { H } & 4.326365 & -0.491558 & 3.222013\end{array}$

$\begin{array}{llll}C & 6.393412 & 0.282755 & 1.565092\end{array}$

$\begin{array}{llll}\text { H } & 6.807412 & -0.163319 & 2.480759\end{array}$

\begin{tabular}{llll} 
H & 6.807412 & $\mathbf{- 0 . 1 6 3 3 1 9}$ & $\mathbf{2 . 4 8 0 7 5 9}$ \\
\hline & 4.211327 & -2.243288 & -2.320135
\end{tabular}

$\begin{array}{llll}\text { H } & 4.211327 & -2.243288 & -2.320135\end{array}$ 


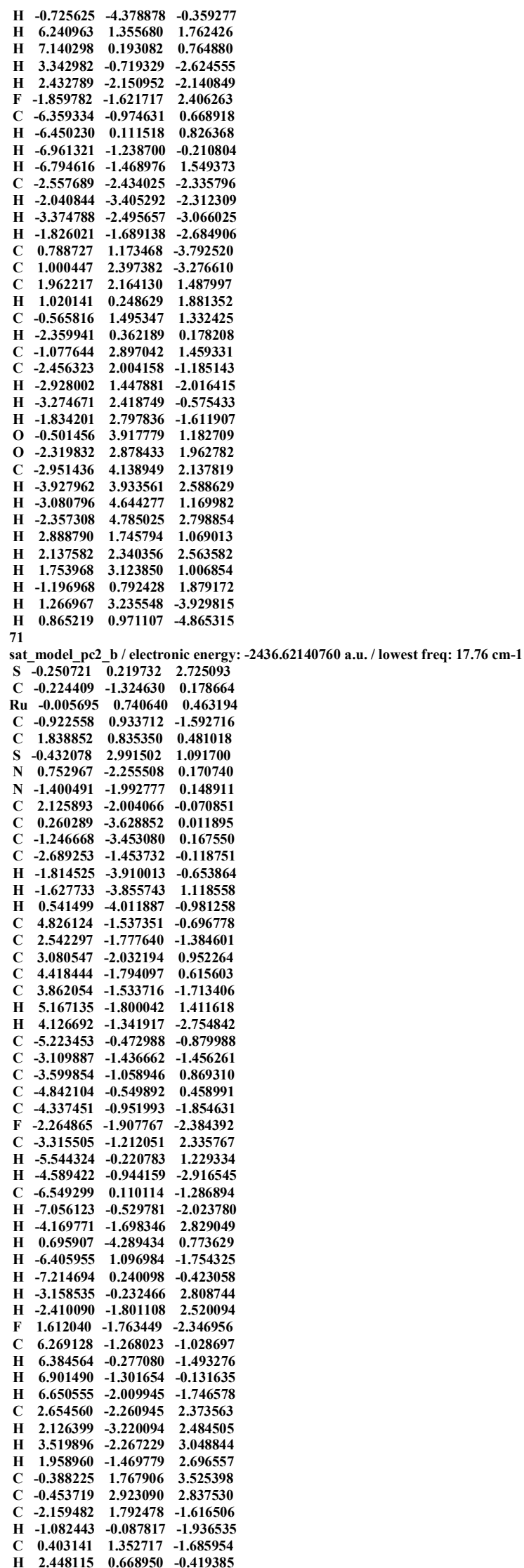


C $\quad \begin{array}{llll}0.851743 & 2.761542 & -1.827074\end{array}$

$\begin{array}{llll}C & \mathbf{2 . 6 8 4 8 5 6} & \mathbf{1 . 3 5 2 3 0 6} & \mathbf{1 . 5 8 9 7 1}\end{array}$

H $3.589595 \quad 0.731978 \quad 1.700426$

$\begin{array}{lllll}\text { H } & \mathbf{3 . 0 3 1 3 4 5} & \mathbf{2 . 3 4 8 0 9 4} & \mathbf{1 . 2 5 1 5 3 8}\end{array}$

$\begin{array}{llll}\text { H } & 2.173091 & 1.472982 & 2.550281\end{array}$

$\begin{array}{llll}0 & 2.173091 & 1.472982 & 2.550281\end{array}$

\begin{tabular}{llll}
\hline & 0.161186 & 3.733762 & -2.022403
\end{tabular}

$\begin{array}{llll}\text { O } & 2.190427 & 2.838715 & -1.74607\end{array}$

$\begin{array}{llll}\text { C } & 2.756191 & 4.137546 & -1.802965 \\ \text { H } & \mathbf{3 . 8 3 7 5 7 7} & \mathbf{4 . 0 0 6 0 0 6} & -\mathbf{1 . 6 8 7 4 6 3}\end{array}$

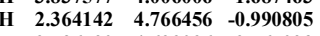

$\begin{array}{llll}\text { H } & \mathbf{2 . 5 3 6 7 9 0} & \mathbf{4 . 6 2 2 0 2 6} & \mathbf{- 2 . 7 6 5 0 9 3}\end{array}$

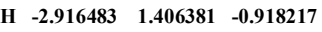

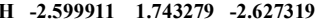

$\begin{array}{llll}\text { H } & -1.944158 & 2.836825 & -1.374997\end{array}$

H $\quad \begin{array}{llll}1.136323 & 0.629754 & -2.046615\end{array}$

\begin{tabular}{lllll}
\hline H & $-\mathbf{0 . 4 1 9 7 5 1}$ & $\mathbf{1 . 7 5 2 9 6 4}$ & $\mathbf{4 . 6 1 9 6 3 4}$
\end{tabular}

\begin{tabular}{cccc}
\hline & -0.539129 & 3.882301 & 3.358765
\end{tabular}

56

sat_model_14e1 / electronic energy: -2091.16423387 a.u. / lowest freq: $21.22 \mathrm{~cm}-1$

$\begin{array}{llll}S & -0.664109 & 1.799643 & 1.689243\end{array}$

$\begin{array}{llll}C & 0.227217 & -0.862908 & 0.290244\end{array}$

$\begin{array}{lllll}\text { Ru } & -0.211197 & 1.016768 & -0.407680\end{array}$

$\begin{array}{llll}\text { C } & \mathbf{1 . 4 2 4 5 0 0} & \mathbf{1 . 7 3 3 3 3 1} & \mathbf{- 0 . 5 9 2 1 7 1}\end{array}$

$\begin{array}{llll}\text { S } & -1.816541 & 2.506135 & -1.237129\end{array}$

$\begin{array}{lllll}\text { N } & 1.394537 & -1.490357 & 0.508440\end{array}$

$\begin{array}{lllll}\text { N } & 1.394537 & -1.490357 & \mathbf{0 . 5 0 8 4 4 0}\end{array}$

$\begin{array}{llll}\mathrm{N} & -0.762821 & -1.739599 & 0.560071\end{array}$

$\begin{array}{llll}\text { C } & -0.7628215 & -1.739599 & 0.560071 \\ C & \mathbf{0 . 9 5 8 7 9 9} & \mathbf{0 . 3 0 9 2 0 0}\end{array}$

$\begin{array}{llll}C & 1.230066 & -2.870100 & 0.985071\end{array}$

$\begin{array}{lllll}C & -0.273548 & -3.096949 & 0.823069\end{array}$

$\begin{array}{llll}\text { C } & -2.121849 & -1.504494 & 0.220277\end{array}$

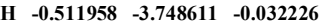

$\begin{array}{llll}\text { H } & -0.741515 & -3.519510 & 1.722198\end{array}$

$\begin{array}{llll}\text { H } & \mathbf{1 . 8 3 3 5 2 1} & \mathbf{- 3 . 5 5 9 1 0 0} & \mathbf{0 . 3 7 9 4 8 5}\end{array}$

C $5.296721-0.025149-0.184392$

$\begin{array}{llll}\text { C } & 5.296721 & -0.025149 & -0.184392\end{array}$

$\begin{array}{llll}C & 3.376869 & -1.295342 & -0.859417\end{array}$

$\begin{array}{llll}\text { C } & 3.324750 & -0.154868 & 1.266493\end{array}$

$\begin{array}{llll}C & 4.615864 & 0.308757 & 0.990896\end{array}$

$\begin{array}{llll}C & 4.660794 & -0.850792 & -1.119572\end{array}$

H $\quad \begin{array}{llll}5.109873 & 0.948403 & 1.726569\end{array}$

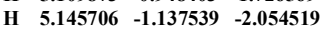

$\begin{array}{llll}\text { C } & -4.775562 & -1.028160 & -0.572668\end{array}$

$\begin{array}{llll}C & -2.504038 & -1.562566 & -1.121264\end{array}$

$\begin{array}{llll}C & -3.092840 & -1.236139 & 1.197007\end{array}$

$\begin{array}{llll}C & -3.092840 & -1.236139 & 1.197007\end{array}$

$\begin{array}{llll}C & -4.404104 & -1.000505 & \mathbf{0 . 7 7 6 9 5 8} \\ C & -3.803572 & -1.322995 & -1.533072\end{array}$

$\begin{array}{llll}\text { C } & -3.803572 & -1.322995 & -1.533072\end{array}$

\begin{tabular}{llll} 
F & -1.567554 & -1.3229708 & -2.034834 \\
\hline
\end{tabular}

$\begin{array}{llll}\text { C } & -2.709277 & -1.176576 & 2.647422\end{array}$

$\begin{array}{llll}\text { H } & -5.163703 & -0.780341 & 1.531268\end{array}$

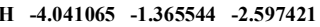

$\begin{array}{llll}C & -6.188122 & -0.714724 & -0.986696\end{array}$

$\begin{array}{lllll}\text { H } & -6.453201 & -1.217490 & -1.926953\end{array}$

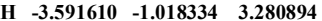

H $1.560893 \quad 2.946664 \quad 2.032594$

- $1.5606220 .369025-1.145266$

$\begin{array}{llll}1 & -6.306022 & 0.369025 & -1.145266\end{array}$

$\begin{array}{llll}\text { H } & -6.908420 & -1.015852 & \mathbf{- 0 . 2 1 3 5 4 9}\end{array}$

$\begin{array}{llll}\text { H } & \mathbf{- 2 . 0 0 5 5 4 6} & \mathbf{- 0 . 3 4 4 6 2 4} & \mathbf{2 . 8 0 8 2 3 3}\end{array}$

$\begin{array}{llll}\text { H } & -2.212824 & -2.102676 & 2.973809\end{array}$

$\begin{array}{llll}\text { F } & \mathbf{2 . 7 5 1 1 9 3} & \mathbf{- 2 . 0 6 2 1 4 4} & \mathbf{- 1 . 7 5 7 2 6 2}\end{array}$

$\begin{array}{lllll}C & 6.691453 & 0.476110 & -0.447685\end{array}$

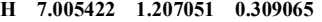

$\begin{array}{llll}\text { H } & 7.413207 & -0.355226 & -0.437624\end{array}$

$\begin{array}{lllll}\text { H } & 6.758790 & \mathbf{0 . 9 5 3 2 2 0} & -1.436573\end{array}$

$\begin{array}{llll}C & 2.711588 & 0.215799 & 2.534555\end{array}$

\begin{tabular}{llll}
$C$ & 2.611588 & 0.215799 & 2.534555 \\
\hline
\end{tabular}

\begin{tabular}{cccc}
$\mathrm{C}$ & $\mathbf{1 . 8 1 4 0 1 6}$ & $\mathbf{0 . 9 4 7 2 5 0}$ & $\mathbf{2 . 3 2 8 8 4 2}$ \\
\hline & 2.131703 & -0.659479 & $\mathbf{2}$
\end{tabular}

$\begin{array}{lllll}\text { H } & \mathbf{2 . 1 3 1 7 0 3} & \mathbf{- 0 . 6 5 9 4 7 9} & \mathbf{2 . 9 9 6 2 9 8}\end{array}$

$\begin{array}{llll}\text { H } & 3.305502 & \mathbf{0 . 6 5 5 8 3 8} & 3.262345\end{array}$

$\begin{array}{llll}\text { C } & -1.811696 & 3.103380 & 1.419988\end{array}$

$\begin{array}{llll}C & -2.286639 & 3.405858 & 0.200804\end{array}$

$\begin{array}{llll}\text { H } & 1.902682 & 2.357650 & 0.182268\end{array}$

$\begin{array}{llll}\text { C } & 2.143647 & 1.677115 & -1.906194\end{array}$

$\begin{array}{lllll}\text { H } & \mathbf{2 . 1 5 8 5 7 3} & \mathbf{2 . 6 8 0 1 6 7} & \mathbf{- 2 . 3 6 6 1 6 3}\end{array}$

$\begin{array}{lllll}\text { H } & 3.191768 & 1.374421 & -1.749241\end{array}$

H $1.688401-0.975310-2.624380$

\begin{tabular}{lllll}
$H$ & 1.688401 & 0.975310 & -2.624380 \\
\hline & -2.999965 & 4.223089 & 0.057677
\end{tabular}

$\begin{array}{llll}H & -2.999965 & 4.223089 & 0.057677\end{array}$

$\begin{array}{llll}\text { H } & -2.122544 & 3.655846 & 2.312637\end{array}$

71

sat_model_pc1_c / electronic energy: -2436.61997971 a.u. / lowest freq: $24.33 \mathrm{~cm}-1$ $\begin{array}{llll}S & -0.265995 & 1.049811 & 2.482565\end{array}$

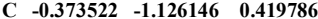

$\begin{array}{lllll}\text { Ru } & -\mathbf{0 . 1 5 6 2 5 0} & \mathbf{0 . 9 3 8 7 9 4} & \mathbf{0 . 1 5 7 0 0 8}\end{array}$

$\begin{array}{llll}\text { C } & -1.927029 & 1.382767 & -0.118350\end{array}$

$\begin{array}{llll}C & 0.932364 & 0.565594 & -1.763452\end{array}$

$\begin{array}{llll}\text { C } & 0.932364 & 0.565594 & -1.763452\end{array}$

$\begin{array}{lllll}\mathrm{S} & 0.761048 & 3.123222 & \mathbf{0 . 2 5 3 9 8}\end{array}$

$\begin{array}{lllll}\text { N } & 0.638954 & \mathbf{- 1 . 9 7 6 1 3 0} & \mathbf{0 . 7 0 2 6 1 0}\end{array}$

$\begin{array}{llll}\text { N } & -1.518666 & -1.834175 & 0.532141\end{array}$

$\begin{array}{llll}C & 2.033474 & -1.778456 & 0.497228\end{array}$

$\begin{array}{llll}C & 0.189201 & -3.325587 & 1.069503\end{array}$

$\begin{array}{llll}C & -1.309043 & -3.262915 & 0.793904\end{array}$

$\begin{array}{llll}\text { C } & -2.800996 & -1.416939 & 0.097379\end{array}$

H $-1.602573-3.850278-0.089858$

$\begin{array}{llll}H & -1.917493 & -3.593345 & 1.646918\end{array}$

$\begin{array}{lll}-1.917493 & -3.593345 & 1.646918\end{array}$

$\begin{array}{rrrr}\text { H } & 0.703995 & -4.079889 & 0.459478 \\ \text { C } & 4.792555 & -1.635103 & -0.070243\end{array}$ 
$\begin{array}{llll}C & \mathbf{2 . 5 6 3 1 6 2} & -\mathbf{2} .188557 & \mathbf{- 0 . 7 3 3 4 2 9}\end{array}$ $\begin{array}{llll}\text { C } & 2.919448 & -1.348043 & 1.49226\end{array}$

$\begin{array}{llll}C & 4.283072 & -1.264193 & 1.17315\end{array}$

$\begin{array}{llll}C & 3.904926 & -2.120648 & -1.038434\end{array}$

$\begin{array}{llll}\text { H } & 4.973365 & -0.911602 & 1.944130\end{array}$

$\begin{array}{llll}\text { H } & 4.245894 & -2.436208 & -2.026038\end{array}$

$\begin{array}{llll}C & -5.316781 & -0.651857 & -0.898842\end{array}$

$\begin{array}{llll}C & -3.116297 & -1.531813 & -1.257744\end{array}$

$\begin{array}{llll}C & -3.779362 & -0.956733 & 0.987446\end{array}$

$\begin{array}{llll}C & \mathbf{- 5 . 0 2 1 8 5 9} & \mathbf{- 0 . 5 7 7 8 7 6} & \mathbf{0 . 4 6 5 9 4 2}\end{array}$

$\begin{array}{llll}C & -4.340640 & -1.147982 & -1.772336\end{array}$

$\begin{array}{llll}\text { F } & -2.176390 & -2.005289 & -2.085637\end{array}$

$\begin{array}{llll}C & -3.474544 & \mathbf{- 0 . 8 3 3 4 7 1} & \mathbf{2 . 4 5 1 7 7 9} \\ \mathrm{H} & -\mathbf{5 . 7 8 5 2 9 5} & -0.206472 & \mathbf{1 . 1 5 4 3 8 2}\end{array}$

H $-4.522996-1242203-2844297$

$\begin{array}{llll}\text { H } & -4.522996 & -1.242203 & -2.844297 \\ C & -6.646292 & -0.192189 & -1.434105\end{array}$

$\begin{array}{llll}\text { C } & -6.646292 & -0.192189 & -1.434105\end{array}$

\begin{tabular}{llll} 
H & -7.036781 & -0.893499 & -2.185449 \\
\hline
\end{tabular}

$\begin{array}{llll}\text { H } & -4.376676 & -0.574220 & \mathbf{3 . 0 2 0 8 7 5}\end{array}$

$\begin{array}{llll}\text { H } & 0.420105 & -3.519604 & \mathbf{2 . 1 2 8 1 5 9}\end{array}$

$\begin{array}{llll}\text { H } & -6.543904 & 0.789082 & -1.923752\end{array}$

$\begin{array}{lllll}\text { H } & -7.389943 & -0.091462 & -0.632328\end{array}$

$\begin{array}{llll}\text { H } & -2.713356 & -0.054272 & 2.619650\end{array}$

$\begin{array}{llll}\text { H } & -\mathbf{3 . 0 6 5 0 7 9} & -\mathbf{1 . 7 7 0 3 7 7} & \mathbf{2 . 8 5 8 0 2 4}\end{array}$

$\begin{array}{llll}\text { F } & 1.708800 & -2.659291 & -1.655042\end{array}$

$\begin{array}{llll}C & 6.263626 & -1.543349 & -0.375420\end{array}$

H $6.808231-1.020500$

$\begin{array}{lllll}\text { H } & 6.704142 & \mathbf{- 2 . 5 4 6 4 5 5} & \mathbf{- 0 . 4 8 6 8 9 5}\end{array}$

$\begin{array}{llll}\text { H } & 6.436153 & -1.008017 & -1.321091\end{array}$

$\begin{array}{llll}\text { C } & 2.462761 & -1.011110 & 2.882312\end{array}$

$\begin{array}{llll}\text { H } & 1.467054 & -1.417939 & 3.095921\end{array}$

H $3.177271 \quad-1.402031 \quad 3.620430$

$\begin{array}{llll}\text { H } & 2.390285 & 0.077909 & 3.014470\end{array}$

$\begin{array}{llll}\text { C } & \mathbf{0 . 1 3 2 3 1 6} & \mathbf{2 . 7 0 6 1 4 2} & \mathbf{2 . 8 6 8 3 5 7}\end{array}$

$\begin{array}{llll}C & 0.570523 & 3.568758 & \mathbf{1 . 9 3 1 2 7 5}\end{array}$

$\begin{array}{llll}C & 0.571171 & 2.58758 & 1.931275\end{array}$

\begin{tabular}{llll} 
C & -2.711741 & 2.382976 & 0.656523 \\
\hline
\end{tabular}

\begin{tabular}{lllll} 
H & -2.518889 & 1.017343 & -0.973683 \\
\hline & -0.364935 & 0.891937 & -2.147910
\end{tabular}

$\begin{array}{lllll}\text { C } & -0.364935 & 0.891937 & -2.147910\end{array}$

$\begin{array}{lllll}\text { H } & \mathbf{1 . 1 9 4 9 5 5} & \mathbf{- 0 . 4 8 7 9 1 5} & \mathbf{- 1 . 7 0 9 3 8 8}\end{array}$

$\begin{array}{llll}C & 2.118335 & 1.448350 & -1.952311\end{array}$

$\begin{array}{lllll}C & -0.793868 & 2.164868 & -2.824826\end{array}$

$\begin{array}{llll}\text { H } & -0.214536 & 3.032331 & -2.493193\end{array}$

$\begin{array}{llll}\text { H } & -0.651192 & 2.056031 & -3.912922\end{array}$

$\begin{array}{lllll}\text { H } & -1.863680 & 2.355656 & -2.655278\end{array}$

$\begin{array}{lllll}\text { O } & 2.179059 & 2.437535 & -2.643683\end{array}$

\begin{tabular}{lllll}
\hline & 3.168509 & 0.970792 & -1.276415
\end{tabular}

$\begin{array}{llll}C & 4.351042 & 1.747858 & -1.296895\end{array}$

$\begin{array}{llll}\text { H } & \mathbf{5 . 0 7 8 0 1 3} & \mathbf{1 . 2 1 0 6 8 1} & \mathbf{- 0 . 6 7 7 9 1 2}\end{array}$

$\begin{array}{lllll}\text { H } & 4.161300 & 2.745303 & -0.873602\end{array}$

$\begin{array}{lllll}\text { H } & 4.735239 & 1.861181 & -2.321049\end{array}$

$\begin{array}{llll}\text { H } & -\mathbf{3 . 6 8 4 0 0 3} & \mathbf{1 . 9 4 6 0 9 1} & \mathbf{0 . 9 4 2 9 5 5}\end{array}$

$\begin{array}{llll}\text { H } & -\mathbf{2} .942198 & \mathbf{3 . 2 1 0 5 6 9} & \mathbf{- 0 . 0 4 0 7 5 2}\end{array}$

$\begin{array}{llll}\text { H } & -2.202239 & \mathbf{2 . 7 9 3 7 4 6} & \mathbf{1 . 5 3 5 2 2 6}\end{array}$

\begin{tabular}{llll}
\hline & -1.023166 & 0.037993 & -2.329240
\end{tabular}

\begin{tabular}{rrrrr}
\hline H & -1.023166 & 0.037993 & -2.329240 \\
\hline & 0.836724 & 4.597875 & 2.194144
\end{tabular}

\begin{tabular}{cccc} 
H & $\mathbf{0 . 8 3 6 7 2 4}$ & $\mathbf{4 . 5 9 7 8 7 5}$ & $\mathbf{2 . 1 9 4 1 4 4}$ \\
\hline
\end{tabular}

$\begin{array}{rrrr}\text { H } & 0.021896 & 3.008059 & 3.914783\end{array}$

sat_model_ts1_c / electronic energy: $\mathbf{- 2 4 3 6 . 6 1 0 9 0 7 2 0}$ a.u. / lowest freq: $-\mathbf{1 3 3 . 4 7} \mathrm{cm}-\mathbf{1}$

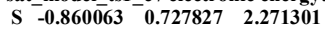

$\begin{array}{llll}C & -0.378613 & -1.155188 & -0.080026\end{array}$

$\begin{array}{lllll}\text { Ru } & -0.106573 & \mathbf{0 . 9 0 9 1 2 8} & \mathbf{0 . 1 0 2 0 2 1}\end{array}$

$\begin{array}{lrrr}C & -1.612110 & \mathbf{1 . 2 7 6 7 2 8} & \mathbf{- 0 . 9 2 1 4 5 0}\end{array}$

$\begin{array}{llll}C & 1.375834 & 0.913146 & -1.467833\end{array}$

$\begin{array}{llll}\mathrm{S} & \mathbf{0 . 6 3 3 4 5 5} & 3.109999 & \mathbf{0 . 7 3 9 6 9 2}\end{array}$

$\begin{array}{llll}\text { N } & 0.613660 & -2.077713 & -0.052752\end{array}$

$\begin{array}{llll}\text { N } & -1.537108 & -1.859301 & -0.142694\end{array}$

$\begin{array}{llll}C & \mathbf{2 . 0 1 5 6 6 8} & \mathbf{- 1 . 8 8 8 8 0 7} & \mathbf{0 . 0 8 7 6 3 7}\end{array}$

$\begin{array}{llll}C & 0.151883 & -3.468505 & -0.137484\end{array}$

$\begin{array}{llll}C & -1.361729 & -3.313501 & -0.210667\end{array}$

$\begin{array}{llll}C & -2.872012 & -1.376307 & -0.148119\end{array}$

$\begin{array}{llll}\text { H } & -1.787301 & -3.700034 & -1.148277\end{array}$

$\begin{array}{llll}\text { H } & -1.883385 & -3.798214 & 0.628066\end{array}$

$\begin{array}{lllll}\text { H } & 0.575298 & -3.952431 & -1.029024\end{array}$

$\begin{array}{llll}C & 4.826721 & -1.751020 & 0.239155\end{array}$

$\begin{array}{llll}C & \mathbf{2 . 8 2 5 4 1 2} & \mathbf{2} .037054 & \mathbf{- 1 . 0 4 1 8 9 1}\end{array}$

$\begin{array}{llll}C & \mathbf{2 . 6 3 2 5 8 6} & -\mathbf{1 . 7 3 2 3 7 2} & \mathbf{1 . 3 3 6 2 2 1}\end{array}$

$\begin{array}{llll}C & 4.030166 & -1.647647 & 1.380583\end{array}$

$\begin{array}{llll}C & 4.202402 & -1.961026 & -0.995488\end{array}$

$\begin{array}{llll}\text { H } & 4.511654 & -1.512113 & 2.352473\end{array}$

$\begin{array}{llll}\text { H } & 4.773078 & -2.062412 & -1.920370\end{array}$

$\begin{array}{llll}\text { C } & \mathbf{- 5 . 5 3 7 5 0 4} & \mathbf{- 0 . 4 7 2 3 5 0} & \mathbf{- 0 . 3 0 4 3 6 1}\end{array}$

$\begin{array}{llll}C & -3.493814 & -1.120246 & -1.373715\end{array}$

$\begin{array}{llll}C & -3.637220 & -1.278346 & 1.022631\end{array}$

$\begin{array}{llll}C & -3.637220 & -1.278346 & 1.022631 \\ C & -4.951010 & -0.802016 & 0.918804\end{array}$

$\begin{array}{llll}\text { C } & -4.791075 & -\mathbf{0 . 6 5 7 8 6 8} & -\mathbf{1 . 4 7 5 5 4 0}\end{array}$

$\begin{array}{llll}\text { F } & -2.778316 & -1.303217 & -2.492574\end{array}$

$\begin{array}{llll}\text { C } & -3.116709 & -1.750076 & 2.352130\end{array}$

$\begin{array}{llll}\text { H } & -5.539151 & -0.700308 & 1.834611\end{array}$

$\begin{array}{llll}\text { H } & -5.207741 & -0.447283 & -2.462088\end{array}$

$\begin{array}{lllll}\text { C } & \mathbf{- 6 . 9 3 7 4 5 0} & 0.075117 & -0.378597\end{array}$

$\begin{array}{llll}\text { H } & -7.507204 & -0.396337 & -1.192267\end{array}$

$\begin{array}{llll}\text { H } & -3.592603 & -2.710227 & 2.609146\end{array}$

H $-0.484827-4.028780-0.748948$

$\begin{array}{rrrr}\text { H } & 0.484827 & -4.028780 & 0.748948 \\ \text { H } & -6.915613 & 1.158084 & -0.578318\end{array}$ 


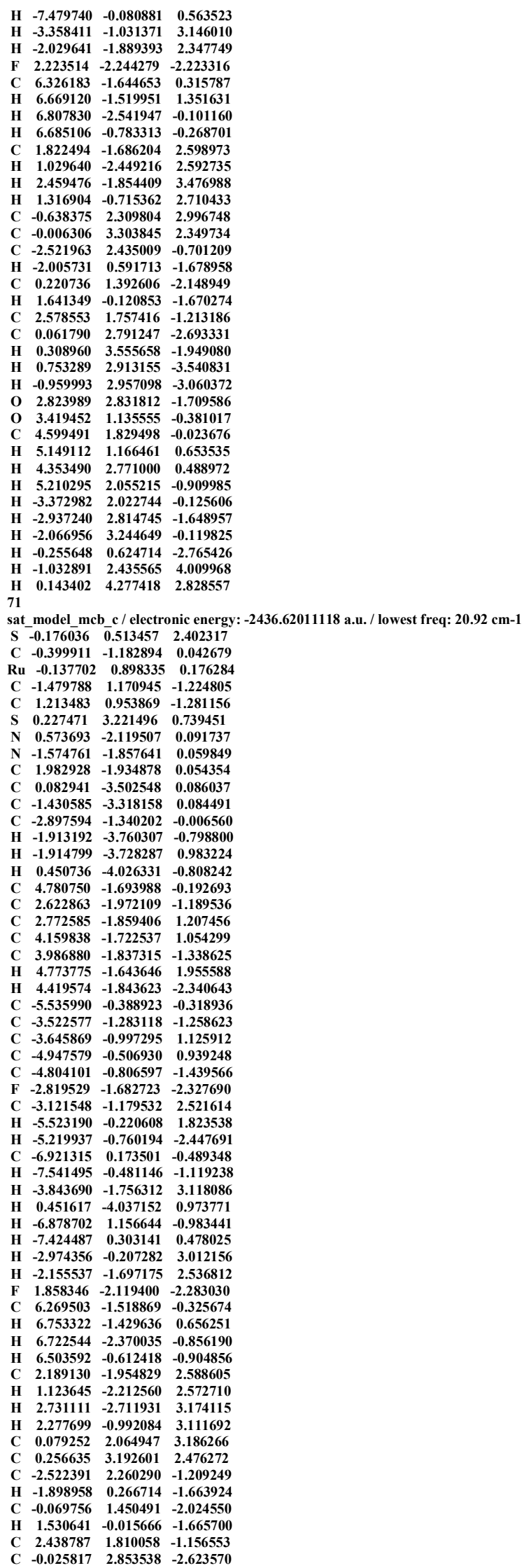


H $\quad 0.076866 \quad 3.633073-1.859129$ $\begin{array}{lllll}H & 0.830762 & \mathbf{2 . 9 4 0 8 6 2} & \mathbf{- 3 . 3 0 0 9 2}\end{array}$ $\begin{array}{llll}\text { H } & \mathbf{- 0 . 9 4 5 9 9 6} & \mathbf{3 . 0 4 1 4 7 2} & \mathbf{- 3 . 1 9 2 8 2 9}\end{array}$

$\begin{array}{lllll}0 & \mathbf{2 . 7 4 3 3 2 0} & \mathbf{2 . 7 8 3 6 5 1} & -1.801904\end{array}$

$\begin{array}{lllll}0 & 3.254312 & 1.267340 & -0.245653\end{array}$

C $4.5080631 .894066-0.047083$

$\begin{array}{llll}C & 4.508063 & 1.894066 & \mathbf{0 . 0 4 7 0 8 3}\end{array}$

$\begin{array}{llll}\text { H } & 4.375738 & 2.912924 & 0.34461\end{array}$

$\begin{array}{lllll}\text { H } & \mathbf{5 . 0 7 6 7 3 1} & \mathbf{1 . 9 4 4 1 6 5} & \mathbf{- 0 . 9 8 7 0 8}\end{array}$

H $\quad \mathbf{5 . 0 4 3 2 1 3} \quad \mathbf{1 . 2 7 7 4 3 4} \quad \mathbf{0 . 6 8 3 7 2 3}$

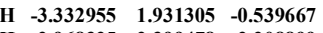

H $-2.968325 \quad 2.399478-2.208899$

$\begin{array}{llll}\text { H } & -2.137689 & 3.217541 & -0.840192\end{array}$

\begin{tabular}{cccc} 
H & $-\mathbf{0 . 1 8 5 9 4 1}$ & $\mathbf{0 . 7 0 5 0 5 1}$ & $-\mathbf{2 . 8 2 2 6 1 7}$ \\
\hline & $\mathbf{0 . 4 2 5 7 9 1}$ & $\mathbf{4} 148663$ & $\mathbf{2 . 9 8 2 4 5 3}$
\end{tabular}

$\begin{array}{llll}\text { H } & 0.091994 & 2.048068 & 4.280109\end{array}$

71

sat_model_ts2_c / electronic energy: $\mathbf{- 2 4 3 6 . 6 0 3 4 0 3 0 0}$ a.u. / lowest freq: $\mathbf{- 1 8 2 . 7 6} \mathbf{c m}-\mathbf{1}$

$\begin{array}{llll}C & 0.278856 & -1.301545 & -0.179841\end{array}$

$\begin{array}{lllll}\text { Ru } & \mathbf{0 . 1 3 2 8 3 7} & \mathbf{0 . 7 5 9 0 8 8} & \mathbf{0 . 2 0 5 3 7 6}\end{array}$

$\begin{array}{lllll}\text { C } & 0.609541 & 0.217434 & 2.232621\end{array}$

$\begin{array}{llll}\text { C } & -1.707230 & 0.870595 & 0.397558\end{array}$

$\begin{array}{lllll}\text { S } & 0.465474 & 3.115333 & 0.586071\end{array}$

$\begin{array}{llll}N & -0.670176 & -2.251853 & -0.316932\end{array}$

N $1.467069-1.948973-\mathbf{- 0 . 2 6 8 5 2 6}$

$\begin{array}{lll}C & -2.072480 & -2.066 \\ C & -0.153353 & -3.614\end{array}$

$\begin{array}{llll}\text { C } & -0.153353 & -3.614499 & -0.486812\end{array}$

$\begin{array}{llll}\text { C } & \mathbf{1 . 3 5 6 2 2 5} & \mathbf{- 3 . 4 0 1 3 3 9} & \mathbf{- 0 . 4 3 6 7 0 2}\end{array}$

$\begin{array}{llll}C & 2.769967 & -1.384869 & -0.225545\end{array}$

$\begin{array}{llll}\text { H } & 1.832702 & -3.913592 & 0.411854\end{array}$

$\begin{array}{llll}\text { H } & 1.864320 & -3.717723 & -1.359557\end{array}$

$\begin{array}{llll}\text { H } & -0.520601 & -4.261048 & 0.322743\end{array}$

$\begin{array}{llll}\text { C } & -4.854203 & -1.684576 & -0.212897\end{array}$

$\begin{array}{llll}C & -2801543 & -2.363872 & 0.824452\end{array}$

C $-2.749125-1.612335-1.467564$

$\begin{array}{llll}C & -2.749125 & -1.612335 & -1.467564 \\ C & -4.132711 & -1.421014 & -1.381493\end{array}$

$\begin{array}{llll}C & -4.132711 & -1.421014 & -1.381493\end{array}$

$\begin{array}{llll}C & -4.168766 & -2.174031 & 0.906570\end{array}$

$\begin{array}{lllll}\text { H } & -4.663873 & -1.046482 & -2.259974\end{array}$

$\begin{array}{lllll}\text { H } & -4.684529 & -2.398684 & 1.841930\end{array}$

$\begin{array}{llll}\text { C } & 5.370923 & \mathbf{- 0 . 3 1 1 5 7 8} & \mathbf{- 0 . 0 4 5 4 7 0}\end{array}$

$\begin{array}{llll}\text { C } & 3.441494 & \mathbf{- 1 . 2 8 5 9 2 6} & \mathbf{0 . 9 9 5 4 4 2}\end{array}$

$\begin{array}{llll}C & 3.461895 & -1.041797 & -1.398616\end{array}$

$\begin{array}{llll}\text { C } & 4.742664 & -0.493073 & -1.280669\end{array}$

$\begin{array}{llll}C & 4.708109 & -0.740463 & 1.109138\end{array}$

$\begin{array}{llll}\text { F } & \mathbf{2 . 8 4 9 9 8 9} & \mathbf{- 1 . 7 6 3 8 9 5} & \mathbf{2 . 0 9 6 1 6 9}\end{array}$

C $2.886031-1.322771 \quad-2.758740$

H $\quad \mathbf{5 . 2 7 1 1 8 3}-\mathbf{- 0 . 2 0 3 8 6 5}-\mathbf{- 2 . 1 9 2 8 1 3}$

$\begin{array}{llll}\text { H } & \mathbf{5 . 1 6 8 7 9 2} & \mathbf{- 0 . 6 6 9 2 7 0} & \mathbf{2 . 0 9 6 1 0 7}\end{array}$

$\begin{array}{llll}\text { C } & \mathbf{6 . 7 2 7 6 0 4} & \mathbf{0 . 3 3 2 6 7 6} & \mathbf{0 . 0 4 9 2 7 8}\end{array}$

$\begin{array}{llll}\text { H } & 7.319964 & \mathbf{- 0 . 0 9 6 1 0 5} & \mathbf{0 . 8 6 9 6 6 6}\end{array}$

$\begin{array}{llll}\text { H } & 3.335364 & -2.243300 & -3.166335\end{array}$

$\begin{array}{llll}\text { H } & -\mathbf{0 . 4 9 9 1 8 9} & \mathbf{- 4 . 0 3 1 8 4 8} & \mathbf{- 1 . 4 4 4 3 3 3}\end{array}$

\begin{tabular}{lllll} 
H & 6.623153 & 1.411631 & 0.245424 \\
\hline
\end{tabular}

$\begin{array}{llll}\text { H } & 7.290883 & 0.218707 & -0.886959\end{array}$

$\begin{array}{llll}\text { H } & \mathbf{3 . 1 0 8 3 4 7} & \mathbf{- 0 . 5 0 4 2 1 6} & \mathbf{- 3 . 4 5 5 7 4 1}\end{array}$

$\begin{array}{llll}\text { H } & \mathbf{1 . 7 9 7 9 1 1} & \mathbf{- 1 . 4 5 3 6 6 7} & \mathbf{- 2 . 7 2 9 0 7 3}\end{array}$

$\begin{array}{llll}\text { F } & -2.133512 & -2.802894 & 1.898742\end{array}$

$\begin{array}{llll}\text { C } & \mathbf{- 6 . 3 4 1 5 4 2} & \mathbf{- 1 . 4 6 4 4 9 7} & \mathbf{- 0 . 1 4 5 6 2 7}\end{array}$

H $\quad-6.723445-1.010109-1.069300$

$\begin{array}{llll}\text { H } & -6.868962 & -2.418020 & 0.010182\end{array}$

$\begin{array}{llll}\text { H } & -6.602731 & -0.805505 & 0.695671\end{array}$

$\begin{array}{llll}C & -1.988578 & -1.283379 & -2.719251\end{array}$

$\begin{array}{llll}\text { H } & -1.249916 & -2.062787 & -2.957769\end{array}$

$\begin{array}{llll}\text { H } & -\mathbf{2 . 6 6 8 3 3 5} & -\mathbf{1 . 1 7 3 0 0 0} & \mathbf{- 3 . 5 7 3 8 8 8}\end{array}$

$\begin{array}{llll}\text { H } & -1.430513 & -0.342382 & -2.592104\end{array}$

$\begin{array}{llll}C & 0.992899 & 2.914242 & -2.065474\end{array}$

$\begin{array}{lllll}C & 0.830350 & 3.723962 & -1.005258\end{array}$

$\begin{array}{llll}C & \mathbf{1 . 6 4 6 8 2 6} & \mathbf{1 . 0 3 8 5 7 1} & \mathbf{2 . 9 6 4 4 8 5}\end{array}$

$\begin{array}{lllll}\text { H } & 0.856114 & \mathbf{- 0 . 8 4 5 9 6 1} & \mathbf{2 . 2 1 8 4 2 3}\end{array}$

$\begin{array}{lllll}\text { C } & -0.793127 & 0.479517 & \mathbf{2 . 3 4 7 9 6 9}\end{array}$

$\begin{array}{lllll}\text { H } & -2.424161 & 0.051470 & 0.530775\end{array}$

$\begin{array}{lllll}\text { C } & -2.473461 & 2.150050 & 0.190371\end{array}$

$\begin{array}{llll}C & -2.473461 & 2.150050 & 0.190371\end{array}$

\begin{tabular}{llll} 
C & -1.314176 & 1.612575 & 3.193774 \\
\hline
\end{tabular}

$\begin{array}{lllll}\mathrm{H} & \mathbf{- 0 . 8 2 4 5 6 2} & 2.565417 & 2.956842\end{array}$

$\begin{array}{rrrr}H & -2.392788 & 1.751292 & \mathbf{3 . 0 6 7 1 0 8}\end{array}$

$\begin{array}{llll}\text { H } & -1.103652 & 1.370905 & 4.248599\end{array}$

$\begin{array}{lllll}\text { O } & -\mathbf{3 . 1 5 7 4 5 1} & \mathbf{2 . 6 9 0 8 0 6} & \mathbf{1 . 0 2 7 5 8 8}\end{array}$

$\begin{array}{lllll}O & -2.398413 & 2.556113 & -1.072098\end{array}$

$\begin{array}{lllll}\text { C } & -\mathbf{3 . 0 7 8 8 8 2} & \mathbf{3 . 7 5 9 0 1 4} & \mathbf{- 1 . 3 9 7 4 5 1}\end{array}$

$\begin{array}{llll}\text { H } & -2.663326 & 4.598053 & -0.821701\end{array}$

H $-4.154263 \quad 3.673015-1.186171$

H $-2.014094 \quad 3.921581 \quad-2.467596$

\begin{tabular}{llll} 
H & -2.914094 & 3.921581 & -2.467596 \\
\hline & 2.626562 & 0.957795 & 2.473405
\end{tabular}

\begin{tabular}{cccc} 
H & $\mathbf{2 . 6 2 6 5 6 2}$ & $\mathbf{0 . 9 5 7 7 9 5}$ & $\mathbf{2 . 4 7 3 4 0 5}$ \\
\hline & 1.759146 & $\mathbf{0 . 6 5 0 2 6 7}$ & 3.991631
\end{tabular}

$\begin{array}{lllll} & & 1.759146 & 0.650267 & 3.99163\end{array}$

H $1.391865 \quad 2.102907 \quad 3.019343$

$\begin{array}{llll}\text { H } & -\mathbf{1 . 4 1 2 3 4 0} & \mathbf{- 0 . 4 2 0 9 8 2} & \mathbf{2 . 3 6 8 7 0 8}\end{array}$

$\begin{array}{llll}\text { H } & \mathbf{1 . 2 3 1 5 6 9} & \mathbf{3 . 3 0 2 9 4 7} & \mathbf{- 3 . 0 6 0 3 8 0}\end{array}$

H $\quad 0.942511 \quad 4.808365 \quad-1.108907$

71

sat_model_pc2_c / electronic energy: -2436.61584799 a.u. / lowest freq: $16.17 \mathrm{~cm}-1$

S $-0.538309-1.784736-1.454798$

$\begin{array}{llll}C & 0.118647 & -1.191855 & -0.389087\end{array}$

$\begin{array}{llll}\text { Ru } & 0.203149 & 0.655141 & 0.570113\end{array}$ 
$\begin{array}{llll}C & 0.766729 & -0.682793 & 2.419885\end{array}$ $\begin{array}{llll}C & -1.564958 & 1.135755 & 0.58024\end{array}$ $\begin{array}{llll}\text { S } & 1.417899 & 2.387849 & \mathbf{1 . 5 5 6 6 0 8}\end{array}$ $\begin{array}{llll}N & -0.939261 & -1.991775 & -0.615317\end{array}$ $\begin{array}{llll}N & 1.214036 & -1.861728 & -0.803290\end{array}$ C $-2.306857-1.676604-0.430944$ $\begin{array}{llll}C & -2.306857 & -1.676604 & -0.430944\end{array}$ $\begin{array}{llll}C & -0.584549 & -3.303248 & -1.173121\end{array}$

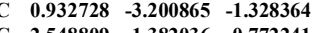
$\begin{array}{llll}C & 2.548809 & -1.382036 & -0.772241\end{array}$

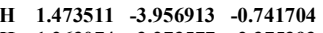
$\begin{array}{lllll}\text { H } & \mathbf{1 . 2 6 3 0 7 4} & -\mathbf{3 . 2 7 3 5 7 7} & \mathbf{- 2 . 3 7 5 3 9 3}\end{array}$ $\begin{array}{llll}\text { H } & -0.890377 & -4.098254 & -0.478322\end{array}$ $\begin{array}{llll}\text { C } & -\mathbf{5 . 0 2 7 8 0 4} & -\mathbf{1 . 1 2 6 8 7 5} & \mathbf{- 0 . 0 1 5 0 6 7}\end{array}$ $\begin{array}{llll}C & -2.970875 & -2.124776 & 0.710426\end{array}$ C $-3.024033-0.986037-1.415710$ C $-4.375972-0.716841-1.181176$ $\begin{array}{llll}C & -4.375972 & -0.716841 & -1.181176\end{array}$ $\begin{array}{llll}C & -4.306105 & -1.852104 & 0.942381\end{array}$ $\begin{array}{llll}\mathrm{C} & -4.935202 & -0.156209 & -1.933908\end{array}$ $\begin{array}{llll}\text { H } & -4.772580 & -2.210715 & 1.861796\end{array}$ $\begin{array}{llll}C & 5.192919 & -0.436811 & -0.580864\end{array}$ $\begin{array}{llll}C & 3.362146 & -1.697883 & 0.318246\end{array}$ $\begin{array}{llll}C & 3.106508 & -0.670129 & -1.846141\end{array}$ $\begin{array}{llll}C & 4.414403 & -0.193000 & -1.716215\end{array}$ $\begin{array}{llll}\text { C } & 4.657314 & -1.228027 & 0.441193\end{array}$ $\begin{array}{llll}\text { F } & 2.872977 & -\mathbf{2 . 5 0 3 0 7 9} & \mathbf{1 . 2 6 7 9 5 5}\end{array}$ $\begin{array}{llll}C & \mathbf{2 . 3 6 0 6 1 4} & \mathbf{- 0 . 4 9 1 7 1 2} & \mathbf{- 3 . 1 3 8 8 0 0}\end{array}$ $\begin{array}{llll}\text { H } & 4.842692 & 0.383841 & -2.539973\end{array}$

$\begin{array}{lllll}\text { H } & 5.235882 & -1.490397 & 1.328853\end{array}$

$\begin{array}{llll}\text { C } & 6.574919 & \mathbf{0 . 1 4 3 9 8 5} & \mathbf{- 0 . 4 5 1 3 7 7}\end{array}$ $\begin{array}{llll}\text { H } & \mathbf{7 . 2 2 6 4 0 6} & \mathbf{- 0 . 4 9 7 8 2 0} & \mathbf{0 . 1 5 7 7 5 6}\end{array}$ H $2.723456-1.228018 \quad-3.874713$ H $\begin{array}{rrrr}-1.103806 & \mathbf{- 3 . 4 6 2 4 3 4} & \mathbf{- 2 . 1 2 9 3 5 0}\end{array}$ H $\quad 6.526648 \quad 1.128750 \quad 0.040030$

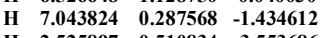
$\begin{array}{lllll}\text { H } & \mathbf{2 . 5 2 5 8 9 7} & \mathbf{0 . 5 1 0 8 3 4} & \mathbf{- 3 . 5 5 3 6 8 6}\end{array}$ $\begin{array}{llll}H & 1.280103 & -0.630645 & -3.016314\end{array}$ $\begin{array}{llll}\text { F } & -\mathbf{2 . 2 7 7 8 8 0} & \mathbf{- 2 . 8 3 2 9 4 1} & \mathbf{1 . 6 1 1 7 7 9} \\ \text { C } & -6.483916 & \mathbf{- 0 . 8 2 1 9 0 6} & \mathbf{0 . 2 1 5 5 6 0}\end{array}$ $\begin{array}{lllll}\text { H } & -6.880818 & -0.149930 & -0.556927\end{array}$ $\begin{array}{lllll}\text { H } & -7.082434 & -1.746067 & 0.204547\end{array}$

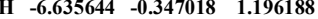
$\begin{array}{llll}C & -2.328064 & -0.541238 & -2.669451\end{array}$ $\begin{array}{llll}\text { H } & -\mathbf{1 . 8 3 4 3 6 1} & -\mathbf{1 . 3 8 7 0 6 7} & \mathbf{- 3 . 1 7 2 4 3 0}\end{array}$

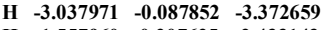
\begin{tabular}{lllll}
\hline & -1.557960 & 0.207625 & -2.433143
\end{tabular}

$\begin{array}{lllll}C & 1.272839 & 3.298414 & \mathbf{- 0 . 9 9 3 6 7 9}\end{array}$

$\begin{array}{lllll}\text { C } & 1.648072 & 3.550284 & 0.272522\end{array}$

$\begin{array}{llll}C & 1.979521 & -0.292790 & 3.221996\end{array}$ $\begin{array}{llll}\text { H } & 0.853517 & -1.661547 & 1.943837\end{array}$ $\begin{array}{llll}C & -0.512260 & -0.207815 & 2.627091\end{array}$ $\begin{array}{llll}\text { H } & -2.344026 & 0.703237 & 1.233095\end{array}$ $\begin{array}{llll}\text { C } & -2.095131 & \mathbf{2 . 3 4 7 6 7 6} & -0.117417\end{array}$ $\begin{array}{llll}C & -0.906259 & 0.811856 & 3.660006\end{array}$ \begin{tabular}{llll}
$C$ & -0.906259 & 0.811856 & 3.660006 \\
\hline & -0.126013 & 1.562641 & 3.833794
\end{tabular} \begin{tabular}{llll}
$\mathrm{H}$ & -0.126013 & 1.562641 & $\mathbf{3 . 8 3 3 7 9 4}$ \\
\hline
\end{tabular} \begin{tabular}{rrrr}
\hline & -1.821097 & 1.343701 & $\mathbf{3 . 3 6 1 6 9 3}$ \\
\hline
\end{tabular} $\begin{array}{lllll}\text { H } & -1.119767 & \mathbf{0 . 3 0 2 6 4 3} & 4.614959 \\ \text { O } & -1.969535 & \mathbf{3 . 4 6 8 6 7 6} & \mathbf{0 . 3 1 7 8 6 2}\end{array}$ $\begin{array}{lllll}\text { O } & -2.805661 & 2.064498 & -1.207973\end{array}$ $\begin{array}{lllll}C & -3.288103 & 3.163724 & -1.962412\end{array}$ $\begin{array}{lllll}\text { H } & -2.447332 & 3.754818 & -2.354364\end{array}$

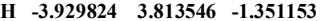
$\begin{array}{llll}\text { H } & -3.863227 & \mathbf{2 . 7 3 6 7 0 5} & \mathbf{- 2 . 7 9 1 4 4 4}\end{array}$ $\begin{array}{llll}\text { H } & \mathbf{2 . 8 8 8 2 6 7} & \mathbf{- 0 . 2 6 2 5 8 1} & \mathbf{2 . 6 0 6 9 6 4}\end{array}$ $\begin{array}{ccccc}\text { H } & 2.888267 & -0.262581 & 2.606964 \\ \text { H } & \mathbf{2 . 1 3 3 6 6 0} & \mathbf{- 1 . 0 5 6 8 4 8} & \mathbf{4 . 0 0 2 8 6 1}\end{array}$ $\begin{array}{rrrr}\text { H } & 2.133660 & -1.056848 & 4.002861 \\ \text { H } & 1.871953 & \mathbf{0 . 6 8 1 2 3 6} & \mathbf{3 . 7 1 3 5 7 8}\end{array}$ $\begin{array}{crrrr}\text { H } & \mathbf{1 . 8 7 1 9 5 3} & \mathbf{0 . 6 8 1 2 3 6} & \mathbf{3 . 7 1 3 5 7 8} \\ \text { H } & \mathbf{- 1 . 3 2 4 0 1 1} & \mathbf{- 0 . 8 6 0 7 9 7} & \mathbf{2 . 3 0 2 2 3 6}\end{array}$ $\begin{array}{lllll}\text { H } & 1.420878 & 4.034822 & -1.789952\end{array}$ $\begin{array}{llll}\text { H } & 2.113346 & 4.500410 & 0.552516\end{array}$ 59

作 $\begin{array}{llll}S & 0.971826 & -1.090987 & 2.351225\end{array}$

$\begin{array}{llll}C & 0.004101 & 1.042128 & 0.214726\end{array}$

$\begin{array}{llll}\text { Ru } & 0.248244 & -0.986908 & 0.171411\end{array}$ $\begin{array}{llll}C & -1.394242 & -1.744541 & 0.342234\end{array}$ $\begin{array}{llll}C & 1.942441 & -2.407633 & -0.391189\end{array}$ $\begin{array}{llll}\text { N } & -1.116437 & 1.774244 & 0.290278\end{array}$ N $\begin{array}{cccc}1.049524 & 1.877286 & 0.090570\end{array}$ $\begin{array}{llll}\text { C } & -2.445088 & 1.285730 & 0.365946\end{array}$ $\begin{array}{llll}\text { C } & -\mathbf{0 . 8 5 0 7 7 7} & \mathbf{3 . 2 2 0 0 3 0} & \mathbf{0 . 2 5 0 8 4 8}\end{array}$

C $\quad 0.636022 \quad 3.272540 \quad-0.103943$

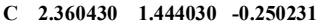

$\begin{array}{llll}\text { H } & 0.808818 & 3.563708 & -1.151510\end{array}$

$\begin{array}{cccc}\text { H } & \mathbf{0 . 8 0 8 8 1 8} & 3.563708 & -1.151510 \\ \text { H } & \mathbf{1 . 2 1 0 4 2 8} & \mathbf{3 . 9 4 5 0 8 6} & \mathbf{0 . 5 4 6 3 3 1}\end{array}$

$\begin{array}{llll}\text { H } & -1.486018 & \mathbf{3 . 7 0 5 3 3 3} & \mathbf{- 0 . 5 0 1 2 4 7}\end{array}$

$\begin{array}{llll}C & -5.088623 & 0.335392 & 0.373681\end{array}$

$\begin{array}{llll}C & -3.216444 & 1.271652 & -0.796753\end{array}$

$\begin{array}{llll}C & -3.012552 & 0.848016 & 1.570163\end{array}$

$\begin{array}{llll}C & -4.326134 & 0.369580 & 1.545901\end{array}$

$\begin{array}{llll}\text { C } & -4.521200 & 0.812490 & \mathbf{- 0 . 8 1 2 2 1 4}\end{array}$

$\begin{array}{lllll}\text { H } & -4.769411 & 0.010032 & 2.477916\end{array}$

$\begin{array}{lllll}\text { H } & -\mathbf{5 . 0 7 7 7 4 4} & 0.818087 & -1.751158\end{array}$

$\begin{array}{llll}C & 4.899069 & 0.540049 & -1.036647\end{array}$

$\begin{array}{llll}C & 4.899069 & 0.540049 & -1.036647 \\ \text { C } & \mathbf{2 . 6 2 8 6 0 2} & \mathbf{1 . 0 8 7 2 8 0} & -1.572951\end{array}$ 
$\begin{array}{llll}C & 3.393466 & 1.396794 & 0.697486\end{array}$ $\begin{array}{llll}\text { C } & 4.644199 & 0.932216 & 0.28256\end{array}$

$\begin{array}{llll}\text { C } & 3.871623 & 0.637244 & -1.979696\end{array}$

$\begin{array}{llll}\text { F } & \mathbf{1 . 6 3 7 9 7 6} & \mathbf{1 . 1 7 5 2 8 7} & \mathbf{- 2 . 4 6 4 4 4 9}\end{array}$

$\begin{array}{llll}\text { C } & 3.144652 & 1.818040 & 2.116834\end{array}$

$\begin{array}{llll}\text { H } & 5.450780 & 0.876897 & 1.018044\end{array}$

$\begin{array}{llll}\text { H } & 4.021014 & \mathbf{0 . 3 5 5 8 7 9} & \mathbf{- 3 . 0 2 3 4 0 3}\end{array}$

$\begin{array}{llll}C & 6.245630 & -0.004269 & -1.430487\end{array}$

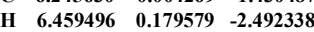

$\begin{array}{llll}\text { H } & 4.081609 & 1.840639 & 2.68832\end{array}$

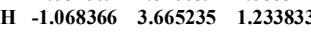

H $6.274744-1.093894-1.270002$

$\begin{array}{llll}\text { H } & 7.050349 & \mathbf{0 . 4 3 9 9 2 4} & \mathbf{- 0 . 8 2 8 3 3 8}\end{array}$

$\begin{array}{llll}\text { H } & \mathbf{2 . 4 5 2 5 4 6} & \mathbf{1 . 1 0 9 5 0 3} & \mathbf{2 . 5 9 8 9 8 6}\end{array}$

$\begin{array}{lllll}\text { H } & 2.684582 & 2.816181 & 2.166612\end{array}$

$\begin{array}{llll}\text { F } & -2.660123 & 1.704043 & -1.932735\end{array}$

$\begin{array}{llll}C & -6.482965 & -0.229934 & 0.366449\end{array}$

$\begin{array}{llll}\text { H } & -6.897181 & -0.299577 & 1.381190\end{array}$

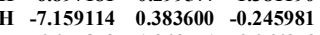

$\begin{array}{llll}\text { H } & -6.477853 & -1.243551 & -0.064972\end{array}$

$\begin{array}{llll}\text { C } & -2.203234 & 0.855577 & 2.834197\end{array}$

H $\quad-\mathbf{1 . 6 5 7 5 5 5} \quad \mathbf{1 . 8 0 2 1 0 6} \quad \mathbf{2 . 9 5 8 6 9 2}$

H $\quad \begin{array}{llll}-2.842526 & 0.703084 & 3.713123\end{array}$

$\begin{array}{llll}\text { H } & -1.452251 & 0.050695 & 2.811061\end{array}$

$\begin{array}{llll}C & 2.324679 & -2.197455 & \mathbf{2 . 2 8 5 7 8 5}\end{array}$

$\begin{array}{llll}C & 2.729089 & -2.769706 & 1.13895\end{array}$

$\begin{array}{llll}\text { H } & -1.941744 & -2.232142 & 1.15408\end{array}$

C $-\mathbf{- 1 . 8 2 0 1 4 8}-\mathbf{- 1 . 6 8 5 4 7 4}-\mathbf{- 1 . 0 4 7 0 0 8}$

$\begin{array}{llll}0 & -0.985084 & -1.236394 & -1.847040\end{array}$

$\begin{array}{llll}0 & \mathbf{- 3 . 0 3 3 4 5 2} & \mathbf{- 2 . 0 4 8 3 5 2} & \mathbf{- 1 . 4 0 0 8 3 6}\end{array}$

$\begin{array}{lllll}C & -3.399708 & -1.851930 & -2.766194\end{array}$

$\begin{array}{lllll}\text { H } & -2.801945 & -2.500871 & -3.420331\end{array}$

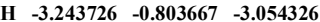

H $-4.460023 \quad-2.113909-2.837356$

$\begin{array}{lllll}\text { H } & \mathbf{2 . 8 2 4 1 6 7} & \mathbf{- 2 . 4 0 4 9 7 9} & \mathbf{3 . 2 3 7 1 4 6}\end{array}$

$\begin{array}{llll} & 3.559238 & -3.480672 \quad 1.106820\end{array}$

71

sat_model_ts_PTR_c / electronic energy: $\mathbf{- 2 4 3 6 . 6 0 7 9 8 5 8 3}$ a.u. / lowest freq: $\mathbf{- 1 4 4 . 2 8} \mathbf{c m - 1}$

$\begin{array}{lllll}\mathrm{Ru} & -\mathbf{0 . 2 1 8 7 3 7} & \mathbf{0 . 3 9 6 0 1 4} & \mathbf{0 . 6 5 2 0 7 9}\end{array}$

$\begin{array}{llll}S & 0.263205 & -0.640991 & 2.644890\end{array}$

$\begin{array}{llll}\text { C } & \mathbf{- 0 . 7 4 4 8 4 1} & \mathbf{0 . 0 5 5 0 4 8} & \mathbf{3 . 8 3 2 7 9 5}\end{array}$

$\begin{array}{llll}\text { C } & -1.640498 & 1.037611 & 3.500005\end{array}$

$\begin{array}{llll}\text { S } & -1.760168 & 1.642493 & 1.912838\end{array}$

$\begin{array}{llll}C & 0.494971 & -1.255235 & -0.368098\end{array}$

(156273 -0.757739

N $1.700500-1.764438-0.678911$

$\begin{array}{llll}C & 0.123846 & -\mathbf{3 . 3 4 1 6 3 3} & -\mathbf{1 . 4 1 3 4 8 4}\end{array}$

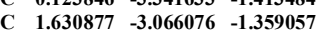

$\begin{array}{llll}C & -1.824706 & -1.906678 & -0.675676\end{array}$

$\begin{array}{llll}\text { C } & -2.454007 & -1.278486 & -1.757742\end{array}$

$\begin{array}{llll}C & -2.592790 & -2.256900 & 0.444987\end{array}$

$\begin{array}{llll}C & -3.786540 & -0.922454 & -1.736283\end{array}$

$\begin{array}{llll}C & \mathbf{- 3 . 9 4 3 5 0 9} & \mathbf{- 1 . 8 8 5 5 8 0} & \mathbf{0 . 4 6 3 4 4 6}\end{array}$

$\begin{array}{llll}C & -3.94509 & -1.885580 & 0.463446\end{array}$

$\begin{array}{llll}C & -4.549052 & -1.210057 & -0.597164\end{array}$

H $-4.213301-0.409598-2.59116$

$\begin{array}{llll}\text { H } & -4.539617 & -2.136959 & 1.344664\end{array}$

$\begin{array}{llll}\text { C } & \mathbf{- 5 . 9 8 8 3 9 2} & \mathbf{- 0 . 7 7 6 3 3 2} & -\mathbf{0 . 5 2 7 7 8 0}\end{array}$

$\begin{array}{llll}\text { F } & -1.722986 & -1.021118 & -2.845445\end{array}$

$\begin{array}{llll}C & -2.014394 & -3.038412 & 1.591523\end{array}$

$\begin{array}{llll}\text { H } & -2.183275 & -2.515490 & \mathbf{2 . 5 4 3 4 7 9}\end{array}$

$\begin{array}{llll}\text { H } & -2.504265 & -4.021885 & 1.659201\end{array}$

$\begin{array}{llll}\text { H } & -0.934753 & -3.193127 & 1.480513\end{array}$

$\begin{array}{llll}C & 2.950058 & -1.110661 & -0.534133\end{array}$

$\begin{array}{llll}C & 3.446760 & -0.381341 & -1.620115\end{array}$

$\begin{array}{llll}C & 3.724735 & -1.199503 & 0.628334\end{array}$

$\begin{array}{llll}C & 4.631885 & 0.323553 & -1.566743\end{array}$

$\begin{array}{llrr}C & 4.928595 & -0.482583 & 0.679626\end{array}$

$\begin{array}{llll}C & \mathbf{5 . 3 9 0 4 2 0} & \mathbf{0 . 2 8 7 5 8 2} & \mathbf{- 0 . 3 8 8 0 7 0}\end{array}$

$\begin{array}{llll}\text { H } & 4.954004 & 0.893975 & -2.439885\end{array}$

$\begin{array}{llll}\text { H } & \mathbf{5 . 5 2 4 4 3 6} & \mathbf{- 0 . 5 3 4 4 8 8} & \mathbf{1 . 5 9 4 7 1 0}\end{array}$

$\begin{array}{llll}C & 6.679607 & 1.059064 & -0.300771\end{array}$

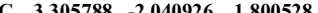

C $3.166961-1.418817 \quad 2.696327$

\begin{tabular}{llll} 
H & 3.166961 & -1.418817 & 2.696327 \\
\hline
\end{tabular}

$\begin{array}{llll}\text { H } & 2.361192 & -2.566695 & 1.616534\end{array}$

$\begin{array}{llll}\text { H } & 4.083753 & -2.784661 & \mathbf{2 . 0 2 8 6 2 7}\end{array}$

$\begin{array}{lllll}F & 2.723538 & -0.360215 & -2.746676\end{array}$

$\begin{array}{llll}C & 1.353636 & 1.651711 & 0.578643\end{array}$

$\begin{array}{llll}\text { H } & 2.299514 & 1.103371 & 0.695528\end{array}$

$\begin{array}{llll}\text { C } & -\mathbf{0 . 5 1 9 6 5 6} & \mathbf{1 . 4 4 2 3 0 1} & \mathbf{- 1 . 1 5 4 8 9 6}\end{array}$

$\begin{array}{llll}C & 0.973906 & 1.867453 & -0.910500\end{array}$

$\begin{array}{lllll}\text { H } & -0.622444 & 0.829419 & -2.054760\end{array}$

$\begin{array}{llll}\text { H } & 1.557818 & 1.158263 & -1.502438\end{array}$

$\begin{array}{lll}1.557818 & 1.158263 & -1.502438\end{array}$

$\begin{array}{llll}\text { C } 1.325473 & 3.265639 & -1.436756\end{array}$

$\begin{array}{lllll}\text { H } & -6.519441 & -1.271196 & 0.296482\end{array}$

$\begin{array}{lllll}\text { H } & -6.516745 & -0.996768 & -1.466637\end{array}$

$\begin{array}{lllll}\text { H } & -6.051391 & 0.311119 & -0.364041\end{array}$

$\begin{array}{llll}\text { H } & 7.389311 & 0.725188 & -1.072801\end{array}$

$\begin{array}{lllll}\text { H } & 7.157013 & 0.935204 & 0.680259\end{array}$

$\begin{array}{llll}\text { H } & 6.502843 & \mathbf{2 . 1 3 2 9 0 6} & \mathbf{- 0 . 4 6 3 6 9 8}\end{array}$

\begin{tabular}{lllll}
\hline & 0.761699 & 4.053984 & -0.925729
\end{tabular}

$\begin{array}{lllll}\text { H } & \mathbf{1 . 0 9 1 9 6 7} & \mathbf{3 . 3 2 0 4 6 7} & \mathbf{- 2 . 5 1 1 2 1 3}\end{array}$

$\begin{array}{llll}\text { H } & \mathbf{2 . 4 0 2 9 6 4} & 3.452552 & -1.307610\end{array}$

$\begin{array}{llll}\text { C } & \mathbf{1 . 3 4 1 3 7 6} & \mathbf{2 . 8 7 2 0 2 8} & \mathbf{1 . 4 7 4 4 0 5}\end{array}$ 


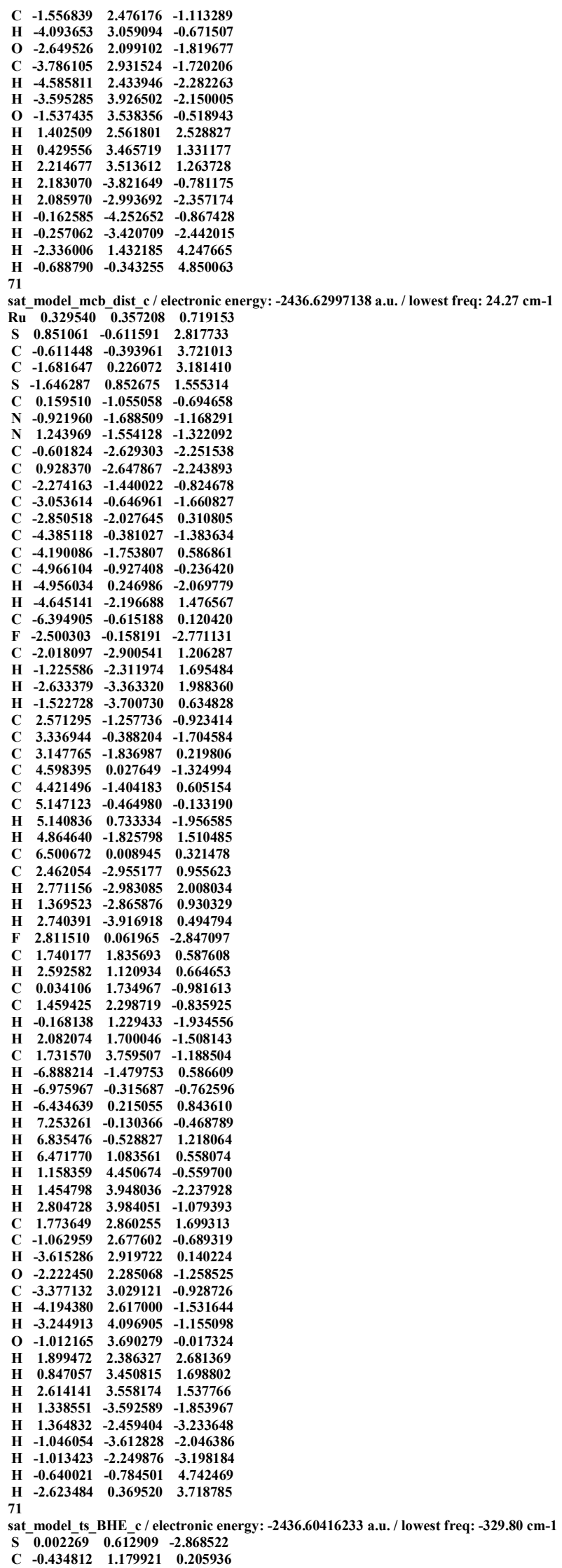


$\begin{array}{llll}\text { Ru } & 0.119690 & -0.524487 & -0.775041\end{array}$

$\begin{array}{lrrr}\text { C } & -0.933819 & -2.263950 & -1.376680\end{array}$

$\begin{array}{llll}\text { C } & -0.039889 & -1.863812 & 0.921667\end{array}$

$\begin{array}{llll}C & 2.253265 & -0.966757 & -1.323181\end{array}$

$\begin{array}{lllll}\text { N } & \mathbf{0 . 5 0 4 7 6 9} & \mathbf{2 . 0 7 3 1 8 0} & \mathbf{0 . 5 8 1 3 7 8}\end{array}$

$\begin{array}{rrrrr}N & -1.639288 & 1.722263 & 0.47928 \\ C & 1.887045 & 1.785285 & 0.721577\end{array}$

$\begin{array}{llll}\text { C } & 1.887045 & 1.785285 & 0.721577\end{array}$

$\begin{array}{lllll}C & -0.049581 & 3.252204 & 1.252612\end{array}$

$\begin{array}{llll}\text { C } & -1.547259 & 3.086726 & 1.016923\end{array}$

$\begin{array}{llll}C & -2.920410 & 1.114253 & 0.421999\end{array}$

$\begin{array}{llll}C & 4.584027 & 1.127566 & 1.105809\end{array}$

$\begin{array}{llll}C & 2.295544 & 1.012984 & 1.808709\end{array}$

$\begin{array}{lllll}\text { C } & 2.843155 & 2.284295 & -0.170908\end{array}$

$\begin{array}{llll}\text { C } & 4.181109 & 1.941092 & 0.041177\end{array}$

$\begin{array}{llll}\text { C } & 3.619803 & 0.669773 & 2.009739\end{array}$

$\begin{array}{cccc}\text { H } & 4.933875 & \mathbf{2 . 3 0 7 6 2 9} & -\mathbf{0 . 6 6 1 6 5 4}\end{array}$

$\begin{array}{llll}\text { H } & \mathbf{3 . 8 8 4 0 1 4} & \mathbf{0 . 0 4 4 7 4 0} & \mathbf{2 . 8 6 4 2 8} 2\end{array}$

$\begin{array}{llll}\text { C } & -5.534722 & 0.069365 & 0.558518\end{array}$

$\begin{array}{llll}C & \mathbf{- 3 . 4 8 2 6 7 0} & \mathbf{0 . 6 8 7 4 6 1} & \mathbf{1 . 6 2 9 3 1 9}\end{array}$

$\begin{array}{llll}C & -3.695377 & 1.039720 & -0.745390\end{array}$

$\begin{array}{llll}C & -4.984007 & 0.500923 & -0.651602\end{array}$

$\begin{array}{llll}\text { C } & -4.764499 & 0.178757 & 1.721089\end{array}$

$\begin{array}{llll}\text { F } & -\mathbf{2 . 7 3 2 7 0 1} & \mathbf{0 . 7 6 8 0 5 2} & \mathbf{2 . 7 3 3 1 9 2}\end{array}$

$\begin{array}{llll}C & -3.161986 & 1.505401 & -2.066866\end{array}$

$\begin{array}{llll}\text { H } & \mathbf{- 5 . 5 8 5 8 2 7} & \mathbf{0 . 4 3 4 7 6 6} & \mathbf{- 1 . 5 6 1 7 7 5}\end{array}$

\begin{tabular}{llll}
\hline & -5.142463 & -0.141806 & $\mathbf{2 . 6 9 3 4 6 5}$
\end{tabular}

$\begin{array}{llll}\text { C } & \mathbf{- 6 . 9 1 3 1 9 7} & \mathbf{- 0 . 5 3 1 6 2 6} & \mathbf{0 . 6 1 1 0 4 0}\end{array}$

$\begin{array}{llll}\text { H } & -\mathbf{7 . 3 9 7 1 7 0} & \mathbf{- 0 . 3 4 4 4 5 5} & \mathbf{1 . 5 7 9 4 5 3}\end{array}$

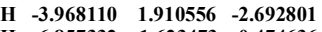

$\begin{array}{llll}\text { H } & -6.857332 & -1.623473 & 0.474636\end{array}$

$\begin{array}{llll}\text { H } & -7.556139 & -0.131759 & -0.185053\end{array}$

$\begin{array}{llll}\text { H } & -2.703529 & 0.664631 & -2.609702\end{array}$

$\begin{array}{lllll}\text { H } & -2.381467 & 2.268379 & -1.949630\end{array}$

$\begin{array}{llll}\text { F } & \mathbf{1 3 6 7 4 9 9} & \mathbf{0 . 6 0 6 1 2 0} & \mathbf{2 . 6 7 6 8 7 0}\end{array}$

$\begin{array}{cccc}\text { F } & 1.367499 & 0.606120 & 2.676870 \\ \text { C } & 6.024191 & 0.718323 & 1.262811\end{array}$

\begin{tabular}{llll}
$C$ & 6.024191 & 0.718323 & 1.262811 \\
\hline$H$ & 6.705179 & 1.459520 & 0.822055
\end{tabular}

\begin{tabular}{ccccc} 
H & 6.705179 & $\mathbf{1 . 4 5 9 5 2 0}$ & $\mathbf{0 . 8 2 2 0 5 5}$ \\
\hline & 6.290786 & $\mathbf{0 . 5 8 4 0 3 2}$ & $\mathbf{2 . 3 2 0 5 5 4}$
\end{tabular}

$\begin{array}{rrrrr}\text { H } & 6.290786 & \mathbf{0 . 5 8 4 0 3 2} & \mathbf{2 . 3 2 0 5 5 4} \\ \text { H } & \mathbf{6 . 2 0 3 0 3 5} & \mathbf{- 0 . 2 4 2 2 1 9} & \mathbf{0 . 7 5 3 2 6 6}\end{array}$

$\begin{array}{llll}\text { C } & 2.424335 & 3.148998 & -1.325551\end{array}$

$\begin{array}{llll}\text { H } & 2.167522 & 4.165017 & -0.984205\end{array}$

H $\quad 3.232773 \quad 3.237426 \quad-2.062901$

H $\quad 1.539011 \quad 2.729021 \quad-1.824328$

$\begin{array}{llll}\text { C } \quad \mathbf{1 . 5 6 4 3 1 3} & \mathbf{0 . 3 5 9 9 7 7} & \mathbf{- 3 . 5 5 1 9 1 1}\end{array}$

C $2.540635-0.327298-2.907931$

$\begin{array}{llll}C & 2.540635 & -0.327298 & -2.907931 \\ C & -0.148546 & -3.387604 & -2.005496\end{array}$

$\begin{array}{lrrr}C & -0.148546 & \mathbf{3} .387604 & \mathbf{- 2 . 0 0 5 4 9 6}\end{array}$

$\begin{array}{llll}C & -1.810146 & -2.018854 & -1.993122\end{array}$

$\begin{array}{llll}\text { C } & -1.220679 & -2.012711 & 0.045164\end{array}$

$\begin{array}{llll}\text { H } & -\mathbf{0 . 2 5 4 4 7 2} & -\mathbf{1 . 3 4 7 9 9 0} & \mathbf{1 . 8 6 4 1 1 5}\end{array}$

$\begin{array}{llll}\text { C } & 1.016952 & -2.864052 & 1.124458\end{array}$

$\begin{array}{llll}\text { C } & -2.599199 & -2.281544 & 0.624452\end{array}$

$\begin{array}{llll}\text { H } & -2.689174 & -3.371255 & 0.749256\end{array}$

$\begin{array}{llll}\text { H } & -2.711348 & -1.817637 & 1.611690\end{array}$

$\begin{array}{llll}\text { H } & -3.409216 & -1.936693 & -0.034784\end{array}$

\begin{tabular}{lrrrr} 
H & -3.409216 & -1.936693 & -0.034784 \\
\hline & 1.107611 & -3.979315 & 0.652627
\end{tabular}

\begin{tabular}{lllll}
\hline & 1.107611 & $\mathbf{3 . 9 7 9 3 1 5}$ & $\mathbf{0 . 6 5 2 6 2 7}$ \\
\hline & 1.945064 & -2394349 & 1.980702
\end{tabular}

$\begin{array}{llll}\text { O } & 1.945064 & -2.394349 & 1.980702\end{array}$

$\begin{array}{llll}\text { C } & 3.047978 & \mathbf{- 3 . 2 3 7 8 6 4} & \mathbf{2 . 2 4 7 5 2 2}\end{array}$

$\begin{array}{ccccc}\text { H } & 3.592004 & -3.479190 & \mathbf{1 . 3 2 2 9 2 7} \\ \text { H } & \mathbf{2 . 7 2 7 3 6 7} & -\mathbf{4 . 1 7 7 1 3 5} & \mathbf{2 . 7 2 2 2 4 1}\end{array}$

$\begin{array}{llll}\text { H } & 3.702561 & \mathbf{- 2 . 6 8 1 9 7 3} & \mathbf{2 . 9 2 8 6 0 2}\end{array}$

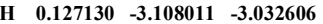

$\begin{array}{lllll}\text { H } & -0.799725 & -4.278338 & -2.066709\end{array}$

H $\quad 0.748063-\mathbf{- 3 . 6 6 0 1 2 3}-1.446508$

H $-1.492207-0.697687-0.244722$

\begin{tabular}{llll} 
H & $\mathbf{- 1 . 4 9 2 2 0 7}$ & $\mathbf{- 0 . 6 9 7 6 8 7}$ & $\mathbf{- 0 . 2 4 4 7 2 2}$ \\
\hline & 0.215097 & $\mathbf{3 . 2 2 7 2 4 0}$ & $\mathbf{2} 321240$
\end{tabular}

\begin{tabular}{ccccc}
\hline & $\mathbf{0 . 2 1 5 0 9 7}$ & $\mathbf{3 . 2 2 7 2 4 0}$ & $\mathbf{2 . 3 2 1 2 4 0}$
\end{tabular}

$\begin{array}{llll}\text { H } & 0.354137 & 4.174801 & 0.814598\end{array}$

$\begin{array}{lllll}\text { H } & -1.944232 & 3.800081 & 0.278453\end{array}$

H $\quad \begin{array}{llll}-2.134471 & 3.177860 & 1.939839\end{array}$

$\begin{array}{llll}\text { H } & \mathbf{3 . 5 2 7 8 6 5} & \mathbf{- 0 . 4 8 3 0 9 7} & \mathbf{- 3 . 3 5 1 3 1 0}\end{array}$

$\begin{array}{lllll}\text { H } & 1.748147 & 0.793285 & -4.540715\end{array}$

71

$\begin{array}{llll}S & 0.143438 & \mathbf{0 . 5 5 7 2 0 3} & \mathbf{2 . 7 2 8 3 2 8}\end{array}$

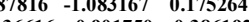

$\begin{array}{llll}\text { C } & -2.289815 & 1.485761 & -0.672886\end{array}$

$\begin{array}{llll}C & 0.079156 & 2.133365 & -1.426718\end{array}$

$\begin{array}{lllll}\text { S } & 2.256725 & 1.280722 & 0.431432\end{array}$

$\begin{array}{lllll}\text { N } & 0.938749 & -1.958385 & 0.029659\end{array}$

$\begin{array}{lrrr}\text { N } & -1.201559 & -1.860192 & 0.385631\end{array}$

$\begin{array}{lllll}\text { C } & \mathbf{2 . 2 9 1 0 0 4} & -1.716066 & -0.316578\end{array}$

$\begin{array}{llll}C & 0.507958 & -3.358396 & -0.028551\end{array}$

C -0.874

$\begin{array}{llll}C & -0.874614 & -3.277702 & 0.576036\end{array}$

$\begin{array}{llll}C & -2.576043 & -1.536389 & 0.237033\end{array}$

$\begin{array}{llll}C & 4.958087 & -1.346096 & -1.123558\end{array}$

$\begin{array}{lllll}\text { C } & 2.610658 & -1.312739 & -1.612844\end{array}$

$\begin{array}{llll}C & 3.326007 & -1.997903 & 0.582700\end{array}$

$\begin{array}{llll}C & 4.644932 & -1.795206 & 0.161130\end{array}$

$\begin{array}{llll}C & 3.913303 & -1.113606 & -2.026433\end{array}$

$\begin{array}{llll}\text { H } & 5.455093 & -1.991329 & 0.868599\end{array}$

$\begin{array}{llll}\text { H } & \mathbf{4 . 1 0 2 6 3 7} & \mathbf{- 0 . 7 7 3 8 4 2} & \mathbf{- 3 . 0 4 6 4 8 3}\end{array}$

$\begin{array}{llll}C & -5.302793 & -1.005603 & -0.298785\end{array}$

C $-3.182292-1.886915-0.978944$

$\begin{array}{llll}C & -3.182292 & -1.886915 & -0.978944 \\ C & -3.391183 & -1.004836 & 1.245030\end{array}$

$\begin{array}{llll}C & -3.391183 & -1.004836 & 1.245030\end{array}$ 


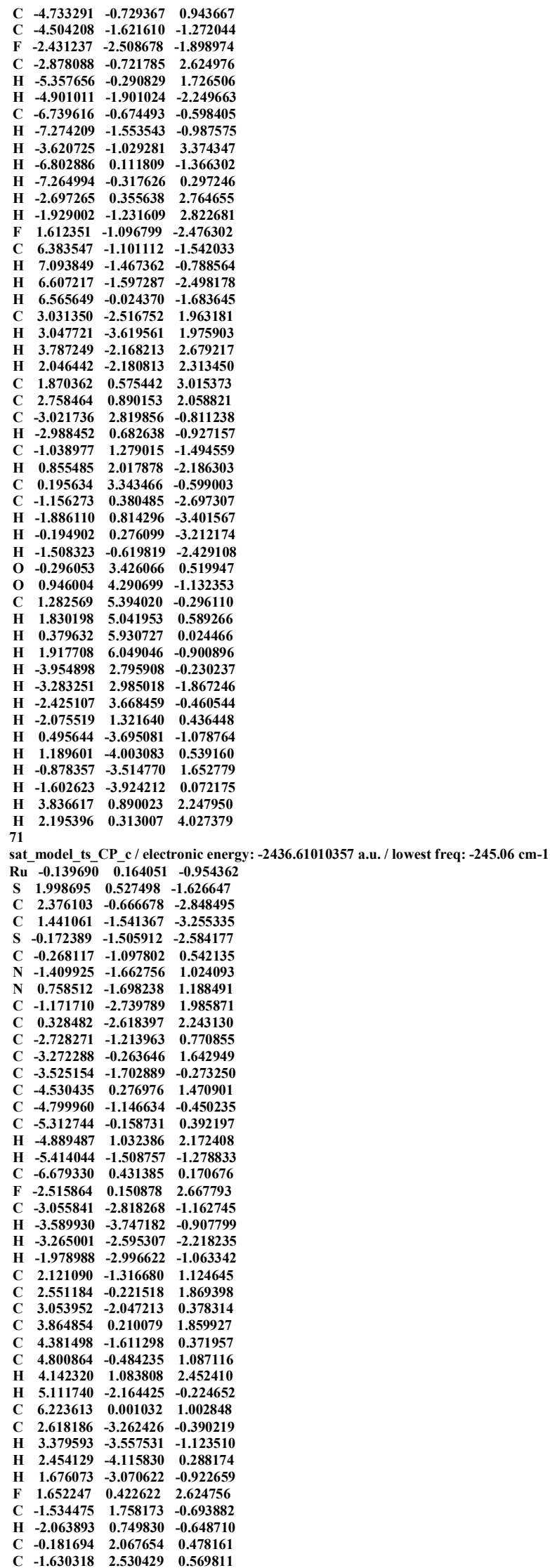


$\begin{array}{lllll}\text { H } & 0.139434 & 1.433062 & 1.302067\end{array}$ $\begin{array}{llll}\text { C } & -2.022069 & 3.990825 & 0.584137\end{array}$ $\begin{array}{llll}\text { H } & -2.119825 & 1.977811 & 1.37413\end{array}$

$\begin{array}{llll}\text { H } & 6.911727 & -0.811094 & 0.730567\end{array}$

\begin{tabular}{llll}
\hline & 6.558549 & 0.437344 & 1.954649
\end{tabular}

$\begin{array}{llll}6.55849 & 0.437344 & 1.954649\end{array}$ H $-6.612183 \quad 1.5184290 .012830$

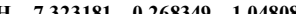
$\begin{array}{llll}4 & -7.323181 & 0.268349 & 1.04808\end{array}$ $\begin{array}{llll}\text { H } & -7.171912 & \mathbf{- 0 . 0 1 2 2 2 9} & \mathbf{- 0 . 7 0 4 5 3 0}\end{array}$ $\begin{array}{llll}\text { H } & -1.489408 & 4.575295 & -0.174503\end{array}$ $\begin{array}{llll}\text { H } & -1.796514 & 4.428103 & 1.567903\end{array}$ H $\quad \begin{array}{cccc}-3.104652 & 4.089820 & 0.413318\end{array}$

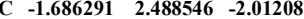

$\begin{array}{lllll}\text { O } & \mathbf{0 . 7 7 1 9 0 9} & \mathbf{3 . 9 2 6 0 3 6} & -\mathbf{0 . 7 3 8 8 3 5}\end{array}$

$\begin{array}{llll}C & 0.880763 & 3.009474 & 0.047166\end{array}$

$\begin{array}{lllll}\text { O } & 2.032605 & 2.725796 & 0.660684\end{array}$

$\begin{array}{llll}\text { C } & \mathbf{3 . 1 7 4 1 3 6} & \mathbf{3 . 4 6 0 6 7 5} & \mathbf{0 . 2 5 2 3 2 5}\end{array}$

$\begin{array}{llll}\text { H } & 3.038723 & 4.535339 & \mathbf{0 . 4 3 9 0 8 5}\end{array}$

$\begin{array}{llll}\text { H } & 4.012008 & 3.076175 & 0.843859\end{array}$

H $\quad 3.368818 \quad 3.299996 \quad-0.817436$

$\begin{array}{lllll}\text { H } & -\mathbf{1 . 5 6 8 9 4 3} & \mathbf{1 . 8 1 0 8 0 0} & \mathbf{- 2 . 8 6 7 3 1 6}\end{array}$

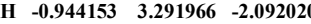

$\begin{array}{llll}\text { H } & -2.691800 & 2.938025 & -2.054612\end{array}$

$\begin{array}{llll}\text { H } & -1.448066 & -3.709690 & \mathbf{1 . 5 4 2 4 0 1}\end{array}$

$\begin{array}{llll}H & -1.771196 & -2.590329 & 2.89418\end{array}$

H $0.552940-2.183858 \quad 3.230269$

$\begin{array}{llll}\text { H } & \mathbf{0 . 8 5 3 8 0 7} & \mathbf{- 3 . 5 7 9 5 6 2} & \mathbf{2 . 1 5 9 8 9 9}\end{array}$

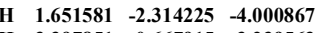

H $3.397851 \quad-0.667915 \quad-3.239563$

71

sat_model_prod_CP_c / electronic energy: $-\mathbf{2 4 3 6 . 6 2 1 9 7 0 7 0}$ a.u. / lowest freq: $16.56 \mathrm{~cm}-1$

Ru $\quad-0.287363-0.020496-0.877912$

$\begin{array}{llll}1.712806 & 0.766962 & -1.585464\end{array}$

C $2.279060-0.396289-2.762542$

C $1.497430-1.416235-3.157661$

C $1.497430-1.416235-3.157661$

S $-0.123308-1.604556-2.520456$

$\begin{array}{llll}C & 0.013193 & -1.232724 & 0.594909\end{array}$

$\begin{array}{llll}\text { N } & -1.025588 & -1.906569 & 1.16068\end{array}$

$\begin{array}{llll}\text { N } & 1.138833 & -1.699412 & 1.183527\end{array}$

$\begin{array}{llll}C & \mathbf{- 0 . 6 1 8 6 2 1} & \mathbf{- 2 . 9 3 1 1 2 0} & \mathbf{2 . 1 1 6 7 2}\end{array}$

$\begin{array}{llll}C & \mathbf{0 . 8 6 5 3 5 3} & \mathbf{- 2 . 6 0 7 5 0 7} & \mathbf{2 . 3 0 0 9 9 8}\end{array}$

$\begin{array}{llll}C & -2.373394 & -1.605401 & 0.86003\end{array}$

$\begin{array}{llll}C & -2.984400 & -0.539312 & 1.528823\end{array}$

$\begin{array}{llll}C & -3.126961 & -2.340780 & -0.071493\end{array}$

$\begin{array}{llll}C & -4.283478 & -0.141137 & 1.268974\end{array}$

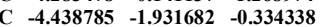

$\begin{array}{llll}\text { C } & -5.026556 & -0.837032 & 0.308224\end{array}$

$\begin{array}{rrrr}\text { H } & -\mathbf{4 . 7 0 4 5 7 8} & \mathbf{0 . 7 0 6 2 0 2} & \mathbf{1 . 8 1 3 3 5 1}\end{array}$

$\begin{array}{llll}\text { H } & \mathbf{5 . 0 2 3 7 4 0} & \mathbf{- 2 . 4 9 2 1 8 1} & \mathbf{- 1 . 0 6 7 9 4 7}\end{array}$

$\begin{array}{llll}C & -6.422261 & -0.393741 & -0.038371\end{array}$

$\begin{array}{llll}\text { F } & -2.267370 & 0.122372 & 2.44419\end{array}$

$\begin{array}{llll}\text { C } & \mathbf{- 2 . 5 4 7 1 0 2} & \mathbf{- 3 . 5 6 3 0 3 6} & \mathbf{- 0 . 7 2 4 2 0 9}\end{array}$

$\begin{array}{llll}\text { H } & -2.656836 & -4.435050 & -0.059179\end{array}$

$\begin{array}{llll}\text { H } & -3.065666 & -3.791751 & -1.664385\end{array}$

$\begin{array}{llll}\text { H } & -1.478135 & -3.431768 & -0.939454\end{array}$

C $2.444571-1.18$

C $2.816967-0.025695-1.685665$

C 2.8169

$\begin{array}{llll}C & 3.386032 & -1.848337 & 0.212023\end{array}$

$\begin{array}{llll}\text { C } & 4.085871 & 0.515207 & 1.576569\end{array}$

$\begin{array}{llll}C & 4.664583 & -1.294928 & 0.097122\end{array}$

$\begin{array}{llll}C & \mathbf{5 . 0 2 8 9 2 3} & \mathbf{- 0 . 1 1 8 2 5 1} & \mathbf{0 . 7 5 9 6 0 6}\end{array}$

$\begin{array}{llll}\text { H } & 4.330517 & \mathbf{1 . 4 2 2 4 5 8} & \mathbf{2} .132369\end{array}$

$\begin{array}{llll}\text { H } & 5.400457 & -1.799719 & -0.534407\end{array}$

$\begin{array}{lllll}\text { C } & 6.399182 & 0.479188 & 0.579837\end{array}$

$\begin{array}{llll}C & 3.011892 & \mathbf{- 3 . 1 2 6 3 5 7} & \mathbf{- 0 . 4 8 4 5 0}\end{array}$

$\begin{array}{llll}\text { H } 3.751316 & -3.387732 & -1.252504\end{array}$

$\begin{array}{llll}\text { H } & \mathbf{2 . 9 6 1 3 9 0} & -\mathbf{3 . 9 6 1 6 1 5} & \mathbf{0 . 2 3 3 2 7 4}\end{array}$

$\begin{array}{llll}\text { H } & 2.026504 & -3.034187 & -0.961757\end{array}$

$\begin{array}{lllll}\text { F } & 1.912467 & 0.567785 & 2.469277\end{array}$

$\begin{array}{llll}\text { C } & -\mathbf{2 . 2 5 7 3 0 2} & \mathbf{2 . 3 4 1 9 1 1} & \mathbf{- 0 . 5 6 2 6 5 5}\end{array}$

$\begin{array}{llll}\text { H } & \mathbf{- 2 . 7 3 7 1 4 3} & \mathbf{1 . 3 5 7 3 5 1} & \mathbf{- 0 . 5 9 9 1 7 9}\end{array}$

$\begin{array}{llll}\text { C } & -0.912114 & 2.312105 & 0.167449\end{array}$

C $-2.180594 \quad 2.910017 \quad 0.809005$

$\begin{array}{lllll}C & -2.180594 & 2.910017 & 0.809005\end{array}$

$\begin{array}{llll}\mathrm{H} & -0.566596 & 1.385558 & 0.685644\end{array}$

$\begin{array}{llll}\text { C } & -2.290130 & 4.386357 & 1.100900\end{array}$

$\begin{array}{llll}\text { H } & -2.583607 & 2.276513 & 1.601884\end{array}$

$\begin{array}{llll}\text { H } & 7.127636 & -0.276077 & 0.254207\end{array}$

H $\quad 6.764523 \quad 0.936156 \quad 1.51058$

$\begin{array}{lllll}\text { H } & 6.374475 & 1.269665 & -0.187197\end{array}$

$\begin{array}{lllll}\text { H } & -6.391230 & 0.393984 & -0.807978\end{array}$

\begin{tabular}{llll}
\hline & -6.391230 & 0.393984 & -0.807978
\end{tabular}

$\begin{array}{lll}-6.9400745 & 0.021713 & 0.837196\end{array}$

\begin{tabular}{llll}
\hline & -7.019745 & -1.224188 & -0.438042 \\
\hline
\end{tabular}

$-1.8349275 .004305-0.317200$

$\begin{array}{llll}H & -1.797365 & 4.628257 & 2.054298\end{array}$

$\begin{array}{lll}-3.350385 & 4.667175 & 1.188309\end{array}$

$\begin{array}{llll}C & -2.498050 & 3.142871 & -1.81851\end{array}$

$\begin{array}{lllll}O & 0.185704 & 4.192599 & -0.88486\end{array}$

$\begin{array}{llll}C & 0.208610 & 3.249473 & -0.129222\end{array}$

$\begin{array}{lllll}\text { O } & 1.269506 & 2.943652 & 0.617145\end{array}$

$\begin{array}{llll}\text { C } & \mathbf{2 . 4 4 2 7 5 6} & \mathbf{3 . 7 1 4 1 7 8} & \mathbf{0 . 4 1 4 4 6 9}\end{array}$

$\begin{array}{llll}C & 2.24784 & 4.783941 & 0.56493\end{array}$

$3.170718 \quad 3.357999$

$\begin{array}{llll}\text { H } & 3.170718 & 3.357999 & 1.15012\end{array}$ 


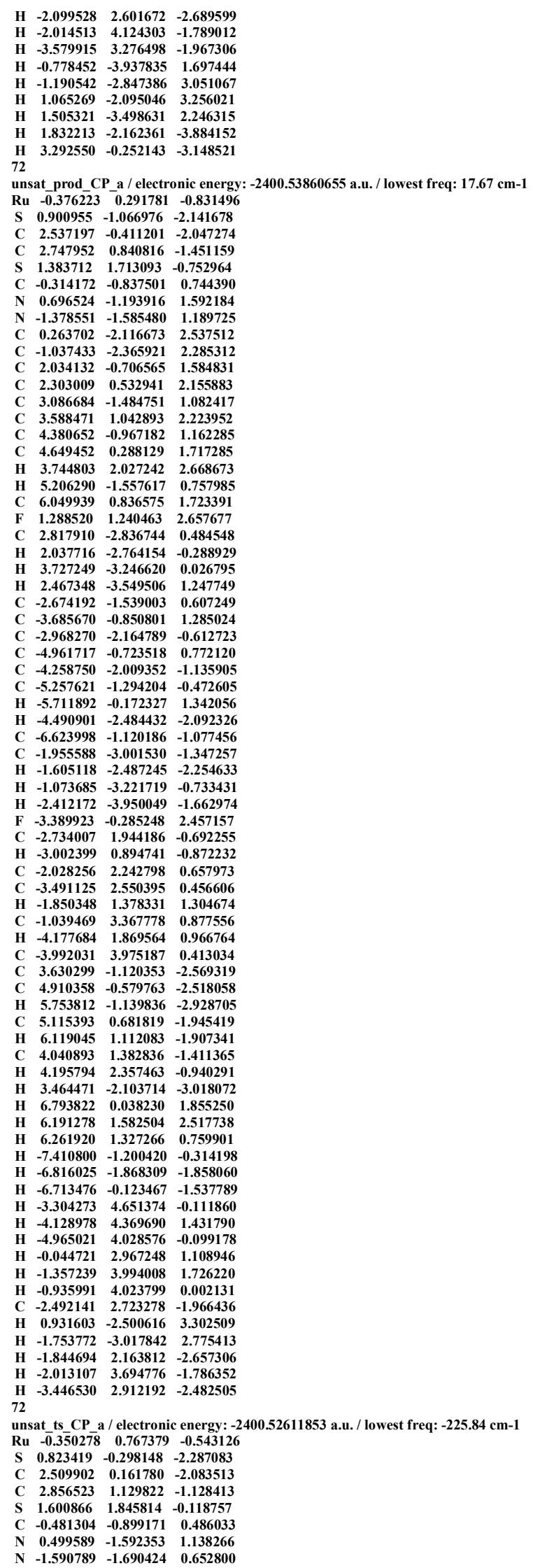


$\begin{array}{llll}C & 0.008189 & -2.763791 & 1.700605\end{array}$ $\begin{array}{llll}C & -1.301418 & -2.829002 & 1.395173\end{array}$ $\begin{array}{llll}C & 1.874600 & -1.241965 & 1.271045\end{array}$

$\begin{array}{llll}C & \mathbf{2 . 2 4 2 8 1 3} & \mathbf{- 0 . 2 9 3 3 7 5} & \mathbf{2 . 2 1 8 7 7 5}\end{array}$

$\begin{array}{llll}\text { C } & 2.864543 & -1.905140 & 0.530089\end{array}$

$\begin{array}{llll}C & 3.566893 & 0.050587 & 2.433767\end{array}$

$\begin{array}{llll}C & 3.566893 & 0.050587 & 2.433767\end{array}$

$\begin{array}{llll}C & 4.196646 & -1.557225 & 0.758206\end{array}$

$\begin{array}{llll}C & 4.565914 & -0.581736 & 1.69008\end{array}$

$\begin{array}{llll}\text { H } & 3.801216 & 0.815622 & 3.175895\end{array}$

$\begin{array}{llll}H & 4.974000 & -2.053192 & 0.172068\end{array}$

C $\quad 6.009124 \quad-0.191091 \quad 1.853550$

$\begin{array}{llll}\text { F } & \mathbf{1 . 2 8 6 6 8 6} & \mathbf{0 . 2 9 6 0 8 3} & \mathbf{2 . 9 3 9 7 3 9}\end{array}$

$\begin{array}{llll}\text { C } & 2.495516 & -2.962307 & -0.471880\end{array}$

H $1.744161 \quad-2.572729 \quad-1.176422$

H $\quad 3.378365-3.278614 \quad-1.042197$

$\begin{array}{llll}\text { H } & \mathbf{2 . 0 6 8 9 5 9} & \mathbf{- 3 . 8 5 0 8 6 9} & \mathbf{0 . 0 1 9 4 2 4}\end{array}$

$\begin{array}{llll}C & -2.899934 & -1.432246 & 0.161182\end{array}$

$\begin{array}{llll}C & -3.878067 & -1.020798 & 1.067827\end{array}$

$\begin{array}{llll}C & -3.249810 & -1.646352 & -1.179683\end{array}$

$\begin{array}{llll}C & \mathbf{5 . 1 8 2 9 4 6} & \mathbf{- 0 . 7 7 1 8 0 1} & \mathbf{0 . 6 8 3 1 2 2}\end{array}$

$\begin{array}{llll}C & -4.568149 & -1.378111 & -1.563730\end{array}$

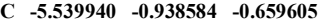

$\begin{array}{llll}\text { H } & \mathbf{5} 5.904602 & \mathbf{- 0 . 4 4 1 1 8 9} & \mathbf{1 . 4 3 2 1 3 8}\end{array}$

$\begin{array}{llll}\text { H } & -\mathbf{4 . 8 4 6 7 8 8} & \mathbf{- 1 . 5 3 4 4 2 6} & \mathbf{- 2 . 6 0 8 7 2 5}\end{array}$

$\begin{array}{llll}C & -6.941881 & -0.633465 & -1.113095\end{array}$

$\begin{array}{llll}C & -2.242633 & -2.135202 & -2.180570\end{array}$

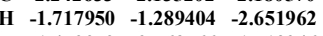

H $\quad-1.472979-2.763766-1.713246$

$\begin{array}{llll}\text { H } & -2.735755 & -2.708092 & -2.976687\end{array}$

$\begin{array}{llll}\text { F } & -3.525201 & -0.843201 & 2.342946\end{array}$

$\begin{array}{lllll}\text { C } & -2.105802 & 1.916581 & -0.438916\end{array}$

$\begin{array}{lllll}\text { H } & -2.438103 & 0.960573 & -0.944331\end{array}$

$\begin{array}{llll}\text { C } & -1.408957 & 1.843129 & \mathbf{1 . 2 9 2 5 1 9}\end{array}$

$\begin{array}{llll}C & -1.408957 & 1.843129 & 1.292519\end{array}$

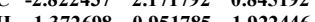

$\begin{array}{llll}\text { C } & -1.372698 & 0.951785 & 1.922446\end{array}$

$\begin{array}{llll}\text { C } & -\mathbf{0 . 5 7 6 5 0 5} & \mathbf{2 . 9 7 8 8 2 3} & \mathbf{1 . 8 4 0 2 4 9}\end{array}$

$\begin{array}{llll}\text { H } & -3.518765 & 1.370645 & 1.102840\end{array}$

$\begin{array}{llll}\text { C } & \mathbf{- 3 . 4 4 4 7 2 9} & \mathbf{3 . 5 2 2 2 5 4} & \mathbf{1 . 1 3 4 7 6 7}\end{array}$

$\begin{array}{llll}C & 3.525187 & -0.440147 & \mathbf{- 2 . 8 4 5 0 8 1}\end{array}$

$\begin{array}{llll}C & 4.855105 & -0.074061 & -2.669047\end{array}$

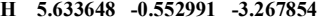

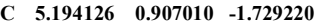

H $6.238103 \quad 1.197004 \quad-1.588264$

C $4.1984111 .500469-0.961829$

$\begin{array}{llll}C & 4.198441 & 1.500469 & \mathbf{- 0 . 9 6 1 8 2 9}\end{array}$

$\begin{array}{lllll}\text { H } & 4.455272 & \mathbf{2 . 2 4 8 6 7 8} & \mathbf{- 0 . 2 0 6 8 8 7}\end{array}$

$\begin{array}{llll}\text { H } & 3.257162 & \mathbf{- 1 . 2 0 5 4 5 1} & \mathbf{- 3 . 5 7 8 4 0 8}\end{array}$

$\begin{array}{rrrrr}\text { H } & 6.673279 & -1.062424 & \mathbf{1 . 7 6 3 2 1 2} \\ \text { H } & 6.192819 & \mathbf{0 . 2 8 9 7 4 5} & \mathbf{2 . 8 2 4 1 5 9}\end{array}$

$\begin{array}{cccc}\text { H } & 6.293903 & 0.522208 & 1.063415\end{array}$

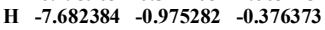

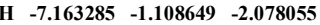

$\begin{array}{llll}\text { H } & -7.077156 & \mathbf{0 . 4 5 2 7 9 4} & -1.235517\end{array}$

\begin{tabular}{llll}
\hline & -2.783645 & $\mathbf{4 3 6 1 9 6 7}$ & $\mathbf{0 . 8 8 2 5 0 8}$
\end{tabular}

$\begin{array}{llll}-3.63076 & 3.605430 & 2.203846\end{array}$

\begin{tabular}{cccc}
\hline H & -3.692076 & 3.605430 & $\mathbf{2 . 2 0 3 8 4 6}$ \\
\hline
\end{tabular}

$\begin{array}{llll}\text { H } & -4.378865 & 3.646109 & \mathbf{0 . 5 6 6 1 0 9}\end{array}$

$\begin{array}{llll}\text { H } & 0.394575 & 2.607325 & 2.189172\end{array}$

$\begin{array}{llll}\text { H } & -1.094837 & 3.447048 & 2.694085\end{array}$

$\begin{array}{llll}\text { H } & \mathbf{- 0 . 3 8 2 1 7 5} & \mathbf{3 . 7 6 7 6 8 6} & \mathbf{1 . 0 9 8 2 4 6}\end{array}$

$\begin{array}{llll}\text { C } & -1.896213 & 3.076172 & -1.394361\end{array}$

$\begin{array}{llll}\text { H } & 0.646026 & -3.431369 & 2.271443\end{array}$

$\begin{array}{llll}\text { H } & -2.061414 & \mathbf{- 3 . 5 6 6 7 6 9} & \mathbf{1 . 6 3 2 1 7 1}\end{array}$

$\begin{array}{llll}H & -1.314528 & 2.788485 & -2.281988\end{array}$

H $-1.3684303 .904010-0.901368$

\begin{tabular}{lllll} 
H & $-\mathbf{1 . 3 6 8 4 3 0}$ & $\mathbf{3 . 9 0 4 0 1 0}$ & $\mathbf{- 0 . 9 0 1 3 6 8}$ \\
\hline & -2.873137 & 3.457178 & -1.735025
\end{tabular}

$\begin{array}{lllll}\text { H } & -2.873137 & 3.457178 & -1.735025\end{array}$

72

unsat_prod_BHE_a / electronic energy: $\mathbf{- 2 4 0 0 . 5 7 1 2 8 1 1 9}$ a.u. / lowest freq: $27.39 \mathrm{~cm}-1$

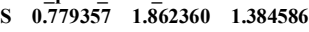

$\begin{array}{llll}C & 0.320361 & \mathbf{- 0 . 6 2 8 5 7 1} & \mathbf{- 0 . 8 3 1 6 0 8}\end{array}$

$\begin{array}{llll}\text { Ru } & 0.258909 & -0.419215 & 1.130281\end{array}$

$\begin{array}{llll}\text { C } & 2.104787 & -1.863731 & 1.653222\end{array}$

$\begin{array}{llll}\text { C } & -0.392163 & -2.357478 & 1.931032\end{array}$

$\begin{array}{llll}\text { S } & -1.940672 & 0.246021 & 1.416827\end{array}$

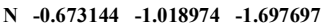

$\begin{array}{llll}N & 1.423400 & -0.531694 & -1.648789\end{array}$

$\begin{array}{llll}C & -2.084130 & -0.957160 & -1.485620\end{array}$

$\begin{array}{llll}C & -0.184371 & -1.196354 & -2.986249\end{array}$

$\begin{array}{llll}C & 1.126534 & -0.893612 & -2.957017\end{array}$

$\begin{array}{llll}\text { C } & \mathbf{2 . 6 7 8 5 2 2} & \mathbf{0 . 0 6 7 9 1 7} & \mathbf{- 1 . 3 3 1 0 2 3}\end{array}$

$\begin{array}{llll}\text { C } & -4.862850 & -0.814920 & -1.105813\end{array}$

$\begin{array}{llll}\text { C } & -2.812477 & -2.067729 & -1.082698\end{array}$

$\begin{array}{llll}C & -2.756628 & 0.248429 & -1.745924\end{array}$

$\begin{array}{llll}C & -1135720 & 0.294459 & -1.554328\end{array}$

$\begin{array}{llll}C & -4.135720 & 0.294459 & -1.554328 \\ C & -4.182979 & -2.012112 & -0.878472\end{array}$

$\begin{array}{llll}C & -4.182979 & -2.012112 & -0.878472\end{array}$

$\begin{array}{llll}\text { H } & -4.658699 & 1.235779 & -1.740485\end{array}$

$\begin{array}{lllll}\text { H } & -4.701692 & -2.912344 & -0.544192\end{array}$

$\begin{array}{lllll}C & 5.181500 & 1.205378 & -0.742462\end{array}$

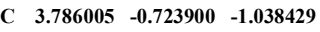

$\begin{array}{llll}C & 2.845755 & 1.458431 & -1.441559\end{array}$

$\begin{array}{lllll}C & 4.094128 & 1.999015 & -1.124790\end{array}$

C $\quad \mathbf{5 . 0 2 0 5 6 0} \quad \mathbf{- 0 . 1 8 2 9 4 4} \quad-0.722199$

$\begin{array}{llll}\text { F } & 3.658909 & -2.055934 & -1.078347\end{array}$

C $1.720962-2.328999-1.923779$

$\begin{array}{llll}\text { C } & 1.720962 & 2.328999 & -1.923779 \\ \text { H } & 4.226310 & 3.081433 & -1.194644\end{array}$ 
$\begin{array}{llll}\text { H } & \mathbf{5 . 8 4 5 9 9 5} & \mathbf{- 0 . 8 5 4 7 3 9} & \mathbf{- 0 . 4 8 0 1 8 2}\end{array}$ $\begin{array}{llll}C & 6.497971 & 1.826610 & -0.362146\end{array}$

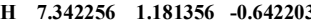
$\begin{array}{lllll}\text { H } & \mathbf{1 . 2 7 7 5 7 8} & \mathbf{1 . 9 3 0 7 5 9} & \mathbf{- 2 . 8 4 8 7 9 5}\end{array}$ $\begin{array}{llll}H & 6.546679 & 1.980549 & 0.727627\end{array}$ $46.631980 \quad 2.806244-0.840841$ \begin{tabular}{llll} 
H & 6.631980 & 2.806244 & -0.840841 \\
\hline
\end{tabular} \begin{tabular}{llll} 
H & 2.075768 & 3.348999 & -2.118198 \\
\hline
\end{tabular} $\begin{array}{llll}0.931156 & 2.378393 & -1.16092\end{array}$ $\begin{array}{llll}\text { F } & -2.184947 & -3.230481 & -0.874628\end{array}$ $\begin{array}{llll}C & -6.341173 & -0.707202 & -0.845368\end{array}$ $\begin{array}{llll}\text { H } & -6.825453 & -0.033402 & -1.566169\end{array}$ $\begin{array}{llll}\text { H } & -6.834671 & -1.687257 & -0.897232\end{array}$ $\begin{array}{llll}\text { H } & -6.520266 & -0.295907 & 0.161002\end{array}$ $\begin{array}{llll}\text { C } & -1.985863 & 1.463750 & -2.17588\end{array}$ $\begin{array}{lllll}\text { H } & -1.475418 & 1.301336 & -3.137956\end{array}$ $\begin{array}{lllll}\text { H } & -2.648795 & 2.332197 & -2.275760\end{array}$ $\begin{array}{llll}\text { H } & -1.217762 & 1.709667 & -1.42828\end{array}$

$\begin{array}{llll}C & -0.749151 & \mathbf{2 . 7 1 1 1 8 7} & \mathbf{1 . 1 6 3 4 0 2}\end{array}$

$\begin{array}{llll}\text { C } & -\mathbf{0 . 7 8 3 0 3 1} & \mathbf{4 . 1 0 1 5 4 2} & \mathbf{0 . 9 6 5 8 0 4}\end{array}$

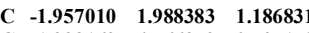
\begin{tabular}{llll}
$C$ & -1.993169 & 4.766379 & 0.79515 \\
\hline
\end{tabular} $\begin{array}{lllll}\text { H } & 0.158558 & 4.656737 & 0.940992\end{array}$ $\begin{array}{lrrr}\text { C } & -3.173100 & 2.670199 & 1.015599\end{array}$ $\begin{array}{lllll}\text { C } & -3.194167 & 4.047304 & 0.823314\end{array}$ $\begin{array}{lllll}\text { H } & -2.002222 & 5.847167 & 0.635274\end{array}$ $\begin{array}{llll}-4.105372 & 2.099053 & 1.021443\end{array}$ $\begin{array}{llll}\text { H } & -4.147734 & 4.562485 & 0.68518\end{array}$

$\begin{array}{llll}\text { H } & \mathbf{2 . 8 9 5 3 2 5} & \mathbf{- 2 . 2 2 2 1 7 7} & \mathbf{0 . 9 8 4 2 7}\end{array}$

C $\quad 0.811740 \quad-2.495957 \quad 1.215641$ $\begin{array}{llll}\text { H } & -1.238999 & -2.846934 & 1.436756\end{array}$ $\begin{array}{llll}C & -0.597170 & -2.199472 & 3.424898\end{array}$ $\begin{array}{llll}C & 0.894704 & -3.434322 & 0.035535\end{array}$ $\begin{array}{llll}\text { H } & 1.280160 & -4.406510 & 0.386079\end{array}$ $\begin{array}{lllll}\text { H } & -0.088628 & -3.598003 & -0.415397\end{array}$ $\begin{array}{llll}H & -0.088628 & -3.598003 & -0.415397\end{array}$ \begin{tabular}{llll}
\hline & 1.584572 & -3.069751 & -0.735363
\end{tabular} $\begin{array}{llll}\text { H } & 2.114861 & -0.721418 & 1.359717\end{array}$ $\begin{array}{lrrr}\text { H } & -\mathbf{0 . 8 3 1 8 5 7} & \mathbf{- 1 . 5 1 0 2 1 6} & \mathbf{- 3 . 7 9 9 0 4 5} \\ \text { H } & \mathbf{1 . 8 8 3 5 5 0} & \mathbf{- 0 . 8 9 0 0 7 9} & \mathbf{- 3 . 7 3 4 8 4 3}\end{array}$

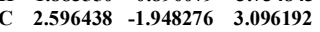
$\begin{array}{lllll}\text { H } & -1.659997 & -\mathbf{2 . 0 1 2 4 8 8} & 3.627852\end{array}$

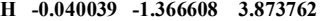
$\begin{array}{llll}\text { H } & -0.302344 & \mathbf{- 3 . 1 2 0 9 8 6} & \mathbf{3 . 9 5 5 0 7 1}\end{array}$ $\begin{array}{llll}\text { H } & \mathbf{1 . 9 8 0 8 0 3} & -1.369231 & 3.792412\end{array}$ $\begin{array}{llll}\text { H } & 3.622968 & -1.560400 & 3.160388\end{array}$ $\begin{array}{llll}\text { H } & \mathbf{2 . 6 0 4 1 1 2} & \mathbf{2} .995350 & \mathbf{3 . 4 3 2 3 2 3}\end{array}$ 72

unsat_ts_BHE_a / electronic energy: $\mathbf{- 2 4 0 0 . 5 0 6 7 5 3 8 3}$ a.u. / lowest freq: $-\mathbf{1 9 . 0 9} \mathrm{cm}-1$ $\begin{array}{llll}S & 1.342491 & 1.889845 & 0.714671\end{array}$

$\begin{array}{llll}C & 0.172441 & -0.836707 & -0.907380\end{array}$

$\begin{array}{llll}\text { Ru } & \mathbf{0 . 0 6 3 0 4 4} & \mathbf{- 0 . 1 4 9 3 2 7} & \mathbf{1 . 0 0 6 1 2 6}\end{array}$

$\begin{array}{llll}C & \mathbf{1 . 1 4 5 6 9 3} & \mathbf{- 0 . 6 6 7 0 1 3} & \mathbf{2 . 7 3 8 0 5 5}\end{array}$

$\begin{array}{llll}C & \mathbf{- 0 . 7 2 3 4 7 2} & \mathbf{- 1 . 9 7 3 7 7 9} & \mathbf{1 . 7 6 6 8 9 5}\end{array}$

$\begin{array}{llll}\text { S } & -1.731633 & 1.973779 & 1.76685 \\ N & 1.120079\end{array}$

N $-0.862007-1.203578-1.716187$

N $-0.862007-1.203578-1.716187$

$\begin{array}{llll}\mathrm{N} & 1.272872 & -0.995161 & -1.695466\end{array}$

$\begin{array}{llll}C & -2.253727 & -1.117077 & -1.420268\end{array}$

$\begin{array}{llll}C & -0.412137 & -1.578160 & -2.972494\end{array}$

$\begin{array}{llll}C & 0.931490 & -1.455936 & -2.956558\end{array}$

$\begin{array}{llll}C & 2.615084 & -0.653540 & -1.357381\end{array}$

$\begin{array}{llll}C & -4.991125 & -1.001739 & -0.844636\end{array}$

$\begin{array}{lllll}\text { C } & \mathbf{- 2 . 9 2 1 2 4 8} & \mathbf{- 2 . 2 1 9 4 4 6} & -0.895597\end{array}$

$\begin{array}{llll}\text { C }-2.962896 & 0.053204 & -1.718346\end{array}$

$\begin{array}{lll}C & -4327340 & 0.086\end{array}$

$\begin{array}{llll}C & -4.327340 & 0.086014 & -1.421270\end{array}$

C $-4.270600-2.173775-0.589222$

$\begin{array}{llll}\text { H } & -4.887522 & 0.998813 & -1.638132\end{array}$

$\begin{array}{llll}-4.747520 & \mathbf{- 3 . 0 5 9 6 2 4} & \mathbf{- 0 . 1 6 6 0 9 4}\end{array}$

$\begin{array}{llll}\text { C } & 5.269973 & -0.069231 & -0.681332\end{array}$

$\begin{array}{llll}C & 3.403938 & -1.574833 & -0.674652\end{array}$

$\begin{array}{llll}C & 3.166573 & 0.566274 & -1.767329\end{array}$

$\begin{array}{lllll}C & 4.492154 & 0.833980 & -1.412380\end{array}$

$\begin{array}{lllll}C & 4.711093 & -1.300263 & -0.316837\end{array}$

$\begin{array}{llll}\text { F } & 2.870733 & -2.761989 & -0.373859\end{array}$

C $2.359121 \quad 1.531362-2.588256$

$\begin{array}{llll}C & 2.359121 & 1.531362 & -2.588256\end{array}$

$\begin{array}{llll}\text { H } & 4.930149 & 1.788335 & -1.714190\end{array}$

$\begin{array}{lllll}\text { H } & 5.283674 & -2.051886 & \mathbf{0 . 2 2 9 5 7 4}\end{array}$

$\begin{array}{llll}\text { C } & 6.683771 & 0.262190 & -0.286094\end{array}$

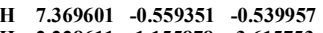

H $\quad \begin{array}{llll}2.228611 & 1.155878 & -3.615753\end{array}$

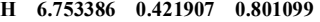

$\begin{array}{llll}\text { H } & 7.037009 & \mathbf{1 . 1 7 4 6 7 8} & \mathbf{- 0 . 7 8 4 2 4 7}\end{array}$

$\begin{array}{lllll}\text { H } & \mathbf{2 . 8 5 6 8 4 3} & \mathbf{2 . 5 0 7 7 1 2} & \mathbf{- 2 . 6 4 3 2 1 1}\end{array}$

$\begin{array}{llll}1.363723 & 1.677812-2.146646\end{array}$

\begin{tabular}{llll}
\hline & 1.363723 & 1.677812 & -2.146646
\end{tabular}

C $-6.450607-0.918769-0.486741$

$\begin{array}{llll}C & -6.450607 & -0.918769 & -0.486741\end{array}$

$\begin{array}{llll}\text { H } & -6.949565 & -0.099431 & -1.021719\end{array}$

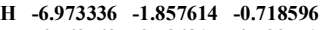

$\begin{array}{lllll}\text { H } & -6.569749 & -0.734815 & 0.592751\end{array}$

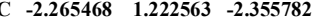

$\begin{array}{llll}\text { H } & -2.019668 & 1.004453 & -3.407418\end{array}$

$\begin{array}{lllll}\text { H } & -2.900113 & 2.117525 & -2.332057\end{array}$

$\begin{array}{llll}\text { H } & -1.325978 & 1.458902 & -1.835711\end{array}$

$\begin{array}{llll}C & 0.325978 & 1.458902 & -1.835711\end{array}$

$\begin{array}{llll}\text { C } & 0.111076 & 3.114415 & 0.539156 \\ \text { C } & \mathbf{0 . 4 5 2 6 6 6} & 4.449764 & \mathbf{0 . 2 2 8 1 5 3}\end{array}$ 
$\begin{array}{lrrr}C & -1.256144 & 2.802257 & 0.703960\end{array}$ $\begin{array}{llll}\text { C } & -\mathbf{0 . 5 2 2 4 1 2} & \mathbf{5 . 4 2 4 1 8 3} & \mathbf{0 . 0 7 9 4 0 9}\end{array}$ $\begin{array}{lllll}\text { H } & 1.507874 & 4.703741 & 0.098670\end{array}$ $\begin{array}{llll}\text { C } & -2.237554 & \mathbf{3 . 7 9 7 2 1 2} & \mathbf{0 . 5 4 8 2 7 4}\end{array}$ $\begin{array}{llll}C & -1.879307 & 5.101063 & \mathbf{0 . 2 3 8 3 6 7}\end{array}$ H $-0.230651-6.448730-0.164721$

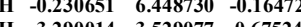
$\begin{array}{llll}\text { H } & -3.290014 & 3.529077 & 0.67524\end{array}$ $\begin{array}{llll}-2.647619 & 5.868160 & 0.118959\end{array}$ H $\quad \begin{array}{llll}2.228256 & -0.493212 & \mathbf{2 . 6 6 3 3 0}\end{array}$ $\begin{array}{llll}C & \mathbf{0 . 7 3 8 9 2 0} & -1.923447 & \mathbf{2 . 0 7 5 7 0 6}\end{array}$ $\begin{array}{llll}\text { H } & -\mathbf{0 . 8 9 7 5 1 0} & \mathbf{- 2 . 7 5 4 6 7 0} & \mathbf{1 . 0 1 4 6 0}\end{array}$ $\begin{array}{llll}C & -1.822843 & -2.048293 & 2.806017\end{array}$ $\begin{array}{llll}\text { C } & \mathbf{1 . 5 7 5 2 0 5} & -\mathbf{3 . 1 9 2 1 2 9} & \mathbf{2 . 2 2 2 2 4}\end{array}$ $\begin{array}{llll}\text { H } & 1.333712 & -3.634178 & 3.200891\end{array}$ $\begin{array}{llll}\text { H } & 1.326486 & \mathbf{3 . 9 2 3 2 8 2} & \mathbf{1 . 4 4 1 8 4 9}\end{array}$ $\begin{array}{llll}1.326486 & -3.923282 & 1.441849\end{array}$ $\begin{array}{llll}\text { H } & 2.653913 & -2.986215 & 2.189860\end{array}$ $\begin{array}{llll}\text { H } & \mathbf{1 . 1 8 0 5 4 8} & \mathbf{- 1 . 5 6 4 0 5 1} & \mathbf{0 . 9 5 7 7 6}\end{array}$ $\begin{array}{llll}\text { H } & -1.097656 & -1.897546 & -3.751129\end{array}$ $\begin{array}{llll}\text { H } & 1.681763 & -1.652382 & -3.715997\end{array}$ $\begin{array}{llll}C & 0.590804 & -0.092148 & 4.020339\end{array}$ $\begin{array}{llll}\text { H } & -2.799030 & -1.978054 & 2.30003\end{array}$

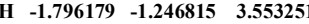
H $\quad-1.808978-3.010702 \quad 3.349979$ $\begin{array}{lllll}\text { H } & -0.496638 & 0.025910 & \mathbf{4} .030365\end{array}$ $\begin{array}{llll}\text { H } & 1.034082 & 0.897281 & 4.196792\end{array}$ $\begin{array}{lrrrr}H & 0.870997 & -0.744584 & 4.867034\end{array}$ 72

unsat_mcb_dist_a / electronic energy: $\mathbf{- 2 4 0 0 . 5 3 6 7 9 8 1 8 ~ a . u . ~ / ~ l o w e s t ~ f r e q : ~} 15.20 \mathrm{~cm}-1$ $\begin{array}{llll}\text { Ru } & 0.349539 & -\mathbf{0 . 6 4 4 0 3 9} & \mathbf{0 . 3 0 5 0 5 8}\end{array}$ S $-0.546226-2.047449-1.433090$

$\begin{array}{llll}C & -2.232777 & -2.180830 & -0.97393\end{array}$

C $-\mathbf{2 . 6 8 0 1 1 3} \quad-\mathbf{1 . 6 6 8 5 5 3} \quad 0.256505$

$\begin{array}{lrrr}\text { S } & -1.565469 & -0.865463 & 1.366002\end{array}$

$\begin{array}{llll}C & 0.593734 & 1.252817 & -0.308537\end{array}$

$\begin{array}{llll}\mathrm{N} & \mathbf{- 0 . 3 3 4 9 1 0} & \mathbf{2 . 2 2 8 8 1 9} & \mathbf{- 0 . 4 8 7 8 4 2}\end{array}$

N $1.764387 \quad 1.818060 \quad-0.714455$

$\begin{array}{lllll}C & \mathbf{0 . 2 5 4 2 0 0} & \mathbf{3 . 3 8 8 6 6 1} & \mathbf{- 0 . 9 8 0 3 4}\end{array}$

$\begin{array}{llll}C & 1.570907 & 3.132522 & -1.122348\end{array}$

$\begin{array}{lllll}C & -1.744695 & 2.076355 & -0.33327\end{array}$

$\begin{array}{llll}\text { C } & -\mathbf{2 . 3 5 3 9 1 6} & \mathbf{2 . 4 8 9 3 4 6} & \mathbf{0 . 8 4 5 2 6 5}\end{array}$

$\begin{array}{llll}C & -2.517836 & 1.517447 & -1.362879\end{array}$

$\begin{array}{llll}C & -3.706576 & \mathbf{2 . 2 9 4 7 2 3} & \mathbf{1 . 0 7 3 4 0 6}\end{array}$

$\begin{array}{llll}\text { C } & -3.878920 & 1.328155 & -1.125232\end{array}$

$\begin{array}{llll}\text { C } & -4.484587 & 1.689369 & 0.083900\end{array}$

$\begin{array}{llll}\text { H } & -4.133981 & 2.610128 & 2.026673\end{array}$

$\begin{array}{llll}\text { H } & -4.487902 & 0.866578 & -1.906607\end{array}$

$\begin{array}{llll}C & -5.950097 & \mathbf{1 . 4 2 8 5 2 0} & \mathbf{0 . 3 0 1 4 5}\end{array}$

$\begin{array}{llll}\text { F } & -1.606863 & 3.073822 & 1.783812\end{array}$

$\begin{array}{llll}\text { C } & -1.888269 & 1.124977 & -2.669150\end{array}$

H $-1.308895 \quad 0.195772 \quad-2.549293$

$\begin{array}{llll}\text { H } & -2.657181 & 0.952472 & \mathbf{- 3 . 4 3 3 1 2 9}\end{array}$

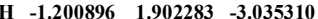

$\begin{array}{llll}\text { C } & \mathbf{2 . 9 9 3 9 2 1} & \mathbf{1 . 1 0 8 4 6 3} & \mathbf{- 0 . 8 2 4 7 6 5}\end{array}$

$\begin{array}{llll}C & 3.981412 & 1.280653 & 0.144012\end{array}$

$\begin{array}{llll}C & 3.212435 & 0.222640 & -1.892338\end{array}$

$\begin{array}{llll}\text { C } & \mathbf{5 . 1 4 6 1 6 9} & \mathbf{0 . 5 3 2 4 2 5} & \mathbf{0 . 1 3 8 0 6 7}\end{array}$

$\begin{array}{llll}C & 4.393471 & -0.522970 & -1.892872\end{array}$

$\begin{array}{llll}C & 5.355618 & -0.400245 & -0.883222\end{array}$

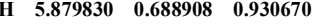

H $4.568153 \quad-1.225516 \quad-2.711318$

$\begin{array}{llll}\text { C } & 6.587352 & -1.264115 & -0.887910\end{array}$

$\begin{array}{llll}C & 2.194436 & 0.076412 & -2.989522\end{array}$

$\begin{array}{llll}\text { H } & 1.328025 & -0.516481 & -2.655703\end{array}$

$\begin{array}{llll}\text { H } & \mathbf{1 . 8 0 8 1 5 9} & \mathbf{1 . 0 5 5 4 8 8} & \mathbf{- 3 . 3 0 8 9 7 1}\end{array}$

$\begin{array}{llll}\text { H } & \mathbf{2 . 6 3 4 3 2 6} & \mathbf{- 0 . 4 2 5 6 9 2} & \mathbf{- 3 . 8 6 0 3 3 3}\end{array}$

$\begin{array}{llll}3.787261 & 2.183610 & 1.104547\end{array}$

$\begin{array}{llll}\text { C } & 1.898847 & -1.731364 & 1.036864\end{array}$

$\begin{array}{llll}\text { H } & \mathbf{2 . 5 7 9 3 4 1} & \mathbf{- 1 . 2 3 3 1 3 7} & \mathbf{0 . 3 0 4 2 4 0}\end{array}$

$\begin{array}{llll}\text { C } & 1.258278 & 0.166782 & 2.100183\end{array}$

$\begin{array}{llll}\text { C } & 2.040742 & -1.122234 & 2.41773\end{array}$

$\begin{array}{llll}\text { H } & 1.978679 & 0.935604 & 1.792647\end{array}$

$\begin{array}{llll}\text { C } & \mathbf{0 . 4 2 4 3 6 3} & \mathbf{0 . 7 7 2 1 9 8} & \mathbf{3 . 2 1 5 5 9 4}\end{array}$

$\begin{array}{llll}\text { H } & 3.102612 & -0.926614 & 2.67511\end{array}$

$\begin{array}{llll}C & \mathbf{1 . 4 4 4 1 9 5} & -\mathbf{1 . 9 8 5 3 8 7} & \mathbf{3 . 5 2 3 3 7 9}\end{array}$

$\begin{array}{llll}C & -3.173678 & -2.790658 & -1.827696\end{array}$

$\begin{array}{llll}\text { C } & -4.511607 & -2.878565 & -1.469659\end{array}$

$\begin{array}{llll}\text { H } & \mathbf{- 5 . 2 2 5 4 2 6} & \mathbf{- 3 . 3 4 7 5 5 8} & \mathbf{- 2 . 1 5 0 9 7 0}\end{array}$

$\begin{array}{lllll}\text { C } & -4.946601 & -2.367457 & -0.237960\end{array}$

$\begin{array}{llll}\text { H } & \mathbf{- 5 . 9 9 9 0 3 8} & \mathbf{- 2 . 4 3 6 3 9 0} & \mathbf{0 . 0 4 6 6 6 9}\end{array}$

$\begin{array}{llll}\text { C } & -4.033360 & -1.768625 & 0.618038\end{array}$

$\begin{array}{llll}\text { H } & -4.362802 & -1.351427 & 1.573620\end{array}$

$\begin{array}{lll}-2.832675 & -3.190187 & -2.786201\end{array}$

$\begin{array}{lll}-2.832675 & -3.190187 & -2.786201\end{array}$

$\begin{array}{llll}-6.562983 & 2.027196 & -0.389825\end{array}$

\begin{tabular}{llll} 
H & -6.256964 & 1.670741 & 1.327693 \\
\hline & -6.178642 & 0.369028 & 0.111644
\end{tabular}

$\begin{array}{llll}\text { H } & -6.178642 & 0.369028 & 0.111644\end{array}$

$\begin{array}{lllll}\text { H } & 7.428743 & -0.770507 & -0.382913\end{array}$

H $6.892603 \quad-1.517496 \quad-1.912398$

H $6.389512 \quad-2.209606 \quad-0.358356$

$\begin{array}{llll}\text { H } & 0.387323 & -2.218675 & 3.315716\end{array}$

H $\quad 1.491032-1.465966 \quad 4.491665$

$\begin{array}{llll}\text { H } & 1.989232 & -2.935550 & \mathbf{3 . 6 2 9 8 6 8}\end{array}$

$\begin{array}{lll}1.981421 & 1.670372 & 2.863853\end{array}$

$\begin{array}{rrrr}\text { H } & -0.101421 & 1.670372 & 2.863853 \\ \text { H } & 1.061046 & \mathbf{1 . 0 7 1 3 0 4} & 4.069275\end{array}$ 
$\begin{array}{llll}\text { H } & \mathbf{- 0 . 3 3 8 4 2 8} & \mathbf{0 . 0 8 2 2 8 5} & \mathbf{3 . 6 0 6 5 8 2}\end{array}$ $\begin{array}{llll}C & 1.923350 & -3.232935 & 0.852890\end{array}$ $\begin{array}{lllll}\text { H } & -0.330148 & 4.281193 & -1.180737\end{array}$ $\begin{array}{lllll}\text { H } & \mathbf{2 . 3 9 2 2 9 4} & \mathbf{3 . 7 4 9 7 4 2} & -1.472493\end{array}$ $\begin{array}{llll}\text { H } & 1.867601 & \mathbf{3 . 5 1 1 8 9 2} & -0.206947\end{array}$ H $1.091433-3.722322-1.379046$ \begin{tabular}{llll}
\hline & 1.091433 & -3.722322 & 1.379046
\end{tabular} \begin{tabular}{lllll}
\hline & 2.862506 & -3.635984 & 1.274360
\end{tabular} 72

unsat_ts_PTR_a / electronic energy: $-\mathbf{2 4 0 0 . 5 0 5 1 9 8 1 3 ~ a . u . ~ / ~ l o w e s t ~ f r e q : ~}-\mathbf{1 2 1 . 2 8} \mathrm{cm}-1$ Ru $\quad 0.227229 \quad-0.854661 \quad-0.102251$

$\begin{array}{llll}\mathrm{S} & -\mathbf{1 . 1 2 1 5 5 5} & -\mathbf{0 . 1 0 8 8 8 4} & -1.809317\end{array}$

$\begin{array}{llll}C & -2.693916 & -0.847606 & -1.560973\end{array}$

$\begin{array}{llll}\text { C } & -2.847736 & -1.832451 & -0.569770\end{array}$

$\begin{array}{llll}\mathrm{S} & -\mathbf{1 . 5 0 8 5 4 5} & \mathbf{- 2 . 2 6 8 6 6 9} & \mathbf{0 . 4 6 7 5 6 6}\end{array}$

$\begin{array}{llll}C & 0.668660 & 1261783 & 0.074906\end{array}$

$\begin{array}{llll}\mathrm{C} & 0.668660 & 1.261783 & 0.074906\end{array}$

$\begin{array}{lrrr}\mathrm{N} & -0.212481 & 2.223506 & 0.459086\end{array}$

$\begin{array}{llll}\mathrm{N} & \mathbf{1 . 8 4 5 9 6 6} & 1.927405 & \mathbf{- 0 . 0 4 1 3 8 6} \\ \mathrm{C} & \mathbf{0 . 4 0 7 8 8 7} & 3.458149 & \mathbf{0 . 5 8 8 3 5 7}\end{array}$

$\begin{array}{lllll}\mathrm{C} & 0.407887 & 3.458149 & 0.588357\end{array}$

$\begin{array}{llll}\text { C } & 1.706389 & 3.274131 & 0.271503\end{array}$

C $\begin{array}{llll}\text { C } & -1.607792 & \mathbf{2 . 0 2 3 8 3 3} & \mathbf{0 . 6 8 2 3 8 2}\end{array}$

$\begin{array}{llll}C & -2.024829 & 1.355999 & 1.829267\end{array}$

$\begin{array}{llll}C & -2.559813 & 2.476474 & \mathbf{- 0 . 2 4 3 2 3 8}\end{array}$

$\begin{array}{llll}\text { C } & \mathbf{- 3 . 3 5 6 1 0 9} & \mathbf{1 . 0 4 1 7 6 2} & \mathbf{2 . 0 4 8 8 8 1}\end{array}$

$\begin{array}{llll}\text { C } & -3.899541 & 2.170197 & -0.005049\end{array}$

$\begin{array}{llll}C & -4.313205 & 1.433270 & 1.110986\end{array}$

\begin{tabular}{llll}
$\mathrm{H}$ & $\mathbf{- 3 . 6 2 6 8 0 8}$ & $\mathbf{0 . 4 8 7 9 3 8}$ & $\mathbf{2 . 9 4 9 3 6 5}$ \\
\hline
\end{tabular}

$\begin{array}{llll}\text { H } & -4.646731 & \mathbf{2 . 4 9 2 9 6 5} & \mathbf{- 0 . 7 3 4 0 1 3}\end{array}$

$\begin{array}{llll}\text { C } & -5.753992 & 1.032064 & 1.270833\end{array}$

$\begin{array}{llll}\text { F } & -1.113478 & 1.016092 & 2.740271\end{array}$

C $-\mathbf{- 2 . 1 4 4 8 3 0} \quad \mathbf{3 . 2 6 0 4 1 1}-\mathbf{- 1 . 4 5 6 9 0 2}$

$\begin{array}{llll}\text { H } & -1.256787 & 2.814039 & -1.925607\end{array}$

$\begin{array}{lllll}\text { H } & -2.952438 & 3.279347 & -2.200129\end{array}$

H $-1.904916 \quad 4.302826 \quad-1.193532$

$\begin{array}{llll}\text { C } & 3.091419 & 1.334326 & -0.392424\end{array}$

$\begin{array}{llll}C & 4.062231 & 1.194042 & 0.599551\end{array}$

$\begin{array}{llll}C & 3.348313 & 0.870153 & -1.689250\end{array}$

$\begin{array}{llll}\text { C } & \mathbf{5 . 2 7 4 0 7 2} & \mathbf{0 . 5 7 2 2 7 3} & \mathbf{0 . 3 5 7 0 4 6}\end{array}$

$\begin{array}{lllll}C & 4.571541 & 0.233961 & -1.921967\end{array}$

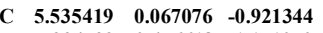

H $\quad 5.994592 \quad 0.476618 \quad 1.171050$

$\begin{array}{llll}\text { H } & 4.779884 & -0.141558 & -2.926894\end{array}$

$\begin{array}{llll}C & 6.819421 & -0.664851 & -1.204537\end{array}$

$\begin{array}{llll}C & 2.331604 & 1.026486 & -2.784008\end{array}$

\begin{tabular}{ccccc} 
H & 1.558669 & 0.245860 & -2.706278 \\
\hline & 1.817055 & 1.995913 & -2.720292
\end{tabular}

\begin{tabular}{lllll}
$H$ & 1.817055 & 1.995913 & -2.720292 \\
\hline
\end{tabular}

$\begin{array}{lllll}\text { H } & \mathbf{2 . 8 0 6 5 6 5} & \mathbf{0 . 9 4 2 8 2 8} & \mathbf{- 3 . 7 6 9 7 6 4}\end{array}$

$\begin{array}{cccc}\text { F } & \mathbf{3 . 7 8 7 3 4 8} & \mathbf{1 . 6 4 2 1 2 4} & \mathbf{1 . 8 2 5 9 6 1} \\ \text { C } & \mathbf{1 . 4 1 3 8 4 8} & \mathbf{- 2 . 4 7 9 4 6 9} & \mathbf{- 0 . 2 0 2 0 8 5}\end{array}$

$\begin{array}{llll}C & 1.413848 & -2.479469 & -0.202085 \\ \text { H } & 1.923803 & -2.406867 & -1.177569\end{array}$

$\begin{array}{llll}\text { C } & \mathbf{1 . 1 0 7 9 5 3} & \mathbf{- 0 . 9 3 2 1 4 4} & \mathbf{1 . 7 7 2 4 2 4}\end{array}$

$\begin{array}{llll}C & 2.170808 & -1.652791 & 0.895041\end{array}$

$\begin{array}{llll}\text { H } & \mathbf{1 . 4 8 8 2 8 2} & \mathbf{0 . 0 3 9 6 1 0} & \mathbf{2 . 1 1 5 9 6 1}\end{array}$

$\begin{array}{llll}C & 0.545905 & -1.697158 & 2.951191\end{array}$

C $2.503241-0.912713 \quad 0.395260$

\begin{tabular}{cccc} 
H & $\mathbf{2 . 8 0 3 2 4 1}$ & $\mathbf{- 0 . 9 1 2 7 1 3}$ & $\mathbf{0 . 3 9 5 2 6 0}$ \\
\hline C & 3.147496 & -2.512948 & $\mathbf{1 . 7 1 4 9 1 2}$
\end{tabular}

$\begin{array}{llll}\text { C } & 3.147496 & -2.512948 & 1.714912\end{array}$

$\begin{array}{llll}C & \mathbf{- 3 . 8 0 1 3 7 2} & \mathbf{- 0 . 4 4 6 0 8 9} & \mathbf{- 2 . 3 2 6 4 6 0}\end{array}$

$\begin{array}{llll}C & -5.042184 & -1.040105 & -2.139312\end{array}$

$\begin{array}{lllll}\text { H } & \mathbf{- 5 . 8 9 6 4 0 5} & \mathbf{- 0 . 7 2 3 5 3 5} & \mathbf{- 2 . 7 4 2 0 3 6}\end{array}$

$\begin{array}{llll}C & -5.191980 & -2.045948 & -1.173055\end{array}$

$\begin{array}{llll}\text { H } & -6.164950 & -2.519450 & -1.021465\end{array}$

$\begin{array}{llll}\text { C } & -4.108547 & -2.437687 & -0.399000\end{array}$

$\begin{array}{llll}\text { H } & -4.227521 & \mathbf{- 3 . 2 1 3 4 7 7} & \mathbf{0 . 3 6 1 9 6 4}\end{array}$

\begin{tabular}{llll}
\hline & -3.672780 & 0.341015 & -3.074218
\end{tabular}

$\begin{array}{llll}H & -6.432911 & 1.300460 & 0.874958\end{array}$

$\begin{array}{llll} & 0.800400 & 0.87458\end{array}$

$\begin{array}{llll}H & -5.940829 & 0.101611 & 0.7350\end{array}$

$\begin{array}{rrrrr}\text { H } & \mathbf{- 5 . 6 3 1 7 6 7} & \mathbf{- 0 . 3 2 0 4 3 7} & \mathbf{- 0 . 5 5 0 2 9 7}\end{array}$

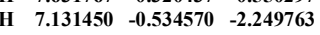

$\begin{array}{lllll}\text { H } & 6.687152 & -1.744523 & -1.030168\end{array}$

$\begin{array}{lllll}\text { H } & 2.633239 & -3.307154 & 2.271753\end{array}$

$\begin{array}{llll}\text { H } & 3.679161 & -1.873624 & 2.435036\end{array}$

$\begin{array}{llll}H & 3.889450 & -2.978178 & 1.049238\end{array}$

\begin{tabular}{llll}
\hline & 3.889450 & -2.978178 & 1.049238 \\
\hline & -0.294286 & -1.141688 & 3.387515
\end{tabular}

\begin{tabular}{llll} 
H & -0.294286 & -1.141688 & 3.387515 \\
\hline & -1.308019 & -1.817494 & 3.741754
\end{tabular}

\begin{tabular}{lllll} 
H & 1.308019 & -1.817494 & 3.741754 \\
\hline
\end{tabular}

$\begin{array}{lllll}\text { H } & \mathbf{0 . 1 7 8 3 4 3} & -2.697735 & \mathbf{2 . 6 8 4 4 0 9} \\ \text { C } & \mathbf{1 . 1 0 9 0 1 0} & -\mathbf{3 . 9 2 3 6 6 1} & \mathbf{0 . 1 2 0 7 2 3}\end{array}$

$\begin{array}{rrrrr}\text { C } & \mathbf{1 . 1 0 9 0 1 0} & \mathbf{- 3 . 9 2 3 6 6 1} & \mathbf{0 . 1 2 0 7 2 3} \\ \mathbf{H} & \mathbf{- 0 . 1 3 6 6 1 0} & \mathbf{4 . 3 4 5 4 4 8} & \mathbf{0 . 8 9 5 7 3 7}\end{array}$

$\begin{array}{lllll}\text { H } & 2.544057 & 3.963651 & 0.236571\end{array}$

$\begin{array}{lllll}\text { H } & 0.432549 & -4.339474 & -0.640021\end{array}$

$\begin{array}{llll}\text { H } & 0.627180 & -4.056869 & 1.098045\end{array}$

\begin{tabular}{llll}
\hline & 2.035445 & -4.523243 & 0.114799
\end{tabular}

H

unsat 14e1 / electronic energy: -2243.45002381 a.u. / lowest freq: $14.79 \mathrm{~cm}-1$

$\begin{array}{llll}S & 0.867262 & -1.380021 & 1.240326\end{array}$

$\begin{array}{llll}C & -0.809241 & 1.071706 & 0.304350\end{array}$

$\begin{array}{llll}\text { Ru } & -0.000196 & -0.541032 & -0.693992\end{array}$

$\begin{array}{llll}C & -1.420298 & -1.627816 & -0.816080\end{array}$

$\begin{array}{lllll}\text { S } & 1.802999 & -1.479683 & -1.833131\end{array}$

\begin{tabular}{llll} 
N & -2.061694 & 1.367141 & 0.737925 \\
\hline
\end{tabular}

$\begin{array}{llll}\text { N } & -0.070108 & 2.154993 & 0.662993\end{array}$

$\begin{array}{llll}\text { C } & \mathbf{- 3 . 2 2 5 9 8 1} & \mathbf{0 . 5 7 9 7 2 3} & \mathbf{0 . 5 1 1 8 5 6}\end{array}$

C $-2.101942 \quad 2.613900 \quad 1349326$

$\begin{array}{llll}C & -2.101942 & 2.613900 & 1.349326 \\ \text { C } & -\mathbf{0 . 8 4 6 9 6 8} & \mathbf{3 . 1 1 1 0 5 6} & \mathbf{1 . 2 9 9 7 5 1}\end{array}$ 
$\begin{array}{llll}\text { C } & 1.309987 & 2.321044 & 0.342302\end{array}$ $\begin{array}{llll}C & -5.555172 & -0.877152 & -0.034960\end{array}$

$\begin{array}{llll}\text { C } & -4.040871 & 0.905715 & -0.570347\end{array}$

$\begin{array}{llll}\text { C } & -\mathbf{3 . 5 7 1 6 8 0} & -\mathbf{0 . 4 8 5 1 4 1} & \mathbf{1 . 3 5 1 9 7 7}\end{array}$

$\begin{array}{llll}C & -4.733507 & -1.202752 & 1.050629\end{array}$

C $-5.198544 \quad 0.201518-0.852182$

$\begin{array}{llll}C & -5.198544 & 0.201518 & -0.852182\end{array}$

$\begin{array}{llll}C & -5.008869 & -2.043777 & 1.691754\end{array}$

$\begin{array}{lll}-5.799992 & 0.490606 & -1.716002\end{array}$

$\begin{array}{llll}C & 3.990983 & 2.627124 & -0.41220\end{array}$

$\begin{array}{llll}C & 1.658880 & 2.625019 & -0.974225\end{array}$

$\begin{array}{llll}\text { C } & 2.309122 & 2.185053 & 1.315135\end{array}$

$\begin{array}{llll}\text { C } & 3.637874 & 2.341633 & 0.911170\end{array}$

$\begin{array}{llll}\text { C } & \mathbf{2 . 9 7 7 5 0 8} & \mathbf{2 . 7 7 0 8 2 7} & \mathbf{- 1 . 3 6 5 3 2 7}\end{array}$

$\begin{array}{llll}\text { F } & 0.685915 & 2.758647 & -1.878503\end{array}$

$\begin{array}{llll}\text { C } & \mathbf{1 . 9 5 3 9 9 0} & \mathbf{1 . 8 5 2 2 1 5} & \mathbf{2 . 7 3 5 9 7 4}\end{array}$

$\begin{array}{llll}\text { H } & 4.426720 & 2.227048 & 1.658410\end{array}$

$\begin{array}{llll}\text { H } & 3.199333 & 2.990426 & -2.411063\end{array}$

$\begin{array}{llll}\text { C } & \mathbf{5 . 4 3 1 2 8 2} & \mathbf{2 . 8 0 1 2 0 2} & \mathbf{- 0 . 8 1 1 7 6 9}\end{array}$

$\begin{array}{lllll}\text { H } & \mathbf{5 . 6 5 6 5 5 7} & \mathbf{3 . 8 6 5 3 9 8} & \mathbf{- 0 . 9 8 4 5 8 4}\end{array}$

$\begin{array}{lllll}\text { H } & \mathbf{2 . 8 5 3 4 6 4} & \mathbf{1 . 8 1 0 7 5 6} & \mathbf{3 . 3 6 3 3 3 2}\end{array}$

$\begin{array}{llll}\text { H } & 5.649375 & 2.264363 & -1.746017\end{array}$

$\begin{array}{lllll}\text { H } & 6.112294 & 2.433665 & -0.03280\end{array}$

$\begin{array}{lllll}\text { H } & 1.456552 & 0.870164 & 2.771475\end{array}$

$\begin{array}{lllll}\text { H } & 1.266506 & \mathbf{2 . 5 9 4 6 1 0} & \mathbf{3 . 1 6 8 0 7 7}\end{array}$

$\begin{array}{llll}\text { F } & -3.672365 & 1.915311 & -1.359911\end{array}$

$\begin{array}{llll}C & -6.813726 & -1.653752 & -0.314145\end{array}$

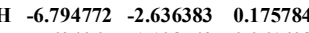

$\begin{array}{llll}\text { H } & -7.694065 & -1.108743 & 0.061608\end{array}$

$\begin{array}{lllll}H & -6.957531 & -1.805000 & -1.393237\end{array}$

$\begin{array}{llll}\text { C } & -2.698026 & -0.847079 & 2.518461\end{array}$

$\begin{array}{llll}\text { H } & -1.750683 & -1.287959 & 2.168984\end{array}$

$\begin{array}{llll}\text { H } & -2.439475 & 0.040988 & 3.114152\end{array}$

$\begin{array}{llll}\text { H } & -3.196846 & -1.573242 & 3.172604\end{array}$

$\begin{array}{llll}C & 2.296496 & -2.304383 & 0.759021\end{array}$

$\begin{array}{llll}C & 2.296406 & -2.304383 & 0.759021\end{array}$

$\begin{array}{llll}C & 3.043581 & -2.984396 & 1.734242\end{array}$

$\begin{array}{llll}\text { C } & 2.700211 & -2.360499 & -0.585950\end{array}$

$\begin{array}{llll}\text { C } & 4.174157 & \mathbf{- 3 . 7 1 4 1 3 1} & \mathbf{1 . 3 8 2 6 2 7}\end{array}$

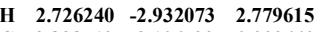

$\begin{array}{lllll}\text { C } & 3.838712 & -3.106799 & -0.929442\end{array}$

$\begin{array}{llll}C & 4.573374 & -3.777421 & 0.043245\end{array}$

$\begin{array}{llll}\text { H } & 4.744915 & -4.236769 & 2.153792\end{array}$

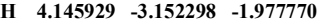

$\begin{array}{llll}\text { H } & \mathbf{5 . 4 5 8 8 8 8} & \mathbf{- 4 . 3 5 0 0 8 5} & \mathbf{- 0 . 2 4 2 1 5 6}\end{array}$

H $\quad-1.641841-2.421866-0.081616$

$\begin{array}{llll}C & -2.273172 & -1.625134 & -2.048811\end{array}$

\begin{tabular}{llll}
\hline & -2.116013 & -2.561468 & -2.611110
\end{tabular}

$\begin{array}{lllll}\text { H } & \mathbf{- 3 . 3 3 9 4 1 8} & \mathbf{- 1 . 5 8 5 1 3 3} & -\mathbf{1 . 7 7 2 4 3 0}\end{array}$

$\begin{array}{llll}\text { H } & -2.060630 & -\mathbf{0 . 7 7 7 4 4 4} & \mathbf{- 2 . 7 2 2 1 1 9}\end{array}$

$\begin{array}{llll}\text { H } & -\mathbf{3 . 0 2 1 3 2 7} & \mathbf{3 . 0 2 4 9 0 0} & \mathbf{1 . 7 5 4 4 8 6}\end{array}$

$\begin{array}{lllll}\text { H } & -0.432398 & 4.051746 & 1.648884\end{array}$

72

unsat_pc1_a / electronic energy: -2400.53840061 a.u. / lowest freq: $13.88 \mathrm{~cm}-1$

$\begin{array}{llll}S & 0.313188 & 1.609144 & -1.337245\end{array}$

C $0.059936-1.269959-0.566042$

C $0.0599360 .1 .26959-0.566042$

Cu $10.0166080 .425351 \quad 0.658720$

$\begin{array}{llll}\text { C } & 1.727316 & 0.407309 & 1.222997\end{array}$

$\begin{array}{llll}C & -1.342357 & -0.832505 & 2.06141\end{array}$

$\begin{array}{llll}\text { S } & -\mathbf{0 . 7 4 0 4 3 5} & 2.491929 & 1.568379\end{array}$

$\begin{array}{llll}N & -0.988014 & -1.879342 & -1.200019\end{array}$

N $1.149878-1.910698-1.077945$

$\begin{array}{llll}C & \mathbf{- 2 . 3 8 1 0 7 9} & \mathbf{- 1 . 6 8 7 2 2 6} & \mathbf{- 0 . 9 4 4 6 4 1}\end{array}$

$\begin{array}{llll}C & -0.554603 & -2.870812 & -2.070097\end{array}$

C $\quad 0.790316 \quad-2.892429-1.991702$

$\begin{array}{llll}C & 2.509727 & -1.718355 & -0.700931\end{array}$

$\begin{array}{llll}C & -5.122989 & -1.508253 & -0.345611\end{array}$

$\begin{array}{llll}C & -\mathbf{3 . 0 0 6 3 1 2} & \mathbf{- 2 . 5 9 8 5 3 3} & \mathbf{- 0 . 0 8 6 1 8 8}\end{array}$

$\begin{array}{llll}C & -3.006312 & -2.598533 & -0.086188 \\ C & -3.154948 & -0.710098 & -1.580973\end{array}$

$\begin{array}{llll}C & -4.515164 & -0.633717 & -1.246814\end{array}$

$\begin{array}{llll}\text { C } & -4.346883 & -2.522370 & 0.230388\end{array}$

$\begin{array}{llll}\text { H } & -5.122121 & 0.135405 & -1.731062\end{array}$

$\begin{array}{lllll}\text { H } & -4.771802 & -3.249772 & 0.924179\end{array}$

$\begin{array}{llll}\text { C } 5.189781 & -1.498366 & 0.102205\end{array}$

C $2.955939-2.280276 \quad 0.494520$

$\begin{array}{llll}C & 2.955939 & -2.280276 & 0.494520 \\ C & 3.419552 & -1.058618 & -1.53789\end{array}$

C $3.419552-1.058618-1.53$

C $4.748562-1.0$

$\begin{array}{llll}\text { C } & 4.269847 & -2.171765 & 0.913665\end{array}$

$\begin{array}{llll}\text { F } & 2.073614 & -2.922215 & 1.263996\end{array}$

$\begin{array}{llll}C & 2.973946 & \mathbf{- 0 . 4 6 3 7 4 9} & \mathbf{- 2 . 8 4 3 0 4 5}\end{array}$

$\begin{array}{llll}\text { H } & 5.464067 & -0.443477 & -1.754395\end{array}$

$\begin{array}{lllll}\text { H } & 4.561867 & -2.616715 & 1.866481\end{array}$

$\begin{array}{llll}\text { C } \quad 6.616907 & -1.335987 & 0.550786\end{array}$

C $7.003422-2.2634390 .996803$

$\begin{array}{lllll}\mathrm{H} & 7.003422 & -2.263439 & 0.996803\end{array}$

$\begin{array}{llll}\text { H } & 2.532399 & -1.224434 & \mathbf{3 . 5 0 4 3 6 9}\end{array}$

$\begin{array}{llll}\text { H } & 6.689360 & -0.548627 & 1.317638\end{array}$

$\begin{array}{llll}\text { H } & 7.271146 & -1.051285 & -0.284006\end{array}$

$\begin{array}{llll}\text { H } & 3.820600 & -\mathbf{0 . 0 0 3 9 4 1} & \mathbf{- 3 . 3 6 8 4 1 3}\end{array}$

$\begin{array}{llll}\text { H } & 2.208325 & 0.308529 & -2.668510\end{array}$

$\begin{array}{llll}\text { F } & -2.261292 & -3.568454 & 0.454929\end{array}$

$\begin{array}{llll}\text { C } & \mathbf{- 6 . 5 7 5 7 9 1} & -\mathbf{1 . 3 6 8 7 1 0} & \mathbf{0 . 0 1 8 4 5 3}\end{array}$

$\begin{array}{llll}\text { H } & -7.120548 & -0.766502 & -0.720824\end{array}$

$\begin{array}{llll}\text { H } & -7.063439 & -2.350514 & 0.099192\end{array}$

$\begin{array}{llll}\text { H } & -6.676802 & -0.870964 & 0.995948\end{array}$

$\begin{array}{rrrr}C & -2.593153 & 0.215830 & -2.620694\end{array}$ 
$\begin{array}{llll}\text { H } & -1.625524 & -0.130971 & -3.002915\end{array}$

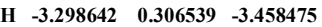
$\begin{array}{llll}\text { H } & -2.425854 & 1.217209 & -2.199705\end{array}$

$\begin{array}{llll}\text { C } & \mathbf{0 . 0 7 9 3 6 7} & \mathbf{3 . 3 1 0 5 9 2} & \mathbf{- 0 . 9 4 3 5 3 0}\end{array}$

C $\quad 0.342758 \quad 4.297801 \quad-1.908963$

$\begin{array}{llll}C & 0.342758 & 4.297801 & -1.908963\end{array}$

$\begin{array}{llll}\text { C } & -0.378960 & 3.694618 & 0.330167 \\ \text { C } & 0.151528 & 5.644983 & -1.624063\end{array}$

$\begin{array}{llll}C & 0.151528 & 5.644983 & -1.624063\end{array}$

$\begin{array}{lllll}\text { H } & 0.702703 & 3.990095 & -2.894662\end{array}$

$\begin{array}{llll}C & -0.564151 & \mathbf{5 . 0 6 0 8 5 9} & \mathbf{0 . 6 0 6 3 7 1}\end{array}$

$\begin{array}{llll}C & -0.305064 & 6.028562 & -0.357729\end{array}$

$\begin{array}{lllll}\text { H } & 0.361158 & 6.398358 & -2.386997\end{array}$

$\begin{array}{llll}\text { H } & -0.919084 & 5.355242 & 1.597789\end{array}$

$\begin{array}{llll}\text { H } & -0.456909 & 7.084848 & -0.123554\end{array}$

$\begin{array}{llll}\text { C } & \mathbf{2 . 7 6 8 5 5 4} & \mathbf{1 . 4 4 2 1 7 1} & \mathbf{0 . 9 6 1 0 5 9}\end{array}$

$\begin{array}{llll}\text { H } & 2.108620 & -0.338083 & 1.942682\end{array}$

$\begin{array}{lrll}C & -0.177600 & -0.600145 & \mathbf{2 . 7 6 3 6 4 4}\end{array}$

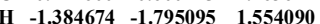

$\begin{array}{llll}\text { C } & -\mathbf{2 . 6 8 5 7 0 8} & \mathbf{- 0 . 2 0 2 3 6 7} & \mathbf{2 . 3 1 5 9 6 1}\end{array}$

$\begin{array}{llll}\text { H } & -3.224923 & -0.022013 & 1.374034\end{array}$

$\begin{array}{llll}\text { H } & -3.300712 & -0.896024 & 2.914397\end{array}$

$\begin{array}{llll}\text { H } & -2.611736 & 0.751513 & 2.851746\end{array}$

$\begin{array}{llll}\text { H } & 3.730224 & 0.952022 & 0.730549\end{array}$

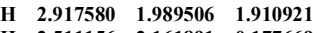

H $2.511156 \quad 2.161891 \quad 0.177669$

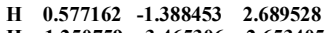

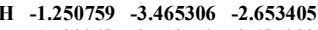

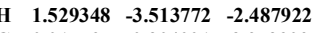

$\begin{array}{llll}C & \mathbf{- 0 . 0 1 7 5 2 5} & \mathbf{0 . 3 0 4 0 0 1} & \mathbf{3 . 9 5 3 2 0 9}\end{array}$

$\begin{array}{lllll}\text { H } & -0.154920 & -0.283554 & 4.876601\end{array}$

$\begin{array}{lllll}\text { H } & 0.992036 & 0.739727 & 3.989013\end{array}$

H $\quad-\mathbf{0 . 7 4 0 4 3 9} \quad 1.128756 \quad 3.963001$

72

unsat_ts1_a / electronic energy: $-\mathbf{2 4 0 0 . 5 2 9 9 4 6 9 8}$ a.u. / lowest freq: $-\mathbf{1 2 2 . 2 1} \mathrm{cm}-1$

$\begin{array}{llll}S & -\mathbf{0 . 6 8 2 5 5 5} & 1.438085 & -1.302558\end{array}$

$\begin{array}{llll}C & 0.100478 & -1.338015 & -0.443561\end{array}$

C $0.100478-1.338015-0.443561$

C $1.590591-0.397282 \quad 1.951440$

$\begin{array}{lrrr}\text { C } & \mathbf{1 . 5 9 0 5 9 1} & \mathbf{- 0 . 3 9 7 2 8 2} & \mathbf{1 . 9 5 1 4 4 0}\end{array}$

$\begin{array}{llll}\text { C } & -1.402942 & -0.086741 & 1.642009\end{array}$

$\begin{array}{llll}\mathrm{S} & \mathbf{0 . 4 5 0 1 1 3} & \mathbf{2 . 6 9 7 8 9 0} & \mathbf{1 . 4 1 4 5 6 0}\end{array}$

$\begin{array}{llll}N & -0.918877 & -2.123767 & -0.913832\end{array}$

N $1.204144-1.896465-1.036659$

$\begin{array}{llll}\text { C } & -\mathbf{2 . 3 1 3 2 0 7} & \mathbf{- 1 . 9 9 5 9 8 8} & \mathbf{- 0 . 6 4 0 8 0 3}\end{array}$

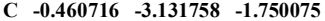

$\begin{array}{llll}\text { C } & 0.876223 & -2.988283 & -1.827555\end{array}$

$\begin{array}{llll}C & 2.569485 & -1.501782 & -0.890308\end{array}$

$\begin{array}{llll}C & -5.046358 & -1.845977 & -0.007571\end{array}$

$\begin{array}{llll}C & \mathbf{- 2 . 8 3 2 5 2 4} & \mathbf{- 2 . 6 1 5 3 3 0} & \mathbf{0 . 4 9 5 5 3 1}\end{array}$

$\begin{array}{llll}C & -3.186185 & -1.357150 & -1.531446\end{array}$

$\begin{array}{llll}C & -4.538918 & -1.281165 & -1.181277\end{array}$

$\begin{array}{llll}C & -4.171013 & -2.540910 & 0.834541\end{array}$

$\begin{array}{llll}\text { H } & \mathbf{- 5 . 2 2 3 0 0 9} & \mathbf{- 0 . 7 6 7 9 1 5} & \mathbf{- 1 . 8 6 1 9 0 6}\end{array}$

$\begin{array}{llll}\text { H } & -\mathbf{4 . 5 1 5 6 2 4} & \mathbf{- 3 . 0 2 4 4 1 0} & \mathbf{1 . 7 5 0 1 4 6}\end{array}$

$\begin{array}{llll}C & 5.292688 & -0.898301 & -0.546152\end{array}$

C $3.370653-2.221635-0.002085$

$\begin{array}{llll}C & 3.370653 & -2.221635 & -0.002085\end{array}$

C $3.154167-0.509722-1.688000$

$\begin{array}{llll}C & 4.508267 & -0.216364 & -1.479558\end{array}$

$\begin{array}{llll}\text { C } & 4.707351 & -1.933362 & 0.192434\end{array}$

$\begin{array}{llll}\text { F } & 2.808207 & -3.214933 & 0.695073\end{array}$

C $\quad 2.371863 \quad 0.186845 \quad-2.762265$

$\begin{array}{llll}\text { H } & 4.968132 & 0.566559 & -2.087429\end{array}$

$\begin{array}{lllll}\text { H } & 5.277727 & -2.516442 & 0.917487\end{array}$

$\begin{array}{llll}\text { C } \quad 6.736723 & -0.537504 & -0.326580\end{array}$

$\begin{array}{lllll}\text { H } & 7.375749 & -1.432631 & -0.334917\end{array}$

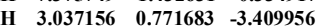

$\begin{array}{llll}\text { H } & 6.866037 & \mathbf{- 0 . 0 5 2 1 4 7} & \mathbf{0 . 6 5 3 4 0 9}\end{array}$

\begin{tabular}{llll}
\hline & $\mathbf{7 . 0 9 9 3 9 8}$ & $\mathbf{0 . 1 5 6 4 7 3}$ & $\mathbf{- 1 . 0 9 6 3 9 4}$
\end{tabular}

$\begin{array}{lllll}\text { H } & 1.615273 & 0.860234 & -2.332344\end{array}$

$\begin{array}{lllll}\text { H } & \mathbf{1 . 8 2 7 5 7 0} & \mathbf{- 0 . 5 3 9 2 7 2} & \mathbf{- 3 . 3 8 5 4 4 1}\end{array}$

$\begin{array}{llll}\text { F } & -1.991551 & -3.279911 & 1.296377\end{array}$

$\begin{array}{llll}\text { C } & \mathbf{- 6 . 5 0 0 1 4 3} & \mathbf{- 1 . 6 9 8 7 7 5} & \mathbf{0 . 3 5 2 1 3 9}\end{array}$

$\begin{array}{llll}\text { H } & -6.668481 & -0.742192 & 0.871955\end{array}$

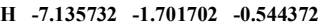

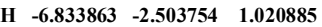

$\begin{array}{lrrrr}\text { H } & -6.833863 & -2.503754 & \mathbf{1 . 0 2 0 8 8 5} \\ \text { C } & -2.721561 & -\mathbf{0 . 8 4 2 7 6 0} & \mathbf{- 2 . 8 6 5 5 9 4}\end{array}$

$\begin{array}{llll}\text { H } & -2.986246 & -1.570272 & -3.649737\end{array}$

$\begin{array}{llll}\text { H } & \mathbf{- 3 . 2 1 3 4 9 3} & \mathbf{0 . 1 0 8 0 3 6} & \mathbf{- 3 . 1 0 9 3 4 9}\end{array}$

$\begin{array}{lllll}\text { H } & -\mathbf{1 . 6 3 8 4 6 7} & -\mathbf{0 . 6 8 0 4 4 5} & \mathbf{- 2 . 8 9 7 6 2 4}\end{array}$

$\begin{array}{llll}\text { C } & \mathbf{- 0 . 7 1 2 5 9 7} & \mathbf{3 . 1 8 5 7 2 2} & \mathbf{- 1 . 0 3 3 0 4 6}\end{array}$

$\begin{array}{llll}C & -1.234204 & 4.029442 & -2.026140\end{array}$

$\begin{array}{llll}C & \mathbf{- 0 . 2 2 4 6 5 9} & \mathbf{3 . 7 3 4 5 0 6} & \mathbf{0 . 1 6 5 4 0 4}\end{array}$

$\begin{array}{llll}C & -1.275951 & 5.407391 & -1.839148\end{array}$

$\begin{array}{llll}C & -1.611879 & 3.588204 & -2.952758\end{array}$

\begin{tabular}{llll} 
H & -1.611879 & 3.588204 & -2.952758 \\
\hline
\end{tabular}

$\begin{array}{llll}\mathrm{C} & -0.273146 & 5.129295 & \mathbf{0 . 3 4 0 9 5 0}\end{array}$

$\begin{array}{llll}C & \mathbf{0 . 7 9 1 7 6 3} & \mathbf{5 . 9 5 8 5 9 2} & \mathbf{- 0 . 6 4 7 6 4 9}\end{array}$

$\begin{array}{lllll}\text { H } & -1.685707 & \mathbf{6 . 0 5 2 3 2 4} & \mathbf{- 2 . 6 1 9 9 3 4}\end{array}$

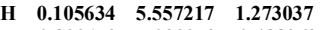

$\begin{array}{lllll}\text { H } & -0.822159 & 7.039253 & -0.488963\end{array}$

$\begin{array}{llll}\text { C } & \mathbf{2 . 8 4 1 7 1 7} & \mathbf{0 . 4 3 1 3 1 1} & \mathbf{2 . 1 2 9 1 0 9}\end{array}$

$\begin{array}{llll}C & 1.793867 & -1.410962 & 1.609181\end{array}$

$\begin{array}{lllll}\text { C } & \mathbf{0 . 4 6 5 4 1 1} & \mathbf{- 0 . 3 2 6 1 2 4} & \mathbf{2 . 8 1 5 3 8 8}\end{array}$

$\begin{array}{llll}\text { H } & -1.648175 & -1.140127 & 1.815347\end{array}$

$\begin{array}{llll}C & -1.6481757 & -1.140127 & 1.815347\end{array}$

$\begin{array}{llll}\text { C } & -2.468727 & 0.819606 & 2.157952 \\ H & -3.294339 & 0.754694 & 1.424452\end{array}$ 


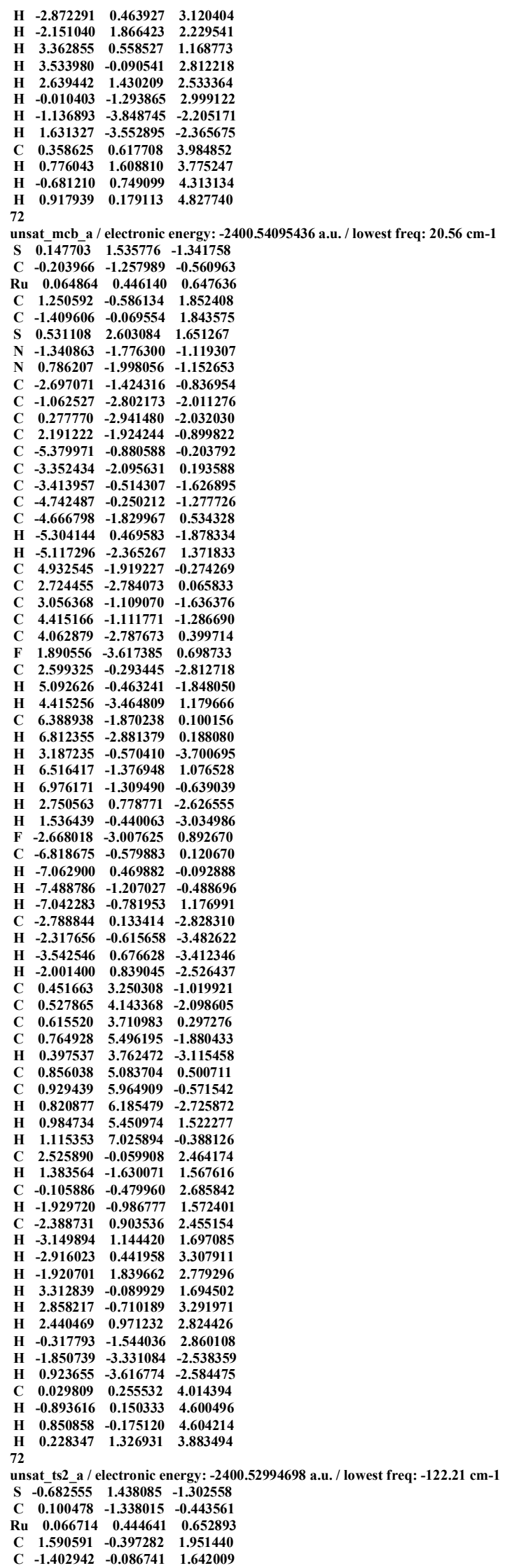


$\begin{array}{lllll}S & 0.450113 & 2.697890 & 1.414560\end{array}$ $\begin{array}{llll}N & -0.918877 & -2.123767 & -0.913832\end{array}$

$\begin{array}{llll}N & 1.204144 & -1.896465 & -1.036659\end{array}$

$\begin{array}{llll}\text { C } & -\mathbf{2 . 3 1 3 2 0 7} & \mathbf{- 1 . 9 9 5 9 8 8} & \mathbf{- 0 . 6 4 0 8 0 3}\end{array}$

$\begin{array}{llll}\text { C } & \mathbf{- 0 . 4 6 0 7 1 6} & \mathbf{- 3 . 1 3 1 7 5 8} & -\mathbf{1 . 7 5 0 0 7 5}\end{array}$

$\begin{array}{llll}C & 0.876223 & -2.988283 & -1.827555\end{array}$

$\begin{array}{llll}C & 0.876223 & -2.988283 & -1.827555 \\ C & 2.569485 & -1.501782 & -0.890308\end{array}$

$\begin{array}{llll}C & 2.569485 & -1.501782 & \mathbf{- 0 . 8 9 0 3 0 8}\end{array}$

$\begin{array}{llll}C & \mathbf{- 5 . 0 4 6 3 5 8} & -1.845977 & \mathbf{- 0 . 0 0 7 5 7 1}\end{array}$

$\begin{array}{llll}C & -2.832524 & -2.615330 & 0.495531\end{array}$

$\begin{array}{llll}C & -3.186185 & -1.357150 & -1.531446\end{array}$

$\begin{array}{llll}C & -4.538918 & -1.281165 & -1.181277\end{array}$

$\begin{array}{llll}C & -4.171013 & -2.540910 & 0.834541\end{array}$

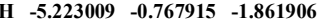

$\begin{array}{llll}H & -\mathbf{4 . 5 1 5 6 2 4} & \mathbf{- 3 . 0 2 4 4 1 0} & \mathbf{1 . 7 5 0 1 4 6}\end{array}$

$\begin{array}{llll}\text { C } & 5.292688 & -0.898301 & -0.546152\end{array}$

$\begin{array}{llll}C & 3.370653 & -2.221635 & -0.002085\end{array}$

$\begin{array}{llll}C & 3.154167 & -0.509722 & -1.688000\end{array}$

$\begin{array}{llll}C & 4.508267 & -0.216364 & -1.479558\end{array}$

$\begin{array}{llll}\text { C } & 4.707351 & -1.933362 & 0.192434\end{array}$

$\begin{array}{llll}\text { F } & \mathbf{2 . 8 0 8 2 0 7} & \mathbf{- 3 . 2 1 4 9 3 3} & \mathbf{0 . 6 9 5 0 7 3}\end{array}$

C $\quad 2.371863 \quad 0.186845 \quad-2.762265$

$\begin{array}{llll}\text { H } & 4.968132 & 0.566559 & -2.087429\end{array}$

$\begin{array}{llll}\text { H } & 5.277727 & -\mathbf{2 . 5 1 6 4 4 2} & \mathbf{0 . 9 1 7 4 8 7}\end{array}$

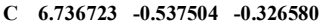

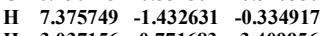

$\begin{array}{llll}\text { H } & 3.037156 & \mathbf{0 . 7 7 1 6 8 3} & \mathbf{- 3 . 4 0 9 9 5 6}\end{array}$

$\begin{array}{llll}\text { H } & 6.866037 & -0.052147 & \mathbf{0 . 6 5 3 4 0 9}\end{array}$

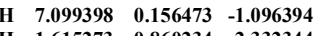

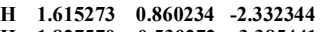

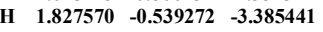

$\begin{array}{llll}\text { F } & -1.991551 & -3.279911 & 1.296377\end{array}$

$\begin{array}{llll}\text { C } & -6.500143 & -1.698775 & 0.352139\end{array}$

$\begin{array}{llll}\text { H } & -6.668481 & -0.742192 & 0.871955\end{array}$

$\begin{array}{llll}\text { H } & -7.135732 & -1.701702 & -0.544372\end{array}$

$\begin{array}{llll}\text { H } & -6.833863 & -2.503754 & 1.020885\end{array}$

$\begin{array}{llll}C & -2.721561 & -0.842760 & -2.865594\end{array}$

$\begin{array}{llll}\text { H } & -\mathbf{2 . 9 8 6 2 4 6} & \mathbf{- 1 . 5 7 0 2 7 2} & \mathbf{- 3 . 6 4 9 7 3 7}\end{array}$

$\begin{array}{llll}\text { H } & -3.213493 & 0.108036 & -3.109349\end{array}$

$\begin{array}{lllll}\text { H } & \mathbf{- 1 . 6 3 8 4 6 7} & \mathbf{- 0 . 6 8 0 4 4 5} & \mathbf{- 2 . 8 9 7 6 2 4}\end{array}$

$\begin{array}{llll}C & \mathbf{- 0 . 7 1 2 5 9 7} & \mathbf{3 . 1 8 5 7 2 2} & \mathbf{- 1 . 0 3 3 0 4 6}\end{array}$

$\begin{array}{llll}C & -1.234204 & 4.029442 & -2.026140\end{array}$

$\begin{array}{llll}C & -0.224659 & 3.734506 & 0.165404\end{array}$

$\begin{array}{llll}\text { C } & -1.275951 & 5.407391 & -1.839148\end{array}$

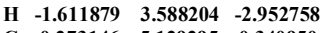

$\begin{array}{llll}\text { C } & -0.273146 & 5.129295 & 0.340950\end{array}$

$\begin{array}{lllll}C & \mathbf{0 . 7 9 1 7 6 3} & \mathbf{5 . 9 5 8 5 9 2} & \mathbf{- 0 . 6 4 7 6 4 9}\end{array}$

$\begin{array}{llll}\text { H } & -1.685707 & \mathbf{6 . 0 5 2 3 2 4} & \mathbf{- 2 . 6 1 9 9 3 4}\end{array}$

$\begin{array}{llll}\text { H } & \mathbf{0 . 1 0 5 6 3 4} & \mathbf{5 . 5 5 7 2 1 7} & \mathbf{1 . 2 7 3 0 3 7}\end{array}$

$\begin{array}{llll}\text { H } & -0.822159 & 7.039253 & -0.488963\end{array}$

$\begin{array}{llll}\text { C } & \mathbf{2 . 8 4 1 7 1 7} & \mathbf{0 . 4 3 1 3 1 1} & \mathbf{2 . 1 2 9 1 0 9}\end{array}$

$\begin{array}{llll}\text { H } & \mathbf{1 . 7 9 3 8 6 7} & \mathbf{- 1 . 4 1 0 9 6 2} & \mathbf{1 . 6 0 9 1 8 1}\end{array}$

$\begin{array}{llll}C & \mathbf{0 . 4 6 5 4 1 1} & \mathbf{- 0 . 3 2 6 1 2 4} & \mathbf{2 . 8 1 5 3 8 8}\end{array}$

$\begin{array}{llll}\text { H } & -1.648175 & -1.140127 & 1.815347\end{array}$

$\begin{array}{llll}\text { C } & -2.468727 & 0.819606 & 2.157952\end{array}$

$\begin{array}{llll}\text { H } & \mathbf{- 3 . 2 9 4 3 3 9} & \mathbf{0 . 7 5 4 6 9 4} & \mathbf{1 . 4 2 4 4 5 2}\end{array}$

$\begin{array}{llll}\text { H } & -2.872291 & \mathbf{0 . 4 6 3 9 2 7} & \mathbf{3 . 1 2 0 4 0 4}\end{array}$

$\begin{array}{llll}\text { H } & -2.151040 & 1.866423 & 2.229541\end{array}$

$\begin{array}{llll}\text { H } & 3.362855 & \mathbf{0 . 5 5 8 5 2 7} & \mathbf{1 . 1 6 8 7 7 3}\end{array}$

$\begin{array}{llll}\text { H } & 3.533980 & -0.090541 & 2.812218\end{array}$

$\begin{array}{llll}\text { H } & 2.639442 & 1.430209 & 2.533364\end{array}$

$\begin{array}{llll}\text { H } & -0.010403 & -1.293865 & 2.999122\end{array}$

H $-1.136893-3.848745-2.205171$

$\begin{array}{llll}H & 1.631327 & -3.552895 & -2.365675\end{array}$

$\begin{array}{llll}\text { C } & \mathbf{0 . 3 5 8 6 2 5} & \mathbf{0 . 6 1 7 7 0 8} & \mathbf{3 . 9 8 4 8 5 2}\end{array}$

$\begin{array}{lllll}\text { H } & 0.776043 & 1.608810 & 3.775247\end{array}$

\begin{tabular}{llll} 
H & -0.681210 & 0.749099 & 4.31313 \\
\hline
\end{tabular}

$\begin{array}{lllll}\text { H } & 0.917939 & 0.179113 & 4.827740\end{array}$

72

unsat_pc2_a / electronic energy: $\mathbf{- 2 4 0 0 . 5 3 8 4 0 0 6 1}$ a.u. / lowest freq: $13.88 \mathrm{~cm}-1$

$\begin{array}{llll}S & 0.313188 & 1.609144 & -1.337245\end{array}$

$\begin{array}{llll}C & 0.059936 & -1.269959 & -0.566042\end{array}$

$\begin{array}{llll}\text { Ru } & -0.016608 & 0.425351 & 0.658720\end{array}$

$\begin{array}{llll}C & 1.727316 & 0.407309 & 1.222997\end{array}$

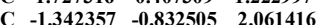

$\begin{array}{llll}\text { S } & \mathbf{- 0 . 7 4 0 4 3 5} & \mathbf{2 . 4 9 1 9 2 9} & \mathbf{1 . 5 6 8 3 7 9}\end{array}$

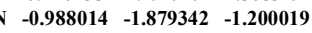

N $1.149878 \quad-1.910698-1.077945$

C $\quad-2.381079-1.687226-0.944641$

$\begin{array}{llll}C & -0.554603 & -2.870812 & -2.070097\end{array}$

C $\quad 0.790316 \quad-2.892429-1.991702$

$\begin{array}{llll}C & 2.509727 & -1.718355 & -0.700931\end{array}$

$\begin{array}{llll}C & -5.122989 & -1.508253 & -0.345611\end{array}$

$\begin{array}{llll}C & \mathbf{- 3 . 0 0 6 3 1 2} & \mathbf{- 2 . 5 9 8 5 3 3} & \mathbf{- 0 . 0 8 6 6 1 8 8}\end{array}$

$\begin{array}{llll}\text { C } & -3.154948 & -0.710098 & -1.580973\end{array}$

$\begin{array}{llll}C & -4.515164 & -0.633717 & -1.246814\end{array}$

$\begin{array}{lllll}\text { C } & -4.346883 & -2.522370 & 0.230388\end{array}$

$\begin{array}{llll}\text { H } & -\mathbf{5 . 1 2 2 1 2 1} & \mathbf{0 . 1 3 5 4 0 5} & -\mathbf{1 . 7 3 1 0 6 2}\end{array}$

$\begin{array}{llll}\text { H } & -4.771802 & -3.249772 & 0.924179\end{array}$

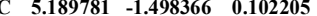

$\begin{array}{llll}C & \mathbf{2} .955939 & \mathbf{- 2} 280276 & \mathbf{0 . 4 9 4 5 2 0}\end{array}$

$\begin{array}{llll}C & 3.419552 & -1.058618 & -1.537891\end{array}$

C $4.748562-0.962992-1.112503$

$\begin{array}{lrrr}\text { C } & 4.748562 & -0.962992 & -1.112503 \\ \text { C } & 4.269847 & -2.171765 & 0.913665\end{array}$ 
$\begin{array}{llll}\text { F } & 2.073614 & -2.922215 & 1.263996\end{array}$

$\begin{array}{llll}C & 2.973946 & -0.463749 & -2.84304\end{array}$

$\begin{array}{llll}\text { H } & 5.464067 & -0.443477 & -1.754395\end{array}$

$\begin{array}{llll}\text { H } & \mathbf{4 . 5 6 1 8 6 7} & \mathbf{- 2 . 6 1 6 7 1 5} & \mathbf{1 . 8 6 6 4 8 1}\end{array}$

C $6.616907-1.335987 \quad 0.550786$

C $7.003422-2.263439-0.996803$

$\begin{array}{llll}\text { H } & 7.003422 & -2.263439 & 0.996803\end{array}$

$\begin{array}{llll}\text { H } & \mathbf{2 . 5 3 2 3 9 9} & \mathbf{- 1 . 2 2 4 4 3 4} & \mathbf{- 3 . 5 0 4 3 6}\end{array}$

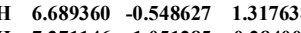

H $7.271146-1.051285-\mathbf{0 . 2 8 4 0 0 6}$

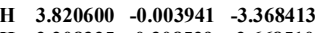

$\begin{array}{llll}\text { H } & \mathbf{2 . 2 0 8 3 2 5} & \mathbf{0 . 3 0 8 5 2 9} & \mathbf{- 2 . 6 6 8 5 1 0}\end{array}$

$\begin{array}{llll}\text { F } & -2.261292 & -3.568454 & 0.454929\end{array}$

$\begin{array}{llll}\text { C } & -6.575791 & -1.368710 & 0.018453\end{array}$

$\begin{array}{llll}\text { H } & -\mathbf{7 . 1 2 0 5 4 8} & \mathbf{- 0 . 7 6 6 5 0 2} & \mathbf{- 0 . 7 2 0 8 2 4}\end{array}$

$\begin{array}{llll}\text { H } & -7.063439 & -2.350514 & 0.099192\end{array}$

$\begin{array}{llll}\text { H } & -6.676802 & -0.870964 & 0.995948\end{array}$

$\begin{array}{llll}C & -2.593153 & 0.215830 & -2.620694\end{array}$

$\begin{array}{lllll}\text { H } & -1.625524 & -0.130971 & -3.002915\end{array}$

H $\quad \begin{array}{llll}-3.298642 & 0.306539 & -3.458475\end{array}$

$\begin{array}{llll}\text { H } & -2.425854 & 1.217209 & -2.199705\end{array}$

$\begin{array}{llll}C & 0.079367 & 3.310592 & -0.943530\end{array}$

$\begin{array}{lllll}C & 0.342758 & 4.297801 & -1.908963\end{array}$

$\begin{array}{llll}\text { C } & \mathbf{- 0 . 3 7 8 9 6 0} & \mathbf{3 . 6 9 4 6 1 8} & \mathbf{0 . 3 3 0 1 6 7}\end{array}$

$\begin{array}{llll}C & 0.151528 & 5.644983 & -1.624063\end{array}$

H $\quad 0.702703 \quad 3.990095 \quad-2.894662$

$\begin{array}{llll}C & \mathbf{- 0 . 5 6 4 1 5 1} & \mathbf{5 . 0 6 0 8 5 9} & \mathbf{0 . 6 0 6 3 7 1}\end{array}$

$\begin{array}{llll}C & -0.305064 & \mathbf{6 . 0 2 8 5 6 2} & -\mathbf{0 . 3 5 7 7 2 9}\end{array}$

$\begin{array}{lllll}\mathrm{H} & \mathbf{0 . 3 6 1 1 5 8} & \mathbf{6 . 3 9 8 3 5 8} & \mathbf{- 2 . 3 8 6 9 9 7}\end{array}$

$\begin{array}{llll}\text { H } & -0.919084 & 5.355242 & 1.597789\end{array}$

$\begin{array}{llll}\text { H } & \mathbf{- 0 . 4 5 6 9 0 9} & \mathbf{7 . 0 8 4 8 4 8} & \mathbf{- 0 . 1 2 3 5 5 4}\end{array}$

$\begin{array}{llll}C & 2.768554 & 1.442171 & 0.961059\end{array}$

$\begin{array}{llll}\text { H } & \mathbf{2 . 1 0 8 6 2 0} & \mathbf{- 0 . 3 3 8 0 8 3} & \mathbf{1 . 9 4 2 6 8 2}\end{array}$

$\begin{array}{lrrr}\text { C } & -\mathbf{0 . 1 7 7 6 0 0} & \mathbf{- 0 . 6 0 0 1 4 5} & \mathbf{2 . 7 6 3 6 4 4}\end{array}$

$\begin{array}{llll}C & -1.384674 & -1.795095 & 1.554090\end{array}$

$\begin{array}{llll}\mathrm{H} & -1.384674 & -1.795095 & 1.554090\end{array}$

\begin{tabular}{llll} 
C & -2.685708 & -0.202367 & 2.315961 \\
\hline
\end{tabular}

$\begin{array}{llll}\text { H } & \mathbf{- 3 . 2 2 4 9 2 3} & \mathbf{- 0 . 0 2 2 0 1 3} & \mathbf{1 . 3 7 4 0 3 4}\end{array}$

$\begin{array}{lllll}\text { H } & -\mathbf{3 . 3 0 0 7 1 2} & \mathbf{- 0 . 8 9 6 0 2 4} & \mathbf{2 . 9 1 4 3 9 7} \\ \text { H } & \mathbf{- 2 . 6 1 1 7 3 6} & \mathbf{0 . 7 5 1 5 1 3} & \mathbf{2 . 8 5 1 7 4 6}\end{array}$

$\begin{array}{lrrrr}\text { H } & -2.611736 & \mathbf{0 . 7 5 1 5 1 3} & \mathbf{2 . 8 5 1 7 4 6} \\ \text { H } & \mathbf{3 . 7 3 0 2 2 4} & \mathbf{0 . 9 5 2 0 2 2} & \mathbf{0 . 7 3 0 5 4 9}\end{array}$

$\begin{array}{llll}\text { H } & 2.917580 & 1.989506 & 1.910921\end{array}$

H $2.511156 \quad 2.161891 \quad 0.177669$

$\begin{array}{llll}\text { H } & 0.577162 & -1.388453 & 2.689528\end{array}$

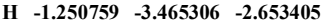

$\begin{array}{llll}\text { H } & 1.529348 & -3.513772 & -2.487922\end{array}$

$\begin{array}{llll}C & -0.017525 & 0.304001 & 3.953209\end{array}$

$\begin{array}{lllll}\text { H } & -0.154920 & -0.283554 & 4.876601\end{array}$

$\begin{array}{llll}\text { H } & 0.992036 & 0.739727 & 3.989013\end{array}$

$\begin{array}{lllll}\text { H } & -0.740439 & 1.128756 & 3.963001\end{array}$

60

unsat_14e1 / electronic energy: $-\mathbf{2 2 4 3 . 4 5 0 0 2 3 8 1}$ a.u. / lowest freq: $14.79 \mathrm{~cm}-1$

$\begin{array}{llll}0.867262 & -1.380021 & 1.240326\end{array}$

$\begin{array}{llll}C & -0.809241 & 1.071706 & 0.304350\end{array}$

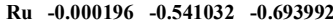

$\begin{array}{llll}C & -1.420298 & -1.627816 & -0.816080\end{array}$

S $1.802999-1.479683-1.833131$

$\begin{array}{llll}\text { N } & -2.061694 & 1.367141 & 0.737925\end{array}$

$\begin{array}{llll}\text { N } & -0.070108 & 2.154993 & 0.662993\end{array}$

$\begin{array}{llll}\text { C } & -\mathbf{3 . 2 2 5 9 8 1} & \mathbf{0 . 5 7 9 7 2 3} & \mathbf{0 . 5 1 1 8 5 6}\end{array}$

$\begin{array}{llll}C & -2.101942 & 2.613900 & 1.349326\end{array}$

$\begin{array}{llll}C & -0.846968 & 3.111056 & 1.299751\end{array}$

$\begin{array}{llll}\text { C } & \mathbf{1 . 3 0 9 9 8 7} & \mathbf{2 . 3 2 1 0 4 4} & \mathbf{0 . 3 4 2 3 0 2}\end{array}$

C $-5.555172-0.877152-0.034960$

$\begin{array}{llll}\text { C } & -4.040871 & 0.905715 & -0.570347\end{array}$

$\begin{array}{llll}C & \mathbf{- 3 . 5 7 1 6 8 0} & \mathbf{- 0 . 4 8 5 1 4 1} & \mathbf{1 . 3 5 1 9 7 7}\end{array}$

$\begin{array}{llll}C & -4.733507 & -1.202752 & 1.050629\end{array}$

$\begin{array}{llll}\text { C } & \mathbf{- 5 . 1 9 8 5 4 4} & \mathbf{0 . 2 0 1 5 1 8} & \mathbf{- 0 . 8 5 2 1 8 2}\end{array}$

$\begin{array}{rrrr}\text { H } & \mathbf{5 . 0 0 8 8 6 9} & \mathbf{- 2 . 0 4 3 7 7 7} & \mathbf{1 . 6 9 1 7 5 4}\end{array}$

$\begin{array}{llll}\text { H } & -5.799992 & 0.490606 & -1.716002\end{array}$

C $\quad \mathbf{3 . 9 9 0 9 8 3} \quad \mathbf{2 . 6 2 7 1 2 4} \quad \mathbf{- 0 . 4 1 2 2 0 0}$

$\begin{array}{llll}\text { C } & \mathbf{1 . 6 5 8 8 8 0} & \mathbf{2 . 6 2 5 0 1 9} & \mathbf{- 0 . 9 7 4 2 2 5}\end{array}$

$\begin{array}{llll}\text { C } & \mathbf{2 . 3 0 9 1 2 2} & \mathbf{2 . 1 8 5 0 5 3} & \mathbf{1 . 3 1 5 1 3 5}\end{array}$

$\begin{array}{llll}\text { C } & 3.637874 & 2.341633 & 0.911170\end{array}$

$\begin{array}{llll}C & 2.977508 & 2770827 & -1.365327\end{array}$

$\begin{array}{llll}\text { C } & 2.977508 & 2.770827 & -1.365327 \\ \text { F } & 0.685915 & 2.758647 & -1.878503\end{array}$

$\begin{array}{lllll} & 0.685915 & 2.758647 & -1.878503\end{array}$

$\begin{array}{llll}\text { C } & 1.953990 & 1.852215 & 2.735974\end{array}$

$\begin{array}{llll}\text { H } & 4.426720 & \mathbf{2 . 2 2 7 0 4 8} & \mathbf{1 . 6 5 8 4 1 0}\end{array}$

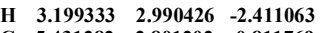

$\begin{array}{llll}\text { C } & 5.431282 & 2.801202 & -0.811769\end{array}$

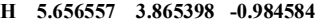

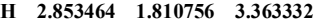

$\begin{array}{llll}\text { H } & 5.649375 & 2.264363 & -1.746017\end{array}$

H $6.112294 \quad 2.433665-0.032808$

\begin{tabular}{llll}
\hline & 6.112294 & $\mathbf{2 . 4 3 3 6 6 5}$ & $\mathbf{- 0 . 0 3 2 8 0 8}$ \\
\hline & 1.456552 & 0.870164 & 2.771475
\end{tabular}

$\begin{array}{llll}\text { H } & \mathbf{1 . 4 5 6 5 5 2} & \mathbf{0 . 8 7 0 1 6 4} & \mathbf{2 . 7 7 1 4 7 5}\end{array}$

$\begin{array}{lllll}\text { H } & 1.266506 & 2.594610 & 3.168077\end{array}$

$\begin{array}{llll}\text { F } & -3.672365 & 1.915311 & -1.359911\end{array}$

$\begin{array}{llll}C & -6.813726 & -1.653752 & -0.314145\end{array}$

$\begin{array}{llll}\text { H } & -6.794772 & -2.636383 & 0.175784\end{array}$

$\begin{array}{llll}\text { H } & -7.694065 & -1.108743 & 0.061608\end{array}$

$\begin{array}{llll}\text { H } & -6.957531 & -1.805000 & -1.393237\end{array}$

$\begin{array}{llll}\text { C } & -\mathbf{2 . 6 9 8 0 2 6} & \mathbf{- 0 . 8 4 7 0 7 9} & \mathbf{2 . 5 1 8 4 6 1}\end{array}$

$\begin{array}{llll}C & -1.750683 & -1.287959 & 2.168984\end{array}$

$\begin{array}{lll}-1.750683 & -1.287959 & 2.168984\end{array}$

$\begin{array}{rrrr}\text { H } & -2.439475 & 0.040988 & 3.114152 \\ \text { H } & -3.196846 & -1.573242 & 3.172604\end{array}$ 
$\begin{array}{llll}\text { C } & \mathbf{2 . 2 9 6 4 9 6} & \mathbf{- 2 . 3 0 4 3 8 3} & \mathbf{0 . 7 5 9 0 2}\end{array}$

$\begin{array}{llll}\text { C } & 3.043581 & -2.984396 & 1.734242\end{array}$

$\begin{array}{llll}C & 2.700211 & -2.360499 & -0.585950\end{array}$

$\begin{array}{llll}C & 4.174157 & -3.714131 & 1.382627\end{array}$

$\begin{array}{llll}\text { H } & \mathbf{2 . 7 2 6 2 4 0} & -\mathbf{2 . 9 3 2 0 7 3} & \mathbf{2 . 7 7 9 6 1 5}\end{array}$

$\begin{array}{llll}\text { C } 3.838712 & -3.106799 & -0.929442\end{array}$

$\begin{array}{llll}\text { C } & 3.838712 & -3.106799 & -0.929442 \\ \text { C } & 4.573374 & -3.777421 & 0.043245\end{array}$

$\begin{array}{llll}\text { C } & 4.573374 & -3.777421 & 0.04324\end{array}$

H $4.744915-4.236769 \quad 2.153792$

$\begin{array}{lrrr}\text { H } & \mathbf{4 . 1 4 5 9 2 9} & \mathbf{- 3 . 1 5 2 2 9 8} & \mathbf{- 1 . 9 7 7 7 7 0} \\ \text { H } & \mathbf{5 . 4 5 8 8 8 8} & \mathbf{- 4 . 3 5 0 0 8 5} & -\mathbf{0 . 2 4 2 1 5 6}\end{array}$

$\begin{array}{llll}\text { H } & -\mathbf{1 . 6 4 1 8 4 1} & \mathbf{- 2 . 4 2 1 8 6 6} & \mathbf{- 0 . 0 8 1 6 1 6}\end{array}$

$\begin{array}{llll}\text { C } & -2.273172 & -1.625134 & -2.048811\end{array}$

H $-2.116013 \quad-2.561468-2.611110$

H $-\mathbf{3 . 3 3 9 4 1 8}-\mathbf{- 1 . 5 8 5 1 3 3}-\mathbf{- 1 . 7 7 2 4 3 0}$

H $\quad-\mathbf{2 . 0 6 0 6 3 0}-\mathbf{- 0 . 7 7 7 4 4 4}-\mathbf{- 2 . 7 2 2 1 1 9}$

$\begin{array}{llll}\text { H } & -\mathbf{3 . 0 2 1 3 2 7} & \mathbf{3 . 0 2 4 9 0 0} & \mathbf{1 . 7 5 4 4 8 6}\end{array}$

\begin{tabular}{cccc}
\hline & -0.432398 & $\mathbf{4 . 0 5 1 7 4 6}$ & $\mathbf{1 . 6 4 8 8 8 4}$
\end{tabular}

75

unsat_pc1_b / electronic energy: $-\mathbf{2 5 8 8 . 9 0 6 4 1 1 4 1}$ a.u. / lowest freq: $-\mathbf{6 . 4 6} \mathrm{cm}-1$

$\begin{array}{llll}S & -0.390071 & 1.332802 & -1.832191\end{array}$

$\begin{array}{llll}C & -0.054528 & -1.428678 & -0.725990\end{array}$

$\begin{array}{llll}\text { Ru } & \mathbf{- 0 . 0 2 5 0 0 0} & \mathbf{0 . 4 2 0 5 2 5} & \mathbf{0 . 2 8 1 5 7 8}\end{array}$

$\begin{array}{llll}\text { C } & \mathbf{1 . 8 0 4 6 7 7} & \mathbf{0 . 6 4 5 9 9 0} & \mathbf{0 . 3 2 5 2 1 7}\end{array}$

$\begin{array}{llll}C & -0.858525 & -0.745718 & \mathbf{2 . 0 2 4 6 8 2}\end{array}$

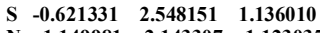

\begin{tabular}{llll} 
N & -1.149981 & -2.143307 & -1.123035 \\
\hline
\end{tabular}

N $0.983759-2.112505-1.280405$

$\begin{array}{llll}C & -2.493317 & -1.963192 & -0.669802\end{array}$

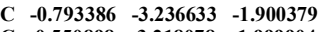

$\begin{array}{llll}C & 0.550898 & -3.218078 & -1.999904\end{array}$

C $\quad 2.367348-1.860614 \quad-1.061119$

$\begin{array}{lllll}\text { C } & \mathbf{- 5 . 0 9 1 1 9 2} & \mathbf{- 1 . 7 4 9 8 4 6} & \mathbf{0 . 3 8 3 9 5 1}\end{array}$

$\begin{array}{lllll}\text { C } & -2.902523 & -2.721165 & 0.434299\end{array}$

$\begin{array}{llll}\text { C } & -3.429056 & -1.155343 & -1.324448\end{array}$

$\begin{array}{llll}C & -4.708272 & -1.047019 & -0.757991\end{array}$

$\begin{array}{llll}\text { C } & -4.167783 & -2.624437 & \mathbf{0 . 9 7 3 4 1 2}\end{array}$

$\begin{array}{llll}\mathrm{H} & \mathbf{- 5 . 4 3 6 3 4 0} & -\mathbf{0 . 3 9 8 5 3 7} & -\mathbf{1 . 2 5 1 8 1 7}\end{array}$

$\begin{array}{llll}\text { H } & -\mathbf{4 . 4 2 0 6 6 5} & \mathbf{- 3 . 2 2 3 0 4 1} & \mathbf{1 . 8 5 0 2 4 1}\end{array}$

$\begin{array}{llll}\text { C } & 5.092838 & -1.482407 & -0.531220\end{array}$

$\begin{array}{llll}C & \mathbf{2 . 9 5 3 3 5 7} & \mathbf{- 2 . 3 6 5 5 0 9} & \mathbf{0 . 0 9 7 7 1 4}\end{array}$

$\begin{array}{llll}C & 3.151400 & -1.172633 & -1.995837\end{array}$

C $\quad 4.507872 \quad-0.999714 \quad-1.708114$

$\begin{array}{llll}\text { C } & 4.294906 & -2.180048 & 0.381891\end{array}$

$\begin{array}{llll}\text { F } & 2.179606 & -3.017282 & 0.969379\end{array}$

$\begin{array}{llll}C & \mathbf{2 . 5 2 9 8 6 9} & \mathbf{- 0 . 6 1 3 3 1 7} & \mathbf{- 3 . 2 4 2 3 8 0}\end{array}$

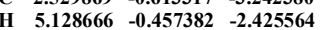

$\begin{array}{llll}\text { H } & 4.702926 & -2.582828 & 1.310446\end{array}$

$\begin{array}{llll}\text { C } & 6.546442 & -1.227539 & -0.237395\end{array}$

$\begin{array}{lllll}\text { H } & 6.957694 & -1.982734 & 0.445989\end{array}$

$\begin{array}{llll}\text { H } & 2.022796 & -1.397438 & -3.824809\end{array}$

$\begin{array}{lllll}\text { H } & 6.671620 & -0.242871 & 0.240647\end{array}$

H $7.144913 \quad-1.224801 \quad-1.158907$

$\begin{array}{llll}\text { H } & 3.287609 & -0.143819 & -3.882209\end{array}$

\begin{tabular}{lll}
-1.2876097 & -0.143819 & -3.882209 \\
\hline & 1.14095 & -2.983123
\end{tabular}

$\begin{array}{llll}\text { H } & 1.769257 & 0.141095 & -2.983123\end{array}$

$\begin{array}{lll}-2.019361 & -3.561331 & 0.985570\end{array}$

$\begin{array}{llll}\text { C } & -6.462591 & -1.589627 & 0.980980\end{array}$

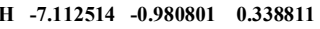

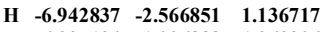

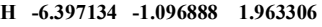

$\begin{array}{lllll}\text { C } & \mathbf{- 3 . 1 4 2 5 4 5} & \mathbf{- 0 . 4 7 3 6 7 6} & \mathbf{- 2 . 6 3 1 7 5 1}\end{array}$

$\begin{array}{lllll}\text { H } & -2.138311 & -0.696787 & -3.008164\end{array}$

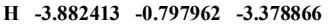

$\begin{array}{lllll}\text { H } & -3.218523 & 0.617224 & -2.527894\end{array}$

$\begin{array}{llll}C & -0.627195 & 3.060358 & -1.582619\end{array}$

$\begin{array}{llll}C & -0.724557 & 3.920387 & -2.690354\end{array}$

$\begin{array}{llll}C & \mathbf{- 0 . 7 1 9 7 5 6} & \mathbf{3 . 5 8 8 6 6 1} & \mathbf{- 0 . 2 8 3 0 5 9}\end{array}$

$\begin{array}{llll}C & -0.916305 & 5.285588 & -2.516319\end{array}$

$\begin{array}{lllll}\text { H } & \mathbf{- 0 . 6 4 4 4 1 5} & \mathbf{3 . 4 9 8 3 9 6} & \mathbf{- 3 . 6 9 5 8 6 4}\end{array}$

$\begin{array}{llll}C & -0.911528 & 4.972965 & -0.121600\end{array}$

$\begin{array}{llll}C & -1.010742 & 5.814403 & -1.223274\end{array}$

$\begin{array}{lllll}\text { H } & -0.988185 & 5.941461 & -3.387049\end{array}$

\begin{tabular}{lllll}
\hline & -0.983174 & 5.382756 & 0.889453
\end{tabular}

$\begin{array}{lllll}\text { H } & -1.159346 & 6.886595 & -1.075858\end{array}$

$\begin{array}{llll}C & 2561900 & 1.761256 & -0.0505055\end{array}$

$\begin{array}{llll}\text { H } & \mathbf{2 . 4 5 7 1 5 2} & \mathbf{0 . 0 5 6 6 9 8} & \mathbf{0 . 9 8 5 5 9 5}\end{array}$

$\begin{array}{llll}\text { C } & 0.435068 & -0.364367 & 2.368487\end{array}$

$\begin{array}{lllll}\text { H } & -\mathbf{0 . 9 3 3 0 1 4} & \mathbf{- 1 . 7 7 3 2 1 1} & \mathbf{1 . 6 6 9 9 9 0}\end{array}$

$\begin{array}{llll}\text { C } & \mathbf{0 . 7 8 6 5 7 7} & \mathbf{0 . 7 0 6 1 3 9} & \mathbf{3 . 3 3 4 8 1 5}\end{array}$

$\begin{array}{llll}\text { C } & \mathbf{- 2 . 1 5 4 1 2 7} & \mathbf{- 0 . 1 7 8 9 3 4} & \mathbf{2 . 5 4 1 1 6 3}\end{array}$

$\begin{array}{llll}\text { H } & -2.906842 & -0.133567 & \mathbf{1 . 7 4 0 1 5 6}\end{array}$

$\begin{array}{llll}\text { H } & -2.547851 & -0.863787 & \mathbf{3 . 3 1 1 8 5 4}\end{array}$

$\begin{array}{llll}4 & -2.030184 & 0.814721 & 2.980494\end{array}$

\begin{tabular}{llll}
\hline H & -2.030184 & 0.814721 & 2.980494 \\
\hline & 0.032695 & 1.320456 & 4.051973
\end{tabular}

\begin{tabular}{llll}
\hline & 0.032695 & 1.320456 & 4.051973
\end{tabular}

$\begin{array}{llll}\text { O } & 2.114141 & 0.908153 & \mathbf{3 . 3 4 8 8 7 6}\end{array}$

$\begin{array}{llll}\text { C } & 2.587965 & 1.950664 & 4.185674\end{array}$

$\begin{array}{lllll}\text { H } & 3.671889 & 1.997791 & 4.034527\end{array}$

H $\quad 2.364439 \quad 1.741034 \quad 5.241532$

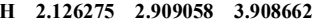

H $\quad \mathbf{3 . 5 8 0 5 4 4} \quad \mathbf{1 . 4 4 1 1 4 3} \quad \mathbf{- 0 . 5 7 5 6 6 5}$

$\begin{array}{lllll}\text { H } & \mathbf{2 . 6 6 4 5 4 3} & \mathbf{2 . 5 2 3 2 3 6} & \mathbf{0 . 4 9 2 6 8 9}\end{array}$

$\begin{array}{llll}\text { H } & 2.059799 & 2.236110 & -1.155042\end{array}$

H $1.232038-1.095136-2.22064$

\begin{tabular}{llll} 
H & 1.232038 & -1.095136 & 2.220644 \\
\hline
\end{tabular}

$\begin{array}{lllll}\text { H } & -1.535742 & -3.922040 & -2.297339\end{array}$ 
$\begin{array}{lllll}\text { H } & 1.245325 & -3.883376 & -2.503417\end{array}$

$\begin{array}{llll}S & 1.193725 & 0.790503 & -1.801804\end{array}$

C $-0.316373-1.531499-0.437388$

Ru $0.163587 \quad 0.408$

$\begin{array}{lllll}\text { Ru } & 0.163587 & \mathbf{0 . 4 0 8 4 9 6} & \mathbf{0 . 2 1 7 4 1 9} \\ \mathrm{C} & 0.042427 & \mathbf{0 . 3 8 8 1 7 9} & \mathbf{2 . 2 1 3 2 8 8}\end{array}$

$\begin{array}{llll}\text { C } & \mathbf{0 . 0 4 2 4 2 7} & \mathbf{- 0 . 3 8 8 1 7 9} & \mathbf{2 . 2 1 3 2 8 8}\end{array}$

$\begin{array}{lrrr}C & -1.557405 & 1.128021 & 0.069342\end{array}$

$\begin{array}{llll}\text { S } & 1.387570 & 2.318777 & 1.001166\end{array}$

N $-1.441171 \quad-2.138668-0.912059$

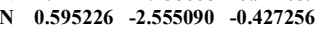

C $-2.704942-1.520705-1.123584$

$\begin{array}{lrrr}C & -1.240366 & -3.486074 & -1.178655 \\ C & 0.046827 & -3.746720 & -0.873003\end{array}$

C $1.964511-2.457648-0.034482$

$\begin{array}{llll}C & 1.964511 & -2.457648 & -0.034482 \\ C & -5.198322 & -0.275592 & -1.399586\end{array}$

$\begin{array}{llll}C & -5.198322 & -0.275592 & -1.399586\end{array}$

$\begin{array}{llll}C & -3.680435 & -1.635777 & -0.134722 \\ C & -2.976701 & -0.801434 & -2.292543\end{array}$

$\begin{array}{llll}\text { C } & -2.976701 & -0.801434 & -2.292543\end{array}$

$\begin{array}{llll}\text { C } & -4.228078 & -0.186066 & -2.403226\end{array}$

$\begin{array}{lllll}C & -4.913527 & -1.020602 & -0.248048\end{array}$

$\begin{array}{lllll}\text { H } & -4.450304 & \mathbf{0 . 3 8 5 8 1 4} & \mathbf{- 3 . 3 0 7 3 9 5}\end{array}$

$\begin{array}{llll}\text { H } & -\mathbf{5 . 6 3 8 1 4 6} & -\mathbf{1 . 1 2 3 7 3 8} & \mathbf{0 . 5 6 1 5 4 4}\end{array}$

$\begin{array}{llll}C & 4.619135 & -2.228882 & 0.855971\end{array}$

$\begin{array}{llll}C & 2.310689 & -2.710450 & 1.294834\end{array}$

$\begin{array}{llll}C & 2.977429 & -2.189830 & -0.965165\end{array}$

$\begin{array}{llll}\text { C } & 4.287827 & -2.062111 & \mathbf{- 0 . 4 9 0 7 7 2}\end{array}$

$\begin{array}{llll}C & 3.607490 & -2.584381 & 1.755795\end{array}$

$\begin{array}{cccc}\text { F } & 1.359010 & -3.111155 & \mathbf{2 . 1 4 3 1 2 6} \\ \text { C } & \mathbf{2 . 7 0 1 7 5 5} & -\mathbf{2 . 1 2 5 2 1 0} & -\mathbf{2 . 4 4 1 3 5 3}\end{array}$

$\begin{array}{llll}\text { C } & \mathbf{2 . 7 0 1 7 5 5} & -2.125210 & -2.441353 \\ \text { H } & 5.078755 & -1.830243 & -1.208301\end{array}$

$\begin{array}{llll}\text { H } & 3.815333 & -2.773720 & 2.810365\end{array}$

$\begin{array}{llll}\text { C } & \mathbf{6 . 0 2 9 0 8 4} & -\mathbf{2 . 0 3 3 8 1 3} & \mathbf{1 . 3 4 3 0 1 9}\end{array}$

H $\quad 6.302476-2.792407 \quad 2.090088$

H $3.016522-3.070789-2.911772$

\begin{tabular}{llll} 
H & 3.016522 & $-\mathbf{3 . 0 7 0 7 8 9}$ & -2.911772 \\
\hline & 6.132652 & -1.047822 & 1.822853
\end{tabular}

\begin{tabular}{llll} 
H & $\mathbf{6 . 1 3 2 6 5 2}$ & $\mathbf{- 1 . 0 4 7 8 2 2}$ & $\mathbf{1 . 8 2 2 8 5 3}$ \\
\hline & 6.751077 & $-\mathbf{0}$
\end{tabular}

$\begin{array}{llll}\text { H } & 6.751077 & -2.080408 & \mathbf{0 . 5 1 6 8 5 1}\end{array}$

$\begin{array}{lllll}\text { H } & 3.267601 & -1.308574 & -2.908136 \\ \text { H } & 1.640035 & -1.968489 & -2.663551\end{array}$

$\begin{array}{llll}\text { F } & \mathbf{- 3 . 3 8 4 8 3 5} & \mathbf{- 2 . 3 2 6 0 7 1} & \mathbf{0 . 9 7 0 7 4 8}\end{array}$

$\begin{array}{llll}C & -6.531446 & 0.408049 & -1.540836\end{array}$

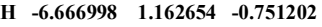

$\begin{array}{llll}\text { H } & -6.625739 & 0.909546 & \mathbf{- 2 . 5 1 2 8 9 3}\end{array}$

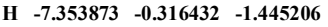

$\begin{array}{llll}\text { C } & -1.931346 & -0.659919 & -3.360963\end{array}$

$\begin{array}{llll}\text { H } & -1.455763 & -1.625626 & -3.587864\end{array}$

$\begin{array}{llll}\text { H } & -2.367235 & -0.258996 & -4.284777\end{array}$

\begin{tabular}{llll}
\hline & -1.132476 & 0.022373 & -3.028606
\end{tabular}

$\begin{array}{llll}C & \mathbf{2 . 0 3 5 2 5 5} & \mathbf{2 . 3 3 9 7 2 1} & \mathbf{- 1 . 6 7 6 6 2 0}\end{array}$

$\begin{array}{lllll}C & \mathbf{2 . 6 3 5 3 7 0} & \mathbf{2 . 8 9 5 4 2 8} & \mathbf{- 2 . 8 1 7 2 5 8}\end{array}$

$\begin{array}{llll}C & \mathbf{2 . 1 1 7 3 1 0} & \mathbf{3 . 0 0 5 7 9 0} & \mathbf{- 0 . 4 4 1 7 8 0}\end{array}$

$\begin{array}{llll}C & 3.317410 & 4.105013 & -2.740875\end{array}$

$\begin{array}{llll}\text { H } & \mathbf{2 . 5 6 0 3 6 8} & \mathbf{2 . 3 6 5 2 2 2} & \mathbf{- 3 . 7 7 0 7 3 9}\end{array}$

$\begin{array}{llll}C & 2.813531 & 4.327477 & -0.380462\end{array}$

$\begin{array}{llll}\text { C } & 2.813531 & 4.227477 & -0.380462 \\ C & 3.406879 & 4.772118 & -1.513492\end{array}$

\begin{tabular}{llll}
$C$ & 3.406879 & 4.772118 & -1.513492 \\
\hline
\end{tabular}

$\begin{array}{llll}\text { H } & 3.778706 & \mathbf{4 . 5 2 9 3 3 0} & \mathbf{- 3 . 6 3 5 5 4 2}\end{array}$

$\begin{array}{lllll}\text { H } & \mathbf{2 . 8 8 0 5 7 4} & \mathbf{4 . 7 4 7 5 2 7} & \mathbf{0 . 5 7 8 7 9 6}\end{array}$

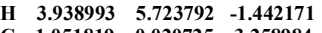

$\begin{array}{llll}C & 1.051819 & 0.020725 & 3.258984\end{array}$

$\begin{array}{llll}\text { H } & -0.031267 & -1.472805 & 2.119647\end{array}$

$\begin{array}{llll}C & -1.229026 & 0.247720 & 2.000098\end{array}$

$\begin{array}{llll}\text { H } & -2.464910 & \mathbf{0 . 5 2 7 6 2 2} & \mathbf{- 0 . 0 5 5 4 1 4} \\ \text { C } & -1.686796 & \mathbf{1 . 4 4 4 4 2 7} & \mathbf{2 . 7 7 7 7 1 7}\end{array}$

C $-1.8658542 .579850-0.085636$

$\begin{array}{llll}C & -1.865854 & \mathbf{2 . 5 7 9 8 5 0} & \mathbf{- 0 . 0 8 5 6 3 6}\end{array}$

$\begin{array}{lllll}\text { H } & \mathbf{- 1 . 9 6 4 5 8 0} & \mathbf{2 . 7 3 6 1 6 5} & \mathbf{- 1 . 1 7 5 8 3 5}\end{array}$

$\begin{array}{llll}\text { H } & \mathbf{- 2 . 8 4 0 8 8 4} & \mathbf{2 . 8 2 8 3 4 6} & \mathbf{0 . 3 6 2 3 6 9}\end{array}$

$\begin{array}{llll}\text { H } & -1.075821 & 3.242334 & \mathbf{0 . 2 8 3 8 7 4}\end{array}$

$\begin{array}{lllll}\text { O } & -1.004337 & 2.247600 & 3.359971\end{array}$

$\begin{array}{lllll}O & -3.024693 & 1.508013 & 2.752136\end{array}$

$\begin{array}{llll}C & -3.623608 & \mathbf{2 . 6 0 2 6 7 0} & \mathbf{3 . 4 3 1 8 9 6}\end{array}$

$\begin{array}{llll}\text { H } & -4.703913 & \mathbf{2 . 4 9 6 1 4 0} & \mathbf{3 . 2 8 8 6 4 9}\end{array}$

H $\quad \mathbf{- 3 . 2 7 6 9 0 9} \quad \mathbf{3 . 5 5 6 4 7 6} \mathbf{3 . 0 0 9 3 7 6}$

$\begin{array}{llll}\text { H } & \mathbf{- 3 . 3 8 0 0 2 6} & \mathbf{2 . 5 7 7 7 5 0} & \mathbf{4 . 5 0 3 2 2 0}\end{array}$

$\begin{array}{lll}-3.380026 & 2.577750 & 4.503220\end{array}$

\begin{tabular}{cccc} 
H & $\mathbf{2 . 0 4 9 7 2 7}$ & $\mathbf{- 0 . 3 4 6 3 6 5}$ & $\mathbf{2 . 9 7 9 4 8 0}$ \\
\hline & 0.782632 & -0.452348 & $\mathbf{4 . 2 1 8 8 7 0}$
\end{tabular}

$\begin{array}{llll}\text { H } & \mathbf{0 . 7 8 2 6 3 2} & \mathbf{- 0 . 4 5 2 3 4 8} & \mathbf{4 . 2 1 8 8 7 0}\end{array}$

$\begin{array}{llll}\text { H } & \mathbf{1 . 1 0 2 0 6 6} & \mathbf{1 . 1 0 3 0 6 2} & \mathbf{3 . 4 0 7 6 3 6}\end{array}$

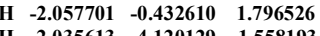

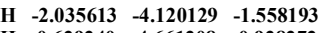

$\begin{array}{llll}\text { H } & 0.629240 & -4.661208 & -0.928272\end{array}$

75

unsat_mcb b / electronic energy: $-\mathbf{2 5 8 8 . 9 0 0 6 4 7 2 9}$ a.u. / lowest freq: $11.17 \mathrm{~cm}-1$

S $\quad \mathbf{1 . 1 3 6 2 9 7} \quad \mathbf{1 . 2 2 9 0 0 6}-\mathbf{- 1 . 6 6 1 7 2 4}$

$\begin{array}{llll}C & -0.317892 & -1.223007 & -0.93211\end{array}$

C $-0.317892-1.223007-0.932111$

$\begin{array}{llll}C & 0.15593 & 0.486525 & 0.232151\end{array}$

$\begin{array}{llll}\text { C } & \mathbf{0 . 2 9 8 8 2 0} & \mathbf{- 0 . 7 1 6 3 4 8} & \mathbf{1 . 8 1 7 9 5 7}\end{array}$

$\begin{array}{llll}C & -1.680213 & 0.913100 & 0.755242\end{array}$

$\begin{array}{llll}\text { S } & 1.211798 & 2.367402 & 1.324389\end{array}$

N $-1.404059-1.577578 \quad-1.675784$

N $\quad 0.591006-2.202048-1.238110$

C $-\mathbf{- 2 . 6 9 2 1 2 1}-\mathbf{- 0 . 9 7 0 5 3 4}-\mathbf{- 1 . 6 2 8 1 3 1}$

C $-1.181582 \quad-2.729920-2.415190$

$\begin{array}{llll}C & 0.078345 & -3.122694 & -2.138481\end{array}$

$\begin{array}{llll}C & \mathbf{1 . 8 7 6 2 2 0} & -\mathbf{2 . 3 9 1 5 4 8} & -\mathbf{0 . 6 4 1 1 1 8}\end{array}$

$\begin{array}{lrrr}C & -5.242723 & 0.158472 & -1.370553\end{array}$ 
$\begin{array}{llll}C & -3.616102 & -1.475380 & -0.712757\end{array}$ $\begin{array}{llll}C & -3.047439 & 0.093132 & -2.46280\end{array}$ $\begin{array}{llll}C & -4.324943 & 0.643440 & -2.307482\end{array}$ $\begin{array}{llll}C & -4.877020 & -0.928620 & -0.566307\end{array}$ $\begin{array}{llll}\text { H } & -4.611517 & 1.482921 & -2.945562\end{array}$ $\begin{array}{llll}H & -5.557189 & -1.345875 & 0.1783\end{array}$ $\begin{array}{llll}H & -5.557189 & -1.345875 & 0.178319 \\ C & 4.314403 & -2.851911 & 0.682555\end{array}$

C $4.314403-2.851911 \quad 0.682555$

C $1.944550-3.1$

$\begin{array}{llll}C & 3.063818 & -1.928036 & -1.217787\end{array}$

$\begin{array}{llll}C & 4.260696 & -2.150583 & -0.521563\end{array}$

$\begin{array}{llll}\text { C } & 3.126696 & -3.390625 & 1.195990\end{array}$

$\begin{array}{lllll}\text { F } & 0.816020 & -3.719185 & 0.983239\end{array}$

$\begin{array}{llll}\text { C } & 3.113866 & -1.283280 & -2.574412\end{array}$

H $\mathbf{5 . 1 8 7 9 4 4} \quad \mathbf{- 1 . 7 6 8 9 6 6} \quad \mathbf{- 0 . 9 5 6 5 3 2}$

H $3.113396 \quad-3.984935 \quad 2.111357$

C $\quad 5.611533-\mathbf{3 . 0 5 3 4 1 6} \quad \mathbf{1 . 4 1 7 4 9 3}$

H $5.784898-4.119680 \quad 1.625529$

H $3.731611-1.901184-\mathbf{3 . 2 4 4 1 8 3}$

$\begin{array}{llll}\text { H } & \mathbf{5 . 5 9 0 7 8 5} & \mathbf{- 2 . 5 3 0 6 1 6} & \mathbf{2 . 3 8 5 9 9 2}\end{array}$

$\begin{array}{lllll}\text { H } & 6.464264 & -2.669366 & 0.842479\end{array}$

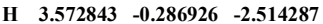

H $\quad 2.121010-1.165753 \quad-3.021990$

$\begin{array}{llll}\text { F } & -3.237726 & -2.485928 & 0.075072\end{array}$

$\begin{array}{llll}\text { C } & -6.601349 & 0.785874 & -1.212518\end{array}$

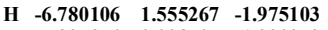

$\begin{array}{llll}\text { H } & -7.395374 & 0.028705 & -1.290959\end{array}$

\begin{tabular}{cccc}
\hline & -6.697142 & 1.257969 & -0.222724
\end{tabular}

$\begin{array}{llll}\text { C } & \mathbf{- 2 . 0 7 3 5 5 4} & \mathbf{0 . 6 3 6 3 8 2} & \mathbf{- 3 . 4 6 7 6 4 6}\end{array}$

$\begin{array}{llll}\text { H } & -1.633660 & -0.170725 & -4.072388\end{array}$

\begin{tabular}{lllll}
\hline & -2.563789 & 1.349700 & -4.142062
\end{tabular}

$\begin{array}{llll}\text { H } & -1.240258 & 1.147684 & -2.961240\end{array}$

$\begin{array}{lllll}\text { C } & \mathbf{2 . 0 4 7 1 7 0} & \mathbf{2 . 6 9 5 8 0 9} & \mathbf{- 1 . 2 7 9 9 0 8}\end{array}$

$\begin{array}{llll}C & 2.749301 & \mathbf{3 . 3 5 3 6 9 3} & \mathbf{- 2 . 3 0 0 3 8 4}\end{array}$

$\begin{array}{llll}C & 2.060583 & 3.192807 & \mathbf{0 . 0 3 4 1 6 2}\end{array}$

$\begin{array}{llll}C & 2.060583 & 3.192807 & 0.034162\end{array}$

$\begin{array}{llll}C & 3.463663 & 4.515266 & \mathbf{- 2 . 0 2 7 9 1 6}\end{array}$

$\begin{array}{llll}\text { H } & 2.727373 & 2.942926 & \mathbf{- 3 . 3 1 3 7 2 3}\end{array}$

$\begin{array}{llll}\text { C } & \mathbf{2 . 7 8 8 2 3 6} & \mathbf{4 . 3 7 2 0 3 2} & \mathbf{0 . 2 9 1 0 1 0}\end{array}$

$\begin{array}{llll}\text { C } & 3.478867 & 5.023966 & -0.723311\end{array}$

$\begin{array}{llll}\text { H } & 4.006314 & 5.024048 & -2.827741\end{array}$

H $2.802066 \quad 4.771472 \quad 1.308519$

$\begin{array}{llll}\text { H } & \mathbf{4 . 0 3 4 1 6 5} & \mathbf{5 . 9 3 7 7 5 4} & \mathbf{- 0 . 4 9 7 8 9 0}\end{array}$

$\begin{array}{llll}C & \mathbf{1 . 2 8 4 2 0 9} & \mathbf{- 0 . 5 5 6 4 1 0} & \mathbf{2 . 9 5 2 3 6 0}\end{array}$

$\begin{array}{llll}\text { H } & 0.160703 & -1.777649 & 1.602293\end{array}$

$\begin{array}{llll}\text { C } & -1.168136 & -0.094089 & 1.926316\end{array}$

$\begin{array}{llll}\text { H } & -2.476295 & \mathbf{0 . 3 9 4 3 3 2} & \mathbf{0 . 2 2 0 6 5 7}\end{array}$

$\begin{array}{llll}C & -1.416175 & 0.567722 & 3.262123\end{array}$

$\begin{array}{llll}C & -2.169938 & \mathbf{2 . 2 9 4 1 8 8} & \mathbf{1 . 1 3 3 8 6 0}\end{array}$

$\begin{array}{llll}\text { H } & -2.362840 & 2.845138 & 0.201149\end{array}$

$\begin{array}{llll}\text { H } & -3.130980 & 2.219757 & 1.671734\end{array}$

$\begin{array}{llll}\text { H } & -1.454425 & 2.865612 & 1.732329\end{array}$

$\begin{array}{lllll}\text { O } & -\mathbf{0 . 7 6 6 3 2 8} & \mathbf{1 . 4 4 2 8 2 5} & \mathbf{3 . 7 6 8 8 1 8}\end{array}$

$\begin{array}{llll}\text { O } & -2.513146 & \mathbf{0 . 0 4 8 6 3 3} & \mathbf{3 . 8 1 7 2 2 2}\end{array}$

$\begin{array}{llll}\text { C } & -2.911492 & 0.596388 & 5.068788\end{array}$

\begin{tabular}{llll}
$C$ & -2.911492 & 0.596388 & 5.068788 \\
\hline & -3.824335 & 0.065337 & 5.357370
\end{tabular}

$\begin{array}{llll}\text { H } & -3.824355 & 0.065337 & \mathbf{5 . 3 5 7 3 7 0}\end{array}$

H $-3.111908 \quad 1.672348 \quad 4.971616$

$\begin{array}{llll}\text { H } & -2.129164 & 0.445491 & 5.825490\end{array}$

$\begin{array}{llll}\text { H } & \mathbf{2 . 2 4 9 6 2 9} & \mathbf{- 0 . 9 6 2 3 0 9} & \mathbf{2 . 6 1 3 6 8 5}\end{array}$

H $0.968429-1.154292 \quad 3.824856$

H $\quad 1.429768 \quad 0.482326 \quad 3.261629$

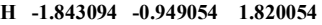

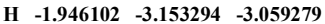

H $\quad 0.662726-3.967745-2.489135$

75

unsat ts2 b / electronic energy: $-\mathbf{2 5 8 8 . 8 9 2 6 1 8 2 4}$ a.u. / lowest freq: $-137.14 \mathrm{~cm}-1$

S $1.193725 \quad 0.790503 \quad-1.801804$

C $-0.316373-1.531499-0.437388$

$\begin{array}{llll}\text { Ru } & 0.163587 & 0.408496 & 0.217419\end{array}$

$\begin{array}{llll}\text { C } & 0.042427 & -0.388179 & 2.213288\end{array}$

$\begin{array}{llll}C & -1.557405 & 1.128021 & 0.069342\end{array}$

$\begin{array}{llll}\text { S } & 1.387570 & \mathbf{2 . 3 1 8 7 7 7} & \mathbf{1 . 0 0 1 1 6 6}\end{array}$

$\begin{array}{llll}\text { N } & -1.441171 & -2.138668 & -0.912059\end{array}$

$\begin{array}{llll}\mathrm{N} & 0.595226 & \mathbf{- 2 . 5 5 5 0 9 0} & \mathbf{- 0 . 4 2 7 2 5 6}\end{array}$

$\begin{array}{llll}\text { C } & -2.704942 & -1.520705 & -1.123584\end{array}$

$\begin{array}{llll}C & -1.240366 & -3.486074 & -1.178655\end{array}$

$\begin{array}{llll}C & \mathbf{0 . 0 4 6 8 2 7} & \mathbf{- 3 . 7 4 6 7 2 0} & \mathbf{- 0 . 8 7 3 0 0 3}\end{array}$

$\begin{array}{llll}C & 1.964511 & -2.457648 & -0.034482\end{array}$

$\begin{array}{llll}C & -5.198322 & -0.275592 & -1.399586\end{array}$

$\begin{array}{llll}C & -\mathbf{3} .680435 & -1.635777 & -0.134722\end{array}$

$\begin{array}{llll}\text { C } & -2.976701 & -0.801434 & -2.292543\end{array}$

$\begin{array}{llll}\text { C } & -4.228078 & -0.186066 & -2.403226\end{array}$

$\begin{array}{llll}C & -4.913527 & -1.020602 & -0.248048\end{array}$

$\begin{array}{llll}C & -4.450304 & 0.385814 & -3.307395\end{array}$

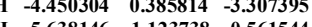

\begin{tabular}{llll}
\hline & -5.638146 & -1.123738 & 0.561544 \\
\hline
\end{tabular}

$\begin{array}{llll}C & 4.619135 & -2.228882 & \mathbf{0 . 8 5 5 9 7 1}\end{array}$

$\begin{array}{llll}C & 2.310689 & -2.710450 & 1.294834\end{array}$

$\begin{array}{llll}C & \mathbf{2 . 9 7 7 4 2 9} & \mathbf{- 2 . 1 8 9 8 3 0} & \mathbf{- 0 . 9 6 5 1 6 5}\end{array}$

$\begin{array}{llll}C & 4.287827 & -2.062111 & -0.490772\end{array}$

$\begin{array}{llll}C & 3.607490 & -2.584381 & 1.755795\end{array}$

$\begin{array}{llll}\text { F } & \mathbf{1 . 3 5 9 0 1 0} & \mathbf{- 3 . 1 1 1 1 5 5} & \mathbf{2 . 1 4 3 1 2 6}\end{array}$

$\begin{array}{llll}C & \mathbf{2 . 7 0 1 7 5 5} & \mathbf{- 2 . 1 2 5 2 1 0} & \mathbf{- 2 . 4 4 1 3 5 3}\end{array}$

$\begin{array}{llll}\text { H } & 5.078755 & -1.830243 & -1.208301\end{array}$

\begin{tabular}{llll}
\hline & 3.078733 & -2.873720 & 2810365
\end{tabular}

$\begin{array}{rrrr}\text { H } & 3.815333 & -2.773720 & 2.810365 \\ \text { C } & 6.029084 & -2.033813 & 1.343019\end{array}$ 
H $\quad \begin{array}{lll}6.302476 & -2.792407 & 2.090088\end{array}$ $\begin{array}{llll}\text { H } & 3.016522 & -3.070789 & -2.911772\end{array}$ H $\quad 6.132652-1.047822 \quad 1.822853$ $\begin{array}{llll}\text { H } & 6.751077 & -2.080408 & 0.516851\end{array}$ H $3.267601-1308574-2.908136$ $1.640035-1.968489-2.663551$ \begin{tabular}{llll} 
H & 1.640035 & -1.968489 & -2.663551 \\
\hline & -3.384835 & -2.326071 & 0.970748
\end{tabular} \begin{tabular}{llll}
\hline & -3.384835 & -2.326071 & 0.970748
\end{tabular} $\begin{array}{llll}\text { C } & -6.531446 & 0.408049 & -1.540836\end{array}$ $\begin{array}{llll}\text { H } & -6.666998 & 1.162654 & -0.751202\end{array}$

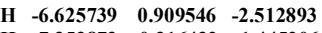
$\begin{array}{lllll}\text { H } & -7.353873 & -0.316432 & -1.445206\end{array}$ $\begin{array}{llll}\text { C } & -1.931346 & -0.659919 & -3.360963\end{array}$ H $-\mathbf{1 . 4 5 5 7 6 3}-\mathbf{- 1 . 6 2 5 6 2 6}-\mathbf{- 3 . 5 8 7 8 6 4}$ $\begin{array}{lll}-1.132476 & 0.022373 & -3.028606\end{array}$ $\begin{array}{llll}\mathrm{H} & -1.132476 & 0.022373 & -3.028606\end{array}$ $\begin{array}{llll}\text { C } & 2.035255 & 2.339721 & -1.676620\end{array}$ $\begin{array}{lllll}C & 2.635370 & 2.895428 & \mathbf{- 2 . 8 1 7 2 5 8}\end{array}$ $\begin{array}{llll}C & 2.117310 & 3.005790 & \mathbf{- 0 . 4 4 1 7 8 0}\end{array}$ $\begin{array}{llll}C & 3.317410 & 4.105013 & -2.740875\end{array}$

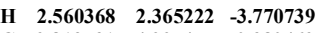
$\begin{array}{llll}C & 2.813531 & 4.227477 & -0.380462\end{array}$ $\begin{array}{lllll}C & 3.406879 & 4.772118 & -1.513492\end{array}$ H $3.778706 \quad 4.529330 \quad-3.635542$ $\begin{array}{lllll}\text { H } & 2.880574 & 4.747527 & 0.578796\end{array}$ H $3.938993 \quad 5.723792 \quad-1.442171$ $\begin{array}{llll}\text { C } & 1.051819 & 0.020725 & \mathbf{3 . 2 5 8 9 8 4}\end{array}$ $\begin{array}{rrrr}\text { H } & \mathbf{- 0 . 0 3 1 2 6 7} & \mathbf{- 1 . 4 7 2 8 0 5} & \mathbf{2 . 1 1 9 6 4 7} \\ \text { C } & \mathbf{- 1 . 2 2 9 0 2 6} & \mathbf{0 . 2 4 7 7 2 0} & \mathbf{2 . 0 0 0 0 9 8}\end{array}$ $\begin{array}{llll}\text { H } & \mathbf{- 2 . 4 6 4 9 1 0} & \mathbf{0 . 5 2 7 6 2 2} & \mathbf{- 0 . 0 5 5 4 1 4}\end{array}$ $\begin{array}{llll}\text { C } & -\mathbf{1 . 6 8 6 7 9 6} & \mathbf{1 . 4 4 4 4 2 7} & \mathbf{2 . 7 7 7 7 1 7}\end{array}$

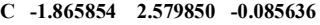

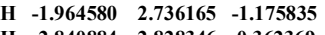
H $\quad \mathbf{- 2 . 8 4 0 8 8 4} \quad \mathbf{2 . 8 2 8 3 4 6} \quad \mathbf{0 . 3 6 2 3 6 9}$

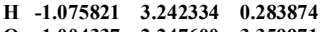
$\begin{array}{llll}\text { O } & -1.004337 & 2.247600 & 3.359971\end{array}$ \begin{tabular}{llll}
\hline & -3.024693 & 1.508013 & 2.752136
\end{tabular} $\begin{array}{llll}\text { C } & \mathbf{- 3 . 6 2 3 6 0 8} & \mathbf{2 . 6 0 2 6 7 0} & \mathbf{3 . 4 3 1 8 9 6}\end{array}$ $\begin{array}{llll}\text { H } & -4.703913 & 2.496140 & 3.288649\end{array}$ H $\quad-3.276909 \quad 3.556476 \quad 3.009376$ H $\quad \begin{array}{rrrr}-3.380026 & 2.577750 & 4.503220\end{array}$ $\begin{array}{llll}\text { H } & 2.049727 & -\mathbf{0 . 3 4 6 3 6 5} & \mathbf{2 . 9 7 9 4 8 0}\end{array}$ $\begin{array}{llll}\text { H } & 0.782632 & -0.452348 & 4.218870\end{array}$ H $\quad \begin{array}{llll}1.102066 & 1.103062 & 3.407636\end{array}$ H $\quad \mathbf{- 2 . 0 5 7 7 0 1}-\mathbf{- 0 . 4 3 2 6 1 0} \mathbf{1 . 7 9 6 5 2 6}$

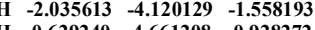
H $\quad 0.629240 \quad-4.661208-0.928272$ 75 unsat_pc2_b / electronic energy: $\mathbf{- 2 5 8 8 . 9 0 6 4 1 1 4 1}$ a.u. / lowest freq: $\mathbf{- 6 . 4 6} \mathrm{cm}-1$ $\begin{array}{llll}\mathrm{S} & \mathbf{- 0 . 3 9 0 0 7 1} & \mathbf{1 . 3 3 2 8 0 2} & \mathbf{- 1 . 8 3 2 1 9}\end{array}$

$\begin{array}{llll}C & -\mathbf{0 . 0 5 4 5 2 8} & -\mathbf{1 . 4 2 8 6 7 8} & \mathbf{- 0 . 8 2 5 9 9 0}\end{array}$

$\begin{array}{lllll}\text { Ru } & -\mathbf{0 . 0 2 5 0 0 0} & \mathbf{0 . 4 2 0 5 2 5} & \mathbf{0 . 2 8 1 5 7 8}\end{array}$

$\begin{array}{lllll}\text { C } & \mathbf{1 . 8 0 4 6 7 7} & \mathbf{0 . 6 4 5 9 9 0} & \mathbf{0 . 3 2 5 2 1 7}\end{array}$

$\begin{array}{llll}C & -0.858525 & -0.745718 & 2.024682\end{array}$

$\begin{array}{llll}C & -0.858525 & 0.745718 & 2.024682\end{array}$

$\begin{array}{llll}\mathrm{S} & -0.621331 & 2.548151 & 1.136010\end{array}$

$\mathrm{N}-1.149981-2.143307-1.123035$

$\begin{array}{llll}\mathrm{N} & 0.983759 & -2.112505 & -1.280405\end{array}$

$\begin{array}{llll}C & -2.493317 & -1.963192 & -0.669802\end{array}$

$\begin{array}{llll}C & -0.793386 & -3.236633 & -1.900379\end{array}$

$\begin{array}{llll}C & 0.550898 & -3.218078 & -1.999904\end{array}$

$\begin{array}{lllll}C & 2.367348 & -1.860614 & -1.061119\end{array}$

$\begin{array}{llll}\text { C } & -5.091192 & -1.749846 & 0.383951\end{array}$

$\begin{array}{llll}C & -2.902523 & -2.721165 & 0.434299\end{array}$

$\begin{array}{llll}\text { C } & -3.429056 & -1.155343 & -1.324448\end{array}$

$\begin{array}{llll}C & -4.708272 & -1.047019 & -0.757991\end{array}$

$\begin{array}{llll}C & -4.167783 & -2.624437 & 0.973412\end{array}$

$\begin{array}{llll}\text { H } & -5.436340 & -\mathbf{0 . 3 9 8 5 3 7} & -\mathbf{1 . 2 5 1 8 1 7}\end{array}$

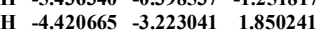

$\begin{array}{llll}\text { C } & \mathbf{5 . 0 9 2 8 3 8} & \mathbf{- 1 . 4 8 2 4 0 7} & \mathbf{- 0 . 5 3 1 2 2 0}\end{array}$

$\begin{array}{llll}C & 2.953357 & -2.365509 & \mathbf{0 . 0 9 7 7 1 4}\end{array}$

$\begin{array}{llll}C & 3.151400 & -1.172633 & -1.995837\end{array}$

$\begin{array}{llll}C & 4.507872 & -0.999714 & -1.708114\end{array}$

$\begin{array}{llll}\text { C } & 4.294906 & -2.180048 & 0.381891\end{array}$

$\begin{array}{llll}\text { F } & 2.179606 & -3.017282 & 0.969379\end{array}$

$\begin{array}{llll}\text { F } & \mathbf{2 . 1 7 9 6 0 6} & \mathbf{- 3 . 0 1 7 2 8 2} & \mathbf{0 . 9 6 9 3 7 9} \\ \text { C } & \mathbf{2 . 5 2 9 8 6 9} & \mathbf{- 0 . 6 1 3 3 1 7} & \mathbf{- 3 . 2 4 2 3 8 0}\end{array}$

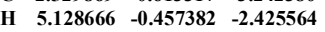

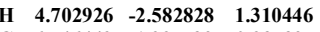

$\begin{array}{llll}C & 6.546442 & -1.227539 & -0.237395\end{array}$

$\begin{array}{lllll}\text { H } & 6.957694 & -1.982734 & 0.445989\end{array}$

H $2.022796 \quad-1.397438 \quad-3.824809$

$\begin{array}{lllll}\text { H } & 6.671620 & -0.242871 & 0.240647\end{array}$

H $7.144913-1.224801-1.158907$

I $3.287609-0.143819-3.882209$

\begin{tabular}{llll} 
H & 3.287609 & -0.143819 & $\mathbf{- 3 . 8 8 2 2 0 9}$ \\
\hline & 1.769257 & 0.141095 & -2.983123
\end{tabular}

$\begin{array}{llll}\text { H } & 1.769257 & 0.141095 & -2.983123\end{array}$

$\begin{array}{llll}F & -2.019361 & -3.561331 & 0.985570\end{array}$

$\begin{array}{llll}\text { C } & -6.462591 & -1.589627 & 0.980980\end{array}$

$\begin{array}{llll}\text { H } & -7.112514 & -0.980801 & 0.338811\end{array}$

$\begin{array}{llll}\text { H } & -6.942837 & -2.566851 & 1.136717\end{array}$

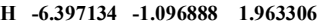

$\begin{array}{llll}\text { C } & \mathbf{- 3 . 1 4 2 5 4 5} & \mathbf{- 0 . 4 7 3 6 7 6} & \mathbf{- 2 . 6 3 1 7 5 1}\end{array}$

$\begin{array}{llll}\text { H } & -2.138311 & -0.696787 & -3.008164\end{array}$

$\begin{array}{llll}\text { H } & -3.882413 & -0.797962 & -3.378866\end{array}$

$\begin{array}{lllll}\text { H } & -3.218523 & 0.617224 & -2.527894\end{array}$

$\begin{array}{llll}\text { C } & -0.627195 & 3.060358 & -1.582619\end{array}$ 


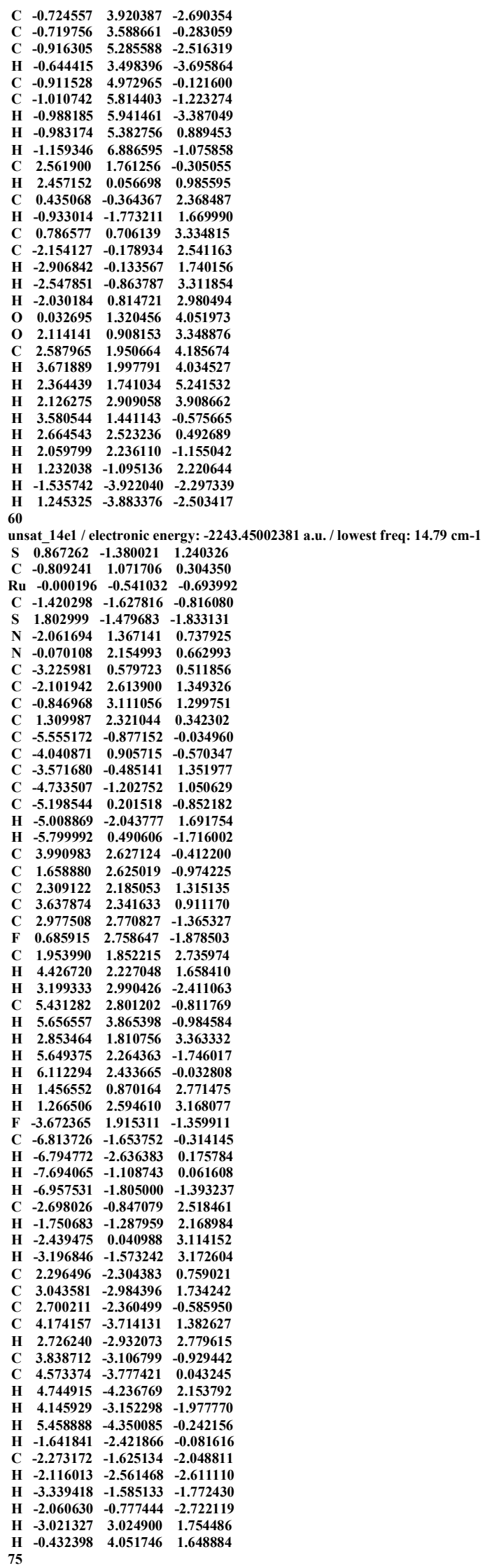


unsat_pc1_c / electronic energy: $-\mathbf{2 5 8 8 . 9 0 7 0 9 5 9 0 ~ a . u . ~ / ~ l o w e s t ~ f r e q : ~} 21.96 \mathrm{~cm}-1$ $\begin{array}{lllll}S & -\mathbf{0 . 5 3 2 3 7 2} & \mathbf{1 . 3 7 9 4 6 2} & -\mathbf{1 . 6 1 1 0 8 6}\end{array}$

$\begin{array}{llll}C & 0.724019 & -1.295938 & -0.609009\end{array}$

$\begin{array}{llll}\text { Ru } & 0.112322 & 0.433620 & 0.419889\end{array}$

$\begin{array}{llll}\text { C } & \mathbf{1 . 7 5 1 5 8 0} & \mathbf{1 . 2 6 4 9 8 2} & \mathbf{0 . 5 2 4 0 1 9}\end{array}$

$\begin{array}{llll}C & -0.270793 & -0.913747 & 2.180045\end{array}$

$\begin{array}{lllll}\mathrm{S} & -1.229547 & 2.099019 & 1.435914\end{array}$

$\begin{array}{llll}\mathrm{N} & \mathbf{- 0 . 0 9 9 4 0 5} & \mathbf{- 2 . 2 6 6 8 0 0} & \mathbf{- 1 . 1 0 4 3 4 3}\end{array}$

$\begin{array}{llll}N & \mathbf{1 . 9 6 8 4 6 5} & \mathbf{- 1 . 7 6 1 2 4 1} & \mathbf{- 0 . 9 1 3 8 9 1}\end{array}$

$\begin{array}{llll}C & -1.526935 & -2.241548 & -1.118035\end{array}$

$\begin{array}{llll}C & 0.615145 & -3.302937 & -1.685966\end{array}$

$\begin{array}{llll}C & 1.920234 & -2.985321 & -1.566123\end{array}$

$\begin{array}{llll}\text { C } & 3.199498 & -1.093669 & -0.663418\end{array}$

C $-\mathbf{- 4 . 3 3 1 3 0 2}-\mathbf{- 2 . 2 4 4 7 4 9}-1.061956$

$\begin{array}{llll}C & -2.229844 & -2.846773 & -0.077105\end{array}$

$\begin{array}{llll}C & -2.231389 & -1.709250 & -2.207452\end{array}$

$\begin{array}{llll}C & -3.627903 & -1.702767 & -2.142511\end{array}$

$\begin{array}{llll}C & -3.611354 & -2.850006 & -0.027625\end{array}$

$\begin{array}{llll}\text { H } & -4.185201 & -1.267936 & -2.975955\end{array}$

$\begin{array}{llll}\text { H } & -4.107875 & -3.322084 & 0.821614\end{array}$

$\begin{array}{llll}\text { C } & 5.612272 & 0.200186 & -0.065762\end{array}$

$\begin{array}{llll}\text { C } & 3.951669 & -1.453403 & 0.450920\end{array}$

$\begin{array}{llll}C & 3.673769 & -0.103087 & -1.534732\end{array}$

$\begin{array}{llll}C & 4.877664 & 0.527680 & -1.212132\end{array}$

$\begin{array}{llll}\text { C } & \mathbf{5 . 1 3 8 8 8 0} & \mathbf{- 0 . 8 1 6 1 0 7} & \mathbf{0 . 7 7 0 5 7 2}\end{array}$

$\begin{array}{llll}\text { F } & 3.493696 & -2.427378 & 1.242658\end{array}$

$\begin{array}{llll}\text { C } & \mathbf{2 . 8 8 7 0 3 3} & \mathbf{0 . 2 8 1 1 7 2} & \mathbf{- 2 . 7 5 4 4 5 1}\end{array}$

$\begin{array}{llll}\text { H } & \mathbf{5 . 2 5 5 1 8 6} & \mathbf{1 . 3 0 5 2 2 1} & \mathbf{- 1 . 8 8 1 0 1 2}\end{array}$

$\begin{array}{llll}\text { H } & 5.682153 & -1.123839 & 1.665584\end{array}$

$\begin{array}{llll}\text { C } & \mathbf{6 . 8 7 8 3 4 4} & \mathbf{0 . 9 4 0 9 9 0} & \mathbf{0 . 2 7 0 0 4 5}\end{array}$

$\begin{array}{llll}\text { H } & 7.536127 & 0.343035 & 0.915038\end{array}$

$\begin{array}{lllll}\text { H } & \mathbf{2 . 5 5 6 0 4 8} & \mathbf{- 0 . 6 0 6 4 7 3} & \mathbf{- 3 . 3 1 4 1 7 9}\end{array}$

$\begin{array}{lllll}\text { H } & \mathbf{6 . 6 4 3 5 3 4} & \mathbf{1 . 8 7 4 5 9 7} & \mathbf{0 . 8 0 5 5 1 0}\end{array}$

$\begin{array}{lllll}\text { H } & \mathbf{7 . 4 3 2 3 5 3} & \mathbf{1 . 2 1 3 9 8 3} & \mathbf{- 0 . 6 3 8 8 4 1}\end{array}$

$\begin{array}{lllll}\text { H } & 3.487624 & 0.910369 & -3.423447\end{array}$

$\begin{array}{llll}\text { H } & 1.980994 & 0.841672 & -2.471034\end{array}$

$\begin{array}{llll}\text { F } & -1.540117 & -\mathbf{3 . 4 6 6 6 5 2} & 0.884019\end{array}$

$\begin{array}{llll}\text { C } & \mathbf{- 5 . 8 3 2 8 6 6} & \mathbf{- 2 . 1 7 1 8 3 7} & \mathbf{- 0 . 9 9 7 6 3 5}\end{array}$

$\begin{array}{llll}\text { H } & -6.144480 & -1.316854 & -0.376429\end{array}$

$\begin{array}{llll}\text { H } & -6.273486 & -2.035384 & -1.994531\end{array}$

$\begin{array}{llll}\text { H } & -6.259779 & -3.078946 & -0.547073\end{array}$

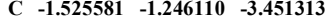

$\begin{array}{llll}\text { H } & -0.477806 & -0.982156 & -3.265904\end{array}$

$\begin{array}{lllll}\text { H } & -1.547124 & -2.050369 & -4.204647\end{array}$

$\begin{array}{llll}\text { H } & -2.024085 & -0.367452 & -3.880237\end{array}$

$\begin{array}{llll}\text { C } & -1.318774 & 2.902910 & -1.204838\end{array}$

$\begin{array}{lllll}C & -1.653080 & 3.814650 & -2.221668\end{array}$

$\begin{array}{llll}C & -1.636650 & 3.207559 & 0.130490\end{array}$

$\begin{array}{llll}C & -2.303898 & 5.005531 & -1.923456\end{array}$

$\begin{array}{lllll}\text { H } & -1.395558 & 3.572759 & -3.256431\end{array}$

$\begin{array}{llll}\text { C } & -2.296476 & 4.416217 & 0.418464\end{array}$

$\begin{array}{llll}C & -2.628584 & 5.306679 & -0.594740\end{array}$

$\begin{array}{llll}\text { H } & -2.557450 & 5.704004 & -2.724183\end{array}$

\begin{tabular}{llll}
$\mathrm{H}$ & -2.557450 & 5.704004 & -2.724183 \\
\hline & -2.541622 & 4.64804 & 1.458250
\end{tabular}

\begin{tabular}{lllll}
\hline & -2.541622 & 4.648404 & 1.458250 \\
\hline & -3.137402 & 6.242311 & -0.351415
\end{tabular}

$\begin{array}{lllll}\text { H } & -3.137402 & 6.242311 & \mathbf{- 0 . 3 5 1 4 1 5}\end{array}$

$\begin{array}{llll}\text { C } & \mathbf{2 . 0 5 6 2 8 2} & \mathbf{2 . 6 5 6 0 8 4} & \mathbf{0 . 0 8 9 5 9 5}\end{array}$

$\begin{array}{llll}\text { H } & 2.631125 & 0.817581 & 1.014911\end{array}$

$\begin{array}{llll}\text { C } & \mathbf{1 . 0 0 0 1 2 5} & \mathbf{- 0 . 4 0 1 8 2 2} & \mathbf{2 . 4 0 7 8 2 5}\end{array}$

$\begin{array}{llll}\text { H } & -0.362554 & -1.894448 & 1.714605\end{array}$

$\begin{array}{llll}C & -1.485222 & -0.535372 & 2.958039\end{array}$

\begin{tabular}{llll}
$C$ & 1.372543 & 0.605301 & 3.459695 \\
\hline
\end{tabular}

$\begin{array}{lllll}\text { H } & 0.589314 & 1.353640 & 3.617960\end{array}$

H $\quad 1.538933 \quad 0.081052 \quad 4.415311$

$\begin{array}{lllll}\text { H } & \mathbf{2 . 3 1 3 0 5 5} & \mathbf{1 . 1 1 2 0 4 6} & \mathbf{3 . 1 9 9 3 9 3}\end{array}$

$\begin{array}{llll}\text { O } & -1.517305 & 0.094604 & 3.987800\end{array}$

$\begin{array}{llll}\text { O } & \mathbf{- 2 . 5 8 1 6 3 9} & \mathbf{- 1 . 0 3 6 8 8 8} & \mathbf{2 . 3 7 8 4 9 0}\end{array}$

$\begin{array}{llll}C & \mathbf{- 3 . 8 2 8 7 8 1} & \mathbf{- 0 . 6 5 0 5 4 5} & \mathbf{2 . 9 2 6 5 8 6}\end{array}$

$\begin{array}{llll}\text { H } & -4.595637 & -1.137840 & 2.313993\end{array}$

$\begin{array}{llll}\text { H } & -3.943734 & 0.441871 & 2.872129\end{array}$

$\begin{array}{llll}\text { H } & \mathbf{- 3 . 9 1 9 6 3 3} & \mathbf{- 0 . 9 7 1 1 4 8} & \mathbf{3 . 9 7 4 1 8 5}\end{array}$

$\begin{array}{llll}\text { H } & \mathbf{2 . 9 7 0 3 9 2} & \mathbf{2 . 6 5 5 5 0 4} & \mathbf{- 0 . 5 2 9 6 9 1}\end{array}$

$\begin{array}{llll}\text { H } & 2.305655 & 3.226236 & 1.004214\end{array}$

$\begin{array}{llll}\text { H } & \mathbf{1 . 2 4 2 6 7 4} & \mathbf{3 . 1 6 8 3 3 7} & \mathbf{- 0 . 4 3 4 8 6 5}\end{array}$

$\begin{array}{llll}\text { H } & 1.823513 & -1.036822 & 2.071937\end{array}$

$\begin{array}{lllll}\text { H } & 0.120091 & -4.161444 & -2.129104\end{array}$

$\begin{array}{llll}\text { H } & 2.821367 & -3.503272 & -1.879013\end{array}$

75

unsat_ts1_c / electronic energy: $\mathbf{- 2 5 8 8 . 8 9 7 9 3 4 2 2}$ a.u. / lowest freq: $\mathbf{- 1 1 5 . 1 5} \mathbf{~ c m}-\mathbf{1}$ $\begin{array}{lllll}\mathrm{S} & \mathbf{0 . 8 9 0 6 6 6} & \mathbf{1 . 3 2 0 9 9 0} & \mathbf{- 1 . 4 5 2 1 6}\end{array}$

$\begin{array}{llll}C & 0.257263 & -1.469206 & -0.395978\end{array}$

$\begin{array}{lllll}\text { Ru } & 0.089791 & 0.415616 & 0.507944\end{array}$

$\begin{array}{llll}\text { C } & \mathbf{1 . 5 7 3 6 7 9} & \mathbf{0 . 1 1 8 8 1 9} & \mathbf{1 . 5 7 7 1 5 5}\end{array}$

$\begin{array}{llll}C & 1.573679 & 0.118819 & 1.577155 \\ C & -1.451781 & -0.327731 & 1.831306\end{array}$

$\begin{array}{llll}\text { C } & -1.451781 & -0.327731 & 1.831306 \\ \text { S } & -\mathbf{0 . 5 3 3 3 5 3} & \mathbf{2 . 6 6 8 3 8 8} & \mathbf{1 . 0 8 3 8 1 2}\end{array}$

$\begin{array}{llll}\mathrm{S} & \mathbf{- 0 . 5 3 3 3 5 3} & \mathbf{2 . 6 6 8 3 8 8} & \mathbf{1 . 0 8 3 8 1 2} \\ \mathrm{N} & \mathbf{- 0 . 7 5 8 0 0 3} & \mathbf{- 2 . 1 9 9 5 4 7} & \mathbf{- 0 . 9 5 4 7 6 1}\end{array}$

$\begin{array}{lrrr}\mathbf{N} & -\mathbf{0 . 7 5 8 0 0 3} & \mathbf{- 2 . 1 9 9 5 4 7} & \mathbf{- 0 . 9 5 4 7 6 1} \\ \mathbf{N} & \mathbf{1 . 3 6 6 0 1 0} & \mathbf{- 2 . 2 1 2 3 0 9} & \mathbf{- 0 . 7 0 6 9 4 0}\end{array}$

$\begin{array}{lrrr}C & -2.155074 & -1.897649 & -0.992393\end{array}$

$\begin{array}{llll}C & -0.293497 & -3.354207 & -1.568306\end{array}$

$\begin{array}{llll}\text { C } & \mathbf{1 . 0 4 4 0 4 1} & \mathbf{- 3 . 3 6 2 2 4 6} & \mathbf{- 1 . 4 1 2 1 8 9}\end{array}$

$\begin{array}{llll}C & 2.730949 & -1.906374 & -0.426058\end{array}$

$\begin{array}{llll}C & -4.938193 & -1.531142 & -1.061433\end{array}$

$\begin{array}{llll}\text { C } & -2.999911 & -2.550714 & -0.093959\end{array}$

$\begin{array}{llll}C & -2.711209 & -1.089112 & -1.990423\end{array}$ 
$\begin{array}{llll}C & -4.099958 & -0.910774 & -1.989586\end{array}$ $\begin{array}{llll}C & -4.369070 & -2.376439 & -0.102247\end{array}$ $\begin{array}{llll}\text { H } & -4.540515 & -0.270842 & -2.758156\end{array}$

$\begin{array}{llll}\text { H } & -4.976923 & -2.897843 & 0.639222\end{array}$

$\begin{array}{llll}\text { C } 5.415733 & -1.395600 & 0.221393\end{array}$

$\begin{array}{llll}C & 5.485759 & -2.345735 & 0.777033\end{array}$

$\begin{array}{llll}C & 3.285759 & -2.345735 & 0.777033 \\ C & 3.550846 & -1.274093 & -1.368089\end{array}$

$\begin{array}{llll}\text { C } & 3.550846 & -1.274093 & -1.368089\end{array}$

$\begin{array}{llll}C & 4.879720 & -1.012437 & -1.010055\end{array}$

$\begin{array}{llll}C & 4.598854 & -2.092117 & 1.121643\end{array}$

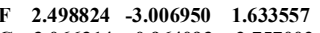

$\begin{array}{llll}C & 3.066314 & -0.964093 & -2.757002\end{array}$

H $\quad \begin{array}{llll}5.520467 & -0.500384 & -1.732182\end{array}$

$\begin{array}{lllll}\text { H } & 4.972694 & -2.438330 & 2.086889\end{array}$

$\begin{array}{llll}\text { C } & 6.838609 & -1.072744 & 0.589221\end{array}$

$\begin{array}{llll}\text { H } & 7.343730 & -1.947827 & 1.022925\end{array}$

H $3.394363 \quad-1.760566 \quad-3.444439$

$\begin{array}{lllll}\text { H } & 6.867294 & -0.271281 & 1.343802\end{array}$

$\begin{array}{llll}\text { H } & 7.413145 & \mathbf{- 0 . 7 3 5 1 6 8} & \mathbf{- 0 . 2 8 3 4 4 8}\end{array}$

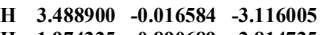

H $\quad 1.974225 \quad-0.890689-2.814725$

$\begin{array}{llll}\text { F } & -2.446651 & -3.361194 & 0.817242\end{array}$

$\begin{array}{llll}C & -6.426668 & -1.310230 & -1.080668\end{array}$

$\begin{array}{lllll}\text { H } & -6.766631 & -0.869794 & -0.130779\end{array}$

H $\quad-6.723307-0.635746-1.894880$

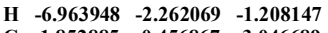

$\begin{array}{llll}C & -1.852885 & -0.456867 & -3.046689\end{array}$

H $-\mathbf{- 1 . 1 2 6 2 1 4}-\mathbf{- 1 . 1 7 7 1 8 2}-\mathbf{- 3 . 4 5 2 6 9 5}$

$\begin{array}{llll}\text { H } & -2.468462 & -0.080297 & -3.873457\end{array}$

$\begin{array}{lllll}\text { H } & -1.269527 & 0.379378 & -2.632603\end{array}$

$\begin{array}{llll}C & 0.793548 & 3.079544 & -1.295230\end{array}$

$\begin{array}{llll}C & 1.339549 & 3.892462 & -2.300994\end{array}$

$\begin{array}{lllll}C & 0.167285 & 3.665960 & \mathbf{- 0 . 1 8 2 6 3 9}\end{array}$

$\begin{array}{llll}C & 1.265807 & 5.278174 & \mathbf{- 2 . 2 1 0 7 3 7}\end{array}$

$\begin{array}{llll}\text { H } & 1.823947 & \mathbf{3 . 4 2 1 5 9 7} & \mathbf{3 . 1 6 0 8 5 4}\end{array}$

$\begin{array}{llll}\text { C } & 1.823947 & 3.421597 & -3.160854 \\ C & 0.098357 & 5.069144 & -0.105655\end{array}$

\begin{tabular}{llll} 
C & 0.098357 & 5.069144 & -0.105655 \\
\hline & 0.640477 & 5.867755 & -1.106058
\end{tabular}

$\begin{array}{llll}\text { C } & \mathbf{0 . 6 4 0 4 7 7} & \mathbf{5 . 8 6 7 7 5 5} & \mathbf{- 1 . 1 0 6 0 5 8}\end{array}$

$\begin{array}{rrrrr}\text { H } & \mathbf{1 . 6 9 4 8 0 3} & \mathbf{5 . 9 0 0 0 1 4} & \mathbf{- 2 . 9 9 9 7 5 4} \\ \text { H } & \mathbf{- 0 . 3 8 8 9 3 4} & \mathbf{5 . 5 2 6 9 2 9} & \mathbf{0 . 7 5 9 1 8 9}\end{array}$

$\begin{array}{lllll}\text { H } & 0.577371 & 6.955471 & -1.024635\end{array}$

$\begin{array}{llll}C & \mathbf{2 . 5 4 4 8 4 7} & \mathbf{1 . 1 7 1 3 0 4} & \mathbf{1 . 9 8 4 7 9 8}\end{array}$

$\begin{array}{llll}\text { H } & 1.901102 & -0.879125 & 1.887062\end{array}$

$\begin{array}{llll}\text { C } & -0.319655 & -0.319872 & 2.686333\end{array}$

$\begin{array}{llll}\text { H } & -1.756960 & -1.303525 & 1.462520\end{array}$

$\begin{array}{llll}\mathrm{C} & -\mathbf{2 . 6 1 3 2 6 2} & \mathbf{0 . 5 9 2 9 8 2} & \mathbf{2 . 0 0 5 2 9 0}\end{array}$

$\begin{array}{llll}\text { C } & -2.613262 & \mathbf{0 . 5 9 2 9 8 2} & \mathbf{2 . 0 0 5 2 9 0} \\ \text { C } & -\mathbf{0 . 1 2 6 3 4 7} & \mathbf{0 . 6 0 3 8 1 8} & \mathbf{3 . 8 6 2 3 3 0}\end{array}$

$\begin{array}{llll}\text { H } & \mathbf{- 0 . 3 0 4 6 4 5} & \mathbf{1 . 6 5 3 2 9 7} & \mathbf{3 . 6 0 3 6 4 6}\end{array}$

\begin{tabular}{lllll}
\hline H & $-\mathbf{0 . 8 4 9 2 6 5}$ & $\mathbf{0 . 3 2 3 7 7 4}$ & $\mathbf{4 . 6 4 3 6 1 2}$
\end{tabular}

$\begin{array}{lllll}\text { H } & 0.884208 & 0.500926 & 4.280108\end{array}$

\begin{tabular}{llll}
\hline & -2.862205 & 1.261014 & 2.980330
\end{tabular}

$\begin{array}{llll}\text { O } & -3.408891 & 0.547389 & 0.933682\end{array}$

$\begin{array}{llll}\text { C } & \mathbf{- 4 . 5 4 5 8 9 4} & \mathbf{1 . 3 9 0 8 1 0} & \mathbf{0 . 9 4 0 7 6 6}\end{array}$

$\begin{array}{llll}\text { H } & -\mathbf{5 . 0 3 3 6 2 9} & \mathbf{1 . 2 4 9 3 2 6} & \mathbf{- 0 . 0 2 9 9 0 7}\end{array}$

\begin{tabular}{llll}
\hline & -4.246493 & 2.441650 & 1.063314
\end{tabular}

\begin{tabular}{llll}
$H$ & -4.246493 & 2.441650 & 1.063314 \\
\hline & -5.235020 & 1.117151 & 1.753012
\end{tabular}

$\begin{array}{llll}\text { H } & -5.235020 & 1.117151 & 1.753012\end{array}$

$\begin{array}{ccccc}\text { H } & 3.390040 & 1.075968 & 1.276091\end{array}$

$\begin{array}{lllll}\text { H } & \mathbf{2 . 9 5 2 5 7 7} & \mathbf{0 . 9 8 0 2 2 5} & \mathbf{2 . 9 9 0 6 3 9}\end{array}$

$\begin{array}{llll}\text { H } & 2.142902 & 2.188102 & 1.911331\end{array}$

$\begin{array}{llll}\text { H } & 0.118354 & -1.312517 & 2.824348\end{array}$

$\begin{array}{llll}\text { H } & -0.966793 & -4.051425 & -2.057032\end{array}$

$\begin{array}{llll}\text { H } & 1.803062 & -4.069040 & -1.732586\end{array}$

75

unsat_mcb_c / electronic energy: -2588.90650174 a.u. / lowest freq: $18.52 \mathrm{~cm}-1$

$\begin{array}{llll}S & 0.458328 & 1.386048 & -1.504955\end{array}$

$\begin{array}{llll}C & 0.458328 & -1.386048 & -1.504955 \\ C & 0.391199 & -1.397435 & -0.544701\end{array}$

$\begin{array}{rrrr}C & 0.31159 & -1.397435 & -0.544701 \\ \text { Ru } & 0.145509 & 0.413615 & 0.508916\end{array}$

$\begin{array}{rrrrr}\text { Ru } & \mathbf{0 . 1 4 5 5 0 9} & \mathbf{0 . 4 1 3 6 1 5} & \mathbf{0 . 5 0 8 9 1 6} \\ \text { C } & \mathbf{1 . 4 1 5 4 3 7} & \mathbf{- 0 . 1 7 4 5 7 6} & \mathbf{1 . 8 6 3 6 7 2}\end{array}$

$\begin{array}{rrrr}\text { C } & \mathbf{1 . 4 1 5 4 3 7} & \mathbf{- 0 . 1 7 4 5 7 6} & \mathbf{1 . 8 6 3 6 7 2} \\ \mathrm{C} & \mathbf{- 1 . 2 7 6 4 4 9} & \mathbf{- 0 . 3 5 3 8 4 0} & \mathbf{1 . 6 7 0 0 1 9}\end{array}$

$\begin{array}{llll}\mathrm{S} & \mathbf{- 0 . 2 8 6 6 9 2} & \mathbf{2 . 6 3 4 9 2 7} & \mathbf{1 . 3 5 4 6 2 0}\end{array}$

N $\quad-\mathbf{0 . 5 6 9 8 5 6}-2.171503-1.135700$

$\begin{array}{llll}N & 1.554215 & -1.991637 & -0.956259\end{array}$

$\begin{array}{llll}\text { C } & -1.991226 & -2.035781 & -1.045381\end{array}$

$\begin{array}{llll}C & -0.019826 & -3.210201 & -1.872128\end{array}$

C $\quad \begin{array}{llll}\text { C.318451 } & -3.096889 & -1.758895\end{array}$

$\begin{array}{llll}C & 2890993 & -1.605701 & -0.629438\end{array}$

$\begin{array}{llll}C & -4.786335 & -1.931400 & -0.799726\end{array}$

$\begin{array}{llll}C & -2.663487 & -2.754905 & -0.057359\end{array}$

$\begin{array}{llll}C & -2.730477 & -1.303350 & -1.981445\end{array}$

$\begin{array}{llll}C & -4.120948 & -1.259630 & -1.827423\end{array}$

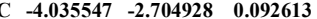

$\begin{array}{llll}\text { H } & -4.704759 & -\mathbf{0 . 6 7 8 8 8 7} & -2.545868\end{array}$

$\begin{array}{llll}\text { H } & -4.503627 & -3.267280 & 0.902454\end{array}$

$\begin{array}{llll}C & 5.514152 & -0.952783 & 0.141396\end{array}$

$\begin{array}{llll}\text { C } & 5.514152 & -0.952783 & 0.141396 \\ C & 3.492303 & -2.191853 & 0.487745\end{array}$

$\begin{array}{llll}C & 3.492303 & -2.191853 & 0.487745\end{array}$

$\begin{array}{llll}\text { C } & 3.645292 & \mathbf{- 0 . 7 4 7 3 0 3} & \mathbf{- 1 . 4 3 6 8 2 6}\end{array}$

$\begin{array}{llll}C & 4.942860 & -0.423505 & -1.016422\end{array}$

$\begin{array}{llll}\text { C } & 4.771968 & -1.872410 & 0.894256\end{array}$

$\begin{array}{llll}\text { F } & \mathbf{2 . 7 8 3 7 4 1} & \mathbf{- 3 . 0 8 0 4 1 6} & \mathbf{1 . 1 9 4 0 3 2}\end{array}$

$\begin{array}{llll}\text { C } & 3.144967 & -0.230895 & -2.757016\end{array}$

$\begin{array}{llll}\text { H } & 5.531347 & 0.258997 & -1.634945\end{array}$

$\begin{array}{llll}\text { H } & \mathbf{5 . 1 7 9 2 0 5} & \mathbf{- 2 . 3 4 3 9 2 9} & \mathbf{1 . 7 9 0 2 4 3}\end{array}$

$\begin{array}{llll}C & 6.893496 & -0.548027 & 0.585767\end{array}$

$\begin{array}{llll}C & 7.449907 & -1.404716 & 0.591355\end{array}$

\begin{tabular}{lrrrr} 
H & 7.449907 & -1.404716 & 0.991355 \\
\hline
\end{tabular}

$\begin{array}{llll}\text { H } & 3.794026 & -0.605771 & -3.563165\end{array}$ 
$\begin{array}{llll}\text { H } & 6.830216 & 0.210182 & 1.382336\end{array}$ H $7.471026-0.114923 \quad-0.24183$

$\begin{array}{lllll}\text { H } & 3.175312 & 0.866834 & -2.783618\end{array}$

$\begin{array}{llll}\text { H } & \mathbf{2 . 1 1 5 3 0 3} & \mathbf{- 0 . 5 4 0 7 6 2} & \mathbf{- 2 . 9 6 8 1 2 8}\end{array}$

$\begin{array}{llll}\text { F } & -1.937622 & -3.494706 & 0.790434\end{array}$

C $-6.278209-1.824688-0.636897$

\begin{tabular}{llll} 
C & -6.278209 & -1.824688 & -0.636897 \\
\hline & -6.745531 & -1.353692 & -1.511963
\end{tabular}

$\begin{array}{llll}\text { H } & -6.745531 & -1.353692 & -1.511963\end{array}$

\begin{tabular}{llll} 
H & -6.735641 & -2.813688 & -0.488213 \\
\hline
\end{tabular}

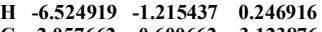

$\begin{array}{llll}\text { C } & -2.057662 & -0.600662 & -3.123876\end{array}$

$\begin{array}{llll}\text { H } & -1.338188 & -1.260724 & -3.631702\end{array}$

$\begin{array}{llll}\text { H } & -2.796203 & -0.260357 & -3.860644\end{array}$

\begin{tabular}{llll} 
H & -1.492218 & 0.271029 & -2.762352 \\
\hline & 0.213342 & 3.128940 & -1306117
\end{tabular}

$\begin{array}{llll}C & 0.348061 & 3.964991 & -2.424321 \\ C & -0.112203 & 3.667278 & -0.050572\end{array}$

$\begin{array}{llll}\text { C } & \mathbf{- 0 . 1 1 2 2 0 3} & 3.667278 & \mathbf{- 0 . 0 5 0 5 7 2}\end{array}$

\begin{tabular}{llll}
$C$ & 0.163601 & 5.337818 & $\mathbf{- 2 . 3 0 4 1 4 2}$ \\
\hline & 0.597361 & 3.523859 & -3.393327
\end{tabular}

$\begin{array}{rrrr}\text { H } & \mathbf{0 . 5 9 7 3 6 1} & \mathbf{3 . 5 2 3 8 5 9} & \mathbf{- 3 . 3 9 3 3 2 7} \\ \text { C } & \mathbf{- 0 . 2 9 7 2 0 1} & \mathbf{5 . 0 5 9 4 5 1} & \mathbf{0 . 0 5 3 6 7 3}\end{array}$

$\begin{array}{llll}C & -0.160650 & 5.883999 & -1.056416\end{array}$

$\begin{array}{llll}\text { H } & \mathbf{0 . 2 6 9 5 7 2} & \mathbf{5 . 9 8 2 2 2 5} & \mathbf{- 3 . 1 7 9 5 3 3}\end{array}$

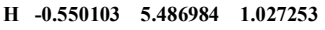

$\begin{array}{llll}\text { H } & -0.308672 & 6.961242 & -0.950199\end{array}$

$\begin{array}{llll}\text { C } & \mathbf{2 . 4 2 4 1 1 9} & \mathbf{0 . 7 3 6 9 8 6} & \mathbf{2 . 5 1 6 3 3 3}\end{array}$

$\begin{array}{llll}\text { H } & \mathbf{1 . 8 4 4 0 9 1} & -1.166780 & \mathbf{1 . 7 2 5 7 3 6}\end{array}$

$\begin{array}{llll}\mathrm{H} & -1.606070 & -1.359110 & 1.409686\end{array}$

$\begin{array}{llll}\text { C } & -2.497964 & \mathbf{0 . 4 5 4 7 3 5} & \mathbf{1 . 9 9 1 6 5 6}\end{array}$

C $\begin{array}{llll}\text { C.126634 } & 0.388395 & 3.914722\end{array}$

$\begin{array}{llll}\text { H } & -\mathbf{0 . 1 9 7 3 3 3} & \mathbf{1 . 4 6 8 6 2 2} & \mathbf{3 . 7 3 9 5 9 2}\end{array}$

$\begin{array}{llll}H & -1.020745 & 0.086114 & 4.471575\end{array}$

$\begin{array}{llll}\text { H } & \mathbf{0 . 7 5 9 3 1 5} & \mathbf{0 . 1 8 9 6 4 8} & \mathbf{4 . 5 3 2 2 8 1}\end{array}$

$\begin{array}{llll}\text { O } & -2.846128 & 0.898832 & 3.058041\end{array}$

$\begin{array}{llll}0 & -3.247997 & 0.548151 & 0.888839\end{array}$

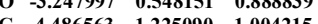

$\begin{array}{llll}C & -3.486563 & 1.225090 & 1.004215\end{array}$

\begin{tabular}{llll} 
H & -4.327224 & 2.284519 & 1.251642 \\
\hline
\end{tabular}

$\begin{array}{llll}\text { H } & \mathbf{- 5 . 1 1 6 2 4 7} & \mathbf{0 . 7 6 6 6 3 8} & \mathbf{1 . 7 8 0 5 0 9} \\ \text { H } & \mathbf{- 4 . 9 7 1 5 2 9} & \mathbf{1 . 1 3 7 0 8 5} & \mathbf{0 . 0 2 5 6 3 2}\end{array}$

$\begin{array}{crrrr}\text { H } & -4.971529 & 1.137085 & 0.025632 \\ \text { H } & 3.262056 & \mathbf{0 . 8 5 7 9 6 2} & \mathbf{1 . 8 1 1 6 7 8}\end{array}$

\begin{tabular}{llll}
\hline & $\mathbf{2 . 8 3 6 1 1 0}$ & $\mathbf{0 . 2 8 0 3 7 2}$ & $\mathbf{3 . 4 3 2 0 6 8}$
\end{tabular}

H $\quad \begin{array}{llll}2.022774 & 1.729843 & 2.746795\end{array}$

H $\quad 0.061338-1.478190 \quad 2.830273$

H $-0.640561-3.925272-2.403111$

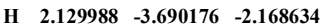

75

unsat ts2 c / electronic energy: $-\mathbf{2 5 8 8 . 8 9 1 5 6 3 4 0}$ a.u. / lowest freq: $-\mathbf{1 8 9 . 1 7} \mathrm{cm}-1$

S $\quad \begin{array}{llll}\mathbf{0 . 9 9 4 2 6 8} & \mathbf{1 . 0 6 8 3 7 7} & \mathbf{- 1 . 4 2 6 6 4 5}\end{array}$

$\begin{array}{llll}C & -0.089418 & -1.650731 & -0.427799\end{array}$

$\begin{array}{llll}\text { Ru } & \mathbf{0 . 1 1 6 9 0 6} & \mathbf{0 . 2 1 6 7 8 6} & \mathbf{0 . 5 1 7 6 5 3}\end{array}$

$\begin{array}{llll}C & 0.354038 & -0.917785 & 2.325374\end{array}$

$\begin{array}{llll}\text { C } & -1.684895 & 0.596569 & 0.699845\end{array}$

$\begin{array}{lllll}C & 0.838156 & 2.268872 & 1.543031\end{array}$

$\begin{array}{llll}\mathrm{N} & -1.164304 & -2.352881 & -0.886880\end{array}$

$\begin{array}{llll}\mathrm{N} & -1.164304 & -2.352881 & -0.886880\end{array}$

C $-2.955114-2.499613-0.682811$

$\begin{array}{llll}C & -2.516704 & -1.912103 & -0.879566\end{array}$

$\begin{array}{llll}C & -0.804637 & -3.591160 & -1.400109\end{array}$

$\begin{array}{lllll}\text { C } & 0.534591 & -3.681807 & -1.271676\end{array}$

$\begin{array}{llll}C & \mathbf{2 . 3 3 8 1 4 1} & \mathbf{- 2 . 2 1 7 0 7 6} & \mathbf{- 0 . 4 7 3 5 9 8}\end{array}$

$\begin{array}{llll}C & \mathbf{- 5 . 1 6 2 6 4 2} & \mathbf{- 1 . 0 0 9 7 2 6} & \mathbf{- 0 . 7 0 4 3 1 9}\end{array}$

$\begin{array}{llll}\text { C } & -\mathbf{3 . 3 6 2 5 6 8} & \mathbf{- 2 . 3 5 6 2 0 1} & \mathbf{0 . 1 3 5 4 2 4}\end{array}$

$\begin{array}{llll}C & -2.997926 & -1.031062 & -1.854558\end{array}$

$\begin{array}{llll}C & -4.320767 & -0.591136 & -1.740004\end{array}$

$\begin{array}{llll}C & -4.669703 & -1.517007 & 0.242567\end{array}$

$\begin{array}{llll}C & -4.669703 & -1.917007 & 0.242567\end{array}$

$\begin{array}{llll}\text { H } & -4.703084 & 0.111441 & -2.484324\end{array}$

$\begin{array}{lrrr}\text { H } & \mathbf{- 5 . 2 8 7 3 7 9} & \mathbf{- 2 . 2 7 6 5 4 0} & \mathbf{1 . 0 6 7 4 1 4} \\ \text { C } & \mathbf{5 . 0 4 6 6 3 1} & -1.657155 & \mathbf{0 . 0 1 5 6 7 5}\end{array}$

$\begin{array}{llll}\text { C } \mathbf{5 . 0 4 6 6 3 1} & -1.657155 & \mathbf{0 . 0 1 5 6 7 5}\end{array}$

$\begin{array}{llll}\text { C } & \mathbf{2 . 9 3 8 2 0 0} & \mathbf{- 2 . 5 4 6 3 9 0} & \mathbf{0 . 7 4 1 4 8 9}\end{array}$

$\begin{array}{llll}C & 3.125777 & -1.686210 & -1.505589\end{array}$

$\begin{array}{llll}C & 4.466193 & -1.402121 & -1.230736\end{array}$

$\begin{array}{llll}C & 4.265870 & -2.260248 & 1.007157\end{array}$

$\begin{array}{llll}\text { F } & 2.217463 & -3.183542 & 1.667819\end{array}$

$\begin{array}{llll}\text { C } \quad 2.574385 & -1.502703 & -2.892265\end{array}$

$\begin{array}{llll}\text { H } & \mathbf{5 . 0 8 0 5 4 9} & \mathbf{- 0 . 9 6 9 9 5 3} & \mathbf{- 2 . 0 2 4 4 4 0}\end{array}$

$\begin{array}{llll}\text { H } & 4.679387 & \mathbf{- 2 . 5 2 4 1 3 6} & \mathbf{1 . 9 8 2 1 1 6}\end{array}$

$\begin{array}{llll}\text { C } & 6.479238 & -1.291472 & 0.294011\end{array}$

$\begin{array}{lllll}\text { H } & 6.952072 & -2.012444 & 0.975407\end{array}$

$\begin{array}{lllll}\text { H } & \mathbf{2 . 7 6 9 1 1 8} & \mathbf{- 2 . 4 0 9 2 9 4} & \mathbf{- 3 . 4 8 8 3 5 2}\end{array}$

$\begin{array}{llll}\text { H } & 6.532866 & -0.300757 & 0.772570\end{array}$

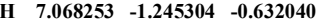

$\begin{array}{lllll}\text { H } & 3.057835 & -\mathbf{0 . 6 5 5 6 2 7} & \mathbf{- 3 . 3 9 5 5 6 2}\end{array}$

$\begin{array}{lllll}\text { H } & 1.492302 & -1.324662 & -2.891547\end{array}$

$\begin{array}{llll}\text { F } & \mathbf{- 2 . 8 6 8 6 3 6} & \mathbf{- 3 . 1 9 2 0 0 7} & \mathbf{1 . 0 5 3 6 3 0}\end{array}$

$\begin{array}{llll}\text { F } & -2.868636 & -3.192007 & 1.053630 \\ \text { C } & -6.576106 & -0.505326 & -0.596299\end{array}$

$\begin{array}{rrrr}C & -6.576106 & -0.505326 & -0.596299 \\ \text { H } & -6.803597 & \mathbf{0 . 2 2 4 5 7 2} & -1.384138\end{array}$

$\begin{array}{rrrrr}\text { H } & -6.803597 & \mathbf{0 . 2 2 4 5 7 2} & -1.384138 \\ \text { H } & -\mathbf{7 . 2 9 5 2 1 2} & \mathbf{- 1 . 3 3 4 4 5 5} & \mathbf{- 0 . 6 7 7 5 0 7}\end{array}$

$\begin{array}{rrrrr}\text { H } & -7.295212 & \mathbf{- 1 . 3 3 4 4 5 5} & \mathbf{- 0 . 6 7 7 5 0 7} \\ \text { H } & \mathbf{- 6 . 7 4 2 9 2 9} & \mathbf{- 0 . 0 2 2 8 7 7} & \mathbf{0 . 3 7 8 2 8 7}\end{array}$

$\begin{array}{lllll}\text { C } & -\mathbf{2 . 0 9 5 8 6 0} & \mathbf{- 0 . 5 3 8 9 4 6} & \mathbf{- 2 . 9 4 8 5 5 4}\end{array}$

$\begin{array}{llll}\text { H } & -1.534741 & -1.366885 & -3.407127\end{array}$

$\begin{array}{lllll}\text { H } & -2.671997 & -0.031522 & -3.732641\end{array}$

$\begin{array}{llll}\text { H } & -1.355180 & 0.170506 & -2.545406\end{array}$

$\begin{array}{llll}C & 1340021 & 2.774566 & -1.113634\end{array}$

C $1.691781 \quad 3.608542-2.185847$

$\begin{array}{rrrr}C & 1.691781 & 3.608542 & -2.185847 \\ \text { C } & \mathbf{1 . 2 5 3 2 5 9} & \mathbf{3 . 3 0 2 5 2 5} & \mathbf{0 . 1 8 5 7 4 0}\end{array}$ 
$\begin{array}{llll}C & 1.952862 & 4.958997 & -1.978632\end{array}$ H $\quad 1.755573 \quad 3.183027 \quad-3.191150$

$\begin{array}{llll}\text { C } & 1.521375 & 4.669088 & 0.380891\end{array}$

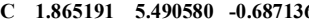

$\begin{array}{llll}\text { H } & 2.222351 & 5.598573 & -2.822280\end{array}$

$1.4486945 .082515 \quad 1.390290$

$\begin{array}{llll}\mathrm{H} & 1.448694 & 5.082515 & 1.390290\end{array}$

$\begin{array}{lllll}H & 2.063118 & 6.551078 & -0.514169\end{array}$

$\begin{array}{llll}C & 1.453969 & \mathbf{- 0 . 5 1 5 6 2 3} & \mathbf{3 . 2 8 0 2 8} 2\end{array}$

$\begin{array}{lllll}\text { H } & 0.428295 & -1.963237 & 2.021045\end{array}$

$\begin{array}{llll}C & -0.994631 & -0.459063 & 2.473033\end{array}$

$\begin{array}{llll}\text { H } & -2.537203 & -0.083298 & 0.582194\end{array}$

$\begin{array}{llll}\text { C } & -2.194778 & 2.008549 & 0.845728\end{array}$

$\begin{array}{llll}\text { C } & -\mathbf{1 . 3 8 5 9 7 8} & \mathbf{0 . 4 7 6 7 9 2} & \mathbf{3 . 5 8 7 6 6 6}\end{array}$

$\begin{array}{llll}\text { H } & -\mathbf{0 . 7 5 3 5 4 1} & \mathbf{1 . 3 7 3 2 6 2} & \mathbf{3 . 6 1 3 5 0 7}\end{array}$

$\begin{array}{llll}\text { H } & -\mathbf{2 . 4 2 6 5 9 2} & \mathbf{0 . 8 0 4 4 9 5} & \mathbf{3 . 4 9 5 1 5 2}\end{array}$

$\begin{array}{llll}\text { H } & -1.261228 & -0.061947 & 4.541066\end{array}$

$\begin{array}{llll}\text { O } & -2.861098 & 2.407247 & 1.771303\end{array}$

$\begin{array}{llll}O & -1.898469 & 2.740593 & -0.220995\end{array}$

$\begin{array}{llll}C & -2.251018 & 4.115662 & -0.178050\end{array}$

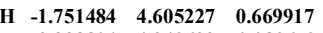

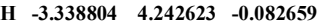

H $\quad-1.895874 \quad 4.549007 \quad-1.118952$

$\begin{array}{llll}\text { H } & 2.440488 & -\mathbf{0 . 6 4 2 6 2 9} & \mathbf{2 . 8 1 2 5 7 0}\end{array}$

$\begin{array}{llll}\text { H } & 1.421455 & -1.170898 & 4.167817\end{array}$

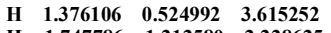

H $-1.747786-1.212580 \quad 2.228625$

$\begin{array}{llll}\text { H } & -1.540112 & -4.280733 & -1.802693\end{array}$

H $\quad 1.233715 \quad-4.466356 \quad-1.543911$

75

unsat_pc2_c / electronic energy: $-\mathbf{2 5 8 8 . 9 0 1 8 3 1 2 7}$ a.u. / lowest freq: $18.09 \mathrm{~cm}-1$

$\begin{array}{lllll}S & 0.847159 & 1.292908 & -1.207672\end{array}$

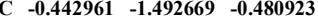

$\begin{array}{llll}\text { Ru } & 0.080021 & 0.163303 & 0.689479\end{array}$

$\begin{array}{llll}\text { C } & 0.133122 & -1.432719 & 2.434700\end{array}$

$\begin{array}{llll}\text { C } & -1.489764 & 1.096363 & 0.670673\end{array}$

$\begin{array}{llll}C & 1.625808 & 1.427904 & 1.890218\end{array}$

$\begin{array}{llll}\mathrm{N} & -\mathbf{1 . 6 5 8 3 5 4} & \mathbf{- 1 . 9 7 6 2 0 9} & \mathbf{- 0 . 8 4 9 9 6 6}\end{array}$

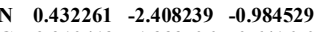

$\begin{array}{llll}C & -2.910413 & -1.333706 & -0.641670\end{array}$

$\begin{array}{llll}C & -1.544069 & -3.165415 & -1.557248\end{array}$

$\begin{array}{llll}C & -0.225128 & -3.437869 & -1.640232\end{array}$

C $\quad \begin{array}{llll}\text { 1.854183 } & -2.323461 & -0.904155\end{array}$

$\begin{array}{llll}C & -5.362589 & -0.078373 & -0.156558\end{array}$

$\begin{array}{llll}C & -3.687547 & -1.683622 & 0.456343\end{array}$

$\begin{array}{llll}C & -3.374784 & -0.377008 & -1.554442\end{array}$

$\begin{array}{llll}C & -4.598255 & 0.237240 & -1.287029\end{array}$

$\begin{array}{llll}\text { C } & -4.897924 & -1.063150 & 0.720484\end{array}$

$\begin{array}{rrrrr}C & -4.963289 & 0.998657 & -1.980296\end{array}$

$\begin{array}{llll}\text { H } & -\mathbf{5 . 4 6 4 4 1 9} & \mathbf{- 1 . 3 5 8 8 2 9} & \mathbf{1 . 6 0 5 1 2 6}\end{array}$

$\begin{array}{llll}C & 4.633737 & -2.122744 & -0.609325\end{array}$

$\begin{array}{llll}\text { C } & \mathbf{2 . 5 0 7 6 4 2} & \mathbf{- 2 . 8 9 8 7 3 7} & \mathbf{0 . 1 8 4 9 8 2}\end{array}$

$\begin{array}{llll}C & 2.602253 & -1.708449 & -1.916930\end{array}$

C $3.984883-1.6098$

\begin{tabular}{lllll}
$C$ & 3.984883 & -1.609871 & -1.737048 \\
\hline
\end{tabular}

$\begin{array}{lllll}C & 3.876558 & -2.797147 & 0.355127\end{array}$

$\begin{array}{llll}\text { F } & 1.786774 & -3.585221 & 1.074918\end{array}$

$\begin{array}{llll}\text { C } & \mathbf{1 . 9 5 7 3 4 2} & \mathbf{- 1 . 2 3 7 0 7 1} & \mathbf{- 3 . 1 8 9 7 0 7}\end{array}$

$\begin{array}{lllll}\text { H } & 4.576399 & -1.114134 & -2.510683\end{array}$

$\begin{array}{lllll}\text { H } & 4.337957 & \mathbf{- 3 . 2 5 2 2 3 0} & \mathbf{1 . 2 3 3 2 1 5}\end{array}$

$\begin{array}{llll}\text { C } & 6.116673 & -1.948648 & -0.425181\end{array}$

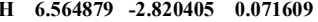

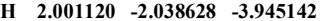

$\begin{array}{lllll}\text { H } & 6.319753 & -1.067927 & 0.204439\end{array}$

\begin{tabular}{lllll} 
H & 6.624083 & -1.793815 & -1.386957 \\
\hline & 2.483006 & -0.362062 & -3.592579
\end{tabular}

$\begin{array}{llll}\text { H } & 2.483006 & \mathbf{0 . 3 6 2 0 6 2} & \mathbf{- 3 . 5 9 2 5 7 9}\end{array}$

$\begin{array}{llll}\text { H } & 0.905573 & -0.963270 & -3.044324\end{array}$

\begin{tabular}{cccc}
\hline F & -3.239386 & -2.634486 & 1.281840
\end{tabular}

$\begin{array}{llll}\text { C } & -6.660869 & 0.637080 & 0.105144\end{array}$

$\begin{array}{lllll}\text { H } & -6.479431 & 1.705083 & 0.300644\end{array}$

$\begin{array}{llll}\text { H } & -7.327523 & 0.574740 & -0.767700\end{array}$

$\begin{array}{llll}\text { H } & -7.186912 & 0.216833 & 0.972359\end{array}$

$\begin{array}{llll}\text { C } & -2.551654 & -0.032230 & -2.761910\end{array}$

$\begin{array}{llll}\text { H } & -2.304900 & -0.932783 & -3.345066\end{array}$

\begin{tabular}{llll}
\hline & -3.089227 & 0.667720 & -3.413457
\end{tabular}

\begin{tabular}{llll} 
H & $\mathbf{- 3 . 0 8 9 2 2 7}$ & $\mathbf{0 . 6 6 7 7 2 0}$ & $\mathbf{- 3 . 4 1 3 4 5 7}$ \\
\hline
\end{tabular}

$\begin{array}{llll}4 & -1.608202 & 0.444736 & -2.456593 \\ C & 1.901751 & 2.565953 & -0.607033\end{array}$

$\begin{array}{llll}C & 1.901751 & 2.565953 & -0.607033\end{array}$

$\begin{array}{llll}C & 2.424177 & 3.525159 & -1.491629\end{array}$

$\begin{array}{llll}\text { C } & 2.247040 & 2.622312 & 0.753114\end{array}$

C $3.279304 \quad 4.520906 \quad-1.034801$

$\begin{array}{lllll}\text { H } & \mathbf{2 . 1 5 0 2 2 5} & \mathbf{3 . 4 7 6 2 8 7} & \mathbf{- 2 . 5 4 9 0 4 2}\end{array}$

$\begin{array}{llll}\text { C } & 3.107764 & 3.636543 & 1.205218\end{array}$

$\begin{array}{llll}\text { C } & 3.623086 & \mathbf{4 . 5 7 7 3 0 3} & \mathbf{0 . 3 2 1 2 5 8}\end{array}$

$\begin{array}{llll}\text { C } & 3.676733 & 5.259281 & -1.735168\end{array}$

$\begin{array}{llll}C & 3.676733 & 5.259281 & -1.735168\end{array}$

$\begin{array}{llll}\text { H } & 3.367192 & 3.678281 & \mathbf{2 . 2 6 6 4 1 3}\end{array}$

$\begin{array}{lllll}\text { H } & 4.292264 & \mathbf{5 . 3 5 9 0 6 2} & \mathbf{0 . 6 8 7 8 9 9}\end{array}$

$\begin{array}{llll}\text { C } & 1.370449 & -1.451345 & 3.290486\end{array}$

$\begin{array}{llll}\text { H } & -0.022298 & -2.348375 & 1.860022\end{array}$

$\begin{array}{lllll}\text { C } & -0.974554 & -0.643094 & 2.647513\end{array}$

$\begin{array}{llll}\text { H } & -2.400925 & 0.838009 & 1.239819\end{array}$

$\begin{array}{llll}\text { C } & -1.624507 & \mathbf{2 . 4 5 8 3 9 8} & \mathbf{0 . 0 6 3 8 6 0}\end{array}$

$\begin{array}{llll}\text { C } & -1.133068 & 0.370422 & 3.746536\end{array}$

$\begin{array}{llll}\text { H } & -0.184543 & 0.845717 & 4.02434\end{array}$

$\begin{array}{llll} & -1.184543 & 0.845717 & 4.024346\end{array}$

$\begin{array}{rrrr}\text { H } & -1.832524 & 1.165737 & 3.452041 \\ \text { H } & -1.553265 & -\mathbf{0 . 1 2 1 4 5 5} & 4.639772\end{array}$ 


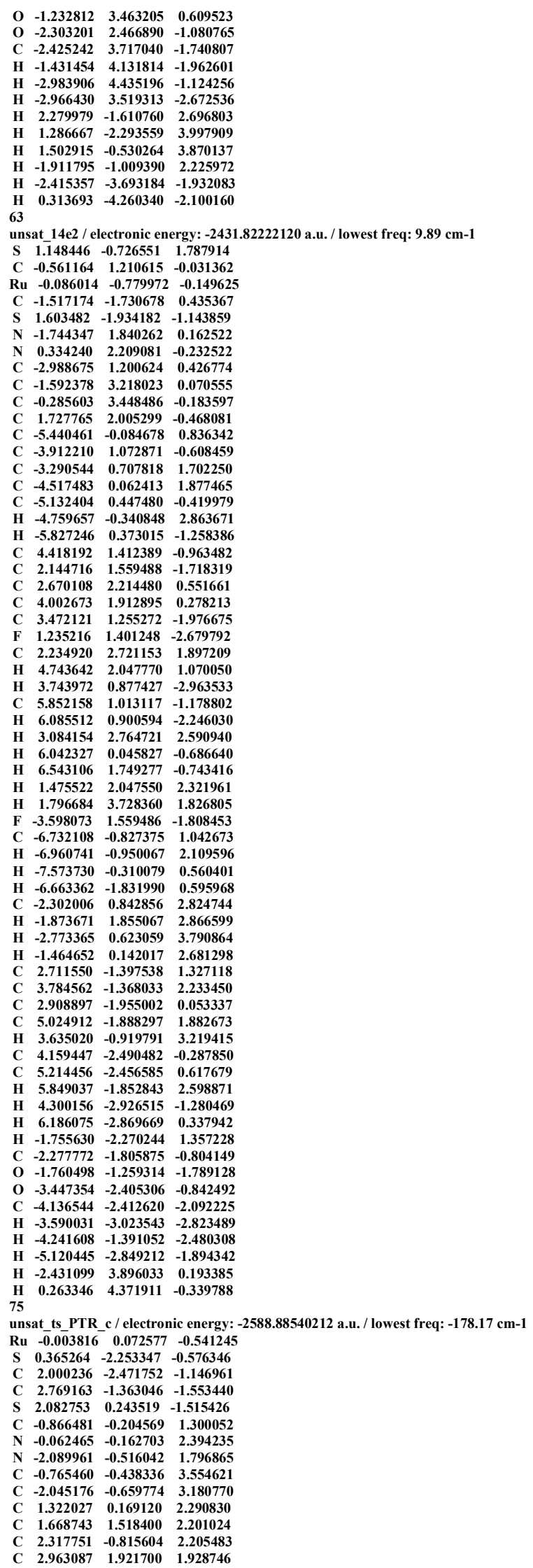


$\begin{array}{llll}\text { C } & 3.621837 & -0.395901 & 1.935987\end{array}$ $\begin{array}{llll}C & 3.955512 & 0.951631 & 1.763127\end{array}$

$\begin{array}{llll}\text { H } & 3.174583 & 2.987068 & 1.830737\end{array}$

$\begin{array}{llll}\text { H } & 4.402180 & -1.154537 & 1.83538\end{array}$

$\begin{array}{llll}\text { C } & \mathbf{5 . 3 5 4 2 5 1} & \mathbf{1 . 3 4 5 7 2 7} & \mathbf{1 . 3 7 5 1 9 2}\end{array}$

F 0.71724

$\begin{array}{llll}\text { C } & 0.717245 & 2.432366 & \mathbf{2 . 3 8 6 2 2}\end{array}$

$\begin{array}{llll}\text { C } 2.013712 & -2.267771 & \mathbf{2 . 4 4 3 3 4}\end{array}$

$\begin{array}{llll}\text { H } & 0.967629 & -2.510146 & \mathbf{2 . 2 2 1 6 6 2}\end{array}$

$\begin{array}{llll}\text { H } & \mathbf{2 . 6 4 9 1 0 9} & \mathbf{- 2 . 9 0 3 8 1 2} & \mathbf{1 . 8 1 2 3 0 8}\end{array}$

$\begin{array}{llll}\text { H } & 2.213952 & -2.522240 & 3.496736\end{array}$

$\begin{array}{llll}C & -3.296462 & -0.624850 & 1.044160\end{array}$

$\begin{array}{llll}\text { C } & -4.119236 & 0.499836 & 0.953292\end{array}$

$\begin{array}{llll}\text { C } & \mathbf{- 3 . 6 8 6 3 0 7} & \mathbf{- 1 . 8 1 7 1 4 4} & \mathbf{0 . 4 2 4 5 1 0}\end{array}$

$\begin{array}{llll}C & -5.290673 & 0.498456 & 0.221166\end{array}$

C $-4.877265-1.812193-0.312480$

$\begin{array}{llll}C & -4.877265 & -1.812193 & -0.312480\end{array}$

$\begin{array}{llll}C & -5.679980 & -0.675586 & -0.436362\end{array}$

$\begin{array}{llll}\text { H } & \mathbf{- 5 . 8 8 5 9 0 5} & 1.411761 & \mathbf{0 . 1 6 9 1 0 0}\end{array}$

$\begin{array}{llll}H & -5.186479 & -2.737029 & -0.805665\end{array}$

$\begin{array}{llll}C & -6.931823 & -0.694425 & -1.271025\end{array}$

$\begin{array}{llll}C & -2.852686 & -3.062320 & 0.514998\end{array}$

$\begin{array}{llll}\text { H } & -2.178869 & -3.137251 & -0.352110\end{array}$

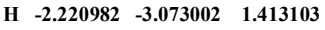

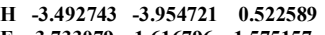

$\begin{array}{llll}\text { F } & -3.733079 & 1.616796 & 1.575157\end{array}$

C $-1.476774 \quad 0.109160 \quad-1.848352$

$\begin{array}{llll}\text { H } & -2.299674 & -0.587562 & -1.624512\end{array}$

$\begin{array}{llll}C & -0.399813 & \mathbf{2 . 1 1 5 6 4 0} & \mathbf{- 0 . 5 1 8 8 6 5}\end{array}$

$\begin{array}{llll}C & -1.693438 & 1.537107 & -1.193001\end{array}$

$\begin{array}{llll}\text { H } & -\mathbf{0 . 6 1 0 6 5 1} & \mathbf{2 . 5 5 5 4 9 5} & \mathbf{0 . 4 5 9 6 4 0}\end{array}$

$\begin{array}{llll}\text { H } & -2.429855 & 1.440333 & -0.394674\end{array}$

$\begin{array}{llll}C & -2.303059 & 2.508151 & -2.224423\end{array}$

$\begin{array}{llll}C & \mathbf{2 . 5 8 7 9 0 2} & \mathbf{- 3 . 7 5 4 3 2 1} & \mathbf{- 1 . 1 5 8 9 1 2}\end{array}$

$\begin{array}{llll}C & 3.903712 & -3.927239 & -1.557156\end{array}$

H $4.345134-4.926445-1.557698$

$\begin{array}{llll}\text { H } & 4.345134 & -4.926445 & -1.557698 \\ C & 4.671788 & -2.818623 & -1.949962\end{array}$

$\begin{array}{llll}C & 4.671788 & -2.818623 & -1.949962\end{array}$

$\begin{array}{llll}\text { H } & \mathbf{5 . 7 1 1 5 2 0} & \mathbf{- 2 . 9 5 2 5 4 6} & \mathbf{- 2 . 2 5 6 6 0 7}\end{array}$

$\begin{array}{llll}C & 4.109697 & -1.552188 & -1.944370\end{array}$

$\begin{array}{llll}\text { H } & 4.701299 & -0.683765 & -2.245343\end{array}$

$\begin{array}{llll}\text { H } & 1.990536 & -4.612502 & -0.840542\end{array}$

$\begin{array}{llll}\text { H } & 6.102851 & \mathbf{0 . 7 2 1 2 2 6} & \mathbf{1 . 8 8 2 6 9 7}\end{array}$

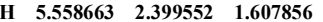

\begin{tabular}{llll}
\hline & 5.490873 & 1.209076 & 0.290478
\end{tabular}

\begin{tabular}{lllll}
\hline & -7.757374 & -0.174979 & -0.763840
\end{tabular}

$\begin{array}{llll}\text { H } & -7.250148 & -1.721601 & -1.493327\end{array}$

H $-6.760342-0.180238-2.229773$

$\begin{array}{llll}4 & -1.611742 & 2.715529 & -3.047106\end{array}$

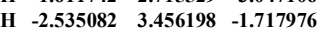

H $\quad \begin{array}{llll}-3.237150 & 2.087760 & -2.624401\end{array}$

$\begin{array}{llll}\text { C } & -1.119189 & 0.089245 & -3.316225\end{array}$

$\begin{array}{llll}C & 0.518047 & 2.958762 & -1.300442\end{array}$

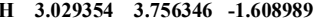

\begin{tabular}{lllll}
\hline & $\mathbf{1 . 2 8 2 8 3 9}$ & $\mathbf{3 . 7 1 0 1 4 9}$ & $\mathbf{- 0 . 4 8 2 5 7 2}$
\end{tabular}

$\begin{array}{llll}\text { C } & 2.286672 & 4.434157 & -1.088963\end{array}$

\begin{tabular}{llll}
$C$ & 2.336672 & 4.434157 & -1.088963 \\
\hline
\end{tabular}

$\begin{array}{llll}\text { H } & 2.861433 & 4.952462 & \mathbf{- 0 . 2 7 7 9 2 5}\end{array}$

H $1.953258-5.168050-1.812998$

$\begin{array}{lllll}\text { O } & 0.664023 & 2.987894 & -2.506568\end{array}$

$\begin{array}{lllll}\text { H } & -0.779149 & -0.922534 & -3.582694\end{array}$

H $\quad \begin{array}{llll}-0.322299 & 0.807326 & -3.547751\end{array}$

$\begin{array}{llll}\text { H } & -\mathbf{2 . 0 0 1 5 0 8} & \mathbf{0 . 3 2 1 1 5 8} & \mathbf{- 3 . 9 3 6 0 8 7}\end{array}$

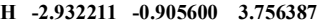

$\begin{array}{llll}\text { H } & -0.287871 & -0.449254 & 4.529284\end{array}$

75

unsat mcb dist c / electronic energy: -2588.91306940 a.u. / lowest freq: $12.54 \mathrm{~cm}-1$

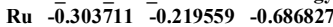

S $-\mathbf{0 . 0 5 8 6 4 8}-\mathbf{2 5 9 0 2 2 3}-\mathbf{- 0 . 6 6 7 6 6 1}$

$\begin{array}{llll}C & \mathbf{1 . 6 4 6 5 0 0} & \mathbf{- 2 . 8 4 1 6 5 4} & \mathbf{- 0 . 9 8 7 9 3 0}\end{array}$

$\begin{array}{llll}\text { C } & \mathbf{2 . 4 8 7 1 7 5} & -1.745984 & -1.237521\end{array}$

$\begin{array}{llll}S & 1.844611 & -0.105222 & -1.260052\end{array}$

$\begin{array}{llll}C & -0.629426 & 0.076893 & 1.276342\end{array}$

$\begin{array}{llll}\mathrm{N} & \mathbf{0 . 2 9 4 2 4 3} & \mathbf{0 . 3 5 6 3 1 5} & \mathbf{2 . 2 3 0 1 1 8}\end{array}$

$\begin{array}{llll}\text { N } & -1.797655 & -0.049138 & 1.963724\end{array}$

$\begin{array}{lllll}\text { C } & -\mathbf{0 . 2 8 7 5 8 8} & \mathbf{0 . 4 0 6 7 5 1} & \mathbf{3 . 4 8 8 9 7 4}\end{array}$

$\begin{array}{llll}C & -1.603147 & 0.158385 & 3.321850\end{array}$

$\begin{array}{llll}C & \mathbf{1 . 6 7 4 8 2 9} & \mathbf{0 . 6 3 7 7 9 1} \mathbf{2 . 0 0 8 2 8 5}\end{array}$

$\begin{array}{llll}C & \mathbf{2 . 0 5 2 5 0 6} & \mathbf{1 . 9 6 1 3 9 1} & \mathbf{1 . 8 0 8 5 7 0}\end{array}$

$\begin{array}{llll}C & \mathbf{2 . 6 3 7 2 5 1} & \mathbf{- 0 . 3 7 9 6 1 0} & \mathbf{2 . 0 0 6 3 6 6}\end{array}$

$\begin{array}{llll}C & 3.369651 & 2.307418 & 1.558407\end{array}$

$\begin{array}{llll}\text { C } & 3.961529 & -0.020351 & 1.753323\end{array}$

$\begin{array}{llll}\text { C } & 4.341868 & 1.305357 & 1.510778\end{array}$

$\begin{array}{lllll}\text { H } & \mathbf{3 . 6 1 7 7 5 5} & \mathbf{3 . 3 5 7 7 2 7} & \mathbf{1 . 3 9 8 0 4 1}\end{array}$

$\begin{array}{llll}\text { H } & 4.720487 & -0.806016 & 1.726537\end{array}$

$\begin{array}{llll}\text { C } & 5.769983 & 1.639951 & 1.173439\end{array}$

$\begin{array}{lllll}\text { F } & \mathbf{1 . 1 1 9 5 8 7} & \mathbf{2 . 9 0 8 5 8 7} & \mathbf{1 . 8 8 1 7 1 2}\end{array}$

$\begin{array}{llll}\text { C } \quad \mathbf{2 . 2 4 4 7 6 4} & \mathbf{- 1 . 8 0 5 2 7 7} & \mathbf{2 . 2 7 0 8 4}\end{array}$

H $\quad \mathbf{1 . 3 4 1 6 7 0}-\mathbf{- 2 . 0 8 2 3 0 4} \quad \mathbf{1 . 7 0 7 9 4 4}$

$\begin{array}{llll}\text { H } & \mathbf{3 . 0 5 0 4 5 7} & \mathbf{- 2 . 4 9 1 0 9 6} & \mathbf{1 . 9 7 8 8 2 9}\end{array}$

$\begin{array}{llll}\text { H } & 2.031975 & -1.959327 & 3.341012\end{array}$

$\begin{array}{llll}\text { C } & \mathbf{- 3 . 0 7 4 0 2 2} & \mathbf{- 0 . 3 0 9 9 9 5} & \mathbf{1 . 3 8 7 3 1}\end{array}$

$\begin{array}{llll}C & -3.895488 & \mathbf{0 . 7 7 9 2 2 8} & \mathbf{1 . 0 8 7 5 1 2}\end{array}$

$\begin{array}{llll}C & -3.524428 & -1.610921 & 1.13388\end{array}$

$\begin{array}{llll}\text { C } & -5.121101 & 0.624799 & 0.470586\end{array}$

$\begin{array}{llll}C & -4.766676 & -1.757793 & 0.504194\end{array}$

$\begin{array}{llll}C & -5.566392 & -0.666484 & 0.156286\end{array}$ 


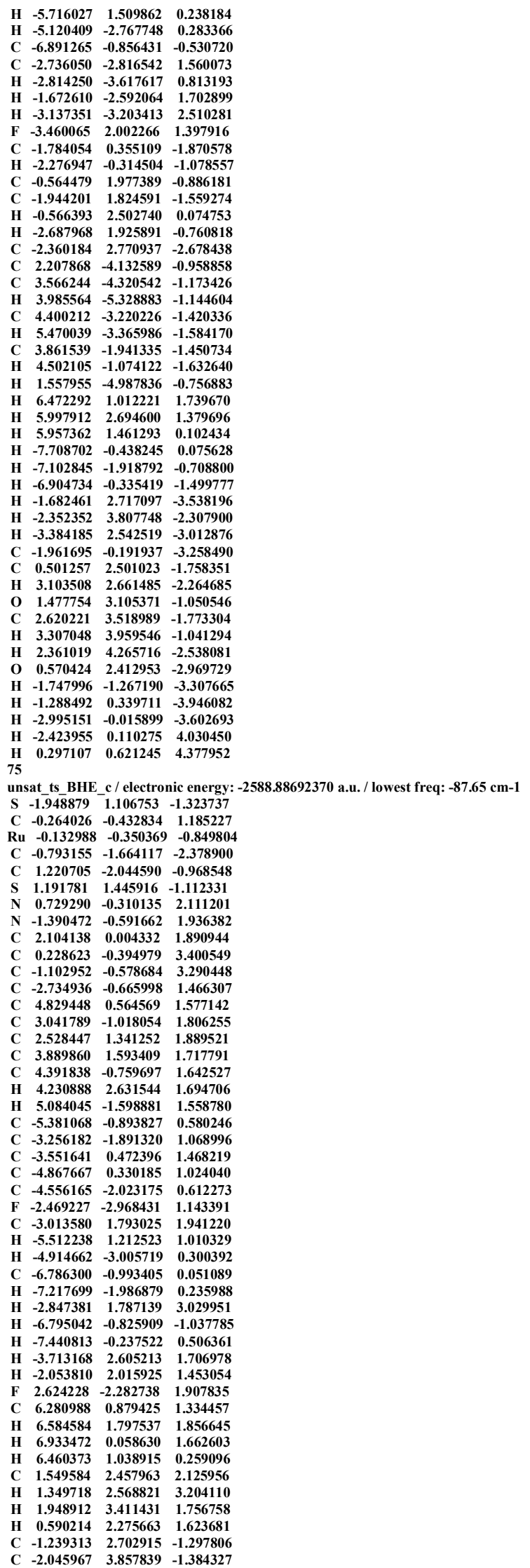


$\begin{array}{llll}C & 0.156343 & 2.864680 & -1.185038\end{array}$ $\begin{array}{llll}C & -1.481827 & 5.123903 & -1.349490\end{array}$ $\begin{array}{llll}\text { H } & -\mathbf{3 . 1 2 8 8 3 9} & 3.738686 & -1.47332\end{array}$

$\begin{array}{llll}C & 0.717896 & 4.153860 & -1.147542\end{array}$

$\begin{array}{llll}C & -0.091551 & 5.277292 & -1.228117\end{array}$

H $-2.1252006 .004967-1.411292$

$\begin{array}{llll}\mathrm{H} & -2.125200 & 6.004967 & -1.411292\end{array}$

$\begin{array}{llll}\mathrm{H} & 1.801856 & 4.25331 & \mathbf{- 1 . 0 5 1 4 4 8}\end{array}$

$\begin{array}{llll}0.352413 & 6.274570 & -1.19495\end{array}$

$\begin{array}{llll}\text { C } & -0.239996 & -1.212828 & -3.708163\end{array}$

$\begin{array}{llll}\text { H } & -1.875381 & -1.843785 & -2.426303\end{array}$

$\begin{array}{llll}C & \mathbf{- 0 . 1 4 9 1 5 2} & \mathbf{- 2 . 4 6 9 0 2 6} & \mathbf{- 1 . 3 3 6 5 7 4}\end{array}$

$\begin{array}{lllll}\text { H } & 1.540315 & -2.431287 & 0.003012\end{array}$

$\begin{array}{llll}\text { C } & 2.361940 & -2.026571 & -1.890991\end{array}$

$\begin{array}{llll}C & -0.657238 & -3.862019 & -0.990314\end{array}$

$\begin{array}{llll}\text { H } & \mathbf{- 0 . 1 9 7 6 2 0} & \mathbf{- 4 . 5 5 7 8 5 1} & \mathbf{- 1 . 7 0 7 8 4 2}\end{array}$

$\begin{array}{llll}\text { H } & -0.355159 & -4.156872 & 0.023141\end{array}$

$\begin{array}{llll}\text { H } & -1.748893 & -3.938454 & -1.076731\end{array}$

\begin{tabular}{lllll}
\hline & $\mathbf{2 . 3 9 1 4 8 2}$ & $\mathbf{- 2 . 3 3 2 2 4 6}$ & $\mathbf{- 3 . 0 6 5 9 4 8}$
\end{tabular}

$\begin{array}{lllll}\text { O } & 3.474601 & -1.633982 & -1.240778\end{array}$

C $4.668919-1.556877 \quad-1.991831$

$\begin{array}{lllll}\text { H } & 4.584323 & -0.807917 & -2.793046\end{array}$

$\begin{array}{llll}\text { H } & 4.920693 & -2.527810 & -2.443185\end{array}$

H $5.453487-1.256802 \quad-1.287134$

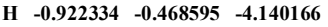

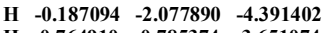

H $\quad 0.764910 \quad-0.785374-3.651074$

$\begin{array}{lllll}\text { H } & -0.840317 & -1.852645 & -0.373909\end{array}$

$\begin{array}{lllll}\text { H } & 0.875999 & -0.316045 & 4.268268\end{array}$

$\begin{array}{llll}\text { H } & -1.877764 & -0.702438 & 4.040502\end{array}$

75

unsat prod BHE c / electronic energy: - 2588.94034948 a.u. / lowest freq: $15.22 \mathrm{~cm}-1$

$\begin{array}{llll}S & -0.975033 & 0.983968 & -2.003868\end{array}$

$\begin{array}{llll}C & -0.460275 & 0.081669 & 1.142825\end{array}$

Ru $\quad-0.219972-0.761676-0.634800$

$\begin{array}{llll}C & -1.894565 & -2.516452 & -0.347574\end{array}$

$\begin{array}{llll}C & 0.691306 & -2.713054 & -0.196149\end{array}$

$\begin{array}{lllll}\text { S } & \mathbf{1 . 9 0 2 6 4 2} & \mathbf{0 . 0 0 7 9 0 8} & -1.138928\end{array}$

N $\begin{array}{cccc}0.471863 & 0.308786 & 2.125429\end{array}$

$\begin{array}{llll}\mathrm{N} & -1.641162 & 0.452542 & 1.740412\end{array}$

C $\quad \begin{array}{llll}\mathbf{1 . 8 8 9 2 0 8} & \mathbf{0 . 4 2 3 8 8 0} & \mathbf{1 . 9 8 0 4 6 5}\end{array}$

$\begin{array}{llll}\mathrm{C} & \mathbf{- 0 . 1 2 4 5 9 2} & \mathbf{0 . 7 5 9 9 7 7} & \mathbf{3 . 2 9 5 9 4 7}\end{array}$

$\begin{array}{llll}C & -1.445529 & 0.850817 & 3.056635\end{array}$

$\begin{array}{llll}\text { C } & -2.909674 & 0.640730 & 1.112463\end{array}$

$\begin{array}{llll}\text { C } & \mathbf{4 . 6 7 4 2 5 4} & 0.694879 & 1.732279\end{array}$

$\begin{array}{llll}C & \mathbf{2 . 7 4 1 0 7 6} & \mathbf{- 0 . 6 4 5 0 2 0} & \mathbf{2 . 2 2 3 7 1 9}\end{array}$

$\begin{array}{llll}\text { C } & \mathbf{2 . 4 3 5 3 0 9} & \mathbf{1 . 6 7 4 6 9 1} & \mathbf{1 . 6 4 7 7 2 9}\end{array}$

$\begin{array}{llll}\text { C } & 3.819599 & 1.785707 & 1.532402\end{array}$

$\begin{array}{llll}\text { C } & 4.116213 & -0.532577 & 2.092829\end{array}$

H $4.246621 \quad 2.754036 \quad 1.26015$

$\begin{array}{llll}\text { H } & 4.735132 & -1.411850 & 2.28003\end{array}$

$\begin{array}{lllll}\text { C } & -5.437628 & 0.996377 & -0.062282\end{array}$

$\begin{array}{llll}\text { C } & -3.913603 & -0.311983 & 1.249165\end{array}$

$\begin{array}{llll}C & \mathbf{- 3 . 2 0 8 3 3 4} & \mathbf{1 . 8 5 9 3 2 5} & \mathbf{0 . 4 7 7 4 4 6}\end{array}$

C $-3.208334 \quad 1.8593250 .477446$

$\begin{array}{llll}C & -4.463082 & 2.001346 & -0.115488\end{array}$

$\begin{array}{llll}C & -5.157250 & -0.168295 & 0.654989\end{array}$

$\begin{array}{llll}\text { C } & -5.680744 & -1.398496 & \mathbf{1 . 9 9 6 7 0 2}\end{array}$

$\begin{array}{llll}\text { C } & -2.222998 & 2.992455 & 0.502306\end{array}$

$\begin{array}{llll}\text { H } & -4.694766 & 2.938693 & -0.627196\end{array}$

$\begin{array}{llll}\text { H } & \mathbf{5 . 8 9 6 8 6 7} & \mathbf{- 0 . 9 6 1 5 4 3} & \mathbf{0 . 7 7 6 1 8 6}\end{array}$

$\begin{array}{llll}\text { C } & \mathbf{- 6 . 7 6 0 0 1 9} & \mathbf{1 . 1 7 5 6 9 9} & \mathbf{- 0 . 7 5 7 0 7 1}\end{array}$

$\begin{array}{lllll}\text { H } & -7.509130 & \mathbf{0 . 4 6 0 0 8 4} & \mathbf{- 0 . 3 9 2 7 3 2}\end{array}$

$\begin{array}{llll}\text { H } & -1.977991 & \mathbf{3} 270987 & \mathbf{1 . 5 3 9 3 1 8}\end{array}$

$\begin{array}{lllll}\text { H } & -6.643876 & 1.018941 & -1.84\end{array}$

\begin{tabular}{lll}
-6.643876 & 1.018941 & -1.841013 \\
\hline
\end{tabular}

\begin{tabular}{llll}
\hline & -7.150041 & 2.193498 & -0.613825 \\
\hline
\end{tabular}

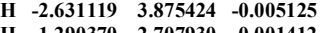

$\begin{array}{llll}H & -1.290370 & 2.707930 & -0.001412\end{array}$

$\begin{array}{llll}\text { F } & 2.232707 & -1.826411 & 2.592407\end{array}$

$\begin{array}{llll}\text { C } & 6.159178 & 0.833788 & 1.533148\end{array}$

$\begin{array}{llll}\text { H } & 6.500052 & 1.853270 & 1.760991\end{array}$

H $6.720007 \quad 0.128523 \quad 2.161792$

$\begin{array}{lllll}\text { H } & 6.421310 & 0.624245 & 0.483708\end{array}$

$\begin{array}{llll}\text { C } \quad 1.533852 & \mathbf{2 . 8 4 7 5 1 7} & \mathbf{1 . 3 8 6 9 1 6}\end{array}$

\begin{tabular}{llll}
\hline & 0.950750 & 3.118431 & 2.280848
\end{tabular}

\begin{tabular}{ccccc}
$\mathrm{H}$ & $\mathbf{0 . 9 5 0 7 5 0}$ & $\mathbf{3 . 1 1 8 4 3 1}$ & $\mathbf{2 . 2 8 0 8 4 8}$ \\
\hline & $\mathbf{2 . 1 1 2 5 2 4}$ & $\mathbf{3} 323763$ & $\mathbf{1 . 0 6 9 3 7 4}$
\end{tabular}

$\begin{array}{llll}\text { H } & 2.112524 & 3.723763 & \mathbf{1 . 0 6 9 3 7 4}\end{array}$

$\begin{array}{lllll}H & 0.821303 & 2.607476 & \mathbf{0 . 5 8 4 8 7 8}\end{array}$

$\begin{array}{lllll}\text { C } & 0.400930 & 2.074069 & -2.149096\end{array}$

$\begin{array}{llll}C & 0.240235 & 3.379935 & -2.640890\end{array}$

$\begin{array}{llll}C & \mathbf{1 . 6 8 3 0 4 8} & \mathbf{1 . 6 3 0 4 1 0} & -1.776851\end{array}$

$\begin{array}{lllll}C & 1.333966 & \mathbf{4 . 2 3 0 3 7 2} & \mathbf{- 2 . 7 6 4 6 2 7}\end{array}$

$\begin{array}{lllll}\text { H } & -\mathbf{0 . 7 5 9 2 8 6} & \mathbf{3 . 7 2 3 5 4 5} & \mathbf{- 2 . 9 2 0 3 6 4}\end{array}$

$\begin{array}{llll}\text { C } & \mathbf{2 . 7 8 1 0 9 7} & \mathbf{2 . 4 9 6 0 0 0} & -1.907875\end{array}$

C $2.610459 \quad 3.784923 \quad-2.400013$

$\begin{array}{llll}C & 2.610459 & 3.784923 & -2.400013\end{array}$

$\begin{array}{llll}\mathrm{H} & 1.192947 & \mathbf{5 . 2 4 5 0 1 6} & \mathbf{- 3 . 1 4 4 2 4 0}\end{array}$

$\begin{array}{llll}H \quad 3.771100 & 2.145600 & -1.604097\end{array}$

$\begin{array}{llll}\text { H } & 3.471541 & 4.451134 & -2.491351\end{array}$

$\begin{array}{llll}\text { C } & -2.306132 & -3.593358 & -1.347787\end{array}$

$\begin{array}{llll}\text { H } & -2.681377 & -2.421441 & 0.405972\end{array}$

$\begin{array}{llll}\text { C } & -0.589424 & -2.606255 & 0.396273\end{array}$

$\begin{array}{llll}\text { H } & \mathbf{1 . 5 1 9 3 0 6} & -2.790146 & \mathbf{0 . 5 1 1 2 1 8}\end{array}$

$\begin{array}{llll}C & 1.077289 & -3.124720 & -1.562709\end{array}$

$\begin{array}{llll}C & -0.709308 & -2.820591 & 1.885267\end{array}$

$\begin{array}{llll}C & -0.7990695 & -3.871685 & 2.063124\end{array}$

$\begin{array}{lrrrr}\text { H } & -0.990695 & -3.871685 & 2.063124 \\ \text { H } & 0.234026 & -2.623602 & 2.401885\end{array}$ 


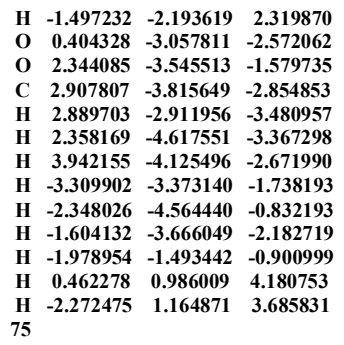

unsat_ts_CP_c / electronic energy: $\mathbf{- 2 5 8 8 . 8 9 5 9 5 1 3 8}$ a.u. / lowest freq: $\mathbf{- 2 3 0 . 8 4} \mathbf{c m}-1$

$\begin{array}{lllll}R u & -\mathbf{0 . 3 8 3 4 6 8} & \mathbf{0 . 2 5 3 2 8 7} & -\mathbf{0 . 8 4 5 2 1 1}\end{array}$

$\begin{array}{lllll}S & 1.560216 & 1.424592 & -0.815614\end{array}$

$\begin{array}{llll}\text { C } & \mathbf{2 . 8 1 5 4 6 5} & \mathbf{0 . 4 0 3 0 7 0} & \mathbf{- 1 . 5 1 2 5 3 9}\end{array}$

$\begin{array}{llll}C & \mathbf{2 . 4 6 9 8 2 8} & \mathbf{- 0 . 8 4 0 0 3 9} & \mathbf{- 2 . 0 6 3 4 1 2}\end{array}$

S $0.782814-1.345564 \quad-2.078581$

$\begin{array}{llll}C & -0.447151 & -0.839789 & 0.789819\end{array}$

$\begin{array}{llll}\text { N } & -1.549314 & -1.473565 & 1.308311\end{array}$

N $0.556319-1.213245 \quad 1.641784$

$\begin{array}{llll}\text { C } & -1.237404 & -2.198614 & 2.451182\end{array}$

$\begin{array}{lllll}C & 0.081909 & -2.030918 & 2.659198\end{array}$

$\begin{array}{llll}C & -2.864010 & -1.471909 & 0.766968\end{array}$

$\begin{array}{llll}C & -3.848895 & -0.718778 & 1.407932\end{array}$

$\begin{array}{llll}C & -3.213025 & -2.271134 & -0.331231\end{array}$

$\begin{array}{llll}\text { C } & -5.160807 & -0.696229 & \mathbf{0 . 9 7 0 8 4 3}\end{array}$

$\begin{array}{llll}C & -4.537589 & -2.226717 & -0.779768\end{array}$

$\begin{array}{llll}\text { C } & -5.518096 & -1.452809 & -0.151261\end{array}$

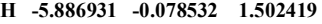

H $-\mathbf{- 4 . 8 1 5 2 6 6}-\mathbf{- 2 . 8 3 5 1 8 3}-\mathbf{- 1 . 6 4 3 9 7 6}$

$\begin{array}{llll}\text { C } & -6.940325 & -1.447751 & -0.642047\end{array}$

$\begin{array}{llll}\text { F } & -3.494559 & 0.021154 & 2.460783\end{array}$

$\begin{array}{llll}\text { C } & -2.197380 & -3.143372 & -1.013181\end{array}$

$\begin{array}{llll}\text { H } & -\mathbf{2 . 6 8 6 0 0 5} & \mathbf{- 3 . 9 9 8 7 4 2} & \mathbf{- 1 . 4 9 7 2 5 3}\end{array}$

$\begin{array}{lllll}\text { H } & -1.652594 & -2.583743 & -1.789099\end{array}$

$\begin{array}{llll}\text { H } & -1.444636 & -3.518894 & -0.306543\end{array}$

$\begin{array}{llll}C & 1.948235 & -0.906813 & 1.570340\end{array}$

$\begin{array}{llll}\text { C } & 2.409859 & \mathbf{0 . 2 8 7 6 8 9} & \mathbf{2 . 1 1 4 9 2 2}\end{array}$

$\begin{array}{llll}C & 2.865653 & -1.847118 & 1.080130\end{array}$

$\begin{array}{llll}\text { C } & 3.757003 & 0.606495 & 2.139797\end{array}$

$\begin{array}{llll}C & 4.222951 & -1.520150 & 1.115668\end{array}$

$\begin{array}{llll}C & 4.684753 & -0.303498 & 1.625940\end{array}$

$\begin{array}{llll}\text { H } & 4.066267 & 1.564323 & 2.562009\end{array}$

$\begin{array}{lllll}\text { H } & 4.944067 & -2.238099 & 0.717931\end{array}$

$\begin{array}{llll}\text { C } & 6.148657 & 0.038818 & 1.587743\end{array}$

$\begin{array}{lllll}\text { C } & 2.398187 & -3.176886 & \mathbf{0 . 5 5 9 8 3 4}\end{array}$

$\begin{array}{llll}\text { H } & 3.226736 & -3.718310 & 0.085335\end{array}$

$\begin{array}{llll}\text { H } & 1.999937 & -3.803945 & 1.373312\end{array}$

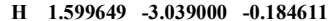

$\begin{array}{llll}\text { F } & 1.526649 & 1.134008 & \mathbf{2} .640207\end{array}$

F 1.526649031 .134008 2.640207

$\begin{array}{llll}\text { C } & -2.223803 & 1.267813 & -1.318089\end{array}$

$\begin{array}{lllll}\text { H } & -2.481709 & 0.170420 & -1.333468\end{array}$

$\begin{array}{llll}\text { C } & -1.667540 & 1.943802 & \mathbf{0 . 2 7 8 7 9 6}\end{array}$

$\begin{array}{lllll}C & -3.050128 & 2.036177 & -0.354492\end{array}$

$\begin{array}{llll}\text { H } & -1.655631 & 1.409404 & 1.228143\end{array}$

$\begin{array}{lllll}\text { C } & -3.691697 & 3.355322 & -0.725024\end{array}$

$\begin{array}{llll}\text { H } & -3.748850 & 1.406983 & 0.200011\end{array}$

$\begin{array}{llll}C & 4.155033 & 0.817370 & -1.508331\end{array}$

$\begin{array}{llll}C & 5.146024 & -0.000827 & -2.037739\end{array}$

$\begin{array}{lllll}\text { H } & 6.187640 & 0.329167 & -2.028807\end{array}$

$\begin{array}{llll}C & 4.807564 & -1.251712 & -2.568017\end{array}$

$\begin{array}{llll}\text { H } & \mathbf{5 . 5 8 3 7 1 5} & -1.904674 & -2.974250\end{array}$

$\begin{array}{llll}\text { C } & \mathbf{3 . 4 8 0 1 1 7} & -\mathbf{1 . 6 6 6 2 0 2} & \mathbf{- 2 . 5 8 0 8 8 9}\end{array}$

$\begin{array}{lllll}\text { H } & 3.211848 & -2.641635 & -2.995489\end{array}$

$\begin{array}{llll}\text { H } & 4.412464 & 1.784763 & -1.068489\end{array}$

$\begin{array}{lllll}\text { H } & 6.773778 & \mathbf{- 0 . 8 6 2 3 2 5} & \mathbf{1 . 6 5 7 6 3 8}\end{array}$

$\begin{array}{lllll}\text { H } & 6.427427 & 0.723797 & \mathbf{2 . 4 0 0 5 8 6}\end{array}$

$\begin{array}{lllll}\text { H } & 6.390269 & 0.534441 & 0.633809\end{array}$

$\begin{array}{llll}\text { H } & -7.328339 & -0.421902 & -0.721154\end{array}$

$\begin{array}{llll}\text { H } & -7.591961 & -1.990675 & 0.060085\end{array}$

$\begin{array}{llll}\text { H } & -7.028125 & -1.928878 & -1.625000\end{array}$

$\begin{array}{llll}\text { H } & -3.009241 & 4.020098 & -1.265715\end{array}$

$\begin{array}{llll}\text { H } & -\mathbf{4 . 0 2 1 2 7 0} & \mathbf{3 . 8 7 7 4 3 3} & \mathbf{0 . 1 8 5 4 4 5}\end{array}$

$\begin{array}{llll}\text { H } & -4.580145 & 3.174291 & -1.348390\end{array}$

$\begin{array}{lrrr}C & -1.919297 & 1.886075 & -2.667790\end{array}$

$\begin{array}{llll}\text { H } & 0.740771 & -2.409422 & 3.434484\end{array}$

$\begin{array}{llll}\text { H } & -1.989641 & -2.758460 & 2.997987\end{array}$

$\begin{array}{lllll}\text { O } & \mathbf{- 0 . 7 1 6 4 4 2} & 3.987575 & -0.584088\end{array}$

$\begin{array}{llll}C & 0.706324 & 3.153300 & 0.290057\end{array}$

$\begin{array}{lllll}C & -0.806324 & 3.153300 & 0.290057\end{array}$

$\begin{array}{llll}\mathrm{C} & -0.124181 & 3.229237 & \mathbf{1 . 4 3 3 0 4 8}\end{array}$

$\begin{array}{llll}\mathrm{C} & \mathbf{0 . 8 3 8 0 3 3} & 4.265386 & \mathbf{1 . 5 4 1 2 3 9}\end{array}$

$\begin{array}{ccccc}\text { H } & \mathbf{0 . 3 6 3 5 9 7} & \mathbf{5 . 2 5 3 6 0 0} & \mathbf{1 . 4 5 9 3 0 0}\end{array}$

$\begin{array}{llll}\text { H } & \mathbf{1 . 3 0 1 6 1 7} & \mathbf{4 . 1 4 6 9 7 0} & \mathbf{2 . 5 2 6 1 1 6}\end{array}$

$\begin{array}{llll}\text { H } & 1.598495 & 4.162736 & 0.754186\end{array}$

$\begin{array}{lllll}\text { H } & -1.252613 & 1.257554 & -3.274559\end{array}$

$\begin{array}{lllll}\text { H } & -1.453883 & 2.870573 & -2.537002\end{array}$

$\begin{array}{llll}H & -2.865016 & 2.013194 & -3.219456\end{array}$

75

unsat prod CP c / electronic energy: $-\mathbf{2 5 8 8 . 9 0 9 2 8 5 2 5}$ a.u. / lowest freq: $13.65 \mathrm{~cm}-1$ Ru $\quad-\mathbf{0 . 3 1 9 9 5 7} \quad-\mathbf{0 . 0 3 8 1 3 7} \quad-\mathbf{0 . 8 5 2 8 7 3}$ 
$\begin{array}{lllll}S & 1.345242 & 1.486601 & -0.836210\end{array}$ $\begin{array}{llll}C & 2.748284 & 0.673786 & -1.530379\end{array}$

$\begin{array}{llll}C & \mathbf{2 . 6 0 1 8 8 7} & \mathbf{- 0 . 6 0 4 7 0 4} & \mathbf{- 2 . 0 8 9 4 5}\end{array}$

$\begin{array}{llll}\text { S } & 1.006433 & -1.358918 & -2.138815\end{array}$

$\begin{array}{llll}C & -0.199487 & -1.089961 & 0.775686\end{array}$

$\begin{array}{llll}\text { N } & -1.241514 & -1.822297 & 1.290768\end{array}$

$\begin{array}{llll}\text { N } & 0.840419 & -1.390084 & 1.610086\end{array}$

$\begin{array}{llll}C & \mathbf{- 0 . 8 5 9 2 8 4} & \mathbf{- 2 . 5 4 0 6 8 2} & \mathbf{2 . 4 1 5 0 1 1}\end{array}$

$\begin{array}{llll}C & 0.446052 & -2.265475 & 2.614743\end{array}$

$\begin{array}{llll}C & -2.555594 & -1.822858 & 0.74814\end{array}$

$\begin{array}{llll}C & -3.554777 & -1.109210 & 1.417631\end{array}$

$\begin{array}{llll}\text { C } & \mathbf{- 2 . 8 7 7 8 2 3} & \mathbf{- 2 . 5 1 9 6 1 9} & \mathbf{- 0 . 4 2 4 4 2 4}\end{array}$

$\begin{array}{llll}C & -4.853724 & -1.047729 & 0.951614 \\ \text { C } & -4.191067 & -2.428480 & -0.904142\end{array}$

C -5.18

$\begin{array}{llll}\text { C } & -\mathbf{5 . 1 8 3 0 6 8} & \mathbf{- 1 . 7 0 2 0 2 9} & \mathbf{- 0 . 2 4 2 8 2 2}\end{array}$

$\begin{array}{llll}\text { H } & \mathbf{- 5 . 5 9 6 0 7 0} & -\mathbf{0 . 4 8 2 3 3 9} & \mathbf{1 . 5 1 8 0 6 1}\end{array}$

$\begin{array}{llll}\text { H } & \mathbf{- 4 . 4 4 5 6 1 5} & \mathbf{- 2 . 9 5 9 4 5 7} & \mathbf{- 1 . 8 2 4 7 2 6} \\ \text { C } & \mathbf{- 6 . 5 7 5 3 8 4} & \mathbf{- 1 . 5 9 4 4 9 1} & \mathbf{- 0 . 8 0 1 6 8 5}\end{array}$

$\begin{array}{lllll}\text { C } & \mathbf{- 6 . 5 7 5 3 8 4} & \mathbf{- 1 . 5 9 4 4 9 1} & \mathbf{- 0 . 8 0 1 6 8 5} \\ \text { F } & \mathbf{- 3 . 2 2 4 4 3 7} & \mathbf{- 0 . 4 5 4 5 0 8} & \mathbf{2 . 5 3 2 1 3 7}\end{array}$

$\begin{array}{llll}\text { C } & -1.859727 & -3.345485 & -1.163813\end{array}$

H $-2.306193 \quad-4.295295 \quad-1.488714$

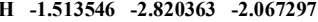

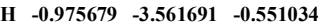

$\begin{array}{llll}\text { C } & \mathbf{2 . 1 6 5 9 0 0} & -\mathbf{0 . 8 7 1 6 3 8} & \mathbf{1 . 5 4 8 7 2 2}\end{array}$

$\begin{array}{llll}\text { C } & 2.420183 & 0.383657 & \mathbf{2 . 0 9 2 8 4 4}\end{array}$

$\begin{array}{llll}C & 3.223538 & -1.632160 & 1.031034\end{array}$

$\begin{array}{llll}\text { C } & 3.692003 & 0.930499 & \mathbf{2 . 1 0 6 8 9 9}\end{array}$

$\begin{array}{llll}\text { C } & 4.504683 & -1.077404 & 1.057809\end{array}$

$\begin{array}{llll}C & 4.756371 & 0.196097 & 1.577943\end{array}$

H $\quad 3.836665 \quad 1.925308 \quad 2.532114$

$\begin{array}{llll}\text { H } & 5.333499 & -1.654470 & 0.641071\end{array}$

$\begin{array}{llll}\text { C } & 6.140084 & 0.783368 & 1.529092\end{array}$

$\begin{array}{llll}\text { C } & 2.975541 & -3.005746 & \mathbf{0 . 4 7 5 3 6 5}\end{array}$

$\begin{array}{llll}\text { H } & 3.890261 & -3.415185 & 0.027929\end{array}$

\begin{tabular}{cccc} 
H & 3.890261 & $\mathbf{- 3 . 4 1 5 1 8 5}$ & $\mathbf{0 . 0 2 7 9 2 9}$ \\
\hline
\end{tabular}

\begin{tabular}{llll} 
H & 2.637318 & -3.699949 & 1.260811 \\
\hline & 2.192823 & -2.968809 & -0.297917
\end{tabular}

$\begin{array}{llll}\text { H } & 2.192823 & -2.968809 & -0.297917\end{array}$

$\begin{array}{ccccc}\text { F } & 1.407071 & \mathbf{1 . 0 6 4 8 0 0} & \mathbf{2 . 6 2 7 1 8 4} \\ \text { C } & -3.058660 & \mathbf{1 . 5 3 6 3 2 9} & -\mathbf{1 . 1 1 0 9 3 7}\end{array}$

$\begin{array}{llll}\text { H } & \mathbf{- 3 . 2 2 4 9 0 6} & \mathbf{0 . 4 5 4 1 4 0} & \mathbf{- 1 . 1 8 0 2 4 1}\end{array}$

$\begin{array}{llll}\text { C } & -2.308041 & 1.935373 & 0.172469\end{array}$

$\begin{array}{llll}\text { C } & \mathbf{- 3 . 8 1 1 1 1 8} & \mathbf{2 . 1 9 0 3 5 9} & \mathbf{- 0 . 0 0 8 6 7 8}\end{array}$

$\begin{array}{llll}\text { H } & -2.064811 & 1.151327 & 0.896155\end{array}$

$\begin{array}{llll}C & -4.384580 & 3.582246 & -0.115984\end{array}$

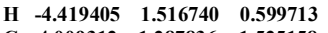

$\begin{array}{llll}C & 4.009312 & 1.287836 & -1.525158\end{array}$

$\begin{array}{llll}\text { C } & \mathbf{5 . 1 1 2 8 4 1} & \mathbf{0 . 6 3 5 0 4 4} & \mathbf{- 2 . 0 6 1 0 9 8}\end{array}$

$\begin{array}{lllll}\text { H } & \mathbf{6 . 0 9 0 7 4 0} & \mathbf{1 . 1 2 2 2 8 4} & \mathbf{- 2 . 0 5 2 8 4 3}\end{array}$

$\begin{array}{llll}\text { C } & 4.971582 & -\mathbf{0 . 6 5 0 4 0 5} & \mathbf{- 2 . 5 9 8 8 8 2}\end{array}$

H $5.839075-1.171176-3.011009$

$\begin{array}{llll}\text { C } & \mathbf{3 . 7 2 4 6 1 8} & \mathbf{- 1 . 2 6 4 6 5 3} & \mathbf{- 2 . 6 1 2 3 6 4}\end{array}$

$\begin{array}{llll}\text { H } & 3.607097 & \mathbf{- 2 . 2 6 7 2 7 4} & \mathbf{- 3 . 0 3 2 8 3 7}\end{array}$

$\begin{array}{lllll}\text { H } & 4.116701 & 2.281061 & -1.080574\end{array}$

$\begin{array}{llll}H & 6.910215 & 0.006350 & 1.634551\end{array}$

H $6.91021541 .535424 \quad 2.316086$

\begin{tabular}{ccccc}
\hline H & 6.290424 & $\mathbf{1 . 5 3 5 4 2 4}$ & $\mathbf{2 . 3 1 6 0 8 6}$ \\
\hline & 6.301082 & $\mathbf{1 . 2 7 6 5 8 8}$ & $\mathbf{0 . 5 5 7 1 5 3}$
\end{tabular}

$\begin{array}{llll}\text { H } & 6.301082 & 1.276588 & \mathbf{0 . 5 5 7 1 5 3}\end{array}$

$\begin{array}{llll}H & -6.720950 & -0.608930 & -1.271669\end{array}$

$\begin{array}{llll}\text { H } & -7.331880 & -1.694828 & -0.010473\end{array}$

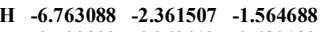

$\begin{array}{llll}\text { H } & -3.733833 & 4.260412 & -0.680183\end{array}$

$\begin{array}{llll}\text { H } & -4.537576 & 4.014526 & 0.884115\end{array}$

$\begin{array}{lllll}\text { H } & -5.362689 & 3.540318 & -0.618597\end{array}$

$\begin{array}{llll}\text { C } & -2.813947 & 2.189425 & -2.450072\end{array}$

$\begin{array}{llll}\text { H } & 1.140252 & -2.602163 & 3.378597\end{array}$

\begin{tabular}{llll} 
H & 1.140252 & -2.602163 & 3.378597 \\
\hline & -1.552540 & -3.173547 & 2.960258
\end{tabular}

\begin{tabular}{lllll}
\hline & -1.552540 & -3.173547 & 2.960258
\end{tabular}

$\begin{array}{llll}O & -1.333668 & 4.050502 & -0.479023\end{array}$

$\begin{array}{llll}\text { C } & -1.388655 & 3.101502 & 0.269083\end{array}$

$\begin{array}{lllll}\text { O } & -\mathbf{0 . 6 2 5 4 2 8} & \mathbf{2 . 9 8 9 7 3 1} & \mathbf{1 . 3 5 7 0 2 4}\end{array}$

$\begin{array}{lllll}\text { C } & 0.338433 & 4.006994 & 1.580487\end{array}$

$\begin{array}{lllll}\text { H } & -0.142121 & 4.993011 & 1.651128\end{array}$

\begin{tabular}{lllll}
\hline & $\mathbf{0 . 8 3 1 7 4 0}$ & $\mathbf{3 . 7 5 4 0 7 5}$ & $\mathbf{2 . 5 2 4 1 3 8}$
\end{tabular}

$\begin{array}{lllll}\text { H } & \mathbf{1 . 0 7 5 7 6 9} & \mathbf{4 . 0 1 5 6 1 7} & \mathbf{0 . 7 6 5 6 7 2}\end{array}$

$\begin{array}{llll}H & 1.971910 & 1.704649 & -2.966000\end{array}$

\begin{tabular}{llll}
\hline & -1.971910 & 1.704649 & -2.966000 \\
\hline & -2.573102 & 3.253385 & -2.351479
\end{tabular}

\begin{tabular}{llll} 
H & -2.573102 & 3.253385 & -2.351479 \\
\hline & -3.707995 & 2.080356 & -3.082450
\end{tabular}

$\begin{array}{llll}-3.5707995 & 2.080356 & -3.082450\end{array}$

66

unsat_model_prod_CP_a / electronic energy: -2247.03640565 a.u. / lowest freq: $22.80 \mathrm{~cm}-1$

$\begin{array}{lllll}\text { Ru } & \mathbf{0 . 1 1 4 3 8 7} & \mathbf{0 . 2 3 3 1 2 3} & \mathbf{1 . 0 1 1 1 0 5}\end{array}$

S $\quad \begin{array}{llll}0.801214 & -1.191992 & 2.536177\end{array}$

$\begin{array}{llll}\text { C } & -2.415560 & -0.569039 & 2.814839\end{array}$

$\begin{array}{llll}\text { C } & -2.799465 & 0.608072 & 2.291780\end{array}$

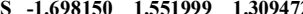

C $-0.168949-0.883326-0.549151$

C $-0.1689293-0.883326-0.549151$

$-1.283293-1.220284-1.264166$

N $0.819785-1.643503-1.126501$

$\begin{array}{llll}C & \mathbf{- 0 . 9 8 7 8 5 6} & \mathbf{- 2 . 1 4 8 4 5 3} & \mathbf{- 2 . 2 5 6 9 4 7}\end{array}$

$\begin{array}{llll}C & 0.331491 & -2.416329 & -2.171051\end{array}$

C $-\mathbf{2 . 5 8 4 4 2 3}-\mathbf{- 0 . 6 6 7 5 0 4}-1.106111$

$\begin{array}{llll}C & -2.856744 & 0.579163 & -1.661061\end{array}$

$\begin{array}{lllll}\text { C } & \mathbf{- 3 . 6 0 1 2 0 8} & \mathbf{- 1 . 3 7 4 7 8 9} & \mathbf{- 0 . 4 5 3 3 9 1}\end{array}$

$\begin{array}{llll}C & -4.108350 & 1.163026 & -1.572544\end{array}$

$\begin{array}{llll}C & -4.864391 & -0.783518 & -0.374103\end{array}$

C $-5.134392 \quad 0.478232-0.915299$

$\begin{array}{llll}C & -5.134392 & 0.478232 & -0.915299\end{array}$

$\begin{array}{llll}\text { H } & -4.266078 & 2.149136 & -2.012749\end{array}$ 


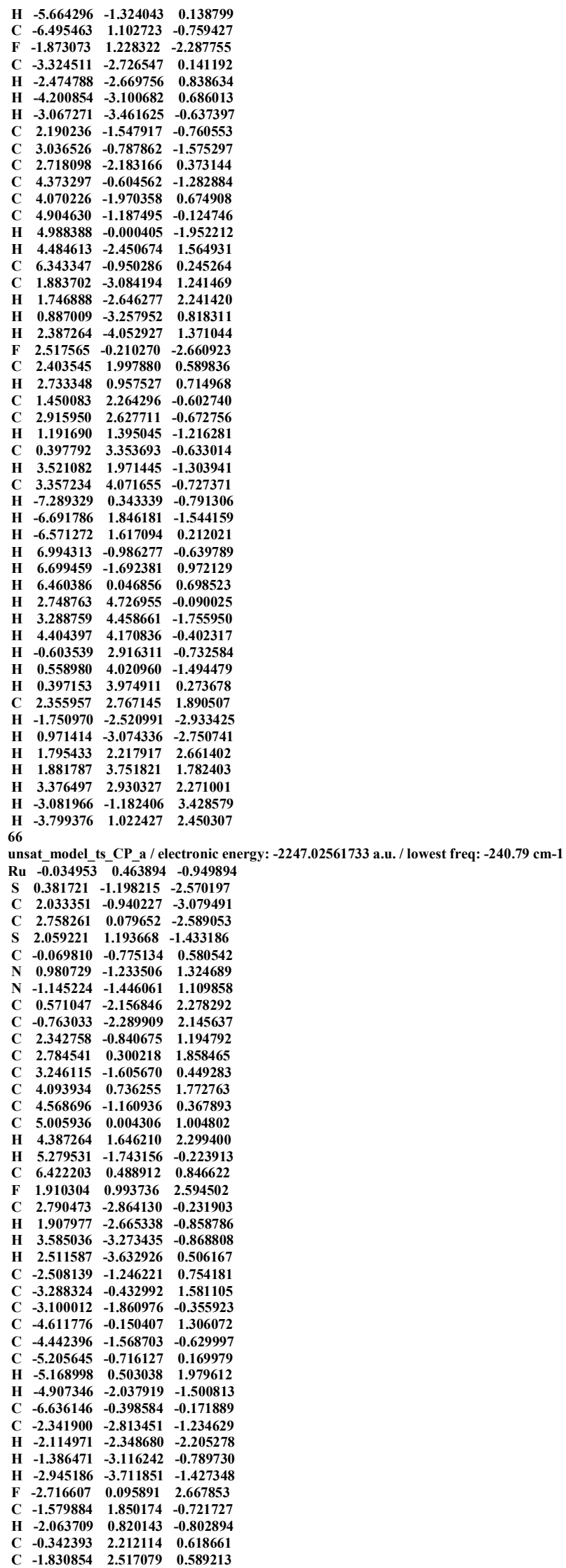




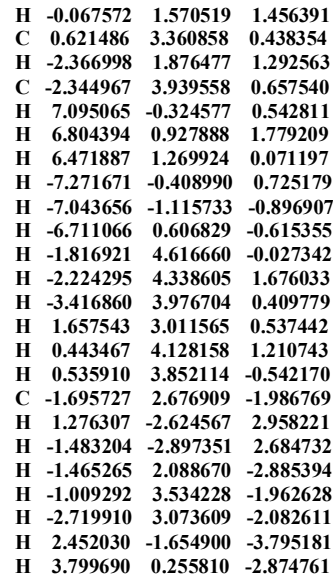

66

unsat model_prod_BHE_a / electronic energy: - 2247.07148971 a.u. / lowest freq: $32.20 \mathrm{~cm}-1$

$S-1.073295-0.154624-2.550443$

$\begin{array}{llll}C & -0.031998 & -0.413480 & -0.721589\end{array}$

$\begin{array}{llll}\text { Ru } & -0.096877 & 0.939301 & 0.717183\end{array}$

$\begin{array}{llll}\text { C } & -\mathbf{1 . 6 7 1 4 9 3} & \mathbf{2 . 4 0 6 1 2 0} & \mathbf{- 0 . 3 3 1 4 1 9}\end{array}$

$\begin{array}{lllll}C & 0.863494 & 2.728216 & -0.137687\end{array}$

$\begin{array}{lllll}\text { S } & 1.920307 & 0.534759 & 1.783219\end{array}$

$\begin{array}{lllll}N & 1.045864 & -0.895040 & -1.425461\end{array}$

N $-1.101250 \quad-1.017541 \quad-1.343081$

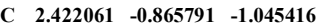

C $0.645736-1.730732-2.461978$

$\begin{array}{llll}\text { C } & -0.696932 & -1.809035 & -2.410770\end{array}$

$\begin{array}{llll}C & -2.448269 & -1.047139 & -0.876993\end{array}$

C $\quad \mathbf{5 . 1 4 8 1 6 5} \quad \mathbf{- 0 . 8 8 2 6 2 0}-\mathbf{- 0 . 3 5 7 7 8 5}$

$\begin{array}{llll}C & 3.300491 & 0.080399 & -1.555787\end{array}$

$\begin{array}{llll}C & 2.916074 & -1.868354 & -0.194022\end{array}$

$\begin{array}{llll}C & 4.271806 & -1.860918 & 0.127911\end{array}$

$\begin{array}{llll}C & 4.645271 & 0.095647 & -1.216113\end{array}$

$\begin{array}{lllll}\text { H } & 4.655892 & -2.634789 & 0.797479\end{array}$

$\begin{array}{llll}\text { H } & \mathbf{5 . 2 8 5 3 5 3} & \mathbf{0 . 8 7 2 2 7 3} & -1.638605\end{array}$

$\begin{array}{llll}C & -5.119731 & -1.098585 & -0.006020\end{array}$

C $\quad \mathbf{- 3 . 4 1 9 9 4 1}-\mathbf{- 0 . 2 4 5 0 1 2}-\mathbf{- 1 . 4 6 9 0 1 6}$

$\begin{array}{llll}C & -2.832740 & -1.970531 & 0.108248\end{array}$

$\begin{array}{llll}C & -4.162146 & -1.963783 & 0.535666\end{array}$

$\begin{array}{llll}C & -4.736802 & -0.240583 & -1.040959\end{array}$

$\begin{array}{llll}\text { F } & -3.073614 & 0.542270 & -2.494823\end{array}$

$\begin{array}{llll}\text { C } & -1.843443 & -2.969637 & 0.636528\end{array}$

$\begin{array}{llll}\mathrm{H} & -4.463500 & -2.669596 & \mathbf{1 . 3 1 3 4 1 3}\end{array}$

$\begin{array}{lll}-5.450416 & 0.424452 & -1.530503\end{array}$

\begin{tabular}{lll}
-5.450416 & 0.424152 & -1.530503 \\
\hline
\end{tabular}

$\begin{array}{llll}C & -6.531686 & -1.084936 & 0.513875\end{array}$

$\begin{array}{lllll}\text { H } & -\mathbf{7 . 2 5 2 1 8 3} & -\mathbf{0 . 8 5 3 2 3 9} & \mathbf{- 0 . 2 8 2 7 7 0}\end{array}$

$\begin{array}{llll}\text { H } & -1.488060 & -3.626170 & -0.173717\end{array}$

$\begin{array}{llll}\text { H } & -6.642271 & -0.314904 & 1.294018\end{array}$

$\begin{array}{llll}\text { H } & -6.802612 & -2.050589 & 0.961968\end{array}$

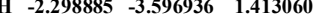

$\begin{array}{llll}H & -0.971906 & -2.462490 & 1.070921\end{array}$

$\begin{array}{llll}\text { F } & \mathbf{2 . 8 5 2 6 5 9} & \mathbf{1 . 0 0 9 4 9 0} & \mathbf{- 2 . 4 0 6 8 1 9}\end{array}$

$\begin{array}{llll}\text { C } \quad 6.593878 & -0.870122 & 0.059975\end{array}$

$\begin{array}{llll}\text { C } & 6.593878 & -0.870122 & 0.059975\end{array}$

\begin{tabular}{ll}
0.154531 \\
\hline
\end{tabular}

$\begin{array}{llll}\text { H } & \mathbf{7 . 2 1 7 1 1 5} & \mathbf{- 0 . 3 1 6 4 2 2} & \mathbf{- 0 . 6 5 5 3 4 7}\end{array}$

$\begin{array}{llll}\text { H } & 6.701913 & -0.383149 & 1.042270\end{array}$

$\begin{array}{llll}\text { C } & 1.978932 & -2.879332 & 0.400987\end{array}$

$\begin{array}{llll}\text { H } & 1.323064 & -3.332355 & -0.357041\end{array}$

$\begin{array}{llll}\text { H } & 2.532923 & -3.681088 & 0.905751\end{array}$

$\begin{array}{lllll}\text { H } & 1.336884 & \mathbf{- 2 . 3 8 3 8 0 6} & \mathbf{1 . 1 4 7 0 3 4}\end{array}$

$\begin{array}{llll}C & 0.269333 & -0.841095 & 3.434808\end{array}$

$\begin{array}{llll}C & 1.541011 & -0.539681 & 3.106479\end{array}$

$\begin{array}{llll}C & 1.54011 & -0.539681 & 3.106479\end{array}$

$\begin{array}{llll}\text { H } & -2.362468 & 2.183550 & -1.153056\end{array}$

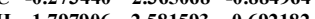

$\begin{array}{llll}\text { H } & \mathbf{1 . 7 9 7 9 0 6} & 2.581593 & \mathbf{- 0 . 6 9 2 1 8 2}\end{array}$

$\begin{array}{llll}\text { C } & 0.993946 & 3.782123 & 0.943771\end{array}$

$\begin{array}{llll}C & -0.158733 & 2.069443 & -2.361548\end{array}$

$\begin{array}{llll}\text { H } & \mathbf{- 0 . 3 9 8 6 1 8} & \mathbf{2 . 9 8 9 0 8 2} & \mathbf{- 2 . 9 2 1 4 3 7}\end{array}$

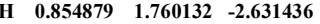

$\begin{array}{llll}\text { H } & -0.866518 & 1.296742 & -2.686124\end{array}$

$\begin{array}{lllll}\text { H } & -1.867877 & 1.452972 & 0.337007\end{array}$

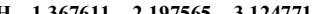

\begin{tabular}{llll}
\hline & 1.367611 & -2.197565 & -3.124771 \\
\hline
\end{tabular}

$\begin{array}{llll}H & -1.410121 & -2.353191 & -3.022072\end{array}$

$\begin{array}{llll}\text { C } & -2.199435 & 3.605851 & \mathbf{0 . 4 5 1 8 0 3}\end{array}$

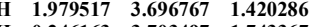

$\begin{array}{lllll}\text { H } & 0.246163 & 3.703497 & 1.743367\end{array}$

\begin{tabular}{lllll}
\hline & 0.909249 & 4.791613 & 0.505295
\end{tabular}

$\begin{array}{llll}\text { H } & -1.728603 & 3.715449 & 1.434688\end{array}$

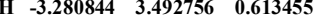

$\begin{array}{llll}\text { H } & -2.032862 & \mathbf{4 . 5 3 3 5 0 7} & -0.114906\end{array}$

$\begin{array}{llll}\text { H } & \mathbf{2 . 3 9 0 5 2 5} & -\mathbf{0 . 9 4 6 7 3 9} & \mathbf{3 . 6 6 4 7 9 3}\end{array}$

$0.032781-1.499271-4.276644$

66 
unsat_model_ts_BHE_a / electronic energy: $-\mathbf{2 2 4 7 . 0 1 4 1 2 9 9 6}$ a.u. / lowest freq: $-\mathbf{7 3 . 8 7} \mathbf{c m - 1}$ $\begin{array}{llll}S & -0.115016 & 1.265225 & 2.488854\end{array}$

$\begin{array}{llll}C & -0.214068 & -1.030461 & 0.023937\end{array}$

$\begin{array}{lllll}\text { Ru } & 0.143703 & 1.000753 & 0.122487\end{array}$

$\begin{array}{llll}\text { C } & -\mathbf{0 . 7 8 0 1 7 3} & \mathbf{2 . 7 8 7 0 4 7} & \mathbf{- 0 . 5 6 0 4 8 4}\end{array}$

$\begin{array}{llll}C & 0.086561 & 1.024968 & -2.021605\end{array}$

$\begin{array}{llll}\text { S } & \mathbf{2 . 2 3 3 9 1 3} & \mathbf{1 . 7 0 0 5 6 2} & \mathbf{0 . 4 4 6 2 1 2}\end{array}$

$\begin{array}{llll}\mathrm{N} & \mathbf{0 . 7 8 3 6 1 8} & -\mathbf{1 . 9 5 3 2 3 0} & \mathbf{- 0 . 0 6 5 0 0 9}\end{array}$

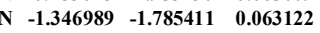

C $2.174749-1.668072-0.171324$

$\begin{array}{llll}C & 0.284869 & -3.247091 & -0.076576\end{array}$

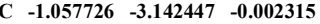

$\begin{array}{llll}\text { C } & -2.687614 & -1.309688 & 0.136786\end{array}$

$\begin{array}{llll}C & 4.893338 & -1.082037 & \mathbf{- 0 . 4 7 5 0 5 5}\end{array}$

$\begin{array}{llll}C & 2.726734 & -1.469804 & -1.433180\end{array}$

$\begin{array}{llll}C & 2.984139 & -1.596214 & 0.967298\end{array}$

$\begin{array}{llll}\text { C } & 4.339441 & -1.308981 & 0.789177\end{array}$

$\begin{array}{llll}C & 4.066982 & -1.168859 & -1.600915\end{array}$

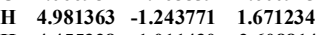

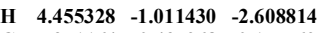

$\begin{array}{llll}C & -5.351164 & -0.437968 & 0.157769\end{array}$

$\begin{array}{llll}\text { C } & -3.417781 & -1.193329 & -1.042903\end{array}$

$\begin{array}{llll}\text { C } & \mathbf{- 3 . 3 0 9 6 6 9} & \mathbf{- 1 . 0 5 5 9 7 4} & \mathbf{1 . 3 6 6 8 1 2}\end{array}$

$\begin{array}{llll}\text { C } & -4.631996 & -0.604906 & 1.347639\end{array}$

$\begin{array}{llll}\text { C } & -4.731329 & -0.756646 & -1.054137\end{array}$

$\begin{array}{llll}\text { F } & -2.818151 & -1.510954 & -2.193629\end{array}$

$\begin{array}{llll}C & -2.585439 & -1.322355 & 2.654787\end{array}$

$\begin{array}{llll}\text { H } & \mathbf{- 5 . 1 2 4 0 2 9} & \mathbf{- 0 . 3 9 1 7 7 5} & \mathbf{2 . 2 9 9 7 3 6}\end{array}$

H $\quad \begin{array}{llll}-5.253641 & -\mathbf{0 . 6 6 6 7 0 7} & \mathbf{- 2 . 0 0 8 1 2 7}\end{array}$

$\begin{array}{llll}\text { C } & -6.761391 & 0.087012 & 0.174900\end{array}$

$\begin{array}{llll}\text { H } & -7.372936 & -0.380827 & -0.609181\end{array}$

$\begin{array}{llll}\text { H } & -2.395865 & -\mathbf{2 . 4 0 1 4 2 8} & \mathbf{2 . 7 7 0 4 2 5}\end{array}$

$\begin{array}{llll}\text { H } & -6.766186 & 1.173513 & -0.007238\end{array}$

$\begin{array}{llll}H & -7.743187 & -0.088508 & 1.146268\end{array}$

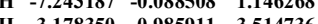

$\begin{array}{llll}\text { H } & -3.178350 & -0.985911 & 3.514736\end{array}$

$\begin{array}{llll}\text { H } & -1.612720 & -0.812214 & 2.684479\end{array}$

$\begin{array}{llll}\text { F } & \mathbf{1 . 9 3 6 2 3 6} & -1.575769 & \mathbf{- 2 . 5 0 2 0 0 5} \\ \text { C } & 6.345546 & -0.718524 & 0.63006\end{array}$

$\begin{array}{llll}C & 6.345546 & -0.718524 & -0.630066\end{array}$

H $6.931502 \quad-1.019153 \quad 0.249148$

H $6.784484-1.190471-1.520512$

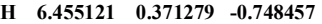

$\begin{array}{llll}\text { C } & \mathbf{2 . 3 8 2 2 0 4} & \mathbf{- 1 . 7 7 7 7 1 2} & \mathbf{2 . 3 3 0 9 1 9}\end{array}$

$\begin{array}{lllll}\text { H } & \mathbf{1 . 8 7 2 1 3 8} & \mathbf{- 2 . 7 4 9 1 2 3} & \mathbf{2 . 4 2 0 5 3 9}\end{array}$

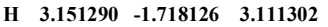

$\begin{array}{llll}\text { H } & \mathbf{1 . 6 3 1 2 4 9} & \mathbf{- 0 . 9 9 3 3 8 8} & \mathbf{2 . 5 1 8 9 4 8}\end{array}$

$\begin{array}{llll}C & 1.412406 & \mathbf{1 . 8 6 5 6 6 2} & \mathbf{2 . 9 9 7 4 1 3}\end{array}$

$\begin{array}{llll}\text { C } & \mathbf{2 . 4 3 4 8 0 1} & \mathbf{2 . 0 6 9 8 8 9} & \mathbf{2 . 1 2 0 9 2 0}\end{array}$

$\begin{array}{llll}\text { H } & -1.628637 & 3.050256 & 0.087982\end{array}$

$\begin{array}{llll}\text { C } & -1.128867 & 1.699198 & -1.491304\end{array}$

$\begin{array}{lllll}\text { H } & -0.196446 & 0.066253 & -2.481437\end{array}$

$\begin{array}{llll}C & 1.160659 & 1.681830 & -2.868862\end{array}$

$\begin{array}{llll}C & -2.469163 & 1.694983 & -2.215399\end{array}$

$\begin{array}{llll}C & -2.4691632 & 1.694983 & -2.215399\end{array}$

$\begin{array}{llll}\mathrm{H} & -2.427032 & 2.478888 & -2.987570\end{array}$

\begin{tabular}{llll}
\hline & -2.652082 & 0.736771 & -2.715687
\end{tabular}

$\begin{array}{llll}H & -3.307213 & 1.920946 & -1.539287\end{array}$

$\begin{array}{lllll}\text { H } & -1.485029 & 0.882024 & -0.527681\end{array}$

$\begin{array}{lllll}\text { H } & 0.938353 & -4.111535 & -0.141065\end{array}$

$\begin{array}{llll}\text { H } & \mathbf{- 1 . 8 4 0 1 0 4} & \mathbf{- 3 . 8 9 4 6 7 4} & \mathbf{0 . 0 0 8 4 0 8}\end{array}$

$\begin{array}{lllll}C & 0.007606 & 4.029133 & -0.906274\end{array}$

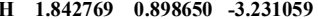

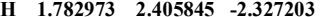

$\begin{array}{lllll}\text { H } & 0.746903 & 2.190008 & -3.758940\end{array}$

\begin{tabular}{lllll} 
H & $\mathbf{0 . 9 0 8 3 9 9}$ & $\mathbf{3 . 8 4 4 4 0 5}$ & -1.495952 \\
\hline & 0.306755 & 4.550780 & 0.014115
\end{tabular}

\begin{tabular}{lllll}
\hline & 0.306755 & 4.550780 & 0.014115 \\
\hline
\end{tabular}

$\begin{array}{lrrrr}\text { H } & \mathbf{- 0 . 6 3 9 1 0 6} & \mathbf{4 . 7 1 7 4 7 5} & \mathbf{- 1 . 4 8 0 4 5 9}\end{array}$

\begin{tabular}{llll}
\hline & 3.401485 & 2.466413 & $\mathbf{2 . 4 4 3 9 2 0}$
\end{tabular}

$\begin{array}{llll}H & 1.546066 & 2.078887 & 4.063122\end{array}$

66

unsat_model_mcb_dist a / electronic energy: $-\mathbf{2 2 4 7 . 0 3 9 9 6 1 9 8}$ a.u. / lowest freq: $22.55 \mathrm{~cm}-1$ Ru $\quad-\mathbf{0 . 2 0 9 4 1 6} \quad 0.875714 \quad-0.034655$

$\begin{array}{lllll}S & -0.082712 & 2.077585 & -2.107973\end{array}$

C $2.2323432 .727997-0.880$

C $1.722345-1.779819-0.49211$

71557

N $1.149670-1.900651-0.217244$

N $\quad-0.993642 \quad-2.009462-0.308070$

$\begin{array}{lllll}C & 0.817304 & -3.243478 & -0.369658\end{array}$

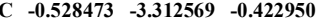

$\begin{array}{lrrr}C & 2.499800 & -1.449022 & -0.159370\end{array}$

$\begin{array}{llll}\text { C } & 3.215046 & -1.604269 & \mathbf{1 . 0 2 4 9 2 4}\end{array}$

C $3.123811-0.890246-1.281303$

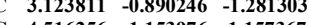

$\begin{array}{llll}C & 4.516256 & -1.153976 & 1.157367\end{array}$

$\begin{array}{llll}\text { C } & 4.439857 & \mathbf{- 0 . 4 4 2 6 0 8} & -1.142063\end{array}$

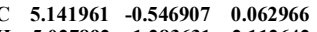

$\begin{array}{llll}\text { H } & 5.027892 & -1.283631 & 2.112642\end{array}$

$\begin{array}{lllll}\text { H } & 4.931688 & 0.008030 & -2.007638\end{array}$

$\begin{array}{llll}\text { C } & 6.538775 & -0.002154 & 0.194273\end{array}$

$\begin{array}{llll}\text { F } & \mathbf{2 . 6 2 0 4 7 9} & \mathbf{- 2 . 2 0 9 1 0 7} & \mathbf{2 . 0 5 7 0 1 5}\end{array}$

$\begin{array}{llll}\text { C } \quad \mathbf{2 . 3 8 2 8 7 0} & -\mathbf{0 . 7 5 9 0 7 1} & \mathbf{- 2 . 5 7 9 8 8 3}\end{array}$

$\begin{array}{llll}\mathrm{H} & 1.613218 & 0.025979 & -2.505386\end{array}$

H $3.067975-0.494272-3.395139$

\begin{tabular}{llll}
\hline & 3.067975 & -0.494272 & $\mathbf{3 . 3 9 5 1 3 9}$
\end{tabular}

H $1.869691 \quad-1.696197 \quad-2.843946$ 


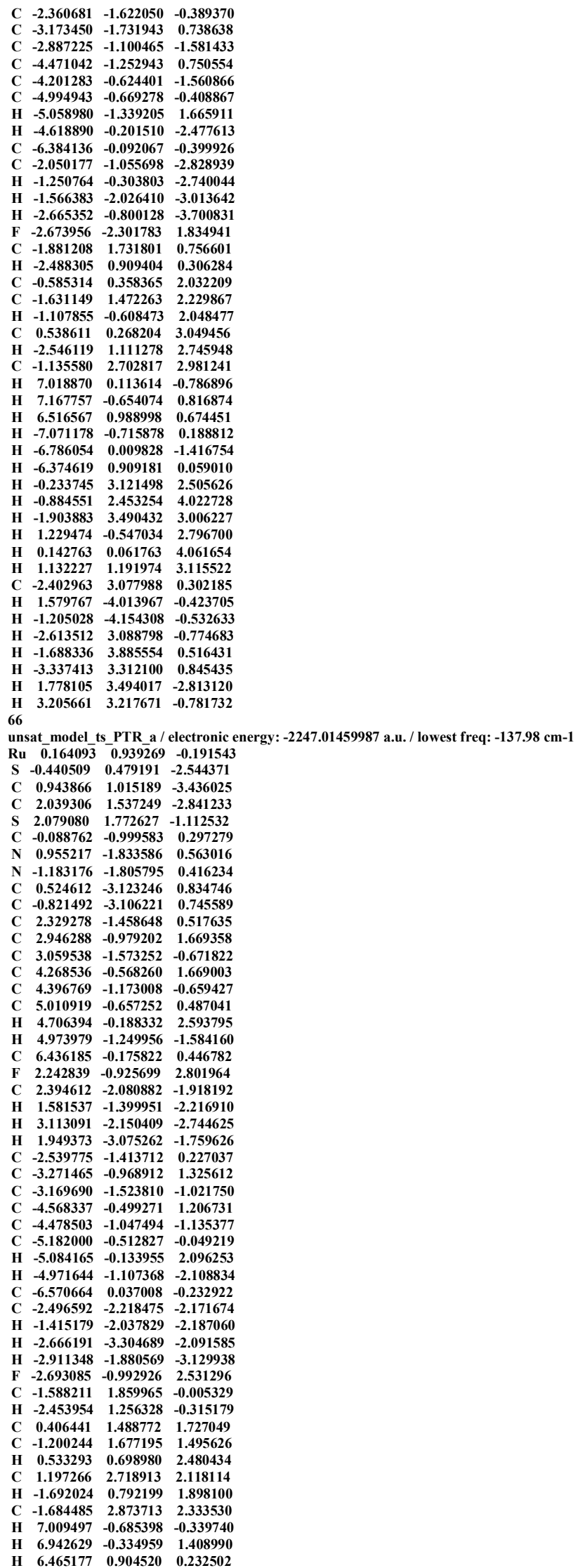




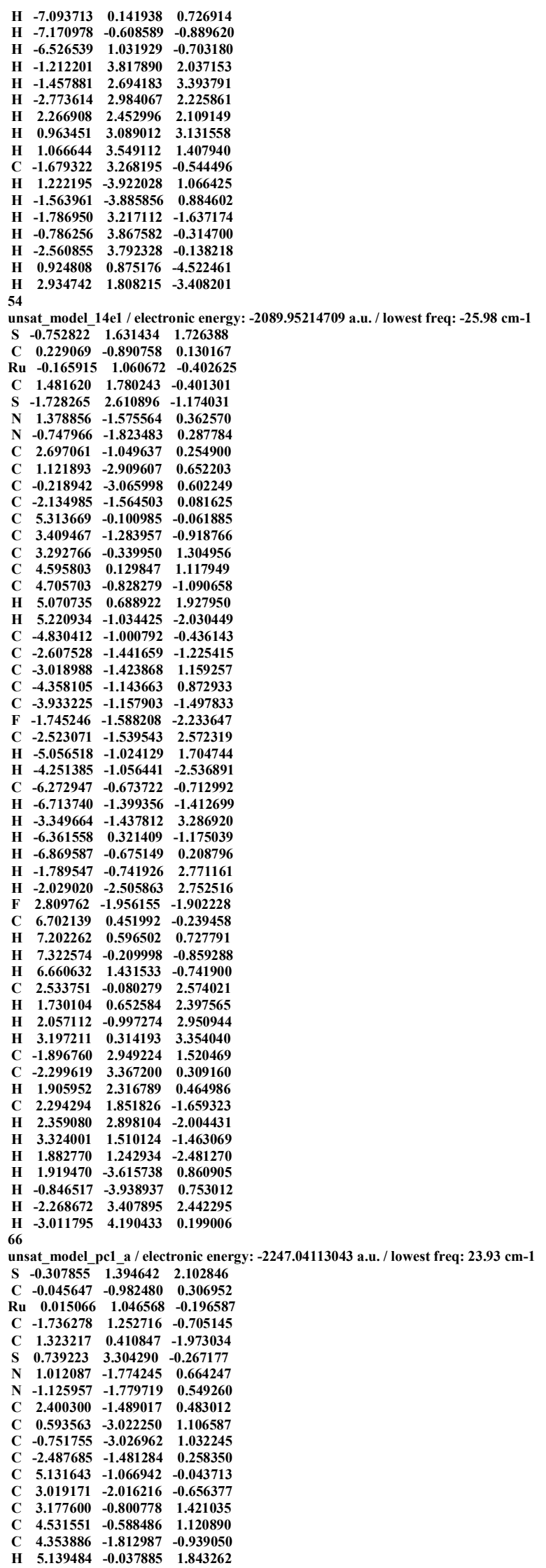


$\begin{array}{llll}4.774022 & -2.233018 & -1.854497\end{array}$

$\begin{array}{llll}C & -5.167290 & -1.014942 & -0.435419\end{array}$

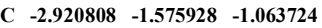

$\begin{array}{llll}\text { C } & \mathbf{- 3 . 4 1 0 4 0 1} & \mathbf{- 1 . 1 7 9 7 4 7} & \mathbf{1 . 2 6 8 2 9 4}\end{array}$

$\begin{array}{llll}C & -4.739035 & -0.950710 & 0.894650\end{array}$

C $-4.734077-1.337517-1.426939$

$\begin{array}{llll}C & -4.234077 & -1.337517 & -1.426939\end{array}$

$\begin{array}{llll}\text { F } & -2.026368 & -1.884438 & -2.005648\end{array}$

$\begin{array}{llll}\text { C } & \mathbf{- 2 . 9 7 6 6 2 0} & -1.086907 & \mathbf{2 . 7 0 2 9 0 6}\end{array}$

H $\quad-\mathbf{5 . 4 6 5 0 5 3} \quad-\mathbf{- 0 . 7 0 7 8 0 0} \quad \mathbf{1 . 6 7 4 1 5 5}$

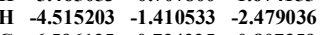

$\begin{array}{llll}C & -6.596125 & -0.724225 & -0.807359\end{array}$

$\begin{array}{llll}\text { H } & -6.972360 & -1.453537 & -1.539118\end{array}$

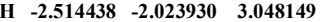

$\begin{array}{llll}\text { H } & -6.678596 & 0.273000 & -1.267620\end{array}$

\begin{tabular}{llll}
\hline & -7.252832 & -0.743192 & 0.072703
\end{tabular}

\begin{tabular}{llll}
\hline & -3.831470 & -0.866293 & 3.354745
\end{tabular}

H $-2.228248-0.287129 \quad 2.820374$

$\begin{array}{llll}\text { F } & \mathbf{2 . 2 7 2 6 3 4} & \mathbf{- 2 . 7 2 9 5 6 1} & -\mathbf{1 . 5 0 6 3 6 2}\end{array}$

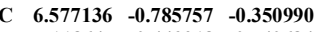

$\begin{array}{llll}\text { H } & 7.118647 & \mathbf{- 0 . 4 4 2 9 1 8} & \mathbf{0 . 5 4 0 6 8 4}\end{array}$

H $7.081726-1.679080-0.745909$

$\begin{array}{lllll}\text { H } & 6.657065 & 0.001614 & -1.117072\end{array}$

$\begin{array}{llll}\text { C } & \mathbf{2 . 6 2 7 7 9 1} & \mathbf{- 0 . 3 2 7 4 4 3} & \mathbf{2 . 7 3 5 4 7 3}\end{array}$

H $\quad \begin{array}{llll}1.654534 & -0.778088 & 2.962022\end{array}$

H $3.333624 \quad-0.570563 \quad 3.542276$

$\begin{array}{llll}\text { H } & 2.476591 & 0.761029 & 2.724692\end{array}$

$\begin{array}{lrrr}\text { C } & \mathbf{- 0 . 0 5 9 0 0 8} & \mathbf{3 . 1 1 1 2 0 1} & \mathbf{2 . 3 3 0 2 2 7}\end{array}$

$\begin{array}{lllll}\text { C } & \mathbf{0 . 3 7 4 6 3 7} & \mathbf{3 . 9 0 6 8 4 2} & \mathbf{1 . 3 3 5 4 0 0}\end{array}$

$\begin{array}{llll}C & -2.752098 & 2.139556 & -0.068227\end{array}$

$\begin{array}{llll}\text { H } & -2.149251 & 0.808376 & -1.627994\end{array}$

$\begin{array}{llll}\text { C } & 0.137638 & 0.866909 & -2.517815\end{array}$

$\begin{array}{llll}\text { H } & \mathbf{1 . 3 8 4 3 4 2} & \mathbf{- 0 . 6 7 0 0 4 3} & \mathbf{- 1 . 8 5 6 3 5 8}\end{array}$

$\begin{array}{llll}\text { C } & \mathbf{2 . 6 5 9 8 1 3} & \mathbf{1 . 1 0 2 8 7 9} & \mathbf{- 2 . 0 3 0 9 8 6}\end{array}$

$\begin{array}{llll}\text { H } & 3.236745 & 0.934516 & -1.109678\end{array}$

$\begin{array}{lllll}\text { H } & 3.246973 & 0.679356 & -2.864043\end{array}$

$\begin{array}{llll}\text { H } & \mathbf{2 . 5 7 0 2 9 7} & \mathbf{2 . 1 8 5 5 0 6} & \mathbf{- 2 . 1 8 1 4 6 3}\end{array}$

$\begin{array}{llll}\text { H } & -\mathbf{3 . 6 6 2 6 2 7} & \mathbf{1 . 5 5 0 9 4 5} & \mathbf{0 . 1 4 4 7 0 8}\end{array}$

$\begin{array}{llll}\text { H } & -3.043702 & 2.900982 & -0.814939\end{array}$

$\begin{array}{llll}\text { H } & -2.409511 & 2.642753 & 0.842309\end{array}$

$\begin{array}{llll}\text { H } & \mathbf{- 0 . 6 0 8 3 4 1} & \mathbf{0 . 0 9 4 8 1 0} & \mathbf{- 2 . 7 2 7 4 6 2}\end{array}$

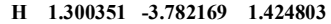

$\begin{array}{llll}\text { H } & -1.482909 & -3.794581 & 1.266067\end{array}$

$\begin{array}{llll}\text { C } & -\mathbf{0 . 0 4 7 6 8 8} & \mathbf{2} .136707 & \mathbf{- 3 . 3 0 1 6 8 5}\end{array}$

H $0.096763 \quad 1.926641-4.375083$

\begin{tabular}{lllll}
$\mathrm{H}$ & $\mathbf{0 . 0 9 6 7 6 3}$ & 1.926641 & -4.375083 \\
\hline & -1.065747 & 2.535808 & -3.179218
\end{tabular}

H $\quad-1.065747 \quad 2.535808-3.179218$

$\begin{array}{lllll}\text { H } & \mathbf{0 . 6 5 8 2 3 4} & 2.923967 & \mathbf{- 3 . 0 1 0 1 5 0}\end{array}$

$\begin{array}{lrrr}\text { H } & -\mathbf{0 . 2 6 4 2 3 4} & 3.512588 & 3.328100 \\ \text { H } & 0.530438 & 4.978259 & 1.499912\end{array}$

66

unsat_model_ts1_a / electronic energy: $-\mathbf{2 2 4 7 . 0 3 2 0 6 4 8 2}$ a.u. / lowest freq: $-\mathbf{1 3 1 . 0 3} \mathbf{c m}-1$

$\begin{array}{lllll}S & -\mathbf{0 . 5 0 8 6 4 6} & \mathbf{1 . 0 6 1 9 2 7} & \mathbf{- 2 . 1 8 0 6 3 4}\end{array}$

$\begin{array}{llll}C & \mathbf{- 0 . 0 5 4 6 3 6} & \mathbf{- 1 . 0 2 0 2 7 5} & \mathbf{- 0 . 0 7 3 8 9 0}\end{array}$

$\begin{array}{llll}\text { Ru } & 0.053658 & 1.065614 & 0.070186\end{array}$

$\begin{array}{llll}\text { C } & 1.455736 & 0.817133 & 1.703707\end{array}$

$\begin{array}{llll}C & -1.484081 & 1.164429 & \mathbf{1 . 0 9 8 5 4 4}\end{array}$

$\begin{array}{lllll}\mathrm{S} & \mathbf{0 . 6 1 5 8 1 8} & \mathbf{3 . 3 8 5 6 9 0} & \mathbf{- 0 . 2 8 3 9 5 1}\end{array}$

$\begin{array}{llll}\mathrm{N} & -1.135735 & -1.854564 & -0.196210\end{array}$

$\begin{array}{llll}N & \mathbf{1 . 0 0 0 7 7 3} & -\mathbf{1 . 8 7 3 3 5 3} & \mathbf{- 0 . 2 7 5 4 9 2}\end{array}$

$\begin{array}{llll}C & -2.516911 & -1.518344 & -0.076072\end{array}$

$\begin{array}{llll}C & -0.760775 & -3.165721 & -0.453703\end{array}$

$\begin{array}{lllll}C & 0.584971 & -3.177349 & -0.503625\end{array}$

$\begin{array}{llll}C & \mathbf{2 . 3 9 5 0 0 8} & \mathbf{- 1 . 5 6 5 0 2 0} & \mathbf{- 0 . 2 3 2 6 8 0}\end{array}$

$\begin{array}{llll}C & -5.238123 & -0.921602 & 0.292092\end{array}$

$\begin{array}{llll}C & -3.094157 & -1.502708 & 1.193561\end{array}$

$\begin{array}{llll}\text { C } & \mathbf{- 3 . 3 2 9 7 3 3} & -1.316195 & -\mathbf{1 . 1 9 9 2 3 9}\end{array}$

$\begin{array}{llll}C & -3.676900 & -1.001684 & -0.985346\end{array}$

$\begin{array}{llll}\text { C } & -4.426189 & -1.195644 & 1.398852\end{array}$

$\begin{array}{llll}\text { H } & -\mathbf{5 . 3 1 2 8 2 8} & -\mathbf{0 . 8 2 4 2 1 6} & -\mathbf{1 . 8 5 6 3 5 6}\end{array}$

$\begin{array}{llll}\text { H } & -4.816630 & -1.173823 & 2.417753\end{array}$

$\begin{array}{llll}\text { C } & 5.157101 & -1.090847 & -0.021337\end{array}$

$\begin{array}{llll}C & 3.094064 & -1.834974 & 0.944426\end{array}$

$\begin{array}{llll}C & 3.101294 & -1.129548 & -1.362118\end{array}$

$\begin{array}{llll}C & 4.472131 & -0.881018 & -1.221432\end{array}$

$\begin{array}{llll}\text { C } & 4.449077 & -1.595812 & 1.074661\end{array}$

$\begin{array}{llll}\text { F } & \mathbf{2 . 4 1 5 8 3 3} & \mathbf{- 2 . 3 3 0 2 4 7} & \mathbf{1 . 9 8 5 4 5 3}\end{array}$

$\begin{array}{llll}\text { C } & \mathbf{2 . 4 2 2 7 0 2} & \mathbf{- 0 . 9 8 6 3 3 5} & \mathbf{- 2 . 6 9 2 4 7 9}\end{array}$

$\begin{array}{llll}\text { H } & \mathbf{5 . 0 2 6 5 8 7} & \mathbf{- 0 . 5 2 6 0 3 3} & -\mathbf{2 . 0 9 3 5 6 7}\end{array}$

$\begin{array}{lllll}\text { H } & 4.936397 & -1.802997 & 2.028869\end{array}$

$\begin{array}{llll}\text { C } & 6.622410 & -0.773061 & 0.103163\end{array}$

\begin{tabular}{llll} 
H & 7.148426 & $\mathbf{- 1 . 5 3 4 4 3 5}$ & $\mathbf{0 . 6 9 6 4 2 4}$ \\
\hline
\end{tabular}

$\begin{array}{llll}\text { H } & 3.151489 & -0.745772 & -3.476797\end{array}$

$\begin{array}{lllll}\text { H } & 6.760632 & 0.194092 & 0.612038\end{array}$

$\begin{array}{llll}\text { H } & 7.103577 & \mathbf{- 0 . 7 0 2 9 1 0} & \mathbf{- 0 . 8 8 1 5 9 8}\end{array}$

$\begin{array}{llll}\text { H } & \mathbf{1 . 6 5 4 5 1 6} & \mathbf{- 0 . 1 9 9 1 5 8} & \mathbf{- 2 . 6 6 6 3 2 6}\end{array}$

$\begin{array}{llll}\text { H } & 1.910829 & -1.921289 & -2.969399\end{array}$

$\begin{array}{llll}\text { F } & -\mathbf{2 . 3 1 5 5 0 0} & \mathbf{- 1 . 7 6 9 2 2 3} & \mathbf{2 . 2 4 8 5 0 6}\end{array}$

$\begin{array}{llll}\text { C } & -6.680498 & -0.537619 & 0.483085\end{array}$

$\begin{array}{lllll}\text { H } & -6.768626 & 0.547698 & 0.650528\end{array}$

$\begin{array}{llll}\text { H } & -7.281636 & -0.785770 & -0.402272\end{array}$

$\begin{array}{llll}\text { H } & -7.117905 & -1.041630 & \mathbf{1 . 3 5 6 0 8 5}\end{array}$

$\begin{array}{llll}\text { C }-2.809161 & -1.517185 & -2.594786\end{array}$

C $-3.021162-2.548332-2.921340$

$\begin{array}{llll}\text { H } & -3.021162 & -2.548332 & -2.921340 \\ \text { H } & -3.304514 & -0.834673 & -3.297837\end{array}$ 
$\begin{array}{llll}\text { H } & -1.728514 & -1.349474 & -2.664304\end{array}$

$\begin{array}{llll}C & -0.325179 & 2.717595 & -2.739548\end{array}$

C $\quad \mathbf{0 . 1 4 2 2 6 2} \quad 3.687626-1.936739$

C $\quad \mathbf{2 . 7 8 7 6 4 0} \quad \mathbf{1 . 5 1 7 1 6 4} \quad \mathbf{1 . 5 5 7 4 4 8}$

$\begin{array}{lllll}\text { H } & 1.554842 & -0.249570 & 1.899997\end{array}$

\begin{tabular}{llll} 
C & $\mathbf{0 . 3 2 8 2 2 9}$ & $\mathbf{1 . 3 9 3 0 2 1}$ & $\mathbf{2 . 3 5 2 2 9 8}$ \\
\hline
\end{tabular}

$\begin{array}{llll}\text { H } & -\mathbf{1 . 8 2 2 3 3 4} & \mathbf{0 . 3 3 8 8 1 4} & \mathbf{1 . 7 3 4 3 4 1}\end{array}$

$\begin{array}{llll}\text { C } & -\mathbf{2 . 4 8 5 5 2 7} & \mathbf{2 . 2 6 9 0 7 7} & \mathbf{1 . 0 7 3 9 0 2}\end{array}$

$\begin{array}{llll}\text { H } & -3.364660 & \mathbf{1 . 8 6 8 4 6 9} & \mathbf{0 . 5 3 5 6 7 8}\end{array}$

$\begin{array}{llll}\text { H } & -2.831730 & 2.513756 & \mathbf{2 . 0 9 2 4 5 0}\end{array}$

$\begin{array}{llll}\text { H } & -2.130474 & 3.168739 & 0.559444\end{array}$

$\begin{array}{llll}\text { H } & 3.338052 & 1.126561 & \mathbf{0 . 6 8 8 9 4 5}\end{array}$

\begin{tabular}{llll} 
H & 3.405669 & $\mathbf{1 . 3 2 5 5 1 3}$ & $\mathbf{2 . 4 5 1 9 2 9}$ \\
\hline & $\mathbf{2 . 6 8 3 0 3}$ & $\mathbf{2 . 6 0 0 6 4 0}$ & $\mathbf{1 . 4 2 6 5 7 1}$
\end{tabular}

$\begin{array}{llll}\text { H } & 2.686303 & 2.600640 & 1.451929\end{array}$

\begin{tabular}{llll} 
H & -0.247116 & 0.679663 & 2.950117 \\
\hline & -1.494266 & -3.957056 & -0.573115
\end{tabular}

\begin{tabular}{lllll} 
H & $\mathbf{- 1 . 4 9 4 2 6 6}$ & $\mathbf{- 3 . 9 5 7 0 5 6}$ & $\mathbf{- 0 . 5 7 3 1 1 5}$ \\
\hline & $\mathbf{1 . 2 9 3 7 6 4}$ & $\mathbf{3 . 9 8 1 2 2 8}$ & $\mathbf{0 . 6 7 6 8 9 4}$
\end{tabular}

$\begin{array}{ccccc}\text { H } & \mathbf{1 . 2 9 3 7 6 4} & \mathbf{- 3 . 9 8 1 2 2 8} & \mathbf{- 0 . 6 7 6 8 9 4} \\ \mathrm{C} & \mathbf{0 . 3 1 2 1 4 9} & \mathbf{2 . 7 8 4 4 0 0} & \mathbf{2 . 9 3 1 4 7 6}\end{array}$

\begin{tabular}{llll} 
C & $\mathbf{0 . 3 1 2 1 4 9}$ & $\mathbf{2 . 7 8 4 4 0 0}$ & $\mathbf{2 . 9 3 1 4 7 6}$ \\
\hline & $\mathbf{0 . 3 6 4 4 9 2}$ & 3.508901 & $\mathbf{2 . 3 2 2 9 9 5}$
\end{tabular}

$\begin{array}{cccc}\text { H } & \mathbf{0 . 8 6 4 4 9 2} & \mathbf{3 . 5 0 8 9 0 1} & \mathbf{2 . 3 2 2 9 9 5} \\ \text { H } & \mathbf{- 0 . 7 0 9 8 6 1} & \mathbf{3 . 1 6 3 1 5 3} & \mathbf{3 . 0 6 7 7 3 3}\end{array}$

\begin{tabular}{lllll}
\hline H & $\mathbf{0 . 7 8 4 2 1 8}$ & $\mathbf{2 . 7 4 2 3 1 5}$ & $\mathbf{3 . 9 2 7 0 9 2}$
\end{tabular}

$\begin{array}{lllll}\text { H } & 0.254134 & 4.713095 & -2.305374\end{array}$

$\begin{array}{llll}\text { H } & -\mathbf{0 . 6 1 3 0 3 6} & \mathbf{2 . 9 1 1 6 8 6} & \mathbf{- 3 . 7 7 7 6 5 4}\end{array}$

66

unsat model mcb a / electronic energy: $-\mathbf{2 2 4 7 . 0 4 3 8 6 7 0 5}$ a.u. / lowest freq: $15.95 \mathrm{~cm}-1$

$\begin{array}{llll}S & -0.082691 & 0.932518 & 2.278211\end{array}$

$\begin{array}{llll}\text { C } & 0.035578 & -1.030288 & 0.110196\end{array}$

$\begin{array}{lllll}\text { Ru } & -\mathbf{0 . 0 2 4 0 9 4} & \mathbf{1 . 0 7 1 1 6 7} & \mathbf{0 . 0 1 1 5 6 8}\end{array}$

$\begin{array}{llll}\text { C } & -1.343763 & 0.987869 & -1.458046\end{array}$

$\begin{array}{llll}C & 1.362847 & 1.090753 & -1.394465\end{array}$

$\begin{array}{lllll}\text { S } & -0.124011 & 3.469395 & 0.333012\end{array}$

$\begin{array}{llll}\mathrm{N} & 1.117294 & -1.860662 & 0.242337\end{array}$

$\begin{array}{lrrrr}\text { N } & -1.021284 & -1.891967 & 0.249487\end{array}$

$\begin{array}{llll}C & \mathbf{2 5 0 2 2 5 3} & -1.518200 & 0.149674\end{array}$

$\begin{array}{llll}C & 2.502253 & -1.518200 & 0.149674 \\ C & 0.740839 & -3.180824 & 0.43558\end{array}$

\begin{tabular}{lllll}
$C$ & 0.740839 & -3.180824 & $\mathbf{0 . 4 4 3 5 5 8}$ \\
\hline & -0.606620 & -3.200367 & $\mathbf{0 . 4 4 7 5 5 3}$
\end{tabular}

$\begin{array}{llll}C & 0.740839 & -3.180824 & 0.443558 \\ C & -0.606620 & -3.200367 & \mathbf{0 . 4 4 7 5 5 3}\end{array}$

$\begin{array}{llll}C & \mathbf{- 2 . 4 1 3 6 3 3} & \mathbf{- 1 . 5 8 3 4 9 0} & \mathbf{0 . 1 5 3 5 8 7}\end{array}$

$\begin{array}{lrrr}C & \mathbf{5 . 2 3 1 4 0 2} & \mathbf{- 0 . 9 3 1 2 9 4} & \mathbf{- 0 . 1 7 3 6 7 2}\end{array}$

$\begin{array}{llll}\text { C } & 3.122689 & -1.599736 & -1.095805\end{array}$

$\begin{array}{llll}\text { C } & 3.276665 & -1.225990 & 1.281461\end{array}$

$\begin{array}{llll}\text { C } & 4.627998 & -0.920272 & 1.088136\end{array}$

$\begin{array}{llll}C & 4.459668 & -1.296948 & -1.280976\end{array}$

H $\quad \begin{array}{llll}\text { H.235390 } & -0.678324 & 1.963924\end{array}$

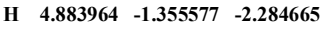

$\begin{array}{llll}\text { C } & \mathbf{- 5 . 1 4 7 4 7 5} & -\mathbf{1 . 0 3 5 2 6 3} & \mathbf{- 0 . 2 1 1 7 5 2}\end{array}$

$\begin{array}{llll}C & -3.028886 & -1.722870 & -1.094368\end{array}$

$\begin{array}{llll}\text { C } & -3.195989 & -1.249278 & 1.263415\end{array}$

$\begin{array}{llll}\text { C } & -4.551767 & -0.961543 & 1.046980\end{array}$

$\begin{array}{llll}C & -4.364320 & -1.444276 & -1.300004\end{array}$

$\begin{array}{lllll}\text { F } & -2.278436 & -2.125524 & -2.126414\end{array}$

$\begin{array}{llll}\text { C } & -2.656552 & -1.252028 & 2.665615\end{array}$

$\begin{array}{llll}\text { H } & \mathbf{- 5 . 1 6 3 8 9 6} & \mathbf{- 0 . 6 8 4 1 5 8} & \mathbf{1 . 9 0 9 1 9 9}\end{array}$

$\begin{array}{llll}\text { H } & -5.782784 & -1.549733 & -2.302419\end{array}$

$\begin{array}{llll}H & -4.782784 & -1.549733 & -2.302419 \\ \text { C } & -6.597181 & -0.687041 & -0.414317\end{array}$

$\begin{array}{llll}\text { C } & -6.597181 & -0.687041 & -0.414317\end{array}$

$\begin{array}{llll}\text { H } & -7.105382 & -1.441647 & \mathbf{- 1 . 0 3 1 9 5 7}\end{array}$

$\begin{array}{llll}\text { H } & -3.262702 & -1.925169 & 3.290215\end{array}$

$\begin{array}{llll}\text { H } & -6.690382 & 0.278957 & -0.934906\end{array}$

$\begin{array}{llll}\text { H } & -7.128057 & -\mathbf{0 . 6 0 5 1 4 5} & \mathbf{0 . 5 4 3 3 9 3}\end{array}$

$\begin{array}{llll}\text { H } & -2.706667 & -0.245894 & 3.104023\end{array}$

$\begin{array}{llll}\text { H } & -1.610614 & -1.576090 & 2.710458\end{array}$

$\begin{array}{llll}\text { F } & 2.385537 & -1.967029 & -2.150026\end{array}$

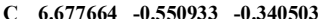

H $6.769877 \quad 0.533598 \quad-0.510980$

$\begin{array}{lllll}\text { H } & \mathbf{6 . 7 6 9 8 7 7} & \mathbf{0 . 5 3 3 5 9 8} & \mathbf{- 0 . 5 1 0 9 8 0}\end{array}$

\begin{tabular}{llll}
\hline & 7.260848 & -0.793796 & $\mathbf{0 . 5 5 8 4 8 0}$
\end{tabular}

$\begin{array}{llll}\text { H } & 7.131844 & -1.060400 & -1.201446\end{array}$

$\begin{array}{llll}\text { C } & 2.688445 & -1.288793 & 2.660919\end{array}$

$\begin{array}{llll}\text { H } & 2.198632 & -2.259338 & 2.834389\end{array}$

$\begin{array}{llll}\text { H } & 3.467548 & -1.155017 & 3.422192\end{array}$

$\begin{array}{llll}\text { H } & 1.923118 & -0.510980 & 2.797084\end{array}$

$\begin{array}{llll}C & -0.152516 & 2.579232 & 2.892792\end{array}$

$\begin{array}{llll}C & -0.173369 & 3.635812 & 2.062223\end{array}$

$\begin{array}{llll}C & -2.533020 & 1.907455 & -1.598603\end{array}$

$\begin{array}{llll}C & -2.533020 & 1.907455 & -1.598603\end{array}$

$\begin{array}{lllll}\text { H } & -1.623728 & -0.020293 & -1.763549\end{array}$

$\begin{array}{llll}\text { C } & 0.013748 & 1.361873 & -2.215696\end{array}$

$\begin{array}{lllll}\text { H } & 1.731823 & 0.109350 & -1.688164\end{array}$

$\begin{array}{lllll}C & \mathbf{2 . 4 8 5 7 1 1} & \mathbf{2 . 0 9 5 8 1 7} & \mathbf{- 1 . 4 9 2 4 4 3}\end{array}$

$\begin{array}{lllll}\text { H } & \mathbf{3 . 2 6 4 5 0 7} & \mathbf{1 . 8 0 8 1 8 4} & \mathbf{- 0 . 7 6 9 0 4 0}\end{array}$

$\begin{array}{lllll}\text { H } & \mathbf{2 . 9 4 9 7 4 5} & \mathbf{2 . 0 6 7 6 8 7} & \mathbf{- 2 . 4 9 3 7 7 3}\end{array}$

H $\quad 2.173176 \quad 3.121394 \quad-1.266018$

H $\quad-\mathbf{3 . 3 1 0 7 7 7} \quad \mathbf{1 . 5 6 9 6 5 7} \quad \mathbf{- 0 . 8 9 5 8 8 6}$

$\begin{array}{llll}\text { H } & -2.963180 & 1.840770 & -2.613223\end{array}$

$\begin{array}{llll}4 & -2.299774 & 2.953253 & -1.369888\end{array}$

\begin{tabular}{llll} 
H & -2.299774 & 2.953253 & -1.369888 \\
\hline & 0.064316 & 0.541513 & -2.945250
\end{tabular}

$\begin{array}{llll}H & 0.064316 & 0.541513 & -2.945250\end{array}$

$\begin{array}{lllll}\text { H } & \mathbf{1 . 4 7 4 0 6 2} & \mathbf{- 3 . 9 7 2 9 2 8} & \mathbf{0 . 5 6 0 6 5 2}\end{array}$

$\begin{array}{lllll}\text { H } & -1.316248 & -4.012659 & 0.570546\end{array}$

$\begin{array}{llll}C & \mathbf{- 0 . 0 1 8 5 8 4} & \mathbf{2 . 7 0 3 2 4 2} & \mathbf{- 2 . 9 3 9 5 9 3}\end{array}$

$\begin{array}{lllll}\text { H } & \mathbf{0 . 8 8 0 8 4 2} & \mathbf{2 . 8 2 0 2 4 3} & \mathbf{- 3 . 5 6 0 0 6 1}\end{array}$

$\begin{array}{lrlll}\text { H } & -\mathbf{0 . 8 9 1 0 9 0} & \mathbf{2 . 7 5 3 9 9 4} & \mathbf{- 3 . 6 0 5 9 1 1}\end{array}$

$\begin{array}{llll}\text { H } & \mathbf{- 0 . 0 6 7 0 2 4} & \mathbf{3 . 5 5 4 0 8 1} & \mathbf{- 2 . 2 4 8 3 3 4}\end{array}$

$\begin{array}{llll}\text { H } & -0.183262 & 2.683553 & 3.981398\end{array}$

$\begin{array}{llll}H & -0.223714 & 4.654667 & 2.460969\end{array}$

unsat_model_ts2_a / electronic energy: $-\mathbf{2 2 4 7 . 0 3 2 0 6 4 8 2}$ a.u. / lowest freq: $-\mathbf{1 3 1 . 0 3} \mathrm{cm}-1$ 


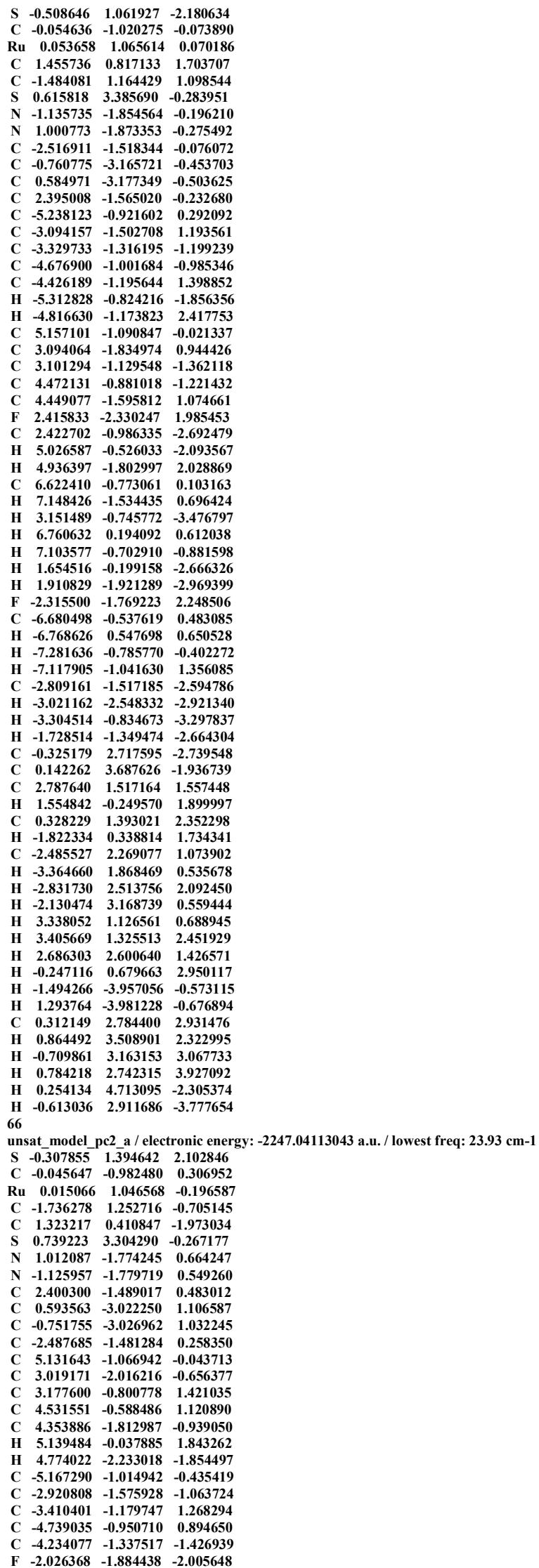




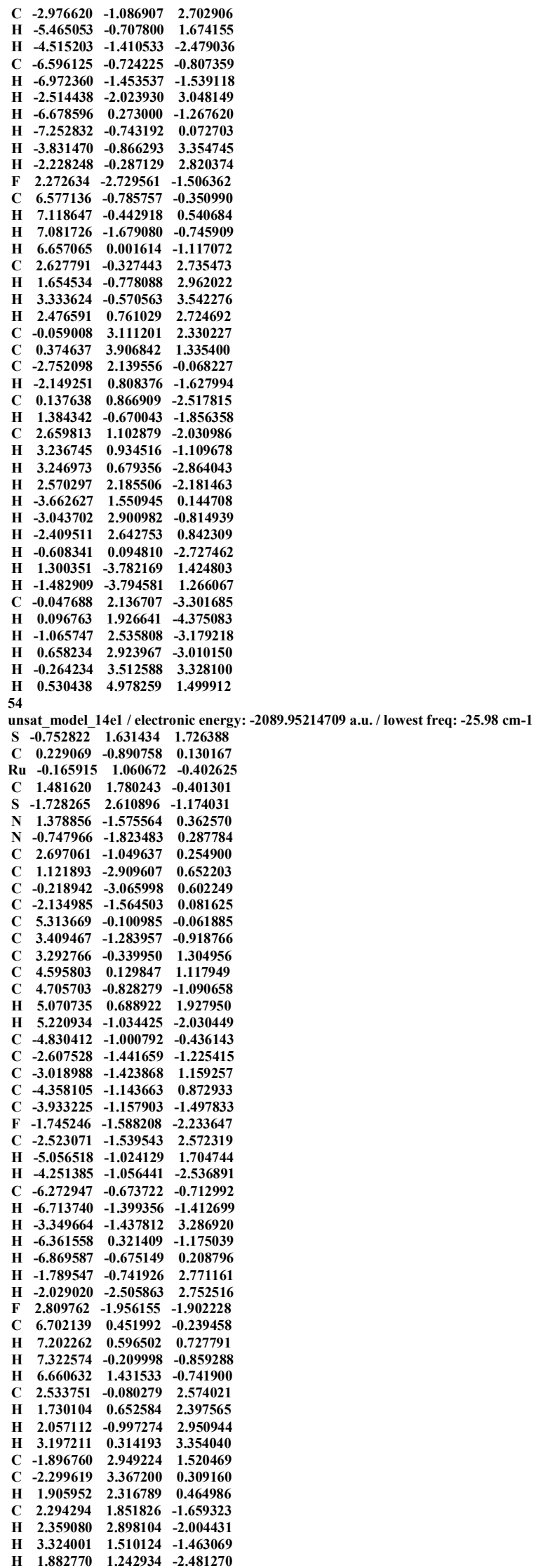


$\begin{array}{llll}\text { H } & 1.919470 & -3.615738 & 0.860905\end{array}$ \begin{tabular}{llll} 
H & -0.846517 & -3.938937 & 0.753012 \\
\hline
\end{tabular} $\begin{array}{llll}\text { H } & -\mathbf{2 . 2 6 8 6 7 2} & \mathbf{3 . 4 0 7 8 9 5} & \mathbf{2 . 4 4 2 2 9 5}\end{array}$ $\begin{array}{llll}\text { H } & -3.011795 & 4.190433 & 0.199006\end{array}$

unsat_model_pc1_b / electronic energy: - 2435.41092684 a.u. / lowest freq: $13.56 \mathrm{~cm}-1$ $\begin{array}{llll}S & 0.970333 & 0.089419 & 2.402385\end{array}$

$\begin{array}{llll}C & 0.289678 & -1.278651 & -0.268377\end{array}$

$\begin{array}{llll}\text { Ru } & 0.043098 & 0.719348 & 0.357628\end{array}$

$\begin{array}{llll}C & -1.729148 & 0.625328 & 0.866428\end{array}$

$\begin{array}{llll}C & 0.371895 & 1.146695 & -1.841503\end{array}$

$\begin{array}{lllll}\text { S } & 0.424974 & 2.954521 & 1.049571\end{array}$

N $1.457814 \quad-1.911970 \quad-0.587861$

$\begin{array}{llll}N & -0.650763 & -2.240383 & -0.482610\end{array}$

$\begin{array}{llll}C & 2.773610 & -1.364861 & -0.504118\end{array}$

$\begin{array}{llll}C & 1.240849 & -3.220540 & -0.993646\end{array}$

$\begin{array}{llll}C & -0.089722 & -3.429017 & -0.927745\end{array}$

$\begin{array}{llll}C & -2.051283 & -2.097713 & -0.283270\end{array}$

$\begin{array}{lrrr}C & \mathbf{5 . 3 5 2 4 7 4} & \mathbf{- 0 . 2 5 5 1 2 3} & \mathbf{- 0 . 4 3 6 3 8 6}\end{array}$

$\begin{array}{llll}C & 3.314351 & -0.717071 & -1.615660\end{array}$

$\begin{array}{llll}C & 3.569257 & -1.553350 & 0.633808\end{array}$

$\begin{array}{llll}C & 4.842105 & -0.973508 & 0.648050\end{array}$

$\begin{array}{llll}C & 4.575021 & -0.149010 & -1.595246\end{array}$

H $\quad 5.459446 \quad-1.098140 \quad 1.541125$

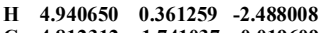

$\begin{array}{llll}\text { C } & -4.812312 & -1.741037 & 0.019609\end{array}$

$\begin{array}{llll}\text { C } & -2.853002 & -1.743977 & -1.365216\end{array}$

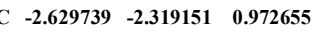

C $-\mathbf{- 4 . 0 1 0 0 2 1}-\mathbf{- 2 . 1 3 5 5 8 1} \mathbf{1 . 0 9 6 4 9 3}$

C $-4.216697-1.551821-1.232814$

$\begin{array}{llll}\text { F } & -2.272517 & -1.557401 & -2.553665\end{array}$

$\begin{array}{llll}\text { C } & -1.772447 & -2.692152 & 2.148108\end{array}$

$\begin{array}{llll}\text { H } & -4.473881 & \mathbf{- 2 . 2 9 7 3 4 1} & \mathbf{2 . 0 7 2 7 0 9}\end{array}$

$\begin{array}{llll}\text { H } & -4.799620 & -1.256009 & -2.106755\end{array}$

$\begin{array}{llll}C & -6.285726 & -1.496205 & 0.202032\end{array}$

C $-6.856886-1.813231-0.681665$

H $-1.100113-3.528753-1.905765$

H $-6.476739-0.421425 \quad 0.34983$

\begin{tabular}{lll}
0.349839 \\
\hline
\end{tabular}

$\begin{array}{llll}H & -6.675738 & -2.027416 & 1.080541\end{array}$

\begin{tabular}{llll}
\hline & -2.389808 & -2.979728 & 3.008570
\end{tabular}

$\begin{array}{llll}\text { H } & -1.136142 & -1.841479 & 2.444217\end{array}$

$\begin{array}{llll}2.599163 & -0.668026 & -2.74242\end{array}$

$\begin{array}{llll}\text { C } & 6.707181 & 0.396020 & \mathbf{- 0 . 3 7 0 5 0 0}\end{array}$

$\begin{array}{llll}\text { H } & 7.321378 & -0.033527 & 0.432170\end{array}$

H $7.247644 \quad 0.288789-1.321678$

\begin{tabular}{lllll}
\hline & 6.601089 & 1.474210 & $-\mathbf{0 . 1 7 2 2 8 0}$
\end{tabular}

$\begin{array}{llll}\text { C } & 3.121207 & -\mathbf{2 . 4 2 1 3 8 9} & \mathbf{1 . 7 7 6 3 9 4}\end{array}$

$\begin{array}{llll}\text { H } & 2.032005 & -2.539991 & 1.811710\end{array}$

$\begin{array}{llll}\text { H } & 3.573367 & -3.421380 & 1.674086\end{array}$

$\begin{array}{llll}\text { H } & 3.446801 & -1.999142 & 2.735871\end{array}$

C $\quad \mathbf{1 . 1 7 4 2 7 0} \quad \mathbf{1 . 5 8 7 5 6 7} \quad \mathbf{3 . 2 8 1 0 8 6}$

$\begin{array}{llll}C & \mathbf{0 . 9 3 8 7 1 5} & \mathbf{2 . 7 8 3 3 1 7} & \mathbf{2 . 7 1 0 0 3 7}\end{array}$

$\begin{array}{llll}C & -2.271262 & 0.952128 & \mathbf{2 . 2 1 2 6 2 2}\end{array}$

\begin{tabular}{llll}
$C$ & -271262 & 0.952128 & 2.212622 \\
\hline
\end{tabular}

$\begin{array}{lllll}\mathrm{C} & -2.548235 & 0.475987 & 0.147536\end{array}$

$\begin{array}{llll}C & -0.970547 & 1.398269 & -1.564921\end{array}$

$\begin{array}{lllll}\mathrm{C} & \mathbf{0 . 5 6 6 5 3 5} & \mathbf{0 . 1 7 1 1 9 1} & \mathbf{- 2 . 2 9 0 1 2 0}\end{array}$

$\begin{array}{llll}C & -1.587576 & 2.743514 & -1.461508\end{array}$

$\begin{array}{llll}C & 1.451624 & 2.158910 & -2.119579\end{array}$

$\begin{array}{llll}\text { H } & \mathbf{2 . 4 1 2 2 7 0} & \mathbf{1 . 8 3 5 6 3 2} & -1.694592\end{array}$

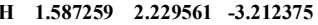

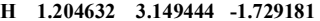

\begin{tabular}{llllll}
\hline & -1.066433 & 3.811091 & -1.683224
\end{tabular}

$\begin{array}{llll}0 & -2.878727 & 2.639788 & -1.101315\end{array}$

$\begin{array}{llll}0 & -2.878727 & 2.639788 & -1.101315 \\ C & -3.575932 & 3.857707 & -0.898130\end{array}$

$\begin{array}{lllll}\text { H } & -4.597078 & 3.584721 & \mathbf{- 0 . 6 1 0 0 3 9}\end{array}$

$\begin{array}{llll}\text { H } & -3.592390 & 4.460208 & -1.817551\end{array}$

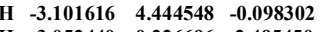

$\begin{array}{llll}\text { H } & -3.052440 & 0.226696 & 2.495450\end{array}$

$\begin{array}{llll}\text { H } & -2.780200 & 1.928526 & 2.097369\end{array}$

$\begin{array}{llll}\text { H } & -1.520368 & 1.044679 & 3.004546\end{array}$

$\begin{array}{llll}\text { H } & -1.692509 & 0.614986 & -1.795748\end{array}$

$\begin{array}{llll}\text { H } & 2.058234 & -3.872015 & -1.286798\end{array}$

\begin{tabular}{lrrr} 
H & -0.695681 & -4.301102 & -1.152737 \\
\hline & 1.511035 & 1.505472 & 4.319489
\end{tabular}

\begin{tabular}{llll}
\hline & 1.511035 & 1.505472 & 4.319489
\end{tabular}

$\begin{array}{llll}H & 1.077033 & 3.712924 & 3.272050\end{array}$

69

unsat_model_ts1_b / electronic energy: -2435.39520421 a.u. / lowest freq: $\mathbf{- 1 3 8 . 9 7} \mathbf{c m}-1$ S $1.217733-0.111855 \quad-2.349132$

C $\quad 0.212373-1.261989 \quad 0.355580$

$\begin{array}{lllll}\text { Ru } & 0.235520 & 0.689790 & -0.430865\end{array}$

C $-1.5997910 .826289-0.786458$

C $-1.5997150 .826289-0.786458$

N

$\begin{array}{lllll}\mathrm{N} & -0.759618 & -2.185946 & 0.60819\end{array}$

N $\begin{array}{ccc}1.335648 & -1.866817 & 0.858815\end{array}$

$\begin{array}{llll}C & -2.130188 & -2.081471 & 0.24265\end{array}$

$\begin{array}{llll}C & -0.263588 & -3.313340 & 1.248587\end{array}$

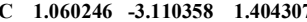

$\begin{array}{llll}C & \mathbf{2 . 6 6 9 8 8 6} & \mathbf{- 1 . 3 7 0 9 5 1} & \mathbf{0 . 7 5 4 4 6 9}\end{array}$

$\begin{array}{llll}C & -4.832364 & -1.736645 & -0.40848\end{array}$

$\begin{array}{llll}C & -3.058786 & -1.691402 & \mathbf{1 . 2 0 5 4 8 0}\end{array}$

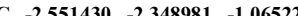

$\begin{array}{llll}C & -2.551430 & -2.348981 & -1.065228 \\ C & -3.905392 & -2.166288 & -1.364173\end{array}$

$\begin{array}{llll}C & -3.905392 & -2.166288 & -1.364173\end{array}$ 


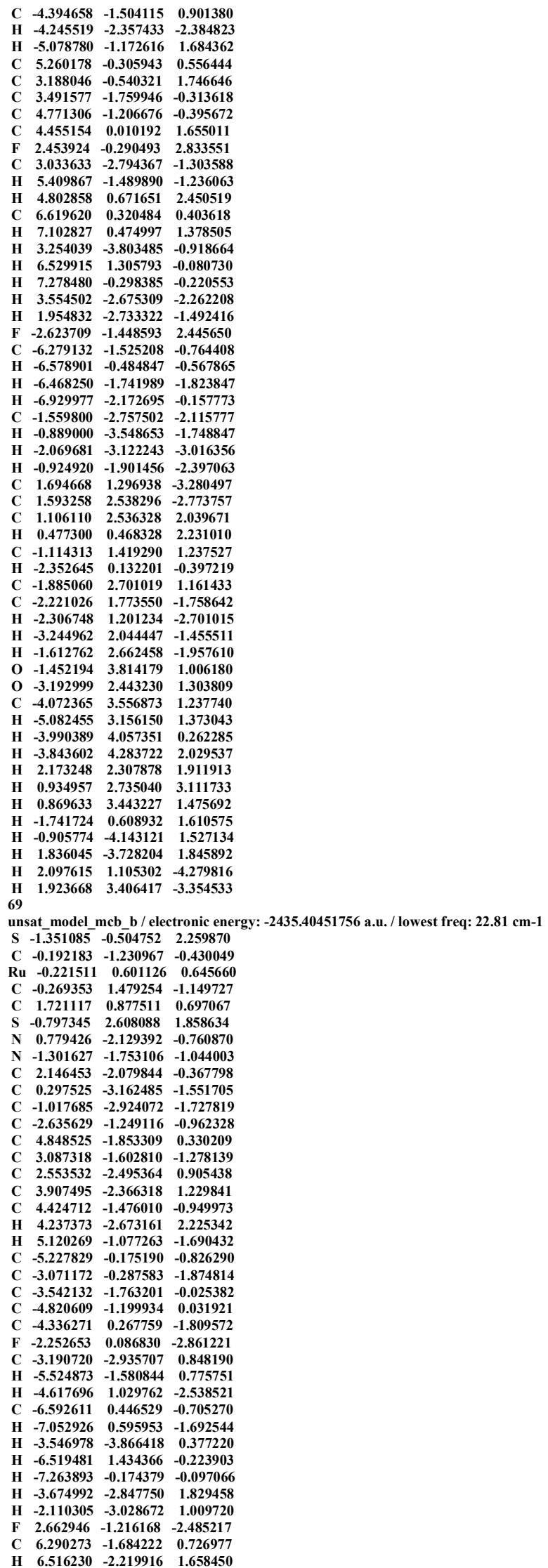


$\begin{array}{llll}\text { H } & 6.966073 & -2.050228 & -0.059165\end{array}$ $\begin{array}{llll}\text { H } & 6.519419 & -0.619049 & 0.886844\end{array}$

$\begin{array}{llll}\text { C } & \mathbf{1 . 5 4 9 4 1 8} & \mathbf{- 3 . 0 1 0 3 1 4} & \mathbf{1 . 8 9 5 6 0 8}\end{array}$

$\begin{array}{llll}\text { H } & \mathbf{0 . 8 9 6 5 9 0} & \mathbf{- 3 . 7 7 2 3 0 1} & \mathbf{1 . 4 4 3 8 2 6}\end{array}$

$\begin{array}{llll}\text { H } & \mathbf{2 . 0 4 8 3 6 6} & \mathbf{3 . 4 5 0 6 8 0} & \mathbf{2 . 7 6 7 9 9 9}\end{array}$

C $0.897550-2.192646 \quad 2.241836$

\begin{tabular}{lrrr}
\hline H & 0.897550 & -2.192646 & $\mathbf{2 . 2 4 1 8 3 6}$ \\
\hline C & -1.929350 & 0.689494 & 3.408183
\end{tabular}

$\begin{array}{llll}C & -1.929350 & 0.689494 & 3.408183 \\ C\end{array}$

$\begin{array}{llll}\text { C } & -1.681501 & 1.998845 & 3.223409\end{array}$

$\begin{array}{llll}C & -0.978583 & 2.770123 & -1.490268\end{array}$

$\begin{array}{lllll}\text { H } & -0.348296 & 0.790180 & -1.995838\end{array}$

$\begin{array}{llll}\text { C } & \mathbf{1 . 2 8 1 8 7 0} & \mathbf{1 . 5 0 5 3 3 1} & \mathbf{- 0 . 7 2 9 4 5 6}\end{array}$

$\begin{array}{lllll}\text { H } & 2.339412 & 0.005784 & 0.483245\end{array}$

$\begin{array}{llll}C & \mathbf{1 . 8 5 5 0 1 4} & \mathbf{2 . 8 9 7 7 7 7} & \mathbf{- 0 . 8 5 6 9 4 9}\end{array}$

$\begin{array}{llll}C & \mathbf{2 . 4 4 9 2 7 6} & \mathbf{1 . 7 4 7 0 7 7} & \mathbf{1 . 7 0 0 2 4 0}\end{array}$

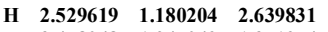

H $3.478048 \quad 1.945042 \quad 1.351274$

$\begin{array}{llll}\text { H } & 1.945357 & \mathbf{2 . 6 9 4 2 7 6} & \mathbf{1 . 9 0 9 2 6 9}\end{array}$

$\begin{array}{lllll}\text { O } & \mathbf{1 . 5 1 2 2 1 8} & \mathbf{3 . 8 8 2 4 4 2} & \mathbf{- 0 . 2 5 8 2 6 0}\end{array}$

$\begin{array}{lllll}\text { O } & \mathbf{2 . 8 4 3 6 6 7} & \mathbf{2 . 8 8 9 9 0 6} & \mathbf{- 1 . 7 5 4 0 6 4}\end{array}$

$\begin{array}{llll}C & 3.497560 & 4.131545 & -1.988539\end{array}$

$\begin{array}{llll}\text { H } & 4.264485 & 3.934620 & -2.744683\end{array}$

H $\quad 3.961321 \quad 4.505993 \quad-1.065419$

$\begin{array}{llll}\text { H } & 2.783621 & 4.880619 & -2.358056\end{array}$

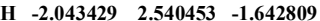

\begin{tabular}{llll} 
H & $-\mathbf{0 . 5 9 7 3 1 0}$ & $\mathbf{3 . 1 8 3 6 8 5}$ & $\mathbf{- 2 . 4 4 0 3 0 2}$ \\
\hline & -0.896880 & $\mathbf{3} .531809$ & -0.709362
\end{tabular}

$\begin{array}{llll}\mathrm{H} & -0.896880 & 3.531809 & -0.709362\end{array}$

$\begin{array}{lllll}\text { H } & \mathbf{1 . 7 7 3 4 7 7} & \mathbf{0 . 8 5 8 6 4 4} & \mathbf{- 1 . 4 6 2 0 2 6}\end{array}$

$\begin{array}{lllll}\text { H } & \mathbf{0 . 9 4 2 1 2 0} & \mathbf{- 3 . 9 6 0 8 5 3} & \mathbf{- 1 . 9 0 6 0 7 6}\end{array}$

$\begin{array}{llll}\text { H } & -\mathbf{1 . 7 8 3 2 2 5} & -\mathbf{3 . 4 7 2 5 6 2} & -\mathbf{2 . 2 6 7 8 7 7}\end{array}$

$\begin{array}{lllll}\text { H } & -2.499534 & 0.306908 & 4.259447\end{array}$

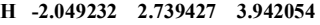

69

unsat model ts2 b / electronic energy: $\mathbf{- 2 4 3 5 . 3 9 5 2 0 4 2 1}$ a.u. / lowest freq: $-\mathbf{1 3 8 . 9 7} \mathbf{c m - 1}$

$\begin{array}{lllll}S & 1.217733 & -0.111855 & -2.349132\end{array}$

$\begin{array}{llll}\text { C } & 0.212373 & -1.261989 & 0.355580\end{array}$

$\begin{array}{llll}\text { Ru } & \mathbf{0 . 2 3 5 5 2 0} & \mathbf{0 . 6 8 9 7 9 0} & \mathbf{- 0 . 4 3 0 8 6 5}\end{array}$

$\begin{array}{llll}C & 0.269186 & 1.350421 & 1.621621\end{array}$

$\begin{array}{llll}C & -1.599791 & 0.826289 & -0.786458\end{array}$

$\begin{array}{lllll}\mathrm{S} & \mathbf{0 . 9 7 0 8 1 5} & 2.849014 & \mathbf{- 1 . 1 7 5 5 0 5}\end{array}$

$\begin{array}{llll}\mathrm{N} & -\mathbf{0 . 7 5 9 6 1 8} & -\mathbf{2 . 1 8 5 9 4 6} & \mathbf{0 . 6 0 8 1 9 1}\end{array}$

$\begin{array}{llll}\text { N } & 1.335648 & -1.866817 & 0.858815\end{array}$

$\begin{array}{llll}\text { C } & -2.130188 & -2.081471 & 0.242651\end{array}$

$\begin{array}{llll}\text { C } & \mathbf{- 0 . 2 6 3 5 8 8} & -\mathbf{3 . 3 1 3 3 4 0} & \mathbf{1 . 2 4 8 5 8 7}\end{array}$

$\begin{array}{llll}\text { C } & \mathbf{1 . 0 6 0 2 4 6} & -\mathbf{3 . 1 1 0 3 5 8} & \mathbf{1 . 4 0 4 3 0 7}\end{array}$

$\begin{array}{llll}C & 2.669886 & -1.370951 & 0.754469\end{array}$

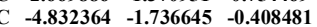

$\begin{array}{llll}C & -3.058786 & -1.691402 & 1.205480\end{array}$

$\begin{array}{llll}C & -2.551430 & -2.348981 & -1.065228\end{array}$

$\begin{array}{llll}C & -3.905392 & -2.166288 & -1.364173\end{array}$

$\begin{array}{llll}C & -4.394658 & -1.504115 & 0.901380\end{array}$

\begin{tabular}{lllll} 
H & -4.245519 & -2.357433 & -2.384823 \\
\hline
\end{tabular}

$\begin{array}{llll}\text { H } & -5.078780 & -1.172616 & 1.684362\end{array}$

$\begin{array}{llll}\text { C } & 5.260178 & -0.305943 & 0.556444\end{array}$

$\begin{array}{llll}C & 3.188046 & -0.540321 & 1.746646\end{array}$

$\begin{array}{llll}C & 3.491577 & -1.759946 & \mathbf{- 0 . 3 1 3 6 1 8}\end{array}$

$\begin{array}{llll}C & 4.771306 & -1.206676 & -0.395672\end{array}$

$\begin{array}{llll}\text { C } & 4.455154 & \mathbf{0 . 0 1 0 1 9 2} & \mathbf{1 . 6 5 5 0 1 1}\end{array}$

$\begin{array}{llll}\text { F } & \mathbf{2 . 4 5 3 9 2 4} & \mathbf{- 0 . 2 9 0 4 9 3} & \mathbf{2 . 8 3 3 5 5 1}\end{array}$

$\begin{array}{llll}\text { C } & 3.033633 & -2.794367 & -1.303588\end{array}$

$\begin{array}{llll}\text { H } & 5.409867 & -1.489890 & -1.236063\end{array}$

$\begin{array}{llll}\text { H } & 4.802858 & 0.671651 & 2.450519\end{array}$

$\begin{array}{lllll}\text { C } & 6.619620 & 0.320484 & 0.403618\end{array}$

$\begin{array}{llll}C & 7.619620 & 0.320484 & 0.403618\end{array}$

\begin{tabular}{ccccc} 
H & 7.102827 & 0.474997 & $\mathbf{1 . 3 7 8 5 0 5}$ \\
\hline & 3.254039 & -3.803485 & -0.918664
\end{tabular}

$\begin{array}{llll}\text { H } & 3.254039 & \mathbf{- 3 . 8 0 3 4 8 5} & \mathbf{- 0 . 9 1 8 6 6 4}\end{array}$

$\begin{array}{llll}\text { H } & 6.529915 & \mathbf{1 . 3 0 5 7 9 3} & \mathbf{- 0 . 0 8 0 7 3 0}\end{array}$

$\begin{array}{llll}\text { H } & 7.278480 & -0.298385 & -0.220553\end{array}$

$\begin{array}{lllll}\text { H } & 3.554502 & -2.675309 & -2.262208\end{array}$

$\begin{array}{llll}\text { H } & 1.954832 & -2.733322 & -1.492416\end{array}$

$\begin{array}{llll}\text { F } & -2.623709 & -1.448593 & 2.445650\end{array}$

$\begin{array}{llll}\text { C } & -6.279132 & -1.525208 & -0.764408\end{array}$

$\begin{array}{llll}\text { H } & -6.578901 & -0.484847 & -0.567865\end{array}$

$\begin{array}{llll}\text { H } & -6.468250 & -1.741989 & -1.823847\end{array}$

H $\quad-6.929977-2.172695 \quad-0.157773$

$\begin{array}{lll}-1.559800 & -2.757502 & -2.115777\end{array}$

$\begin{array}{llll}\text { H } & -0.889000 & -3.548653 & -1.748847\end{array}$

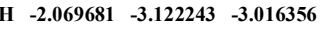

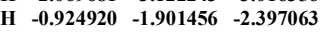

$\begin{array}{llll}C & \mathbf{1 . 6 9 4 6 6 8} & \mathbf{1 . 2 9 6 9 3 8} & \mathbf{- 3 . 2 8 0 4 9 7}\end{array}$

$\begin{array}{llll}C & \mathbf{1 . 5 9 3 2 5 8} & \mathbf{2 . 5 3 8 2 9 6} & \mathbf{- 2 . 7 7 3 7 5 7}\end{array}$

$\begin{array}{llll}\text { C } & 1.106110 & \mathbf{2 . 5 3 6 3 2 8} & \mathbf{2 . 0 3 9 6 7 1}\end{array}$

$\begin{array}{llll}\text { H } & 0.477300 & 0.468328 & 2.231010\end{array}$

\begin{tabular}{llll}
\hline & 0.473013 & 1.419290 & 2.231010
\end{tabular}

\begin{tabular}{llll}
$C$ & -1.114313 & 1.419290 & 1.237527 \\
\hline & -2.352645 & 0.132201 & -0.397219
\end{tabular}

\begin{tabular}{llll}
$\mathrm{H}$ & -2.352645 & 0.132201 & -0.397219 \\
\hline
\end{tabular}

$\begin{array}{llll}\text { C } & -1.885060 & 2.701019 & 1.161433\end{array}$

$\begin{array}{llll}C & -2.221026 & 1.773550 & -1.758642\end{array}$

$\begin{array}{llll}\text { H } & -2.306748 & 1.201234 & -2.701015\end{array}$

$\begin{array}{llll}\text { H } & -3.244962 & 2.044447 & -1.455511\end{array}$

H $\quad-1.612762 \quad 2.662458 \quad-1.957610$

$\begin{array}{lllll}\text { O } & -1.452194 & 3.814179 & 1.006180\end{array}$

$\begin{array}{llll}\text { O } & -\mathbf{3 . 1 9 2 9 9 9} & \mathbf{2 . 4 4 3 2 3 0} & \mathbf{1 . 3 0 3 8 0 9}\end{array}$

$\begin{array}{llll}\text { C } & -4.072365 & 3.556873 & 1.237740\end{array}$

$\begin{array}{llll}\mathrm{H}-5.082455 & 3.156150 & 1.373043\end{array}$

\begin{tabular}{lllll}
\hline & -5.082455 & 3.156150 & 1.373043
\end{tabular}

$\begin{array}{llll}\text { H } & -3.990389 & 4.057351 & 0.262285\end{array}$ 
$\begin{array}{rrrr}\text { H } & -\mathbf{3 . 8 4 3 6 0 2} & \mathbf{4 . 2 8 3 7 2 2} & \mathbf{2 . 0 2 9 5 3 7} \\ \text { H } & \mathbf{2 . 1 7 3 2 4 8} & \mathbf{2 . 3 0 7 8 7 8} & \mathbf{1 . 9 1 1 9 1 3} \\ \text { H } & \mathbf{0 . 9 3 4 9 5 7} & \mathbf{2 . 7 3 5 0 4 0} & \mathbf{3 . 1 1 1 7 3 3} \\ \text { H } & \mathbf{0 . 8 6 9 6 3 3} & \mathbf{3 . 4 4 3 2 2 7} & \mathbf{1 . 4 7 5 6 9 2} \\ \text { H } & -\mathbf{1 . 7 4 1 7 2 4} & \mathbf{0 . 6 0 8 9 3 2} & \mathbf{1 . 6 1 0 5 7 5} \\ \text { H } & -\mathbf{0 . 9 0 5 7 7 4} & -\mathbf{4 . 1 4 3 1 2 1} & \mathbf{1 . 5 2 7 1 3 4} \\ \text { H } & \mathbf{1 . 8 3 6 0 4 5} & \mathbf{- 3 . 7 2 8 2 0 4} & \mathbf{1 . 8 4 5 8 9 2} \\ \text { H } & \mathbf{2 . 0 9 7 6 1 5} & \mathbf{1 . 1 0 5 3 0 2} & \mathbf{- 4 . 2 7 9 8 1 6} \\ \text { H } & \mathbf{1 . 9 2 3 6 6 8} & \mathbf{3 . 4 0 6 4 1 7} & \mathbf{- 3 . 3 5 4 5 3 3}\end{array}$

unsat_model_pc2_b / electronic energy: $-\mathbf{2 4 3 5 . 4 1 0 9 2 6 8 4}$ a.u. / lowest freq: $13.56 \mathrm{~cm}-1$ $\begin{array}{lllll}\text { S } & \mathbf{0 . 9 7 0 3 3 3} & \mathbf{0 . 0 8 9 4 1 9} & \mathbf{2 . 4 0 2 3 8 5}\end{array}$

$\begin{array}{llll}C & 0.289678 & -1.278651 & -0.268377\end{array}$

$\begin{array}{lllll}\text { Ru } & 0.043098 & 0.719348 & \mathbf{0 . 3 5 7 6 2 8}\end{array}$

$\begin{array}{llll}\text { C } & -1.729148 & 0.625328 & 0.866428\end{array}$

$\begin{array}{llll}C & 0.371895 & 1.146695 & -1.841503\end{array}$

$\begin{array}{lllll}\text { S } & \mathbf{0 . 4 2 4 9 7 4} & \mathbf{2 . 9 5 4 5 2 1} & \mathbf{1 . 0 4 9 5 7 1}\end{array}$

$\begin{array}{cccc}\mathrm{N} & \mathbf{1 . 4 5 7 8 1 4} & \mathbf{- 1 . 9 1 1 9 7 0} & \mathbf{- 0 . 5 8 7 8 6 1}\end{array}$

$\begin{array}{lrrr}N & -0.650763 & -2.240383 & -0.482610\end{array}$

$\begin{array}{llll}C & \mathbf{2 . 7 7 3 6 1 0} & -\mathbf{1 . 3 6 4 8 6 1} & \mathbf{- 0 . 5 0 4 1 1 8}\end{array}$

$\begin{array}{llll}C & 1.240849 & -3.220540 & -0.993646\end{array}$

$\begin{array}{llll}C & -0.089722 & -3.429017 & -0.927745\end{array}$

$\begin{array}{llll}C & -2.051283 & -2.097713 & -0.283270\end{array}$

$\begin{array}{llll}C & 5.352474 & -0.255123 & -0.436386\end{array}$

$\begin{array}{llll}\text { C } & 3.314351 & -0.717071 & -1.615660\end{array}$

$\begin{array}{llll}C & 3.569257 & -1.553350 & 0.633808\end{array}$

$\begin{array}{llll}C & 4.842105 & -0.973508 & 0.648050\end{array}$

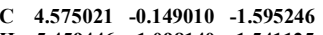

H $\quad 5.459446 \quad-1.098140 \quad 1.541125$

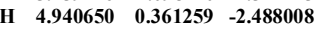

$\begin{array}{llll}\text { C } & -4.812312 & -1.741037 & 0.019609\end{array}$

$\begin{array}{llll}\text { C } & -2.853002 & -1.743977 & -1.365216\end{array}$

$\begin{array}{llll}C & -2.629739 & -2.319151 & 0.972655\end{array}$

$\begin{array}{llll}\text { C } & -4.010021 & -2.135581 & 1.096493\end{array}$

$\begin{array}{llll}C & -4.216697 & -1.551821 & -1.232814\end{array}$

$\begin{array}{llll}\text { F } & -2.272517 & -1.557401 & -2.553665\end{array}$

C $-\mathbf{- 1 . 7 7 2 4 4 7} \quad \mathbf{- 2 . 6 9 2 1 5 2} \quad \mathbf{2 . 1 4 8 1 0 8}$

$\begin{array}{llll}\text { H } & -4.473881 & -2.297341 & \mathbf{2 . 0 7 2 7 0 9}\end{array}$

H $\quad-4.799620-1.256009-2.106755$

$\begin{array}{llll}\text { C } & -6.285726 & -1.496205 & 0.202032\end{array}$

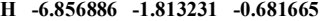

$\begin{array}{llll}\text { H } & -1.100113 & -3.528753 & 1.905765\end{array}$

$\begin{array}{llll}\text { H } & -6.476739 & -0.421425 & 0.349839\end{array}$

\begin{tabular}{llll} 
H & $\mathbf{- 6 . 6 7 5 7 3 8}$ & $\mathbf{- 2 . 0 2 7 4 1 6}$ & $\mathbf{1 . 0 8 0 5 4 1}$ \\
\hline & -2.389808 & -2.979728 & $\mathbf{3} 008570$
\end{tabular}

$\begin{array}{llll}\text { H } & \mathbf{- 2 . 3 8 9 8 0 8} & \mathbf{- 2 . 9 7 9 7 2 8} & \mathbf{3 . 0 0 8 5 7 0}\end{array}$

$\begin{array}{llll}\text { H } & -1.136142 & -1.841479 & 2.444217\end{array}$

$\begin{array}{llll}\text { F } & 2.599163 & -0.668026 & -2.742421\end{array}$

$\begin{array}{llll}C & 6.707181 & 0.396020 & -0.370500\end{array}$

$\begin{array}{llll}\text { H } & 7.321378 & -0.033527 & 0.432170\end{array}$

$\begin{array}{lllll}\text { H } & 7.247644 & 0.288789 & -1.321678\end{array}$

$\begin{array}{llll}H & 6.601089 & 1.474210 & -0.172280\end{array}$

$\begin{array}{llll}\text { C } & 3.121207 & -2.421389 & 1.776394\end{array}$

$\begin{array}{llll}\text { H } & 2.032005 & -2539991 & 1.811710\end{array}$

C $2.032005-2.539991-1.811710$

$\begin{array}{llll}\text { H } & 3.573367 & -3.421380 & 1.674086\end{array}$

$\begin{array}{llll}\text { H } 3.446801 & -1.999142 & \mathbf{2 . 7 3 5 8 7 1}\end{array}$

$\begin{array}{llll}\text { C } & \mathbf{1 . 1 7 4 2 7 0} & \mathbf{1 . 5 8 7 5 6 7} & \mathbf{3 . 2 8 1 0 8 6}\end{array}$

$\begin{array}{llll}\text { C } & \mathbf{0 . 9 3 8 7 1 5} & \mathbf{2 . 7 8 3 3 1 7} & \mathbf{2 . 7 1 0 0 3 7}\end{array}$

$\begin{array}{llll}\text { C } & -2.271262 & 0.952128 & \mathbf{2 . 2 1 2 6 2 2}\end{array}$

$\begin{array}{llll}\text { H } & -2.548235 & 0.475987 & 0.147536\end{array}$

$\begin{array}{llll}\text { C } & -0.970547 & 1.398269 & -1.564921\end{array}$

$\begin{array}{llll}\text { H } & 0.566535 & 0.171191 & \mathbf{- 2 . 2 9 0 1 2 0}\end{array}$

C $-1.587576 \quad 2.743514-1.461508$

C $1.451624 \quad 2.158910-2.119579$

$\begin{array}{llll}C & 1.451624 & 2.158910 & -2.119579\end{array}$

$\begin{array}{llll}\text { H } & 2.412270 & 1.835632 & -1.694592\end{array}$

$\begin{array}{llll}H & 1.587259 & 2.229561 & \mathbf{- 3 . 2 1 2 3 7 5}\end{array}$

$\begin{array}{rrrrr}\text { H } & \mathbf{1 . 2 0 4 6 3 2} & \mathbf{3 . 1 4 9 4 4 4} & \mathbf{- 1 . 7 2 9 1 8 1} \\ \text { O } & -\mathbf{1 . 0 6 6 4 3 3} & \mathbf{3 . 8 1 1 0 9 1} & \mathbf{- 1 . 6 8 3 2 2 4}\end{array}$

\begin{tabular}{lllll|}
\hline & -1.066433 & 3.811091 & -1.683224 \\
O & -2.878727 & 2.639788 & -1.101315
\end{tabular}

\begin{tabular}{lllll}
\hline & -2.878727 & $\mathbf{2 . 6 3 9 7 8 8}$ & -1.101315 \\
$C$ & $\mathbf{- 3 . 5 7 5 9 3 2}$ & $\mathbf{3 . 8 5 7 7 0 7}$ & $\mathbf{- 0 . 8 9 8 1 3 0}$
\end{tabular}

$\begin{array}{llll}\text { H } & -4.597078 & 3.584721 & -0.610039\end{array}$

$\begin{array}{lllll}\text { H } & -3.592390 & 4.460208 & -1.817551\end{array}$

\begin{tabular}{llll}
\hline & -3.101616 & 4.444548 & -0.098302
\end{tabular}

$\begin{array}{lll}-3.01616 & 4.44548 & -0.098302\end{array}$

$\begin{array}{llll}\text { H } & \mathbf{- 3 . 0 5 2 4 4 0} & \mathbf{0 . 2 2 6 6 9 6} & \mathbf{2 . 4 9 5 4 5 0}\end{array}$

\begin{tabular}{llll}
\hline & -2.780200 & 1.928526 & $\mathbf{2 . 0 9 7 3 6 9}$
\end{tabular}

$\begin{array}{rrrr}H & -1.520368 & 1.044679 & \mathbf{3 . 0 0 4 5 4 6}\end{array}$

$\begin{array}{llll}\text { H } & -1.692509 & 0.614986 & -1.795748\end{array}$

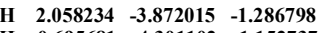

$\begin{array}{llll}\text { H } & -0.695681 & -4.301102 & -1.152737\end{array}$

$\begin{array}{llll}\text { H } & \mathbf{1 . 5 1 1 0 3 5} & \mathbf{1 . 5 0 5 4 7 2} & 4.319489\end{array}$

H $\quad 1.077033 \quad 3.712924 \quad 3.272050$

54

unsat model 14e1 / electronic energy: $\mathbf{- 2 0 8 9 . 9 5 2 1 4 7 0 9}$ a.u. / lowest freq: $\mathbf{- 2 5 . 9 8} \mathbf{~ c m - 1}$

$\begin{array}{lllll}S & -0.752822 & 1.631434 & 1.726388\end{array}$

$\begin{array}{llll}C & 0.229069 & -0.890758 & 0.130167\end{array}$

$\begin{array}{llll}\text { Ru } & -0.165915 & 1.060672 & -0.402625\end{array}$

$\begin{array}{llll}C & \mathbf{1 . 4 8 1 6 2 0} & \mathbf{1 . 7 8 0 2 4 3} & \mathbf{- 0 . 4 0 1 3 0 1}\end{array}$

$\begin{array}{lrrr}C & -1.728265 & 2.610896 & -1.174031\end{array}$

$\begin{array}{llll}\text { N } & 1.378856 & -1.575564 & 0.362570\end{array}$

$\begin{array}{lllll}\text { N } & \mathbf{- 0 . 7 4 7 9 6 6} & \mathbf{- 1 . 8 2 3 4 8 3} & \mathbf{0 . 2 8 7 7 8 4}\end{array}$

$\begin{array}{llll}\text { C } & 2.697061 & -1.049637 & 0.254900\end{array}$

$\begin{array}{llll}C & 2.121893 & -2.909607 & 0.652203\end{array}$

C $-0.218942-3.065998 \quad 0.602249$

$\begin{array}{llll}C & -0.218942 & -3.065998 & 0.602249 \\ \text { C } & -2.134985 & -1.564503 & 0.081625\end{array}$ 
$\begin{array}{llll}C & 5.313669 & -0.100985 & -0.061885\end{array}$ $\begin{array}{llll}C & 3.409467 & -1.283957 & -0.918766\end{array}$

$\begin{array}{llll}C & 3.292766 & -0.339950 & 1.30495\end{array}$

$\begin{array}{llll}C & 4.595803 & 0.129847 & 1.117949\end{array}$

$\begin{array}{llll}C & 4.705703 & -0.828279 & -1.090658\end{array}$

$5.070735-0.688922 \quad 1.927950$

$\begin{array}{llll}\mathrm{H} & 5.070735 & 0.688922 & 1.927950\end{array}$

$\begin{array}{llll}H & 5.220934 & -1.034425 & -2.030449\end{array}$

$792-0.436143$

$\begin{array}{llll}C & -2.607528 & -1.441659 & -1.225415\end{array}$

$\begin{array}{llll}C & -3.018988 & -1.423868 & 1.159257\end{array}$

$\begin{array}{llll}C & -4.358105 & -1.143663 & 0.872933\end{array}$

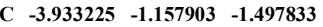

$\begin{array}{llll}\text { F } & -1.745246 & -1.588208 & -2.233647\end{array}$

$\begin{array}{llll}C & -2.523071 & -1.539543 & \mathbf{2 . 5 7 2 3 1 9}\end{array}$

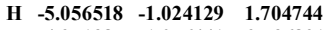

$\begin{array}{llll}\text { H } & -4.251385 & -1.056441 & -2.536891\end{array}$

$\begin{array}{llll}\text { C } & -6.272947 & -0.673722 & -0.712992\end{array}$

-1.412699
-199356

$\begin{array}{llll}\text { H } & -3.349664 & -1.437812 & 3.286920\end{array}$

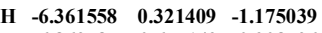

$\begin{array}{llll}\text { H } & -6.869587 & -0.675149 & 0.208796\end{array}$

$\begin{array}{llll}\text { H } & -1.789547 & -0.741926 & 2.771161\end{array}$

H $\quad \begin{array}{rrrr}-2.029020 & -2.505863 & 2.752516\end{array}$

$\begin{array}{llll}\text { F } & 2.809762 & -1.956155 & -1.902228\end{array}$

$\begin{array}{llll}\text { C } & 6.702139 & 0.451992 & -0.239458\end{array}$

$\begin{array}{llll}\text { H } & 7.202262 & 0.596502 & 0.727791\end{array}$

$\begin{array}{llll}\text { H } & 7.322574 & -0.209998 & -0.859288\end{array}$

$\begin{array}{llll}\text { H } & 6.660632 & 1.431533 & -0.741900\end{array}$

$\begin{array}{llll}C & 2.533751 & -0.080279 & \mathbf{2 . 5 7 4 0 2 1}\end{array}$

$\begin{array}{lllll}\text { H } & 1.730104 & 0.652584 & 2.397565\end{array}$

$\begin{array}{llll}\text { H } & \mathbf{2 . 0 5 7 1 1 2} & \mathbf{- 0 . 9 9 7 2 7 4} & \mathbf{2 . 9 5 0 9 4 4}\end{array}$

H $3.197211 \quad 0.314193 \quad 3.354040$

$\begin{array}{llll}C & -1.896760 & \mathbf{2 . 9 4 9 2 2 4} & \mathbf{1 . 5 2 0 4 6 9}\end{array}$

C $-2.299619 \quad 3.367200 \quad 0.309160$

$\begin{array}{llll}C & -2.299619 & 3.367200 & 0.309160\end{array}$

$\begin{array}{lllll}\text { H } & 1.905952 & 2.316789 & 0.464986\end{array}$

$\begin{array}{llll}C & 2.294294 & 1.851826 & -1.659323\end{array}$

$\begin{array}{lllll}\text { H } & \mathbf{2 . 3 5 9 0 8 0} & \mathbf{2 . 8 9 8 1 0 4} & \mathbf{- 2 . 0 0 4 4 3 1}\end{array}$

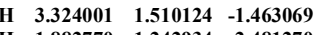

H $\quad \begin{array}{llll}\mathbf{1 . 8 8 2 7 7 0} & 1.242934 & -2.481270\end{array}$

$\begin{array}{lllll}\text { H } & 1.919470 & -3.615738 & 0.860905\end{array}$

\begin{tabular}{cccc}
\hline H & $\mathbf{- 0 . 8 4 6 5 1 7}$ & $\mathbf{- 3 . 9 3 8 9 3 7}$ & $\mathbf{0 . 7 5 3 0 1 2}$ \\
\hline & -2.268672 & $\mathbf{3 . 4 0 7 8 9 5}$ & $\mathbf{2 . 4 4 2 2 9 5}$
\end{tabular}

$\begin{array}{llll}-3.011795 & 4.190433 & 0.199006\end{array}$

unsat_model_pc1_c / electronic energy: $-\mathbf{2 4 3 5 . 4 1 0 2 8 3 4 8}$ a.u. / lowest freq: $20.04 \mathrm{~cm}-1$

$\begin{array}{lll}0.320835 & -0.027810 & 2.539889\end{array}$

$\begin{array}{llll}C & -0.356098 & -1.181224 & -0.268184\end{array}$

$\begin{array}{rrrr}\text { Ru } & -0.099069 & \mathbf{0 . 7 9 6 2 7 3} & \mathbf{0 . 4 0 1 9 3 8}\end{array}$

$\begin{array}{llll}\text { C } & -1.874567 & 1.214404 & 0.683763\end{array}$

$\begin{array}{llll}C & 0.579032 & 1.265339 & -1.675750\end{array}$

$\begin{array}{lllll}\text { S } & \mathbf{0 . 8 5 6 8 3 0} & \mathbf{2 . 8 2 2 3 8 2} & 1.170289\end{array}$

N $0.632410-2.092973-0.513629$

$\begin{array}{llll}\text { N } & -1.491395 & -1.865681 & -0.589479\end{array}$

$\begin{array}{llll}C & 2.031596 & -1.919203 & -0.285872\end{array}$

$\begin{array}{llll}C & 0.122671 & \mathbf{- 3 . 2 9 5 3 2 5} & \mathbf{- 0 . 9 8 0 3 3 8}\end{array}$

$\begin{array}{lrrr}C & -1.217170 & -3.153014 & -1.028770\end{array}$

C $-\mathbf{2 . 8 2 4 6 2 4}-\mathbf{- 1 . 3 8 1 5 7 6}-\mathbf{- 0 . 4 8 0 9 0 0}$

$\begin{array}{llll}C & 4.796400 & -1.576849 & 0.057048\end{array}$

$\begin{array}{llll}C & 2.840849 & -1.465074 & -1.327161\end{array}$

$\begin{array}{llll}C & 2.622133 & -2.300263 & 0.927293\end{array}$

C $\quad 3.997179-\mathbf{2 . 0 9 7 2 2 5} \quad \mathbf{1 . 0 7 9 5 1 0}$

C $4.202201-1.281711-1.173579$

$\begin{array}{llll}\text { H } & 4.461577 & -2.370315 & 2.030311\end{array}$

$\begin{array}{llll}\text { H } & 4.782419 & -\mathbf{0 . 9 0 7 3 4 3} & -\mathbf{2 . 0 1 8 5 0 1}\end{array}$

$\begin{array}{lrrr}C & -\mathbf{5 . 4 2 8 0 2 0} & -\mathbf{0 . 3 5 1 4 8 9} & \mathbf{- 0 . 3 5 4 3 4 2}\end{array}$

$\begin{array}{llll}C & \mathbf{- 3 . 4 5 5 6 1 6} & \mathbf{- 0 . 8 7 6 3 8 7} & \mathbf{- 1 . 6 1 6 2 1 1}\end{array}$

$\begin{array}{llll}C & \mathbf{- 3 . 5 1 4 6 6 7} & \mathbf{- 1 . 4 2 8 1 8 8} & \mathbf{0 . 7 3 6 1 0 6}\end{array}$

$\begin{array}{llll}C & -4.811525 & -0.904570 & 0.772282\end{array}$

$\begin{array}{llll}C & -4.733770 & -0.350537 & -1.570814\end{array}$

$\begin{array}{lllll}\text { F } & -2.785665 & -0.880919 & -2.772187\end{array}$

$\begin{array}{llll}\text { C } & -2.860161 & -1.986709 & 1.966292\end{array}$

$\begin{array}{llll}\text { H } & -5.356145 & -0.925522 & 1.719461\end{array}$

$\begin{array}{lll}-5.356145 & -0.925522 & 1.719461\end{array}$

$\begin{array}{llll}\text { H } & -5.176149 & 0.053484 & -2.483066\end{array}$

$\begin{array}{llll}C & -6.809663 & 0.239265 & -0.275676\end{array}$

$\begin{array}{llll}\text { H } & -7.467786 & -0.189357 & -1.045748\end{array}$

$\begin{array}{llll}\text { H } & -2.361469 & -2.944785 & 1.756951\end{array}$

$\begin{array}{llll}\text { H } & -6.773517 & \mathbf{1 . 3 2 6 2 6 2} & \mathbf{- 0 . 4 4 5 9 8 8}\end{array}$

$\begin{array}{llll}\text { H } & -7.266887 & \mathbf{0 . 0 6 4 4 2 9} & \mathbf{0 . 7 0 7 0 2 2}\end{array}$

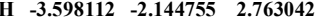

$\begin{array}{llll}\text { H } & -2.087276 & -1.294742 & 2.339479\end{array}$

$\begin{array}{llll}\text { F } & 2.281151 & -1.230530 & -2.516686\end{array}$

$\begin{array}{llll}\text { C } & 6.265377 & -1.327916 & 0.267696\end{array}$

$\begin{array}{cccc}\text { H } & 6.446369 & -\mathbf{0 . 2 5 8 7 7 0} & \mathbf{0 . 4 6 2 0 6 9}\end{array}$

$\begin{array}{llll}\text { H } & 6.650728 & -1.892657 & 1.127206\end{array}$

$\begin{array}{lllll}\text { H } & 6.848933 & -1.603249 & -0.622468\end{array}$

$\begin{array}{lllll}\text { C } & 1.835878 & -2.995978 & 2.003517\end{array}$

$\begin{array}{lllll}\text { H } & 0.759324 & -2.804681 & 1.926097\end{array}$

\begin{tabular}{cccc}
\hline & 1.997129 & -4.083694 & 1.927667
\end{tabular}

$\begin{array}{llll}\text { H } & \mathbf{2 . 1 6 6 3 1 5} & \mathbf{- 2 . 6 7 3 2 7 6} & \mathbf{2 . 9 9 9 3 5 5}\end{array}$

$\begin{array}{llll}C & 0.813964 & 1.368643 & 3.466928\end{array}$

$\begin{array}{llll}\text { C } & \mathbf{1 . 0 4 9 1 0 9} & \mathbf{2 . 5 5 9 9 6 0} & \mathbf{2 . 8 8 4 3 3 6}\end{array}$

$\begin{array}{llll}C & 1.049109 & 2.559960 & 2.884336\end{array}$

\begin{tabular}{llll}
$C$ & -2.448576 & 1.762005 & 1.943037 \\
\hline
\end{tabular}

$\begin{array}{llll}\text { H } & -2.641614 & 1.190179 & -0.107127\end{array}$ 


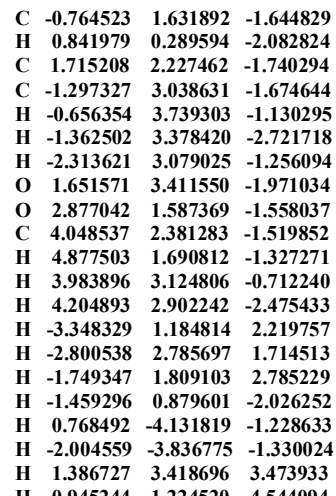

$\begin{array}{cccc}\mathrm{H} & 0.945244 & 1.224520 & 4.544094\end{array}$

unsat model ts1 c / electronic energy: $\mathbf{- 2 4 3 5 . 4 0 0 6 3 9 8 7}$ a.u. / lowest freq: $-\mathbf{1 3 8 . 7 7} \mathbf{c m}-1$

$\begin{array}{lllll}S & \mathbf{0 . 8 7 6 9 9 5} & \mathbf{0 . 7 0 7 7 8 5} & -\mathbf{2 . 2 6 4 2 3 0}\end{array}$

$\begin{array}{llll}C & 0.376891 & -1.157519 & 0.091878\end{array}$

$\begin{array}{llll}\text { Ru } & \mathbf{0 . 1 1 3 2 9 2} & \mathbf{0 . 9 1 4 4 9 2} & \mathbf{- 0 . 0 9 9 7 5 7}\end{array}$

$\begin{array}{llll}\text { C } & \mathbf{1 . 6 1 2 5 2 7} & \mathbf{1 . 2 7 1 0 1 8} & \mathbf{0 . 9 3 4 7 9 7}\end{array}$

$\begin{array}{lrrr}C & -1.387061 & 0.913891 & 1.450986\end{array}$

$\begin{array}{lllll}\mathrm{S} & \mathbf{- 0 . 6 0 6 4 0 3} & \mathbf{3 . 1 1 0 5 5 3} & \mathbf{- 0 . 7 5 6 6 8 7}\end{array}$

$\begin{array}{llll}\mathrm{N} & \mathbf{- 0 . 5 9 6 9 5 1} & \mathbf{- 2 . 1 2 0 3 1 7} & \mathbf{0 . 0 4 2 1 5 2}\end{array}$

$\begin{array}{llll}\mathrm{N} & \mathbf{1 . 5 2 7 4 8 3} & -1.901927 & 0.152964\end{array}$

C $-\mathbf{- 2 . 0 1 0 6 9 0}-\mathbf{- 1 . 9 4 6 4 8 3}-\mathbf{- 0 . 0 7 6 8 5 5}$

$\begin{array}{llll}\text { C } & \mathbf{- 0 . 0 6 7 7 9 6} & \mathbf{- 3 . 4 0 2 4 1 4} & \mathbf{0 . 0 8 7 6 7 2}\end{array}$

$\begin{array}{llll}\text { C } & \mathbf{1 . 2 6 9 8 4 2} & \mathbf{- 3 . 2 6 4 4 4 5} & \mathbf{0 . 1 5 7 8 4 5}\end{array}$

$\begin{array}{llll}\text { C } & 2.874876 & -1.432061 & 0.165287\end{array}$

$\begin{array}{llll}C & -4.810770 & -1.787928 & -0.191833\end{array}$

$\begin{array}{llll}C & -2.795341 & -2.071745 & 1.069716\end{array}$

$\begin{array}{llll}C & -2.633688 & -1.796380 & -1.320927\end{array}$

$\begin{array}{llll}C & -4.030400 & -1.705085 & -1.347044\end{array}$

$\begin{array}{llll}C & -4.172705 & -1.984594 & 1.037709\end{array}$

$\begin{array}{llll}\text { H } & -4.524195 & -1.576080 & -2.313359\end{array}$

$\begin{array}{llll}\text { H } & -4.732645 & -2.067705 & 1.970824\end{array}$

$\begin{array}{llll}\text { C } & \mathbf{5 . 5 2 8 5 1 1} & \mathbf{- 0 . 5 2 4 1 6 5} & \mathbf{0 . 3 2 1 3 1 4}\end{array}$

$\begin{array}{llll}C & 3.479970 & -1.156950 & 1.392789\end{array}$

$\begin{array}{llll}C & 3.635398 & -1.344772 & -1.006903\end{array}$

$\begin{array}{llll}C & 4.948282 & -\mathbf{0 . 8 6 7 3 3 0} & \mathbf{- 0 . 9 0 1 4 5 9}\end{array}$

$\begin{array}{llll}\text { C } & \mathbf{4 . 7 7 7 2 8 8} & \mathbf{- 0 . 6 9 3 8 3 6} & \mathbf{1 . 4 9 1 8 3 4}\end{array}$

$\begin{array}{llll}\text { F } & \mathbf{2 . 7 5 7 3 5 0} & \mathbf{- 1 . 3 2 4 3 2 1} & \mathbf{2 . 5 0 6 4 7 7}\end{array}$

$\begin{array}{llll}C & 3.115864 & -1.830693 & -2.331684\end{array}$

$\begin{array}{llll}\text { H } & 5.540470 & -0.775406 & -1.815423\end{array}$

$\begin{array}{llll}\text { H } & \mathbf{5 . 1 9 0 1 3 5} & \mathbf{- 0 . 4 7 0 8 2 8} & \mathbf{2 . 4 7 7 1 3 0}\end{array}$

$\begin{array}{llll}\text { C } & 6.928797 & \mathbf{0 . 0 2 1 8 2 1} & \mathbf{0 . 3 9 5 5 5 1}\end{array}$

$\begin{array}{llll}\text { H } & \mathbf{7 . 4 9 6 5 6 8} & \mathbf{- 0 . 4 4 8 4 6 4} & \mathbf{1 . 2 1 1 2 0 4}\end{array}$

$\begin{array}{llll}\text { H } & 3.547044 & -2.821459 & -2.547805\end{array}$

$\begin{array}{llll}\text { H } & \mathbf{6 . 9 0 7 2 3 4} & 1.105137 & \mathbf{0 . 5 9 2 8 5 5}\end{array}$

$\begin{array}{llll}\text { H } & \mathbf{7 . 4 7 1 8 5 3} & \mathbf{- 0 . 1 3 7 2 1 7} & \mathbf{- 0 . 5 4 5 4 8 9}\end{array}$

$\begin{array}{llll}\text { H } & 3.412909 & -1.150504 & -3.140457\end{array}$

$\begin{array}{llll}\text { H } & 2.023799 & -1.920228 & -2.349180\end{array}$

$\begin{array}{llll}\text { F } & -2.176056 & -2.263861 & 2.241181\end{array}$

$\begin{array}{llll}C & -6.310105 & -1.671393 & -0.249056\end{array}$

$\begin{array}{llll}\text { H } & -6.654932 & -0.809204 & 0.342343\end{array}$

$\begin{array}{llll}\text { H } & -6.665881 & -1.542590 & -1.279963\end{array}$

$\begin{array}{lllll}\text { H } & -6.791393 & -2.566712 & 0.172464\end{array}$

$\begin{array}{llll}\text { C } & -1.828756 & -1.770407 & -2.587308\end{array}$

$\begin{array}{llll}\text { H } & -1.139603 & -2.628012 & -2.633645\end{array}$

$\begin{array}{llll}\text { H } & -2.484232 & -1.804170 & -3.466865\end{array}$

$\begin{array}{llll}\text { H } & -1.208914 & -0.862533 & -2.643105\end{array}$

$\begin{array}{lllll}C & 0.664031 & 2.283436 & -3.005988\end{array}$

$\begin{array}{lllll}C & 0.035456 & 3.285924 & -2.368423\end{array}$

$\begin{array}{llll}\text { C } & \mathbf{2 . 5 3 8 1 3 6} & \mathbf{2 . 4 1 8 1 5 8} & \mathbf{0 . 7 2 1 1 7 0}\end{array}$

$\begin{array}{llll}\text { H } & 1.990261 & 0.585754 & 1.701331\end{array}$

$\begin{array}{llll}C & \mathbf{- 0 . 2 4 5 5 3 9} & \mathbf{1 . 4 0 4 4 6 8} & \mathbf{2 . 1 4 4 8 8 2}\end{array}$

$\begin{array}{llll}\text { H } & -1.650340 & -0.120335 & 1.655170\end{array}$

$\begin{array}{llll}C & -2.590694 & 1.747681 & 1.169993\end{array}$

$\begin{array}{llll}C & \mathbf{- 0 . 1 0 0 4 4 8} & \mathbf{2 . 8 0 7 4 9 7} & \mathbf{2 . 6 8 0 3 5 2}\end{array}$

$\begin{array}{llll}\text { H } & -\mathbf{0 . 3 6 2 5 6 0} & 3.565668 & 1.935119\end{array}$

$\begin{array}{llll}\text { H } & \mathbf{- 0 . 7 8 7 9 7 2} & \mathbf{2 . 9 2 4 4 8 1} & \mathbf{3 . 5 3 2 0 6 8}\end{array}$

$\begin{array}{llll}\text { H } & 0.921146 & 2.988443 & 3.040752\end{array}$

$\begin{array}{lllll}\text { O } & -2.851616 & 2.824852 & 1.651649\end{array}$

$\begin{array}{llll}\text { O } & -3.413739 & 1.111099 & 0.330481\end{array}$

$\begin{array}{llll}C & -4.591412 & 1.794566 & -0.054627\end{array}$

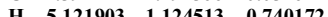

\begin{tabular}{llll} 
H & -5.121903 & 1.124513 & -0.740172 \\
\hline
\end{tabular}

$\begin{array}{cccc}\text { H } & -4.341701 & \mathbf{2 . 7 3 5 5 1 8} & \mathbf{- 0 . 5 6 6 3 9 0}\end{array}$

$\begin{array}{llll}\text { H } & \mathbf{- 5 . 2 2 2 4 5 0} & \mathbf{2 . 0 1 9 6 0 5} & \mathbf{0 . 8 1 7 5 9 1}\end{array}$

$\begin{array}{lllll}\text { H } & 3.402718 & 1.992172 & 0.176731\end{array}$

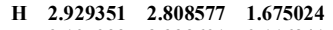

$\begin{array}{ccccc}\text { H } & 2.107099 & 3.223601 & 0.116341\end{array}$

\begin{tabular}{lllll}
\hline H & $\mathbf{0 . 2 2 9 0 2 0}$ & $\mathbf{0 . 6 4 3 5 0 9}$ & $\mathbf{2 . 7 7 1 3 7 4}$
\end{tabular}

$\begin{array}{llll}\text { H } & -0.701422 & -4.283610 & 0.062753\end{array}$

$\begin{array}{llll}\text { H } & \mathbf{2 . 0 6 8 1 7 2} & \mathbf{- 3 . 9 9 8 2 6 1} & \mathbf{0 . 2 0 7 6 1 1}\end{array}$

$\begin{array}{lllll}H & 1.061516 & \mathbf{2 . 3 9 9 2 1 4} & \mathbf{- 4 . 0 1 9 2 1 3}\end{array}$

$\begin{array}{lllll}\text { H } & -0.108786 & 4.256245 & -2.855416\end{array}$

69 
unsat_model_mcb_c / electronic energy: $-\mathbf{2 4 3 5 . 4 1 0 1 1 0 2 5}$ a.u. / lowest freq: $19.05 \mathrm{~cm}-1$ $\begin{array}{llll}S & -0.512007 & 0.566042 & 2.360737\end{array}$

$\begin{array}{llll}C & -0.376099 & -1.185627 & 0.003600\end{array}$

$\begin{array}{llll}\text { Ru } & -\mathbf{0 . 1 5 0 8 5 1} & \mathbf{0 . 9 0 5 4 8 9} & \mathbf{0 . 1 5 6 2 0 8}\end{array}$

$\begin{array}{llll}C & -1.403517 & 1.969949 & -1.316047\end{array}$

$\begin{array}{llll}C & -1.403517 & 1.169949 & -1.316047\end{array}$

\begin{tabular}{llll}
$C$ & 1.293504 & 0.947142 & -1.219304 \\
\hline & 0.212682 & 3.222498 & 0.730157
\end{tabular}

$\begin{array}{lllll}\mathrm{S} & 0.212682 & 3.222498 & \mathbf{0 . 7 3 0 1 5 7}\end{array}$

$\begin{array}{llll}\mathrm{N} & \mathbf{0 . 5 9 0 9 1 1} & -2.152172 & \mathbf{0 . 0 5 6 5 4 3}\end{array}$

\begin{tabular}{lrrr} 
N & -1.534380 & -1.917427 & 0.009683 \\
\hline
\end{tabular}

$\begin{array}{llll}\text { C } & \mathbf{2 . 0 1 0 4 4 8} & \mathbf{- 1 . 9 7 9 2 2 9} & \mathbf{0 . 0 4 8 9 8 5}\end{array}$

$\begin{array}{llll}C & 0.049972 & -3.429257 & 0.082253\end{array}$

$\begin{array}{llll}C & -1.289172 & -3.280975 & 0.052587\end{array}$

C $\quad-\mathbf{2 . 8 7 4 5 6 8}-\mathbf{- 1 . 4 2 5 5 2 3}-\mathbf{- 0 . 0 5 2 2 8 5}$

$\begin{array}{llll}C & 4.802509 & -1.742053 & -0.116613\end{array}$

$\begin{array}{llll}C & 2.673410 & -2.020036 & -1.177207\end{array}$

$\begin{array}{llll}C & 2.756911 & -1.886342 & 1.228991\end{array}$

$\begin{array}{llll}C & 4.146331 & -1.759249 & 1.115946\end{array}$

$\begin{array}{llll}C & \mathbf{4 . 0 4 4 1 4 7} & -\mathbf{1 . 8 8 8 8 0 7} & \mathbf{- 1 . 2 8 3 6 1 1}\end{array}$

$\begin{array}{llll}\text { H } & 4.735393 & -1.675142 & \mathbf{2 . 0 3 2 6 5 8}\end{array}$

$\begin{array}{llll}\text { H } & 4.505774 & -1.901878 & -2.272403\end{array}$

$\begin{array}{llll}C & -5.502104 & -0.469231 & -0.343367\end{array}$

$\begin{array}{llll}C & -3.465872 & -1.281776 & -1.310262\end{array}$

$\begin{array}{llll}\text { C } & -\mathbf{3 . 6 4 2 4 6 3} & -1.183342 & 1.092047\end{array}$

$\begin{array}{llll}\text { C } & -4.941551 & -\mathbf{0 . 6 8 6 5 8 1} & 0.915811\end{array}$

$\begin{array}{llll}C & -4.747266 & -0.796956 & -1.477487\end{array}$

$\begin{array}{llll}\text { F } & -2.746431 & -1.614234 & -2.388488\end{array}$

$\begin{array}{llll}\text { C } & -\mathbf{3 . 1 5 7 6 6 3} & \mathbf{- 1 . 5 0 8 1 4 8} & \mathbf{2 . 4 7 7 3 5 2}\end{array}$

$\begin{array}{llll}\text { H } & \mathbf{- 5 . 5 3 9 0 7 9} & \mathbf{- 0 . 4 7 7 5 7 5} & \mathbf{1 . 8 0 6 9 7 0}\end{array}$

$\begin{array}{llll}\text { H } & -5.146205 & -0.681616 & -2.486731\end{array}$

$\begin{array}{llll}\text { C } & \mathbf{- 6 . 8 8 2 2 8 2} & \mathbf{0 . 1 1 0 9 2 1} & \mathbf{- 0 . 4 9 3 8 1 2}\end{array}$

$\begin{array}{lllll}\text { H } & -7.425743 & -0.360440 & -1.324841\end{array}$

$\begin{array}{llll}\text { H } & -\mathbf{3 . 8 0 5 2 6 6} & -\mathbf{2 . 2 8 3 1 1 8} & \mathbf{2 . 9 1 5 3 9 1}\end{array}$

$\begin{array}{llll}\text { H } & -6.819672 & 1.189165 & -0.710390\end{array}$

$\begin{array}{llll}\text { H } & -7.471237 & -\mathbf{0 . 0 1 1 2 6 2} & \mathbf{0 . 4 2 5 0 5 7}\end{array}$

H $-3.205766 \quad-0.622428 \quad 3.125246$

$\begin{array}{llll}\text { H } & -2.123989 & -1.871913 & 2.488270\end{array}$

$\begin{array}{llll}\text { F } & \mathbf{1 . 9 3 9 5 1 2} & \mathbf{- 2 . 1 6 0 9 7 3} & \mathbf{- 2 . 2 8 7 9 5 7}\end{array}$

$\begin{array}{lllll}C & \mathbf{6 . 2 9 2 8 6 7} & \mathbf{- 1 . 5 5 5 2 8 1} & \mathbf{- 0 . 2 0 5 8 2 3}\end{array}$

$\begin{array}{lllll}\text { H } & 6.772266 & -1.660668 & 0.776561\end{array}$

$\begin{array}{lllll}\text { H } & 6.745444 & -2.283598 & -0.894362\end{array}$

$\begin{array}{llll}\text { H } & 6.529440 & -0.551287 & -0.592056\end{array}$

$\begin{array}{llll}\text { C } & \mathbf{2 . 0 9 1 1 5 6} & \mathbf{- 1 . 9 3 7 1 0 4} & \mathbf{2 . 5 7 2 6 5 8}\end{array}$

$\begin{array}{lllll}\text { H } & 1.379724 & -2.774402 & 2.635430\end{array}$

$\begin{array}{lllll}\text { H } & 2.834688 & -2.051396 & 3.371644\end{array}$

$\begin{array}{llll}\text { H } & 1.515942 & -1.016763 & 2.753630\end{array}$

$\begin{array}{llll}\text { C } & \mathbf{- 0 . 3 1 8 5 9 7} & \mathbf{2 . 1 2 3 5 4 0} & \mathbf{3 . 1 5 1 2 4 9}\end{array}$

$\begin{array}{llll}C & \mathbf{- 0 . 0 1 2 6 8 7} & \mathbf{3 . 2 3 0 6 7 7} & \mathbf{2 . 4 5 2 7 5 8}\end{array}$

$\begin{array}{llll}C & -2.426485 & 2.277281 & -1.371466\end{array}$

$\begin{array}{lllll}\text { H } & -1.812907 & 0.265702 & -1.766017\end{array}$

$\begin{array}{llll}C & 0.055983 & 1.420320 & -2.044350\end{array}$

$\begin{array}{ccccc}\text { H } & \mathbf{1 . 6 3 9 0 2 8} & \mathbf{- 0 . 0 2 6 8 1 7} & \mathbf{- 1 . 5 6 4 0 1 4} \\ \mathrm{C} & 2.503613 & \mathbf{1 . 8 0 9 1 1 3} & \mathbf{1 . 0 2 4 6 9 7}\end{array}$

C $0.503613 \quad 1.80113-1.024697$

\begin{tabular}{llll} 
C & 0.138219 & 2.810819 & -2.668585 \\
\hline
\end{tabular}

$\begin{array}{llll}\mathrm{C} & 0.181113 & 3.604059 & -1.912597\end{array}$

$\begin{array}{lllll}\text { H } & 1.043184 & 2.889418 & -3.281161\end{array}$

$\begin{array}{lllll}\text { H } & -\mathbf{0 . 7 3 7 3 0 7} & \mathbf{2 . 9 8 2 7 4 7} & \mathbf{- 3 . 3 0 8 9 0 8}\end{array}$

$\begin{array}{lllll}\text { O } & 2.824363 & 2.808085 & -1.621122 \\ \text { O } & 3.285076 & \mathbf{1 . 2 4 2 4 3 2} & -\mathbf{0 . 0 9 7 0 5 8}\end{array}$

$\begin{array}{llll}C & 4.519693 & 1.876555 & 0.181702\end{array}$

$\begin{array}{llll}\text { H } & 4.356545 & 2.879842 & 0.601139\end{array}$

$\begin{array}{llll}\text { H } & \mathbf{5 . 1 3 1 5 7 1} & \mathbf{1 . 9 6 5 2 2 9} & \mathbf{- 0 . 7 2 7 8 4 4}\end{array}$

$\begin{array}{llll}\text { H } & \mathbf{5 . 0 2 7 9 1 8} & \mathbf{1 . 2 4 1 6 3 0} & \mathbf{0 . 9 1 6 2 3 4}\end{array}$

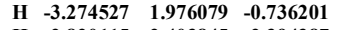

$\begin{array}{lllll}\text { H } & \mathbf{- 2 . 8 2 0 1 1 5} & \mathbf{2 . 4 0 3 8 4 5} & \mathbf{- 2 . 3 9 4 3 8 7}\end{array}$

\begin{tabular}{llll} 
H & -2.043727 & 3.234978 & -1.002595 \\
\hline
\end{tabular}

$\begin{array}{llll}\text { H } & -\mathbf{0 . 0 1 8 3 9 7} & \mathbf{0 . 6 5 6 3 9 1} & -\mathbf{2 . 8 2 9 8 7 7}\end{array}$

$\begin{array}{llll}\text { H } & 0.676508 & -4.315186 & 0.117739\end{array}$

$\begin{array}{llll}\text { H } & -2.094603 & -4.008692 & 0.057886\end{array}$

$\begin{array}{llll}\text { H } & 0.110763 & 4.192797 & 2.960770\end{array}$

$\begin{array}{llll}\text { H } & -0.459671 & 2.129242 & 4.236005\end{array}$

69

unsat model ts2 c / electronic energy: $\mathbf{- 2 4 3 5 . 3 9 2 8 8 1 4 8}$ a.u. / lowest freq: $\mathbf{- 1 7 3 . 1 2} \mathbf{c m - 1}$

$\begin{array}{llll}S & 0.875011 & 1.121940 & -1.924216\end{array}$

$\begin{array}{llll}C & 0.285583 & -1.310853 & -0.144812\end{array}$

$\begin{array}{lllll}\text { Ru } & \mathbf{0 . 1 3 3 4 3 6} & \mathbf{0 . 7 6 0 3 2 4} & \mathbf{0 . 2 1 4 4 5 3}\end{array}$

$\begin{array}{llll}\text { C } & \mathbf{0 . 6 0 0 7 4 1} & \mathbf{0 . 2 5 5 1 3 3} & \mathbf{2 . 2 5 4 0 2 0}\end{array}$

$\begin{array}{llll}C & -1.709820 & 0.850055 & 0.383264\end{array}$

$\begin{array}{lllll}\text { S } & 0.443117 & 3.121654 & 0.543064\end{array}$

$\begin{array}{lllll}\mathrm{N} & \mathbf{- 0 . 6 4 5 3 7 0} & \mathbf{- 2 . 2 9 5 3 8 0} & -\mathbf{0 . 3 0 4 5 1 5}\end{array}$

$\begin{array}{llll}N & 1.466768 & -2.003362 & -0.223561\end{array}$

$\begin{array}{llll}C & -2.056778 & -2.122720 & -0.317219\end{array}$

$\begin{array}{llll}C & -0.069520 & -3.547555 & -0.466739\end{array}$

$\begin{array}{llll}C & 1.264383 & -3.360928 & -0.416759\end{array}$

$\begin{array}{llll}C & \mathbf{2 . 7 8 2 6 8 0} & \mathbf{- 1 . 4 5 0 9 2 5} & \mathbf{- 0 . 1 9 7 5 5 0}\end{array}$

$\begin{array}{llll}C & \mathbf{- 4 . 8 2 5 2 4 1} & \mathbf{- 1 . 7 1 6 7 5 6} & \mathbf{- 0 . 2 0 0 3 5 4}\end{array}$

$\begin{array}{llll}C & -2.774788 & -2.393258 & 0.845346\end{array}$

$\begin{array}{llll}C & -2.724164 & -1.673552 & -1.463031\end{array}$

$\begin{array}{llll}\text { C } & -4.105057 & -1.473009 & -1.375534\end{array}$

$\begin{array}{llll}\text { C } & -4.141454 & -2.191041 & 0.925479\end{array}$

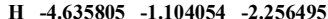

$\begin{array}{llll}\text { H } & -4.655782 & -2.394535 & \mathbf{1 . 8 6 6 2 4 8}\end{array}$

$\begin{array}{llll}C & 5.362423 & -0.348253 & -0.070814\end{array}$

$\begin{array}{llll}\text { C } & 5.362423 & -0.348253 & -0.070814 \\ \text { C } & 3.443903 & -1.292587 & 1.019604\end{array}$

$\begin{array}{llll}\text { C } & 3.443903 & -1.292587 & 1.019604\end{array}$ 
$\begin{array}{llll}C & 3.457580 & -1.148088 & -1.389014\end{array}$ $\begin{array}{llll}C & 4.733671 & -0.585768 & -1.297187\end{array}$ $\begin{array}{llll}C & 4.706760 & -0.732155 & 1.103203\end{array}$

$\begin{array}{llll}\text { F } & 2.854479 & -1.721993 & \mathbf{2 . 1 3 8 1 2 7}\end{array}$

C $2.864673-1.490305-2.727506$

H $5.257135-0.328453-2.221339$

$\begin{array}{lll}5.257135 & -0.328453 & -2.221339 \\ C & -169532-0.614221 & 2.084630\end{array}$

$\begin{array}{llll}H & 5.169532 & -0.614221 & 2.084630\end{array}$

$\begin{array}{llll}\text { C } & 6.713610 & 0.310591 & \mathbf{- 0 . 0 0 6 5 1}\end{array}$

$\begin{array}{llll}\text { H } & 7.322105 & -0.099229 & 0.811755\end{array}$

$\begin{array}{llll}\text { H } & 3.156236 & -2.514914 & -3.011206\end{array}$

$\begin{array}{llll}\text { H } & 6.600166 & 1.391092 & 0.175129\end{array}$

$\begin{array}{llll}\text { H } & \mathbf{7 . 2 6 4 1 5 3} & \mathbf{0 . 1 8 7 6 9 8} & \mathbf{- 0 . 9 4 8 9 7 6}\end{array}$

$\begin{array}{lllll}\text { H } & 3.232751 & -\mathbf{0 . 8 0 6 7 1 1} & \mathbf{- 3 . 5 0 3 3 5 0}\end{array}$

$\begin{array}{llll}\text { H } & 1.769088 & -1.439292 & -2.721031\end{array}$

$\begin{array}{llll}\text { F } & -2.105349 & -2.812695 & 1.922949\end{array}$

$\begin{array}{llll}\text { C } & -6.314080 & -1.505077 & -0.147412\end{array}$

$\begin{array}{llll}\text { H } & -6.649037 & -0.818508 & -0.936279\end{array}$

$\begin{array}{llll}\text { H } & -6.841792 & -\mathbf{2 . 4 6 1 6 9 8} & -\mathbf{0 . 2 8 7 8 5 6}\end{array}$

$\begin{array}{llll}\text { H } & -6.624361 & -1.097173 & 0.824714\end{array}$

$\begin{array}{llll}C & -1.958751 & -1.362723 & -2.716305\end{array}$

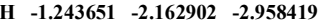

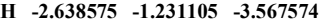

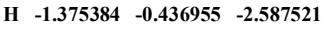

$\begin{array}{llll}\text { C } & 1.024667 & \mathbf{2 . 8 6 2 6 3 3} & \mathbf{- 2 . 0 9 2 1 5 5}\end{array}$

$\begin{array}{lllll}C & 0.836082 & 3.695845 & -1.054561\end{array}$

$\begin{array}{lllll}C & \mathbf{1 . 6 3 6 4 8 1} & \mathbf{1 . 0 9 2 4 5 7} & \mathbf{2 . 9 6 9 3 8 4}\end{array}$

\begin{tabular}{cccc}
\hline & 0.848342 & -0.808092 & $\mathbf{2 . 2 5 8 2 4 0}$
\end{tabular}

$\begin{array}{lrrr}\text { C } & \mathbf{- 0 . 8 0 1 3 6 5} & \mathbf{0 . 5 1 5 9 2 8} & \mathbf{2 . 3 5 8 3 9 8}\end{array}$

$\begin{array}{llll}\text { H } & -2.418007 & 0.025911 & 0.533929\end{array}$

$\begin{array}{llll}C & -2.488639 & 2.115030 & 0.141327\end{array}$

$\begin{array}{lllll}\text { C } & -1.332845 & 1.668204 & 3.170739\end{array}$

$\begin{array}{llll}\text { H } & -\mathbf{0 . 8 4 3 0 8 0} & \mathbf{2 . 6 1 6 2 1 8} & \mathbf{2 . 9 1 5 0 1 0}\end{array}$

$\begin{array}{llll}\text { H } & -2.410497 & 1.801457 & \mathbf{3 . 0 2 9 7 7 0}\end{array}$

$\begin{array}{llll}\text { H } & -1.132802 & 1.453437 & 4.233401\end{array}$

$\begin{array}{lllll}\text { O } & -3.184649 & 2.665371 & 0.962238\end{array}$

\begin{tabular}{llll}
\hline & -2.404915 & 2.498319 & -1.127704
\end{tabular}

$\begin{array}{llll}\text { C } & -\mathbf{3 . 0 9 1 8 6 0} & \mathbf{3 . 6 8 9 8 6 5} & -1.480731\end{array}$

$\begin{array}{lllll}\text { H } & -2.687784 & 4.542293 & -0.916616\end{array}$

H $\quad-4.168293 \quad 3.599140 \quad-1.277016$

$\begin{array}{llll}\text { H } & -2.919254 & 3.834723 & -2.552168\end{array}$

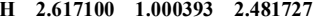

$\begin{array}{llll}\text { H } & 1.747213 & 0.727117 & 4.005081\end{array}$

$\begin{array}{llll}\text { H } & 1.381634 & \mathbf{2 . 1 5 7 8 5 7} & \mathbf{2 . 9 9 9 9 2 6}\end{array}$

\begin{tabular}{cccc} 
H & -1.419460 & -0.384994 & 2.393466 \\
\hline & -0.668989 & -4.442563 & -0.600884
\end{tabular}

\begin{tabular}{llll}
\hline & -0.668989 & -4.442563 & $\mathbf{- 0 . 6 0 0 8 8 4}$ \\
\hline & $\mathbf{2 0 9 2 0 2 8}$ & $-\mathbf{4 0 5 8 3 8 2}$ & -0.500978
\end{tabular}

$\begin{array}{llll}\text { H } & \mathbf{2 . 0 9 2 0 2 8} & \mathbf{- 4 . 0 5 8 3 8 2} & \mathbf{- 0 . 5 0 0 9 7 8}\end{array}$

$\begin{array}{lllll}\text { H } & \mathbf{0 . 9 4 4 0 3 0} & 4.778281 & -1.180597\end{array}$

$\begin{array}{llll}H & 1.281111 & 3.229116 & -3.091059\end{array}$

69

unsat_model_pc2_c / electronic energy: - $\mathbf{2 4 3 5 . 4 0 4 5 6 7 9 2}$ a.u. / lowest freq: $17.54 \mathrm{~cm}-1$

$\begin{array}{llll}0.548478 & 1.742326 & -1.465618\end{array}$

$\begin{array}{llll}C & 0.132175 & -1.194580 & -0.383838\end{array}$

$\begin{array}{llll}\text { Ru } & 0.191892 & 0.660271 & 0.581507\end{array}$

$\begin{array}{lrrr}C & 0.758238 & -\mathbf{0 . 6 5 0 2 9 4} & \mathbf{2 . 4 3 8 6 8 8}\end{array}$

$\begin{array}{lrrr}C & -1.583310 & 1.112199 & 0.565234\end{array}$

$\begin{array}{llll}\text { S } & 1.355704 & \mathbf{2 . 4 3 3 7 2 5} & \mathbf{1 . 5 4 6 0 5 8}\end{array}$

N $-\mathbf{- 0 . 9 0 9 3 0 5}-\mathbf{- 2 . 0 2 5 9 8 6}-\mathbf{- 0 . 6 5 3 1 4 8}$

N $\begin{array}{llll}\text { N } & 1.218236 & -1.891916 & -0.823664\end{array}$

C $-\mathbf{2 . 2 8 7 5 4 3}-\mathbf{- 1 . 7 3 7 9 8 5}-\mathbf{- 0 . 4 4 9 7 4 6}$

$\begin{array}{llll}\text { C } & \mathbf{- 0 . 4 8 2 6 3 4} & \mathbf{- 3 . 2 1 0 7 8 6} & \mathbf{- 1 . 2 3 8 5 4 7}\end{array}$

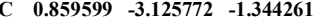

C $2.566811-1.430397-0.786308$

$\begin{array}{llll}C & -4.994544 & -1.204953 & 0.011759\end{array}$

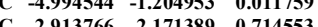

$\begin{array}{llll}C & -3.022788 & -1.066208 & -1.433401\end{array}$

$\begin{array}{llll}C & -4.371176 & -0.809546 & -1.176843\end{array}$

$\begin{array}{llll}C & -4.248536 & -1.906074 & 0.966353\end{array}$

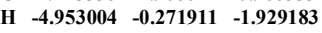

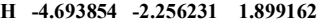

$\begin{array}{llll}\text { C } & \mathbf{5 . 2 0 0 5 1 0} & \mathbf{- 0 . 4 8 9 3 1 9} & \mathbf{- 0 . 5 7 1 1 1 4}\end{array}$

$\begin{array}{llll}C & 3.354483 & -1.732317 & 0.324444\end{array}$

$\begin{array}{llll}C & 3.128455 & -0.725621 & -1.859830\end{array}$

$\begin{array}{llll}C & 4.437110 & -0.254206 & -1.718796\end{array}$

$\begin{array}{llll}C & 4.649323 & -1.263748 & 0.455555\end{array}$

$\begin{array}{llll}\text { F } & 2.844760 & -2.514391 & 1.279059\end{array}$

$\begin{array}{llll}\text { C } & \mathbf{2 . 3 8 8 6 1 0} & \mathbf{- 0 . 5 4 6 0 8 0} & \mathbf{- 3 . 1 5 5 6 7 2}\end{array}$

$\begin{array}{llll}\text { H } & 4.876872 & 0.313792 & -2.542369\end{array}$

$\begin{array}{llll}\text { H } & 5.215147 & -1.514218 & 1.354663\end{array}$

$\begin{array}{llll}\text { C } & 6.583257 & 0.087000 & -0.431862\end{array}$

$\begin{array}{llll}\text { H } & 7.228204 & -0.556807 & 0.182000\end{array}$

$\begin{array}{lllll}\text { H } & 2.717075 & -1.313910 & -3.874963\end{array}$

H $6.533721-1.071654 \quad 0.059544$

\begin{tabular}{ccccc}
\hline & 6.533721 & $\mathbf{1 . 0 7 1 6 5 4}$ & $\mathbf{0 . 0 5 9 5 4 4}$ \\
\hline & 7.059298 & $\mathbf{0 . 2 2 9 2 8}$ & -1.411696
\end{tabular}

$\begin{array}{ccccc}\text { H } & \mathbf{7 . 0 5 9 2 9 8} & \mathbf{0 . 2 2 9 2 8 2} & \mathbf{- 1 . 4 1 1 6 9 6}\end{array}$

$\begin{array}{lllll}\text { H } & 2.597938 & \mathbf{0 . 4 3 9 4 1 5} & \mathbf{- 3 . 5 9 0 7 8 9}\end{array}$

$\begin{array}{lllll}\text { H } & \mathbf{1 . 3 0 2 9 5 3} & -\mathbf{0 . 6 3 4 3 5 7} & -\mathbf{3 . 0 3 1 2 8 5}\end{array}$

$\begin{array}{llll}\text { F } & -2.195245 & -2.854402 & 1.611188\end{array}$

$\begin{array}{llll}\text { C } & -6.437304 & -0.865228 & 0.273258\end{array}$

$\begin{array}{llll}\text { H } & -6.515116 & 0.115867 & 0.768411\end{array}$

$\begin{array}{llll}\text { H } & \mathbf{- 7 . 0 0 9 5 8 9} & \mathbf{- 0 . 8 0 8 4 6 8} & \mathbf{- 0 . 6 6 2 8 7 9}\end{array}$

$\begin{array}{llll}\text { H } & -6.913093 & -1.605955 & 0.930476\end{array}$

$\begin{array}{llll}\text { C }-2.350968 & -0.636088 & -2.704990\end{array}$

\begin{tabular}{llll} 
H & -1.873225 & -1.489931 & -3.209749 \\
\hline
\end{tabular}

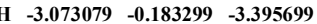




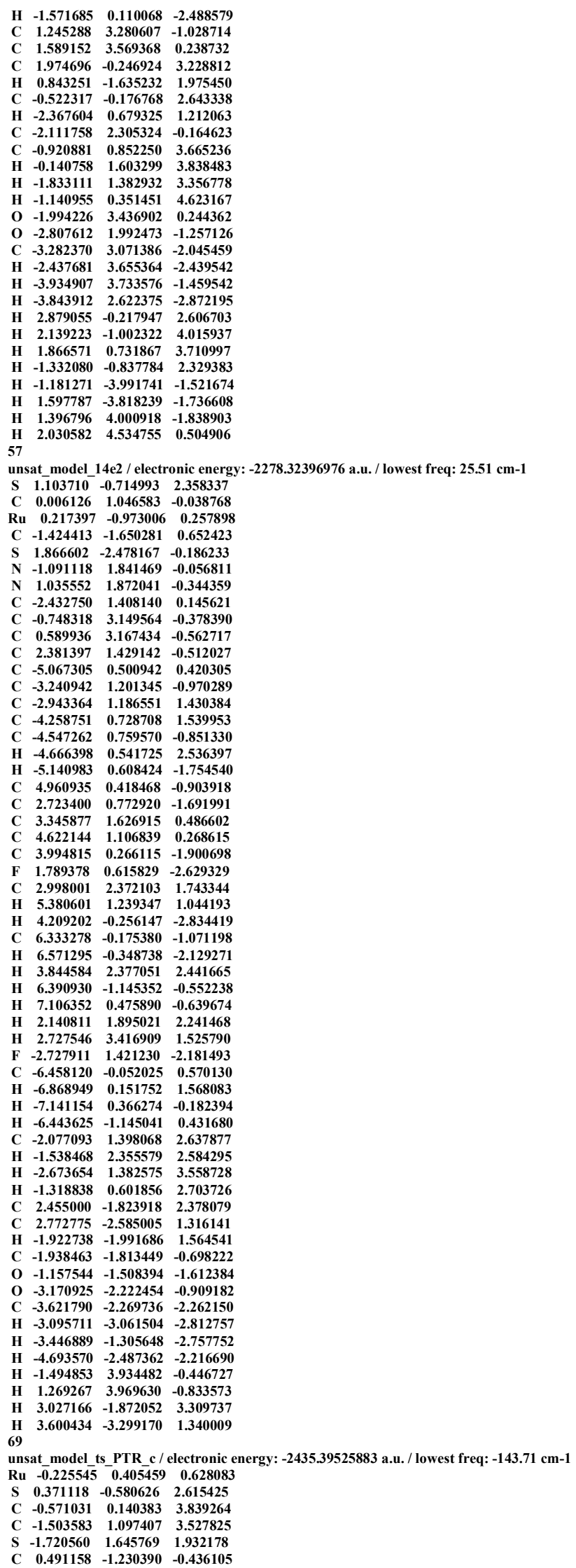




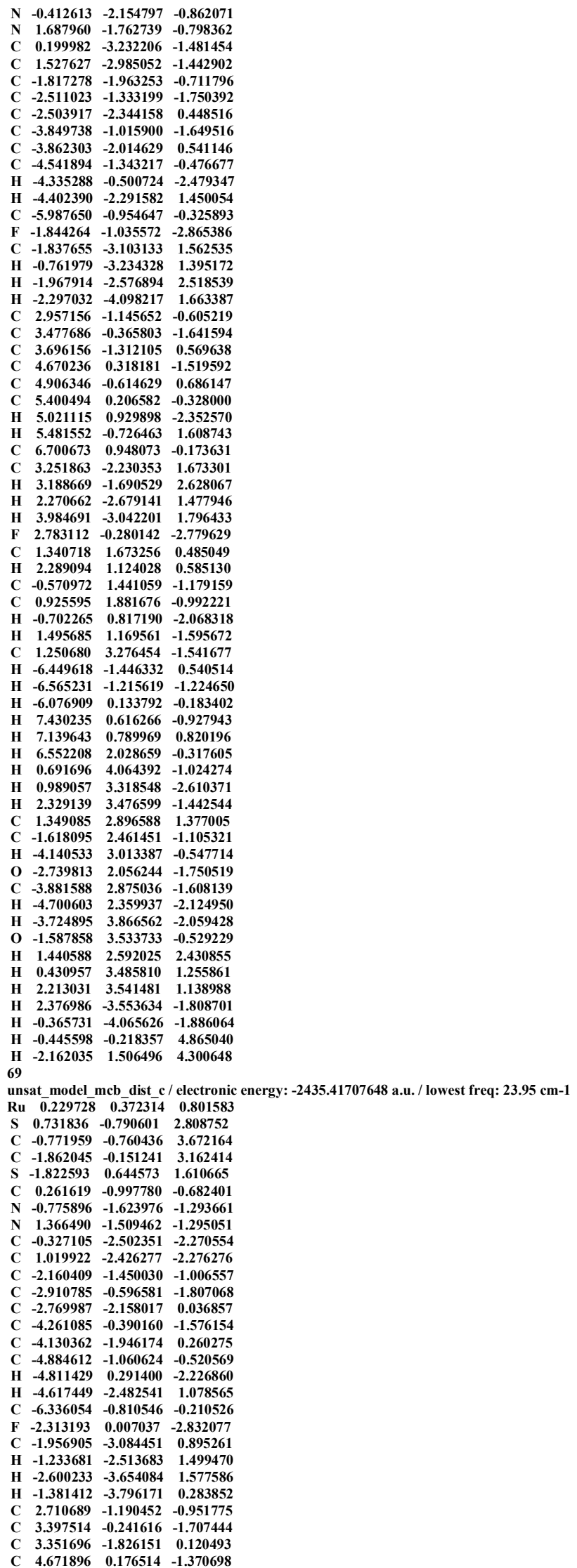


$\begin{array}{llll}C & 4.638912 & -1.396311 & 0.455957\end{array}$ $\begin{array}{llll}C & 5.302614 & -0.391696 & -0.257139\end{array}$ $\begin{array}{llll}\text { H } & 5.160364 & 0.938365 & -1.980689\end{array}$

$\begin{array}{llll}\text { H } & 5.142837 & -1.869090 & 1.302224\end{array}$

$\begin{array}{llll}C & 6.670058 & 0.079731 & 0.156822\end{array}$

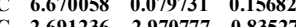

$\begin{array}{llll}\text { C } & 2.691236 & -2.970777 & 0.835272\end{array}$

$\begin{array}{llll}\text { H } & 3.216597 & -3.205184 & 1.769513\end{array}$

$\begin{array}{llll}\text { H } & \mathbf{1 . 6 4 3 8 8 9} & \mathbf{- 2 . 7 4 9 8 4 6} & \mathbf{1 . 0 7 9 2 7}\end{array}$

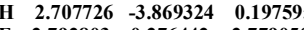

$\begin{array}{llll}\text { F } & 2.792803 & 0.276442 & -2.779050\end{array}$

$\begin{array}{llll}\text { C } & \mathbf{1 . 5 7 9 7 1 4} & \mathbf{1 . 8 2 8 4 1 5} & \mathbf{0 . 6 3 9 6 4 5}\end{array}$

$\begin{array}{lllll}\text { H } & 2.206612 & 0.873222 & 0.589846\end{array}$

$\begin{array}{lrrrr}\text { C } & \mathbf{- 0 . 1 3 1 6 8 4} & \mathbf{1 . 7 9 7 1 1 0} & \mathbf{- 0 . 8 5 6 5 2 8}\end{array}$

$\begin{array}{llll}C & \mathbf{1 . 2 8 5 4 9 3} & \mathbf{2 . 3 9 3 2 3 6} & \mathbf{- 0 . 7 3 4 1 6 2}\end{array}$

$\begin{array}{lllll}\text { H } & -0.361281 & 1.324105 & -1.818188\end{array}$

$\begin{array}{llll}\text { H } & \mathbf{1 . 9 0 4 9 7 2} & \mathbf{1 . 8 5 4 0 1 1} & -1.460131\end{array}$

$\begin{array}{llll}C & 1.508048 & 3.880747 & -0.981982\end{array}$

$\begin{array}{llll}\text { H } & -6.815486 & -1.704554 & 0.211832\end{array}$

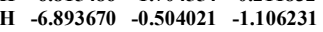

$\begin{array}{llll}\text { H } & -6.430820 & -0.003055 & 0.533195\end{array}$

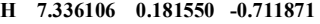

$\begin{array}{llll}\text { H } & 7.133194 & -\mathbf{0 . 6 1 1 7 0 1} & \mathbf{0 . 8 7 2 9 9 9}\end{array}$

$\begin{array}{lllll}\text { H } & 6.603048 & 1.068479 & 0.637007\end{array}$

$\begin{array}{lllll}\text { H } & 0.935943 & 4.506415 & -0.287318\end{array}$

$\begin{array}{lllll}\text { H } & 1.191647 & 4.136509 & -2.005273\end{array}$

$\begin{array}{lllll} & & \mathbf{2} & & \\ \mathrm{H} & \mathbf{5} 77048 & \mathbf{4 . 1 2 9 1 7 8} & \mathbf{- 0 . 8 9 0 6 1 6}\end{array}$

$\begin{array}{llll}C & \mathbf{1 . 9 9 2 7 4 0} & \mathbf{2 . 7 1 3 4 9 3} & \mathbf{1 . 7 8 2 2 5 1}\end{array}$

$\begin{array}{llll}\text { C } & -1.225286 & \mathbf{2 . 7 0 2 7 6 5} & \mathbf{- 0 . 4 6 1 5 1 6}\end{array}$

$\begin{array}{lllll}\text { H } & -3.730749 & 2.922994 & 0.437706\end{array}$

$\begin{array}{lllll}\text { O } & -2.392400 & 2.360892 & -1.047181\end{array}$

$\begin{array}{llll}\text { C } & -\mathbf{3 . 5 4 2 4 0 2} & \mathbf{3 . 0 7 1 7 3 5} & \mathbf{- 0 . 6 3 5 9 0 8}\end{array}$

$\begin{array}{llll}\text { H } & -4.380087 & 2.667352 & -1.216095\end{array}$

\begin{tabular}{llll}
\hline & -3.437016 & 4.149099 & -0.830333
\end{tabular}

$\begin{array}{lllll}\text { O } & -1.163414 & 3.645309 & 0.305433\end{array}$

$\begin{array}{lllll}\text { H } & \mathbf{2 . 1 3 0 8 4 9} & \mathbf{2 . 1 4 7 3 2 7} & \mathbf{2 . 7 1 1 6 8 5}\end{array}$

$\begin{array}{llll}\text { H } & 1.218574 & 3.477890 & 1.941743\end{array}$

$\begin{array}{llll}\text { H } & \mathbf{2 . 9 3 2 0 7 8} & \mathbf{3 . 2 3 8 6 4 7} & \mathbf{1 . 5 3 7 5 0 4}\end{array}$

H $\quad \begin{array}{llll}1.769220 & -2.931648 & -2.877399\end{array}$

H $-1.016854-\mathbf{3 . 0 9 3 1 7 3}-2.864819$

$\begin{array}{llll}\text { H } & -0.807040 & -1.263531 & 4.642994\end{array}$

H $-2.822411-0.119621 \quad 3.685121$

69

unsat model ts BHE c/ electronic energy: $\mathbf{- 2 4 3 5 . 3 9 2 9 0 7 2 3 ~ a . u . ~ / ~ l o w e s t ~ f r e q : ~}-\mathbf{1 9 0 . 8 7} \mathbf{c m - 1}$

$\begin{array}{llll}S & -1.964144 & -0.567012 & -2.088214\end{array}$

$\begin{array}{llll}C & -0.308230 & 0.901764 & 0.626646\end{array}$

$\begin{array}{llll}\text { Ru } & -0.230723 & -0.812908 & -0.508569\end{array}$

$\begin{array}{llll}C & \mathbf{- 0 . 8 3 3 1 2 9} & \mathbf{- 2 . 8 3 1 0 8 7} & \mathbf{- 0 . 1 8 7 4 5 1}\end{array}$

$\begin{array}{llll}C & 1.050711 & -1.734594 & 0.976111\end{array}$

$\begin{array}{llll}\text { S } & 1.158021 & -0.248824 & -2.182803\end{array}$

$\begin{array}{llll}N & 0.694852 & 1.718206 & 1.057458\end{array}$

$\begin{array}{llll}\mathrm{N} & -1.432432 & 1.474932 & 1.143533\end{array}$

$\begin{array}{llll}\text { C } & \mathbf{2 . 0 7 0 2 9 8} & \mathbf{1 . 6 9 0 4 2 1} & \mathbf{0 . 6 7 7 7 7 2}\end{array}$

$\begin{array}{llll}\text { C } & \mathbf{0 . 2 0 3 9 8 9} & \mathbf{2 . 7 6 0 0 8 4} & \mathbf{1 . 8 2 8 6 5 0}\end{array}$

$\begin{array}{llll}\text { C } & -1.134862 & 2.603785 & 1.887520\end{array}$

$\begin{array}{llll}\text { C } & -2.779918 & 1.111054 & 0.852099\end{array}$

\begin{tabular}{llll} 
C & $\mathbf{4 . 7 9 9 7 2 8}$ & $\mathbf{1 . 7 5 0 3 1 6}$ & $\mathbf{0 . 0 5 3 5 5 5}$ \\
\hline
\end{tabular}

$\begin{array}{llll}\text { C } & 3.011661 & 1.116134 & 1.522183\end{array}$

$\begin{array}{llll}C & \mathbf{2 . 4 9 0 8 0 3} & \mathbf{2 . 3 6 8 0 2 4} & \mathbf{- 0 . 4 7 7 6 5 6}\end{array}$

$\begin{array}{llll}C & 3.853112 & \mathbf{2 . 3 6 7 8 6 2} & \mathbf{- 0 . 7 7 5 5 3 0}\end{array}$

$\begin{array}{llll}\text { C } & 4.365182 & 1.134560 & 1.227478\end{array}$

$\begin{array}{llll}\text { H } & 4.191904 & 2.877784 & -1.680780\end{array}$

$\begin{array}{llll}\text { H } & \mathbf{5 . 0 6 1 2 9 4} & \mathbf{0 . 6 5 5 0 5 7} & \mathbf{1 . 9 1 7 5 2 5}\end{array}$

$\begin{array}{llll}C & -5.447088 & 0.417867 & 0.353412\end{array}$

$\begin{array}{llll}C & -3.438818 & 0.180834 & 1.645996\end{array}$

$\begin{array}{llll}C & \mathbf{- 3 . 4 6 1 8 4 9} & \mathbf{1 . 7 4 0 0 1 0} & \mathbf{- 0 . 1 9 8 8 4 5}\end{array}$

$\begin{array}{lllll}C & -4.791199 & \mathbf{1 . 3 8 0 2 8 8} & \mathbf{- 0 . 4 2 3 7 1 7}\end{array}$

$\begin{array}{llll}C & -4.753335 & -0.185261 & \mathbf{1 . 4 0 6 9 0 9}\end{array}$

$\begin{array}{llll}\text { F } & -\mathbf{2 . 7 7 7 8 7 1} & \mathbf{- 0 . 3 6 4 3 5 0} & \mathbf{2 . 6 6 9 8 8 2}\end{array}$

$\begin{array}{lllll}\text { C } & \mathbf{- 2 . 7 5 7 2 3 2} & \mathbf{2 . 7 5 3 0 3 8} & \mathbf{- 1 . 0 5 5 8 7 7}\end{array}$

$\begin{array}{lllll}\text { H } & \mathbf{- 5 . 3 3 0 9 0 6} & \mathbf{1 . 8 5 8 5 3 5} & \mathbf{- 1 . 2 4 4 7 7 0}\end{array}$

$\begin{array}{lllll}\text { H } & \mathbf{- 5 . 2 2 3 4 4 7} & -0.927815 & \mathbf{2} .054018\end{array}$

$\begin{array}{llll}\text { C } & -6.865367 & 0.020262 & 0.044231\end{array}$

H $-7.372832 \quad-0.385207 \quad 0.930062$

\begin{tabular}{llll} 
H & -6.865367 & 0.020262 & 0.044231 \\
\hline & -7.372832 & -0.385207 & $\mathbf{0 . 9 3 0 0 6 2}$
\end{tabular}

\begin{tabular}{llll}
\hline & -2.402103 & 3.608516 & -0.460951
\end{tabular}

$\begin{array}{lllll}\text { H } & \mathbf{- 6 . 8 8 0 9 7 4} & \mathbf{- 0 . 7 5 8 4 6 5} & \mathbf{- 0 . 7 3 4 7 0 6}\end{array}$

$\begin{array}{lllll}\text { H } & -7.445855 & 0.873980 & -0.332182\end{array}$

$\begin{array}{lllll}\text { H } & -\mathbf{3 . 4 2 3 1 8 5} & \mathbf{3 . 1 3 2 2 2 4} & \mathbf{- 1 . 8 4 1 0 4 8}\end{array}$

$\begin{array}{llll}\text { H } & -1.881103 & 2.292721 & -1.538121\end{array}$

$\begin{array}{llll}\text { F } & \mathbf{2 . 5 9 8 3 1 2} & \mathbf{0 . 5 5 3 4 4 3} & \mathbf{2 . 6 5 9 8 1 0}\end{array}$

$\begin{array}{llll}\text { C } & 6.255905 & 1.737380 & -0.326923\end{array}$

$\begin{array}{lllll}\text { H } & 6.560017 & \mathbf{2 . 6 9 3 2 3 4} & -0.776599\end{array}$

H $\quad 6.898839 \quad \mathbf{1 . 5 3 9 5 6 6}$

\begin{tabular}{lllll}
\hline & 6.898839 & 1.539566 & $\mathbf{0 . 5 4 1 5 1 4}$ \\
\hline & 6.448028 & 0.949600 & -1.072875
\end{tabular}

$\begin{array}{llll}\text { H } & 6.448028 & 0.949600 & -1.072875 \\ \text { C } & 1.498096 & 3.113829 & -1.325816\end{array}$

$\begin{array}{llll}\text { C } & \mathbf{1 . 4 9 8 0 9 6} & \mathbf{3 . 1 1 3 8 2 9} & \mathbf{- 1 . 3 2 5 8 1 6}\end{array}$

$\begin{array}{lllll}\text { H } & 1.083497 & \mathbf{3 . 9 7 2 8 7 0} & \mathbf{- 0 . 7 7 4 1 7 8}\end{array}$

H $\quad \mathbf{1 . 9 7 2 7 4 5} \quad 3.493786 \quad-2.239364$

$\begin{array}{lllll}\text { H } & 0.658026 & \mathbf{2 . 4 6 8 5 7 0} & -\mathbf{1 . 6 1 8 5 2 2}\end{array}$

$\begin{array}{llll}\text { C } & -1.168021 & 0.007067 & -3.501478\end{array}$

$\begin{array}{lllll}\text { C } & 0.180663 & 0.177790 & \mathbf{- 3 . 5 4 5 7 6 4}\end{array}$

$\begin{array}{llll}C & -0.195257 & -3.800910 & -1.152821\end{array}$

H $-1.920950-2.979281-0.140245$

C $-0.313870-2.296203-1.075994$

\begin{tabular}{llll}
$C$ & -0.313870 & -2.296203 & 1.075994 \\
\hline & 1.310282 & -1.071316 & 1.807339
\end{tabular}

$\begin{array}{llll}\text { H } & 1.310282 & -1.071316 & 1.807339\end{array}$ 


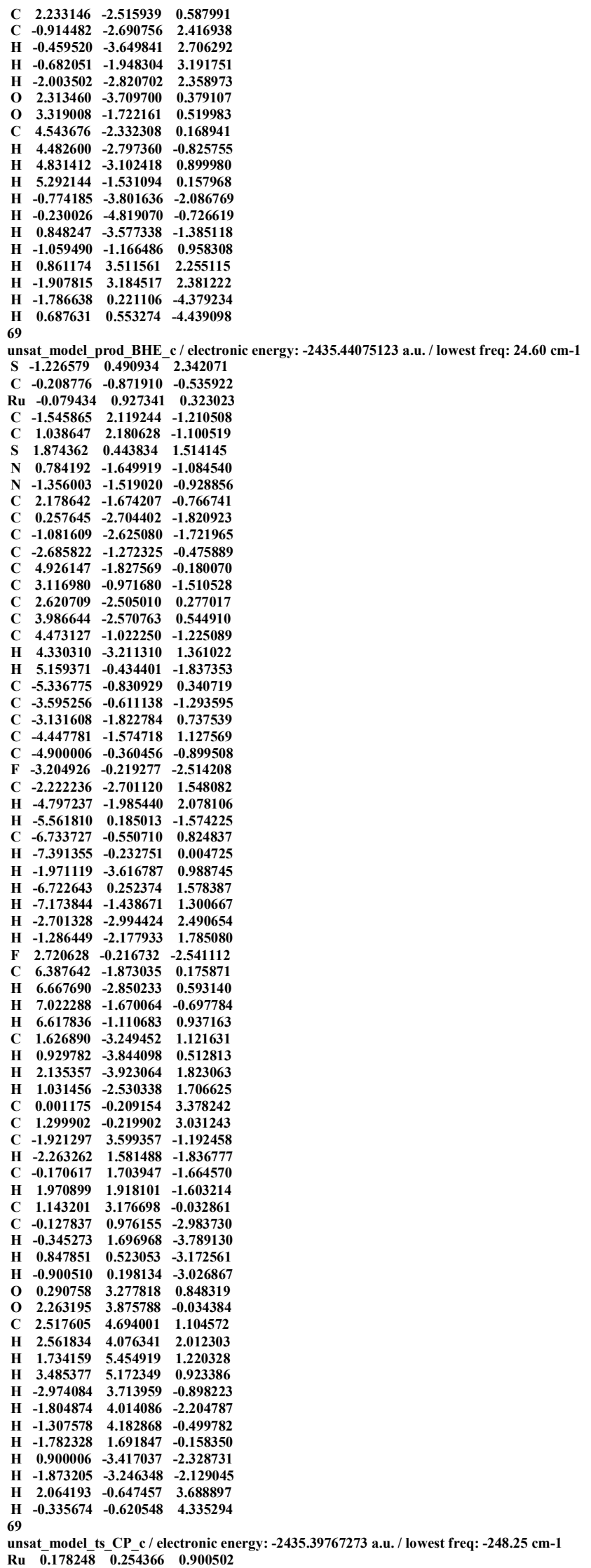


$\begin{array}{llll}S & -1.933406 & 0.244977 & 1.745430\end{array}$

$\begin{array}{llll}C & -1.991531 & -0.972618 & 3.000807\end{array}$

$\begin{array}{llll}C & -0.885230 & -1.660323 & 3.33269\end{array}$

$\begin{array}{llll}C & 0.633803 & -1.350203 & 2.525422\end{array}$

$\begin{array}{llll}C & 0.350311 & -0.931677 & -0.67825 \\ C\end{array}$

N $1.492754-1.428480-1.256776$

$\begin{array}{llll}\mathrm{N} & 1.492754 & -1.428480 & -1.256776 \\ \mathrm{~N}-0.645955 & -1.529961 & -1.396923\end{array}$

C $1.204156-2.304429$

$\begin{array}{llll}C & 1.204156 & -2.304429 & -2.294326\end{array}$

$\begin{array}{llll}C & -0.139258 & -2.368908 & -2.380793\end{array}$

$\begin{array}{llll}C & 2.822903 & -1.066722 & -0.904929\end{array}$

$\begin{array}{llll}C & 3.410047 & 0.021849 & -1.545880\end{array}$

$\begin{array}{llll}C & 3.568955 & -1.802736 & 0.027677\end{array}$

$\begin{array}{llll}C & 4.671017 & 0.484834 & -1.211717\end{array}$

$\begin{array}{llll}C & 4.842642 & -1.333109 & 0.359503\end{array}$

$\begin{array}{llll}\text { C } & 5.400581 & -0.191122 & -0.228323\end{array}$

$\begin{array}{llll}\text { H } & 5.072247 & 1.359366 & -1.726580\end{array}$

$\begin{array}{lrrr}\text { H } & 5.425444 & -1.885745 & 1.100628\end{array}$

$\begin{array}{llll}\text { C } & 6.783307 & 0.266895 & 0.150611\end{array}$

$\begin{array}{llll}\text { F } & \mathbf{2 . 7 1 8 0 0 8} & \mathbf{0 . 6 3 4 4 5 9} & \mathbf{- 2 . 5 1 2 6 2 7}\end{array}$

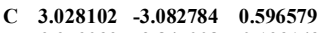

$\begin{array}{lllll}\text { H } & 2.952039 & -3.847008 & -0.193143\end{array}$

$\begin{array}{llll}\text { H } & 3.688198 & -3.469642 & 1.383380\end{array}$

$\begin{array}{llll}\text { H } & \mathbf{2 . 0 2 5 9 3 2} & \mathbf{- 2 . 9 3 8 5 4 2} & \mathbf{1 . 0 2 4 0 6 7}\end{array}$

C $-2.046062-1.360656-1.205133$

$\begin{array}{llll}C & -2.702361 & -0.336988 & -1.877962\end{array}$

$\begin{array}{llll}\text { C } & -2.770325 & -2.238834 & -0.390744\end{array}$

$\begin{array}{llll}C & -4.063468 & -0.128043 & -1.740164\end{array}$

$\begin{array}{llll}C & -4.143821 & -2.027419 & -0.260069\end{array}$

$\begin{array}{llll}C & -4.801785 & -0.976223 & -0.909938\end{array}$

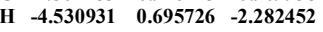

$\begin{array}{llll}\text { H } & -4.719478 & -2.697859 & 0.383022\end{array}$

$\begin{array}{lllll}\text { C } & -6.272199 & -0.735914 & -0.695863\end{array}$

$\begin{array}{llll}C & -2.062843 & -3.358937 & 0.317019\end{array}$

$\begin{array}{llll}\text { H } & -2.740993 & -3.879370 & 1.005003\end{array}$

\begin{tabular}{llll}
$\mathrm{H}$ & -2.740993 & -3.879370 & 1.005003 \\
\hline & -1.671446 & -4.095722 & -0.402564
\end{tabular}

\begin{tabular}{llll}
\hline & -1.671446 & -4.095722 & -0.402564 \\
\hline
\end{tabular}

\begin{tabular}{lllll}
\hline & -1.209713 & -2.972897 & 0.894998
\end{tabular}

\begin{tabular}{rrrr}
\hline & -1.990779 & 0.453309 & -2.687134
\end{tabular}

$\begin{array}{llll}\text { C } & 1.297335 & 2.009728 & 0.531020\end{array}$

$\begin{array}{llll}\text { H } & 1.938591 & 1.058893 & 0.448451\end{array}$

$\begin{array}{lllll}C & \mathbf{- 0 . 1 7 3 6 6 8} & \mathbf{2 . 1 3 8 4 4 4} & \mathbf{- 0 . 5 1 4 6 4 4}\end{array}$

$\begin{array}{lllll}C & 1.195839 & \mathbf{2 . 7 7 0 8 2 4} & \mathbf{- 0 . 7 3 8 6 6 9}\end{array}$

$\begin{array}{lllll}\text { H } & -0.486942 & 1.461909 & -1.307633\end{array}$

$\begin{array}{llll}\text { C } & \mathbf{1 . 4 0 0 3 3 5} & \mathbf{4 . 2 6 8 3 2 3} & -0.787638\end{array}$

$\begin{array}{lllll}\text { H } & 1.678977 & 2.267349 & -1.577889\end{array}$

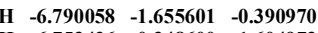

$\begin{array}{llll}\text { H } & -6.753426 & -0.348600 & -1.604973\end{array}$

$\begin{array}{llll}\text { H } & -6.423005 & 0.010397 & 0.100781\end{array}$

$\begin{array}{llll}\text { H } & 6.922690 & 1.337823 & -0.049653\end{array}$

$\begin{array}{llll}\text { H } & 7.541513 & \mathbf{- 0 . 2 8 2 5 3 4} & \mathbf{- 0 . 4 2 9 7 9 6}\end{array}$

$\begin{array}{lllll}\text { H } & \mathbf{6 . 9 8 4 5 1 5} & \mathbf{0 . 0 8 1 5 0 3} & \mathbf{1 . 2 1 4 7 3 3}\end{array}$

$\begin{array}{lllll}\text { H } & 0.861129 & 4.790693 & 0.010943\end{array}$

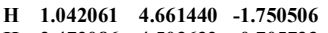

$\begin{array}{llll}H & 2.472086 & 4.503632 & -0.705733\end{array}$

$\begin{array}{llll}\text { C } & \mathbf{1 . 4 8 1 1 2 1} & \mathbf{2 . 7 7 2 6 3 1} & \mathbf{1 . 8 2 5 4 5 5}\end{array}$

$\begin{array}{llll}\text { H } & -0.791571 & -2.927131 & -3.045011\end{array}$

$\begin{array}{lllll}\text { H } & \mathbf{1 . 9 8 7 7 8 9} & \mathbf{- 2 . 7 8 8 4 5 8} & \mathbf{- 2 . 8 6 8 9 8 4}\end{array}$

$\begin{array}{lllll}\text { O } & -1.240545 & 3.870885 & 0.791314\end{array}$

\begin{tabular}{llll}
\hline & -1.302534 & 2.936029 & 0.021769
\end{tabular}

$\begin{array}{llll}\text { O } & -2.461019 & 2.488081 & -0.468771\end{array}$

$\begin{array}{llll}\text { C } & \mathbf{- 3 . 6 4 6 7 8 7} & \mathbf{3 . 0 6 7 0 3 1} & \mathbf{0 . 0 4 8 1 9 7}\end{array}$

$\begin{array}{llll}\text { H } & -3.689816 & 4.143164 & -0.171963\end{array}$

H $-4.477252 \quad 2.547485-0.442413$

H $-3.705771 \quad 2.917590 \quad 1.135440$

$\begin{array}{llll}H & -3.705771 & 2.917590 & 1.135440\end{array}$

$\begin{array}{llll}H & 1.507185 & 2.101997 & \mathbf{2 . 6 9 3 5 4 8}\end{array}$

\begin{tabular}{lllll} 
H & $\mathbf{0 . 6 6 8 6 5 4}$ & $\mathbf{3 . 4 9 6 7 6 2}$ & $\mathbf{1 . 9 5 9 9 4 7}$ \\
\hline & $\mathbf{2}$ & $\mathbf{4}$ &
\end{tabular}

$\begin{array}{lrrrr}\text { H } & 2.434737 & 3.323205 & 1.777675 \\ \text { H } & -\mathbf{2 . 9 6 0 7 3 7} & -1.145161 & 3.478041\end{array}$

$\begin{array}{llll}\text { H } & \mathbf{- 0 . 8 9 0 7 3 4} & \mathbf{- 2 . 4 4 0 5 8 7} & \mathbf{4 . 0 9 9 6 2 5}\end{array}$

69

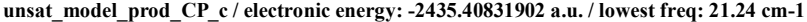

$\begin{array}{llll}\text { Ru } & \mathbf{0 . 2 6 8 0 4 0} & -\mathbf{0 . 0 1 7 7 9 2} & \mathbf{0 . 8 7 3 5 1 4}\end{array}$

$\begin{array}{llll}S & -1.732200 & 0.833385 & 1.507919\end{array}$

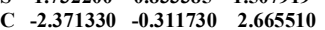

$\begin{array}{llll}C & -2.371330 & -0.311730 & 2.665510 \\ C & -1.631658 & -1.349956 & 3.093397\end{array}$

$\begin{array}{llll}\text { S } & 0.007146 & -1.577463 & \mathbf{2 . 5 1 5 3 0 5}\end{array}$

$\begin{array}{llll}C & -0.051107 & -1.191503 & -0.640060\end{array}$

$\begin{array}{llll}\mathrm{N} & 0.957220 & -1.901804 & -1.235819\end{array}$

$\begin{array}{llll}N & -1.169195 & -1.614867 & -1.298473\end{array}$

$\begin{array}{llll}C & 0.481795 & -2.736048 & -2.237832\end{array}$

$\begin{array}{llll}C & -0.855313 & -2.554723 & -2.275030\end{array}$

$\begin{array}{llll}C & 2.320043 & -1.680683 & -0.903595\end{array}$

$\begin{array}{llll}C & 2.990631 & -0.623559 & -1.523355\end{array}$

$\begin{array}{llll}C & 3.002301 & -2.488523 & 0.017576\end{array}$

$\begin{array}{llll}C & 4.303605 & -0.309275 & -1.225729\end{array}$

$\begin{array}{llll}C & 4.329406 & -2.160643 & 0.317756\end{array}$

$\begin{array}{llll}C & 4.988639 & -1.081055 & -0.278905\end{array}$

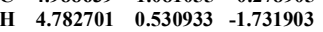

$\begin{array}{lllll}H & 4.868280 & -2.776930 & 1.041271\end{array}$

$\begin{array}{llll}\text { C } & 6.411209 & \mathbf{- 0 . 7 4 2 9 8 0} & \mathbf{0 . 0 7 6 0 9 7}\end{array}$

$\begin{array}{llll}\text { F } & \mathbf{2 . 3 2 3 9 1 5} & \mathbf{0 . 1 0 6 1 3 4} & \mathbf{- 2 . 4 2 2 1 9 3}\end{array}$

$\begin{array}{llll}\text { C } \quad 2.324435 & -3.679727 & 0.632126\end{array}$

C $2.051332-4.413562-0.141775$

$\begin{array}{rrrr}\text { H } & 2.051332 & -4.413562 & -0.141775 \\ \text { H } & \mathbf{2 . 9 8 4 2 4 7} & -4.174133 & 1.356151\end{array}$ 
$\begin{array}{llll}\text { H } & 1.397199 & -3.384974 & 1.146839\end{array}$

$\begin{array}{llll}C & -2.492067 & -1.146185 & -1.074822\end{array}$

$\begin{array}{llll}\text { C } & -2.906155 & 0.023599 & -1.705521\end{array}$

$\begin{array}{llll}C & -3.377029 & -1.850005 & -0.252303\end{array}$

$\begin{array}{llll}C & -4.177860 & 0.536864 & -1.523345\end{array}$

C $-4.659251-1.324256-0.070565$

$\begin{array}{llll}C & -5.071749 & -0.136932 & -0.683907\end{array}$

$\begin{array}{llll}\text { H } & -\mathbf{4 . 4 6 1 8 5 7} & \mathbf{1 . 4 5 6 8 6 1} & \mathbf{- 2 . 0 3 7 6 5 4}\end{array}$

H $\quad \mathbf{- 5 . 3 5 7 1 8 6}-\mathbf{- 1 . 8 5 8 9 1 6} \quad \mathbf{0 . 5 7 8 7 6 6}$

$\begin{array}{llll}C & -6.442284 & 0.431510 & -0.429419\end{array}$

$\begin{array}{llll}C & -2.941573 & -3.131845 & 0.399458\end{array}$

H $\quad-3.671278 \quad-3.456521 \quad 1.152130$

H $\quad-2.839114-3.935547 \quad-0.347376$

$\begin{array}{llll}\text { H } & -1.965852 & -\mathbf{3 . 0 0 5 8 0 4} & \mathbf{0 . 8 9 0 9 9 0}\end{array}$

$\begin{array}{llll}\text { F } & -2.045066 & 0.658536 & -2.500964\end{array}$

$\begin{array}{llll}C & 2.362776 & 2.266156 & 0.649073\end{array}$

$\begin{array}{llll}\text { H } & 2.802156 & 1.262412 & 0.697092\end{array}$

$\begin{array}{llll}\text { C } & \mathbf{1 . 0 7 2 2 5 8} & \mathbf{2 . 3 0 6 1 1 9} & \mathbf{- 0 . 1 7 5 4 8 1}\end{array}$

C $\quad \mathbf{2 . 4 0 4 9 4 0} \quad \mathbf{2 . 8 6 5 5 4 9} \quad \mathbf{- 0 . 7 1 0 7 7 7}$

$\begin{array}{lllll}\text { H } & 0.733038 & 1.399547 & -0.723057\end{array}$

$\begin{array}{llll}C & 2.597754 & 4.341482 & -0.958264\end{array}$

$\begin{array}{llll}\text { H } & 2.838024 & \mathbf{2 . 2 3 2 6 2 2} & -\mathbf{1 . 4 8 8 4 4 0}\end{array}$

$\begin{array}{lllll}\text { H } & -7.140211 & -0.340610 & -0.077857\end{array}$

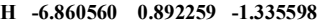

$\begin{array}{lllll}\text { H } & -6.393168 & 1.213889 & 0.344674\end{array}$

$\begin{array}{llll}\text { H } & 6.467330 & 0.249860 & 0.548011\end{array}$

$\begin{array}{llll}\text { H } & \mathbf{7 . 0 4 3 9 3 1} & \mathbf{- 0 . 7 1 3 0 2 2} & \mathbf{- 0 . 8 2 3 4 6 4}\end{array}$

H $\quad 6.836346 \quad-1.476271 \quad 0.773869$

$\begin{array}{lllll}\text { H } & \mathbf{2 . 1 0 3 1 0 7} & \mathbf{4 . 9 6 1 5 5 1} & \mathbf{- 0 . 2 0 0 6 3 5}\end{array}$

H $2.195608 \quad 4.622904 \quad-1.942807$

$\begin{array}{llll}\text { H } & 3.672162 & 4.579058 & -0.951268\end{array}$

$\begin{array}{llll}\text { C } & \mathbf{2 . 5 4 0 6 5 7} & \mathbf{3 . 0 3 3 8 0 6} & \mathbf{1 . 9 3 5 7 2 7}\end{array}$

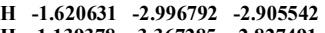

$\begin{array}{lllll}\text { H } & 1.139378 & -3.367285 & -2.827491\end{array}$

\begin{tabular}{lrrr}
\hline & -0.024662 & $\mathbf{4 . 2 2 8 6 6 7}$ & $\mathbf{0 . 8 0 1 2 9 3}$
\end{tabular}

\begin{tabular}{llll}
\hline & -0.024662 & 4.228667 & 0.801293 \\
C & -0.037322 & 3.271248 & 0.063422
\end{tabular}

$\begin{array}{rrrr}\text { C } & -\mathbf{0 . 0 3 7 3 2 2} & \mathbf{3 . 2 7 1 2 4 8} & \mathbf{0 . 0 6 3 4 2 2} \\ \text { O } & \mathbf{- 1 . 0 7 5 7 6 2} & \mathbf{2 . 9 6 8 4 8 6} & \mathbf{- 0 . 7 1 5 6 6 9}\end{array}$

$\begin{array}{llll}\text { O } & \mathbf{- 1 . 0 7 5 7 6 2} & \mathbf{2 . 9 6 8 4 8 6} & \mathbf{- 0 . 7 1 5 6 6 9} \\ \text { C } & -\mathbf{2 . 2 4 1 1 5 0} & \mathbf{3 . 7 6 4 9 9 5} & \mathbf{- 0 . 5 8 2 6 6 6}\end{array}$

$\begin{array}{llll}\text { H } & -2.013715 & 4.827246 & -0.748580\end{array}$

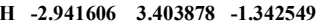

H $\quad-2.677955 \quad 3.636314 \quad 0.417923$

$\begin{array}{llll}\text { H } & \mathbf{2 . 0 8 6 4 5 5} & \mathbf{2 . 4 7 7 3 2 8} & \mathbf{2 . 7 6 9 1 9 2}\end{array}$

$\begin{array}{llll}\text { H } & 2.069770 & 4.021348 & 1.900771\end{array}$

$3.613430 \quad 3.151754 \quad 2.149689$

\begin{tabular}{llll}
$\mathrm{H}$ & 3.613430 & 3.151754 & $\mathbf{2 . 1 4 9 6 8 9}$ \\
\hline $\mathrm{H}$ & -2.010053 & -2.083708 & 3.81096
\end{tabular}

$\begin{array}{llll}\text { H } & -2.010053 & -2.083708 & 3.810969 \\ \text { H } & -3.394240 & -0.140360 & 3.013513\end{array}$

\section{X-ray Structure of Ru-1c}

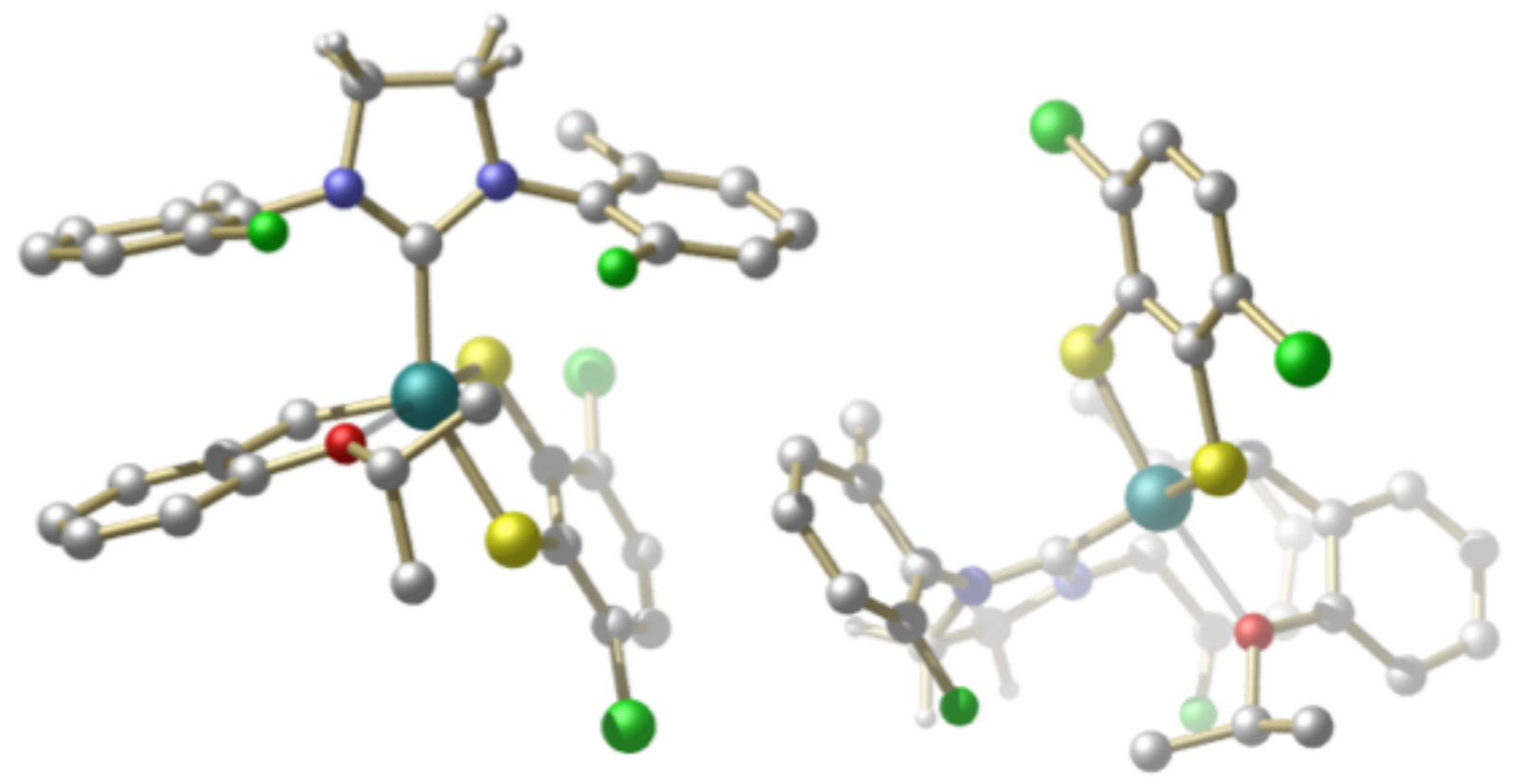

Table 1. Crystal data and structure refinement for $3\left(\mathrm{C}_{33} \mathrm{H}_{30} \mathrm{Cl}_{2} \mathrm{~F}_{2} \mathrm{~N}_{2} \mathrm{ORuS}_{2}\right) \mathrm{CH}_{2} \mathrm{Cl}_{2}(\mathbf{R u}-\mathbf{1 c})$. 
Identification code

Empirical formula

Formula weight

Temperature

Wavelength

Crystal system

Space group

Unit cell dimensions

Volume

Z

Density (calculated)

Absorption coefficient

$\mathrm{F}(000)$

Crystal size

Theta range for data collection

Index ranges

Reflections collected

Independent reflections

Completeness to theta $=66.704^{\circ}$

Absorption correction

Max. and min. transmission

Refinement method

Data / restraints / parameters

Goodness-of-fit on $\mathrm{F}^{2}$

Final $\mathrm{R}$ indices [I $>2$ sigma(I)]

$\mathrm{R}$ indices (all data)

Extinction coefficient

Largest diff. peak and hole
3(C33H30Cl2F2N2ORuS2)CH2Cl2

C100 H92 Cl8 F6 N6 O3 Ru3 S6

2318.96

$100(2) \mathrm{K}$

$1.54178 \AA$

Monoclinic

$\mathrm{P} 2 / \mathrm{n}$

$\mathrm{a}=12.6425(3) \AA$

$\mathrm{a}=90^{\circ}$

$\mathrm{b}=20.1905(4) \AA$

$\mathrm{b}=93.2110(10)^{\circ}$.

$\mathrm{c}=38.4232(7) \AA$

$\mathrm{g}=90^{\circ}$.

9792.4(4) $\AA^{3}$

4

$1.573 \mathrm{Mg} / \mathrm{m}^{3}$

$7.444 \mathrm{~mm}^{-1}$

4704

$0.320 \times 0.180 \times 0.150 \mathrm{~mm}^{3}$

2.303 to $66.704^{\circ}$.

$-15<=\mathrm{h}<=15,-21<=\mathrm{k}<=23,-44<=\mathrm{l}<=45$

63548

17210 [R(int $)=0.0497]$

$99.3 \%$

Semi-empirical from equivalents

0.7533 and 0.5593

Full-matrix least-squares on $\mathrm{F}^{2}$

$17210 / 1253$ / 1231

1.131

$\mathrm{R} 1=0.0662, \mathrm{wR} 2=0.1724$

$\mathrm{R} 1=0.0742, \mathrm{wR} 2=0.1776$

$\mathrm{n} / \mathrm{a}$

2.229 and -1.140 e. $\AA^{-3}$

Table 2. Atomic coordinates $\left(\times 10^{4}\right)$ and equivalent isotropic displacement parameters $\left(\AA^{2} \mathrm{x} \quad 10^{3}\right)$ for $3\left(\mathrm{C}_{33} \mathrm{H}_{30} \mathrm{Cl}_{2} \mathrm{~F}_{2} \mathrm{~N}_{2} \mathrm{ORuS}_{2}\right) \mathrm{CH}_{2} \mathrm{Cl}_{2}(\mathrm{Ru}-1 \mathrm{c})$. U(eq) is defined as one third of the trace of the orthogonalized $\mathrm{U}^{\mathrm{ij}}$ tensor.

\begin{tabular}{|c|c|c|c|c|}
\hline & $\mathrm{x}$ & $\mathrm{y}$ & $\mathrm{z}$ & $\mathrm{U}(\mathrm{eq})$ \\
\hline $\operatorname{Ru}(1)$ & $6819(1)$ & $3872(1)$ & $8314(1)$ & $27(1)$ \\
\hline $\mathrm{Cl}(1)$ & $2912(2)$ & $4134(1)$ & $7706(1)$ & $51(1)$ \\
\hline $\mathrm{Cl}(2)$ & $6388(2)$ & $6302(1)$ & $7741(1)$ & $61(1)$ \\
\hline $\mathrm{S}(1)$ & $5141(1)$ & $3815(1)$ & $8081(1)$ & $31(1)$ \\
\hline $\mathrm{S}(2)$ & $6869(2)$ & 4953(1) & $8142(1)$ & $37(1)$ \\
\hline $\mathrm{F}(1)$ & $8934(4)$ & $2709(3)$ & $9023(1)$ & $53(1)$ \\
\hline $\mathrm{F}(2)$ & $6253(3)$ & $4336(2)$ & $9205(1)$ & $45(1)$ \\
\hline $\mathrm{O}(1)$ & $8618(4)$ & $3906(2)$ & $8476(1)$ & $36(1)$ \\
\hline $\mathrm{N}(1)$ & $6887(4)$ & 2599(3) & $8760(1)$ & $26(1)$ \\
\hline $\mathrm{N}(2)$ & 5543(4) & $3211(3)$ & $8875(1)$ & $27(1)$ \\
\hline $\mathrm{C}(1)$ & $6397(5)$ & $3175(3)$ & $8677(2)$ & $27(1)$ \\
\hline$C(2)$ & $6312(6)$ & $2185(3)$ & $8997(2)$ & $34(2)$ \\
\hline$C(3)$ & $5543(5)$ & $2671(3)$ & $9138(2)$ & $29(1)$ \\
\hline
\end{tabular}




\begin{tabular}{|c|c|c|c|c|}
\hline $\mathrm{C}(4)$ & $7722(6)$ & 2284(3) & $8584(2)$ & $32(1)$ \\
\hline $\mathrm{C}(5)$ & $8739(6)$ & $2314(4)$ & $8735(2)$ & $39(2)$ \\
\hline$C(6)$ & $9584(7)$ & $1985(5)$ & $8608(2)$ & $54(2)$ \\
\hline$C(7)$ & $9361(8)$ & $1585(5)$ & $8323(2)$ & $59(2)$ \\
\hline $\mathrm{C}(8)$ & $8389(8)$ & $1522(4)$ & $8168(2)$ & $53(2)$ \\
\hline $\mathrm{C}(9)$ & $7496(7)$ & $1887(4)$ & $8292(2)$ & $41(2)$ \\
\hline$C(10)$ & $6401(7)$ & $1854(4)$ & $8133(2)$ & $49(2)$ \\
\hline $\mathrm{C}(11)$ & $4846(5)$ & $3751(3)$ & $8919(2)$ & $30(1)$ \\
\hline$C(12)$ & $3767(6)$ & $3701(4)$ & $8826(2)$ & $35(1)$ \\
\hline$C(13)$ & $3110(6)$ & $4224(4)$ & $8914(2)$ & $47(2)$ \\
\hline$C(14)$ & $3500(7)$ & $4776(4)$ & $9081(2)$ & $49(2)$ \\
\hline$C(15)$ & $4566(7)$ & $4825(4)$ & $9181(2)$ & $46(2)$ \\
\hline$C(16)$ & $5218(6)$ & $4311(3)$ & $9098(2)$ & $35(1)$ \\
\hline$C(17)$ & $3333(6)$ & $3106(4)$ & $8631(2)$ & $45(2)$ \\
\hline$C(18)$ & $7401(5)$ & $3348(3)$ & $7987(2)$ & $29(1)$ \\
\hline$C(19)$ & $8533(5)$ & $3274(3)$ & $7972(2)$ & $31(1)$ \\
\hline$C(20)$ & $9184(6)$ & $3579(4)$ & $8230(2)$ & $35(1)$ \\
\hline$C(21)$ & $10279(6)$ & $3538(5)$ & $8231(2)$ & $48(2)$ \\
\hline$C(22)$ & $10723(6)$ & $3165(5)$ & $7971(2)$ & $52(2)$ \\
\hline$C(23)$ & $10102(6)$ & $2856(4)$ & $7714(2)$ & $46(2)$ \\
\hline$C(24)$ & $9015(6)$ & 2906(4) & $7713(2)$ & $38(2)$ \\
\hline$C(25)$ & $9204(7)$ & $4327(4)$ & $8732(2)$ & $46(2)$ \\
\hline$C(26)$ & $9546(8)$ & 4957(4) & $8553(2)$ & $54(2)$ \\
\hline$C(27)$ & $8477(7)$ & $4456(5)$ & $9016(2)$ & $53(2)$ \\
\hline$C(28)$ & $4894(6)$ & 4579(4) & $7879(2)$ & $35(1)$ \\
\hline$C(29)$ & $3924(7)$ & $4711(4)$ & $7701(2)$ & $42(2)$ \\
\hline$C(30)$ & $3737(7)$ & $5316(4)$ & $7523(2)$ & $51(2)$ \\
\hline$C(31)$ & $4509(8)$ & $5783(4)$ & $7536(2)$ & $51(2)$ \\
\hline$C(32)$ & $5446(8)$ & $5675(4)$ & $7719(2)$ & $46(2)$ \\
\hline$C(33)$ & $5672(7)$ & $5075(4)$ & $7895(2)$ & $40(2)$ \\
\hline $\mathrm{Ru}(2)$ & $3658(1)$ & $2668(1)$ & $6251(1)$ & $24(1)$ \\
\hline $\mathrm{Cl}(3)$ & $4354(2)$ & $424(1)$ & $5557(1)$ & $40(1)$ \\
\hline $\mathrm{Cl}(4)$ & $2150(1)$ & 2931(1) & $4916(1)$ & $37(1)$ \\
\hline $\mathrm{S}(3)$ & $3848(1)$ & $1619(1)$ & $6056(1)$ & $27(1)$ \\
\hline $\mathrm{S}(4)$ & $2843(1)$ & $2919(1)$ & $5722(1)$ & $26(1)$ \\
\hline$F(3)$ & $4753(5)$ & $3787(3)$ & $7179(1)$ & $65(1)$ \\
\hline $\mathrm{O}(2)$ & $3664(4)$ & $3773(3)$ & $6388(1)$ & $42(1)$ \\
\hline $\mathrm{N}(3)$ & $4363(5)$ & $2518(3)$ & 7031(1) & $36(1)$ \\
\hline $\mathrm{N}(4)$ & 2791(5) & $2132(3)$ & $6920(2)$ & $40(1)$ \\
\hline$C(34)$ & $3625(5)$ & 2391(3) & $6776(2)$ & $29(1)$ \\
\hline$C(35)$ & $4032(7)$ & $2312(5)$ & $7378(2)$ & $48(2)$ \\
\hline$C(36)$ & $2876(8)$ & $2139(6)$ & $7306(2)$ & $65(3)$ \\
\hline$C(37)$ & $5425(6)$ & $2720(4)$ & $6992(2)$ & $46(2)$ \\
\hline$C(38)$ & $5594(7)$ & $3425(5)$ & $7092(2)$ & $55(2)$ \\
\hline$C(39)$ & $6531(8)$ & $3694(5)$ & $7090(2)$ & $61(2)$ \\
\hline$C(40)$ & $7350(7)$ & $3289(5)$ & $6981(2)$ & $56(2)$ \\
\hline $\mathrm{C}(41)$ & $7259(9)$ & $2633(6)$ & $6888(3)$ & $72(3)$ \\
\hline$C(42)$ & $6205(7)$ & $2326(5)$ & $6896(3)$ & $62(2)$ \\
\hline $\mathrm{C}(43)$ & $5935(10)$ & $1629(5)$ & $6801(3)$ & $78(3)$ \\
\hline
\end{tabular}




\begin{tabular}{|c|c|c|c|c|}
\hline C(44) & $1863(6)$ & $1866(5)$ & $6750(2)$ & $47(2)$ \\
\hline$C(45)$ & $1637(15)$ & $1226(8)$ & $6645(9)$ & $52(3)$ \\
\hline$C(46)$ & $647(15)$ & $1100(12)$ & $6506(5)$ & $59(3)$ \\
\hline $\mathrm{C}(47)$ & $-122(18)$ & $1584(15)$ & $6469(7)$ & $69(4)$ \\
\hline $\mathrm{C}(48)$ & $40(12)$ & 2216(12) & $6569(5)$ & $65(3)$ \\
\hline C(49) & $1076(13)$ & $2366(10)$ & $6717(7)$ & $58(3)$ \\
\hline$C(50)$ & $2478(16)$ & 731(9) & $6704(6)$ & $55(4)$ \\
\hline $\mathrm{F}(4)$ & $1246(11)$ & $3016(7)$ & $6841(4)$ & $71(3)$ \\
\hline$C(45 X)$ & 1948(17) & $1162(8)$ & $6685(10)$ & $52(3)$ \\
\hline$C(46 X)$ & 1091(16) & $794(12)$ & $6546(5)$ & $59(3)$ \\
\hline$C(47 X)$ & $146(17)$ & $1109(14)$ & $6456(6)$ & $69(4)$ \\
\hline$C(48 X)$ & $17(19)$ & $1760(15)$ & $6508(8)$ & $65(3)$ \\
\hline$C(49 X)$ & $867(12)$ & 2115(12) & $6672(7)$ & $58(3)$ \\
\hline$C(50 X)$ & 2929(17) & $770(12)$ & $6777(7)$ & $55(4)$ \\
\hline$F(4 X)$ & $823(13)$ & 2766(9) & $6765(5)$ & $74(4)$ \\
\hline $\mathrm{C}(51)$ & $5047(5)$ & 2881(4) & $6217(2)$ & $31(1)$ \\
\hline$C(52)$ & $5407(6)$ & $3557(4)$ & $6252(2)$ & $36(1)$ \\
\hline$C(53)$ & $6441(7)$ & $3751(5)$ & $6201(2)$ & $50(2)$ \\
\hline$C(54)$ & $6765(9)$ & $4386(5)$ & $6252(2)$ & $63(2)$ \\
\hline$C(55)$ & 6054(9) & $4843(5)$ & $6352(2)$ & $63(2)$ \\
\hline$C(56)$ & $4980(8)$ & $4686(4)$ & $6407(2)$ & $54(2)$ \\
\hline$C(57)$ & $4674(6)$ & $4035(4)$ & $6349(2)$ & $38(2)$ \\
\hline$C(58)$ & $2796(8)$ & $4232(5)$ & $6449(2)$ & $65(2)$ \\
\hline C(59) & 2636(14) & $4318(8)$ & 6811(3) & $54(3)$ \\
\hline $\mathrm{C}(60)$ & $1862(12)$ & $4082(8)$ & $6226(4)$ & $56(3)$ \\
\hline$C(58 X)$ & $2796(8)$ & $4232(5)$ & $6449(2)$ & $65(2)$ \\
\hline$C(59 X)$ & $2120(16)$ & $3871(9)$ & $6669(5)$ & $54(3)$ \\
\hline$C(60 X)$ & $2440(17)$ & $4567(10)$ & $6147(4)$ & $56(3)$ \\
\hline $\mathrm{C}(61)$ & $3517(5)$ & $1658(3)$ & $5608(2)$ & $25(1)$ \\
\hline$C(62)$ & $3723(5)$ & $1125(3)$ & $5383(2)$ & $29(1)$ \\
\hline$C(63)$ & $3463(5)$ & $1146(4)$ & $5031(2)$ & $33(1)$ \\
\hline$C(64)$ & $2970(6)$ & $1701(4)$ & $4886(2)$ & $33(1)$ \\
\hline$C(65)$ & $2775(5)$ & $2232(3)$ & $5101(2)$ & $28(1)$ \\
\hline$C(66)$ & $3050(5)$ & $2227(3)$ & $5459(2)$ & $23(1)$ \\
\hline $\operatorname{Ru}(3)$ & $8359(1)$ & $3186(1)$ & $4779(1)$ & $28(1)$ \\
\hline $\mathrm{Cl}(5)$ & $7051(2)$ & $3439(1)$ & $3415(1)$ & $63(1)$ \\
\hline $\mathrm{Cl}(6)$ & $11199(2)$ & $4608(1)$ & $4087(1)$ & $61(1)$ \\
\hline $\mathrm{S}(5)$ & $7512(1)$ & $3269(1)$ & $4243(1)$ & $33(1)$ \\
\hline$S(6)$ & $9656(1)$ & $3860(1)$ & $4576(1)$ & $37(1)$ \\
\hline $\mathrm{F}(5)$ & $7600(4)$ & 2071(2) & $5775(1)$ & $46(1)$ \\
\hline$F(6)$ & $7462(3)$ & $4321(2)$ & $5420(1)$ & $42(1)$ \\
\hline $\mathrm{O}(3)$ & $9407(4)$ & $3005(2)$ & $5283(1)$ & $34(1)$ \\
\hline $\mathrm{N}(5)$ & $6574(4)$ & $2498(3)$ & $5176(1)$ & $28(1)$ \\
\hline $\mathrm{N}(6)$ & $6187(4)$ & $3503(3)$ & $5023(1)$ & $28(1)$ \\
\hline $\mathrm{C}(67)$ & $6931(5)$ & $3032(3)$ & $5012(2)$ & $26(1)$ \\
\hline $\mathrm{C}(68)$ & $5480(5)$ & $2580(3)$ & $5282(2)$ & $31(1)$ \\
\hline $\mathrm{C}(69)$ & $5316(5)$ & $3323(3)$ & $5246(2)$ & $31(1)$ \\
\hline$C(70)$ & $7003(5)$ & $1846(3)$ & $5194(2)$ & $28(1)$ \\
\hline$C(71)$ & $7491(5)$ & $1626(3)$ & $5502(2)$ & $33(1)$ \\
\hline
\end{tabular}




\begin{tabular}{|c|c|c|c|c|}
\hline$C(72)$ & $7872(6)$ & $1000(4)$ & $5554(2)$ & $40(2)$ \\
\hline$C(73)$ & 7734(6) & $560(4)$ & $5279(2)$ & $43(2)$ \\
\hline $\mathrm{C}(74)$ & $7252(5)$ & $757(4)$ & 4961(2) & $38(2)$ \\
\hline$C(75)$ & $6843(5)$ & 1398(3) & $4915(2)$ & $30(1)$ \\
\hline$C(76)$ & $6277(6)$ & 1611(4) & $4587(2)$ & $35(2)$ \\
\hline C(77) & $6331(5)$ & 4173(3) & $4919(2)$ & $31(1)$ \\
\hline$C(78)$ & $5770(6)$ & $4440(4)$ & $4634(2)$ & $40(2)$ \\
\hline C(79) & $5985(7)$ & 5099(4) & $4544(2)$ & $48(2)$ \\
\hline$C(80)$ & $6710(7)$ & $5466(4)$ & $4740(2)$ & $48(2)$ \\
\hline $\mathrm{C}(81)$ & $7234(6)$ & $5215(4)$ & $5032(2)$ & $43(2)$ \\
\hline $\mathrm{C}(82)$ & 7004(6) & $4580(4)$ & $5120(2)$ & $35(1)$ \\
\hline C(83) & 4975(7) & 4030(4) & $4421(2)$ & $49(2)$ \\
\hline$C(84)$ & $8742(5)$ & $2316(3)$ & $4732(2)$ & $28(1)$ \\
\hline C(85) & $9462(5)$ & 2004(3) & 4991(2) & $30(1)$ \\
\hline$C(86)$ & $9859(5)$ & $1362(3)$ & $4950(2)$ & $32(1)$ \\
\hline C(87) & $10535(5)$ & 1086(4) & $5206(2)$ & $40(2)$ \\
\hline C(88) & $10803(5)$ & $1436(4)$ & $5503(2)$ & $39(2)$ \\
\hline C(89) & $10417(5)$ & 2078(4) & $5553(2)$ & $36(2)$ \\
\hline$C(90)$ & 9794(5) & 2364(3) & $5286(2)$ & $31(1)$ \\
\hline $\mathrm{C}(91)$ & $9875(6)$ & 3453(4) & $5559(2)$ & $44(2)$ \\
\hline$C(92)$ & 9171(6) & $3434(4)$ & $5865(2)$ & $43(2)$ \\
\hline C(93) & $10056(6)$ & $4119(4)$ & $5415(2)$ & $47(2)$ \\
\hline$C(94)$ & $8389(6)$ & $3693(3)$ & $3980(2)$ & $36(1)$ \\
\hline C(95) & $8199(6)$ & $3771(4)$ & $3621(2)$ & $43(2)$ \\
\hline$C(96)$ & $8881(7)$ & 4111(4) & $3411(2)$ & $50(2)$ \\
\hline C(97) & 9792(7) & $4383(4)$ & $3564(2)$ & $51(2)$ \\
\hline C(98) & $10004(6)$ & $4305(4)$ & 3914(2) & $44(2)$ \\
\hline C(99) & $9329(6)$ & 3959(3) & $4133(2)$ & $38(2)$ \\
\hline $\mathrm{C}(1 \mathrm{~S})$ & 9064(11) & $4867(7)$ & $7532(3)$ & $93(4)$ \\
\hline $\mathrm{Cl}(1 \mathrm{~S})$ & $8332(3)$ & $5016(2)$ & $7137(1)$ & $91(1)$ \\
\hline $\mathrm{Cl}(2 \mathrm{~S})$ & 10289(3) & $5266(3)$ & $7537(1)$ & $121(2)$ \\
\hline
\end{tabular}

Table 3. Bond lengths $(\AA)$ and angles $\left.\left({ }^{\circ}\right]\right)$ for $3\left(\mathrm{C}_{33} \mathrm{H}_{30} \mathrm{Cl}_{2} \mathrm{~F}_{2} \mathrm{~N}_{2} \mathrm{ORuS}_{2}\right) \mathrm{CH}_{2} \mathrm{Cl}_{2}$ (Ru-1c).

\begin{tabular}{ll}
\hline $\mathrm{Ru}(1)-\mathrm{C}(18)$ & $1.830(7)$ \\
$\mathrm{Ru}(1)-\mathrm{C}(1)$ & $2.072(6)$ \\
$\mathrm{Ru}(1)-\mathrm{S}(1)$ & $2.2591(18)$ \\
$\mathrm{Ru}(1)-\mathrm{S}(2)$ & $2.2829(17)$ \\
$\mathrm{Ru}(1)-\mathrm{O}(1)$ & $2.325(5)$ \\
$\mathrm{Cl}(1)-\mathrm{C}(29)$ & $1.731(9)$ \\
$\mathrm{Cl}(2)-\mathrm{C}(32)$ & $1.737(9)$ \\
$\mathrm{S}(1)-\mathrm{C}(28)$ & $1.747(7)$ \\
$\mathrm{S}(2)-\mathrm{C}(33)$ & $1.757(8)$ \\
$\mathrm{F}(1)-\mathrm{C}(5)$ & $1.376(9)$ \\
$\mathrm{F}(2)-\mathrm{C}(16)$ & $1.350(9)$ \\
$\mathrm{O}(1)-\mathrm{C}(20)$ & $1.384(9)$ \\
$\mathrm{O}(1)-\mathrm{C}(25)$ & $1.469(8)$ \\
$\mathrm{N}(1)-\mathrm{C}(1)$ & $1.348(8)$ \\
$\mathrm{N}(1)-\mathrm{C}(4)$ & $1.434(9)$
\end{tabular}




\begin{tabular}{|c|c|}
\hline $\mathrm{N}(1)-\mathrm{C}(2)$ & $1.462(8)$ \\
\hline $\mathrm{N}(2)-\mathrm{C}(1)$ & $1.357(8)$ \\
\hline $\mathrm{N}(2)-\mathrm{C}(11)$ & $1.418(8)$ \\
\hline $\mathrm{N}(2)-\mathrm{C}(3)$ & $1.487(8)$ \\
\hline$C(2)-C(3)$ & $1.503(9)$ \\
\hline $\mathrm{C}(2)-\mathrm{H}(2 \mathrm{~A})$ & 0.9900 \\
\hline $\mathrm{C}(2)-\mathrm{H}(2 \mathrm{~B})$ & 0.9900 \\
\hline $\mathrm{C}(3)-\mathrm{H}(3 \mathrm{~A})$ & 0.9900 \\
\hline $\mathrm{C}(3)-\mathrm{H}(3 \mathrm{~B})$ & 0.9900 \\
\hline$C(4)-C(5)$ & $1.382(11)$ \\
\hline $\mathrm{C}(4)-\mathrm{C}(9)$ & $1.394(10)$ \\
\hline$C(5)-C(6)$ & $1.369(11)$ \\
\hline$C(6)-C(7)$ & $1.378(13)$ \\
\hline $\mathrm{C}(6)-\mathrm{H}(6)$ & 0.9500 \\
\hline$C(7)-C(8)$ & $1.342(14)$ \\
\hline $\mathrm{C}(7)-\mathrm{H}(7)$ & 0.9500 \\
\hline $\mathrm{C}(8)-\mathrm{C}(9)$ & $1.451(12)$ \\
\hline $\mathrm{C}(8)-\mathrm{H}(8)$ & 0.9500 \\
\hline$C(9)-C(10)$ & $1.483(12)$ \\
\hline $\mathrm{C}(10)-\mathrm{H}(10 \mathrm{~A})$ & 0.9800 \\
\hline $\mathrm{C}(10)-\mathrm{H}(10 \mathrm{~B})$ & 0.9800 \\
\hline $\mathrm{C}(10)-\mathrm{H}(10 \mathrm{C})$ & 0.9800 \\
\hline$C(11)-C(16)$ & $1.390(10)$ \\
\hline$C(11)-C(12)$ & $1.395(10)$ \\
\hline$C(12)-C(13)$ & $1.397(11)$ \\
\hline$C(12)-C(17)$ & $1.503(11)$ \\
\hline$C(13)-C(14)$ & $1.365(13)$ \\
\hline $\mathrm{C}(13)-\mathrm{H}(13)$ & 0.9500 \\
\hline$C(14)-C(15)$ & $1.383(12)$ \\
\hline $\mathrm{C}(14)-\mathrm{H}(14)$ & 0.9500 \\
\hline$C(15)-C(16)$ & $1.374(11)$ \\
\hline $\mathrm{C}(15)-\mathrm{H}(15)$ & 0.9500 \\
\hline $\mathrm{C}(17)-\mathrm{H}(17 \mathrm{~A})$ & 0.9800 \\
\hline $\mathrm{C}(17)-\mathrm{H}(17 \mathrm{~B})$ & 0.9800 \\
\hline $\mathrm{C}(17)-\mathrm{H}(17 \mathrm{C})$ & 0.9800 \\
\hline$C(18)-C(19)$ & $1.444(9)$ \\
\hline $\mathrm{C}(18)-\mathrm{H}(18)$ & 0.9500 \\
\hline C(19)-C(20) & $1.396(10)$ \\
\hline C(19)-C(24) & $1.407(10)$ \\
\hline$C(20)-C(21)$ & $1.387(11)$ \\
\hline$C(21)-C(22)$ & $1.393(12)$ \\
\hline $\mathrm{C}(21)-\mathrm{H}(21)$ & 0.9500 \\
\hline $\mathrm{C}(22)-\mathrm{C}(23)$ & $1.377(12)$ \\
\hline $\mathrm{C}(22)-\mathrm{H}(22)$ & 0.9500 \\
\hline $\mathrm{C}(23)-\mathrm{C}(24)$ & $1.378(11)$ \\
\hline $\mathrm{C}(23)-\mathrm{H}(23)$ & 0.9500 \\
\hline $\mathrm{C}(24)-\mathrm{H}(24)$ & 0.9500 \\
\hline$C(25)-C(27)$ & $1.490(11)$ \\
\hline$C(25)-C(26)$ & $1.522(11)$ \\
\hline
\end{tabular}




\begin{tabular}{|c|c|}
\hline $\mathrm{C}(25)-\mathrm{H}(25)$ & 1.0000 \\
\hline $\mathrm{C}(26)-\mathrm{H}(26 \mathrm{~A})$ & 0.9800 \\
\hline $\mathrm{C}(26)-\mathrm{H}(26 \mathrm{~B})$ & 0.9800 \\
\hline $\mathrm{C}(26)-\mathrm{H}(26 \mathrm{C})$ & 0.9800 \\
\hline $\mathrm{C}(27)-\mathrm{H}(27 \mathrm{~A})$ & 0.9800 \\
\hline $\mathrm{C}(27)-\mathrm{H}(27 \mathrm{~B})$ & 0.9800 \\
\hline $\mathrm{C}(27)-\mathrm{H}(27 \mathrm{C})$ & 0.9800 \\
\hline $\mathrm{C}(28)-\mathrm{C}(29)$ & $1.396(11)$ \\
\hline$C(28)-C(33)$ & $1.403(11)$ \\
\hline$C(29)-C(30)$ & $1.413(11)$ \\
\hline$C(30)-C(31)$ & $1.356(13)$ \\
\hline $\mathrm{C}(30)-\mathrm{H}(30)$ & 0.9500 \\
\hline $\mathrm{C}(31)-\mathrm{C}(32)$ & $1.362(13)$ \\
\hline $\mathrm{C}(31)-\mathrm{H}(31)$ & 0.9500 \\
\hline $\mathrm{C}(32)-\mathrm{C}(33)$ & $1.409(11)$ \\
\hline $\mathrm{Ru}(2)-\mathrm{C}(51)$ & $1.820(7)$ \\
\hline $\mathrm{Ru}(2)-\mathrm{C}(34)$ & $2.096(6)$ \\
\hline $\mathrm{Ru}(2)-\mathrm{S}(3)$ & $2.2639(16)$ \\
\hline $\mathrm{Ru}(2)-\mathrm{S}(4)$ & $2.2831(15)$ \\
\hline $\mathrm{Ru}(2)-\mathrm{O}(2)$ & $2.293(5)$ \\
\hline $\mathrm{Cl}(3)-\mathrm{C}(62)$ & $1.739(7)$ \\
\hline $\mathrm{Cl}(4)-\mathrm{C}(65)$ & $1.750(7)$ \\
\hline$S(3)-C(61)$ & $1.750(6)$ \\
\hline$S(4)-C(66)$ & $1.754(6)$ \\
\hline $\mathrm{F}(3)-\mathrm{C}(38)$ & $1.348(11)$ \\
\hline $\mathrm{O}(2)-\mathrm{C}(57)$ & $1.397(9)$ \\
\hline $\mathrm{O}(2)-\mathrm{C}(58)$ & $1.465(10)$ \\
\hline N(3)-C(34) & $1.339(9)$ \\
\hline N(3)-C(37) & $1.419(10)$ \\
\hline $\mathrm{N}(3)-\mathrm{C}(35)$ & $1.479(9)$ \\
\hline N(4)-C(34) & $1.326(9)$ \\
\hline N(4)-C(44) & $1.416(10)$ \\
\hline $\mathrm{N}(4)-\mathrm{C}(36)$ & $1.479(9)$ \\
\hline$C(35)-C(36)$ & $1.513(12)$ \\
\hline $\mathrm{C}(35)-\mathrm{H}(35 \mathrm{~A})$ & 0.9900 \\
\hline $\mathrm{C}(35)-\mathrm{H}(35 \mathrm{~B})$ & 0.9900 \\
\hline $\mathrm{C}(36)-\mathrm{H}(36 \mathrm{~A})$ & 0.9900 \\
\hline $\mathrm{C}(36)-\mathrm{H}(36 \mathrm{~B})$ & 0.9900 \\
\hline $\mathrm{C}(37)-\mathrm{C}(42)$ & $1.335(13)$ \\
\hline $\mathrm{C}(37)-\mathrm{C}(38)$ & $1.486(13)$ \\
\hline $\mathrm{C}(38)-\mathrm{C}(39)$ & $1.303(13)$ \\
\hline$C(39)-C(40)$ & $1.402(14)$ \\
\hline C(39)-H(39) & 0.9500 \\
\hline$C(40)-C(41)$ & $1.374(15)$ \\
\hline $\mathrm{C}(40)-\mathrm{H}(40)$ & 0.9500 \\
\hline $\mathrm{C}(41)-\mathrm{C}(42)$ & $1.472(14)$ \\
\hline $\mathrm{C}(41)-\mathrm{H}(41)$ & 0.9500 \\
\hline$C(42)-C(43)$ & $1.490(15)$ \\
\hline $\mathrm{C}(43)-\mathrm{H}(43 \mathrm{~A})$ & 0.9800 \\
\hline
\end{tabular}




\begin{tabular}{|c|c|}
\hline $\mathrm{C}(43)-\mathrm{H}(43 \mathrm{~B})$ & 0.9800 \\
\hline $\mathrm{C}(43)-\mathrm{H}(43 \mathrm{C})$ & 0.9800 \\
\hline$C(44)-C(45)$ & $1.380(17)$ \\
\hline$C(44)-C(49)$ & $1.417(17)$ \\
\hline$C(45)-C(46)$ & $1.357(19)$ \\
\hline$C(45)-C(50)$ & $1.47(2)$ \\
\hline$C(46)-C(47)$ & $1.38(3)$ \\
\hline $\mathrm{C}(46)-\mathrm{H}(46)$ & 0.9500 \\
\hline$C(47)-C(48)$ & $1.34(3)$ \\
\hline $\mathrm{C}(47)-\mathrm{H}(47)$ & 0.9500 \\
\hline$C(48)-C(49)$ & $1.43(2)$ \\
\hline $\mathrm{C}(48)-\mathrm{H}(48)$ & 0.9500 \\
\hline$C(49)-F(4)$ & $1.41(2)$ \\
\hline $\mathrm{C}(50)-\mathrm{H}(50 \mathrm{~A})$ & 0.9800 \\
\hline $\mathrm{C}(50)-\mathrm{H}(50 \mathrm{~B})$ & 0.9800 \\
\hline $\mathrm{C}(50)-\mathrm{H}(50 \mathrm{C})$ & 0.9800 \\
\hline $\mathrm{C}(51)-\mathrm{C}(52)$ & $1.443(10)$ \\
\hline $\mathrm{C}(51)-\mathrm{H}(51)$ & 0.9500 \\
\hline$C(52)-C(53)$ & $1.388(11)$ \\
\hline$C(52)-C(57)$ & $1.404(11)$ \\
\hline$C(53)-C(54)$ & $1.357(13)$ \\
\hline $\mathrm{C}(53)-\mathrm{H}(53)$ & 0.9500 \\
\hline $\mathrm{C}(54)-\mathrm{C}(55)$ & $1.359(15)$ \\
\hline $\mathrm{C}(54)-\mathrm{H}(54)$ & 0.9500 \\
\hline$C(55)-C(56)$ & $1.422(14)$ \\
\hline $\mathrm{C}(55)-\mathrm{H}(55)$ & 0.9500 \\
\hline$C(56)-C(57)$ & $1.384(11)$ \\
\hline $\mathrm{C}(56)-\mathrm{H}(56)$ & 0.9500 \\
\hline $\mathrm{C}(58)-\mathrm{C}(59)$ & $1.430(15)$ \\
\hline$C(58)-C(60)$ & $1.452(16)$ \\
\hline $\mathrm{C}(58)-\mathrm{H}(58)$ & 1.0000 \\
\hline $\mathrm{C}(59)-\mathrm{H}(59 \mathrm{~A})$ & 0.9800 \\
\hline $\mathrm{C}(59)-\mathrm{H}(59 \mathrm{~B})$ & 0.9800 \\
\hline $\mathrm{C}(59)-\mathrm{H}(59 \mathrm{C})$ & 0.9800 \\
\hline $\mathrm{C}(60)-\mathrm{H}(60 \mathrm{~A})$ & 0.9800 \\
\hline $\mathrm{C}(60)-\mathrm{H}(60 \mathrm{~B})$ & 0.9800 \\
\hline $\mathrm{C}(60)-\mathrm{H}(60 \mathrm{C})$ & 0.9800 \\
\hline$C(61)-C(66)$ & $1.399(9)$ \\
\hline $\mathrm{C}(61)-\mathrm{C}(62)$ & $1.414(9)$ \\
\hline$C(62)-C(63)$ & $1.374(9)$ \\
\hline$C(63)-C(64)$ & $1.384(10)$ \\
\hline $\mathrm{C}(63)-\mathrm{H}(63)$ & 0.9500 \\
\hline$C(64)-C(65)$ & $1.384(10)$ \\
\hline $\mathrm{C}(64)-\mathrm{H}(64)$ & 0.9500 \\
\hline$C(65)-C(66)$ & $1.400(9)$ \\
\hline $\mathrm{Ru}(3)-\mathrm{C}(84)$ & $1.834(7)$ \\
\hline $\mathrm{Ru}(3)-\mathrm{C}(67)$ & $2.083(6)$ \\
\hline $\mathrm{Ru}(3)-\mathrm{S}(5)$ & $2.2738(19)$ \\
\hline $\mathrm{Ru}(3)-\mathrm{S}(6)$ & $2.2994(16)$ \\
\hline
\end{tabular}




\begin{tabular}{|c|c|}
\hline $\mathrm{Ru}(3)-\mathrm{O}(3)$ & $2.312(5)$ \\
\hline $\mathrm{Cl}(5)-\mathrm{C}(95)$ & $1.749(9)$ \\
\hline $\mathrm{Cl}(6)-\mathrm{C}(98)$ & $1.728(9)$ \\
\hline $\mathrm{S}(5)-\mathrm{C}(94)$ & $1.763(7)$ \\
\hline $\mathrm{S}(6)-\mathrm{C}(99)$ & $1.741(8)$ \\
\hline $\mathrm{F}(5)-\mathrm{C}(71)$ & $1.382(8)$ \\
\hline $\mathrm{F}(6)-\mathrm{C}(82)$ & $1.366(8)$ \\
\hline $\mathrm{O}(3)-\mathrm{C}(90)$ & $1.382(8)$ \\
\hline $\mathrm{O}(3)-\mathrm{C}(91)$ & $1.493(9)$ \\
\hline $\mathrm{N}(5)-\mathrm{C}(67)$ & $1.340(8)$ \\
\hline $\mathrm{N}(5)-\mathrm{C}(70)$ & $1.424(8)$ \\
\hline$N(5)-C(68)$ & $1.473(8)$ \\
\hline $\mathrm{N}(6)-\mathrm{C}(67)$ & $1.341(8)$ \\
\hline $\mathrm{N}(6)-\mathrm{C}(77)$ & $1.423(9)$ \\
\hline $\mathrm{N}(6)-\mathrm{C}(69)$ & $1.479(8)$ \\
\hline$C(68)-C(69)$ & $1.519(9)$ \\
\hline $\mathrm{C}(68)-\mathrm{H}(68 \mathrm{~A})$ & 0.9900 \\
\hline $\mathrm{C}(68)-\mathrm{H}(68 \mathrm{~B})$ & 0.9900 \\
\hline $\mathrm{C}(69)-\mathrm{H}(69 \mathrm{~A})$ & 0.9900 \\
\hline $\mathrm{C}(69)-\mathrm{H}(69 \mathrm{~B})$ & 0.9900 \\
\hline $\mathrm{C}(70)-\mathrm{C}(71)$ & $1.377(10)$ \\
\hline $\mathrm{C}(70)-\mathrm{C}(75)$ & $1.408(9)$ \\
\hline $\mathrm{C}(71)-\mathrm{C}(72)$ & $1.363(10)$ \\
\hline $\mathrm{C}(72)-\mathrm{C}(73)$ & $1.384(12)$ \\
\hline $\mathrm{C}(72)-\mathrm{H}(72)$ & 0.9500 \\
\hline $\mathrm{C}(73)-\mathrm{C}(74)$ & $1.392(11)$ \\
\hline $\mathrm{C}(73)-\mathrm{H}(73)$ & 0.9500 \\
\hline$C(74)-C(75)$ & $1.401(10)$ \\
\hline $\mathrm{C}(74)-\mathrm{H}(74)$ & 0.9500 \\
\hline$C(75)-C(76)$ & $1.478(10)$ \\
\hline $\mathrm{C}(76)-\mathrm{H}(76 \mathrm{~A})$ & 0.9800 \\
\hline $\mathrm{C}(76)-\mathrm{H}(76 \mathrm{~B})$ & 0.9800 \\
\hline $\mathrm{C}(76)-\mathrm{H}(76 \mathrm{C})$ & 0.9800 \\
\hline $\mathrm{C}(77)-\mathrm{C}(78)$ & $1.382(10)$ \\
\hline $\mathrm{C}(77)-\mathrm{C}(82)$ & $1.385(10)$ \\
\hline $\mathrm{C}(78)-\mathrm{C}(79)$ & $1.405(11)$ \\
\hline $\mathrm{C}(78)-\mathrm{C}(83)$ & $1.507(12)$ \\
\hline $\mathrm{C}(79)-\mathrm{C}(80)$ & $1.371(13)$ \\
\hline $\mathrm{C}(79)-\mathrm{H}(79)$ & 0.9500 \\
\hline $\mathrm{C}(80)-\mathrm{C}(81)$ & $1.370(12)$ \\
\hline $\mathrm{C}(80)-\mathrm{H}(80)$ & 0.9500 \\
\hline $\mathrm{C}(81)-\mathrm{C}(82)$ & $1.362(10)$ \\
\hline $\mathrm{C}(81)-\mathrm{H}(81)$ & 0.9500 \\
\hline $\mathrm{C}(83)-\mathrm{H}(83 \mathrm{~A})$ & 0.9800 \\
\hline $\mathrm{C}(83)-\mathrm{H}(83 \mathrm{~B})$ & 0.9800 \\
\hline $\mathrm{C}(83)-\mathrm{H}(83 \mathrm{C})$ & 0.9800 \\
\hline $\mathrm{C}(84)-\mathrm{C}(85)$ & $1.455(10)$ \\
\hline $\mathrm{C}(84)-\mathrm{H}(84)$ & 0.9500 \\
\hline$C(85)-C(90)$ & $1.391(10)$ \\
\hline
\end{tabular}




\begin{tabular}{|c|c|}
\hline$C(85)-C(86)$ & $1.402(9)$ \\
\hline $\mathrm{C}(86)-\mathrm{C}(87)$ & $1.384(10)$ \\
\hline $\mathrm{C}(86)-\mathrm{H}(86)$ & 0.9500 \\
\hline $\mathrm{C}(87)-\mathrm{C}(88)$ & $1.368(11)$ \\
\hline $\mathrm{C}(87)-\mathrm{H}(87)$ & 0.9500 \\
\hline $\mathrm{C}(88)-\mathrm{C}(89)$ & $1.403(11)$ \\
\hline $\mathrm{C}(88)-\mathrm{H}(88)$ & 0.9500 \\
\hline $\mathrm{C}(89)-\mathrm{C}(90)$ & $1.383(10)$ \\
\hline C(89)-H(89) & 0.9500 \\
\hline C(91)-C(93) & $1.478(11)$ \\
\hline $\mathrm{C}(91)-\mathrm{C}(92)$ & $1.514(11)$ \\
\hline $\mathrm{C}(91)-\mathrm{H}(91)$ & 1.0000 \\
\hline $\mathrm{C}(92)-\mathrm{H}(92 \mathrm{~A})$ & 0.9800 \\
\hline $\mathrm{C}(92)-\mathrm{H}(92 \mathrm{~B})$ & 0.9800 \\
\hline $\mathrm{C}(92)-\mathrm{H}(92 \mathrm{C})$ & 0.9800 \\
\hline $\mathrm{C}(93)-\mathrm{H}(93 \mathrm{~A})$ & 0.9800 \\
\hline $\mathrm{C}(93)-\mathrm{H}(93 \mathrm{~B})$ & 0.9800 \\
\hline $\mathrm{C}(93)-\mathrm{H}(93 \mathrm{C})$ & 0.9800 \\
\hline$C(94)-C(95)$ & $1.393(11)$ \\
\hline C(94)-C(99) & $1.403(11)$ \\
\hline $\mathrm{C}(95)-\mathrm{C}(96)$ & $1.394(10)$ \\
\hline $\mathrm{C}(96)-\mathrm{C}(97)$ & $1.377(13)$ \\
\hline $\mathrm{C}(96)-\mathrm{H}(96)$ & 0.9500 \\
\hline $\mathrm{C}(97)-\mathrm{C}(98)$ & $1.368(13)$ \\
\hline $\mathrm{C}(97)-\mathrm{H}(97)$ & 0.9500 \\
\hline $\mathrm{C}(98)-\mathrm{C}(99)$ & $1.415(10)$ \\
\hline $\mathrm{C}(1 \mathrm{~S})-\mathrm{Cl}(2 \mathrm{~S})$ & $1.745(12)$ \\
\hline $\mathrm{C}(1 \mathrm{~S})-\mathrm{Cl}(1 \mathrm{~S})$ & $1.756(12)$ \\
\hline C(1S)-H(1S1) & 0.9900 \\
\hline $\mathrm{C}(1 \mathrm{~S})-\mathrm{H}(1 \mathrm{~S} 2)$ & 0.9900 \\
\hline $\mathrm{C}(18)-\mathrm{Ru}(1)-\mathrm{C}(1)$ & $101.5(3)$ \\
\hline $\mathrm{C}(18)-\mathrm{Ru}(1)-\mathrm{S}(1)$ & $95.9(2)$ \\
\hline $\mathrm{C}(1)-\mathrm{Ru}(1)-\mathrm{S}(1)$ & $87.76(18)$ \\
\hline $\mathrm{C}(18)-\mathrm{Ru}(1)-\mathrm{S}(2)$ & $109.5(2)$ \\
\hline $\mathrm{C}(1)-\mathrm{Ru}(1)-\mathrm{S}(2)$ & 148.99(19) \\
\hline $\mathrm{S}(1)-\mathrm{Ru}(1)-\mathrm{S}(2)$ & $88.52(7)$ \\
\hline $\mathrm{C}(18)-\mathrm{Ru}(1)-\mathrm{O}(1)$ & $77.0(2)$ \\
\hline $\mathrm{C}(1)-\mathrm{Ru}(1)-\mathrm{O}(1)$ & $97.2(2)$ \\
\hline $\mathrm{S}(1)-\mathrm{Ru}(1)-\mathrm{O}(1)$ & 171.99(13) \\
\hline $\mathrm{S}(2)-\mathrm{Ru}(1)-\mathrm{O}(1)$ & $90.41(13)$ \\
\hline $\mathrm{C}(28)-\mathrm{S}(1)-\mathrm{Ru}(1)$ & $105.8(3)$ \\
\hline $\mathrm{C}(33)-\mathrm{S}(2)-\mathrm{Ru}(1)$ & $104.7(3)$ \\
\hline $\mathrm{C}(20)-\mathrm{O}(1)-\mathrm{C}(25)$ & $118.2(6)$ \\
\hline $\mathrm{C}(20)-\mathrm{O}(1)-\mathrm{Ru}(1)$ & $109.8(4)$ \\
\hline $\mathrm{C}(25)-\mathrm{O}(1)-\mathrm{Ru}(1)$ & $130.1(5)$ \\
\hline $\mathrm{C}(1)-\mathrm{N}(1)-\mathrm{C}(4)$ & $127.9(5)$ \\
\hline $\mathrm{C}(1)-\mathrm{N}(1)-\mathrm{C}(2)$ & $113.7(5)$ \\
\hline $\mathrm{C}(4)-\mathrm{N}(1)-\mathrm{C}(2)$ & $116.5(5)$ \\
\hline
\end{tabular}




\begin{tabular}{|c|c|}
\hline$C(1)-N(2)-C(11)$ & $128.8(5)$ \\
\hline$C(1)-N(2)-C(3)$ & $111.9(5)$ \\
\hline $\mathrm{C}(11)-\mathrm{N}(2)-\mathrm{C}(3)$ & $117.4(5)$ \\
\hline $\mathrm{N}(1)-\mathrm{C}(1)-\mathrm{N}(2)$ & $106.5(5)$ \\
\hline $\mathrm{N}(1)-\mathrm{C}(1)-\mathrm{Ru}(1)$ & $127.7(5)$ \\
\hline $\mathrm{N}(2)-\mathrm{C}(1)-\mathrm{Ru}(1)$ & $125.8(5)$ \\
\hline $\mathrm{N}(1)-\mathrm{C}(2)-\mathrm{C}(3)$ & $101.9(5)$ \\
\hline $\mathrm{N}(1)-\mathrm{C}(2)-\mathrm{H}(2 \mathrm{~A})$ & 111.4 \\
\hline $\mathrm{C}(3)-\mathrm{C}(2)-\mathrm{H}(2 \mathrm{~A})$ & 111.4 \\
\hline $\mathrm{N}(1)-\mathrm{C}(2)-\mathrm{H}(2 \mathrm{~B})$ & 111.4 \\
\hline $\mathrm{C}(3)-\mathrm{C}(2)-\mathrm{H}(2 \mathrm{~B})$ & 111.4 \\
\hline $\mathrm{H}(2 \mathrm{~A})-\mathrm{C}(2)-\mathrm{H}(2 \mathrm{~B})$ & 109.2 \\
\hline $\mathrm{N}(2)-\mathrm{C}(3)-\mathrm{C}(2)$ & $102.1(5)$ \\
\hline $\mathrm{N}(2)-\mathrm{C}(3)-\mathrm{H}(3 \mathrm{~A})$ & 111.4 \\
\hline $\mathrm{C}(2)-\mathrm{C}(3)-\mathrm{H}(3 \mathrm{~A})$ & 111.4 \\
\hline $\mathrm{N}(2)-\mathrm{C}(3)-\mathrm{H}(3 \mathrm{~B})$ & 111.4 \\
\hline $\mathrm{C}(2)-\mathrm{C}(3)-\mathrm{H}(3 \mathrm{~B})$ & 111.4 \\
\hline $\mathrm{H}(3 \mathrm{~A})-\mathrm{C}(3)-\mathrm{H}(3 \mathrm{~B})$ & 109.2 \\
\hline$C(5)-C(4)-C(9)$ & $120.5(7)$ \\
\hline $\mathrm{C}(5)-\mathrm{C}(4)-\mathrm{N}(1)$ & $118.3(6)$ \\
\hline $\mathrm{C}(9)-\mathrm{C}(4)-\mathrm{N}(1)$ & $120.7(7)$ \\
\hline$C(6)-C(5)-F(1)$ & $117.3(7)$ \\
\hline$C(6)-C(5)-C(4)$ & $123.7(8)$ \\
\hline$F(1)-C(5)-C(4)$ & $119.0(6)$ \\
\hline$C(5)-C(6)-C(7)$ & $116.0(9)$ \\
\hline $\mathrm{C}(5)-\mathrm{C}(6)-\mathrm{H}(6)$ & 122.0 \\
\hline $\mathrm{C}(7)-\mathrm{C}(6)-\mathrm{H}(6)$ & 122.0 \\
\hline$C(8)-C(7)-C(6)$ & $123.4(9)$ \\
\hline $\mathrm{C}(8)-\mathrm{C}(7)-\mathrm{H}(7)$ & 118.3 \\
\hline $\mathrm{C}(6)-\mathrm{C}(7)-\mathrm{H}(7)$ & 118.3 \\
\hline$C(7)-C(8)-C(9)$ & $121.0(8)$ \\
\hline $\mathrm{C}(7)-\mathrm{C}(8)-\mathrm{H}(8)$ & 119.5 \\
\hline $\mathrm{C}(9)-\mathrm{C}(8)-\mathrm{H}(8)$ & 119.5 \\
\hline$C(4)-C(9)-C(8)$ & $115.3(8)$ \\
\hline$C(4)-C(9)-C(10)$ & $120.1(7)$ \\
\hline$C(8)-C(9)-C(10)$ & $124.6(7)$ \\
\hline $\mathrm{C}(9)-\mathrm{C}(10)-\mathrm{H}(10 \mathrm{~A})$ & 109.5 \\
\hline $\mathrm{C}(9)-\mathrm{C}(10)-\mathrm{H}(10 \mathrm{~B})$ & 109.5 \\
\hline $\mathrm{H}(10 \mathrm{~A})-\mathrm{C}(10)-\mathrm{H}(10 \mathrm{~B})$ & 109.5 \\
\hline $\mathrm{C}(9)-\mathrm{C}(10)-\mathrm{H}(10 \mathrm{C})$ & 109.5 \\
\hline $\mathrm{H}(10 \mathrm{~A})-\mathrm{C}(10)-\mathrm{H}(10 \mathrm{C})$ & 109.5 \\
\hline $\mathrm{H}(10 \mathrm{~B})-\mathrm{C}(10)-\mathrm{H}(10 \mathrm{C})$ & 109.5 \\
\hline$C(16)-C(11)-C(12)$ & $118.9(7)$ \\
\hline$C(16)-C(11)-N(2)$ & $119.3(6)$ \\
\hline $\mathrm{C}(12)-\mathrm{C}(11)-\mathrm{N}(2)$ & $121.3(6)$ \\
\hline $\mathrm{C}(11)-\mathrm{C}(12)-\mathrm{C}(13)$ & $118.0(7)$ \\
\hline$C(11)-C(12)-C(17)$ & $120.6(7)$ \\
\hline$C(13)-C(12)-C(17)$ & $121.3(7)$ \\
\hline$C(14)-C(13)-C(12)$ & $121.8(8)$ \\
\hline
\end{tabular}




\begin{tabular}{|c|c|}
\hline $\mathrm{C}(14)-\mathrm{C}(13)-\mathrm{H}(13)$ & 119.1 \\
\hline $\mathrm{C}(12)-\mathrm{C}(13)-\mathrm{H}(13)$ & 119.1 \\
\hline$C(13)-C(14)-C(15)$ & $120.6(8)$ \\
\hline $\mathrm{C}(13)-\mathrm{C}(14)-\mathrm{H}(14)$ & 119.7 \\
\hline $\mathrm{C}(15)-\mathrm{C}(14)-\mathrm{H}(14)$ & 119.7 \\
\hline$C(16)-C(15)-C(14)$ & $118.0(8)$ \\
\hline $\mathrm{C}(16)-\mathrm{C}(15)-\mathrm{H}(15)$ & 121.0 \\
\hline $\mathrm{C}(14)-\mathrm{C}(15)-\mathrm{H}(15)$ & 121.0 \\
\hline $\mathrm{F}(2)-\mathrm{C}(16)-\mathrm{C}(15)$ & $119.0(7)$ \\
\hline $\mathrm{F}(2)-\mathrm{C}(16)-\mathrm{C}(11)$ & $118.4(6)$ \\
\hline$C(15)-C(16)-C(11)$ & $122.5(7)$ \\
\hline $\mathrm{C}(12)-\mathrm{C}(17)-\mathrm{H}(17 \mathrm{~A})$ & 109.5 \\
\hline $\mathrm{C}(12)-\mathrm{C}(17)-\mathrm{H}(17 \mathrm{~B})$ & 109.5 \\
\hline $\mathrm{H}(17 \mathrm{~A})-\mathrm{C}(17)-\mathrm{H}(17 \mathrm{~B})$ & 109.5 \\
\hline $\mathrm{C}(12)-\mathrm{C}(17)-\mathrm{H}(17 \mathrm{C})$ & 109.5 \\
\hline $\mathrm{H}(17 \mathrm{~A})-\mathrm{C}(17)-\mathrm{H}(17 \mathrm{C})$ & 109.5 \\
\hline $\mathrm{H}(17 \mathrm{~B})-\mathrm{C}(17)-\mathrm{H}(17 \mathrm{C})$ & 109.5 \\
\hline $\mathrm{C}(19)-\mathrm{C}(18)-\mathrm{Ru}(1)$ & $121.7(5)$ \\
\hline $\mathrm{C}(19)-\mathrm{C}(18)-\mathrm{H}(18)$ & 119.2 \\
\hline $\mathrm{Ru}(1)-\mathrm{C}(18)-\mathrm{H}(18)$ & 119.2 \\
\hline$C(20)-C(19)-C(24)$ & $118.3(6)$ \\
\hline$C(20)-C(19)-C(18)$ & $118.1(6)$ \\
\hline$C(24)-C(19)-C(18)$ & $123.6(6)$ \\
\hline $\mathrm{O}(1)-\mathrm{C}(20)-\mathrm{C}(21)$ & $125.5(7)$ \\
\hline $\mathrm{O}(1)-\mathrm{C}(20)-\mathrm{C}(19)$ & $112.9(6)$ \\
\hline$C(21)-C(20)-C(19)$ & $121.6(7)$ \\
\hline$C(20)-C(21)-C(22)$ & $118.2(7)$ \\
\hline $\mathrm{C}(20)-\mathrm{C}(21)-\mathrm{H}(21)$ & 120.9 \\
\hline $\mathrm{C}(22)-\mathrm{C}(21)-\mathrm{H}(21)$ & 120.9 \\
\hline $\mathrm{C}(23)-\mathrm{C}(22)-\mathrm{C}(21)$ & $121.5(8)$ \\
\hline $\mathrm{C}(23)-\mathrm{C}(22)-\mathrm{H}(22)$ & 119.2 \\
\hline $\mathrm{C}(21)-\mathrm{C}(22)-\mathrm{H}(22)$ & 119.2 \\
\hline $\mathrm{C}(22)-\mathrm{C}(23)-\mathrm{C}(24)$ & $119.8(8)$ \\
\hline $\mathrm{C}(22)-\mathrm{C}(23)-\mathrm{H}(23)$ & 120.1 \\
\hline $\mathrm{C}(24)-\mathrm{C}(23)-\mathrm{H}(23)$ & 120.1 \\
\hline$C(23)-C(24)-C(19)$ & $120.6(7)$ \\
\hline $\mathrm{C}(23)-\mathrm{C}(24)-\mathrm{H}(24)$ & 119.7 \\
\hline $\mathrm{C}(19)-\mathrm{C}(24)-\mathrm{H}(24)$ & 119.7 \\
\hline $\mathrm{O}(1)-\mathrm{C}(25)-\mathrm{C}(27)$ & $106.5(6)$ \\
\hline $\mathrm{O}(1)-\mathrm{C}(25)-\mathrm{C}(26)$ & 109.1(6) \\
\hline$C(27)-C(25)-C(26)$ & $112.8(8)$ \\
\hline $\mathrm{O}(1)-\mathrm{C}(25)-\mathrm{H}(25)$ & 109.5 \\
\hline $\mathrm{C}(27)-\mathrm{C}(25)-\mathrm{H}(25)$ & 109.5 \\
\hline $\mathrm{C}(26)-\mathrm{C}(25)-\mathrm{H}(25)$ & 109.5 \\
\hline $\mathrm{C}(25)-\mathrm{C}(26)-\mathrm{H}(26 \mathrm{~A})$ & 109.5 \\
\hline $\mathrm{C}(25)-\mathrm{C}(26)-\mathrm{H}(26 \mathrm{~B})$ & 109.5 \\
\hline $\mathrm{H}(26 \mathrm{~A})-\mathrm{C}(26)-\mathrm{H}(26 \mathrm{~B})$ & 109.5 \\
\hline $\mathrm{C}(25)-\mathrm{C}(26)-\mathrm{H}(26 \mathrm{C})$ & 109.5 \\
\hline $\mathrm{H}(26 \mathrm{~A})-\mathrm{C}(26)-\mathrm{H}(26 \mathrm{C})$ & 109.5 \\
\hline
\end{tabular}




\begin{tabular}{|c|c|}
\hline $\mathrm{H}(26 \mathrm{~B})-\mathrm{C}(26)-\mathrm{H}(26 \mathrm{C})$ & 109.5 \\
\hline $\mathrm{C}(25)-\mathrm{C}(27)-\mathrm{H}(27 \mathrm{~A})$ & 109.5 \\
\hline $\mathrm{C}(25)-\mathrm{C}(27)-\mathrm{H}(27 \mathrm{~B})$ & 109.5 \\
\hline $\mathrm{H}(27 \mathrm{~A})-\mathrm{C}(27)-\mathrm{H}(27 \mathrm{~B})$ & 109.5 \\
\hline $\mathrm{C}(25)-\mathrm{C}(27)-\mathrm{H}(27 \mathrm{C})$ & 109.5 \\
\hline $\mathrm{H}(27 \mathrm{~A})-\mathrm{C}(27)-\mathrm{H}(27 \mathrm{C})$ & 109.5 \\
\hline $\mathrm{H}(27 \mathrm{~B})-\mathrm{C}(27)-\mathrm{H}(27 \mathrm{C})$ & 109.5 \\
\hline$C(29)-C(28)-C(33)$ & $118.6(7)$ \\
\hline $\mathrm{C}(29)-\mathrm{C}(28)-\mathrm{S}(1)$ & 121.1(6) \\
\hline $\mathrm{C}(33)-\mathrm{C}(28)-\mathrm{S}(1)$ & $120.2(6)$ \\
\hline$C(28)-C(29)-C(30)$ & $121.4(8)$ \\
\hline $\mathrm{C}(28)-\mathrm{C}(29)-\mathrm{Cl}(1)$ & $119.7(6)$ \\
\hline $\mathrm{C}(30)-\mathrm{C}(29)-\mathrm{Cl}(1)$ & $118.9(7)$ \\
\hline$C(31)-C(30)-C(29)$ & $119.0(8)$ \\
\hline $\mathrm{C}(31)-\mathrm{C}(30)-\mathrm{H}(30)$ & 120.5 \\
\hline $\mathrm{C}(29)-\mathrm{C}(30)-\mathrm{H}(30)$ & 120.5 \\
\hline$C(30)-C(31)-C(32)$ & $120.6(8)$ \\
\hline $\mathrm{C}(30)-\mathrm{C}(31)-\mathrm{H}(31)$ & 119.7 \\
\hline $\mathrm{C}(32)-\mathrm{C}(31)-\mathrm{H}(31)$ & 119.7 \\
\hline$C(31)-C(32)-C(33)$ & $122.3(9)$ \\
\hline $\mathrm{C}(31)-\mathrm{C}(32)-\mathrm{Cl}(2)$ & $118.8(6)$ \\
\hline $\mathrm{C}(33)-\mathrm{C}(32)-\mathrm{Cl}(2)$ & $118.9(7)$ \\
\hline$C(28)-C(33)-C(32)$ & $118.0(8)$ \\
\hline $\mathrm{C}(28)-\mathrm{C}(33)-\mathrm{S}(2)$ & $120.3(6)$ \\
\hline $\mathrm{C}(32)-\mathrm{C}(33)-\mathrm{S}(2)$ & $121.6(7)$ \\
\hline $\mathrm{C}(51)-\mathrm{Ru}(2)-\mathrm{C}(34)$ & $101.8(3)$ \\
\hline $\mathrm{C}(51)-\mathrm{Ru}(2)-\mathrm{S}(3)$ & $94.3(2)$ \\
\hline $\mathrm{C}(34)-\mathrm{Ru}(2)-\mathrm{S}(3)$ & $94.43(19)$ \\
\hline $\mathrm{C}(51)-\mathrm{Ru}(2)-\mathrm{S}(4)$ & $105.8(2)$ \\
\hline $\mathrm{C}(34)-\mathrm{Ru}(2)-\mathrm{S}(4)$ & $152.07(19)$ \\
\hline$S(3)-R u(2)-S(4)$ & $87.95(6)$ \\
\hline $\mathrm{C}(51)-\mathrm{Ru}(2)-\mathrm{O}(2)$ & $78.2(3)$ \\
\hline $\mathrm{C}(34)-\mathrm{Ru}(2)-\mathrm{O}(2)$ & $92.2(2)$ \\
\hline $\mathrm{S}(3)-\mathrm{Ru}(2)-\mathrm{O}(2)$ & $170.93(15)$ \\
\hline $\mathrm{S}(4)-\mathrm{Ru}(2)-\mathrm{O}(2)$ & $89.05(14)$ \\
\hline $\mathrm{C}(61)-\mathrm{S}(3)-\mathrm{Ru}(2)$ & $105.1(2)$ \\
\hline $\mathrm{C}(66)-\mathrm{S}(4)-\mathrm{Ru}(2)$ & $105.2(2)$ \\
\hline $\mathrm{C}(57)-\mathrm{O}(2)-\mathrm{C}(58)$ & $118.4(7)$ \\
\hline $\mathrm{C}(57)-\mathrm{O}(2)-\mathrm{Ru}(2)$ & 109.6(4) \\
\hline $\mathrm{C}(58)-\mathrm{O}(2)-\mathrm{Ru}(2)$ & $131.2(6)$ \\
\hline $\mathrm{C}(34)-\mathrm{N}(3)-\mathrm{C}(37)$ & $127.1(6)$ \\
\hline $\mathrm{C}(34)-\mathrm{N}(3)-\mathrm{C}(35)$ & $112.6(6)$ \\
\hline $\mathrm{C}(37)-\mathrm{N}(3)-\mathrm{C}(35)$ & $119.6(6)$ \\
\hline $\mathrm{C}(34)-\mathrm{N}(4)-\mathrm{C}(44)$ & $127.9(6)$ \\
\hline $\mathrm{C}(34)-\mathrm{N}(4)-\mathrm{C}(36)$ & $113.5(6)$ \\
\hline $\mathrm{C}(44)-\mathrm{N}(4)-\mathrm{C}(36)$ & $118.6(6)$ \\
\hline $\mathrm{N}(4)-\mathrm{C}(34)-\mathrm{N}(3)$ & $108.0(6)$ \\
\hline $\mathrm{N}(4)-\mathrm{C}(34)-\mathrm{Ru}(2)$ & $124.5(5)$ \\
\hline $\mathrm{N}(3)-\mathrm{C}(34)-\mathrm{Ru}(2)$ & $127.0(5)$ \\
\hline
\end{tabular}




\begin{tabular}{|c|c|}
\hline $\mathrm{N}(3)-\mathrm{C}(35)-\mathrm{C}(36)$ & $102.7(6)$ \\
\hline $\mathrm{N}(3)-\mathrm{C}(35)-\mathrm{H}(35 \mathrm{~A})$ & 111.2 \\
\hline $\mathrm{C}(36)-\mathrm{C}(35)-\mathrm{H}(35 \mathrm{~A})$ & 111.2 \\
\hline $\mathrm{N}(3)-\mathrm{C}(35)-\mathrm{H}(35 \mathrm{~B})$ & 111.2 \\
\hline $\mathrm{C}(36)-\mathrm{C}(35)-\mathrm{H}(35 \mathrm{~B})$ & 111.2 \\
\hline $\mathrm{H}(35 \mathrm{~A})-\mathrm{C}(35)-\mathrm{H}(35 \mathrm{~B})$ & 109.1 \\
\hline $\mathrm{N}(4)-\mathrm{C}(36)-\mathrm{C}(35)$ & $101.6(6)$ \\
\hline $\mathrm{N}(4)-\mathrm{C}(36)-\mathrm{H}(36 \mathrm{~A})$ & 111.4 \\
\hline $\mathrm{C}(35)-\mathrm{C}(36)-\mathrm{H}(36 \mathrm{~A})$ & 111.4 \\
\hline $\mathrm{N}(4)-\mathrm{C}(36)-\mathrm{H}(36 \mathrm{~B})$ & 111.4 \\
\hline $\mathrm{C}(35)-\mathrm{C}(36)-\mathrm{H}(36 \mathrm{~B})$ & 111.4 \\
\hline $\mathrm{H}(36 \mathrm{~A})-\mathrm{C}(36)-\mathrm{H}(36 \mathrm{~B})$ & 109.3 \\
\hline $\mathrm{C}(42)-\mathrm{C}(37)-\mathrm{N}(3)$ & $125.1(9)$ \\
\hline $\mathrm{C}(42)-\mathrm{C}(37)-\mathrm{C}(38)$ & $123.0(8)$ \\
\hline $\mathrm{N}(3)-\mathrm{C}(37)-\mathrm{C}(38)$ & $111.8(8)$ \\
\hline $\mathrm{C}(39)-\mathrm{C}(38)-\mathrm{F}(3)$ & $120.4(10)$ \\
\hline C(39)-C(38)-C(37) & $121.0(10)$ \\
\hline $\mathrm{F}(3)-\mathrm{C}(38)-\mathrm{C}(37)$ & $118.6(8)$ \\
\hline C(38)-C(39)-C(40) & $116.5(10)$ \\
\hline C(38)-C(39)-H(39) & 121.7 \\
\hline $\mathrm{C}(40)-\mathrm{C}(39)-\mathrm{H}(39)$ & 121.7 \\
\hline$C(41)-C(40)-C(39)$ & $125.9(10)$ \\
\hline $\mathrm{C}(41)-\mathrm{C}(40)-\mathrm{H}(40)$ & 117.1 \\
\hline $\mathrm{C}(39)-\mathrm{C}(40)-\mathrm{H}(40)$ & 117.1 \\
\hline $\mathrm{C}(40)-\mathrm{C}(41)-\mathrm{C}(42)$ & $117.6(11)$ \\
\hline $\mathrm{C}(40)-\mathrm{C}(41)-\mathrm{H}(41)$ & 121.2 \\
\hline $\mathrm{C}(42)-\mathrm{C}(41)-\mathrm{H}(41)$ & 121.2 \\
\hline$C(37)-C(42)-C(41)$ & $116.0(10)$ \\
\hline$C(37)-C(42)-C(43)$ & $117.9(9)$ \\
\hline$C(41)-C(42)-C(43)$ & $126.1(10)$ \\
\hline $\mathrm{C}(42)-\mathrm{C}(43)-\mathrm{H}(43 \mathrm{~A})$ & 109.5 \\
\hline $\mathrm{C}(42)-\mathrm{C}(43)-\mathrm{H}(43 \mathrm{~B})$ & 109.5 \\
\hline $\mathrm{H}(43 \mathrm{~A})-\mathrm{C}(43)-\mathrm{H}(43 \mathrm{~B})$ & 109.5 \\
\hline $\mathrm{C}(42)-\mathrm{C}(43)-\mathrm{H}(43 \mathrm{C})$ & 109.5 \\
\hline $\mathrm{H}(43 \mathrm{~A})-\mathrm{C}(43)-\mathrm{H}(43 \mathrm{C})$ & 109.5 \\
\hline $\mathrm{H}(43 \mathrm{~B})-\mathrm{C}(43)-\mathrm{H}(43 \mathrm{C})$ & 109.5 \\
\hline $\mathrm{C}(45)-\mathrm{C}(44)-\mathrm{N}(4)$ & $130.0(10)$ \\
\hline$C(45)-C(44)-C(49)$ & $120.5(12)$ \\
\hline $\mathrm{N}(4)-\mathrm{C}(44)-\mathrm{C}(49)$ & $109.3(11)$ \\
\hline$C(46)-C(45)-C(44)$ & $117.4(16)$ \\
\hline$C(46)-C(45)-C(50)$ & $125.3(16)$ \\
\hline$C(44)-C(45)-C(50)$ & $117.2(14)$ \\
\hline$C(45)-C(46)-C(47)$ & $123(2)$ \\
\hline $\mathrm{C}(45)-\mathrm{C}(46)-\mathrm{H}(46)$ & 118.7 \\
\hline $\mathrm{C}(47)-\mathrm{C}(46)-\mathrm{H}(46)$ & 118.7 \\
\hline$C(48)-C(47)-C(46)$ & $123.1(18)$ \\
\hline $\mathrm{C}(48)-\mathrm{C}(47)-\mathrm{H}(47)$ & 118.4 \\
\hline $\mathrm{C}(46)-\mathrm{C}(47)-\mathrm{H}(47)$ & 118.4 \\
\hline$C(47)-C(48)-C(49)$ & $116(2)$ \\
\hline
\end{tabular}




\begin{tabular}{|c|c|}
\hline $\mathrm{C}(47)-\mathrm{C}(48)-\mathrm{H}(48)$ & 122.1 \\
\hline $\mathrm{C}(49)-\mathrm{C}(48)-\mathrm{H}(48)$ & 122.1 \\
\hline $\mathrm{F}(4)-\mathrm{C}(49)-\mathrm{C}(44)$ & $122.7(14)$ \\
\hline $\mathrm{F}(4)-\mathrm{C}(49)-\mathrm{C}(48)$ & $116.8(15)$ \\
\hline $\mathrm{C}(44)-\mathrm{C}(49)-\mathrm{C}(48)$ & $120.5(18)$ \\
\hline $\mathrm{C}(45)-\mathrm{C}(50)-\mathrm{H}(50 \mathrm{~A})$ & 109.5 \\
\hline $\mathrm{C}(45)-\mathrm{C}(50)-\mathrm{H}(50 \mathrm{~B})$ & 109.5 \\
\hline $\mathrm{H}(50 \mathrm{~A})-\mathrm{C}(50)-\mathrm{H}(50 \mathrm{~B})$ & 109.5 \\
\hline $\mathrm{C}(45)-\mathrm{C}(50)-\mathrm{H}(50 \mathrm{C})$ & 109.5 \\
\hline $\mathrm{H}(50 \mathrm{~A})-\mathrm{C}(50)-\mathrm{H}(50 \mathrm{C})$ & 109.5 \\
\hline $\mathrm{H}(50 \mathrm{~B})-\mathrm{C}(50)-\mathrm{H}(50 \mathrm{C})$ & 109.5 \\
\hline $\mathrm{C}(52)-\mathrm{C}(51)-\mathrm{Ru}(2)$ & $121.1(5)$ \\
\hline $\mathrm{C}(52)-\mathrm{C}(51)-\mathrm{H}(51)$ & 119.4 \\
\hline $\mathrm{Ru}(2)-\mathrm{C}(51)-\mathrm{H}(51)$ & 119.4 \\
\hline$C(53)-C(52)-C(57)$ & $119.0(8)$ \\
\hline$C(53)-C(52)-C(51)$ & $123.2(8)$ \\
\hline$C(57)-C(52)-C(51)$ & $117.8(6)$ \\
\hline$C(54)-C(53)-C(52)$ & $121.7(10)$ \\
\hline $\mathrm{C}(54)-\mathrm{C}(53)-\mathrm{H}(53)$ & 119.1 \\
\hline $\mathrm{C}(52)-\mathrm{C}(53)-\mathrm{H}(53)$ & 119.1 \\
\hline$C(53)-C(54)-C(55)$ & 118.9(9) \\
\hline $\mathrm{C}(53)-\mathrm{C}(54)-\mathrm{H}(54)$ & 120.5 \\
\hline $\mathrm{C}(55)-\mathrm{C}(54)-\mathrm{H}(54)$ & 120.5 \\
\hline $\mathrm{C}(54)-\mathrm{C}(55)-\mathrm{C}(56)$ & $122.9(8)$ \\
\hline $\mathrm{C}(54)-\mathrm{C}(55)-\mathrm{H}(55)$ & 118.6 \\
\hline $\mathrm{C}(56)-\mathrm{C}(55)-\mathrm{H}(55)$ & 118.6 \\
\hline$C(57)-C(56)-C(55)$ & $116.7(9)$ \\
\hline $\mathrm{C}(57)-\mathrm{C}(56)-\mathrm{H}(56)$ & 121.7 \\
\hline $\mathrm{C}(55)-\mathrm{C}(56)-\mathrm{H}(56)$ & 121.7 \\
\hline $\mathrm{C}(56)-\mathrm{C}(57)-\mathrm{O}(2)$ & $126.2(8)$ \\
\hline$C(56)-C(57)-C(52)$ & $120.9(8)$ \\
\hline $\mathrm{O}(2)-\mathrm{C}(57)-\mathrm{C}(52)$ & $112.9(6)$ \\
\hline$C(59)-C(58)-C(60)$ & $116.5(12)$ \\
\hline $\mathrm{C}(59)-\mathrm{C}(58)-\mathrm{O}(2)$ & 112.3(9) \\
\hline $\mathrm{C}(60)-\mathrm{C}(58)-\mathrm{O}(2)$ & $111.5(9)$ \\
\hline $\mathrm{C}(59)-\mathrm{C}(58)-\mathrm{H}(58)$ & 105.2 \\
\hline $\mathrm{C}(60)-\mathrm{C}(58)-\mathrm{H}(58)$ & 105.2 \\
\hline $\mathrm{O}(2)-\mathrm{C}(58)-\mathrm{H}(58)$ & 105.2 \\
\hline $\mathrm{C}(58)-\mathrm{C}(59)-\mathrm{H}(59 \mathrm{~A})$ & 109.5 \\
\hline $\mathrm{C}(58)-\mathrm{C}(59)-\mathrm{H}(59 \mathrm{~B})$ & 109.5 \\
\hline H(59A)-C(59)-H(59B) & 109.5 \\
\hline $\mathrm{C}(58)-\mathrm{C}(59)-\mathrm{H}(59 \mathrm{C})$ & 109.5 \\
\hline $\mathrm{H}(59 \mathrm{~A})-\mathrm{C}(59)-\mathrm{H}(59 \mathrm{C})$ & 109.5 \\
\hline H(59B)-C(59)-H(59C) & 109.5 \\
\hline $\mathrm{C}(58)-\mathrm{C}(60)-\mathrm{H}(60 \mathrm{~A})$ & 109.5 \\
\hline $\mathrm{C}(58)-\mathrm{C}(60)-\mathrm{H}(60 \mathrm{~B})$ & 109.5 \\
\hline $\mathrm{H}(60 \mathrm{~A})-\mathrm{C}(60)-\mathrm{H}(60 \mathrm{~B})$ & 109.5 \\
\hline $\mathrm{C}(58)-\mathrm{C}(60)-\mathrm{H}(60 \mathrm{C})$ & 109.5 \\
\hline $\mathrm{H}(60 \mathrm{~A})-\mathrm{C}(60)-\mathrm{H}(60 \mathrm{C})$ & 109.5 \\
\hline
\end{tabular}




\begin{tabular}{|c|c|}
\hline $\mathrm{H}(60 \mathrm{~B})-\mathrm{C}(60)-\mathrm{H}(60 \mathrm{C})$ & 109.5 \\
\hline$C(66)-C(61)-C(62)$ & $117.5(6)$ \\
\hline $\mathrm{C}(66)-\mathrm{C}(61)-\mathrm{S}(3)$ & $120.7(5)$ \\
\hline $\mathrm{C}(62)-\mathrm{C}(61)-\mathrm{S}(3)$ & $121.7(5)$ \\
\hline$C(63)-C(62)-C(61)$ & $122.4(6)$ \\
\hline $\mathrm{C}(63)-\mathrm{C}(62)-\mathrm{Cl}(3)$ & $118.9(5)$ \\
\hline $\mathrm{C}(61)-\mathrm{C}(62)-\mathrm{Cl}(3)$ & $118.7(5)$ \\
\hline$C(62)-C(63)-C(64)$ & $119.9(6)$ \\
\hline $\mathrm{C}(62)-\mathrm{C}(63)-\mathrm{H}(63)$ & 120.0 \\
\hline $\mathrm{C}(64)-\mathrm{C}(63)-\mathrm{H}(63)$ & 120.0 \\
\hline$C(65)-C(64)-C(63)$ & $118.6(6)$ \\
\hline $\mathrm{C}(65)-\mathrm{C}(64)-\mathrm{H}(64)$ & 120.7 \\
\hline $\mathrm{C}(63)-\mathrm{C}(64)-\mathrm{H}(64)$ & 120.7 \\
\hline$C(64)-C(65)-C(66)$ & $122.5(6)$ \\
\hline $\mathrm{C}(64)-\mathrm{C}(65)-\mathrm{Cl}(4)$ & $118.2(5)$ \\
\hline $\mathrm{C}(66)-\mathrm{C}(65)-\mathrm{Cl}(4)$ & $119.2(5)$ \\
\hline$C(61)-C(66)-C(65)$ & $119.0(6)$ \\
\hline $\mathrm{C}(61)-\mathrm{C}(66)-\mathrm{S}(4)$ & $119.5(5)$ \\
\hline $\mathrm{C}(65)-\mathrm{C}(66)-\mathrm{S}(4)$ & $121.5(5)$ \\
\hline $\mathrm{C}(84)-\mathrm{Ru}(3)-\mathrm{C}(67)$ & $98.0(2)$ \\
\hline$C(84)-R u(3)-S(5)$ & $95.4(2)$ \\
\hline $\mathrm{C}(67)-\mathrm{Ru}(3)-\mathrm{S}(5)$ & $91.39(18)$ \\
\hline $\mathrm{C}(84)-\mathrm{Ru}(3)-\mathrm{S}(6)$ & $109.64(18)$ \\
\hline $\mathrm{C}(67)-\mathrm{Ru}(3)-\mathrm{S}(6)$ & $152.31(18)$ \\
\hline$S(5)-R u(3)-S(6)$ & $87.60(7)$ \\
\hline $\mathrm{C}(84)-\mathrm{Ru}(3)-\mathrm{O}(3)$ & $77.8(2)$ \\
\hline $\mathrm{C}(67)-\mathrm{Ru}(3)-\mathrm{O}(3)$ & $95.0(2)$ \\
\hline $\mathrm{S}(5)-\mathrm{Ru}(3)-\mathrm{O}(3)$ & $171.30(13)$ \\
\hline $\mathrm{S}(6)-\mathrm{Ru}(3)-\mathrm{O}(3)$ & $89.50(12)$ \\
\hline $\mathrm{C}(94)-\mathrm{S}(5)-\mathrm{Ru}(3)$ & $106.0(3)$ \\
\hline $\mathrm{C}(99)-\mathrm{S}(6)-\mathrm{Ru}(3)$ & $105.3(3)$ \\
\hline $\mathrm{C}(90)-\mathrm{O}(3)-\mathrm{C}(91)$ & $115.8(6)$ \\
\hline $\mathrm{C}(90)-\mathrm{O}(3)-\mathrm{Ru}(3)$ & $110.0(4)$ \\
\hline $\mathrm{C}(91)-\mathrm{O}(3)-\mathrm{Ru}(3)$ & $133.3(4)$ \\
\hline $\mathrm{C}(67)-\mathrm{N}(5)-\mathrm{C}(70)$ & $129.0(5)$ \\
\hline $\mathrm{C}(67)-\mathrm{N}(5)-\mathrm{C}(68)$ & $112.7(5)$ \\
\hline $\mathrm{C}(70)-\mathrm{N}(5)-\mathrm{C}(68)$ & $116.8(5)$ \\
\hline $\mathrm{C}(67)-\mathrm{N}(6)-\mathrm{C}(77)$ & $124.4(5)$ \\
\hline $\mathrm{C}(67)-\mathrm{N}(6)-\mathrm{C}(69)$ & $112.9(5)$ \\
\hline$C(77)-N(6)-C(69)$ & $120.4(5)$ \\
\hline $\mathrm{N}(5)-\mathrm{C}(67)-\mathrm{N}(6)$ & $107.5(5)$ \\
\hline $\mathrm{N}(5)-\mathrm{C}(67)-\mathrm{Ru}(3)$ & $130.2(5)$ \\
\hline $\mathrm{N}(6)-\mathrm{C}(67)-\mathrm{Ru}(3)$ & $122.3(4)$ \\
\hline $\mathrm{N}(5)-\mathrm{C}(68)-\mathrm{C}(69)$ & $102.2(5)$ \\
\hline $\mathrm{N}(5)-\mathrm{C}(68)-\mathrm{H}(68 \mathrm{~A})$ & 111.3 \\
\hline $\mathrm{C}(69)-\mathrm{C}(68)-\mathrm{H}(68 \mathrm{~A})$ & 111.3 \\
\hline $\mathrm{N}(5)-\mathrm{C}(68)-\mathrm{H}(68 \mathrm{~B})$ & 111.3 \\
\hline $\mathrm{C}(69)-\mathrm{C}(68)-\mathrm{H}(68 \mathrm{~B})$ & 111.3 \\
\hline $\mathrm{H}(68 \mathrm{~A})-\mathrm{C}(68)-\mathrm{H}(68 \mathrm{~B})$ & 109.2 \\
\hline
\end{tabular}




\begin{tabular}{|c|c|}
\hline $\mathrm{N}(6)-\mathrm{C}(69)-\mathrm{C}(68)$ & $101.2(5)$ \\
\hline $\mathrm{N}(6)-\mathrm{C}(69)-\mathrm{H}(69 \mathrm{~A})$ & 111.5 \\
\hline $\mathrm{C}(68)-\mathrm{C}(69)-\mathrm{H}(69 \mathrm{~A})$ & 111.5 \\
\hline $\mathrm{N}(6)-\mathrm{C}(69)-\mathrm{H}(69 \mathrm{~B})$ & 111.5 \\
\hline $\mathrm{C}(68)-\mathrm{C}(69)-\mathrm{H}(69 \mathrm{~B})$ & 111.5 \\
\hline $\mathrm{H}(69 \mathrm{~A})-\mathrm{C}(69)-\mathrm{H}(69 \mathrm{~B})$ & 109.3 \\
\hline$C(71)-C(70)-C(75)$ & $119.0(6)$ \\
\hline $\mathrm{C}(71)-\mathrm{C}(70)-\mathrm{N}(5)$ & $119.5(6)$ \\
\hline$C(75)-C(70)-N(5)$ & $121.2(6)$ \\
\hline$C(72)-C(71)-C(70)$ & $124.3(7)$ \\
\hline$C(72)-C(71)-F(5)$ & $118.2(6)$ \\
\hline$C(70)-C(71)-F(5)$ & $117.5(6)$ \\
\hline$C(71)-C(72)-C(73)$ & $117.0(7)$ \\
\hline $\mathrm{C}(71)-\mathrm{C}(72)-\mathrm{H}(72)$ & 121.5 \\
\hline $\mathrm{C}(73)-\mathrm{C}(72)-\mathrm{H}(72)$ & 121.5 \\
\hline $\mathrm{C}(72)-\mathrm{C}(73)-\mathrm{C}(74)$ & $121.2(7)$ \\
\hline $\mathrm{C}(72)-\mathrm{C}(73)-\mathrm{H}(73)$ & 119.4 \\
\hline $\mathrm{C}(74)-\mathrm{C}(73)-\mathrm{H}(73)$ & 119.4 \\
\hline $\mathrm{C}(73)-\mathrm{C}(74)-\mathrm{C}(75)$ & $120.9(7)$ \\
\hline $\mathrm{C}(73)-\mathrm{C}(74)-\mathrm{H}(74)$ & 119.5 \\
\hline $\mathrm{C}(75)-\mathrm{C}(74)-\mathrm{H}(74)$ & 119.5 \\
\hline $\mathrm{C}(74)-\mathrm{C}(75)-\mathrm{C}(70)$ & $117.5(7)$ \\
\hline$C(74)-C(75)-C(76)$ & $122.2(6)$ \\
\hline$C(70)-C(75)-C(76)$ & $120.3(6)$ \\
\hline $\mathrm{C}(75)-\mathrm{C}(76)-\mathrm{H}(76 \mathrm{~A})$ & 109.5 \\
\hline $\mathrm{C}(75)-\mathrm{C}(76)-\mathrm{H}(76 \mathrm{~B})$ & 109.5 \\
\hline $\mathrm{H}(76 \mathrm{~A})-\mathrm{C}(76)-\mathrm{H}(76 \mathrm{~B})$ & 109.5 \\
\hline $\mathrm{C}(75)-\mathrm{C}(76)-\mathrm{H}(76 \mathrm{C})$ & 109.5 \\
\hline $\mathrm{H}(76 \mathrm{~A})-\mathrm{C}(76)-\mathrm{H}(76 \mathrm{C})$ & 109.5 \\
\hline $\mathrm{H}(76 \mathrm{~B})-\mathrm{C}(76)-\mathrm{H}(76 \mathrm{C})$ & 109.5 \\
\hline $\mathrm{C}(78)-\mathrm{C}(77)-\mathrm{C}(82)$ & $118.8(7)$ \\
\hline $\mathrm{C}(78)-\mathrm{C}(77)-\mathrm{N}(6)$ & $121.6(7)$ \\
\hline $\mathrm{C}(82)-\mathrm{C}(77)-\mathrm{N}(6)$ & $119.5(6)$ \\
\hline $\mathrm{C}(77)-\mathrm{C}(78)-\mathrm{C}(79)$ & $117.8(8)$ \\
\hline $\mathrm{C}(77)-\mathrm{C}(78)-\mathrm{C}(83)$ & $120.9(7)$ \\
\hline $\mathrm{C}(79)-\mathrm{C}(78)-\mathrm{C}(83)$ & $121.3(7)$ \\
\hline $\mathrm{C}(80)-\mathrm{C}(79)-\mathrm{C}(78)$ & $120.6(7)$ \\
\hline $\mathrm{C}(80)-\mathrm{C}(79)-\mathrm{H}(79)$ & 119.7 \\
\hline $\mathrm{C}(78)-\mathrm{C}(79)-\mathrm{H}(79)$ & 119.7 \\
\hline $\mathrm{C}(81)-\mathrm{C}(80)-\mathrm{C}(79)$ & $121.9(7)$ \\
\hline $\mathrm{C}(81)-\mathrm{C}(80)-\mathrm{H}(80)$ & 119.0 \\
\hline $\mathrm{C}(79)-\mathrm{C}(80)-\mathrm{H}(80)$ & 119.0 \\
\hline $\mathrm{C}(82)-\mathrm{C}(81)-\mathrm{C}(80)$ & $116.8(8)$ \\
\hline $\mathrm{C}(82)-\mathrm{C}(81)-\mathrm{H}(81)$ & 121.6 \\
\hline $\mathrm{C}(80)-\mathrm{C}(81)-\mathrm{H}(81)$ & 121.6 \\
\hline $\mathrm{C}(81)-\mathrm{C}(82)-\mathrm{F}(6)$ & $118.9(7)$ \\
\hline $\mathrm{C}(81)-\mathrm{C}(82)-\mathrm{C}(77)$ & $123.7(7)$ \\
\hline $\mathrm{F}(6)-\mathrm{C}(82)-\mathrm{C}(77)$ & $117.5(6)$ \\
\hline $\mathrm{C}(78)-\mathrm{C}(83)-\mathrm{H}(83 \mathrm{~A})$ & 109.5 \\
\hline
\end{tabular}




\begin{tabular}{|c|c|}
\hline $\mathrm{C}(78)-\mathrm{C}(83)-\mathrm{H}(83 \mathrm{~B})$ & 109.5 \\
\hline $\mathrm{H}(83 \mathrm{~A})-\mathrm{C}(83)-\mathrm{H}(83 \mathrm{~B})$ & 109.5 \\
\hline $\mathrm{C}(78)-\mathrm{C}(83)-\mathrm{H}(83 \mathrm{C})$ & 109.5 \\
\hline $\mathrm{H}(83 \mathrm{~A})-\mathrm{C}(83)-\mathrm{H}(83 \mathrm{C})$ & 109.5 \\
\hline $\mathrm{H}(83 \mathrm{~B})-\mathrm{C}(83)-\mathrm{H}(83 \mathrm{C})$ & 109.5 \\
\hline $\mathrm{C}(85)-\mathrm{C}(84)-\mathrm{Ru}(3)$ & $120.3(5)$ \\
\hline $\mathrm{C}(85)-\mathrm{C}(84)-\mathrm{H}(84)$ & 119.8 \\
\hline $\mathrm{Ru}(3)-\mathrm{C}(84)-\mathrm{H}(84)$ & 119.8 \\
\hline $\mathrm{C}(90)-\mathrm{C}(85)-\mathrm{C}(86)$ & $118.7(6)$ \\
\hline $\mathrm{C}(90)-\mathrm{C}(85)-\mathrm{C}(84)$ & $118.8(6)$ \\
\hline $\mathrm{C}(86)-\mathrm{C}(85)-\mathrm{C}(84)$ & $122.5(6)$ \\
\hline $\mathrm{C}(87)-\mathrm{C}(86)-\mathrm{C}(85)$ & $120.3(7)$ \\
\hline $\mathrm{C}(87)-\mathrm{C}(86)-\mathrm{H}(86)$ & 119.9 \\
\hline $\mathrm{C}(85)-\mathrm{C}(86)-\mathrm{H}(86)$ & 119.9 \\
\hline $\mathrm{C}(88)-\mathrm{C}(87)-\mathrm{C}(86)$ & $119.9(7)$ \\
\hline $\mathrm{C}(88)-\mathrm{C}(87)-\mathrm{H}(87)$ & 120.0 \\
\hline $\mathrm{C}(86)-\mathrm{C}(87)-\mathrm{H}(87)$ & 120.0 \\
\hline $\mathrm{C}(87)-\mathrm{C}(88)-\mathrm{C}(89)$ & $121.2(7)$ \\
\hline $\mathrm{C}(87)-\mathrm{C}(88)-\mathrm{H}(88)$ & 119.4 \\
\hline $\mathrm{C}(89)-\mathrm{C}(88)-\mathrm{H}(88)$ & 119.4 \\
\hline $\mathrm{C}(90)-\mathrm{C}(89)-\mathrm{C}(88)$ & $118.3(7)$ \\
\hline $\mathrm{C}(90)-\mathrm{C}(89)-\mathrm{H}(89)$ & 120.9 \\
\hline $\mathrm{C}(88)-\mathrm{C}(89)-\mathrm{H}(89)$ & 120.9 \\
\hline $\mathrm{O}(3)-\mathrm{C}(90)-\mathrm{C}(89)$ & $125.7(7)$ \\
\hline $\mathrm{O}(3)-\mathrm{C}(90)-\mathrm{C}(85)$ & $113.0(6)$ \\
\hline$C(89)-C(90)-C(85)$ & $121.3(7)$ \\
\hline $\mathrm{C}(93)-\mathrm{C}(91)-\mathrm{O}(3)$ & $110.4(7)$ \\
\hline $\mathrm{C}(93)-\mathrm{C}(91)-\mathrm{C}(92)$ & $115.2(7)$ \\
\hline $\mathrm{O}(3)-\mathrm{C}(91)-\mathrm{C}(92)$ & $108.1(6)$ \\
\hline $\mathrm{C}(93)-\mathrm{C}(91)-\mathrm{H}(91)$ & 107.7 \\
\hline $\mathrm{O}(3)-\mathrm{C}(91)-\mathrm{H}(91)$ & 107.7 \\
\hline $\mathrm{C}(92)-\mathrm{C}(91)-\mathrm{H}(91)$ & 107.7 \\
\hline $\mathrm{C}(91)-\mathrm{C}(92)-\mathrm{H}(92 \mathrm{~A})$ & 109.5 \\
\hline $\mathrm{C}(91)-\mathrm{C}(92)-\mathrm{H}(92 \mathrm{~B})$ & 109.5 \\
\hline $\mathrm{H}(92 \mathrm{~A})-\mathrm{C}(92)-\mathrm{H}(92 \mathrm{~B})$ & 109.5 \\
\hline $\mathrm{C}(91)-\mathrm{C}(92)-\mathrm{H}(92 \mathrm{C})$ & 109.5 \\
\hline $\mathrm{H}(92 \mathrm{~A})-\mathrm{C}(92)-\mathrm{H}(92 \mathrm{C})$ & 109.5 \\
\hline $\mathrm{H}(92 \mathrm{~B})-\mathrm{C}(92)-\mathrm{H}(92 \mathrm{C})$ & 109.5 \\
\hline $\mathrm{C}(91)-\mathrm{C}(93)-\mathrm{H}(93 \mathrm{~A})$ & 109.5 \\
\hline $\mathrm{C}(91)-\mathrm{C}(93)-\mathrm{H}(93 \mathrm{~B})$ & 109.5 \\
\hline $\mathrm{H}(93 \mathrm{~A})-\mathrm{C}(93)-\mathrm{H}(93 \mathrm{~B})$ & 109.5 \\
\hline $\mathrm{C}(91)-\mathrm{C}(93)-\mathrm{H}(93 \mathrm{C})$ & 109.5 \\
\hline $\mathrm{H}(93 \mathrm{~A})-\mathrm{C}(93)-\mathrm{H}(93 \mathrm{C})$ & 109.5 \\
\hline H(93B)-C(93)-H(93C) & 109.5 \\
\hline C(95)-C(94)-C(99) & $117.8(7)$ \\
\hline $\mathrm{C}(95)-\mathrm{C}(94)-\mathrm{S}(5)$ & $122.9(6)$ \\
\hline $\mathrm{C}(99)-\mathrm{C}(94)-\mathrm{S}(5)$ & $119.3(6)$ \\
\hline $\mathrm{C}(94)-\mathrm{C}(95)-\mathrm{C}(96)$ & $123.4(8)$ \\
\hline $\mathrm{C}(94)-\mathrm{C}(95)-\mathrm{Cl}(5)$ & $119.8(6)$ \\
\hline
\end{tabular}




$\begin{array}{ll}\mathrm{C}(96)-\mathrm{C}(95)-\mathrm{Cl}(5) & 116.8(7) \\ \mathrm{C}(97)-\mathrm{C}(96)-\mathrm{C}(95) & 118.7(8) \\ \mathrm{C}(97)-\mathrm{C}(96)-\mathrm{H}(96) & 120.7 \\ \mathrm{C}(95)-\mathrm{C}(96)-\mathrm{H}(96) & 120.7 \\ \mathrm{C}(98)-\mathrm{C}(97)-\mathrm{C}(96) & 119.1(7) \\ \mathrm{C}(98)-\mathrm{C}(97)-\mathrm{H}(97) & 120.5 \\ \mathrm{C}(96)-\mathrm{C}(97)-\mathrm{H}(97) & 120.5 \\ \mathrm{C}(97)-\mathrm{C}(98)-\mathrm{C}(99) & 123.4(8) \\ \mathrm{C}(97)-\mathrm{C}(98)-\mathrm{Cl}(6) & 117.1(6) \\ \mathrm{C}(99)-\mathrm{C}(98)-\mathrm{Cl}(6) & 119.4(7) \\ \mathrm{C}(94)-\mathrm{C}(99)-\mathrm{C}(98) & 117.7(7) \\ \mathrm{C}(94)-\mathrm{C}(99)-\mathrm{S}(6) & 121.1(5) \\ \mathrm{C}(98)-\mathrm{C}(99)-\mathrm{S}(6) & 121.3(7) \\ \mathrm{Cl}(2 \mathrm{~S})-\mathrm{C}(1 \mathrm{~S})-\mathrm{Cl}(1 \mathrm{~S}) & 110.8(6) \\ \mathrm{Cl}(2 \mathrm{~S})-\mathrm{C}(1 \mathrm{~S})-\mathrm{H}(1 \mathrm{~S} 1) & 109.5 \\ \mathrm{Cl}(1 \mathrm{~S})-\mathrm{C}(1 \mathrm{~S})-\mathrm{H}(1 \mathrm{~S} 1) & 109.5 \\ \mathrm{Cl}(2 \mathrm{~S})-\mathrm{C}(1 \mathrm{~S})-\mathrm{H}(1 \mathrm{~S} 2) & 109.5 \\ \mathrm{Cl}(1 \mathrm{~S})-\mathrm{C}(1 \mathrm{~S})-\mathrm{H}(1 \mathrm{~S} 2) & 109.5 \\ \mathrm{H}(1 \mathrm{~S} 1)-\mathrm{C}(1 \mathrm{~S})-\mathrm{H}(1 \mathrm{~S} 2) & 108.1\end{array}$

Symmetry transformations used to generate equivalent atoms:

Table 4. Anisotropic displacement parameters $\left(\AA^{2} \mathrm{x} 10^{3}\right)$ for $3\left(\mathrm{C}_{33} \mathrm{H}_{30} \mathrm{Cl}_{2} \mathrm{~F}_{2} \mathrm{~N}_{2} \mathrm{ORuS}_{2}\right) \mathrm{CH}_{2} \mathrm{Cl}_{2}$ (Ru-1c). The anisotropic displacement factor exponent takes the form: $-2 p^{2}\left[h^{2} a^{* 2} U^{11}+\ldots+2 h k a^{*} b^{*} U^{12}\right]$

\begin{tabular}{lcccccc}
\hline & $\mathrm{U}^{11}$ & $\mathrm{U}^{22}$ & $\mathrm{U}^{33}$ & $\mathrm{U}^{23}$ & $\mathrm{U}^{13}$ & $\mathrm{U}^{12}$ \\
\hline $\mathrm{Ru}(1)$ & $37(1)$ & $22(1)$ & $23(1)$ & $2(1)$ & $2(1)$ & $-7(1)$ \\
$\mathrm{Cl}(1)$ & $42(1)$ & $64(1)$ & $47(1)$ & $4(1)$ & $4(1)$ & $16(1)$ \\
$\mathrm{Cl}(2)$ & $106(2)$ & $34(1)$ & $44(1)$ & $7(1)$ & $17(1)$ & $-4(1)$ \\
$\mathrm{S}(1)$ & $37(1)$ & $28(1)$ & $28(1)$ & $4(1)$ & $2(1)$ & $1(1)$ \\
$\mathrm{S}(2)$ & $56(1)$ & $23(1)$ & $32(1)$ & $3(1)$ & $9(1)$ & $-7(1)$ \\
$\mathrm{F}(1)$ & $48(3)$ & $70(3)$ & $40(2)$ & $-12(2)$ & $-5(2)$ & $-2(2)$ \\
$\mathrm{F}(2)$ & $43(2)$ & $42(3)$ & $49(2)$ & $-13(2)$ & $-1(2)$ & $-10(2)$ \\
$\mathrm{O}(1)$ & $35(2)$ & $40(3)$ & $32(2)$ & $0(2)$ & $-4(2)$ & $-17(2)$ \\
$\mathrm{N}(1)$ & $33(3)$ & $23(3)$ & $24(2)$ & $0(2)$ & $2(2)$ & $-1(2)$ \\
$\mathrm{N}(2)$ & $32(3)$ & $21(3)$ & $28(3)$ & $0(2)$ & $5(2)$ & $-1(2)$ \\
$\mathrm{C}(1)$ & $35(3)$ & $25(3)$ & $20(3)$ & $-1(2)$ & $-4(2)$ & $-6(2)$ \\
$\mathrm{C}(2)$ & $46(4)$ & $21(3)$ & $35(3)$ & $5(3)$ & $16(3)$ & $1(3)$ \\
$\mathrm{C}(3)$ & $33(3)$ & $27(3)$ & $29(3)$ & $4(2)$ & $11(3)$ & $0(3)$ \\
$\mathrm{C}(4)$ & $45(3)$ & $26(3)$ & $27(3)$ & $2(2)$ & $11(2)$ & $-6(3)$ \\
$\mathrm{C}(5)$ & $44(3)$ & $44(4)$ & $30(3)$ & $7(3)$ & $9(3)$ & $2(3)$ \\
$\mathrm{C}(6)$ & $52(5)$ & $60(5)$ & $52(4)$ & $5(4)$ & $15(4)$ & $12(4)$ \\
$\mathrm{C}(7)$ & $69(5)$ & $50(5)$ & $59(5)$ & $0(4)$ & $17(4)$ & $14(4)$ \\
$\mathrm{C}(8)$ & $82(5)$ & $42(5)$ & $36(4)$ & $-6(3)$ & $18(4)$ & $-3(4)$ \\
$\mathrm{C}(9)$ & $66(4)$ & $28(4)$ & $31(3)$ & $-1(3)$ & $13(3)$ & $-4(3)$ \\
$\mathrm{C}(10)$ & $74(5)$ & $42(5)$ & $30(4)$ & $-1(3)$ & $-2(3)$ & $-28(4)$ \\
$\mathrm{C}(11)$ & $35(3)$ & $28(3)$ & $27(3)$ & $2(2)$ & $1(2)$ & $1(2)$ \\
$\mathrm{C}(12)$ & $37(3)$ & $31(3)$ & $39(4)$ & $9(3)$ & $2(3)$ & $-4(3)$ \\
& & & & & \\
& & & & & \\
& & & & & \\
\end{tabular}




\begin{tabular}{|c|c|c|c|c|c|c|}
\hline$C(13)$ & $38(4)$ & $46(4)$ & $57(5)$ & $17(3)$ & 7(3) & 7(3) \\
\hline C(14) & $48(4)$ & $39(4)$ & 63(5) & 7(3) & $16(4)$ & $11(3)$ \\
\hline$C(15)$ & $60(4)$ & $28(4)$ & $52(5)$ & $-3(3)$ & $8(4)$ & 2(3) \\
\hline$C(16)$ & $43(3)$ & $27(3)$ & $36(4)$ & $0(3)$ & 9(3) & $-4(3)$ \\
\hline $\mathrm{C}(17)$ & $41(4)$ & $47(4)$ & $46(4)$ & $8(3)$ & $-7(3)$ & $-16(3)$ \\
\hline C(18) & $32(3)$ & $32(3)$ & 23(3) & 3(3) & $-2(2)$ & $-9(3)$ \\
\hline$C(19)$ & $32(3)$ & $28(3)$ & $31(3)$ & $6(2)$ & $-1(2)$ & $-5(2)$ \\
\hline$C(20)$ & $34(3)$ & $42(4)$ & 29(3) & 7(3) & $2(2)$ & $-13(3)$ \\
\hline$C(21)$ & $35(3)$ & $66(5)$ & $41(4)$ & $0(4)$ & $-3(3)$ & $-11(3)$ \\
\hline $\mathrm{C}(22)$ & $30(4)$ & $72(6)$ & $54(4)$ & 1(4) & $5(3)$ & 1(4) \\
\hline$C(23)$ & $41(4)$ & $48(5)$ & $49(4)$ & $-1(3)$ & 11(3) & $-4(3)$ \\
\hline$C(24)$ & $39(3)$ & $36(4)$ & $39(4)$ & $-1(3)$ & $-1(3)$ & $-1(3)$ \\
\hline$C(25)$ & $52(4)$ & $59(5)$ & $28(3)$ & $-5(3)$ & $3(3)$ & $-29(4)$ \\
\hline$C(26)$ & $69(6)$ & $51(5)$ & $43(4)$ & $-9(4)$ & $6(4)$ & $-39(4)$ \\
\hline$C(27)$ & $51(5)$ & $70(6)$ & $39(4)$ & $-9(4)$ & 7(3) & $-32(4)$ \\
\hline$C(28)$ & 49(3) & $35(3)$ & 21(3) & $1(3)$ & 11(3) & $15(3)$ \\
\hline$C(29)$ & $57(4)$ & $40(4)$ & 29(3) & $0(3)$ & $12(3)$ & $20(3)$ \\
\hline$C(30)$ & $64(5)$ & $52(4)$ & $36(4)$ & 1(3) & $8(4)$ & $26(3)$ \\
\hline$C(31)$ & $75(5)$ & $40(4)$ & $39(4)$ & $6(3)$ & 19(3) & $25(3)$ \\
\hline$C(32)$ & $80(5)$ & $29(4)$ & $31(4)$ & $1(3)$ & $12(3)$ & $11(3)$ \\
\hline C(33) & $66(4)$ & $32(3)$ & $22(3)$ & $-2(3)$ & $10(3)$ & $8(3)$ \\
\hline $\mathrm{Ru}(2)$ & $22(1)$ & $24(1)$ & $24(1)$ & $-2(1)$ & $3(1)$ & $-1(1)$ \\
\hline $\mathrm{Cl}(3)$ & $50(1)$ & $27(1)$ & $42(1)$ & $-7(1)$ & $-7(1)$ & $6(1)$ \\
\hline $\mathrm{Cl}(4)$ & $46(1)$ & $35(1)$ & $30(1)$ & $7(1)$ & $-4(1)$ & $-1(1)$ \\
\hline $\mathrm{S}(3)$ & $34(1)$ & $22(1)$ & $26(1)$ & $0(1)$ & $0(1)$ & $2(1)$ \\
\hline$S(4)$ & $28(1)$ & $24(1)$ & $25(1)$ & 1(1) & 2(1) & 1(1) \\
\hline $\mathrm{F}(3)$ & $78(3)$ & $64(3)$ & $54(3)$ & $-14(3)$ & 4(3) & 7(3) \\
\hline $\mathrm{O}(2)$ & $46(3)$ & $32(3)$ & $48(3)$ & $-17(2)$ & $4(2)$ & $7(2)$ \\
\hline $\mathrm{N}(3)$ & $40(3)$ & $42(3)$ & $25(3)$ & $4(2)$ & $-1(2)$ & $-2(2)$ \\
\hline $\mathrm{N}(4)$ & $37(3)$ & $57(4)$ & $27(3)$ & $-2(3)$ & $8(2)$ & $-5(3)$ \\
\hline$C(34)$ & $31(3)$ & $29(3)$ & 27(3) & $-2(2)$ & $5(2)$ & 1(3) \\
\hline$C(35)$ & $54(4)$ & $63(6)$ & $26(3)$ & $1(3)$ & $-2(3)$ & $-11(4)$ \\
\hline$C(36)$ & $57(5)$ & $110(9)$ & $28(4)$ & 2(4) & $7(3)$ & $-16(5)$ \\
\hline $\mathrm{C}(37)$ & $43(3)$ & $59(4)$ & $33(4)$ & $11(3)$ & $-12(3)$ & $-10(3)$ \\
\hline C(38) & $54(4)$ & $73(5)$ & $36(4)$ & $12(4)$ & $-9(3)$ & $-4(3)$ \\
\hline $\mathrm{C}(39)$ & $66(5)$ & $64(6)$ & $52(5)$ & 3(4) & $-8(4)$ & $-11(4)$ \\
\hline$C(40)$ & $50(4)$ & $76(5)$ & 41(4) & $8(4)$ & $-10(4)$ & $-1(4)$ \\
\hline $\mathrm{C}(41)$ & $65(5)$ & $81(6)$ & $68(6)$ & $6(5)$ & $-8(5)$ & $-6(5)$ \\
\hline$C(42)$ & $38(4)$ & $78(5)$ & $68(6)$ & $24(5)$ & $-11(4)$ & $8(3)$ \\
\hline $\mathrm{C}(43)$ & $98(8)$ & $57(5)$ & $81(7)$ & $17(5)$ & $10(6)$ & $38(5)$ \\
\hline C(44) & $34(3)$ & $83(4)$ & 26(3) & 2(3) & $9(3)$ & $-12(3)$ \\
\hline$C(45)$ & $42(8)$ & $87(5)$ & $27(7)$ & $-6(5)$ & $10(8)$ & $-27(4)$ \\
\hline$C(46)$ & $41(8)$ & $104(9)$ & $33(6)$ & $-2(7)$ & $10(6)$ & $-27(5)$ \\
\hline $\mathrm{C}(47)$ & $44(7)$ & $119(9)$ & $44(7)$ & $8(8)$ & $7(6)$ & $-19(6)$ \\
\hline $\mathrm{C}(48)$ & $36(5)$ & $117(9)$ & $43(7)$ & $18(8)$ & $7(4)$ & $-12(6)$ \\
\hline$C(49)$ & $32(5)$ & $103(8)$ & $40(7)$ & $10(8)$ & $11(5)$ & $-7(5)$ \\
\hline$C(50)$ & $54(10)$ & $65(7)$ & $47(9)$ & $-4(6)$ & $17(9)$ & $-25(7)$ \\
\hline $\mathrm{F}(4)$ & $52(8)$ & $94(8)$ & $69(8)$ & $10(6)$ & $16(6)$ & $5(6)$ \\
\hline$C(45 \mathrm{X})$ & $42(8)$ & $87(5)$ & $27(7)$ & $-6(5)$ & $10(8)$ & $-27(4)$ \\
\hline
\end{tabular}




\begin{tabular}{|c|c|c|c|c|c|c|}
\hline$C(46 X)$ & $41(8)$ & $104(9)$ & $33(6)$ & $-2(7)$ & $10(6)$ & $-27(5)$ \\
\hline$C(47 X)$ & $44(7)$ & $119(9)$ & $44(7)$ & $8(8)$ & $7(6)$ & $-19(6)$ \\
\hline$C(48 X)$ & $36(5)$ & $117(9)$ & $43(7)$ & $18(8)$ & $7(4)$ & $-12(6)$ \\
\hline$C(49 X)$ & $32(5)$ & $103(8)$ & $40(7)$ & $10(8)$ & $11(5)$ & $-7(5)$ \\
\hline$C(50 X)$ & $54(10)$ & $65(7)$ & $47(9)$ & $-4(6)$ & $17(9)$ & $-25(7)$ \\
\hline$F(4 X)$ & $49(9)$ & $98(9)$ & $74(10)$ & $15(8)$ & $3(7)$ & $23(7)$ \\
\hline$C(51)$ & $31(3)$ & $40(3)$ & $23(3)$ & $-5(3)$ & 2(2) & $-1(3)$ \\
\hline$C(52)$ & $41(3)$ & $44(4)$ & $22(3)$ & $-2(3)$ & 2(3) & $-12(3)$ \\
\hline$C(53)$ & $49(4)$ & $71(5)$ & $31(4)$ & $2(4)$ & $6(3)$ & $-27(4)$ \\
\hline C(54) & $74(6)$ & $73(5)$ & 41(4) & $8(4)$ & $-5(4)$ & $-41(4)$ \\
\hline$C(55)$ & $93(6)$ & $48(5)$ & $45(5)$ & $9(4)$ & $-22(4)$ & $-38(4)$ \\
\hline$C(56)$ & $87(5)$ & $34(4)$ & $38(4)$ & $-2(3)$ & $-16(4)$ & $-7(4)$ \\
\hline $\mathrm{C}(57)$ & $52(4)$ & $33(3)$ & $28(3)$ & $-1(3)$ & $0(3)$ & $-9(3)$ \\
\hline$C(58)$ & $72(5)$ & $69(6)$ & $56(4)$ & $-6(4)$ & $17(4)$ & $35(4)$ \\
\hline C(59) & $73(7)$ & $44(6)$ & $45(5)$ & $-11(4)$ & $8(5)$ & $22(5)$ \\
\hline $\mathrm{C}(60)$ & $65(7)$ & $54(8)$ & $50(6)$ & $-5(5)$ & $14(5)$ & $31(5)$ \\
\hline $\mathrm{C}(58 \mathrm{X})$ & $72(5)$ & $69(6)$ & $56(4)$ & $-6(4)$ & $17(4)$ & $35(4)$ \\
\hline$C(59 X)$ & $73(7)$ & $44(6)$ & $45(5)$ & $-11(4)$ & $8(5)$ & $22(5)$ \\
\hline$C(60 X)$ & $65(7)$ & $54(8)$ & $50(6)$ & $-5(5)$ & $14(5)$ & $31(5)$ \\
\hline$C(61)$ & $23(3)$ & $28(3)$ & $26(3)$ & $1(2)$ & $4(2)$ & $-7(2)$ \\
\hline$C(62)$ & $29(3)$ & $26(3)$ & $32(3)$ & $0(2)$ & 4(2) & $-7(2)$ \\
\hline$C(63)$ & $34(3)$ & $34(3)$ & $31(3)$ & $-7(3)$ & $3(3)$ & $-6(3)$ \\
\hline$C(64)$ & $38(4)$ & $35(3)$ & $26(3)$ & $0(2)$ & $3(3)$ & $-6(3)$ \\
\hline$C(65)$ & $24(3)$ & $31(3)$ & $31(3)$ & 6(2) & $4(2)$ & $-6(2)$ \\
\hline$C(66)$ & $19(3)$ & $26(3)$ & $25(3)$ & $-1(2)$ & $8(2)$ & $-5(2)$ \\
\hline $\mathrm{Ru}(3)$ & $28(1)$ & $18(1)$ & $38(1)$ & $3(1)$ & $14(1)$ & $-2(1)$ \\
\hline $\mathrm{Cl}(5)$ & $49(1)$ & $86(2)$ & $56(1)$ & $29(1)$ & $6(1)$ & $3(1)$ \\
\hline $\mathrm{Cl}(6)$ & $60(1)$ & $37(1)$ & $88(2)$ & $1(1)$ & $38(1)$ & $-15(1)$ \\
\hline $\mathrm{S}(5)$ & $32(1)$ & $30(1)$ & $38(1)$ & $8(1)$ & $16(1)$ & $2(1)$ \\
\hline$S(6)$ & $35(1)$ & $23(1)$ & $53(1)$ & $5(1)$ & $18(1)$ & $-6(1)$ \\
\hline $\mathrm{F}(5)$ & $53(3)$ & $49(3)$ & $37(2)$ & $-3(2)$ & $-3(2)$ & $-4(2)$ \\
\hline $\mathrm{F}(6)$ & $45(2)$ & $35(2)$ & $46(2)$ & $2(2)$ & $0(2)$ & $4(2)$ \\
\hline $\mathrm{O}(3)$ & $38(3)$ & $23(2)$ & $40(2)$ & $-2(2)$ & $9(2)$ & $-5(2)$ \\
\hline $\mathrm{N}(5)$ & $28(3)$ & $22(2)$ & $34(3)$ & $6(2)$ & $12(2)$ & $-2(2)$ \\
\hline $\mathrm{N}(6)$ & $28(3)$ & $26(3)$ & $31(3)$ & $6(2)$ & $12(2)$ & $3(2)$ \\
\hline $\mathrm{C}(67)$ & $26(3)$ & $20(3)$ & $31(3)$ & 2(2) & $11(2)$ & $-1(2)$ \\
\hline$C(68)$ & $27(3)$ & $32(3)$ & $36(3)$ & 7(3) & $15(3)$ & 1(3) \\
\hline $\mathrm{C}(69)$ & $29(3)$ & $31(3)$ & $35(3)$ & $6(3)$ & $17(3)$ & $3(3)$ \\
\hline$C(70)$ & $28(3)$ & $22(3)$ & $34(3)$ & $4(2)$ & $9(2)$ & $-3(2)$ \\
\hline $\mathrm{C}(71)$ & $31(3)$ & $32(3)$ & $35(3)$ & $5(2)$ & $7(3)$ & $-5(3)$ \\
\hline$C(72)$ & $31(4)$ & $36(3)$ & $53(4)$ & $14(3)$ & $0(3)$ & $-5(3)$ \\
\hline$C(73)$ & $41(4)$ & $26(3)$ & $62(4)$ & $12(3)$ & $5(3)$ & $-5(3)$ \\
\hline$C(74)$ & $28(3)$ & $25(3)$ & $62(4)$ & $0(3)$ & $9(3)$ & $-7(3)$ \\
\hline$C(75)$ & $28(3)$ & $25(3)$ & $39(3)$ & $1(2)$ & $7(3)$ & $-13(2)$ \\
\hline$C(76)$ & $40(4)$ & $32(4)$ & $33(3)$ & $-2(3)$ & $5(3)$ & $0(3)$ \\
\hline$C(77)$ & $39(3)$ & $27(3)$ & $28(3)$ & $3(2)$ & $17(2)$ & $6(2)$ \\
\hline$C(78)$ & $49(4)$ & $38(4)$ & $33(3)$ & $7(3)$ & $13(3)$ & $16(3)$ \\
\hline$C(79)$ & $69(5)$ & $38(4)$ & $38(4)$ & $10(3)$ & $10(3)$ & $26(3)$ \\
\hline $\mathrm{C}(80)$ & $71(5)$ & $24(4)$ & $49(4)$ & $9(3)$ & $23(3)$ & $14(3)$ \\
\hline
\end{tabular}




\begin{tabular}{lcccccc}
$\mathrm{C}(81)$ & $48(4)$ & $31(3)$ & $52(4)$ & $1(3)$ & $17(3)$ & $3(3)$ \\
$\mathrm{C}(82)$ & $36(4)$ & $31(3)$ & $39(3)$ & $7(3)$ & $13(3)$ & $5(3)$ \\
$\mathrm{C}(83)$ & $59(5)$ & $52(5)$ & $34(4)$ & $-2(3)$ & $-1(3)$ & $21(4)$ \\
$\mathrm{C}(84)$ & $21(3)$ & $26(3)$ & $39(3)$ & $7(3)$ & $17(2)$ & $-5(2)$ \\
$\mathrm{C}(85)$ & $28(3)$ & $20(3)$ & $42(3)$ & $5(2)$ & $12(2)$ & $-4(2)$ \\
$\mathrm{C}(86)$ & $29(3)$ & $25(3)$ & $42(4)$ & $7(3)$ & $14(3)$ & $0(2)$ \\
$\mathrm{C}(87)$ & $26(3)$ & $41(4)$ & $54(4)$ & $9(3)$ & $14(3)$ & $8(3)$ \\
$\mathrm{C}(88)$ & $24(3)$ & $40(4)$ & $52(4)$ & $13(3)$ & $7(3)$ & $-2(3)$ \\
$\mathrm{C}(89)$ & $20(3)$ & $42(4)$ & $47(4)$ & $1(3)$ & $6(3)$ & $-6(3)$ \\
$\mathrm{C}(90)$ & $24(3)$ & $26(3)$ & $45(3)$ & $3(2)$ & $12(2)$ & $-3(2)$ \\
$\mathrm{C}(91)$ & $37(4)$ & $33(4)$ & $61(5)$ & $-10(3)$ & $5(3)$ & $-8(3)$ \\
$\mathrm{C}(92)$ & $39(4)$ & $41(4)$ & $50(4)$ & $-3(3)$ & $3(3)$ & $5(3)$ \\
$\mathrm{C}(93)$ & $41(4)$ & $34(4)$ & $68(5)$ & $-11(4)$ & $17(4)$ & $-9(3)$ \\
$\mathrm{C}(94)$ & $37(3)$ & $22(3)$ & $50(3)$ & $14(3)$ & $19(3)$ & $11(3)$ \\
$\mathrm{C}(95)$ & $40(4)$ & $36(4)$ & $55(4)$ & $16(3)$ & $21(3)$ & $11(3)$ \\
$\mathrm{C}(96)$ & $53(4)$ & $47(5)$ & $53(4)$ & $24(4)$ & $30(3)$ & $18(3)$ \\
$\mathrm{C}(97)$ & $57(4)$ & $31(4)$ & $68(4)$ & $16(4)$ & $37(3)$ & $8(3)$ \\
$\mathrm{C}(98)$ & $43(4)$ & $22(4)$ & $68(4)$ & $6(3)$ & $28(3)$ & $9(3)$ \\
$\mathrm{C}(99)$ & $38(3)$ & $19(3)$ & $59(4)$ & $8(3)$ & $25(3)$ & $11(3)$ \\
$\mathrm{C}(1 \mathrm{~S})$ & $114(9)$ & $116(11)$ & $51(6)$ & $18(6)$ & $12(5)$ & $-46(8)$ \\
$\mathrm{Cl}(1 \mathrm{~S})$ & $96(2)$ & $103(2)$ & $73(2)$ & $22(2)$ & $-1(2)$ & $-32(2)$ \\
$\mathrm{Cl}(2 \mathrm{~S})$ & $77(2)$ & $198(5)$ & $89(2)$ & $5(3)$ & $16(2)$ & $-22(2)$ \\
& & & & & \\
\hline
\end{tabular}


Table 5. Hydrogen coordinates $\left(\times 10^{4}\right)$ and isotropic displacement parameters $\left(\AA^{2} \times 10^{3}\right)$ for $3\left(\mathrm{C}_{33} \mathrm{H}_{30} \mathrm{Cl}_{2} \mathrm{~F}_{2} \mathrm{~N}_{2} \mathrm{ORuS}_{2}\right) \mathrm{CH}_{2} \mathrm{Cl}_{2}\left(\mathbf{R u}^{-}\right.$ 1c).

\begin{tabular}{|c|c|c|c|c|}
\hline & $\mathrm{x}$ & $\mathrm{y}$ & $\mathrm{z}$ & $\mathrm{U}(\mathrm{eq})$ \\
\hline $\mathrm{H}(2 \mathrm{~A})$ & 6792 & 2001 & 9186 & 40 \\
\hline $\mathrm{H}(2 \mathrm{~B})$ & 5939 & 1817 & 8872 & 40 \\
\hline $\mathrm{H}(3 \mathrm{~A})$ & 4829 & 2473 & 9151 & 35 \\
\hline $\mathrm{H}(3 \mathrm{~B})$ & 5790 & 2832 & 9372 & 35 \\
\hline $\mathrm{H}(6)$ & 10281 & 2031 & 8711 & 65 \\
\hline $\mathrm{H}(7)$ & 9925 & 1340 & 8232 & 70 \\
\hline $\mathrm{H}(8)$ & 8285 & 1235 & 7973 & 64 \\
\hline $\mathrm{H}(10 \mathrm{~A})$ & 6013 & 1497 & 8243 & 74 \\
\hline $\mathrm{H}(10 \mathrm{~B})$ & 6424 & 1766 & 7883 & 74 \\
\hline $\mathrm{H}(10 \mathrm{C})$ & 6041 & 2277 & 8169 & 74 \\
\hline $\mathrm{H}(13)$ & 2371 & 4195 & 8856 & 56 \\
\hline $\mathrm{H}(14)$ & 3036 & 5130 & 9130 & 59 \\
\hline $\mathrm{H}(15)$ & 4839 & 5203 & 9303 & 56 \\
\hline $\mathrm{H}(17 \mathrm{~A})$ & 3191 & 3220 & 8385 & 67 \\
\hline H(17B) & 2675 & 2964 & 8732 & 67 \\
\hline $\mathrm{H}(17 \mathrm{C})$ & 3853 & 2745 & 8650 & 67 \\
\hline $\mathrm{H}(18)$ & 6946 & 3119 & 7822 & 35 \\
\hline $\mathrm{H}(21)$ & 10715 & 3759 & 8403 & 57 \\
\hline $\mathrm{H}(22)$ & 11472 & 3122 & 7972 & 63 \\
\hline $\mathrm{H}(23)$ & 10423 & 2610 & 7537 & 55 \\
\hline $\mathrm{H}(24)$ & 8588 & 2690 & 7536 & 46 \\
\hline $\mathrm{H}(25)$ & 9844 & 4084 & 8829 & 56 \\
\hline $\mathrm{H}(26 \mathrm{~A})$ & 9937 & 5241 & 8723 & 81 \\
\hline $\mathrm{H}(26 \mathrm{~B})$ & 10002 & 4844 & 8364 & 81 \\
\hline $\mathrm{H}(26 \mathrm{C})$ & 8918 & 5194 & 8457 & 81 \\
\hline $\mathrm{H}(27 \mathrm{~A})$ & 8836 & 4737 & 9195 & 80 \\
\hline $\mathrm{H}(27 \mathrm{~B})$ & 7839 & 4681 & 8920 & 80 \\
\hline $\mathrm{H}(27 \mathrm{C})$ & 8278 & 4035 & 9122 & 80 \\
\hline $\mathrm{H}(30)$ & 3081 & 5395 & 7398 & 61 \\
\hline $\mathrm{H}(31)$ & 4396 & 6190 & 7415 & 61 \\
\hline $\mathrm{H}(35 \mathrm{~A})$ & 4117 & 2678 & 7549 & 58 \\
\hline $\mathrm{H}(35 \mathrm{~B})$ & 4442 & 1923 & 7466 & 58 \\
\hline $\mathrm{H}(36 \mathrm{~A})$ & 2706 & 1701 & 7403 & 78 \\
\hline $\mathrm{H}(36 \mathrm{~B})$ & 2407 & 2478 & 7401 & 78 \\
\hline H(39) & 6652 & 4140 & 7160 & 74 \\
\hline $\mathrm{H}(40)$ & 8030 & 3486 & 6970 & 67 \\
\hline $\mathrm{H}(41)$ & 7855 & 2387 & 6822 & 86 \\
\hline $\mathrm{H}(43 \mathrm{~A})$ & 6576 & 1356 & 6821 & 118 \\
\hline $\mathrm{H}(43 \mathrm{~B})$ & 5635 & 1613 & 6560 & 118 \\
\hline $\mathrm{H}(43 \mathrm{C})$ & 5415 & 1458 & 6958 & 118 \\
\hline $\mathrm{H}(46)$ & 477 & 663 & 6432 & 71 \\
\hline $\mathrm{H}(47)$ & -799 & 1465 & 6368 & 83 \\
\hline $\mathrm{H}(48)$ & -500 & 2542 & 6543 & 78 \\
\hline
\end{tabular}




\begin{tabular}{|c|c|c|c|c|}
\hline $\mathrm{H}(50 \mathrm{~A})$ & 2911 & 715 & 6500 & 82 \\
\hline $\mathrm{H}(50 \mathrm{~B})$ & 2160 & 295 & 6739 & 82 \\
\hline $\mathrm{H}(50 \mathrm{C})$ & 2924 & 853 & 6910 & 82 \\
\hline $\mathrm{H}(46 \mathrm{X})$ & 1156 & 329 & 6514 & 71 \\
\hline $\mathrm{H}(47 \mathrm{X})$ & -427 & 859 & 6354 & 83 \\
\hline $\mathrm{H}(48 \mathrm{X})$ & -628 & 1976 & 6437 & 78 \\
\hline $\mathrm{H}(50 \mathrm{D})$ & 2986 & 691 & 7029 & 82 \\
\hline $\mathrm{H}(50 \mathrm{E})$ & 3551 & 1017 & 6708 & 82 \\
\hline $\mathrm{H}(50 \mathrm{~F})$ & 2893 & 345 & 6653 & 82 \\
\hline $\mathrm{H}(51)$ & 5544 & 2540 & 6175 & 38 \\
\hline $\mathrm{H}(53)$ & 6934 & 3430 & 6130 & 60 \\
\hline $\mathrm{H}(54)$ & 7477 & 4508 & 6218 & 76 \\
\hline $\mathrm{H}(55)$ & 6285 & 5287 & 6386 & 76 \\
\hline $\mathrm{H}(56)$ & 4496 & 5011 & 6479 & 64 \\
\hline $\mathrm{H}(58)$ & 3045 & 4673 & 6367 & 78 \\
\hline $\mathrm{H}(59 \mathrm{~A})$ & 3315 & 4417 & 6936 & 81 \\
\hline H(59B) & 2143 & 4685 & 6842 & 81 \\
\hline $\mathrm{H}(59 \mathrm{C})$ & 2341 & 3910 & 6905 & 81 \\
\hline $\mathrm{H}(60 \mathrm{~A})$ & 2063 & 4034 & 5984 & 84 \\
\hline $\mathrm{H}(60 \mathrm{~B})$ & 1545 & 3668 & 6303 & 84 \\
\hline $\mathrm{H}(60 \mathrm{C})$ & 1347 & 4442 & 6240 & 84 \\
\hline $\mathrm{H}(58 \mathrm{X})$ & 3124 & 4584 & 6603 & 78 \\
\hline $\mathrm{H}(59 \mathrm{D})$ & 2547 & 3678 & 6864 & 81 \\
\hline $\mathrm{H}(59 \mathrm{E})$ & 1591 & 4171 & 6758 & 81 \\
\hline $\mathrm{H}(59 \mathrm{~F})$ & 1761 & 3516 & 6534 & 81 \\
\hline $\mathrm{H}(60 \mathrm{D})$ & 3043 & 4776 & 6041 & 84 \\
\hline $\mathrm{H}(60 \mathrm{E})$ & 2099 & 4253 & 5982 & 84 \\
\hline $\mathrm{H}(60 \mathrm{~F})$ & 1928 & 4908 & 6207 & 84 \\
\hline $\mathrm{H}(63)$ & 3622 & 781 & 4888 & 40 \\
\hline $\mathrm{H}(64)$ & 2771 & 1717 & 4644 & 39 \\
\hline $\mathrm{H}(68 \mathrm{~A})$ & 5410 & 2434 & 5526 & 37 \\
\hline $\mathrm{H}(68 \mathrm{~B})$ & 4971 & 2332 & 5126 & 37 \\
\hline $\mathrm{H}(69 \mathrm{~A})$ & 4615 & 3429 & 5131 & 38 \\
\hline $\mathrm{H}(69 \mathrm{~B})$ & 5389 & 3549 & 5475 & 38 \\
\hline $\mathrm{H}(72)$ & 8218 & 871 & 5769 & 48 \\
\hline $\mathrm{H}(73)$ & 7973 & 117 & 5307 & 52 \\
\hline $\mathrm{H}(74)$ & 7199 & 453 & 4772 & 46 \\
\hline $\mathrm{H}(76 \mathrm{~A})$ & 6318 & 1260 & 4412 & 53 \\
\hline $\mathrm{H}(76 \mathrm{~B})$ & 6605 & 2015 & 4502 & 53 \\
\hline $\mathrm{H}(76 \mathrm{C})$ & 5533 & 1698 & 4630 & 53 \\
\hline H(79) & 5624 & 5292 & 4345 & 58 \\
\hline $\mathrm{H}(80)$ & 6854 & 5907 & 4670 & 57 \\
\hline $\mathrm{H}(81)$ & 7732 & 5473 & 5168 & 52 \\
\hline $\mathrm{H}(83 \mathrm{~A})$ & 5239 & 3575 & 4403 & 73 \\
\hline $\mathrm{H}(83 \mathrm{~B})$ & 4870 & 4221 & 4187 & 73 \\
\hline $\mathrm{H}(83 \mathrm{C})$ & 4300 & 4026 & 4534 & 73 \\
\hline $\mathrm{H}(84)$ & 8469 & 2067 & 4537 & 33 \\
\hline $\mathrm{H}(86)$ & 9662 & 1116 & 4746 & 38 \\
\hline $\mathrm{H}(87)$ & 10814 & 654 & 5176 & 48 \\
\hline
\end{tabular}




\begin{tabular}{lrrrr}
$\mathrm{H}(88)$ & 11259 & 1240 & 5679 & 47 \\
$\mathrm{H}(89)$ & 10578 & 2311 & 5764 & 43 \\
$\mathrm{H}(91)$ & 10581 & 3268 & 5640 & 52 \\
$\mathrm{H}(92 \mathrm{~A})$ & 9096 & 2975 & 5943 & 65 \\
$\mathrm{H}(92 \mathrm{~B})$ & 9489 & 3701 & 6057 & 65 \\
$\mathrm{H}(92 \mathrm{C})$ & 8472 & 3613 & 5794 & 65 \\
$\mathrm{H}(93 \mathrm{~A})$ & 10363 & 4408 & 5599 & 71 \\
$\mathrm{H}(93 \mathrm{~B})$ & 10544 & 4087 & 5226 & 71 \\
$\mathrm{H}(93 \mathrm{C})$ & 9380 & 4305 & 5323 & 71 \\
$\mathrm{H}(96)$ & 8721 & 4153 & 3168 & 60 \\
$\mathrm{H}(97)$ & 10266 & 4622 & 3428 & 61 \\
$\mathrm{H}(1 \mathrm{~S} 1)$ & 8661 & 5030 & 7728 & 112 \\
$\mathrm{H}(1 \mathrm{~S} 2)$ & 9173 & 4385 & 7562 & 112 \\
\hline
\end{tabular}

Table 6. Torsion angles $\left(^{\circ}\right)$ for $3\left(\mathrm{C}_{33} \mathrm{H}_{30} \mathrm{Cl}_{2} \mathrm{~F}_{2} \mathrm{~N}_{2} \mathrm{ORuS}_{2}\right) \mathrm{CH}_{2} \mathrm{Cl}_{2}(\mathbf{R u}-\mathbf{1 c})$.

\begin{tabular}{|c|c|}
\hline $\mathrm{C}(4)-\mathrm{N}(1)-\mathrm{C}(1)-\mathrm{N}(2)$ & $169.0(6)$ \\
\hline $\mathrm{C}(2)-\mathrm{N}(1)-\mathrm{C}(1)-\mathrm{N}(2)$ & $5.7(7)$ \\
\hline $\mathrm{C}(4)-\mathrm{N}(1)-\mathrm{C}(1)-\mathrm{Ru}(1)$ & $-9.8(9)$ \\
\hline $\mathrm{C}(2)-\mathrm{N}(1)-\mathrm{C}(1)-\mathrm{Ru}(1)$ & $-173.1(5)$ \\
\hline $\mathrm{C}(11)-\mathrm{N}(2)-\mathrm{C}(1)-\mathrm{N}(1)$ & $171.3(6)$ \\
\hline $\mathrm{C}(3)-\mathrm{N}(2)-\mathrm{C}(1)-\mathrm{N}(1)$ & $8.0(7)$ \\
\hline $\mathrm{C}(11)-\mathrm{N}(2)-\mathrm{C}(1)-\mathrm{Ru}(1)$ & $-9.8(9)$ \\
\hline $\mathrm{C}(3)-\mathrm{N}(2)-\mathrm{C}(1)-\mathrm{Ru}(1)$ & $-173.1(4)$ \\
\hline $\mathrm{C}(1)-\mathrm{N}(1)-\mathrm{C}(2)-\mathrm{C}(3)$ & $-16.3(7)$ \\
\hline $\mathrm{C}(4)-\mathrm{N}(1)-\mathrm{C}(2)-\mathrm{C}(3)$ & $178.4(6)$ \\
\hline$C(1)-N(2)-C(3)-C(2)$ & $-17.5(7)$ \\
\hline $\mathrm{C}(11)-\mathrm{N}(2)-\mathrm{C}(3)-\mathrm{C}(2)$ & 177.1(6) \\
\hline $\mathrm{N}(1)-\mathrm{C}(2)-\mathrm{C}(3)-\mathrm{N}(2)$ & $18.7(7)$ \\
\hline $\mathrm{C}(1)-\mathrm{N}(1)-\mathrm{C}(4)-\mathrm{C}(5)$ & $104.5(8)$ \\
\hline $\mathrm{C}(2)-\mathrm{N}(1)-\mathrm{C}(4)-\mathrm{C}(5)$ & $-92.6(8)$ \\
\hline $\mathrm{C}(1)-\mathrm{N}(1)-\mathrm{C}(4)-\mathrm{C}(9)$ & $-83.4(9)$ \\
\hline $\mathrm{C}(2)-\mathrm{N}(1)-\mathrm{C}(4)-\mathrm{C}(9)$ & $79.5(8)$ \\
\hline$C(9)-C(4)-C(5)-C(6)$ & $2.0(12)$ \\
\hline $\mathrm{N}(1)-\mathrm{C}(4)-\mathrm{C}(5)-\mathrm{C}(6)$ & $174.1(7)$ \\
\hline$C(9)-C(4)-C(5)-F(1)$ & $-179.8(6)$ \\
\hline $\mathrm{N}(1)-\mathrm{C}(4)-\mathrm{C}(5)-\mathrm{F}(1)$ & $-7.7(10)$ \\
\hline $\mathrm{F}(1)-\mathrm{C}(5)-\mathrm{C}(6)-\mathrm{C}(7)$ & $178.9(7)$ \\
\hline $\mathrm{C}(4)-\mathrm{C}(5)-\mathrm{C}(6)-\mathrm{C}(7)$ & $-2.9(13)$ \\
\hline$C(5)-C(6)-C(7)-C(8)$ & $1.5(14)$ \\
\hline$C(6)-C(7)-C(8)-C(9)$ & $0.6(15)$ \\
\hline$C(5)-C(4)-C(9)-C(8)$ & $0.2(10)$ \\
\hline $\mathrm{N}(1)-\mathrm{C}(4)-\mathrm{C}(9)-\mathrm{C}(8)$ & $-171.6(6)$ \\
\hline$C(5)-C(4)-C(9)-C(10)$ & $179.6(7)$ \\
\hline $\mathrm{N}(1)-\mathrm{C}(4)-\mathrm{C}(9)-\mathrm{C}(10)$ & $7.7(10)$ \\
\hline$C(7)-C(8)-C(9)-C(4)$ & $-1.5(12)$ \\
\hline$C(7)-C(8)-C(9)-C(10)$ & $179.2(8)$ \\
\hline$C(1)-N(2)-C(11)-C(16)$ & $-68.9(9)$ \\
\hline $\mathrm{C}(3)-\mathrm{N}(2)-\mathrm{C}(11)-\mathrm{C}(16)$ & $93.6(8)$ \\
\hline
\end{tabular}




\begin{tabular}{|c|c|}
\hline $\mathrm{C}(1)-\mathrm{N}(2)-\mathrm{C}(11)-\mathrm{C}(12)$ & $119.1(8)$ \\
\hline $\mathrm{C}(3)-\mathrm{N}(2)-\mathrm{C}(11)-\mathrm{C}(12)$ & $-78.3(8)$ \\
\hline$C(16)-C(11)-C(12)-C(13)$ & $0.7(10)$ \\
\hline $\mathrm{N}(2)-\mathrm{C}(11)-\mathrm{C}(12)-\mathrm{C}(13)$ & $172.6(6)$ \\
\hline$C(16)-C(11)-C(12)-C(17)$ & $179.7(7)$ \\
\hline $\mathrm{N}(2)-\mathrm{C}(11)-\mathrm{C}(12)-\mathrm{C}(17)$ & $-8.3(10)$ \\
\hline$C(11)-C(12)-C(13)-C(14)$ & $1.1(12)$ \\
\hline$C(17)-C(12)-C(13)-C(14)$ & $-178.0(7)$ \\
\hline $\mathrm{C}(12)-\mathrm{C}(13)-\mathrm{C}(14)-\mathrm{C}(15)$ & $-2.3(13)$ \\
\hline$C(13)-C(14)-C(15)-C(16)$ & $1.7(13)$ \\
\hline$C(14)-C(15)-C(16)-F(2)$ & $-177.3(7)$ \\
\hline$C(14)-C(15)-C(16)-C(11)$ & $0.1(12)$ \\
\hline$C(12)-C(11)-C(16)-F(2)$ & $176.2(6)$ \\
\hline $\mathrm{N}(2)-\mathrm{C}(11)-\mathrm{C}(16)-\mathrm{F}(2)$ & $4.1(10)$ \\
\hline$C(12)-C(11)-C(16)-C(15)$ & $-1.3(11)$ \\
\hline $\mathrm{N}(2)-\mathrm{C}(11)-\mathrm{C}(16)-\mathrm{C}(15)$ & $-173.4(7)$ \\
\hline$C(1)-R u(1)-C(18)-C(19)$ & $-100.1(5)$ \\
\hline$S(1)-R u(1)-C(18)-C(19)$ & $171.1(5)$ \\
\hline$S(2)-R u(1)-C(18)-C(19)$ & $80.5(5)$ \\
\hline $\mathrm{O}(1)-\mathrm{Ru}(1)-\mathrm{C}(18)-\mathrm{C}(19)$ & $-5.2(5)$ \\
\hline $\mathrm{Ru}(1)-\mathrm{C}(18)-\mathrm{C}(19)-\mathrm{C}(20)$ & $4.2(9)$ \\
\hline $\mathrm{Ru}(1)-\mathrm{C}(18)-\mathrm{C}(19)-\mathrm{C}(24)$ & $-176.4(5)$ \\
\hline $\mathrm{C}(25)-\mathrm{O}(1)-\mathrm{C}(20)-\mathrm{C}(21)$ & $9.5(11)$ \\
\hline $\mathrm{Ru}(1)-\mathrm{O}(1)-\mathrm{C}(20)-\mathrm{C}(21)$ & $175.7(7)$ \\
\hline $\mathrm{C}(25)-\mathrm{O}(1)-\mathrm{C}(20)-\mathrm{C}(19)$ & $-171.3(6)$ \\
\hline $\mathrm{Ru}(1)-\mathrm{O}(1)-\mathrm{C}(20)-\mathrm{C}(19)$ & $-5.1(7)$ \\
\hline $\mathrm{C}(24)-\mathrm{C}(19)-\mathrm{C}(20)-\mathrm{O}(1)$ & $-177.8(6)$ \\
\hline $\mathrm{C}(18)-\mathrm{C}(19)-\mathrm{C}(20)-\mathrm{O}(1)$ & $1.6(9)$ \\
\hline$C(24)-C(19)-C(20)-C(21)$ & $1.5(11)$ \\
\hline$C(18)-C(19)-C(20)-C(21)$ & $-179.1(7)$ \\
\hline $\mathrm{O}(1)-\mathrm{C}(20)-\mathrm{C}(21)-\mathrm{C}(22)$ & $177.2(7)$ \\
\hline$C(19)-C(20)-C(21)-C(22)$ & $-2.0(12)$ \\
\hline$C(20)-C(21)-C(22)-C(23)$ & $1.7(14)$ \\
\hline $\mathrm{C}(21)-\mathrm{C}(22)-\mathrm{C}(23)-\mathrm{C}(24)$ & $-1.0(14)$ \\
\hline$C(22)-C(23)-C(24)-C(19)$ & $0.5(12)$ \\
\hline$C(20)-C(19)-C(24)-C(23)$ & $-0.7(11)$ \\
\hline$C(18)-C(19)-C(24)-C(23)$ & $179.9(7)$ \\
\hline $\mathrm{C}(20)-\mathrm{O}(1)-\mathrm{C}(25)-\mathrm{C}(27)$ & $-164.4(7)$ \\
\hline $\mathrm{Ru}(1)-\mathrm{O}(1)-\mathrm{C}(25)-\mathrm{C}(27)$ & $32.7(9)$ \\
\hline $\mathrm{C}(20)-\mathrm{O}(1)-\mathrm{C}(25)-\mathrm{C}(26)$ & $73.6(9)$ \\
\hline $\mathrm{Ru}(1)-\mathrm{O}(1)-\mathrm{C}(25)-\mathrm{C}(26)$ & $-89.3(7)$ \\
\hline $\mathrm{Ru}(1)-\mathrm{S}(1)-\mathrm{C}(28)-\mathrm{C}(29)$ & $178.8(5)$ \\
\hline $\mathrm{Ru}(1)-\mathrm{S}(1)-\mathrm{C}(28)-\mathrm{C}(33)$ & $-1.5(6)$ \\
\hline $\mathrm{C}(33)-\mathrm{C}(28)-\mathrm{C}(29)-\mathrm{C}(30)$ & $3.4(10)$ \\
\hline $\mathrm{S}(1)-\mathrm{C}(28)-\mathrm{C}(29)-\mathrm{C}(30)$ & $-176.9(5)$ \\
\hline $\mathrm{C}(33)-\mathrm{C}(28)-\mathrm{C}(29)-\mathrm{Cl}(1)$ & $-175.0(5)$ \\
\hline $\mathrm{S}(1)-\mathrm{C}(28)-\mathrm{C}(29)-\mathrm{Cl}(1)$ & $4.7(8)$ \\
\hline $\mathrm{C}(28)-\mathrm{C}(29)-\mathrm{C}(30)-\mathrm{C}(31)$ & $-1.9(11)$ \\
\hline $\mathrm{Cl}(1)-\mathrm{C}(29)-\mathrm{C}(30)-\mathrm{C}(31)$ & $176.5(6)$ \\
\hline
\end{tabular}




\begin{tabular}{|c|c|}
\hline$C(29)-C(30)-C(31)-C(32)$ & $-0.8(12)$ \\
\hline$C(30)-C(31)-C(32)-C(33)$ & $1.8(12)$ \\
\hline $\mathrm{C}(30)-\mathrm{C}(31)-\mathrm{C}(32)-\mathrm{Cl}(2)$ & $-177.4(6)$ \\
\hline $\mathrm{C}(29)-\mathrm{C}(28)-\mathrm{C}(33)-\mathrm{C}(32)$ & $-2.3(10)$ \\
\hline $\mathrm{S}(1)-\mathrm{C}(28)-\mathrm{C}(33)-\mathrm{C}(32)$ & $178.0(5)$ \\
\hline$C(29)-C(28)-C(33)-S(2)$ & $175.6(5)$ \\
\hline$S(1)-C(28)-C(33)-S(2)$ & $-4.1(8)$ \\
\hline $\mathrm{C}(31)-\mathrm{C}(32)-\mathrm{C}(33)-\mathrm{C}(28)$ & $-0.3(11)$ \\
\hline $\mathrm{Cl}(2)-\mathrm{C}(32)-\mathrm{C}(33)-\mathrm{C}(28)$ & $179.0(5)$ \\
\hline$C(31)-C(32)-C(33)-S(2)$ & $-178.1(6)$ \\
\hline $\mathrm{Cl}(2)-\mathrm{C}(32)-\mathrm{C}(33)-\mathrm{S}(2)$ & $1.2(9)$ \\
\hline $\mathrm{Ru}(1)-\mathrm{S}(2)-\mathrm{C}(33)-\mathrm{C}(28)$ & $7.3(6)$ \\
\hline $\mathrm{Ru}(1)-\mathrm{S}(2)-\mathrm{C}(33)-\mathrm{C}(32)$ & $-174.9(5)$ \\
\hline $\mathrm{C}(44)-\mathrm{N}(4)-\mathrm{C}(34)-\mathrm{N}(3)$ & $-173.7(8)$ \\
\hline $\mathrm{C}(36)-\mathrm{N}(4)-\mathrm{C}(34)-\mathrm{N}(3)$ & $6.9(10)$ \\
\hline $\mathrm{C}(44)-\mathrm{N}(4)-\mathrm{C}(34)-\mathrm{Ru}(2)$ & $14.1(12)$ \\
\hline $\mathrm{C}(36)-\mathrm{N}(4)-\mathrm{C}(34)-\mathrm{Ru}(2)$ & $-165.3(7)$ \\
\hline $\mathrm{C}(37)-\mathrm{N}(3)-\mathrm{C}(34)-\mathrm{N}(4)$ & $171.6(7)$ \\
\hline $\mathrm{C}(35)-\mathrm{N}(3)-\mathrm{C}(34)-\mathrm{N}(4)$ & $1.9(9)$ \\
\hline $\mathrm{C}(37)-\mathrm{N}(3)-\mathrm{C}(34)-\mathrm{Ru}(2)$ & $-16.4(11)$ \\
\hline $\mathrm{C}(35)-\mathrm{N}(3)-\mathrm{C}(34)-\mathrm{Ru}(2)$ & $173.9(6)$ \\
\hline $\mathrm{C}(34)-\mathrm{N}(3)-\mathrm{C}(35)-\mathrm{C}(36)$ & $-9.3(10)$ \\
\hline $\mathrm{C}(37)-\mathrm{N}(3)-\mathrm{C}(35)-\mathrm{C}(36)$ & $-179.9(8)$ \\
\hline$C(34)-N(4)-C(36)-C(35)$ & $-12.3(11)$ \\
\hline$C(44)-N(4)-C(36)-C(35)$ & $168.3(8)$ \\
\hline $\mathrm{N}(3)-\mathrm{C}(35)-\mathrm{C}(36)-\mathrm{N}(4)$ & $11.9(10)$ \\
\hline $\mathrm{C}(34)-\mathrm{N}(3)-\mathrm{C}(37)-\mathrm{C}(42)$ & $-75.2(11)$ \\
\hline $\mathrm{C}(35)-\mathrm{N}(3)-\mathrm{C}(37)-\mathrm{C}(42)$ & $93.9(10)$ \\
\hline $\mathrm{C}(34)-\mathrm{N}(3)-\mathrm{C}(37)-\mathrm{C}(38)$ & $108.2(8)$ \\
\hline $\mathrm{C}(35)-\mathrm{N}(3)-\mathrm{C}(37)-\mathrm{C}(38)$ & $-82.7(9)$ \\
\hline $\mathrm{C}(42)-\mathrm{C}(37)-\mathrm{C}(38)-\mathrm{C}(39)$ & $-0.1(13)$ \\
\hline $\mathrm{N}(3)-\mathrm{C}(37)-\mathrm{C}(38)-\mathrm{C}(39)$ & $176.5(8)$ \\
\hline $\mathrm{C}(42)-\mathrm{C}(37)-\mathrm{C}(38)-\mathrm{F}(3)$ & $178.9(8)$ \\
\hline $\mathrm{N}(3)-\mathrm{C}(37)-\mathrm{C}(38)-\mathrm{F}(3)$ & $-4.5(10)$ \\
\hline $\mathrm{F}(3)-\mathrm{C}(38)-\mathrm{C}(39)-\mathrm{C}(40)$ & $-177.2(7)$ \\
\hline$C(37)-C(38)-C(39)-C(40)$ & $1.8(13)$ \\
\hline$C(38)-C(39)-C(40)-C(41)$ & $-2.6(14)$ \\
\hline$C(39)-C(40)-C(41)-C(42)$ & $1.5(15)$ \\
\hline $\mathrm{N}(3)-\mathrm{C}(37)-\mathrm{C}(42)-\mathrm{C}(41)$ & $-177.2(8)$ \\
\hline$C(38)-C(37)-C(42)-C(41)$ & $-1.0(13)$ \\
\hline $\mathrm{N}(3)-\mathrm{C}(37)-\mathrm{C}(42)-\mathrm{C}(43)$ & $4.0(13)$ \\
\hline$C(38)-C(37)-C(42)-C(43)$ & $-179.8(8)$ \\
\hline$C(40)-C(41)-C(42)-C(37)$ & $0.3(14)$ \\
\hline$C(40)-C(41)-C(42)-C(43)$ & $179.0(9)$ \\
\hline$C(34)-N(4)-C(44)-C(45)$ & $91(2)$ \\
\hline$C(36)-N(4)-C(44)-C(45)$ & $-90(2)$ \\
\hline $\mathrm{C}(34)-\mathrm{N}(4)-\mathrm{C}(44)-\mathrm{C}(49)$ & $-93.9(14)$ \\
\hline$C(36)-N(4)-C(44)-C(49)$ & $85.4(14)$ \\
\hline $\mathrm{N}(4)-\mathrm{C}(44)-\mathrm{C}(45)-\mathrm{C}(46)$ & $175.8(16)$ \\
\hline
\end{tabular}




\begin{tabular}{|c|c|}
\hline$C(49)-C(44)-C(45)-C(46)$ & $1(4)$ \\
\hline $\mathrm{N}(4)-\mathrm{C}(44)-\mathrm{C}(45)-\mathrm{C}(50)$ & $-2(4)$ \\
\hline$C(49)-C(44)-C(45)-C(50)$ & $-177(2)$ \\
\hline$C(44)-C(45)-C(46)-C(47)$ & $0(4)$ \\
\hline$C(50)-C(45)-C(46)-C(47)$ & $178(3)$ \\
\hline$C(45)-C(46)-C(47)-C(48)$ & $-1(4)$ \\
\hline$C(46)-C(47)-C(48)-C(49)$ & $0(3)$ \\
\hline$C(45)-C(44)-C(49)-F(4)$ & $176(2)$ \\
\hline $\mathrm{N}(4)-\mathrm{C}(44)-\mathrm{C}(49)-\mathrm{F}(4)$ & $0(2)$ \\
\hline$C(45)-C(44)-C(49)-C(48)$ & $-1(3)$ \\
\hline $\mathrm{N}(4)-\mathrm{C}(44)-\mathrm{C}(49)-\mathrm{C}(48)$ & $-176.9(17)$ \\
\hline $\mathrm{C}(47)-\mathrm{C}(48)-\mathrm{C}(49)-\mathrm{F}(4)$ & $-177(2)$ \\
\hline $\mathrm{C}(47)-\mathrm{C}(48)-\mathrm{C}(49)-\mathrm{C}(44)$ & $0(3)$ \\
\hline $\mathrm{C}(34)-\mathrm{Ru}(2)-\mathrm{C}(51)-\mathrm{C}(52)$ & $-95.5(5)$ \\
\hline $\mathrm{S}(3)-\mathrm{Ru}(2)-\mathrm{C}(51)-\mathrm{C}(52)$ & $169.0(5)$ \\
\hline$S(4)-R u(2)-C(51)-C(52)$ & $79.9(5)$ \\
\hline $\mathrm{O}(2)-\mathrm{Ru}(2)-\mathrm{C}(51)-\mathrm{C}(52)$ & $-5.7(5)$ \\
\hline $\mathrm{Ru}(2)-\mathrm{C}(51)-\mathrm{C}(52)-\mathrm{C}(53)$ & $-174.9(6)$ \\
\hline $\mathrm{Ru}(2)-\mathrm{C}(51)-\mathrm{C}(52)-\mathrm{C}(57)$ & $6.6(9)$ \\
\hline $\mathrm{C}(57)-\mathrm{C}(52)-\mathrm{C}(53)-\mathrm{C}(54)$ & $1.6(11)$ \\
\hline $\mathrm{C}(51)-\mathrm{C}(52)-\mathrm{C}(53)-\mathrm{C}(54)$ & $-176.9(7)$ \\
\hline $\mathrm{C}(52)-\mathrm{C}(53)-\mathrm{C}(54)-\mathrm{C}(55)$ & $-0.5(13)$ \\
\hline$C(53)-C(54)-C(55)-C(56)$ & $0.1(13)$ \\
\hline$C(54)-C(55)-C(56)-C(57)$ & $-0.7(12)$ \\
\hline $\mathrm{C}(55)-\mathrm{C}(56)-\mathrm{C}(57)-\mathrm{O}(2)$ & $179.9(7)$ \\
\hline$C(55)-C(56)-C(57)-C(52)$ & $1.8(11)$ \\
\hline $\mathrm{C}(58)-\mathrm{O}(2)-\mathrm{C}(57)-\mathrm{C}(56)$ & $8.9(11)$ \\
\hline $\mathrm{Ru}(2)-\mathrm{O}(2)-\mathrm{C}(57)-\mathrm{C}(56)$ & $179.6(6)$ \\
\hline $\mathrm{C}(58)-\mathrm{O}(2)-\mathrm{C}(57)-\mathrm{C}(52)$ & $-172.9(6)$ \\
\hline $\mathrm{Ru}(2)-\mathrm{O}(2)-\mathrm{C}(57)-\mathrm{C}(52)$ & $-2.2(7)$ \\
\hline$C(53)-C(52)-C(57)-C(56)$ & $-2.2(11)$ \\
\hline $\mathrm{C}(51)-\mathrm{C}(52)-\mathrm{C}(57)-\mathrm{C}(56)$ & $176.4(7)$ \\
\hline $\mathrm{C}(53)-\mathrm{C}(52)-\mathrm{C}(57)-\mathrm{O}(2)$ & $179.5(6)$ \\
\hline $\mathrm{C}(51)-\mathrm{C}(52)-\mathrm{C}(57)-\mathrm{O}(2)$ & $-2.0(9)$ \\
\hline $\mathrm{C}(57)-\mathrm{O}(2)-\mathrm{C}(58)-\mathrm{C}(59)$ & $-94.1(12)$ \\
\hline $\mathrm{Ru}(2)-\mathrm{O}(2)-\mathrm{C}(58)-\mathrm{C}(59)$ & $97.6(12)$ \\
\hline $\mathrm{C}(57)-\mathrm{O}(2)-\mathrm{C}(58)-\mathrm{C}(60)$ & $133.2(10)$ \\
\hline $\mathrm{Ru}(2)-\mathrm{O}(2)-\mathrm{C}(58)-\mathrm{C}(60)$ & $-35.1(13)$ \\
\hline $\mathrm{Ru}(2)-\mathrm{S}(3)-\mathrm{C}(61)-\mathrm{C}(66)$ & $-10.7(5)$ \\
\hline $\mathrm{Ru}(2)-\mathrm{S}(3)-\mathrm{C}(61)-\mathrm{C}(62)$ & $168.3(5)$ \\
\hline$C(66)-C(61)-C(62)-C(63)$ & $-1.3(9)$ \\
\hline$S(3)-C(61)-C(62)-C(63)$ & $179.7(5)$ \\
\hline $\mathrm{C}(66)-\mathrm{C}(61)-\mathrm{C}(62)-\mathrm{Cl}(3)$ & $177.3(4)$ \\
\hline $\mathrm{S}(3)-\mathrm{C}(61)-\mathrm{C}(62)-\mathrm{Cl}(3)$ & $-1.8(7)$ \\
\hline$C(61)-C(62)-C(63)-C(64)$ & $-0.8(10)$ \\
\hline $\mathrm{Cl}(3)-\mathrm{C}(62)-\mathrm{C}(63)-\mathrm{C}(64)$ & $-179.3(5)$ \\
\hline $\mathrm{C}(62)-\mathrm{C}(63)-\mathrm{C}(64)-\mathrm{C}(65)$ & $1.6(10)$ \\
\hline$C(63)-C(64)-C(65)-C(66)$ & $-0.4(10)$ \\
\hline $\mathrm{C}(63)-\mathrm{C}(64)-\mathrm{C}(65)-\mathrm{Cl}(4)$ & $-179.9(5)$ \\
\hline
\end{tabular}




\begin{tabular}{|c|c|}
\hline$C(62)-C(61)-C(66)-C(65)$ & $2.5(8)$ \\
\hline$S(3)-C(61)-C(66)-C(65)$ & $-178.5(5)$ \\
\hline$C(62)-C(61)-C(66)-S(4)$ & $-176.5(4)$ \\
\hline$S(3)-C(61)-C(66)-S(4)$ & $2.5(7)$ \\
\hline$C(64)-C(65)-C(66)-C(61)$ & $-1.7(9)$ \\
\hline $\mathrm{Cl}(4)-\mathrm{C}(65)-\mathrm{C}(66)-\mathrm{C}(61)$ & $177.8(5)$ \\
\hline$C(64)-C(65)-C(66)-S(4)$ & $177.3(5)$ \\
\hline $\mathrm{Cl}(4)-\mathrm{C}(65)-\mathrm{C}(66)-\mathrm{S}(4)$ & $-3.2(7)$ \\
\hline $\mathrm{Ru}(2)-\mathrm{S}(4)-\mathrm{C}(66)-\mathrm{C}(61)$ & $7.0(5)$ \\
\hline $\mathrm{Ru}(2)-\mathrm{S}(4)-\mathrm{C}(66)-\mathrm{C}(65)$ & $-172.0(4)$ \\
\hline $\mathrm{C}(70)-\mathrm{N}(5)-\mathrm{C}(67)-\mathrm{N}(6)$ & $170.8(6)$ \\
\hline $\mathrm{C}(68)-\mathrm{N}(5)-\mathrm{C}(67)-\mathrm{N}(6)$ & $5.0(8)$ \\
\hline$C(70)-N(5)-C(67)-R u(3)$ & $-10.3(11)$ \\
\hline $\mathrm{C}(68)-\mathrm{N}(5)-\mathrm{C}(67)-\mathrm{Ru}(3)$ & $-176.0(5)$ \\
\hline $\mathrm{C}(77)-\mathrm{N}(6)-\mathrm{C}(67)-\mathrm{N}(5)$ & $170.9(6)$ \\
\hline $\mathrm{C}(69)-\mathrm{N}(6)-\mathrm{C}(67)-\mathrm{N}(5)$ & $7.9(8)$ \\
\hline$C(77)-N(6)-C(67)-R u(3)$ & $-8.2(9)$ \\
\hline $\mathrm{C}(69)-\mathrm{N}(6)-\mathrm{C}(67)-\mathrm{Ru}(3)$ & $-171.2(5)$ \\
\hline $\mathrm{C}(67)-\mathrm{N}(5)-\mathrm{C}(68)-\mathrm{C}(69)$ & $-14.9(7)$ \\
\hline$C(70)-N(5)-C(68)-C(69)$ & $177.5(6)$ \\
\hline $\mathrm{C}(67)-\mathrm{N}(6)-\mathrm{C}(69)-\mathrm{C}(68)$ & $-16.5(7)$ \\
\hline $\mathrm{C}(77)-\mathrm{N}(6)-\mathrm{C}(69)-\mathrm{C}(68)$ & $179.7(6)$ \\
\hline $\mathrm{N}(5)-\mathrm{C}(68)-\mathrm{C}(69)-\mathrm{N}(6)$ & $17.3(7)$ \\
\hline$C(67)-N(5)-C(70)-C(71)$ & $107.8(8)$ \\
\hline $\mathrm{C}(68)-\mathrm{N}(5)-\mathrm{C}(70)-\mathrm{C}(71)$ & $-86.9(8)$ \\
\hline $\mathrm{C}(67)-\mathrm{N}(5)-\mathrm{C}(70)-\mathrm{C}(75)$ & $-78.8(9)$ \\
\hline $\mathrm{C}(68)-\mathrm{N}(5)-\mathrm{C}(70)-\mathrm{C}(75)$ & $86.5(7)$ \\
\hline$C(75)-C(70)-C(71)-C(72)$ & $2.5(10)$ \\
\hline $\mathrm{N}(5)-\mathrm{C}(70)-\mathrm{C}(71)-\mathrm{C}(72)$ & $176.0(6)$ \\
\hline$C(75)-C(70)-C(71)-F(5)$ & $-177.6(6)$ \\
\hline $\mathrm{N}(5)-\mathrm{C}(70)-\mathrm{C}(71)-\mathrm{F}(5)$ & $-4.1(9)$ \\
\hline$C(70)-C(71)-C(72)-C(73)$ & $-1.3(11)$ \\
\hline $\mathrm{F}(5)-\mathrm{C}(71)-\mathrm{C}(72)-\mathrm{C}(73)$ & $178.8(6)$ \\
\hline $\mathrm{C}(71)-\mathrm{C}(72)-\mathrm{C}(73)-\mathrm{C}(74)$ & $1.7(11)$ \\
\hline$C(72)-C(73)-C(74)-C(75)$ & $-3.2(11)$ \\
\hline$C(73)-C(74)-C(75)-C(70)$ & $4.2(10)$ \\
\hline$C(73)-C(74)-C(75)-C(76)$ & $-176.7(6)$ \\
\hline$C(71)-C(70)-C(75)-C(74)$ & $-3.8(9)$ \\
\hline $\mathrm{N}(5)-\mathrm{C}(70)-\mathrm{C}(75)-\mathrm{C}(74)$ & $-177.2(6)$ \\
\hline$C(71)-C(70)-C(75)-C(76)$ & $177.1(6)$ \\
\hline$N(5)-C(70)-C(75)-C(76)$ & $3.7(9)$ \\
\hline $\mathrm{C}(67)-\mathrm{N}(6)-\mathrm{C}(77)-\mathrm{C}(78)$ & $114.1(8)$ \\
\hline $\mathrm{C}(69)-\mathrm{N}(6)-\mathrm{C}(77)-\mathrm{C}(78)$ & $-84.1(8)$ \\
\hline $\mathrm{C}(67)-\mathrm{N}(6)-\mathrm{C}(77)-\mathrm{C}(82)$ & $-69.6(9)$ \\
\hline $\mathrm{C}(69)-\mathrm{N}(6)-\mathrm{C}(77)-\mathrm{C}(82)$ & $92.2(8)$ \\
\hline $\mathrm{C}(82)-\mathrm{C}(77)-\mathrm{C}(78)-\mathrm{C}(79)$ & $5.6(10)$ \\
\hline $\mathrm{N}(6)-\mathrm{C}(77)-\mathrm{C}(78)-\mathrm{C}(79)$ & $-178.1(6)$ \\
\hline $\mathrm{C}(82)-\mathrm{C}(77)-\mathrm{C}(78)-\mathrm{C}(83)$ & $-176.0(6)$ \\
\hline $\mathrm{N}(6)-\mathrm{C}(77)-\mathrm{C}(78)-\mathrm{C}(83)$ & $0.3(10)$ \\
\hline
\end{tabular}




\begin{tabular}{|c|c|}
\hline $\mathrm{C}(77)-\mathrm{C}(78)-\mathrm{C}(79)-\mathrm{C}(80)$ & $-1.6(11)$ \\
\hline $\mathrm{C}(83)-\mathrm{C}(78)-\mathrm{C}(79)-\mathrm{C}(80)$ & $-180.0(7)$ \\
\hline $\mathrm{C}(78)-\mathrm{C}(79)-\mathrm{C}(80)-\mathrm{C}(81)$ & $-1.5(12)$ \\
\hline $\mathrm{C}(79)-\mathrm{C}(80)-\mathrm{C}(81)-\mathrm{C}(82)$ & $0.4(12)$ \\
\hline $\mathrm{C}(80)-\mathrm{C}(81)-\mathrm{C}(82)-\mathrm{F}(6)$ & $-176.4(6)$ \\
\hline $\mathrm{C}(80)-\mathrm{C}(81)-\mathrm{C}(82)-\mathrm{C}(77)$ & $3.9(11)$ \\
\hline $\mathrm{C}(78)-\mathrm{C}(77)-\mathrm{C}(82)-\mathrm{C}(81)$ & $-7.1(10)$ \\
\hline $\mathrm{N}(6)-\mathrm{C}(77)-\mathrm{C}(82)-\mathrm{C}(81)$ & $176.6(6)$ \\
\hline $\mathrm{C}(78)-\mathrm{C}(77)-\mathrm{C}(82)-\mathrm{F}(6)$ & $173.2(6)$ \\
\hline $\mathrm{N}(6)-\mathrm{C}(77)-\mathrm{C}(82)-\mathrm{F}(6)$ & $-3.1(9)$ \\
\hline$C(67)-R u(3)-C(84)-C(85)$ & $-96.4(5)$ \\
\hline $\mathrm{S}(5)-\mathrm{Ru}(3)-\mathrm{C}(84)-\mathrm{C}(85)$ & $171.4(4)$ \\
\hline $\mathrm{S}(6)-\mathrm{Ru}(3)-\mathrm{C}(84)-\mathrm{C}(85)$ & $82.0(5)$ \\
\hline $\mathrm{O}(3)-\mathrm{Ru}(3)-\mathrm{C}(84)-\mathrm{C}(85)$ & $-3.0(4)$ \\
\hline $\mathrm{Ru}(3)-\mathrm{C}(84)-\mathrm{C}(85)-\mathrm{C}(90)$ & $4.4(8)$ \\
\hline $\mathrm{Ru}(3)-\mathrm{C}(84)-\mathrm{C}(85)-\mathrm{C}(86)$ & $-173.7(5)$ \\
\hline$C(90)-C(85)-C(86)-C(87)$ & $2.4(9)$ \\
\hline $\mathrm{C}(84)-\mathrm{C}(85)-\mathrm{C}(86)-\mathrm{C}(87)$ & $-179.5(6)$ \\
\hline $\mathrm{C}(85)-\mathrm{C}(86)-\mathrm{C}(87)-\mathrm{C}(88)$ & $1.3(10)$ \\
\hline $\mathrm{C}(86)-\mathrm{C}(87)-\mathrm{C}(88)-\mathrm{C}(89)$ & $-1.0(10)$ \\
\hline $\mathrm{C}(87)-\mathrm{C}(88)-\mathrm{C}(89)-\mathrm{C}(90)$ & $-2.9(10)$ \\
\hline $\mathrm{C}(91)-\mathrm{O}(3)-\mathrm{C}(90)-\mathrm{C}(89)$ & $12.2(9)$ \\
\hline $\mathrm{Ru}(3)-\mathrm{O}(3)-\mathrm{C}(90)-\mathrm{C}(89)$ & $-177.6(5)$ \\
\hline $\mathrm{C}(91)-\mathrm{O}(3)-\mathrm{C}(90)-\mathrm{C}(85)$ & $-169.9(5)$ \\
\hline $\mathrm{Ru}(3)-\mathrm{O}(3)-\mathrm{C}(90)-\mathrm{C}(85)$ & $0.3(6)$ \\
\hline $\mathrm{C}(88)-\mathrm{C}(89)-\mathrm{C}(90)-\mathrm{O}(3)$ & $-175.6(6)$ \\
\hline $\mathrm{C}(88)-\mathrm{C}(89)-\mathrm{C}(90)-\mathrm{C}(85)$ & $6.7(10)$ \\
\hline $\mathrm{C}(86)-\mathrm{C}(85)-\mathrm{C}(90)-\mathrm{O}(3)$ & $175.5(5)$ \\
\hline $\mathrm{C}(84)-\mathrm{C}(85)-\mathrm{C}(90)-\mathrm{O}(3)$ & $-2.7(8)$ \\
\hline $\mathrm{C}(86)-\mathrm{C}(85)-\mathrm{C}(90)-\mathrm{C}(89)$ & $-6.5(9)$ \\
\hline $\mathrm{C}(84)-\mathrm{C}(85)-\mathrm{C}(90)-\mathrm{C}(89)$ & $175.3(6)$ \\
\hline $\mathrm{C}(90)-\mathrm{O}(3)-\mathrm{C}(91)-\mathrm{C}(93)$ & $139.5(6)$ \\
\hline $\mathrm{Ru}(3)-\mathrm{O}(3)-\mathrm{C}(91)-\mathrm{C}(93)$ & $-27.8(9)$ \\
\hline $\mathrm{C}(90)-\mathrm{O}(3)-\mathrm{C}(91)-\mathrm{C}(92)$ & $-93.8(7)$ \\
\hline $\mathrm{Ru}(3)-\mathrm{O}(3)-\mathrm{C}(91)-\mathrm{C}(92)$ & $98.9(7)$ \\
\hline $\mathrm{Ru}(3)-\mathrm{S}(5)-\mathrm{C}(94)-\mathrm{C}(95)$ & $172.3(5)$ \\
\hline $\mathrm{Ru}(3)-\mathrm{S}(5)-\mathrm{C}(94)-\mathrm{C}(99)$ & $-6.8(6)$ \\
\hline C(99)-C(94)-C(95)-C(96) & $-1.5(11)$ \\
\hline $\mathrm{S}(5)-\mathrm{C}(94)-\mathrm{C}(95)-\mathrm{C}(96)$ & $179.4(6)$ \\
\hline $\mathrm{C}(99)-\mathrm{C}(94)-\mathrm{C}(95)-\mathrm{Cl}(5)$ & $179.0(5)$ \\
\hline $\mathrm{S}(5)-\mathrm{C}(94)-\mathrm{C}(95)-\mathrm{Cl}(5)$ & $-0.2(9)$ \\
\hline$C(94)-C(95)-C(96)-C(97)$ & $0.1(12)$ \\
\hline $\mathrm{Cl}(5)-\mathrm{C}(95)-\mathrm{C}(96)-\mathrm{C}(97)$ & $179.7(6)$ \\
\hline $\mathrm{C}(95)-\mathrm{C}(96)-\mathrm{C}(97)-\mathrm{C}(98)$ & $0.9(12)$ \\
\hline $\mathrm{C}(96)-\mathrm{C}(97)-\mathrm{C}(98)-\mathrm{C}(99)$ & $-0.4(12)$ \\
\hline $\mathrm{C}(96)-\mathrm{C}(97)-\mathrm{C}(98)-\mathrm{Cl}(6)$ & $175.7(6)$ \\
\hline C(95)-C(94)-C(99)-C(98) & $1.8(10)$ \\
\hline $\mathrm{S}(5)-\mathrm{C}(94)-\mathrm{C}(99)-\mathrm{C}(98)$ & $-179.0(5)$ \\
\hline $\mathrm{C}(95)-\mathrm{C}(94)-\mathrm{C}(99)-\mathrm{S}(6)$ & $-177.7(5)$ \\
\hline
\end{tabular}


$\mathrm{S}(5)-\mathrm{C}(94)-\mathrm{C}(99)-\mathrm{S}(6)$

$\mathrm{Cl}(6)-\mathrm{C}(98)-\mathrm{C}(99)-\mathrm{S}(6)$

$\mathrm{Ru}(3)-\mathrm{S}(6)-\mathrm{C}(99)-\mathrm{C}(94)$

Symmetry transformations used to generate equivalent atoms:

\section{X-ray Structure of Ru-1d}
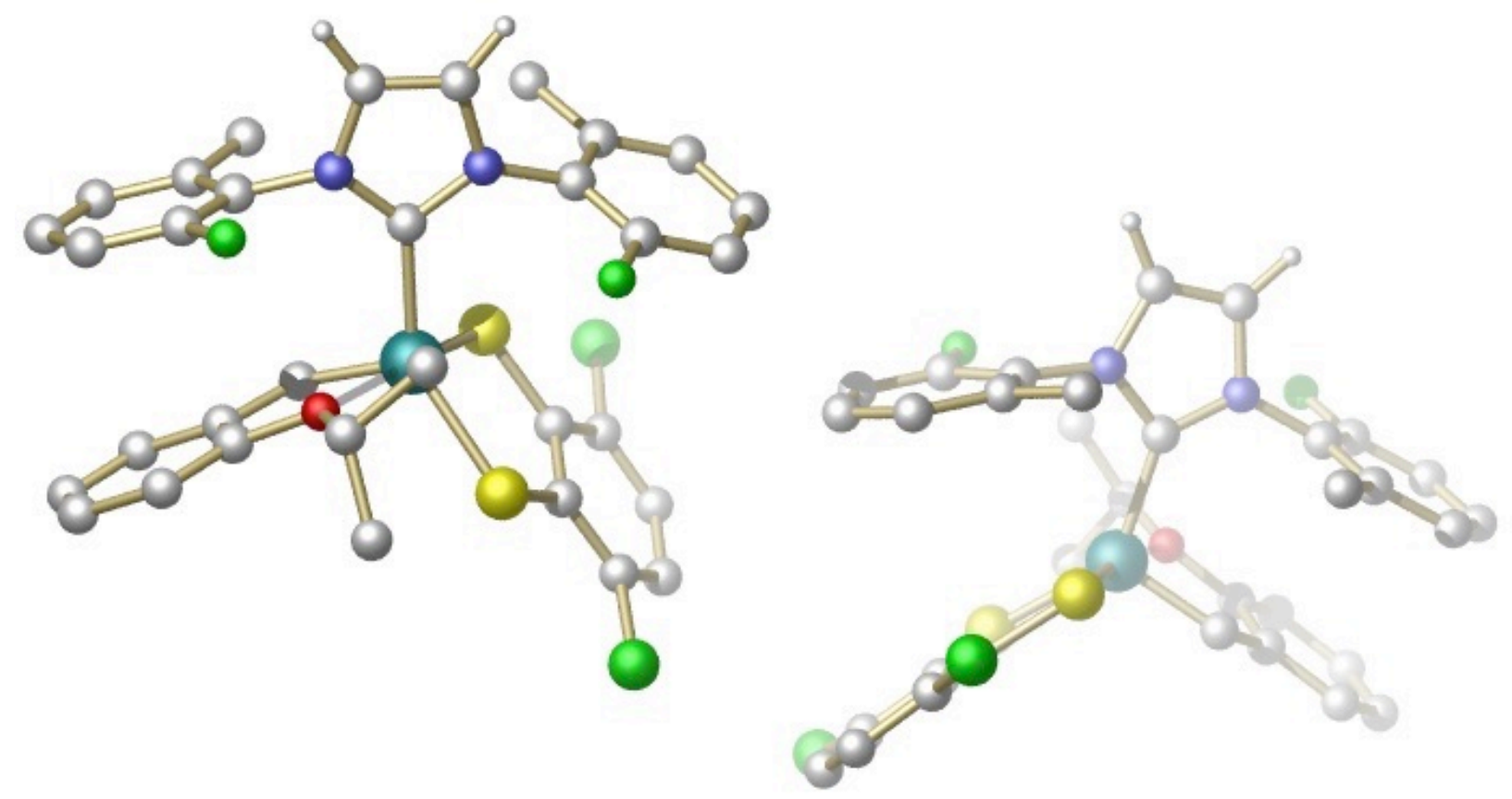

Table 7. Crystal data and structure refinement for $\mathrm{C}_{33} \mathrm{H}_{28} \mathrm{Cl}_{2} \mathrm{~F}_{2} \mathrm{~N}_{2} \mathrm{ORuS}_{2}$ (Ru-1d).

Identification code

Empirical formula

Formula weight

Temperature

Wavelength

Crystal system

Space group

Unit cell dimensions

Volume

$\mathrm{Z}$

Density (calculated)

Absorption coefficient

$\mathrm{F}(000)$
C33.50 H28 Cl2 F2 N2 O1.50 Ru S2

C33.50 H28 Cl2 F2 N2 O1.50 Ru S2

758.68

100(2) K

$1.54178 \AA$

Monoclinic

$\mathrm{P} 2{ }_{1} / \mathrm{c}$

$\mathrm{a}=15.3836(8) \AA$

$\mathrm{a}=90^{\circ}$.

$\mathrm{b}=17.6296(9) \AA$

$\mathrm{c}=24.9348(13) \AA$

6440.4(6) $\AA^{3}$

8

$1.565 \mathrm{Mg} / \mathrm{m}^{3}$

$7.049 \mathrm{~mm}^{-1}$

3080 
Crystal size

Theta range for data collection

Index ranges

Reflections collected

Independent reflections

Completeness to theta $=67.679^{\circ}$

Refinement method

Data / restraints / parameters

Goodness-of-fit on $\mathrm{F}^{2}$

Final R indices [I $>2$ sigma(I)]

$\mathrm{R}$ indices (all data)

Extinction coefficient

Largest diff. peak and hole

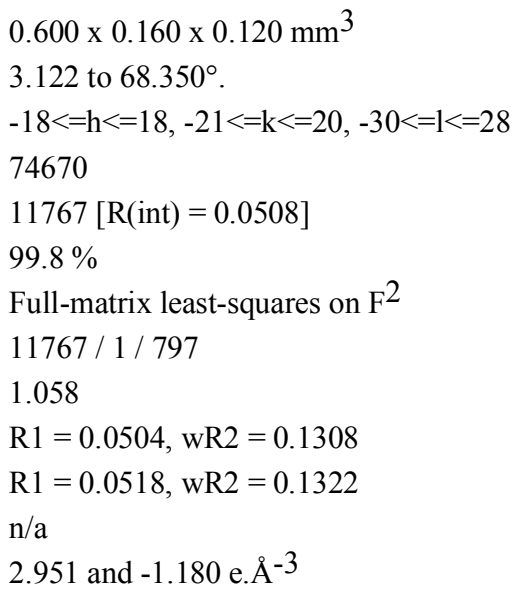

$0.600 \times 0.160 \times 0.120 \mathrm{~mm}^{3}$

74670

$99.8 \%$

Full-matrix least-squares on $\mathrm{F}^{2}$

11767 / 1 / 797

$\mathrm{R} 1=0.0518, \mathrm{wR} 2=0.1322$

2.951 and -1.180 e. $\AA^{-3}$

Table 8. Atomic coordinates $\left(\times 10^{4}\right)$ and equivalent isotropic displacement parameters $\left(\AA^{2} \times 10^{3}\right)$ for $\mathrm{C}_{33} \mathrm{H}_{28} \mathrm{Cl}_{2} \mathrm{~F}_{2} \mathrm{~N}_{2} \mathrm{ORuS} \mathrm{S}_{2}\left(\mathbf{R u}_{-}\right.$ 1d). $U\left(\right.$ eq) is defined as one third of the trace of the orthogonalized $U^{i j}$ tensor.

\begin{tabular}{|c|c|c|c|c|}
\hline & $\mathrm{x}$ & $\mathrm{y}$ & $\mathrm{z}$ & $\mathrm{U}(\mathrm{eq})$ \\
\hline $\mathrm{Ru}(1)$ & $5157(1)$ & $2453(1)$ & $7108(1)$ & $20(1)$ \\
\hline$S(1)$ & $5564(1)$ & $2589(1)$ & $6318(1)$ & $28(1)$ \\
\hline $\mathrm{S}(2)$ & $4159(1)$ & $1542(1)$ & $6661(1)$ & $33(1)$ \\
\hline $\mathrm{Cl}(1)$ & $5690(1)$ & 2493(1) & $5074(1)$ & $61(1)$ \\
\hline $\mathrm{Cl}(2)$ & $3151(2)$ & $260(1)$ & $5788(1)$ & $89(1)$ \\
\hline $\mathrm{F}(1)$ & $5441(3)$ & $3622(2)$ & $8726(1)$ & $56(1)$ \\
\hline $\mathrm{F}(2)$ & $2848(2)$ & $3662(2)$ & $6655(1)$ & $46(1)$ \\
\hline $\mathrm{O}(1)$ & $4918(2)$ & $2209(2)$ & $7953(1)$ & $25(1)$ \\
\hline $\mathrm{N}(1)$ & $5655(3)$ & 4011(2) & $7700(2)$ & $31(1)$ \\
\hline $\mathrm{N}(2)$ & $4640(2)$ & $4128(2)$ & $6895(2)$ & $27(1)$ \\
\hline$C(1)$ & $5196(3)$ & $3605(2)$ & $7239(2)$ & $24(1)$ \\
\hline$C(2)$ & $5384(4)$ & $4767(3)$ & $7651(2)$ & $42(1)$ \\
\hline$C(3)$ & $4747(4)$ & $4836(3)$ & $7147(2)$ & $39(1)$ \\
\hline$C(4)$ & $6410(4)$ & $3737(3)$ & $8155(2)$ & $34(1)$ \\
\hline$C(5)$ & $7270(4)$ & $3681(3)$ & $8084(2)$ & $39(1)$ \\
\hline$C(6)$ & $7987(4)$ & $3418(3)$ & $8542(3)$ & $55(2)$ \\
\hline$C(7)$ & $7839(5)$ & $3237(4)$ & $9048(2)$ & $60(2)$ \\
\hline$C(8)$ & $6998(5)$ & $3297(3)$ & $9117(2)$ & $56(2)$ \\
\hline$C(9)$ & $6296(4)$ & $3551(3)$ & $8668(2)$ & $43(1)$ \\
\hline$C(10)$ & $7403(3)$ & $3890(3)$ & $7532(2)$ & $41(1)$ \\
\hline $\mathrm{C}(11)$ & 4084(3) & $3989(3)$ & $6326(2)$ & $31(1)$ \\
\hline$C(12)$ & $3192(3)$ & $3715(3)$ & $6221(2)$ & $37(1)$ \\
\hline$C(13)$ & $2668(4)$ & $3502(3)$ & $5695(2)$ & $45(1)$ \\
\hline$C(14)$ & $3035(4)$ & $3582(3)$ & $5256(2)$ & $47(1)$ \\
\hline$C(15)$ & $3887(4)$ & 3893(3) & $5342(2)$ & $42(1)$ \\
\hline$C(16)$ & $4428(3)$ & 4113(3) & $5877(2)$ & $35(1)$ \\
\hline$C(17)$ & $5352(4)$ & $4460(3)$ & $5967(2)$ & $44(1)$ \\
\hline$C(18)$ & $4944(3)$ & $1909(3)$ & $5847(2)$ & $35(1)$ \\
\hline$C(19)$ & $5027(4)$ & $1838(4)$ & $5305(2)$ & $49(1)$ \\
\hline$C(20)$ & $4602(4)$ & $1279(4)$ & $4935(2)$ & $59(2)$ \\
\hline
\end{tabular}




\begin{tabular}{|c|c|c|c|c|}
\hline$C(21)$ & $4062(5)$ & $788(4)$ & $5096(2)$ & $67(2)$ \\
\hline $\mathrm{C}(22)$ & 3909(4) & $857(3)$ & $5625(2)$ & $57(2)$ \\
\hline$C(23)$ & $4346(4)$ & $1421(3)$ & 6003(2) & $40(1)$ \\
\hline C(24) & $6287(3)$ & 2113(2) & 7541(2) & $25(1)$ \\
\hline$C(25)$ & $6412(3)$ & $1815(2)$ & $8101(2)$ & $25(1)$ \\
\hline$C(26)$ & $7229(3)$ & 1501(3) & $8442(2)$ & $30(1)$ \\
\hline $\mathrm{C}(27)$ & 7292(3) & $1224(3)$ & $8976(2)$ & $35(1)$ \\
\hline$C(28)$ & 6543(3) & $1263(3)$ & $9170(2)$ & $36(1)$ \\
\hline C(29) & $5727(3)$ & $1578(3)$ & $8846(2)$ & $32(1)$ \\
\hline$C(30)$ & $5672(3)$ & $1858(2)$ & $8319(2)$ & $24(1)$ \\
\hline $\mathrm{C}(31)$ & 4070(3) & $2252(3)$ & $8113(2)$ & $32(1)$ \\
\hline C(32) & $3605(3)$ & $1485(3)$ & $8021(2)$ & $38(1)$ \\
\hline$C(33)$ & $3505(3)$ & $2869(3)$ & $7756(2)$ & $38(1)$ \\
\hline $\mathrm{Ru}(2)$ & $-65(1)$ & $6342(1)$ & $7230(1)$ & $19(1)$ \\
\hline$S(3)$ & $164(1)$ & $6403(1)$ & $6378(1)$ & $25(1)$ \\
\hline $\mathrm{S}(4)$ & $-1262(1)$ & $7149(1)$ & 6897(1) & $23(1)$ \\
\hline $\mathrm{Cl}(3)$ & $135(1)$ & 6891(1) & $5150(1)$ & $44(1)$ \\
\hline $\mathrm{Cl}(4)$ & $-2652(1)$ & 8379(1) & 6163(1) & $46(1)$ \\
\hline $\mathrm{F}(3)$ & $604(2)$ & $5044(2)$ & $8848(1)$ & $46(1)$ \\
\hline $\mathrm{F}(4)$ & $-2020(2)$ & $5013(2)$ & 6736(1) & $38(1)$ \\
\hline $\mathrm{N}(3)$ & $807(2)$ & $4829(2)$ & 7797(2) & $28(1)$ \\
\hline $\mathrm{N}(4)$ & $-195(2)$ & $4636(2)$ & $7000(2)$ & $27(1)$ \\
\hline $\mathrm{O}(2)$ & $-163(2)$ & 6461(2) & 8131(1) & $22(1)$ \\
\hline C(34) & $259(3)$ & $5206(2)$ & 7343(2) & $24(1)$ \\
\hline$C(35)$ & $682(4)$ & $4050(3)$ & $7739(2)$ & $41(1)$ \\
\hline$C(36)$ & 49(4) & 3927(3) & $7242(2)$ & $40(1)$ \\
\hline C(37) & 1523(3) & $5165(2)$ & $8246(2)$ & $28(1)$ \\
\hline C(38) & 1413(3) & $5259(3)$ & 8768(2) & $34(1)$ \\
\hline C(39) & 2077(4) & $5567(3)$ & $9215(2)$ & $45(1)$ \\
\hline $\mathrm{C}(40)$ & 2882(4) & $5778(3)$ & $9127(2)$ & $49(1)$ \\
\hline$C(41)$ & $3026(3)$ & $5669(3)$ & $8612(2)$ & $44(1)$ \\
\hline $\mathrm{C}(42)$ & 2349(3) & $5351(3)$ & $8161(2)$ & $36(1)$ \\
\hline C(43) & $2500(3)$ & 5199(3) & $7605(2)$ & $44(1)$ \\
\hline C(44) & $-763(3)$ & $4714(2)$ & $6424(2)$ & $29(1)$ \\
\hline$C(45)$ & $-384(3)$ & $4595(3)$ & 5991(2) & $34(1)$ \\
\hline C(46) & $-960(3)$ & $4677(3)$ & $5439(2)$ & $37(1)$ \\
\hline C(47) & $-1866(3)$ & 4877(3) & $5328(2)$ & $35(1)$ \\
\hline $\mathrm{C}(48)$ & $-2234(3)$ & 4992(3) & $5764(2)$ & $33(1)$ \\
\hline C(49) & $-1672(3)$ & 4903(2) & $6307(2)$ & $29(1)$ \\
\hline $\mathrm{C}(50)$ & $613(3)$ & $4411(3)$ & $6110(2)$ & $46(1)$ \\
\hline C(51) & $-607(3)$ & 7086(2) & 5997(2) & $26(1)$ \\
\hline $\mathrm{C}(52)$ & $-621(3)$ & $7318(3)$ & $5457(2)$ & $33(1)$ \\
\hline $\mathrm{C}(53)$ & $-1208(4)$ & $7865(3)$ & $5156(2)$ & $44(1)$ \\
\hline C(54) & $-1826(4)$ & $8198(3)$ & $5383(2)$ & $46(1)$ \\
\hline C(55) & $-1854(3)$ & 7970(3) & $5906(2)$ & $36(1)$ \\
\hline C(56) & $-1243(3)$ & $7423(2)$ & $6222(2)$ & $26(1)$ \\
\hline C(57) & 1015(3) & $6820(2)$ & $7597(2)$ & $23(1)$ \\
\hline C(58) & 1181(3) & 7093(2) & $8169(2)$ & $22(1)$ \\
\hline C(59) & 1939(3) & $7537(2)$ & $8449(2)$ & $28(1)$ \\
\hline
\end{tabular}




\begin{tabular}{lrrrr}
$\mathrm{C}(60)$ & $2051(3)$ & $7802(3)$ & $8987(2)$ & $32(1)$ \\
$\mathrm{C}(61)$ & $1416(3)$ & $7622(3)$ & $9251(2)$ & $34(1)$ \\
$\mathrm{C}(62)$ & $654(3)$ & $7177(3)$ & $8988(2)$ & $30(1)$ \\
$\mathrm{C}(63)$ & $547(3)$ & $6917(2)$ & $8449(2)$ & $22(1)$ \\
$\mathrm{C}(64)$ & $-911(3)$ & $6288(3)$ & $8364(2)$ & $30(1)$ \\
$\mathrm{C}(65)$ & $-1512(3)$ & $6975(3)$ & $8321(2)$ & $40(1)$ \\
$\mathrm{C}(66)$ & $-1411(3)$ & $5612(3)$ & $8042(2)$ & $37(1)$ \\
$\mathrm{O}(1 \mathrm{~T})$ & $8944(4)$ & $5116(4)$ & $9609(2)$ & $16(2)$ \\
$\mathrm{C}(1 \mathrm{~T})$ & $9407(6)$ & $4447(6)$ & $9705(4)$ & $28(2)$ \\
$\mathrm{O}(1 \mathrm{~S})$ & $9182(7)$ & $4850(7)$ & $9633(3)$ & $32(2)$ \\
$\mathrm{C}(1 \mathrm{~S})$ & $9659(7)$ & $4227(6)$ & $9902(5)$ & $18(3)$ \\
\hline
\end{tabular}

Table 9. Bond lengths $(\AA)$ and angles $\left(^{\circ}\right)$ for $\mathrm{C}_{33} \mathrm{H}_{28} \mathrm{Cl}_{2} \mathrm{~F}_{2} \mathrm{~N}_{2} \mathrm{ORuS} \mathrm{S}_{2}$ (Ru-1d).

\begin{tabular}{ll}
\hline $\mathrm{Ru}(1)-\mathrm{C}(24)$ & $1.846(4)$ \\
$\mathrm{Ru}(1)-\mathrm{C}(1)$ & $2.055(4)$ \\
$\mathrm{Ru}(1)-\mathrm{S}(1)$ & $2.2543(10)$ \\
$\mathrm{Ru}(1)-\mathrm{S}(2)$ & $2.2656(11)$ \\
$\mathrm{Ru}(1)-\mathrm{O}(1)$ & $2.288(3)$ \\
$\mathrm{S}(1)-\mathrm{C}(18)$ & $1.745(5)$ \\
$\mathrm{S}(2)-\mathrm{C}(23)$ & $1.763(6)$ \\
$\mathrm{Cl}(1)-\mathrm{C}(19)$ & $1.750(7)$ \\
$\mathrm{Cl}(2)-\mathrm{C}(22)$ & $1.709(7)$ \\
$\mathrm{F}(1)-\mathrm{C}(9)$ & $1.372(7)$ \\
$\mathrm{F}(2)-\mathrm{C}(12)$ & $1.344(6)$ \\
$\mathrm{O}(1)-\mathrm{C}(30)$ & $1.385(5)$ \\
$\mathrm{O}(1)-\mathrm{C}(31)$ & $1.478(5)$ \\
$\mathrm{N}(1)-\mathrm{C}(1)$ & $1.356(6)$ \\
$\mathrm{N}(1)-\mathrm{C}(2)$ & $1.390(6)$ \\
$\mathrm{N}(1)-\mathrm{C}(4)$ & $1.438(6)$ \\
$\mathrm{N}(2)-\mathrm{C}(1)$ & $1.367(6)$ \\
$\mathrm{N}(2)-\mathrm{C}(3)$ & $1.384(6)$ \\
$\mathrm{N}(2)-\mathrm{C}(11)$ & $1.438(6)$ \\
$\mathrm{C}(2)-\mathrm{C}(3)$ & $1.343(8)$ \\
$\mathrm{C}(2)-\mathrm{H}(2 \mathrm{~A})$ & 0.9500 \\
$\mathrm{C}(3)-\mathrm{H}(3 \mathrm{~A})$ & 0.9500 \\
$\mathrm{C}(4)-\mathrm{C}(9)$ & $1.382(7)$ \\
$\mathrm{C}(4)-\mathrm{C}(5)$ & $1.391(8)$ \\
$\mathrm{C}(5)-\mathrm{C}(6)$ & $1.403(8)$ \\
$\mathrm{C}(5)-\mathrm{C}(10)$ & $1.498(7)$ \\
$\mathrm{C}(6)-\mathrm{C}(7)$ & $1.386(9)$ \\
$\mathrm{C}(6)-\mathrm{H}(6 \mathrm{~A})$ & 0.9500 \\
$\mathrm{C}(7)-\mathrm{C}(8)$ & $1.360(10)$ \\
$\mathrm{C}(7)-\mathrm{H}(7 \mathrm{~A})$ & 0.9500 \\
$\mathrm{C}(8)-\mathrm{C}(9)$ & $1.373(8)$ \\
$\mathrm{C}(8)-\mathrm{H}(8 \mathrm{~A})$ & 0.9500 \\
$\mathrm{C}(10)-\mathrm{H}(10 \mathrm{~A})$ & 0.9800 \\
$\mathrm{C}(10)-\mathrm{H}(10 \mathrm{~B})$ & 0.9800 \\
$\mathrm{C}(10)-\mathrm{H}(10 \mathrm{C})$ & \\
&
\end{tabular}




\begin{tabular}{|c|c|}
\hline$C(11)-C(16)$ & $1.394(7)$ \\
\hline$C(11)-C(12)$ & $1.403(7)$ \\
\hline$C(12)-C(13)$ & $1.366(8)$ \\
\hline$C(13)-C(14)$ & $1.383(8)$ \\
\hline $\mathrm{C}(13)-\mathrm{H}(13 \mathrm{~A})$ & 0.9500 \\
\hline$C(14)-C(15)$ & $1.376(8)$ \\
\hline $\mathrm{C}(14)-\mathrm{H}(14 \mathrm{~A})$ & 0.9500 \\
\hline$C(15)-C(16)$ & $1.394(7)$ \\
\hline $\mathrm{C}(15)-\mathrm{H}(15 \mathrm{~A})$ & 0.9500 \\
\hline$C(16)-C(17)$ & $1.500(7)$ \\
\hline $\mathrm{C}(17)-\mathrm{H}(17 \mathrm{~A})$ & 0.9800 \\
\hline $\mathrm{C}(17)-\mathrm{H}(17 \mathrm{~B})$ & 0.9800 \\
\hline $\mathrm{C}(17)-\mathrm{H}(17 \mathrm{C})$ & 0.9800 \\
\hline C(18)-C(19) & $1.400(7)$ \\
\hline$C(18)-C(23)$ & $1.399(8)$ \\
\hline C(19)-C(20) & $1.373(9)$ \\
\hline$C(20)-C(21)$ & $1.343(11)$ \\
\hline $\mathrm{C}(20)-\mathrm{H}(20)$ & 0.9500 \\
\hline $\mathrm{C}(21)-\mathrm{C}(22)$ & $1.415(10)$ \\
\hline $\mathrm{C}(21)-\mathrm{H}(21)$ & 0.9500 \\
\hline$C(22)-C(23)$ & $1.393(7)$ \\
\hline$C(24)-C(25)$ & $1.447(6)$ \\
\hline $\mathrm{C}(24)-\mathrm{H}(24 \mathrm{~A})$ & 0.9500 \\
\hline$C(25)-C(26)$ & $1.400(6)$ \\
\hline$C(25)-C(30)$ & $1.407(6)$ \\
\hline$C(26)-C(27)$ & $1.392(6)$ \\
\hline $\mathrm{C}(26)-\mathrm{H}(26 \mathrm{~A})$ & 0.9500 \\
\hline $\mathrm{C}(27)-\mathrm{C}(28)$ & $1.382(7)$ \\
\hline $\mathrm{C}(27)-\mathrm{H}(27 \mathrm{~A})$ & 0.9500 \\
\hline $\mathrm{C}(28)-\mathrm{C}(29)$ & $1.386(7)$ \\
\hline $\mathrm{C}(28)-\mathrm{H}(28 \mathrm{~A})$ & 0.9500 \\
\hline$C(29)-C(30)$ & $1.380(6)$ \\
\hline $\mathrm{C}(29)-\mathrm{H}(29 \mathrm{~A})$ & 0.9500 \\
\hline $\mathrm{C}(31)-\mathrm{C}(33)$ & $1.502(6)$ \\
\hline $\mathrm{C}(31)-\mathrm{C}(32)$ & $1.514(7)$ \\
\hline $\mathrm{C}(31)-\mathrm{H}(31 \mathrm{~A})$ & 1.0000 \\
\hline $\mathrm{C}(32)-\mathrm{H}(32 \mathrm{~A})$ & 0.9800 \\
\hline $\mathrm{C}(32)-\mathrm{H}(32 \mathrm{~B})$ & 0.9800 \\
\hline $\mathrm{C}(32)-\mathrm{H}(32 \mathrm{C})$ & 0.9800 \\
\hline $\mathrm{C}(33)-\mathrm{H}(33 \mathrm{~A})$ & 0.9800 \\
\hline $\mathrm{C}(33)-\mathrm{H}(33 \mathrm{~B})$ & 0.9800 \\
\hline $\mathrm{C}(33)-\mathrm{H}(33 \mathrm{C})$ & 0.9800 \\
\hline $\mathrm{Ru}(2)-\mathrm{C}(57)$ & $1.838(4)$ \\
\hline $\mathrm{Ru}(2)-\mathrm{C}(34)$ & $2.063(4)$ \\
\hline $\mathrm{Ru}(2)-\mathrm{S}(3)$ & $2.2610(10)$ \\
\hline $\mathrm{Ru}(2)-\mathrm{S}(4)$ & $2.2738(10)$ \\
\hline $\mathrm{Ru}(2)-\mathrm{O}(2)$ & $2.305(3)$ \\
\hline$S(3)-C(51)$ & $1.753(4)$ \\
\hline$S(4)-C(56)$ & $1.759(4)$ \\
\hline
\end{tabular}




\begin{tabular}{|c|c|}
\hline $\mathrm{Cl}(3)-\mathrm{C}(52)$ & $1.747(5)$ \\
\hline $\mathrm{Cl}(4)-\mathrm{C}(55)$ & $1.710(5)$ \\
\hline $\mathrm{F}(3)-\mathrm{C}(38)$ & $1.373(6)$ \\
\hline $\mathrm{F}(4)-\mathrm{C}(49)$ & $1.348(5)$ \\
\hline $\mathrm{N}(3)-\mathrm{C}(34)$ & $1.360(5)$ \\
\hline $\mathrm{N}(3)-\mathrm{C}(35)$ & $1.389(6)$ \\
\hline $\mathrm{N}(3)-\mathrm{C}(37)$ & $1.439(5)$ \\
\hline $\mathrm{N}(4)-\mathrm{C}(34)$ & $1.366(5)$ \\
\hline $\mathrm{N}(4)-\mathrm{C}(36)$ & $1.389(6)$ \\
\hline $\mathrm{N}(4)-\mathrm{C}(44)$ & $1.441(5)$ \\
\hline $\mathrm{O}(2)-\mathrm{C}(63)$ & $1.393(5)$ \\
\hline $\mathrm{O}(2)-\mathrm{C}(64)$ & $1.470(5)$ \\
\hline$C(35)-C(36)$ & $1.340(7)$ \\
\hline $\mathrm{C}(35)-\mathrm{H}(35 \mathrm{~A})$ & 0.9500 \\
\hline $\mathrm{C}(36)-\mathrm{H}(36 \mathrm{~A})$ & 0.9500 \\
\hline$C(37)-C(38)$ & $1.373(7)$ \\
\hline$C(37)-C(42)$ & $1.389(7)$ \\
\hline C(38)-C(39) & $1.373(7)$ \\
\hline$C(39)-C(40)$ & $1.372(9)$ \\
\hline C(39)-H(39A) & 0.9500 \\
\hline$C(40)-C(41)$ & $1.381(9)$ \\
\hline $\mathrm{C}(40)-\mathrm{H}(40 \mathrm{~A})$ & 0.9500 \\
\hline$C(41)-C(42)$ & $1.396(7)$ \\
\hline $\mathrm{C}(41)-\mathrm{H}(41 \mathrm{~A})$ & 0.9500 \\
\hline$C(42)-C(43)$ & $1.499(7)$ \\
\hline $\mathrm{C}(43)-\mathrm{H}(43 \mathrm{~A})$ & 0.9800 \\
\hline $\mathrm{C}(43)-\mathrm{H}(43 \mathrm{~B})$ & 0.9800 \\
\hline $\mathrm{C}(43)-\mathrm{H}(43 \mathrm{C})$ & 0.9800 \\
\hline C(44)-C(49) & $1.379(6)$ \\
\hline $\mathrm{C}(44)-\mathrm{C}(45)$ & $1.392(7)$ \\
\hline$C(45)-C(46)$ & $1.398(7)$ \\
\hline$C(45)-C(50)$ & $1.506(7)$ \\
\hline$C(46)-C(47)$ & $1.381(7)$ \\
\hline $\mathrm{C}(46)-\mathrm{H}(46 \mathrm{~A})$ & 0.9500 \\
\hline $\mathrm{C}(47)-\mathrm{C}(48)$ & $1.385(7)$ \\
\hline $\mathrm{C}(47)-\mathrm{H}(47 \mathrm{~A})$ & 0.9500 \\
\hline $\mathrm{C}(48)-\mathrm{C}(49)$ & $1.376(6)$ \\
\hline $\mathrm{C}(48)-\mathrm{H}(48 \mathrm{~A})$ & 0.9500 \\
\hline $\mathrm{C}(50)-\mathrm{H}(50 \mathrm{~A})$ & 0.9800 \\
\hline $\mathrm{C}(50)-\mathrm{H}(50 \mathrm{~B})$ & 0.9800 \\
\hline $\mathrm{C}(50)-\mathrm{H}(50 \mathrm{C})$ & 0.9800 \\
\hline$C(51)-C(52)$ & $1.400(6)$ \\
\hline$C(51)-C(56)$ & $1.401(6)$ \\
\hline$C(52)-C(53)$ & $1.376(7)$ \\
\hline$C(53)-C(54)$ & $1.378(8)$ \\
\hline $\mathrm{C}(53)-\mathrm{H}(53 \mathrm{~A})$ & 0.9500 \\
\hline$C(54)-C(55)$ & $1.377(7)$ \\
\hline $\mathrm{C}(54)-\mathrm{H}(54 \mathrm{~A})$ & 0.9500 \\
\hline$C(55)-C(56)$ & $1.408(6)$ \\
\hline
\end{tabular}




\begin{tabular}{|c|c|}
\hline$C(57)-C(58)$ & $1.452(5)$ \\
\hline $\mathrm{C}(57)-\mathrm{H}(57 \mathrm{~A})$ & 0.9500 \\
\hline$C(58)-C(63)$ & $1.398(6)$ \\
\hline $\mathrm{C}(58)-\mathrm{C}(59)$ & $1.400(6)$ \\
\hline $\mathrm{C}(59)-\mathrm{C}(60)$ & $1.382(6)$ \\
\hline $\mathrm{C}(59)-\mathrm{H}(59 \mathrm{~A})$ & 0.9500 \\
\hline$C(60)-C(61)$ & $1.371(7)$ \\
\hline $\mathrm{C}(60)-\mathrm{H}(60 \mathrm{~A})$ & 0.9500 \\
\hline$C(61)-C(62)$ & $1.397(7)$ \\
\hline $\mathrm{C}(61)-\mathrm{H}(61 \mathrm{~A})$ & 0.9500 \\
\hline$C(62)-C(63)$ & $1.381(6)$ \\
\hline $\mathrm{C}(62)-\mathrm{H}(62 \mathrm{~A})$ & 0.9500 \\
\hline$C(64)-C(65)$ & $1.507(7)$ \\
\hline$C(64)-C(66)$ & $1.510(6)$ \\
\hline $\mathrm{C}(64)-\mathrm{H}(64 \mathrm{~A})$ & 1.0000 \\
\hline $\mathrm{C}(65)-\mathrm{H}(65 \mathrm{~A})$ & 0.9800 \\
\hline $\mathrm{C}(65)-\mathrm{H}(65 \mathrm{~B})$ & 0.9800 \\
\hline $\mathrm{C}(65)-\mathrm{H}(65 \mathrm{C})$ & 0.9800 \\
\hline $\mathrm{C}(66)-\mathrm{H}(66 \mathrm{~A})$ & 0.9800 \\
\hline $\mathrm{C}(66)-\mathrm{H}(66 \mathrm{~B})$ & 0.9800 \\
\hline $\mathrm{C}(66)-\mathrm{H}(66 \mathrm{C})$ & 0.9800 \\
\hline $\mathrm{O}(1 \mathrm{~T})-\mathrm{C}(1 \mathrm{~T})$ & $1.361(10)$ \\
\hline $\mathrm{O}(1 \mathrm{~T})-\mathrm{H}(1 \mathrm{~T})$ & 0.8481 \\
\hline $\mathrm{C}(1 \mathrm{~T})-\mathrm{H}(1 \mathrm{~T} 1)$ & 0.9800 \\
\hline $\mathrm{C}(1 \mathrm{~T})-\mathrm{H}(1 \mathrm{~T} 2)$ & 0.9800 \\
\hline $\mathrm{C}(1 \mathrm{~T})-\mathrm{H}(1 \mathrm{~T} 3)$ & 0.9800 \\
\hline $\mathrm{O}(1 \mathrm{~S})-\mathrm{C}(1 \mathrm{~S})$ & $1.376(12)$ \\
\hline $\mathrm{O}(1 \mathrm{~S})-\mathrm{H}(1 \mathrm{~S})$ & 0.9138 \\
\hline C(1S)-H(1S1) & 0.9800 \\
\hline $\mathrm{C}(1 \mathrm{~S})-\mathrm{H}(1 \mathrm{~S} 2)$ & 0.9800 \\
\hline C(1S)-H(1S3) & 0.9800 \\
\hline $\mathrm{C}(24)-\mathrm{Ru}(1)-\mathrm{C}(1)$ & $104.58(18)$ \\
\hline $\mathrm{C}(24)-\mathrm{Ru}(1)-\mathrm{S}(1)$ & $94.74(13)$ \\
\hline $\mathrm{C}(1)-\mathrm{Ru}(1)-\mathrm{S}(1)$ & $91.79(12)$ \\
\hline $\mathrm{C}(24)-\mathrm{Ru}(1)-\mathrm{S}(2)$ & $115.69(13)$ \\
\hline $\mathrm{C}(1)-\mathrm{Ru}(1)-\mathrm{S}(2)$ & $139.58(12)$ \\
\hline $\mathrm{S}(1)-\mathrm{Ru}(1)-\mathrm{S}(2)$ & $88.43(4)$ \\
\hline $\mathrm{C}(24)-\mathrm{Ru}(1)-\mathrm{O}(1)$ & $77.68(15)$ \\
\hline $\mathrm{C}(1)-\mathrm{Ru}(1)-\mathrm{O}(1)$ & $92.55(14)$ \\
\hline $\mathrm{S}(1)-\mathrm{Ru}(1)-\mathrm{O}(1)$ & $172.02(8)$ \\
\hline $\mathrm{S}(2)-\mathrm{Ru}(1)-\mathrm{O}(1)$ & $92.59(8)$ \\
\hline $\mathrm{C}(18)-\mathrm{S}(1)-\mathrm{Ru}(1)$ & $105.94(18)$ \\
\hline $\mathrm{C}(23)-\mathrm{S}(2)-\mathrm{Ru}(1)$ & 105.41(19) \\
\hline $\mathrm{C}(30)-\mathrm{O}(1)-\mathrm{C}(31)$ & $118.5(3)$ \\
\hline $\mathrm{C}(30)-\mathrm{O}(1)-\mathrm{Ru}(1)$ & $110.7(2)$ \\
\hline $\mathrm{C}(31)-\mathrm{O}(1)-\mathrm{Ru}(1)$ & $129.9(2)$ \\
\hline $\mathrm{C}(1)-\mathrm{N}(1)-\mathrm{C}(2)$ & $111.6(4)$ \\
\hline $\mathrm{C}(1)-\mathrm{N}(1)-\mathrm{C}(4)$ & $125.4(4)$ \\
\hline
\end{tabular}




\begin{tabular}{|c|c|}
\hline $\mathrm{C}(2)-\mathrm{N}(1)-\mathrm{C}(4)$ & $122.6(4)$ \\
\hline $\mathrm{C}(1)-\mathrm{N}(2)-\mathrm{C}(3)$ & $111.0(4)$ \\
\hline $\mathrm{C}(1)-\mathrm{N}(2)-\mathrm{C}(11)$ & $125.0(4)$ \\
\hline $\mathrm{C}(3)-\mathrm{N}(2)-\mathrm{C}(11)$ & $123.7(4)$ \\
\hline $\mathrm{N}(1)-\mathrm{C}(1)-\mathrm{N}(2)$ & $103.9(4)$ \\
\hline $\mathrm{N}(1)-\mathrm{C}(1)-\mathrm{Ru}(1)$ & $129.7(3)$ \\
\hline $\mathrm{N}(2)-\mathrm{C}(1)-\mathrm{Ru}(1)$ & $125.8(3)$ \\
\hline $\mathrm{C}(3)-\mathrm{C}(2)-\mathrm{N}(1)$ & $106.4(4)$ \\
\hline $\mathrm{C}(3)-\mathrm{C}(2)-\mathrm{H}(2 \mathrm{~A})$ & 126.8 \\
\hline $\mathrm{N}(1)-\mathrm{C}(2)-\mathrm{H}(2 \mathrm{~A})$ & 126.8 \\
\hline $\mathrm{C}(2)-\mathrm{C}(3)-\mathrm{N}(2)$ & $107.2(4)$ \\
\hline $\mathrm{C}(2)-\mathrm{C}(3)-\mathrm{H}(3 \mathrm{~A})$ & 126.4 \\
\hline $\mathrm{N}(2)-\mathrm{C}(3)-\mathrm{H}(3 \mathrm{~A})$ & 126.4 \\
\hline$C(9)-C(4)-C(5)$ & $119.7(5)$ \\
\hline $\mathrm{C}(9)-\mathrm{C}(4)-\mathrm{N}(1)$ & $120.7(5)$ \\
\hline $\mathrm{C}(5)-\mathrm{C}(4)-\mathrm{N}(1)$ & $119.6(4)$ \\
\hline$C(4)-C(5)-C(6)$ & $117.5(5)$ \\
\hline$C(4)-C(5)-C(10)$ & $120.1(5)$ \\
\hline$C(6)-C(5)-C(10)$ & $122.4(5)$ \\
\hline$C(7)-C(6)-C(5)$ & $120.8(6)$ \\
\hline $\mathrm{C}(7)-\mathrm{C}(6)-\mathrm{H}(6 \mathrm{~A})$ & 119.6 \\
\hline $\mathrm{C}(5)-\mathrm{C}(6)-\mathrm{H}(6 \mathrm{~A})$ & 119.6 \\
\hline$C(8)-C(7)-C(6)$ & $121.5(6)$ \\
\hline $\mathrm{C}(8)-\mathrm{C}(7)-\mathrm{H}(7 \mathrm{~A})$ & 119.2 \\
\hline $\mathrm{C}(6)-\mathrm{C}(7)-\mathrm{H}(7 \mathrm{~A})$ & 119.2 \\
\hline $\mathrm{C}(7)-\mathrm{C}(8)-\mathrm{C}(9)$ & $117.7(6)$ \\
\hline $\mathrm{C}(7)-\mathrm{C}(8)-\mathrm{H}(8 \mathrm{~A})$ & 121.1 \\
\hline $\mathrm{C}(9)-\mathrm{C}(8)-\mathrm{H}(8 \mathrm{~A})$ & 121.1 \\
\hline $\mathrm{C}(8)-\mathrm{C}(9)-\mathrm{F}(1)$ & $119.0(5)$ \\
\hline $\mathrm{C}(8)-\mathrm{C}(9)-\mathrm{C}(4)$ & $122.8(6)$ \\
\hline $\mathrm{F}(1)-\mathrm{C}(9)-\mathrm{C}(4)$ & $118.2(5)$ \\
\hline $\mathrm{C}(5)-\mathrm{C}(10)-\mathrm{H}(10 \mathrm{~A})$ & 109.5 \\
\hline $\mathrm{C}(5)-\mathrm{C}(10)-\mathrm{H}(10 \mathrm{~B})$ & 109.5 \\
\hline $\mathrm{H}(10 \mathrm{~A})-\mathrm{C}(10)-\mathrm{H}(10 \mathrm{~B})$ & 109.5 \\
\hline $\mathrm{C}(5)-\mathrm{C}(10)-\mathrm{H}(10 \mathrm{C})$ & 109.5 \\
\hline $\mathrm{H}(10 \mathrm{~A})-\mathrm{C}(10)-\mathrm{H}(10 \mathrm{C})$ & 109.5 \\
\hline $\mathrm{H}(10 \mathrm{~B})-\mathrm{C}(10)-\mathrm{H}(10 \mathrm{C})$ & 109.5 \\
\hline$C(16)-C(11)-C(12)$ & $119.7(4)$ \\
\hline $\mathrm{C}(16)-\mathrm{C}(11)-\mathrm{N}(2)$ & $120.6(4)$ \\
\hline $\mathrm{C}(12)-\mathrm{C}(11)-\mathrm{N}(2)$ & $119.8(4)$ \\
\hline $\mathrm{F}(2)-\mathrm{C}(12)-\mathrm{C}(13)$ & $119.5(5)$ \\
\hline $\mathrm{F}(2)-\mathrm{C}(12)-\mathrm{C}(11)$ & $118.5(4)$ \\
\hline$C(13)-C(12)-C(11)$ & $122.0(5)$ \\
\hline $\mathrm{C}(12)-\mathrm{C}(13)-\mathrm{C}(14)$ & $117.9(5)$ \\
\hline $\mathrm{C}(12)-\mathrm{C}(13)-\mathrm{H}(13 \mathrm{~A})$ & 121.0 \\
\hline $\mathrm{C}(14)-\mathrm{C}(13)-\mathrm{H}(13 \mathrm{~A})$ & 121.0 \\
\hline$C(15)-C(14)-C(13)$ & $121.1(5)$ \\
\hline $\mathrm{C}(15)-\mathrm{C}(14)-\mathrm{H}(14 \mathrm{~A})$ & 119.4 \\
\hline $\mathrm{C}(13)-\mathrm{C}(14)-\mathrm{H}(14 \mathrm{~A})$ & 119.4 \\
\hline
\end{tabular}




\begin{tabular}{|c|c|}
\hline$C(14)-C(15)-C(16)$ & $121.5(5)$ \\
\hline $\mathrm{C}(14)-\mathrm{C}(15)-\mathrm{H}(15 \mathrm{~A})$ & 119.3 \\
\hline $\mathrm{C}(16)-\mathrm{C}(15)-\mathrm{H}(15 \mathrm{~A})$ & 119.3 \\
\hline$C(15)-C(16)-C(11)$ & $117.5(5)$ \\
\hline$C(15)-C(16)-C(17)$ & $121.2(5)$ \\
\hline$C(11)-C(16)-C(17)$ & $121.2(4)$ \\
\hline $\mathrm{C}(16)-\mathrm{C}(17)-\mathrm{H}(17 \mathrm{~A})$ & 109.5 \\
\hline $\mathrm{C}(16)-\mathrm{C}(17)-\mathrm{H}(17 \mathrm{~B})$ & 109.5 \\
\hline $\mathrm{H}(17 \mathrm{~A})-\mathrm{C}(17)-\mathrm{H}(17 \mathrm{~B})$ & 109.5 \\
\hline $\mathrm{C}(16)-\mathrm{C}(17)-\mathrm{H}(17 \mathrm{C})$ & 109.5 \\
\hline $\mathrm{H}(17 \mathrm{~A})-\mathrm{C}(17)-\mathrm{H}(17 \mathrm{C})$ & 109.5 \\
\hline $\mathrm{H}(17 \mathrm{~B})-\mathrm{C}(17)-\mathrm{H}(17 \mathrm{C})$ & 109.5 \\
\hline$C(19)-C(18)-C(23)$ & $118.3(5)$ \\
\hline C(19)-C(18)-S(1) & $121.3(5)$ \\
\hline$C(23)-C(18)-S(1)$ & $120.4(4)$ \\
\hline$C(20)-C(19)-C(18)$ & $123.3(6)$ \\
\hline $\mathrm{C}(20)-\mathrm{C}(19)-\mathrm{Cl}(1)$ & $117.2(5)$ \\
\hline $\mathrm{C}(18)-\mathrm{C}(19)-\mathrm{Cl}(1)$ & $119.5(4)$ \\
\hline$C(21)-C(20)-C(19)$ & $118.2(6)$ \\
\hline $\mathrm{C}(21)-\mathrm{C}(20)-\mathrm{H}(20)$ & 120.9 \\
\hline $\mathrm{C}(19)-\mathrm{C}(20)-\mathrm{H}(20)$ & 120.9 \\
\hline$C(20)-C(21)-C(22)$ & $121.1(6)$ \\
\hline $\mathrm{C}(20)-\mathrm{C}(21)-\mathrm{H}(21)$ & 119.4 \\
\hline $\mathrm{C}(22)-\mathrm{C}(21)-\mathrm{H}(21)$ & 119.4 \\
\hline$C(23)-C(22)-C(21)$ & $120.6(7)$ \\
\hline $\mathrm{C}(23)-\mathrm{C}(22)-\mathrm{Cl}(2)$ & $120.1(5)$ \\
\hline $\mathrm{C}(21)-\mathrm{C}(22)-\mathrm{Cl}(2)$ & $119.3(5)$ \\
\hline$C(22)-C(23)-C(18)$ & $118.3(5)$ \\
\hline $\mathrm{C}(22)-\mathrm{C}(23)-\mathrm{S}(2)$ & $122.3(5)$ \\
\hline $\mathrm{C}(18)-\mathrm{C}(23)-\mathrm{S}(2)$ & $119.5(4)$ \\
\hline $\mathrm{C}(25)-\mathrm{C}(24)-\mathrm{Ru}(1)$ & $120.5(3)$ \\
\hline $\mathrm{C}(25)-\mathrm{C}(24)-\mathrm{H}(24 \mathrm{~A})$ & 119.8 \\
\hline $\mathrm{Ru}(1)-\mathrm{C}(24)-\mathrm{H}(24 \mathrm{~A})$ & 119.8 \\
\hline$C(26)-C(25)-C(30)$ & $117.9(4)$ \\
\hline$C(26)-C(25)-C(24)$ & $124.1(4)$ \\
\hline$C(30)-C(25)-C(24)$ & $118.0(4)$ \\
\hline$C(27)-C(26)-C(25)$ & $120.5(4)$ \\
\hline $\mathrm{C}(27)-\mathrm{C}(26)-\mathrm{H}(26 \mathrm{~A})$ & 119.7 \\
\hline $\mathrm{C}(25)-\mathrm{C}(26)-\mathrm{H}(26 \mathrm{~A})$ & 119.7 \\
\hline$C(28)-C(27)-C(26)$ & $119.7(4)$ \\
\hline $\mathrm{C}(28)-\mathrm{C}(27)-\mathrm{H}(27 \mathrm{~A})$ & 120.1 \\
\hline $\mathrm{C}(26)-\mathrm{C}(27)-\mathrm{H}(27 \mathrm{~A})$ & 120.1 \\
\hline$C(27)-C(28)-C(29)$ & $121.2(4)$ \\
\hline $\mathrm{C}(27)-\mathrm{C}(28)-\mathrm{H}(28 \mathrm{~A})$ & 119.4 \\
\hline $\mathrm{C}(29)-\mathrm{C}(28)-\mathrm{H}(28 \mathrm{~A})$ & 119.4 \\
\hline$C(30)-C(29)-C(28)$ & $118.8(4)$ \\
\hline $\mathrm{C}(30)-\mathrm{C}(29)-\mathrm{H}(29 \mathrm{~A})$ & 120.6 \\
\hline $\mathrm{C}(28)-\mathrm{C}(29)-\mathrm{H}(29 \mathrm{~A})$ & 120.6 \\
\hline $\mathrm{C}(29)-\mathrm{C}(30)-\mathrm{O}(1)$ & $125.7(4)$ \\
\hline
\end{tabular}




\begin{tabular}{|c|c|}
\hline$C(29)-C(30)-C(25)$ & $121.8(4)$ \\
\hline $\mathrm{O}(1)-\mathrm{C}(30)-\mathrm{C}(25)$ & $112.5(3)$ \\
\hline $\mathrm{O}(1)-\mathrm{C}(31)-\mathrm{C}(33)$ & $106.1(3)$ \\
\hline $\mathrm{O}(1)-\mathrm{C}(31)-\mathrm{C}(32)$ & $109.1(4)$ \\
\hline $\mathrm{C}(33)-\mathrm{C}(31)-\mathrm{C}(32)$ & $113.3(4)$ \\
\hline $\mathrm{O}(1)-\mathrm{C}(31)-\mathrm{H}(31 \mathrm{~A})$ & 109.4 \\
\hline $\mathrm{C}(33)-\mathrm{C}(31)-\mathrm{H}(31 \mathrm{~A})$ & 109.4 \\
\hline $\mathrm{C}(32)-\mathrm{C}(31)-\mathrm{H}(31 \mathrm{~A})$ & 109.4 \\
\hline $\mathrm{C}(31)-\mathrm{C}(32)-\mathrm{H}(32 \mathrm{~A})$ & 109.5 \\
\hline $\mathrm{C}(31)-\mathrm{C}(32)-\mathrm{H}(32 \mathrm{~B})$ & 109.5 \\
\hline $\mathrm{H}(32 \mathrm{~A})-\mathrm{C}(32)-\mathrm{H}(32 \mathrm{~B})$ & 109.5 \\
\hline $\mathrm{C}(31)-\mathrm{C}(32)-\mathrm{H}(32 \mathrm{C})$ & 109.5 \\
\hline $\mathrm{H}(32 \mathrm{~A})-\mathrm{C}(32)-\mathrm{H}(32 \mathrm{C})$ & 109.5 \\
\hline $\mathrm{H}(32 \mathrm{~B})-\mathrm{C}(32)-\mathrm{H}(32 \mathrm{C})$ & 109.5 \\
\hline $\mathrm{C}(31)-\mathrm{C}(33)-\mathrm{H}(33 \mathrm{~A})$ & 109.5 \\
\hline $\mathrm{C}(31)-\mathrm{C}(33)-\mathrm{H}(33 \mathrm{~B})$ & 109.5 \\
\hline $\mathrm{H}(33 \mathrm{~A})-\mathrm{C}(33)-\mathrm{H}(33 \mathrm{~B})$ & 109.5 \\
\hline $\mathrm{C}(31)-\mathrm{C}(33)-\mathrm{H}(33 \mathrm{C})$ & 109.5 \\
\hline $\mathrm{H}(33 \mathrm{~A})-\mathrm{C}(33)-\mathrm{H}(33 \mathrm{C})$ & 109.5 \\
\hline $\mathrm{H}(33 \mathrm{~B})-\mathrm{C}(33)-\mathrm{H}(33 \mathrm{C})$ & 109.5 \\
\hline$C(57)-R u(2)-C(34)$ & $103.51(17)$ \\
\hline $\mathrm{C}(57)-\mathrm{Ru}(2)-\mathrm{S}(3)$ & $94.00(12)$ \\
\hline $\mathrm{C}(34)-\mathrm{Ru}(2)-\mathrm{S}(3)$ & $94.19(12)$ \\
\hline $\mathrm{C}(57)-\mathrm{Ru}(2)-\mathrm{S}(4)$ & $113.79(13)$ \\
\hline $\mathrm{C}(34)-\mathrm{Ru}(2)-\mathrm{S}(4)$ & $142.38(12)$ \\
\hline $\mathrm{S}(3)-\mathrm{Ru}(2)-\mathrm{S}(4)$ & $88.15(4)$ \\
\hline $\mathrm{C}(57)-\mathrm{Ru}(2)-\mathrm{O}(2)$ & $77.98(14)$ \\
\hline $\mathrm{C}(34)-\mathrm{Ru}(2)-\mathrm{O}(2)$ & $92.37(13)$ \\
\hline $\mathrm{S}(3)-\mathrm{Ru}(2)-\mathrm{O}(2)$ & $170.66(8)$ \\
\hline $\mathrm{S}(4)-\mathrm{Ru}(2)-\mathrm{O}(2)$ & $90.73(7)$ \\
\hline $\mathrm{C}(51)-\mathrm{S}(3)-\mathrm{Ru}(2)$ & $105.58(15)$ \\
\hline$C(56)-S(4)-R u(2)$ & $105.88(15)$ \\
\hline $\mathrm{C}(34)-\mathrm{N}(3)-\mathrm{C}(35)$ & $111.5(4)$ \\
\hline $\mathrm{C}(34)-\mathrm{N}(3)-\mathrm{C}(37)$ & $125.5(3)$ \\
\hline $\mathrm{C}(35)-\mathrm{N}(3)-\mathrm{C}(37)$ & $122.4(4)$ \\
\hline $\mathrm{C}(34)-\mathrm{N}(4)-\mathrm{C}(36)$ & $111.8(3)$ \\
\hline $\mathrm{C}(34)-\mathrm{N}(4)-\mathrm{C}(44)$ & $126.3(3)$ \\
\hline $\mathrm{C}(36)-\mathrm{N}(4)-\mathrm{C}(44)$ & $121.4(4)$ \\
\hline $\mathrm{C}(63)-\mathrm{O}(2)-\mathrm{C}(64)$ & $117.8(3)$ \\
\hline $\mathrm{C}(63)-\mathrm{O}(2)-\mathrm{Ru}(2)$ & $109.6(2)$ \\
\hline $\mathrm{C}(64)-\mathrm{O}(2)-\mathrm{Ru}(2)$ & $131.1(2)$ \\
\hline $\mathrm{N}(3)-\mathrm{C}(34)-\mathrm{N}(4)$ & $103.3(3)$ \\
\hline $\mathrm{N}(3)-\mathrm{C}(34)-\mathrm{Ru}(2)$ & $130.5(3)$ \\
\hline $\mathrm{N}(4)-\mathrm{C}(34)-\mathrm{Ru}(2)$ & $125.0(3)$ \\
\hline $\mathrm{C}(36)-\mathrm{C}(35)-\mathrm{N}(3)$ & 107.1(4) \\
\hline $\mathrm{C}(36)-\mathrm{C}(35)-\mathrm{H}(35 \mathrm{~A})$ & 126.4 \\
\hline $\mathrm{N}(3)-\mathrm{C}(35)-\mathrm{H}(35 \mathrm{~A})$ & 126.5 \\
\hline $\mathrm{C}(35)-\mathrm{C}(36)-\mathrm{N}(4)$ & $106.3(4)$ \\
\hline $\mathrm{C}(35)-\mathrm{C}(36)-\mathrm{H}(36 \mathrm{~A})$ & 126.9 \\
\hline
\end{tabular}




\begin{tabular}{|c|c|}
\hline $\mathrm{N}(4)-\mathrm{C}(36)-\mathrm{H}(36 \mathrm{~A})$ & 126.9 \\
\hline $\mathrm{C}(38)-\mathrm{C}(37)-\mathrm{C}(42)$ & $120.0(4)$ \\
\hline $\mathrm{C}(38)-\mathrm{C}(37)-\mathrm{N}(3)$ & $120.1(4)$ \\
\hline $\mathrm{C}(42)-\mathrm{C}(37)-\mathrm{N}(3)$ & $119.8(4)$ \\
\hline $\mathrm{C}(39)-\mathrm{C}(38)-\mathrm{F}(3)$ & $118.4(5)$ \\
\hline C(39)-C(38)-C(37) & $122.7(5)$ \\
\hline $\mathrm{F}(3)-\mathrm{C}(38)-\mathrm{C}(37)$ & $118.9(4)$ \\
\hline$C(38)-C(39)-C(40)$ & $117.5(5)$ \\
\hline C(38)-C(39)-H(39A) & 121.2 \\
\hline $\mathrm{C}(40)-\mathrm{C}(39)-\mathrm{H}(39 \mathrm{~A})$ & 121.2 \\
\hline$C(39)-C(40)-C(41)$ & $121.3(5)$ \\
\hline $\mathrm{C}(39)-\mathrm{C}(40)-\mathrm{H}(40 \mathrm{~A})$ & 119.4 \\
\hline $\mathrm{C}(41)-\mathrm{C}(40)-\mathrm{H}(40 \mathrm{~A})$ & 119.3 \\
\hline$C(40)-C(41)-C(42)$ & $120.8(5)$ \\
\hline $\mathrm{C}(40)-\mathrm{C}(41)-\mathrm{H}(41 \mathrm{~A})$ & 119.6 \\
\hline $\mathrm{C}(42)-\mathrm{C}(41)-\mathrm{H}(41 \mathrm{~A})$ & 119.6 \\
\hline$C(37)-C(42)-C(41)$ & $117.7(5)$ \\
\hline$C(37)-C(42)-C(43)$ & $120.4(4)$ \\
\hline$C(41)-C(42)-C(43)$ & $121.9(5)$ \\
\hline $\mathrm{C}(42)-\mathrm{C}(43)-\mathrm{H}(43 \mathrm{~A})$ & 109.5 \\
\hline $\mathrm{C}(42)-\mathrm{C}(43)-\mathrm{H}(43 \mathrm{~B})$ & 109.5 \\
\hline $\mathrm{H}(43 \mathrm{~A})-\mathrm{C}(43)-\mathrm{H}(43 \mathrm{~B})$ & 109.5 \\
\hline $\mathrm{C}(42)-\mathrm{C}(43)-\mathrm{H}(43 \mathrm{C})$ & 109.5 \\
\hline $\mathrm{H}(43 \mathrm{~A})-\mathrm{C}(43)-\mathrm{H}(43 \mathrm{C})$ & 109.5 \\
\hline $\mathrm{H}(43 \mathrm{~B})-\mathrm{C}(43)-\mathrm{H}(43 \mathrm{C})$ & 109.5 \\
\hline C(49)-C(44)-C(45) & $120.6(4)$ \\
\hline $\mathrm{C}(49)-\mathrm{C}(44)-\mathrm{N}(4)$ & $120.2(4)$ \\
\hline$C(45)-C(44)-N(4)$ & $119.2(4)$ \\
\hline$C(44)-C(45)-C(46)$ & $117.3(4)$ \\
\hline$C(44)-C(45)-C(50)$ & $121.5(4)$ \\
\hline$C(46)-C(45)-C(50)$ & $121.2(4)$ \\
\hline$C(47)-C(46)-C(45)$ & $121.5(5)$ \\
\hline $\mathrm{C}(47)-\mathrm{C}(46)-\mathrm{H}(46 \mathrm{~A})$ & 119.3 \\
\hline $\mathrm{C}(45)-\mathrm{C}(46)-\mathrm{H}(46 \mathrm{~A})$ & 119.3 \\
\hline$C(46)-C(47)-C(48)$ & $120.5(4)$ \\
\hline $\mathrm{C}(46)-\mathrm{C}(47)-\mathrm{H}(47 \mathrm{~A})$ & 119.7 \\
\hline $\mathrm{C}(48)-\mathrm{C}(47)-\mathrm{H}(47 \mathrm{~A})$ & 119.7 \\
\hline$C(49)-C(48)-C(47)$ & $118.1(4)$ \\
\hline $\mathrm{C}(49)-\mathrm{C}(48)-\mathrm{H}(48 \mathrm{~A})$ & 120.9 \\
\hline $\mathrm{C}(47)-\mathrm{C}(48)-\mathrm{H}(48 \mathrm{~A})$ & 120.9 \\
\hline $\mathrm{F}(4)-\mathrm{C}(49)-\mathrm{C}(48)$ & $118.8(4)$ \\
\hline $\mathrm{F}(4)-\mathrm{C}(49)-\mathrm{C}(44)$ & $119.2(4)$ \\
\hline$C(48)-C(49)-C(44)$ & $121.9(4)$ \\
\hline $\mathrm{C}(45)-\mathrm{C}(50)-\mathrm{H}(50 \mathrm{~A})$ & 109.5 \\
\hline $\mathrm{C}(45)-\mathrm{C}(50)-\mathrm{H}(50 \mathrm{~B})$ & 109.5 \\
\hline $\mathrm{H}(50 \mathrm{~A})-\mathrm{C}(50)-\mathrm{H}(50 \mathrm{~B})$ & 109.5 \\
\hline $\mathrm{C}(45)-\mathrm{C}(50)-\mathrm{H}(50 \mathrm{C})$ & 109.5 \\
\hline $\mathrm{H}(50 \mathrm{~A})-\mathrm{C}(50)-\mathrm{H}(50 \mathrm{C})$ & 109.5 \\
\hline $\mathrm{H}(50 \mathrm{~B})-\mathrm{C}(50)-\mathrm{H}(50 \mathrm{C})$ & 109.5 \\
\hline
\end{tabular}




\begin{tabular}{|c|c|}
\hline$C(52)-C(51)-C(56)$ & $117.0(4)$ \\
\hline $\mathrm{C}(52)-\mathrm{C}(51)-\mathrm{S}(3)$ & $122.1(4)$ \\
\hline$C(56)-C(51)-S(3)$ & $120.9(3)$ \\
\hline$C(53)-C(52)-C(51)$ & $123.0(5)$ \\
\hline $\mathrm{C}(53)-\mathrm{C}(52)-\mathrm{Cl}(3)$ & $118.4(4)$ \\
\hline $\mathrm{C}(51)-\mathrm{C}(52)-\mathrm{Cl}(3)$ & $118.5(4)$ \\
\hline$C(52)-C(53)-C(54)$ & $119.6(5)$ \\
\hline $\mathrm{C}(52)-\mathrm{C}(53)-\mathrm{H}(53 \mathrm{~A})$ & 120.2 \\
\hline $\mathrm{C}(54)-\mathrm{C}(53)-\mathrm{H}(53 \mathrm{~A})$ & 120.2 \\
\hline$C(55)-C(54)-C(53)$ & $119.2(5)$ \\
\hline $\mathrm{C}(55)-\mathrm{C}(54)-\mathrm{H}(54 \mathrm{~A})$ & 120.4 \\
\hline $\mathrm{C}(53)-\mathrm{C}(54)-\mathrm{H}(54 \mathrm{~A})$ & 120.4 \\
\hline$C(54)-C(55)-C(56)$ & $121.6(5)$ \\
\hline $\mathrm{C}(54)-\mathrm{C}(55)-\mathrm{Cl}(4)$ & $117.7(4)$ \\
\hline $\mathrm{C}(56)-\mathrm{C}(55)-\mathrm{Cl}(4)$ & $120.7(4)$ \\
\hline$C(51)-C(56)-C(55)$ & $119.6(4)$ \\
\hline $\mathrm{C}(51)-\mathrm{C}(56)-\mathrm{S}(4)$ & $119.0(3)$ \\
\hline$C(55)-C(56)-S(4)$ & $121.4(4)$ \\
\hline $\mathrm{C}(58)-\mathrm{C}(57)-\mathrm{Ru}(2)$ & $120.2(3)$ \\
\hline $\mathrm{C}(58)-\mathrm{C}(57)-\mathrm{H}(57 \mathrm{~A})$ & 119.9 \\
\hline $\mathrm{Ru}(2)-\mathrm{C}(57)-\mathrm{H}(57 \mathrm{~A})$ & 119.9 \\
\hline$C(63)-C(58)-C(59)$ & $118.5(4)$ \\
\hline$C(63)-C(58)-C(57)$ & $118.5(4)$ \\
\hline$C(59)-C(58)-C(57)$ & $123.0(4)$ \\
\hline$C(60)-C(59)-C(58)$ & $120.8(4)$ \\
\hline $\mathrm{C}(60)-\mathrm{C}(59)-\mathrm{H}(59 \mathrm{~A})$ & 119.6 \\
\hline $\mathrm{C}(58)-\mathrm{C}(59)-\mathrm{H}(59 \mathrm{~A})$ & 119.6 \\
\hline $\mathrm{C}(61)-\mathrm{C}(60)-\mathrm{C}(59)$ & $119.4(4)$ \\
\hline $\mathrm{C}(61)-\mathrm{C}(60)-\mathrm{H}(60 \mathrm{~A})$ & 120.3 \\
\hline $\mathrm{C}(59)-\mathrm{C}(60)-\mathrm{H}(60 \mathrm{~A})$ & 120.3 \\
\hline$C(60)-C(61)-C(62)$ & $121.6(4)$ \\
\hline $\mathrm{C}(60)-\mathrm{C}(61)-\mathrm{H}(61 \mathrm{~A})$ & 119.2 \\
\hline $\mathrm{C}(62)-\mathrm{C}(61)-\mathrm{H}(61 \mathrm{~A})$ & 119.2 \\
\hline$C(63)-C(62)-C(61)$ & $118.4(4)$ \\
\hline $\mathrm{C}(63)-\mathrm{C}(62)-\mathrm{H}(62 \mathrm{~A})$ & 120.8 \\
\hline $\mathrm{C}(61)-\mathrm{C}(62)-\mathrm{H}(62 \mathrm{~A})$ & 120.8 \\
\hline $\mathrm{C}(62)-\mathrm{C}(63)-\mathrm{O}(2)$ & $125.8(4)$ \\
\hline $\mathrm{C}(62)-\mathrm{C}(63)-\mathrm{C}(58)$ & $121.3(4)$ \\
\hline $\mathrm{O}(2)-\mathrm{C}(63)-\mathrm{C}(58)$ & $112.9(3)$ \\
\hline $\mathrm{O}(2)-\mathrm{C}(64)-\mathrm{C}(65)$ & $110.0(4)$ \\
\hline $\mathrm{O}(2)-\mathrm{C}(64)-\mathrm{C}(66)$ & $106.6(3)$ \\
\hline$C(65)-C(64)-C(66)$ & $113.2(4)$ \\
\hline $\mathrm{O}(2)-\mathrm{C}(64)-\mathrm{H}(64 \mathrm{~A})$ & 109.0 \\
\hline $\mathrm{C}(65)-\mathrm{C}(64)-\mathrm{H}(64 \mathrm{~A})$ & 109.0 \\
\hline $\mathrm{C}(66)-\mathrm{C}(64)-\mathrm{H}(64 \mathrm{~A})$ & 108.9 \\
\hline $\mathrm{C}(64)-\mathrm{C}(65)-\mathrm{H}(65 \mathrm{~A})$ & 109.5 \\
\hline $\mathrm{C}(64)-\mathrm{C}(65)-\mathrm{H}(65 \mathrm{~B})$ & 109.5 \\
\hline $\mathrm{H}(65 \mathrm{~A})-\mathrm{C}(65)-\mathrm{H}(65 \mathrm{~B})$ & 109.5 \\
\hline $\mathrm{C}(64)-\mathrm{C}(65)-\mathrm{H}(65 \mathrm{C})$ & 109.5 \\
\hline
\end{tabular}




$\begin{array}{ll}\text { H(65A)-C(65)-H(65C) } & 109.5 \\ \text { H(65B)-C(65)-H(65C) } & 109.5 \\ \text { C(64)-C(66)-H(66A) } & 109.5 \\ \text { C(64)-C(66)-H(66B) } & 109.5 \\ \text { H(66A)-C(66)-H(66B) } & 109.5 \\ \text { C(64)-C(66)-H(66C) } & 109.5 \\ \text { H(66A)-C(66)-H(66C) } & 109.5 \\ \text { H(66B)-C(66)-H(66C) } & 109.5 \\ \text { C(1T)-O(1T)-H(1T) } & 110.4 \\ \text { O(1T)-C(1T)-H(1T1) } & 109.4 \\ \text { O(1T)-C(1T)-H(1T2) } & 109.5 \\ \text { H(1T1)-C(1T)-H(1T2) } & 109.5 \\ \text { O(1T)-C(1T)-H(1T3) } & 109.5 \\ \text { H(1T1)-C(1T)-H(1T3) } & 109.5 \\ \text { H(1T2)-C(1T)-H(1T3) } & 109.5 \\ \text { C(1S)-O(1S)-H(1S) } & 117.5 \\ \text { O(1S)-C(1S)-H(1S1) } & 109.7 \\ \text { O(1S)-C(1S)-H(1S2) } & 109.2 \\ \text { H(1S1)-C(1S)-H(1S2) } & 109.5 \\ \text { O(1S)-C(1S)-H(1S3) } & 109.5 \\ \text { H(1S1)-C(1S)-H(1S3) } & 109.5 \\ \text { H(1S2)-C(1S)-H(1S3) } & 109.5 \\ & \end{array}$

Symmetry transformations used to generate equivalent atoms:

Table 10. Anisotropic displacement parameters $\left(\AA^{2} \times 10^{3}\right)$ for $\mathrm{C}_{33} \mathrm{H}_{28} \mathrm{Cl}_{2} \mathrm{~F}_{2} \mathrm{~N}_{2} \mathrm{ORuS} 2(\mathbf{R u}-\mathbf{1 d})$. The anisotropic displacement factor exponent takes the form: $-2 p^{2}\left[h^{2} a^{* 2} U^{11}+\ldots+2 h k a^{*} b^{*} U^{12}\right]$.

\begin{tabular}{lcccccc}
\hline & $\mathrm{U}^{11}$ & $\mathrm{U}^{22}$ & $\mathrm{U}^{33}$ & $\mathrm{U}^{23}$ & $\mathrm{U}^{13}$ & $\mathrm{U}^{12}$ \\
\hline $\mathrm{Ru}(1)$ & $21(1)$ & $19(1)$ & $17(1)$ & $0(1)$ & $3(1)$ & $0(1)$ \\
$\mathrm{S}(1)$ & $31(1)$ & $33(1)$ & $18(1)$ & $2(1)$ & $7(1)$ & $7(1)$ \\
$\mathrm{S}(2)$ & $38(1)$ & $24(1)$ & $26(1)$ & $4(1)$ & $-8(1)$ & $-7(1)$ \\
$\mathrm{Cl}(1)$ & $49(1)$ & $112(1)$ & $22(1)$ & $3(1)$ & $11(1)$ & $22(1)$ \\
$\mathrm{Cl}(2)$ & $135(2)$ & $54(1)$ & $41(1)$ & $10(1)$ & $-29(1)$ & $-48(1)$ \\
$\mathrm{F}(1)$ & $78(2)$ & $61(2)$ & $38(2)$ & $-4(1)$ & $32(2)$ & $-10(2)$ \\
$\mathrm{F}(2)$ & $36(2)$ & $59(2)$ & $44(2)$ & $14(1)$ & $15(1)$ & $4(1)$ \\
$\mathrm{O}(1)$ & $22(1)$ & $31(2)$ & $22(1)$ & $4(1)$ & $7(1)$ & $1(1)$ \\
$\mathrm{N}(1)$ & $41(2)$ & $26(2)$ & $26(2)$ & $-4(2)$ & $12(2)$ & $-7(2)$ \\
$\mathrm{N}(2)$ & $33(2)$ & $22(2)$ & $28(2)$ & $0(1)$ & $11(2)$ & $1(1)$ \\
$\mathrm{C}(1)$ & $28(2)$ & $24(2)$ & $24(2)$ & $0(2)$ & $12(2)$ & $-3(2)$ \\
$\mathrm{C}(2)$ & $64(3)$ & $26(2)$ & $38(3)$ & $-9(2)$ & $18(2)$ & $-3(2)$ \\
$\mathrm{C}(3)$ & $55(3)$ & $23(2)$ & $43(3)$ & $-1(2)$ & $20(2)$ & $6(2)$ \\
$\mathrm{C}(4)$ & $49(3)$ & $27(2)$ & $23(2)$ & $-5(2)$ & $5(2)$ & $-10(2)$ \\
$\mathrm{C}(5)$ & $46(3)$ & $33(3)$ & $34(3)$ & $-2(2)$ & $5(2)$ & $-16(2)$ \\
$\mathrm{C}(6)$ & $47(3)$ & $48(3)$ & $58(4)$ & $-3(3)$ & $-4(3)$ & $-15(3)$ \\
$\mathrm{C}(7)$ & $74(4)$ & $55(4)$ & $35(3)$ & $2(3)$ & $-7(3)$ & $-23(3)$ \\
$\mathrm{C}(8)$ & $86(5)$ & $47(3)$ & $26(3)$ & $0(2)$ & $5(3)$ & $-14(3)$ \\
$\mathrm{C}(9)$ & $62(3)$ & $40(3)$ & $26(2)$ & $-11(2)$ & $15(2)$ & $-14(2)$ \\
& & & & &
\end{tabular}




\begin{tabular}{|c|c|c|c|c|c|c|}
\hline$C(10)$ & $36(3)$ & $47(3)$ & 41(3) & $3(2)$ & $16(2)$ & $-12(2)$ \\
\hline$C(11)$ & $38(2)$ & $26(2)$ & $28(2)$ & $3(2)$ & $8(2)$ & $8(2)$ \\
\hline $\mathrm{C}(12)$ & $37(3)$ & $35(3)$ & $37(3)$ & $11(2)$ & $9(2)$ & $11(2)$ \\
\hline$C(13)$ & $36(3)$ & $48(3)$ & $44(3)$ & $15(2)$ & 1(2) & $3(2)$ \\
\hline C(14) & 49(3) & 48(3) & $34(3)$ & $9(2)$ & $0(2)$ & $8(2)$ \\
\hline$C(15)$ & $47(3)$ & $42(3)$ & $33(3)$ & $11(2)$ & $7(2)$ & $10(2)$ \\
\hline$C(16)$ & $42(3)$ & $29(2)$ & $34(2)$ & $6(2)$ & $10(2)$ & $9(2)$ \\
\hline$C(17)$ & $53(3)$ & $39(3)$ & $43(3)$ & $8(2)$ & $21(2)$ & $1(2)$ \\
\hline C(18) & 41(3) & $34(2)$ & 19(2) & $-2(2)$ & $-6(2)$ & $19(2)$ \\
\hline C(19) & $43(3)$ & $70(4)$ & $24(2)$ & $-7(2)$ & $-4(2)$ & $28(3)$ \\
\hline C(20) & 53(4) & 79(5) & 31(3) & $-14(3)$ & $-7(3)$ & $23(3)$ \\
\hline$C(21)$ & $85(5)$ & $56(4)$ & $30(3)$ & $-20(3)$ & $-26(3)$ & $28(4)$ \\
\hline C(22) & $68(4)$ & $36(3)$ & $40(3)$ & $-2(2)$ & $-25(3)$ & $7(3)$ \\
\hline$C(23)$ & $45(3)$ & $30(2)$ & $28(2)$ & $-1(2)$ & $-14(2)$ & $15(2)$ \\
\hline$C(24)$ & $22(2)$ & $29(2)$ & 22(2) & $-2(2)$ & $6(2)$ & $-1(2)$ \\
\hline$C(25)$ & $27(2)$ & $23(2)$ & $22(2)$ & $-1(2)$ & $5(2)$ & $0(2)$ \\
\hline$C(26)$ & $28(2)$ & $37(2)$ & $25(2)$ & $4(2)$ & $9(2)$ & $4(2)$ \\
\hline C(27) & $32(2)$ & $48(3)$ & 22(2) & $10(2)$ & 2(2) & $6(2)$ \\
\hline$C(28)$ & $39(3)$ & $46(3)$ & 21(2) & $11(2)$ & $8(2)$ & $0(2)$ \\
\hline C(29) & $34(2)$ & $38(2)$ & $26(2)$ & $5(2)$ & $12(2)$ & $-1(2)$ \\
\hline C(30) & $26(2)$ & $23(2)$ & $23(2)$ & 1(2) & $5(2)$ & $-1(2)$ \\
\hline $\mathrm{C}(31)$ & $25(2)$ & $39(3)$ & $36(2)$ & $10(2)$ & $16(2)$ & $3(2)$ \\
\hline C(32) & $23(2)$ & $40(3)$ & $52(3)$ & $14(2)$ & $13(2)$ & $1(2)$ \\
\hline C(33) & $30(2)$ & $40(3)$ & 48(3) & $12(2)$ & $18(2)$ & $6(2)$ \\
\hline $\mathrm{Ru}(2)$ & 21(1) & 19(1) & $16(1)$ & 1(1) & $5(1)$ & $0(1)$ \\
\hline$S(3)$ & $30(1)$ & $27(1)$ & $18(1)$ & $0(1)$ & $9(1)$ & $0(1)$ \\
\hline $\mathrm{S}(4)$ & 23(1) & $24(1)$ & 21(1) & $0(1)$ & $3(1)$ & $1(1)$ \\
\hline $\mathrm{Cl}(3)$ & 49(1) & 64(1) & $22(1)$ & $3(1)$ & $15(1)$ & $2(1)$ \\
\hline $\mathrm{Cl}(4)$ & $40(1)$ & $54(1)$ & $40(1)$ & $7(1)$ & $4(1)$ & $14(1)$ \\
\hline $\mathrm{F}(3)$ & $44(2)$ & $60(2)$ & $31(1)$ & $10(1)$ & $7(1)$ & $-5(1)$ \\
\hline $\mathrm{F}(4)$ & $34(1)$ & $53(2)$ & $27(1)$ & $5(1)$ & $10(1)$ & $-2(1)$ \\
\hline $\mathrm{N}(3)$ & $31(2)$ & 21(2) & $26(2)$ & $3(1)$ & $-1(2)$ & $-2(1)$ \\
\hline $\mathrm{N}(4)$ & $29(2)$ & $23(2)$ & $22(2)$ & $0(1)$ & $-2(1)$ & $-1(1)$ \\
\hline $\mathrm{O}(2)$ & 23(1) & $27(1)$ & $16(1)$ & $-1(1)$ & $7(1)$ & $-4(1)$ \\
\hline C(34) & $23(2)$ & $25(2)$ & $22(2)$ & $1(2)$ & $4(2)$ & $0(2)$ \\
\hline C(35) & $49(3)$ & 21(2) & $39(3)$ & $4(2)$ & $-8(2)$ & $-3(2)$ \\
\hline$C(36)$ & $48(3)$ & $21(2)$ & 39(3) & $4(2)$ & $-7(2)$ & $-2(2)$ \\
\hline C(37) & $28(2)$ & $20(2)$ & $27(2)$ & 2(2) & $-6(2)$ & $1(2)$ \\
\hline$C(38)$ & $34(2)$ & $33(2)$ & 29(2) & $7(2)$ & $-1(2)$ & $2(2)$ \\
\hline C(39) & $51(3)$ & $40(3)$ & $30(2)$ & 2(2) & $-9(2)$ & $7(2)$ \\
\hline$C(40)$ & $45(3)$ & $35(3)$ & $46(3)$ & $-3(2)$ & $-18(2)$ & $1(2)$ \\
\hline C(41) & $31(2)$ & $32(3)$ & $59(3)$ & $2(2)$ & $-3(2)$ & $-1(2)$ \\
\hline $\mathrm{C}(42)$ & $30(2)$ & $25(2)$ & 43(3) & $4(2)$ & $-2(2)$ & $5(2)$ \\
\hline C(43) & $35(3)$ & 48(3) & $51(3)$ & $-1(2)$ & $18(2)$ & $2(2)$ \\
\hline C(44) & $36(2)$ & $22(2)$ & $22(2)$ & $-1(2)$ & $-2(2)$ & $-2(2)$ \\
\hline C(45) & $35(2)$ & $31(2)$ & $32(2)$ & $-8(2)$ & $4(2)$ & $3(2)$ \\
\hline$C(46)$ & $45(3)$ & $38(3)$ & $27(2)$ & $-10(2)$ & $8(2)$ & $1(2)$ \\
\hline $\mathrm{C}(47)$ & $40(3)$ & $33(2)$ & 23(2) & $-3(2)$ & $-3(2)$ & $1(2)$ \\
\hline C(48) & $28(2)$ & $35(2)$ & 29(2) & $2(2)$ & $1(2)$ & $-1(2)$ \\
\hline
\end{tabular}




\begin{tabular}{llllccc}
$\mathrm{C}(49)$ & $34(2)$ & $29(2)$ & $22(2)$ & $1(2)$ & $6(2)$ & $-5(2)$ \\
$\mathrm{C}(50)$ & $38(3)$ & $61(3)$ & $37(3)$ & $-15(2)$ & $7(2)$ & $10(2)$ \\
$\mathrm{C}(51)$ & $31(2)$ & $26(2)$ & $17(2)$ & $1(2)$ & $1(2)$ & $-9(2)$ \\
$\mathrm{C}(52)$ & $36(2)$ & $41(3)$ & $22(2)$ & $-1(2)$ & $7(2)$ & $-3(2)$ \\
$\mathrm{C}(53)$ & $48(3)$ & $56(3)$ & $23(2)$ & $6(2)$ & $3(2)$ & $2(3)$ \\
$\mathrm{C}(54)$ & $46(3)$ & $47(3)$ & $34(3)$ & $12(2)$ & $-6(2)$ & $7(2)$ \\
$\mathrm{C}(55)$ & $34(2)$ & $41(3)$ & $27(2)$ & $1(2)$ & $-1(2)$ & $-3(2)$ \\
$\mathrm{C}(56)$ & $29(2)$ & $26(2)$ & $18(2)$ & $1(2)$ & $0(2)$ & $-4(2)$ \\
$\mathrm{C}(57)$ & $21(2)$ & $28(2)$ & $21(2)$ & $1(2)$ & $9(2)$ & $1(2)$ \\
$\mathrm{C}(58)$ & $24(2)$ & $21(2)$ & $21(2)$ & $1(2)$ & $7(2)$ & $2(2)$ \\
$\mathrm{C}(59)$ & $29(2)$ & $30(2)$ & $28(2)$ & $-1(2)$ & $10(2)$ & $-2(2)$ \\
$\mathrm{C}(60)$ & $32(2)$ & $34(2)$ & $29(2)$ & $-6(2)$ & $6(2)$ & $-4(2)$ \\
$\mathrm{C}(61)$ & $37(2)$ & $39(3)$ & $23(2)$ & $-9(2)$ & $6(2)$ & $0(2)$ \\
$\mathrm{C}(62)$ & $33(2)$ & $37(2)$ & $22(2)$ & $-2(2)$ & $11(2)$ & $-1(2)$ \\
$\mathrm{C}(63)$ & $25(2)$ & $21(2)$ & $19(2)$ & $-1(2)$ & $3(2)$ & $1(2)$ \\
$\mathrm{C}(64)$ & $25(2)$ & $43(3)$ & $24(2)$ & $1(2)$ & $11(2)$ & $-6(2)$ \\
$\mathrm{C}(65)$ & $30(2)$ & $58(3)$ & $37(3)$ & $-10(2)$ & $17(2)$ & $-3(2)$ \\
$\mathrm{C}(66)$ & $33(2)$ & $50(3)$ & $32(2)$ & $-1(2)$ & $15(2)$ & $-13(2)$ \\
& & & & & & \\
\hline
\end{tabular}

Table 11. Hydrogen coordinates $\left(\times 10^{4}\right)$ and isotropic displacement parameters $\left(\AA^{2} \times 10^{3}\right)$ for $\mathrm{C}_{33} \mathrm{H}_{28} \mathrm{Cl}_{2} \mathrm{~F}_{2} \mathrm{~N}_{2} \mathrm{ORuS} \mathrm{S}_{2}(\mathbf{R u}-\mathbf{1 d})$.

\begin{tabular}{|c|c|c|c|c|}
\hline & $\mathrm{x}$ & $\mathrm{y}$ & $\mathrm{z}$ & $\mathrm{U}(\mathrm{eq})$ \\
\hline $\mathrm{H}(2 \mathrm{~A})$ & 5605 & 5157 & 7921 & 50 \\
\hline $\mathrm{H}(3 \mathrm{~A})$ & 4430 & 5286 & 6992 & 47 \\
\hline $\mathrm{H}(6 \mathrm{~A})$ & 8581 & 3364 & 8505 & 67 \\
\hline $\mathrm{H}(7 \mathrm{~A})$ & 8337 & 3067 & 9354 & 72 \\
\hline $\mathrm{H}(8 \mathrm{~A})$ & 6900 & 3167 & 9464 & 67 \\
\hline $\mathrm{H}(10 \mathrm{~A})$ & 8044 & 3818 & 7554 & 61 \\
\hline $\mathrm{H}(10 \mathrm{~B})$ & 7017 & 3567 & 7233 & 61 \\
\hline $\mathrm{H}(10 \mathrm{C})$ & 7233 & 4423 & 7446 & 61 \\
\hline $\mathrm{H}(13 \mathrm{~A})$ & 2070 & 3305 & 5632 & 54 \\
\hline $\mathrm{H}(14 \mathrm{~A})$ & 2693 & 3419 & 4889 & 56 \\
\hline $\mathrm{H}(15 \mathrm{~A})$ & 4112 & 3960 & 5030 & 50 \\
\hline $\mathrm{H}(17 \mathrm{~A})$ & 5628 & 4576 & 6367 & 65 \\
\hline $\mathrm{H}(17 \mathrm{~B})$ & 5743 & 4102 & 5845 & 65 \\
\hline $\mathrm{H}(17 \mathrm{C})$ & 5290 & 4928 & 5746 & 65 \\
\hline $\mathrm{H}(20)$ & 4688 & 1241 & 4574 & 71 \\
\hline $\mathrm{H}(21)$ & 3778 & 388 & 4850 & 80 \\
\hline $\mathrm{H}(24 \mathrm{~A})$ & 6791 & 2133 & 7396 & 29 \\
\hline $\mathrm{H}(26 \mathrm{~A})$ & 7745 & 1476 & 8309 & 36 \\
\hline $\mathrm{H}(27 \mathrm{~A})$ & 7847 & 1008 & 9205 & 42 \\
\hline $\mathrm{H}(28 \mathrm{~A})$ & 6588 & 1071 & 9534 & 43 \\
\hline $\mathrm{H}(29 \mathrm{~A})$ & 5215 & 1602 & 8983 & 38 \\
\hline $\mathrm{H}(31 \mathrm{~A})$ & 4222 & 2396 & 8519 & 38 \\
\hline $\mathrm{H}(32 \mathrm{~A})$ & 3042 & 1511 & 8127 & 57 \\
\hline $\mathrm{H}(32 \mathrm{~B})$ & 3459 & 1343 & 7624 & 57 \\
\hline
\end{tabular}




\begin{tabular}{|c|c|c|c|c|}
\hline $\mathrm{H}(32 \mathrm{C})$ & 4015 & 1105 & 8255 & 57 \\
\hline $\mathrm{H}(33 \mathrm{~A})$ & 2930 & 2922 & 7845 & 57 \\
\hline $\mathrm{H}(33 \mathrm{~B})$ & 3841 & 3349 & 7832 & 57 \\
\hline $\mathrm{H}(33 \mathrm{C})$ & 3374 & 2738 & 7357 & 57 \\
\hline $\mathrm{H}(35 \mathrm{~A})$ & 989 & 3674 & 8002 & 50 \\
\hline $\mathrm{H}(36 \mathrm{~A})$ & -187 & 3450 & 7086 & 48 \\
\hline $\mathrm{H}(39 \mathrm{~A})$ & 1984 & 5632 & 9571 & 54 \\
\hline $\mathrm{H}(40 \mathrm{~A})$ & 3349 & 6003 & 9426 & 59 \\
\hline $\mathrm{H}(41 \mathrm{~A})$ & 3593 & 5813 & 8564 & 53 \\
\hline $\mathrm{H}(43 \mathrm{~A})$ & 3115 & 5364 & 7619 & 65 \\
\hline $\mathrm{H}(43 \mathrm{~B})$ & 2435 & 4655 & 7523 & 65 \\
\hline $\mathrm{H}(43 \mathrm{C})$ & 2047 & 5480 & 7309 & 65 \\
\hline $\mathrm{H}(46 \mathrm{~A})$ & -723 & 4594 & 5134 & 45 \\
\hline $\mathrm{H}(47 \mathrm{~A})$ & -2240 & 4937 & 4949 & 42 \\
\hline $\mathrm{H}(48 \mathrm{~A})$ & -2857 & 5128 & 5690 & 39 \\
\hline $\mathrm{H}(50 \mathrm{~A})$ & 750 & 4347 & 5753 & 69 \\
\hline $\mathrm{H}(50 \mathrm{~B})$ & 983 & 4826 & 6325 & 69 \\
\hline $\mathrm{H}(50 \mathrm{C})$ & 755 & 3940 & 6328 & 69 \\
\hline $\mathrm{H}(53 \mathrm{~A})$ & -1188 & 8011 & 4793 & 53 \\
\hline $\mathrm{H}(54 \mathrm{~A})$ & -2229 & 8581 & 5181 & 56 \\
\hline $\mathrm{H}(57 \mathrm{~A})$ & 1465 & 6889 & 7412 & 27 \\
\hline $\mathrm{H}(59 \mathrm{~A})$ & 2381 & 7657 & 8266 & 34 \\
\hline $\mathrm{H}(60 \mathrm{~A})$ & 2564 & 8106 & 9173 & 39 \\
\hline $\mathrm{H}(61 \mathrm{~A})$ & 1496 & 7804 & 9621 & 40 \\
\hline $\mathrm{H}(62 \mathrm{~A})$ & 219 & 7056 & 9176 & 36 \\
\hline $\mathrm{H}(64 \mathrm{~A})$ & -646 & 6147 & 8769 & 36 \\
\hline $\mathrm{H}(65 \mathrm{~A})$ & -2009 & 6855 & 8477 & 60 \\
\hline $\mathrm{H}(65 \mathrm{~B})$ & -1150 & 7396 & 8533 & 60 \\
\hline $\mathrm{H}(65 \mathrm{C})$ & -1770 & 7121 & 7924 & 60 \\
\hline $\mathrm{H}(66 \mathrm{~A})$ & -1918 & 5476 & 8184 & 56 \\
\hline $\mathrm{H}(66 \mathrm{~B})$ & -1649 & 5739 & 7641 & 56 \\
\hline $\mathrm{H}(66 \mathrm{C})$ & -989 & 5182 & 8092 & 56 \\
\hline $\mathrm{H}(1 \mathrm{~T})$ & 9062 & 5363 & 9911 & 23 \\
\hline $\mathrm{H}(1 \mathrm{~T} 1)$ & 10052 & 4543 & 9906 & 42 \\
\hline $\mathrm{H}(1 \mathrm{~T} 2)$ & 9355 & 4199 & 9345 & 42 \\
\hline H(1T3) & 9148 & 4117 & 9934 & 42 \\
\hline $\mathrm{H}(1 \mathrm{~S})$ & 9545 & 5157 & 9557 & 48 \\
\hline $\mathrm{H}(1 \mathrm{~S} 1)$ & 10219 & 4169 & 9797 & 28 \\
\hline $\mathrm{H}(1 \mathrm{~S} 2)$ & 9281 & 3772 & 9791 & 28 \\
\hline $\mathrm{H}(1 \mathrm{~S} 3)$ & 9815 & 4296 & 10311 & 28 \\
\hline
\end{tabular}

Table 12. Torsion angles $\left(^{\circ}\right)$ for $\mathrm{C}_{33} \mathrm{H}_{28} \mathrm{Cl}_{2} \mathrm{~F}_{2} \mathrm{~N}_{2} \mathrm{ORuS}$ ( Ru-1d).

\begin{tabular}{lc}
\hline $\mathrm{C}(2)-\mathrm{N}(1)-\mathrm{C}(1)-\mathrm{N}(2)$ & $1.1(5)$ \\
$\mathrm{C}(4)-\mathrm{N}(1)-\mathrm{C}(1)-\mathrm{N}(2)$ & $-171.5(4)$ \\
$\mathrm{C}(2)-\mathrm{N}(1)-\mathrm{C}(1)-\mathrm{Ru}(1)$ & $-170.2(3)$ \\
$\mathrm{C}(4)-\mathrm{N}(1)-\mathrm{C}(1)-\mathrm{Ru}(1)$ & $17.2(6)$ \\
$\mathrm{C}(3)-\mathrm{N}(2)-\mathrm{C}(1)-\mathrm{N}(1)$ & $-1.2(5)$ \\
$\mathrm{C}(11)-\mathrm{N}(2)-\mathrm{C}(1)-\mathrm{N}(1)$ & $173.0(4)$
\end{tabular}




\begin{tabular}{|c|c|}
\hline$C(3)-N(2)-C(1)-R u(1)$ & $170.6(3)$ \\
\hline $\mathrm{C}(11)-\mathrm{N}(2)-\mathrm{C}(1)-\mathrm{Ru}(1)$ & $-15.2(6)$ \\
\hline $\mathrm{C}(1)-\mathrm{N}(1)-\mathrm{C}(2)-\mathrm{C}(3)$ & $-0.6(6)$ \\
\hline $\mathrm{C}(4)-\mathrm{N}(1)-\mathrm{C}(2)-\mathrm{C}(3)$ & $172.2(4)$ \\
\hline $\mathrm{N}(1)-\mathrm{C}(2)-\mathrm{C}(3)-\mathrm{N}(2)$ & $-0.2(6)$ \\
\hline $\mathrm{C}(1)-\mathrm{N}(2)-\mathrm{C}(3)-\mathrm{C}(2)$ & $0.9(6)$ \\
\hline $\mathrm{C}(11)-\mathrm{N}(2)-\mathrm{C}(3)-\mathrm{C}(2)$ & $-173.4(4)$ \\
\hline $\mathrm{C}(1)-\mathrm{N}(1)-\mathrm{C}(4)-\mathrm{C}(9)$ & $-105.1(5)$ \\
\hline $\mathrm{C}(2)-\mathrm{N}(1)-\mathrm{C}(4)-\mathrm{C}(9)$ & $83.1(6)$ \\
\hline $\mathrm{C}(1)-\mathrm{N}(1)-\mathrm{C}(4)-\mathrm{C}(5)$ & $76.8(6)$ \\
\hline $\mathrm{C}(2)-\mathrm{N}(1)-\mathrm{C}(4)-\mathrm{C}(5)$ & $-95.0(6)$ \\
\hline$C(9)-C(4)-C(5)-C(6)$ & $1.2(7)$ \\
\hline $\mathrm{N}(1)-\mathrm{C}(4)-\mathrm{C}(5)-\mathrm{C}(6)$ & $179.3(4)$ \\
\hline $\mathrm{C}(9)-\mathrm{C}(4)-\mathrm{C}(5)-\mathrm{C}(10)$ & $-179.3(5)$ \\
\hline $\mathrm{N}(1)-\mathrm{C}(4)-\mathrm{C}(5)-\mathrm{C}(10)$ & $-1.2(7)$ \\
\hline$C(4)-C(5)-C(6)-C(7)$ & $-1.1(8)$ \\
\hline $\mathrm{C}(10)-\mathrm{C}(5)-\mathrm{C}(6)-\mathrm{C}(7)$ & $179.4(5)$ \\
\hline$C(5)-C(6)-C(7)-C(8)$ & $0.8(9)$ \\
\hline$C(6)-C(7)-C(8)-C(9)$ & $-0.6(9)$ \\
\hline $\mathrm{C}(7)-\mathrm{C}(8)-\mathrm{C}(9)-\mathrm{F}(1)$ & $-179.6(5)$ \\
\hline $\mathrm{C}(7)-\mathrm{C}(8)-\mathrm{C}(9)-\mathrm{C}(4)$ & $0.7(8)$ \\
\hline $\mathrm{C}(5)-\mathrm{C}(4)-\mathrm{C}(9)-\mathrm{C}(8)$ & $-1.1(8)$ \\
\hline $\mathrm{N}(1)-\mathrm{C}(4)-\mathrm{C}(9)-\mathrm{C}(8)$ & $-179.1(5)$ \\
\hline $\mathrm{C}(5)-\mathrm{C}(4)-\mathrm{C}(9)-\mathrm{F}(1)$ & $179.2(4)$ \\
\hline $\mathrm{N}(1)-\mathrm{C}(4)-\mathrm{C}(9)-\mathrm{F}(1)$ & $1.1(7)$ \\
\hline $\mathrm{C}(1)-\mathrm{N}(2)-\mathrm{C}(11)-\mathrm{C}(16)$ & $-89.9(5)$ \\
\hline $\mathrm{C}(3)-\mathrm{N}(2)-\mathrm{C}(11)-\mathrm{C}(16)$ & $83.6(6)$ \\
\hline $\mathrm{C}(1)-\mathrm{N}(2)-\mathrm{C}(11)-\mathrm{C}(12)$ & $89.0(5)$ \\
\hline $\mathrm{C}(3)-\mathrm{N}(2)-\mathrm{C}(11)-\mathrm{C}(12)$ & $-97.5(5)$ \\
\hline$C(16)-C(11)-C(12)-F(2)$ & $-174.4(4)$ \\
\hline $\mathrm{N}(2)-\mathrm{C}(11)-\mathrm{C}(12)-\mathrm{F}(2)$ & $6.7(6)$ \\
\hline$C(16)-C(11)-C(12)-C(13)$ & $5.6(7)$ \\
\hline $\mathrm{N}(2)-\mathrm{C}(11)-\mathrm{C}(12)-\mathrm{C}(13)$ & $-173.3(4)$ \\
\hline $\mathrm{F}(2)-\mathrm{C}(12)-\mathrm{C}(13)-\mathrm{C}(14)$ & $178.4(4)$ \\
\hline $\mathrm{C}(11)-\mathrm{C}(12)-\mathrm{C}(13)-\mathrm{C}(14)$ & $-1.6(8)$ \\
\hline$C(12)-C(13)-C(14)-C(15)$ & $-2.5(8)$ \\
\hline$C(13)-C(14)-C(15)-C(16)$ & $2.5(8)$ \\
\hline$C(14)-C(15)-C(16)-C(11)$ & $1.5(7)$ \\
\hline $\mathrm{C}(14)-\mathrm{C}(15)-\mathrm{C}(16)-\mathrm{C}(17)$ & $-179.3(5)$ \\
\hline$C(12)-C(11)-C(16)-C(15)$ & $-5.4(7)$ \\
\hline $\mathrm{N}(2)-\mathrm{C}(11)-\mathrm{C}(16)-\mathrm{C}(15)$ & $173.6(4)$ \\
\hline$C(12)-C(11)-C(16)-C(17)$ & $175.4(4)$ \\
\hline $\mathrm{N}(2)-\mathrm{C}(11)-\mathrm{C}(16)-\mathrm{C}(17)$ & $-5.6(7)$ \\
\hline $\mathrm{Ru}(1)-\mathrm{S}(1)-\mathrm{C}(18)-\mathrm{C}(19)$ & $179.5(3)$ \\
\hline $\mathrm{Ru}(1)-\mathrm{S}(1)-\mathrm{C}(18)-\mathrm{C}(23)$ & $-0.3(4)$ \\
\hline $\mathrm{C}(23)-\mathrm{C}(18)-\mathrm{C}(19)-\mathrm{C}(20)$ & $-4.8(7)$ \\
\hline $\mathrm{S}(1)-\mathrm{C}(18)-\mathrm{C}(19)-\mathrm{C}(20)$ & $175.3(4)$ \\
\hline $\mathrm{C}(23)-\mathrm{C}(18)-\mathrm{C}(19)-\mathrm{Cl}(1)$ & $174.3(3)$ \\
\hline $\mathrm{S}(1)-\mathrm{C}(18)-\mathrm{C}(19)-\mathrm{Cl}(1)$ & $-5.5(6)$ \\
\hline
\end{tabular}




\begin{tabular}{|c|c|}
\hline$C(18)-C(19)-C(20)-C(21)$ & $1.7(8)$ \\
\hline $\mathrm{Cl}(1)-\mathrm{C}(19)-\mathrm{C}(20)-\mathrm{C}(21)$ & $-177.5(5)$ \\
\hline$C(19)-C(20)-C(21)-C(22)$ & $2.1(9)$ \\
\hline$C(20)-C(21)-C(22)-C(23)$ & $-2.6(9)$ \\
\hline $\mathrm{C}(20)-\mathrm{C}(21)-\mathrm{C}(22)-\mathrm{Cl}(2)$ & $175.3(5)$ \\
\hline $\mathrm{C}(21)-\mathrm{C}(22)-\mathrm{C}(23)-\mathrm{C}(18)$ & $-0.6(7)$ \\
\hline $\mathrm{Cl}(2)-\mathrm{C}(22)-\mathrm{C}(23)-\mathrm{C}(18)$ & $-178.5(4)$ \\
\hline $\mathrm{C}(21)-\mathrm{C}(22)-\mathrm{C}(23)-\mathrm{S}(2)$ & $178.7(4)$ \\
\hline $\mathrm{Cl}(2)-\mathrm{C}(22)-\mathrm{C}(23)-\mathrm{S}(2)$ & $0.8(6)$ \\
\hline $\mathrm{C}(19)-\mathrm{C}(18)-\mathrm{C}(23)-\mathrm{C}(22)$ & $4.1(7)$ \\
\hline $\mathrm{S}(1)-\mathrm{C}(18)-\mathrm{C}(23)-\mathrm{C}(22)$ & $-176.1(4)$ \\
\hline $\mathrm{C}(19)-\mathrm{C}(18)-\mathrm{C}(23)-\mathrm{S}(2)$ & $-175.3(3)$ \\
\hline $\mathrm{S}(1)-\mathrm{C}(18)-\mathrm{C}(23)-\mathrm{S}(2)$ & $4.6(5)$ \\
\hline $\mathrm{Ru}(1)-\mathrm{S}(2)-\mathrm{C}(23)-\mathrm{C}(22)$ & $174.4(4)$ \\
\hline $\mathrm{Ru}(1)-\mathrm{S}(2)-\mathrm{C}(23)-\mathrm{C}(18)$ & $-6.3(4)$ \\
\hline $\mathrm{C}(1)-\mathrm{Ru}(1)-\mathrm{C}(24)-\mathrm{C}(25)$ & $96.1(3)$ \\
\hline $\mathrm{S}(1)-\mathrm{Ru}(1)-\mathrm{C}(24)-\mathrm{C}(25)$ & $-170.8(3)$ \\
\hline $\mathrm{S}(2)-\mathrm{Ru}(1)-\mathrm{C}(24)-\mathrm{C}(25)$ & $-80.3(3)$ \\
\hline $\mathrm{O}(1)-\mathrm{Ru}(1)-\mathrm{C}(24)-\mathrm{C}(25)$ & $6.7(3)$ \\
\hline $\mathrm{Ru}(1)-\mathrm{C}(24)-\mathrm{C}(25)-\mathrm{C}(26)$ & $175.7(3)$ \\
\hline $\mathrm{Ru}(1)-\mathrm{C}(24)-\mathrm{C}(25)-\mathrm{C}(30)$ & $-5.6(5)$ \\
\hline $\mathrm{C}(30)-\mathrm{C}(25)-\mathrm{C}(26)-\mathrm{C}(27)$ & $1.6(7)$ \\
\hline $\mathrm{C}(24)-\mathrm{C}(25)-\mathrm{C}(26)-\mathrm{C}(27)$ & $-179.7(4)$ \\
\hline $\mathrm{C}(25)-\mathrm{C}(26)-\mathrm{C}(27)-\mathrm{C}(28)$ & $-0.4(7)$ \\
\hline $\mathrm{C}(26)-\mathrm{C}(27)-\mathrm{C}(28)-\mathrm{C}(29)$ & $-0.3(8)$ \\
\hline $\mathrm{C}(27)-\mathrm{C}(28)-\mathrm{C}(29)-\mathrm{C}(30)$ & $-0.2(8)$ \\
\hline $\mathrm{C}(28)-\mathrm{C}(29)-\mathrm{C}(30)-\mathrm{O}(1)$ & $-177.9(4)$ \\
\hline $\mathrm{C}(28)-\mathrm{C}(29)-\mathrm{C}(30)-\mathrm{C}(25)$ & $1.5(7)$ \\
\hline $\mathrm{C}(31)-\mathrm{O}(1)-\mathrm{C}(30)-\mathrm{C}(29)$ & $-4.3(6)$ \\
\hline $\mathrm{Ru}(1)-\mathrm{O}(1)-\mathrm{C}(30)-\mathrm{C}(29)$ & $-174.4(4)$ \\
\hline $\mathrm{C}(31)-\mathrm{O}(1)-\mathrm{C}(30)-\mathrm{C}(25)$ & $176.3(4)$ \\
\hline $\mathrm{Ru}(1)-\mathrm{O}(1)-\mathrm{C}(30)-\mathrm{C}(25)$ & $6.2(4)$ \\
\hline$C(26)-C(25)-C(30)-C(29)$ & $-2.1(6)$ \\
\hline $\mathrm{C}(24)-\mathrm{C}(25)-\mathrm{C}(30)-\mathrm{C}(29)$ & 179.1(4) \\
\hline $\mathrm{C}(26)-\mathrm{C}(25)-\mathrm{C}(30)-\mathrm{O}(1)$ & $177.3(4)$ \\
\hline $\mathrm{C}(24)-\mathrm{C}(25)-\mathrm{C}(30)-\mathrm{O}(1)$ & $-1.5(5)$ \\
\hline $\mathrm{C}(30)-\mathrm{O}(1)-\mathrm{C}(31)-\mathrm{C}(33)$ & $161.2(4)$ \\
\hline $\mathrm{Ru}(1)-\mathrm{O}(1)-\mathrm{C}(31)-\mathrm{C}(33)$ & $-30.9(5)$ \\
\hline $\mathrm{C}(30)-\mathrm{O}(1)-\mathrm{C}(31)-\mathrm{C}(32)$ & $-76.4(5)$ \\
\hline $\mathrm{Ru}(1)-\mathrm{O}(1)-\mathrm{C}(31)-\mathrm{C}(32)$ & $91.6(4)$ \\
\hline $\mathrm{C}(35)-\mathrm{N}(3)-\mathrm{C}(34)-\mathrm{N}(4)$ & $-1.0(5)$ \\
\hline $\mathrm{C}(37)-\mathrm{N}(3)-\mathrm{C}(34)-\mathrm{N}(4)$ & $170.4(4)$ \\
\hline $\mathrm{C}(35)-\mathrm{N}(3)-\mathrm{C}(34)-\mathrm{Ru}(2)$ & $167.0(4)$ \\
\hline $\mathrm{C}(37)-\mathrm{N}(3)-\mathrm{C}(34)-\mathrm{Ru}(2)$ & $-21.6(7)$ \\
\hline $\mathrm{C}(36)-\mathrm{N}(4)-\mathrm{C}(34)-\mathrm{N}(3)$ & $1.5(5)$ \\
\hline $\mathrm{C}(44)-\mathrm{N}(4)-\mathrm{C}(34)-\mathrm{N}(3)$ & $-170.1(4)$ \\
\hline$C(36)-N(4)-C(34)-R u(2)$ & $-167.3(4)$ \\
\hline $\mathrm{C}(44)-\mathrm{N}(4)-\mathrm{C}(34)-\mathrm{Ru}(2)$ & $21.0(6)$ \\
\hline $\mathrm{C}(34)-\mathrm{N}(3)-\mathrm{C}(35)-\mathrm{C}(36)$ & $0.1(6)$ \\
\hline
\end{tabular}




\begin{tabular}{|c|c|}
\hline$C(37)-N(3)-C(35)-C(36)$ & $-171.6(5)$ \\
\hline $\mathrm{N}(3)-\mathrm{C}(35)-\mathrm{C}(36)-\mathrm{N}(4)$ & $0.8(6)$ \\
\hline $\mathrm{C}(34)-\mathrm{N}(4)-\mathrm{C}(36)-\mathrm{C}(35)$ & $-1.5(6)$ \\
\hline $\mathrm{C}(44)-\mathrm{N}(4)-\mathrm{C}(36)-\mathrm{C}(35)$ & $170.6(5)$ \\
\hline $\mathrm{C}(34)-\mathrm{N}(3)-\mathrm{C}(37)-\mathrm{C}(38)$ & $107.7(5)$ \\
\hline $\mathrm{C}(35)-\mathrm{N}(3)-\mathrm{C}(37)-\mathrm{C}(38)$ & $-81.7(6)$ \\
\hline $\mathrm{C}(34)-\mathrm{N}(3)-\mathrm{C}(37)-\mathrm{C}(42)$ & $-76.3(6)$ \\
\hline$C(35)-N(3)-C(37)-C(42)$ & $94.2(6)$ \\
\hline $\mathrm{C}(42)-\mathrm{C}(37)-\mathrm{C}(38)-\mathrm{C}(39)$ & $3.2(7)$ \\
\hline $\mathrm{N}(3)-\mathrm{C}(37)-\mathrm{C}(38)-\mathrm{C}(39)$ & $179.2(4)$ \\
\hline $\mathrm{C}(42)-\mathrm{C}(37)-\mathrm{C}(38)-\mathrm{F}(3)$ & $-177.5(4)$ \\
\hline $\mathrm{N}(3)-\mathrm{C}(37)-\mathrm{C}(38)-\mathrm{F}(3)$ & $-1.5(6)$ \\
\hline $\mathrm{F}(3)-\mathrm{C}(38)-\mathrm{C}(39)-\mathrm{C}(40)$ & $180.0(4)$ \\
\hline $\mathrm{C}(37)-\mathrm{C}(38)-\mathrm{C}(39)-\mathrm{C}(40)$ & $-0.8(7)$ \\
\hline $\mathrm{C}(38)-\mathrm{C}(39)-\mathrm{C}(40)-\mathrm{C}(41)$ & $-1.3(8)$ \\
\hline$C(39)-C(40)-C(41)-C(42)$ & $1.0(8)$ \\
\hline $\mathrm{C}(38)-\mathrm{C}(37)-\mathrm{C}(42)-\mathrm{C}(41)$ & $-3.4(6)$ \\
\hline $\mathrm{N}(3)-\mathrm{C}(37)-\mathrm{C}(42)-\mathrm{C}(41)$ & $-179.4(4)$ \\
\hline $\mathrm{C}(38)-\mathrm{C}(37)-\mathrm{C}(42)-\mathrm{C}(43)$ & $175.6(4)$ \\
\hline $\mathrm{N}(3)-\mathrm{C}(37)-\mathrm{C}(42)-\mathrm{C}(43)$ & $-0.4(6)$ \\
\hline $\mathrm{C}(40)-\mathrm{C}(41)-\mathrm{C}(42)-\mathrm{C}(37)$ & $1.4(7)$ \\
\hline $\mathrm{C}(40)-\mathrm{C}(41)-\mathrm{C}(42)-\mathrm{C}(43)$ & $-177.7(5)$ \\
\hline $\mathrm{C}(34)-\mathrm{N}(4)-\mathrm{C}(44)-\mathrm{C}(49)$ & $-85.8(6)$ \\
\hline $\mathrm{C}(36)-\mathrm{N}(4)-\mathrm{C}(44)-\mathrm{C}(49)$ & $103.3(5)$ \\
\hline $\mathrm{C}(34)-\mathrm{N}(4)-\mathrm{C}(44)-\mathrm{C}(45)$ & $94.4(5)$ \\
\hline $\mathrm{C}(36)-\mathrm{N}(4)-\mathrm{C}(44)-\mathrm{C}(45)$ & $-76.6(6)$ \\
\hline$C(49)-C(44)-C(45)-C(46)$ & $0.2(7)$ \\
\hline $\mathrm{N}(4)-\mathrm{C}(44)-\mathrm{C}(45)-\mathrm{C}(46)$ & $-179.9(4)$ \\
\hline$C(49)-C(44)-C(45)-C(50)$ & $178.2(5)$ \\
\hline $\mathrm{N}(4)-\mathrm{C}(44)-\mathrm{C}(45)-\mathrm{C}(50)$ & $-2.0(7)$ \\
\hline$C(44)-C(45)-C(46)-C(47)$ & $0.7(7)$ \\
\hline$C(50)-C(45)-C(46)-C(47)$ & $-177.2(5)$ \\
\hline $\mathrm{C}(45)-\mathrm{C}(46)-\mathrm{C}(47)-\mathrm{C}(48)$ & $-0.9(8)$ \\
\hline $\mathrm{C}(46)-\mathrm{C}(47)-\mathrm{C}(48)-\mathrm{C}(49)$ & $0.2(7)$ \\
\hline $\mathrm{C}(47)-\mathrm{C}(48)-\mathrm{C}(49)-\mathrm{F}(4)$ & $179.9(4)$ \\
\hline $\mathrm{C}(47)-\mathrm{C}(48)-\mathrm{C}(49)-\mathrm{C}(44)$ & $0.8(7)$ \\
\hline $\mathrm{C}(45)-\mathrm{C}(44)-\mathrm{C}(49)-\mathrm{F}(4)$ & $179.9(4)$ \\
\hline $\mathrm{N}(4)-\mathrm{C}(44)-\mathrm{C}(49)-\mathrm{F}(4)$ & $0.0(6)$ \\
\hline $\mathrm{C}(45)-\mathrm{C}(44)-\mathrm{C}(49)-\mathrm{C}(48)$ & $-1.0(7)$ \\
\hline $\mathrm{N}(4)-\mathrm{C}(44)-\mathrm{C}(49)-\mathrm{C}(48)$ & $179.2(4)$ \\
\hline $\mathrm{Ru}(2)-\mathrm{S}(3)-\mathrm{C}(51)-\mathrm{C}(52)$ & $175.5(3)$ \\
\hline $\mathrm{Ru}(2)-\mathrm{S}(3)-\mathrm{C}(51)-\mathrm{C}(56)$ & $-4.8(4)$ \\
\hline $\mathrm{C}(56)-\mathrm{C}(51)-\mathrm{C}(52)-\mathrm{C}(53)$ & $1.6(7)$ \\
\hline $\mathrm{S}(3)-\mathrm{C}(51)-\mathrm{C}(52)-\mathrm{C}(53)$ & $-178.7(4)$ \\
\hline $\mathrm{C}(56)-\mathrm{C}(51)-\mathrm{C}(52)-\mathrm{Cl}(3)$ & $-177.9(3)$ \\
\hline $\mathrm{S}(3)-\mathrm{C}(51)-\mathrm{C}(52)-\mathrm{Cl}(3)$ & $1.8(5)$ \\
\hline $\mathrm{C}(51)-\mathrm{C}(52)-\mathrm{C}(53)-\mathrm{C}(54)$ & $-1.0(8)$ \\
\hline $\mathrm{Cl}(3)-\mathrm{C}(52)-\mathrm{C}(53)-\mathrm{C}(54)$ & $178.5(4)$ \\
\hline $\mathrm{C}(52)-\mathrm{C}(53)-\mathrm{C}(54)-\mathrm{C}(55)$ & $-1.0(8)$ \\
\hline
\end{tabular}




\begin{tabular}{|c|c|}
\hline $\mathrm{C}(53)-\mathrm{C}(54)-\mathrm{C}(55)-\mathrm{C}(56)$ & $2.4(8)$ \\
\hline $\mathrm{C}(53)-\mathrm{C}(54)-\mathrm{C}(55)-\mathrm{Cl}(4)$ & $-178.2(4)$ \\
\hline $\mathrm{C}(52)-\mathrm{C}(51)-\mathrm{C}(56)-\mathrm{C}(55)$ & $-0.2(6)$ \\
\hline $\mathrm{S}(3)-\mathrm{C}(51)-\mathrm{C}(56)-\mathrm{C}(55)$ & $-179.9(3)$ \\
\hline $\mathrm{C}(52)-\mathrm{C}(51)-\mathrm{C}(56)-\mathrm{S}(4)$ & $179.5(3)$ \\
\hline $\mathrm{S}(3)-\mathrm{C}(51)-\mathrm{C}(56)-\mathrm{S}(4)$ & $-0.2(5)$ \\
\hline $\mathrm{C}(54)-\mathrm{C}(55)-\mathrm{C}(56)-\mathrm{C}(51)$ & $-1.8(7)$ \\
\hline $\mathrm{Cl}(4)-\mathrm{C}(55)-\mathrm{C}(56)-\mathrm{C}(51)$ & $178.8(3)$ \\
\hline$C(54)-C(55)-C(56)-S(4)$ & $178.5(4)$ \\
\hline $\mathrm{Cl}(4)-\mathrm{C}(55)-\mathrm{C}(56)-\mathrm{S}(4)$ & $-0.9(6)$ \\
\hline $\mathrm{Ru}(2)-\mathrm{S}(4)-\mathrm{C}(56)-\mathrm{C}(51)$ & $5.0(4)$ \\
\hline $\mathrm{Ru}(2)-\mathrm{S}(4)-\mathrm{C}(56)-\mathrm{C}(55)$ & $-175.3(3)$ \\
\hline $\mathrm{C}(34)-\mathrm{Ru}(2)-\mathrm{C}(57)-\mathrm{C}(58)$ & $-97.4(3)$ \\
\hline $\mathrm{S}(3)-\mathrm{Ru}(2)-\mathrm{C}(57)-\mathrm{C}(58)$ & $167.3(3)$ \\
\hline $\mathrm{S}(4)-\mathrm{Ru}(2)-\mathrm{C}(57)-\mathrm{C}(58)$ & $77.6(3)$ \\
\hline $\mathrm{O}(2)-\mathrm{Ru}(2)-\mathrm{C}(57)-\mathrm{C}(58)$ & $-7.8(3)$ \\
\hline $\mathrm{Ru}(2)-\mathrm{C}(57)-\mathrm{C}(58)-\mathrm{C}(63)$ & $6.3(5)$ \\
\hline $\mathrm{Ru}(2)-\mathrm{C}(57)-\mathrm{C}(58)-\mathrm{C}(59)$ & $-172.4(3)$ \\
\hline $\mathrm{C}(63)-\mathrm{C}(58)-\mathrm{C}(59)-\mathrm{C}(60)$ & $-0.7(6)$ \\
\hline $\mathrm{C}(57)-\mathrm{C}(58)-\mathrm{C}(59)-\mathrm{C}(60)$ & $178.0(4)$ \\
\hline $\mathrm{C}(58)-\mathrm{C}(59)-\mathrm{C}(60)-\mathrm{C}(61)$ & $0.5(7)$ \\
\hline$C(59)-C(60)-C(61)-C(62)$ & $-0.2(7)$ \\
\hline$C(60)-C(61)-C(62)-C(63)$ & $0.0(7)$ \\
\hline $\mathrm{C}(61)-\mathrm{C}(62)-\mathrm{C}(63)-\mathrm{O}(2)$ & $179.1(4)$ \\
\hline $\mathrm{C}(61)-\mathrm{C}(62)-\mathrm{C}(63)-\mathrm{C}(58)$ & $-0.1(6)$ \\
\hline $\mathrm{C}(64)-\mathrm{O}(2)-\mathrm{C}(63)-\mathrm{C}(62)$ & $5.4(6)$ \\
\hline $\mathrm{Ru}(2)-\mathrm{O}(2)-\mathrm{C}(63)-\mathrm{C}(62)$ & $172.9(4)$ \\
\hline $\mathrm{C}(64)-\mathrm{O}(2)-\mathrm{C}(63)-\mathrm{C}(58)$ & $-175.3(3)$ \\
\hline $\mathrm{Ru}(2)-\mathrm{O}(2)-\mathrm{C}(63)-\mathrm{C}(58)$ & $-7.8(4)$ \\
\hline$C(59)-C(58)-C(63)-C(62)$ & $0.5(6)$ \\
\hline $\mathrm{C}(57)-\mathrm{C}(58)-\mathrm{C}(63)-\mathrm{C}(62)$ & $-178.2(4)$ \\
\hline $\mathrm{C}(59)-\mathrm{C}(58)-\mathrm{C}(63)-\mathrm{O}(2)$ & $-178.8(4)$ \\
\hline $\mathrm{C}(57)-\mathrm{C}(58)-\mathrm{C}(63)-\mathrm{O}(2)$ & $2.4(5)$ \\
\hline $\mathrm{C}(63)-\mathrm{O}(2)-\mathrm{C}(64)-\mathrm{C}(65)$ & $74.3(4)$ \\
\hline $\mathrm{Ru}(2)-\mathrm{O}(2)-\mathrm{C}(64)-\mathrm{C}(65)$ & $-90.0(4)$ \\
\hline $\mathrm{C}(63)-\mathrm{O}(2)-\mathrm{C}(64)-\mathrm{C}(66)$ & $-162.5(4)$ \\
\hline $\mathrm{Ru}(2)-\mathrm{O}(2)-\mathrm{C}(64)-\mathrm{C}(66)$ & $33.2(5)$ \\
\hline
\end{tabular}

Symmetry transformations used to generate equivalent atoms:

\section{References}

1. Johns, A. M.; Ahmed, T. S.; Jackson, B. W.; Grubbs, G. H.; Pederson, R. L. High trans kinetic selectivity in ruthenium-based olefin cross-metathesis through stereoretention. Org. Lett. 2016, 18, 772-775.

2. Nookaraju, U.; Kumar, P. Total synthesis of (+)-petromyroxol viatandem $\alpha$-aminoxylation-allylation and asymmetric dihydroxylation-S $\mathrm{S}_{\mathrm{N}}$ cyclization approach. $R S C A d v .2015,5,63311-63317$. 
3. Koh, M. J.; Khan, R. K. M.; Torker, S.; Yu, M.; Mikus, M.; Hoveyda, A. H. High-value alcohols and higheroxidation-state compounds by catalytic $Z$-selective cross-metathesis. Nature 2015, 517, 181-186.

4. Murphy, S. K.; Coulter, M. M.; Dong, V. M. $\beta$-hydroxy ketones prepared by regioselective hydroacylation. Chem. Sci. 2012, 3, 355-358.

5. Kroll, J. H.; Donahue, N. M.; Cee, V. J.; Demerjian, K. L.; Anderson, J. G. Gas-phase ozonolysis of alkenes: formation of $\mathrm{OH}$ from anti carbonyl oxides. J. Am. Chem. Soc. 2002, 124, 8518-8519.

6. Fukuda, H.; Nishiyama, Y.; Nakamura, S.; Ohno, Y.; Eguchi, T.; Iwabuchi, Y.; Usui, T.; Kanoh, N. Synthesis and structure-activity relationship of vicenistatin, a cytotoxic 20 -membered macrolactam glycoside. Chem. Asian. J. 2012, 7, 2872-2881.

7. Panarese, J. D. \& Waters, S. P. Enantioselective formal total synthesis of (+)-aspergillide C. Org. Lett. 2009, 11, 5086-5088.

8. Sammakia, T.; John, D. M.; Kim, G.; Berliner, M. A. Remote asymmetric induction in an intramolecular ionic Diels-Alder reaction: Application to the total synthesis of (+)-dihydrocompactin. J. Am. Chem. Soc. 2005, 127, 6504-6505.

9. Zhu, C.; Shen, X.; Nelson, S. G. Cinchona alkaloid-Lewis acid catalyst systems for enantioselective ketene-aldehyde cycloadditions. J. Am. Chem. Soc. 2004, 126, 5352-5353.

10. Kündig, E. P.; Seidel, T. M.; Jia, Y. -X.; Bernardinelli, G. Bulky chiral carbene ligands and their application in the palladium-catalyzed asymmetric intramolecular $\alpha$-arylation of amides. Angew. Chem., Int. Ed. 2007, 46, 84848487.

11. Dible, B. R.; Cowley, R. E.; Holland, P. L. Remote substitution on $N$-heterocyclic carbenes heightens the catalytic reactivity of their palladium complexes. Organometallics 2011, 30, 5123-5132.

12. Peter, D.; Bruckner, R. A new approach to models of the 4,5-dihydroxycyclopentenone core of the kodaistatins A-D: elucidation of the diol configuration in kodaistatin A. Chem. Eur. J. 2017, 23, 12104-12109.

13. Tommasi, S.; Perrone, S.; Rosato, F.; Salomone, A.; Troisi, L. One-pot ester synthesis from allyl and benzyl halides and alcohols by palladium-catalyzed carbonylation. Synthesis 2012, 44, 423-430.

14. Qi, L.; Mui, Y. F.; Lo, S. W.; Lui, M. Y.; Akien, G. R.; Horváth, I. T. Catalytic conversion of fructose, glucose, and sucrose to 5-(hydroxymethyl)furfural and levulinic and formic acids in $\gamma$-valerolactone as a green solvent. ACS Catal. 2014, 4, 1470-1477.

15. Victorio, C.; Javier, F.; Jose, G. Selective ruthenium-catalyzed hydration of nitriles to amides in pure aqueous medium under neutral conditions. Chem. Eur. J. 2008, 14, 6601-6605.

16. Khan, R. K.; M., Toker, S.; Hoveyda, A. H. Readily accessible and easily modifiable Ru-based catalysts for efficient and $Z$-selective ring-opening metathesis polymerization and ring-opening/cross-metathesis. J. Am. Chem. Soc. 2013, 135, 10258-10261.

17. Liu, J.; Zhang, L.; He, J.; He, L.; Ma, B.; Pan, X.; She, X. Facile total synthesis of the antimalarial nonenolide. Tetrahedron: Asymmetry 2008, 19, 906-911. 
18. Chatterjee, S.; Ghadigaonkar, S.; Sur, P.; Sharma, A.; Chattopadhyay, S. A chemoenzymatic synthesis of hept-6ene-2,5-diol stereomers: Application to asymmetric synthesis of decarestrictine L, pyrenophorol, and stagonolide E. J. Org. Chem. 2014, 79, 8067-8076.

19. Hoveyda, H. R.; Vezina, M. Synthesis of unsaturated amino alcohols through unexpectedly selective Rucatalyzed cross-metathesis reactions. Org. Lett. 2005, 7, 2113-2116.

20. For reviews on the application of DFT calculations to transition metal chemistry, see: (a) Cramer, C. J.; Truhlar, D. G. Density functional theory for transition metals and transition metal chemistry. Phys. Chem. Chem. Phys. 2009, 11, 10757-10816. (b) Grimme, S.; Ehrlich, S.; Goerigk, L. Effect of the damping function in dispersion corrected density functional theory. J. Comp. Chem. 2011, 32, 1456-1465. (c) Peverati, R.; Truhlar, D. G. Quest for a universal density functional: the accuracy of density functionals across a broad spectrum of databases in chemistry and physics. Phil. Trans. R. Soc. A. 2014, 372, 20120476. For recent comparisons of density funcionals in benchmark studies, see: (d) Mardirossian, N.; Head-Gordon, M. J. Chem. Theory Comput. 2016, 12, 4303-4325. (e) Mardirossian, N.; Head-Gordon, M. How accurate are the minnesota density functionals for noncovalent interactions, isomerization energies, thermochemistry, and barrier heights involving molecules composed of main-group elements? J. Chem. Phys. 2016, 144, 214110. (f) Brauer, B.; Kesharwani, M. K.; Kozuch, S.; Martin, J. M. The S66x8 benchmark for noncovalent interactions revisited: explicitly correlated ab initio methods and density functional theory. Phys. Chem. Chem. Phys. 2016, 18, 20905-20925. (g) Weymuth, T.; Couzijn, E. P. A.; Chen, P.; Reiher, M. New benchmark set of transition-metal coordination reactions for the assessment of density functionals. J. Chem. Theory Comput. 2014, 10, 3092-3103. (h) Zhang, W.; Truhlar, D. G.; Tang M. Tests of exchange-correlation functional approximations against reliable experimental data for average bond energies of $3 \mathrm{~d}$ transition metal compounds. $J$. Chem. Theory Comput. 2013, 9, 3965-3977. (i) Yu, H. S.; He, X.; Li, S. L; Truhlar, D. G. MN15: A Kohn-Sham global-hybrid exchange-correlation density functional with broad accuracy for multi-reference and single-reference systems and noncovalent interactions. Chem. Sci. 2016, 7, 5032-5051. (j) Steinmetz, M.; Grimme S. Benchmark study of the performance of density functional theory for bond activations with (Ni,Pd)-based transition-metal catalysts. ChemistryOpen 2013, 2, 115-124. (k) Goerigk, L.; Kruse H.; Grimme, S. Benchmarking density functional methods against the S66 and S66x8 datasets for non-covalent interactions. ChemPhysChem 2011, 12, 3421-3433.

21. Frisch, M. J.; Trucks, G. W.; Schlegel, H. B.; Scuseria, G. E.; Robb, M. A.; Cheeseman, J. R.; Scalmani, G.; Barone, V.; Mennucci, B.; Petersson, G. A.; Nakatsuji, H.; Caricato, M.; Li, X.; Hratchian, H. P.; Izmaylov, A. F.; Bloino, J.; Zheng, G.; Sonnenberg, J. L.; Hada, M.; Ehara, M.; Toyota, K.; Fukuda, R.; Hasegawa, J.; Ishida, M.; Nakajima, T.; Honda, Y.; Kitao, O.; Nakai, H.; Vreven, T.; Montgomery, Jr., J. A.; Peralta, J. E.; Ogliaro, F.; Bearpark, M.; Heyd, J. J.; Brothers, E.; Kudin, K. N.; Staroverov, V. N.; Kobayashi, R.; Normand, J.; Raghavachari, K.; Rendell, A.; Burant, J. C.; Iyengar, S. S.; Tomasi, J.; Cossi, M.; Rega, N.; Millam, J. M.; Klene, M.; Knox, J. E.; Cross, J. B.; Bakken, V.; Adamo, C.; Jaramillo, J.; Gomperts, R.; Stratmann, R. E.; Yazyev, O.; Austin, A. J.; Cammi, R.; Pomelli, C.; Ochterski, J. W.; Martin, R. L.; Morokuma, K.; Zakrzewski, V. G.; Voth, G. A.; Salvador, P.; Dannenberg, J. J.; Dapprich, S.; Daniels, A. D.; Farkas, Ö.; Foresman, J. B.; Ortiz, J. V.; Cioslowski, J.; Fox, D. Gaussian 09, 2009, Revision D.01, Gaussian, Inc., Wallingford CT. 
22. Weigend, F.; Ahlrichs, R. Balanced basis sets of split valence, triple zeta valence and quadruple zeta valence quality for H to Rn: Design and assessment of accuracy. Phys. Chem. Chem. Phys. 2005, 7, 3297-3305.

23. Scalmani, G.; Frisch, M. J. Continuous surface charge polarizable continuum models of solvation. I. General formalism. J. Chem. Phys. 2010, 132, 114110.

24. (a) Page, M.; McIver Jr., J. W. On evaluating the reaction path Hamiltonian. J. Chem. Phys. 1988, 88, 922-935.

(b) Page, M.; Doubleday Jr. C.; McIver Jr., J. W. Following steepest descent reaction paths. The use of higher energy derivatives with ab initio electronic structure methods. J. Chem. Phys. 1990, 93, 5634-5642.

25. Marenich, A. V.; Cramer, C. J.; Truhlar, D. G. Universal solvation model based on solute electron density and on a continuum model of the solvent defined by the bulk dielectric constant and atomic surface tensions. J. Phys. Chem. B 2009, 113, 6378-6396.

26. For selected examples highlighting the importance of including treatment of dispersion interactions in modeling olefin metathesis reactions promoted by Ru carbene complexes, see: (a) Torker, S.; Merki, D.; Chen, P. Gas-Phase Thermochemistry of Ruthenium Carbene Metathesis Catalysts. J. Am. Chem. Soc. 2008, 130, 4808-4814. (b) Minenkov, Y.; Occhipinti, G.; Singstad, A.; Jensen, V. R. The accuracy of DFT-optimized geometries of functional transition metal compounds: a validation study of catalysts for olefin metathesis and other reactions in the homogeneous phase. Dalton Trans. 2012, 41, 5526-5541. (c) Minenkov, Y.; Occhipinti, G.; Jensen, V. R. Complete reaction pathway of ruthenium-catalyzed olefin metathesis of ethyl vinyl ether: kinetics and mechanistic insight from DFT. Organometallics 2013, 32, 2099-2111. (d) Khan, R. K. M.; Torker, S.; Hoveyda, A. H. Reactivity and selectivity differences between catecholate and catechothiolate $\mathrm{Ru}$ complexes. Implications regarding design of stereoselective olefin metathesis catalysts. J. Am. Chem. Soc. 2014, 136, 14337-14340. (e) Torker, S.; Koh, M. J.; Khan, R. K. M.; Hoveyda, A. H. Regarding a persisting puzzle in olefin metathesis with Ru complexes: why are transformations of alkenes with a small substituent $Z$-selective? Organometallics 2016, 35, 543-562. (f) Mikus, M. S.; Torker, S.; Hoveyda, A. H. Controllable ROMP tacticity by harnessing the fluxionality of stereogenic-at-ruthenium complexes. Angew. Chem., Int. Ed. 2016, 55, 4997-5002.

27. Zhao, Y. \& Truhlar, D. G. Density functionals with broad applicability in chemistry. Acc. Chem. Res. 2008, 41, $157-167$.

28. Chai, J.-D. \& Head-Gordon, M. Long-range corrected hybrid density functionals with damped atom-atom dispersion corrections. Phys. Chem. Chem. Phys. 2008, 10, 6615-6620.

29. Adamo, C.; Barone, V. Toward reliable density functional methods without adjustable parameters: The PBE0 mode. J. Chem. Phys. 1999, 110, 6158-6169.

30. Lichtenberger, D. L. \& Gladysz, J. A. New author guidelines for 2014: A format for computational structural data that can be opened with freely available programs such as "Mercury"1. Organometallics 2014, 33, 835-835. The "coordinates.xyz" file can be generated by copying all the coordinates in Section 7 into a text file without empty lines and changing the extension to ".xyz". 
31. For reviews on the properties of NHC ligands, see: (a) Diez-Gonzalez, S.; Nolan, S. P. Stereoelectronic parameters associated with N-heterocyclic carbene (NHC) ligands: A quest for understanding. Coord. Chem. Rev. 2007, 251, 874-883. (b) Dröge, T.; Glorius, F. The measure of all rings-N-heterocyclic carbenes. Angew. Chem., Int. Ed. 2010, 49, 6940-6952. (c) Nelson, D. J.; Nolan, S. P. Quantifying and understanding the electronic properties of $N$-heterocyclic carbenes. Chem. Soc. Rev. 2013, 42, 6723-6753. (d) Hopkinson, M. N.; Richter, C.; Schedler, M.; Glorius, F. An overview of N-heterocyclic carbenes. Nature 2014, 510, 485-496. (e) Couzijn, E. P. A.; Lai, Y.-Y.; Limacher, A.; Chen. P. Intuitive quantifiers of charge flows in coordinate bonding. Organometallics 2017, 36, 3205-3214.

32. Dorta, R.; Stevens, E. D.; Scott, N. M.; Costabile, C.; Cavallo, L.; Hoff, C. D.; Nolan, S. P. Steric and electronic properties of N-heterocyclic carbenes (NHC): A detailed study on their interaction with Ni(CO)4. J. Am. Chem. Soc. 2005, 127, 2485-2495.

33. Magill, A. M.; Cavell, K. J.; Yates, B. F. Basicity of nucleophilic carbenes in aqueous and nonaqueous solventstheoretical predictions. J. Am. Chem. Soc. 2004, 126, 8717-8724.

34. Back, O.; Henry-Ellinger, M.; Martin, C. D.; Martin, D.; Bertrand, G. ${ }^{31}$ P NMR Chemical shifts of carbenephosphinidene adducts as an indicator of the $\pi$-accepting properties of carbenes. Angew. Chem., Int. Ed. 2013, 52, 2939-2943.

35. Liske, A.; Verlinden, K.; Buhl, H.; Schaper, K.; Ganter, C. Determining the $\pi$-acceptor properties of Nheterocyclic carbenes by measuring the ${ }^{77} \mathrm{Se}$ NMR chemical shifts of their selenium adducts. Organometallics 2013, $32,5269-5272$.

36. Leuthäußer S.; Schwarz, D.; Plenio, H. Tuning the electronic properties of N-heterocyclic carbenes. Chem. Eur. J. 2007, 13, 7195-7203.

37 Süßner, M.; Plenio H. $\pi$-Face donor properties of N-heterocyclic carbenes. Chem. Commun. 2005, 5417-5419.

38. Clavier, H.; Nolan, S. P. Percent buried volume for phosphine and $N$-heterocyclic carbene ligands: steric properties in organometallic chemistry. Chem. Commun. 2010, 46, 841-861.

39. Gusev, D. G. Electronic and steric parameters of $76 \mathrm{~N}$-heterocyclic carbenes in $\mathrm{Ni}(\mathrm{CO})_{3}(\mathrm{NHC})$. Organometallics 2009, 28, 6458-6461.

40. For a review on dispersion interactions, see: Wagner, J. P.; Schreiner, P. R. London dispersion in molecular chemistry-reconsidering steric effects. Angew. Chem., Int. Ed. 2015, 54, 12274-12296.

41. For studies regarding the attenuation of dispersion interactions in solution, see: (a) Yang, L.; Adam, C.; Nichol, G. S.; Cockroft, S. L. How much do van der Waals dispersion forces contribute to molecular recognition in solution? Nat. Chem. 2013, 5, 1006-1010. (b) Pollice, R.; Bot, M.; Kobylianskii, I. J.; Shenderovich, I.; Chen, P. Attenuation of London dispersion in dichloromethane solutions. J. Am. Chem. Soc. 2017, 139, 13126-13140.

42. For a review on aryl-aryl interactions, see: Hunter, C. A., Lawson, K. R.; Perkins, J.; Urch, C. J. Aromatic interactions. J. Chem. Soc., Perkin Trans. 2001, 2, 651-669. 